
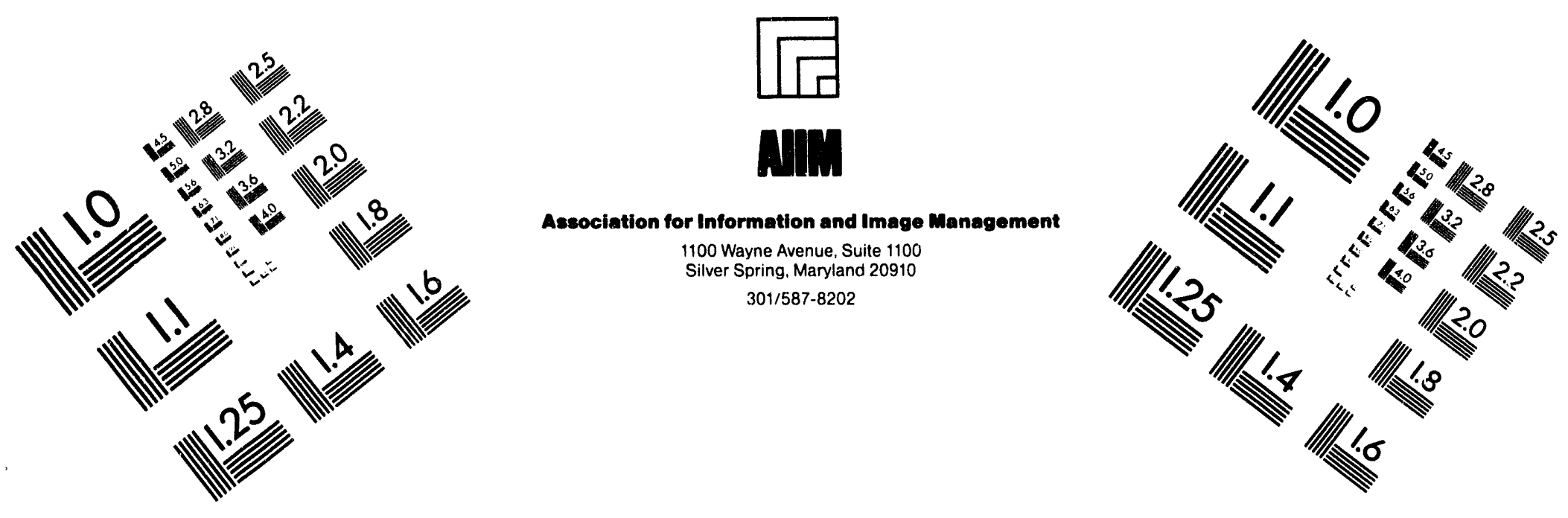

\title{
Centimeter
}

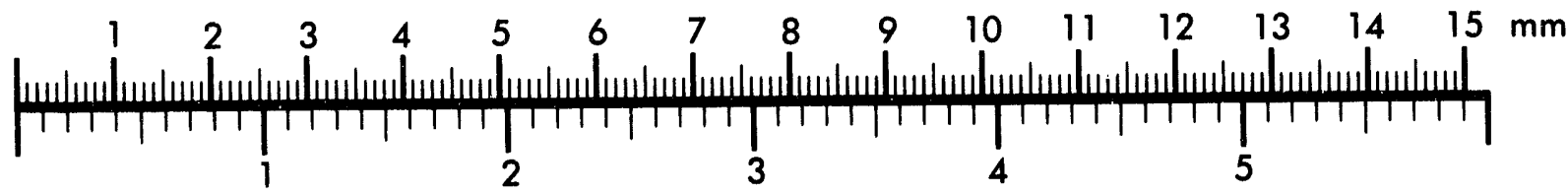

Inches
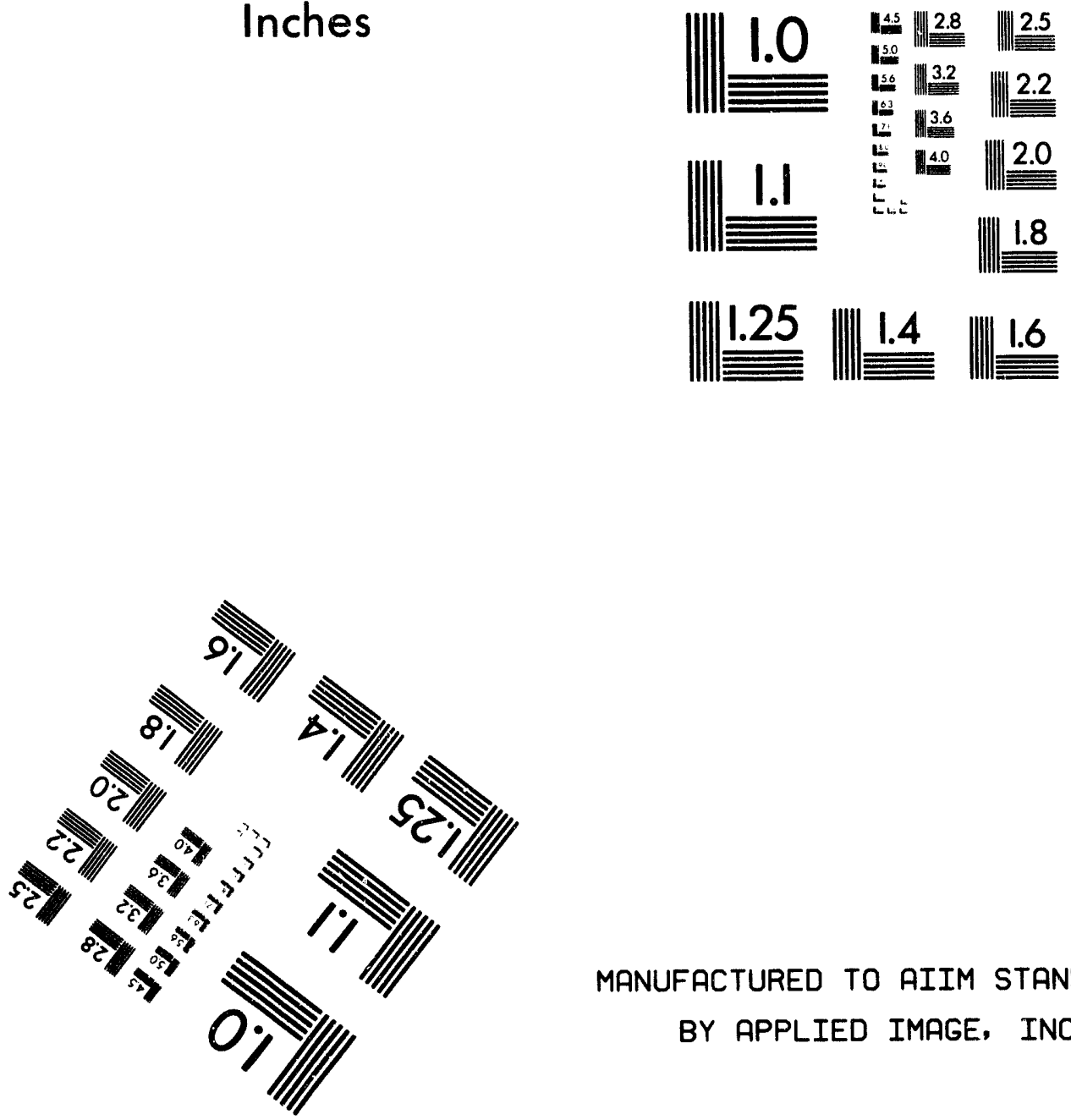

MANUFACTURED TO AIIM STANDARDS

BY APPLIED IMAGE, INC.

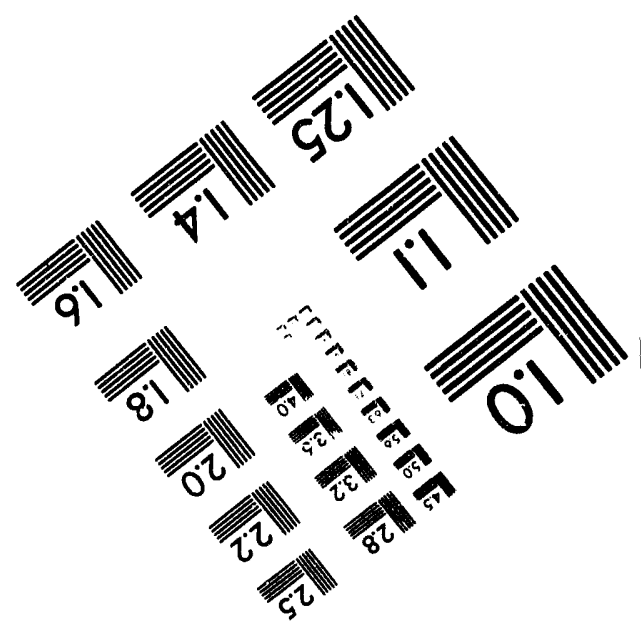



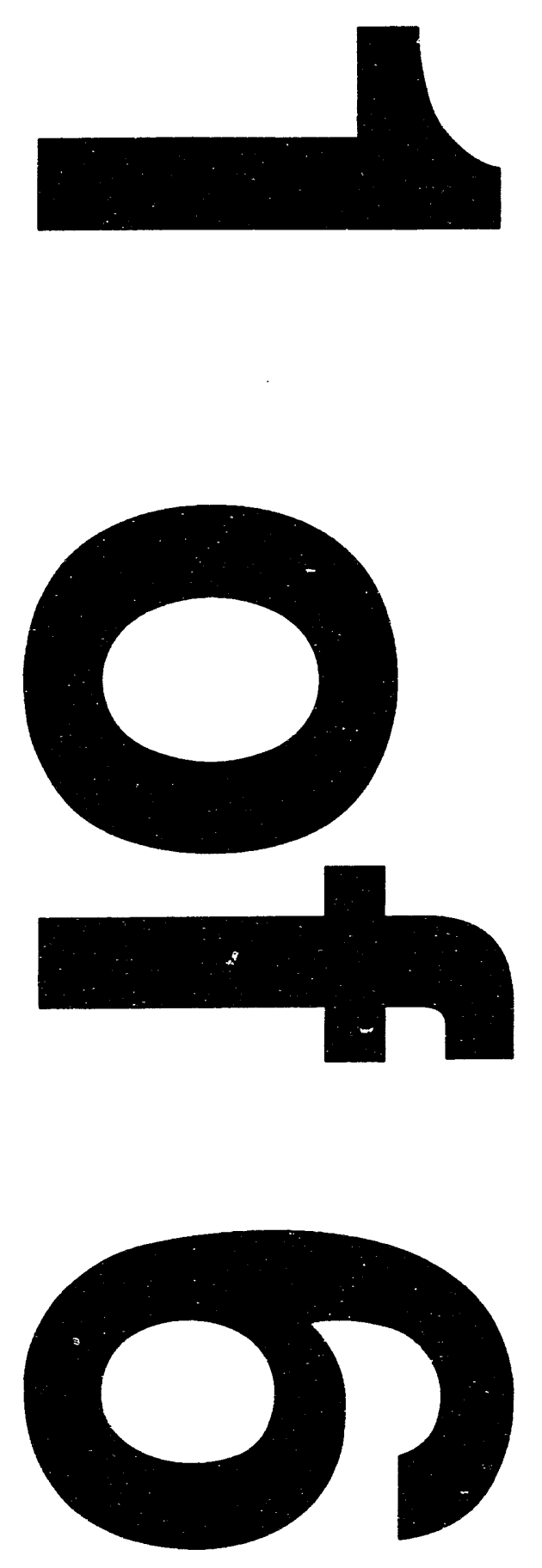


\section{Climatological Data Summary 1993 with Historical Data}

D. J. Hoitink

K. W. Burk

June 1994

Prepared for

the U.S. Department of Energy

under Contract DE-AC06-76RLO 1830

Pacific Northwest Laboratory

Richland, Washington 99352

\%ำ Battelle

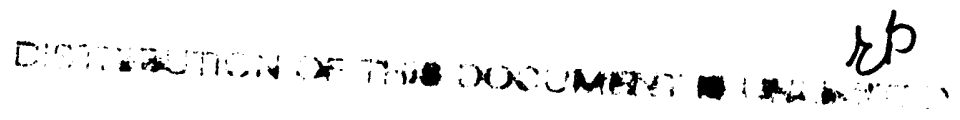




\section{PREFACE}

This document uses English units (such as, mph, inches, and ${ }^{\circ} \mathrm{F}$ ) when presenting all information. The decision to use English units was based on several factors. First, measurements made at the Hanford Meteorology Station are recorded and stored in English units. Second, previous Hanford climatological summaries were published using English units. Third, English units are still the standard in the National Oceanic and Atmospheric Administration (specifically, National Climatic Data Center and National Weather Service) reporting and publications. Finally, most users of this document are more familiar with meteorological parameters in English units, rather than their metric equivalents. For example, most users of this document think of a pleasant summer day being $86^{\circ} \mathrm{F}$ rather than $30^{\circ} \mathrm{C}$.

Throughout this document the term "normal" is used to indicate climatological normal which is defined as an average value over a period of years of any meteorological element such as temperature, pressure, and rainfall. The accepted convention uses a 30 -year time period, ending with the first year of each new decade (such as 1951-1980, 1961-1990, 1971-2000). The current time period used for clinatological normals is 1961-1990.

Some useful conversions between English units and metric equivalents are:

$$
\begin{aligned}
& 1 \text { foot }=0.3048 \text { meter } \\
& 1 \text { mile }=1.609 \text { kilometer } \\
& 1 \text { inch }=2.54 \text { centimeters }
\end{aligned}
$$

1 mile per hour $=0.447$ meters $/$ second

${ }^{\circ} \mathrm{F}=\left(9 / 5 \times{ }^{\circ} \mathrm{C}\right)+32$

${ }^{\circ} \mathrm{C}=5 / 9 \times\left({ }^{\circ} \mathrm{F}-32\right)$ 


\section{SUMMARY}

This document presents the climatological data summary for calendar year 1993. It presents updated historical climatologies for temperature, wind, precipitation, and other miscellaneous meteorological parameters from the Hanford Meteorology Station (HMS) and Hanford Meteorological Monitoring Network. It also presents climatological normal and extreme values of temperature and precipitation for the HMS.

Previous documents (Stone et a1. 1983, for example) have included climatological data collected at the old Hanford Townsite, located approximately 10 miles east-northeast of the present HMS. The records for these two different sites (HMS and Hanford Townsite) have been frequently interchanged as if representing the same location. With the exception of Section 2.0 , the remainder of this document uses data only from the HMS, with a period of record beginning December 7, 1944. 


\section{ACKNOWLEDGMENTS}

The authors would like to thank S. A. Stage, who developed the software used for the wind rose plots throughout this document. We would also like to thank the staff of the Hanford Meteorology Station, both past and present, without whose weather observations over the past 50 years this document would not have been possible. 


\section{CONTENTS}

PREFACE

i i i

SUMMARY

ACKNOWLEDGMENTS

vi i

1.0 INTRODUCTION

2.0 CALENDAR YEAR 1993 SUMMARY

3.0 TEMPERATURE CLIMATOLOGY . . . . . . . . . . . . . . . 3.1

3.1 MONTHLY, SEASONAL, AND ANNUAL AVERAGE TEMPERATURES • • . • 3.1

3.2 DAYS WITH MAXIMUM TEMPERATURES $\geq 100^{\circ} \mathrm{F}, \geq 90^{\circ} \mathrm{F}$, AND $\leq 32 \circ \mathrm{F}$. . . . . . . . . . . . . . . . . 3.1

3.3 DAYS WITH MINIMUM TEMPERATURES $\leq 32^{\circ} \mathrm{F}$ OR $\leq 0^{\circ} \mathrm{F} \ldots \ldots . . . .9$

3.4 MONTHLY EXTREME DAILY MAXIMUM AND MINIMUM TEMPERATURES . • 3.12

3.5 NORMAL AND EXTREME DAILY TEMPERATURES . . . . . . . . . . 3.12

4.0 PRECIPITATION CLIMATOLOGY . . . . . . . . . . . . . . . 4.1

4.1 MONTHLY AND ANNUAL TOTALS . . . . . . . . . . . . 4.1

4.2 SEASONAL PRECIPITATION . . . . . . . . . . . . . 4.1

4.3 AVERAGE NUMBER OF DAYS WITH SPECIFIED AMOUNTS

OF PRECIPITATION . . . . . . . . . . . . . . 4.1

4.4 TOTAL TIME WITH PRECIPITATION OBSERVED . . . . . . . . . . . 4.4

4.5 NOTABLE WET PERIODS . . . . . . . . . . . . . . 4.5

4.6 NOTABLE DRY PERIODS .................. 4.5

4.7 SNOWFALL ....................... . . 4.6

4.8 NORMAL AND MAXIMUM DAILY PRECIPITATION . . . . . . . . . 4.9 
5.0 WIND CLIMATOLOGY . . . . . . . . . . . . . 5.1

5.1 MONTHLY AND ANNUAL PREVAILING WIND DIRECTIONS, AVERAGE SPEEDS, AND PEAK GUSTS ................... 5.1

5.2 NUMBER OF DAYS WITH PEAK GUSTS ABOVE OR BELOW SPECIFIC THRESHOLDS ..................... 5.1

5.3 PERCENT FREQUENCY OF MONTHLY AND ANNUAL WIND DIRECTION AND SPEED AT 50 FEET ......................... 5.1

5.4 COMPOSITE WIND ROSES AND JOINT FREQUENCY DISTRIBUTIONS FOR THE HANFORD METEOROLOGICAL MONITORING NETWORK . . . 5.5

5.5 MONTHLY AND ANNUAL WIND ROSES AND WIND SPEED HISTOGRAMS FOR THE HANFORD METEOROLOGICAL MONITORING NETWORK $\ldots . .5 .5$

6.0 MISCELLANEOUS CLIMATOLOGICAL STATISTICS . . . . . . . . 6.1

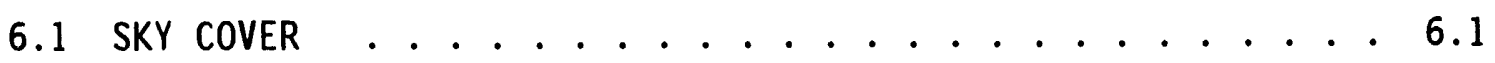

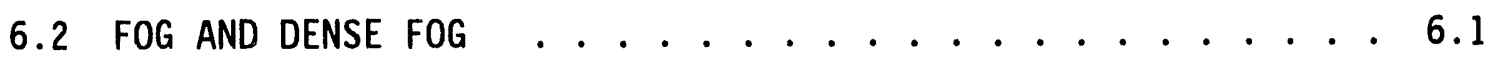

6.3 PSYCHROMETRIC DATA . . . . . . . . . . . . 6.3

6.4 SOLAR RADIATION . . . . . . . . . . . . . . 6.4

6.5 THUNDERSTORMS . . . . . . . . . . . . 6.5

6.6 DUST AND BLOWING DUST $\ldots \ldots \ldots . \ldots . \ldots$

6.7 GLAZE . . . . . . . . . . . . . . 6.6

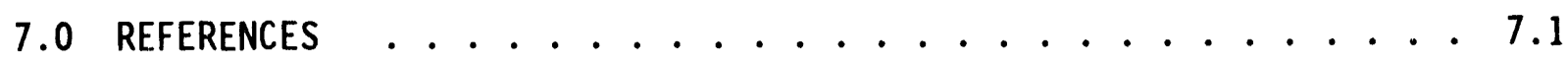

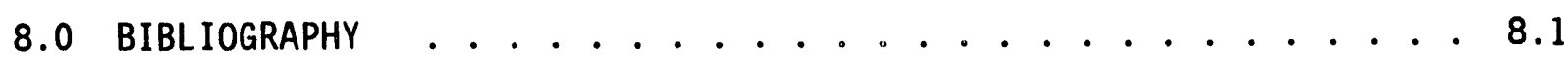

APPENDIX A - 1993 WIND CLIMATOLOGY . . . . . . . . . . A.1

APPENDIX B - WIND CLIMATOLOGY .............. B. 


\section{FIGURES}

1.1 Map of the Hanford Site and Surrounding Areas . . . . . . . . 1.2

2.1 Hanford Meteorological Monitoring Network Wind Roses for 1993 . . 2.12

2.2 Observed Daily Temperatures for 1993 with Normal Temperatures . . 2.14

5.1 Hanford Meteorological Monitoring Network Wind Roses for the Period from 1982 Through 1993 . . . . . . . . . . . 5.6

A.1 Wind Rose and Wind Speed Histogram . . . . . . . . . . . . A.1

B.1 Wind Rose and Wind Speed Histogram . . . . . . . . . . . . B.1

B.2 Wind Rose and Wind Speed Histogram . . . . . . . . . . . . B.325

\section{TABLES}

1.1 Site Numbers, Names, and Codes for the Hanford Meteorological

Monitoring Network .. . . . . . . . . . . . . . 1.1

2.1 Daily Temperature Records for 1993 . . . . . . . . . . . . 2.2

2.2 Summary of Miscellaneous Meteorological Parameters for 1993 • • 2.7

2.3 1993 Monthly and Annual Average Temperature from the Hanford Meteorological Monitoring Network . . . . . . . . . . 2.9

2.4 1993 Monthly and Annual Precipitation from the Hanford Meteorological Monitoring Network .. . . . . . . . . 2.10

2.5 1993 Monthly and Annual Average Wind Speed from the Hanford Meteorological Monitoring Network ............. 2.11

3.1 Monthly and Annual Average Temperatures . . . . . . . . . . 3.2

3.2 Seasonal Average Temperatures . . . . . . . . . . . . 3.3

3.3 Monthly and Seasonal Number of Days with Maximum Temperature Above or Below Certain Thresholds . . . . . . . . . . . 3.4

3.4 Days With Maximum Temperatures $z 104 \circ \mathrm{F}$. . . . . . . . . . . 3.7

3.5 Record of Annual First and Last Dates with Maximum Temperature
$\geq 90^{\circ} \mathrm{F}$ and Minimum Temperatures $\leq 32 \circ \mathrm{F}$. . . . . . . . . . 3.8

3.6 Monthly and Annual Maximum Temperatures . . . . . . . . . . . 3.10 
3.7 Monthly and Seasonal Number of Days with Minimum Temperature Below Certain Thresholds ............... 3.11

3.8 Days with Minimum Temperatures $\leq$ 0 0 F . . . . . . . 3.13

3.9 Monthly and Annual Minimum Temperatures . . . . . . . . . . 3.14

3.10 Monthly Normal Temperatures and Monthly Extremes of Maximum and Minimum Temperatures .............. . 3.15

3.11 Normal and Extreme Daily Maximum and Minimum Temperatures . . . 3.16

4.1 Monthly and Annual Precipitation . . . . . . . . . . . 4.2

4.2 Seasonal Precipitation ............... 4. . 4.3

4.3 Average Number of Days with Precipitation of Specified Amount . . 4.4

4.4 Monthly and Annual Averages and Extremes in Total Time with Precipitation Observed: July 1946 Through June 1971, July 1974 Through December $1993 \ldots \ldots$. . . . . . . . . 4.4

4.5 Monthly and Seasonal Snowfall Including First and Last Dates of Both Trace and Measurable Snowfalls . . . . . . . . 4.7

4.6 Miscellaneous Snowfall Statistics, 1946 Through 1993 . . . . 4.10

4.7 Normal and Maximum Daily Precipitation . . . . . . . . . 4.11

5.1 Monthly and Annual Prevailing Wind Directions, Average Speeds, and Peak Gusts at 50 Feet (1945 Through 1993) . . . . . . . 5.2

5.2 Number of Days with Peak Gusts at 50 Feet Above or Below Specific Thresholds (1945 Through 1993) ............ 5.3

5.3 Percent Frequency of Monthly and Annual Wind Direction and Wind Speed at 50 Feet (1955 Through 1993) . . . . . . . 5.4

6.1 Average Sky Cover (1946 Through 1993) and Number of Days Clear, Partly Cloudy, and Cloudy (1954 Through 1993)

6.2 Monthly and Annual Number of Days with Fog and Dense Fog (1945 Through 1993) ............... 6.3

6.3 Monthly Averages of Psychrometric Data (1950 Through 1993) . . . 6.4

6.4 Solar Radiation - Average and Extreme Daily Values (1953 Through 1993) .................... 6.5

6.5 Average Number of Days of Various Meteorological Phenomenon (1945 Through 1993) ................. 6.6 
A.1 1993 Joint Frequency Distributions for Meteorological Monitoring Network Wind Stations

B.1 1982-1993 Joint Frequency Distributions for Meteorological

Monitoring Network Wind Stations

B. 373 


\subsection{INTRODUCTION}

This document presents the calendar year 1993 climatological data summary for the Hanford Meteorology Station (HMS), and additional climatologies for temperature, wind, precipitation, and other meteorological parameters for the HMS and the automated stations of the Hanford Meteorological Monitoring Network (HMMN). Climatological normal and extreme values for temperature and precipitation are also presented. Currently 26 monitoring stations are within and located near the U.S. Department of Energy's (DOE's) Hanford Site in southeastern Washington State (Table 1.1 and Figure 1.1). A detailed description of each of the monitoring sites, including photographs of the topography surrounding each site, is provided in Glantz and Islam (1988).

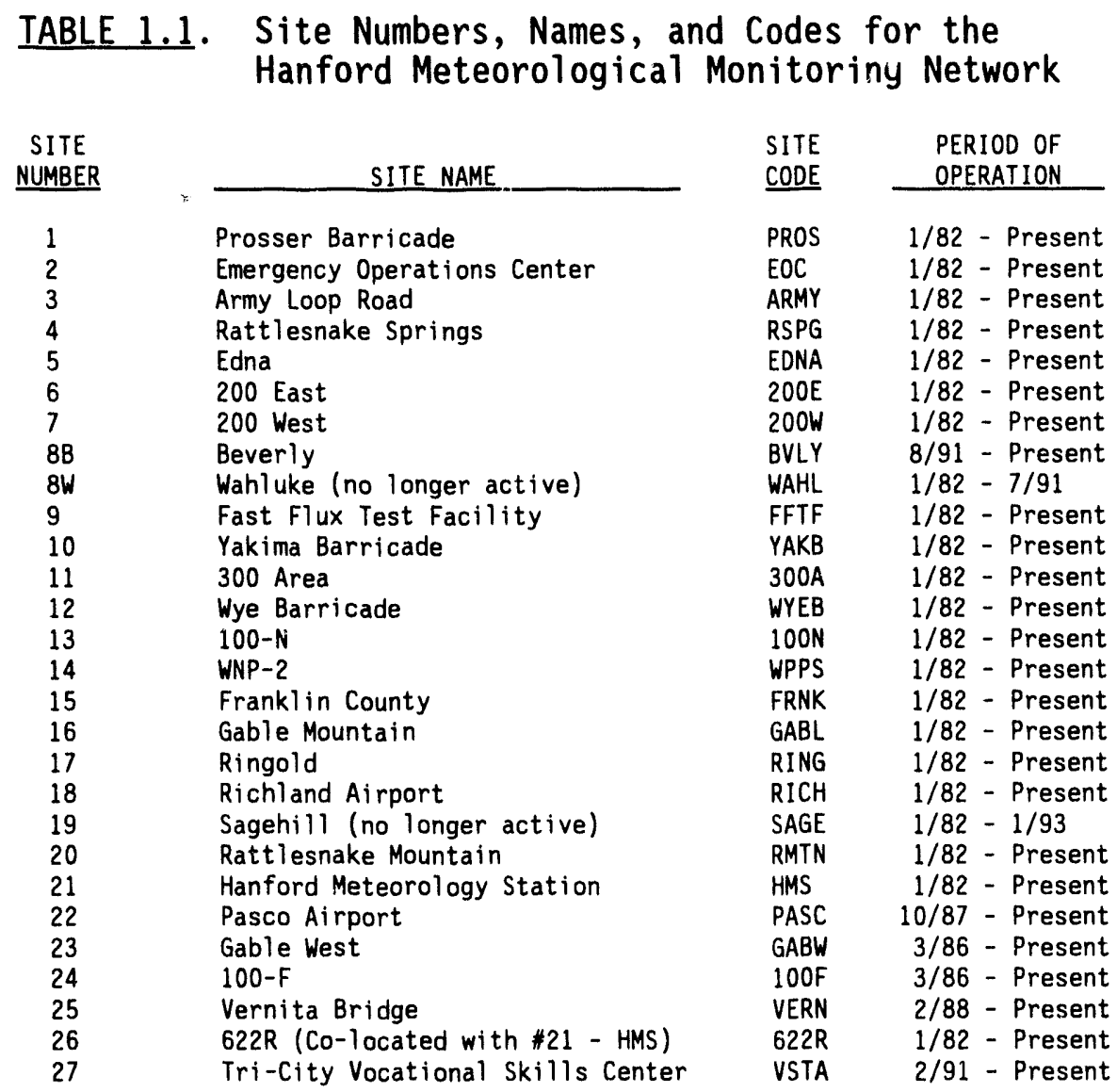




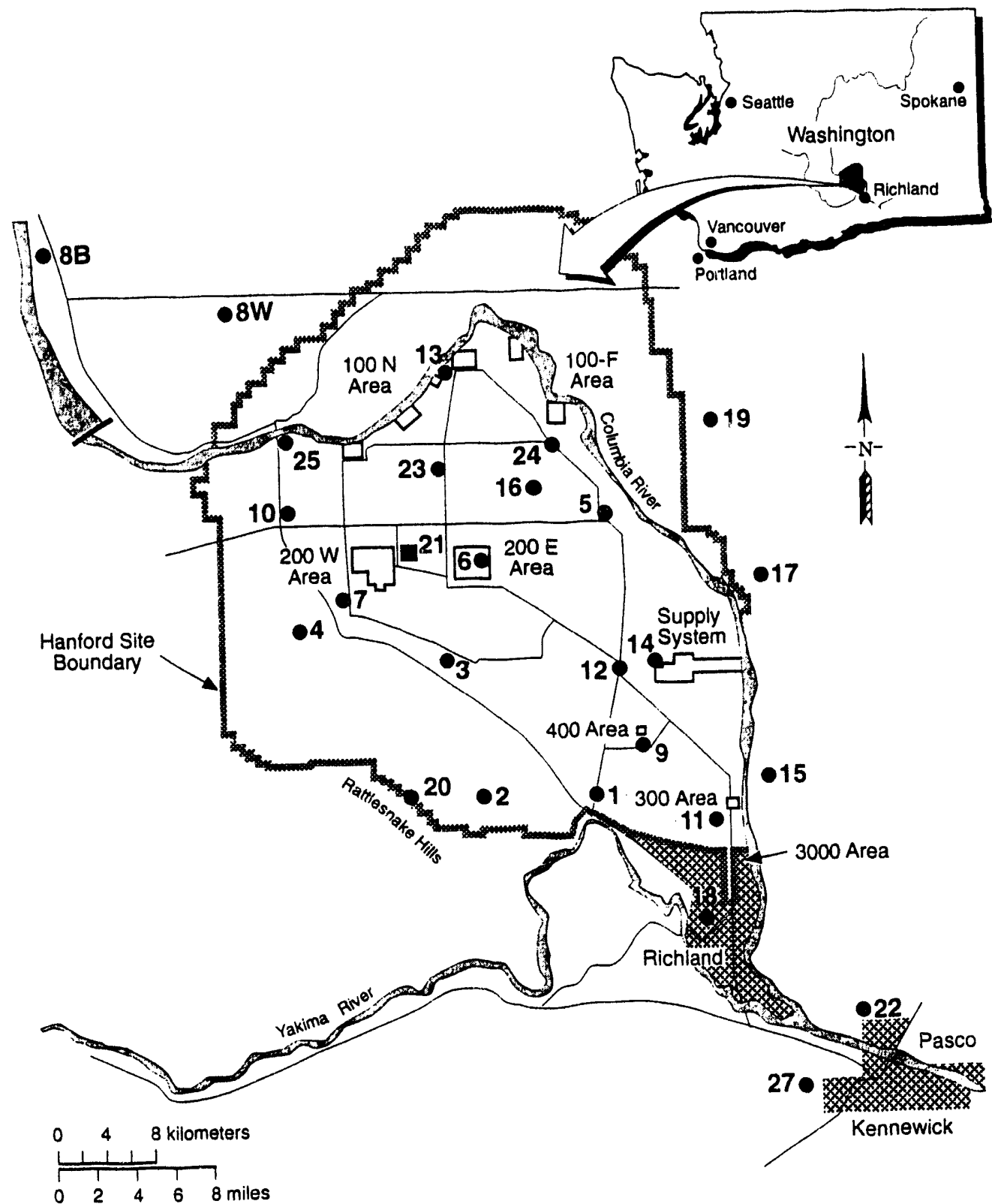

FIGURE 1.1. Map of the Hanford Site and Surrounding Areas (See text for discussion of symbols and numbers)

The location of the Hanford Meteorology Station is indicated by a black square. Other meteorological monitoring locations are denoted by black circles. Station identification numbers are shown in bold type beside the square and circles. (In Table 1.1, names are provided for each of the numbered monitoring locations.) 
Operation of the HMS is a function of the Meteorological and Climatological Services Project which is funded by the U.S. DOE, Richland Operations office. This project, managed by the Pacific Northwest Laboratory, ${ }^{(a)}$ is responsible for providing DOE and Hanford Site contractors with orgoing meteorological and climatological services support, primarily for emergency response activities, Hanford Site work scheduling, and general site safety. Detailed, real-time meteorological data are needed in the event of a release of hazardous material to the atmosphere from any uf the Hanford Site facilities. These data can be used to model atmospheric dispersion and to estimate the environmental impacts of the release. Meteorological data and weather forecasts are also necessary to assure that operations and activities on the Hanford Site are conducted safely, particuiarly where specific weather conditions might impact those operations or activities. The climatological database is also used in environmental studies, environmental impact reports, facility design, and planning operations.

During the period from April 1912 through March 1943, cooperative observers for the U.S. Weather Bureau (now the National Weather Service) recorded daily maximum and minimum temperatures, and precipitation, including measurements of unmelted snow at the Hanford Townsite, about 10 miles eastnortheast of the present iMS. From late-1943 until mid-1944, the U.S. Weather Bureau recorded some meteorological operations in Richland. Then, in 1944 as part of the Manhattan Project, the Hanford Meteorology Station was estab1ished. Hourly observations began on December 7, 1944.

The HMS and its $410-\mathrm{ft}(125-\mathrm{m})$ instrument tower are located near the center of the Hanford Site, between the 200 West and 200 East Areas (Figure 1.1, No 21). Hourly observations of wind direction, wind speed, and air temperature are made at multiple levels on the 410-ft tower. A variety of other meteorological parameters are also measured or observed. These parameters include present weather, dew point temperature, relative humidity, precipitation, atmospheric pressure, cloud cover, visibility, and solar radiation. Several climatological summaries of data collected at the HMS, at

(a) The Pacific Northwest Laboratory is operated by Battelle Memorial Institute for the U.S. Department of Energy under Contract DE-AC06-76RLO 1830. 
the old Hanford Townsite, and Richland monitoring locations have been published over the past 30 years (Jenne and Kerns 1959, Stone et a1. 1972, and Stone et al. 1983).

Beginning with calendar year 1994 monthly summaries and with this

document, historical data from the old Hanford Townsite and Richland will no longer be included in these reports. The climatological data record from the HMS is now sufficiently long to stand alone.

The sections in this document are organized as follows:

- Section 2.0 - Calendar Year 1993 Summary

- Section 3.0 - Temperature Climatology

- Section 4.0 - Precipitation Climatology

- Section 5.0 - Wind Climatology

- Section 6.0 - Miscellaneous Climatological Statistics

- Section 7.0 - References

- Section 8.0 - Bibliography. 


\subsection{CALENDAR YEAR 1993 SUMMARY $(a)$}

Calendar year 1993 is the first year since 1985 with a below-normal average annual temperature. The average temperature for 1993 was $51.8^{\circ} \mathrm{F}, 1.5^{\circ}$ below normal $\left(53.3^{\circ} \mathrm{F}\right)$. The warmest year on record is 1992 , which averaged $56.4^{\circ} \mathrm{F}$; the coldest is 1985 , which averaged $49.6^{\circ} \mathrm{F}$.

Eight months during 1993 were cooler than normal, six of which were at least $2.0^{\circ}$ below normal, and four of which were more than $5.0^{\circ}$ below normal. July 1993 was a record cold month, averaging $70.5^{\circ} \mathrm{F}, 5.7^{\circ}$ below normal $\left(76.2^{\circ} \mathrm{F}\right)$. Four months were warmer than normal, three of which were at least $3.0^{\circ}$ above normal. May had the greatest positive departure $\left(+5.2^{\circ}\right)$, and February the greatest negative $\left(-7.2^{\circ}\right)$.

Precipitation for calendar year 1993 totaled 7.83 inches, $125 \%$ of normal (6.26 inches). Calendar year snowfall totaled 36.2 inches, the third highest calendar year snowfall total on record. The highest was 45.1 inches in 1916. Normal yearly snowfall is 13.8 inches.

January 1993 was much colder and wetter than normal. The average temperature for the month was $24.8^{\circ} \mathrm{F}, 6.5^{\circ}$ below normal. It was the coldest month at the HMS since December 1990, which averaged $24.1^{\circ} \mathrm{F}$. Precipitation for the month totaled 1.30 inches, $165 \%$ of normal. Snowfall for January was 17.1 inches, the second highest monthly snowfall on record, compared to a normal January snowfall of 3.9 inches. January 1950 recorded 23.4 inches, the 1argest January snowfall on record.

February 1993 was another cold month, averaging $30.8^{\circ} \mathrm{F}, 7.2^{\circ}$ below normal. The period from February 15 to 28 averaged $18.1^{\circ}$ below normal, with numerous record low temperatures (see Table 2.1). The minimum temperature of $3^{\circ} \mathrm{F}$ on February 28 was the coldest temperature ever recorded that 1 ate in the winter season. February precipitation totaled 1.17 inches, $189 \%$ of normal

(a) Note: During 1993, the climatological database included data from the old Hanford Townsite, with the record beginning in 1912. Therefore, the calendar year 1993 summary includes some references to that data. 
IABLE 2.1. Daily Temperature Records for 1993 (Previous record and year of occurrence in parentheses)

\begin{tabular}{|c|c|c|c|c|}
\hline \multirow[b]{2}{*}{ DATE } & \multicolumn{2}{|c|}{ MAXIMUM } & \multicolumn{2}{|c|}{ MINIMUM } \\
\hline & $\mathrm{HIGH}$ & LOW & $\mathrm{HIGH}$ & LOW \\
\hline $\operatorname{Jan} 13$ & & & & $-4^{\star}(-4,1949)$ \\
\hline Feb 22 & & & & $11(14,1918)$ \\
\hline Feb 24 & & & & $11^{*}(11,1962)$ \\
\hline Feb 25 & & $28(34,1962)$ & & $4(14,1962)$ \\
\hline Feb 26 & & $28^{\star}(28,1955)$ & & $10(12,1962)$ \\
\hline Feb 27 & & $26(30,1955)$ & & \\
\hline Feb 28 & & $25(27,1962)$ & & $3(12,1960)$ \\
\hline Mar 1 & & $26(34,1967)$ & & \\
\hline May 3 & & $58(60,1942)$ & & \\
\hline May 12 & $100(98,1931)$ & & & \\
\hline May 19 & $92(91,1912)$ & & & \\
\hline Jun 22 & & $71(73,1963)$ & & \\
\hline Jul 13 & & $77^{*}(77,1983)$ & & \\
\hline Jul 17 & & $73(77,1987+)$ & & \\
\hline Aug 25 & & & & $43(46,1925)$ \\
\hline Sep 29 & $92(91,1990+)$ & & & \\
\hline Sep 30 & $88 *(88,1991+)$ & & $64(58,1989+)$ & \\
\hline Oct 27 & & $43^{\star}(43,1956)$ & & \\
\hline Dec 10 & $67(64,1946)$ & & & \\
\hline
\end{tabular}

(0.62 of an inch). Snowfall for February totaled 12.4 inches, compared to a February normal of 2.0 inches, and was the fourth highest February snowfall on record.

February 1916 holds the record snowfall with 26.0 inches. The 10.2-inch snowfall that occurred between 6 p.m. on February 18 and 6 p.m. on February 19 was a new record $24-\mathrm{hr}$ snowfall. The previous record was 8.8 inches on November 21 and 22, 1985.

The winter of 1992-93 (December 1992, January and February 1993) averaged $28.5^{\circ} \mathrm{F}, 5.1^{\circ}$ below normal $\left(33.6^{\circ} \mathrm{F}\right)$. The coldest winter on record averaged $24.2 \circ \mathrm{F}$ in 1948-49, and the warmest was $41.1^{\circ} \mathrm{F}$ in 1933-34. Winter season snowfall totaled 56.1 inches, the most snowy winter on record. The previous record was 43.6 inches during the winter of 1915-1916, and normal is 13.8 inches. Other snowfall records for the winter of 1992-1993 include: 
- largest December snowfall with 21.0 inches (previous record 19.1 inches in 1964)

- serond most snowy January with 17.1 inches (record January was 23.4 inches in 1950)

- fourth highest February snowfall with 12.4 inches (record February was 26.0 inches in 1916)

- third most snowy March with 3.5 inches (record March was 4.2 inches in 1951)

- most days with $\geq 1$ inch on ground: 71 (previous record 62 days in 1985-1980)

- most days with $\geq 6$ inches on grourid: 41 (previous record 35 days in 1964-1965)

- most days with $\geq 12$ inches on ground: 9 (previous record 4 days in 1964-1965)

- greatest 24-hr snowfall: 10.2 inches on February 18 and 19 (previous record 8.8 inches on November 21 and 22, 1985).

March 1993 continued the 1993 pattern of cool months. The average temperature of $43.2^{\circ} \mathrm{F}$ was $2.4^{\circ}$ below normal. It was the foggiest March on record, both for number of days with fog $(10)$ and total duration of fog (129.1 hours). The previous records were 9 days in 1989, and 35.1 hours total duration in 1978. 1993 al so had a record number of cloudy days in March (24); the previous record was 23 days in March 1977. Precipitation for March 1993 totaled 0.67 inch, $143 \%$ of normal $(0.47 \mathrm{inch})$. March snowfall totaled 3.5 inches, the third snowiest March on record. Normal March snowfall is 0.3 inch.

April 1993 temperatures were nearly normal. The average temperature of $52.5^{\circ} \mathrm{F}$ was $0.2^{\circ}$ below normal. No prolonged periods of either above or below normal temperatures prevailed. April precipitation totaled 0.71 inch, $173 \%$ of normal ( $0.41 \mathrm{inch})$. A thunderstorm on April 29, although dropping only 0.01 inch of rain at the HMS, left as much as 0.40 inch of precipitation at other Tri-Cities locations, with some reports of one-half inch hail.

May 1993 was the seventh warmest May on record. The average temperature of $66.5^{\circ} \mathrm{F}$ was $5.2^{\circ}$ above normal. The warmest May occurred in 1947 and 
averaged $68.8^{\circ} \mathrm{F}$. Precipitation for May totaled 0.60 inch, $118 \%$ of normal $(0.51$ inch), and was the eighth consecutive month with above normal precipitation.

The spring season (March, April, and May) was slightly warmer and much wetter than normal. The temperature averaged $54.1^{\circ} \mathrm{F}, 0.9^{\circ}$ above normal $\left(53.2^{\circ} \mathrm{F}\right)$. The warmest springs occurred in 1992 and 1947 with average temperatures of $58.2^{\circ} \mathrm{F}$, while the coolest, in 1955, averaged $48.0^{\circ} \mathrm{F} .1993$ springtime precipitation totaled 1.98 inches, $142 \%$ of normal ( 1.39 inches). Tre wettest spring, in 1957, received 3.06 inches, while the driest, in 1968, received only 0.09 inch.

June 1993 was slightly cooler than normal, averaging $68.4^{\circ} \mathrm{F}, 1.3^{\circ}$ below normal $(69.7 \circ \mathrm{F})$. Precipitation for June totaled only 0.12 inch, $32 \%$ of normal (0.38 inch).

July 1993 was the coolest July on record. The average temperature of $70.5^{\circ} \mathrm{F}$, was $5.7^{\circ}$ below normal $\left(76.2^{\circ} \mathrm{F}\right)$. The previous coolest July was $70.6^{\circ} \mathrm{F}$ in 1986; the warmest, in 1985, averaged $82.2^{\circ} \mathrm{F}$. Only three days during July 1993 had average temperatures that were above normal. July 1993 was the wettest July on record. Precipitation for the month totaled 1.76 inches, 978\% of normal ( $0.18 \mathrm{inch}$ ). The previous wettest July, in 1966, received 0.81 inch. The $24-\mathrm{hr}$ precipitation total of 1.39 inches on July 16-17 was a record 24-hr precipitation total for the month. The previous record was 0.59 inch in July 1947. The most precipitation in a $24-\mathrm{hr}$ period at the HMS was 1.91 inches on October 1 and 2, 1957.

August 1993 was cooler than normal, averaging $73.1^{\circ} \mathrm{F}, 2.0^{\circ}$ below normal $\left(75.1^{\circ} \mathrm{F}\right)$. Precipitation for the month totaled 0.24 inch, $89 \%$ of normal (0.27 inch).

The summer of 1993 (June, July, and August) was the sixth coolest summer on record. The average summer-season temperature of $70.7 \circ \mathrm{F}$ was $3.0^{\circ}$ below normal $\left(73.7^{\circ} \mathrm{F}\right)$. The coolest summer was in 1980 and averaged $70.2^{\circ} \mathrm{F}$; while the warmest, in 1958, averaged $78.2^{\circ} \mathrm{F}$. Only three days in 1993 had a maximum temperature $\geq 100^{\circ} \mathrm{F}$. Precipitation for the summer of 1993 totaled 
2.12 inches, $255 \%$ of normal ( 0.83 incn). The wettest summer, in 1950 , received 2.92 inches; while the driest, in 1919, received only a trace.

September 1993 was slightly warmer than normal. The average temperature of $66.5^{\circ} \mathrm{F}$ was $0.8^{\circ}$ above normal $\left(65.7^{\circ} \mathrm{F}\right)$. Precipitation for september 1993 totaled only 0.04 inch, $13 \%$ of normal ( 0.31 inch).

October 1993 was warmer than normal, averaging $55.4^{\circ} \mathrm{F}, 2.5^{\circ}$ above normal $(52.9 \circ \mathrm{F})$. Only eight days during the month had average temperatures below normal. Precipitation for the month totaled 0.09 inch, $24 \%$ of normal (0.38 inch).

November 1993 was much cooler than normal. The average temperature of $34.6^{\circ} \mathrm{F}$ was $5.6^{\circ}$ below normal $\left(40.2^{\circ} \mathrm{F}\right)$. The period from November 22 through 28 averaged $17.6^{\circ} \mathrm{F}$ below normal, due to an Arctic air mass that moved southward from Canada. The minimum temperature of $-1 \circ \mathrm{F}$ on November 24 was only the third time the month of November has recorded sub-zero temperatures. Sub-zero readings also occurred in 1955 and 1985. November 1993 was the coldest November since 1985, which was the coldest November on record, averaging only 24.8० F. November 1993 was dry, receiving only 0.19 inch of precipitation, $21 \%$ of normal ( 0.91 inch). Snowfall totaled 1.4 inches, compared to a November normal $0^{\circ} 1.8$ inches. The peak wind gust of $67 \mathrm{mph}$ on November 3 was the strongest gust recorded at the HMS since a gust of $73 \mathrm{mph}$ occurred on January 28, 1990, and the strongest gust ever recorded in November. The previous November peak gust was $66 \mathrm{mph}$ on November 14, 1981, with the all-time peak gust at the HMS being $80 \mathrm{mph}$ on January 11, 1972.

The autumn season (September, October, and November) was slightly cooler and much drier than normal. The average temperature of $52.2 \circ \mathrm{F}$ compares to an autumn normal of $52.9 \circ \mathrm{F}$. The warmest was $57.1^{\circ}$ in 1990 ; the coolest, $44.5^{\circ} \mathrm{F}$ in 1985. Autumn precipitation totaled 0.32 inches, only $20 \%$ of normal (1.60 inches). The wettest autumn was 4.79 inches in 1973; the driest, 0.04 inch in 1976 .

December 1993 was warmer than normal, averaging $35.4^{\circ} \mathrm{F}, 4.0^{\circ}$ above normal $\left(31.4^{\circ} \mathrm{F}\right)$. The weather during the second half of the month was dominated by a strong high pressure ridge which trapped cooler air near the 
surface. Maximum temperatures during the period from December 16 through 31 had a range of on $1 y 6^{\circ}$, from $31^{\circ} \mathrm{F}$ to $37^{\circ} \mathrm{F}$. December 1993 precipitation totaled 0.94 inch, $91 \%$ of normal (1.03 inches). December snowfall totaled 1.8 inches, compared to a December normal of 5.7 inches.

Following are some additional statistics for 1993:

\begin{tabular}{|c|c|c|c|}
\hline CATEGORY & 1993 & NORMAL & RECORD \\
\hline Cays with maximum temperatures $\geq 100^{\circ} \mathrm{F}$ & 3 & 12 & 32 \\
\hline Days with riaximum temperatures $\geq 90^{\circ} \mathrm{F}$ & 43 & 51 & 85 \\
\hline Days with minimum temperatures $\leq 32^{\circ} \mathrm{F}$ & 114 & 107 & 139 \\
\hline Days with minimum temperatures $\leq 0^{\circ} \mathrm{F}$ & 3 & 2 & 18 \\
\hline Days with thunderstorms & 13 & 10 & 23 \\
\hline Days with fog (visibility $\leq 6 \mathrm{mi}$ & 67 & 45 & 76 \\
\hline Days with dense fog (visibility $\leq 1 / 4 \mathrm{mi}$ ) & 29 & 24 & 42 \\
\hline Days with peak wind gusts $\leq 12 \mathrm{mph}$ & 75 & 50 & 87 \\
\hline Days with peak wind gusts $\geq 25 \mathrm{mph}$ & 139 & 155 & 190 \\
\hline Days with peak wind gusts $\geq 40 \mathrm{mph}$ & 23 & 26 & 57 \\
\hline Days with peak wind gusts $\geq 50 \mathrm{mph}$ & 6 & 5 & 18 \\
\hline
\end{tabular}

Table 2.1 lists the daily temperature records for 1993, with the previous record and year of occurrence. Table 2.2 lists the monthly and annual totals for numerous meteorological parameters for 1993. Tables 2.3, 2.4, and 2.5 list 1993 monthly and annual average temperature ( $\left.{ }^{\circ} F\right)$, monthly and arinual total precipitation (inches), and monthly and annual average wind speed (mph), respectively from the HMMN.

Figure 2.1 depicts the wind roses from the HMMN for 1993. Wind arrows indicate direction from which wind blows. Length of arrow is proportional to frequency of occurrence. Figure A.1(a) in Appendix A depicts 1993 wind roses from the individual HMMN stations (see Table 1.1 and Figure 1.1 for names and locations). Each petal of the wind rose represents the proportional amount of time that the wind blew from that direction. The width of the petal segments correspond to each wind speed categnry represented in Figure $A .1(b)$ in Appendix A. Starting from the center of the rose, the narrowest segment 


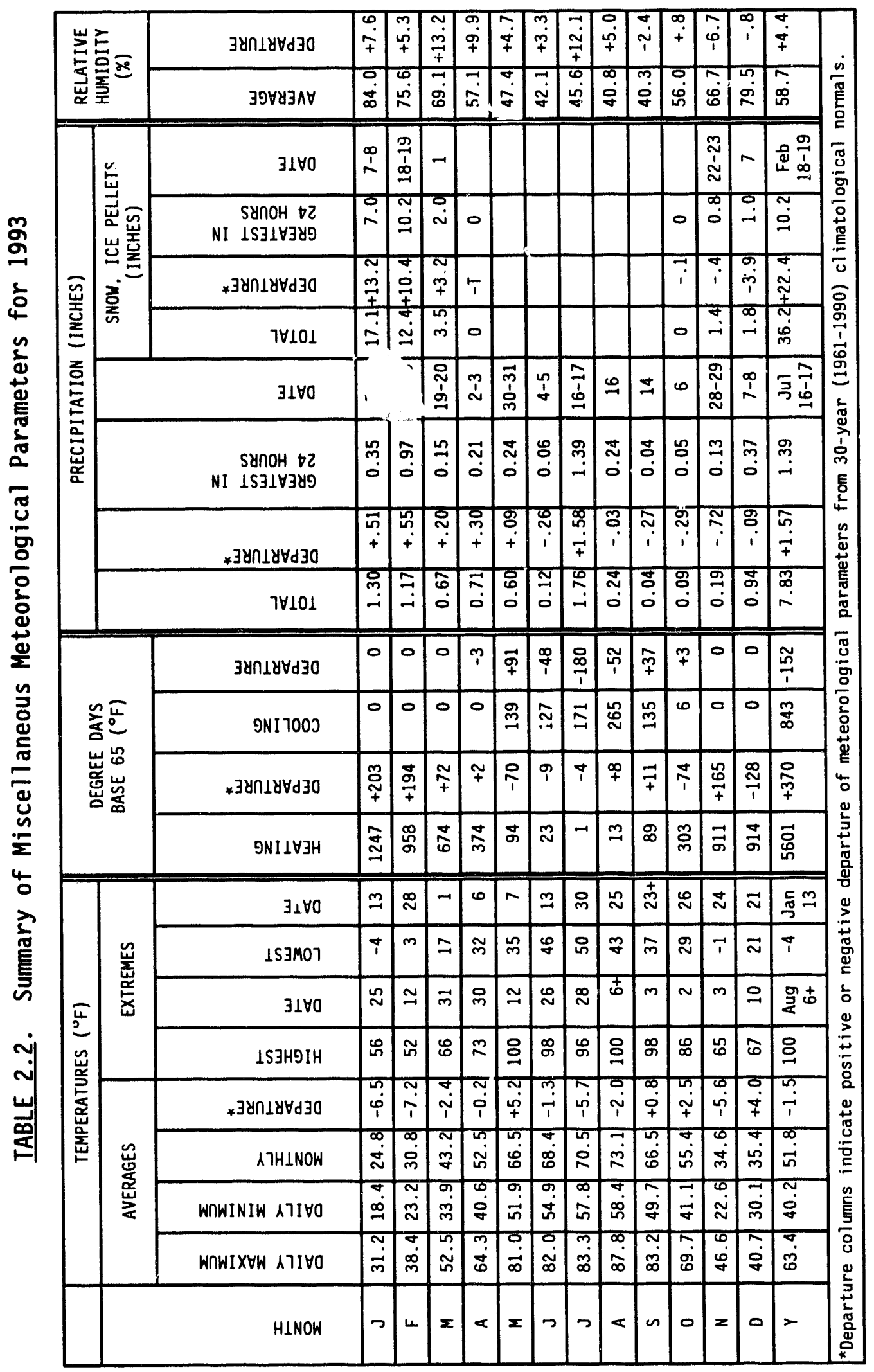

2.7 


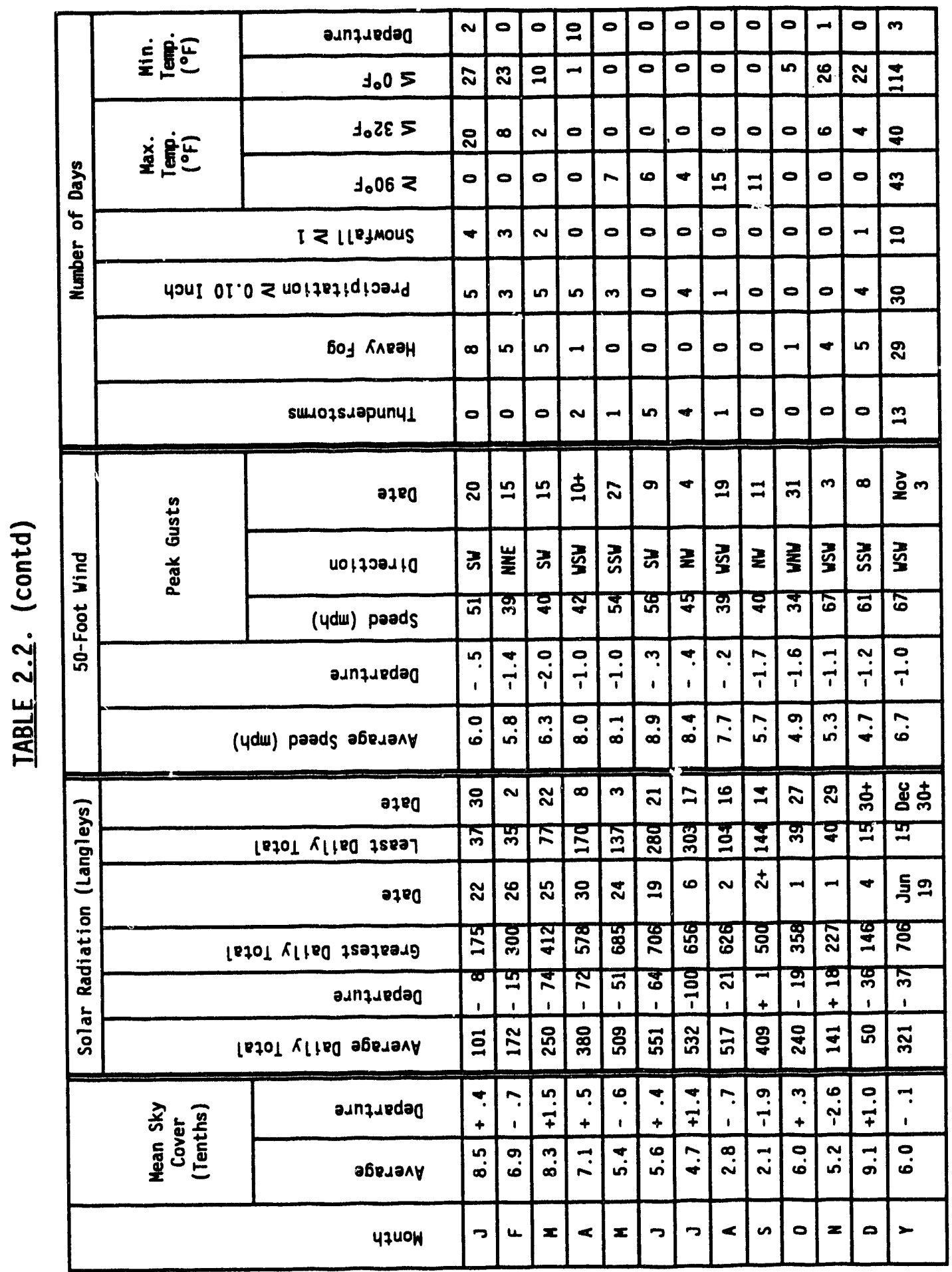


IABLE 2.3. 1993 Monthly and Annual Average Temperature ( $\left.{ }^{\circ} \mathrm{F}\right)$

from the Hanford Meteorological Monitoring Network

\begin{tabular}{|c|c|c|c|c|c|c|c|c|c|c|c|c|c|}
\hline STATION & JAN & FEB & MAR & APR & MAY & JUN & JUL & AUG & SEP & $\mathrm{OCT}$ & NOV & DEC & ANNUAL \\
\hline 1 PROS & 24.5 & 29.4 & 41.6 & 51.7 & 65.5 & 67.6 & 68.8 & 71.9 & 64.6 & 52.1 & 33.4 & 35.5 & 50.6 \\
\hline 2 EOC & 24.6 & 29.7 & 42.2 & 51.2 & 65.1 & 66.3 & 67.8 & 72.1 & 67.7 & 55.4 & 35.9 & 34.5 & 51.0 \\
\hline S ARMY & 25.0 & 29.4 & 42.4 & 52.3 & 66.1 & 68.2 & 69.8 & 72.6 & 65.8 & 53.1 & 33.1 & 35.7 & 51.1 \\
\hline 4 RSPG & 25.5 & 30.1 & 42.1 & 52.0 & 63.7 & 65.4 & 69.0 & 71.7 & 65.7 & 53.5 & 33.2 & 35.5 & 50.4 \\
\hline 5 EDNA & 24.5 & 30.0 & 41.5 & 51.4 & 65.3 & 67.9 & 69.1 & 72.8 & 64.7 & 52.2 & 32.4 & 35.8 & 50.5 \\
\hline $6200 E$ & 24.7 & 30.8 & 42.6 & 52.7 & 66.8 & 68.3 & 69.5 & 73.2 & 67.7 & 55.2 & 35.4 & 35.7 & 51.8 \\
\hline $7200 \mathrm{~W}$ & 25.4 & 29.3 & 41.4 & 51.6 & 64.8 & 67.8 & 69.3 & 71.0 & 64.0 & 51.5 & 32.5 & 34.9 & 50.4 \\
\hline B BVLY & 26.1 & 31.4 & 42.4 & 52.4 & 66.8 & 67.7 & 69.7 & 72.7 & 66.6 & 55.0 & 34.9 & 36.0 & 51.8 \\
\hline 9 FFTF & 23.9 & 29.9 & 42.2 & 52.1 & 65.7 & 57.5 & 68.8 & 72.4 & 66.3 & 54.0 & 34.3 & 35.7 & 51.0 \\
\hline 10 YAKD & 24.9 & 30.2 & 42.3 & 51.8 & 65.9 & 67.8 & 69.3 & 73.0 & 66.9 & 54.4 & 33.6 & 35.3 & 51.3 \\
\hline $11300 \mathrm{~A}$ & 24.6 & 30.1 & 42.3 & 52.5 & 65.4 & 67.3 & 69.0 & 71.7 & 64.9 & 53.1 & 34.4 & 36.7 & 51.0 \\
\hline 12 WYEB & 24.1 & 29.8 & 42.0 & 51.9 & 65.7 & 67.8 & 69.3 & 72.5 & 66.2 & 53.6 & 33.5 & 35.5 & 51.0 \\
\hline $13100 \mathrm{~N}$ & 24.5 & 30.7 & 41.8 & 51.1 & 65.3 & 67.5 & 68.7 & 71.8 & 65.0 & 53.3 & 33.5 & 35.3 & 50.7 \\
\hline 14 WPPS & 24.0 & 29.6 & 41.4 & 51.4 & 64.8 & 67.3 & 68.8 & 71.7 & 64.8 & 52.5 & 32.6 & 35.8 & 50.5 \\
\hline 15 FRNK & 23.5 & 29.3 & 41.8 & 50.8 & 63.3 & 65.0 & 66.3 & 69.5 & 64.1 & 53.1 & 34.3 & 34.7 & 49.6 \\
\hline $16 \mathrm{GABL}$ & 24.4 & 30.1 & 42.2 & 51.3 & 65.9 & 66.6 & 67.7 & 72.6 & 68.6 & 56.4 & 36.2 & 34.5 & 51.3 \\
\hline 17 RING & 24.7 & 29.8 & 41.9 & 51.2 & 64.3 & 65.4 & 67.0 & 69.9 & 63.1 & 52.2 & 32.9 & 35.4 & 49.8 \\
\hline $18 \mathrm{RICH}$ & 25.0 & 30.6 & 43.1 & 53.3 & 66.0 & 67.6 & 69.2 & 72.2 & 66.1 & 54.6 & 35.9 & 36.7 & 51.7 \\
\hline 20 RMTN & 21.2 & 24.5 & 36.5 & 40.9 & 57.3 & 56.3 & 57.8 & 63.3 & 61.7 & 51.2 & 31.4 & 28.4 & 44.2 \\
\hline 21 HMS & 23.8 & 29.6 & 41.6 & 51.7 & 65.4 & 67.5 & 68.8 & 72.2 & 66.1 & 53.3 & 32.7 & 34.5 & 50.6 \\
\hline 22 PASC & 24.9 & 30.4 & 43.3 & 53.8 & 66.4 & 68.0 & 69.8 & 72.0 & 65.2 & 53.8 & 35.0 & 37.1 & 51.7 \\
\hline $23 \mathrm{GABW}$ & 24.4 & 29.8 & 41.2 & 51.4 & 65.2 & 67.9 & 69.2 & 71.7 & 64.4 & 51.8 & 31.9 & 35.0 & 50.4 \\
\hline $24100 F$ & 24.7 & 30.4 & 41.7 & 51.6 & 65.5 & 68.0 & 69.1 & 72.2 & 65.0 & 52.7 & 32.8 & 35.8 & 50.9 \\
\hline 25 VERN & 24.8 & 30.0 & 41.5 & 51.2 & 65.0 & 67.2 & 69.1 & 72.3 & 66.0 & 54.8 & 34.8 & 36.6 & 51.2 \\
\hline $26622 R$ & 24.7 & 30.6 & 42.6 & 52.3 & 66.1 & 67.8 & 69.2 & 72.8 & 67.2 & 54.9 & 34.6 & 35.7 & 51.8 \\
\hline 27 VSTA & 25.3 & 30.7 & 43.3 & 53.2 & 65.5 & 67.1 & 68.6 & 71.5 & 65.4 & 54.0 & 36.1 & 36.7 & 51.4 \\
\hline
\end{tabular}


IABLE 2.4. 1993 Monthly and Annual Precipitation (Inches) from the Hanford Meteorological Monitoring Network

\begin{tabular}{|c|c|c|c|c|c|c|c|c|c|c|c|c|c|}
\hline STATION & JAN & FEB & MAR & APR & MAY & JUN & JUL & $A \cup G$ & SEP & OCT & NOV & DEC & YEAR \\
\hline 1 PROS & .51 & .35 & .90 & .53 & .62 & .38 & .99 & .29 & .10 & .03 & .12 & 1.25 & 6.07 \\
\hline $2 \mathrm{EOC}$ & 1.30 & .18 & 1.50 & .91 & .63 & .58 & 1.48 & .19 & .14 & .05 & .22 & 1.48 & 8.66 \\
\hline 3 ARMY & .34 & .23 & .58 & .63 & .57 & .25 & 1.06 & .21 & .04 & .03 & .00 & .00 & 3.94 \\
\hline 4 RSF'G $^{\prime}$ & 1.26 & .55 & .83 & .59 & .58 & .09 & 1.76 & .09 & .01 & .08 & .20 & .72 & 6.76 \\
\hline $6200 E$ & .32 & .11 & .55 & .73 & .44 & .13 & 2.14 & .30 & .01 & .06 & .12 & .67 & 5.58 \\
\hline $7200 \mathrm{~W}$ & .43 & .06 & .15 & .34 & .47 & .67 & 1.77 & .25 & .03 & .06 & .20 & 1.10 & 4.93 \\
\hline 8 BVLY & .60 & .25 & .38 & .61 & .39 & .85 & 1.69 & .00 & .11 & .06 & .18 & .75 & 5.88 \\
\hline 10 YAKB & .43 & .27 & .51 & .44 & .32 & .06 & 2.32 & .05 & .02 & .13 & .15 & .84 & 5.54 \\
\hline $11300 \mathrm{~A}$ & .28 & .12 & .65 & .90 & .73 & .42 & .73 & .84 & .03 & .01 & .17 & .85 & 5.73 \\
\hline 12 WYEB & .44 & .14 & .89 & .78 & .56 & .45 & .77 & .24 & .10 & .03 & .09 & .66 & 5.15 \\
\hline 14 WPPS & .47 & .16 & .81 & 1.00 & .65 & .59 & .80 & .26 & .14 & .04 & .11 & .52 & 5.55 \\
\hline 17 RING & .88 & .58 & .44 & .47 & .87 & .43 & .75 & .55 & .10 & .04 & .19 & .88 & 6.18 \\
\hline 20 RMTN & .51 & .49 & .78 & .59 & .57 & .52 & .86 & .23 & .15 & .02 & .19 & .47 & 5.38 \\
\hline 22 PASC & .57 & .10 & .61 & .73 & .60 & .48 & .34 & .92 & .01 & .09 & .18 & .72 & 5.35 \\
\hline $24100 F$ & .84 & .35 & .70 & .94 & .74 & .16 & 1.84 & .38 & .04 & .10 & .27 & .97 & 7.33 \\
\hline $26622 R$ & 1.30 & 1.17 & .67 & .71 & .60 & .12 & 1.76 & .24 & .04 & .09 & .19 & .94 & 7.83 \\
\hline 27 VSTA & .63 & .30 & .69 & .59 & .46 & .07 & .21 & 1.02 & .01 & .08 & .11 & .77 & 4.94 \\
\hline
\end{tabular}


IABLE 2.5. 1993 Monthly and Annual Average Wind Speed (mph) from the Hanford Meteorological Monitoring Network

STATION JAN FEB MAR APR MAY JUN JUL AUG SEP OCT NOV DEC ANNUAL

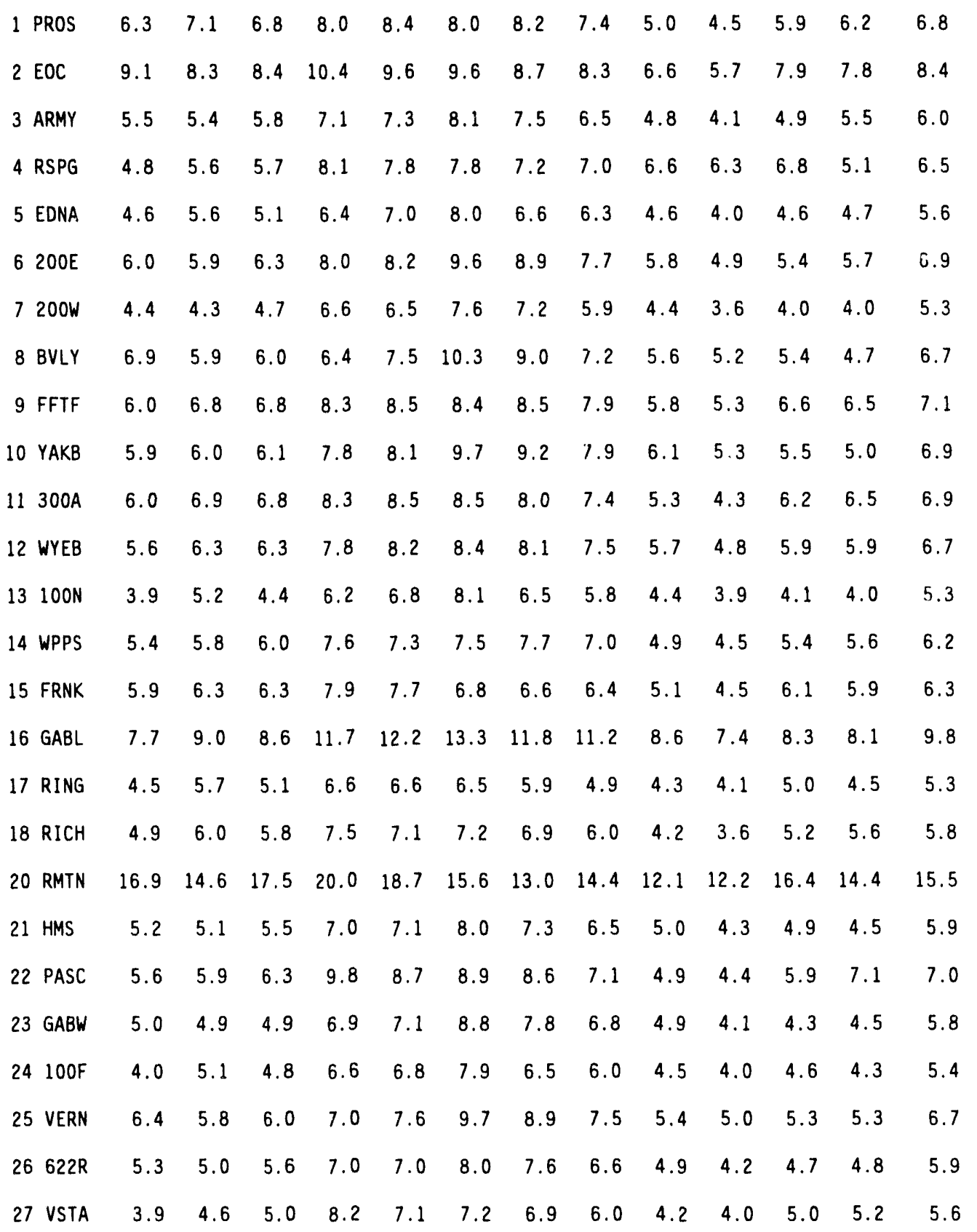




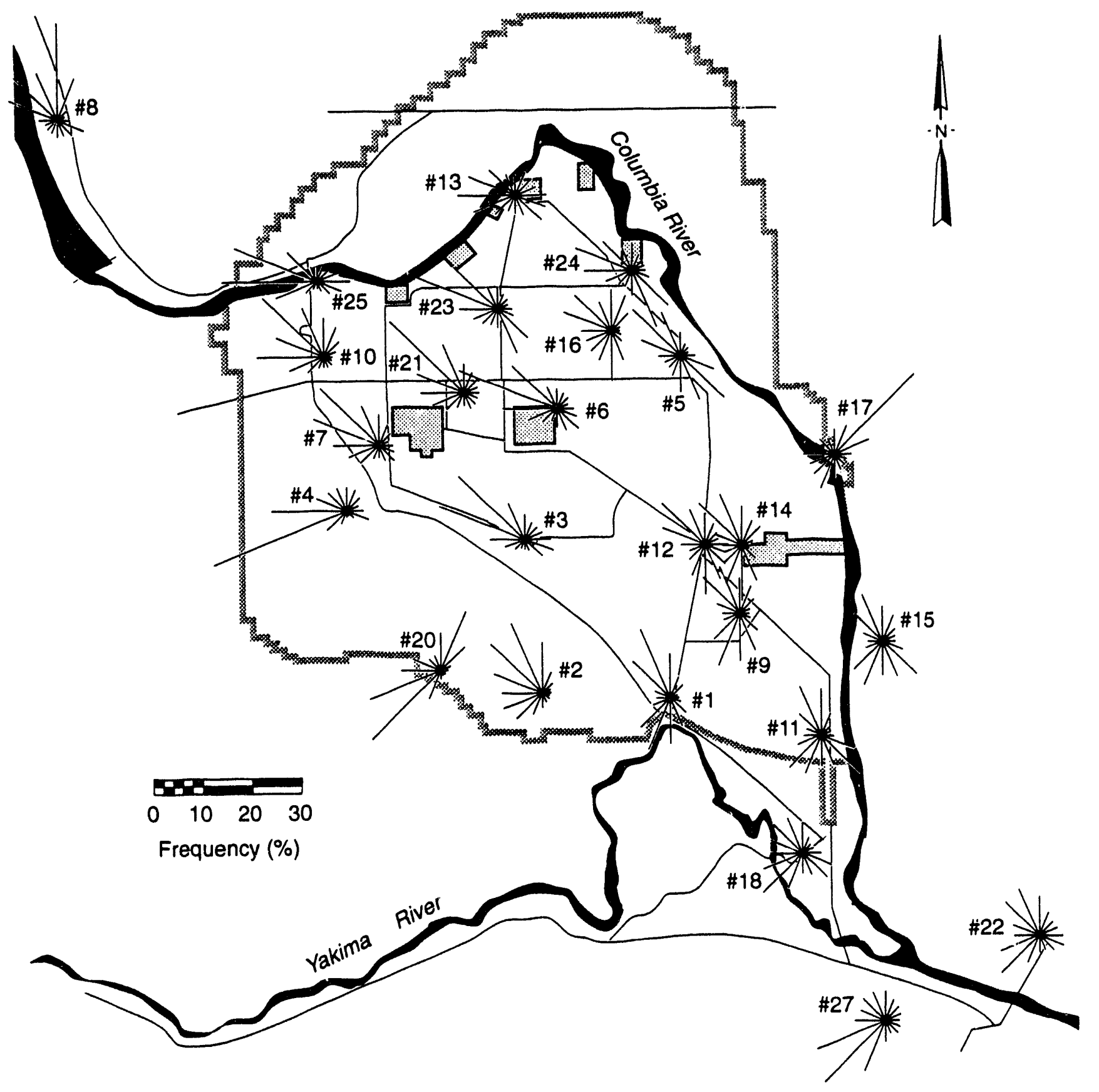

FIGURE 2.1. Hinford Meteorological Monitoring Network Wind Roses for 1993 
represents winds in the 1 - to 3 -mph class, the next widest segment represents the 4- to 7-mph class, and so forth. The length of each of these segments is proportional to the frequency of occurrence for each speed class.

Figure $A .1(b)$ is a wind speed histogram representing the proportional amount of time in each speed class. Table A.l in Appendix A lists joint frequency distributions of wind direction versus wind speed class for the individual HMMN stations.

Figure 2.2 graphically depicts the 1993 daily observed maximum and minimum temperatures, and the normal maximum, minimum, and mean daily temperatures for the HMS. 


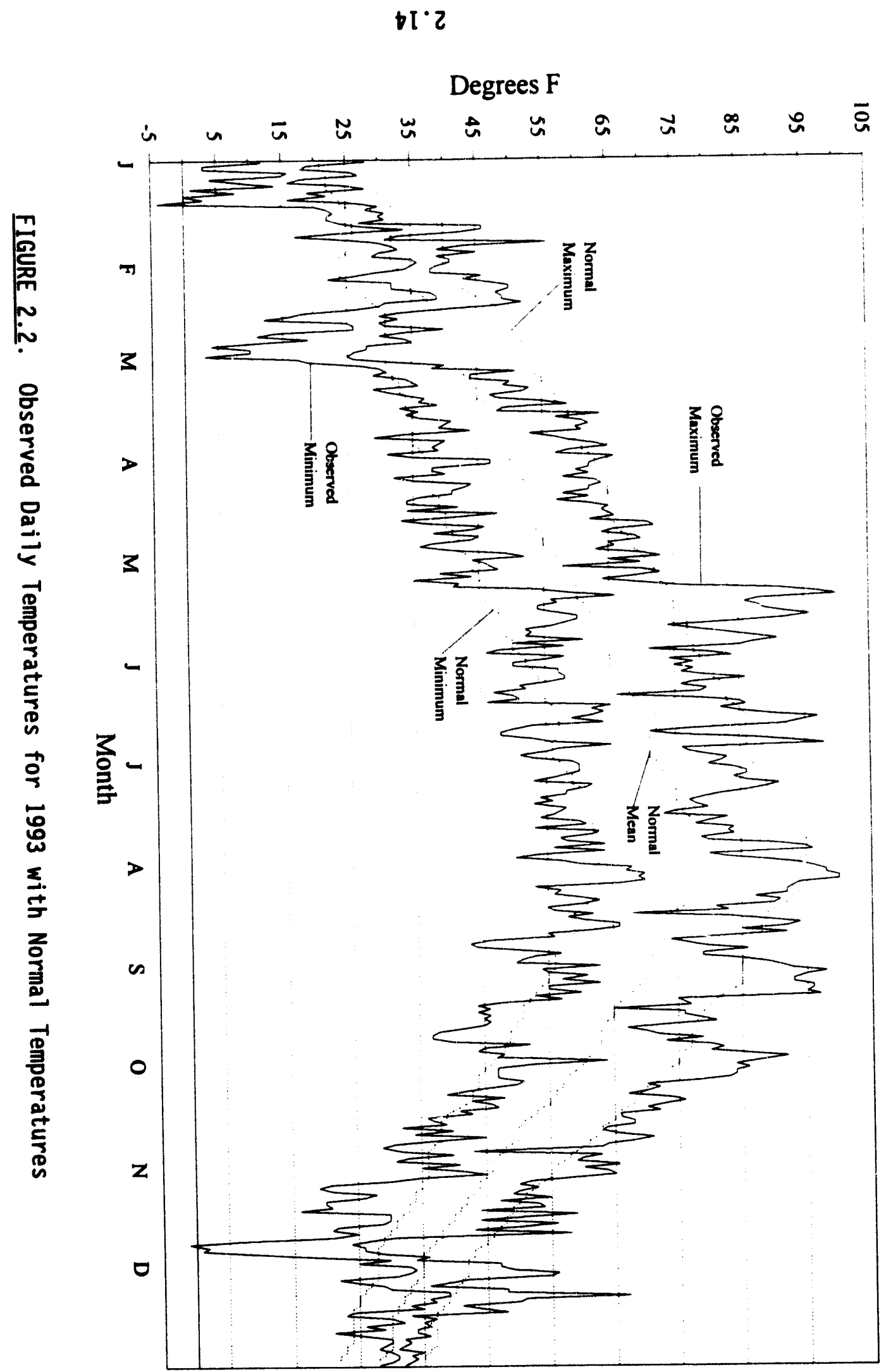




\subsection{IEMPERATURE CLIMATOLOGY}

\subsection{MONTHLY, SEASONAL, AND ANNUAL AVERAGE TEMPERATURES}

Monthly, seasonal, and annual average temperatures, computed from observed daily maximum and minimum temperatures for the period from 1945 through 1993, are presented in Tables 3.1 and 3.2. In these tables, the highest and lowest values, representing the warmest and coldest month, season, or year, are underlined in each column. Averages, based upon the entire period of record, as well as the climatological normal temperatures, averages based upon the period from 1961 to 1990, are indicated at the bottom of each table.

As indicated in Table 3.1, a much wider range and variability in temperatures is found during the late fall and winter months (November through February) than during the rest of the year. The range of average monthly temperatures for January is from $12.1^{\circ} \mathrm{F}(1950)$ to $42.5^{\circ} \mathrm{F}(1953), 30.4^{\circ}$; for November 21.7०; February 18.9०; and December $17.5^{\circ}$; whereas for the rest of the year the monthly range is from a low of $10.5^{\circ}$ in April, to a high of $13.8^{\circ}$ in June. The coldest month ever was January $1950\left(12.1^{\circ} \mathrm{F}\right)$ and the hottest was July $1985\left(82.2^{\circ} \mathrm{F}\right)$. As shown in Table 3.2, seasonal ranges are from $8.0^{\circ}$ during the summer (June, July, and August) to $16.4^{\circ}$ in winter (December, January, and February). The coidest season was the winter of 1948 to $1949\left(24.2^{\circ} \mathrm{F}\right)$, and the hottest was the summer of $1958\left(78.2^{\circ} \mathrm{F}\right)$.

\subsection{DAYS WITH MAXIMUM TEMPERATURES $\geq 100^{\circ} \mathrm{F}, \geq 90^{\circ} \mathrm{F}$, AND $\leq 32^{\circ} \mathrm{F}$}

Table 3.3 contains the number of days each year with maximum temperatures in the categories $\geq 100^{\circ} \mathrm{F}, \geq 90^{\circ} \mathrm{F}$, and $\leq 32^{\circ} \mathrm{F}$.

Maximum temperatures $\geq 100 \circ \mathrm{F}$ have occurred as early as May 5 (1966) and as late as September 6 (1955). The annual number of days with maximum temperatures in this category has ranged from 1 (1954) to 28 (1958). The greatest number of consecutive days with maximum temperatures $\geq 100^{\circ} \mathrm{F}$ is 11 , and has occurred three times: July 22 through August 1, 1962; August 10 through 20, 1967; and August 6 through 16, 1981. 


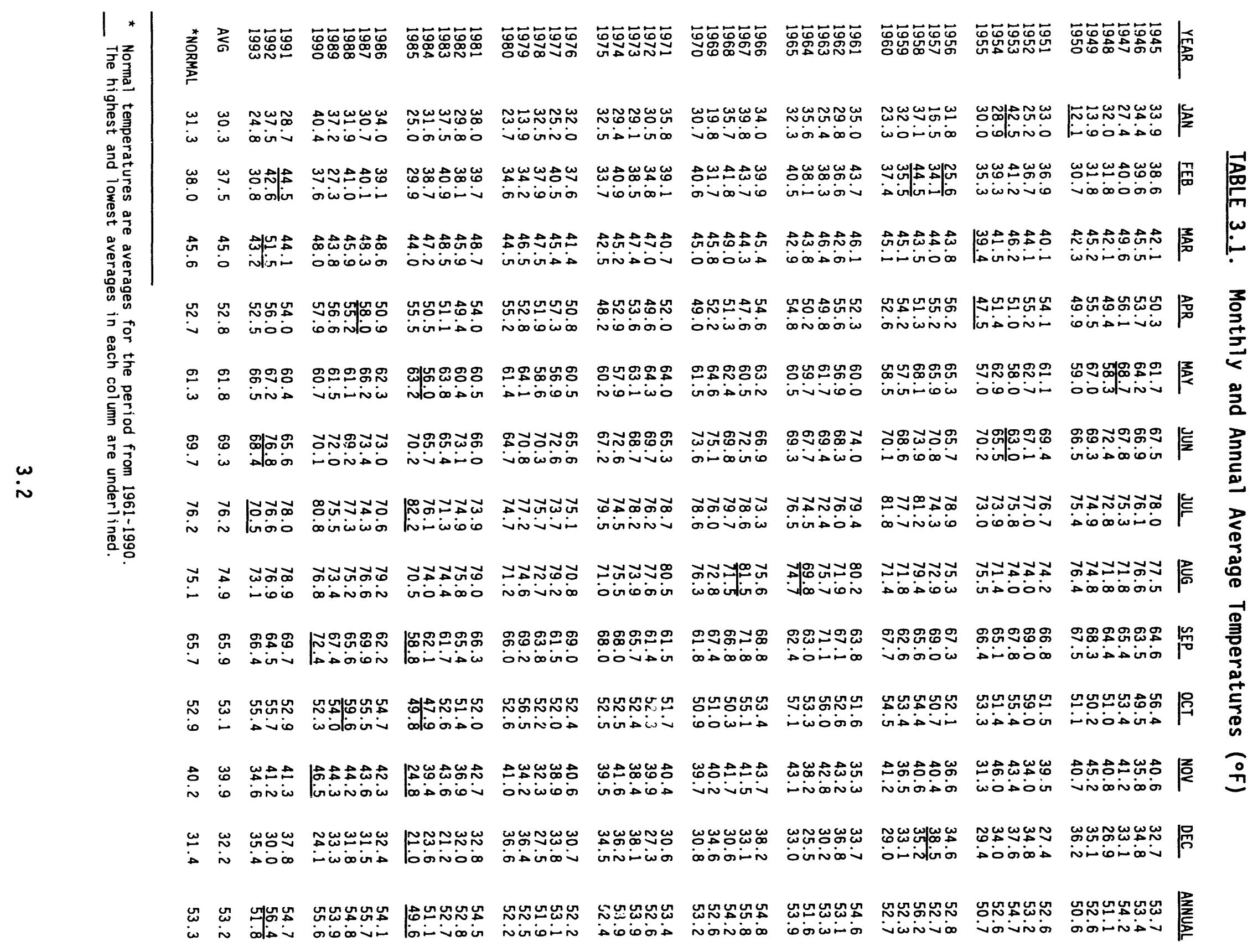


IABLE 3.2. Seasonal Average Temperatures $\left({ }^{\circ} \mathrm{F}\right)$

\begin{tabular}{|c|c|c|c|c|}
\hline YEAR & $\begin{array}{l}\text { WINTER } \\
\text { DECC-FEB } \\
\end{array}$ & $\begin{array}{c}\text { SPRING } \\
\text { MAR-MAY } \\
\end{array}$ & $\begin{array}{c}\text { SUMMER } \\
\text { SUN-AUG }\end{array}$ & $\begin{array}{c}\text { AUTUMN } \\
\text { SEP-NOV } \\
\end{array}$ \\
\hline 1945 & ---- & 51.4 & 74.3 & 53.9 \\
\hline $\begin{array}{l}1946 \\
1947 \\
1948 \\
1949 \\
1950\end{array}$ & $\begin{array}{l}35.6 \\
34.1 \\
32.3 \\
24.2 \\
26.0\end{array}$ & $\begin{array}{l}54.5 \\
58.1 \\
49.9 \\
55.9 \\
50.4\end{array}$ & $\begin{array}{l}73.2 \\
71.6 \\
72.3 \\
73.0 \\
72.8\end{array}$ & $\begin{array}{l}49.6 \\
53.3 \\
52.1 \\
54.6 \\
53.1\end{array}$ \\
\hline $\begin{array}{l}1951 \\
1952 \\
1953 \\
1954 \\
1955\end{array}$ & $\begin{array}{l}35.4 \\
29.8 \\
39.5 \\
35.3 \\
33.1\end{array}$ & $\begin{array}{l}51.8 \\
54.0 \\
51.7 \\
51.9 \\
48.0 \\
\end{array}$ & $\begin{array}{l}73.4 \\
72.7 \\
70.9 \\
70.3 \\
72.9\end{array}$ & $\begin{array}{l}52.6 \\
54.0 \\
55.5 \\
54.2 \\
50.3\end{array}$ \\
\hline $\begin{array}{l}1956 \\
1957 \\
1958 \\
1959 \\
1960\end{array}$ & $\begin{array}{l}28.9 \\
28.4 \\
40.0 \\
34.2 \\
31.3\end{array}$ & $\begin{array}{l}55.1 \\
55.0 \\
54.3 \\
52.3 \\
52.1\end{array}$ & $\begin{array}{l}73.3 \\
72.7 \\
78.2 \\
72.7 \\
74.4\end{array}$ & $\begin{array}{l}52.0 \\
53.4 \\
53.5 \\
50.8 \\
54.5\end{array}$ \\
\hline $\begin{array}{l}1961 \\
1962 \\
1963 \\
1964 \\
1965\end{array}$ & $\begin{array}{l}35.9 \\
33.4 \\
33.5 \\
34.6 \\
32.8\end{array}$ & $\begin{array}{l}52.8 \\
51.7 \\
52.6 \\
51.2 \\
52.7\end{array}$ & $\begin{array}{l}77.9 \\
72.1 \\
72.5 \\
70.7 \\
73.5\end{array}$ & $\begin{array}{l}50.2 \\
54.3 \\
56.6 \\
51.5 \\
54.2\end{array}$ \\
\hline $\begin{array}{l}1966 \\
1967 \\
1968 \\
1969 \\
1970\end{array}$ & $\begin{array}{l}35.6 \\
40.6 \\
36.9 \\
27.4 \\
35.3\end{array}$ & $\begin{array}{l}54.4 \\
50.8 \\
54.2 \\
54.2 \\
51.8\end{array}$ & $\begin{array}{l}71.9 \\
77.5 \\
73.7 \\
74.6 \\
76.2\end{array}$ & $\begin{array}{l}55.3 \\
56.1 \\
52.9 \\
52.9 \\
50.8\end{array}$ \\
\hline $\begin{array}{l}1971 \\
1972 \\
1973 \\
1974 \\
1975\end{array}$ & $\begin{array}{l}35.2 \\
32.0 \\
31.6 \\
36.1 \\
34.1\end{array}$ & $\begin{array}{l}52.2 \\
53.6 \\
54.7 \\
52.0 \\
50.3\end{array}$ & $\begin{array}{l}74.8 \\
74.5 \\
73.5 \\
74.2 \\
72.6\end{array}$ & $\begin{array}{l}51.2 \\
55.2 \\
52.2 \\
54.0 \\
53.3\end{array}$ \\
\hline $\begin{array}{l}1976 \\
1977 \\
1978 \\
1979 \\
1980\end{array}$ & $\begin{array}{l}34.7 \\
32.1 \\
34.7 \\
25.2 \\
31.6\end{array}$ & $\begin{array}{l}50.9 \\
53.2 \\
52.7 \\
54.5 \\
53.7\end{array}$ & $\begin{array}{l}70.5 \\
75.2 \\
72.9 \\
74.2 \\
70.2 \\
\end{array}$ & $\begin{array}{l}54.0 \\
50.8 \\
49.4 \\
53.3 \\
53.2\end{array}$ \\
\hline $\begin{array}{l}1981 \\
1982 \\
1983 \\
1984 \\
1985\end{array}$ & $\begin{array}{l}38.1 \\
33.6 \\
36.8 \\
30.5 \\
26.2\end{array}$ & $\begin{array}{l}54.4 \\
51.9 \\
54.5 \\
51.2 \\
54.2\end{array}$ & $\begin{array}{l}73.0 \\
74.6 \\
70.4 \\
71.9 \\
74.3\end{array}$ & $\begin{array}{l}53.7 \\
51.2 \\
52.6 \\
49.8 \\
44.5 \\
\end{array}$ \\
\hline $\begin{array}{l}1986 \\
1987 \\
1988 \\
1989 \\
1990\end{array}$ & $\begin{array}{l}31.4 \\
34.4 \\
34.8 \\
32.1 \\
37.1\end{array}$ & $\begin{array}{l}53.9 \\
57.5 \\
54.1 \\
54.0 \\
55.5\end{array}$ & $\begin{array}{l}74.3 \\
74.8 \\
73.9 \\
73.6 \\
75.9\end{array}$ & $\begin{array}{l}53.1 \\
56.3 \\
56.5 \\
55.2 \\
57.1 \\
\end{array}$ \\
\hline $\begin{array}{l}1991 \\
1992 \\
1993 \\
\text { AVG } \\
\text { * NORMAL }\end{array}$ & $\begin{array}{l}32.4 \\
39.3 \\
28.5 \\
33.3 \\
33.6\end{array}$ & $\begin{array}{l}52.8 \\
58.2 \\
54.1 \\
53.2 \\
53.2\end{array}$ & $\begin{array}{l}74.2 \\
76.8 \\
70.7 \\
73.5 \\
73.7\end{array}$ & $\begin{array}{l}54.6 \\
53.8 \\
52.1 \\
53.0 \\
52.9\end{array}$ \\
\hline
\end{tabular}

* Normal temperatures are averages for the period from 1961-1990. The highest and lowest averages in each column are underlined. 


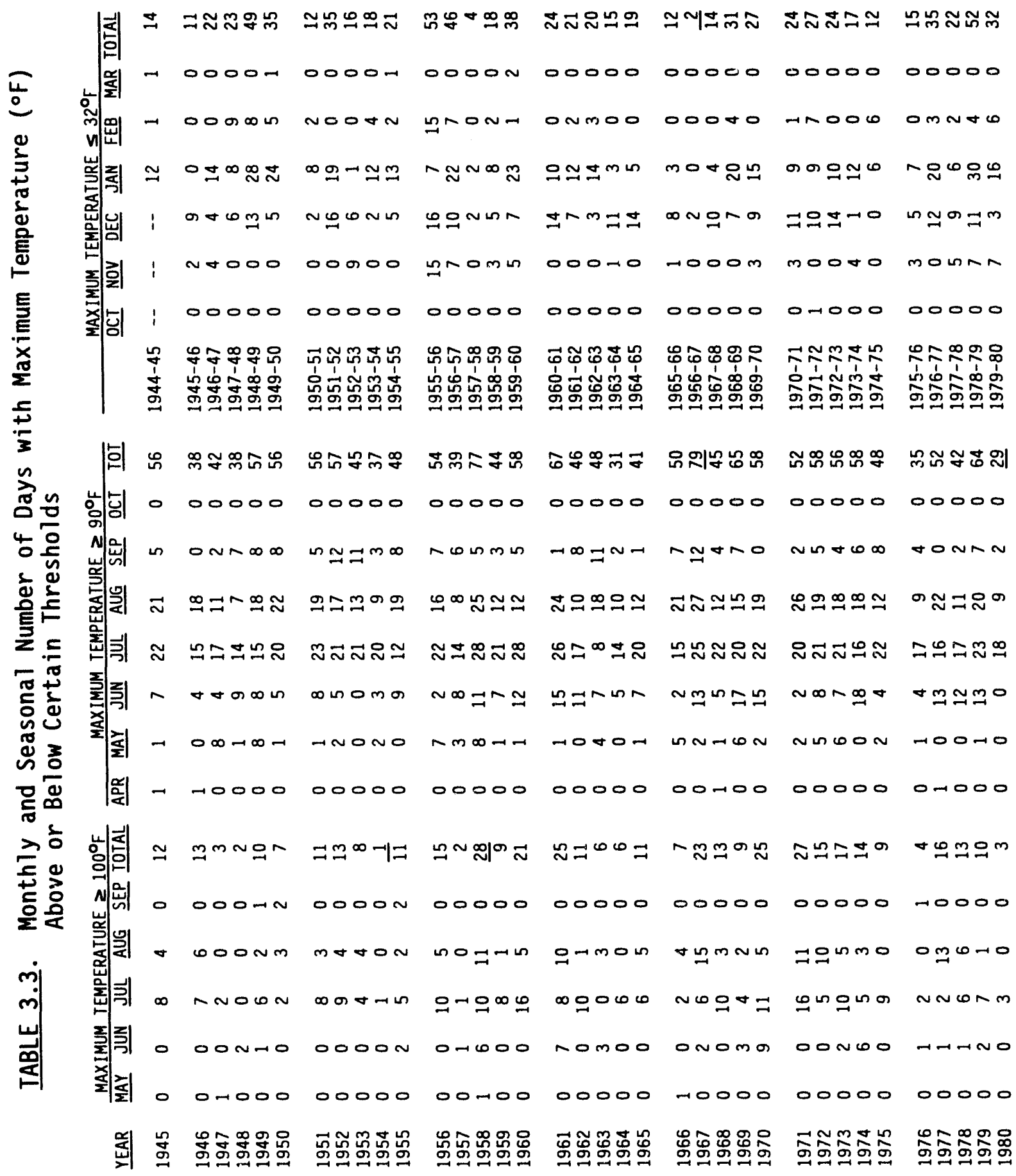




\section{TABLE 3.3. (contd)}

MAXIMUM TEMPERATURE $>100^{\circ} \mathrm{F}$ MAXIMUM TEMPERATURE $>90^{\circ} \mathrm{F}$ YEAR MAY JUN JUL AUG SEP TOTAL APR MAY JUN JUL AUG SEP OCT TOT

TEMPERATURE $\leq 32^{\circ} \mathrm{F}$

\begin{tabular}{|c|c|c|c|c|c|c|c|c|c|c|c|c|c|c|c|c|c|c|c|c|c|c|}
\hline $\begin{array}{l}1981 \\
1982 \\
1983 \\
1984 \\
1985\end{array}$ & $\begin{array}{l}0 \\
0 \\
1 \\
0 \\
0\end{array}$ & $\begin{array}{l}0 \\
2 \\
0 \\
0 \\
1\end{array}$ & $\begin{array}{r}3 \\
5 \\
1 \\
3 \\
15\end{array}$ & $\begin{array}{r}13 \\
3 \\
0 \\
3 \\
0\end{array}$ & $\begin{array}{l}0 \\
0 \\
0 \\
0 \\
0\end{array}$ & $\begin{array}{r}16 \\
10 \\
2 \\
6 \\
16\end{array}$ & $\begin{array}{l}1 \\
0 \\
0 \\
0 \\
0\end{array}$ & $\begin{array}{l}0 \\
0 \\
8 \\
1 \\
3\end{array}$ & $\begin{array}{r}4 \\
15 \\
2 \\
4 \\
10\end{array}$ & $\begin{array}{r}19 \\
16 \\
9 \\
21 \\
30\end{array}$ & $\begin{array}{r}22 \\
17 \\
13 \\
16 \\
7\end{array}$ & $\begin{array}{r}11 \\
5 \\
0 \\
4 \\
0\end{array}$ & $\begin{array}{l}0 \\
0 \\
0 \\
0 \\
0\end{array}$ & $\begin{array}{l}57 \\
53 \\
32 \\
46 \\
50\end{array}$ & $\begin{array}{l}1980-81 \\
1981-82 \\
1982-83 \\
1983-84 \\
1984-85\end{array}$ & $\begin{array}{l}0 \\
0 \\
0 \\
0 \\
0\end{array}$ & $\begin{array}{l}1 \\
0 \\
3 \\
0 \\
2\end{array}$ & $\begin{array}{r}6 \\
8 \\
10 \\
25 \\
18\end{array}$ & $\begin{array}{r}0 \\
10 \\
5 \\
12 \\
29\end{array}$ & $\begin{array}{l}2 \\
2 \\
0 \\
1 \\
9\end{array}$ & 0 & $\begin{array}{r}9 \\
20 \\
18 \\
38 \\
58 \\
\end{array}$ \\
\hline $\begin{array}{l}1989 \\
1990\end{array}$ & $\begin{array}{l}3 \\
1 \\
0 \\
0 \\
0\end{array}$ & $\begin{array}{l}1 \\
5 \\
0 \\
0 \\
0\end{array}$ & $\begin{array}{r}0 \\
3 \\
8 \\
2 \\
11\end{array}$ & $\begin{array}{l}6 \\
4 \\
3 \\
2 \\
9\end{array}$ & $\begin{array}{l}0 \\
1 \\
3 \\
0 \\
0\end{array}$ & $\begin{array}{r}14 \\
4 \\
20\end{array}$ & $\begin{array}{l}0 \\
2 \\
0 \\
0 \\
0\end{array}$ & $\begin{array}{l}6 \\
6 \\
4 \\
0 \\
1\end{array}$ & $\begin{array}{r}11 \\
15 \\
11 \\
13 \\
8\end{array}$ & $\begin{array}{r}9 \\
14 \\
19 \\
20 \\
24\end{array}$ & $\begin{array}{r}27 \\
19 \\
20 \\
9 \\
15\end{array}$ & $\begin{array}{r}3 \\
12 \\
7 \\
3 \\
12\end{array}$ & $\begin{array}{l}0 \\
0 \\
0 \\
0 \\
0\end{array}$ & $\begin{array}{l}56 \\
68 \\
61 \\
45 \\
60\end{array}$ & & & $\begin{array}{r}15 \\
0 \\
0 \\
0 \\
2\end{array}$ & $\begin{array}{r}25 \\
7 \\
16 \\
11 \\
6\end{array}$ & $\begin{array}{r}5 \\
9 \\
11 \\
2 \\
0\end{array}$ & $\begin{array}{l}1 \\
8 \\
1\end{array}$ & 0 & $\begin{array}{r}46 \\
16 \\
28 \\
22 \\
9\end{array}$ \\
\hline $\begin{array}{l}1991 \\
1992 \\
1993 \\
1994\end{array}$ & $\begin{array}{r}0 \\
0 \\
1 \\
--\end{array}$ & $\begin{array}{r}0 \\
7 \\
0 \\
--\end{array}$ & $\begin{array}{r}4 \\
5 \\
0 \\
--\end{array}$ & $\begin{array}{r}8 \\
9 \\
2 \\
--\end{array}$ & $\begin{array}{r}0 \\
0 \\
0 \\
--\end{array}$ & $\begin{array}{r}12 \\
21 \\
3 \\
--\end{array}$ & $\begin{array}{r}0 \\
0 \\
0 \\
--\end{array}$ & $\begin{array}{r}0 \\
8 \\
7 \\
--\end{array}$ & $\begin{array}{r}1 \\
16 \\
6 \\
--\end{array}$ & $\begin{array}{r}25 \\
15 \\
4 \\
--\end{array}$ & $\begin{array}{l}23 \\
17 \\
15 \\
-\end{array}$ & $\begin{array}{r}5 \\
3 \\
11 \\
--\end{array}$ & $\begin{array}{r}0 \\
0 \\
0 \\
--\end{array}$ & -- & & 0 & $\begin{array}{l}0 \\
0 \\
1 \\
6\end{array}$ & $\begin{array}{r}15 \\
3 \\
11 \\
4\end{array}$ & $\begin{array}{r}13 \\
0 \\
20 \\
--\end{array}$ & $\begin{array}{r}0 \\
0 \\
8 \\
--\end{array}$ & $\begin{array}{r}0 \\
0 \\
2 \\
-\end{array}$ & $\begin{array}{r}28 \\
3 \\
42 \\
10\end{array}$ \\
\hline $\begin{array}{l}\text { VGG } \\
\text { NORM }\end{array}$ & $\begin{array}{l}0 \\
0\end{array}$ & $\begin{array}{l}1 \\
2\end{array}$ & $\begin{array}{l}6 \\
6\end{array}$ & $\begin{array}{l}4 \\
5\end{array}$ & $\begin{array}{l}0 \\
0\end{array}$ & $\begin{array}{l}12 \\
13\end{array}$ & $\begin{array}{l}0 \\
0\end{array}$ & $\begin{array}{l}3 \\
2\end{array}$ & $\begin{array}{l}8 \\
9\end{array}$ & $\begin{array}{l}19 \\
19\end{array}$ & $\begin{array}{l}16 \\
17\end{array}$ & $\begin{array}{l}5 \\
5\end{array}$ & $\begin{array}{l}0 \\
0\end{array}$ & $\begin{array}{l}51 \\
52\end{array}$ & $\begin{array}{l}\text { AVG } \\
\text { NORMAL }\end{array}$ & & $\begin{array}{l}2 \\
2\end{array}$ & $\begin{array}{r}9 \\
10\end{array}$ & $\begin{array}{l}11 \\
10\end{array}$ & $\begin{array}{l}3 \\
2\end{array}$ & ) & $\begin{array}{l}5 \\
4\end{array}$ \\
\hline
\end{tabular}

* Normal temperatures are averages for the period from 1961-1990.

Greatest and least seasonal totals are underlined. 
One particularly notable period of above-normal temperatures occurred July 15 through August 13, 1971. This 30-day period included 27 days with maximum temperatures $\geq 100^{\circ} \mathrm{F}$ in 3 separate periods of 9 consecutive days each. The lowest maxinum temperature during the 30 -day period was $98^{\circ} \mathrm{F}$ and the highest was $112^{\circ} \mathrm{F}$. The average maximum temperature during this period was $104.7^{\circ} \mathrm{F}$.

Table 3.4 lists the dates of all occurrences of maximum temperatures $\geq 104^{\circ} \mathrm{F}$.

Maximum temperatures $\geq 90^{\circ} \mathrm{F}$ occur an average of 51 times per year, and vary from a low of 29 (1980) to a high of 79 (1967). The earliest occurrences have varied from April 24 (1977) through July 2 (1953), with an average annual date of May 21 (see Table 3.5). The latest annual occurrence of maximum temperatures $\geq 90^{\circ} \mathrm{F}$ has varied from August 17 (1983) through September 29 (1993 and earlier years). The average date for the period from 1946 through 1993 is September 14. The longest period of consecutive maximum temperatures $\geq 90^{\circ} \mathrm{F}$ is 32 days from July 13 through August 13, 1971.

The average seasonal number of days with maximum temperatures $\leq 32^{\circ} \mathrm{F}$ is 25. The earliest seasonal occurrence of a day with a maximum temperature $\leq 32^{\circ} \mathrm{F}$ has been October 30 (1971) and the latest March 11 (1950). The number of winter days with maximum temperatures $\leq 32^{\circ} \mathrm{F}$ has varied from 2 (winter of 1966-1967) to 58 (winter of 1984-1985). The greatest consecutive number of days with maximum temperatures $\leq 32^{\circ} \mathrm{F}$ is 29 days, from December 30,1984 through January 27, 1985. During the period from December 27, 1978 through February 4, 1979 (40 days), only 1 maximum temperature $>32^{\circ} \mathrm{F}$ occurred. The average maximum temperature for that period was $21^{\circ} \mathrm{F}$.

Table 3.6 lists the monthly and annual monthly maximum temperatures. 
IABLE 3.4. Days With Maximum Temperatures $\geq 104^{\circ} \mathrm{F}$

TEMP

DATE(S) OF OCCURRENCE

\begin{tabular}{|c|c|c|c|c|c|}
\hline $8 / 4 / 61$ & & & & & \\
\hline $8 / 9 / 71$ & & & & & \\
\hline $6 / 23 / 92$ & $7 / 31 / 71$ & & & & \\
\hline $\begin{array}{l}7 / 12 / 90 \\
7 / 17 / 60\end{array}$ & $7 / 20 / 79$ & $7 / 9 / 75$ & $8 / 8 / 72$ & $7 / 6 / 68$ & $7 / 18 / 60$ \\
\hline $8 / 14 / 92$ & $7 / 11 / 90$ & $7 / 19 / 79$ & $8 / 7 / 72$ & $8 / 10 / 71$ & $8 / 1 / 71$ \\
\hline $\begin{array}{l}6 / 24 / 92 \\
8 / 11 / 71 \\
8 / 18 / 67\end{array}$ & $\begin{array}{l}8 / 5 / 90 \\
7 / 27 / 71 \\
8 / 17 / 67\end{array}$ & $\begin{array}{l}7 / 18 / 79 \\
7 / 19 / 71 \\
8 / 16 / 67\end{array}$ & $\begin{array}{l}7 / 27 / 75 \\
7 / 28 / 68 \\
7 / 31 / 65\end{array}$ & $\begin{array}{l}7 / 5 / 75 \\
7 / 8 / 68 \\
7 / 13 / 61\end{array}$ & $\begin{array}{l}8 / 12 / 71 \\
7 / 4 / 68 \\
6 / 17 / 61\end{array}$ \\
\hline $\begin{array}{l}8 / 13 / 92 \\
7 / 28 / 82 \\
7 / 28 / 71 \\
7 / 28 / 58\end{array}$ & $\begin{array}{ll}8 / & 1 / 92 \\
8 / & 8 / 81 \\
7 / & 5 / 68 \\
7 / 14 / 55\end{array}$ & $\begin{array}{l}7 / 31 / 92 \\
7 / 17 / 79 \\
8 / 3 / 61\end{array}$ & $\begin{array}{l}6 / 25 / 92 \\
8 / 18 / 77 \\
7 / 22 / 59\end{array}$ & $\begin{array}{l}7 / 14 / 87 \\
8 / 8 / 71 \\
7 / 20 / 59\end{array}$ & $\begin{array}{l}7 / 29 / 82 \\
7 / 30 / 71 \\
7 / 19 / 59\end{array}$ \\
\hline $\begin{array}{l}8 / 18 / 92 \\
7 / 25 / 84 \\
7 / 10 / 75 \\
8 / 1 / 65 \\
7 / 9 / 52\end{array}$ & $\begin{array}{l}7 / 18 / 92 \\
7 / 22 / 80 \\
7 / 29 / 73 \\
7 / 12 / 64\end{array}$ & $\begin{array}{l}6 / 22 / 92 \\
8 / 9 / 78 \\
7 / 15 / 73 \\
7 / 24 / 62\end{array}$ & $\begin{array}{l}9 / 1 / 87 \\
7 / 23 / 78 \\
8 / 6 / 72 \\
6 / 16 / 61\end{array}$ & $\begin{array}{l}6 / 30 / 87 \\
8 / 17 / 77 \\
7 / 20 / 71 \\
6 / 22 / 58\end{array}$ & $\begin{array}{l}7 / 9 / 85 \\
8 / 13 / 77 \\
7 / 4 / 70 \\
7 / 19 / 56\end{array}$ \\
\hline $\begin{array}{l}7 / 17 / 92 \\
7 / 20 / 85 \\
8 / 4 / 78 \\
7 / 19 / 70 \\
8 / 30 / 67 \\
8 / 2 / 61 \\
7 / 30 / 52\end{array}$ & $\begin{array}{l}7 / 3 / 91 \\
7 / 27 / 82 \\
8 / 3 / 78 \\
7 / 16 / 70 \\
8 / 20 / 67 \\
8 / 11 / 60 \\
7 / 10 / 52\end{array}$ & $\begin{array}{l}7 / 22 / 90 \\
7 / 26 / 82 \\
7 / 4 / 75 \\
7 / 8 / 70 \\
8 / 19 / 67 \\
7 / 19 / 60\end{array}$ & $\begin{array}{l}7 / 15 / 90 \\
8 / 16 / 81 \\
7 / 21 / 71 \\
7 / 27 / 68 \\
8 / 15 / 67 \\
7 / 7 / 60\end{array}$ & $\begin{array}{l}7 / 26 / 88 \\
8 / 13 / 81 \\
7 / 18 / 71 \\
7 / 7 / 68 \\
8 / 13 / 67 \\
7 / 13 / 55\end{array}$ & $\begin{array}{l}8 / 9 / 87 \\
8 / 12 / 81 \\
8 / 23 / 70 \\
7 / 3 / 68 \\
7 / 3 / 67 \\
8 / 4 / 52\end{array}$ \\
\hline $\begin{array}{l}7 / 10 / 90 \\
7 / 29 / 85 \\
8 / 10 / 81 \\
8 / 20 / 77 \\
7 / 19 / 73 \\
7 / 16 / 71 \\
8 / 11 / 67 \\
6 / 18 / 61 \\
8 / 11 / 58 \\
7 / 21 / 56 \\
7 / 31 / 49\end{array}$ & $\begin{array}{l}7 / 25 / 88 \\
7 / 21 / 85 \\
8 / 7 / 81 \\
8 / 12 / 77 \\
6 / 22 / 73 \\
7 / 9 / 70 \\
7 / 12 / 67 \\
8 / 10 / 60 \\
7 / 17 / 58 \\
7 / 22 / 55 \\
7 / 30 / 49\end{array}$ & $\begin{array}{l}7 / 21 / 88 \\
7 / 8 / 85 \\
7 / 27 / 81 \\
7 / 30 / 74 \\
8 / 28 / 72 \\
7 / 3 / 70 \\
7 / 30 / 65 \\
7 / 16 / 60 \\
7 / 11 / 58 \\
8 / 15 / 53 \\
7 / 15 / 49\end{array}$ & $\begin{array}{l}7 / 20 / 88 \\
7 / 4 / 85 \\
7 / 4 / 81 \\
7 / 28 / 74 \\
8 / 9 / 72 \\
6 / 23 / 70 \\
7 / 25 / 62 \\
7 / 18 / 59 \\
8 / 22 / 56 \\
7 / 23 / 51 \\
6 / 29 / 48\end{array}$ & $\begin{array}{l}5 / 31 / 86 \\
8 / 7 / 82 \\
8 / 8 / 78 \\
8 / 1 / 73 \\
8 / 13 / 71 \\
6 / 21 / 70 \\
7 / 23 / 62 \\
8 / 25 / 58 \\
7 / 24 / 56 \\
7 / 17 / 51 \\
8 / 20 / 46\end{array}$ & $\begin{array}{l}5 / 30 / 86 \\
8 / 11 / 81 \\
7 / 25 / 78 \\
7 / 27 / 73 \\
8 / 7 / 71 \\
8 / 31 / 67 \\
8 / 14 / 61 \\
8 / 24 / 58 \\
7 / 23 / 56 \\
8 / 1 / 49\end{array}$ \\
\hline
\end{tabular}


TABLE 3.5. Record of Annual First and Last Dates with Maximum Temperatures $\geq 90^{\circ} \mathrm{F}$ and Minimum Temperatures $\leq 32^{\circ} \mathrm{F}$

\begin{tabular}{|c|c|c|c|c|c|}
\hline YEAR & $\begin{array}{l}\text { MAXIMUM } \\
\text { FIRST IN } \\
\text { SPRING } \\
\end{array}$ & $\begin{array}{l}\frac{290^{\circ} \mathrm{F}}{\text { LAST IN }} \\
\text { SUMMER }\end{array}$ & $\begin{array}{l}\text { MINIMUM } \\
\text { LAST IN } \\
\text { SPRING }\end{array}$ & $\begin{array}{c}P \leq 32^{\circ} \mathrm{F} \\
\text { FIRST IN } \\
\text { FALL } \\
\end{array}$ & $\begin{array}{c}\text { GROWING* } \\
\text { DAYS } \\
\end{array}$ \\
\hline 1945 & MAY 30 & SEP 14 & APR 10 & OCT 18 & 190 \\
\hline $\begin{array}{l}1946 \\
1947 \\
1948 \\
1949 \\
1950\end{array}$ & $\begin{array}{lr}\text { APR } & 25 \\
\text { MAY } & 6 \\
\text { MAY } & 26 \\
\text { MAY } & 8 \\
\text { MAY } & 26\end{array}$ & $\begin{array}{ll}\text { AUG } & 24 \\
\text { SEP } & 12 \\
\text { SEP } & 13 \\
\text { SEP } & 27 \\
\text { SEP } & 23\end{array}$ & $\begin{array}{lr}\text { APR } & 7 \\
\text { APR } & 7 \\
\text { MAY } & 2 \\
\text { MAY } & 3 \\
\text { APR } & 27\end{array}$ & $\begin{array}{lr}\text { OCT } & 11 \\
\text { NOV } & 4 \\
\text { OCT } & 17 \\
\text { OCT } & 8 \\
\text { NOV } & 8\end{array}$ & $\begin{array}{l}186 \\
210 \\
167 \\
157 \\
194\end{array}$ \\
\hline $\begin{array}{l}1951 \\
1952 \\
1953 \\
1954 \\
1955\end{array}$ & $\begin{array}{rr}\text { MAY } & 22 \\
\text { MAY } & 24 \\
\text { JUL } & 2 \\
\text { MAY } & 17 \\
\text { JUN } & 6\end{array}$ & $\begin{array}{ll}\text { SEP } & 19 \\
\text { SEP } & 26 \\
\text { SEP } & 15 \\
\text { SEP } & 10 \\
\text { SEP } & 10\end{array}$ & $\begin{array}{lr}\text { APR } & 21 \\
\text { APR } & 29 \\
\text { APR } & 15 \\
\text { MAY } & 1 \\
\text { MAY } & 14\end{array}$ & $\begin{array}{lr}\text { OCT } & 15 \\
\text { NOV } & 1 \\
\text { OCT } & 24 \\
\text { OCT } & 1 \\
\text { OCT } & 31\end{array}$ & $\begin{array}{l}176 \\
185 \\
191 \\
152 \\
169\end{array}$ \\
\hline $\begin{array}{l}1956 \\
1957 \\
1958 \\
1959 \\
1960\end{array}$ & $\begin{array}{ll}\text { MAY } & 16 \\
\text { MAY } & 29 \\
\text { MAY } & 18 \\
\text { MAY } & 13 \\
\text { MAY } & 10\end{array}$ & $\begin{array}{ll}\text { SEP } & 19 \\
\text { SEP } & 15 \\
\text { SEP } & 10 \\
\text { SEP } & 13 \\
\text { SEP } & 18\end{array}$ & $\begin{array}{lr}\text { APR } & 6 \\
\text { MAR } & 26 \\
\text { MAR } & 19 \\
\text { MAY } & 5 \\
\text { APR } & 21\end{array}$ & $\begin{array}{ll}\text { OCT } & 22 \\
\text { OCT } & 22 \\
\text { OCT } & 21 \\
\text { OCT } & 30 \\
\text { OCT } & 11\end{array}$ & $\begin{array}{l}198 \\
209 \\
215 \\
177 \\
172\end{array}$ \\
\hline $\begin{array}{l}1961 \\
1962 \\
1963 \\
1964 \\
1965\end{array}$ & $\begin{array}{lr}\text { MAY } & 25 \\
\text { JUN } & 8 \\
\text { MAY } & 20 \\
\text { JUN } & 23 \\
\text { MAY } & 28\end{array}$ & $\begin{array}{lr}\text { SEP } & 4 \\
\text { SEP } & 26 \\
\text { SEP } & 29 \\
\text { SEP } & 24 \\
\text { SEP } & 1\end{array}$ & $\begin{array}{lr}\text { APR } & 19 \\
\text { MAY } & 4 \\
\text { APR } & 16 \\
\text { APR } & 19 \\
\text { MAY } & 5\end{array}$ & $\begin{array}{ll}\text { OCT } & 20 \\
\text { NOV } & 12 \\
\text { OCT } & 25 \\
\text { OCT } & 16 \\
\text { OCT } & 16\end{array}$ & $\begin{array}{l}183 \\
191 \\
191 \\
179 \\
163\end{array}$ \\
\hline $\begin{array}{l}1966 \\
1967 \\
1968 \\
1969 \\
1970\end{array}$ & $\begin{array}{lr}\text { MAY } & 3 \\
\text { MAY } & 20 \\
\text { APR } & 29 \\
\text { MAY } & 9 \\
\text { MAY } & 16\end{array}$ & $\begin{array}{lr}\text { SEP } & 22 \\
\text { SEP } & 28 \\
\text { SEP } & 9 \\
\text { SEP } & 12 \\
\text { AUG } & 31\end{array}$ & $\begin{array}{ll}\text { APR } & 19 \\
\text { APR } & 28 \\
\text { APR } & 22 \\
\text { APR } & 26 \\
\text { MAY } & 11\end{array}$ & $\begin{array}{lr}\text { OCT } & 14 \\
\text { OCT } & 26 \\
\text { OCT } & 21 \\
\text { OCT } & 13 \\
\text { OCT } & 7\end{array}$ & $\begin{array}{l}177 \\
180 \\
181 \\
169 \\
148\end{array}$ \\
\hline $\begin{array}{l}1971 \\
1972 \\
1973 \\
1974 \\
1975\end{array}$ & $\begin{array}{ll}\text { MAY } & 11 \\
\text { MAY } & 13 \\
\text { MAY } & 13 \\
\text { JUN } & 10 \\
\text { MAY } & 30\end{array}$ & $\begin{array}{ll}\text { SEP } & 10 \\
\text { SEP } & 16 \\
\text { SEP } & 11 \\
\text { SEP } & 25 \\
\text { SEP } & 15\end{array}$ & $\begin{array}{lr}\text { APR } & 22 \\
\text { APR } & 30 \\
\text { APR } & 8 \\
\text { MAY } & 16 \\
\text { APR } & 29\end{array}$ & $\begin{array}{lr}\text { OCT } & 16 \\
\text { SEP } & 25 \\
\text { OCT } & 4 \\
\text { OCT } & 6 \\
\text { OCT } & 23\end{array}$ & $\begin{array}{l}176 \\
147 \\
178 \\
142 \\
176\end{array}$ \\
\hline $\begin{array}{l}1976 \\
1977 \\
1978 \\
1979 \\
1980\end{array}$ & $\begin{array}{lr}\text { MAY } & 16 \\
\text { APR } & 24 \\
\text { JUN } & 2 \\
\text { MAY } & 22 \\
\text { JUL } & 1\end{array}$ & $\begin{array}{lr}\text { SEP } & 29 \\
\text { AUG } & 22 \\
\text { SEP } & 3 \\
\text { SEP } & 20 \\
\text { SEP } & 6\end{array}$ & $\begin{array}{ll}\text { APR } & 23 \\
\text { APR } & 14 \\
\text { APR } & 23 \\
\text { APR } & 19 \\
\text { APR } & 11\end{array}$ & $\begin{array}{lr}\text { OCT } & 19 \\
\text { OCT } & 27 \\
\text { OCT } & 7 \\
\text { OCT } & 31 \\
\text { OCT } & 22\end{array}$ & $\begin{array}{l}178 \\
195 \\
166 \\
194 \\
193\end{array}$ \\
\hline $\begin{array}{l}1981 \\
1982 \\
1983 \\
1984 \\
1985\end{array}$ & $\begin{array}{ll}\text { APR } & 30 \\
\text { JUN } & 10 \\
\text { MAY } & 23 \\
\text { MAY } & 29 \\
\text { MAY } & 18\end{array}$ & $\begin{array}{lr}\text { SEP } & 18 \\
\text { SEP } & 8 \\
\text { AUG } & 17 \\
\text { SEP } & 18 \\
\text { AUG } & 29\end{array}$ & $\begin{array}{ll}\text { APR } 13 \\
\text { APR } 21 \\
\text { APR } 16 \\
\text { APR } 13 \\
\text { APR } 21\end{array}$ & $\begin{array}{lr}\text { OCT } & 14 \\
\text { OCT } & 18 \\
\text { OCT } & 11 \\
\text { OCT } & 14 \\
\text { OCT } & 7\end{array}$ & $\begin{array}{l}183 \\
179 \\
177 \\
183 \\
168\end{array}$ \\
\hline $\begin{array}{l}1986 \\
1987 \\
1988 \\
1989 \\
1990\end{array}$ & $\begin{array}{lr}\text { MAY } & 25 \\
\text { APR } & 27 \\
\text { MAY } & 11 \\
\text { JUN } & 1 \\
\text { MAY } & 5\end{array}$ & $\begin{array}{lr}\text { SEP } & 4 \\
\text { SEP } & 23 \\
\text { SEP } & 14 \\
\text { SEP } & 24 \\
\text { SEP } & 29\end{array}$ & $\begin{array}{lr}\text { APR } & 30 \\
\text { APR } & 20 \\
\text { APR } & 9 \\
\text { MAR } & 30 \\
\text { MAR } & 27\end{array}$ & $\begin{array}{rr}\text { NOV } & 9 \\
\text { OCT } & 16 \\
\text { OCT } & 27 \\
\text { OCT } & 29 \\
\text { OCT } & 17\end{array}$ & $\begin{array}{l}192 \\
178 \\
200 \\
212 \\
203\end{array}$ \\
\hline
\end{tabular}


TABLE 3.5. (contd)

\begin{tabular}{|c|c|c|c|c|c|}
\hline YEAR & $\begin{array}{l}\text { MAXIMUM } \\
\text { FIRST IN } \\
\text { SPRING }\end{array}$ & $\begin{array}{l}\frac{20^{\circ} \mathrm{F}}{\text { LAST IN }} \\
\text { SUMMER } \\
\end{array}$ & $\begin{array}{l}\text { MINIMUM } \\
\text { LAST IN } \\
\text { SPRING }\end{array}$ & $\begin{array}{c}P \leq 32^{\circ} \mathrm{F} \\
\begin{array}{c}\text { FIRST IN } \\
\text { FALL }\end{array} \\
\end{array}$ & $\begin{array}{l}\text { GROWING* } \\
\text { DAYS }\end{array}$ \\
\hline $\begin{array}{l}1991 \\
1992 \\
1993\end{array}$ & $\begin{array}{lr}\text { JUN } & 10 \\
\text { MAY } & 4 \\
\text { MAY } & 10\end{array}$ & $\begin{array}{ll}\text { SEP } & 26 \\
\text { SEP } & 3 \\
\text { SEP } & 29+\end{array}$ & $\begin{array}{ll}\text { APR } & 8 \\
\text { APR } & 8 \\
\text { APR } & 6\end{array}$ & $\begin{array}{ll}\text { OCT } & 22 \\
\text { OCT } & 15 \\
\text { OCT } 20\end{array}$ & $\begin{array}{l}196 \\
189 \\
196\end{array}$ \\
\hline $\begin{array}{l}\text { AVERAGE } \\
\text { NORMAL }\end{array}$ & $\begin{array}{ll}\text { MAY } & 21 \\
\text { MAY } & 21\end{array}$ & $\begin{array}{ll}\text { SEP } 14 \\
\text { SEP } 14\end{array}$ & $\begin{array}{l}\text { APR } 20 \\
\text { APR } 21\end{array}$ & $\begin{array}{ll}\text { OCT } & 19 \\
\text { OCT } & 18\end{array}$ & $\begin{array}{l}181 \\
179\end{array}$ \\
\hline
\end{tabular}

* Days between last freezing temperature in Spring, and first freezing temperature in Fall.

+ Also in previous years.

The earliest and latest dates in each column are underlined.

Only six days were recorded when the daily maximum temperature was $\leq 0^{\circ} \mathrm{F}$. These days are:

\begin{tabular}{|c|c|}
\hline DATE & MAX. TEMP. \\
\hline Jan. 31,1950 & $-2^{\circ} \mathrm{F}$ \\
\hline Feb. 1,1950 & $-3 \circ \mathrm{F}$ \\
\hline Feb. 2,1950 & $-3 \circ F$ \\
\hline
\end{tabular}

\begin{tabular}{cc} 
DATE & MAX. TEMP. \\
\hline Jan. 27, 1957 & 0०F \\
Dec. 29, 1968 & $-2 \circ \mathrm{F}$ \\
Dec. 30, 1968 & $-2 \circ \mathrm{F}$
\end{tabular}

\subsection{DAYS WITH MINIMUM TEMPERATURES $\leq 32^{\circ} \mathrm{F} O R \leq 0^{\circ} \mathrm{F}$}

The monthly and seasonal number of days with minimum temperatures at or below $32^{\circ} \mathrm{F}$ or $0^{\circ} \mathrm{F}$ are listed in Table 3.7 .

The seasonal average number of days with minimum temperatures $\leq 32^{\circ} \mathrm{F}$ is 106 ; however, the number ranges from 70 (winter of 1991-1992) to 143 (winter of 1984-1985). The greatest consecutive number of days with minimum temperatures of $\leq 32^{\circ} \mathrm{F}$ is 93 days, from November 9, 1978 through February 9, 1979.

The first autumn temperature $\leq 32^{\circ} \mathrm{F}$ has occurred as early as September 25 (1972), and as late as November 12 (1962). The average date is 0ctober 19 (Table 3.5). The last date in spring for minimum temperatures $\leq 32^{\circ} \mathrm{F}$ has varied from March 19 (1958) to May 16 (1974), with an average date of April 20. The average number of days between last freezing temperature in the spring and first freezing temperature in the fall is 181 days. 
TABLE 3.6. Monthly and Annual Maximum Temperatures $\left({ }^{\circ} \mathrm{F}\right)$

YEAR JAN FEB MAR APR MAY JUN JUL AUG SEP OCT NOV DEC ANNUAL

\begin{tabular}{|c|c|c|c|c|c|c|c|c|c|c|c|c|c|}
\hline 1945 & 61 & 65 & 72 & 76 & 90 & 98 & 104 & 105 & 93 & 84 & 72 & 57 & 105 \\
\hline $\begin{array}{l}1946 \\
1947 \\
1948 \\
1949 \\
1950\end{array}$ & $\begin{array}{l}57 \\
57 \\
60 \\
48 \\
50\end{array}$ & $\begin{array}{l}60 \\
68 \\
64 \\
56 \\
63\end{array}$ & $\begin{array}{l}76 \\
76 \\
73 \\
64 \\
64\end{array}$ & $\begin{array}{l}91 \\
88 \\
76 \\
84 \\
78\end{array}$ & $\begin{array}{r}89 \\
101 \\
91 \\
98 \\
90\end{array}$ & $\begin{array}{r}98 \\
97 \\
104 \\
102 \\
99\end{array}$ & $\begin{array}{r}105 \\
103 \\
98 \\
104 \\
102\end{array}$ & $\begin{array}{r}104 \\
98 \\
97 \\
104 \\
103\end{array}$ & $\begin{array}{r}89 \\
94 \\
98 \\
100 \\
102\end{array}$ & $\begin{array}{l}75 \\
83 \\
78 \\
74 \\
76\end{array}$ & $\begin{array}{l}64 \\
63 \\
57 \\
65 \\
62\end{array}$ & $\begin{array}{l}64 \\
55 \\
52 \\
60 \\
55\end{array}$ & $\begin{array}{l}105 \\
103 \\
104 \\
104 \\
103\end{array}$ \\
\hline $\begin{array}{l}1951 \\
1952 \\
1953 \\
1954 \\
1955\end{array}$ & $\begin{array}{l}55 \\
50 \\
63 \\
59 \\
50\end{array}$ & $\begin{array}{l}65 \\
55 \\
65 \\
63 \\
58\end{array}$ & $\begin{array}{l}65 \\
70 \\
69 \\
65 \\
63\end{array}$ & $\begin{array}{l}82 \\
89 \\
78 \\
83 \\
77\end{array}$ & $\begin{array}{l}94 \\
92 \\
88 \\
98 \\
86\end{array}$ & $\begin{array}{r}97 \\
94 \\
86 \\
94 \\
102\end{array}$ & $\begin{array}{l}104 \\
106 \\
103 \\
100 \\
107\end{array}$ & $\begin{array}{r}101 \\
105 \\
104 \\
99 \\
101\end{array}$ & $\begin{array}{r}97 \\
97 \\
97 \\
92 \\
101\end{array}$ & $\begin{array}{l}79 \\
85 \\
81 \\
73 \\
75\end{array}$ & $\begin{array}{l}60 \\
62 \\
65 \\
62 \\
66\end{array}$ & $\begin{array}{l}58 \\
54 \\
59 \\
54 \\
56\end{array}$ & $\begin{array}{l}104 \\
106 \\
104 \\
100 \\
107\end{array}$ \\
\hline $\begin{array}{l}1956 \\
1957 \\
1958 \\
1959 \\
1960\end{array}$ & $\begin{array}{l}59 \\
48 \\
60 \\
59 \\
55\end{array}$ & $\begin{array}{l}56 \\
65 \\
63 \\
60 \\
55\end{array}$ & $\begin{array}{l}64 \\
66 \\
63 \\
65 \\
83\end{array}$ & $\begin{array}{l}85 \\
89 \\
78 \\
79 \\
82\end{array}$ & $\begin{array}{r}96 \\
97 \\
101 \\
91 \\
90\end{array}$ & $\begin{array}{r}95 \\
100 \\
106 \\
97 \\
96\end{array}$ & $\begin{array}{l}106 \\
102 \\
107 \\
107 \\
110\end{array}$ & $\begin{array}{r}104 \\
96 \\
104 \\
103 \\
105\end{array}$ & $\begin{array}{l}94 \\
98 \\
97 \\
92 \\
94\end{array}$ & $\begin{array}{l}79 \\
73 \\
89 \\
77 \\
82\end{array}$ & $\begin{array}{l}64 \\
60 \\
67 \\
70 \\
63\end{array}$ & $\begin{array}{l}59 \\
59 \\
60 \\
64 \\
52\end{array}$ & $\begin{array}{l}106 \\
102 \\
107 \\
107 \\
110\end{array}$ \\
\hline $\begin{array}{l}1961 \\
1962 \\
1963 \\
1964 \\
1965\end{array}$ & $\begin{array}{l}60 \\
63 \\
56 \\
57 \\
60\end{array}$ & $\begin{array}{l}64 \\
60 \\
64 \\
60 \\
67\end{array}$ & $\begin{array}{l}68 \\
70 \\
70 \\
74 \\
71\end{array}$ & $\begin{array}{l}75 \\
85 \\
72 \\
73 \\
82\end{array}$ & $\begin{array}{l}94 \\
81 \\
93 \\
88 \\
91\end{array}$ & $\begin{array}{r}108 \\
98 \\
102 \\
95 \\
96\end{array}$ & $\begin{array}{r}108 \\
106 \\
96 \\
106 \\
108\end{array}$ & $\begin{array}{r}113 \\
100 \\
101 \\
97 \\
106\end{array}$ & $\begin{array}{l}90 \\
97 \\
98 \\
90 \\
91\end{array}$ & $\begin{array}{l}81 \\
76 \\
83 \\
80 \\
84\end{array}$ & $\begin{array}{l}58 \\
67 \\
61 \\
60 \\
64\end{array}$ & $\begin{array}{l}56 \\
56 \\
57 \\
57 \\
56\end{array}$ & $\begin{array}{l}113 \\
106 \\
102 \\
106 \\
108\end{array}$ \\
\hline $\begin{array}{l}1966 \\
1967 \\
1968 \\
1969 \\
1970\end{array}$ & $\begin{array}{l}56 \\
62 \\
66 \\
44 \\
56\end{array}$ & $\begin{array}{l}59 \\
67 \\
64 \\
46 \\
60\end{array}$ & $\begin{array}{l}78 \\
65 \\
68 \\
74 \\
67\end{array}$ & $\begin{array}{l}81 \\
71 \\
90 \\
80 \\
71\end{array}$ & $\begin{array}{r}100 \\
92 \\
90 \\
95 \\
92\end{array}$ & $\begin{array}{r}95 \\
101 \\
99 \\
103 \\
104\end{array}$ & $\begin{array}{l}100 \\
105 \\
110 \\
101 \\
106\end{array}$ & $\begin{array}{l}102 \\
108 \\
102 \\
102 \\
105\end{array}$ & $\begin{array}{l}99 \\
98 \\
97 \\
96 \\
89\end{array}$ & $\begin{array}{l}82 \\
78 \\
73 \\
74 \\
86\end{array}$ & $\begin{array}{l}64 \\
65 \\
60 \\
63 \\
63\end{array}$ & $\begin{array}{l}56 \\
62 \\
59 \\
54 \\
58\end{array}$ & $\begin{array}{l}102 \\
108 \\
110 \\
103 \\
106\end{array}$ \\
\hline $\begin{array}{l}1971 \\
1972 \\
1973 \\
1974 \\
1975\end{array}$ & $\begin{array}{l}72 \\
59 \\
51 \\
61 \\
56\end{array}$ & $\begin{array}{l}66 \\
68 \\
61 \\
59 \\
58\end{array}$ & $\begin{array}{l}65 \\
76 \\
68 \\
69 \\
65\end{array}$ & $\begin{array}{l}76 \\
78 \\
80 \\
77 \\
75\end{array}$ & $\begin{array}{l}92 \\
96 \\
98 \\
86 \\
90\end{array}$ & $\begin{array}{r}99 \\
98 \\
104 \\
103 \\
95\end{array}$ & $\begin{array}{l}111 \\
103 \\
106 \\
104 \\
110\end{array}$ & $\begin{array}{r}112 \\
110 \\
104 \\
103 \\
98\end{array}$ & $\begin{array}{l}91 \\
95 \\
98 \\
92 \\
96\end{array}$ & $\begin{array}{l}85 \\
83 \\
76 \\
80 \\
82\end{array}$ & $\begin{array}{l}64 \\
58 \\
62 \\
64 \\
75\end{array}$ & $\begin{array}{l}50 \\
65 \\
58 \\
60 \\
62\end{array}$ & $\begin{array}{l}112 \\
110 \\
106 \\
104 \\
110\end{array}$ \\
\hline $\begin{array}{l}1976 \\
1977 \\
1978 \\
1979 \\
1980\end{array}$ & $\begin{array}{l}59 \\
61 \\
51 \\
37 \\
51\end{array}$ & $\begin{array}{l}59 \\
70 \\
57 \\
62 \\
59\end{array}$ & $\begin{array}{l}69 \\
73 \\
74 \\
76 \\
68\end{array}$ & $\begin{array}{l}80 \\
94 \\
76 \\
83 \\
87\end{array}$ & $\begin{array}{l}90 \\
82 \\
87 \\
94 \\
87\end{array}$ & $\begin{array}{r}100 \\
100 \\
101 \\
102 \\
88\end{array}$ & $\begin{array}{l}102 \\
101 \\
106 \\
110 \\
106\end{array}$ & $\begin{array}{r}98 \\
107 \\
106 \\
101 \\
98\end{array}$ & $\begin{array}{r}102 \\
87 \\
90 \\
96 \\
95\end{array}$ & $\begin{array}{l}84 \\
75 \\
81 \\
84 \\
89\end{array}$ & $\begin{array}{l}71 \\
68 \\
69 \\
59 \\
65\end{array}$ & $\begin{array}{l}57 \\
64 \\
54 \\
59 \\
69\end{array}$ & $\begin{array}{l}102 \\
107 \\
106 \\
110 \\
106\end{array}$ \\
\hline $\begin{array}{l}1981 \\
1982 \\
1983 \\
1984 \\
1985\end{array}$ & $\begin{array}{l}55 \\
57 \\
61 \\
60 \\
36\end{array}$ & $\begin{array}{l}66 \\
68 \\
62 \\
62 \\
60\end{array}$ & $\begin{array}{l}70 \\
71 \\
64 \\
67 \\
68\end{array}$ & $\begin{array}{l}91 \\
81 \\
77 \\
79 \\
82\end{array}$ & $\begin{array}{r}89 \\
88 \\
103 \\
94 \\
95\end{array}$ & $\begin{array}{r}96 \\
102 \\
92 \\
96 \\
102\end{array}$ & $\begin{array}{l}104 \\
107 \\
100 \\
106 \\
106\end{array}$ & $\begin{array}{r}107 \\
104 \\
99 \\
103 \\
97\end{array}$ & $\begin{array}{l}99 \\
94 \\
87 \\
92 \\
86\end{array}$ & $\begin{array}{l}83 \\
75 \\
78 \\
81 \\
74\end{array}$ & $\begin{array}{l}65 \\
63 \\
67 \\
61 \\
66\end{array}$ & $\begin{array}{l}58 \\
62 \\
46 \\
52 \\
39\end{array}$ & $\begin{array}{l}107 \\
107 \\
103 \\
106 \\
106\end{array}$ \\
\hline $\begin{array}{l}1986 \\
1987 \\
1988 \\
1989 \\
1990\end{array}$ & $\begin{array}{l}57 \\
55 \\
54 \\
67 \\
60\end{array}$ & $\begin{array}{l}72 \\
60 \\
71 \\
53 \\
64\end{array}$ & $\begin{array}{l}74 \\
70 \\
71 \\
67 \\
76\end{array}$ & $\begin{array}{l}84 \\
93 \\
83 \\
80 \\
81\end{array}$ & $\begin{array}{r}104 \\
102 \\
94 \\
88 \\
94\end{array}$ & $\begin{array}{r}103 \\
106 \\
99 \\
97 \\
96\end{array}$ & $\begin{array}{r}99 \\
107 \\
105 \\
101 \\
110\end{array}$ & $\begin{array}{l}103 \\
105 \\
102 \\
103 \\
108\end{array}$ & $\begin{array}{r}95 \\
106 \\
102 \\
94 \\
98\end{array}$ & $\begin{array}{l}84 \\
87 \\
88 \\
80 \\
80\end{array}$ & $\begin{array}{l}63 \\
66 \\
69 \\
73 \\
68\end{array}$ & $\begin{array}{l}52 \\
59 \\
57 \\
58 \\
57\end{array}$ & $\begin{array}{l}104 \\
107 \\
105 \\
103 \\
110\end{array}$ \\
\hline $\begin{array}{l}1991 \\
1992 \\
1993\end{array}$ & $\begin{array}{l}59 \\
60 \\
56\end{array}$ & $\begin{array}{l}66 \\
62 \\
52\end{array}$ & $\begin{array}{l}69 \\
78 \\
66\end{array}$ & $\begin{array}{l}82 \\
85 \\
73\end{array}$ & $\begin{array}{r}83 \\
98 \\
100\end{array}$ & $\begin{array}{r}93 \\
111 \\
98\end{array}$ & $\begin{array}{r}105 \\
107 \\
96\end{array}$ & $\begin{array}{l}103 \\
109 \\
100\end{array}$ & $\begin{array}{l}95 \\
91 \\
98\end{array}$ & $\begin{array}{l}88 \\
87 \\
86\end{array}$ & $\begin{array}{l}65 \\
62 \\
65\end{array}$ & $\begin{array}{l}59 \\
53 \\
67\end{array}$ & $\begin{array}{l}105 \\
111 \\
100\end{array}$ \\
\hline
\end{tabular}


TABLE 3.7. Monthly and Seasonal Number of Days with Minimum Temperature $\left({ }^{\circ} \mathrm{F}\right)$ Below Certain Thresholds

MINIMUM TEMPERATURE $\leq 32^{\circ} \mathrm{F}$

SEASON SEP OCT NOV DEC JAN FEB MAR APR MAY TOTAL

\begin{tabular}{|c|c|c|c|c|c|c|c|c|c|c|c|c|c|c|c|}
\hline $1944-45$ & -- & -- & -- & $\cdots$ & 27 & 18 & 12 & 6 & 0 & 63 & - & -- & 0 & 0 & 0 \\
\hline $1945-46$ & 0 & 5 & 14 & 25 & 27 & 20 & 10 & 2 & 0 & 103 & 0 & 0 & 0 & 0 & 0 \\
\hline $1946-47$ & 0 & 8 & 23 & 24 & 27 & 19 & 6 & 1 & 0 & 108 & 0 & 0 & 1 & 0 & 1 \\
\hline $1947-48$ & 0 & 0 & 11 & 26 & 25 & 24 & 21 & 7 & 1 & 115 & 0 & 0 & 0 & 0 & 0 \\
\hline $1948-49$ & 0 & 8 & 15 & 30 & 31 & 25 & 11 & 4 & 1 & 125 & 0 & 2 & 9 & 0 & 11 \\
\hline $1949-50$ & 0 & 10 & 4 & 25 & 30 & 22 & 18 & 4 & 0 & 113 & 0 & 0 & 14 & 4 & $\underline{18}$ \\
\hline $1950-51$ & 0 & 0 & 13 & 19 & 26 & 25 & 21 & 2 & 0 & 106 & 0 & 0 & 0 & 0 & 0 \\
\hline $1951-52$ & 0 & 6 & 19 & 26 & 31 & 24 & 20 & 6 & 0 & 132 & 0 & 0 & 0 & 0 & 0 \\
\hline $1952-53$ & 0 & 0 & 25 & 19 & 9 & 15 & 12 & 4 & 0 & 84 & 0 & 0 & 0 & 0 & 0 \\
\hline $1953-54$ & 0 & 1 & 14 & 22 & 23 & 16 & 19 & 4 & 1 & 100 & 0 & 0 & 2 & 0 & 2 \\
\hline $1954-55$ & 0 & 6 & 6 & 26 & 30 & 25 & 22 & 10 & 1 & 126 & 0 & 0 & 0 & 0 & 0 \\
\hline $1955-56$ & 0 & 1 & 22 & 28 & 25 & 26 & 14 & 2 & 0 & 118 & 1 & 0 & 2 & 3 & 6 \\
\hline $1956-57$ & 0 & 3 & 18 & 21 & 31 & 23 & 11 & 0 & 0 & 107 & 0 & 0 & 12 & 1 & 13 \\
\hline $1957-58$ & 0 & 2 & 17 & 16 & 19 & 5 & 16 & 0 & 0 & 75 & 0 & 0 & 0 & 0 & 0 \\
\hline $1958-59$ & 0 & 4 & 14 & 24 & 25 & 24 & 14 & 2 & 1 & 108 & 0 & 0 & 2 & 0 & 2 \\
\hline $1959-60$ & 0 & 2 & 24 & 26 & 31 & 21 & 10 & 4 & 0 & 118 & 0 & 0 & 1 & 0 & 1 \\
\hline $1960-61$ & 0 & 4 & 15 & 29 & 23 & 10 & 7 & 5 & 0 & 93 & 0 & 0 & 0 & 0 & 0 \\
\hline $1961-62$ & 0 & 7 & 28 & 26 & 27 & 17 & 19 & 0 & 1 & 125 & 0 & 0 & 1 & 0 & 1 \\
\hline $1962-63$ & 0 & 0 & 13 & 17 & 27 & 17 & 11 & 2 & 0 & 87 & 0 & 0 & 2 & 0 & 2 \\
\hline $1963-64$ & 0 & 5 & 8 & 31 & 26 & 26 & 16 & 4 & 0 & 116 & 0 & 0 & 0 & 0 & 0 \\
\hline $1964-65$ & 0 & 5 & 13 & 29 & 25 & 18 & 19 & 1 & 1 & 111 & 0 & 2 & 0 & 0 & 2 \\
\hline $1965-66$ & 0 & 1 & 8 & 25 & 26 & 22 & 13 & 3 & 0 & 98 & 0 & 0 & 0 & 0 & 0 \\
\hline $1966-67$ & 0 & 3 & 11 & 18 & 20 & 17 & 18 & 9 & 0 & 96 & 0 & 0 & 0 & 0 & 0 \\
\hline $1967-68$ & 0 & 1 & 17 & 25 & 23 & 13 & 6 & 5 & 0 & 90 & 0 & 0 & 0 & 0 & 0 \\
\hline $1968-69$ & 0 & 4 & 8 & 24 & 30 & 25 & 15 & 1 & 0 & 107 & 0 & 4 & 5 & 1 & 10 \\
\hline $1969-70$ & 0 & 5 & 19 & 21 & 28 & 13 & 16 & 7 & 1 & 110 & 0 & 0 & 0 & 0 & 0 \\
\hline $1970-71$ & 0 & 8 & 14 & 28 & 24 & 19 & 20 & 7 & 0 & 120 & 0 & 0 & 0 & 0 & 0 \\
\hline $1971-72$ & 0 & 9 & 18 & 27 & 25 & 23 & 13 & 6 & 0 & 121 & 0 & 0 & 3 & 1 & 4 \\
\hline $1972-73$ & 3 & 6 & 13 & 23 & 30 & 23 & 10 & 4 & 0 & 112 & 0 & 7 & 1 & 0 & 8 \\
\hline $1973-74$ & 0 & 4 & 14 & 16 & 19 & 15 & 12 & 0 & 1 & 81 & 0 & 0 & 8 & 0 & 8 \\
\hline $1974-75$ & 0 & 4 & 12 & 26 & 29 & 24 & 17 & 7 & 0 & 119 & 0 & 0 & 0 & 0 & 0 \\
\hline $1975-76$ & 0 & 2 & 23 & 28 & 30 & 22 & 19 & 6 & 0 & 130 & 0 & 0 & 0 & 0 & 0 \\
\hline $1976-77$ & 0 & 8 & 17 & 30 & 30 & 19 & 14 & 1 & 0 & 119 & 0 & 0 & 0 & 0 & 0 \\
\hline $1977-78$ & 0 & 3 & 18 & 25 & 22 & 17 & 11 & 4 & 0 & 100 & 0 & 1 & 2 & 0 & 3 \\
\hline $1978-79$ & 0 & 7 & 26 & 31 & 31 & 21 & 13 & 2 & 0 & 131 & 0 & 3 & 8 & 2 & 13 \\
\hline $1979-80$ & 0 & 1 & 23 & 22 & 31 & 22 & 13 & 3 & 0 & 115 & 0 & 0 & 1 & 0 & 1 \\
\hline $1980-81$ & 0 & 4 & 16 & 16 & 17 & 17 & 11 & 6 & 0 & 87 & 0 & 0 & 0 & 0 & 0 \\
\hline $1981-82$ & 0 & 5 & 13 & 23 & 27 & 17 & 12 & 12 & 0 & 109 & 0 & 0 & 2 & 0 & 2 \\
\hline $1982-83$ & 0 & 4 & 21 & 26 & 20 & 13 & 4 & 9 & 0 & 97 & 0 & 0 & 0 & 0 & 0 \\
\hline $1983-84$ & 0 & 3 & 11 & 31 & 26 & 17 & 5 & 2 & 0 & 95 & 0 & 4 & 0 & 0 & 4 \\
\hline $1984-85$ & 0 & 14 & 20 & 31 & 31 & 25 & 20 & 2 & 0 & 143 & 0 & 4 & 0 & 3 & 7 \\
\hline $1985-86$ & 0 & 7 & 23 & 31 & 23 & 17 & 8 & 4 & 0 & 113 & 5 & 1 & 0 & 0 & 6 \\
\hline $1986-87$ & 0 & 0 & 11 & 29 & 25 & 17 & 9 & 2 & 0 & 93 & 0 & 0 & 0 & 0 & 0 \\
\hline $1987-88$ & 0 & 3 & 11 & 25 & 29 & 22 & 13 & 2 & 0 & 105 & 0 & 0 & 0 & 0 & 0 \\
\hline $1988-89$ & 0 & 1 & 12 & 23 & 24 & 25 & 11 & 0 & 0 & 96 & 0 & 0 & 0 & 4 & 4 \\
\hline $1989-90$ & 0 & 2 & 11 & 25 & 18 & 20 & 11 & 0 & 0 & 87 & 0 & 0 & 0 & 0 & 0 \\
\hline
\end{tabular}


IABLE 3.7. (contd)

\begin{tabular}{|c|c|c|c|c|c|c|c|c|c|c|c|c|c|c|c|}
\hline \multirow[b]{2}{*}{ SEASON } & \multicolumn{10}{|c|}{ MINIMUM TEMPERATURE $\leq 32^{\circ} \mathrm{F}$} & \multicolumn{5}{|c|}{ MINIMUM TEMPERATURE $\leq 0^{\circ} \mathrm{F}$} \\
\hline & SEP & OCT & NOV & DEC & JAN & FEB & MAR & APR & MAY & TOTAL & NOV & DEC & JAN & FEB & TOTAL \\
\hline $1990-91$ & 0 & 2 & 11 & 27 & 27 & 14 & 14 & 2 & 0 & 97 & 0 & 8 & 0 & 0 & 8 \\
\hline $1991-92$ & 0 & 6 & 8 & 18 & 22 & 11 & 3 & 2 & 0 & 70 & 0 & 0 & 0 & 0 & $0^{\star \star}$ \\
\hline $1992-93$ & 0 & 1 & 9 & 29 & 27 & 23 & 10 & 1 & 0 & $1 \overline{00}$ & 0 & 0 & 2 & 0 & $\sqrt{2}$ \\
\hline $1993-94$ & 0 & 5 & 26 & 22 & -- & -- & -- & -- & -- & 53 & 1 & 0 & - & -- & 1 \\
\hline AVERAGE & 0 & 4 & 16 & 25 & 26 & 19 & 13 & 4 & 0 & 106 & 0 & 1 & 2 & 0 & 3 \\
\hline *NORMAL & 0 & 4 & 15 & 25 & 26 & 19 & 13 & 4 & 0 & 107 & 0 & 1 & 1 & 0 & 3 \\
\hline
\end{tabular}

\footnotetext{
* Normals are averages for the period from 1961-1990.

* Greatest and least seasonal totals are underlined.
}

The daily minimum temperature is $\leq 0 \circ \mathrm{F}$ (Table 3.7 ), an average of three days per winter season; however, nearly half of all winters have no minimum temperatures in this category. The most of those days in any season was 18 (winter of 1949-1950) and the least of those days was 0 (as recently as the winter of 1991-1992). The greatest number of consecutive days with minimum temperatures $\leq 00 \mathrm{~F}$ is 11 days, from January 25 through February 4, 1950. During this same period, four consecutive days had minimum temperatures $\leq-20^{\circ} \mathrm{F}$. Table 3.8 ists all days with minimum temperatures $\leq 0^{\circ} \mathrm{F}$. Table 3.9 Tists monthly and annual minimum temperatures.

\subsection{MONTHLY EXTREME DAILY MAXIMUM AND MINIMUM TEMPERATURES}

Monthly extreme daily maximum and minimum temperatures are presented in Table 3.10. Note that a greater temperature range exists during the winter months than in summer. February temperatures have ranged from $72^{\circ} \mathrm{F}$ to $-23^{\circ} \mathrm{F}$, a range of $95^{\circ}$, while July temperatures have ranged from $111^{\circ} \mathrm{F}$ to $39^{\circ} \mathrm{F}$, a range of $82^{\circ}$. August also has an $82^{\circ}$ range, from $113^{\circ} \mathrm{F}$ to $41^{\circ} \mathrm{F}$.

\subsection{NORMAL AND EXTREME DAILY TEMPERATURES}

Table 3.11 lists the normal and extreme daily maximum and minimum temperatures. Climatological normals are computed every 10 years, and are based upon a 30-year period, ending with the first year of each new decade. 
IABLE 3.8. Days With Minimum Temperatures $\leq 0^{\circ} \mathrm{F}$

\begin{tabular}{|c|c|c|c|c|c|c|}
\hline IEMP & & & $\operatorname{AIE}(S)$ OF 0 & SURRENCE & & \\
\hline-23 & $2 / 3 / 50$ & $2 / 1 / 50$ & & & & \\
\hline-22 & $1 / 26 / 57$ & & & & & \\
\hline-21 & $1 / 27 / 57$ & $2 / 2 / 5 . J$ & $1 / 31 / 50$ & & & \\
\hline .18 & $1 / 29 / 50$ & & & & & \\
\hline-14 & $12 / 30 / 68$ & $1 / 29 / 57$ & $1 / 28 / 57$ & & & \\
\hline-13 & $11 / 23 / 85$ & $12 / 22 / 83$ & $1 / 9 / 74$ & $12 / 16 / 64$ & $1 / 20 / 50$ & \\
\hline-12 & $12 / 22 / 90$ & $11 / 24 / 85$ & $2 / 1 / 79$ & $12 / 17 / 64$ & $1 / 25 / 57$ & \\
\hline-11 & $1 / 1 / 79$ & $1 / 17 / 50$ & $1 / 14 / 50$ & $1 / 25 / 49$ & & \\
\hline-10 & $\begin{array}{r}12 / 29 / 90 \\
2 / 2 / 56\end{array}$ & $\begin{array}{r}12 / 21 / 90 \\
2 / 1 / 56\end{array}$ & $2 / 2 / 79$ & $12 / 30 / 78$ & $1 / 6 / 74$ & $12 / 29 / 68$ \\
\hline-9 & $12 / 23 / 83$ & $1 / 6 / 79$ & $12 / 31 / 78$ & $1 / 2 / 78$ & $1 / 8 / 74$ & \\
\hline-8 & $\begin{array}{r}12 / 1 / 85 \\
1 / 16 / 50\end{array}$ & $1 / 6 / 82$ & $1 / 7 / 74$ & $12 / 10 / 72$ & $1 / 23 / 69$ & $1 / 30 / 57$ \\
\hline-7 & $1 / 7 / 79$ & $1 / 31 / 56$ & $1 / 28 / 50$ & $1 / 5 / 50$ & & \\
\hline-6 & $\begin{array}{r}11 / 22 / 85 \\
1 / 28 / 69\end{array}$ & $\begin{array}{l}1 / 31 / 79 \\
1 / 18 / 57\end{array}$ & $\begin{array}{l}1 / 5 / 74 \\
1 / 20 / 54\end{array}$ & $\begin{array}{r}12 / 13 / 72 \\
1 / 4 / 50\end{array}$ & $\begin{array}{r}12 / 8 / 72 \\
1 / 24 / 49\end{array}$ & $\begin{array}{l}1 / 29 / 69 \\
1 / 11 / 49\end{array}$ \\
\hline-5 & $\begin{array}{l}2 / 5 / 89 \\
1 / 15 / 50\end{array}$ & $2 / 4 / 85$ & $1 / 1 / 78$ & $1 / 10 / 74$ & $12 / 12 / 72$ & $12 / 9 / 72$ \\
\hline-4 & $\begin{array}{l}1 / 13 / 93 \\
1 / 11 / 74 \\
1 / 12 / 49\end{array}$ & $\begin{array}{l}12 / 23 / 90 \\
12 / 11 / 72\end{array}$ & $\begin{array}{l}2 / 4 / 89 \\
1 / 28 / 72\end{array}$ & $\begin{array}{c}12 / 19 / 84 \\
1 / 12 / 63\end{array}$ & $\begin{array}{r}12 / 21 / 83 \\
1 / 28 / 49\end{array}$ & $\begin{array}{l}1 / 27 / 79 \\
1 / 13 / 49\end{array}$ \\
\hline-3 & $\begin{array}{r}2 / 6 / 89 \\
12 / 29 / 78 \\
1 / 11 / 63\end{array}$ & $\begin{array}{c}11 / 25 / 85 \\
12 / 31 / 77 \\
1 / 17 / 57\end{array}$ & $\begin{array}{l}213 / 85 \\
1 / 31 / 69\end{array}$ & $\begin{array}{r}12 / 18 / 84 \\
1 / 30 / 69\end{array}$ & $\begin{array}{r}1 / 10 / 80 \\
12 / 31 / 68\end{array}$ & $\begin{array}{r}1 / 8 / 79 \\
12 / 28 / 68\end{array}$ \\
\hline-2 & $\begin{array}{r}12 / 31 / 90 \\
1 / 4 / 74 \\
1 / 10 / 49\end{array}$ & $\begin{array}{l}12 / 30 / 90 \\
12 / 14 / 72 \\
12 / 27 / 48\end{array}$ & $\begin{array}{r}12 / 20 / 90 \\
1 / 22 / 62\end{array}$ & $\begin{array}{c}12 / 21 / 84 \\
1 / 31 / 57\end{array}$ & $\begin{array}{c}12 / 20 / 84 \\
1 / 19 / 57\end{array}$ & $\begin{array}{l}1 / 5 / 79 \\
1 / 20 / 49\end{array}$ \\
\hline-1 & $\begin{array}{r}11 / 24 / 93 \\
1 / 18 / 60 \\
11 / 14 / 55\end{array}$ & $\begin{array}{r}11 / 26 / 85 \\
1 / 4 / 59 \\
2 / 4 / 50\end{array}$ & $\begin{array}{l}1 / 8 / 73 \\
2 / 2 / 57 \\
1 / 25 / 50\end{array}$ & $\begin{array}{l}2 / 3 / 72 \\
1 / 16 / 57 \\
1 / 13 / 50\end{array}$ & $\begin{array}{l}1 / 26 / 72 \\
2 / 3 / 56\end{array}$ & $\begin{array}{l}2 / 1 / 69 \\
1 / 30 / 56\end{array}$ \\
\hline 0 & $\begin{array}{l}1 / 11 / 93 \\
1 / 28 / 79 \\
1 / 26 / 50\end{array}$ & $\begin{array}{r}12 / 24 / 90 \\
1 / 27 / 72 \\
1 / 4 / 49\end{array}$ & $\begin{array}{c}2 / 2 / 89 \\
1 / 3 / 59 \\
12 / 26 / 48\end{array}$ & $\begin{array}{l}2 / 6 / 85 \\
1 / 24 / 57 \\
1 / 15 / 47\end{array}$ & $\begin{array}{r}12 / 27 / 83 \\
1 / 21 / 54\end{array}$ & $\begin{array}{l}1 / 7 / 82 \\
1 / 27 / 50\end{array}$ \\
\hline
\end{tabular}


TABLE 3.9. Monthly and Annual Minimum Temperatures ( ${ }^{\circ} \mathrm{F}$ )

\begin{tabular}{|c|c|c|c|c|c|c|c|c|c|c|c|c|c|}
\hline YEAR & JAN & FEB & MAR & APR & MAY & JUN & JUL & AUG & $\underline{\text { SEP }}$ & $\underline{O C T}$ & NOV & DEC & ANNUAL \\
\hline 1945 & 21 & 14 & 10 & 28 & 38 & 46 & 53 & 47 & 35 & 26 & 16 & 13 & 10 \\
\hline $\begin{array}{l}1946 \\
1947 \\
1948 \\
1949 \\
1950\end{array}$ & $\begin{array}{r}18 \\
0 \\
14 \\
-11 \\
-21\end{array}$ & $\begin{array}{r}18 \\
11 \\
1 \\
3 \\
-23\end{array}$ & $\begin{array}{l}25 \\
23 \\
13 \\
27 \\
20\end{array}$ & $\begin{array}{l}30 \\
32 \\
28 \\
30 \\
27\end{array}$ & $\begin{array}{l}33 \\
42 \\
32 \\
31 \\
38\end{array}$ & $\begin{array}{l}44 \\
45 \\
51 \\
42 \\
44\end{array}$ & $\begin{array}{l}50 \\
53 \\
49 \\
49 \\
49\end{array}$ & $\begin{array}{l}49 \\
50 \\
47 \\
47 \\
51\end{array}$ & $\begin{array}{l}35 \\
40 \\
34 \\
38 \\
38\end{array}$ & $\begin{array}{l}21 \\
34 \\
22 \\
23 \\
34\end{array}$ & $\begin{array}{l}16 \\
22 \\
20 \\
28 \\
21\end{array}$ & $\begin{array}{r}6 \\
16 \\
-2 \\
10 \\
22\end{array}$ & $\begin{array}{r}6 \\
0 \\
-2 \\
-11 \\
-23\end{array}$ \\
\hline $\begin{array}{l}1951 \\
1952 \\
1953 \\
1954 \\
1955\end{array}$ & $\begin{array}{r}6 \\
5 \\
24 \\
-6 \\
18\end{array}$ & $\begin{array}{l}18 \\
16 \\
20 \\
17 \\
15\end{array}$ & $\begin{array}{r}22 \\
24 \\
23 \\
18 \\
5\end{array}$ & $\begin{array}{l}26 \\
27 \\
27 \\
26 \\
26\end{array}$ & $\begin{array}{l}37 \\
37 \\
36 \\
28 \\
31\end{array}$ & $\begin{array}{l}41 \\
42 \\
40 \\
41 \\
42\end{array}$ & $\begin{array}{l}51 \\
49 \\
52 \\
45 \\
43\end{array}$ & $\begin{array}{l}47 \\
46 \\
51 \\
48 \\
48\end{array}$ & $\begin{array}{l}39 \\
42 \\
37 \\
36 \\
37\end{array}$ & $\begin{array}{l}27 \\
34 \\
30 \\
26 \\
32\end{array}$ & $\begin{array}{r}23 \\
7 \\
24 \\
23 \\
-1\end{array}$ & $\begin{array}{r}4 \\
17 \\
20 \\
14 \\
9\end{array}$ & $\begin{array}{r}4 \\
5 \\
20 \\
-6 \\
-1\end{array}$ \\
\hline $\begin{array}{l}1956 \\
1957 \\
1958 \\
1959 \\
1960\end{array}$ & $\begin{array}{r}-7 \\
-22 \\
16 \\
-1 \\
-1\end{array}$ & $\begin{array}{r}-10 \\
-1 \\
29 \\
19 \\
10\end{array}$ & $\begin{array}{l}15 \\
28 \\
23 \\
25 \\
13\end{array}$ & $\begin{array}{l}28 \\
34 \\
34 \\
30 \\
30\end{array}$ & $\begin{array}{l}38 \\
48 \\
38 \\
30 \\
33\end{array}$ & $\begin{array}{l}40 \\
48 \\
47 \\
41 \\
46\end{array}$ & $\begin{array}{l}54 \\
51 \\
49 \\
49 \\
52\end{array}$ & $\begin{array}{l}49 \\
52 \\
53 \\
49 \\
41\end{array}$ & $\begin{array}{l}39 \\
36 \\
34 \\
41 \\
40\end{array}$ & $\begin{array}{l}31 \\
32 \\
30 \\
26 \\
30\end{array}$ & $\begin{array}{r}15 \\
20 \\
9 \\
6 \\
22\end{array}$ & $\begin{array}{r}2 \\
23 \\
21 \\
14 \\
14\end{array}$ & $\begin{array}{r}-10 \\
-22 \\
9 \\
-1 \\
-1\end{array}$ \\
\hline $\begin{array}{l}1961 \\
1962 \\
1963 \\
1964 \\
1965\end{array}$ & $\begin{array}{l}16 \\
-2 \\
-4 \\
15 \\
10\end{array}$ & $\begin{array}{r}27 \\
7 \\
8 \\
19 \\
18\end{array}$ & $\begin{array}{l}25 \\
15 \\
22 \\
15 \\
14\end{array}$ & $\begin{array}{l}31 \\
33 \\
28 \\
30 \\
32\end{array}$ & $\begin{array}{l}38 \\
31 \\
36 \\
35 \\
32\end{array}$ & $\begin{array}{l}44 \\
37 \\
45 \\
45 \\
48\end{array}$ & $\begin{array}{l}50 \\
42 \\
49 \\
50 \\
50\end{array}$ & $\begin{array}{l}56 \\
49 \\
49 \\
44 \\
42\end{array}$ & $\begin{array}{l}36 \\
40 \\
45 \\
39 \\
33\end{array}$ & $\begin{array}{l}26 \\
34 \\
23 \\
30 \\
30\end{array}$ & $\begin{array}{l}10 \\
16 \\
17 \\
20 \\
26\end{array}$ & $\begin{array}{r}3 \\
16 \\
7 \\
-13 \\
10\end{array}$ & $\begin{array}{r}3 \\
-2 \\
-4 \\
-13 \\
10\end{array}$ \\
\hline $\begin{array}{l}1966 \\
1967 \\
1968 \\
1969 \\
1970\end{array}$ & $\begin{array}{r}17 \\
23 \\
10 \\
-8 \\
8\end{array}$ & $\begin{array}{l}19 \\
20 \\
15 \\
-1 \\
21\end{array}$ & $\begin{array}{l}19 \\
20 \\
25 \\
22 \\
24\end{array}$ & $\begin{array}{l}26 \\
27 \\
23 \\
31 \\
26\end{array}$ & $\begin{array}{l}37 \\
34 \\
33 \\
38 \\
30\end{array}$ & $\begin{array}{l}38 \\
47 \\
42 \\
52 \\
46\end{array}$ & $\begin{array}{l}48 \\
52 \\
51 \\
53 \\
50\end{array}$ & $\begin{array}{l}50 \\
56 \\
47 \\
45 \\
52\end{array}$ & $\begin{array}{l}43 \\
43 \\
39 \\
41 \\
34\end{array}$ & $\begin{array}{l}29 \\
30 \\
30 \\
29 \\
23\end{array}$ & $\begin{array}{l}22 \\
17 \\
23 \\
19 \\
11\end{array}$ & $\begin{array}{r}22 \\
6 \\
-14 \\
19 \\
8\end{array}$ & $\begin{array}{r}17 \\
6 \\
-14 \\
-8 \\
8\end{array}$ \\
\hline $\begin{array}{l}1971 \\
1972 \\
1973 \\
1974 \\
1975\end{array}$ & $\begin{array}{r}8 \\
-4 \\
-1 \\
-13 \\
14\end{array}$ & $\begin{array}{l}15 \\
-1 \\
21 \\
23 \\
10\end{array}$ & $\begin{array}{l}15 \\
24 \\
26 \\
21 \\
19\end{array}$ & $\begin{array}{l}27 \\
26 \\
27 \\
33 \\
21\end{array}$ & $\begin{array}{l}36 \\
36 \\
34 \\
32 \\
33\end{array}$ & $\begin{array}{l}44 \\
45 \\
45 \\
41 \\
38\end{array}$ & $\begin{array}{l}44 \\
50 \\
46 \\
48 \\
53\end{array}$ & $\begin{array}{l}51 \\
49 \\
46 \\
48 \\
46\end{array}$ & $\begin{array}{l}38 \\
30 \\
43 \\
40 \\
44\end{array}$ & $\begin{array}{l}13 \\
20 \\
31 \\
29 \\
26\end{array}$ & $\begin{array}{l}21 \\
24 \\
16 \\
24 \\
15\end{array}$ & $\begin{array}{r}5 \\
-8 \\
14 \\
17 \\
14\end{array}$ & $\begin{array}{r}5 \\
-8 \\
-1 \\
-13 \\
10\end{array}$ \\
\hline $\begin{array}{l}1976 \\
1977 \\
1978 \\
1979 \\
1980\end{array}$ & $\begin{array}{r}16 \\
4 \\
-9 \\
-11 \\
-3\end{array}$ & $\begin{array}{r}10 \\
21 \\
17 \\
-12 \\
19\end{array}$ & $\begin{array}{l}11 \\
24 \\
25 \\
20 \\
25\end{array}$ & $\begin{array}{l}25 \\
31 \\
30 \\
29 \\
28\end{array}$ & $\begin{array}{l}35 \\
34 \\
37 \\
38 \\
38\end{array}$ & $\begin{array}{l}37 \\
39 \\
44 \\
45 \\
40\end{array}$ & $\begin{array}{l}47 \\
49 \\
50 \\
39 \\
47\end{array}$ & $\begin{array}{l}44 \\
48 \\
47 \\
53 \\
42\end{array}$ & $\begin{array}{l}42 \\
36 \\
41 \\
42 \\
41\end{array}$ & $\begin{array}{l}28 \\
28 \\
21 \\
32 \\
30\end{array}$ & $\begin{array}{r}13 \\
9 \\
7 \\
13 \\
18\end{array}$ & $\begin{array}{r}12 \\
-3 \\
-10 \\
19 \\
9\end{array}$ & $\begin{array}{r}10 \\
-3 \\
-10 \\
-12 \\
-3\end{array}$ \\
\hline $\begin{array}{l}1981 \\
1982 \\
1983 \\
1984 \\
1985\end{array}$ & $\begin{array}{r}23 \\
-8 \\
12 \\
10 \\
5\end{array}$ & $\begin{array}{r}8 \\
9 \\
15 \\
24 \\
-5\end{array}$ & $\begin{array}{l}24 \\
24 \\
29 \\
25 \\
21\end{array}$ & $\begin{array}{l}24 \\
24 \\
27 \\
30 \\
26\end{array}$ & $\begin{array}{l}35 \\
33 \\
37 \\
33 \\
33\end{array}$ & $\begin{array}{l}40 \\
47 \\
40 \\
37 \\
44\end{array}$ & $\begin{array}{l}45 \\
45 \\
49 \\
51 \\
56\end{array}$ & $\begin{array}{l}48 \\
51 \\
50 \\
47 \\
46\end{array}$ & $\begin{array}{l}34 \\
41 \\
35 \\
36 \\
33\end{array}$ & $\begin{array}{l}27 \\
26 \\
29 \\
12 \\
26\end{array}$ & $\begin{array}{r}19 \\
18 \\
22 \\
25 \\
-13\end{array}$ & $\begin{array}{r}8 \\
13 \\
-13 \\
-4 \\
-8\end{array}$ & $\begin{array}{r}8 \\
-8 \\
-13 \\
-4 \\
-13\end{array}$ \\
\hline $\begin{array}{l}1986 \\
1987 \\
1988 \\
1989 \\
1990\end{array}$ & $\begin{array}{r}12 \\
9 \\
14 \\
15 \\
22\end{array}$ & $\begin{array}{r}15 \\
18 \\
9 \\
-5 \\
9\end{array}$ & $\begin{array}{l}29 \\
24 \\
24 \\
14 \\
24\end{array}$ & $\begin{array}{l}28 \\
30 \\
31 \\
35 \\
37\end{array}$ & $\begin{array}{l}37 \\
38 \\
35 \\
39 \\
39\end{array}$ & $\begin{array}{l}43 \\
43 \\
42 \\
46 \\
47\end{array}$ & $\begin{array}{l}48 \\
49 \\
47 \\
49 \\
46\end{array}$ & $\begin{array}{l}54 \\
51 \\
52 \\
52 \\
52\end{array}$ & $\begin{array}{l}38 \\
41 \\
38 \\
44 \\
48\end{array}$ & $\begin{array}{l}33 \\
31 \\
32 \\
27 \\
31\end{array}$ & $\begin{array}{l}16 \\
17 \\
28 \\
21 \\
28\end{array}$ & $\begin{array}{r}18 \\
9 \\
8 \\
19 \\
-12\end{array}$ & $\begin{array}{r}12 \\
9 \\
8 \\
-5 \\
-12\end{array}$ \\
\hline $\begin{array}{l}1991 \\
1992 \\
1993\end{array}$ & $\begin{array}{r}5 \\
19 \\
-4\end{array}$ & $\begin{array}{r}26 \\
22 \\
3\end{array}$ & $\begin{array}{l}22 \\
32 \\
17\end{array}$ & $\begin{array}{l}31 \\
27 \\
32\end{array}$ & $\begin{array}{l}38 \\
37 \\
35\end{array}$ & $\begin{array}{l}44 \\
49 \\
46\end{array}$ & $\begin{array}{l}55 \\
54 \\
50\end{array}$ & $\begin{array}{l}47 \\
43 \\
43\end{array}$ & $\begin{array}{l}42 \\
40 \\
37\end{array}$ & $\begin{array}{l}23 \\
30 \\
29\end{array}$ & $\begin{array}{l}23 \\
17 \\
-1\end{array}$ & $\begin{array}{l}20 \\
12 \\
21\end{array}$ & $\begin{array}{r}5 \\
12 \\
4\end{array}$ \\
\hline
\end{tabular}


TABLE 3.10. Monthly Normal Temperatures ( $\left.{ }^{\circ} \mathrm{F}\right)$ and Monthly Extremes of Maximum and Minimum Temperatures $\left({ }^{\circ} \mathrm{F}\right)$

NORMAL $(1961-1990)$

DAILY EXTREME

MAXIMUM

MONTH

January

February

March

Apri 1

May

June

July

August

September

October

November

December

MAX

38.4

47.3

28

$57.3 \quad 33.9$

$33.9 \quad 45.7$

45.7

$66.0 \quad 39.6$

$\begin{array}{llll}52.7 & 94 & 24 & 1977\end{array}$

$75.2 \quad 47.4$

$\begin{array}{llll}61.2 & 104 & 31 & 1986 \\ & 104 & 30 & 1986\end{array}$

$83.9 \quad 55.3$

69.7

$111 \quad 23 \quad 1992$

$90.2 \quad 59.9 \quad 75.1 \quad 11$

$80.1 \quad 51.2 \quad 65.6 \quad 106$

41961

$65.7 \quad 40$

52.

$\begin{array}{lll}89 & 4 & 1980 \\ 89 & 3 & 1958\end{array}$

$\begin{array}{llllll}38.1 & 24.7 & 31.2 & 69 & 26 & 1980\end{array}$ $\begin{array}{lll}41 & 6 & 1945\end{array}$

$\begin{array}{lll}51 & 11 \quad 1967\end{array}$

19
1986

$80 \quad 24 \quad 1992$

$\begin{array}{lll}37 & 3 & 1962\end{array}$

$\begin{array}{lll}37 & 2 & 1976 \\ 37 & 1 & 1984\end{array}$

$\begin{array}{lllllllll}91.4 & 60.9 & 76.1 & 111 & 31 & 1971 & 59 & 2 & 1966\end{array}$

$\begin{array}{lll}79 & 18 & 1960\end{array}$

$\begin{array}{lll}39 & 2 & 1979\end{array}$

$67 \quad 20 \quad 1959$

$81 \quad 4 \quad 1961$

$\begin{array}{lll}32 & 30 \quad 1971\end{array}$

$\begin{array}{rrr}60 & 24 & 1945 \\ 60 & 15 & 1988 \\ 60 & 2 & 1988\end{array}$

$\begin{array}{lllllllll}48.7 & 31.9 & 40.2 & 75 & 3 & 1975 & 6 & 24 & 1985\end{array}$

$\begin{array}{llllll}60 & 9 & 1989 & -13 & 23 & 1985\end{array}$ $\begin{array}{llllll}56 & 2 & 1975 & -14 & 30 & 1968\end{array}$ $\begin{array}{lll}-2 & 30 & 1968\end{array}$ $\begin{array}{lll}-2 & 29 & 1968\end{array}$ 
IABLE 3.11. Normal and Extreme Daily Maximum and Minimum Temperatures $\left({ }^{\circ} \mathrm{F}\right)$

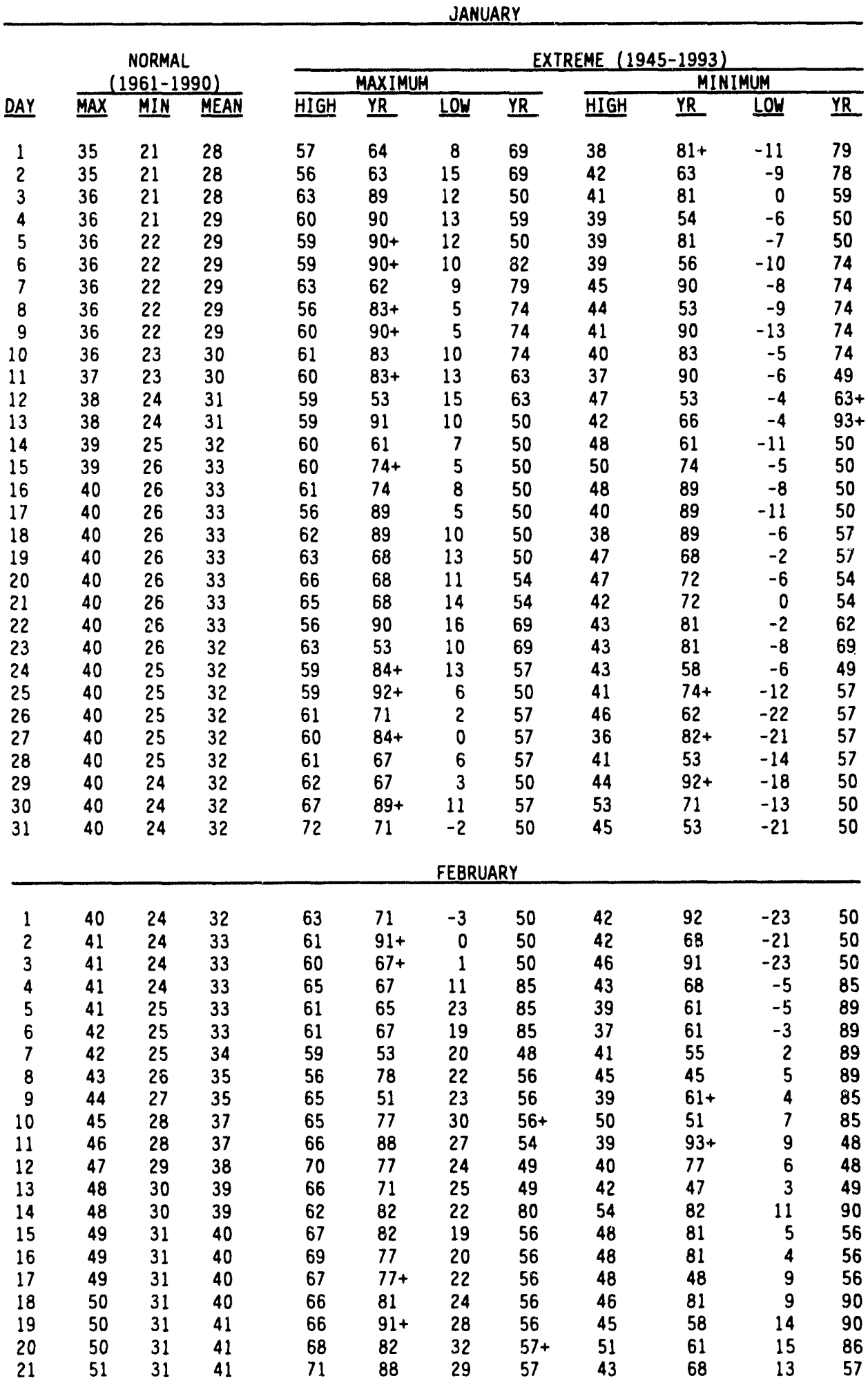


TABLE 3.11. (contd)

NORMAL
DAY MAX MIN MEAN

FEBRUARY (contd)

$\begin{array}{rrllllllllll}22 & 51 & 31 & 41 & 62 & 47 & 29 & 57 & 42 & 58 & 11 & 93 \\ 23 & 51 & 31 & 41 & 68 & 47 & 34 & 93+ & 43 & 83+ & 19 & 93 \\ 24 & 52 & 31 & 41 & 72 & 86 & 32 & 62 & 60 & 86 & 11 & 93+ \\ 25 & 52 & 31 & 42 & 72 & 86 & 28 & 93 & 49 & 86 & 4 & 93 \\ 26 & 52 & 31 & 42 & 65 & 57+ & 28 & 93+ & 46 & 92 & 10 & 93 \\ 27 & 52 & 31 & 42 & 68 & 72 & 26 & 93 & 44 & 92 & 7 & 62 \\ 28 & 52 & 31 & 42 & 67 & 67 & 25 & 93 & 48 & 72 & 3 & 93 \\ 29 & 52 & 31 & 42 & 63 & 88+ & 40 & 60 & 43 & 92 & 12 & 60\end{array}$

MARCH

\begin{tabular}{|c|c|c|c|c|c|c|c|c|c|c|c|}
\hline & \multicolumn{11}{|c|}{ MARCH } \\
\hline 1 & 52 & 31 & 42 & 64 & 88 & 26 & 93 & 41 & 68 & 15 & $71+$ \\
\hline 2 & 52 & 31 & 42 & 66 & 68 & 26 & 60 & 46 & 87 & 14 & 60 \\
\hline 3 & 52 & 31 & 42 & 66 & 68 & 24 & 60 & 50 & 87 & 14 & 89 \\
\hline 4 & 52 & 31 & 42 & 63 & 53 & 26 & 55 & 46 & 87 & 7 & 55 \\
\hline 5 & 53 & 31 & 42 & 68 & 72 & 33 & $89+$ & 50 & 87 & 6 & 55 \\
\hline 6 & 54 & 32 & 43 & 65 & $67+$ & 33 & 57 & 43 & 79 & 18 & 60 \\
\hline 7 & 55 & 32 & 44 & 66 & 53 & 42 & 74 & 42 & $86+$ & 21 & 74 \\
\hline 8 & 55 & 33 & 44 & 67 & 53 & 33 & 51 & 46 & 83 & 20 & 76 \\
\hline 9 & 56 & 33 & 44 & 69 & 53 & 40 & 51 & 47 & 83 & 22 & 51 \\
\hline 10 & 56 & 33 & 45 & 69 & 72 & 40 & 48 & 45 & $87+$ & 13 & 48 \\
\hline 11 & 56 & 33 & 45 & 67 & 89 & 32 & 50 & 42 & $89+$ & 21 & 50 \\
\hline 12 & 57 & 34 & 45 & 68 & 92 & 37 & 51 & 48 & 87 & 15 & 56 \\
\hline 13 & 57 & 34 & 45 & 70 & 92 & 38 & 51 & 44 & $87+$ & 22 & $69+$ \\
\hline 14 & 57 & 34 & 46 & 72 & 92 & 40 & 49 & 45 & 61 & 23 & 53 \\
\hline 15 & 58 & 34 & 46 & 70 & $65+$ & 37 & 49 & 50 & 92 & 23 & 76 \\
\hline 16 & 58 & 34 & 46 & 76 & 72 & 43 & 89 & 45 & 92 & 23 & 55 \\
\hline 17 & 59 & 35 & 47 & 76 & 72 & 38 & 65 & 48 & 69 & 17 & 65 \\
\hline 18 & 59 & 35 & 47 & 76 & 47 & 41 & 65 & 47 & 90 & 14 & 65 \\
\hline 19 & 59 & 35 & 47 & 76 & 47 & 48 & $65+$ & 47 & 47 & 16 & 65 \\
\hline 20 & 59 & 35 & 47 & 76 & 47 & 49 & 50 & 49 & 88 & 22 & 74 \\
\hline 21 & 59 & 35 & 47 & 74 & 60 & 41 & 75 & 46 & 47 & 26 & $82+$ \\
\hline 22 & 59 & 35 & 47 & 74 & $78+$ & 47 & 71 & 47 & 78 & 24 & $64+$ \\
\hline 23 & 59 & 35 & 47 & 77 & 60 & 39 & 64 & 45 & $56+$ & 20 & 48 \\
\hline 24 & 59 & 35 & 47 & 78 & 60 & 38 & 55 & 45 & $60+$ & 15 & 64 \\
\hline 25 & 59 & 35 & 47 & 83 & 60 & 35 & 55 & 48 & 52 & 23 & $64+$ \\
\hline 26 & 60 & 35 & 47 & 71 & $78+$ & 38 & 65 & 49 & $92+$ & 21 & 85 \\
\hline 27 & 60 & 35 & 48 & 73 & $69+$ & 47 & 79 & 46 & 89 & 24 & 75 \\
\hline 28 & 61 & 36 & 48 & 75 & 66 & 42 & 54 & 49 & 78 & 19 & 75 \\
\hline 29 & 61 & 36 & 49 & 78 & 66 & 49 & 54 & 48 & 60 & 18 & 54 \\
\hline 30 & 62 & 37 & 49 & 75 & 92 & 52 & 67 & 47 & $92+$ & 20 & 54 \\
\hline \multirow[t]{2}{*}{31} & 62 & 37 & 49 & 78 & 92 & 51 & 75 & 47 & 61 & 28 & 53 \\
\hline & \multicolumn{11}{|c|}{ APRIL } \\
\hline 1 & 63 & 37 & 50 & 80 & 90 & 45 & 76 & 50 & 59 & 24 & 82 \\
\hline 2 & 63 & 37 & 50 & 83 & 92 & 48 & 82 & 50 & 87 & 25 & 76 \\
\hline 3 & 64 & 37 & 50 & 76 & 77 & 50 & $63+$ & 48 & 77 & 23 & 75 \\
\hline 4 & 64 & 38 & 51 & 82 & 60 & 45 & 75 & 56 & 91 & 27 & 50 \\
\hline 5 & 64 & 38 & 51 & 78 & $77+$ & 51 & 75 & 54 & 60 & 21 & 75 \\
\hline 6 & 65 & 38 & 51 & 82 & 77 & 41 & 45 & 51 & 62 & 28 & $67+$ \\
\hline 7 & 65 & 38 & 52 & 85 & 77 & 45 & 53 & 53 & 60 & 26 & 54 \\
\hline 8 & 65 & 38 & 52 & 81 & 66 & 51 & 53 & 47 & 60 & 27 & $92+$ \\
\hline 9 & 65 & 38 & 52 & 80 & 85 & 48 & 92 & 49 & 74 & 29 & $75+$ \\
\hline 10 & 65 & 38 & 52 & 85 & 68 & 53 & $83+$ & 47 & 57 & 24 & 81 \\
\hline 11 & 65 & 38 & 52 & 79 & 88 & 52 & 83 & 51 & $56+$ & 27 & 83 \\
\hline
\end{tabular}


TABLE 3.11. (contd)

NORMAL

EXTREME (1945-1993)

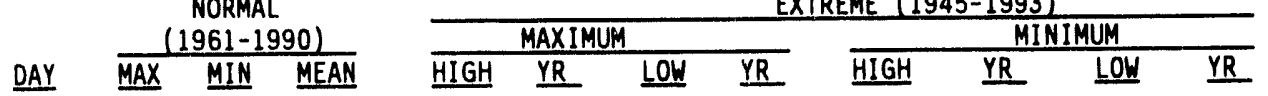

\section{APRIL (contd)}

$\begin{array}{llllllllllll}12 & 66 & 39 & 52 & 83 & 88 & 52 & 68 & 48 & 82+ & 28 & 71+ \\ 13 & 66 & 39 & 52 & 88 & 47 & 54 & 55 & 59 & 88 & 23 & 68 \\ 14 & 66 & 39 & 52 & 85 & 62+ & 47 & 75 & 55 & 85 & 29 & 83 \\ 15 & 66 & 39 & 52 & 82 & 88 & 55 & 75+ & 54 & 87 & 27 & 55 \\ 16 & 66 & 39 & 52 & 83 & 54 & 53 & 63 & 54 & 92 & 28 & 82 \\ 17 & 65 & 40 & 52 & 84 & 49 & 48 & 63 & 52 & 90 & 26 & 55 \\ 18 & 65 & 40 & 52 & 87 & 52 & 51 & 67 & 50 & 83+ & 29 & 68 \\ 19 & 65 & 40 & 52 & 78 & 56 & 50 & 51 & 54 & 89+ & 27 & 66 \\ 20 & 66 & 40 & 53 & 84 & 56 & 45 & 67 & 53 & 90+ & 28 & 82 \\ 21 & 66 & 40 & 53 & 85 & 56 & 52 & 67 & 60 & 56 & 26 & 85+ \\ 22 & 66 & 41 & 54 & 81 & 82+ & 53 & 88 & 52 & 81+ & 28 & 72 \\ 23 & 67 & 41 & 54 & 88 & 81+ & 56 & 79+ & 58 & 77 & 30 & 78 \\ 24 & 68 & 41 & 54 & 94 & 77 & 53 & 75 & 56 & 52 & 28 & 86+ \\ 25 & 68 & 42 & 55 & 89 & 52 & 56 & 58 & 59 & 52 & 31 & 55 \\ 26 & 68 & 42 & 55 & 85 & 92 & 53 & 48 & 57 & 78 & 28 & 48 \\ 27 & 69 & 42 & 55 & 90 & 87 & 50 & 90 & 57 & 92 & 27 & 70 \\ 28 & 69 & 42 & 56 & 93 & 87 & 56 & 67 & 64 & 87 & 27 & 67 \\ 29 & 70 & 43 & 56 & 90 & 68 & 47 & 67 & 60 & 87 & 29 & 52 \\ 30 & 70 & 43 & 57 & 91 & 81 & 56 & 67+ & 56 & 81 & 29 & 86\end{array}$

MAY

$\begin{array}{rrrrrlllllll}1 & 71 & 43 & 57 & 87 & 47 & 53 & 69 & 59 & 77 & 28 & 54 \\ 2 & 71 & 43 & 57 & 89 & 71 & 56 & 88 & 60 & 71 & 32 & 48 \\ 3 & 71 & 44 & 58 & 91 & 66 & 58 & 93 & 60 & 71 & 31 & 49 \\ 4 & 72 & 44 & 58 & 94 & 66 & 56 & 63 & 56 & 71+ & 31 & 62 \\ 5 & 72 & 45 & 58 & 100 & 66 & 52 & 61 & 65 & 66 & 30 & 59 \\ 6 & 73 & 45 & 59 & 98 & 92 & 56 & 86 & 62 & 87 & 34 & 77 \\ 7 & 73 & 45 & 59 & 99 & 87 & 61 & 90 & 66 & 92 & 33 & 84 \\ 8 & 73 & 46 & 59 & 102 & 87 & 56 & 62 & 67 & 87 & 36 & 81 \\ 9 & 74 & 46 & 60 & 97 & 87 & 56 & 48 & 66 & 49 & 34 & 73 \\ 10 & 74 & 46 & 60 & 96 & 49 & 53 & 67 & 66 & 49 & 34 & 70 \\ 11 & 74 & 46 & 60 & 98 & 49 & 51 & 67 & 68 & 49 & 30 & 70 \\ 12 & 74 & 46 & 60 & 100 & 93 & 57 & 70 & 66 & 93 & 34 & 85 \\ 13 & 74 & 46 & 60 & 93 & 73+ & 57 & 55 & 65 & 49 & 34 & 85 \\ 14 & 75 & 47 & 61 & 98 & 73 & 56 & 55 & 61 & 73+ & 31 & 55 \\ 15 & 75 & 47 & 61 & 97 & 73 & 57 & 59 & 64 & 73+ & 35 & 74 \\ 16 & 75 & 47 & 61 & 95 & 73 & 54 & 55 & 60 & 73 & 32 & 74 \\ 17 & 76 & 48 & 62 & 96 & 73 & 61 & 74 & 59 & 85 & 38 & 88+ \\ 18 & 76 & 48 & 62 & 98 & 54 & 62 & 74 & 67 & 56 & 36 & 72 \\ 19 & 77 & 48 & 62 & 92 & 93 & 56 & 62 & 70 & 56 & 33 & 75 \\ 20 & 77 & 48 & 62 & 93 & 47 & 58 & 60 & 59 & 56 & 36 & 71 \\ 21 & 77 & 49 & 63 & 94 & 58 & 62 & 60+ & 59 & 58 & 37 & 74+ \\ 22 & 77 & 49 & 63 & 98 & 58 & 63 & 64 & 64 & 58 & 33 & 60 \\ 23 & 77 & 49 & 63 & 95 & 85+ & 59 & 62 & 66 & 58 & 35 & 64 \\ 24 & 77 & 49 & 63 & 96 & 92 & 54 & 62 & 63 & 81 & 35 & 75 \\ 25 & 77 & 50 & 63 & 98 & 92+ & 62 & 84 & 65 & 83 & 38 & 91+ \\ 26 & 77 & 50 & 64 & 101 & 58+ & 54 & 80 & 69 & 47 & 38 & 78 \\ 27 & 78 & 50 & 64 & 93 & 83 & 62 & 89 & 69 & 58 & 37 & 73 \\ 28 & 78 & 50 & 64 & 99 & 83 & 61 & 89 & 63 & 72+ & 38 & 79+ \\ 29 & 78 & 51 & 64 & 103 & 83 & 68 & 89+ & 71 & 86 & 35 & 76 \\ 30 & 79 & 51 & 65 & 104 & 86 & 62 & 76+ & 68 & 86 & 41 & 55 \\ 31 & 79 & 52 & 65 & 104 & 86 & 54 & 71 & 69 & 86 & 40 & 76+\end{array}$


TABLE 3.11. (contd)

NORMAL

DAY MAX MIN MEAN $\frac{(1961-1990)}{\text { HIGH } \quad \text { MAXIMUM }}$ LOW $\underline{\text { YR }}$ HIGH $\underline{\text { YR }}$ LOW

JUNE

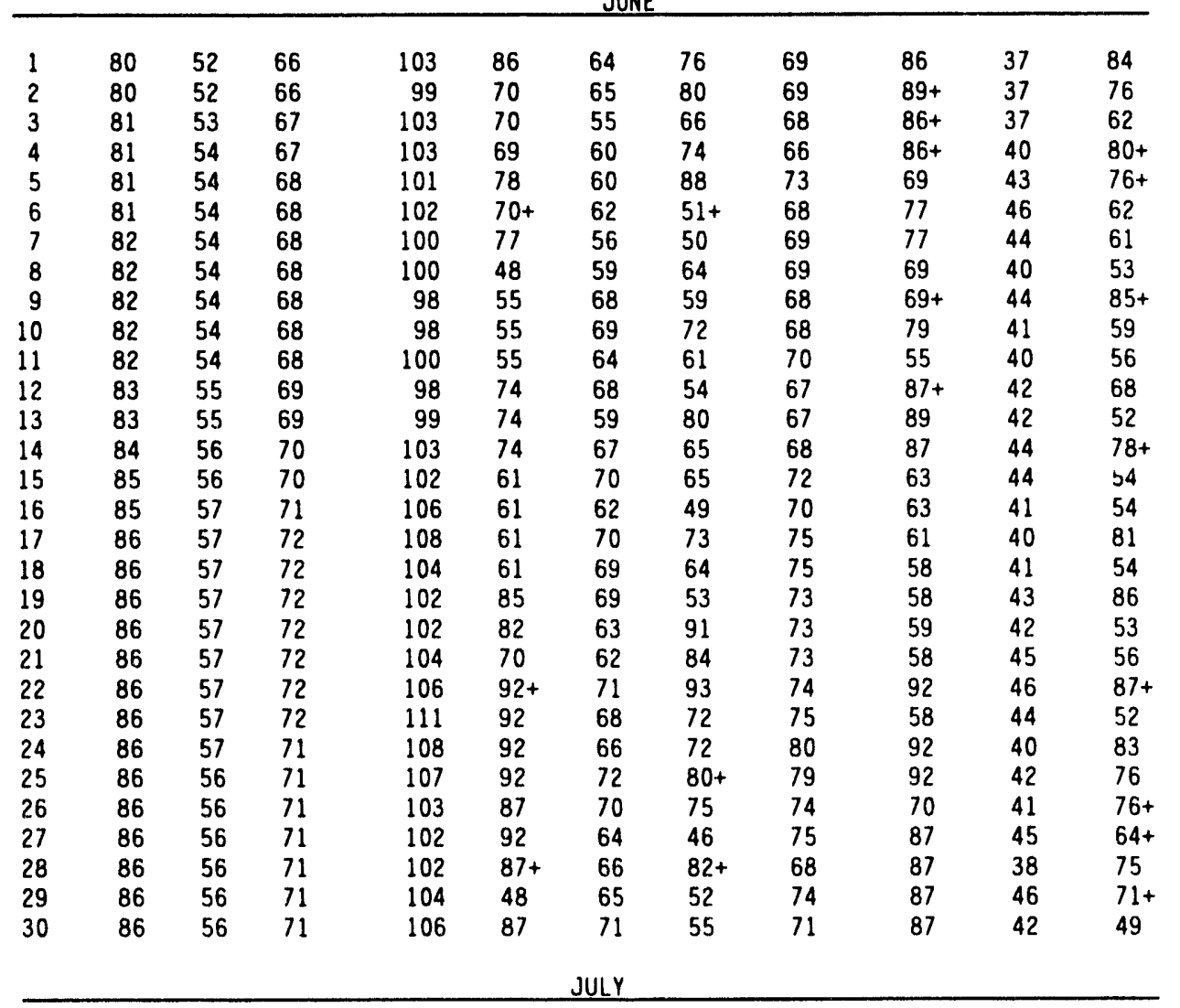

$\begin{array}{llllllllllll}1 & 86 & 57 & 72 & 103 & 87 & 66 & 66 & 75 & 87 & 46 & 73+ \\ 2 & 87 & 57 & 72 & 102 & 67 & 59 & 66 & 66 & 63 & 39 & 79 \\ 3 & 87 & 58 & 72 & 105 & 91+ & 71 & 66 & 70 & 67 & 46 & 90 \\ 4 & 88 & 58 & 73 & 108 & 68 & 71 & 86 & 75 & 70 & 48 & 66 \\ 5 & 88 & 59 & 73 & 108 & 75 & 66 & 51 & 76 & 75 & 47 & 80 \\ 6 & 89 & 59 & 74 & 110 & 68 & 71 & 55 & 76 & 68 & 44 & 71 \\ 7 & 89 & 59 & 74 & 105 & 68+ & 75 & 81 & 73 & 68 & 45 & 71 \\ 8 & 89 & 59 & 74 & 108 & 68 & 71 & 72 & 74 & 85 & 45 & 81 \\ 9 & 89 & 60 & 74 & 110 & 75 & 76 & 55 & 78 & 75 & 50 & 72+ \\ 10 & 90 & 60 & 75 & 106 & 75 & 67 & 74 & 79 & 75 & 49 & 76 \\ 11 & 90 & 60 & 75 & 109 & 90 & 76 & 74 & 78 & 75 & 46 & 81 \\ 12 & 90 & 60 & 75 & 110 & 90 & 75 & 88 & 75 & 90 & 50 & 74 \\ 13 & 90 & 60 & 75 & 108 & 61 & 77 & 93+ & 73 & 90+ & 49 & 76 \\ 14 & 90 & 61 & 76 & 107 & 87+ & 77 & 83 & 78 & 61 & 50 & 83 \\ 15 & 91 & 61 & 76 & 106 & 73 & 71 & 82 & 76 & 55 & 45 & 82 \\ 16 & 91 & 61 & 76 & 105 & 70 & 68 & 86 & 74 & 90 & 48 & 74 \\ 17 & 92 & 61 & 76 & 110 & 60 & 73 & 93 & 77 & 58 & 48 & 86 \\ 18 & 92 & 61 & 77 & 110 & 60 & 78 & 87 & 79 & 60 & 49 & 86 \\ 19 & 92 & 61 & 77 & 109 & 79 & 72 & 49 & 77 & 79 & 51 & 77 \\ 20 & 93 & 62 & 77 & 110 & 79 & 75 & 65+ & 74 & 56 & 53 & 68+ \\ 21 & 93 & 62 & 78 & 105 & 71 & 68 & 65 & 77 & 88 & 49 & 49 \\ 22 & 94 & 62 & 78 & 107 & 59 & 74 & 92 & 75 & 85 & 47 & 82 \\ 23 & 94 & 63 & 78 & 106 & 78 & 69 & 92 & 74 & 62+ & 49 & 63\end{array}$


TABLE 3.11. (contd)

NORMAL

DAY $\frac{(1961-1990)}{\text { MAX }}$

EXTREME (1945-1993)

JULY (contd)

$\begin{array}{llllllllllll}24 & 94 & 63 & 79 & 106 & 62 & 78 & 63 & 75 & 62 & 52 & 52+ \\ 25 & 95 & 63 & 79 & 106 & 84 & 73 & 90 & 77 & 62 & 51 & 49+ \\ 26 & 95 & 63 & 79 & 105 & 88+ & 66 & 55 & 76 & 88 & 54 & 48 \\ 27 & 95 & 64 & 79 & 108 & 75+ & 74 & 48 & 74 & 73+ & 52 & 86 \\ 28 & 95 & 64 & 79 & 108 & 68 & 77 & 50+ & 76 & 68 & 49 & 59 \\ 29 & 95 & 63 & 79 & 107 & 82 & 80 & 93+ & 78 & 82 & 52 & 50 \\ 30 & 95 & 63 & 79 & 107 & 71 & 78 & 75 & 74 & 90 & 49 & 50 \\ 31 & 94 & 63 & 79 & 111 & 71 & 75 & 85 & 74 & 71+ & 53 & 64+\end{array}$

AUGUST

\begin{tabular}{|c|c|c|c|c|c|c|c|c|c|c|}
\hline 1 & 94 & 63 & 78 & 109 & 71 & 77 & $76+$ & 80 & 49 & 51 \\
\hline 2 & 94 & 62 & 78 & 105 & 61 & 75 & 56 & 75 & $77+$ & 46 \\
\hline 3 & 94 & 62 & 78 & 107 & 61 & 77 & 62 & 73 & 86 & 52 \\
\hline 4 & 93 & 62 & 78 & 113 & 61 & 78 & $64+$ & 81 & 61 & 48 \\
\hline 5 & 93 & 62 & 78 & 108 & 90 & 77 & 46 & 72 & 91 & 45 \\
\hline 6 & 94 & 62 & 78 & 106 & 72 & 80 & 48 & 77 & 90 & 49 \\
\hline 7 & 94 & 63 & 78 & 109 & 72 & 70 & 62 & 73 & 71 & 50 \\
\hline 8 & 94 & 63 & 78 & 110 & 72 & 75 & 62 & 79 & $82+$ & 48 \\
\hline 9 & 94 & 63 & 78 & 112 & 71 & 78 & 47 & 78 & 90 & 51 \\
\hline 10 & 94 & 63 & 78 & 109 & 71 & 76 & 85 & 77 & 71 & 52 \\
\hline 11 & 93 & 63 & 78 & 108 & 71 & 79 & 83 & 73 & 58 & 50 \\
\hline 12 & 93 & 62 & 77 & 108 & 71 & 79 & 78 & 77 & 92 & 52 \\
\hline 13 & 92 & 61 & 77 & 107 & 92 & 74 & 68 & 79 & 92 & 49 \\
\hline 14 & 91 & 60 & 76 & 109 & 92 & 70 & 68 & 78 & $92+$ & 49 \\
\hline 15 & 90 & 60 & 75 & 105 & 67 & 72 & 60 & 76 & 45 & 51 \\
\hline 16 & 89 & 59 & 74 & 108 & 67 & 68 & 93 & 70 & 81 & 48 \\
\hline 17 & 89 & 59 & 74 & 108 & 67 & 78 & $72+$ & 68 & 91 & 47 \\
\hline 18 & 89 & 59 & 74 & 108 & 67 & 71 & 80 & 70 & $91+$ & 47 \\
\hline 19 & 88 & 59 & 74 & 105 & 67 & 70 & 68 & 76 & 91 & 46 \\
\hline 20 & 88 & 59 & 74 & 105 & 67 & 67 & 59 & 77 & 82 & 49 \\
\hline 21 & 88 & 59 & 74 & 103 & $91+$ & 70 & 60 & 74 & $82+$ & 47 \\
\hline 22 & 88 & 59 & 73 & 104 & 56 & 70 & 92 & 76 & $61+$ & 41 \\
\hline 23 & 88 & 58 & 73 & 105 & 70 & 69 & 92 & 74 & 61 & 45 \\
\hline 24 & 87 & 58 & 73 & 104 & 58 & 70 & 68 & 71 & 66 & 43 \\
\hline 25 & 87 & 58 & 72 & 104 & 58 & 72 & 77 & 68 & $88+$ & 43 \\
\hline 26 & 87 & 58 & 72 & 100 & 84 & 68 & 56 & 67 & 58 & 44 \\
\hline 27 & 87 & 57 & 72 & 101 & 72 & 73 & 68 & 71 & 67 & 47 \\
\hline 28 & 86 & 57 & 72 & 104 & 72 & 70 & 51 & 74 & 86 & 42 \\
\hline 29 & 86 & 56 & 71 & 102 & 67 & 72 & 51 & 73 & 67 & 42 \\
\hline 30 & 86 & 56 & 71 & 105 & 67 & 68 & 51 & 71 & 67 & 44 \\
\hline 31 & 85 & 56 & 71 & 104 & 67 & 72 & 71 & 73 & 67 & 45 \\
\hline
\end{tabular}

SEPTEMBER

\begin{tabular}{|c|c|c|c|c|c|c|c|c|c|c|c|}
\hline 1 & 85 & 56 & 71 & 106 & 87 & 61 & 71 & 70 & 87 & 44 & 80 \\
\hline 2 & 85 & 56 & 71 & 102 & 50 & 70 & 71 & 70 & 49 & 47 & $75+$ \\
\hline 3 & 85 & 55 & 70 & 101 & 88 & 72 & 70 & 67 & $55+$ & 44 & $80+$ \\
\hline 4 & 85 & 55 & 70 & 102 & 88 & 68 & 59 & 68 & 55 & 44 & 80 \\
\hline 5 & 85 & 55 & 70 & 100 & 55 & 72 & 60 & 68 & 63 & 43 & 69 \\
\hline 6 & 85 & 55 & 70 & 101 & 55 & 72 & 92 & 65 & $57+$ & 44 & 83 \\
\hline 7 & 85 & 54 & 69 & 97 & 58 & 60 & 78 & 72 & 55 & 42 & $92+$ \\
\hline 8 & 84 & 54 & 69 & 99 & 81 & 61 & 85 & 69 & 63 & 42 & $76+$ \\
\hline 9 & 83 & 53 & 68 & 98 & $81+$ & 66 & 85 & 68 & 69 & 40 & 62 \\
\hline 10 & 82 & 53 & 67 & 97 & 93 & 68 & 85 & 65 & 63 & 43 & $82+$ \\
\hline 11 & 81 & 52 & 67 & 98 & $90+$ & 62 & 85 & 66 & 69 & 41 & 88 \\
\hline
\end{tabular}


TABLE 3.11. (contd)

NORMAL

NORMAL
DAY MAX MIN MEAN

SEPTEMBER (contd)

$\begin{array}{llllllllllll}12 & 81 & 52 & 66 & 96 & 69 & 62 & 70 & 67 & 53 & 38 & 49 \\ 13 & 80 & 51 & 66 & 98 & 48 & 59 & 80 & 62 & 60+ & 40 & 74\end{array}$

$\begin{array}{llllllllllll}14 & 80 & 51 & 65 & 93 & 75+ & 62 & 92 & 61 & 90+ & 38 & 70 \\ 15 & 79 & 51 & 65 & 96 & 79+ & 58 & 59 & 60 & 88+ & 35 & 70\end{array}$

$\begin{array}{lllllllllll}16 & 79 & 51 & 65 & 96 & 81+ & 62 & 65+ & 65 & 79 & 35\end{array}$

$\begin{array}{lllllllllll}17 & 79 & 51 & 65 & 97 & 81 & 59 & 86 & 62 & 51 & 33\end{array}$

$\begin{array}{llllllllllll}18 & 78 & 50 & 64 & 98 & 81 & 57 & 83 & 61 & 79 & 34 & 65\end{array}$

$\begin{array}{lllllllllll}19 & 78 & 50 & 64 & 96 & 67 & 62 & 83 & 67 & 56 & 36\end{array}$

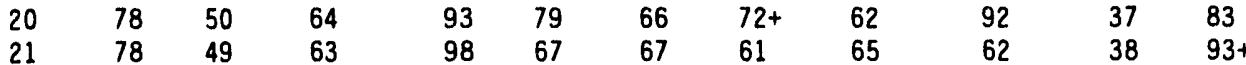

$\begin{array}{llllllllllll}22 & 77 & 49 & 63 & 93 & 66 & 52 & 84 & 68 & 66 & 36 & 81+\end{array}$

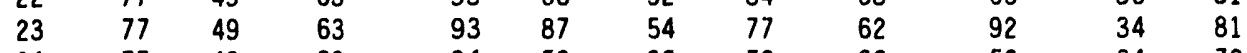

$\begin{array}{lllllllllll}24 & 77 & 49 & 63 & 94 & 52 & 60 & 72 & 60 & 50 & 34\end{array}$

$\begin{array}{lllllllllll}25 & 77 & 48 & 63 & 97 & 52 & 56 & 77 & 66 & 49 & 30\end{array}$

$\begin{array}{lllllllllll}26 & 77 & 48 & 62 & 92 & 52 & 57 & 48 & 61 & 79 & 32\end{array}$

\begin{tabular}{lllllllllll}
27 & 76 & 48 & 62 & 92 & $63+$ & 58 & 77 & 60 & 49 & 30 \\
\hline
\end{tabular}

$\begin{array}{lllllllllll}28 & 76 & 48 & 62 & 92 & 67 & 58 & 77 & 62 & 76 & 33\end{array}$

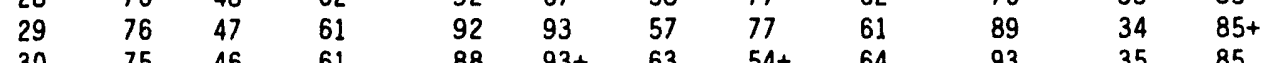

$\begin{array}{llllllllllll}30 & 75 & 46 & 61 & 88 & 93+ & 63 & 54+ & 64 & 93 & 35 & 85\end{array}$

OCTOBER

\begin{tabular}{|c|c|c|c|c|c|c|c|c|c|c|c|}
\hline 1 & 75 & 46 & 60 & 88 & $91+$ & 61 & 59 & 59 & 92 & 30 & 54 \\
\hline 2 & 74 & 45 & 60 & 86 & $93+$ & 56 & 67 & 60 & 88 & 32 & 54 \\
\hline 3 & 73 & 45 & 59 & 89 & 58 & 55 & 50 & 58 & $88+$ & 33 & $73+$ \\
\hline 4 & 73 & 44 & 58 & 89 & 80 & 55 & 50 & 57 & 88 & 32 & 73 \\
\hline 5 & 72 & 44 & 58 & 87 & 58 & 52 & 57 & 52 & $88+$ & 34 & $82+$ \\
\hline 6 & 72 & 44 & 58 & 85 & 80 & 53 & $57+$ & 57 & 60 & 30 & 74 \\
\hline 7 & 71 & 44 & 57 & 86 & 80 & 48 & 57 & 57 & 88 & 29 & 74 \\
\hline 8 & 70 & 44 & 57 & 84 & 65 & 51 & 85 & 58 & 87 & 26 & 85 \\
\hline 9 & 70 & 43 & 56 & 83 & $88+$ & 52 & 58 & 54 & 53 & 26 & 85 \\
\hline 10 & 69 & 43 & 56 & 82 & 88 & 50 & 62 & 54 & 84 & 33 & 59 \\
\hline 11 & 69 & 43 & 56 & 84 & 52 & 52 & $68+$ & 55 & 63 & 30 & 60 \\
\hline 12 & 68 & 43 & 55 & 82 & 91 & 54 & 66 & 56 & 52 & 34 & $85+$ \\
\hline 13 & 68 & 42 & 55 & 78 & $64+$ & 57 & 69 & 55 & 88 & 31 & 69 \\
\hline 14 & 67 & 41 & 54 & 77 & $91+$ & 58 & $90+$ & 59 & 88 & 24 & 69 \\
\hline 15 & 66 & 40 & 53 & 81 & 63 & 53 & 92 & 60 & 88 & 29 & 70 \\
\hline 16 & 66 & 39 & 53 & 79 & 63 & 54 & $92+$ & 54 & 88 & 29 & 84 \\
\hline 17 & 65 & 39 & 52 & 76 & 63 & 49 & 50 & 49 & 55 & 29 & $71+$ \\
\hline 18 & 65 & 38 & 51 & 76 & 73 & 47 & 49 & 50 & 52 & 27 & 49 \\
\hline 19 & 64 & 38 & 51 & 78 & $92+$ & 51 & 84 & 52 & 92 & 27 & $69+$ \\
\hline 20 & 63 & 38 & 50 & 74 & 78 & 45 & 47 & 54 & 73 & 23 & 49 \\
\hline 21 & 63 & 38 & 50 & 73 & $91+$ & 50 & 68 & 54 & 63 & 20 & 84 \\
\hline 22 & 63 & 38 & 50 & 74 & 59 & 46 & 50 & 51 & 52 & 20 & 84 \\
\hline 23 & 62 & 38 & 50 & 73 & $66+$ & 39 & 84 & 51 & 60 & 25 & 84 \\
\hline 24 & 61 & 38 & 50 & 75 & $77+$ & 49 & 57 & 60 & 45 & 26 & 75 \\
\hline 25 & 61 & 37 & 49 & 75 & 55 & 49 & 57 & 51 & 79 & 26 & 78 \\
\hline 26 & 60 & 37 & 48 & 69 & 92 & 50 & 80 & 50 & $86+$ & 21 & 78 \\
\hline 27 & 59 & 36 & 48 & 74 & 85 & 43 & $93+$ & 54 & 81 & 23 & 70 \\
\hline 28 & 58 & 36 & 47 & 68 & $65+$ & 35 & 91 & 52 & 49 & 18 & 71 \\
\hline 29 & 58 & 36 & 47 & 70 & 53 & 42 & $91+$ & 48 & 74 & 13 & 71 \\
\hline 30 & 57 & 36 & 46 & 75 & 67 & 32 & 71 & 49 & $86+$ & 20 & 72 \\
\hline 31 & 56 & 36 & 46 & 75 & 67 & 34 & 84 & 54 & 67 & 12 & 84 \\
\hline
\end{tabular}


TABLE 3.11. (contd)

\begin{tabular}{c} 
NORMAL \\
DAY \\
\cline { 2 - 3 } MAX MIN MEAN
\end{tabular} NOVEMBER

\begin{tabular}{|c|c|c|c|c|c|c|c|c|c|c|c|}
\hline 1 & 56 & 36 & 46 & 70 & 45 & 31 & 84 & 49 & 87 & 22 & 78 \\
\hline 2 & 56 & 36 & 46 & 68 & 75 & 38 & 91 & 51 & 85 & 22 & $89+$ \\
\hline 3 & 55 & 36 & 45 & 75 & 75 & 36 & 73 & 53 & 83 & 24 & 61 \\
\hline 4 & 55 & 36 & 45 & 71 & 75 & 32 & 73 & 46 & $89+$ & 16 & 73 \\
\hline 5 & 54 & 35 & 44 & 63 & 89 & 31 & 73 & 48 & 88 & 20 & $73+$ \\
\hline 6 & 53 & 35 & 44 & 64 & 58 & 32 & 73 & 49 & 89 & 19 & 73 \\
\hline 7 & 53 & 35 & 44 & 69 & 78 & 34 & 45 & 48 & 80 & 19 & $93+$ \\
\hline 8 & 52 & 34 & 43 & 64 & 80 & 30 & 45 & 48 & 89 & 20 & 48 \\
\hline 9 & 51 & 34 & 43 & 73 & 89 & 32 & 45 & 60 & 89 & 18 & 86 \\
\hline 10 & 51 & 34 & 42 & 73 & 89 & 35 & 86 & 56 & 89 & 16 & 86 \\
\hline 11 & 50 & 34 & 42 & 66 & 89 & 32 & 85 & 48 & 89 & 14 & 78 \\
\hline 12 & 50 & 33 & 42 & 65 & 91 & 20 & 55 & 52 & 49 & 6 & 55 \\
\hline 13 & 49 & 33 & 41 & 64 & 87 & 14 & 55 & 46 & 90 & 6 & 59 \\
\hline 14 & 49 & 33 & 41 & 64 & 53 & 19 & 55 & 45 & 53 & -1 & 55 \\
\hline 15 & 49 & 32 & 40 & 65 & 53 & 18 & 55 & 46 & 83 & 1 & 55 \\
\hline 16 & 48 & 32 & 40 & 65 & 76 & 18 & 55 & 49 & 54 & 7 & 59 \\
\hline 17 & 48 & 31 & 40 & 71 & 76 & 22 & 55 & 46 & $83+$ & 10 & 61 \\
\hline 18 & 48 & 31 & 39 & 64 & 46 & 25 & 55 & 47 & 54 & 11 & 55 \\
\hline 19 & 47 & 31 & 39 & 67 & 62 & 22 & 85 & 44 & 54 & 14 & 85 \\
\hline 20 & 47 & 30 & 38 & 65 & 58 & 24 & 85 & 47 & 74 & 3 & 85 \\
\hline 21 & 46 & 30 & 38 & 63 & 58 & 17 & 85 & 46 & 65 & 3 & 85 \\
\hline 22 & 46 & 29 & 38 & 65 & $67+$ & 17 & 85 & 53 & 90 & -6 & 85 \\
\hline 23 & 45 & 29 & 37 & 70 & 59 & 11 & 85 & 54 & 90 & -13 & 85 \\
\hline 24 & 45 & 29 & 37 & 67 & 59 & 6 & 85 & 57 & 90 & -12 & 85 \\
\hline 25 & 44 & 28 & 36 & 65 & 62 & 11 & 85 & 48 & 62 & -3 & 85 \\
\hline 26 & 43 & 28 & 36 & 65 & 49 & 15 & 85 & 45 & 49 & -1 & 85 \\
\hline 27 & 43 & 28 & 35 & 63 & 49 & 12 & 85 & 46 & 49 & 8 & 85 \\
\hline 28 & 43 & 28 & 35 & 62 & 73 & 11 & 85 & 43 & 73 & 7 & 85 \\
\hline 29 & 42 & 28 & 35 & 57 & $90+$ & 14 & 85 & 38 & $73+$ & 8 & 85 \\
\hline 30 & 42 & 28 & 35 & 60 & 51 & 15 & 85 & 41 & 66 & 6 & 85 \\
\hline & \multicolumn{11}{|c|}{ DECEMBER } \\
\hline 1 & 42 & 28 & 35 & 65 & 72 & 14 & 85 & 45 & 81 & -8 & 85 \\
\hline 2 & 42 & 28 & 35 & 64 & 77 & 20 & 85 & 56 & 75 & 8 & 85 \\
\hline 3 & 42 & 28 & 35 & 62 & $82+$ & 27 & $85+$ & 54 & 75 & 8 & 85 \\
\hline 4 & 42 & 28 & 35 & 60 & 75 & 23 & 72 & 41 & 52 & 1 & 72 \\
\hline 5 & 41 & 28 & 34 & 58 & $91+$ & 21 & 72 & 41 & 87 & 7 & 72 \\
\hline 6 & 41 & 27 & 34 & 59 & 87 & 16 & 56 & 43 & 87 & 8 & 56 \\
\hline 7 & 40 & 27 & 33 & 58 & $73+$ & 18 & 56 & 40 & 52 & 2 & 56 \\
\hline 8 & 40 & 26 & 33 & 58 & 89 & 17 & 72 & 48 & 46 & -6 & 72 \\
\hline 9 & 40 & 26 & 33 & 64 & 46 & 13 & 72 & 44 & 56 & -5 & 72 \\
\hline 10 & 39 & 25 & 32 & 67 & 93 & 13 & 72 & 42 & $67+$ & -8 & 72 \\
\hline 11 & 39 & 25 & 32 & 59 & $91+$ & 11 & 72 & 36 & $93+$ & -4 & 72 \\
\hline 12 & 39 & 25 & 32 & 57 & 88 & 22 & 72 & 42 & $77+$ & -5 & 72 \\
\hline 13 & 38 & 25 & 31 & 56 & $88+$ & 13 & 72 & 40 & $73+$ & -6 & 72 \\
\hline 14 & 38 & 24 & 31 & 57 & $79+$ & 15 & 72 & 48 & 79 & -2 & 72 \\
\hline 15 & 37 & 24 & 31 & 64 & 59 & 16 & 72 & 41 & 56 & 2 & 72 \\
\hline 16 & 37 & 24 & 30 & 56 & 73 & 4 & 64 & 43 & 73 & -13 & 64 \\
\hline 17 & 37 & 24 & 30 & 57 & $71+$ & 5 & 64 & 41 & 62 & -12 & 64 \\
\hline 18 & 36 & 24 & 30 & 56 & 56 & 13 & 64 & 38 & 62 & -3 & 84 \\
\hline 19 & 36 & 24 & 30 & 54 & 66 & 17 & 84 & 40 & 66 & -4 & 84 \\
\hline 20 & 37 & 24 & 30 & 57 & 74 & 11 & 84 & 37 & $66+$ & -2 & $93+$ \\
\hline 21 & 37 & 24 & 30 & 61 & 72 & 11 & 90 & 43 & 73 & -10 & $91)$ \\
\hline 22 & 37 & 24 & 30 & 59 & 80 & 7 & 90 & 42 & 72 & -13 & 83 \\
\hline 23 & 37 & 24 & 30 & 57 & 63 & 6 & 83 & 40 & 72 & -9 & 83 \\
\hline
\end{tabular}


IABLE 3.11. (contd)

NORMAL
DAY MAX MIN MEAN

DECEMBER (contd)

$\begin{array}{rrrlllllllll}24 & 37 & 24 & 30 & 55 & 61+ & 15 & 90+ & 39 & 80+ & 0 & 90 \\ 25 & 37 & 23 & 30 & 65 & 80 & 16 & 90 & 41 & 72 & 1 & 90 \\ 26 & 36 & 23 & 30 & 69 & 80 & 20 & 90+ & 53 & 80 & 0 & 48 \\ 27 & 36 & 23 & 29 & 62 & 80+ & 19 & 48 & 40 & 49 & -2 & 48 \\ 28 & 35 & 22 & 29 & 56 & 57+ & 14 & 68 & 38 & 49 & -3 & 68 \\ 29 & 35 & 22 & 28 & 60 & 49 & -2 & 68 & 39 & 62 & -10 & 90+ \\ 30 & 35 & 21 & 28 & 54 & 70 & -2 & 68 & 39 & 88+ & -14 & 68 \\ 31 & 35 & 21 & 28 & 56 & 62 & 4 & 68 & 44 & 80 & -9 & 78\end{array}$

+ Latest of several occurrences.

The current normal period is 1961 through 1990, and the next will be 1971 through 2000. The normal temperatures in Table 3.11 are computed using a 7-day running mean, centered about each day.

Four possible temperature extremes are presented for each day, a record high and low maximum, and a record high and low minimum. These daily records, plus the year of occurrence, are also indicated in Table 3.11. 


\subsection{PRECIPITATION CLIMATOLOGY}

\subsection{MONTHLY AND ANNUAL TOTALS}

Table 4.1 shows monthly and annual precipitation totals for the period of record from 1946 through 1993. Normal monthly precipitation amounts for the period from 1961 to 1990, and averages for the entire period of record are indicated at the bottom of the table. Monthly and annual extremes are underlined in each column. The wettest year on record was 1950 with 11.45 inches of precipitation, while the driest was 1976 with 2.99 inches.

The months of November through February in this period provide 3.35 inches, or 54\%, of the normal annual precipitation. December is the wettest month, receiving 1.03 inches, while July is the driest, receiving only 0.18 inch. The wettest month on record was June 1950 with 2.92 inches, while the driest months, September 1991, August 1988, and August 1955, received no precipitation.

\subsection{SEASONAL PRECIPITATION}

Table 4.2 provides seasonal precipitation information, with normal and average seasonal data at the bottom of the table. The extremes for each season are underlined. The wettest season was the winter of 1958-1959 with 5.06 inches, while the driest, the summer of 1973 , received only 0.03 inch.

\subsection{AVERAGE NUMBER OF DAYS WITH SPECIFIED AMOUNTS OF PRECIPITATION}

Table 4.3 presents information on the average number of days per year with precipitation events in the following categories: $\geq$ Trace, $\geq 0.01$ inch, $\geq 0.10$ inch, $\geq 0.25$ inch, $\geq 0.50$ inch and $\geq 1.00$ inch. A trace, designated with a letter $T$ throughout this document, is less than .005 inch of precipitation. An average of 125 days per year have a trace or more of precipitation; however, only 24 days receive totals of 0.10 inch or more. During the 48-year period of record, only 3 days have had an inch or more of precipitation. 
IABLE 4.1. Monthly and Annual Precipitation (Inches)

\begin{tabular}{|c|c|c|c|c|c|c|c|c|c|c|c|c|c|}
\hline YEAR & JAN & FEB & MAR & APR & MAY & JUN & $\underline{J U L}$ & $\underline{A \cup G}$ & $\underline{\text { SEP }}$ & $\underline{O C T}$ & NOV & DEC & NNUAL \\
\hline 1945 & $-\cdots$ & -..- & $\cdots$ & $-\cdots$ & -.- & --- & --. & $\cdots$ & $\ldots$ & -- & ---- & --- & -- \\
\hline $\begin{array}{l}1946 \\
1947 \\
1948 \\
1949 \\
1950 \\
1951 \\
1952 \\
1953 \\
1954\end{array}$ & $\begin{array}{l}-.-- \\
0.32 \\
1.36 \\
0.13 \\
1.80 \\
0.84 \\
0.65 \\
2.16 \\
1.48 \\
0.56\end{array}$ & $\begin{array}{l}-.- \\
0.27 \\
0.69 \\
0.68 \\
1.06 \\
0.51 \\
0.50 \\
0.25 \\
0.28\end{array}$ & $\begin{array}{l}--- \\
0.42 \\
0.07 \\
1.12 \\
0.87 \\
0.46 \\
0.06 \\
0.17 \\
0.59 \\
0.17\end{array}$ & $\begin{array}{l}\cdots \\
0.70 \\
0.95 \\
0.02 \\
0.47 \\
0.53 \\
0.13 \\
0.77 \\
0.07 \\
0.40\end{array}$ & $\begin{array}{l}0.02 \\
0.02 \\
1.71 \\
0.16 \\
0.27 \\
0.43 \\
0.58 \\
0.28 \\
0.41 \\
0.59\end{array}$ & $\begin{array}{l}1.07 \\
1.47 \\
0.01 \\
2.92 \\
1.38 \\
1.07 \\
0.55 \\
0.10 \\
0.28\end{array}$ & $\begin{array}{l}0.15 \\
0.71 \\
0.40 \\
0.01 \\
0.07 \\
0.37 \\
T \\
T \\
0.22 \\
0.57\end{array}$ & $\begin{array}{l}0.35 \\
0.68 \\
0.39 \\
0.03 \\
T \\
0.15 \\
0.08 \\
0.96 \\
0.42 \\
0\end{array}$ & $\begin{array}{l}0.52 \\
1.34 \\
0.16 \\
0.23 \\
0.01 \\
0.10 \\
0.08 \\
0.13\end{array}$ & $\begin{array}{l}0.65 \\
2.20 \\
0.45 \\
0.10 \\
2.46 \\
0.71 \\
0.04 \\
0.20 \\
0.42 \\
0.40\end{array}$ & $\begin{array}{l}0.66 \\
0.81 \\
0.95 \\
1.47 \\
0.55 \\
0.82 \\
0.20 \\
0.96 \\
0.86\end{array}$ & $\begin{array}{l}0.11 \\
0.75 \\
1.11 \\
j .16 \\
0.97 \\
0.70 \\
0.77 \\
0.49\end{array}$ & $\begin{array}{r}9 . .29 \\
9.71 \\
4.12 \\
11.45 \\
7.00 \\
4.16 \\
6.92 \\
5.71 \\
7.53\end{array}$ \\
\hline $\begin{array}{l}1956 \\
1957 \\
1958 \\
1959\end{array}$ & $\begin{array}{l}1.71 \\
0.48 \\
1.74 \\
2.05 \\
0.51\end{array}$ & $\begin{array}{l}0.56 \\
0.23 \\
1.48 \\
1.17 \\
0.58\end{array}$ & $\begin{array}{l}0.10 \\
1.86 \\
0.46 \\
0.40 \\
0.67\end{array}$ & $\begin{array}{l}T \\
0.38 \\
0.64 \\
0.20 \\
0.53\end{array}$ & $\begin{array}{l}0.22 \\
0.82 \\
0.74 \\
0.50 \\
0.71\end{array}$ & $\begin{array}{l}0.86 \\
0.47 \\
0.81 \\
0.23 \\
0.14\end{array}$ & $\begin{array}{l}T \\
0.05 \\
0.02 \\
T \\
T\end{array}$ & $\begin{array}{l}0.38 \\
0.02 \\
T \\
0.03 \\
0.26\end{array}$ & $\begin{array}{l}0.01 \\
0.34 \\
0.05 \\
1.26 \\
0.23\end{array}$ & $\begin{array}{l}1.03 \\
2.72 \\
0.19 \\
0.56 \\
0.23\end{array}$ & $\begin{array}{l}0.15 \\
0.39 \\
0.77 \\
0.41 \\
0.92\end{array}$ & $\begin{array}{l}.58 \\
.53 \\
.84 \\
.26 \\
.64\end{array}$ & $\begin{array}{l}5.60 \\
8.29 \\
8.74 \\
7.07 \\
5.42\end{array}$ \\
\hline $\begin{array}{l}1961 \\
1962 \\
1963 \\
1964 \\
1965\end{array}$ & $\begin{array}{l}0.33 \\
0.13 \\
0.95 \\
0.37 \\
0.93\end{array}$ & $\begin{array}{l}\frac{2.10}{0.90} \\
0.69 \\
0.01 \\
0.14\end{array}$ & $\begin{array}{l}1.02 \\
0.14 \\
0.53 \\
0.03 \\
0.03\end{array}$ & $\begin{array}{l}0.48 \\
0.34 \\
1.17 \\
0.11 \\
0.09\end{array}$ & $\begin{array}{l}0.80 \\
1.35 \\
0.43 \\
0.04 \\
0.15\end{array}$ & $\begin{array}{l}0.42 \\
0.12 \\
0.28 \\
0.90 \\
0.49\end{array}$ & $\begin{array}{l}0.15 \\
T \\
0.31 \\
0.04 \\
0.11\end{array}$ & $\begin{array}{l}.09 \\
.50 \\
.01 \\
.24 \\
.03\end{array}$ & $\begin{array}{l}T \\
0.38 \\
0.02 \\
0.09 \\
0.11\end{array}$ & $\begin{array}{l}0.07 \\
0.95 \\
0.04 \\
0.28 \\
0.01\end{array}$ & $\begin{array}{l}0.49 \\
0.65 \\
0.74 \\
0.94 \\
1.17\end{array}$ & $\begin{array}{l}0.89 \\
0.60 \\
1.14 \\
2.34 \\
0.39\end{array}$ & $\begin{array}{l}6.84 \\
6.06 \\
6.31 \\
5.39 \\
3.65\end{array}$ \\
\hline $\begin{array}{l}1966 \\
1967 \\
1968 \\
1969 \\
1970\end{array}$ & $\begin{array}{l}0.68 \\
0.32 \\
0.88 \\
1.24 \\
2.47 \\
\end{array}$ & $\begin{array}{l}0.03 \\
T \\
0.58 \\
0.54 \\
0.75\end{array}$ & $\begin{array}{l}0.39 \\
0.14 \\
0.02 \\
0.10 \\
0.27\end{array}$ & $\begin{array}{l}0.03 \\
0.90 \\
0.01 \\
1.22 \\
0.45\end{array}$ & $\begin{array}{l}0.05 \\
0.56 \\
0.06 \\
0.51 \\
0.54\end{array}$ & $\begin{array}{l}0.43 \\
0.57 \\
0.19 \\
0.75 \\
0.25\end{array}$ & $\begin{array}{l}0.81 \\
T \\
0.04 \\
T \\
0.01\end{array}$ & $\begin{array}{l}T \\
T \\
0.51 \\
T \\
T\end{array}$ & $\begin{array}{l}0.27 \\
0.05 \\
0.25 \\
0.48\end{array}$ & $\begin{array}{l}0.39 \\
0.13 \\
0.93\end{array}$ & $\begin{array}{l}2.25 \\
0.16 \\
1.23 \\
0.13 \\
0.71\end{array}$ & $\begin{array}{l}0.60 \\
0.43 \\
1.25 \\
1.29 \\
0.61\end{array}$ & $\begin{array}{l}5.9 \\
3.2 \\
5.9 \\
6.3 \\
6.3\end{array}$ \\
\hline $\begin{array}{l}1972 \\
1973 \\
1974 \\
1975\end{array}$ & $\begin{array}{l}0.78 \\
0.19 \\
0.90 \\
0.90 \\
1.70\end{array}$ & $\begin{array}{l}0.10 \\
0.27 \\
0.21 \\
0.41 \\
0.98\end{array}$ & $\begin{array}{l}1.02 \\
0.58 \\
0.08 \\
0.52 \\
0.33\end{array}$ & $\begin{array}{l}0.07 \\
0.10 \\
T \\
0.46 \\
0.42\end{array}$ & $\begin{array}{l}0.56 \\
2.03 \\
0.24 \\
0.28 \\
0.38\end{array}$ & $\begin{array}{l}0.71 \\
0.66 \\
0.01 \\
0.12\end{array}$ & $\begin{array}{l}0.13 \\
0.16 \\
T \\
0.71 \\
0.32\end{array}$ & $\begin{array}{l}0.09 \\
0.56 \\
0.02 \\
T \\
1.16\end{array}$ & $\begin{array}{l}1.13 \\
0.02 \\
0.43 \\
0.01 \\
0.03\end{array}$ & $\begin{array}{l}0.18 \\
T \\
1.72 \\
0.21 \\
0.87\end{array}$ & $\begin{array}{l}0.46 \\
0.55 \\
2.64 \\
0.71 \\
0.60\end{array}$ & $\begin{array}{l}1.07 \\
1.27 \\
2.02 \\
0.97\end{array}$ & $\begin{array}{l}6.3 \\
6.3 \\
8.2 \\
5.3 \\
7.7\end{array}$ \\
\hline $\begin{array}{l}1976 \\
1977 \\
1978 \\
1979 \\
1980\end{array}$ & $\begin{array}{l}0.56 \\
0.08 \\
1.52 \\
0.54 \\
1.32\end{array}$ & $\begin{array}{l}0.36 \\
0.57 \\
0.92 \\
0.17 \\
1.30\end{array}$ & $\begin{array}{l}0.23 \\
0.41 \\
0.30 \\
0.54 \\
0.30\end{array}$ & $\begin{array}{l}0.41 \\
T \\
0.46 \\
0.52 \\
0.86\end{array}$ & $\begin{array}{l}0.08 \\
0.65 \\
0.41 \\
0.10 \\
1.41\end{array}$ & $\begin{array}{l}0.11 \\
0.37 \\
0.09 \\
T \\
0.96\end{array}$ & $\begin{array}{l}0.13 \\
0.06 \\
0.52 \\
0.09 \\
I^{+}\end{array}$ & $\begin{array}{l}0.96 \\
1.36 \\
0.57 \\
0.38 \\
0.02\end{array}$ & $\begin{array}{l}T \\
0.66 \\
0.11 \\
0.20 \\
0.85\end{array}$ & $\begin{array}{l}0.04 \\
0.15 \\
T \\
0.67 \\
0.33\end{array}$ & $\begin{array}{l}\frac{T}{0.63} \\
1.21 \\
1.36 \\
0.44\end{array}$ & $\begin{array}{l}0.11 \\
1.47 \\
0.26 \\
0.99 \\
1.89\end{array}$ & $\begin{array}{l}2.9 \\
6.4 \\
6.3 \\
5.5 \\
9.6\end{array}$ \\
\hline $\begin{array}{l}1981 \\
1982 \\
1983 \\
1984 \\
1985 \\
1986 \\
1987 \\
1988 \\
1989\end{array}$ & $\begin{array}{l}0.56 \\
0.33 \\
1.44 \\
0.23 \\
0.34 \\
1.76 \\
0.80 \\
0.48 \\
0.21 \\
0.77\end{array}$ & $\begin{array}{l}0.60 \\
0.57 \\
1.36 \\
0.94 \\
0.82 \\
1.37 \\
0.19 \\
\frac{T}{1.67}^{1.67} \\
0.09\end{array}$ & $\begin{array}{l}0.70 \\
0.30 \\
1.00 \\
1.01 \\
0.36 \\
0.76 \\
1.05 \\
0.39 \\
1.56 \\
0.10\end{array}$ & $\begin{array}{l}0.02 \\
0.75 \\
0.42 \\
0.60 \\
0.01 \\
\frac{T}{0.14} \\
1.12 \\
0.84 \\
0.40\end{array}+$ & $\begin{array}{l}0.99 \\
0.28 \\
0.52 \\
0.55 \\
0.12 \\
0.30 \\
0.17 \\
0.33\end{array}$ & $\begin{array}{l}0.43 \\
0.75 \\
0.68 \\
0.99 \\
0.15 \\
\frac{T}{0.11} \\
0.11 \\
0.01 \\
0.36\end{array}+$ & $\begin{array}{l}0.19 \\
0.22 \\
0.31 \\
0.06 \\
0.12 \\
0.21 \\
0.50 \\
0.13 \\
0.01 \\
0.14\end{array}$ & $\begin{array}{l}0.03 \\
0.20 \\
0.12 \\
T \\
0.01 \\
0.02 \\
0.07 \\
\frac{0}{0.26} \\
0.83\end{array}+$ & $\begin{array}{l}0.55 \\
0.46 \\
0.42 \\
0.63 \\
0.96 \\
0.01 \\
0.39 \\
0.03\end{array}$ & $\begin{array}{l}0.39 \\
1.33 \\
0.52 \\
0.07 \\
0.46 \\
0.29 \\
\frac{T}{0.01}^{0.42} \\
0.78\end{array}$ & $\begin{array}{l}1.08 \\
0.91 \\
2.12 \\
1.83 \\
1.24 \\
0.65 \\
0.40 \\
0.82 \\
1.04\end{array}$ & $\begin{array}{l}1.79 \\
2.12 \\
0.57 \\
0.84 \\
0.77 \\
1.63 \\
0.40\end{array}$ & $\begin{array}{r}7.0 \\
7.9 \\
11.0 \\
7.2 \\
5.1 \\
7.0 \\
5.0 \\
4.1\end{array}$ \\
\hline & $\begin{array}{l}0.33 \\
0.44 \\
1.30\end{array}$ & $\begin{array}{l}0.94 \\
1.17\end{array}$ & $\begin{array}{l}1.12 \\
0.09 \\
0.67\end{array}$ & $\begin{array}{l}0.45 \\
0.94 \\
0.71\end{array}$ & $\frac{0.49}{0.60}$ & $\begin{array}{l}1.44 \\
1.14 \\
0.12\end{array}$ & $\begin{array}{l}0.29 \\
0.38 \\
1.76 \\
\end{array}$ & $\begin{array}{l}0.07 \\
0.20 \\
0.24\end{array}$ & $\begin{array}{l}0 \\
0.27 \\
0.04\end{array}$ & $\begin{array}{l}0.53 \\
0.61 \\
0.09\end{array}$ & $\begin{array}{l}1.44 \\
1.07 \\
0.19\end{array}$ & $\begin{array}{l}0.40 \\
1.82 \\
0.94\end{array}$ & $\begin{array}{l}6.7 \\
7.9 \\
7.8\end{array}$ \\
\hline RMA & $\begin{array}{l}0.88 \\
0.79\end{array}$ & $\begin{array}{l}0.63 \\
0.62\end{array}$ & $\begin{array}{l}0.50 \\
0.47\end{array}$ & 0.41 & 0.51 & 0.38 & 0.18 & 0.27 & 0.31 & $\begin{array}{l}0.52 \\
0.39\end{array}$ & 0.91 & $\begin{array}{l}0.94 \\
1.03\end{array}$ & 6. \\
\hline
\end{tabular}

* Normal precipitation figures are averages for the period 1961-1990. Greatest and least values for each column are underlined.

+ Most recent of multiple occurrences. 


\section{TABLE 4.2. Seasonal Precipitation (Inches)}

\begin{tabular}{|c|c|c|c|c|}
\hline YEAR & $\begin{array}{l}\text { WINTER+ } \\
\text { DEC-FEB } \\
\end{array}$ & $\begin{array}{l}\text { SPRING } \\
\text { MAR-MAY }\end{array}$ & $\begin{array}{l}\text { SUMMER } \\
\text { JUN-AUG }\end{array}$ & $\begin{array}{l}\text { AUTUMN } \\
\text { SEP-NOV }\end{array}$ \\
\hline $\begin{array}{l}1945 \\
1946 \\
1947 \\
1948 \\
1949 \\
1950\end{array}$ & $\begin{array}{l}0.70 \\
2.80 \\
1.92 \\
3.02\end{array}$ & $\begin{array}{l}-. . \\
1.14 \\
2.73 \\
1.30 \\
1.61\end{array}$ & $\begin{array}{l}2.46 \\
2.26 \\
0.05 \\
2.99 \\
\end{array}$ & $\begin{array}{l}1.83 \\
4.35 \\
1.56 \\
1.80 \\
3.02\end{array}$ \\
\hline $\begin{array}{l}1951 \\
1952 \\
1953 \\
1954 \\
1955\end{array}$ & $\begin{array}{l}2.32 \\
1.85 \\
3.18 \\
2.25 \\
1.13\end{array}$ & $\begin{array}{l}1.42 \\
0.77 \\
1.22 \\
1.07 \\
1.16\end{array}$ & $\begin{array}{l}1.90 \\
1.15 \\
1.51 \\
0.74 \\
0.85\end{array}$ & $\begin{array}{l}1.63 \\
0.32 \\
1.29 \\
1.79 \\
2.71\end{array}$ \\
\hline $\begin{array}{l}1956 \\
1957 \\
1958 \\
1959 \\
1960\end{array}$ & $\begin{array}{l}4.30 \\
1.29 \\
3.75 \\
5.06 \\
1.35\end{array}$ & $\begin{array}{l}0.32 \\
3.06 \\
1.84 \\
1.10 \\
1.91\end{array}$ & $\begin{array}{l}1.24 \\
0.54 \\
0.83 \\
0.26 \\
0.40\end{array}$ & $\begin{array}{l}1.19 \\
3.45 \\
1.01 \\
2.23 \\
1.38\end{array}$ \\
\hline $\begin{array}{l}1961 \\
1962 \\
1963 \\
1964 \\
1965\end{array}$ & $\begin{array}{l}3.07 \\
1.92 \\
2.24 \\
1.52 \\
3.41\end{array}$ & $\begin{array}{l}2.30 \\
1.83 \\
2.13 \\
0.18 \\
0.27\end{array}$ & $\begin{array}{l}0.66 \\
0.62 \\
0.60 \\
1.18 \\
0.63\end{array}$ & $\begin{array}{l}0.56 \\
1.98 \\
0.80 \\
1.31 \\
1.29\end{array}$ \\
\hline $\begin{array}{l}1966 \\
1967 \\
1968 \\
1969 \\
1970\end{array}$ & $\begin{array}{l}1.10 \\
0.92 \\
1.89 \\
3.03 \\
4.51\end{array}$ & $\begin{array}{l}0.47 \\
1.60 \\
0.09 \\
1.83 \\
1.26\end{array}$ & $\begin{array}{l}1.24 \\
0.57 \\
0.74 \\
0.75 \\
0.26\end{array}$ & $\begin{array}{l}2.91 \\
0.34 \\
2.41 \\
0.71 \\
0.98\end{array}$ \\
\hline $\begin{array}{l}1971 \\
1972 \\
1973 \\
1974 \\
1975\end{array}$ & $\begin{array}{l}1.49 \\
1.53 \\
2.38 \\
3.33 \\
3.65\end{array}$ & $\begin{array}{l}1.65 \\
2.71 \\
0.32 \\
1.26 \\
1.13\end{array}$ & $\begin{array}{l}0.93 \\
1.38 \\
0.03 \\
0.83 \\
1.72\end{array}$ & $\begin{array}{l}1.77 \\
0.57 \\
4.79 \\
0.93 \\
1.50\end{array}$ \\
\hline $\begin{array}{l}1976 \\
1977 \\
1978 \\
1979 \\
1980\end{array}$ & $\begin{array}{l}1.62 \\
0.76 \\
3.91 \\
0.97 \\
3.61\end{array}$ & $\begin{array}{l}0.72 \\
1.06 \\
1.17 \\
1.16 \\
2.57\end{array}$ & $\begin{array}{l}1.20 \\
1.79 \\
1.18 \\
0.47 \\
0.98\end{array}$ & $\begin{array}{l}\frac{0.04}{1.44} \\
1.32 \\
2.23 \\
1.62\end{array}$ \\
\hline $\begin{array}{l}1981 \\
1982 \\
1983 \\
1984 \\
1985\end{array}$ & $\begin{array}{l}3.05 \\
2.35 \\
4.59 \\
3.29 \\
1.73\end{array}$ & $\begin{array}{l}1.71 \\
1.33 \\
1.94 \\
2.16 \\
0.49\end{array}$ & $\begin{array}{l}0.65 \\
1.17 \\
1.11 \\
1.05 \\
0.28\end{array}$ & $\begin{array}{l}2.07 \\
2.79 \\
3.10 \\
2.32 \\
2.33\end{array}$ \\
\hline $\begin{array}{l}1986 \\
1987 \\
1988 \\
1989 \\
1990\end{array}$ & $\begin{array}{l}3.97 \\
1.76 \\
2.11 \\
2.28 \\
1.15\end{array}$ & $\begin{array}{l}1.06 \\
1.36 \\
1.84 \\
2.99 \\
1.36\end{array}$ & $\begin{array}{l}0.23 \\
0.68 \\
0.24 \\
0.28 \\
1.33\end{array}$ & $\begin{array}{l}1.90 \\
0.41 \\
1.22 \\
1.48 \\
0.80\end{array}$ \\
\hline $\begin{array}{l}1991 \\
1992 \\
1993\end{array}$ & $\begin{array}{l}1.24 \\
1.78 \\
4.29\end{array}$ & $\begin{array}{l}2.06 \\
1.03 \\
1.98\end{array}$ & $\begin{array}{l}1.80 \\
1.72 \\
2.12\end{array}$ & $\begin{array}{l}1.97 \\
1.95 \\
0.32\end{array}$ \\
\hline $\begin{array}{l}\text { AVG } \\
\star \text { NORMAL }\end{array}$ & $\begin{array}{l}2.45 \\
2.44\end{array}$ & $\begin{array}{l}1.44 \\
1.40\end{array}$ & $\begin{array}{l}1.01 \\
0.83\end{array}$ & $\begin{array}{l}1.70 \\
1.60\end{array}$ \\
\hline
\end{tabular}

* Normal precipitation figures are averages for the period 1961-1990.

Greatest and least values for each column are underlined.

+ For the Winter season. December is included in the previous year. 
IABLE 4.3. Average Number of Days with Precipitation of Specified Amount

\begin{tabular}{|c|c|c|c|c|c|c|}
\hline MONTH & $\begin{array}{l}\text { TRACE OR } \\
\text { MORE } \\
\end{array}$ & $\begin{array}{l}0.01 \text { INCH } \\
\text { OR MORE }\end{array}$ & $\begin{array}{l}0.10 \text { INCH } \\
\text { OR MORE }\end{array}$ & $\begin{array}{l}0.25 \text { INCH } \\
\text { OR MORE }\end{array}$ & $\begin{array}{l}0.50 \text { INCH } \\
\text { OR MORE }\end{array}$ & $\begin{array}{l}1.00 \text { INCH } \\
\text { OR MORE }\end{array}$ \\
\hline Jan & 16 & 9 & 3 & 1 & * & 0 \\
\hline Feb & 12 & 7 & 2 & $i$ & 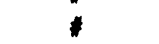 & 0 \\
\hline Mar & 11 & 6 & 2 & 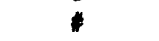 & 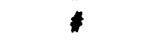 & 0 \\
\hline Apr & 10 & 5 & 2 & $*$ & 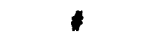 & 0 \\
\hline May & 10 & 5 & 2 & 1 & 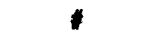 & 0 \\
\hline Jun & 9 & 5 & 2 & 1 & 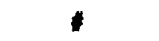 & * \\
\hline Jul & 5 & 2 & $i$ & 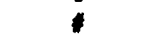 & 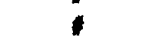 & 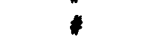 \\
\hline Aug & 6 & 3 & 1 & 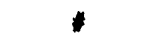 & 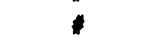 & 0 \\
\hline Sep & 6 & 3 & 1 & 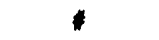 & 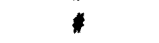 & 0 \\
\hline oct & 9 & 5 & 2 & 1 & 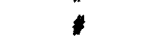 & 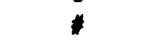 \\
\hline Nov & 14 & 8 & 3 & 1 & 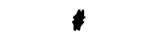 & 0 \\
\hline Dec & 17 & 10 & 3 & 1 & 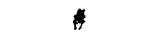 & 0 \\
\hline Annual & 125 & 68 & 24 & 7 & $1^{\star}$ & $\hbar$ \\
\hline
\end{tabular}

* Although the number of days with 0.50 inch or more averages less than $1 / 2$ day for any one month, 61 such days have been recorded during 48 years of record.

* Used to denote an average of less than $1 / 2$ day.

\subsection{TOTAL TIME WITH PRECIPITATION OBSERVED}

The total time during which precipitation was observed at the HMS includes all types of precipitation. These data are presented in Table 4.4. No record was kept for the hours 1600 through 2400 from July 1971 through June 1974; therefore, a 3-year gap exists in the record for those hours.

TABLE 4.4. Monthly and Annual Averages and Extremes in Total Time with Precipitation Observed: July 1946 Through June 1971, July 1974 Through December 1993

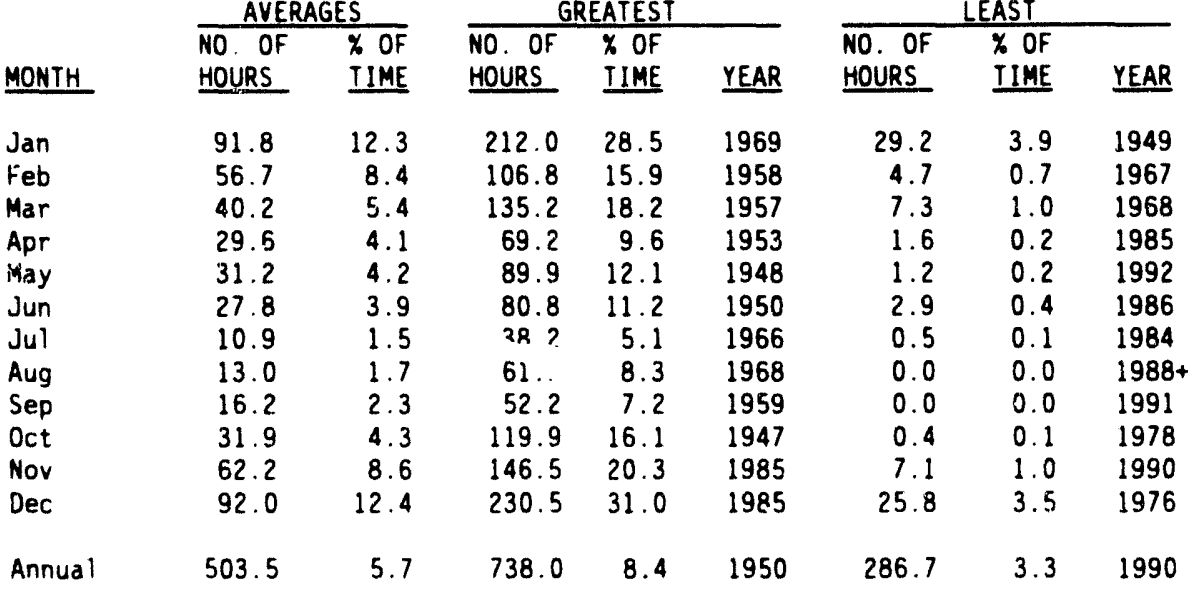

+ Most recent of several occurrences. 
The months of November through February, which contribute more than half of the annual precipitation, have received precipitation more than $10 \%$ of the time (10.4\%), more than three times greater than the other eight months of the year $(3.4 \%)$.

\subsection{NOTABLE WET PERIODS}

Seven periods are listed when precipitation was abnormally high:

\begin{tabular}{|c|c|c|c|c|c|}
\hline \multirow[b]{2}{*}{ PERIOD } & \multirow{2}{*}{\multicolumn{3}{|c|}{$\frac{\text { NÜ̈rīEE OF OF DAYS WITH TRACE OR MORE }}{\text { GREATEST MEASURABLE }}$}} & \multicolumn{2}{|c|}{$\begin{array}{l}\text { TOTAL AMOUNT } \\
\text { (INCHES) } \\
\end{array}$} \\
\hline & & & $\begin{array}{l}\text { MEASURABLE } \\
\text { PRECIP. }\end{array}$ & $\begin{array}{l}\text { WATER } \\
\text { EQUIV. }\end{array}$ & $\begin{array}{l}\text { SNOW- } \\
\text { FALL }\end{array}$ \\
\hline Oct 7 - Nov 4, 1947 & 23 out of 29 & 10 & 17 & 2.21 & 0 \\
\hline $\operatorname{Jan} 3-28,1950$ & 21 out of 26 & 10 & 15 & 1.80 & 23.4 \\
\hline Nov 11 - Dec 19,1950 & 33 out of 39 & 12 & 20 & 1.37 & 3.7 \\
\hline Nov 16 - Dec 22, 1955 & 31 out of 37 & 15 & 24 & 3.19 & 22.7 \\
\hline Oct 31-Dec 7, 1973 & 32 out of 38 & 14 & 20 & 3.45 & 8.1 \\
\hline Nov $15-\operatorname{Dec} 7,1985$ & 17 out of 23 & 8 & 14 & 1.96 & 25.2 \\
\hline $\begin{array}{l}\text { Dec } 27,1992- \\
\operatorname{Jan} 23,1993\end{array}$ & 26 out of 29 & 12 & 19 & 2.02 & 26.8 \\
\hline
\end{tabular}

From a precipitation standpoint, 1973 was an unusual year. Total precipitation for the year was 8.27 inches or $132 \%$ of normal (6.25 inches). The period from March 30 through September 18 was extremely dry, receiving only 0.29 inch of precipitation during that 173-day period, while the period from October 31 through December 7 was a notable wet period (see previous table). During the months of October, November, and December 1973, 6.38 inches of precipitation were recorded, $289 \%$ of normal (2.21 inches) for those months.

\subsection{NOTABLE DRY PERIODS}

The HMS is in a semi-arid region, thus it experiences many dry periods. January, March, and December are the only months that have always received measurable precipitation (1946-1993). A total of 37 months during the period of record have been without measurable precipitation, with the months of July 
and August accounting for 20 of those months. The record number of consecutive days with zero precipitation (not even a trace) occurred in 1988, when the period from July 14 through September 17 (66 days) was totally dry. The following list indicates some long periods with small amounts of precipitation.

NOTABLE DRY PERIODS

\begin{tabular}{|c|c|c|c|c|}
\hline YEAR & FROM & TO & $\begin{array}{l}\text { NUMBER } \\
\text { OF DAYS } \\
\end{array}$ & $\begin{array}{l}\text { TOTAL PRECIP. } \\
\text { (INCHES) }\end{array}$ \\
\hline 1952 & Jun 30 & Nov 10 & 134 & 0.20 \\
\hline 1967 & Jun 22 & Nov 7 & 139 & 0.18 \\
\hline 1968 & Feb 24 & Aug 13 & 172 & 0.32 \\
\hline 1973 & $\operatorname{Mar} 30$ & Sep 18 & 173 & 0.29 \\
\hline 1976 & Aug 26 & Dec 31 & 128 & 0.15 \\
\hline 1985 & Mar 31 & Sep 7 & 161 & 0.43 \\
\hline 1986 & May 6 & Sep 12 & 129 & 0.30 \\
\hline 1987 & JuT 19 & Oct 31 & 105 & 0.08 \\
\hline 1988 & Jun 6 & Sep 17 & 105 & 0.13 \\
\hline
\end{tabular}

The driest year on record (1946 to 1993) was 1976. Total precipitation for that year was 2.99 inches, less than half of normal. During the period from September through December, the total precipitation was 0.15 inch, which was $6 \%$ of normal (2.52 inches) for those months.

\subsection{SNOWFALL}

Snowfall, which includes all frozen precipitation, has varied from a seasonal total of 0.3 inch in 1957-58 to 56.1 inches in 1992-93. Table 4.5 provides information on monthly and seasonal snowfall amounts, as well as the dates and amounts of earliest and latest snowfall each season. The earliest measurable snowfall (0.3 inch) was recorded on October 26, 1957, while the latest measurable snowfall (1.0 inch) was recorded on April 6, 1982. The average date of the first measurable snow is November 30 , and the last measurable snow date is February 13. Normal snowfall for the period 1961 to 1990, and averages for the entire period of record are indicated at the bottom of the table. Monthly and seasonal extremes are underlined in each column. 
TABLE 4.5. Monthly and Seasonal Snowfall (Inches) Including First and Last Dates of Both Trace and Measurable Snowfalls

\begin{tabular}{|c|c|c|c|c|c|c|c|c|c|c|c|c|c|c|c|c|}
\hline SEASON & $\underline{O C T}$ & NOV & $\underline{D E C}$ & JAN & FEB & MAR & APR & TOTAL & \multicolumn{2}{|c|}{ FIRST } & \multicolumn{2}{|c|}{$\begin{array}{c}\text { FIRST } \\
\text { MEASURABLE }\end{array}$} & \multicolumn{2}{|c|}{$\begin{array}{c}\text { LAST } \\
\text { MEASURABLE } \\
\text { DATE AMT }\end{array}$} & \multicolumn{2}{|c|}{$\begin{array}{c}\text { LAST } \\
\text { DATE AMT }\end{array}$} \\
\hline $\begin{array}{l}1945-46 \\
1946-47 \\
1947-48 \\
1948-49 \\
1949-50\end{array}$ & $\begin{array}{l}--- \\
T \\
0 \\
0 \\
T\end{array}$ & $\begin{array}{c}7.2 \\
i \\
1.7 \\
0\end{array}$ & $\begin{array}{l}--. \\
0.5 \\
3.0 \\
8.1 \\
0.7\end{array}$ & $\begin{array}{r}-.- \\
3.3 \\
2.6 \\
1.8 \\
23.4 \\
\end{array}$ & $\begin{array}{l}-.- \\
\top \\
5.5 \\
6.9 \\
3.1\end{array}$ & $\begin{array}{c}T^{\top} \\
0.1 \\
T \\
1.5\end{array}$ & $\begin{array}{l}--- \\
0 \\
T \\
0 \\
T\end{array}$ & $\begin{array}{l}11.0 \\
11.0 \\
18.2 \\
28.7 \\
28.7\end{array}$ & $\begin{array}{l}11 / 14 \\
11 / 7 \\
10 / 18\end{array}$ & $\begin{array}{l}T \\
T \\
T\end{array}$ & $\begin{array}{l}11 / 17^{-} \\
12 / 3 \\
11 / 18 \\
12 / 16\end{array}$ & $\begin{array}{l}- \\
0.2 \\
0.1 \\
0.2 \\
0.1\end{array}$ & $\begin{array}{l}1 / 31 \\
3 / 9 \\
2 / 21 \\
3 / 13\end{array}$ & $\begin{array}{l}- \\
2.2 \\
0.1 \\
0.2 \\
0.3\end{array}$ & $\begin{array}{l}4 / 7 \\
3 / 15 \\
4 / 2\end{array}$ & $\begin{array}{l}T \\
T \\
T\end{array}$ \\
\hline $\begin{array}{l}1950-51 \\
1951-52 \\
1952-53 \\
1953-54 \\
1954-55\end{array}$ & $\begin{array}{l}0 \\
0 \\
0 \\
0 \\
0\end{array}$ & $\begin{array}{l}0.8 \\
0.5 \\
T \\
0 \\
0\end{array}$ & $\begin{array}{l}2.9 \\
4.4 \\
3.1 \\
1.0 \\
1.8\end{array}$ & $\begin{array}{r}5.3 \\
7.5 \\
2.7 \\
14.3 \\
6.0\end{array}$ & $\begin{array}{c}5.3 \\
3.1 \\
0 \\
1.6 \\
2.4\end{array}$ & $\begin{array}{c}4.2 \\
T \\
T \\
T \\
0.7\end{array}$ & $\begin{array}{l}0 \\
0 \\
0 \\
0 \\
T\end{array}$ & $\begin{array}{r}18.5 \\
15.5 \\
5.8 \\
16.9 \\
10.9\end{array}$ & $\begin{array}{l}11 / 19 \\
11 / 22 \\
12 / 3\end{array}$ & $\begin{array}{l}T \\
T\end{array}$ & $\begin{array}{l}11 / 30 \\
11 / 25 \\
12 / 1 \\
12 / 8 \\
12 / 4\end{array}$ & $\begin{array}{l}0.8 \\
0.5 \\
0.3 \\
1.0 \\
1.8\end{array}$ & $\begin{array}{l}3 / 12 \\
2 / 24 \\
1 / 2 \\
2 / 11 \\
3 / 25\end{array}$ & $\begin{array}{l}1.1 \\
0.1 \\
2.7 \\
1.6 \\
0.7\end{array}$ & $\begin{array}{l}3 / 20 \\
3 / 31 \\
3 / 10 \\
4 / 2\end{array}$ & $\begin{array}{l}T \\
T \\
T \\
T\end{array}$ \\
\hline $\begin{array}{l}1955-56 \\
1956-57 \\
1957-58 \\
1958-59 \\
1959-60\end{array}$ & $\begin{array}{c}0 \\
T \\
0.3 \\
0 \\
0\end{array}$ & $\begin{array}{c}12.7 \\
0.1 \\
0 \\
T \\
0.3\end{array}$ & $\begin{array}{c}13.4 \\
2.5 \\
T \\
0.9 \\
1.0\end{array}$ & $\begin{array}{c}10.2 \\
7.9 \\
T \\
4.5 \\
5.9\end{array}$ & $\begin{array}{c}2.2 \\
1.4 \\
0 \\
12.7 \\
T\end{array}$ & $\begin{array}{c}T \\
4.0 \\
T \\
0 \\
1.5\end{array}$ & $\begin{array}{l}0 \\
1 \\
0 \\
0 \\
0\end{array}$ & $\begin{array}{r}38.5 \\
15.9 \\
0.3 \\
18.1 \\
8.7\end{array}$ & $\begin{array}{l}10 / 26 \\
11 / 14 \\
11 / 4\end{array}$ & $\begin{array}{l}T \\
T\end{array}$ & $\begin{array}{l}11 / 2 \\
11 / 26 \\
10 / 26 \\
12 / 6 \\
11 / 15\end{array}$ & $\begin{array}{l}0.2 \\
0.1 \\
0.3 \\
0.4 \\
0.1\end{array}$ & $\begin{array}{l}2 / 23 \\
3 / 6 \\
10 / 26 \\
2 / 19 \\
3 / 5\end{array}$ & $\begin{array}{l}0.1 \\
1.7 \\
0.3 \\
1.2 \\
1.4\end{array}$ & $\begin{array}{l}3 / 26 \\
3 / 12 \\
3 / 16\end{array}$ & $\begin{array}{l}T \\
T \\
T\end{array}$ \\
\hline $\begin{array}{l}1960-61 \\
1961-62 \\
1962-63 \\
1963-64 \\
1964-65\end{array}$ & $\begin{array}{l}0 \\
0 \\
0 \\
0 \\
0\end{array}$ & $\begin{array}{c}0 \\
0.5 \\
T \\
T \\
0.1\end{array}$ & $\begin{array}{c}3.3 \\
\frac{6.1}{T}+ \\
\frac{6.4}{19.1}\end{array}$ & $\begin{array}{l}1.9 \\
0.4 \\
7.1 \\
2.9 \\
6.6\end{array}$ & $\begin{array}{c}0 \\
2.4 \\
0.6 \\
T \\
T\end{array}$ & $\begin{array}{c}1.6 \\
0.9 \\
0 \\
T \\
T\end{array}$ & $\begin{array}{l}0 \\
0 \\
0 \\
T \\
0\end{array}$ & $\begin{array}{r}6.8 \\
10.3 \\
7.7 \\
9.3 \\
25.8\end{array}$ & $\begin{array}{l}12 / 9 \\
11 / 18 \\
11 / 29 \\
11 / 19 \\
11 / 21\end{array}$ & $\begin{array}{l}T \\
T \\
T \\
T \\
T\end{array}$ & $\begin{array}{l}12 / 10 \\
11 / 23 \\
1 / 30 \\
12 / 8 \\
11 / 29\end{array}$ & $\begin{array}{l}0.1 \\
0.1 \\
0.4 \\
4.3 \\
0.1\end{array}$ & $\begin{array}{l}3 / 5 \\
3 / 9 \\
2 / 1 \\
1 / 24 \\
1 / 23\end{array}$ & $\begin{array}{l}1.6 \\
0.1 \\
0.6 \\
1.5 \\
3.1\end{array}$ & $\begin{array}{l}3 / 11 \\
2 / 13 \\
3 / 22 \\
3 / 27\end{array}$ & $\begin{array}{l}T \\
T \\
T \\
T\end{array}$ \\
\hline $\begin{array}{l}1965-66 \\
1966-67 \\
1967-68 \\
1968-69 \\
1969-70\end{array}$ & $\begin{array}{l}0 \\
0 \\
0 \\
0 \\
0\end{array}$ & $\begin{array}{c}T \\
0.4 \\
0 \\
T \\
T\end{array}$ & $\begin{array}{l}6.9 \\
2.8 \\
5.7 \\
9.7 \\
2.7\end{array}$ & $\begin{array}{r}2.6 \\
0.1 \\
4.5 \\
15.9 \\
6.6\end{array}$ & $\begin{array}{c}T \\
0 \\
0.3 \\
2.1 \\
T\end{array}$ & $\begin{array}{l}T \\
0 \\
0 \\
0 \\
0.2\end{array}$ & $\begin{array}{l}0 \\
0 \\
T \\
0 \\
0\end{array}$ & $\begin{array}{r}9.5 \\
3.3 \\
10.5 \\
27.7 \\
9.5\end{array}$ & $\begin{array}{l}11 / 23 \\
12 / 6 \\
11 / 16 \\
11 / 29\end{array}$ & $\begin{array}{l}T \\
T \\
T\end{array}$ & $\begin{array}{l}12 / 23 \\
11 / 11 \\
12 / 9 \\
12 / 19 \\
12 / 8\end{array}$ & $\begin{array}{l}0.6 \\
0.2 \\
0.6 \\
0.1 \\
1.3\end{array}$ & $\begin{array}{l}1 / 22 \\
1 / 26 \\
2 / 17 \\
2 / 23 \\
3 / 1\end{array}$ & $\begin{array}{l}0.2 \\
0.1 \\
0.3 \\
2.0 \\
0.2\end{array}$ & $\begin{array}{l}3 / 21 \\
4 / 16 \\
2 / 28\end{array}$ & $\begin{array}{l}\mathrm{T} \\
\mathrm{T}\end{array}$ \\
\hline $\begin{array}{l}1970-71 \\
1971-72 \\
1972-73 \\
1973-74 \\
1974-75\end{array}$ & $\begin{array}{c}0 \\
0.6 \\
0 \\
\frac{1.5}{0}\end{array}$ & $\begin{array}{c}0.5 \\
T \\
T \\
6.6 \\
0\end{array}$ & $\begin{array}{l}4.4 \\
8.1 \\
7.2 \\
7.5 \\
0.7\end{array}$ & $\begin{array}{l}2.0 \\
4.9 \\
4.3 \\
3.9 \\
2.5\end{array}$ & $\begin{array}{c}T \\
1.4 \\
1.7 \\
0 \\
12.1\end{array}$ & $\begin{array}{c}0.6 \\
0.1 \\
0 \\
T \\
T\end{array}$ & $\begin{array}{l}0 \\
T \\
0 \\
0 \\
T\end{array}$ & $\begin{array}{r}7.5 \\
15.1 \\
13.2 \\
19.5 \\
15.3\end{array}$ & $\begin{array}{l}11 / 22 \\
11 / 27 \\
12 / 2\end{array}$ & $\begin{array}{l}T \\
T \\
T\end{array}$ & $\begin{array}{l}11 / 30 \\
11 / 29 \\
12 / 3 \\
10 / 31 \\
12 / 12\end{array}$ & $\begin{array}{l}0.5 \\
0.1 \\
1.7 \\
1.5 \\
0.3\end{array}$ & $\begin{array}{l}3 / 14 \\
2 / 5 \\
2 / 10 \\
1 / 12 \\
2 / 9\end{array}$ & $\begin{array}{l}0.1 \\
0.1 \\
1.7 \\
2.3 \\
1.7\end{array}$ & $\begin{array}{l}3 / 22 \\
4 / 12 \\
2 / 13 \\
3 / 6 \\
4 / 4\end{array}$ & $\begin{array}{l}T \\
T \\
T \\
T \\
T\end{array}$ \\
\hline
\end{tabular}


IABLE 4.5. (contd)

\begin{tabular}{|c|c|c|c|c|c|c|c|c|c|c|c|c|c|c|c|c|}
\hline \multirow[b]{2}{*}{ SEASON } & \multirow[b]{2}{*}{$\underline{O C T}$} & \multirow[b]{2}{*}{ NOV } & \multirow[b]{2}{*}{$\underline{\mathrm{DEC}}$} & \multirow[b]{2}{*}{$\underline{\text { JAN }}$} & \multirow[b]{2}{*}{ FEB } & \multirow[b]{2}{*}{$\underline{\text { MAR }}$} & \multirow[b]{2}{*}{$\underline{\text { APR }}$} & \multirow[b]{2}{*}{ TOTAL } & \multicolumn{2}{|c|}{ FIRST } & \multicolumn{2}{|c|}{$\begin{array}{c}\text { FIRST } \\
\text { MEASURABLE }\end{array}$} & \multicolumn{2}{|c|}{$\begin{array}{c}\text { LAST } \\
\text { MEASURABLE }\end{array}$} & \multicolumn{2}{|c|}{ LAST } \\
\hline & & & & & & & & & DATE & AMT & DATE & AMT & DATE & $\underline{A M T}$ & DATE & AMI \\
\hline $\begin{array}{l}1975-76 \\
1976-77 \\
1977-78 \\
1978-79 \\
1979-80\end{array}$ & $\begin{array}{l}0 \\
0 \\
0 \\
0 \\
0\end{array}$ & $\begin{array}{c}1.7 \\
0 \\
2.1 \\
10.1 \\
5.6\end{array}$ & $\begin{array}{l}3.8 \\
0.2 \\
3.4 \\
1.4 \\
7.3\end{array}$ & $\begin{array}{r}6.0 \\
2.9 \\
2.9 \\
10.3 \\
8.7\end{array}$ & $\begin{array}{l}0.2 \\
T \\
0.9 \\
0.5 \\
4.5\end{array}$ & $\begin{array}{c}T \\
T \\
T \\
0.1 \\
0.3\end{array}$ & $\begin{array}{l}T \\
0 \\
0 \\
0 \\
0\end{array}$ & $\begin{array}{r}11.7 \\
3.1 \\
9.3 \\
22.6 \\
26.2\end{array}$ & $\begin{array}{l}12 / 4 \\
11 / 15 \\
11 / 15\end{array}$ & $\begin{array}{l}T \\
T \\
T\end{array}$ & $\begin{array}{l}11 / 10 \\
12 / 23 \\
11 / 18 \\
11 / 18 \\
11 / 22\end{array}$ & $\begin{array}{l}0.6 \\
0.2 \\
0.1 \\
5.3 \\
1.4\end{array}$ & $\begin{array}{l}2 / 3 \\
1 / 31 \\
2 / 26 \\
3 / 3 \\
3 / 5\end{array}$ & $\begin{array}{l}0.2 \\
0.2 \\
0.1 \\
0.1 \\
0.3\end{array}$ & $\begin{array}{l}4 / 1 \\
3 / 27 \\
3 / 5\end{array}$ & $\begin{array}{l}T \\
T \\
T\end{array}$ \\
\hline $\begin{array}{l}1980-81 \\
1981-82 \\
1982-83 \\
1983-84 \\
1984-85\end{array}$ & $\begin{array}{l}0 \\
0 \\
0 \\
0 \\
T\end{array}$ & $\begin{array}{c}0.3 \\
0 \\
0.2 \\
1 \\
4.9\end{array}$ & $\begin{array}{r}2.2 \\
12.1 \\
4.6 \\
17.8 \\
5.8\end{array}$ & $\begin{array}{c}T \\
2.4 \\
3.2 \\
1.5 \\
1.3\end{array}$ & $\begin{array}{c}T \\
T \\
2.3 \\
T \\
8.5\end{array}$ & $\begin{array}{c}0 \\
\mathrm{~T} \\
0 \\
0 \\
1.4\end{array}$ & $\begin{array}{l}0 \\
1.0 \\
0 \\
0 \\
0\end{array}$ & $\begin{array}{r}2.5 \\
15.5 \\
10.3 \\
19.3 \\
21.9\end{array}$ & $\begin{array}{l}12 / 3 \\
11 / 12 \\
11 / 28 \\
10 / 23\end{array}$ & $\begin{array}{l}T \\
T \\
T \\
T\end{array}$ & $\begin{array}{l}11 / 14 \\
12 / 13 \\
11 / 26 \\
12 / 2 \\
11 / 24\end{array}$ & $\begin{array}{l}0.3 \\
2.5 \\
0.2 \\
0.5 \\
0.2\end{array}$ & $\begin{array}{l}12 / 6 \\
4 / 6 \\
2 / 9 \\
1 / 21 \\
3 / 4\end{array}$ & $\begin{array}{l}0.3 \\
1.0 \\
0.3 \\
1.5 \\
1.4\end{array}$ & $2 / 13$ & $T$ \\
\hline $\begin{array}{l}1985-86 \\
1986-87 \\
1987-88 \\
1988-89 \\
1989-90\end{array}$ & $\begin{array}{l}0 \\
0 \\
0 \\
0 \\
0\end{array}$ & $\begin{array}{c}\frac{18.3}{0} \\
1.1 \\
0 \\
0\end{array}$ & $\begin{array}{l}7.6 \\
5.1 \\
4.7 \\
3.5 \\
1.4\end{array}$ & $\begin{array}{l}2.7 \\
3.3 \\
5.6 \\
0.2 \\
0.6\end{array}$ & $\begin{array}{c}5.5 \\
0 \\
0 \\
17.0 \\
0.7\end{array}$ & $\begin{array}{c}0 \\
0 \\
0 \\
3.1 \\
T\end{array}$ & $\begin{array}{c}0 \\
0 \\
0.2 \\
T \\
0\end{array}$ & $\begin{array}{r}34.1 \\
8.4 \\
11.6 \\
23.8 \\
2.7\end{array}$ & $12 / 25$ & $T$ & $\begin{array}{l}11 / 10 \\
12 / 4 \\
11 / 30 \\
12 / 18 \\
12 / 26\end{array}$ & $\begin{array}{l}0.6 \\
0.4 \\
1.1 \\
0.3 \\
0.3\end{array}$ & $\begin{array}{l}2 / 21 \\
1 / 26 \\
1 / 20 \\
3 / 5 \\
2 / 17\end{array}$ & $\begin{array}{l}0.9 \\
0.1 \\
0.1 \\
0.2 \\
0.2\end{array}$ & $\begin{array}{l}1 / 31 \\
5 / 18\end{array}$ & $\begin{array}{l}T \\
T\end{array}$ \\
\hline $\begin{array}{l}1990-91 \\
1991-92 \\
1992-93 \\
1993-94\end{array}$ & $\begin{array}{c}0 \\
1.2 \\
0 \\
0 \\
\end{array}$ & $\begin{array}{l}\frac{0}{T}^{+} \\
2.1 \\
1.4\end{array}$ & $\begin{array}{r}6.1 \\
0.6 \\
21.0 \\
1.8\end{array}$ & $\begin{array}{r}3.8 \\
0.3 \\
17.1 \\
0 \\
\end{array}$ & $\begin{array}{c}\frac{0}{T}+ \\
12.4 \\
0.9\end{array}$ & $\begin{array}{c}0.1 \\
0 \\
3.5 \\
0 \\
\end{array}$ & $\begin{array}{l}0 \\
0 \\
0 \\
0 \\
\end{array}$ & $\begin{array}{r}10.0 \\
2.1 \\
56.1 \\
4.1\end{array}$ & & & $\begin{array}{l}12 / 18 \\
10 / 28 \\
11 / 21 \\
11 / 22\end{array}$ & $\begin{array}{l}0.1 \\
0.8 \\
0.2 \\
0.6\end{array}$ & $\begin{array}{l}3 / 2 \\
1 / 5 \\
3 / 3 \\
2 / 26\end{array}$ & $\begin{array}{l}0.1 \\
0.3 \\
1.5 \\
0.3\end{array}$ & $\begin{array}{l}2 / 7 \\
3 / 16\end{array}$ & $\begin{array}{l}T \\
T\end{array}$ \\
\hline $\begin{array}{l}\text { AVE } \\
\text { *NORMAL }\end{array}$ & $\begin{array}{l}0.1 \\
0.1\end{array}$ & $\begin{array}{l}1.5 \\
1.7\end{array}$ & $\begin{array}{l}5.3 \\
5.7\end{array}$ & $\begin{array}{l}5.0 \\
3.9\end{array}$ & $\begin{array}{l}2.5 \\
2.0\end{array}$ & $\begin{array}{l}0.5 \\
0.3\end{array}$ & $\begin{array}{l}T \\
T\end{array}$ & $\begin{array}{l}15.0 \\
13.8\end{array}$ & $\begin{array}{l}11 / 20 \\
11 / 24\end{array}$ & & $\begin{array}{l}11 / 30 \\
12 / 3\end{array}$ & & $\begin{array}{l}2 / 13 \\
2 / 12\end{array}$ & & $\begin{array}{l}3 / 11 \\
3 / 8\end{array}$ & \\
\hline
\end{tabular}

* Normal snowfall figures are averages for 1961-1990.

Greatest and least values for each column are underlined.

+ Most recent of multiple occurrences. 
Table 4.6 lists some miscellaneous snowfall statistics for the HMS for the period from 1946 through 1993. Included in this table are average number of days per month with snow depth above certain threshold values, greatest number of days per month with snow depth above certain threshold values, record number of consecutive days with snow depth above certain threshold values, and record monthly snow depth and 24-hr snowfall amounts. The record snow depth at the HMS is 15.6 inches, recorded in December 1985. The record number of days with snow depth $\geq 6$ inches was 43 days in the winter of 1992-1993.

\subsection{NORMAL AND MAXIMUM DAILY PRECIPITATION}

Table 4.7 contains normal and maximum values of precipitation (minimum values are not needed, because every day of the year has a minimum value of zero). The normal precipitation values are based upon the period from 1961 through 1990, while the daily maximum values are for the entire period of record (1945 through 1993). The maximum daily value for each month is underlined.

Only four days during the year received no precipitation (not even a trace) during the entire period of record. Those days are July 23 and September 6,7 , and 24 . The days with the most measurable precipitation events are December 15 and 19, with 15 events each. Climatologically speaking, the wettest period of the year is from December 14 through 24, each day with a normal precipitation value of $0.04 \mathrm{inch}$, and the driest period is from July 23 through August 11, with each day having a normal value of only a trace. October 1, 1957 recorded the greatest 1-day precipitation, 1.60 inches. 
TABLE 4.6. Miscellaneous Snowfall Statistics, 1946 Through 1993

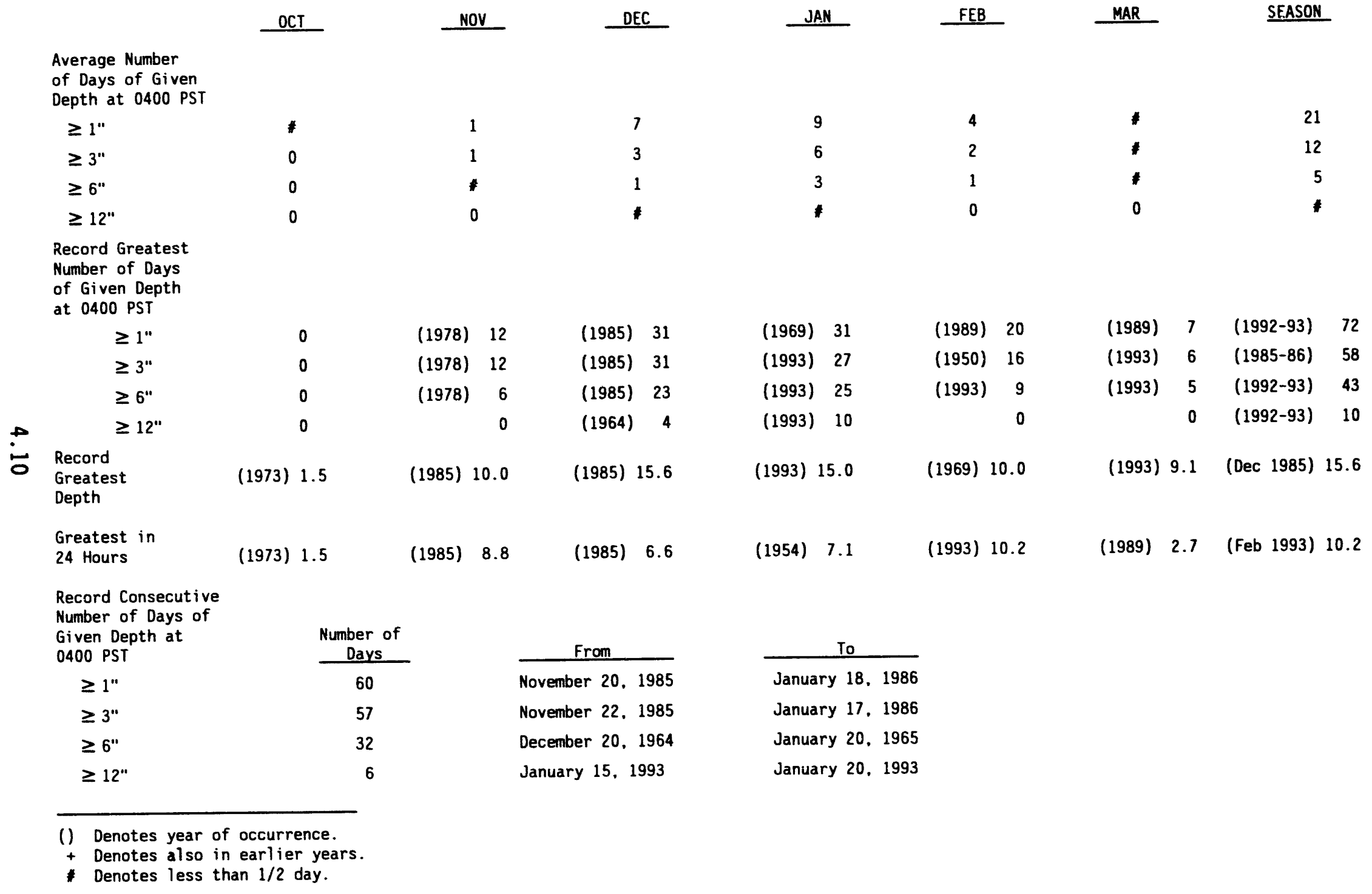


TABLE 4.7. Normal and Maximum Daily Precipitation (Inches)

\begin{tabular}{|c|c|c|c|c|c|}
\hline \multicolumn{5}{|c|}{$\begin{array}{c}\text { NORMAL PERIOD } \\
(1961-1990) \\
\end{array}$} & $\begin{array}{l}\text { HISTORICAL PERIOD } \\
(1945-1993) \\
\end{array}$ \\
\hline & & NUMBER OF YEARS & & & \\
\hline
\end{tabular}

\begin{tabular}{|c|c|c|c|c|c|c|c|}
\hline \multicolumn{8}{|c|}{ JANUARY } \\
\hline 1 & 0.02 & 5 & 10 & 0.20 & 1987 & 0.20 & 1987 \\
\hline 2 & 0.02 & 9 & 9 & 0.17 & 1983 & 0.27 & 1953 \\
\hline 3 & 0.02 & 8 & 7 & 0.28 & 1966 & 0.28 & 1966 \\
\hline 4 & 0.02 & 7 & 10 & 0.17 & 1976 & 0.25 & 1956 \\
\hline 5 & 0.02 & 9 & 8 & 0.23 & 1966 & 0.23 & 1966 \\
\hline 6 & 0.02 & 4 & 8 & 0.50 & 1983 & 0.87 & 1948 \\
\hline 7 & 0.02 & 8 & 5 & 0.31 & 1990 & 0.31 & 1990 \\
\hline 8 & 0.02 & 7 & 9 & 0.25 & 1978 & 0.59 & 1953 \\
\hline 9 & 0.02 & 8 & 9 & 0.20 & $1980+$ & 0.24 & 1953 \\
\hline 10 & 0.03 & 10 & 5 & 0.20 & $1979+$ & 0.21 & 1951 \\
\hline 11 & 0.03 & 4 & 10 & 0.17 & 1980 & 0.48 & 1959 \\
\hline 12 & 0.03 & 9 & 10 & 0.32 & 1973 & 0.58 & 1958 \\
\hline 13 & 0.03 & 10 & 8 & 0.33 & 1980 & 0.37 & 1950 \\
\hline 14 & 0.03 & 10 & 3 & 0.43 & 1978 & 0.43 & 1978 \\
\hline 15 & 0.03 & 7 & 5 & 0.18 & 1968 & 0.34 & 1956 \\
\hline 16 & 0.03 & 10 & 6 & 0.53 & 1971 & 0.70 & 1954 \\
\hline 17 & 0.02 & 5 & 7 & 0.10 & 1986 & 0.18 & 1958 \\
\hline 18 & 0.02 & 7 & 8 & 0.25 & 1974 & 0.25 & 1974 \\
\hline 19 & 0.02 & 8 & 1 & 0.32 & 1970 & 0.39 & 1950 \\
\hline 20 & 0.02 & 5 & 6 & 0.26 & 1985 & 0.32 & 1953 \\
\hline 21 & 0.02 & 6 & 5 & 0.15 & 1984 & 0.16 & 1954 \\
\hline 22 & 0.02 & 6 & 7 & 0.54 & 1970 & 0.54 & 1970 \\
\hline 23 & 0.03 & 8 & 9 & 0.27 & 1965 & 0.27 & 1965 \\
\hline 24 & 0.03 & 8 & 6 & 0.22 & 1970 & 0.22 & 1970 \\
\hline 25 & 0.03 & 6 & 6 & 0.72 & 1975 & 0.72 & 1975 \\
\hline 26 & 0.03 & 8 & 4 & 0.36 & 1970 & 0.36 & 1970 \\
\hline 27 & 0.03 & 6 & 6 & 0.20 & 1981 & 0.32 & 1954 \\
\hline 28 & 0.03 & 6 & 7 & 0.19 & 1986 & 0.19 & 1986 \\
\hline 29 & 0.03 & 9 & 4 & 0.31 & 1965 & 0.33 & 1958 \\
\hline 30 & 0.03 & 8 & 8 & 0.12 & 1986 & 0.12 & 1986 \\
\hline 31 & 0.03 & 9 & 10 & 0.94 & 1963 & $\underline{0.94}$ & $\underline{1963}$ \\
\hline \multicolumn{8}{|c|}{ FEBRUARY } \\
\hline 1 & 0.03 & 8 & 4 & 0.72 & 1961 & 0.72 & 1961 \\
\hline 2 & 0.02 & 5 & 8 & 0.26 & 1963 & 0.26 & 1963 \\
\hline 3 & 0.02 & 5 & 6 & 0.06 & 1990 & 0.12 & 1950 \\
\hline 4 & 0.02 & 5 & 5 & 0.28 & 1975 & 0.28 & 1975 \\
\hline 5 & 0.02 & 7 & 7 & 0.07 & 1978 & 0.15 & 1953 \\
\hline 6 & 0.02 & 10 & 2 & 0.18 & 1961 & 0.18 & 1961 \\
\hline 7 & 0.02 & 5 & 4 & 0.27 & 1985 & 0.27 & 1985 \\
\hline 8 & 0.02 & 5 & 8 & 0.12 & 1985 & 0.12 & 1985 \\
\hline 9 & 0.02 & 9 & 5 & 0.37 & 1962 & 0.43 & 1959 \\
\hline 10 & 0.02 & 5 & 5 & 0.64 & 1961 & 0.64 & 1961 \\
\hline 11 & 0.02 & 10 & 3 & 0.30 & 1969 & 0.30 & 1969 \\
\hline 12 & 0.03 & 9 & 6 & 0.20 & 1986 & 0.42 & 1958 \\
\hline 13 & 0.02 & 10 & 6 & 0.21 & 1981 & 0.21 & 1981 \\
\hline 14 & 0.03 & 5 & 11 & 0.39 & 1986 & 0.39 & 1986 \\
\hline 15 & 0.03 & 12 & 7 & 0.30 & 1970 & 0.30 & 1970 \\
\hline 16 & 0.03 & 7 & 7 & 0.42 & 1989 & 0.42 & 1989 \\
\hline 17 & 0.03 & 10 & 7 & 0.42 & 1989 & 0.42 & 1989 \\
\hline 18 & 0.03 & 9 & 2 & 0.34 & 1983 & 0.34 & $1983+$ \\
\hline 19 & 0.02 & 9 & 5 & 0.14 & 1989 & 0.78 & $\underline{1993}$ \\
\hline 20 & 0.02 & 5 & 3 & 0.18 & 1984 & $\overline{0.18}$ & $\overrightarrow{1984}$ \\
\hline 21 & 0.02 & 8 & 3 & 0.20 & 1986 & 0.36 & 1956 \\
\hline
\end{tabular}


TABLE 4.7. (contd)

\begin{tabular}{|c|c|c|c|c|}
\hline & $\begin{array}{c}\text { NORMAL PERIOL } \\
(1961-1990) \\
\end{array}$ & & & $\begin{array}{l}\text { HISTORICAL PERIOD } \\
(1945-1993) \\
\end{array}$ \\
\hline & NUMBER OF YEARS & & & \\
\hline
\end{tabular}

FEBRUARY (contd)

$\begin{array}{llllllll}22 & 0.02 & 4 & 5 & 0.15 & 1989 & 0.21 & 1949 \\ 23 & 0.02 & 5 & 4 & 0.22 & 1968 & 0.22 & 1968 \\ 24 & 0.02 & 5 & 5 & 0.10 & 198 ! . & 0.33 & 1950 \\ 25 & 0.02 & 9 & 3 & 0.22 & 1983 & 0.25 & 1948 \\ 26 & 0.02 & 6 & 4 & 0.30 & 1976 & 0.30 & 1976 \\ 27 & 0.02 & 9 & 3 & 0.24 & 1980 & 0.24 & 1980 \\ 28 & 0.02 & 7 & 4 & 0.33 & 1977 & 0.33 & 1977 \\ 29 & 0.02 & 1 & 1 & 0.04 & 1984 & 0.04 & 1984\end{array}$

MARCH

\begin{tabular}{|c|c|c|c|c|c|c|c|}
\hline \multicolumn{8}{|c|}{ MARCH } \\
\hline 1 & 0.02 & 8 & 4 & 0.15 & 1972 & 0.15 & 1972 \\
\hline 2 & 0.01 & 4 & 8 & 0.07 & 1989 & 0.20 & $1991+$ \\
\hline 3 & 0.01 & 3 & 7 & 0.15 & 1977 & 0.15 & $1991+$ \\
\hline 4 & 0.01 & 9 & 6 & 0.16 & 1985 & 0.44 & 1957 \\
\hline 5 & 0.01 & 6 & 7 & 0.23 & 1989 & 0.23 & 1989 \\
\hline 6 & 0.02 & 7 & 3 & 0.07 & $1971+$ & 0.24 & 1957 \\
\hline 7 & 0.02 & 6 & 4 & 0.21 & 1986 & 0.21 & 1986 \\
\hline 8 & 0.02 & 8 & 2 & 0.19 & 1988 & 0.23 & 1951 \\
\hline 9 & 0.02 & 6 & 6 & 0.31 & 1989 & 0.31 & 1989 \\
\hline 10 & 0.02 & 11 & 4 & 0.06 & $1980+$ & 0.08 & 1950 \\
\hline 11 & 0.02 & 8 & 6 & 0.24 & 1989 & 0.24 & 1989 \\
\hline 12 & 0.02 & 9 & 6 & 0.42 & 1987 & 0.42 & 1987 \\
\hline 13 & 0.02 & 7 & 8 & 0.35 & 1983 & 0.35 & 1983 \\
\hline 14 & 0.02 & 9 & 3 & 0.14 & 1970 & 0.14 & 1970 \\
\hline 15 & 0.02 & 7 & 4 & 0.18 & 1987 & 0.25 & 1949 \\
\hline 16 & 0.01 & 7 & 5 & 0.22 & 1989 & 0.34 & 1949 \\
\hline 17 & 0.01 & 3 & 7 & 0.05 & 1967 & 0.16 & 1949 \\
\hline 18 & 0.01 & 7 & 5 & 0.04 & 1989 & 0.25 & 1949 \\
\hline 19 & 0.01 & 3 & 4 & 0.12 & 1987 & 0.12 & 1987 \\
\hline 20 & 0.01 & 5 & 4 & 0.43 & 1984 & 0.43 & 1984 \\
\hline 21 & 0.01 & 5 & 2 & 0.10 & $1984+$ & 0.18 & 1958 \\
\hline 22 & 0.02 & 4 & 8 & 0.22 & 1961 & 0.22 & 1961 \\
\hline 23 & 0.02 & 7 & 4 & 0.26 & 1986 & 0.26 & 1986 \\
\hline 24 & 0.02 & 4 & 2 & 0.12 & 1961 & 0.52 & 1991 \\
\hline 25 & 0.02 & 9 & 3 & 0.43 & 1971 & $\overline{0.43}$ & $\overline{1971}$ \\
\hline 26 & 0.02 & 5 & 6 & 0.50 & 1981 & 0.50 & 1981 \\
\hline 27 & 0.02 & 5 & 4 & 0.42 & 1979 & 0.42 & 1979 \\
\hline 28 & 0.02 & 5 & 3 & 0.13 & 1982 & 0.13 & 1982 \\
\hline 29 & 0.01 & 8 & 1 & 0.15 & 1983 & 0.15 & 1983 \\
\hline 30 & 0.01 & 4 & 7 & 0.23 & 1974 & 0.23 & 1974 \\
\hline 31 & 0.01 & 4 & 6 & 0.14 & 1976 & 0.14 & 1976 \\
\hline \multicolumn{8}{|c|}{ APRIL } \\
\hline 1 & 0.01 & 3 & 12 & 0.18 & 1983 & 0.22 & 1958 \\
\hline 2 & 0.01 & 6 & 3 & 0.10 & 1979 & 0.18 & 1948 \\
\hline 3 & 0.01 & 2 & 3 & 0.03 & 1963 & 0.18 & 1947 \\
\hline 4 & 0.01 & 5 & 9 & 0.13 & 1984 & 0.18 & 1948 \\
\hline 5 & 0.01 & 6 & 6 & 0.44 & 1969 & 0.44 & 1969 \\
\hline 6 & 0.01 & 6 & 3 & 0.36 & 1982 & 0.36 & 1982 \\
\hline 7 & 0.01 & 3 & 1 & 0.22 & 1984 & 0.30 & 1953 \\
\hline 8 & 0.01 & 5 & 2 & 0.17 & 1976 & 0.18 & 1991 \\
\hline 9 & 0.01 & 3 & 10 & 0.12 & 1980 & 0.32 & 1992 \\
\hline
\end{tabular}


IABLE 4.7. (contd)

\begin{tabular}{|c|c|c|c|c|c|}
\hline \multirow[b]{3}{*}{ DAY } & \multicolumn{3}{|l|}{$\begin{array}{c}\text { NORMAL PERIOD } \\
(1961-1990)\end{array}$} & \multicolumn{2}{|c|}{$\begin{array}{l}\text { HISTORICAL PERIOD } \\
(1945-1993)\end{array}$} \\
\hline & NUMBER OF YEARS & & & & \\
\hline & W/MEAS. & MAXIMUM & YEAR & MAXIMUM & YEAR \\
\hline
\end{tabular}

APRIL (contd)

$\begin{array}{llllllll}10 & 0.01 & 2 & 6 & 0.03 & 1969 & 0.10 & 1958 \\ 11 & 0.01 & 4 & 9 & 0.23 & 1982 & 0.23 & 1982 \\ 12 & 0.01 & 4 & 5 & 0.08 & 1961 & 0.09 & 1992 \\ 13 & 0.01 & 1 & 3 & 0.01 & 1990 & 0.39 & 1992 \\ 14 & 0.01 & 4 & 4 & 0.17 & 1975+ & 0.17 & 1975+ \\ 15 & 0.01 & 3 & 8 & 0.09 & 1962 & 0.17 & 1991 \\ 16 & 0.02 & 4 & 7 & 0.08 & 1979+ & 0.11 & 1948 \\ 17 & 0.02 & 6 & 6 & 0.36 & 1988 & 0.36 & 1988 \\ 18 & 0.02 & 4 & 6 & 0.31 & 1967 & 0.31 & 1967 \\ 19 & 0.02 & 7 & 9 & 0.41 & 1970 & 0.41 & 1970 \\ 20 & 0.02 & 6 & 6 & 0.56 & 1980 & 0.56 & 1980 \\ 21 & 0.02 & 2 & 7 & 0.07 & 1989 & 0.07 & 1989 \\ 22 & 0.02 & 7 & 6 & 0.12 & 1974 & 0.22 & 1960 \\ 23 & 0.01 & 6 & 5 & 0.22 & 1974 & 0.22 & 1974 \\ 24 & 0.01 & 4 & 8 & 0.22 & 1975 & 0.22 & 1975 \\ 25 & 0.02 & 3 & 6 & 0.35 & 1989 & 0.35 & 1989 \\ 26 & 0.02 & 2 & 7 & 0.04 & 1989 & 0.25 & 1955 \\ 27 & 0.02 & 7 & 5 & 0.28 & 1989+ & 0.28 & 1989+ \\ 28 & 0.02 & 6 & 5 & 0.48 & 1988 & 0.51 & 1951 \\ 29 & 0.02 & 2 & 3 & 0.30 & 1961 & 0.30 & 1961 \\ 30 & 0.02 & 6 & 4 & 0.12 & 1984 & 0.12 & 1984\end{array}$

MAY

\begin{tabular}{rlllllll}
\hline 1 & 0.02 & 5 & 6 & 0.19 & 1984 & 0.19 & 1984 \\
2 & 0.01 & 5 & 1 & 0.17 & 1975 & 0.17 & 1975 \\
3 & 0.01 & 4 & 3 & 0.29 & 1977 & 0.29 & 1977 \\
4 & 0.01 & 6 & 5 & 0.10 & 1967 & 0.10 & 1967 \\
5 & 0.02 & 8 & 3 & 0.28 & 1963 & 0.28 & 1963 \\
6 & 0.02 & 4 & 6 & 0.20 & 1986 & 0.20 & 1986 \\
7 & 0.02 & 3 & 1 & 0.39 & 1983 & 0.39 & 1983 \\
8 & 0.02 & 5 & 7 & 0.55 & 1972 & 0.55 & 1972 \\
9 & 0.02 & 5 & 5 & 0.25 & 1961 & 0.40 & 1948 \\
10 & 0.02 & 6 & 7 & 0.39 & 1961 & 0.39 & 1961 \\
11 & 0.02 & 4 & 4 & 0.19 & 1967 & 0.39 & 1951 \\
12 & 0.02 & 4 & 7 & 0.50 & 1970 & 0.50 & 1970 \\
13 & 0.01 & 4 & 2 & 0.11 & 1985 & 0.15 & 1952 \\
14 & 0.01 & 4 & 6 & 0.25 & 1978 & 0.25 & 1978 \\
15 & 0.01 & 3 & 6 & 0.06 & 1975 & 0.29 & 1955 \\
16 & 0.01 & 0 & 8 & $T$ & $1988+$ & 0.14 & 1991 \\
17 & 0.01 & 4 & 3 & 0.13 & 1982 & 0.25 & 1959 \\
18 & 0.01 & 5 & 2 & 0.13 & 1981 & 0.13 & 1981 \\
19 & 0.01 & 7 & 3 & 0.15 & 1965 & 0.55 & 1948 \\
20 & 0.02 & 4 & 1 & 0.70 & 1972 & 0.70 & 1972 \\
21 & 0.02 & 3 & 4 & 0.69 & 1972 & 0.69 & 1972 \\
22 & 0.02 & 6 & 5 & 0.12 & 1984 & 0.12 & 1984 \\
23 & 0.03 & 5 & 6 & 0.33 & 1990 & 0.33 & 1990 \\
24 & 0.03 & 4 & 5 & 0.51 & 1962 & 0.51 & 1962 \\
25 & 0.02 & 6 & 2 & 0.74 & 1981 & 0.74 & 1981 \\
26 & 0.02 & 4 & 6 & 0.79 & 1980 & 0.79 & 1980 \\
27 & 0.02 & 6 & 2 & 0.11 & 1990 & 0.11 & 1990 \\
28 & 0.02 & 5 & 7 & 0.28 & 1988 & 0.28 & 1988 \\
29 & 0.02 & 5 & 3 & 0.11 & 1961 & 0.11 & 1961 \\
30 & 0.01 & 6 & 1 & 0.14 & 1987 & 0.14 & 1987 \\
31 & 0.02 & 2 & 5 & 0.35 & 1971 & 0.35 & 1971 \\
& & & & & & &
\end{tabular}


TABLE 4.7. (contd)

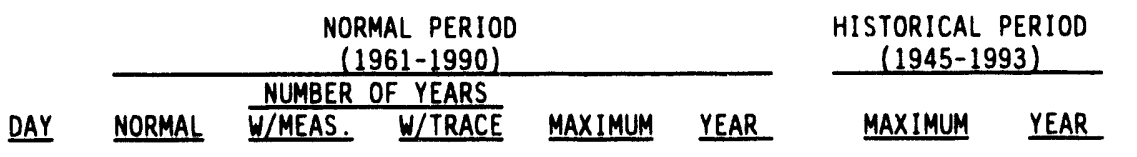

\begin{tabular}{|c|c|c|c|c|c|c|c|}
\hline \multicolumn{8}{|c|}{ JUNE } \\
\hline $\begin{array}{l}1 \\
2 \\
3 \\
4 \\
5 \\
6 \\
7 \\
8 \\
9 \\
10 \\
11 \\
12 \\
13 \\
14 \\
15 \\
16 \\
17 \\
18 \\
19 \\
20 \\
21 \\
22 \\
23 \\
24 \\
25 \\
26 \\
27 \\
28 \\
29 \\
30\end{array}$ & $\begin{array}{l}0.02 \\
0.02 \\
0.02 \\
0.02 \\
0.02 \\
0.02 \\
0.01 \\
0.01 \\
0.02 \\
0.02 \\
0.01 \\
0.01 \\
0.01 \\
0.01 \\
0.01 \\
0.01 \\
0.01 \\
0.01 \\
0.01 \\
0.01 \\
0.01 \\
0.01 \\
0.01 \\
0.01 \\
0.01 \\
0.01 \\
0.01 \\
0.01 \\
0.01 \\
0.01\end{array}$ & $\begin{array}{l}3 \\
5 \\
6 \\
6 \\
4 \\
6 \\
5 \\
6 \\
3 \\
2 \\
3 \\
4 \\
6 \\
4 \\
4 \\
2 \\
2 \\
2 \\
1 \\
5 \\
5 \\
4 \\
7 \\
5 \\
3 \\
2 \\
4 \\
2 \\
4 \\
1\end{array}$ & $\begin{array}{l}5 \\
6 \\
9 \\
3 \\
4 \\
3 \\
5 \\
6 \\
4 \\
7 \\
5 \\
8 \\
4 \\
2 \\
1 \\
5 \\
5 \\
5 \\
3 \\
2 \\
2 \\
7 \\
2 \\
3 \\
4 \\
6 \\
3 \\
8 \\
2 \\
1\end{array}$ & $\begin{array}{l}0.29 \\
0.12 \\
0.30 \\
0.25 \\
0.14 \\
0.36 \\
0.15 \\
0.49 \\
0.07 \\
0.08 \\
0.13 \\
0.60 \\
0.35 \\
0.04 \\
0.15 \\
0.14 \\
0.48 \\
0.07 \\
0.01 \\
0.24 \\
0.32 \\
0.14 \\
0.17 \\
0.21 \\
0.02 \\
0.27 \\
0.37 \\
0.23 \\
0.16 \\
0.06\end{array}$ & $\begin{array}{l}1977 \\
1966 \\
1971 \\
1984 \\
1981 \\
1990 \\
1972 \\
1964 \\
1963 \\
1983 \\
1961 \\
1969 \\
1980 \\
1983 \\
1964 \\
1980 \\
1965 \\
1983 \\
1983 \\
1984 \\
1967 \\
1971 \\
1963 \\
1972 \\
1980 \\
1982 \\
1983 \\
1984 \\
1984 \\
1976\end{array}$ & $\begin{array}{l}0.29 \\
0.12 \\
0.30 \\
0.45 \\
0.49 \\
0.54 \\
0.71 \\
0.49 \\
0.22 \\
0.14 \\
0.39 \\
0.79 \\
0.49 \\
0.37 \\
0.15 \\
0.18 \\
1.09 \\
0.07 \\
0.09 \\
0.24 \\
0.32 \\
0.14 \\
0.17 \\
0.21 \\
0.03 \\
0.27 \\
0.37 \\
0.24 \\
0.53 \\
0.06\end{array}$ & $\begin{array}{l}1977 \\
1966 \\
1971 \\
1951 \\
1991 \\
1951 \\
1947 \\
1964 \\
1948 \\
1956 \\
1950 \\
1992 \\
1948 \\
1956 \\
1964 \\
1948 \\
1950 \\
1983 \\
1991 \\
1984 \\
1967 \\
1971 \\
1963 \\
1972 \\
1954 \\
1982 \\
1983 \\
1992 \\
1991 \\
1976\end{array}$ \\
\hline \multicolumn{8}{|c|}{ JULY } \\
\hline 1 & 001 & 5 & & & & & \\
\hline & 0.01 & 3 & 3 & 0.31 & 1966 & 0.31 & 1966 \\
\hline 2 & 0.01 & 3 & 6 & 0.34 & 1966 & 0.34 & 1966 \\
\hline 3 & 0.01 & 3 & 3 & 0.31 & 1978 & 0.31 & 1978 \\
\hline 4 & 0.01 & 3 & 4 & 0.10 & 1986 & 0.10 & 1986 \\
\hline 5 & 0.01 & 1 & 3 & 0.19 & 1981 & 0.36 & 1951 \\
\hline 6 & 0.01 & 1 & 3 & 0.02 & 1979 & 0.04 & 1993 \\
\hline 7 & 0.01 & 3 & 2 & 0.30 & 1963 & 0.30 & 1963 \\
\hline 8 & 0.01 & 5 & 3 & 0.14 & 1974 & 0.14 & 1974 \\
\hline 9 & 0.01 & 3 & 2 & 0.27 & 1987 & 0.27 & 1987 \\
\hline 10 & 0.01 & 2 & 5 & 0.05 & 1972 & 0.16 & 1954 \\
\hline 11 & 0.01 & 2 & 4 & 0.04 & 1979 & 0.04 & 1979 \\
\hline 12 & $T$ & 0 & 4 & $T$ & $1982+$ & $T$ & $1982+$ \\
\hline 13 & $T$ & 2 & 5 & 0.28 & 1975 & 0.28 & 1975 \\
\hline 14 & $T$ & 1 & 2 & 0.03 & 1966 & 0.05 & 1957 \\
\hline 15 & $T$ & 2 & 2 & 0.04 & 1975 & 0.08 & 1991 \\
\hline 16 & $T$ & 4 & 3 & 0.07 & 1966 & 0.50 & 1993 \\
\hline 17 & $T$ & 3 & 1 & 0.05 & $1987+$ & 0.89 & 1993 \\
\hline 18 & $T$ & 2 & 2 & 0.12 & 1987 & 0.12 & 1987 \\
\hline 19 & $\mathrm{~T}$ & 2 & 2 & 0.45 & 1974 & 0.45 & 1974 \\
\hline 20 & $T$ & 2 & 2 & 0.09 & 1965 & 0.09 & 1965 \\
\hline 21 & $T$ & 1 & 2 & 0.02 & 1965 & 0.02 & 1965 \\
\hline 22 & $T$ & 0 & 2 & $T$ & $1987+$ & $T$ & $1993+$ \\
\hline
\end{tabular}

\subsection{4}


IABLE 4.7. (contd)

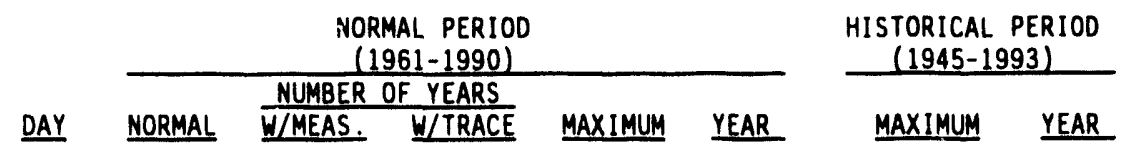

JULY (contd)

$\begin{array}{llllllll}23 & T & 2 & 0 & 0.15 & 1961 & 0.28 & 1992 \\ 24 & T & 2 & 2 & 0.06 & 1990 & 0.07 & 1955 \\ 25 & T & 3 & 2 & 0.23 & 1983 & 0.23 & 1983 \\ 26 & T & 0 & 1 & T & 1978 & 0.22 & 1955 \\ 27 & T & 1 & 1 & 0.02 & 1983 & 0.31 & 1947 \\ 28 & T & 1 & 3 & 0.06 & 1984 & 0.28 & 1947 \\ 29 & T & 1 & 1 & 0.01 & 1964 & 0.01 & 1993+ \\ 30 & T & 0 & 3 & T & 1987+ & T & 1987+ \\ 31 & T & 1 & 2 & 0.12 & 1985 & 0.12 & 1991+\end{array}$

AUGUST

\begin{tabular}{|c|c|c|c|c|c|c|c|}
\hline 1 & $T$ & 2 & 1 & 0.08 & $1976+$ & 0.08 & $1976+$ \\
\hline 2 & $T$ & 1 & 3 & 0.01 & 1976 & 0.01 & 1976 \\
\hline 3 & $T$ & 1 & 2 & 0.29 & 1962 & 0.29 & 1962 \\
\hline 4 & $T$ & 1 & 2 & 0.01 & 1985 & 0.04 & 1948 \\
\hline 5 & $T$ & 0 & 3 & $T$ & $1984+$ & $T$ & $1991+$ \\
\hline 6 & $T$ & 1 & 2 & 0.11 & 1976 & 0.11 & 1976 \\
\hline 7 & $T$ & 2 & 1 & 0.33 & 1976 & 0.33 & 1976 \\
\hline 8 & $T$ & 0 & 4 & $T$ & $1989+$ & 0.08 & 1952 \\
\hline 9 & $\mathrm{~T}$ & 2 & 2 & 0.10 & 1982 & 0.10 & 1982 \\
\hline 10 & $\mathrm{~T}$ & 0 & 3 & $T$ & $1984+$ & 0.01 & 1947 \\
\hline 11 & $\mathrm{~T}$ & 1 & 4 & 0.01 & 1983 & 0.11 & 1947 \\
\hline 12 & 0.01 & 3 & 5 & 0.18 & 1962 & 0.18 & 1962 \\
\hline 13 & 0.01 & 4 & 2 & 0.04 & $1987+$ & 0.04 & $1987+$ \\
\hline 14 & 0.01 & 5 & 3 & 0.09 & 1979 & 0.09 & 1979 \\
\hline 15 & 0.01 & 3 & 1 & 0.42 & 1972 & 0.42 & 1972 \\
\hline 16 & 0.01 & 1 & 1 & 0.01 & 1968 & 0.24 & 1993 \\
\hline 17 & 0.01 & 0 & 3 & $T$ & $1980+$ & $T$ & $1980+$ \\
\hline 18 & 0.01 & 4 & 3 & 0.69 & 1975 & 0.69 & 1975 \\
\hline 19 & 0.01 & 3 & 5 & 0.05 & 1979 & 0.18 & 1954 \\
\hline 20 & 0.01 & 4 & 6 & 0.03 & 1978 & 0.22 & 1953 \\
\hline 21 & 0.02 & 3 & 4 & 0.76 & 1990 & $\underline{0.76}$ & 1990 \\
\hline 22 & 0.01 & 6 & 1 & 0.18 & 1978 & 0.18 & 1978 \\
\hline 23 & 0.02 & 4 & 1 & 0.14 & 1975 & 0.14 & 1975 \\
\hline 24 & 0.02 & 6 & 1 & 0.38 & 1977 & 0.38 & 1977 \\
\hline 25 & 0.01 & 2 & 6 & 0.29 & 1976 & 0.29 & 1976 \\
\hline 26 & 0.01 & 3 & 4 & 0.19 & 1968 & 0.38 & 1953 \\
\hline 27 & 0.01 & 5 & 3 & 0.14 & 1989 & 0.14 & 1989 \\
\hline 28 & 0.01 & 2 & 4 & 0.13 & 1975 & 0.13 & 1975 \\
\hline 29 & 0.01 & 6 & 1 & 0.28 & 1977 & 0.51 & 1947 \\
\hline 30 & 0.01 & 2 & 4 & 0.61 & 1977 & 0.61 & 1977 \\
\hline 31 & 0.01 & $?$ & 3 & 0.02 & 1961 & 0.02 & $1961+$ \\
\hline \multicolumn{8}{|c|}{ SEPTEMBER } \\
\hline 1 & 0.01 & 7 & 2 & 0.43 & 1971 & 0.43 & 1971 \\
\hline 2 & 0.01 & 2 & 4 & 0.17 & 1971 & 0.17 & 1971 \\
\hline 3 & 0.01 & 3 & 1 & 0.13 & 1979 & 0.13 & 1979 \\
\hline 4 & 0.01 & 1 & 1 & 0.02 & 1977 & 0.19 & 1960 \\
\hline 5 & $T$ & 3 & 2 & 0.19 & 1971 & 0.19 & 1971 \\
\hline 6 & $T$ & 3 & 0 & 0.29 & 1971 & 0.29 & 1971 \\
\hline 7 & $T$ & 2 & 0 & 0.04 & 1978 & 0.23 & 1947 \\
\hline 8 & 0.01 & 2 & 3 & 0.10 & 1985 & 0.10 & $19 \ddot{0} 5$ \\
\hline 9 & $T$ & 2 & 4 & 0.07 & 1985 & 0.07 & 1985 \\
\hline
\end{tabular}


TABLE 4.7. (contd)

\begin{tabular}{|c|c|c|c|}
\hline & $\begin{array}{c}\text { NORMAL PERIOD } \\
(1961-1990)\end{array}$ & & $\begin{array}{l}\text { HISTORICAL PERIOD } \\
(1945-1993)\end{array}$ \\
\hline DAY & $\begin{array}{l}\text { NUMBER OF YEARS } \\
\text { W/MEAS. W/TRACE }\end{array}$ & MAXIMUM & MAXIMUM \\
\hline
\end{tabular}

SEPTEMBER (contd)

\begin{tabular}{llllllll}
10 & 0.01 & 3 & 3 & 0.06 & 1985 & 0.06 & 1985 \\
11 & 0.01 & 4 & 1 & 0.10 & 1966 & 0.10 & 1966 \\
12 & 0.01 & 0 & 3 & $T$ & $1980+$ & 0.03 & 1958 \\
13 & 0.01 & 5 & 2 & 0.79 & 1980 & 0.79 & $\frac{1980}{1959}$ \\
14 & 0.01 & 3 & 4 & 0.04 & 1985 & 0.41 & 1986 \\
15 & 0.01 & 2 & 5 & 0.54 & 1986 & 0.54 & 1947 \\
16 & 0.02 & 2 & 7 & 0.03 & 1985 & 0.66 & 1949 \\
17 & 0.01 & 4 & 5 & 0.26 & 1969 & 0.26 & 1969 \\
18 & 0.01 & 4 & 6 & 0.22 & 1983 & 0.41 & 1959 \\
19 & 0.01 & 6 & 4 & 0.26 & 1973 & 0.26 & 1973 \\
20 & 0.01 & 4 & 8 & 0.13 & 1988 & 0.13 & 1988 \\
21 & 0.01 & 2 & 4 & 0.03 & 1971 & 0.03 & 1971 \\
22 & 0.01 & 4 & 4 & 0.20 & 1984 & 0.20 & 1984 \\
23 & 0.01 & 4 & 2 & 0.21 & 1986 & 0.21 & 1986 \\
24 & 0.01 & 4 & 0 & 0.10 & 1977 & 0.10 & 1977 \\
25 & 0.01 & 3 & 3 & 0.25 & 1982 & 0.25 & 1982 \\
26 & 0.01 & 4 & 1 & 0.22 & 1981 & 0.22 & 1981 \\
27 & 0.01 & 4 & 2 & 0.38 & 1981 & 0.43 & 1955 \\
28 & 0.01 & 5 & 3 & 0.34 & 1962 & 0.34 & 1962 \\
29 & 0.01 & 3 & 1 & 0.07 & 1986 & 0.07 & 1986 \\
30 & 0.01 & 2 & 3 & 0.02 & 1969 & 0.03 & $1953+$ \\
& & & 3 & & & & \\
\hline
\end{tabular}

\begin{tabular}{rlllllll}
\hline 1 & $T$ & 2 & 5 & 0.01 & $1969+$ & $\frac{1.60}{1}$ & $\frac{1957}{1957}$ \\
2 & $T$ & 5 & 5 & 0.06 & 1967 & 0.31 & 1950 \\
3 & $T$ & 2 & 1 & 0.04 & 1975 & 0.14 & 1950 \\
4 & $T$ & 1 & 4 & 0.01 & 1970 & 0.15 & 1950 \\
5 & $T$ & 0 & 9 & $T$ & $1981+$ & 0.25 & 19573 \\
6 & $T$ & 5 & 4 & 0.22 & 1973 & 0.22 & 1973 \\
7 & 0.01 & 3 & 3 & 0.25 & 1985 & 0.25 & 1985 \\
8 & 0.01 & 1 & 5 & 0.06 & 1964 & 0.49 & 1950 \\
9 & 0.01 & 5 & 2 & 0.09 & 1968 & 0.32 & 1947 \\
10 & 0.01 & 5 & 4 & 0.09 & 1985 & 0.32 & 1959 \\
11 & 0.01 & 3 & 7 & 0.43 & 1968 & 0.43 & 1968 \\
12 & 0.01 & 6 & 3 & 0.52 & 1962 & 0.52 & 1962 \\
13 & 0.01 & 2 & 3 & 0.04 & 1980 & 0.09 & 1957 \\
14 & 0.01 & 4 & 3 & 0.11 & 1962 & 0.43 & 1950 \\
15 & 0.01 & 1 & 1 & 0.01 & 1980 & 0.15 & 1947 \\
16 & 0.01 & 0 & 1 & $T$ & 1975 & 0.24 & 1947 \\
17 & 0.01 & 2 & 3 & 0.16 & 1968 & 0.23 & 1950 \\
18 & 0.01 & 4 & 3 & 0.28 & 1979 & 0.28 & 1979 \\
19 & 0.02 & 5 & 4 & 0.12 & 1979 & 0.64 & 1947 \\
20 & 0.02 & 5 & 5 & 0.15 & 1968 & 0.37 & 1947 \\
21 & 0.02 & 8 & 3 & 0.45 & 1975 & 0.45 & 1975 \\
22 & 0.02 & 5 & 9 & 0.20 & 1983 & 0.23 & 1957 \\
23 & 0.02 & 4 & 2 & 0.39 & 1973 & 0.39 & 1973 \\
24 & 0.02 & 4 & 5 & 0.11 & 1979 & 0.12 & 1991 \\
25 & 0.02 & 5 & 3 & 0.22 & 1975 & 0.22 & 1975 \\
26 & 0.02 & 6 & 3 & 0.12 & 1989 & 0.18 & 1956 \\
27 & 0.02 & 7 & 4 & 0.09 & 1969 & 0.33 & 1956 \\
28 & 0.02 & 4 & 6 & 0.93 & 1982 & 0.93 & 1982 \\
29 & 0.02 & 7 & 8 & 0.18 & 1986 & 0.38 & 1950 \\
30 & 0.02 & 3 & 7 & 0.52 & 1990 & 0.52 & 1990 \\
31 & 0.02 & 3 & 6 & 0.64 & 1973 & 0.64 & 1973
\end{tabular}


IABLE 4.7. (contd)

\begin{tabular}{cccc} 
NORMAL PERIOD & $(196)-1990)$ & & $\begin{array}{c}\text { HISTORICAL PERIOD } \\
(1945-1993)\end{array}$ \\
\cline { 2 - 4 } DAY NORMAL WUMEAS OF YEARS & W/TRACE & MAXIMUM YEAR & MAXIMUM YEAR
\end{tabular}

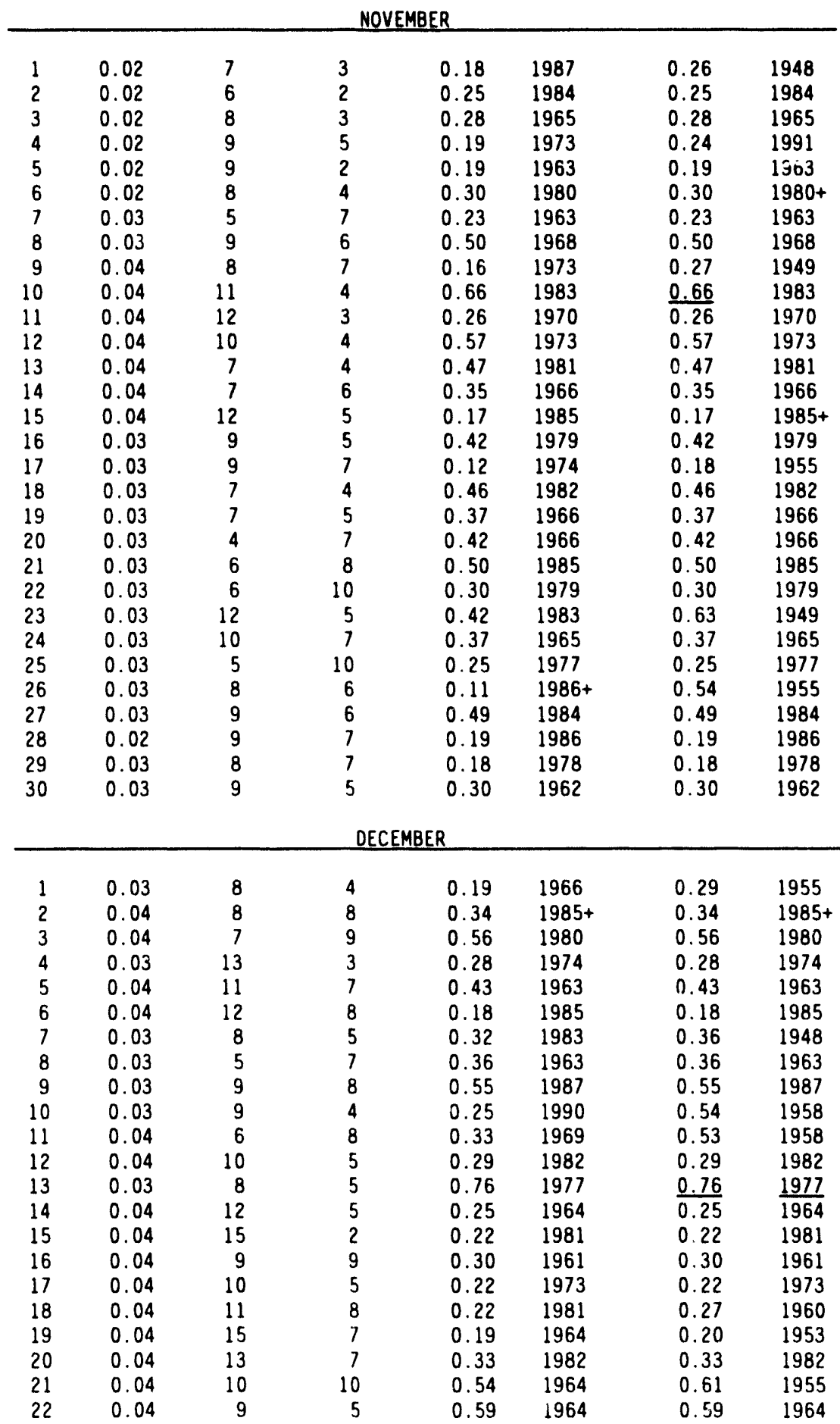

4.17 
IABLE 4.7. (contd)

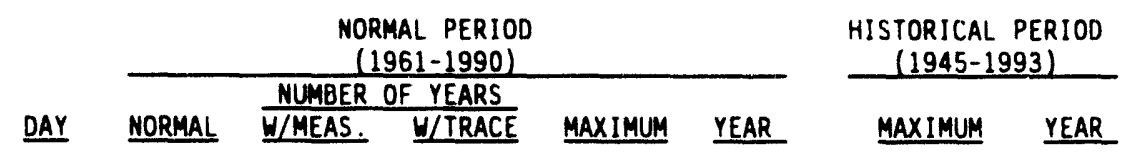

DECEMBER (contd)

$\begin{array}{rrrrrlll}23 & 0.04 & 7 & 7 & 0.31 & 1975 & 0.31 & 1975 \\ 24 & 0.04 & 11 & 8 & 0.36 & 1968 & 0.36 & 1968 \\ 25 & 0.03 & 12 & 6 & 0.26 & 1980 & 0.26 & 1980 \\ 26 & 0.03 & 9 & 6 & 0.19 & 1973 & 0.25 & 1958 \\ 27 & 0.03 & 11 & 7 & 0.36 & 1973 & 0.36 & 1973 \\ 28 & 0.02 & 10 & 11 & 0.09 & 1990+ & 0.10 & 1951 \\ 29 & 0.02 & 9 & 7 & 0.55 & 1983 & 0.55 & 1983 \\ 30 & 0.02 & 5 & 9 & 0.15 & 1979 & 0.15 & 1979 \\ 31 & 0.02 & 4 & 9 & 0.29 & 1968 & 0.29 & 1968\end{array}$

+ Latest of several occurrences. 


\subsection{WIND CLIMATOLOGY}

\subsection{MONTHLY AND ANNUAL PREVAILING WIND DIRECTIONS, AVERAGE SPEEDS, AND PEAK GUSTS}

At the HMS, the prevailing wind direction for every month of the year is either WNW or NW (Table 5.1), and the peak gusts for every month are from the SSW, SW, or WSW. The highest monthly average wind speeds occur in June and the lowest in December. The variability in monthly average wind speeds is much greater in the winter months than during the remainder of the year. The highest January average $(10.3 \mathrm{mph})$ is more than 3.5 times greater than the lowest $(2.9 \mathrm{mph})$, while in June the highest average $(10.7 \mathrm{mph})$ is only 1.4 times greater than the lowest $(7.7 \mathrm{mph})$.

\subsection{NUMBER OF DAYS WITH PEAK GUSTS ABOVE OR BELOW SPECIFIC THRESHOLDS}

Table 5.2 lists the number of days by month and year with peak wind gusts (at 50 feet) above or below specific threshold wind speeds. June and July have the highest average number of days with gusts $\geq 25 \mathrm{mph}$ (nearly $20 e^{-5}=h$ ); however, March has the highest average number of days with gusts $\geq 40 \mathrm{mph}$ ( 3 days), and January the highest average number of days with gusts $\geq 50 \mathrm{mph}(0.9 \mathrm{day})$. January al so has the record highest number of gusts $\geq 40 \mathrm{mph}$ and $50 \mathrm{mph}$ at 11 and 7 days respectively in 1990. Calendar year 1990 recorded the most days with gusts $\geq 40 \mathrm{mph}$ and $50 \mathrm{mph}$ at 57 and 18 days, respectively. Of particular interest is that previous records for these categories were 41 days $\geq 40 \mathrm{mph}$ in 1961, and 10 days $\geq 50 \mathrm{mph}$ in 1972 .

\subsection{PERCENT FREQUENCY OF MONTHLY AND ANNUAL WIND DIRECTION AND SPEED AT 50 FEET}

Table 5.3 presents HMS data on the percent frequency of monthly and annual wind direction and wind speed at 50 feet. This table shows that for every month of the year the prevailing wind direction is either from the WNW or NW. Winds are relatively evenly distributed from the NNE through the SSW at between 2 and $4 \%$ on an annual average for each direction. 
TABLE 5.1. Monthly and Annual Prevailing Wind Directions, Average Speeds (mph), and Peak Gusts (mph) at 50 Feet (1945 Through 1993)

\begin{tabular}{|c|c|c|c|c|c|c|c|c|c|}
\hline \multirow[b]{2}{*}{ MONTH } & \multirow[b]{2}{*}{$\begin{array}{l}\text { PREVAILING } \\
\text { DIRECTION }\end{array}$} & \multirow[b]{2}{*}{$\begin{array}{l}\text { AVERAGE } \\
\text { SPEED } \\
\end{array}$} & \multirow[b]{2}{*}{$\begin{array}{l}\text { HIGHEST } \\
\text { AVERAGE } \\
\end{array}$} & \multirow[b]{2}{*}{ YEAR } & \multirow[b]{2}{*}{$\begin{array}{r}\text { LOWEST } \\
\text { AVERAGE } \\
\end{array}$} & \multirow[b]{2}{*}{$\underline{\text { YEAR }}$} & \multicolumn{3}{|c|}{ PEAK GUSTS } \\
\hline & & & & & & & $\underline{\text { SPEED }}$ & DIRECTION & YEAR \\
\hline JAN & NW & 6.3 & 10.3 & 1972 & 2.9 & 1985 & 80 & SW & 1972 \\
\hline FEB & NW & 7.0 & 10.8 & 1976 & 4.6 & 1963 & 65 & SW & 1971 \\
\hline MAR & WWH & 8.2 & 10.7 & $1977+$ & 5.9 & 1958 & 70 & SW & 1956 \\
\hline APR & WNW & 8.9 & 11.1 & $1972+$ & 7.4 & $1989+$ & 73 & SSW & 1972 \\
\hline MAY & HWW & 8.8 & 10.7 & 1983 & 5.8 & 1957 & 71 & SSW & 1948 \\
\hline JUN & WNW & 9.1 & 10.7 & $1983+$ & 7.7 & $1950+$ & 72 & SW & 1957 \\
\hline JUL & WNW & 8.6 & 10.7 & 1983 & 6.8 & 1955 & 69 & WSW & 1979 \\
\hline AUG & WNW & 7.9 & 9.1 & 1946 & 6.0 & 1956 & 66 & SW & 1961 \\
\hline SEP & WNW & 7.5 & 9.2 & 1961 & 5.4 & 1957 & 65 & SSW & 1953 \\
\hline OCT & WHW & 6.5 & 9.1 & 1946 & 4.4 & 1952 & 63 & SSW & 1950 \\
\hline NOV & NW & 6.2 & 10.0 & 1990 & 2.9 & 1956 & 67 & WSW & 1993 \\
\hline DEC & NW & 5.9 & 8.3 & 1968 & 3.3 & 1985 & 71 & SW & 1955 \\
\hline YEAR & NW & 7.6 & 8.4 & 1983 & 6.2 & 1989 & 80 & SW & 1972 \\
\hline
\end{tabular}

+ Also in earlier years. 
TABLE 5.2. Number of Days With Peak Gusts Above or Below Specific Thresholds (1945 Through 1993)

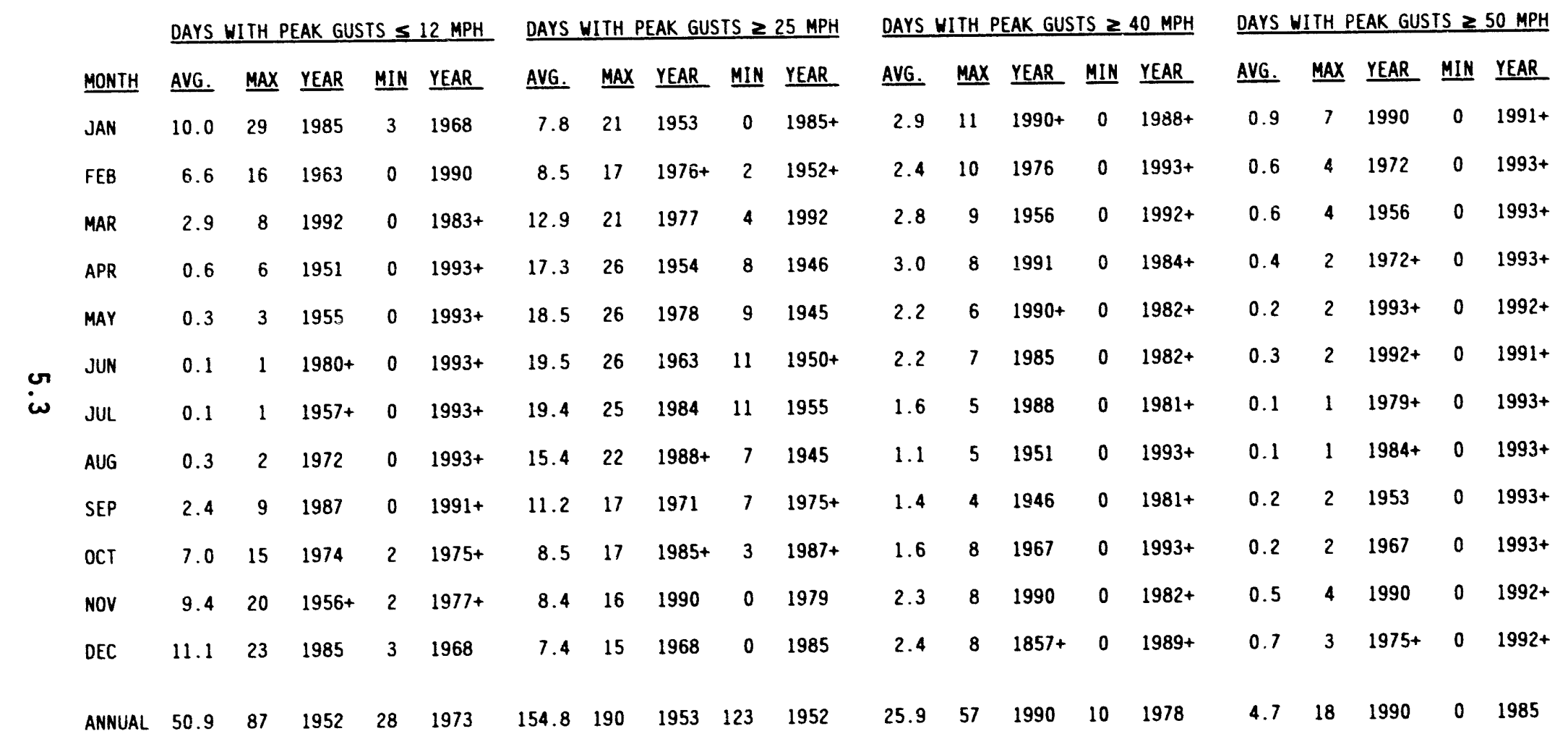

+ Most recent of multiple occurrences. 
TABLE 5.3. Percent Frequency of Monthly and Annual Wind Direction and Wind Speed at 50 Feet (1955 Through 1993)

WIND DIRECTION

\begin{tabular}{|c|c|c|c|c|c|c|c|c|c|c|c|c|c|}
\hline DIRECTION & JAN & FEB & MAR & APR & MAY & JUN & JUL & AUG & SEP & OCT & NOV & DEC & IOTAL \\
\hline N & 4.5 & 5.1 & 4.8 & 4.2 & 3.9 & 3.8 & 4.8 & 4.8 & 6.0 & 5.3 & 4.6 & 4.5 & 4.7 \\
\hline NNE & 3. & 4. & 4.5 & 3.5 & 3. & 3.2 & 4.3 & 4.0 & 5. & 4.2 & 3.5 & 3.1 & 3.9 \\
\hline NE & 3.0 & 3. & 3.4 & 3.4 & 3.4 & 3.1 & 3.7 & 3. & 4. & 3.6 & 2.9 & 2.7 & 3.4 \\
\hline ENE & 2.2 & 2. & 2.0 & 2.2 & 2.4 & 2.1 & 2.5 & 2. & 2. & 2. & 2.5 & 2.4 & 2.3 \\
\hline & 2.6 & 2. & 2.2 & 2.4 & 2. & 2. & 2.8 & 3. & 3. & 2. & 2. & 2.6 & 2.6 \\
\hline ESE & 2.8 & 2. & 2.7 & 2.4 & 2. & 2. & 2. & 3. & 3. & 3. & 3. & 3.1 & 2.9 \\
\hline$E$ & 4. & 3. & 3. & 3.1 & 3. & 3. & 3. & 3. & 3. & 5. & 4.6 & 4.7 & 3.9 \\
\hline SSE & 3.3 & 3. & 3.3 & 2.8 & 2. & 2. & 2.2 & 2. & 2. & 3. & 3.7 & 3.5 & 3.0 \\
\hline 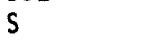 & 3.3 & 3. & 3. & 3.2 & 2. & 2.7 & 2.4 & 2. & 2. & 3.5 & 4. & 3.5 & 3.1 \\
\hline SSW & 4.8 & 4. & 5.1 & 4.4 & 3.4 & 3.4 & 2.6 & 3. & 3.3 & 4.1 & 5. & 4.7 & 4.0 \\
\hline SW & 6.7 & 7.6 & 9.1 & 8.9 & 6.6 & 6.6 & 5.7 & 6.0 & 5.6 & 6.8 & 7. & 6.7 & 7.0 \\
\hline WSW & 6.6 & 7.2 & 10.3 & 11.9 & 10.4 & 9.6 & 8.5 & 8.7 & 9.2 & 8.4 & 7.7 & 7.0 & 8.8 \\
\hline 4 & 6.6 & 8.3 & 9.7 & 11.8 & 11.7 & 11.1 & 9.9 & 10.8 & 11.4 & 10.4 & 8.4 & 7.1 & 9.8 \\
\hline WNW & 14.8 & 14.7 & 14.6 & 16.6 & 18.8 & 19.3 & 19.4 & 18.0 & 15.4 & 13.2 & 12.6 & 13.7 & 15.9 \\
\hline NW & 19.2 & 18. & 14.3 & 14.2 & 17.5 & 19.6 & 20.0 & 17.7 & 14.9 & 13.4 & 15.7 & 18.1 & 16.9 \\
\hline NNK & 7.1 & 6.8 & 5.6 & 4.5 & 4.2 & 4.6 & 5.2 & 5.3 & 5.7 & 6. & 7.0 & 6.9 & 5.8 \\
\hline Calm & 4.7 & 2.7 & .9 & .5 & .5 & .4 & .3 & .5 & 1.1 & 2.5 & 4.3 & 5.4 & 2.0 \\
\hline OTAL & 00.0 & 100.0 & 100.0 & 100.0 & 100.0 & 100.0 & 100.0 & 100.0 & 100.0 & 100.0 & 100.0 & 100.0 & 100.0 \\
\hline
\end{tabular}

WIND SPEED

SPEED $\quad \underline{\text { JAN }}$ FEB MAR APR MAY JUN JUL AUG SEP OCT NOV DEC TOTAL

\begin{tabular}{|c|c|c|c|c|c|c|c|c|c|c|c|c|c|}
\hline Calm & 4.7 & 2.7 & .9 & .5 & .5 & .4 & .3 & .5 & 1.1 & 2.5 & 4.3 & 5.4 & $\begin{array}{r}2.0 \\
20.8\end{array}$ \\
\hline $1-3$ & 31.6 & 25.7 & 17.7 & 13.6 & 12.3 & 10.2 & 11.3 & 14.4 & 19.3 & 28.0 & 30.9 & 34.4 & 20.8 \\
\hline $4-7$ & 33.3 & 34.6 & 35.6 & 33.7 & 34.3 & 35.3 & 38.4 & 41.3 & 39.9 & 38.0 & 34.1 & 32.0 & 35.9 \\
\hline $8-1$ & 19.4 & 23.5 & 27.2 & 28.6 & 30.1 & 29.7 & 29.1 & 27.4 & 25.6 & 20.8 & 19.7 & 18.1 & 24.9 \\
\hline $13-$ & 6.9 & 8.7 & 12.2 & 15.8 & 16.0 & 16.8 & 14.6 & 11.8 & 10.2 & 7.5 & 7.0 & 6.4 & 11.1 \\
\hline $19-$ & 2.6 & 3.2 & 4.6 & 6.1 & 5.7 & 6.4 & 5.4 & 4.0 & 3.3 & 2.5 & 2.7 & 2.5 & 4.1 \\
\hline $25-3$ & 1.1 & 1.3 & 1.5 & 1.6 & 1.0 & 1.2 & .9 & 6 & .6 & .6 & 1.0 & 1.0 & 1.0 \\
\hline 32 & .3 & .3 & .3 & .1 & .0 & .0 & .0 & .0 & .0 & .0 & .2 & .2 & .1 \\
\hline $39-4$ & .0 & .0 & .0 & .0 & .0 & .0 & .0 & .0 & .0 & .0 & .0 & .0 & .0 \\
\hline$>46$ & .0 & .0 & .0 & .0 & .0 & .0 & .0 & .0 & .0 & .0 & .0 & .0 & .0 \\
\hline
\end{tabular}

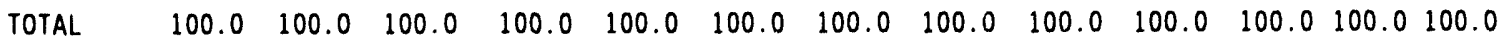

The wind speed class with the highest frequency of occurrence is 4 to $7 \mathrm{mph}$, with winds in that category nearly $36 \%$ of the time. The speed class with the second highest frequency is 8 to $12 \mathrm{mph}$, at nearly $25 \%$. Winds averaging over $25 \mathrm{mph}$ occur only slightly more than $1 \%$ of the time on an annual basis, with the highest frequency occurring in March (1.8\%). 


\subsection{COMPOSITE WIND ROSES AND JOINT FREQUENCY DISTRIBUTIONS FOR THE HANFORD METEOROLOGICAL MONITORING NETWORK}

Figure 5.1 and Table B.1 (Appendix B) contain composite wind roses and joint frequency distributions for the entire Hanford Meteorological Monitoring Network (see Table 1.1 and Figure 1.1) for the period from 1982 through 1993. In Figure 5.1, wind arrows indicate the direction from which the wind blows and are proportional in length to the frequency of occurrence as indicated in Table B.1. Table B.1 presents composite joint frequency distributions by direction and speed class for each of the stations in the network.

\subsection{MONTHLY AND ANNUAL WIND ROSES AND WIND SPEED HISTOGRAMS FOR THE HANFORD METEOROLOGICAL MONITORING NETWORK}

Figures B.1(a) and B.1(b) in Appendix B present monthly wind roses and wind speed histograms for all the Hanford Meteorological Monitoring Network stations for the period from 1982 through 1993. Figure B.1(a) depicts the wind roses for the stations. Each petal of the wind rose represents the proportional amount of time the wind blew from that direction. The widths of the petal segments correspond to the wind speed categories represented in Figure B.1(b). Starting from the center of the rose, the narrowest segment represents winds in the 1-3 mph class, the next widest segment represents the 4-7 mph class, and so forth. The length of each of these segments is proportional to the frequency of occurrence for the speed class. Figure B.1(b) contains wind speed histograms representing the proportional amount of time in each speed class.

Figures B.2(a) and B.2(b) in Appendix B present monthly wind roses and wind speed histograms for the $60 \mathrm{-m}$ level of the $100 \mathrm{~N}, 300$ Area, Fast Flux Test Facility, and HMS towers for the period from 1986 through 1993. 


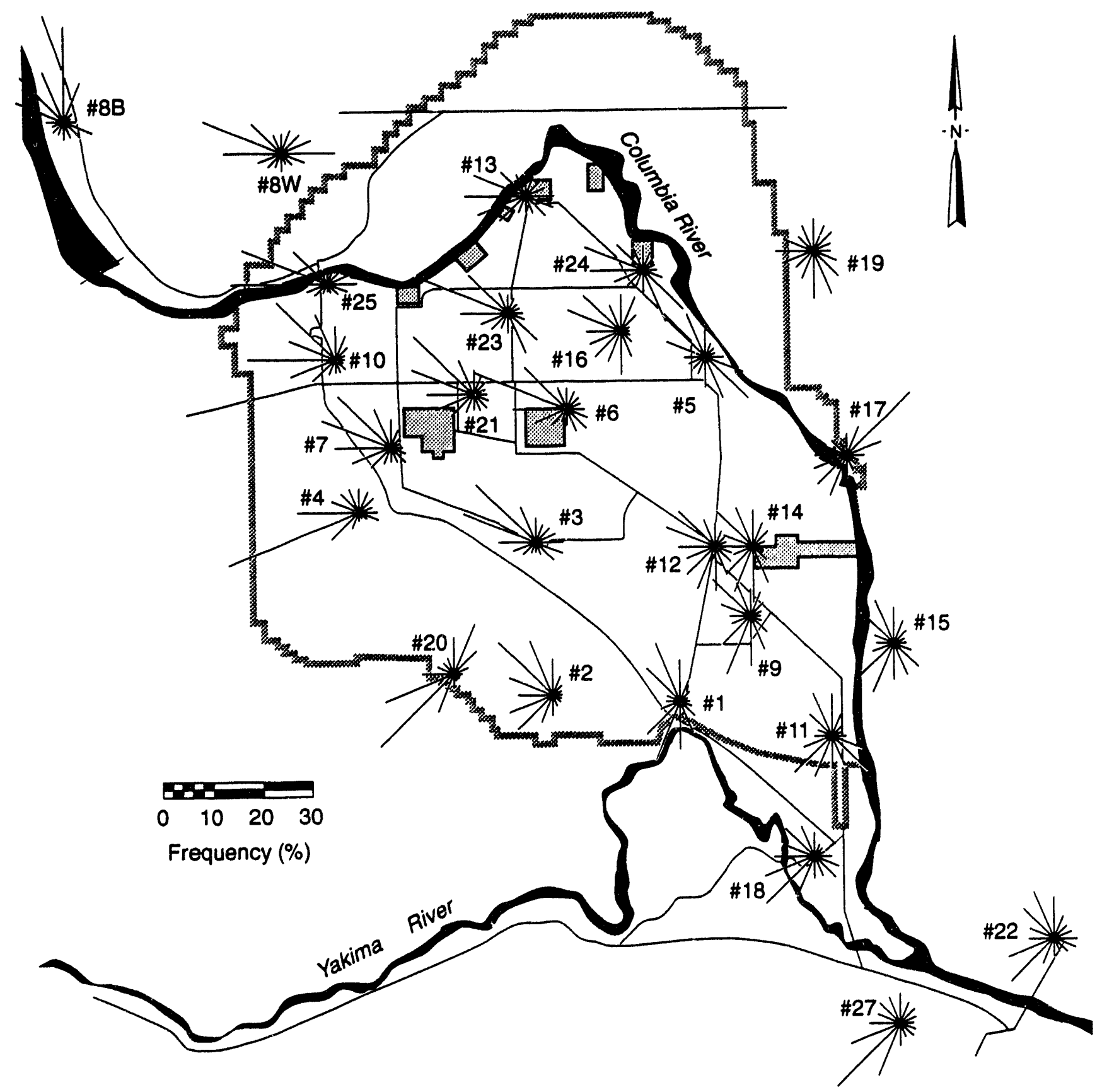

FIGURE 5.1. Hanford Meteorological Monitoring Network Wind Roses for the Period from 1982 Through 1993 


\subsection{MISCELLANEOUS CLIMATOLOGICAL STATISTICS}

\subsection{SKY COVER}

The term sky cover is used to express the portion of the celestial dome that is 1) covered, but not necessarily hidden, by clouds or obscuring phenomena aloft; 2) hidden by an obscuring phenomenon on the ground (such as fog or smoke); 3) or a combination of both 1 and 2 . The sky cover is determined hourly by scanning the sky and estimating the number of tenths that are covered ( 0 denotes clear sky and 10 denotes overcast). Average monthly sunrise to sunset sky covers for the period from 1946 through 1993 are shown in Table 6.1. Also shown in Table 6.1 are the number of clear, partly cloudy, and cloudy days for the period from 1954 through 1993. The number of clear, partly cloudy, and cloudy days is the result of assigning each day to one of the following categories:

\begin{tabular}{|c|c|}
\hline Category & $\begin{array}{c}\text { Average } \\
\text { Sky Cover }\end{array}$ \\
\hline $\begin{array}{l}\text { Clear } \\
\text { Partly cloudy } \\
\text { Cloudy }\end{array}$ & $\begin{array}{l}0-3 \text { tenths } \\
4-7 \text { tenths } \\
\text { 8-10 tenths }\end{array}$ \\
\hline
\end{tabular}

During the period of record (1954 through 1993), an average of 195 sunny days (the sum of the clear and partly cloudy days) have been recorded per year at the HMS.

\subsection{FOG AND DENSE FOG}

Table 6.2 shows the average monthly and annual number of days with fog and dense fog. Fog is reported anytime horizontal visibility is reduced to 6 miles or less, due to the suspension of water droplets in the surface layer of the atmosphere. Dense fog is reported when horizontal visibility is reduced to $1 / 4$ mile or less. Most fog at the HMS is radiation fog, a common type of fog which forms on nights characterized by light wind, clear sky, and 
TABLE 6.1. Average Sky Cover (Sunrise to Sunset) (1946 Through 1993) and Number of Days Clear, Partly Cloudy, and Cloudy (1954 Through 1993)

\begin{tabular}{|c|c|c|c|c|c|c|c|c|c|c|c|c|c|c|c|c|}
\hline & & COVE & $R$ (SCAL & $0-10$ & & & MBER & OF CLEA & R DAY & & PARTLY & & MBER & OF CLOU & JDY DA & \\
\hline MONTH & $\underline{\mathrm{AVG}}$ & $\underline{\operatorname{MAX}}$ & $\underline{\text { YEAR }}$ & MIN & $\underline{\text { YEAR }}$ & $\underline{\mathrm{AVG}}$ & $\underline{\operatorname{MAX}}$ & YEAR & MIN & YEAR & DAYS & $\underline{A V G}$ & $\underline{\operatorname{MAX}}$ & YEAR & MIN & $\underline{\text { YEAR }}$ \\
\hline JAN & 8.0 & 9.2 & 1978 & 4.3 & 1949 & 3 & 9 & 1984 & 0 & $1955+$ & 5 & 23 & 28 & 1978 & 17 & 1963 \\
\hline FEB & 7.5 & 9.3 & 1980 & 6.1 & 1989 & 4 & 9 & $1991+$ & 0 & $1984+$ & 5 & 19 & 26 & $1980+$ & 12 & 1964 \\
\hline MAR & 6.8 & 8.5 & 1978 & 4.9 & 1965 & 6 & 12 & $1979+$ & 1 & $1978+$ & 8 & 17 & 24 & 1993 & 9 & $1979+$ \\
\hline APR & 6.4 & 8.1 & 1963 & 3.7 & 1951 & 6 & 12 & 1962 & 1 & 1963 & 9 & 15 & 21 & $1979+$ & 6 & 1956 \\
\hline MAY & 5.9 & 8.1 & 1993 & 3.6 & 1992 & 8 & 18 & 1992 & 1 & 1977 & 1 & 12 & 19 & $1977+$ & 3 & 1992 \\
\hline JUN & 5.3 & 7.0 & 1950 & 2.8 & 1961 & 10 & 21 & 1961 & 5 & $1972+$ & 10 & 10 & 15 & $1983+$ & 5 & $1979+$ \\
\hline JUL & 3.1 & 5.0 & 1983 & 0.9 & 1953 & 19 & 26 & 1960 & 12 & $1987+$ & 7 & 5 & 12 & 1976 & 0 & $1984+$ \\
\hline AUG & 3.4 & 5.9 & 1968 & 0.6 & 1955 & 18 & 30 & 1955 & 9 & 1978 & 8 & 5 & 13 & $1983+$ & 0 & $1988+$ \\
\hline SEP & 3.9 & 6.7 & 1978 & 1.4 & $1990+$ & 15 & 27 & 1975 & 6 & 1978 & 7 & 8 & 16 & 1977 & 0 & 1990 \\
\hline OCT & 5.7 & 8.0 & 1975 & 3.3 & 1987 & 10 & 20 & 1987 & 1 & 1975 & 8 & 13 & 22 & 1973 & 6 & 1986 \\
\hline NOV & 7.6 & 9.1 & 1972 & 5.2 & 1993 & 5 & 12 & 1993 & 1 & $1973+$ & 5 & 20 & 25 & $1973+$ & 13 & 1993 \\
\hline DEC & 8.1 & 9.3 & 1985 & 6.4 & 1978 & 4 & 9 & 1978 & 1 & $1985+$ & 4 & 23 & 29 & 1985 & 17 & 1978 \\
\hline ANNUAL & 5.9 & 6.6 & $1978+$ & 5.1 & 1949 & 108 & 134 & 1967 & 80 & 1977 & 87 & 170 & 193 & 1978 & 146 & 1991 \\
\hline
\end{tabular}

+ Most recent of multiple occurrences. 
TABLE 6.2. Monthly and Annual Number of Days with Fog and Dense Fog (1945 Through 1993)

\begin{tabular}{|c|c|c|c|c|c|c|c|c|c|c|}
\hline \multirow[b]{2}{*}{ MONTH } & \multicolumn{5}{|c|}{ DAYS WITH FOG (VISIBILITY $\leq 6 \mathrm{MI)}$} & \multicolumn{5}{|c|}{ DAYS WITH DENSE FOG (VISIBILITY $\leq 1 / 4$ MI) } \\
\hline & AVG. & $\underline{\operatorname{MAX}}$ & YEAR & MIN & $\underline{\text { YEAR }}$ & AVG. & $\underline{\operatorname{MAX}}$ & $\underline{\text { YEAR }}$ & MIN & $\underline{\text { YEAR }}$ \\
\hline JAN & 11.0 & 25 & 1979 & 0 & 1949 & 5.8 & 15 & 1976 & 0 & 1949 \\
\hline FEB & 6.6 & 20 & 1963 & 0 & $1988+$ & 3.3 & 11 & 1963 & 0 & $1988+$ \\
\hline MAR & 2.2 & 10 & 1993 & 0 & $1990+$ & 0.9 & 5 & $1993+$ & 0 & $1990+$ \\
\hline APR & 0.4 & 3 & 1992 & 0 & $1987+$ & 0.1 & 1 & $1993+$ & 0 & $1992+$ \\
\hline MAY & 0.2 & 3 & 1948 & 0 & $1993+$ & $<0.1$ & 1 & 1958 & 0 & $1993+$ \\
\hline JUN & 0.1 & 2 & 1971 & 0 & $1993+$ & $<0.1$ & 1 & 1971 & 0 & $1993+$ \\
\hline JUL & $<0.1$ & 1 & 1966 & 0 & $1993+$ & 0 & 0 & & 0 & \\
\hline AUG & 0.1 & 1 & $1985+$ & 0 & $1993+$ & $<0.1$ & 1 & $1985+$ & 0 & $1993+$ \\
\hline SEP & 0.3 & 2 & $1985+$ & 0 & $1993+$ & 0.1 & 1 & $1992+$ & 0 & $1993+$ \\
\hline OCT & 2.0 & 9 & 1962 & 0 & $1989+$ & 1.0 & 7 & 1980 & 0 & $1991+$ \\
\hline NOV & 9.5 & 19 & $1985+$ & 0 & 1990 & 5.4 & 13 & 1965 & 0 & $1990+$ \\
\hline DEC & 14.0 & 25 & $1989+$ & 2 & 1968 & 7.6 & 17 & 1950 & 2 & $1968+$ \\
\hline ANNUAL & 46.4 & 84 & $1985-86$ & 22 & 1948-49 & 24.2 & 42 & $1950-51$ & 9 & $1948-4$ \\
\hline
\end{tabular}

+ Most recent of multiple occurrences.

Longest duration of fog: $113.7 \mathrm{hrs}$, December 16-20, 1985

Longest duration of dense fog: 47.0 hours, December 1957

moist air in the lower levels of the atmosphere. Nearly $90 \%$ of both fog and dense fog at the HMS occurs during the 1 ate fall and winter months, although fog has been observed every month of the year.

\subsection{PSYCHROMETRIC DATA}

Psychrometric data include observations of dry bulb, wet bulb, and dewpoint temperatures and relative humidity. The dry bulb temperature is the temperature of the ambient air; the wet bulb temperature is the lowest temperature to which a parcel of air, under constant pressure, can be cooled by evaporating water into it. The dew point temperature is the temperature to which a given parcel of air, under constant pressure and water-vapor content, must be cooled to attain saturation. Relative humidity is the ratio of the 
actual water-vapor content of the air to the one where saturation would occur if the pressure and temperature remained unchanged.

Table 6.3 presents monthly and annual averages and extremes of dry bulb, wet bulb, dew point temperatures and relative humidity from the HMS for the period from 1950 through 1993.

\subsection{SOLAR RADIATION}

Table 6.4 presents average and extreme daily solar radiation values by month for the period from 1953 through 1993. These data are reported in langleys. (A langley is a unit defined as 1 gram calorie per square centimeter.)

TABLE 6.3. Monthly Averages of Psychrometric Data (1950 Through 1993)* MONTHLY AVERAGES

\begin{tabular}{llllllllllllll} 
CATEGORY & JAN & FEB & MAR & APR & MAY & JUN & JUL & AUG & SEP & OCT & NOV & DEC & YEAR \\
Dry Bulb & 30.6 & 37.4 & 44.9 & 53.1 & 62.1 & 69.9 & 77.0 & 75.6 & 66.2 & 53.1 & 39.9 & 32.3 & 53.5 \\
Wet Bulb & 28 & 34 & 38 & 43 & 49 & 55 & 58 & 58 & 52 & 45 & 36 & 30 & 44 \\
Rel. Hum. & 76.9 & 70.7 & 56.7 & 47.1 & 42.7 & 39.5 & 33.3 & 35.6 & 42.0 & 56.2 & 73.4 & 80.2 & 54.5 \\
Dewpoint & 23.8 & 27.7 & 28.8 & 31.4 & 36.7 & 41.5 & 43.4 & 43.8 & 40.0 & 36.0 & 31.1 & 26.4 & 34.2 \\
& \multicolumn{8}{c}{ EXTREMES OF MONTHLY AVERAGES } \\
\hline
\end{tabular}

\begin{tabular}{|c|c|c|c|c|c|c|c|c|c|c|c|c|c|}
\hline \multicolumn{14}{|l|}{ Dry Bulb } \\
\hline $\begin{array}{l}\text { Highest } \\
\text { Year } \\
\text { Lowest } \\
\text { Year }\end{array}$ & $\begin{array}{l}43.0 \\
1953 \\
12.9 \\
1950\end{array}$ & $\begin{array}{c}44.6 \\
1991 \\
25.8 \\
1956\end{array}$ & $\begin{array}{l}51.6 \\
1992 \\
39.6 \\
1955\end{array}$ & $\begin{array}{c}58.6 \\
1987 \\
48.3 \\
1955\end{array}$ & $\begin{array}{l}68.7 \\
1958 \\
57.0 \\
1984\end{array}$ & $\begin{array}{c}77.3 \\
1992 \\
64.2 \\
1953\end{array}$ & $\begin{array}{l}83.3 \\
1985 \\
71.3 \\
1986\end{array}$ & $\begin{array}{l}82.5 \\
1967 \\
70.6 \\
1964\end{array}$ & $\begin{array}{c}72.7 \\
1990 \\
58.9 \\
1985\end{array}$ & $\begin{array}{l}59.5 \\
1988 \\
48.1 \\
1984\end{array}$ & $\begin{array}{l}46.3 \\
1990 \\
25.7 \\
1985\end{array}$ & $\begin{array}{ll}3 & 38.8 \\
0 & 1953 \\
7 & 21.9 \\
5 & 1985\end{array}$ & $\begin{array}{l}56.6 \\
1992 \\
50.2 \\
1985\end{array}$ \\
\hline \multicolumn{14}{|l|}{ Wet Bulb } \\
\hline $\begin{array}{l}\text { Highest } \\
\text { Year } \\
\text { Lowest } \\
\text { Year }\end{array}$ & $\begin{array}{r}39 \\
1953 \\
12 \\
1950\end{array}$ & $\begin{array}{r}41 \\
1956 \\
23 \\
1956\end{array}$ & $\begin{array}{r}44 \\
1992 \\
33 \\
1955\end{array}$ & $\begin{array}{r}47 \\
1992 \\
39 \\
1955\end{array}$ & $\begin{array}{r}55 \\
1958 \\
45 \\
1959\end{array}$ & $\begin{array}{c}59 \\
1992+19 \\
51 \\
1983+19\end{array}$ & $\begin{array}{r}62 \\
85+1 \\
56 \\
86+1\end{array}$ & $\begin{array}{r}61 \\
1991+ \\
55 \\
1980+\end{array}$ & $\begin{array}{rr}56 & \\
1990+ & 1 \\
48 & \\
1970 & 1\end{array}$ & $\begin{array}{r}50 \\
1988 \\
40 \\
1984\end{array}$ & $\begin{array}{r}42 \\
1954 \\
24 \\
24 \\
1985\end{array}$ & $\begin{array}{r}36 \\
1991+ \\
21 \\
1985\end{array}$ & $\begin{array}{r}47 \\
1992 \\
41 \\
1985\end{array}$ \\
\hline \multicolumn{14}{|l|}{ Rel. Hum. } \\
\hline $\begin{array}{l}\text { Highest } \\
\text { Year } \\
\text { Lowest } \\
\text { Year }\end{array}$ & $\begin{array}{l}88.8 \\
1960 \\
60.0 \\
1963\end{array}$ & $\begin{array}{c}86.9 \\
1963 \\
54.0 \\
1967\end{array}$ & $\begin{array}{l}69.1 \\
1993 \\
44.0 \\
1965\end{array}$ & $\begin{array}{c}64.5 \\
1963 \\
36.9 \\
1966\end{array}$ & $\begin{array}{l}61.9 \\
1948 \\
31.2 \\
1966\end{array}$ & $\begin{array}{c}53.5 \\
1950 \\
30.0 \\
1949\end{array}$ & $\begin{array}{l}45.6 \\
1993 \\
21.9 \\
1959\end{array}$ & $\begin{array}{l}47.8 \\
1976 \\
24.5 \\
1967\end{array}$ & $\begin{array}{c}55.5 \\
1977 \\
33.2 \\
1974\end{array}$ & $\begin{array}{l}74.2 \\
1962 \\
2 \\
42.5 \\
1952\end{array}$ & $\begin{array}{l}88.7 \\
1979 \\
62.8 \\
1976\end{array}$ & $\begin{array}{ll}7 & 90.5 \\
9 & 1950 \\
8 & 69.0 \\
6 & 1968\end{array}$ & $\begin{array}{l}58.9 \\
1978 \\
49.4 \\
1967\end{array}$ \\
\hline \multicolumn{14}{|l|}{ Dewpoint } \\
\hline $\begin{array}{l}\text { Highest } \\
\text { Year } \\
\text { Lowest } \\
\text { Year }\end{array}$ & $\begin{array}{r}34.4 \\
1953 \\
6.5 \\
1950\end{array}$ & $\begin{array}{c}36.7 \\
1992+ \\
17.3 \\
1956\end{array}$ & $\begin{array}{c}37.2 \\
1986 \\
20.8 \\
1965+\end{array}$ & $\begin{array}{r}37.1 \\
1992+ \\
26.0 \\
1982\end{array}$ & $\begin{array}{l}43.8 \\
1957 \\
30.4 \\
1964\end{array}$ & $\begin{array}{c}47.5 \\
1958 \\
37.5 \\
1954\end{array}$ & $\begin{array}{l}50.1 \\
1975 \\
35.4 \\
1959\end{array}$ & $\begin{array}{l}48.4 \\
1976 \\
38.4 \\
1955\end{array}$ & $\begin{array}{cc}4 & 45.4 \\
& 1963 \\
4 & 33.8 \\
& 1970\end{array}$ & $\begin{array}{ll}4 & 43.5 \\
& 1962 \\
8 & 30.2 \\
0 & 1984\end{array}$ & $\begin{array}{ll} & 38.3 \\
2 & 1954 \\
2 & 19.4 \\
4 & 1985\end{array}$ & $\begin{array}{ll}3 & 34.3 \\
4 & 1950 \\
4 & 15.1 \\
5 & 1983\end{array}$ & $\begin{array}{l}37.7 \\
1958 \\
31.5 \\
1955\end{array}$ \\
\hline
\end{tabular}

* Dry bulb wet bulb, and dew point temperatures in ${ }^{\circ} \mathrm{F}$, relative humidity in $\%$.

+ Most recent of multiple occurrences. 
IABLE 6.4. Solar Radiation - Average and Extreme Daily Values (1953 Through 1993)

\begin{tabular}{|c|c|c|c|c|c|c|c|c|c|c|c|c|c|}
\hline & JAN & FEB & MAR & APR & MAY & JUN & JUL & $A \cup G$ & SEP & OCT & NOV & DEC & YEAR \\
\hline AVERAGE & 110 & 188 & 324 & 455 & $55 R$ & 612 & 634 & 541 & 409 & 257 & 126 & 86 & 358 \\
\hline $\begin{array}{l}\text { HIGHEST } \\
\text { YEAR }\end{array}$ & $\begin{array}{c}277 \\
1969\end{array}$ & $\begin{array}{c}422 \\
1958\end{array}$ & $\begin{array}{c}542 \\
1968\end{array}$ & $\begin{array}{c}704 \\
1972\end{array}$ & $\begin{array}{c}838 \\
1977\end{array}$ & $\begin{array}{c}821 \\
1971\end{array}$ & $\begin{array}{c}808 \\
1974\end{array}$ & $\begin{array}{c}721 \\
1957\end{array}$ & $\begin{array}{c}591 \\
1970\end{array}$ & $\begin{array}{c}434 \\
1973\end{array}$ & $\begin{array}{c}295 \\
1971\end{array}$ & $\begin{array}{c}196 \\
1972\end{array}$ & $\begin{array}{c}838 \\
\text { MAY } 1977\end{array}$ \\
\hline $\begin{array}{l}\text { LOWEST } \\
\text { YEAR }\end{array}$ & $\begin{array}{c}16 \\
1976+\end{array}$ & $\begin{array}{c}21 \\
1976\end{array}$ & $\begin{array}{c}44 \\
1979\end{array}$ & $\begin{array}{c}75 \\
1974\end{array}$ & $\begin{array}{c}67 \\
1962\end{array}$ & $\begin{array}{c}92 \\
1992\end{array}$ & $\begin{array}{c}118 \\
1972\end{array}$ & $\begin{array}{c}104 \\
1993\end{array}$ & $\begin{array}{c}61 \\
1957\end{array}$ & $\begin{array}{c}33 \\
1974\end{array}$ & $\begin{array}{c}14 \\
1969\end{array}$ & $\begin{array}{c}9 \\
1973\end{array}$ & $\begin{array}{c}9 \\
\text { DEC } 1973\end{array}$ \\
\hline
\end{tabular}

+ Most recent of multiple occurrences.

The highest daily values occur with a clear sky and clean air, and the lowest daily values commonly occur on days overcast with low stratus clouds. The lowest midday values of hourly solar radiation occurred on May 18, 1980, as the dense ash cloud from the morning eruption of the Mount St. Helens volcano passed over eastern Washington. Hourly .0lar radiation values dropped to zero at 1100 hours and remained at zero for the rest of that day.

\subsection{THUNDERSTORMS}

A thunderstorm day is one in which thunder is heard at the observing station one or more times during a calendar day. If a thunderstorm were to begin just before midnight, and continue until after midnight, it is possible to have two thunderstorm days from a single storm.

Table 6.5 shows that thunderstorms have occurred in every month of the year, except January and November. The thunderstorm season is essentially from April through September. The average number of thunderstorm days per year is 10; however, the total has varied from a low of 3 in 1949, to a high of 23 in 1948. The largest number of thunderstorms in any single month has been 8 in June 1972, July 1983, and August 1953.

Prior to 1975 , wet bulb temperatures $\geq 75^{\circ} \mathrm{F}$ had never been observed at the HMS. On July 8, 9, and 10 of that year, 7 hourly observations were made of wet bulb temperature $\geq 75^{\circ} \mathrm{F}$. 
TABLE 6.5. Average Number of Days of Various Meteorological Phenomenon (1945 Through 1993)

\begin{tabular}{|c|c|c|c|c|c|c|c|c|c|c|c|c|c|}
\hline PHENOMENON & JAN & FEB & MAR & APR & MAY & JUN & JUL & $\underline{A U G}$ & SEP & OCT & NOV & $\underline{D E C}$ & YEAR \\
\hline Thunderstorm & 0 & $\leq .1$ & 0.2 & 0.8 & 1.6 & 2.4 & 2.0 & 2.1 & 0.8 & 0.2 & 0 & 1.1 & 10 \\
\hline Dust or Blowing Dust & 0.4 & 0.1 & 0.5 & 0.6 & 0.7 & 0.4 & 0.4 & 0.3 & 0.5 & 0.3 & 0.2 & 0.2 & \\
\hline Glaze & 2.2 & 0.7 & 0.1 & 0 & 0 & 0 & 0 & 0 & 0 & 0 & 0.8 & 2.5 & \\
\hline
\end{tabular}

\subsection{DUST AND BLOWING DUST}

The criteria for both dust and blowing dust is that horizontal visibility be reduces to 6 miles or less. Dust is carried into the area from a distant source and may occur without strong winds. Blowing dust occurs when dust is being picked up locally and occurs with stronger winds. Both dust and blowing dust have occurred at the HMS, but, in most cases, it is blowing dust. Table 6.5 presents the average number of days per month and year of dust and blowing dust during the period from 1945 through 1993. The average number of days per year with dust or blowing dust is 5 . The greatest number of such days in any year was 20 days in 1980, while the fewest was 0 in 1987 and earlier years. The greatest number of days with dust or blowing dust in any one month was 9 in May 1980.

\subsection{GLAZE}

Glaze is a coating of ice formed when rain or drizzle freezes on contact with any surface having a temperature that is below freezing. Table 6.5 provides data on the number of days per month and year with glaze for the period from 1945 through 1993 . The average number of days with freezing rain or freezing drizzle is 6 . The highest number of days with glaze in any winter season was 18 during the winter of 1969-1970; the least, 1 day during the winter of 1987-1988 and earlier winters. The greatest number of such days in any single month was 9 in January 1970. 


\subsection{REFERENCES}

Glantz, C. S., and M. M. Islam. 1988. The Data Collection Component of the Hanford Meteorology Monitoring Program. PNL-6684, Pacific Northwest Laboratory, Richland, Washington.

Jenne, D. E., and R. E. Kerns. 1959. A Climatological Study of the Hanford Area. HW-57722, General Electric, Hanford Atomic Products Operation, Richland, Washington.

Stone, W. A., D. E. Jenne, and J. M. Thorp. 1972. Climatography of the Hanford Area. BNWL-1605, Pacific Northwest Laboratory, Richland, Washington.

Stone, W. A., J. M. Thorp, O. P. Gifford, and D. J. Hoitink. 1983. Climatological Summary for the Hanford Area. PNL-4622, Pacific Northwest Laboratory, Richland, Washington. 


\subsection{BIBLIOGRAPHY}

Andrews, G. L., and J. W. Buck. 1987. Hanford Meteorological Station Computer Codes: Volume 2 - The PROD Computer Code. PNL-6279 Vol. 2, Pacific Northwest Laboratory, Richland, Washington.

Andrews, G. L., and J.W. Buck. 1987. Hanford Meteorological Station Computer Codes: Volume 4 - The SUM Computer Code. PNL-6279 Vo1. 4, Pacific Northwest Laboratory, Richland, Washington.

Andrews, G. L., and J. W. Buck. 1987. Hanford Meteorological Station Computer Codes: Volume 6 - The SFC Computer Code. PNL-6279 Vol. 6, Pacific Northwest Laboratory, Richland, Washington.

Andrews, G. L., and J.W. Buck. 1988. Hanford Meteorological Station Computer Codes: Volume 7 - The RIVER Computer Code. PNL-6279 Vol. 7, Pacific Northwest Laboratory, Richland, Washington.

Andrews, G. L., and K. W. Burk. 1988. Hanford Meteorological Station Computer Codes: Volume 8 - The REVIEW Computer Code. PNL-6279 Vo1. 8, Pacific Northwest Laboratory, Richland, Washington.

Andrews, G. L. 1988. The Hanford Meteorological Data Collection System and Data Base. PNL-6509, Pacific Northwest Laboratory, Richland, Washington.

Andrews, G. L., and K. W. Burk. 1989. Hanford Meteorological Station Computer Codes: Volume 10 - The ARCHIVE Computer Code. PNL-6279 Vo1. 10, Pacific Northwest Laboratory, Richland, Washington.

Buck, J. W., and G. L. Andrews. 1987. Hanford Meteorological Station Computer Codes: Volume 1 - The GEN Computer Code. PNL-6279 Vol. 1, Pacific Northwest Laboratory, Richland, Washington.

Buck, J. W., and G. L. Andrews. 1987. Hanford Meteorological Station Computer Codes: Volume 3 - The TANK Computer Code. PNL-6279 Vo1. 3, Pacific Northwest Laboratory, Richland, Washington.

Buck, J. W., and G. L. Andrews. 1987. Hanford Meteorological Station Computer Codes: Volume 5 - The TOWER Computer Code. PNL-6279 Vol. 5, Pacific Northwest Laboratory, Richland, Washington.

Burk, K. W., and G. L. Andrews. 1989. Hanford Meteorological Station Computer Codes: Volume 9 - The Quality Assurance Computer Codes. PNL-6279 Vol. 9, Pacific Northwest Laboratory, Richland, Washington. 
Glantz, C. S., M. N. Schwartz, K. W. Burk, R. B. Kasper, M. W. Ligotke, and P. J. Perrault. 1990. Climatological Summary of Wind and Temperature Data for the Hanford Meteorological Monitoring Network. PNL-7471, Pacific Northwest Laboratory, Richland, Washington.

Stage, S. A., J. V. Ramsde11, Jr., C. A. Simonen, K. W. Burk, and L. K. Berg. 1993. Final Report on the Meteorological Database, December $1944-1949$.

PNWD-2200 HEDR, Battelle Pacific Northwest Laboratories, Richland, Washington. 
APPENDIX A

1993 WIND CLIMATOLOGY 


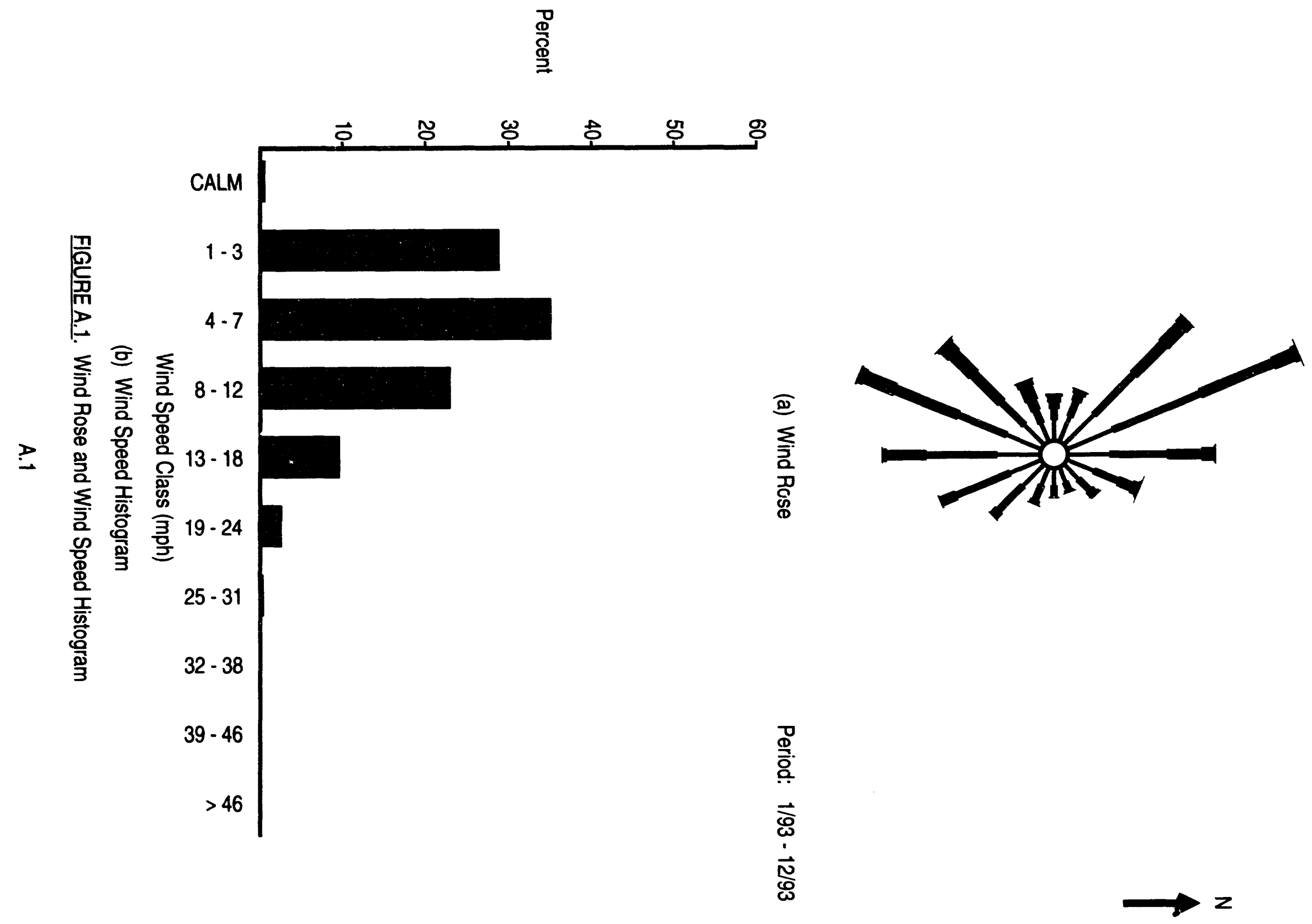



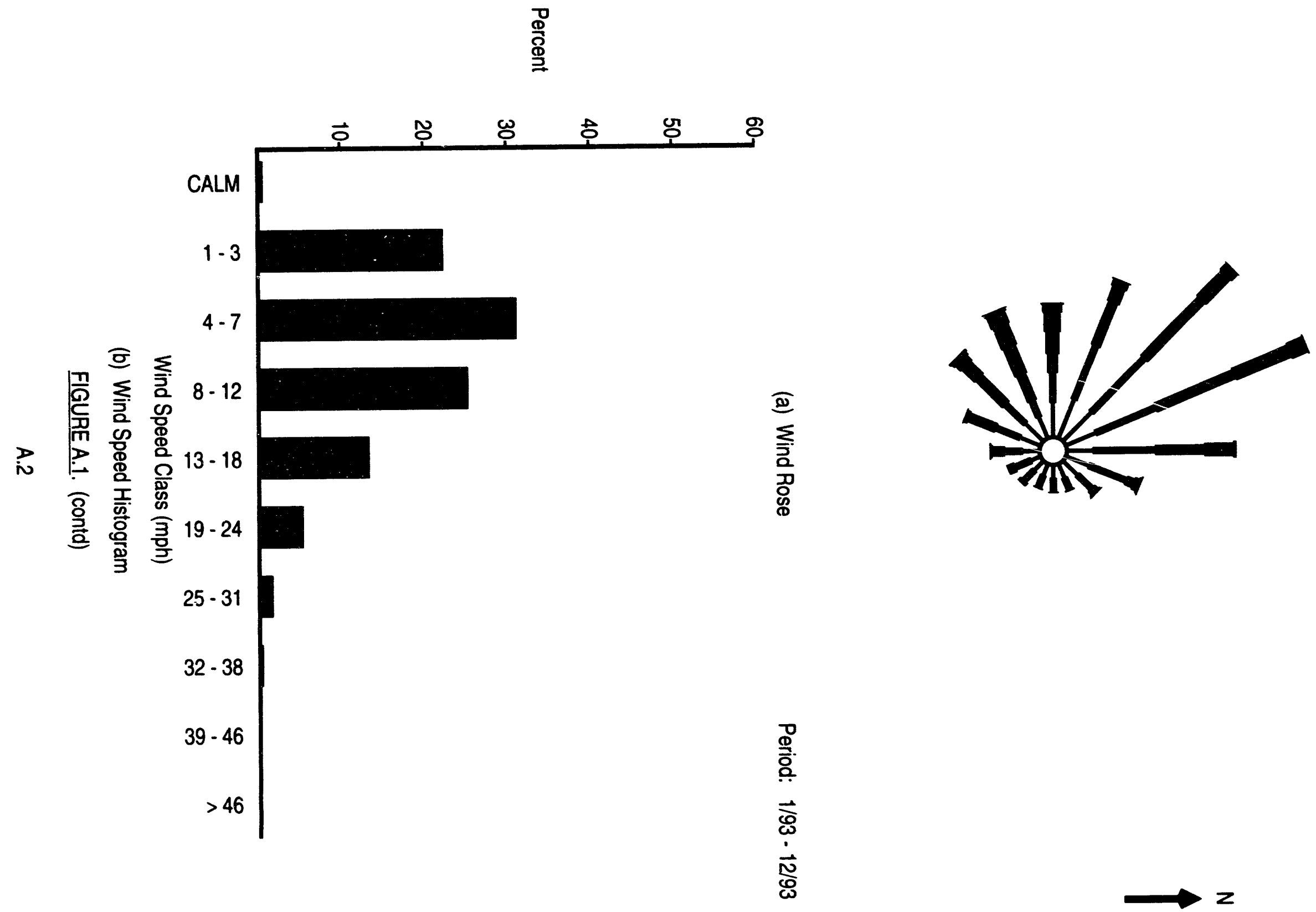

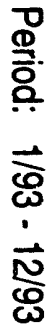




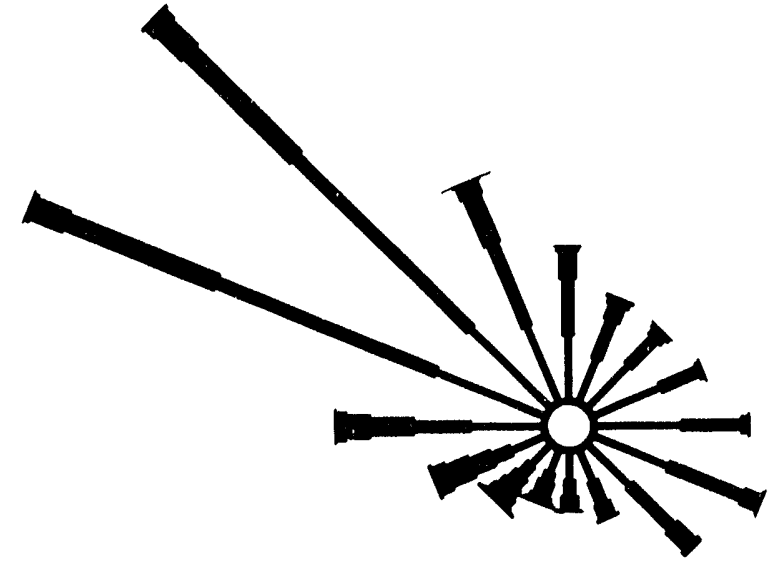

(a) Wind Rose

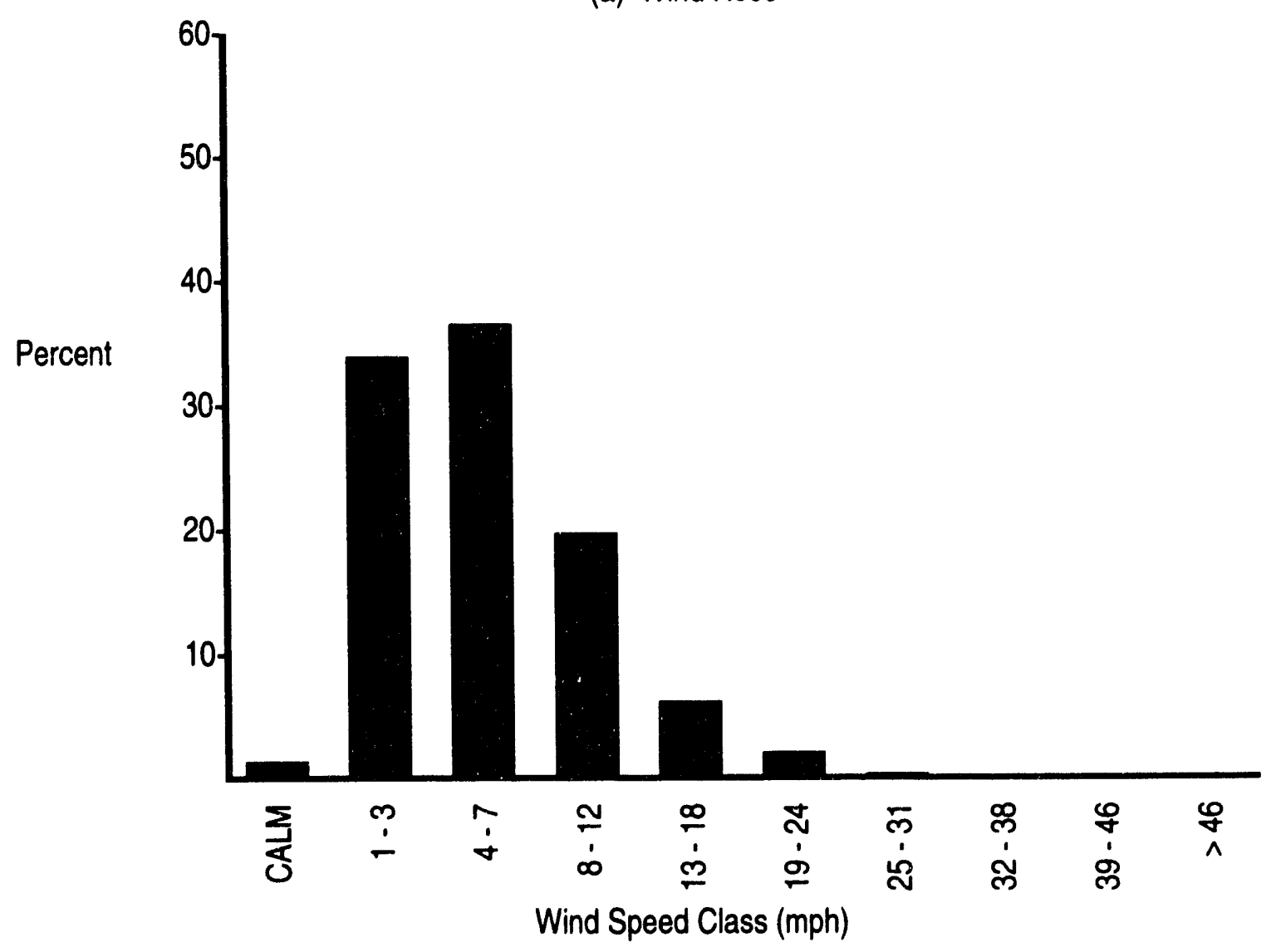

(b) Wind Speed Histogram

FIGURE A.1. (contd) 

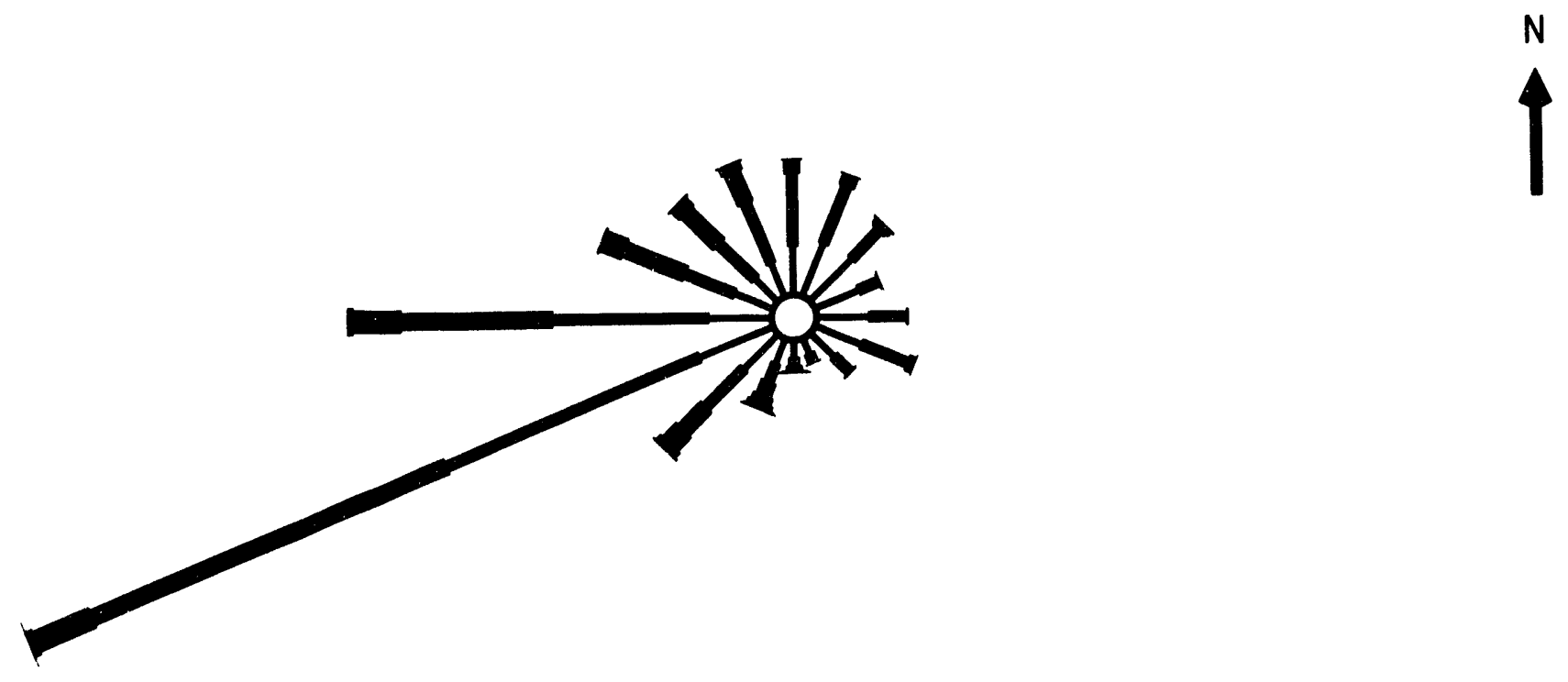

(a) Wind Rose

Period: $1 / 93-12 / 93$

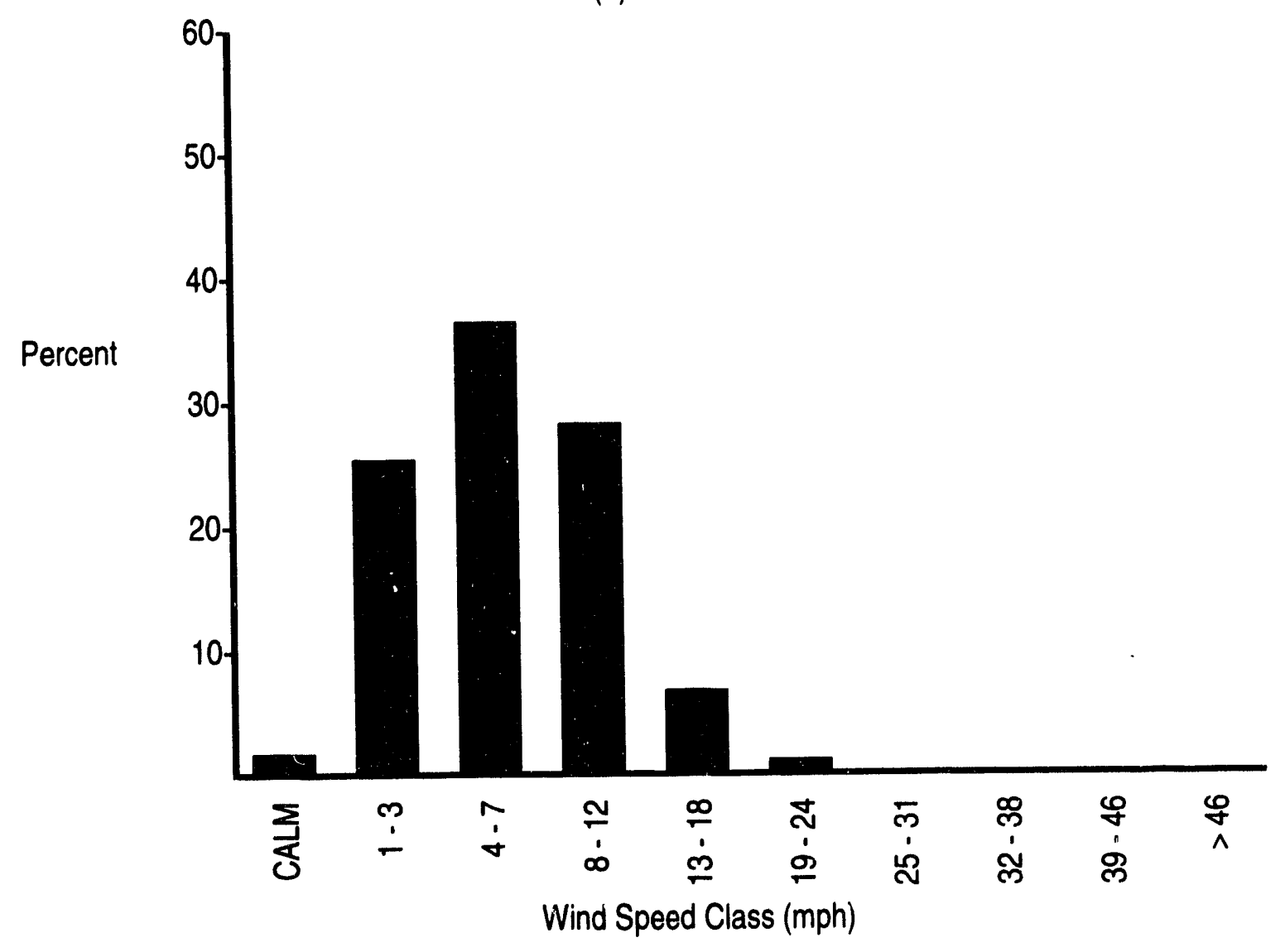

(b) Wind Speed Histogram

FIGURE A.1. (contd) 


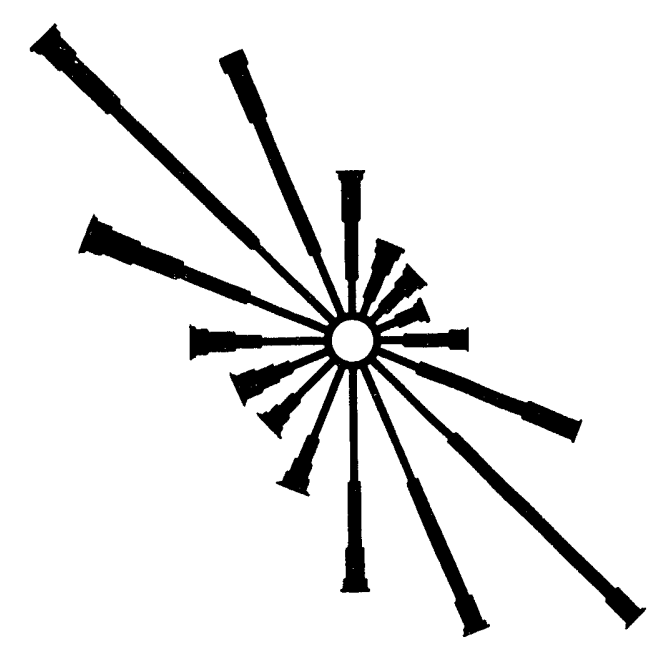

(a) Wind Rose

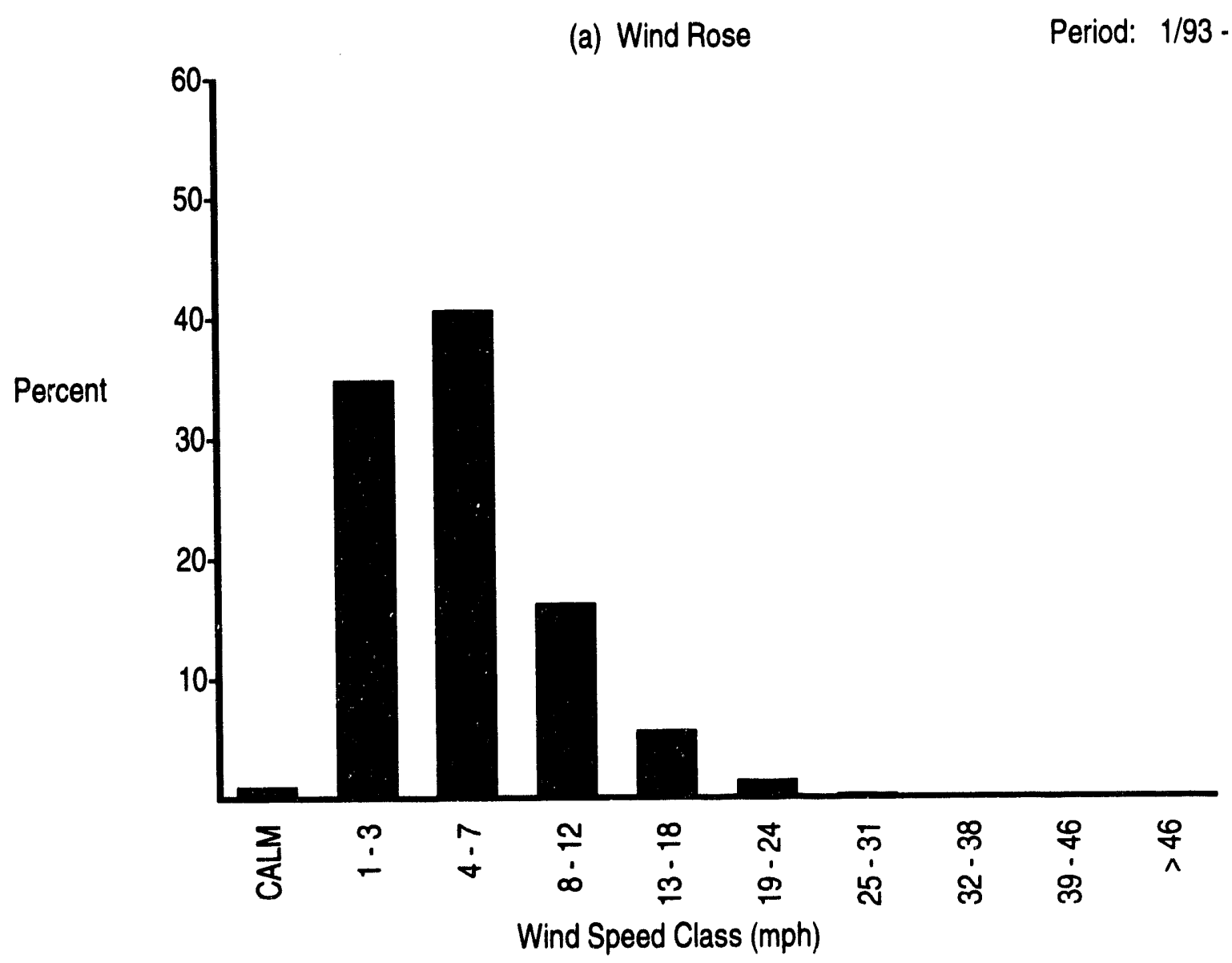

(b) Wind Speed Histogram

FIGURE A.1. (contd) 


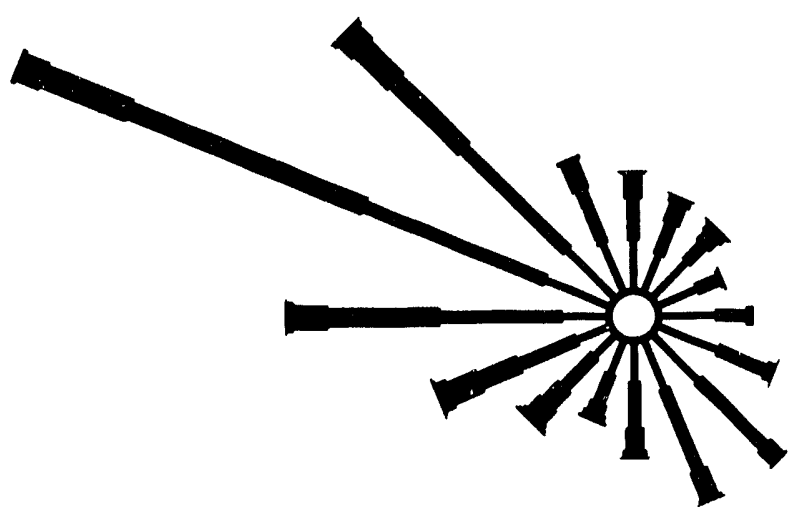

(a) Wind Rose

Period: 1/93-12/93

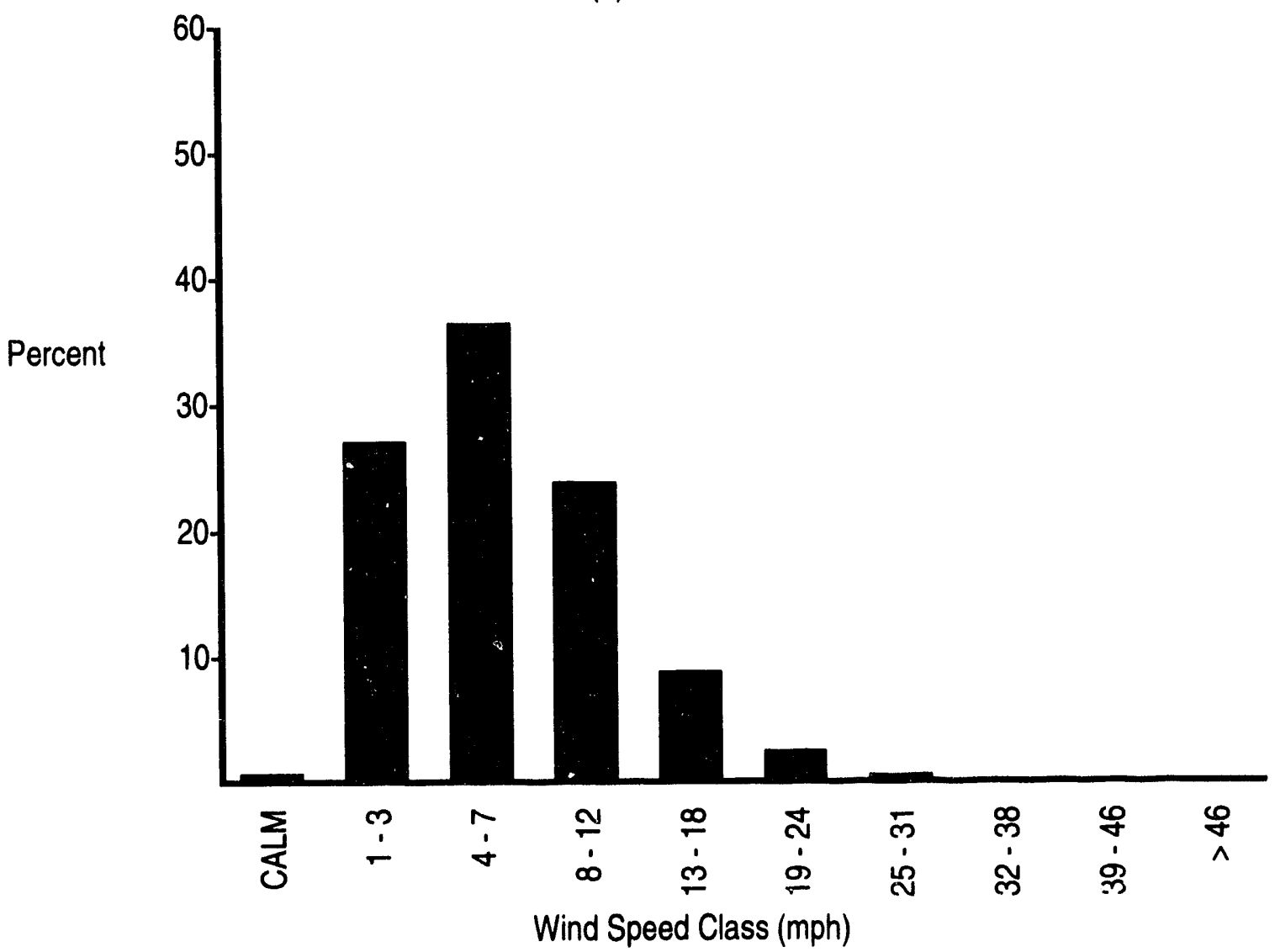

(b) Wind Speed Histogram

FIGURE A.1. (contd) 

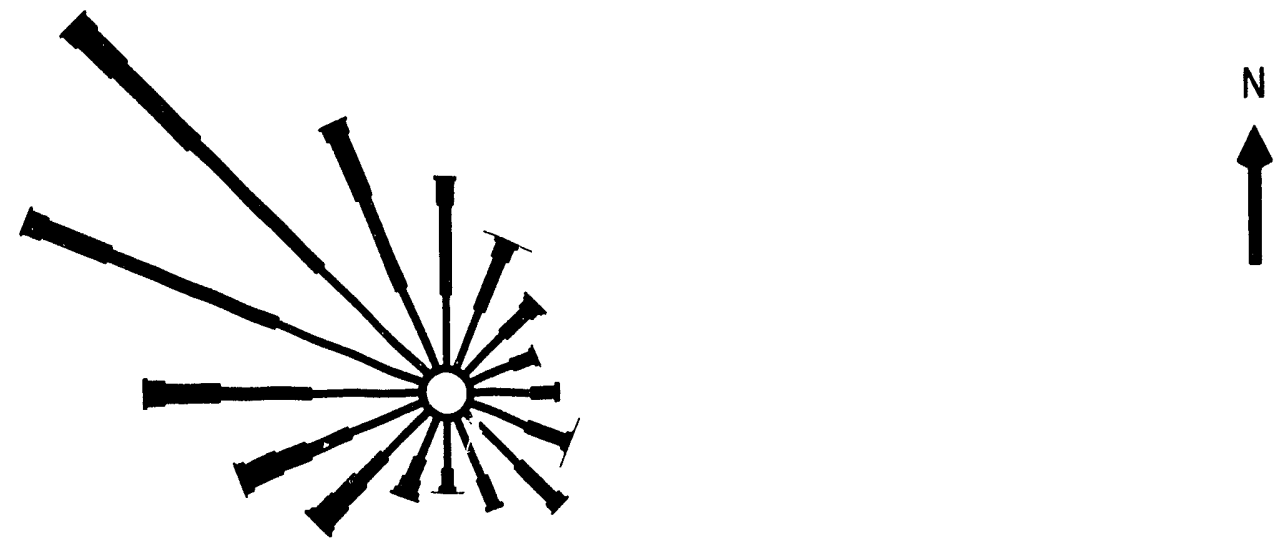

(a) Wind Rose

Period: $1 / 93-12 / 93$

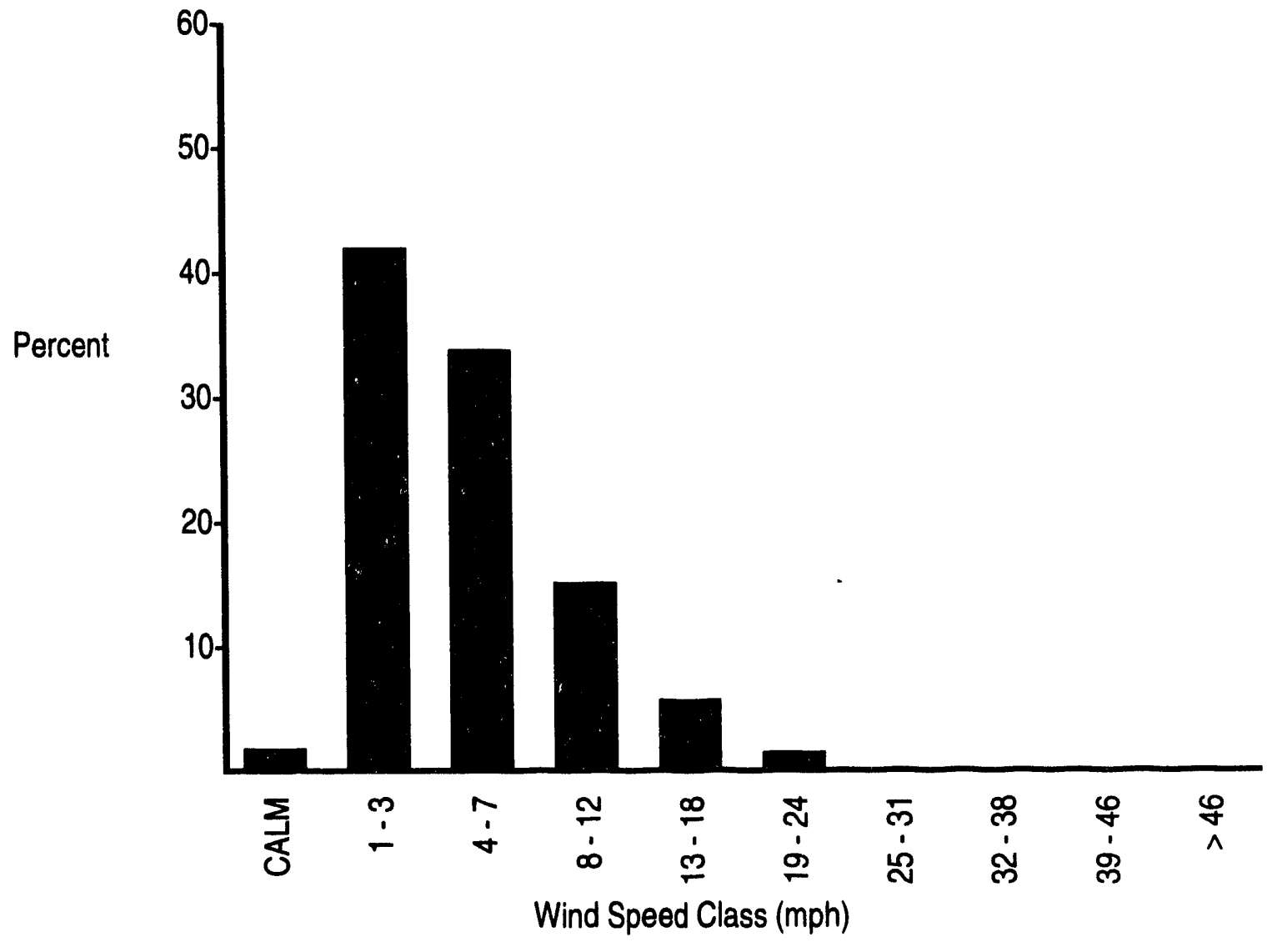

(b) Wind Speed Histogram

FigUREA.1. (contd) 


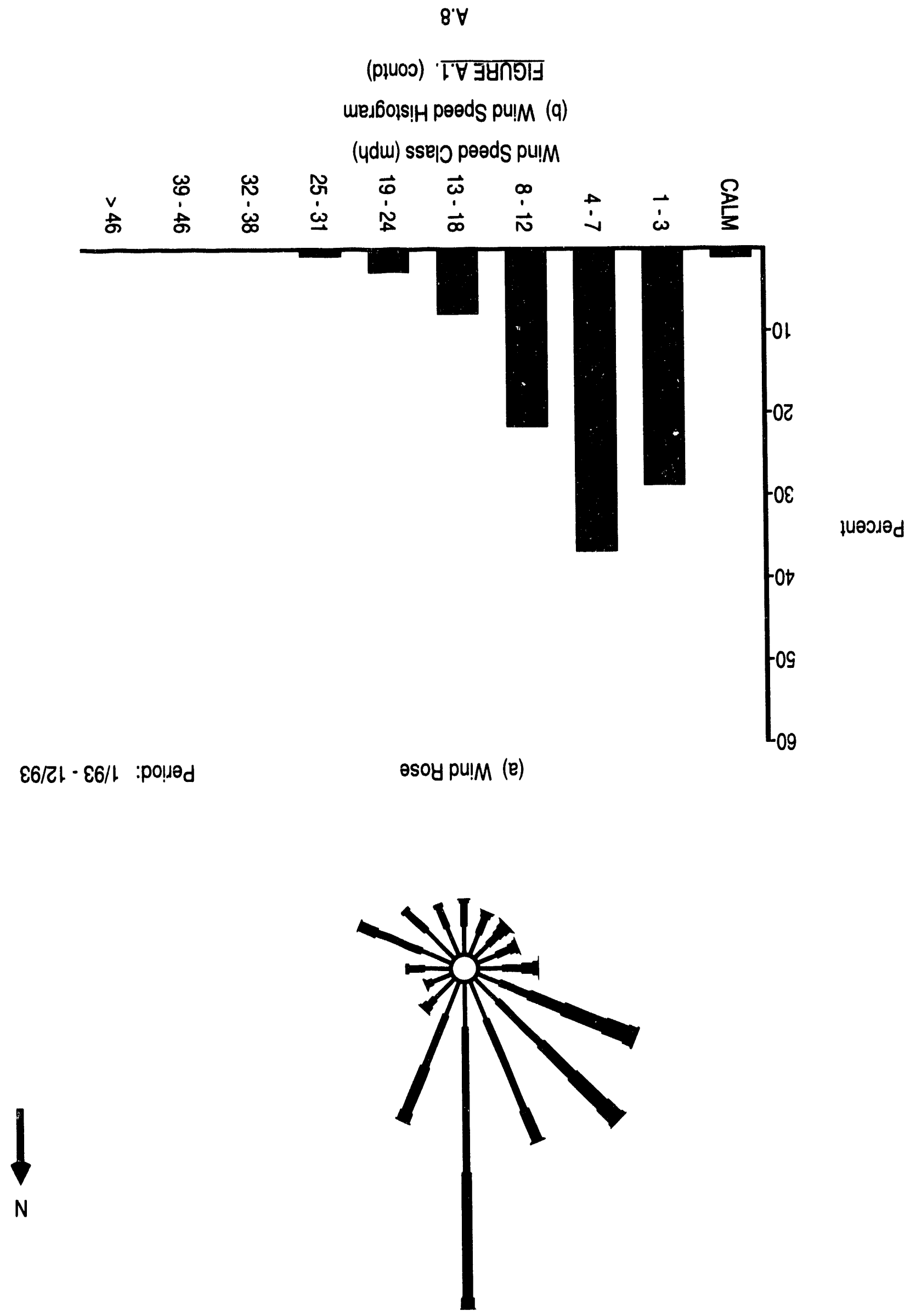




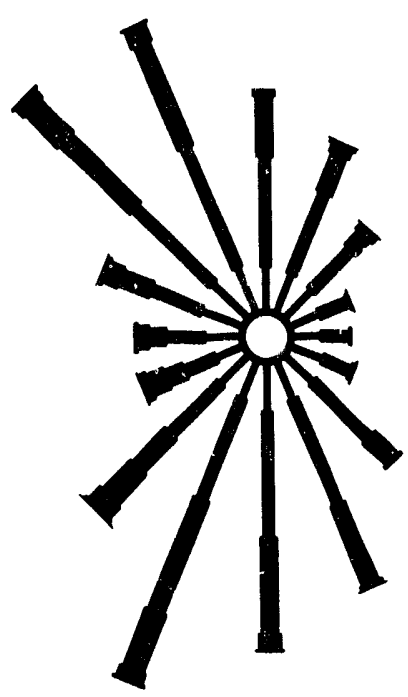

(a) Wind Rose

Period: $1 / 93 \cdot 12 / 93$

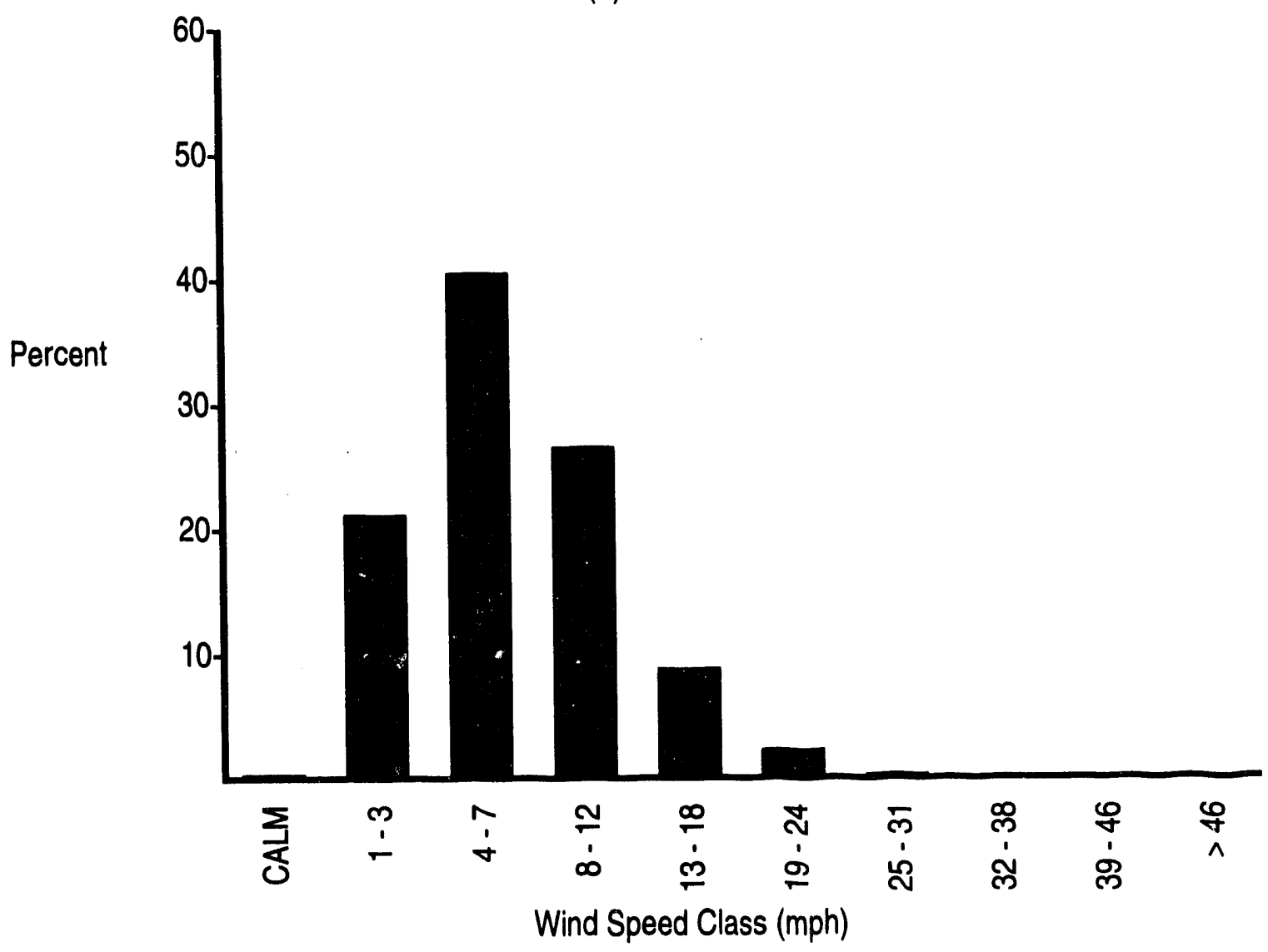

(b) Wind Speed Histogram

FIGURE A.1. (contd) 


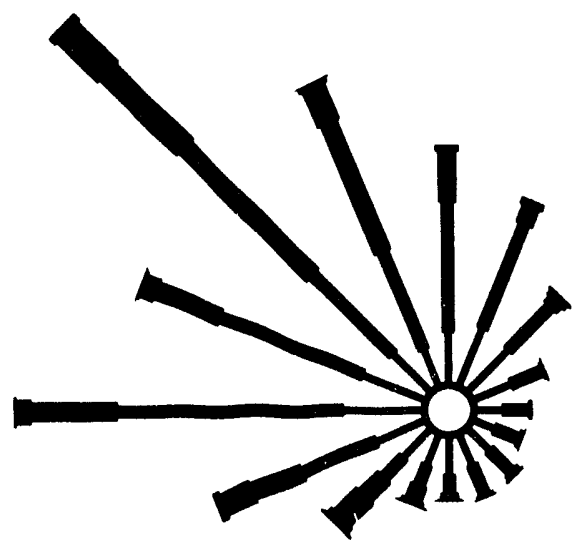

(a) Wind Rose

Period: $1 / 93 \cdot 12 / 93$

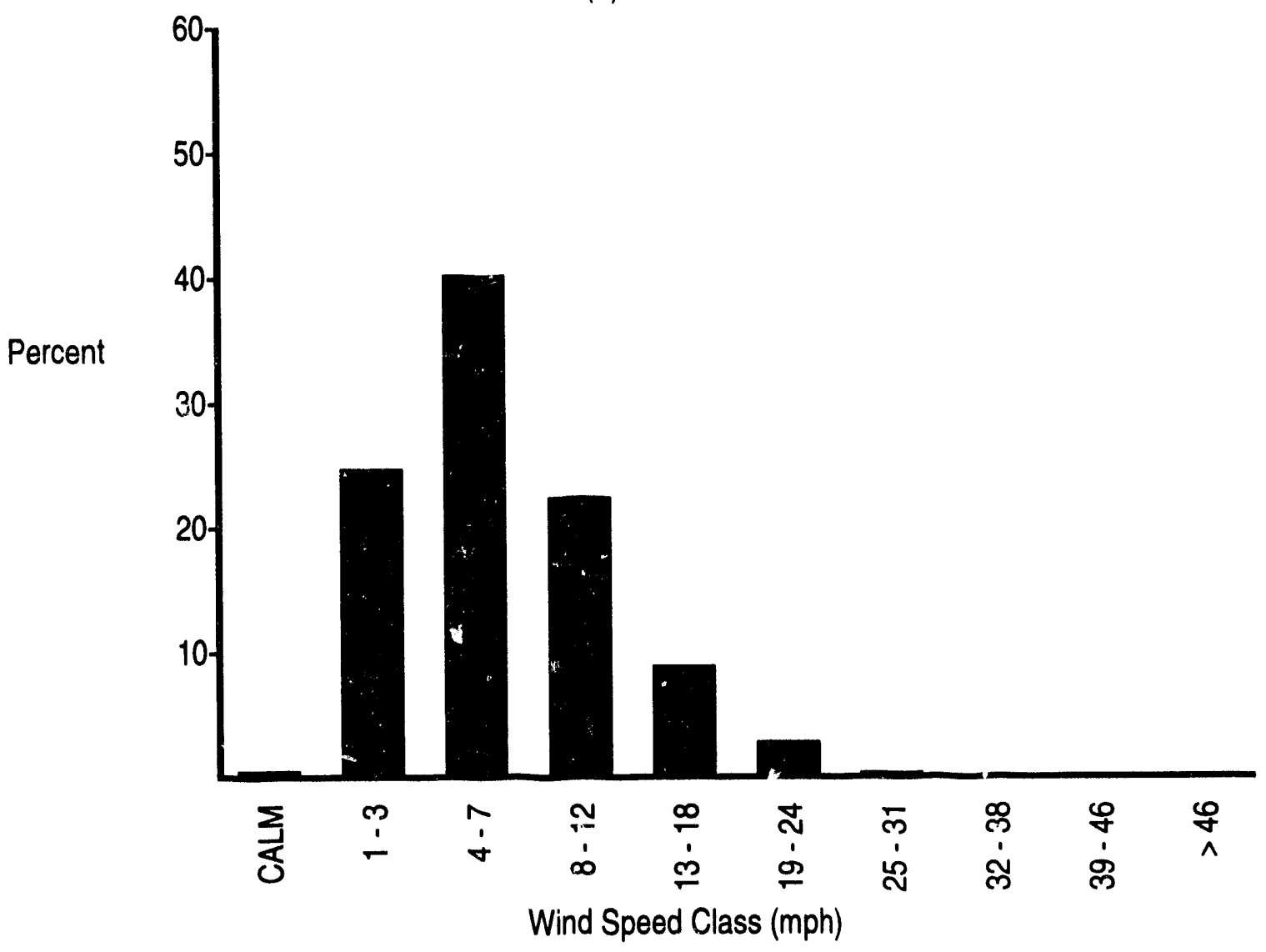

(b) Wind Speed Histogram

FIGUREA.1. (colitd) 

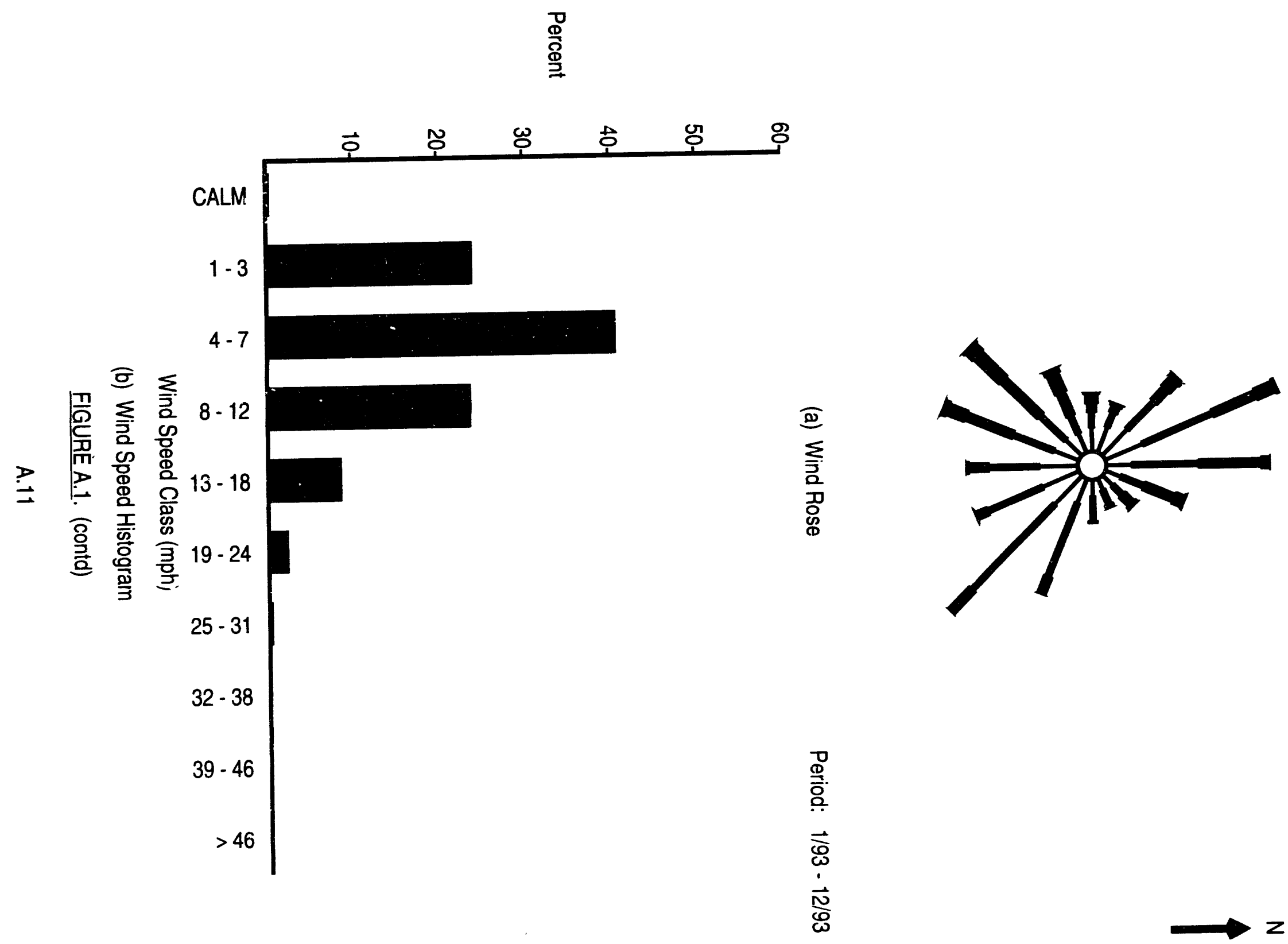

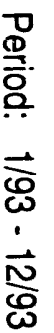



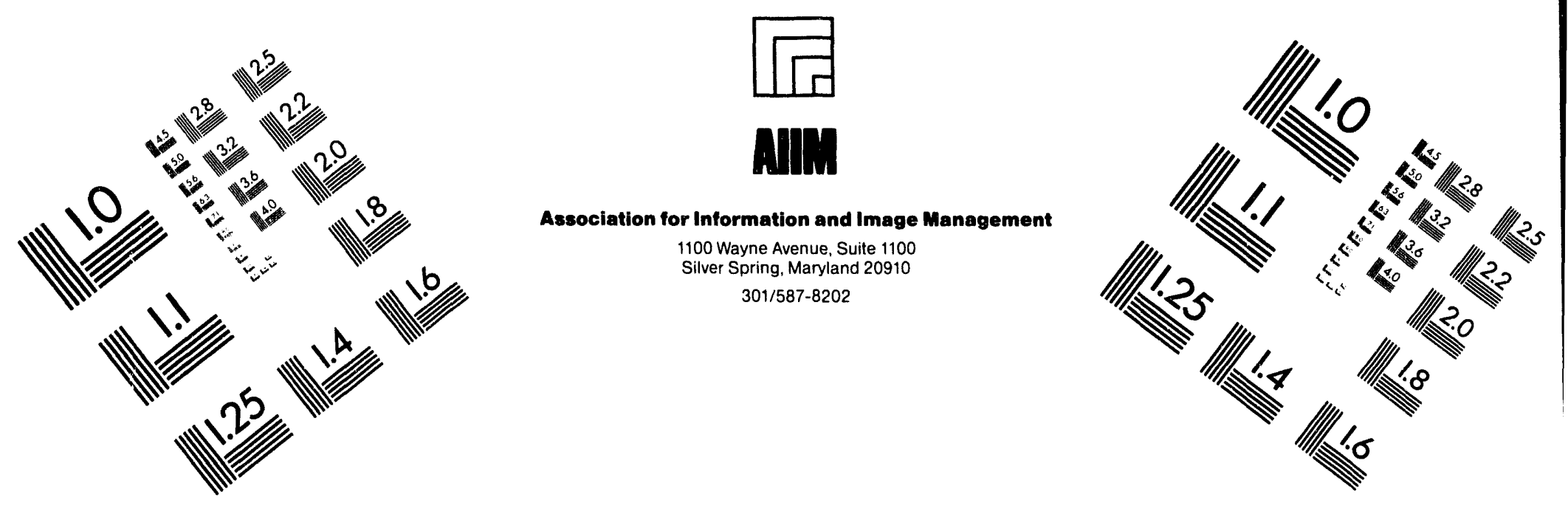

\section{Centimeter}

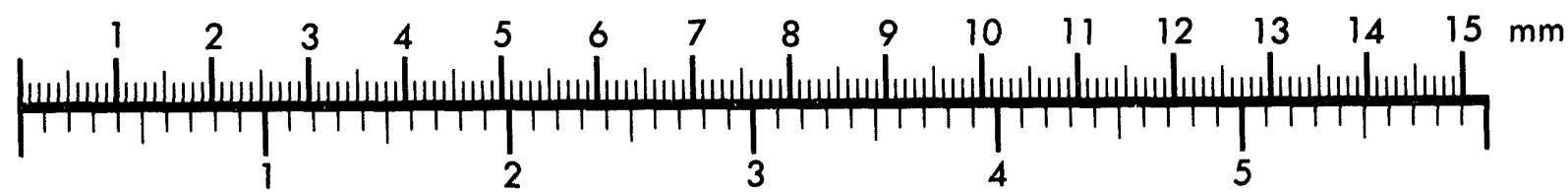
Inches
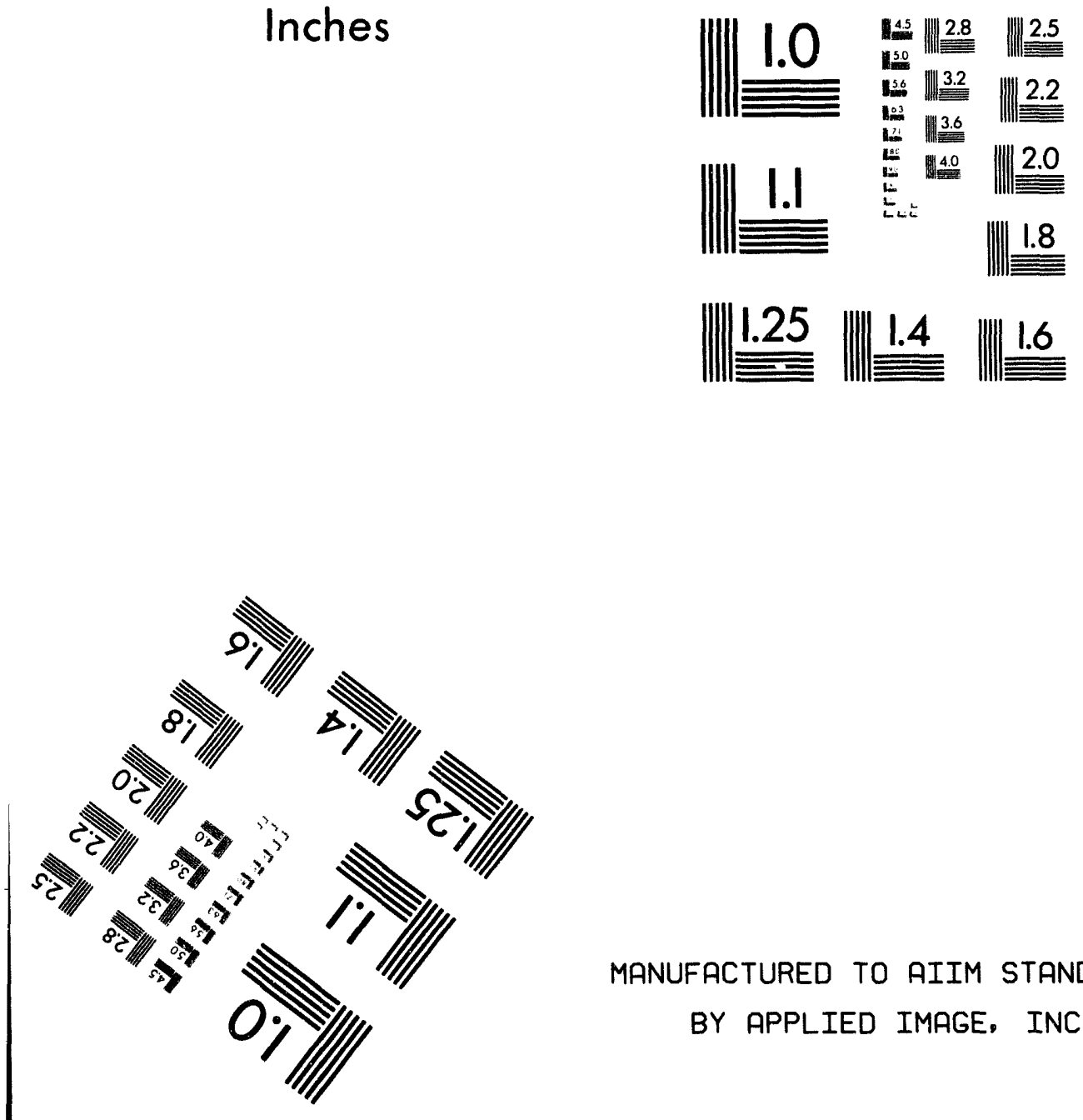

MANUFACTURED TO AIIM STANDARDS

BY APPLIED IMAGE, INC.

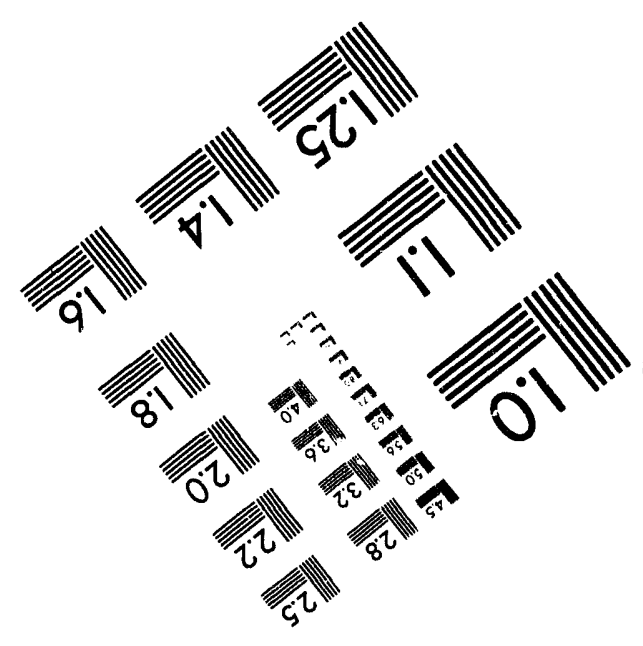



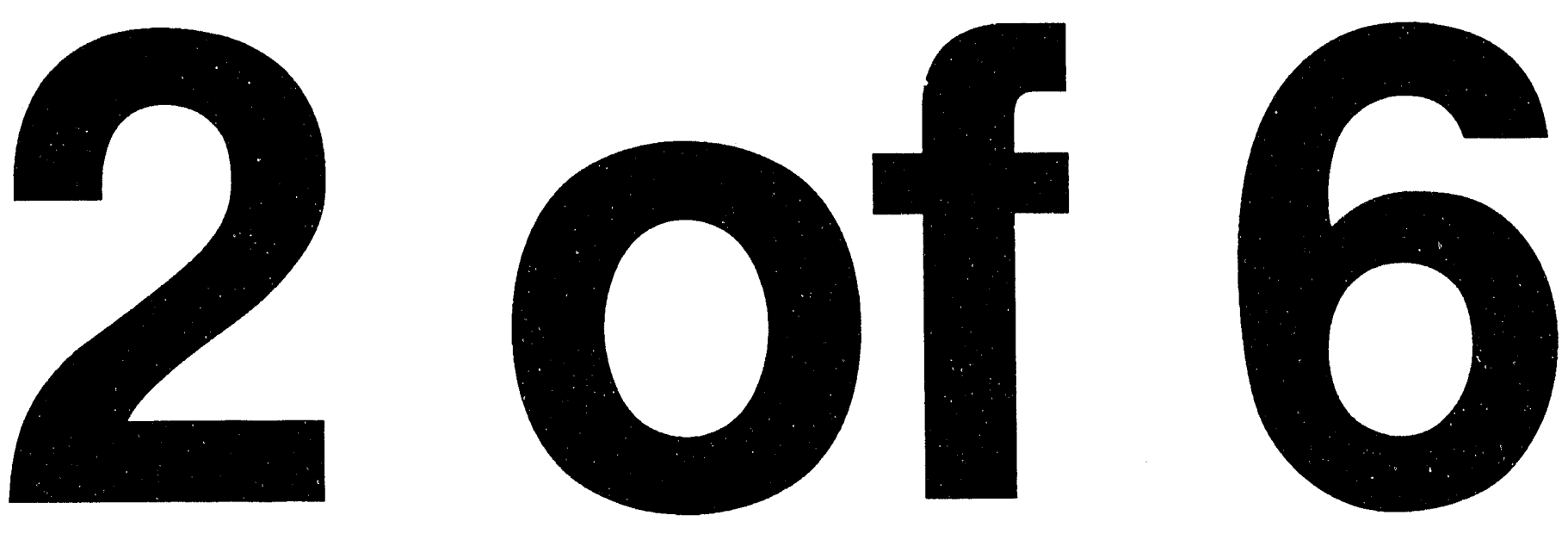


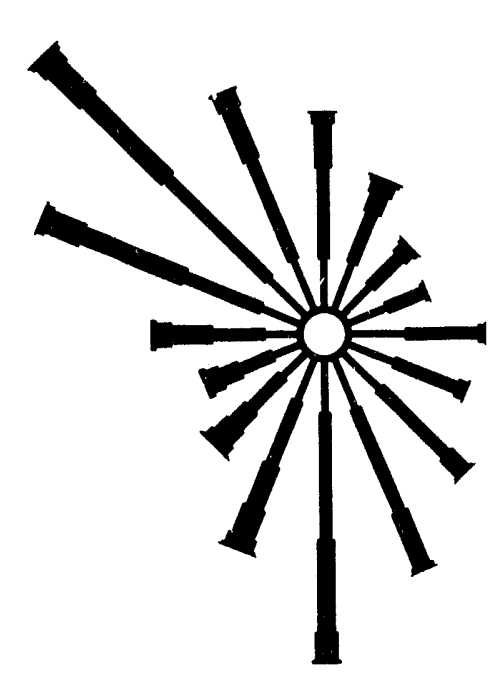

(a) Wind Rose

Period: $1 / 93-12 / 93$

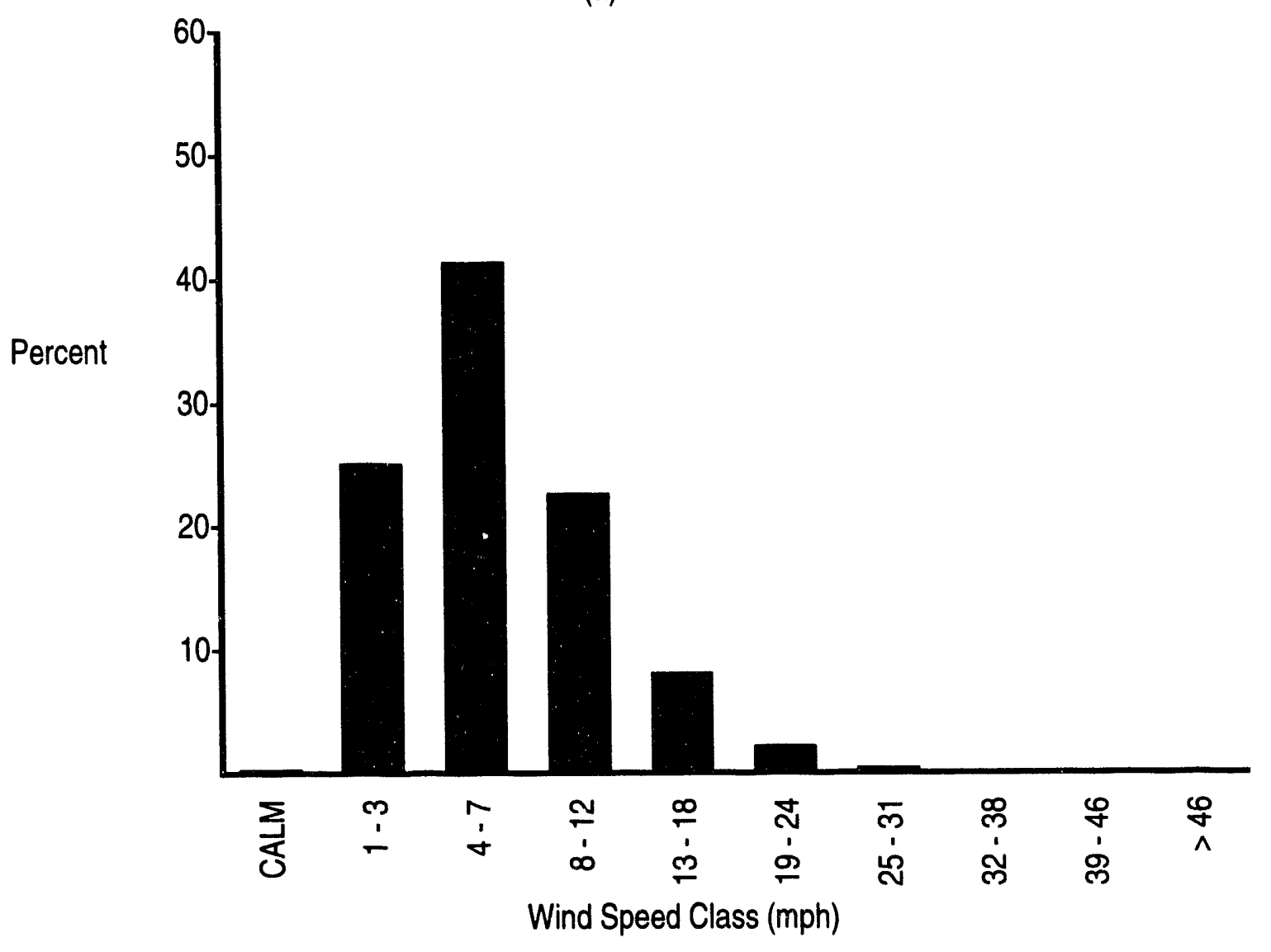

(b) Wind Speed Histogram

FIGURE A.1. (contd) 


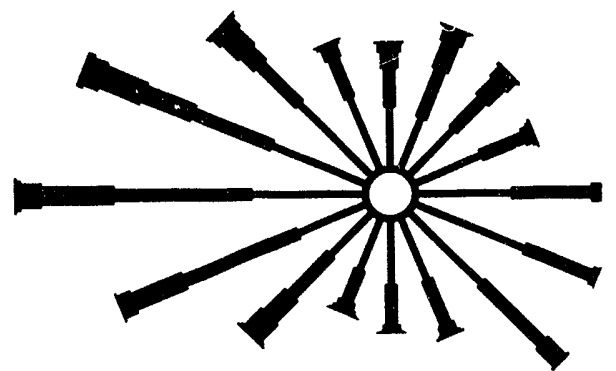

(a) Wind Rose

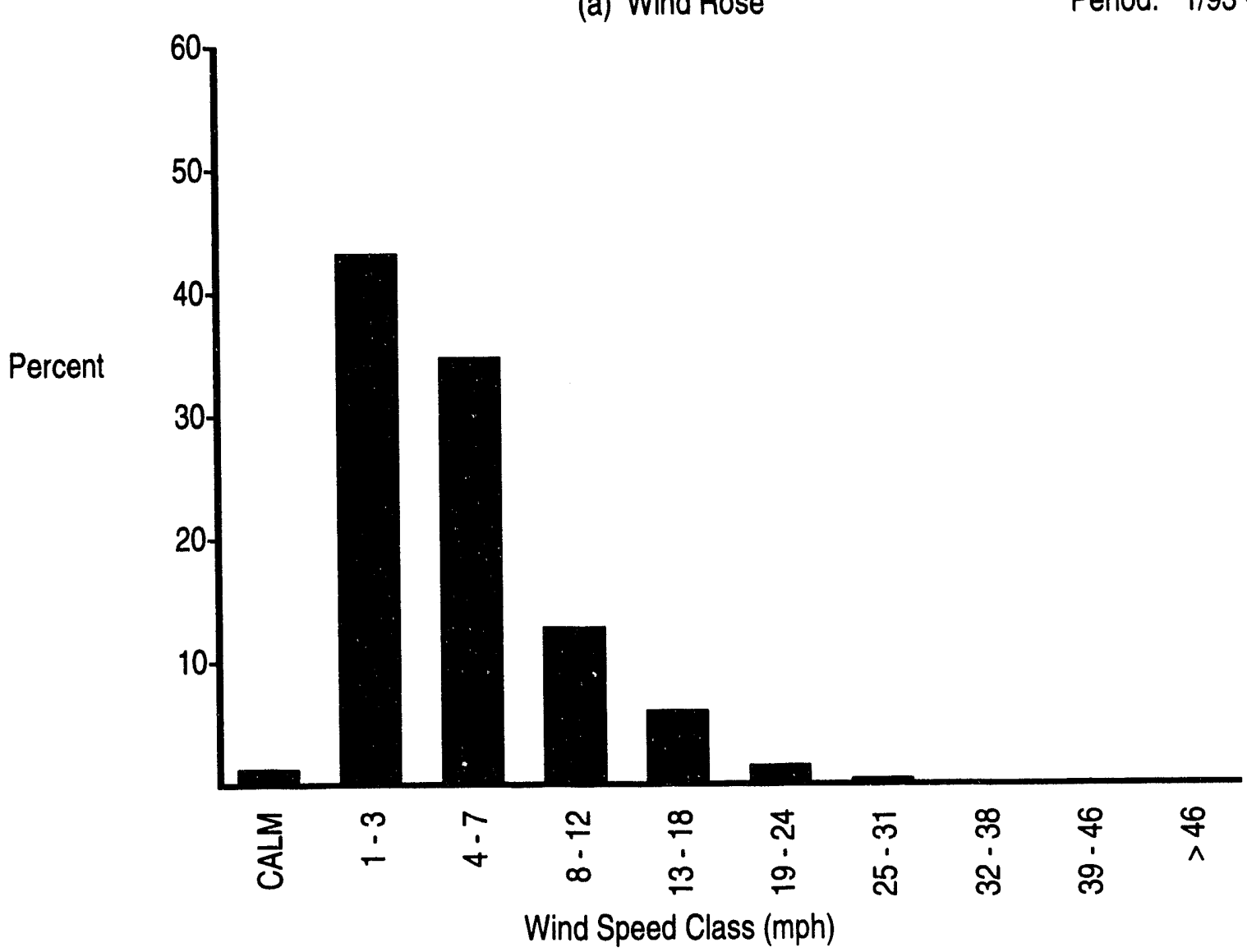

(b) Wind Speed Histogram

FIGURE A.1. (contd) 


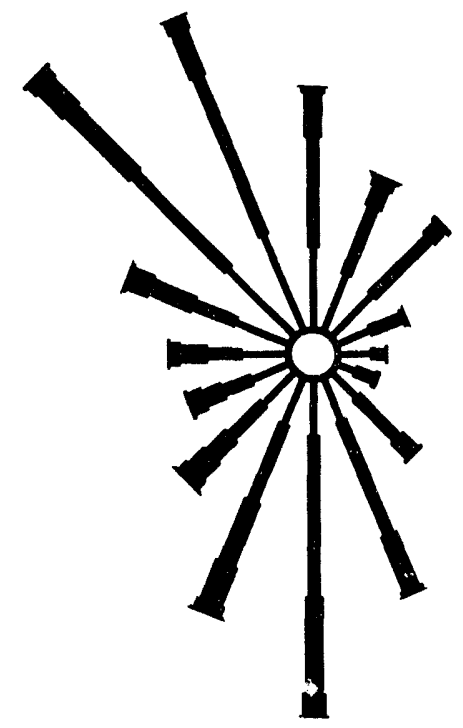

(a) Wind Rose

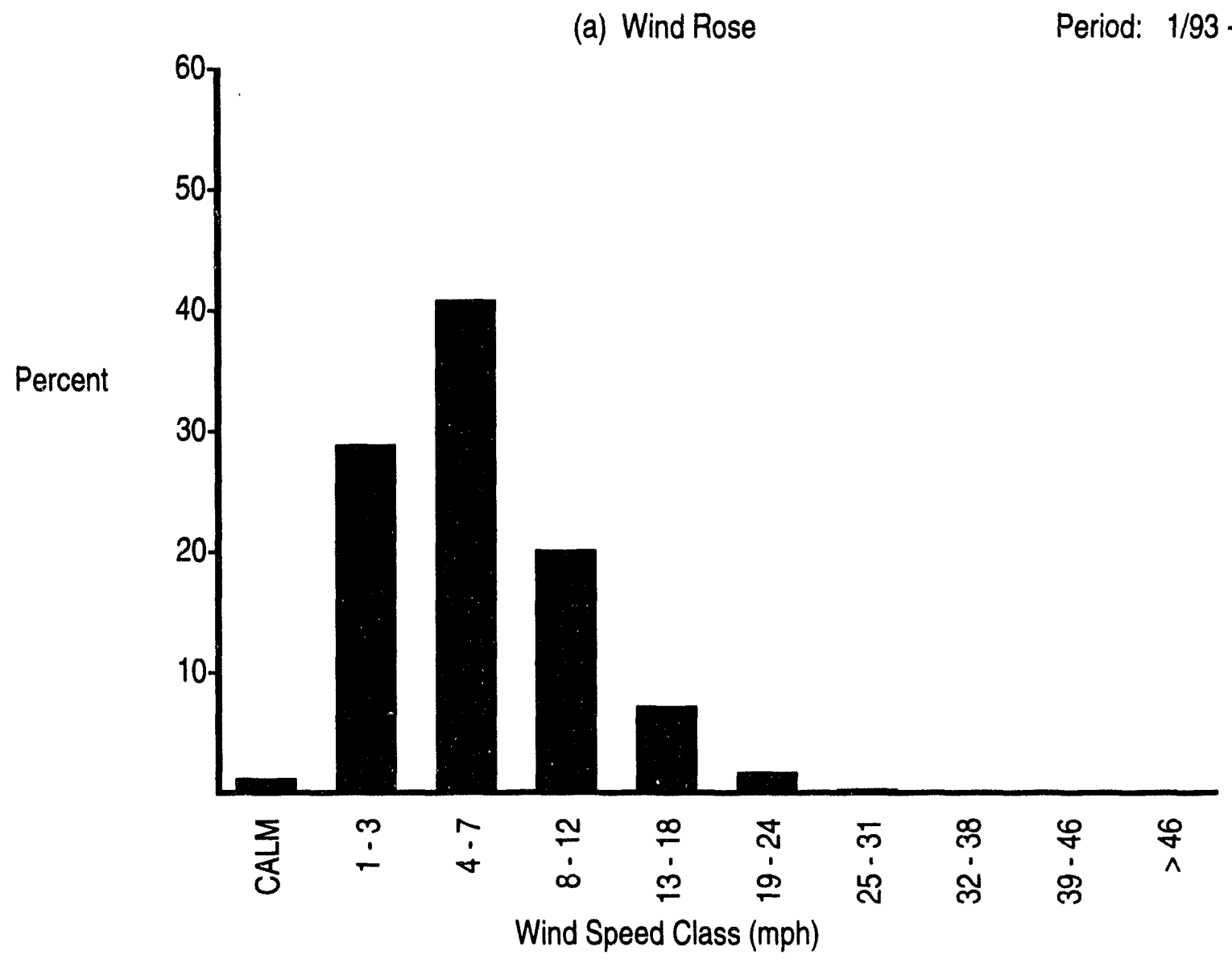

(b) Wind Speed Histogram

FIGURE A.1. (contd) 


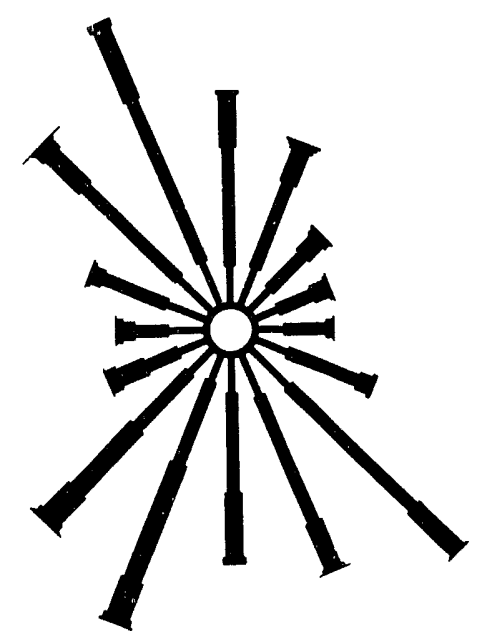

(a) Wind Rose

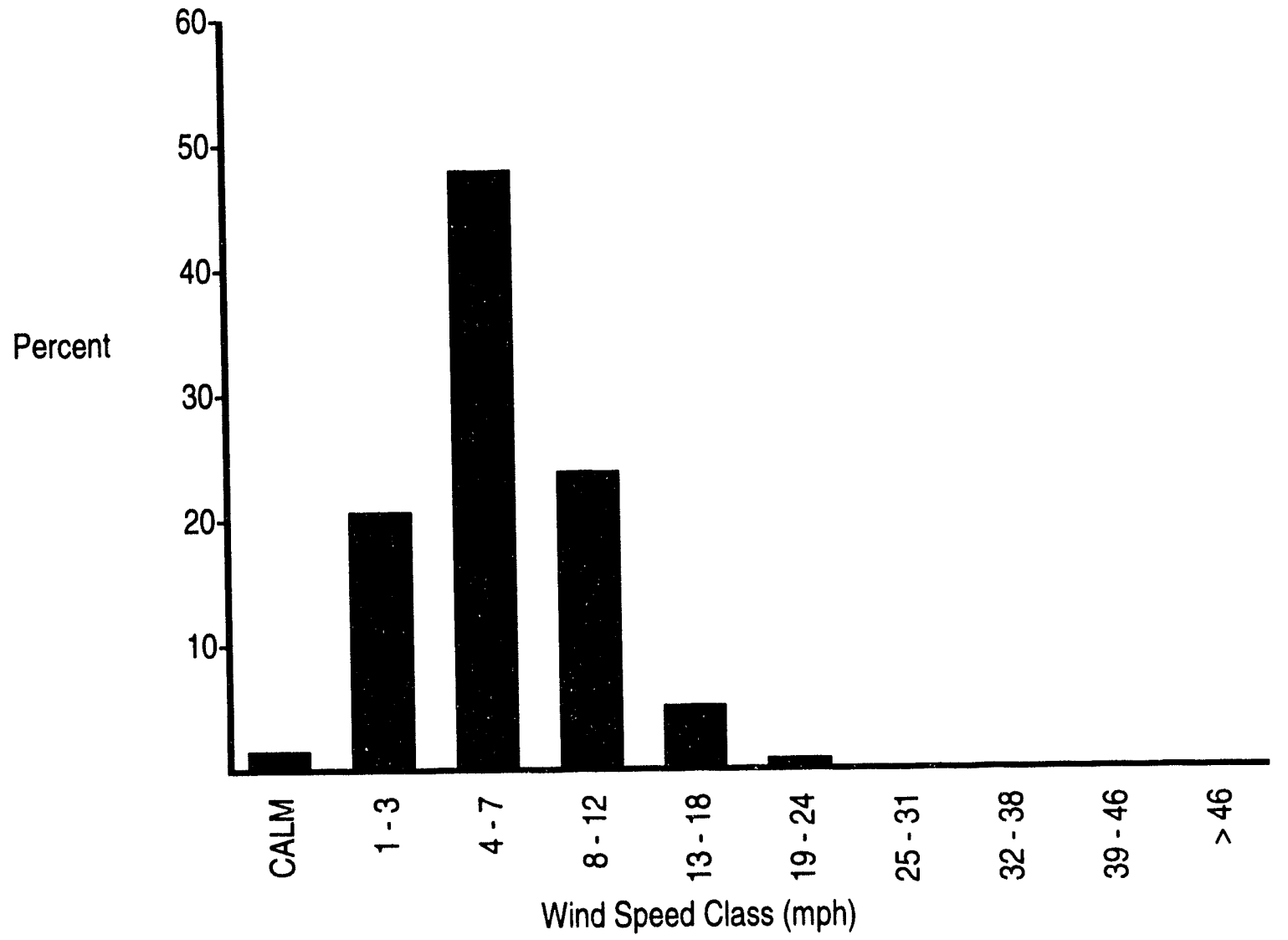

(b) Wind Speed Histogram

FIGURE A.1. (contd) 

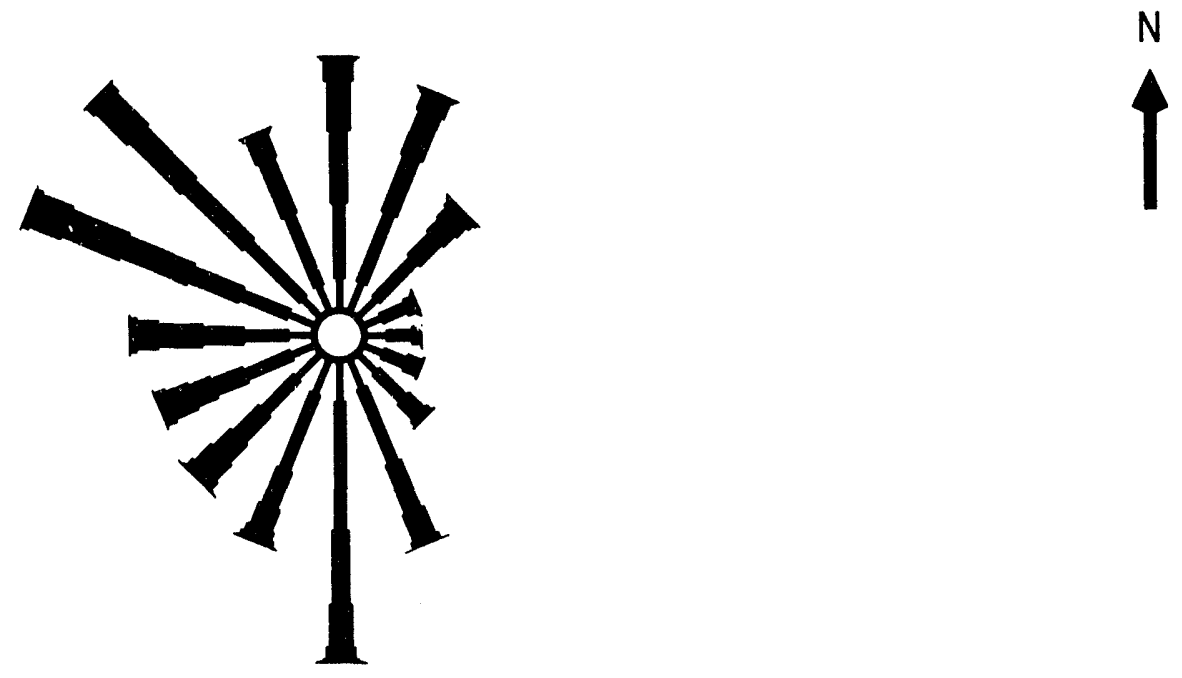

(a) Wind Rose

Period: $1 / 93-12 / 93$

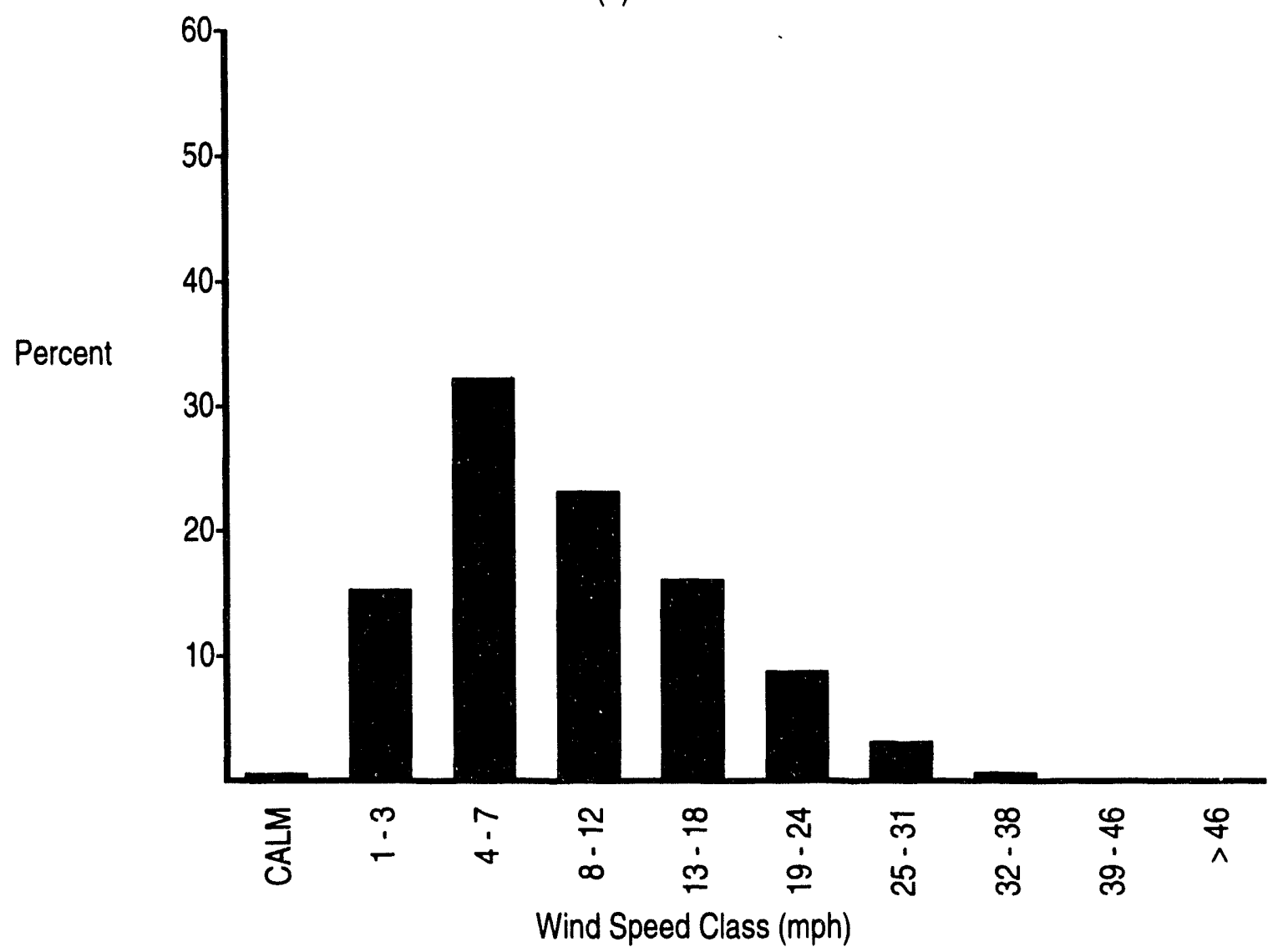

(b) Wind Speed Histogram

FIGURE A.1. (contd) 

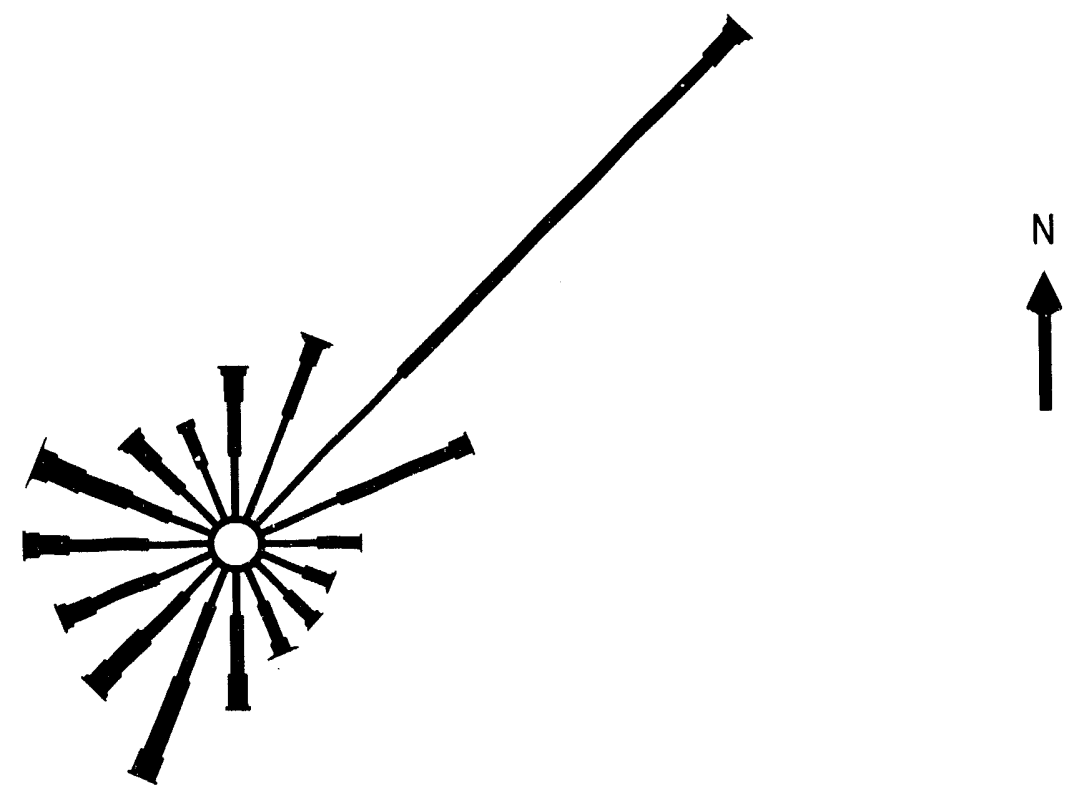

(a) Wind Rose

Period: $\quad 1 / 93-12 / 93$

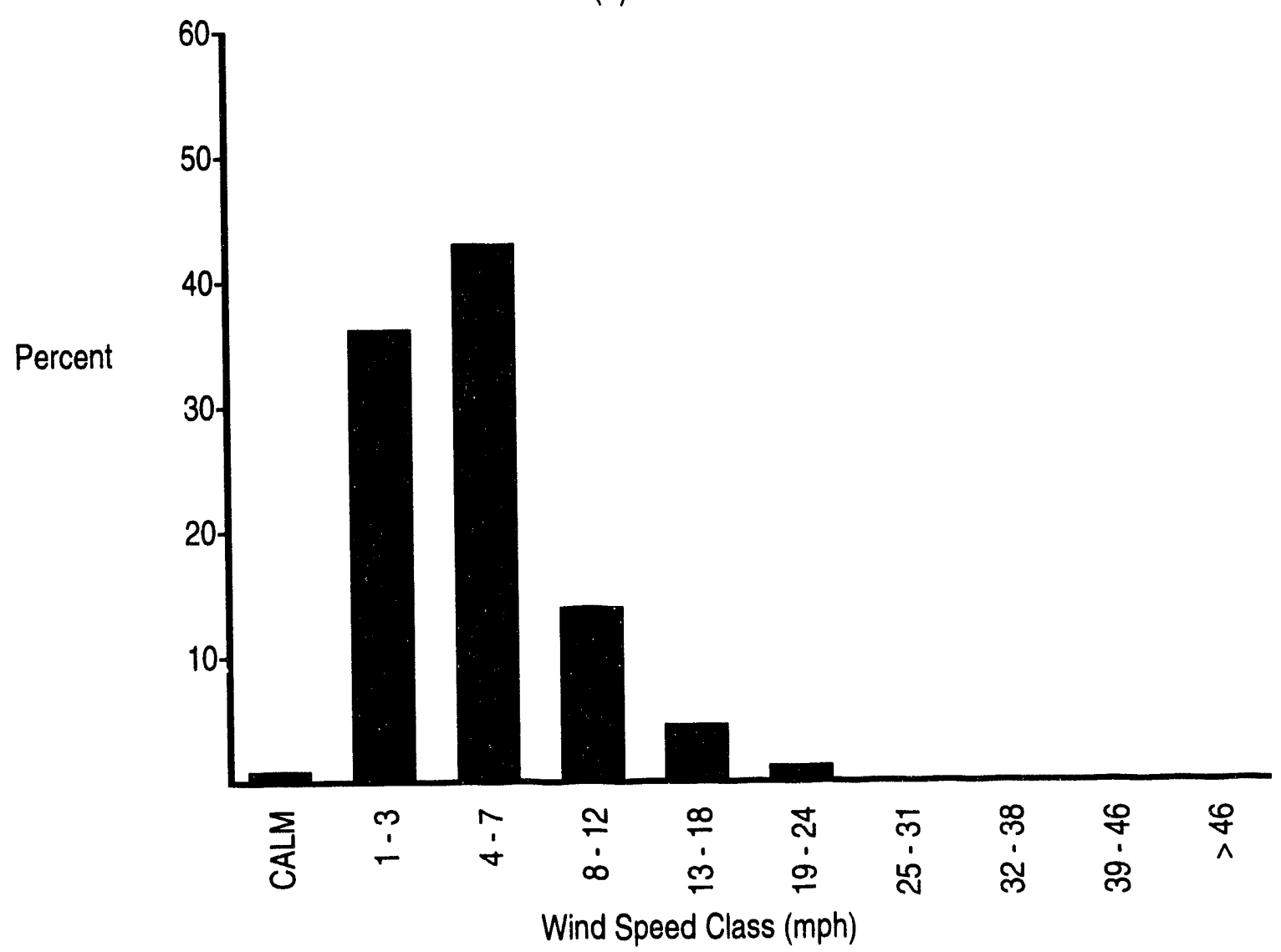

(b) Wind Speed Histogram

FIGURE A.1. (contd) 


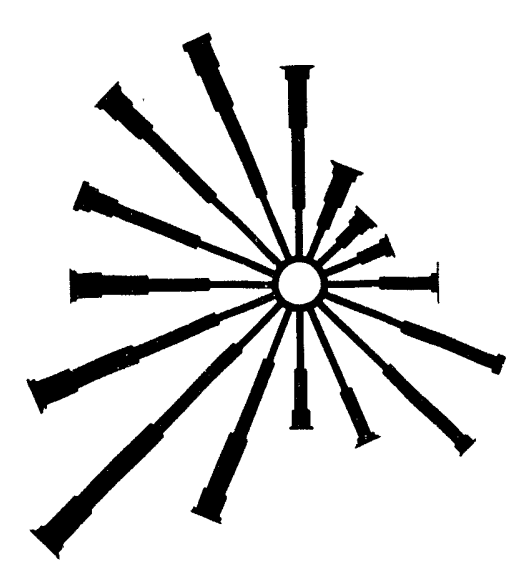

(a) Wind Rose

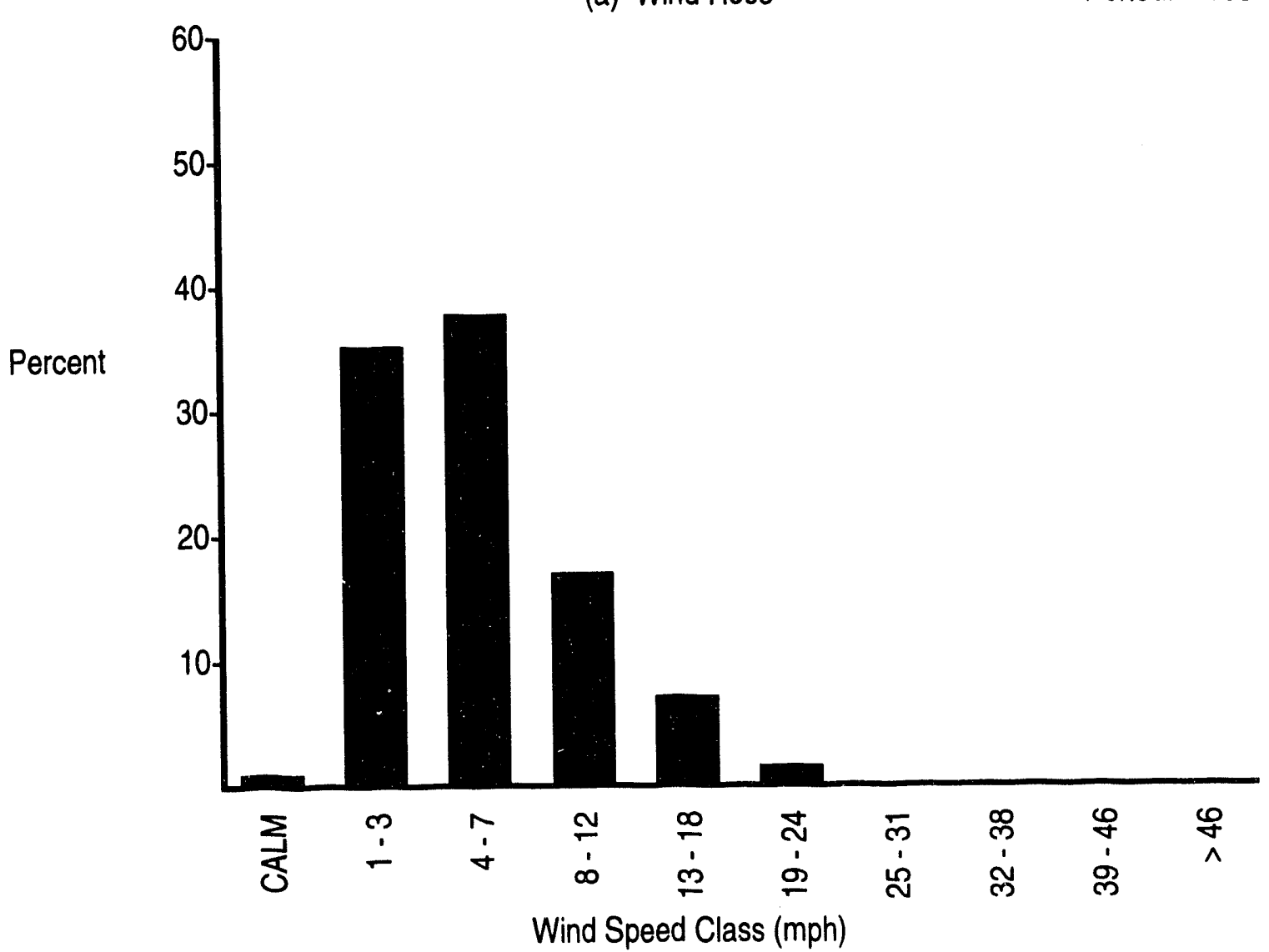

(b) Wind Speed Histogram

FIGURE A.1. (contd)

\section{A.18}




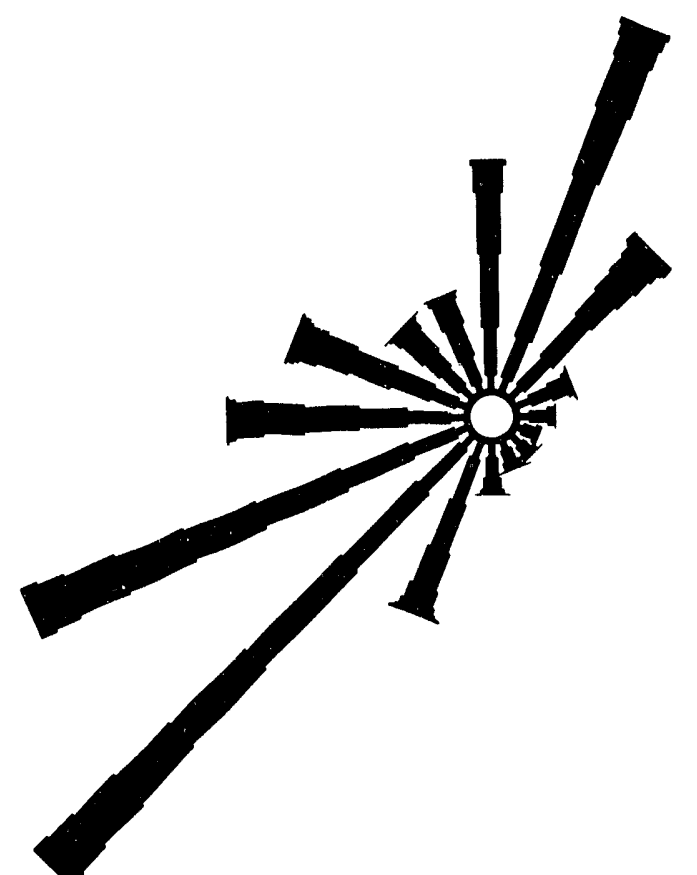

(a) Wind Rose

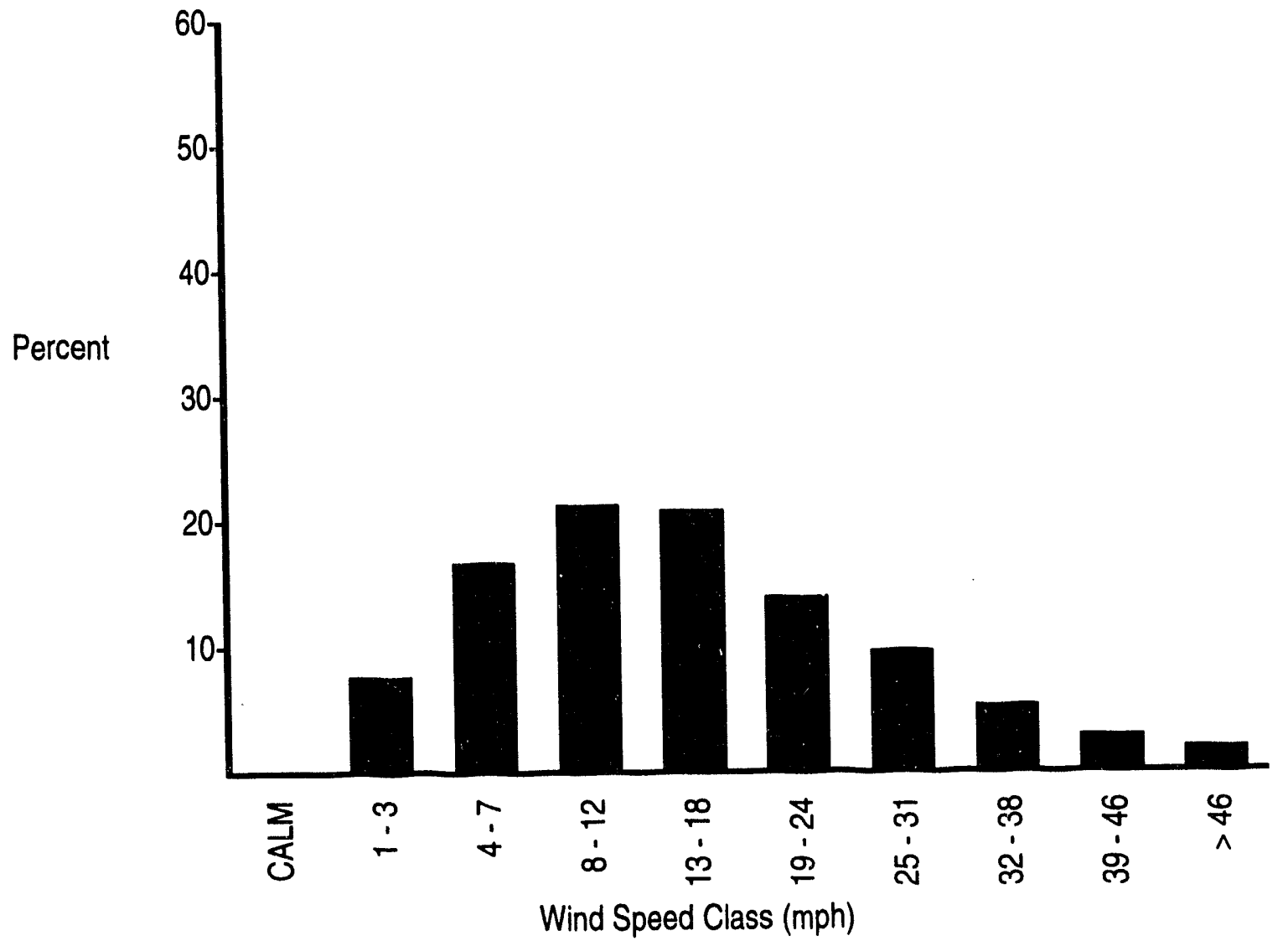

(b) Wind Speed Histogram

FIGURE A.1. (contd) 


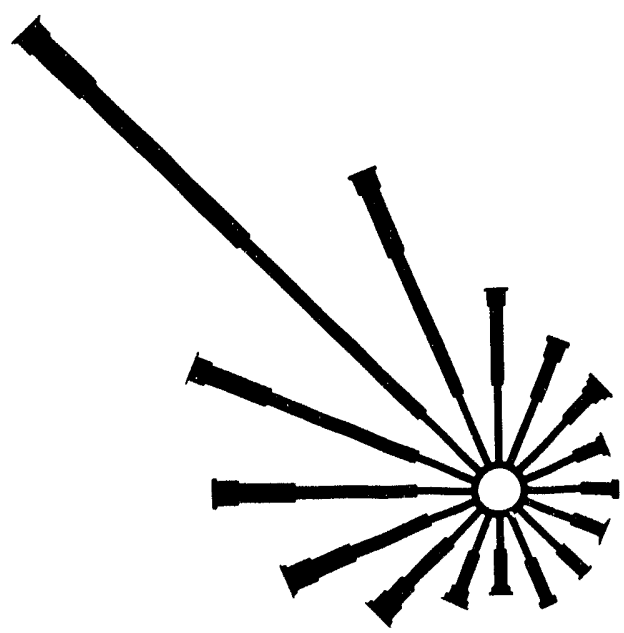

(a) Wind Rose

Period: $1 / 93-12 / 93$

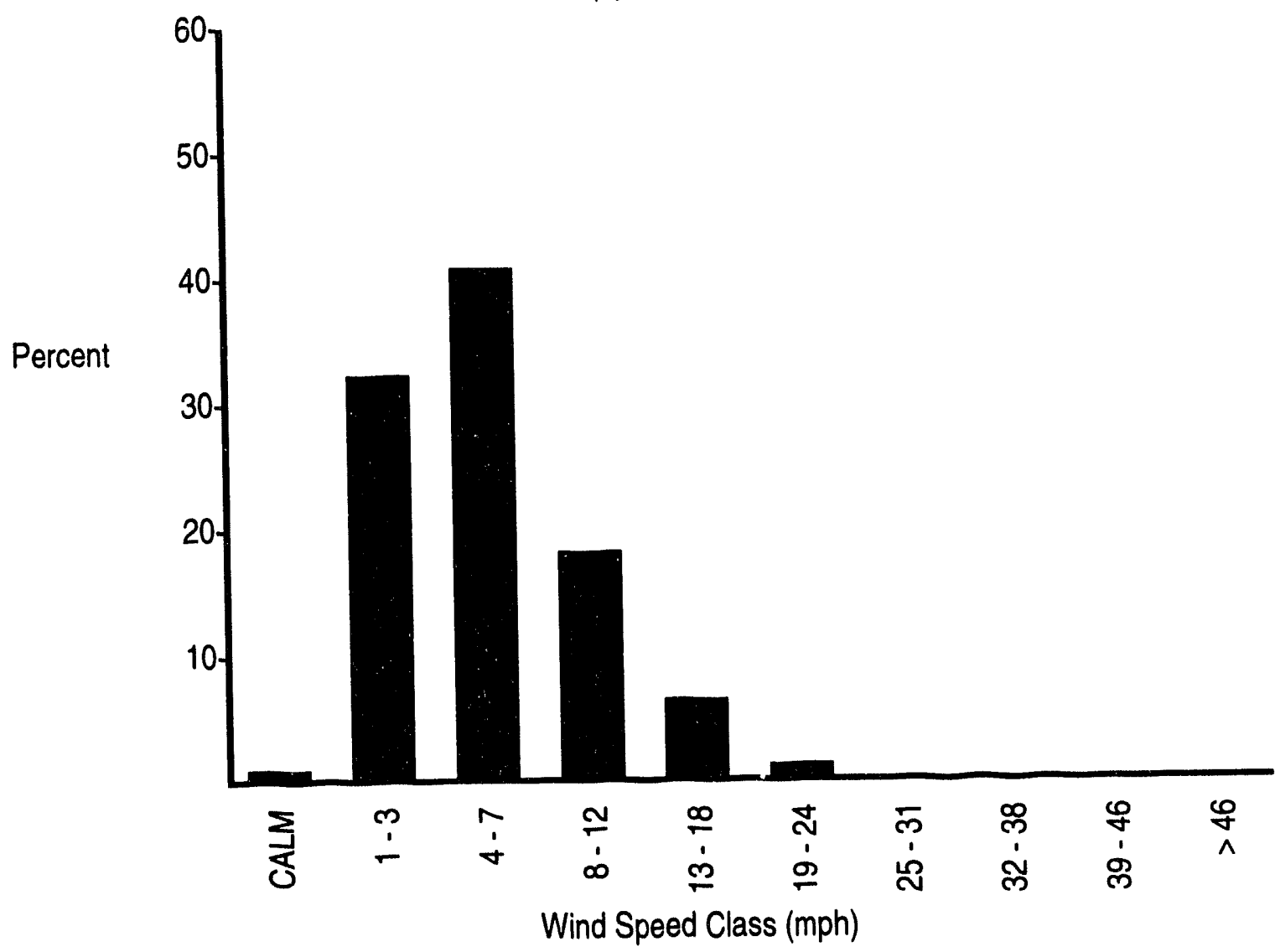

(b) Wind Speed Histogram

FIGURE A.1. (contd) 

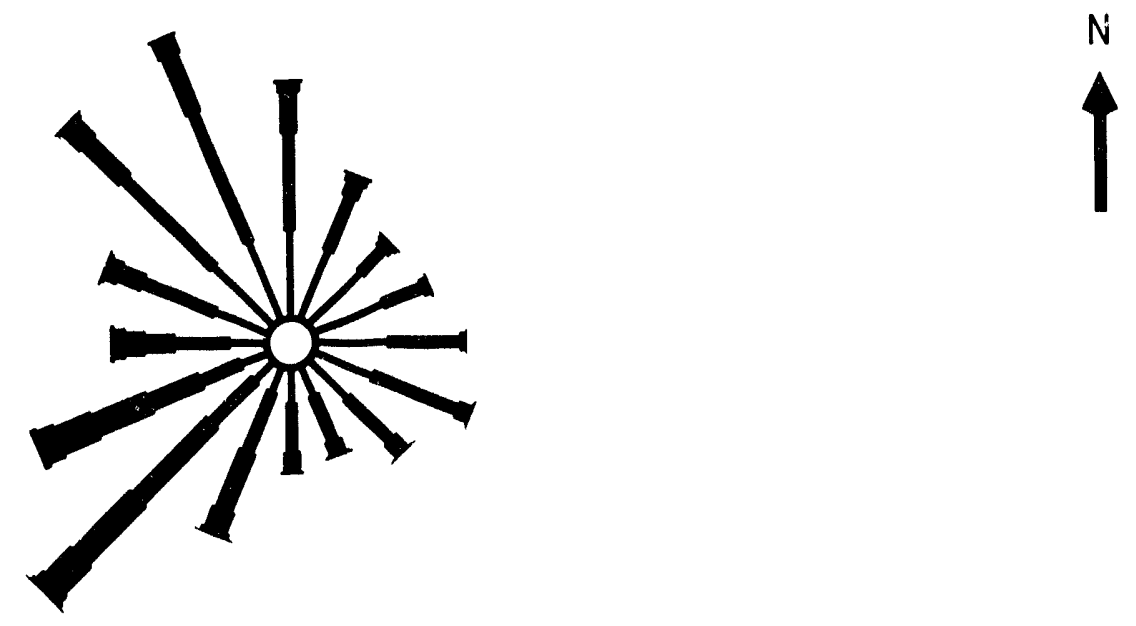

(a) Wind Rose

Period: $1 / 93-12 / 93$

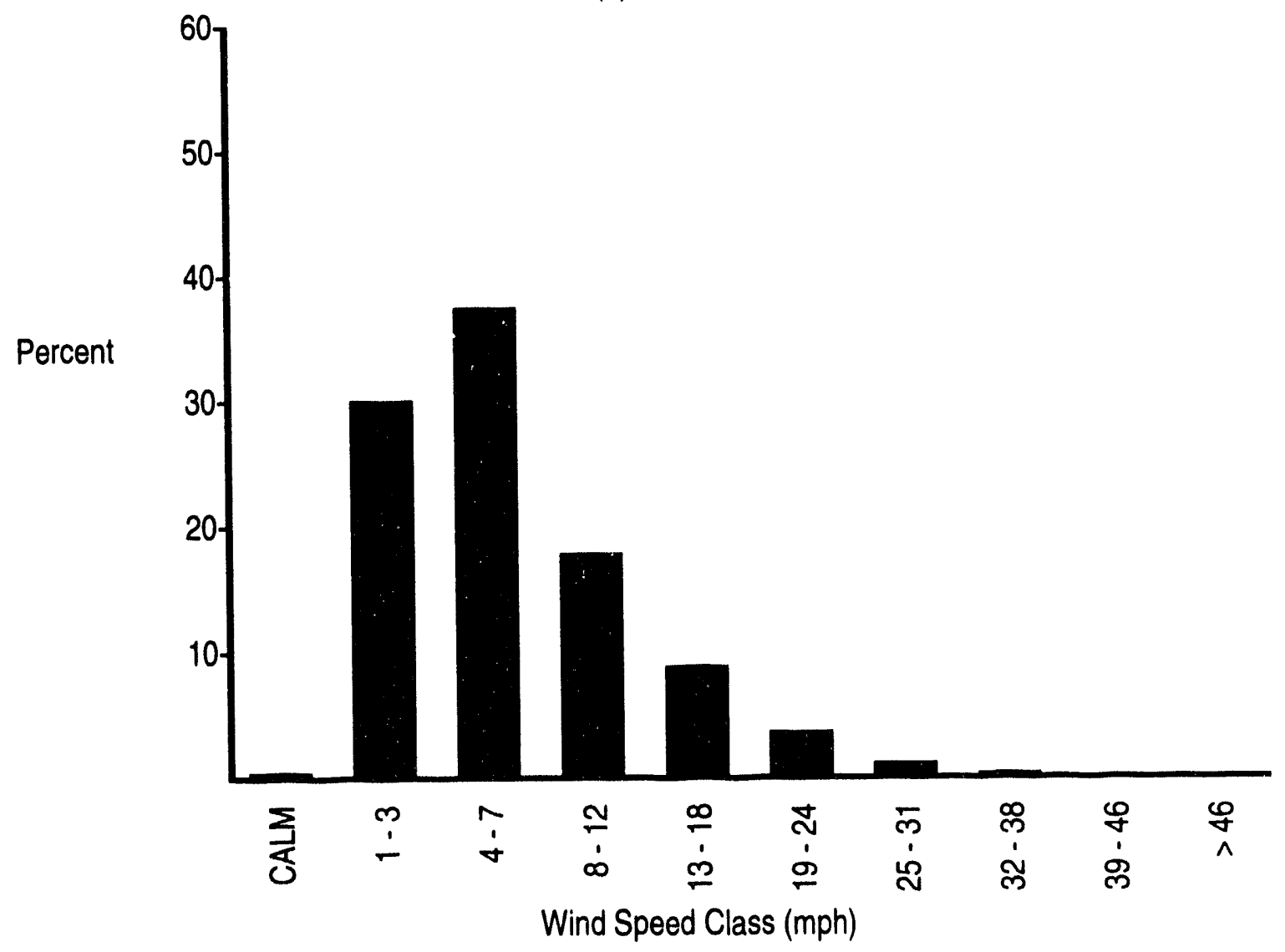

(b) Wind Speed Histogram

FIGURE A.1. (contd) 


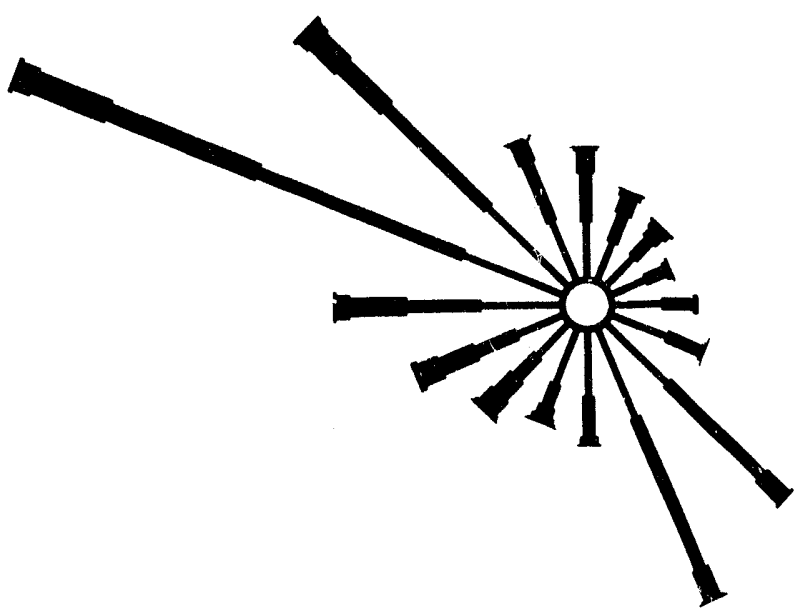

(a) Wind Rose

Period: $1 / 93-12 / 93$

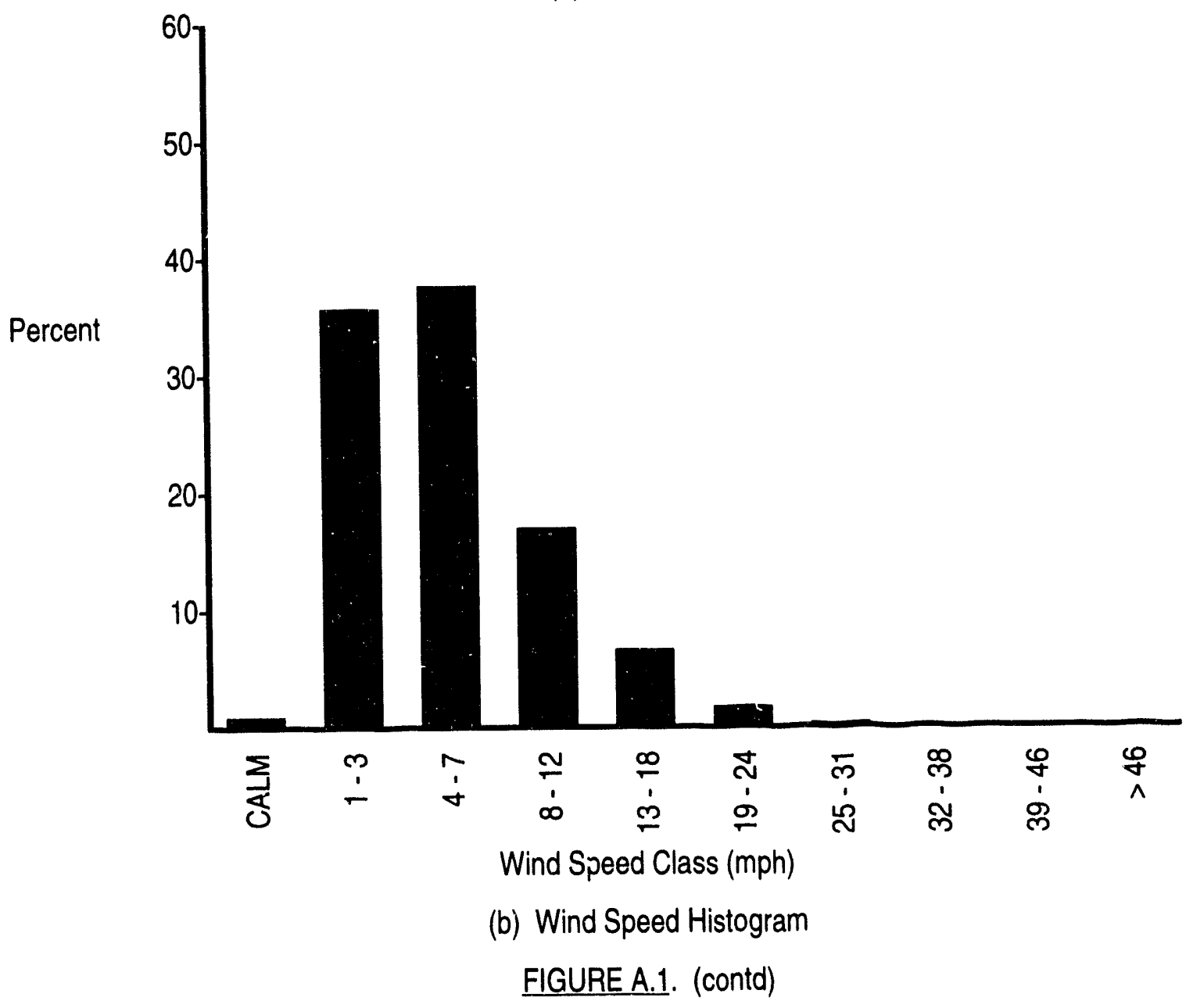




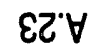

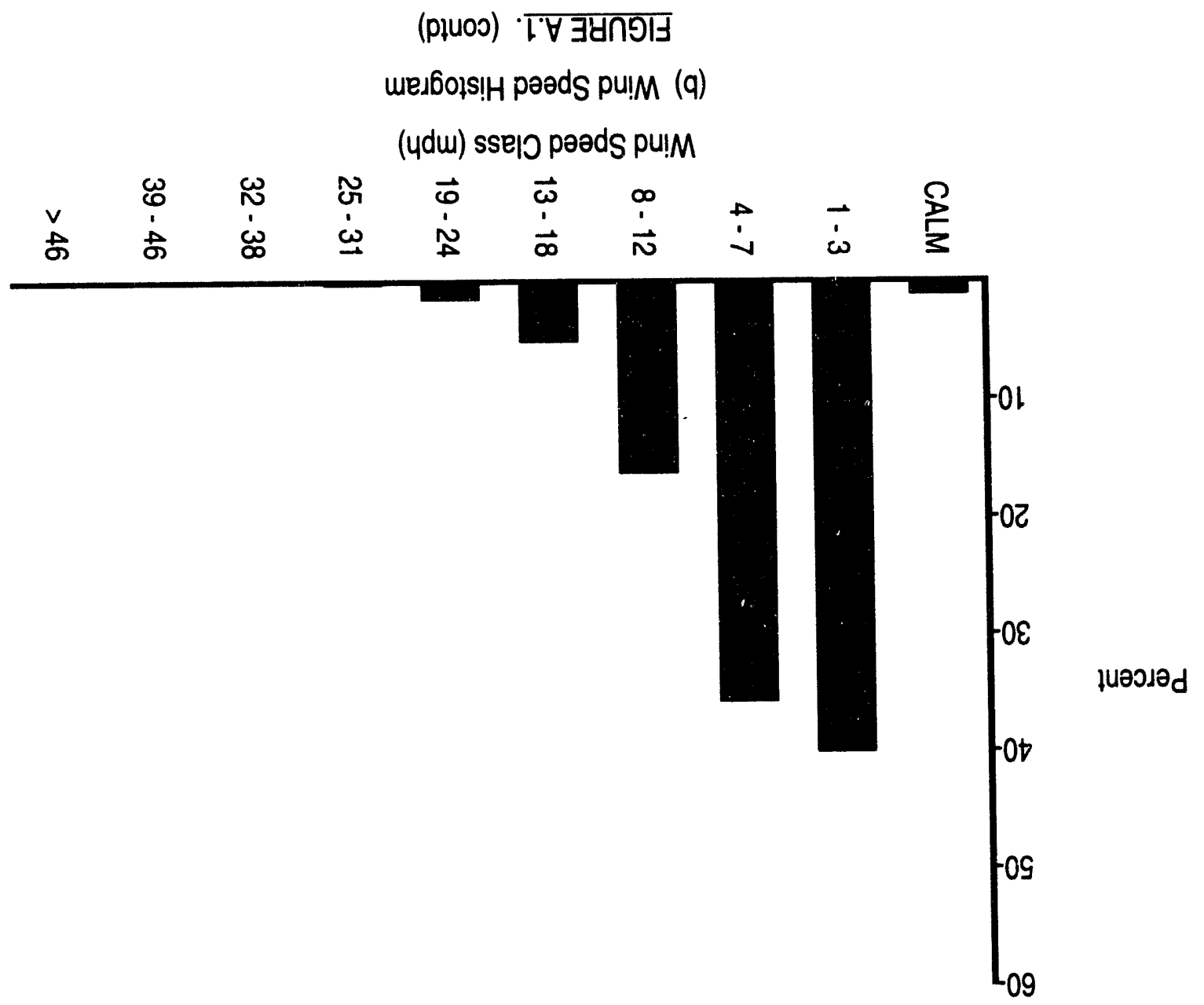

$\varepsilon 6 / 2 /-\varepsilon 6 / /$ :po!lad

әsoy Pu!M (e)

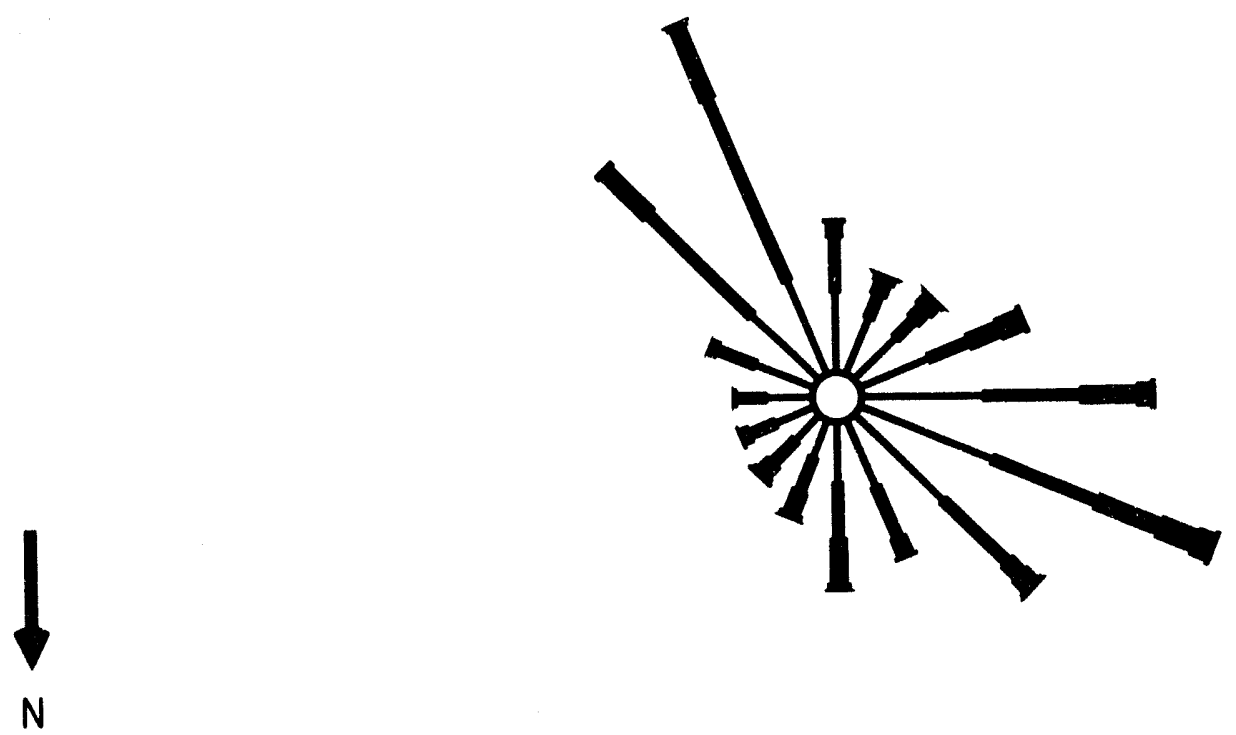




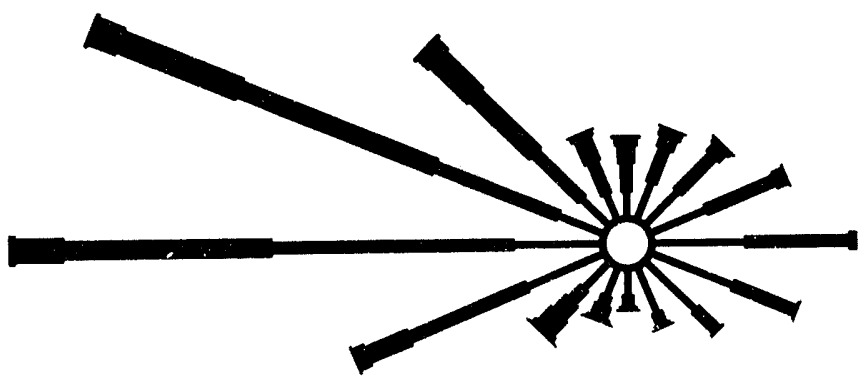

(a) Wind Rose

Period: $1 / 93-12 / 93$

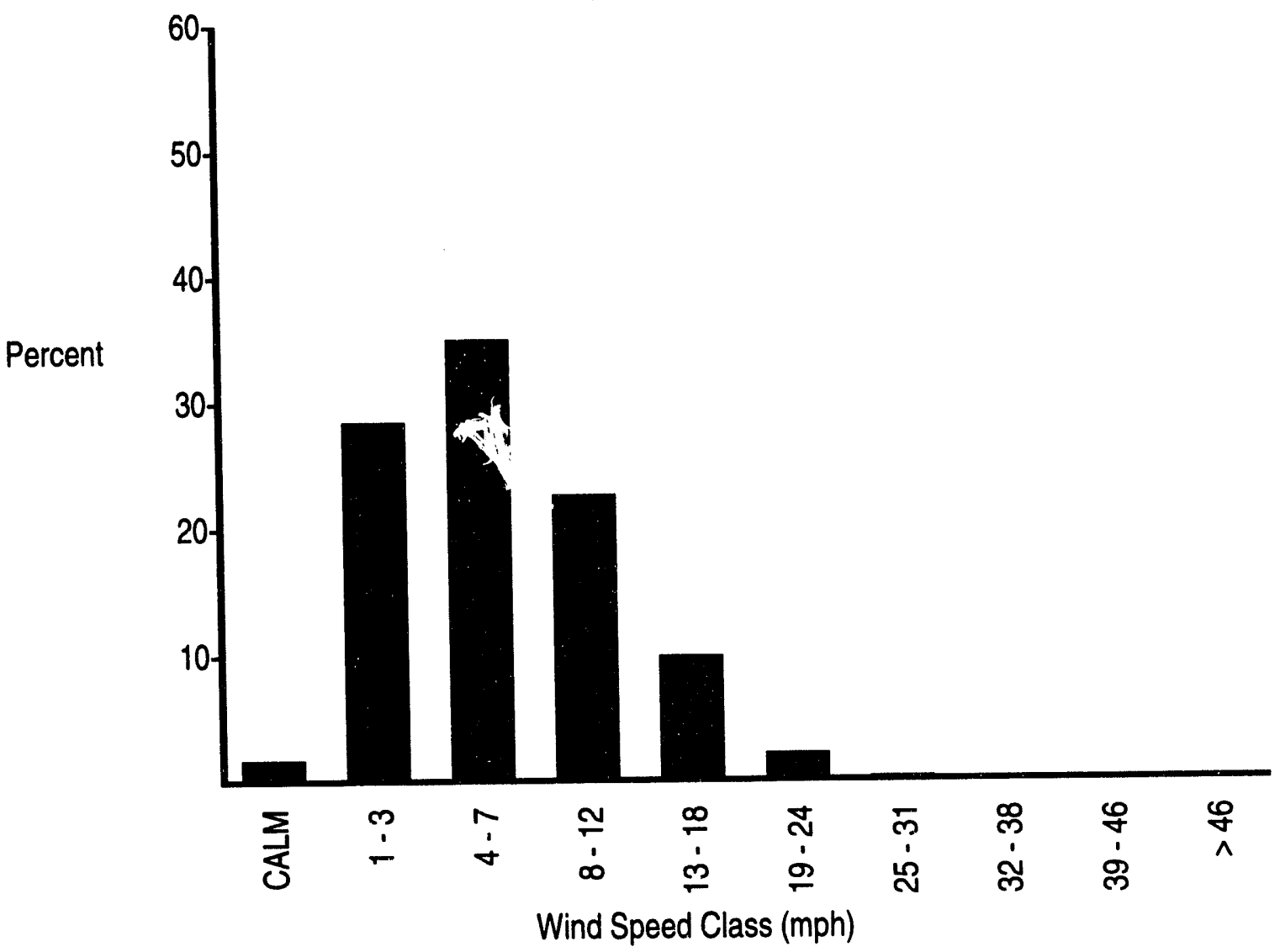

(b) Wind Speed Histogram

FIGURE A.1. (contd) 

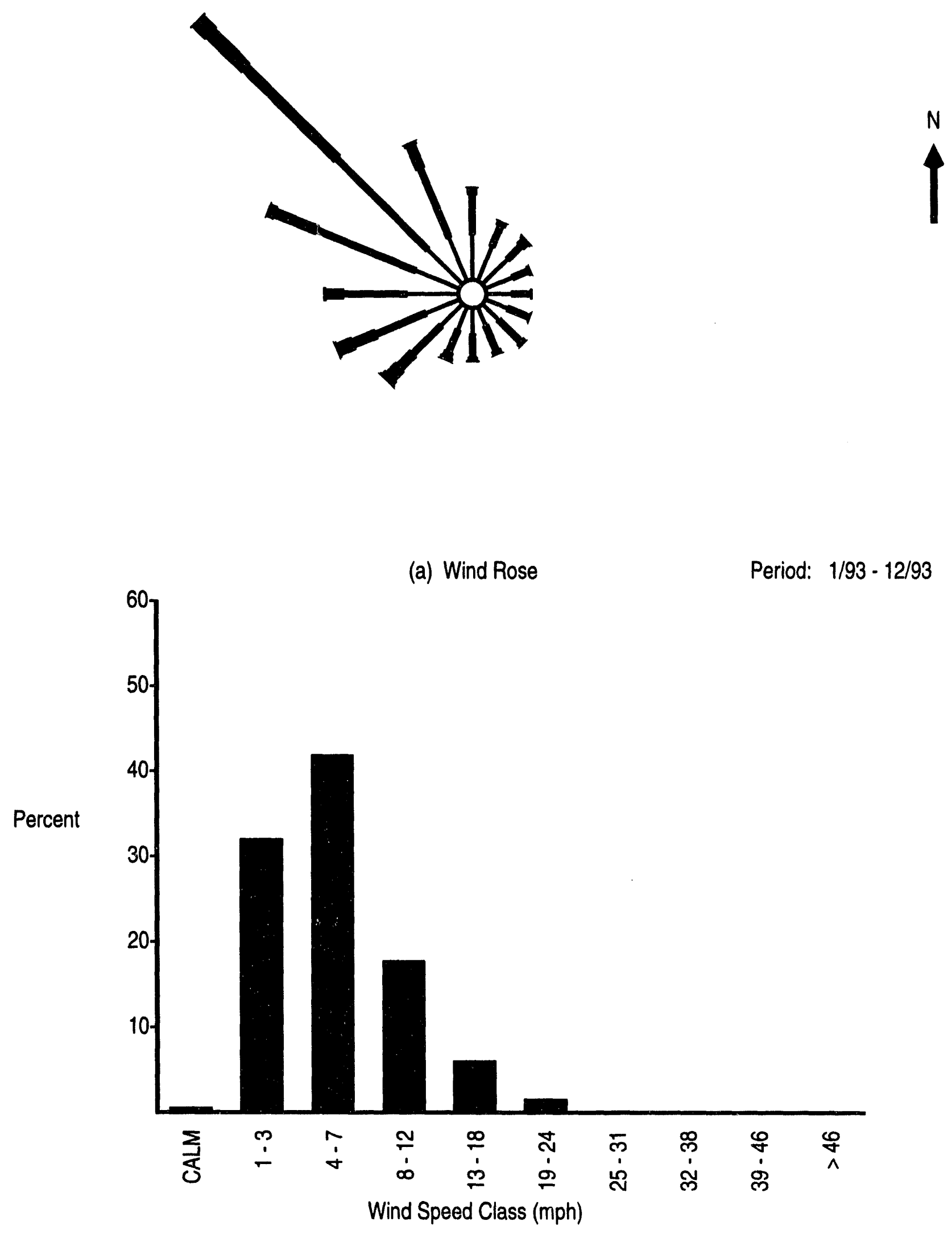

(b) Wind Speed Histogram

FIGURE A.1. (contd) 


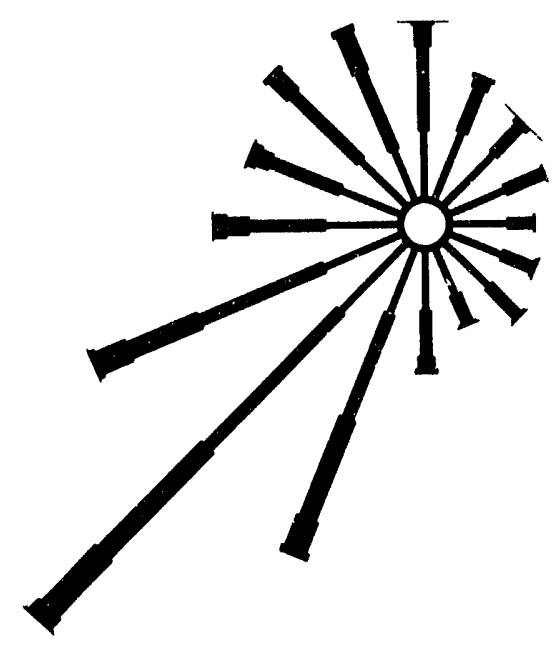

(a) Wind Rose

Period: $1 / 93-12 / 93$

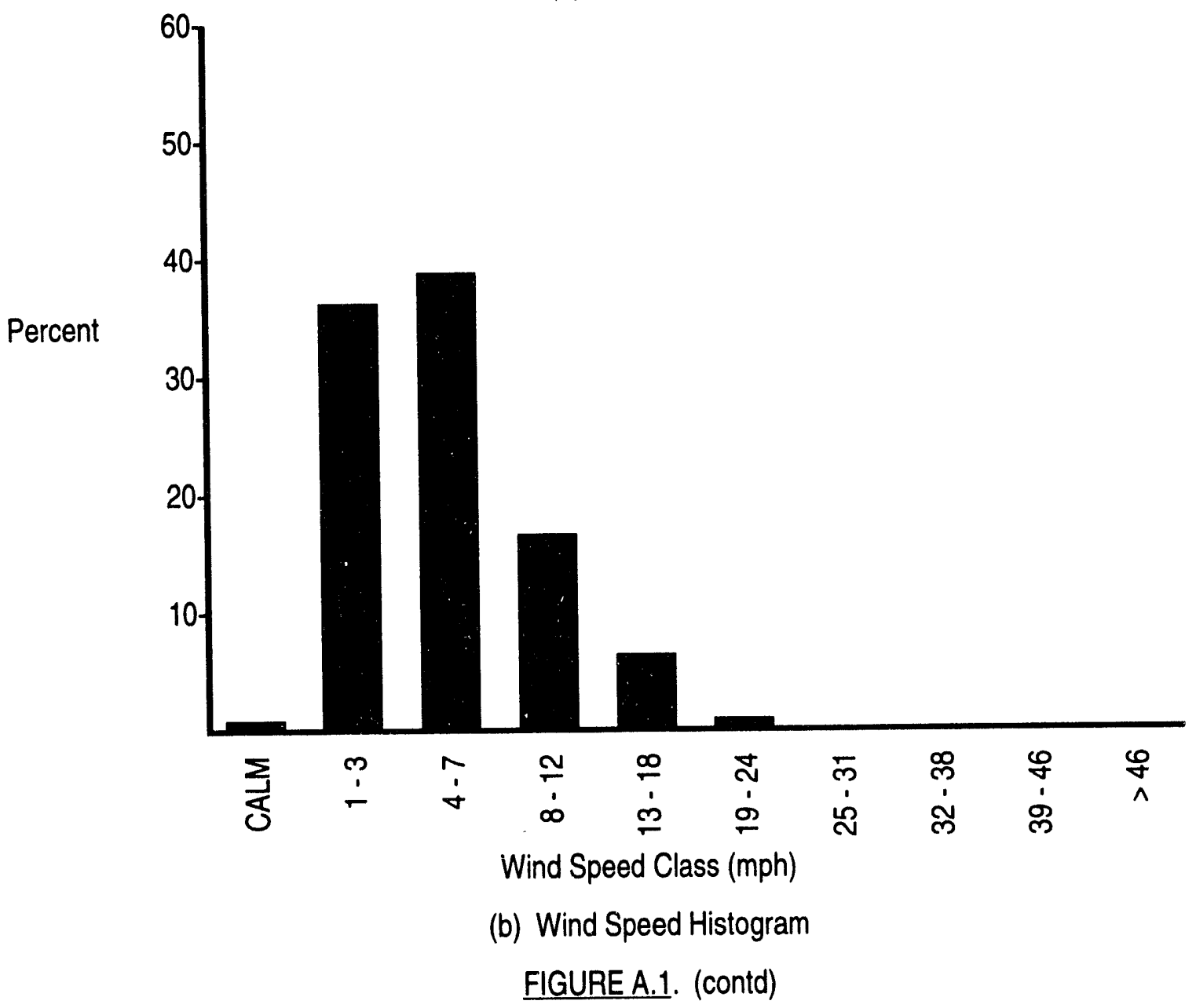




\section{TABLE A.1. 1993 Joint Frequency Distribution (\%) for Meteorological Monitoring Network Winá Stations}

Station: (1) PROS

Begin: $1 / 93$ EIRECTION End: $12 / 93$

Total Hours:

8573

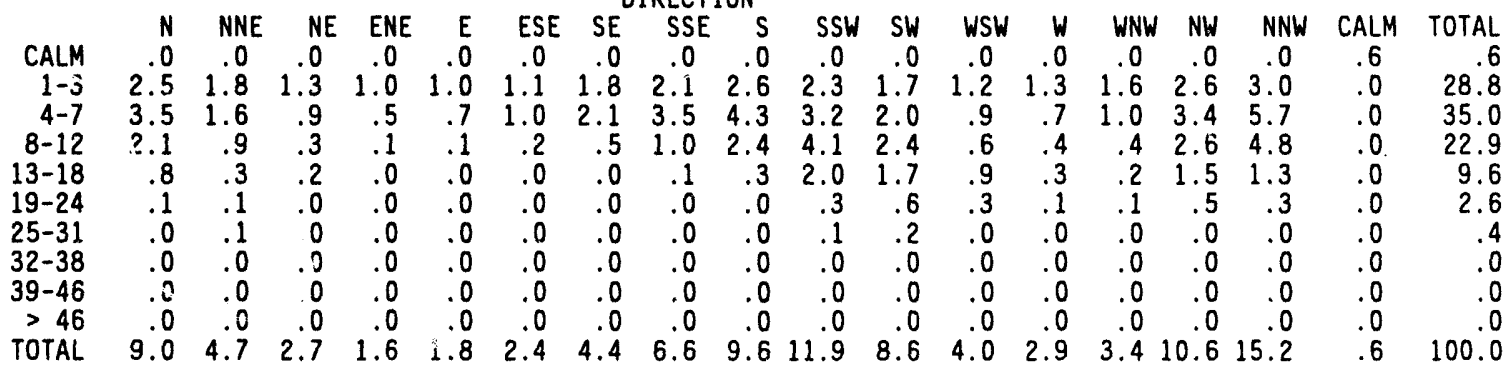

Station: (2) EOC

Begin: $1 / 93$ DIRECTION End: 12/93

Total Hours:

8571

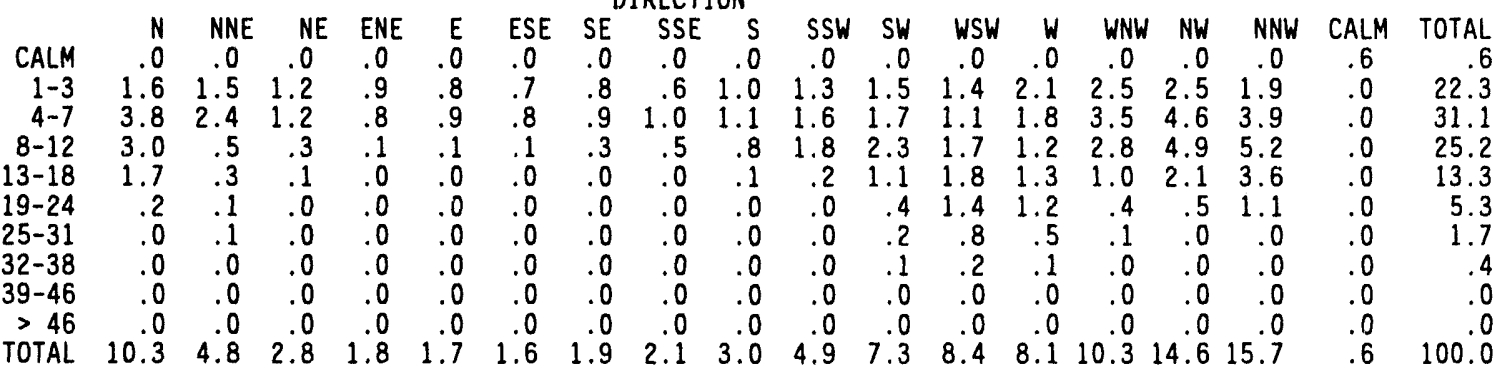

Station: ( 3) ARMY

$$
\text { Begin: } 1 / 93 \text { End: 12/93 }
$$

Total Hours: 8507

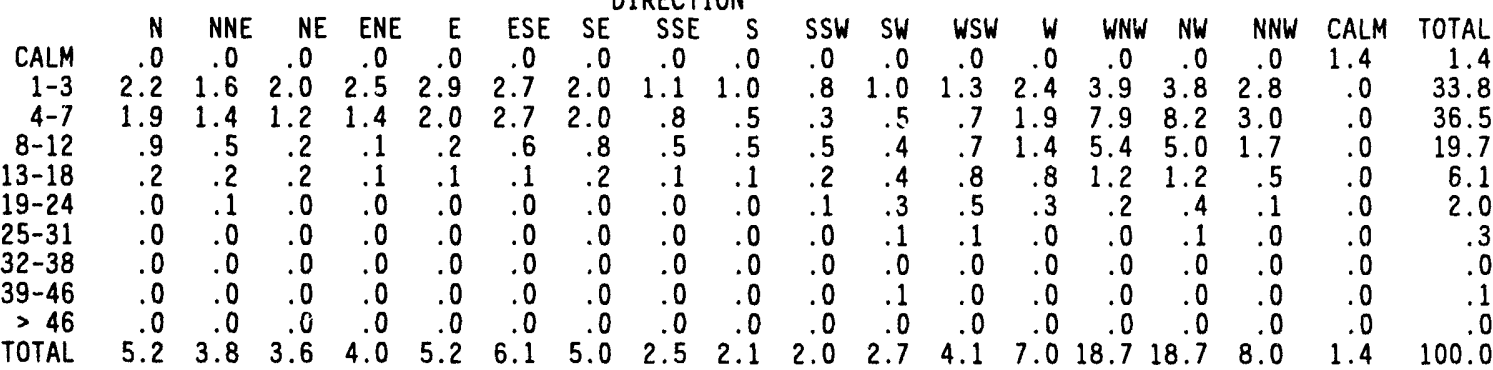

Station: (4) RSPG

\begin{tabular}{|c|c|c|c|c|c|c|c|c|c|c|c|c|c|c|c|c|c|c|}
\hline & & & & & Begi & $n:$ & $/ 93$ & IRCII & $E$ & ad: 1 & $2 / 93$ & & & & Tot & a) & rs: & 841 \\
\hline CALM & $\begin{array}{c}N \\
0\end{array}$ & $\underset{0}{\text { NNE }}$ & $\begin{array}{l}\text { NE } \\
0\end{array}$ & $\begin{array}{c}\text { ENE } \\
0\end{array}$ & $\begin{array}{l}E \\
0\end{array}$ & ESE & SE & SSE & $\begin{array}{l}S \\
0\end{array}$ & $\underset{0}{S S W}$ & SW & WSW & $\begin{array}{l}W \\
3\end{array}$ & WNW & $\begin{array}{r}N W \\
0\end{array}$ & NNW & CALM & \\
\hline $1-3$ & 1.8 & 2.0 & 2.1 & 1.7 & 1.9 & 1.8 & 1.2 & .6 & .6 & .9 & 1.8 & 2.9 & 2.2 & 1.5 & 1.1 & 1.3 & 1.1 & \\
\hline- & 2.6 & 2.3 & 1.4 & .8 & 1.4 & 1.8 & .8 & $A$ & .3 & .1 & 2.0 & 10.1 & 5.7 & 1.9 & 1.8 & 2. & & \\
\hline 1 & .5 & .4 & .1 & .1 & .1 & .3 & .1 & .0 & .2 & .5 & 1.2 & 14.0 & 5.5 & 2.4 & 1.6 & 1.2 & . & \\
\hline $13-18$ & .0 & .0 & .2 & .0 & .0 & .0 & .0 & .0 & .0 & .3 & .7 & 2.2 & 1.7 & .8 & 4 & .3 & & \\
\hline & .0 & .0 & .1 & .0 & .0 & .0 & .0 & .0 & .0 & .2 & .2 & .3 & .2 & .1 & .0 & . & & \\
\hline & .0 & .0 & .0 & .0 & .0 & .0 & .0 & .0 & .0 & . & .1 & .0 & .0 & . & & & & \\
\hline & 0 & .0 & .0 & .0 & .0 & .0 & .0 & .0 & .0 & $\theta$ & .0 & .0 & .0 & . & .0 & & & \\
\hline 39 & & .0 & 0 & 0 & .0 & .0 & .0 & & .0 & 0 & .0 & .0 & .0 & .0 & .0 & . & & \\
\hline $\begin{array}{r}>4 \\
\text { TOTAL }\end{array}$ & $\begin{array}{r}.0 \\
5.0\end{array}$ & $\begin{array}{r}.0 \\
4.8\end{array}$ & $\begin{array}{r}.0 \\
3.9\end{array}$ & .0 & $\begin{array}{r}.0 \\
3.4\end{array}$ & .0 & $\begin{array}{r}.0 \\
2.0\end{array}$ & .0 & .0 & .0 & .0 & .0 & .0 & .0 & .0 & $\begin{array}{r}.0 \\
5.2\end{array}$ & i: & \\
\hline
\end{tabular}


TABLE A.1. (contd)

Station: ( 5) EDNA

Begin: $1 / 93$ DIRECTION End: $12 / 93$

Total Hours: 8466

\begin{tabular}{|c|c|c|c|c|c|c|c|c|c|c|c|c|c|c|c|c|c|c|}
\hline & & & & & o & 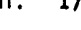 & 0 & RECT & I0 & 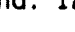 & 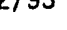 & & & & & & & \\
\hline & $N$ & NNE & $\mathrm{NE}$ & ENE & $E$ & ESE & SE & SSE & s & SSW & SW & WSW & W & WNW & NW & NNW & CALM & TOTAL \\
\hline CALN & .0 & .0 & 0 & 0 & .0 & .0 & .0 & 0 & .0 & & 0 & .0 & .0 & .0 & 0 & 0 & & 1.0 \\
\hline $1-$ & 1.2 & .6 & .0 & .8 & .9 & 1.5 & 3.8 & 4.3 & 3.9 & 2.7 & 1.8 & 1.4 & 2.2 & 2.9 & 3. & 2. & & \\
\hline $4-7$ & 2.0 & 1.0 & .9 & .7 & 1.5 & 3.8 & 7.5 & 4.3 & 2.2 & .9 & 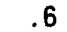 & .5 & .9 & 2.5 & 6 & 4 & & \\
\hline $8-1$ & 1.4 & .6 & .5 & .2 & .6 & 1.8 & 1.1 & .9 & 1. & & & & .9 & 1. & 2 & 1. & & \\
\hline 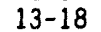 & .2 & .3 & .2 & .1 & .0 &. & .1 & . & . & & & . & $\because$ & $i$ & & & & \\
\hline & .0 & .1 & 0 & .0 & .0 & .8 & .0 & .8 & .1 & .1 & . & . & .1 & -1 & & & & \\
\hline & & .0 & 0 & & .0 & & .0 & .0 & .0 & & .0 & . & .8 & .2 & $\theta$ & . & & 3 \\
\hline & & .0 & & 0 & 0 & 0 & 0 & & & & & & & & & & & \\
\hline & & .0 & .0 & 0 & .0 & .0 & .0 & .0 & .0 & & .1 & . &. & .0 & & .8 & & \\
\hline & & .0 & .0 & .0 & .0 & 0 & .0 & .0 & .0 & & .0 & .0 & .0 & .0 & & & & \\
\hline & 4.8 & 2.6 & 2.3 & 1.8 & 3.0 & 7.2 & 12.4 & 9.7 & 7.5 & 4.5 & 3.2 & 3.4 & 4.6 & 8.7 & 13.7 & $9 . F$ & 1.0 & 100.0 \\
\hline
\end{tabular}

Station: ( 6) 200E

Begin: $1 / 93$ End: 12/93

Total Hours:

8557

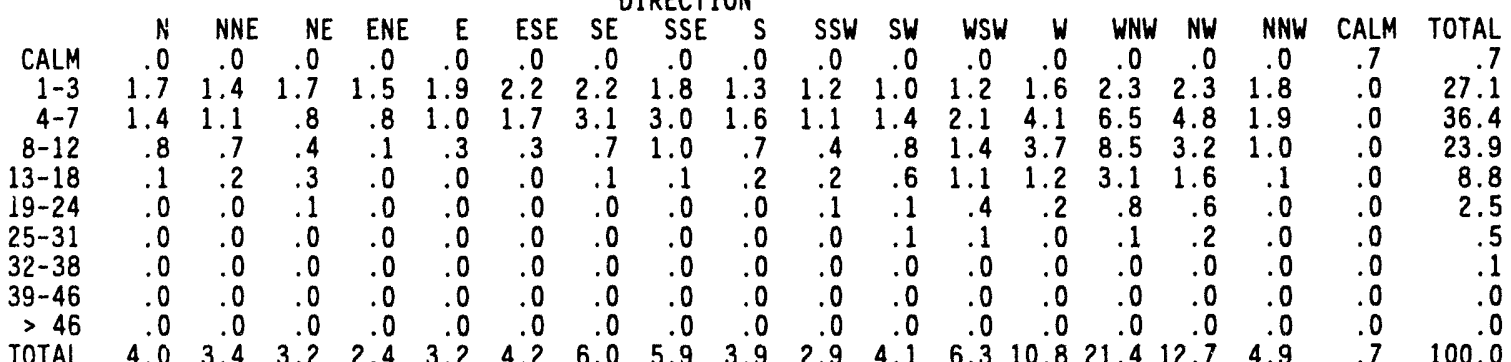

Station: ( 7) 200W

Begin: 1/93 End: 12/93

Total Hours:

8556

N NNE NE ENE E ESE SE SSE S SSW SW WSW W WNW NW NNW CALM TOTAL

$\begin{array}{lllllllllllllllllll}\text { CALM } & .0 & .0 & .0 & .0 & .0 & .0 & .0 & .0 & .0 & .0 & .0 & .0 & .0 & .0 & .0 & .0 & 1.8 & 1.8\end{array}$

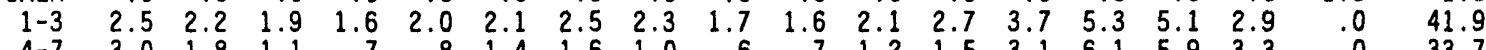

$\begin{array}{lllllllllllllllllll}4.7 & 3.0 & 1.8 & 1.1 & .7 & .8 & 1.4 & 1.6 & 1.0 & .6 & .7 & 1.2 & 1.5 & 3.1 & 6.1 & 5.9 & 3.3 & .0 & 33.7\end{array}$

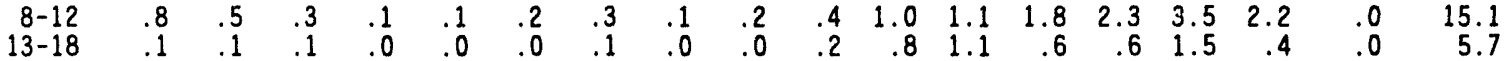

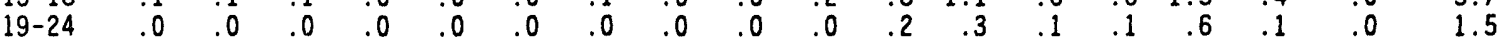

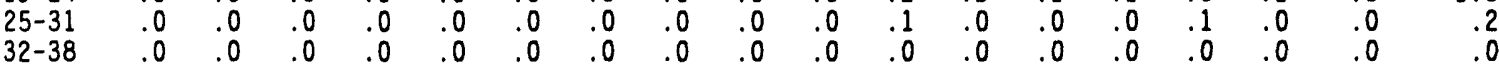

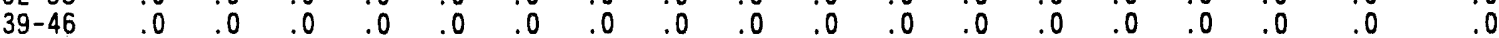

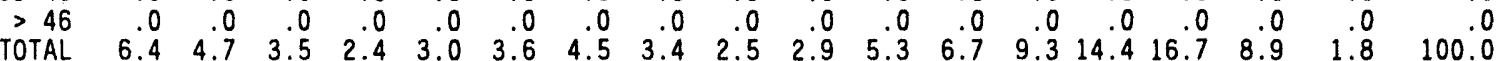

Station: ( 8) BVLY

Begin: $1 / 93$ DIRECTION End: $12 / 93$

Total Hours: $\quad 8520$

CALIM NNE NE ENE E ESE SE SSE S SSW SW WSW W WNW NW NNW CALM TOTAL

$\begin{array}{lllllllllllllllllll}\text { CALM } & .0 & .0 & .0 & .0 & .0 & .0 & .0 & .0 & .0 & .0 & .0 & .0 & .0 & .0 & .0 & .0 & 1.1 & 1.1\end{array}$

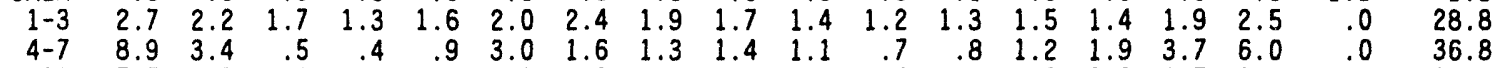

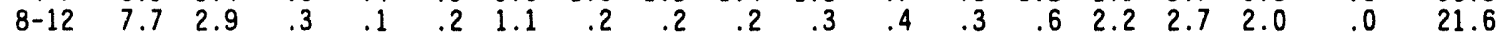

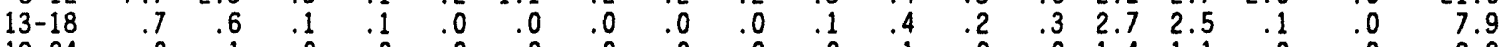

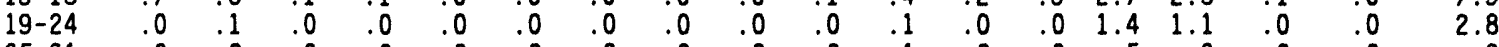

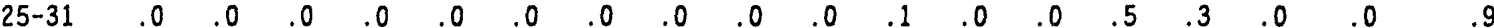

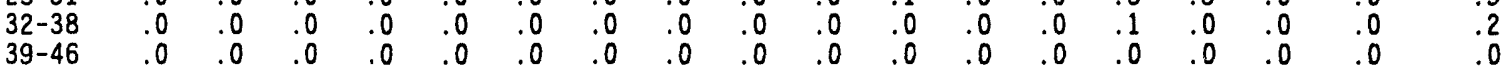

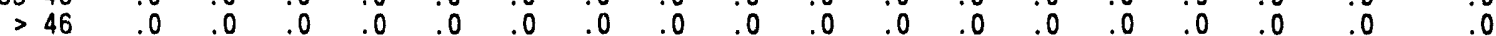

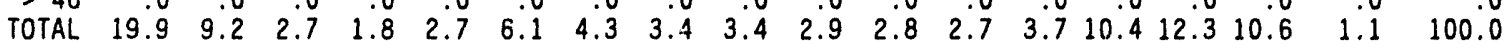


IABLE A.1. (contd)

Station: (9) FFTF

Begin: $1 / 93$

End: $12 / 93$

Total Hours: 8573

DIRECTION End: $12 / 93$

NW NNW CALM TOTAL

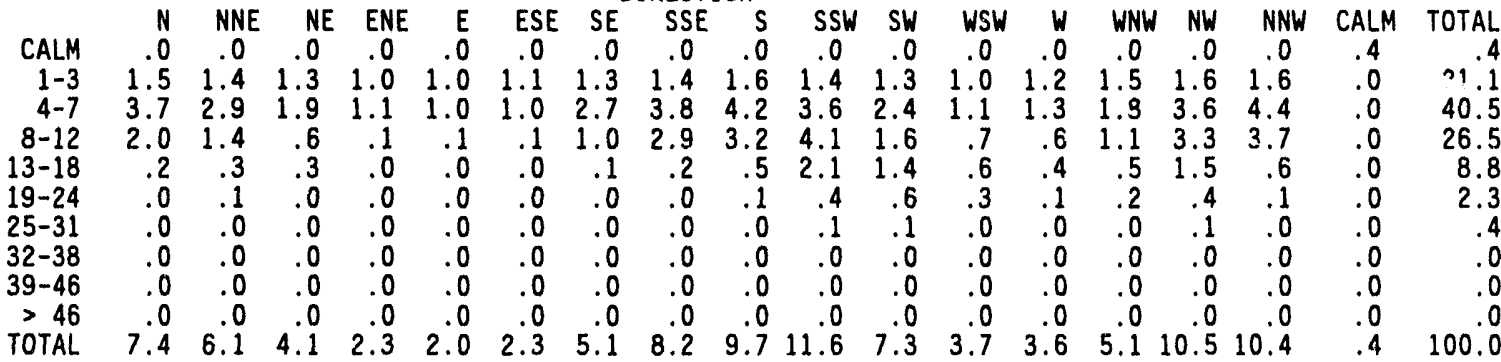

Station: (10) YAKB

Begin: $1 / 93$

End: $12 / 93$

Total Hours:

8500

DIRECTION

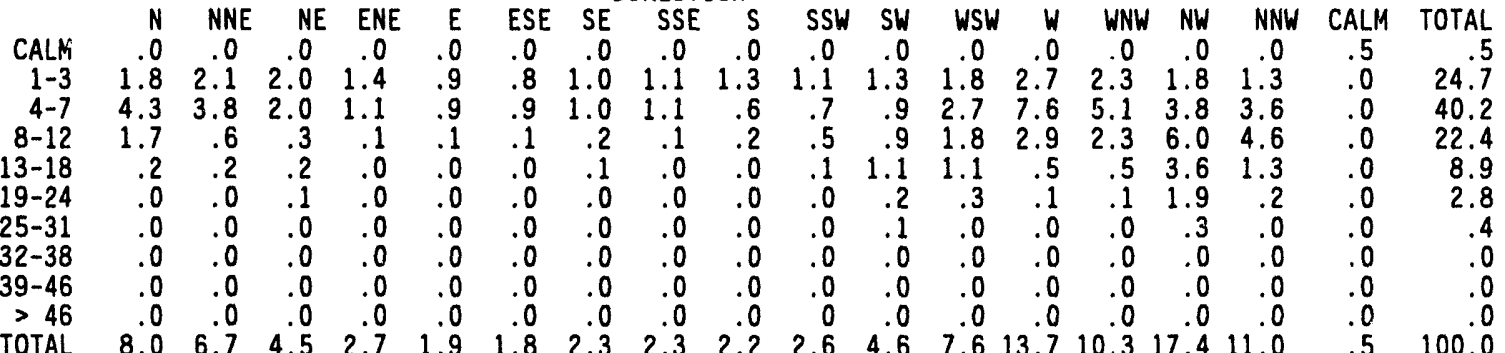

Station: (11) 300A

Begin: $1 / 93$ DIRECTION End: $12 / 93$

Total Hours:

8535

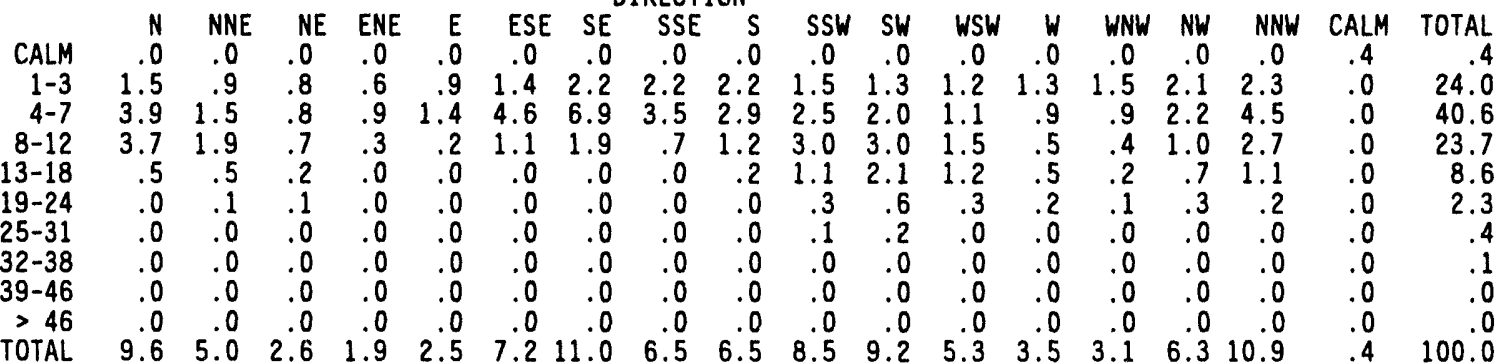

Station: (12) WYEB

Begin: 1/93

End: $12 / 93$

Total Hours:

8574

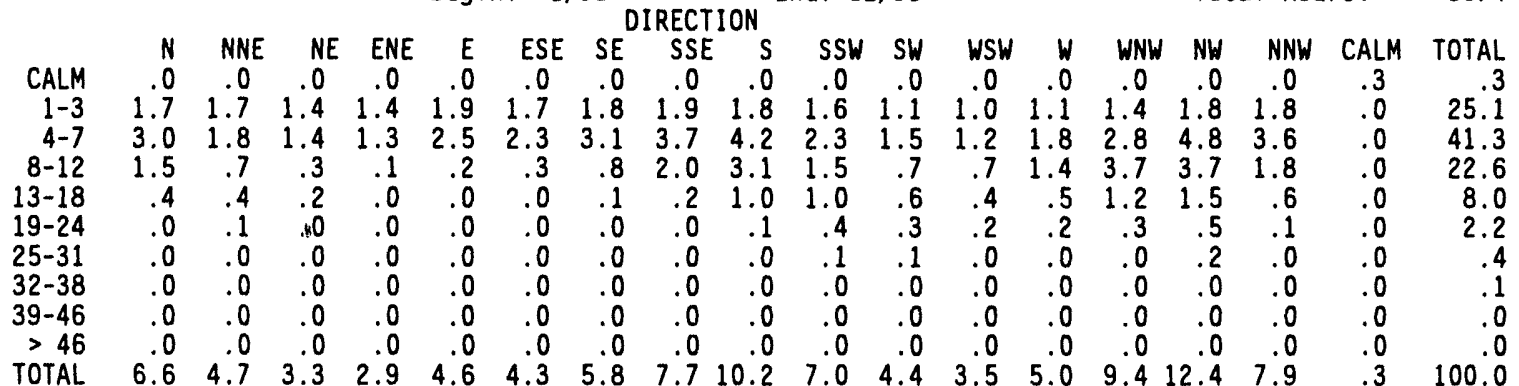




\section{TABLE A.1. (contd)}

Station: (13) $100 \mathrm{~N}$

Begin: 1/93

End: $12 / 93$

Total Hours:

8573

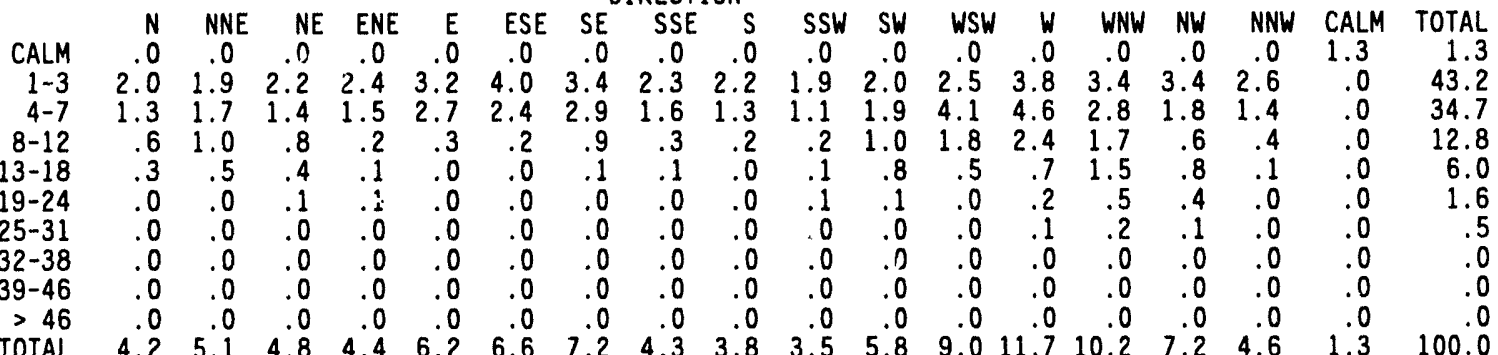

Station: (14) WPPS

Begin: $1 / 93$ DIRECTION End: $12 / 93$

Total Hours:

8536

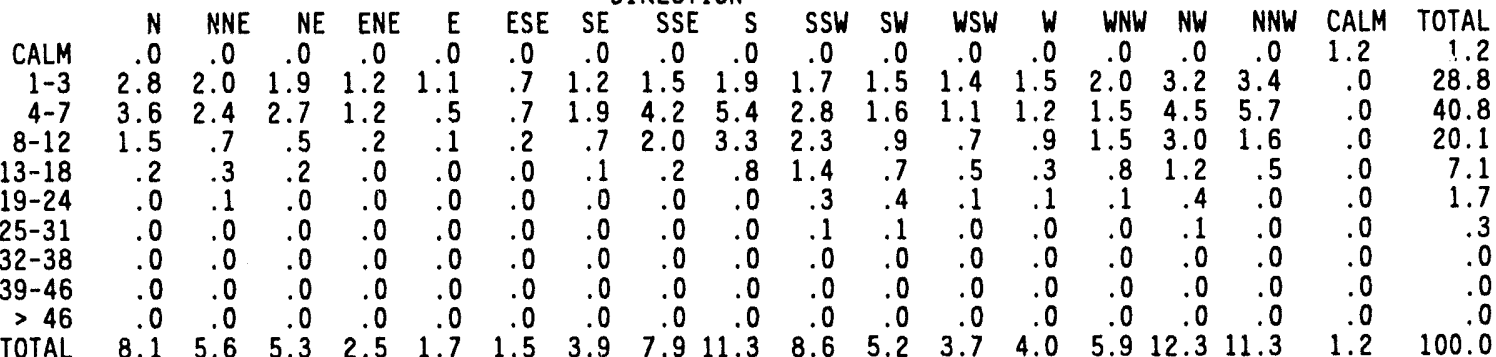

Station: (15) FRNK

Begin: $1 / 93$ DIRECTION End: $12 / 93$

Total Hours:

8573

CALM NNE NE ENE E ESE SE SSE S SSW SW WSW W WNW NW NNW CALM TOTAL

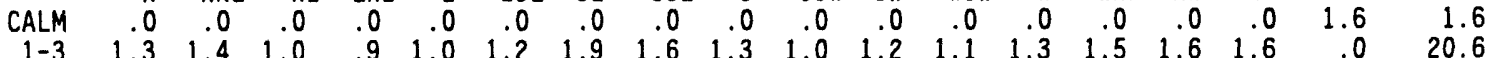

$\begin{array}{lllllllllllllllllll}1-3 & 1.3 & 1.4 & 1.0 & .9 & 1.0 & 1.2 & 1.9 & 1.6 & 1.3 & 1.0 & 1.2 & 1.1 & 1.3 & 1.5 & 1.6 & 1.6 & .0 & 20.6 \\ 4-7 & 4.0 & 3.1 & 1.6 & 1.3 & 1.4 & 2.5 & 5.8 & 4.6 & 3.3 & 3.0 & 2.5 & 1.2 & 1.1 & 2.3 & 4.4 & 5.9 & .0 & 47.9\end{array}$

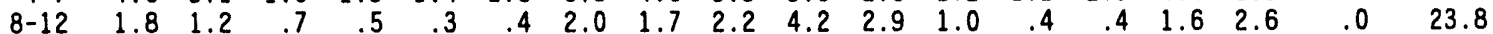

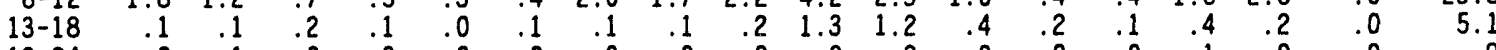

$\begin{array}{lllllllllllllllllll}19-24 & .0 & .1 & .0 & .0 & .0 & .0 & .0 & .0 & .0 & .2 & .2 & .0 & .0 & .0 & .1 & .0 & .0 & .9\end{array}$

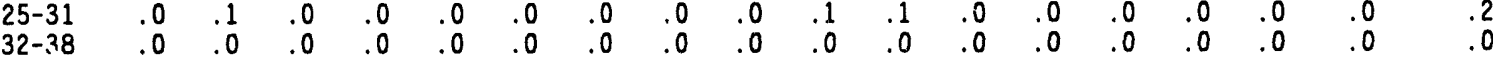

$\begin{array}{lllllllllllllllllll}39-4.5 & .0 & .0 & .0 & .0 & .0 & .0 & .0 & .0 & .0 & .0 & .0 & .0 & .0 & .0 & .0 & .0 & .0 & .0\end{array}$

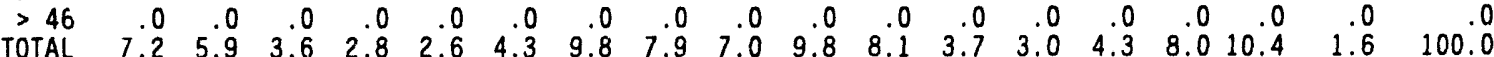

Station: (16) GABL

Begin: $1 / 93$ DIRECTION End: $12 / 93$

Total Hours: 8575

$\begin{array}{rrrrrrrrrrrrrrrrrrr} & \text { N } & \text { NNE } & \text { NE } & \text { ENE } & \text { E } & \text { ESE } & \text { SE } & \text { SSE } & \text { S } & \text { SSW } & \text { SW } & \text { WSW } & \text { W } & \text { WNW } & \text { NW } & \text { NNW } & \text { CALM } & \text { TOTAL } \\ \text { CALM } & .0 & .0 & .0 & .0 & .0 & .0 & .0 & .0 & .0 & .0 & .0 & .0 & .0 & .0 & .0 & .0 & .6 & .6 \\ 1-3 & 1.1 & 1.1 & .9 & .7 & .7 & .7 & .9 & 1.1 & 1.4 & 1.3 & 1.1 & .9 & .8 & 1.0 & .9 & .9 & .0 & 15.3 \\ 4-7 & 2.5 & 2.5 & 1.8 & .9 & .9 & .8 & 1.3 & 2.7 & 4.3 & 2.8 & 1.6 & 1.7 & 1.5 & 1.7 & 2.6 & 2.6 & .0 & 32.2 \\ 8-12 & 2.4 & 2.0 & 1.0 & .3 & .3 & .5 & .7 & 1.8 & 2.5 & 1.3 & 1.2 & 1.1 & 1.4 & 1.8 & 2.8 & 2.0 & .0 & 23.1 \\ 13-18 & 1.6 & 1.6 & .6 & .1 & .0 & .1 & .2 & 1.0 & 1.4 & .8 & .8 & .8 & 1.5 & 2.4 & 2.4 & .8 & .0 & 16.1 \\ 19-24 & .7 & .6 & .5 & .0 & .0 & .0 & .1 & .2 & .4 & .3 & .7 & .8 & .7 & 2.1 & 1.5 & .1 & .0 & 8.7 \\ 25-31 & .1 & .1 & .2 & .0 & .0 & .0 & .0 & .0 & .1 & .2 & .3 & .4 & .2 & 1.1 & .4 & .0 & .0 & 3.1 \\ 32-38 & .0 & .1 & .1 & .0 & .0 & .0 & .0 & .0 & .0 & .0 & .0 & .0 & .0 & .2 & .0 & .0 & .0 & .0 \\ 39-46 & .0 & .0 & .0 & .0 & .0 & .0 & .0 & .0 & .0 & .0 & .0 & .0 & .0 & .0 & .0 & .0 & .0 & .0 \\ >46 & .0 & .0 & .0 & .0 & .0 & .0 & .0 & .0 & .0 & .0 & .0 & .0 & .0 & .0 & .0 & .0 & .0 & .0 \\ \text { TOTAL } & 8.5 & 7.9 & 5.0 & 2.0 & 1.9 & 2.1 & 3.1 & 6.7 & 10.2 & 6.7 & 6.0 & 5.7 & 6.2 & 10.4 & 10.5 & 6.5 & .6 & 100.0\end{array}$ 


\section{TABLE A.1. (contd)}

Station: (17) RING

Begin: $1 / 93$ End: $12 / 93$

Total Hours:

8439

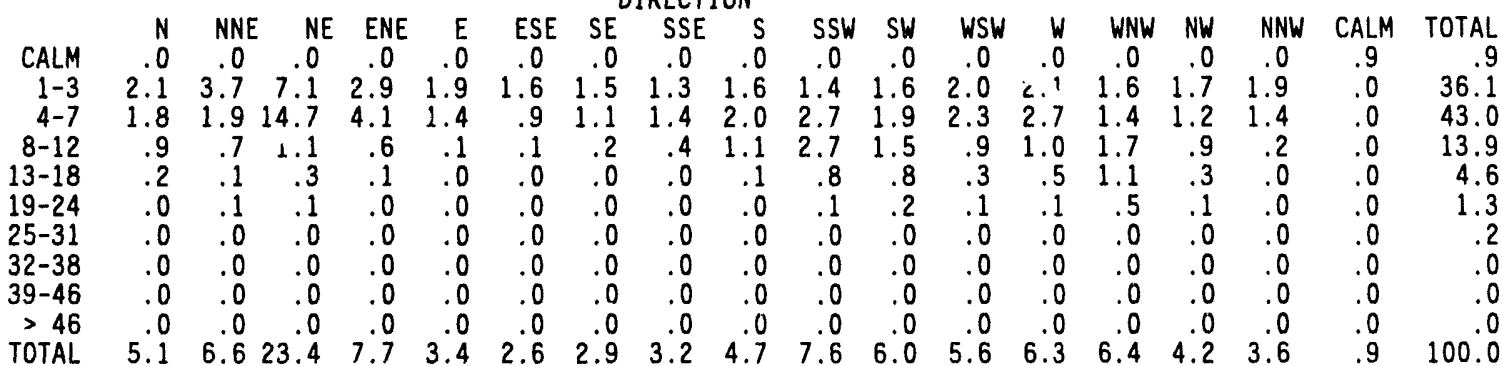

Station: (18) RICH

Begin: 1/93 End: ' '

Total Hours:

8573

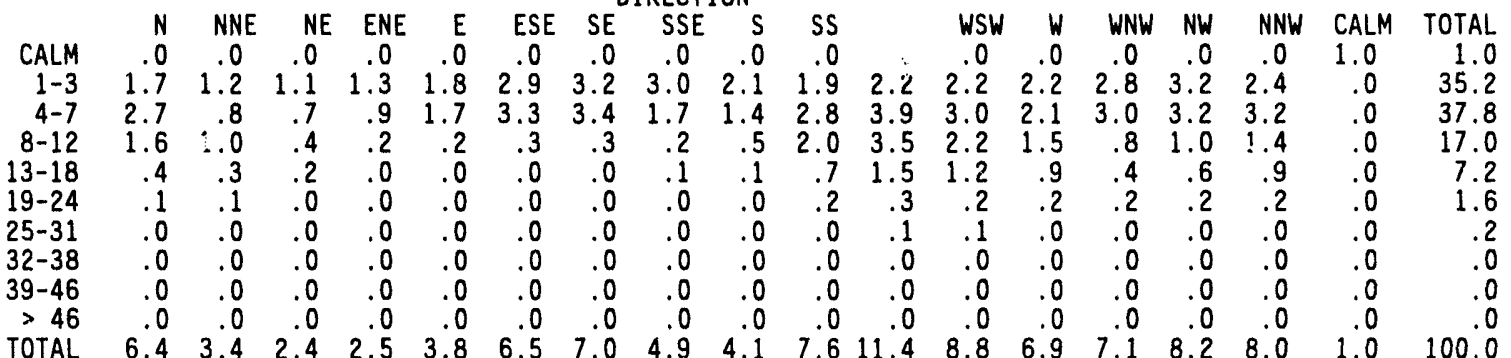

Station: (20) RMTN

Begin: $1 / 93$ DIRECTION End: $12 / 93$

Total Hours: 8186

$\begin{array}{rrrrrrrrrrrrrrrrrrr} & \text { N } & \text { NNE } & \text { NE } & \text { ENE } & \text { E } & \text { ESE } & \text { SE } & \text { SSE } & \text { S } & \text { SSW } & \text { SW } & \text { WSW } & \text { W } & \text { WNW } & \text { NW } & \text { NNW } & \text { CALM } & \text { TOTAL } \\ \text { CALM } & .0 & .0 & .0 & .0 & .0 & .0 & .0 & .0 & .0 & .0 & .0 & .0 & .0 & .0 & .0 & .0 & .2 & .2 \\ 1-3 & .6 & .5 & .5 & .5 & .4 & .3 & .4 & .3 & .5 & .7 & .5 & .5 & .6 & .4 & .5 & .4 & .0 & 7.6 \\ 4-7 & 1.6 & 1.5 & 1.2 & .7 & .6 & .4 & .3 & .4 & .7 & 1.6 & 2.0 & 1.6 & 1.4 & .9 & .8 & .9 & .0 & 16.7 \\ 8-12 & 2.4 & 2.5 & 1.8 & .6 & .3 & .2 & .2 & .3 & .4 & 1.7 & 3.6 & 2.5 & 1.6 & 1.4 & .7 & 1.2 & .0 & 21.2 \\ 13-18 & 2.1 & 3.2 & 1.1 & .2 & .0 & .0 & .0 & .0 & .2 & 1.4 & 4.5 & 3.0 & 1.8 & 1.5 & .9 & .9 & .0 & 20.8 \\ 19-24 & .9 & 2.4 & .8 & .1 & .0 & .0 & .0 & .0 & .0 & .6 & 3.5 & 2.6 & 1.4 & 1.1 & .3 & .2 & .0 & 13.9 \\ 25-31 & .2 & 1.8 & .7 & .0 & .0 & .0 & .0 & .0 & .0 & .2 & 2.6 & 2.5 & .9 & .6 & .1 & .0 & .0 & 9.6 \\ 32-38 & .0 & .7 & .4 & .0 & .0 & .0 & .0 & .0 & .0 & .1 & 1.9 & 1.8 & .3 & .1 & .0 & .0 & .0 & 5.3 \\ 39-46 & .0 & .4 & .1 & .0 & .0 & .0 & .0 & .0 & .0 & .0 & 1.1 & .9 & .1 & .2 & .0 & .0 & .0 & 2.9 \\ >46 & .0 & .2 & .4 & .0 & .0 & .0 & .0 & .0 & .0 & .1 & .6 & .6 & .0 & .1 & .0 & .0 & .0 & 1.9 \\ \text { TOTAL } & 7.8 & 13.2 & 6.9 & 2.1 & 1.3 & .8 & 1.0 & 1.1 & 1.8 & 6.3 & 20.3 & 16.0 & 8.1 & 6.3 & 3.4 & 3.5 & .2 & 100.0\end{array}$

Station: (21) HMS

Begin: 1/93 End: 12/93

Total Hours: $\quad 8552$

$\begin{array}{rrrrrrrrrrrrrrrrrrr} & \text { N } & \text { NNE } & \text { NE } & \text { ENE } & \text { E } & \text { ESE } & \text { SE } & \text { SSE } & \text { S } & \text { SSW } & \text { SW } & \text { WSW } & \text { W } & \text { WNW } & \text { NW } & \text { NNW } & \text { CALM } & \text { TOTAL } \\ \text { CALM } & .0 & .0 & .0 & .0 & .0 & .0 & .0 & .0 & .0 & .0 & .0 & .0 & .0 & .0 & .0 & .0 & .9 & .9 \\ 1-3 & 2.7 & 2.4 & 2.4 & 1.9 & 1.9 & 1.8 & 2.0 & 1.7 & 1.2 & 1.4 & 1.6 & 1.7 & 1.9 & 2.1 & 2.7 & 2.7 & .0 & 32.3 \\ 4-7 & 2.7 & 1.4 & 1.1 & .9 & 1.0 & 1.1 & 1.2 & 1.4 & 1.2 & 1.3 & 2.0 & 2.8 & 4.1 & 5.4 & 8.3 & 5.1 & .0 & 40.8 \\ 8-12 & .5 & .5 & .3 & .2 & .2 & .0 & .0 & .2 & .2 & .3 & .7 & 1.4 & 1.9 & 2.2 & 7.2 & 2.3 & .0 & 18.2 \\ 13-18 & .1 & .2 & .2 & .0 & .0 & .0 & .0 & .0 & .1 & .2 & .6 & .9 & .8 & .5 & 2.3 & .6 & .0 & 6.4 \\ 19-24 & .0 & .0 & .1 & .0 & .0 & .0 & .0 & .0 & .0 & .0 & .2 & .2 & .1 & .0 & .6 & .1 & .0 & 1.3 \\ 25-31 & .0 & .0 & .0 & .0 & .0 & .0 & .0 & .0 & .0 & .0 & .0 & .0 & .0 & .0 & .1 & .0 & .0 & .1 \\ 32-38 & .0 & .0 & .0 & .0 & .0 & .0 & .0 & .0 & .0 & .0 & .0 & .0 & .0 & .0 & .0 & .0 & .0 & .0 \\ 39-46 & .0 & .0 & .0 & .0 & .0 & .0 & .0 & .0 & .0 & .0 & .0 & .0 & .0 & .0 & .0 & .0 & .0 & .0 \\ >46 & .0 & .0 & .0 & .0 & .0 & .0 & .0 & .0 & .0 & .0 & .0 & .0 & .0 & .0 & .0 & .0 & .0 & .0 \\ \text { TOTAL } & 5.9 & 4.6 & 4.1 & 3.0 & 3.1 & 3.0 & 3.2 & 3.4 & 2.7 & 3.3 & 5.0 & 6.9 & 8.8 & 10.2 & 21.2 & 10.7 & .9 & 100.0\end{array}$




\section{TABLE A.1. (contd)}

Station: (22) PASC

Begin: 1/93

End: $12 / 93$

Total Hours:

8536

$\begin{array}{rrrrrrrrrrrrrrrrrrr} & \text { N } & \text { NNE } & \text { NE } & \text { ENE } & \text { E } & \text { ESE } & \text { SE } & \text { SSE } & \text { S } & \text { SSW } & \text { SW } & \text { WSW } & \text { W } & \text { WNW } & \text { NW } & \text { NNW } & \text { CALM } & \text { TOTAL } \\ \text { CALM } & .0 & .0 & .0 & .0 & .0 & .0 & .0 & .0 & .0 & .0 & .0 & .0 & .0 & .0 & .0 & .0 & .5 & .5 \\ 1-3 & 2.9 & 2.4 & 2.5 & 2.5 & 2.4 & 2.1 & 1.7 & 1.1 & 1.2 & .9 & .9 & 1.0 & 1.2 & 1.9 & 2.7 & 2.8 & .0 & 30.1 \\ 4-7 & 3.2 & 2.0 & 1.0 & 1.4 & 2.4 & 3.1 & 2.1 & 1.6 & 1.7 & 2.2 & 2.0 & 1.4 & 1.8 & 2.6 & 4.2 & 4.7 & .0 & 37.5 \\ 8-12 & 1.4 & .5 & .2 & .2 & .2 & .4 & .6 & .4 & .7 & 2.1 & 3.6 & 1.9 & 1.0 & .8 & 1.7 & 2.1 & .0 & 17.8 \\ 13-18 & .3 & .3 & .1 & .1 & .0 & .1 & .0 & .1 & .1 & .7 & 2.7 & 2.0 & .7 & .4 & .8 & .7 & .0 & 8.9 \\ 19-24 & .1 & .1 & .0 & .0 & .0 & .0 & .0 & .0 & .0 & .2 & 1.2 & 1.3 & .4 & .1 & .2 & .1 & .0 & 3.7 \\ 25-31 & .0 & .0 & .0 & .0 & .0 & .0 & .0 & .0 & .0 & .0 & .3 & .6 & .1 & .1 & .0 & .0 & .0 & 1.2 \\ 32-38 & .0 & .0 & .0 & .0 & .0 & .0 & .0 & .0 & .0 & .0 & .2 & .2 & .0 & .0 & .0 & .0 & .0 & .0 \\ 39-46 & .0 & .0 & .0 & .0 & .0 & .0 & .0 & .0 & .0 & .0 & .0 & .0 & .0 & .0 & .0 & .0 & .0 & .0 \\ >46 & .0 & .0 & .0 & .0 & .0 & .0 & .0 & .0 & .0 & .0 & .0 & .0 & .0 & .0 & .0 & .0 & .0 & .0 \\ \text { TOTAL } & 7.9 & 5.2 & 3.9 & 4.2 & 5.0 & 5.6 & 4.4 & 3.2 & 3.6 & 6.1 & 10.9 & 8.4 & 5.2 & 5.9 & 9.6 & 10.2 & .5 & 100.0\end{array}$

Station: (23) GABW

Begin: 1/93 End: $12 / 93$

Total Hours:

$8573^{\circ}$

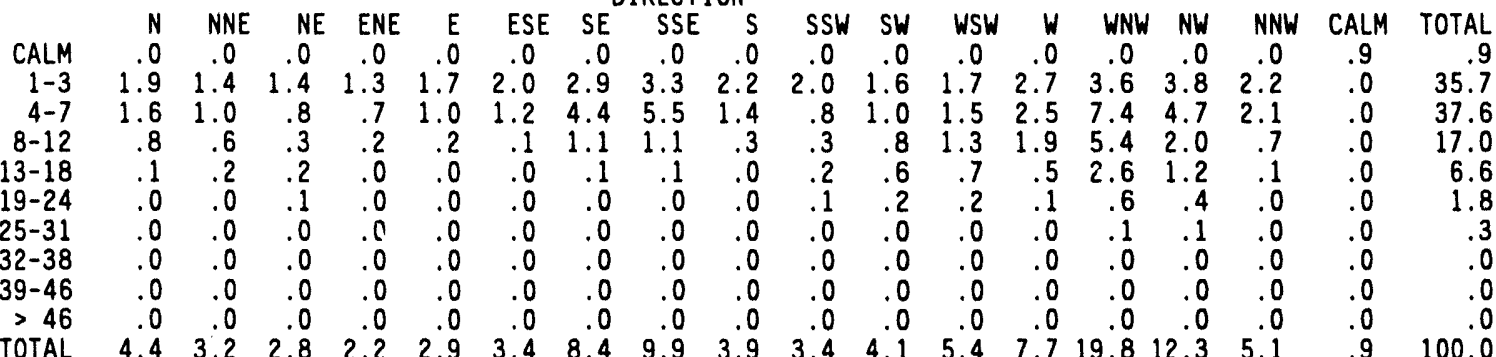

Station: (24) 100F

Begin: $1 / 93$ DIRECTION End: $12 / 93$

Total Hours:

8506

CALM N NNE NE ENE E ESE SE SSE S SSW SW WSW W WNW NW NNW CALM TOTAL

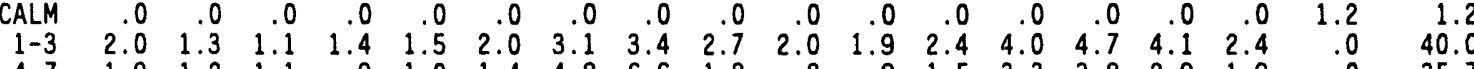

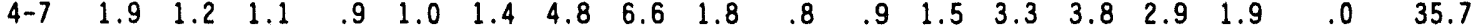

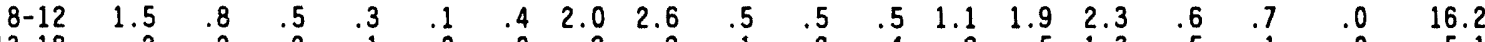

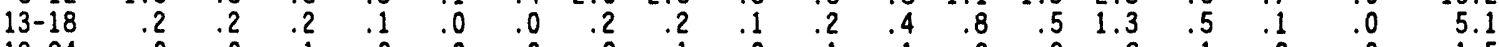

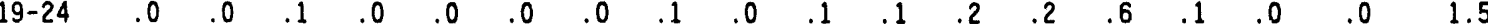

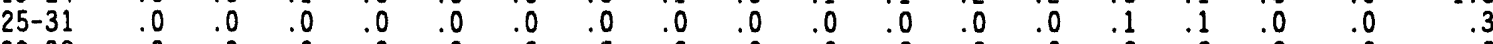

$\begin{array}{lllllllllllllllllll}32-38 & .0 & .0 & .0 & .0 & .0 & .0 & .0 & .0 & .0 & .0 & .0 & .0 & .0 & .0 & .0 & .0 & .0 & .0 \\ 39-46 & 0 & 0 & 0 & 0 & 0 & 0 & 0 & 0 & 0 & 0 & 0 & 0 & 0 & 0 & 0 & .0 & .0 & .0\end{array}$

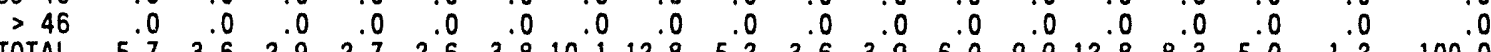

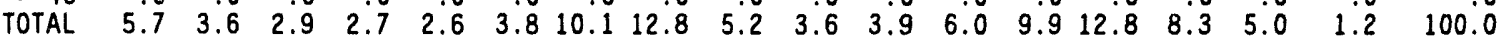

Station: (25) VERN

Begin: $1 / 93 \quad$ End: $12 / 93$

Total Hours:

8532

\begin{tabular}{|c|c|c|c|c|c|c|c|c|c|c|c|c|c|c|c|c|c|c|}
\hline $\begin{array}{r}\text { CALM } \\
1-3 \\
4-7 \\
8-12 \\
3-18 \\
9-24\end{array}$ & $\begin{array}{r}N \\
.0 \\
.9 \\
1.0 \\
.6 \\
.2 \\
.1 \\
.0 \\
.0 \\
.0 \\
.0 \\
2.8\end{array}$ & $\begin{array}{c}\text { NNE } \\
.0 \\
1.3 \\
1.2 \\
.4 \\
.3 \\
.0 \\
.0 \\
.0 \\
.0 \\
.0 \\
3.3\end{array}$ & $\begin{array}{c}\text { NE } \\
.0 \\
1.6 \\
1.6 \\
.3 \\
.2 \\
.1 \\
.0 \\
.0 \\
.0 \\
.0 \\
3.8\end{array}$ & $\begin{array}{c}\text { ENE } \\
.0 \\
2.0 \\
2.5 \\
.4 \\
.0 \\
.0 \\
.0 \\
.0 \\
.0 \\
.0 \\
5.0\end{array}$ & $\begin{array}{r}E \\
.0 \\
3.1 \\
3.5 \\
.3 \\
.0 \\
.0 \\
.0 \\
.0 \\
.0 \\
.0 \\
6.8\end{array}$ & $\begin{array}{c}\text { ESE } \\
.0 \\
2.9 \\
2.3 \\
.1 \\
.0 \\
.0 \\
.0 \\
.0 \\
.0 \\
.0 \\
5.3\end{array}$ & $\begin{array}{r}\text { SE } \\
.0 \\
2.3 \\
1.0 \\
.1 \\
.0 \\
.0 \\
.0 \\
.0 \\
.0 \\
.0 \\
3.4\end{array}$ & $\begin{array}{c}\text { SSE } \\
.0 \\
1.6 \\
.4 \\
.1 \\
.0 \\
.0 \\
.0 \\
.0 \\
.0 \\
.0 \\
2.2\end{array}$ & $\begin{array}{r}S \\
.0 \\
.9 \\
.4 \\
.1 \\
.0 \\
.0 \\
.0 \\
.0 \\
.0 \\
.0 \\
1.4\end{array}$ & $\begin{array}{c}\text { SSW } \\
.0 \\
1.0 \\
.3 \\
.4 \\
.1 \\
.0 \\
.0 \\
.0 \\
.0 \\
.0 \\
1.9\end{array}$ & $\begin{array}{r}\text { SW } \\
.0 \\
1.2 \\
.6 \\
.6 \\
.7 \\
.2\end{array}$ & $\begin{array}{c}\text { WSW } \\
.0 \\
2.8 \\
4.3 \\
1.5 \\
.4 \\
.1 \\
.0\end{array}$ & $\begin{array}{r}W \\
.0 \\
3.0 \\
8.1 \\
6.9 \\
1.7 \\
.2\end{array}$ & $\begin{array}{r}\text { WNW } \\
.0 \\
1.6 \\
4.4 \\
7.0 \\
4.3 \\
1.0 \\
.1\end{array}$ & $\begin{array}{r}N W \\
.0 \\
1.3 \\
2.2 \\
2.8 \\
1.8 \\
.5\end{array}$ & $\begin{array}{c}\text { NNW } \\
.0 \\
.9 \\
1.2 \\
.9 \\
.2 \\
.0 \\
.0 \\
.0 \\
.0 \\
.0 \\
3.2\end{array}$ & $\begin{array}{c}\text { CALM } \\
1.7 \\
.0\end{array}$ & ТОТ \\
\hline
\end{tabular}




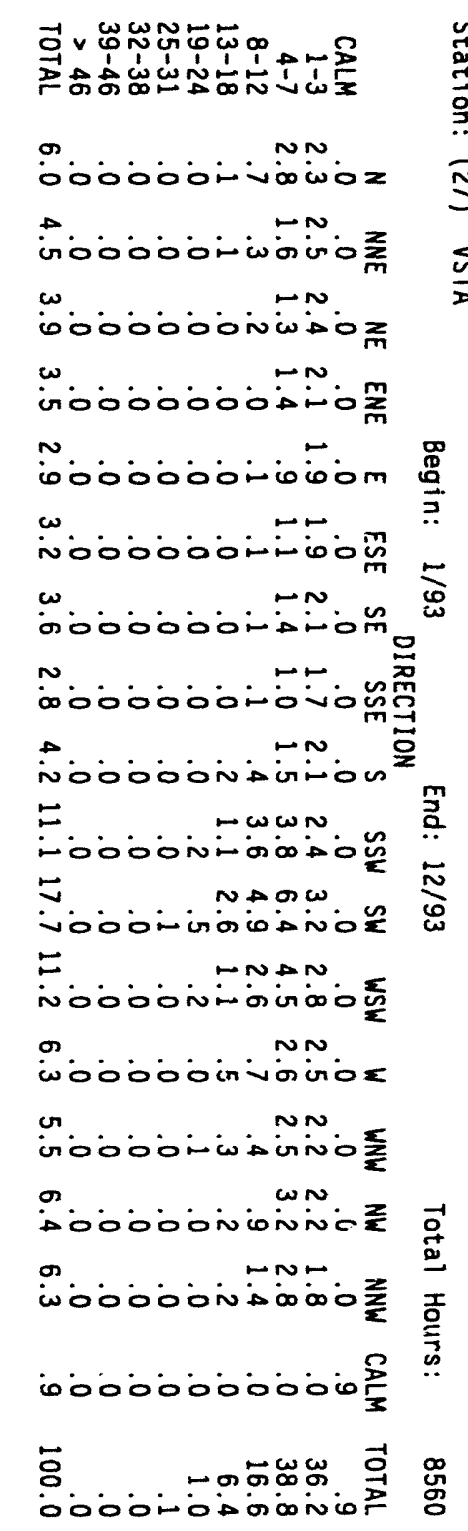

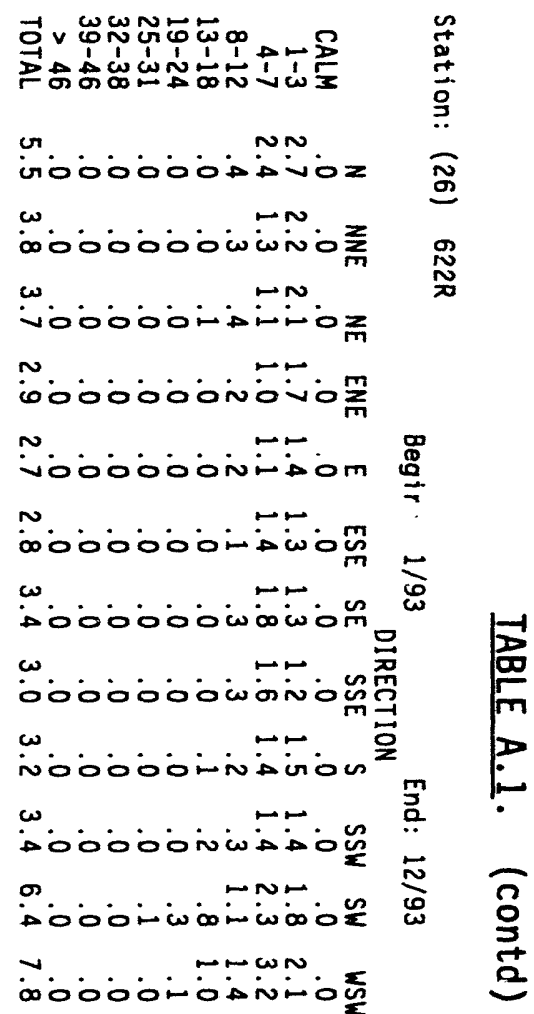

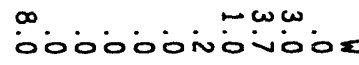

$\approx$

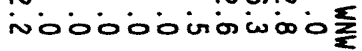

N

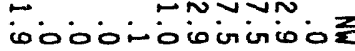

$\infty$.

DAN

Doodonutio

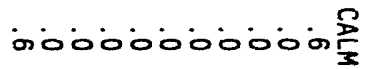

s

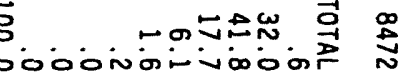


APPENDIX B

WIND CLIMATOLOGY 


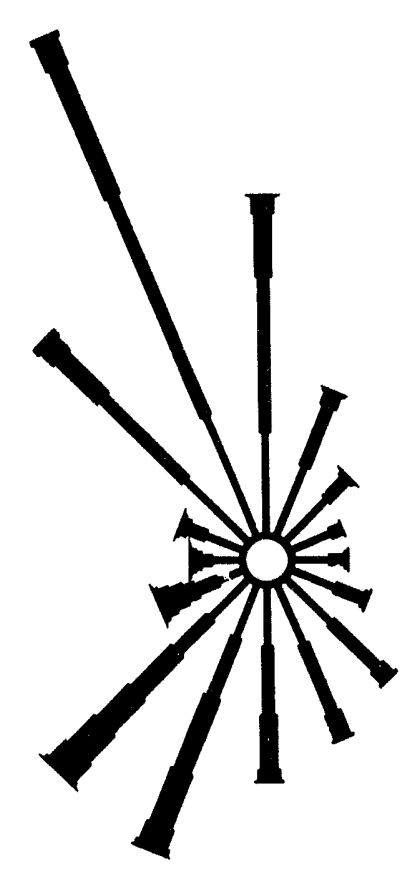

(a) Wind Rose

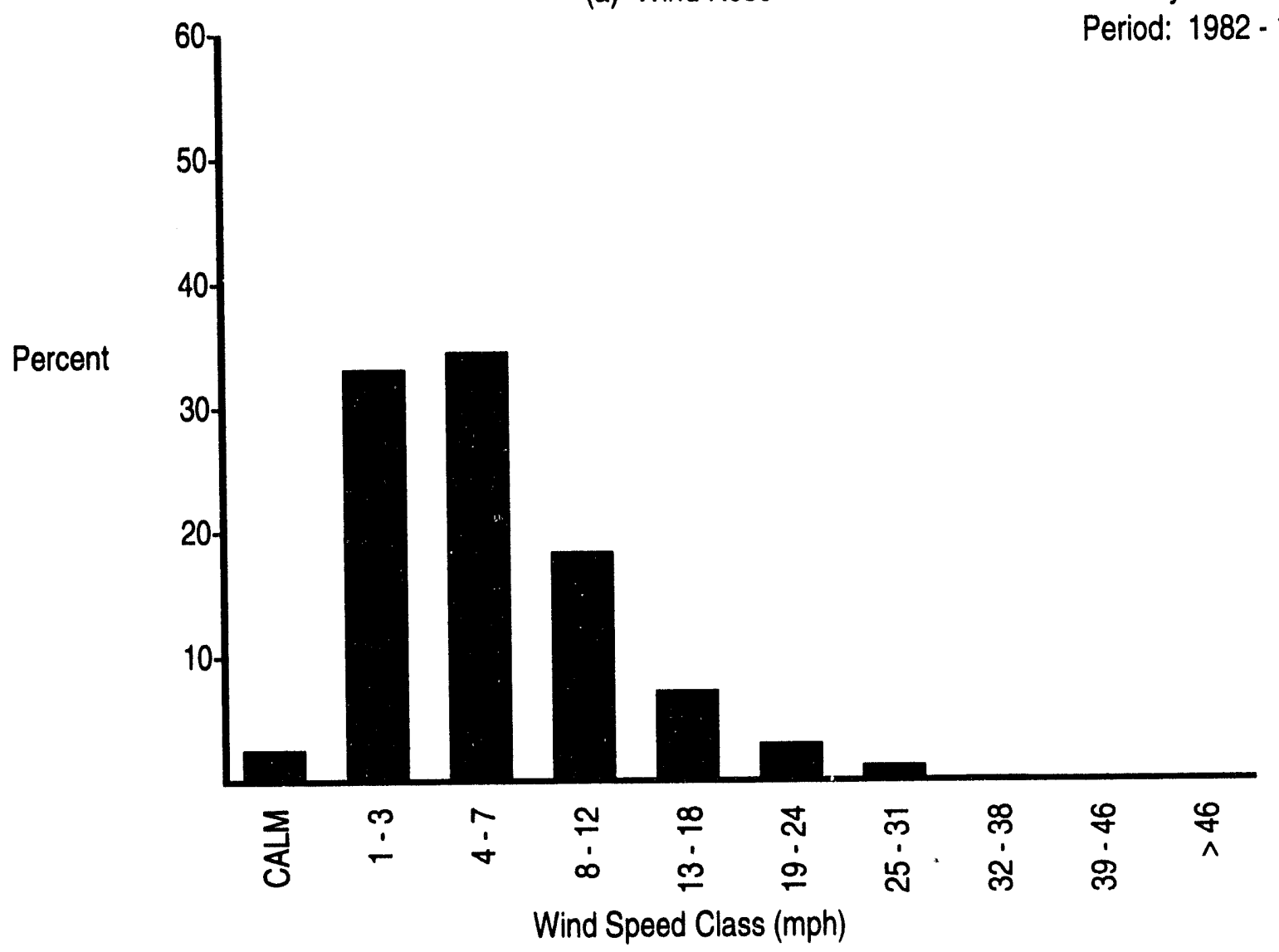

(b) Wind Speed Histogram
January Data

Period: $1982-1993$

FIGURE B.1. Wind Rose and Wind Speed Histogram

B.1 


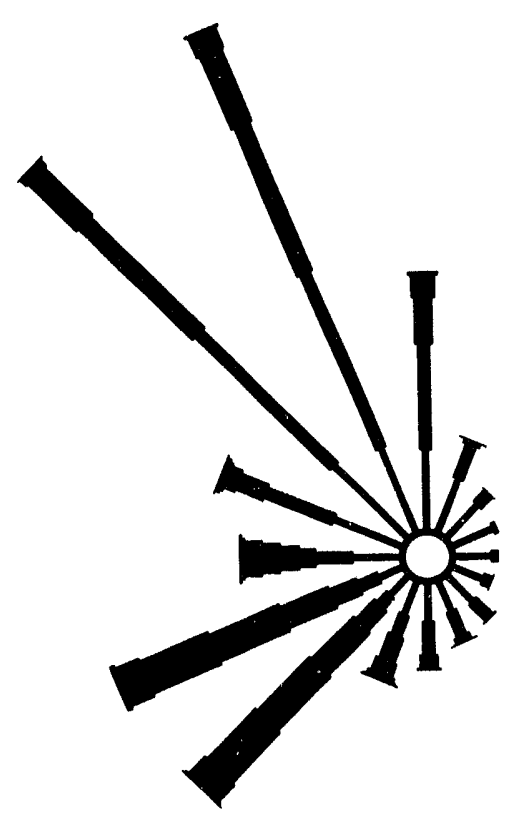

(a) Wind Rose

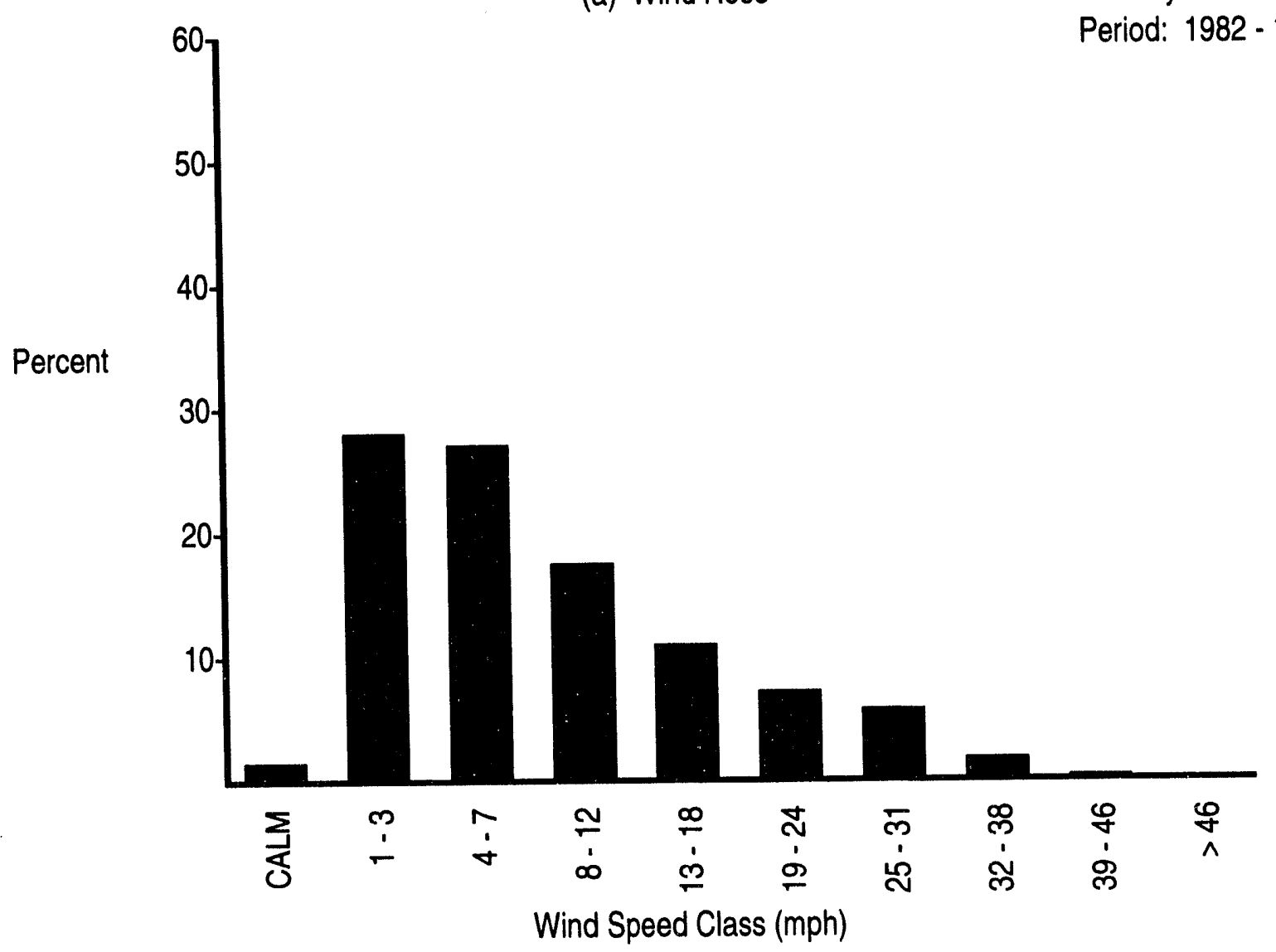

(b) Wind Speed Histogram

FIGURE B.1. (contd) 

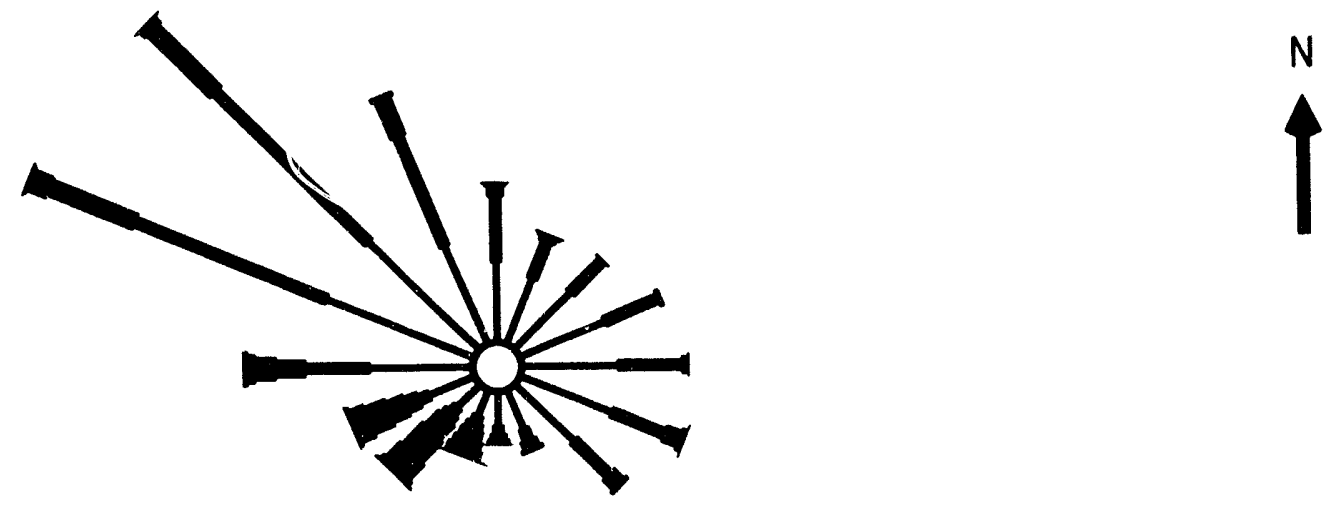

(a) Wind Rose

January Data

Period: $1982-1993$

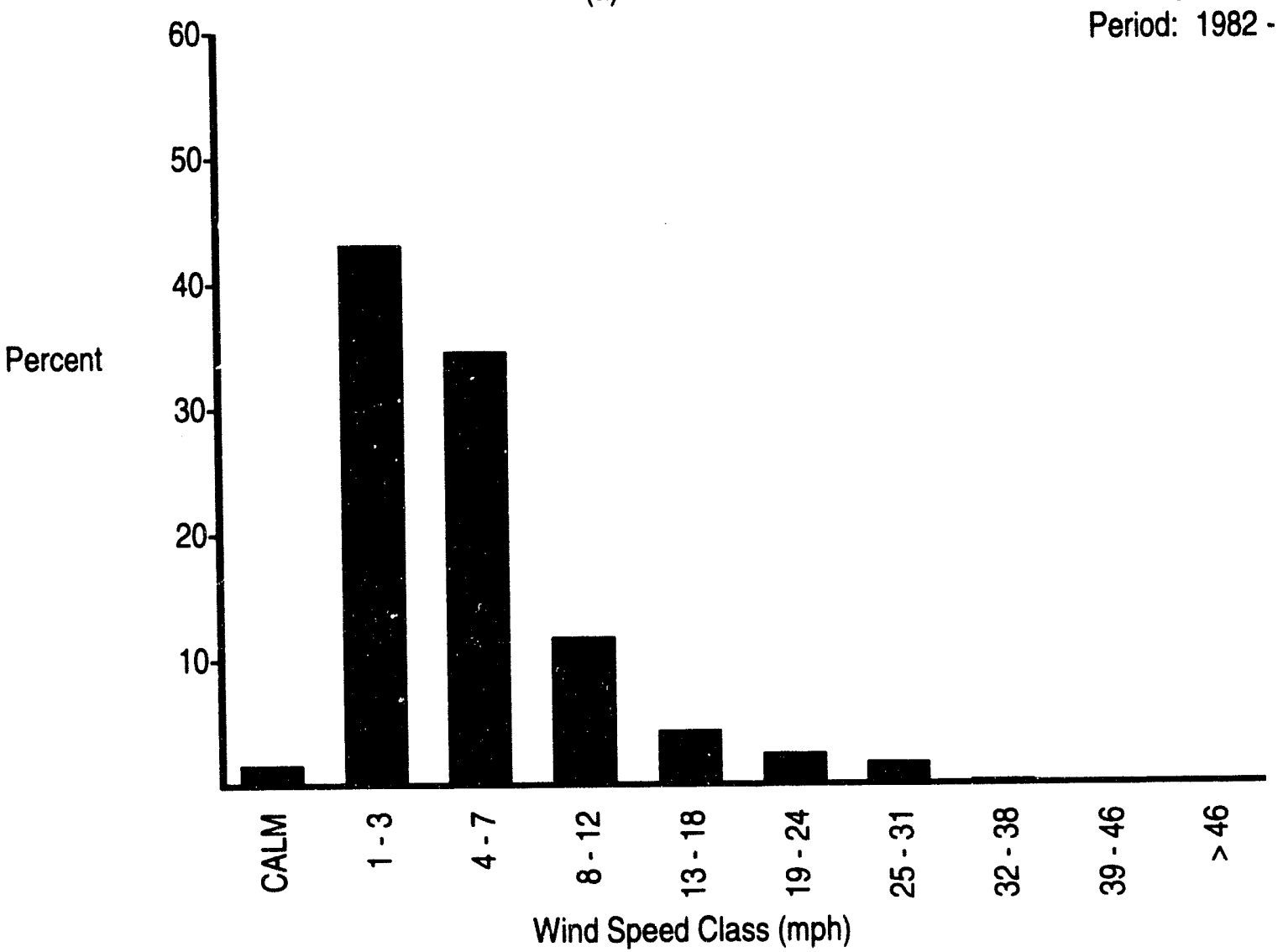

(b) Wind Speed Histogram

FIGURE B.1. (contd) 


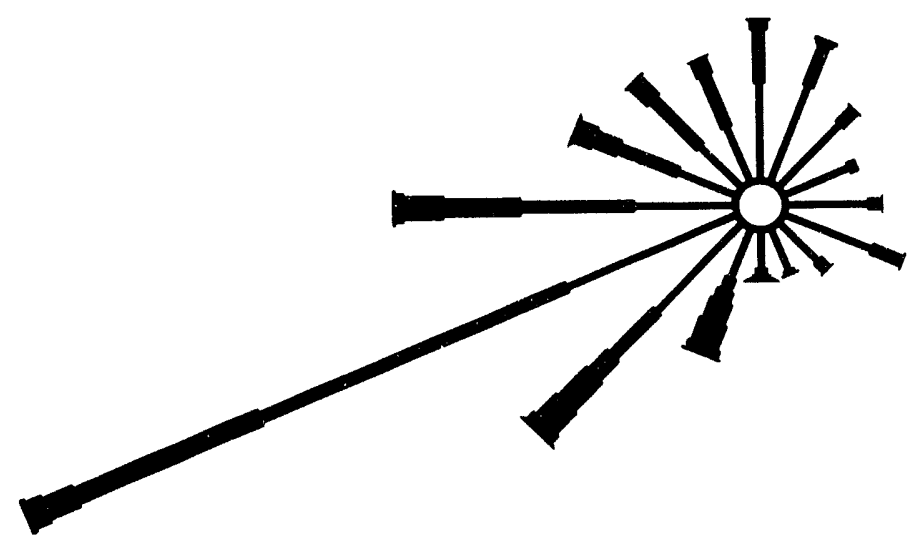

(a) Wind Rose

January Data

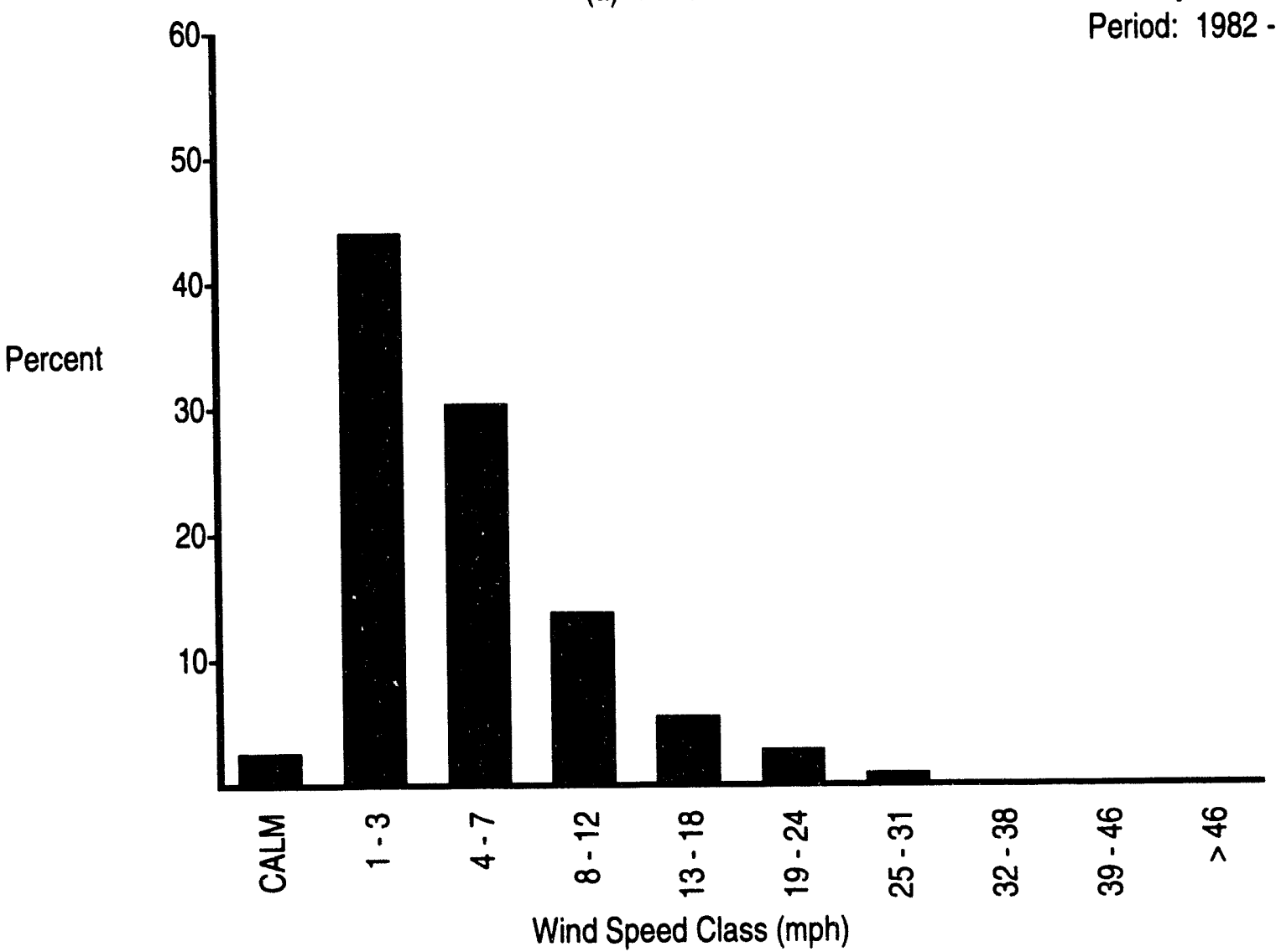

(b) Wind Speed Histogram

FIGURE B.1. (contd) 


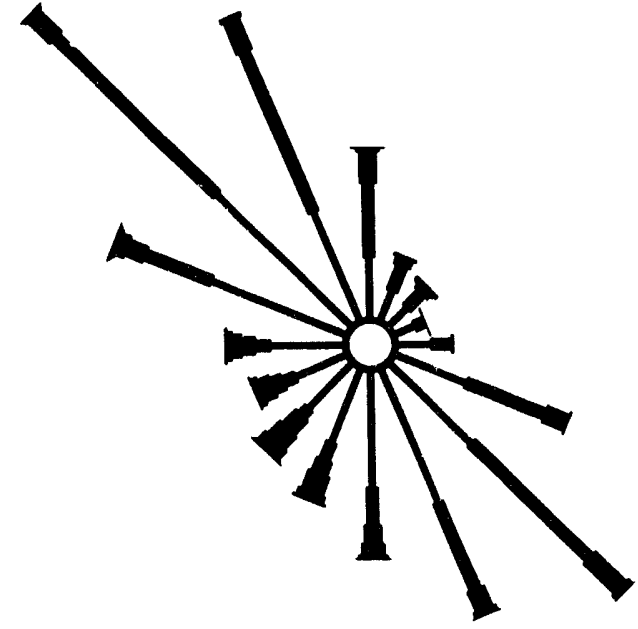

(a) Wind Rose

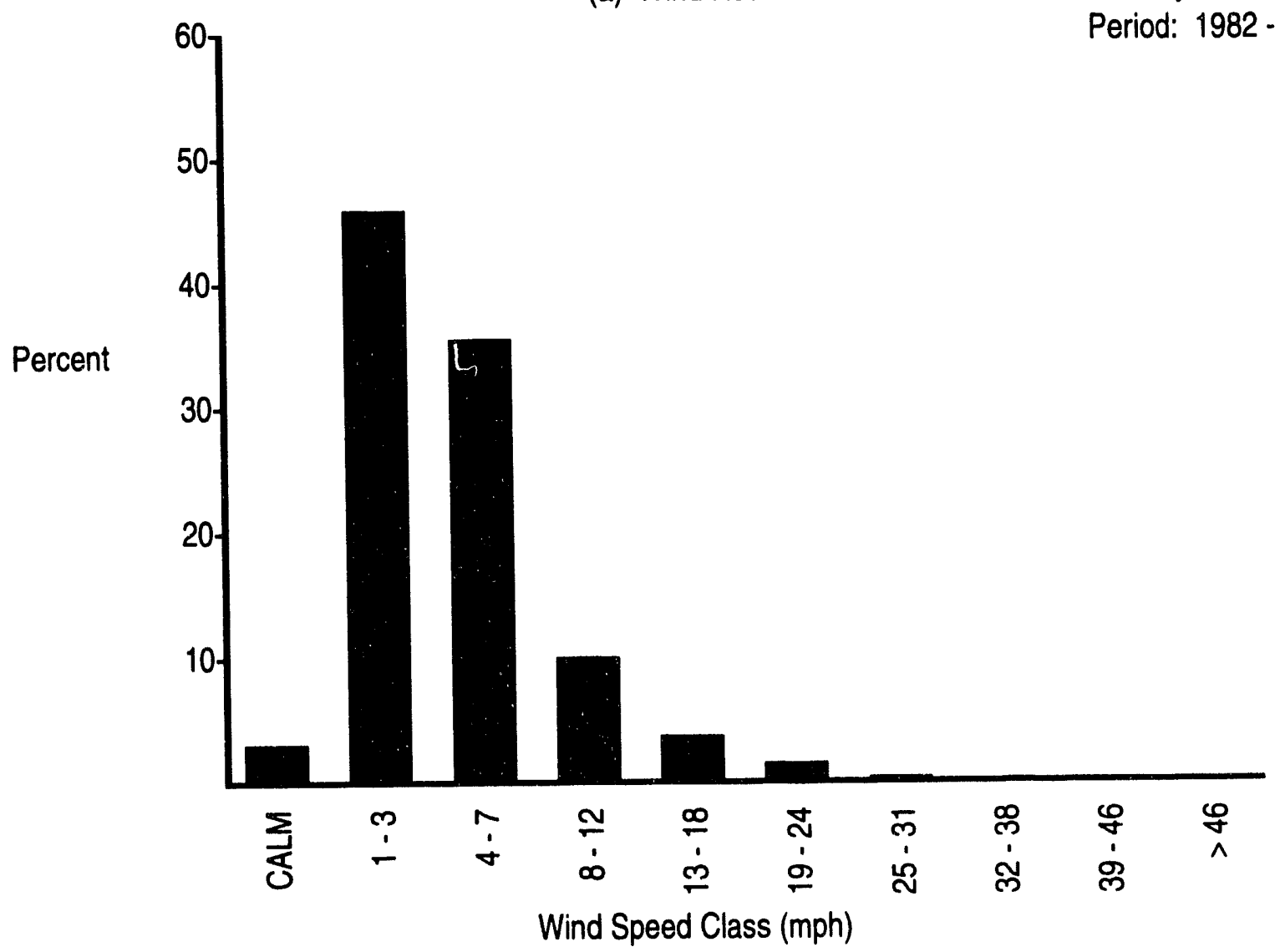

(b) Wind Speed Histogram

FIGURE B.1. (contd)
January Data

Period: 1982 - 1993 


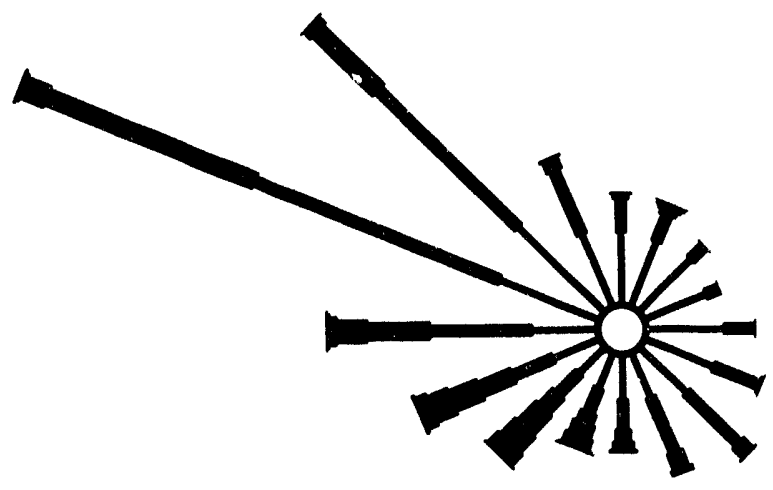

(a) Wind Rose

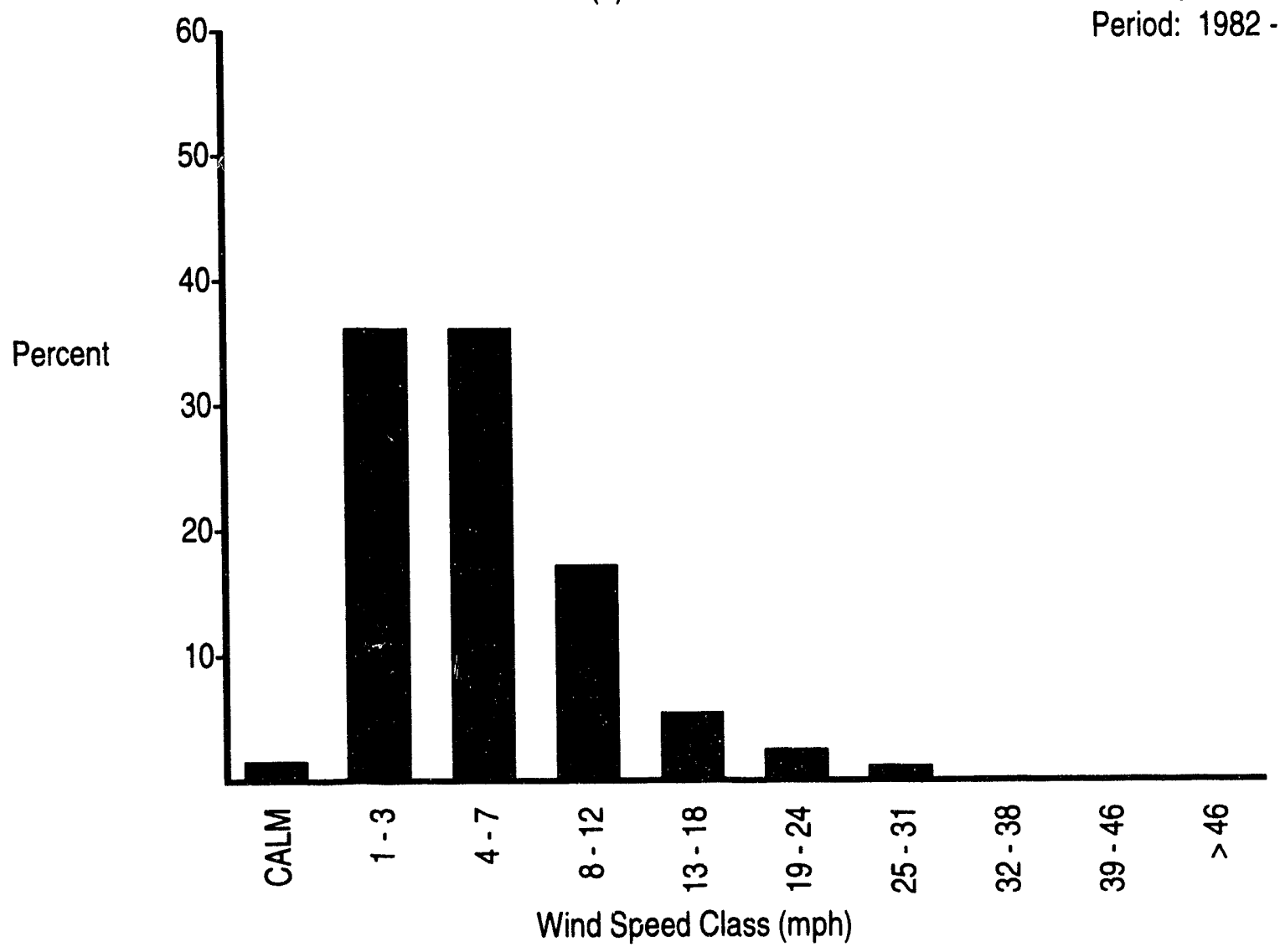

(b) Wind Speed Histogram

FIGURE B.1. (contd) 

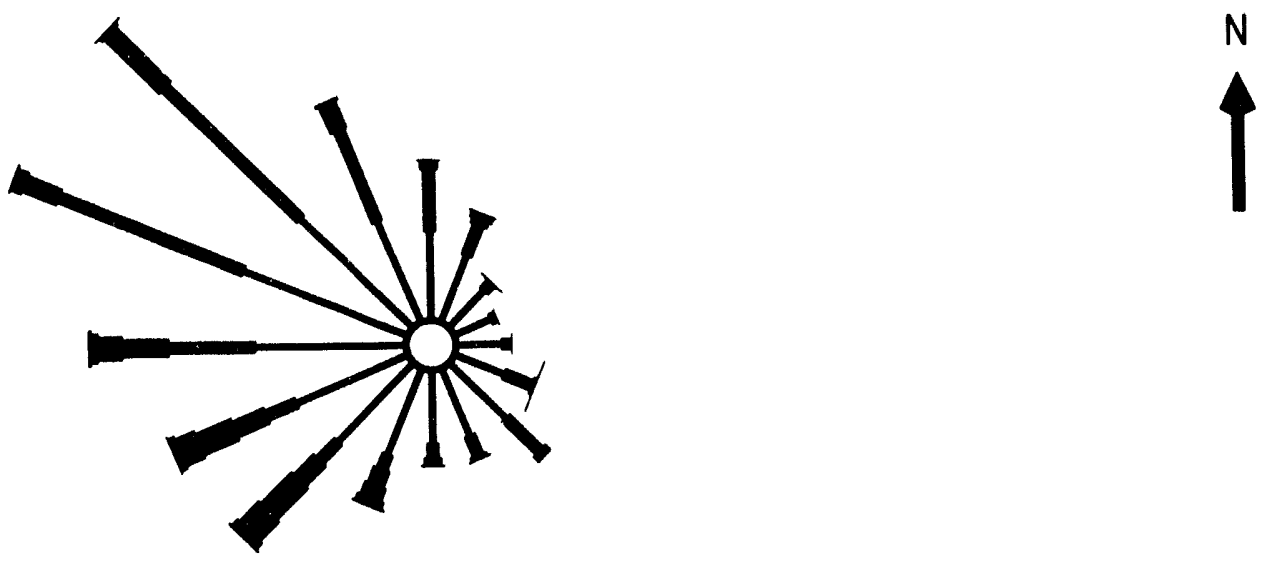

(a) Wind Rose

January Data

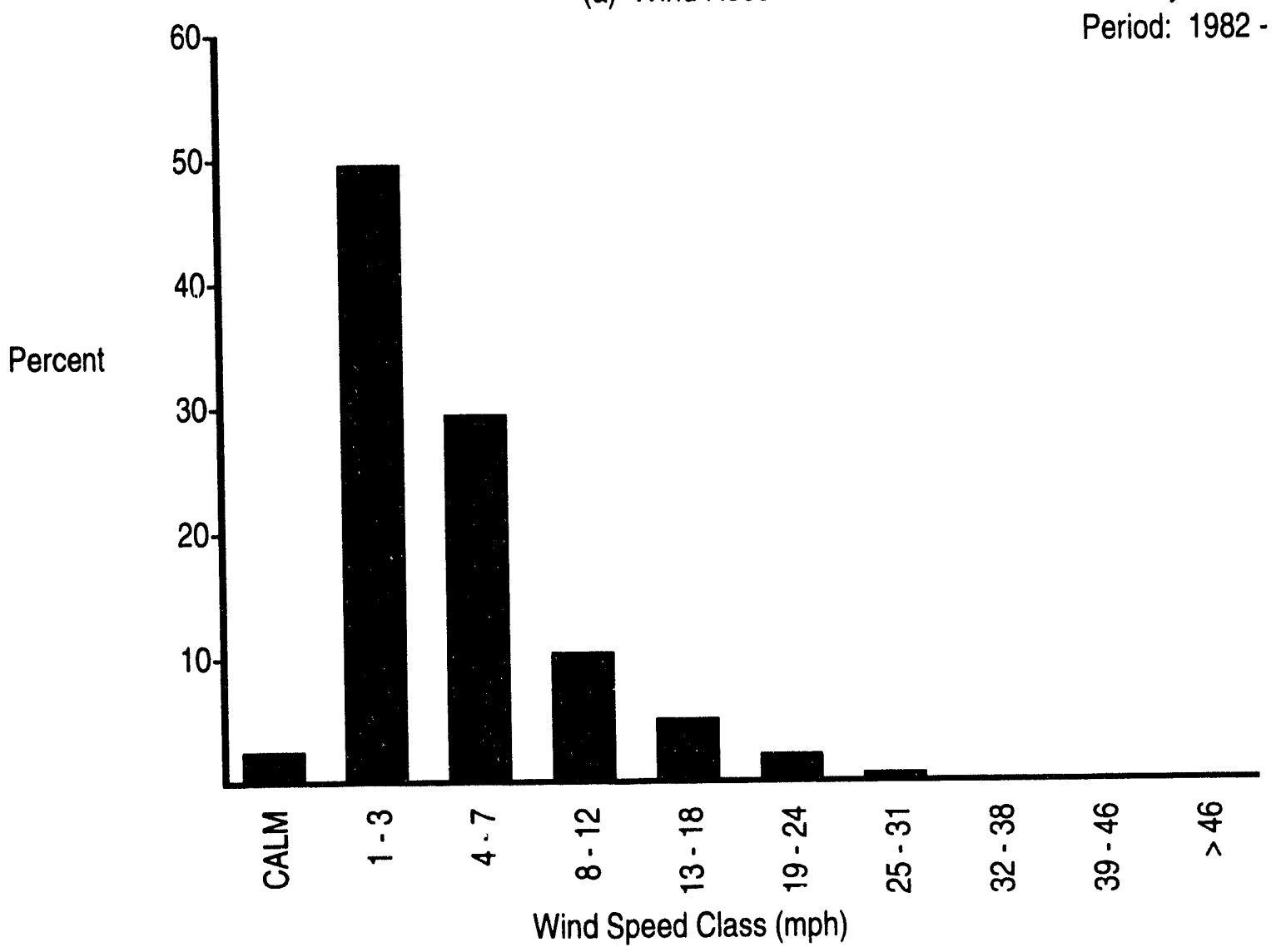

(b) Wind Speed Histogram

FIGURE B.1. (contd) 


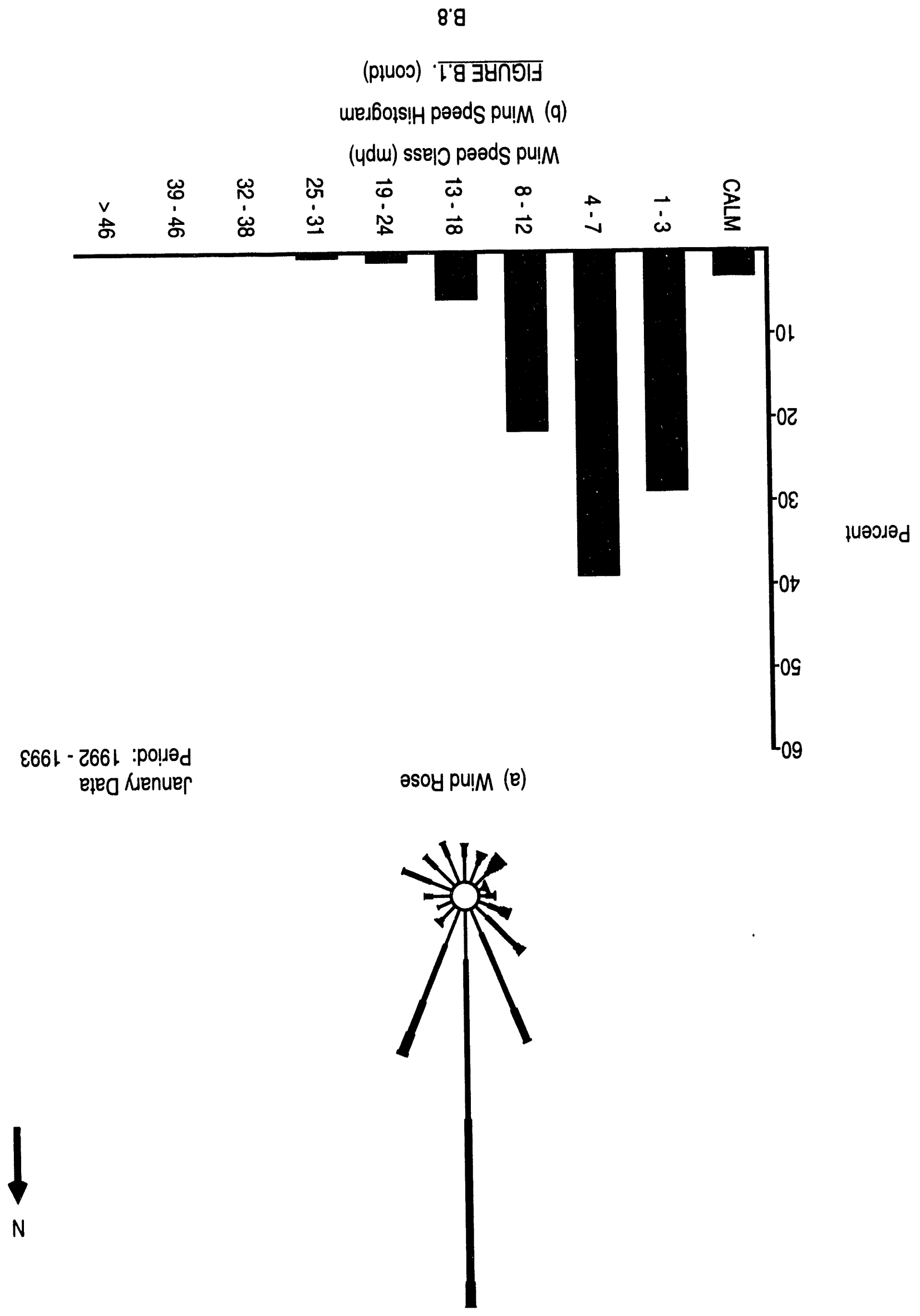




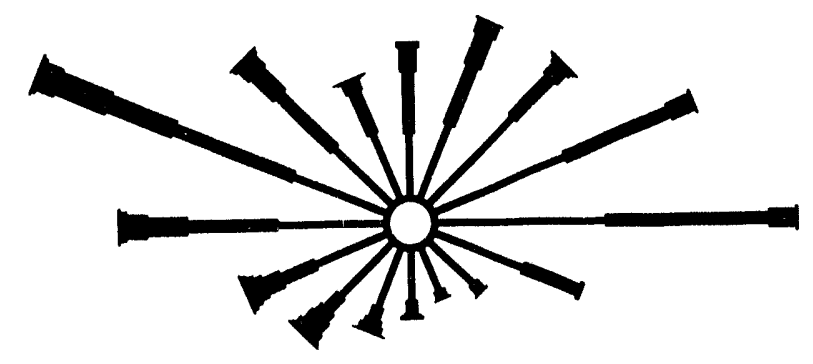

(a) Wind Rose

January Data

Period: $1982-1991$

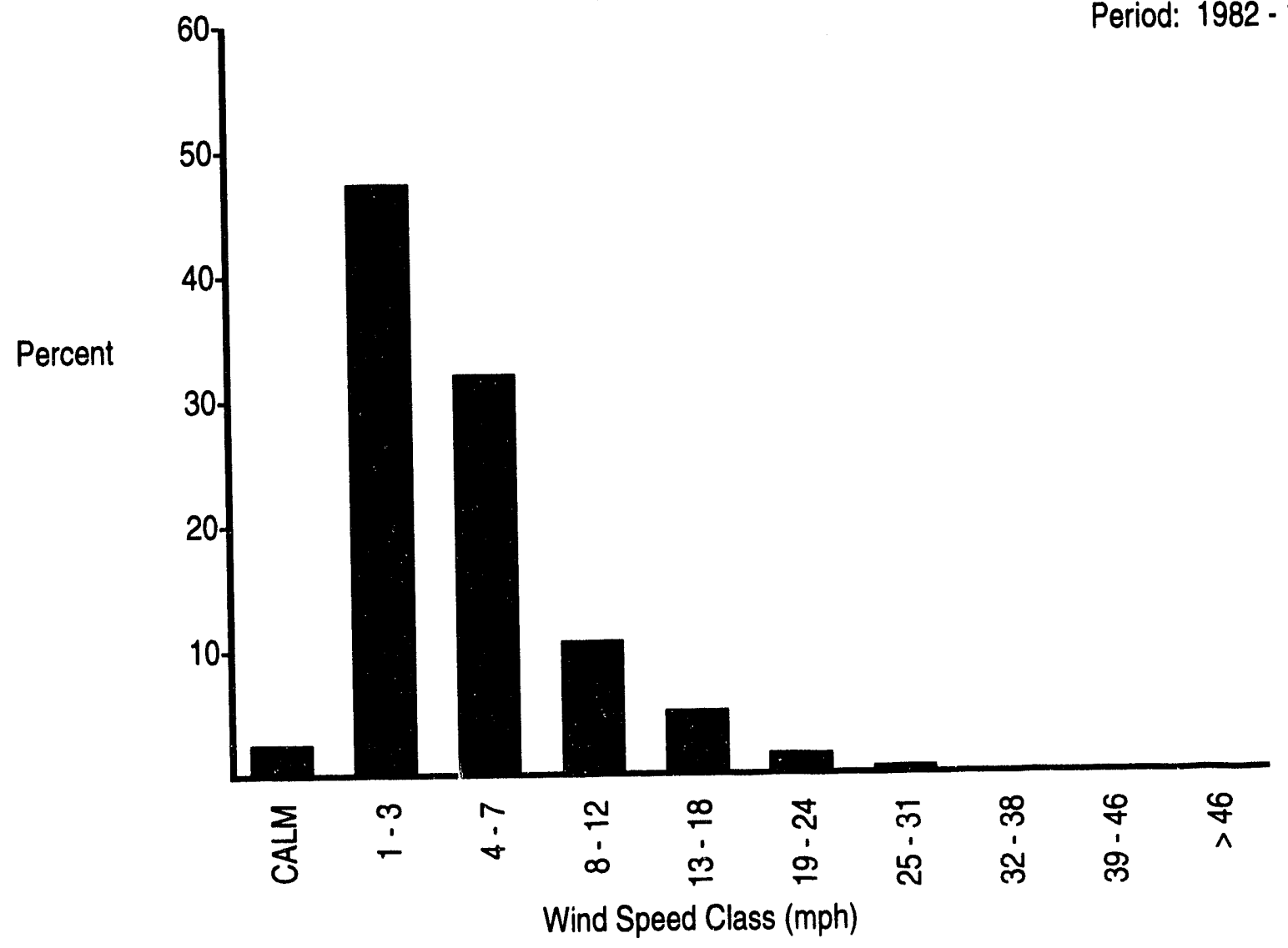

(b) Wind Speed Histogram

FIGURE B.1. (contd) 


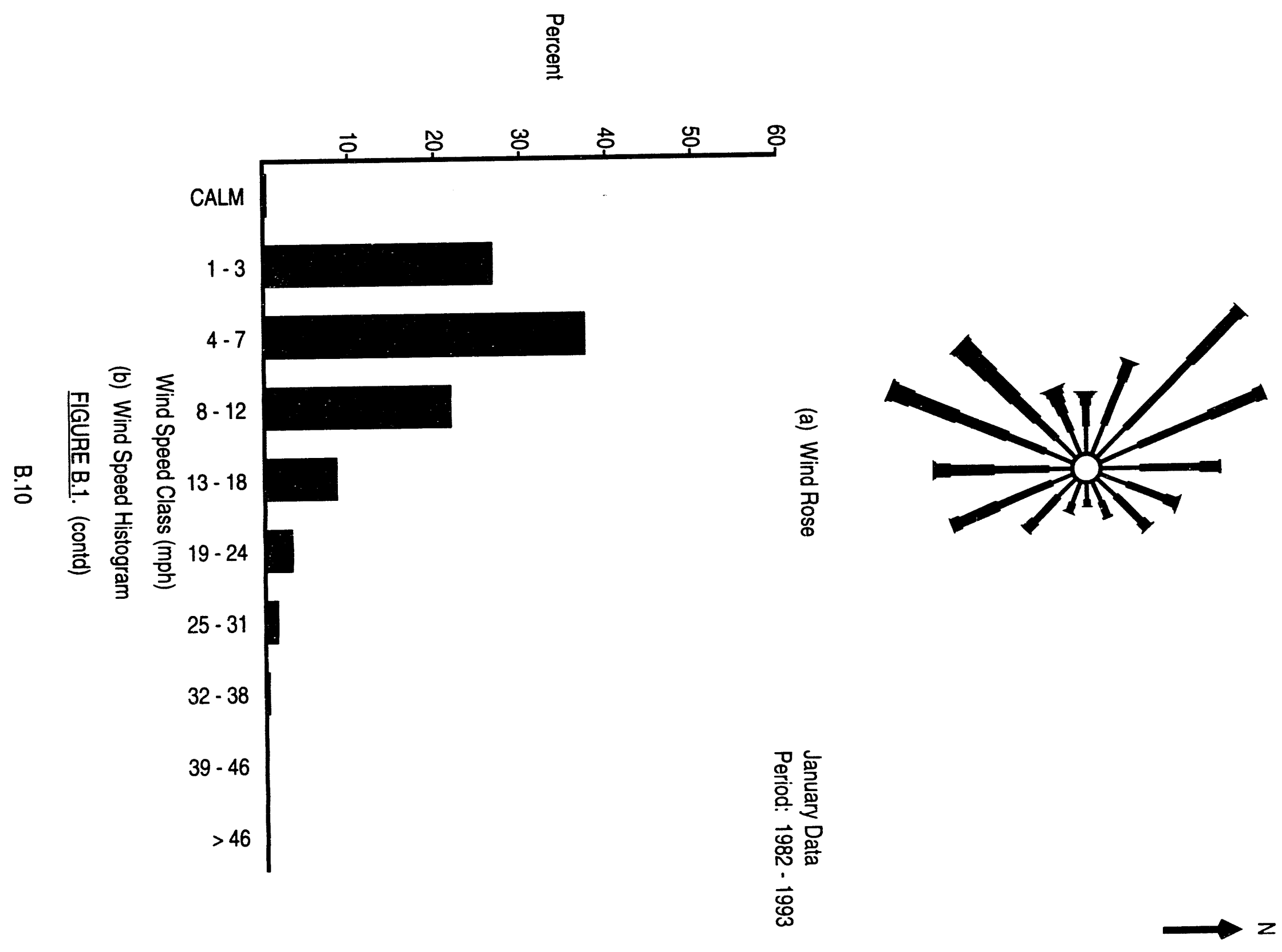




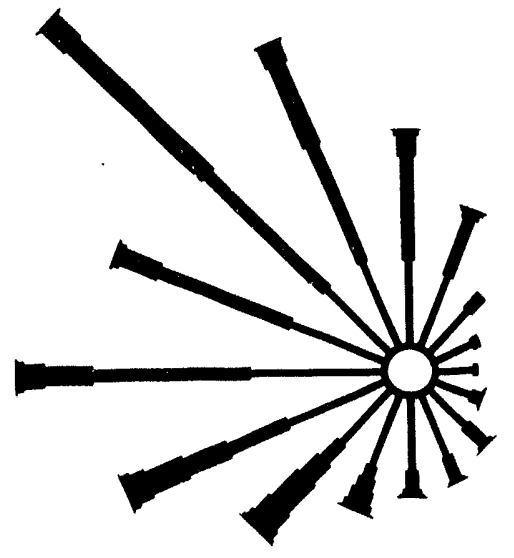

(a) Wind Rose

January Data

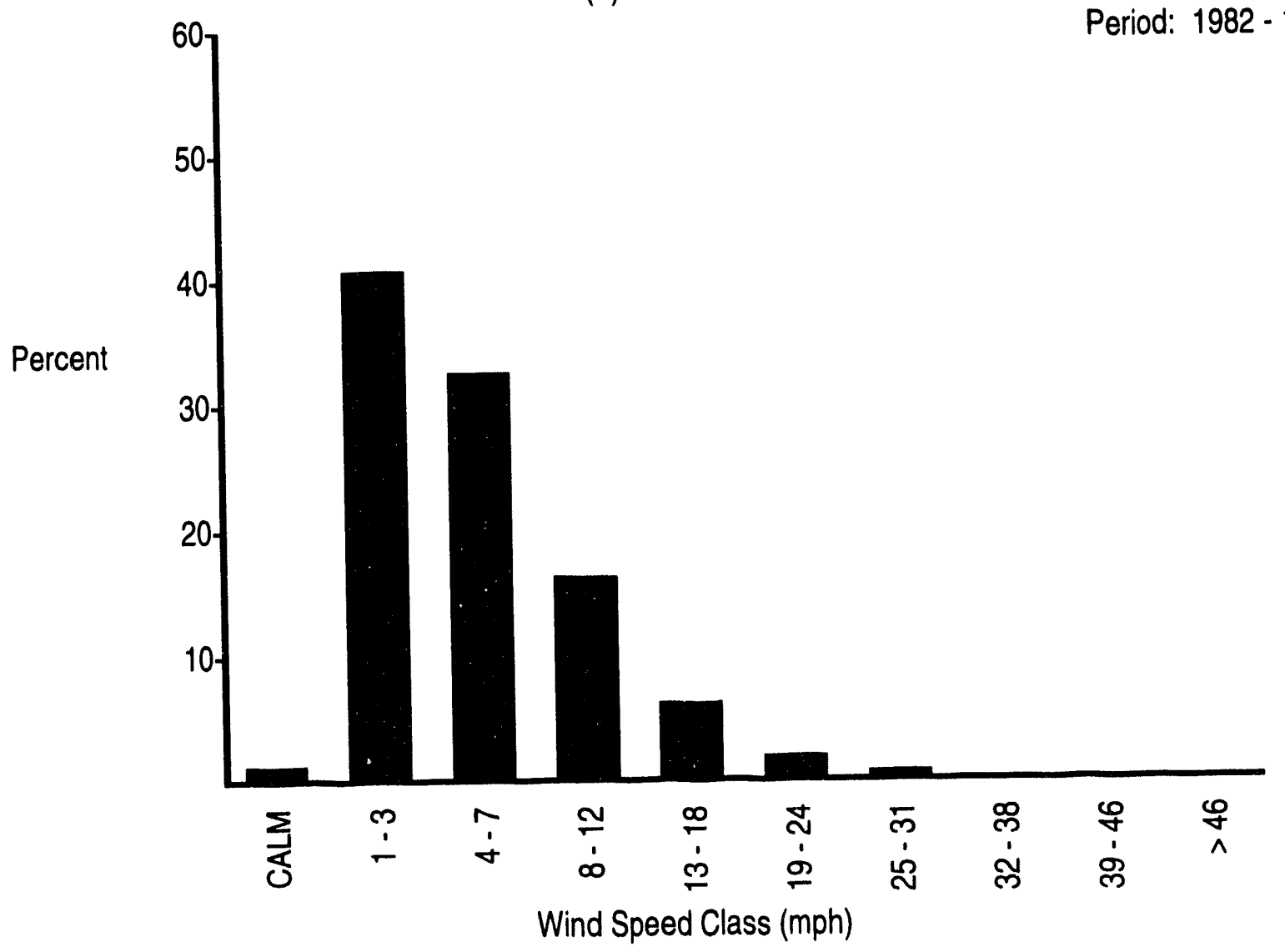

(b) Wind Speed Histogram

FIGURE B.1. (contd) 

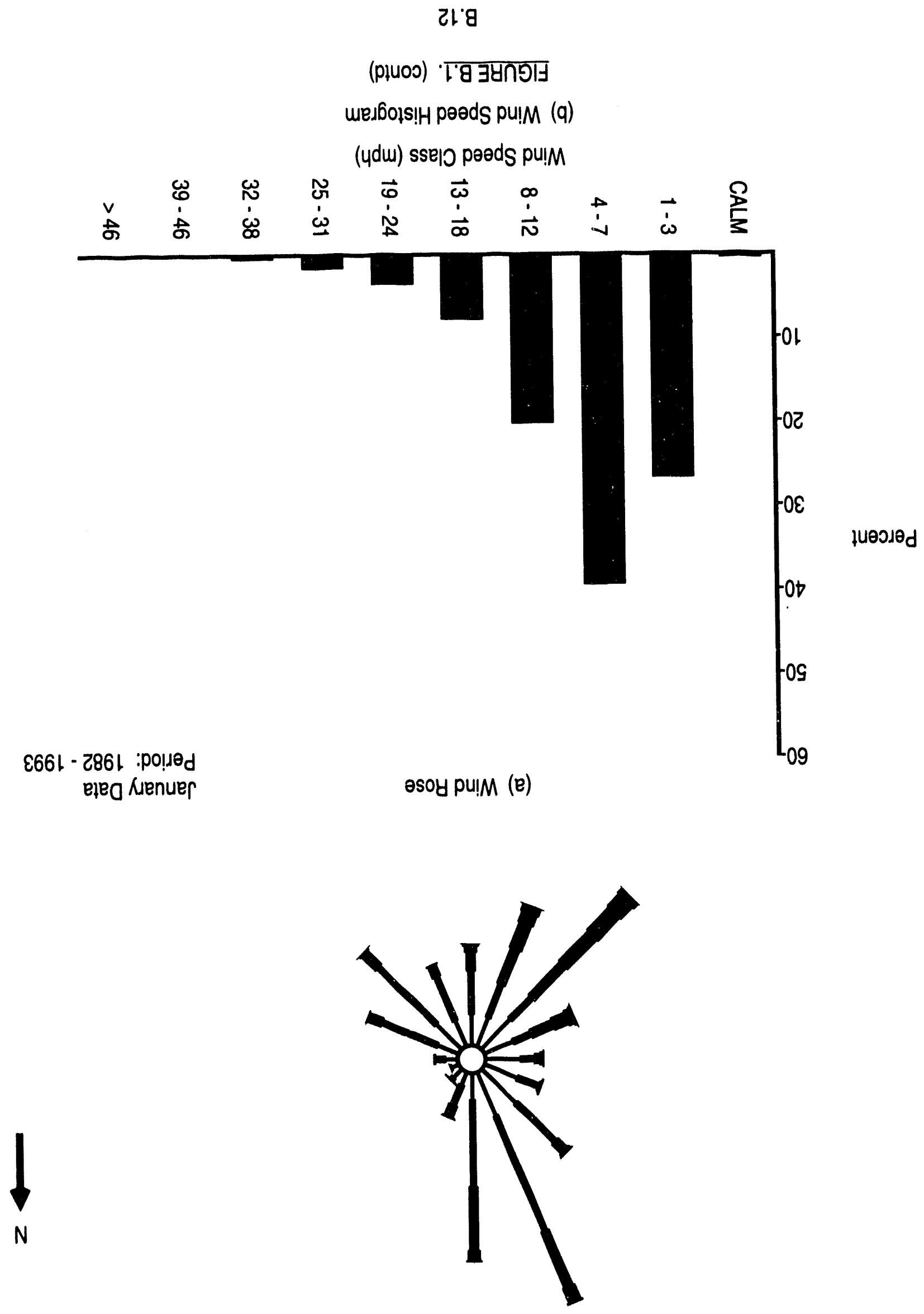


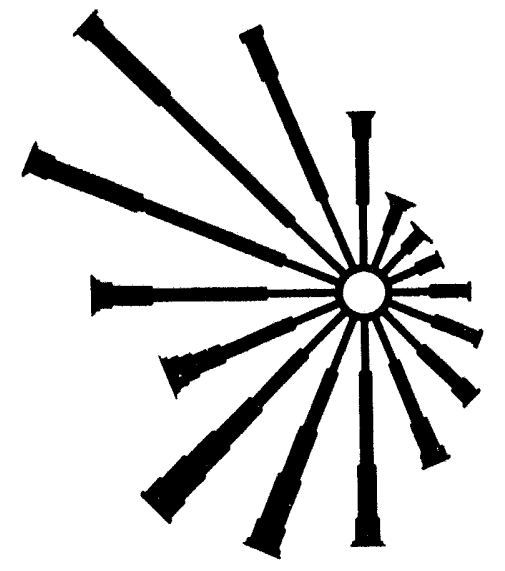

(a) Wind Rose

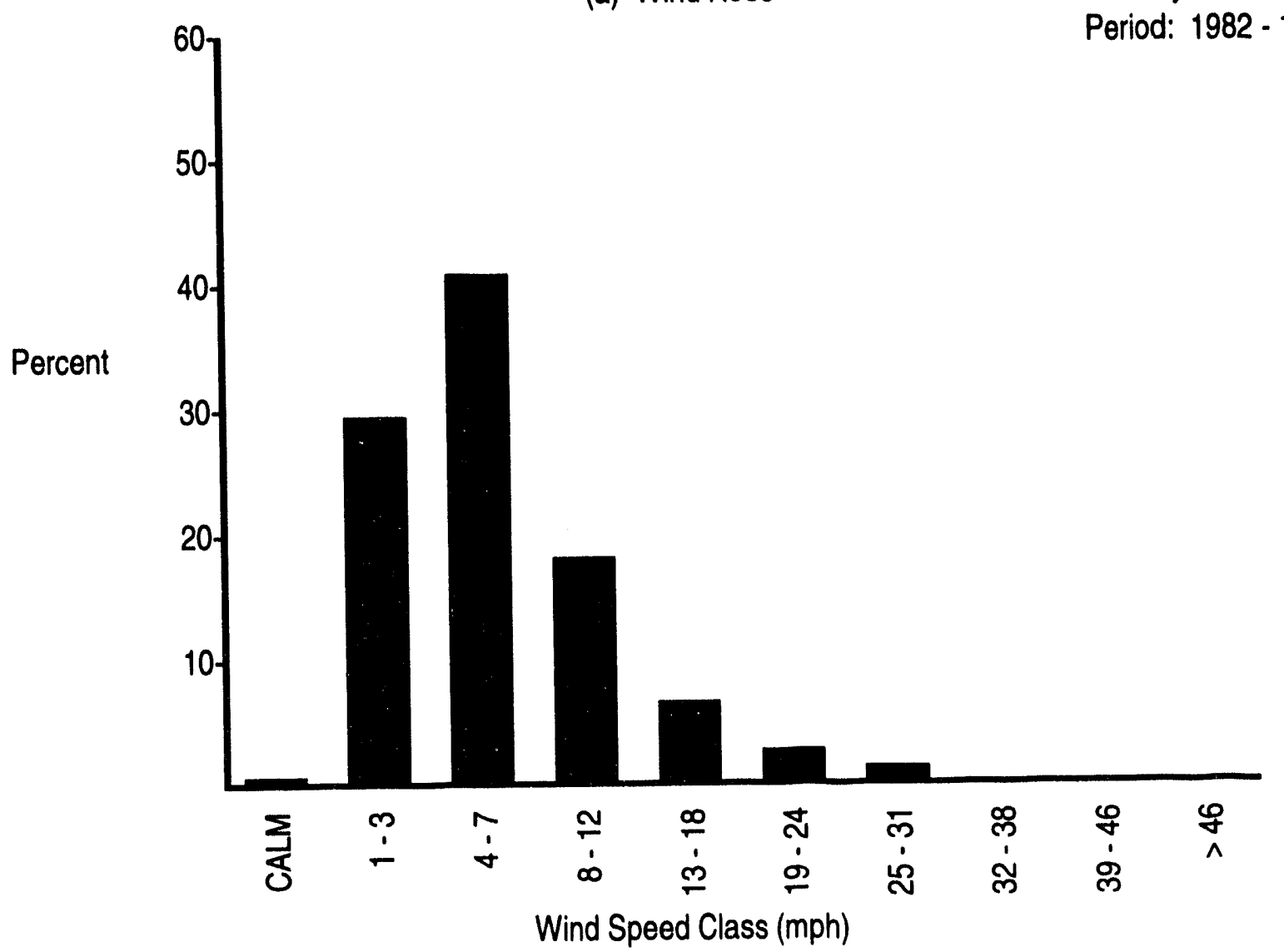

(b) Wind Speed Histogram

FIGURE B.1. (contd)

B.13 


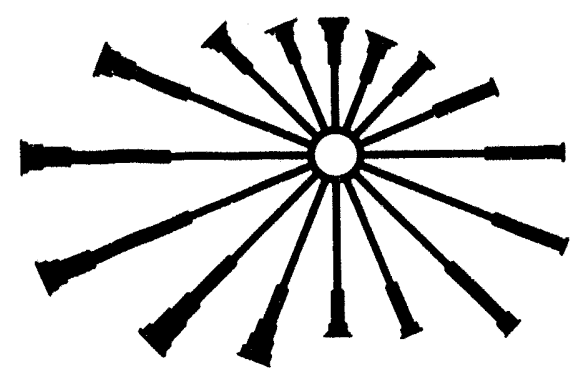

(a) Wind Rose

January Data

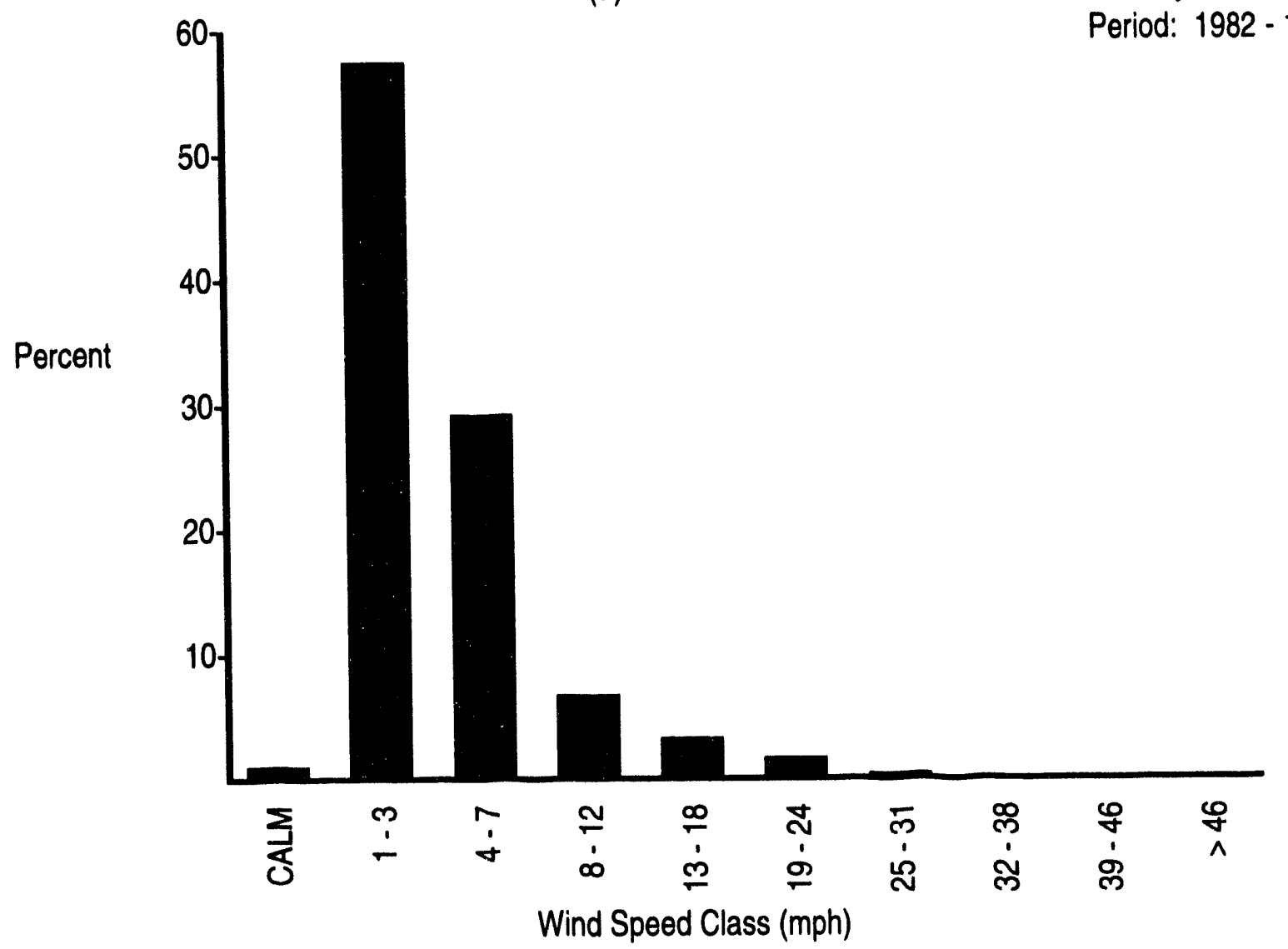

(b) Wind Speed Histogram

FIGURE B.1. (contd) 


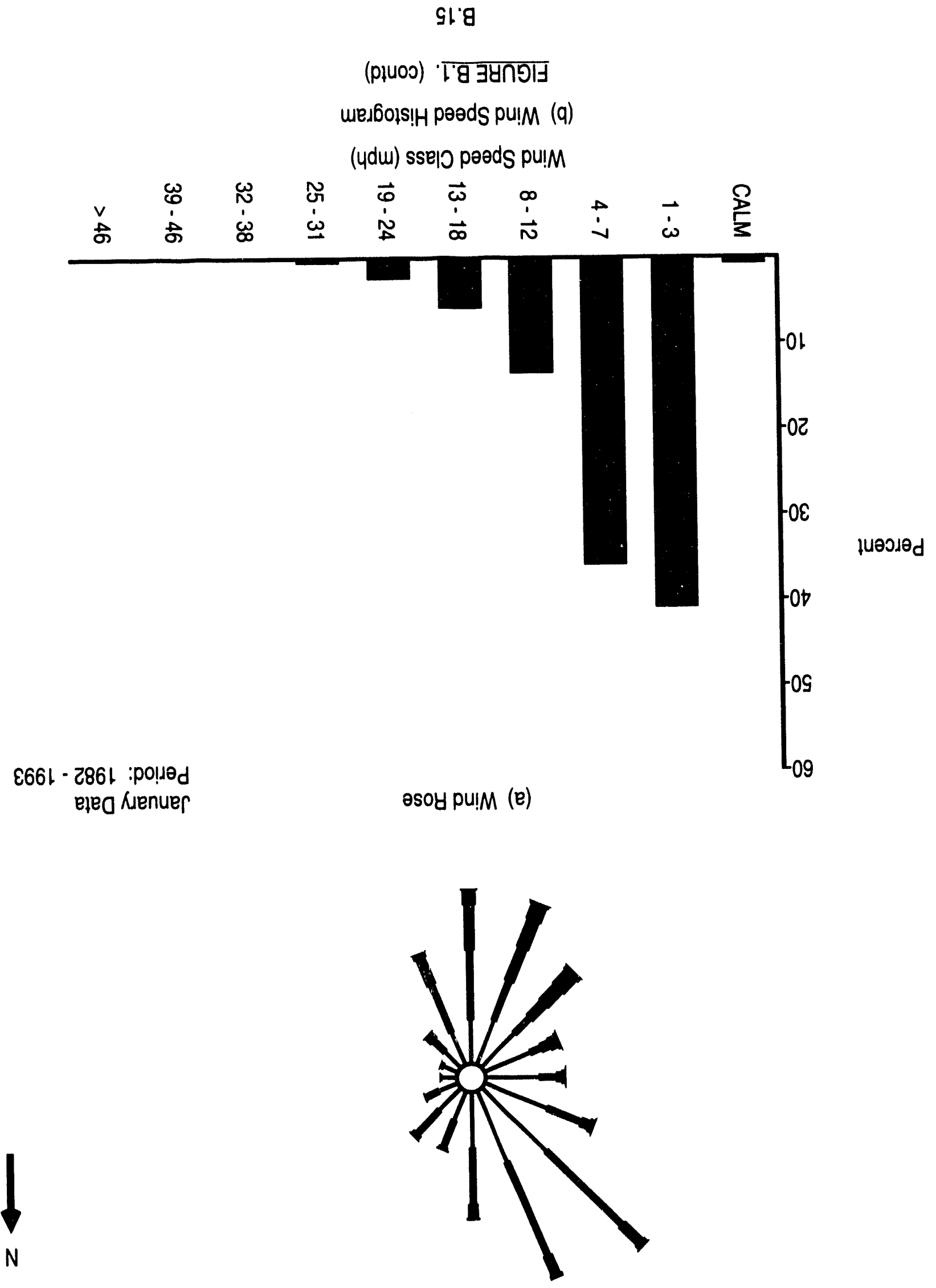




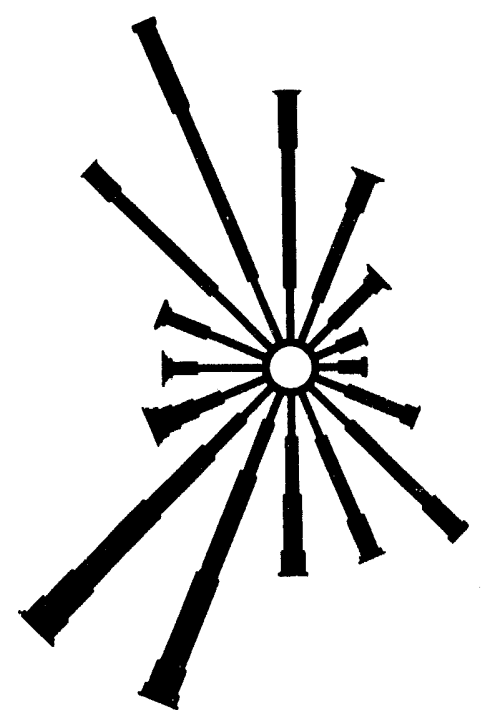

(a) Wind Rose

January Data

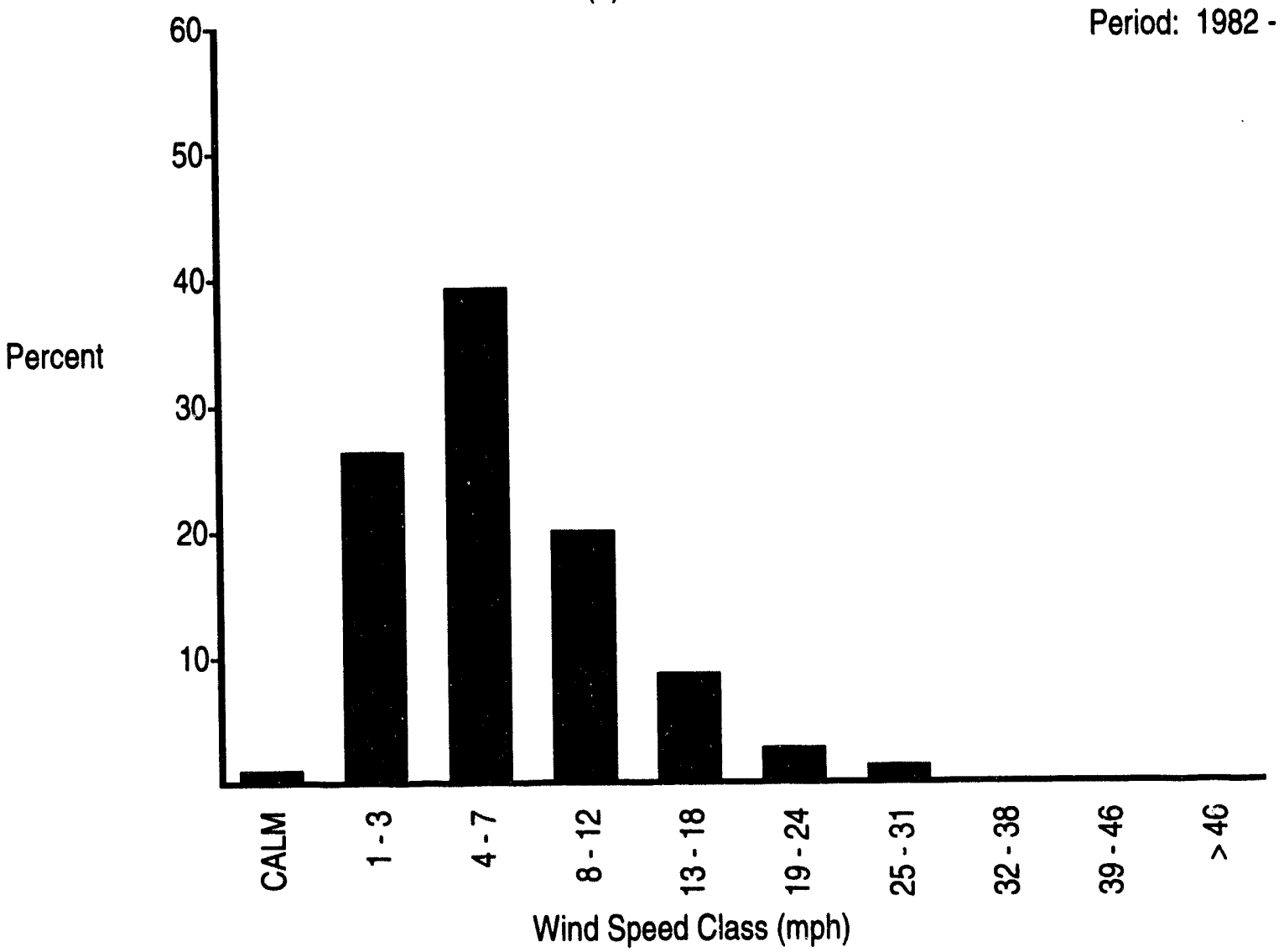

(b) Wind Speed Histogram

FIGURE B.1. (contd)

B. 16 


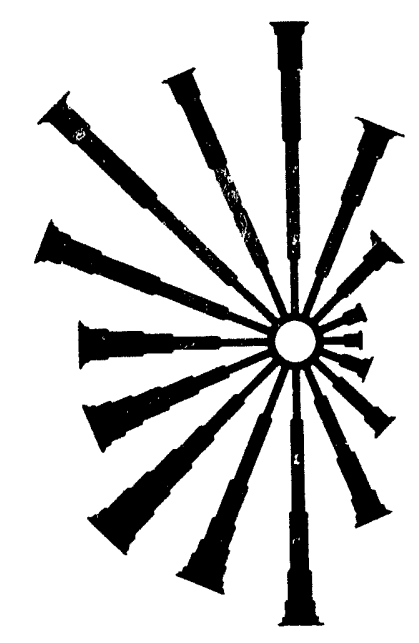

(a) Wind Rose

January Data

Period: 1982 - 1993

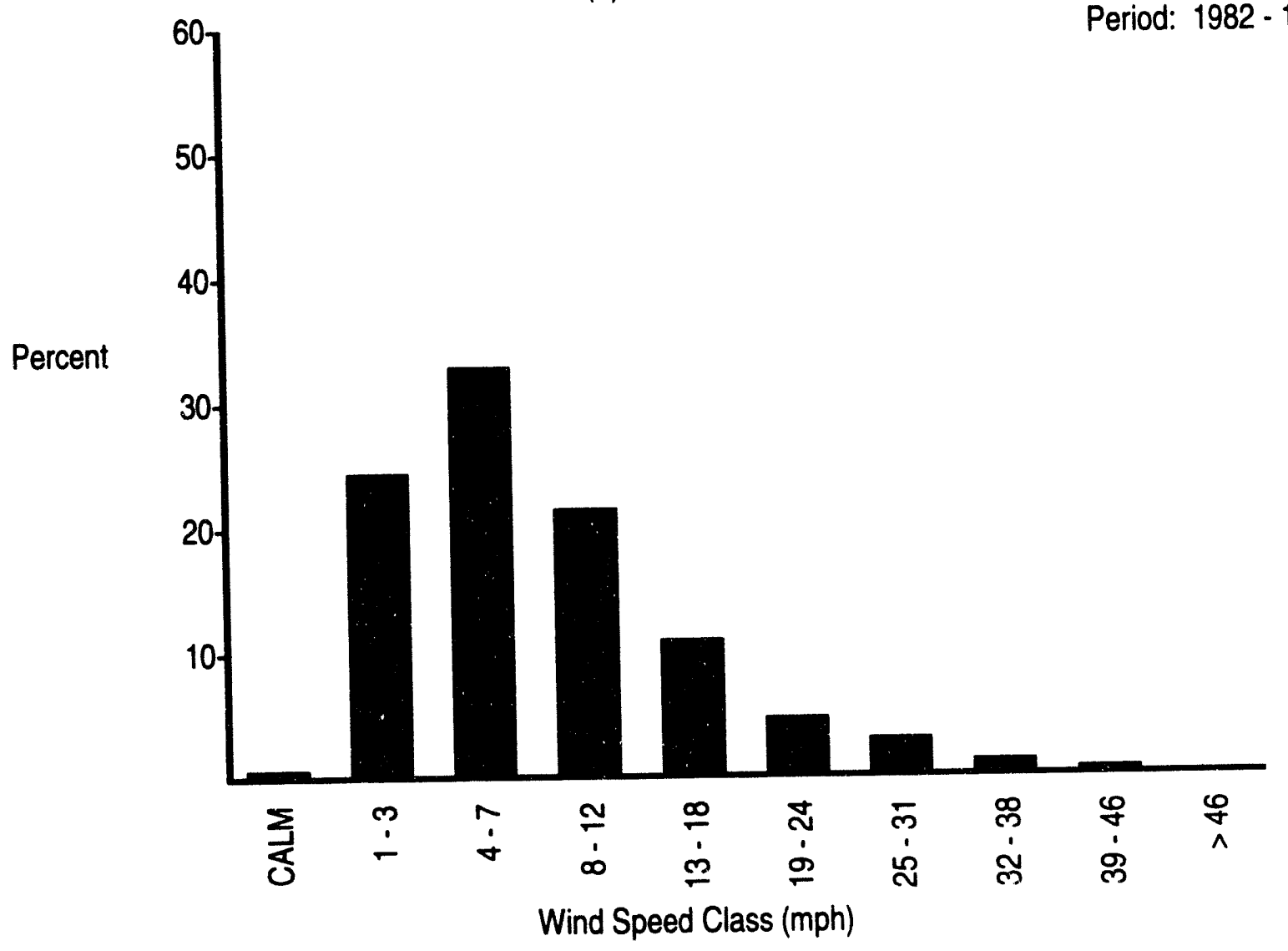

(b) Wind Speed Histogram

FIGURE B.1. (contd) 

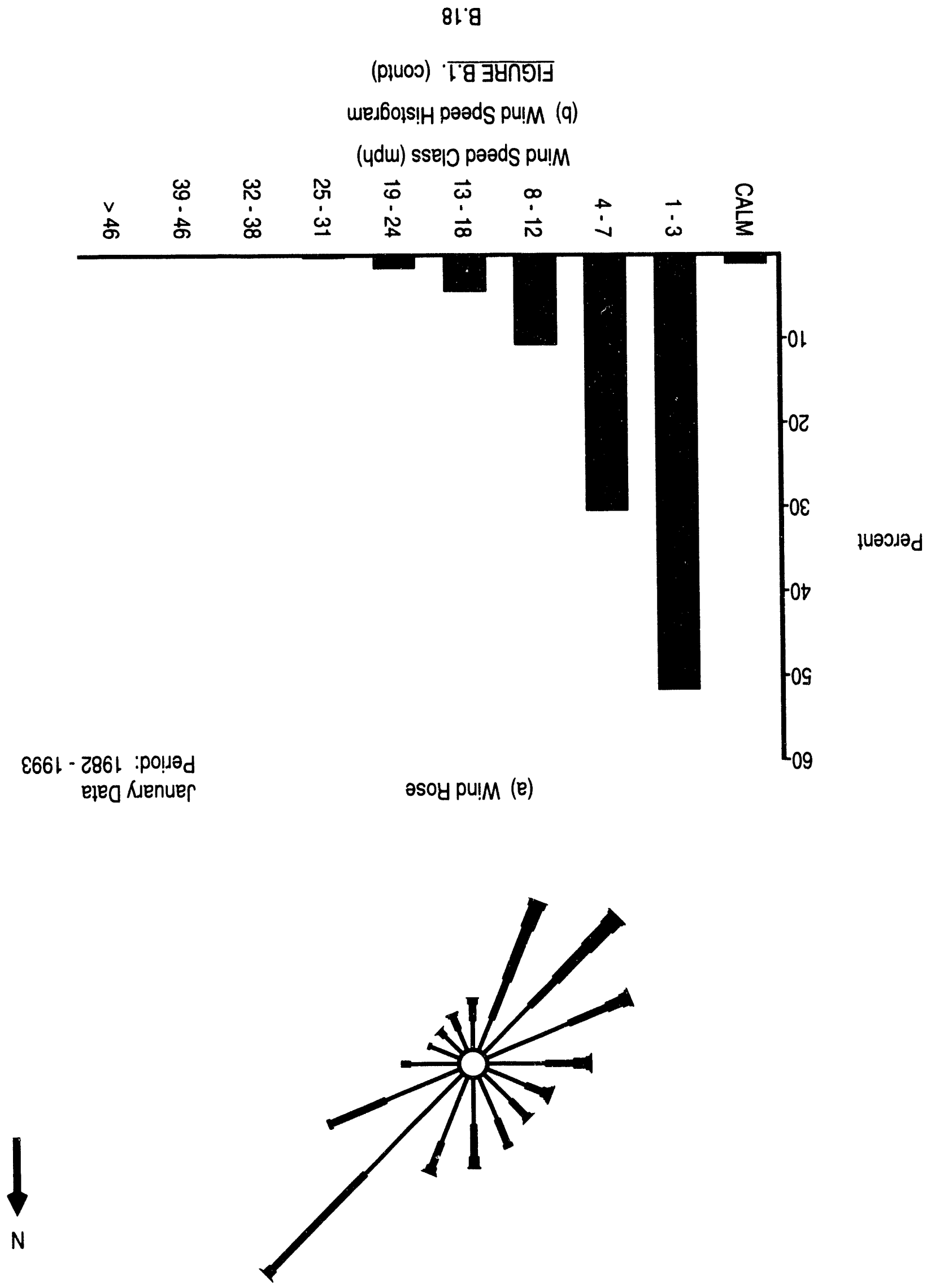


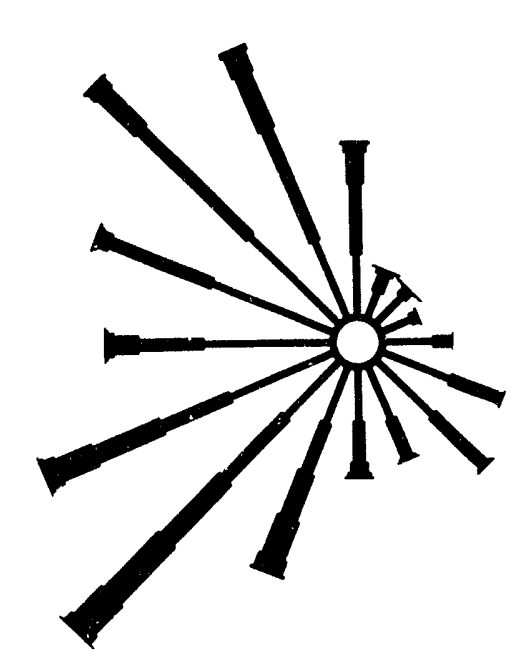

N

(a) Wind Rose

January Data

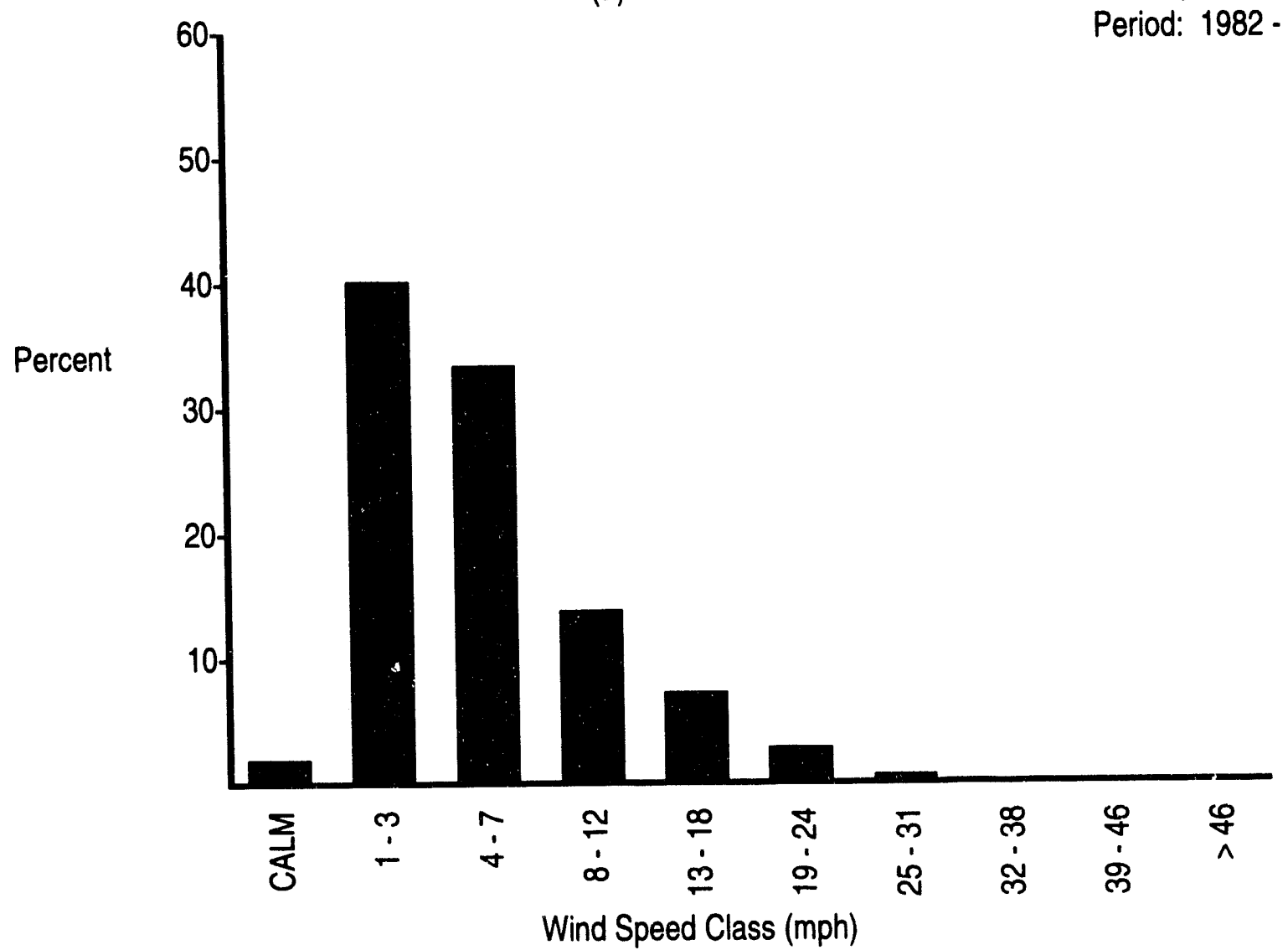

(b) Wind Speed Histogram

FIGURE B.1. (contd)

B. 19 


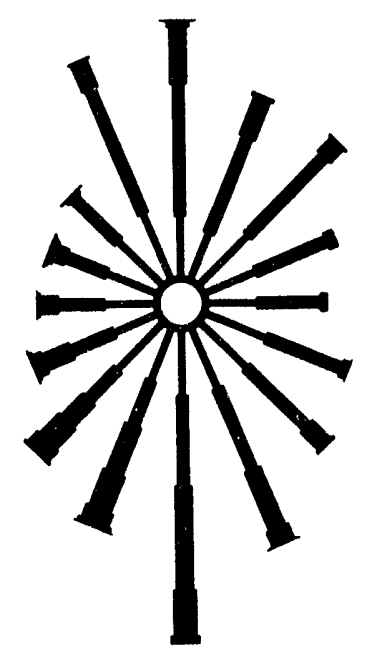

(a) Wind Rose

January Data

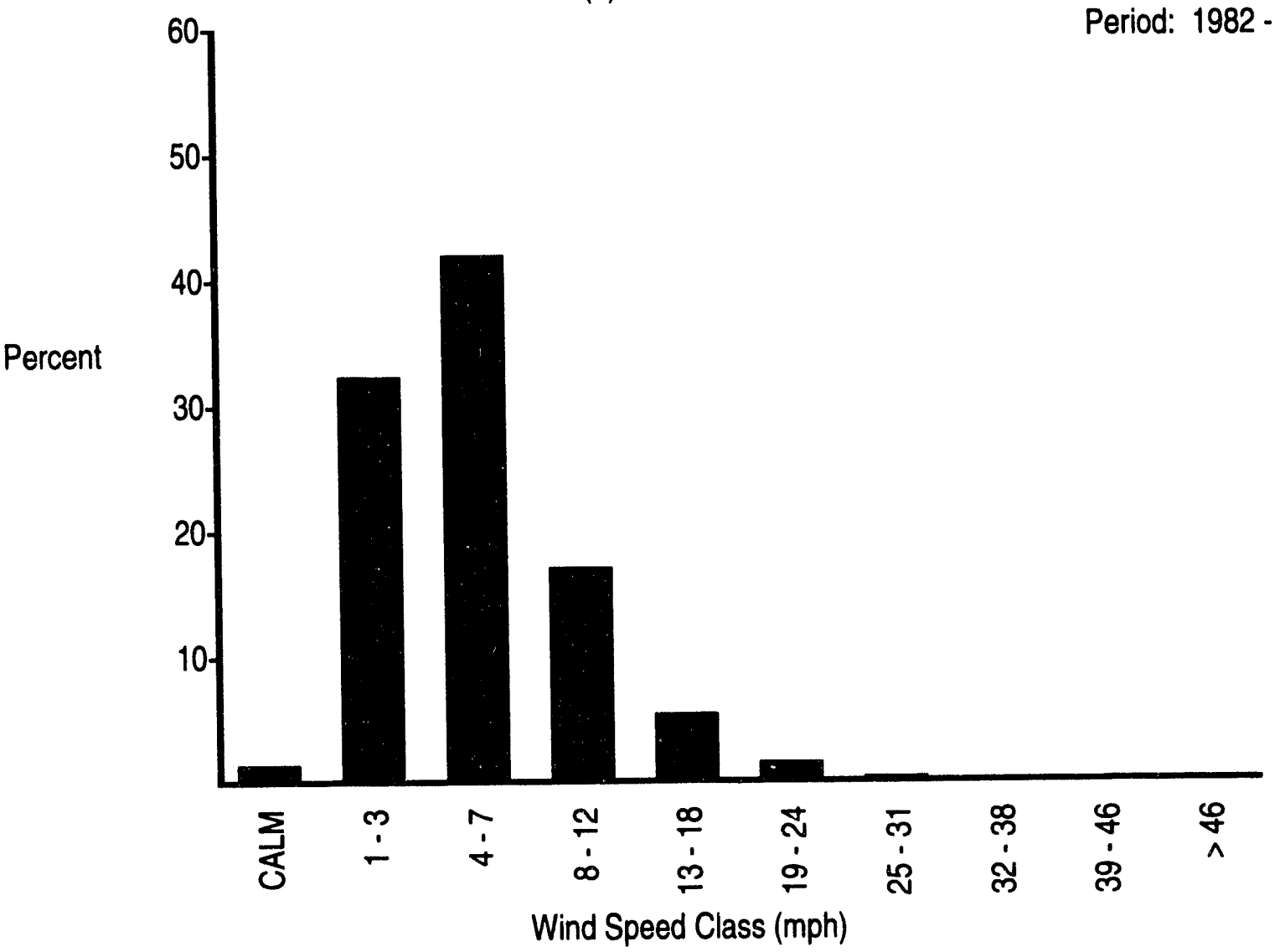

(b) Wind Speed Histogram

FIGURE B.1. (contd) 


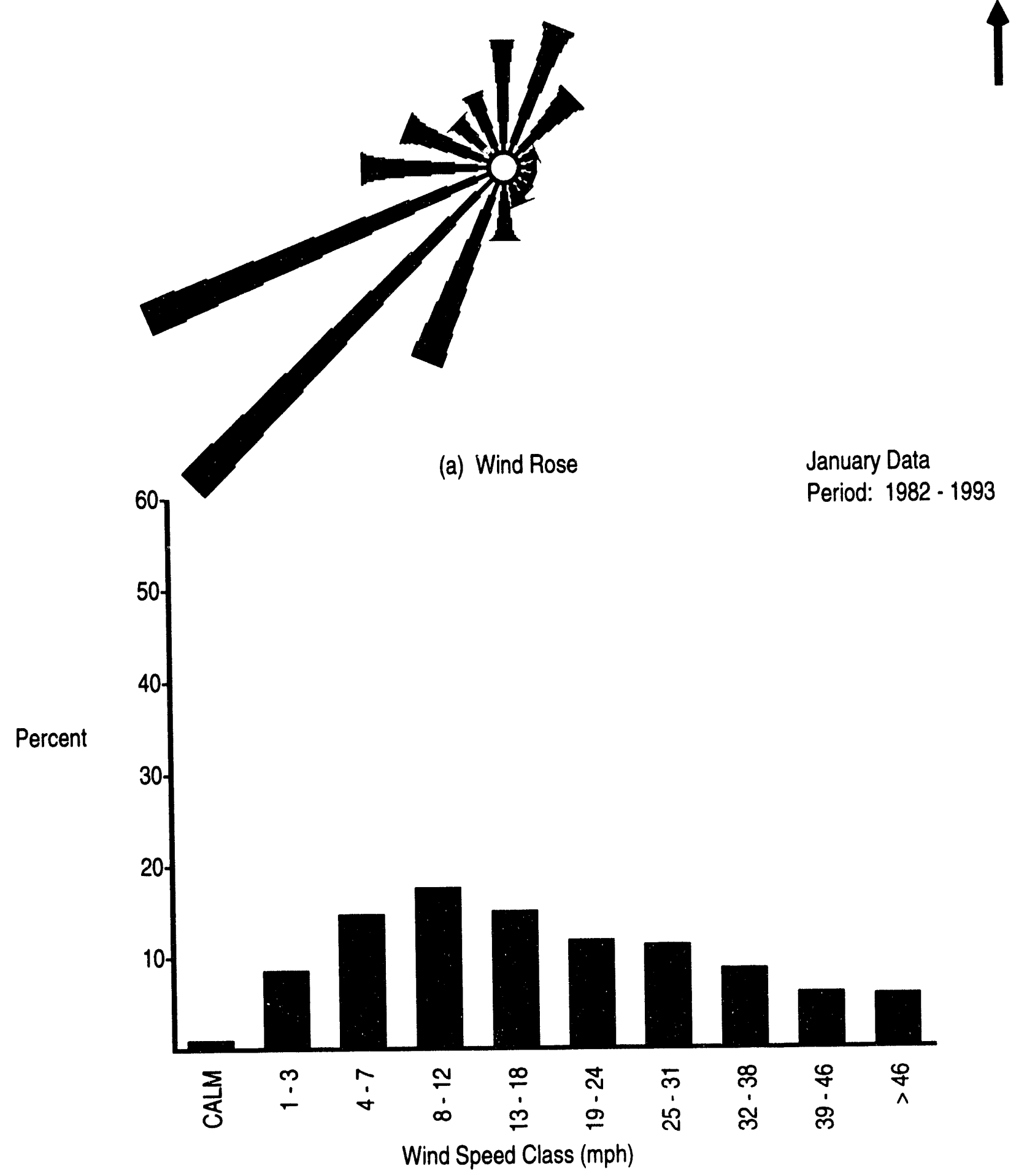

(b) Wind Speed Histogram

FIGURE B.1. (contd) 


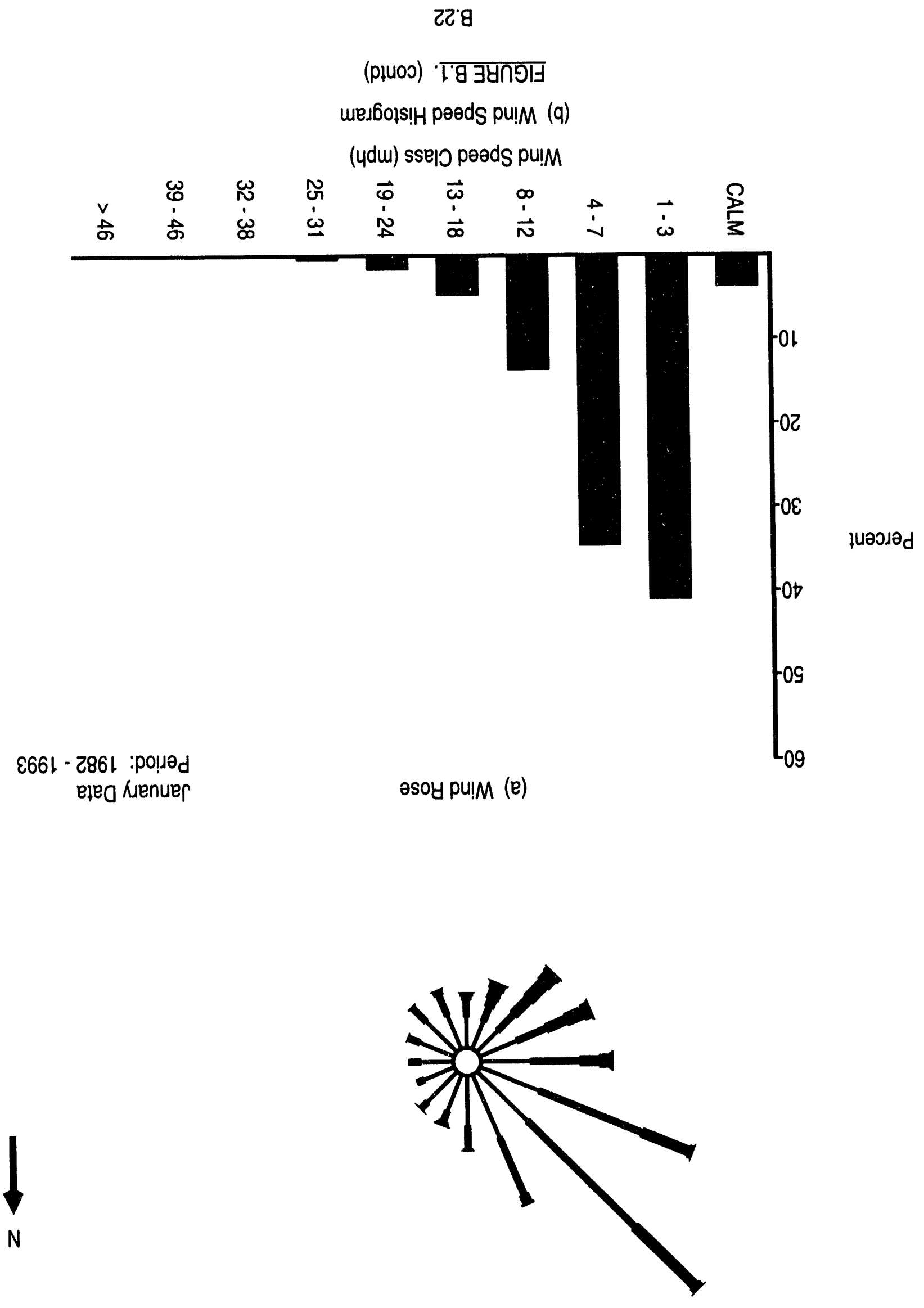



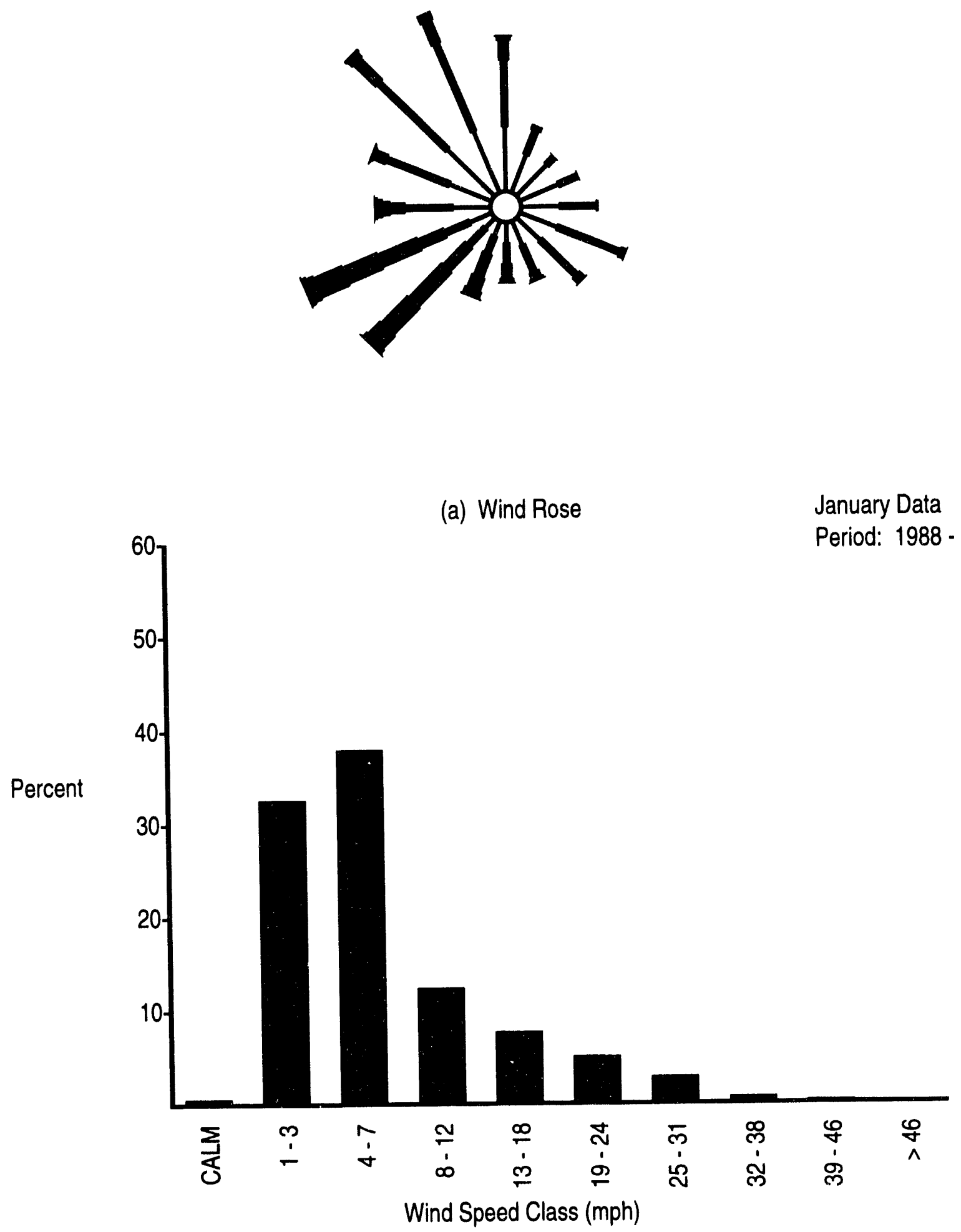

(b) Wind Speed Histogram

FIGURE B.1. (contd) 


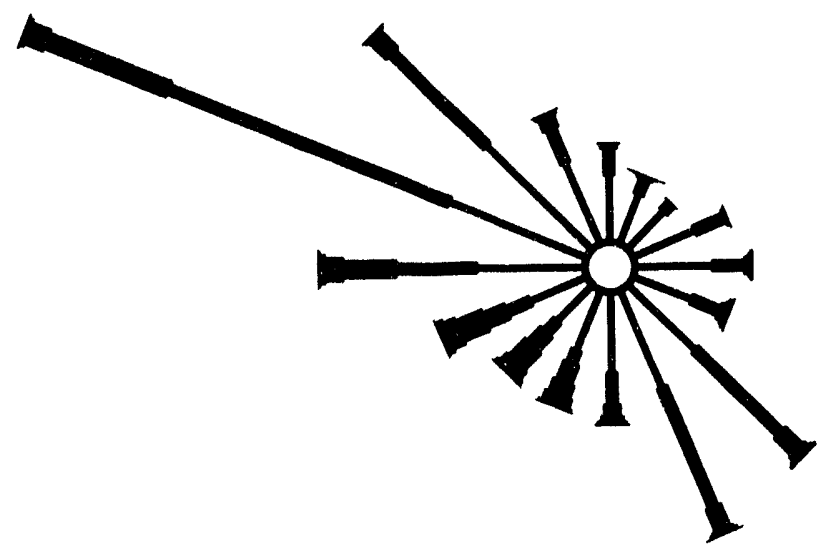

(a) Wind Rose

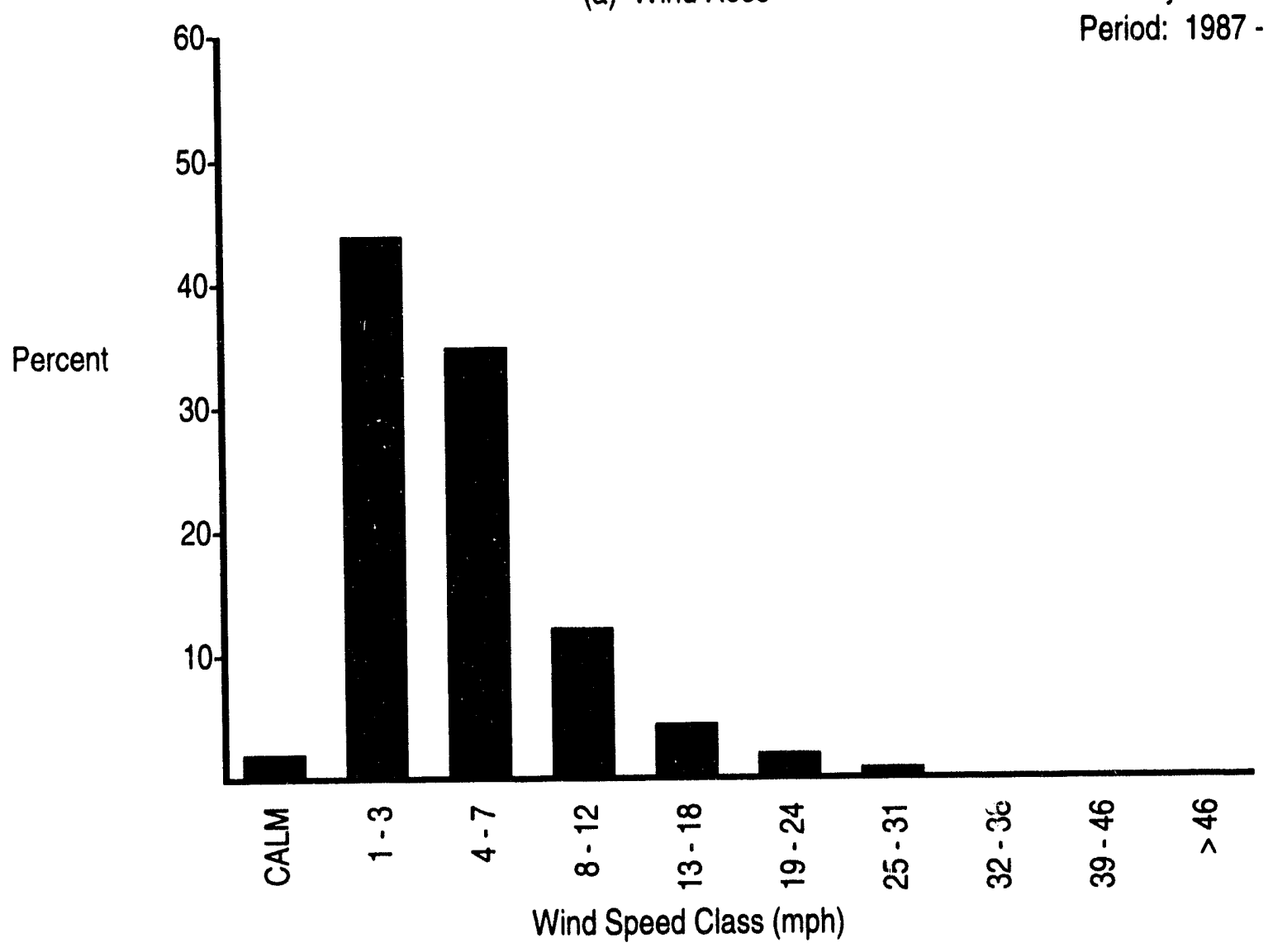

(b) Wind Speed Histogram

FIGURE B.1. (contd)
January Data

Period: 1987 - 1993 


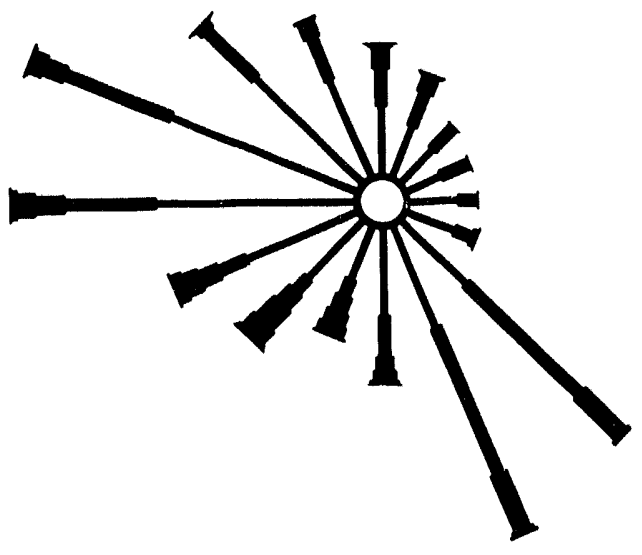

(a) Wind Rose

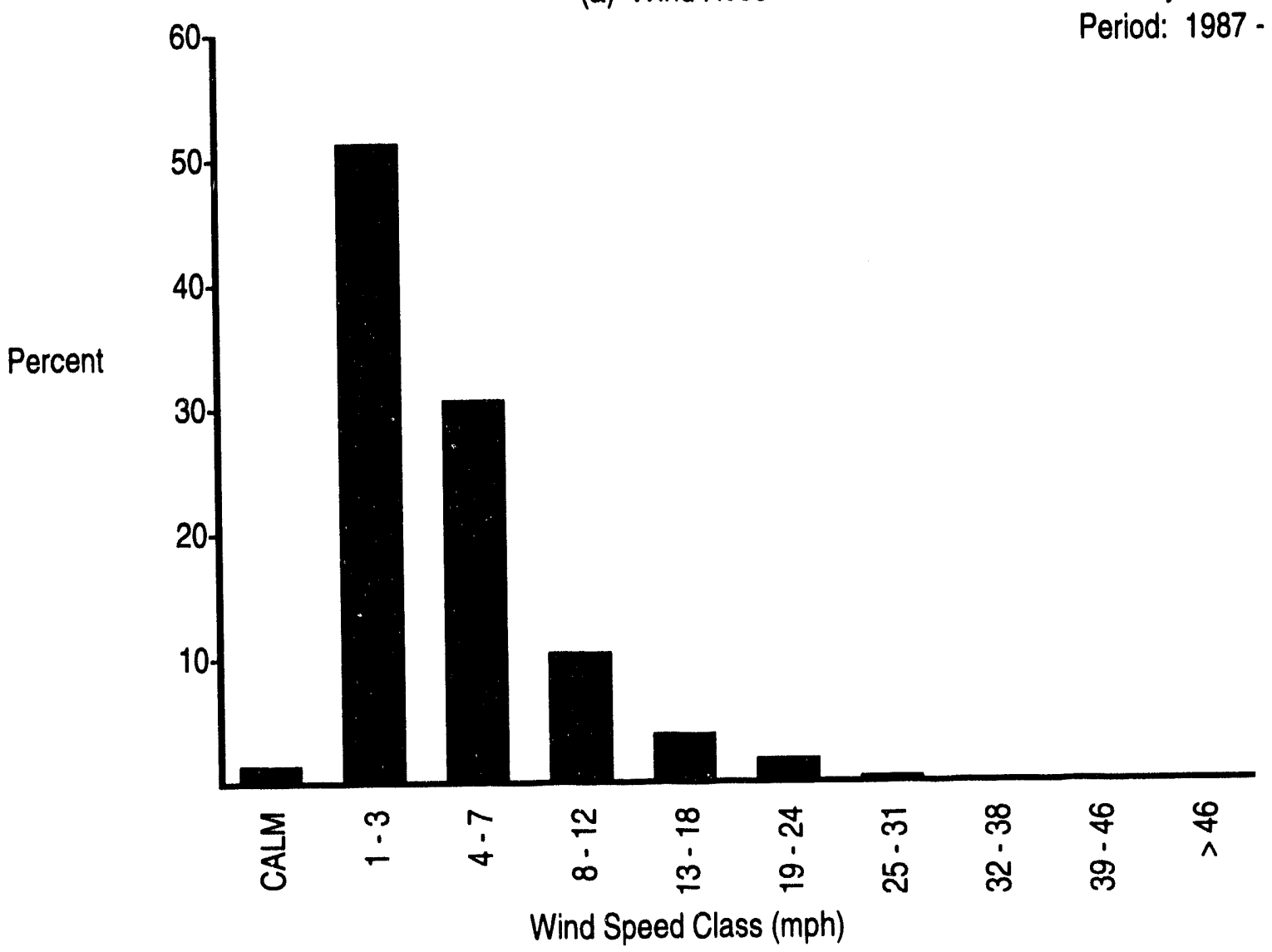

(b) Wind Speed Histogram

FIGURE B.1. (contd) 


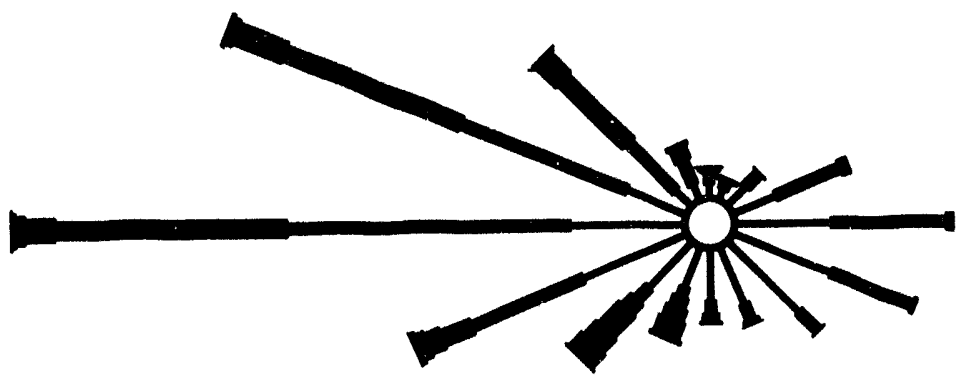

N

(a) Wind Rose

January Data

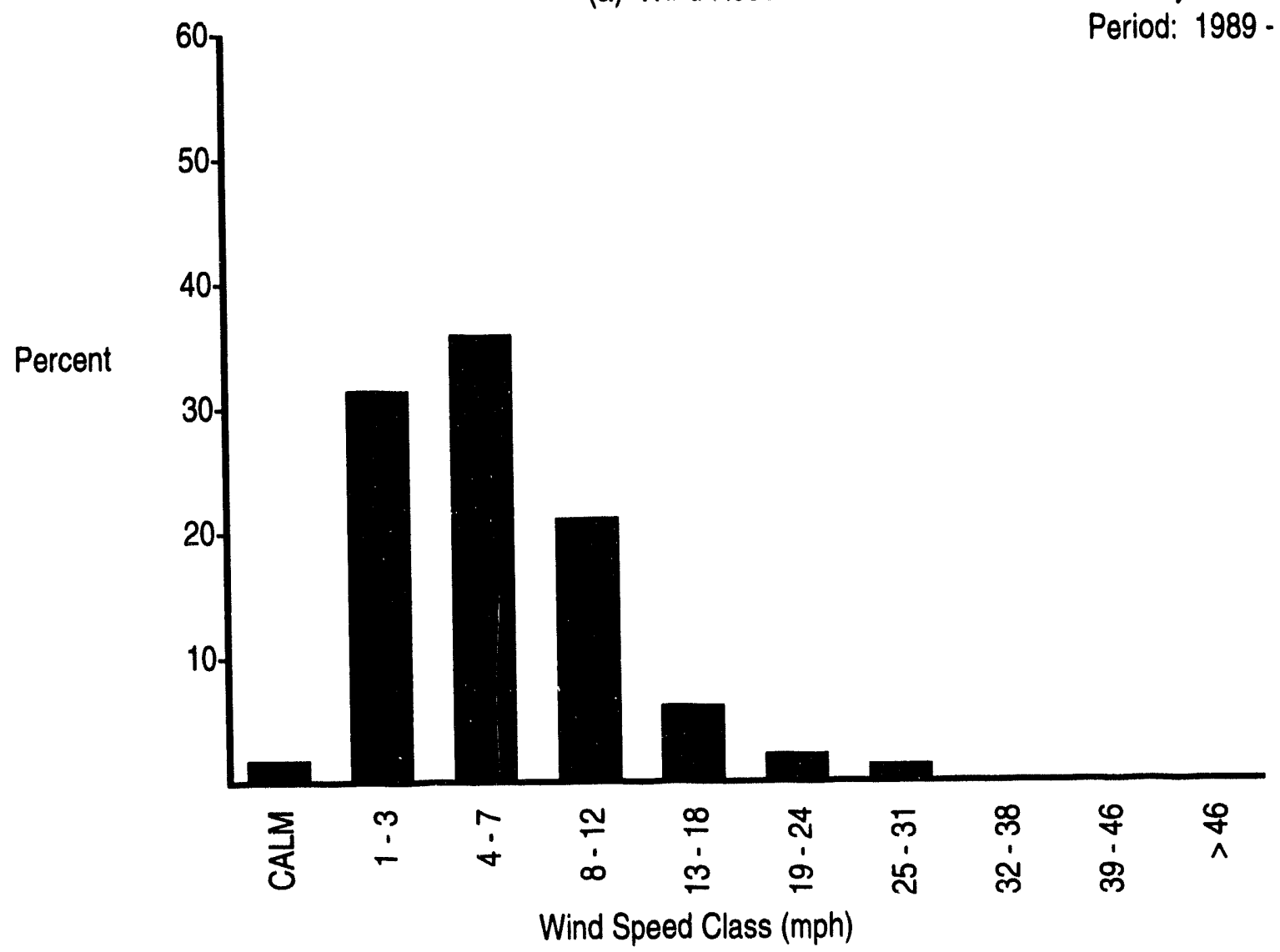

(b) Wind Speed Histogram

FIGURE B.1. (contd) 

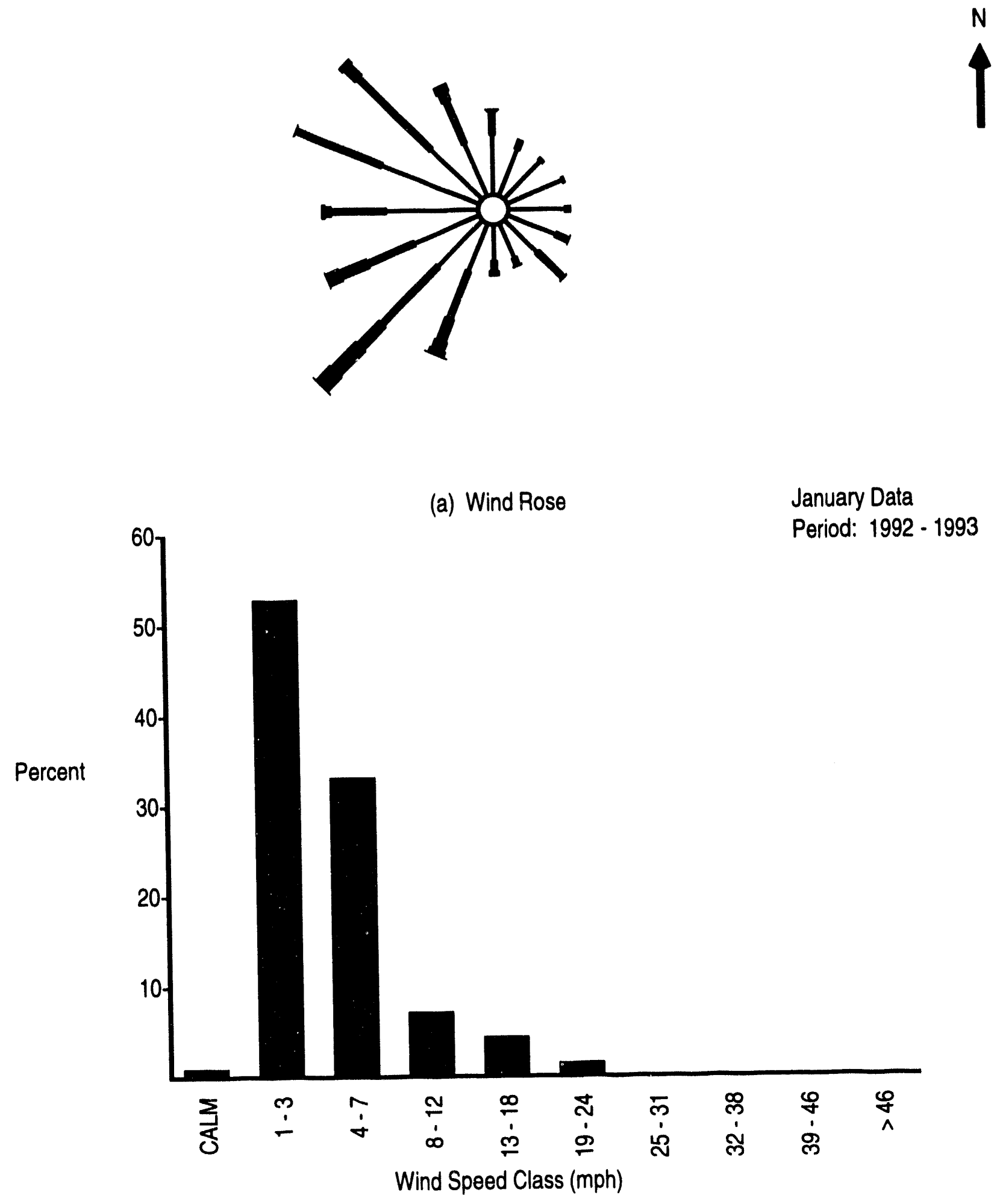

(b) Wind Speed Histogram

FIGURE B.1. (contd) 


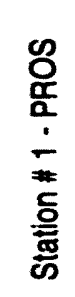

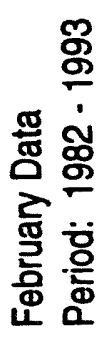

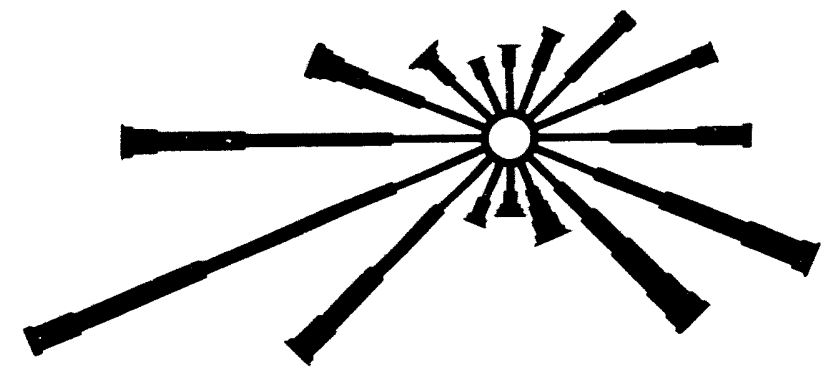

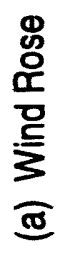

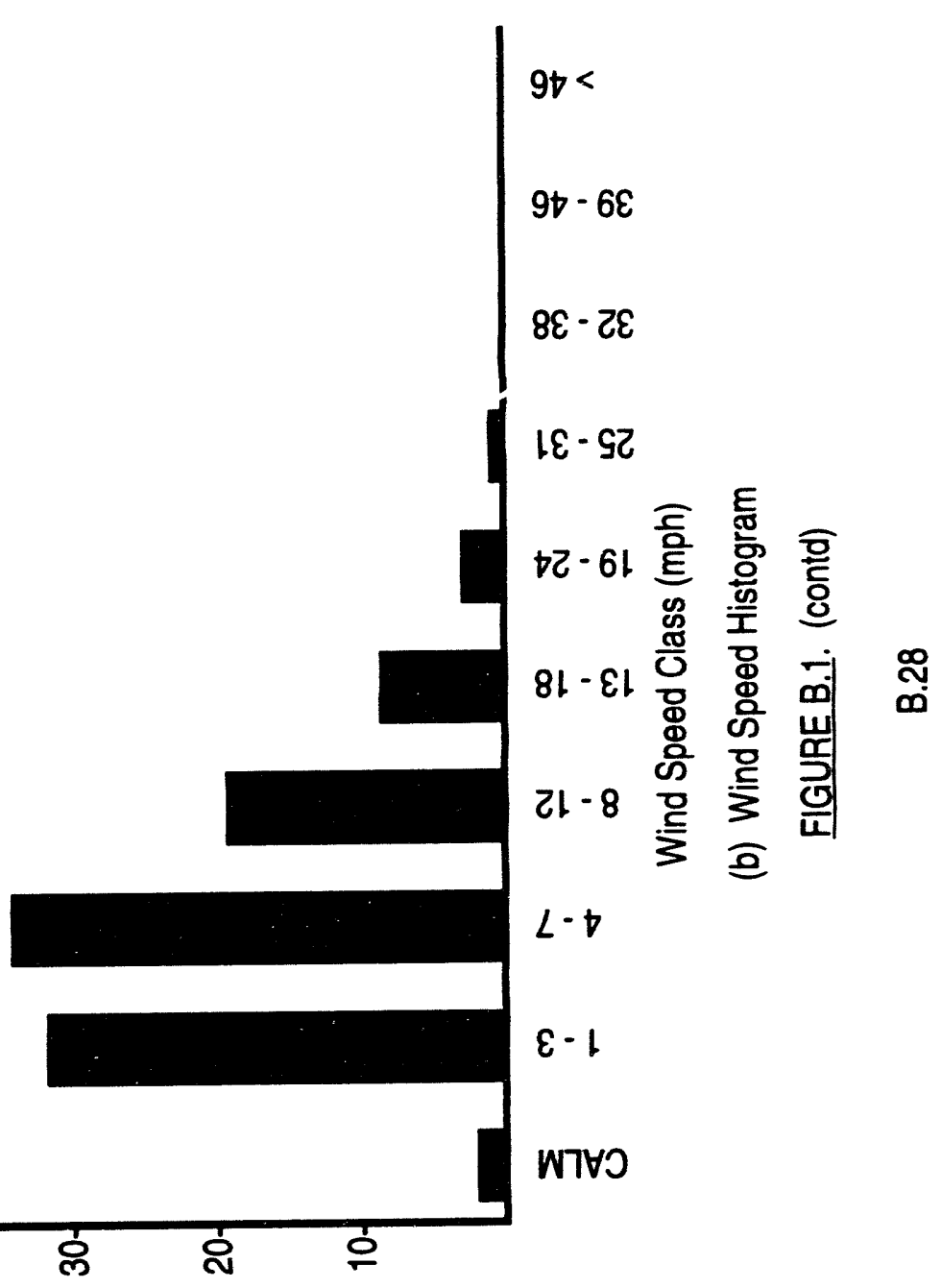

홍 

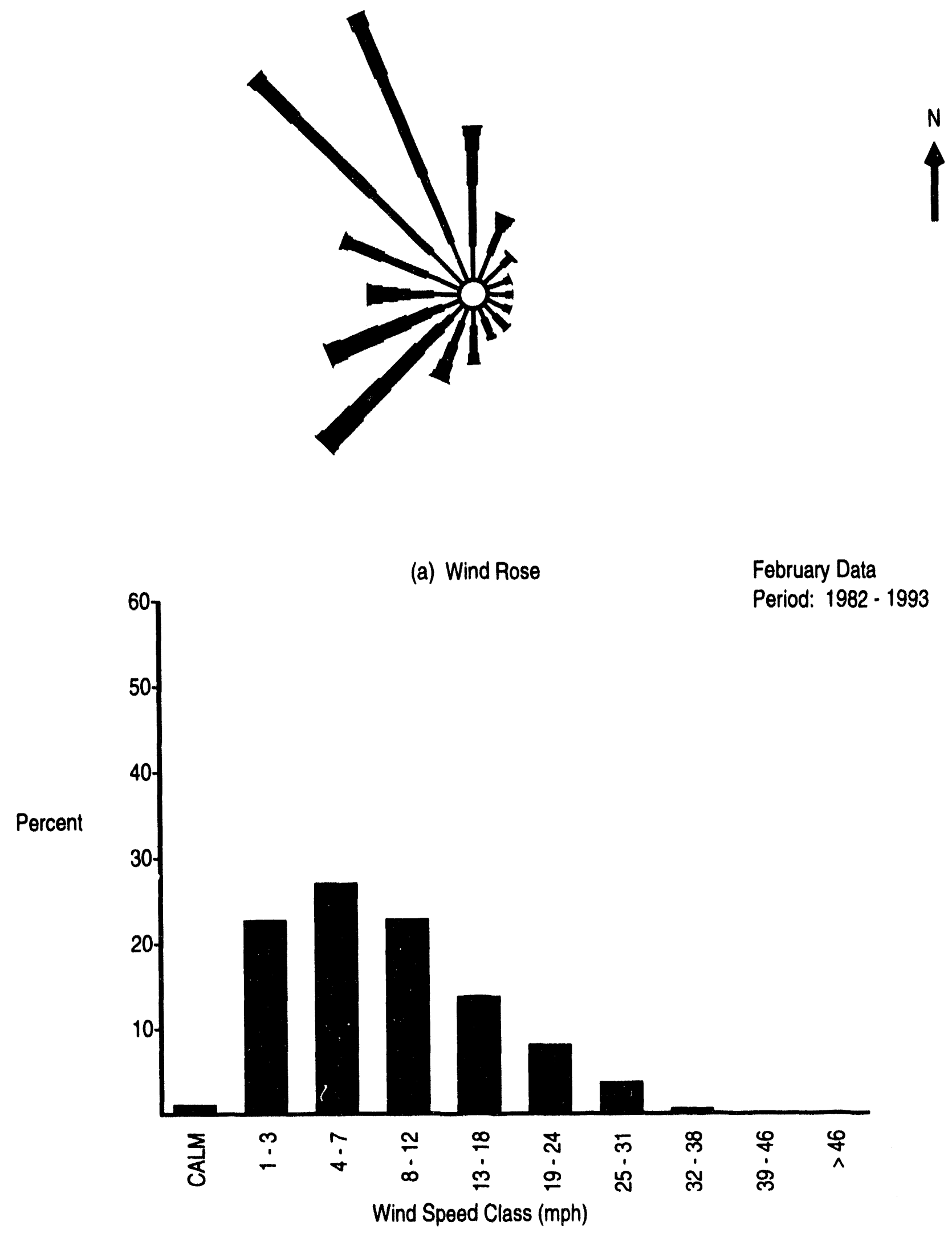

(b) Wind Speed Histogram

FIGURE B.1. (contd) 


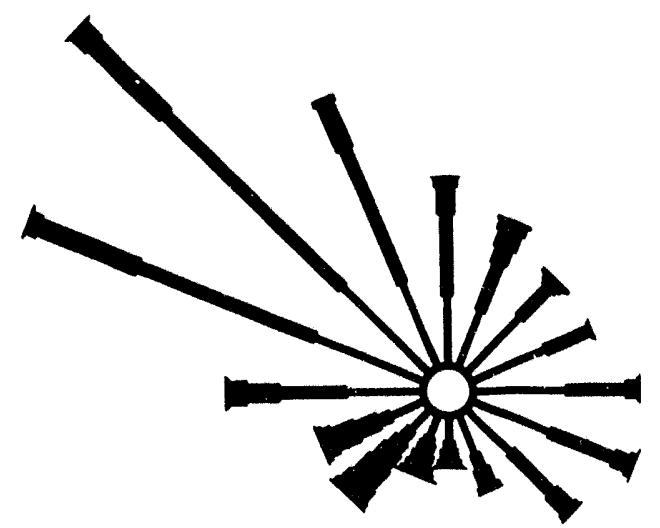

(a) Wind Rose

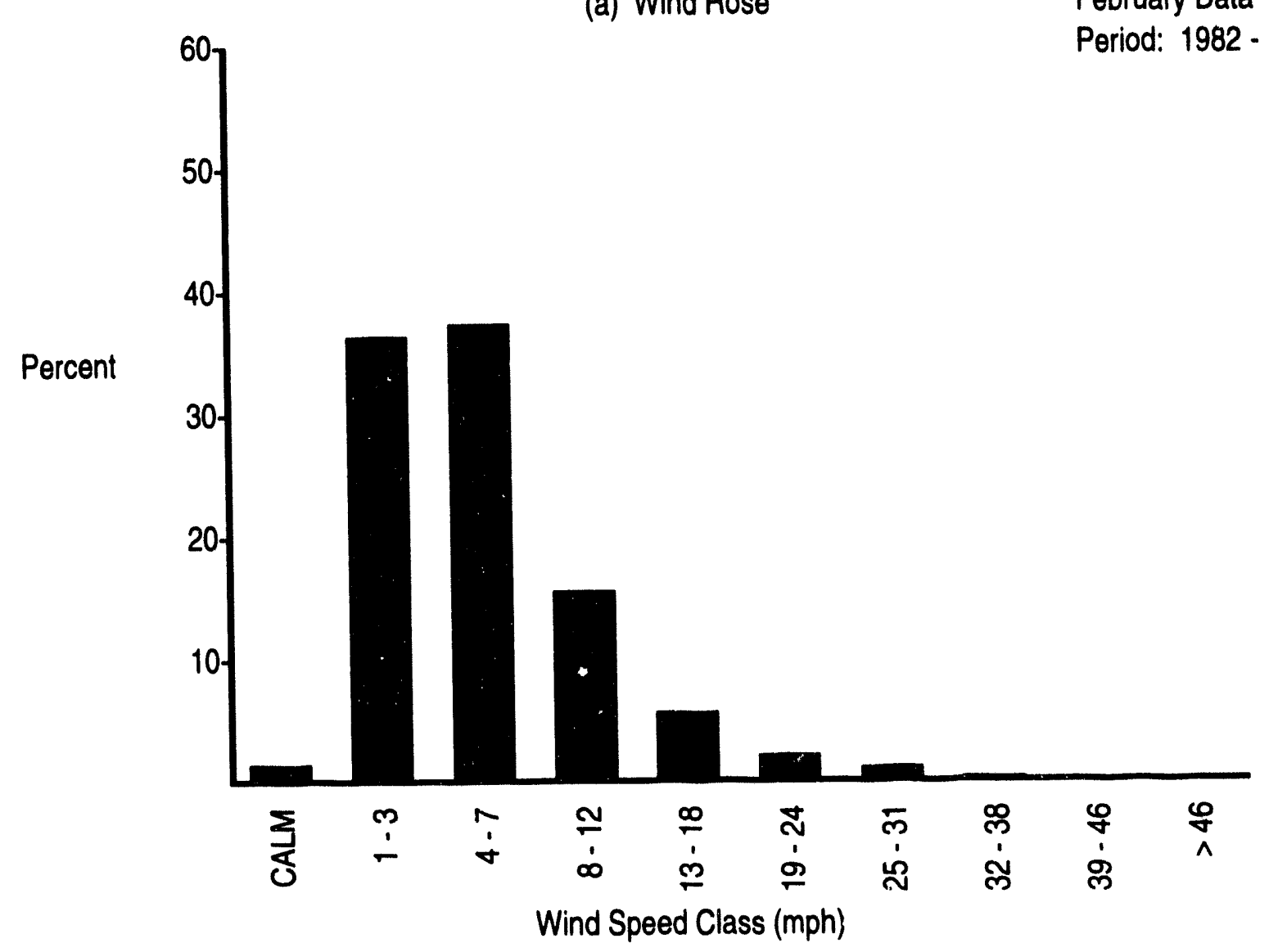

(b) Wind Speed Histogram

FIGURE B.1. (contd)
February Data

Period: 1982 - 1993

\section{B.30}




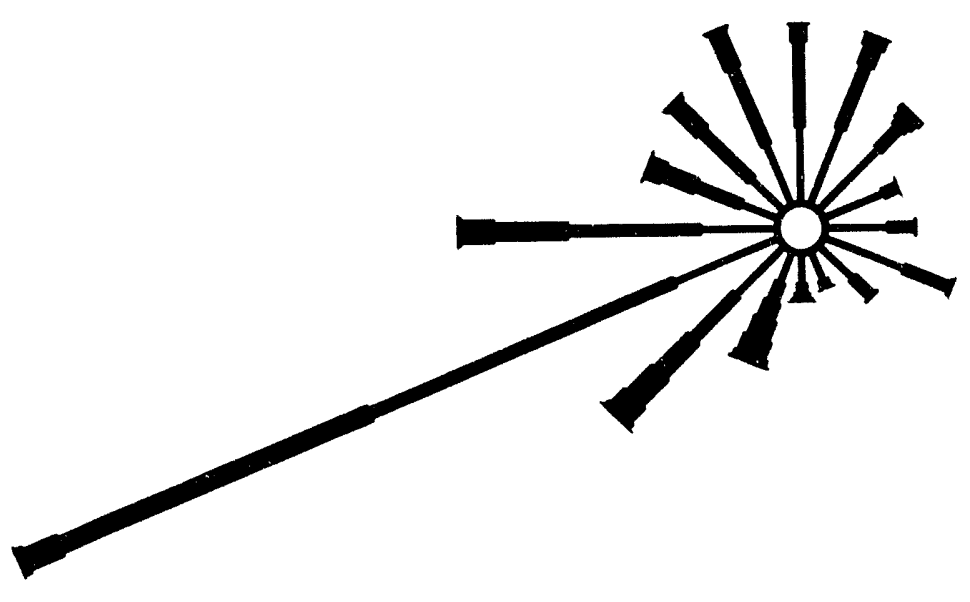

(a) Wind Rose

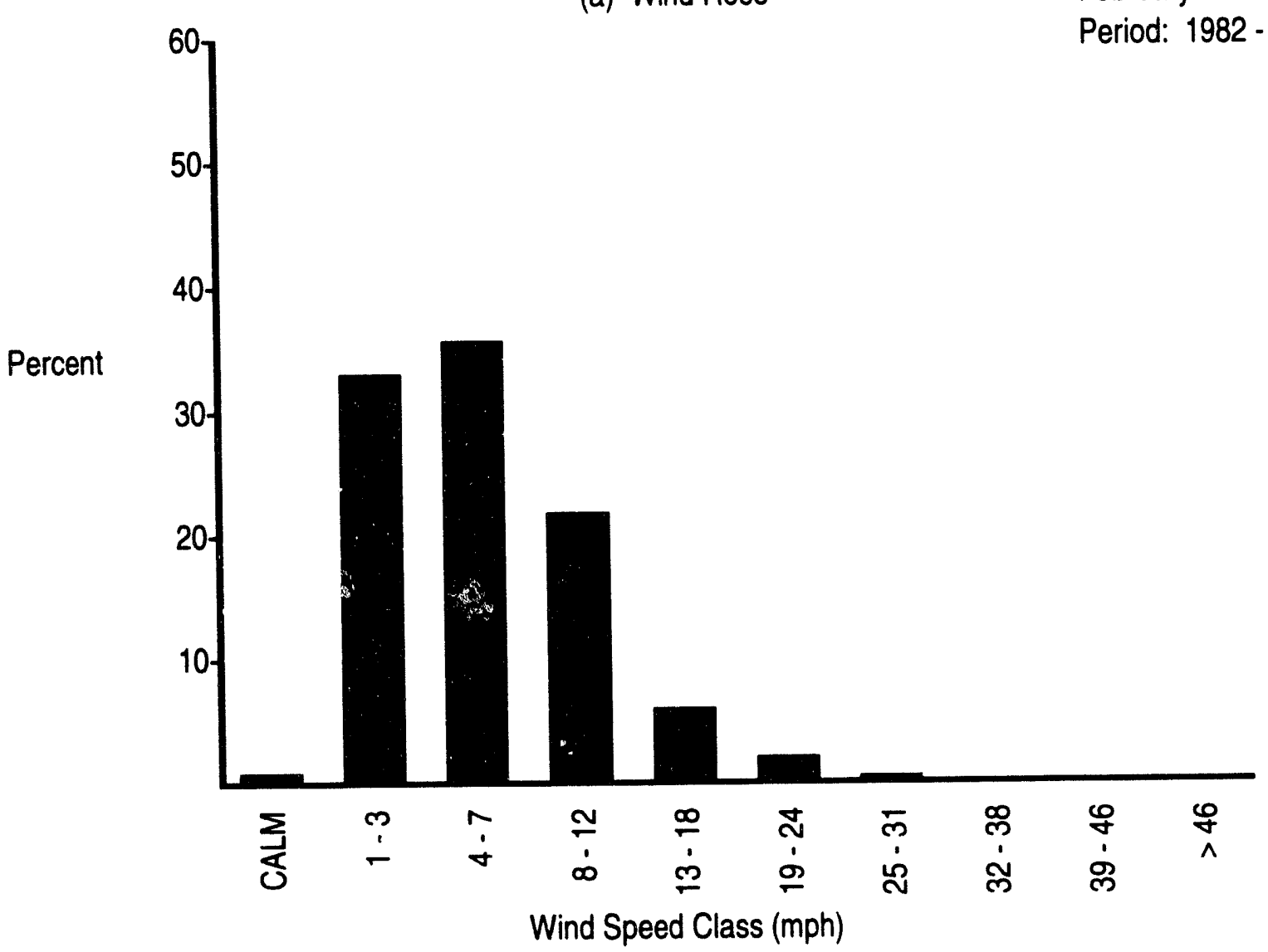

(b) Wind Speed Histogram

FIGURE B.1. (contd)
February Data

Period: 1982 - 1993 


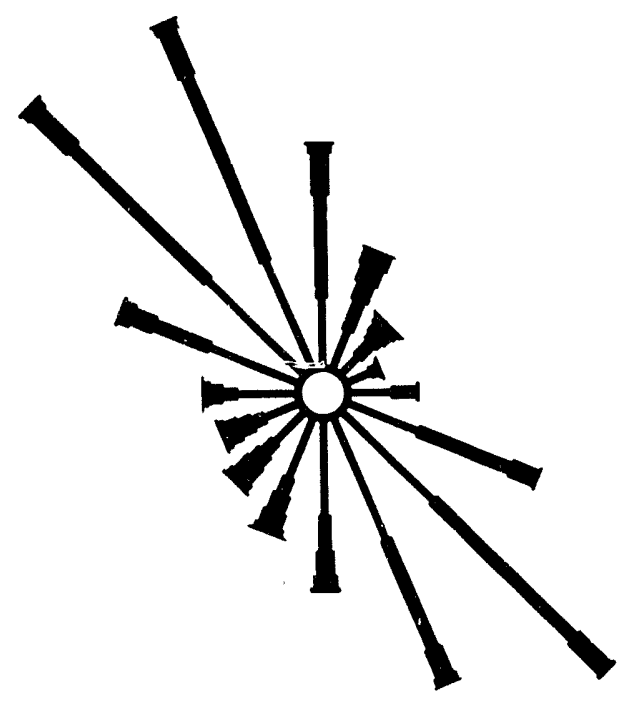

(a) Wind Rose

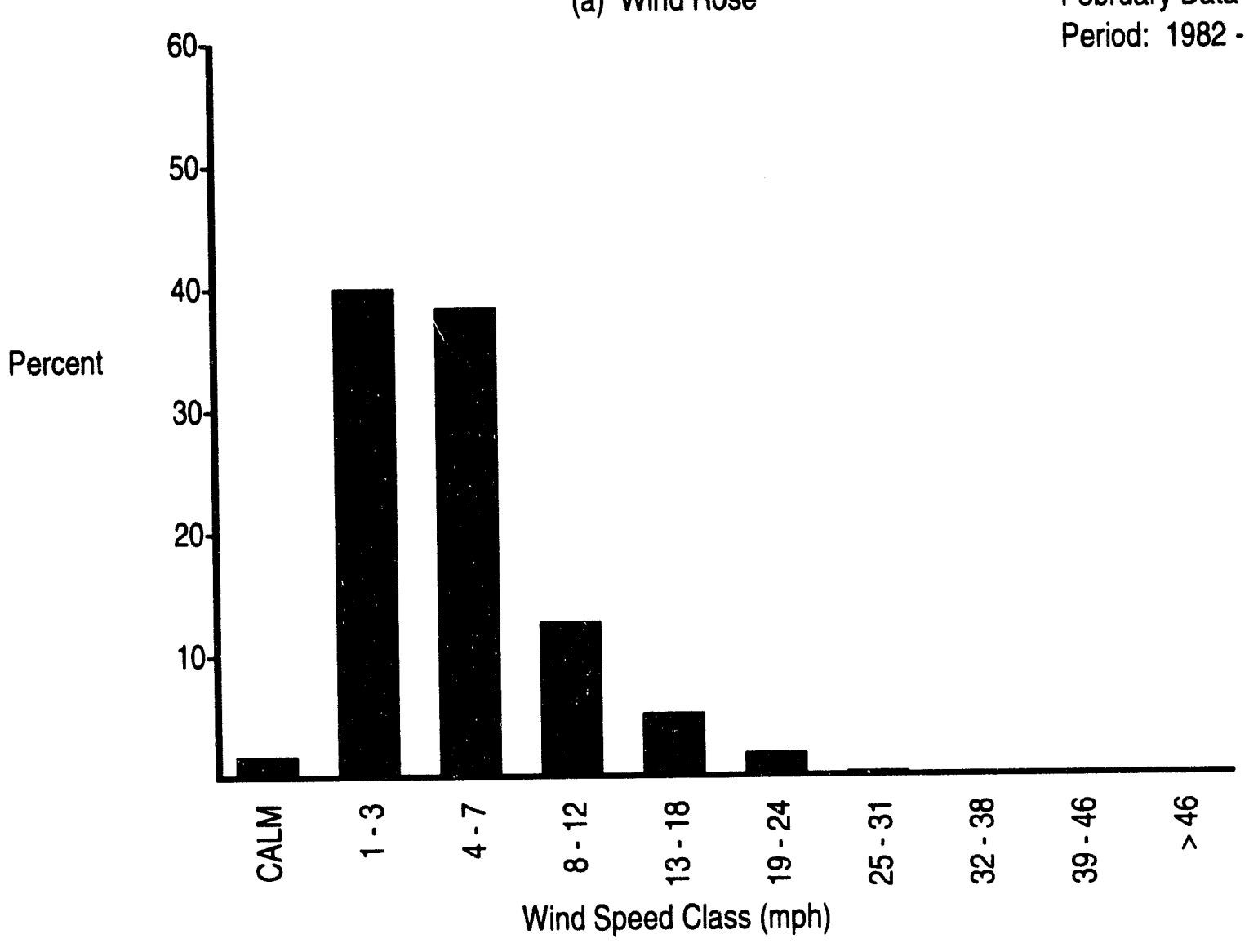

(b) Wind Speed Histogram

FIGURE B.1. (contd)
February Data

Period: 1982 - 1993

\section{B.32}




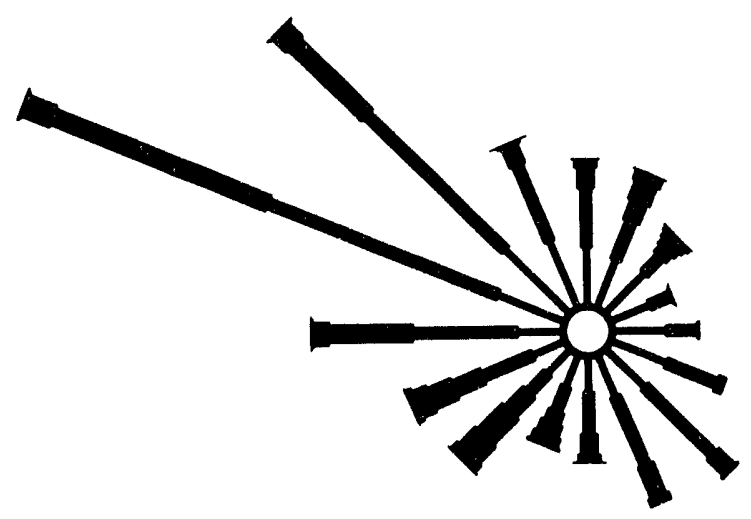

(a) Wind Rose

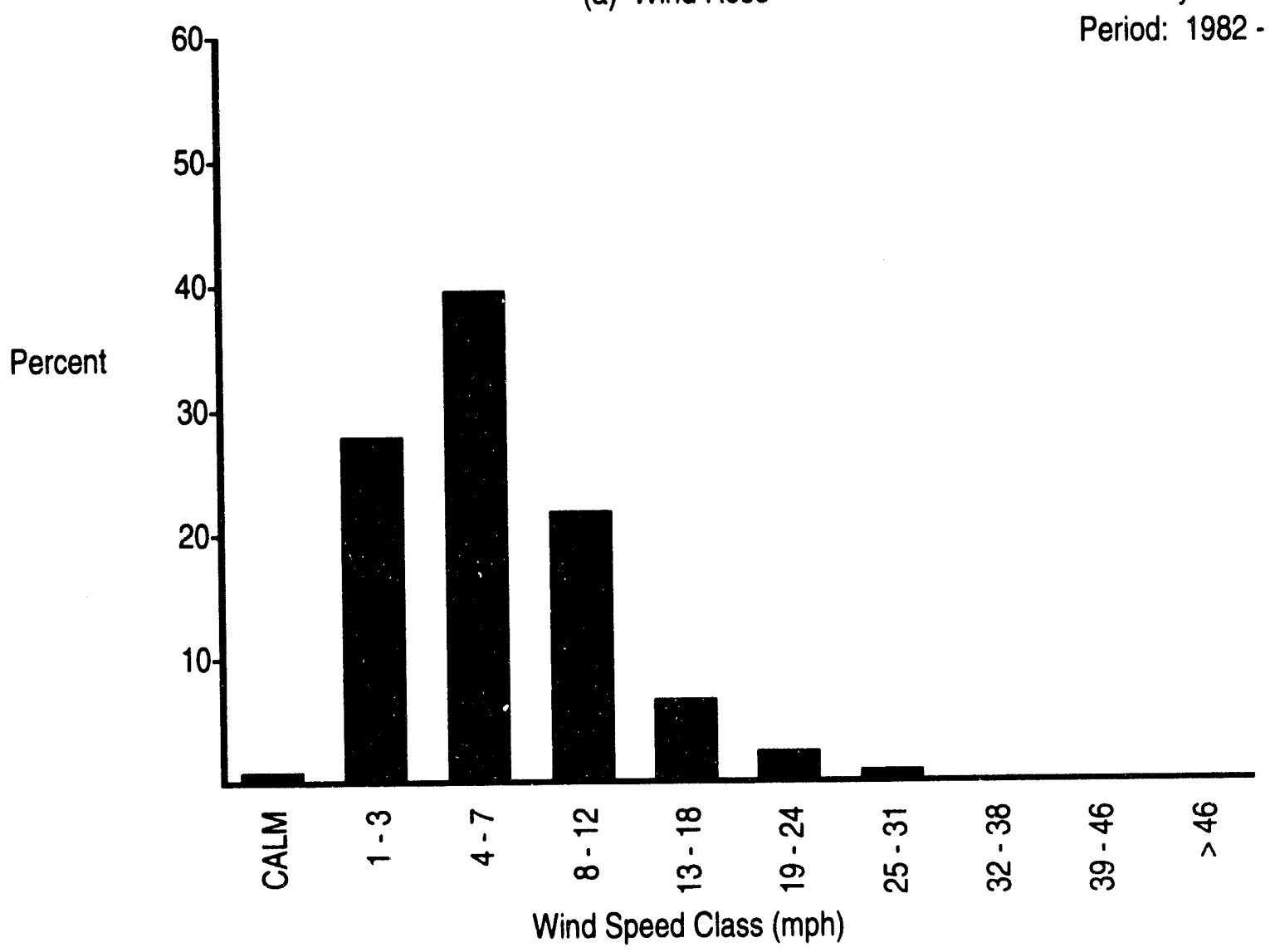

(b) Wind Speed Histogram
February Data

Period: 1982 - 1993

\section{FIGURE B.1. (contd)}




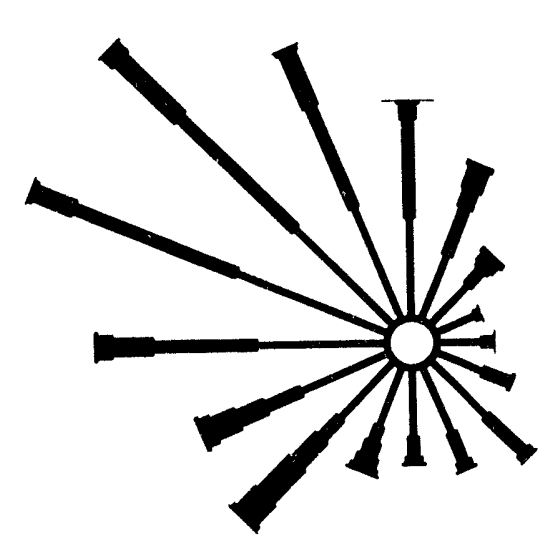

(a) Wind Rose

February Data

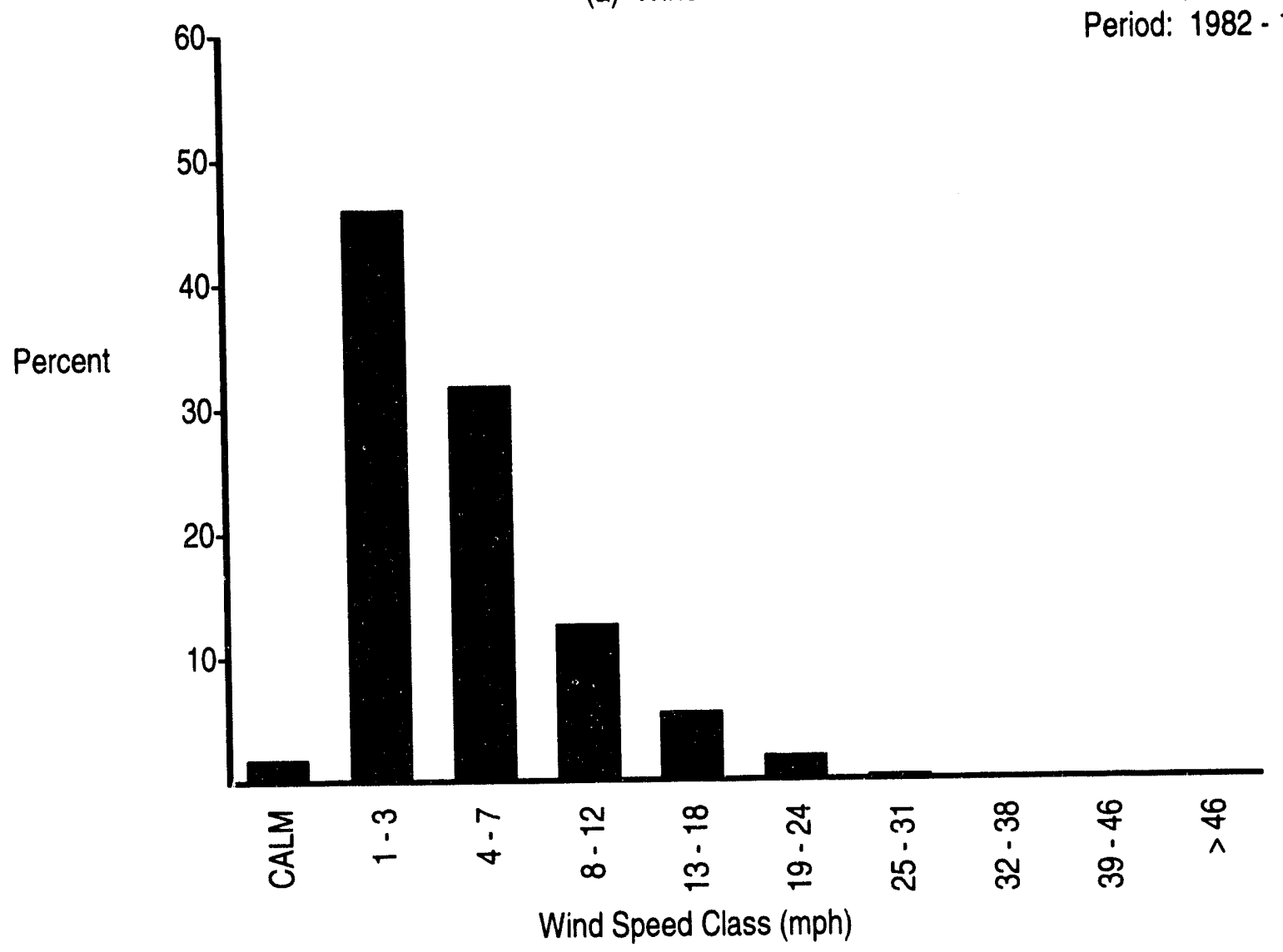

(b) Wind Speed Histogram

FIGURE B.1. (contd) 


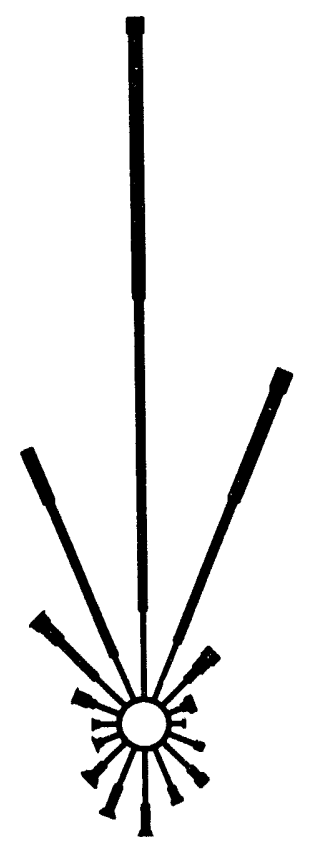

(a) Wind Rose

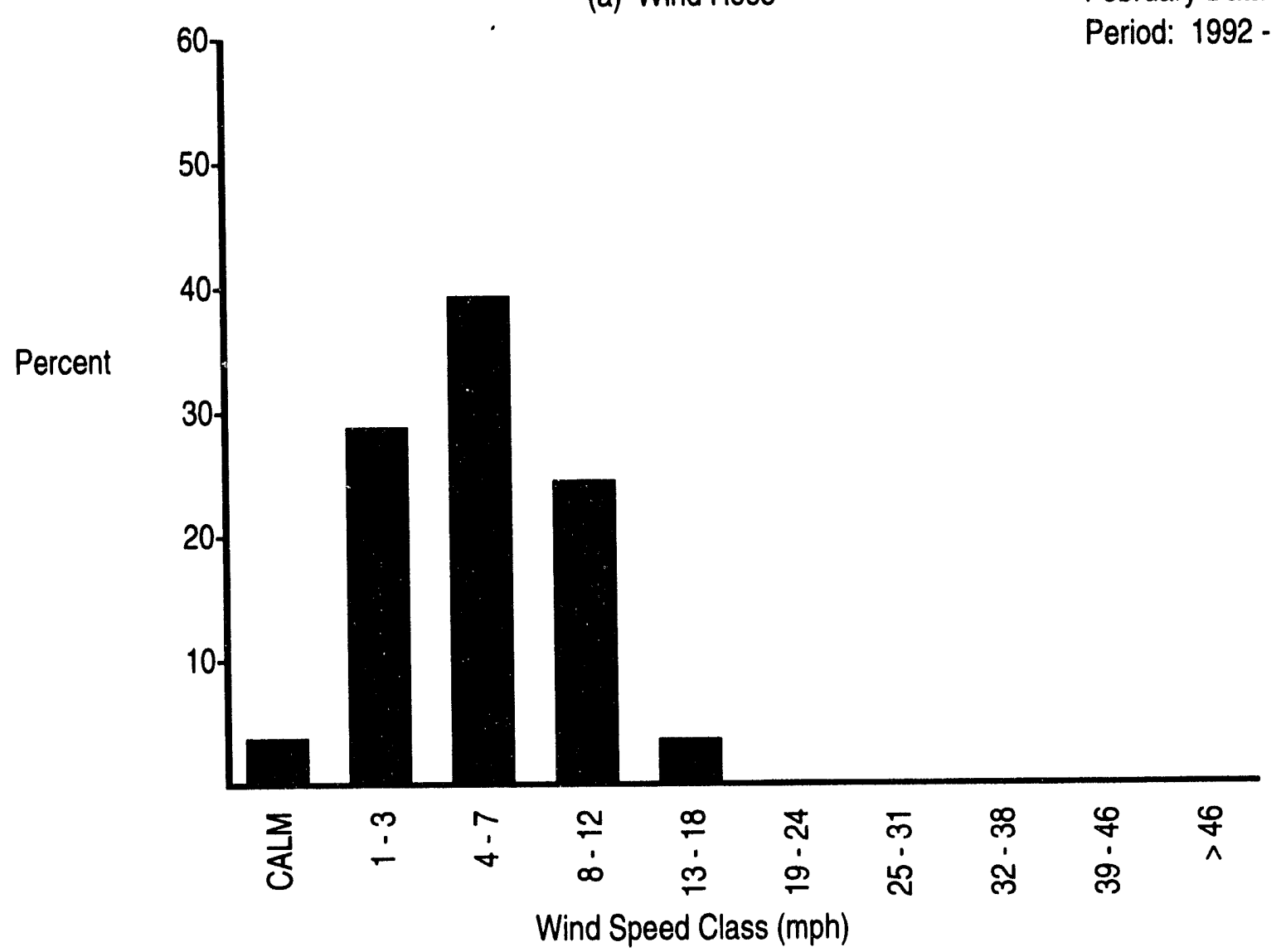

(b) Wind Speed Histogram

FIGURE B.1. (contd)
February Data

Period: 1992 - 1993

\section{B. 35}



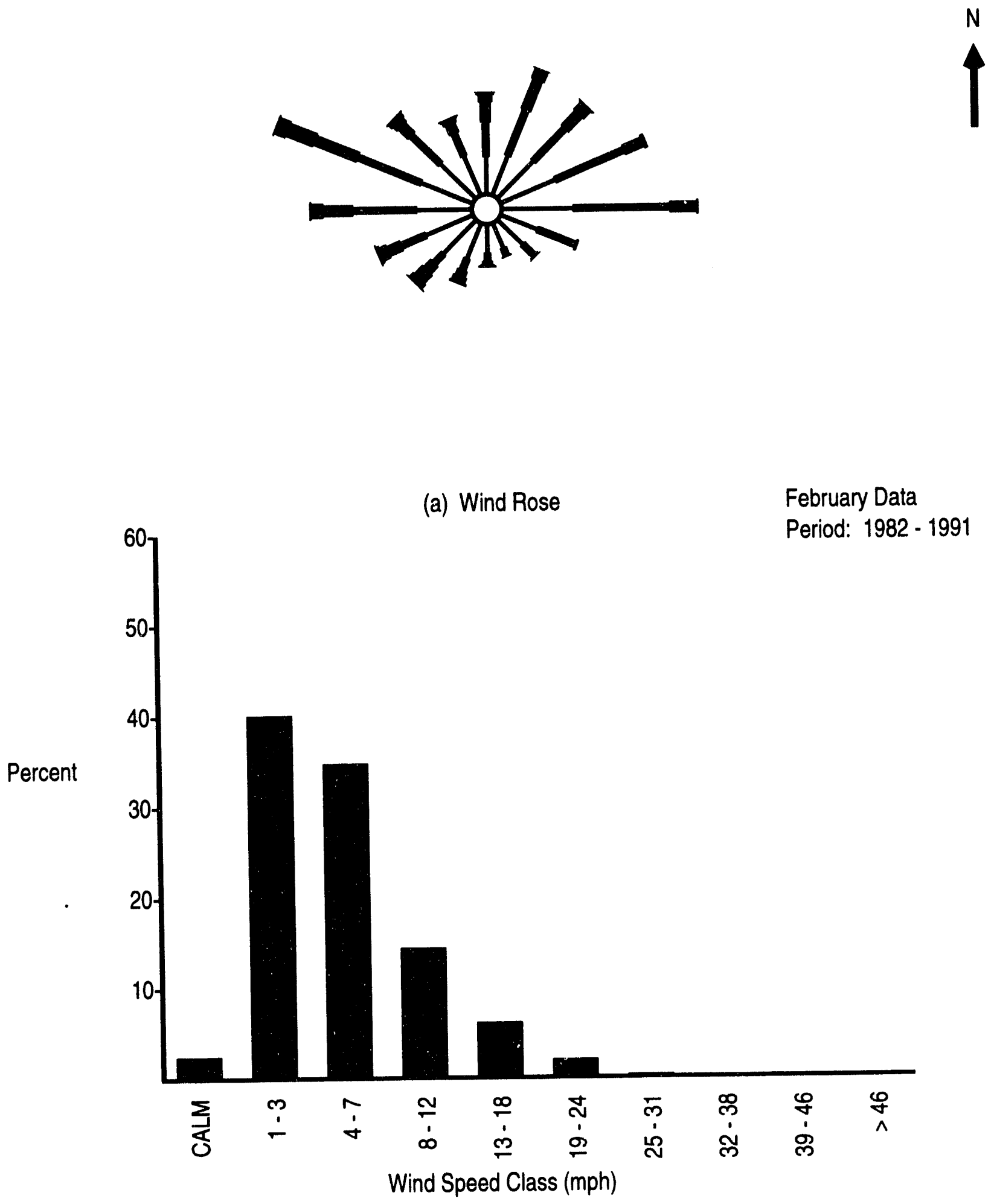

(b) Wind Speed Histogram

FIGURE B.1. (contd) 


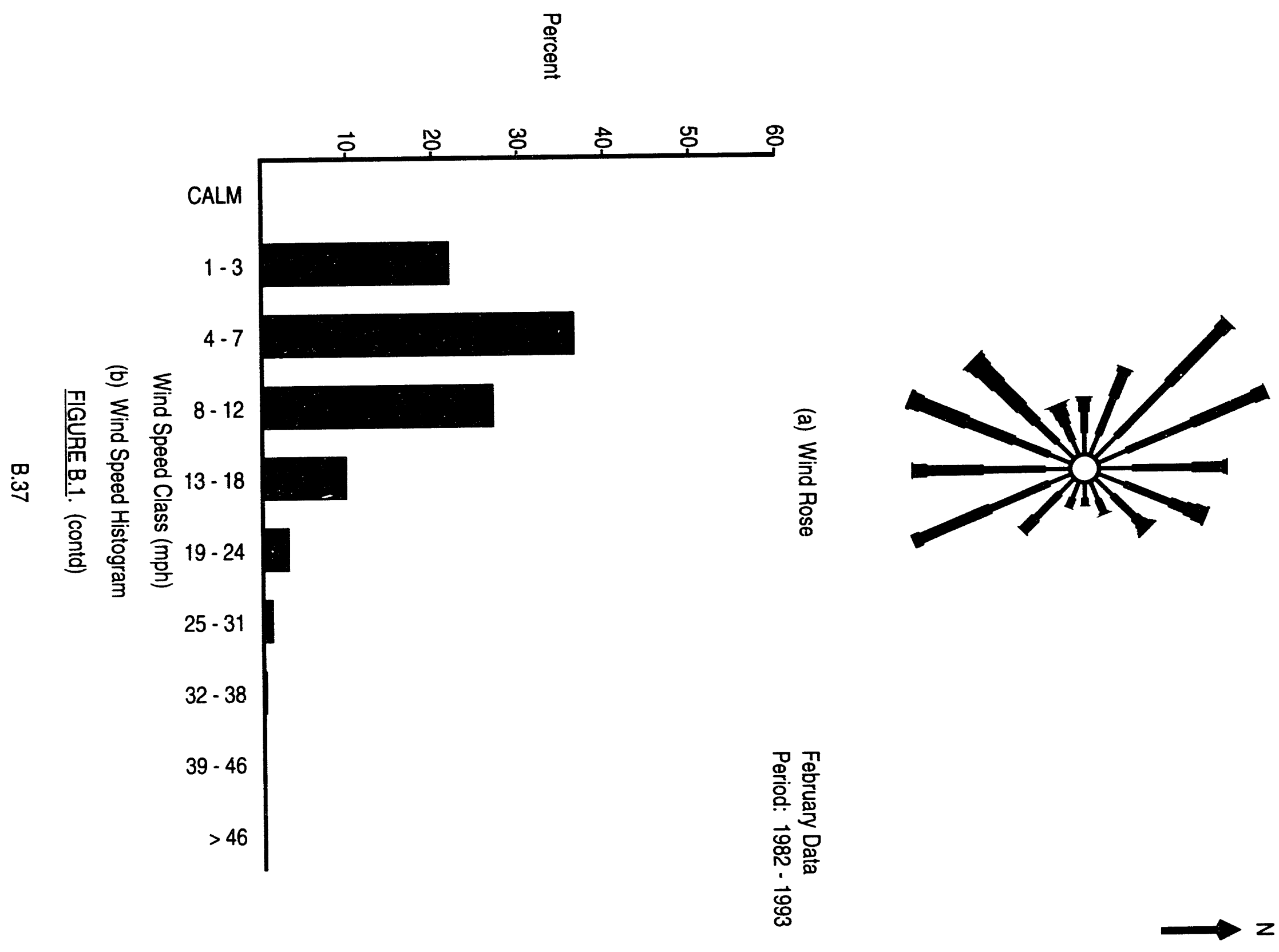




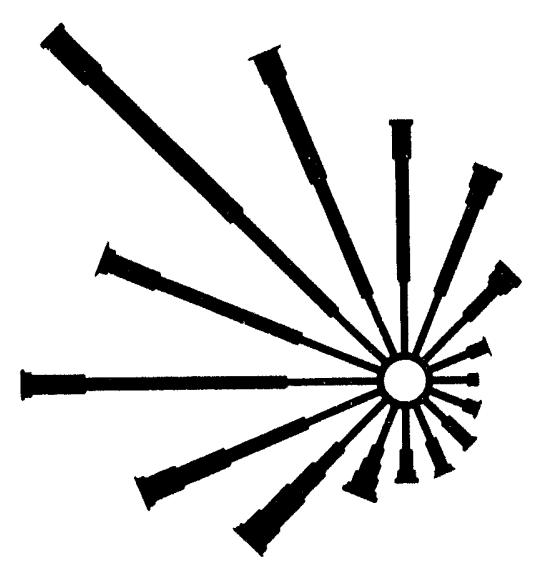

(a) Wind Rose

February Data

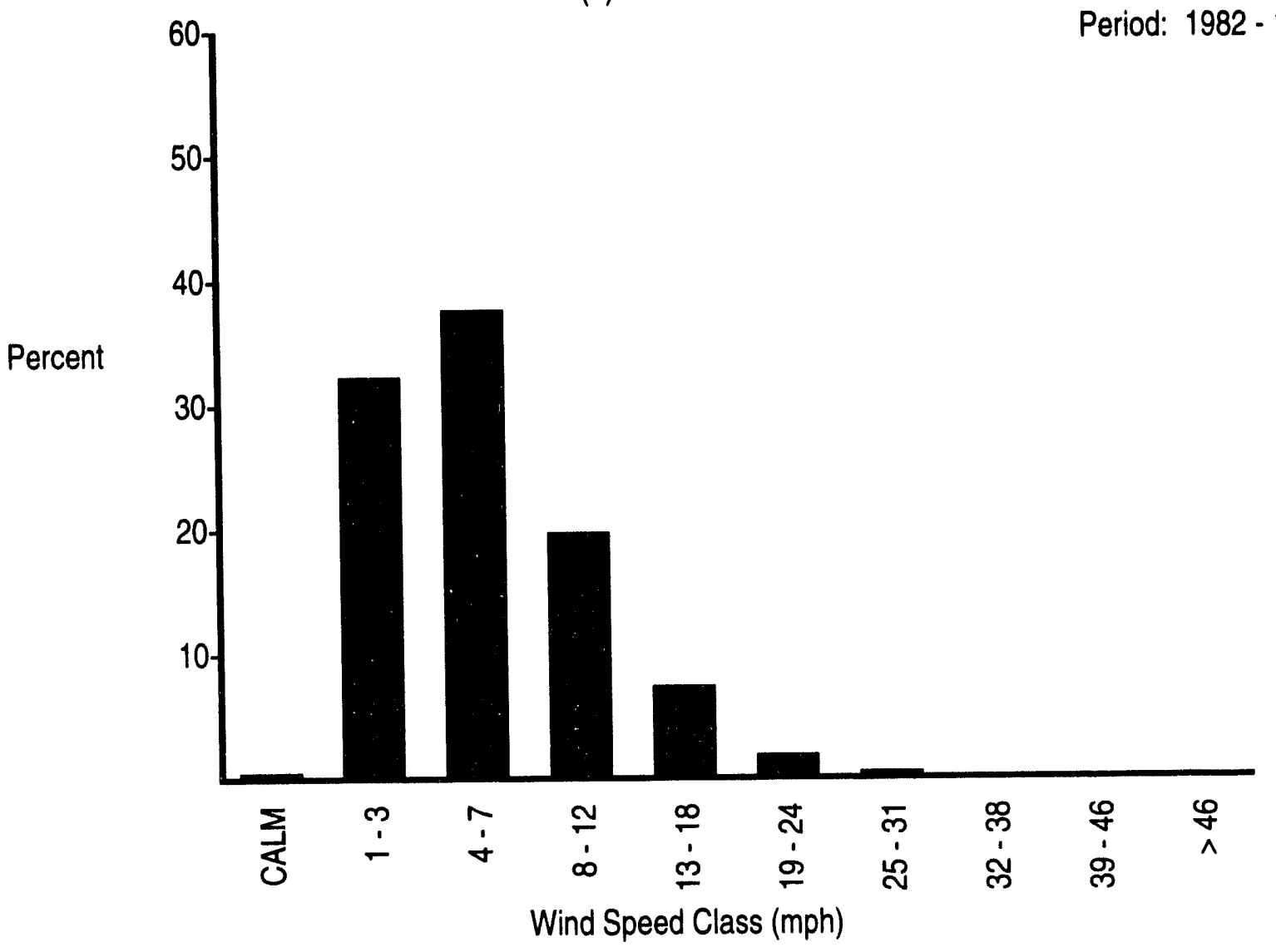

(b) Wind Speed Histogram

FIGURE B.1. (contd) 


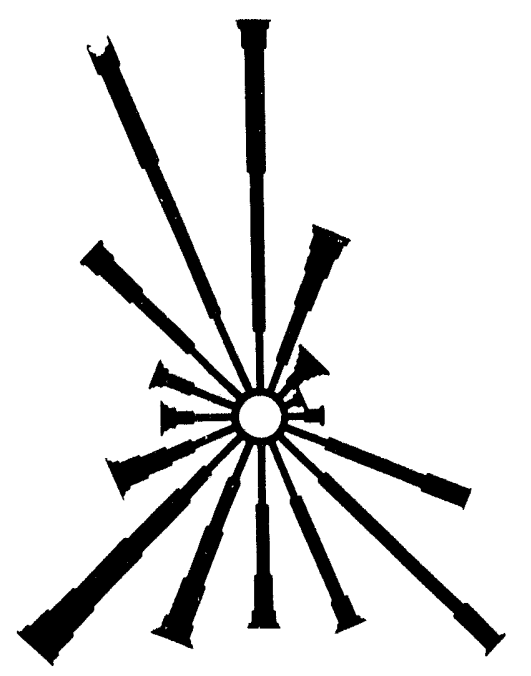

(a) Wind Rose

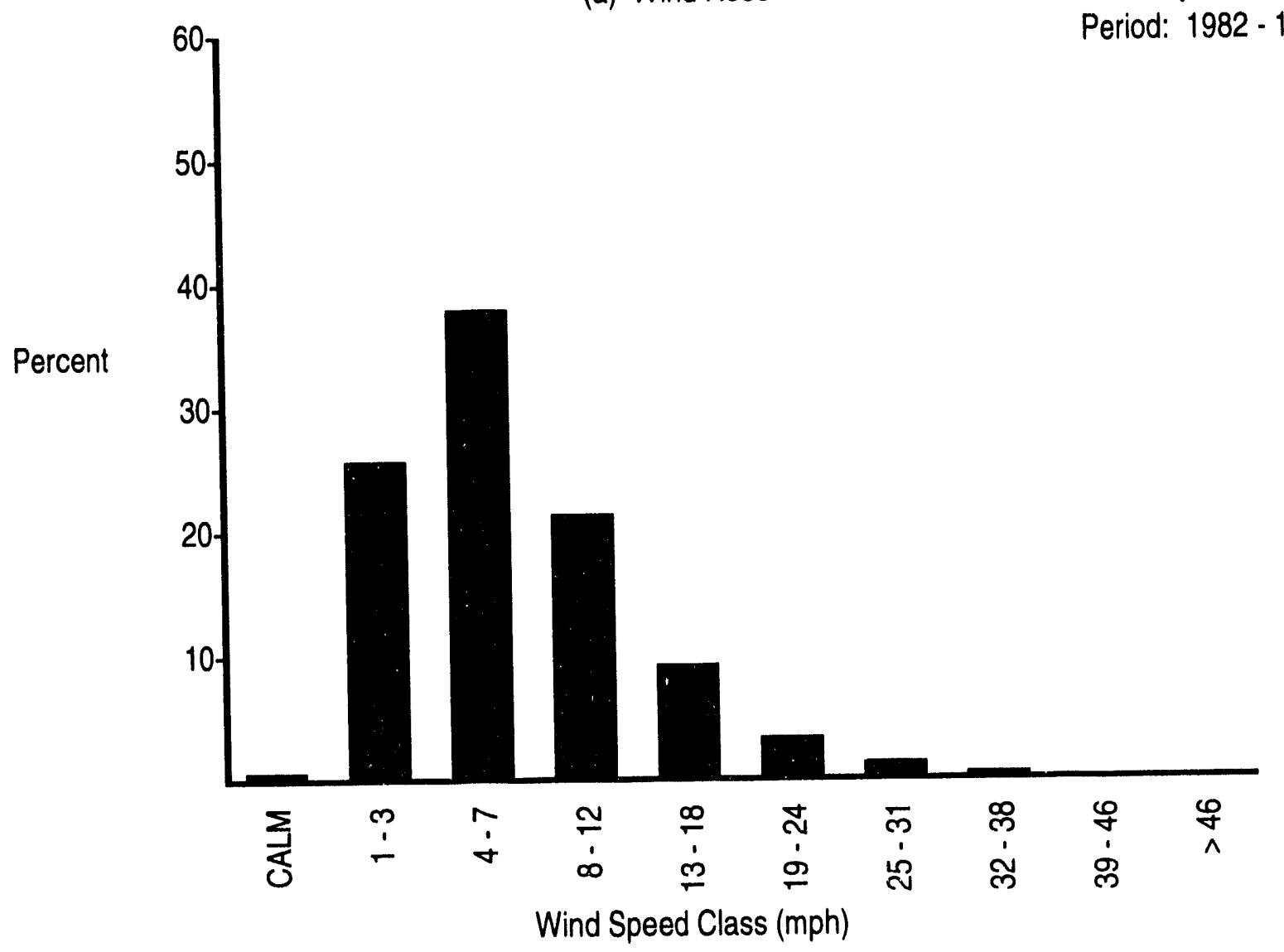

(b) Wind Speed Histogram

FIGURE B.1. (contd)
February Data

Period: 1982 - 1993 


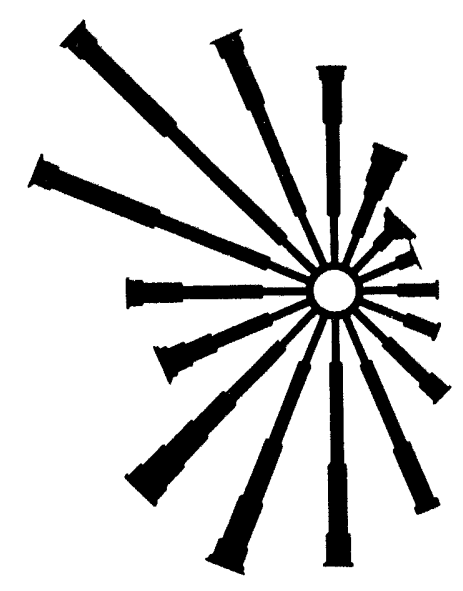

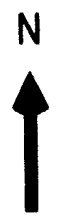

(a) Wind Rose

February Data

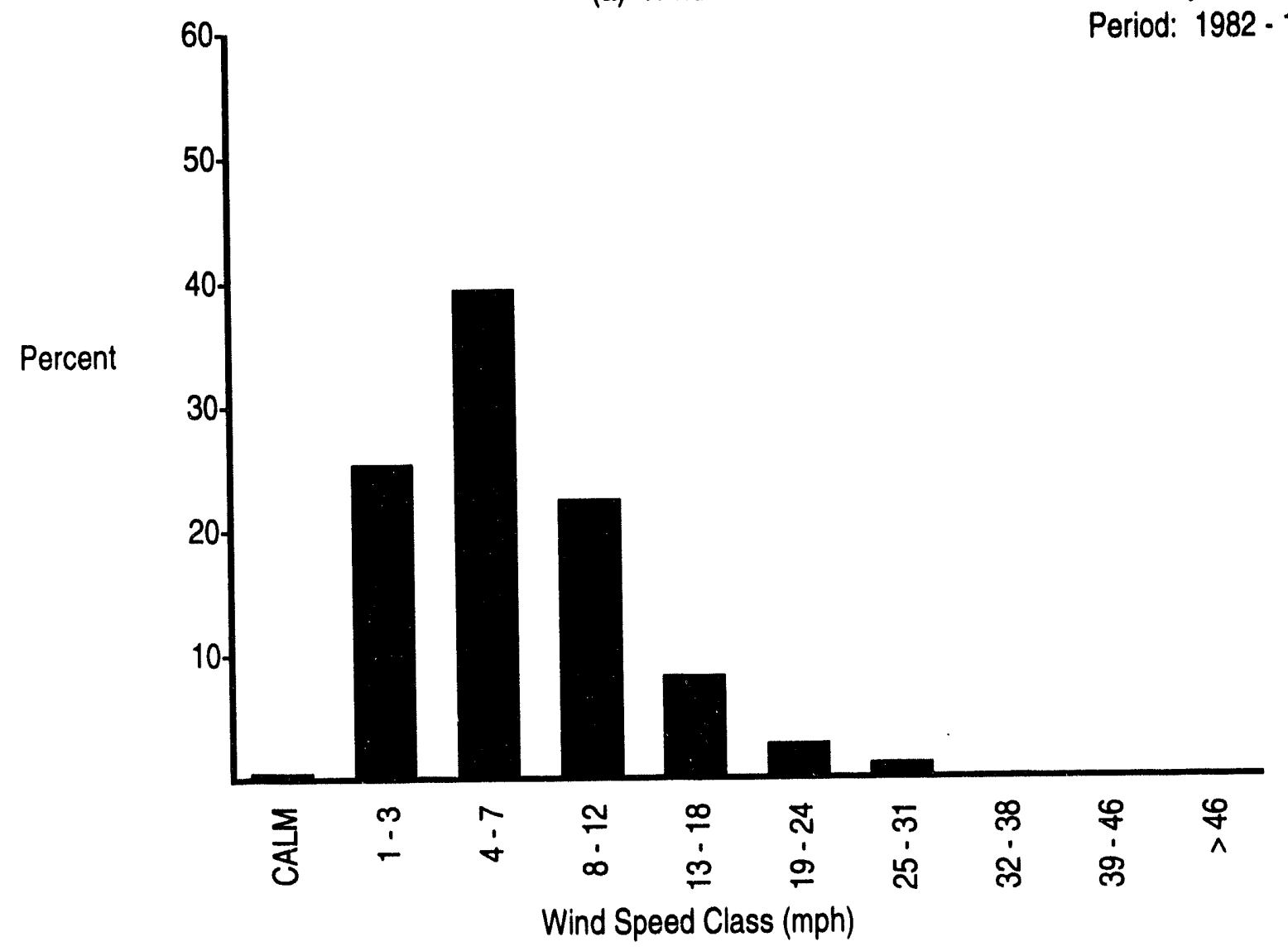

(b) Wind Speed Histogram

FIGURE B.1. (contd) 


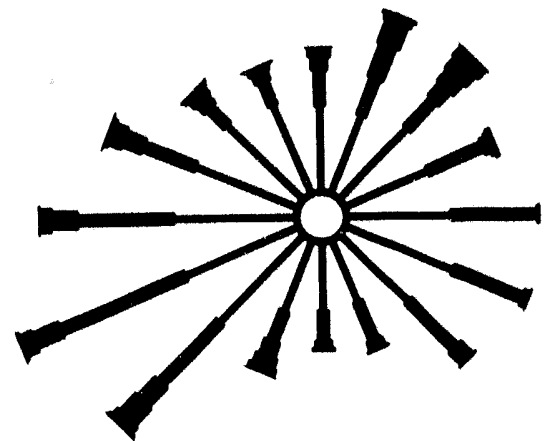

(a) Wind Rose

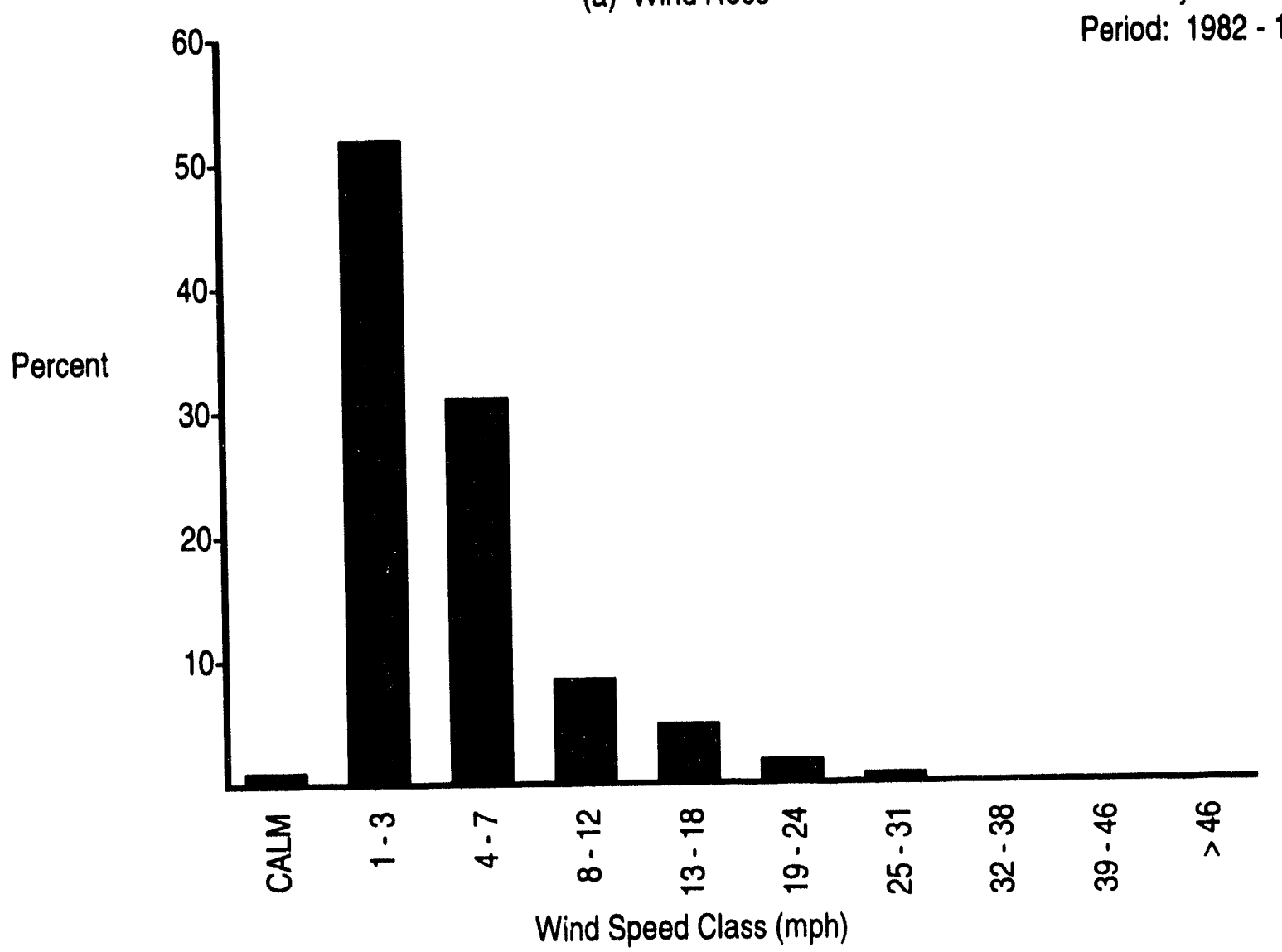

(b) Wind Speed Histogram
February Data

Period: 1982 - 1993

\section{FIGURE B.1. (contd)}




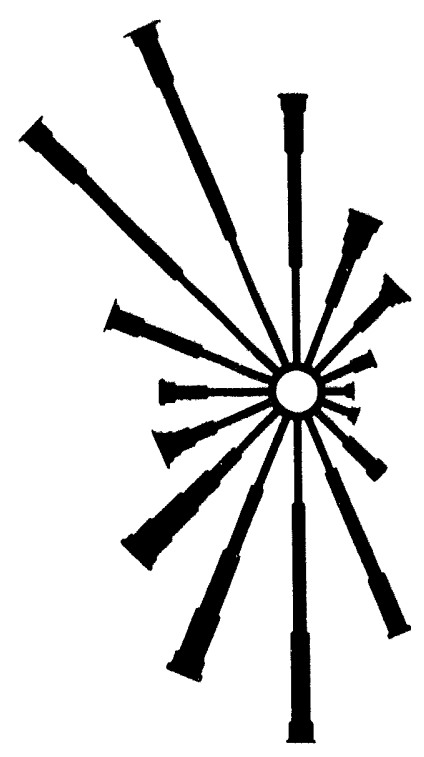

(a) Wind Rose

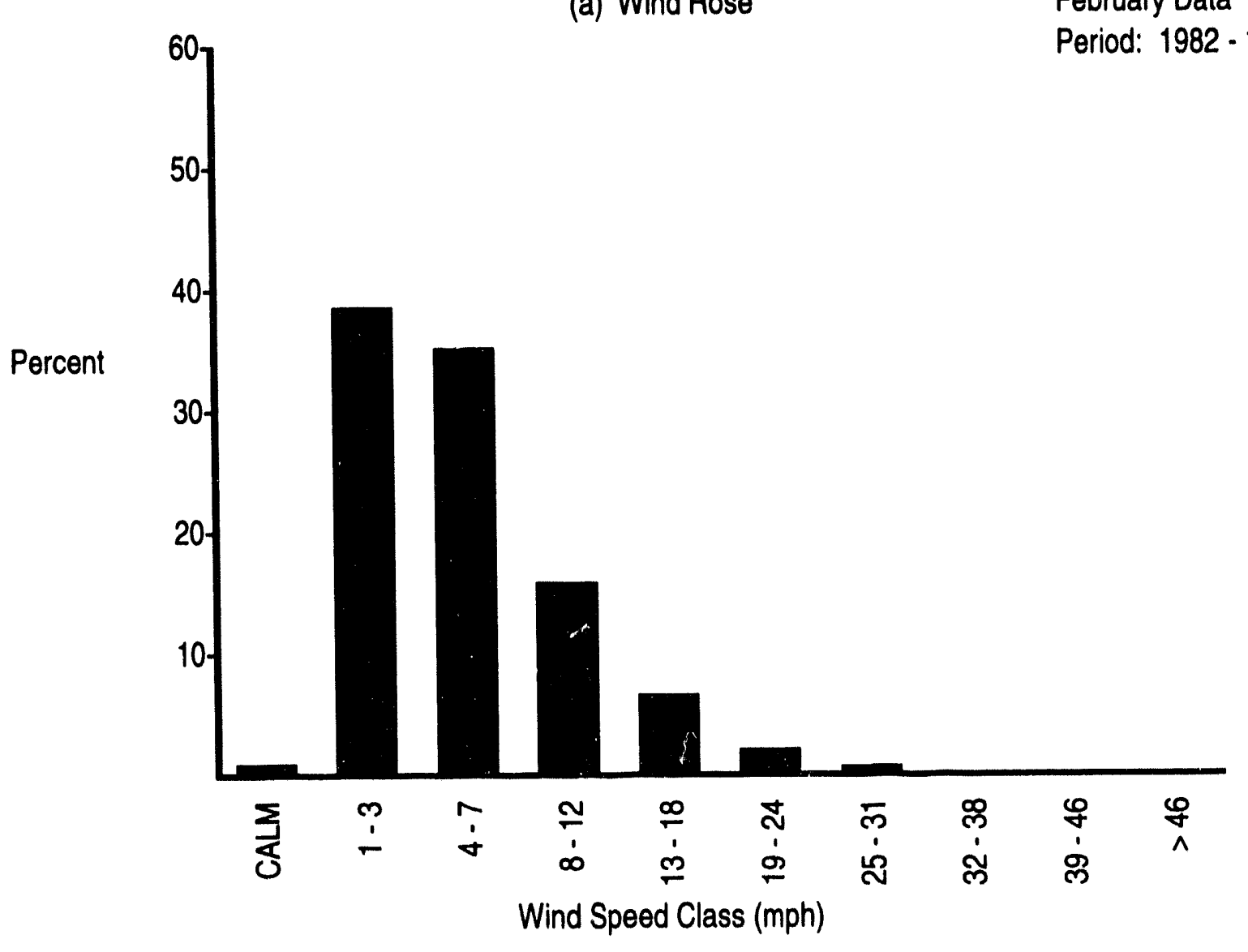

(b) Wind Speed Histogram

FIGURE B.1. (contd) 


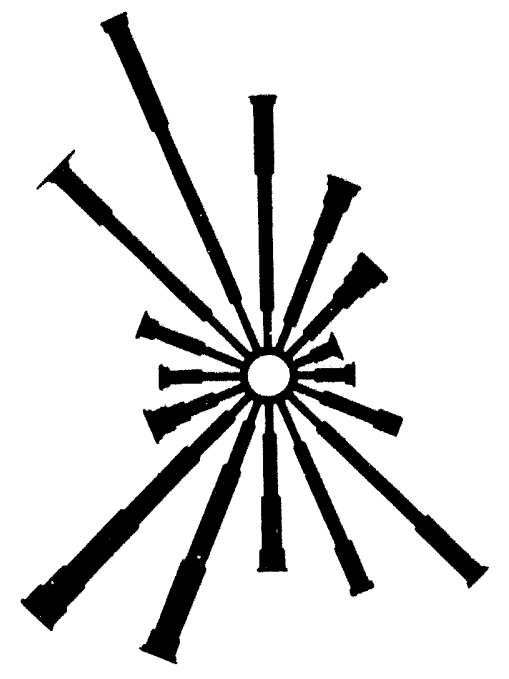

(a) Wind Rose

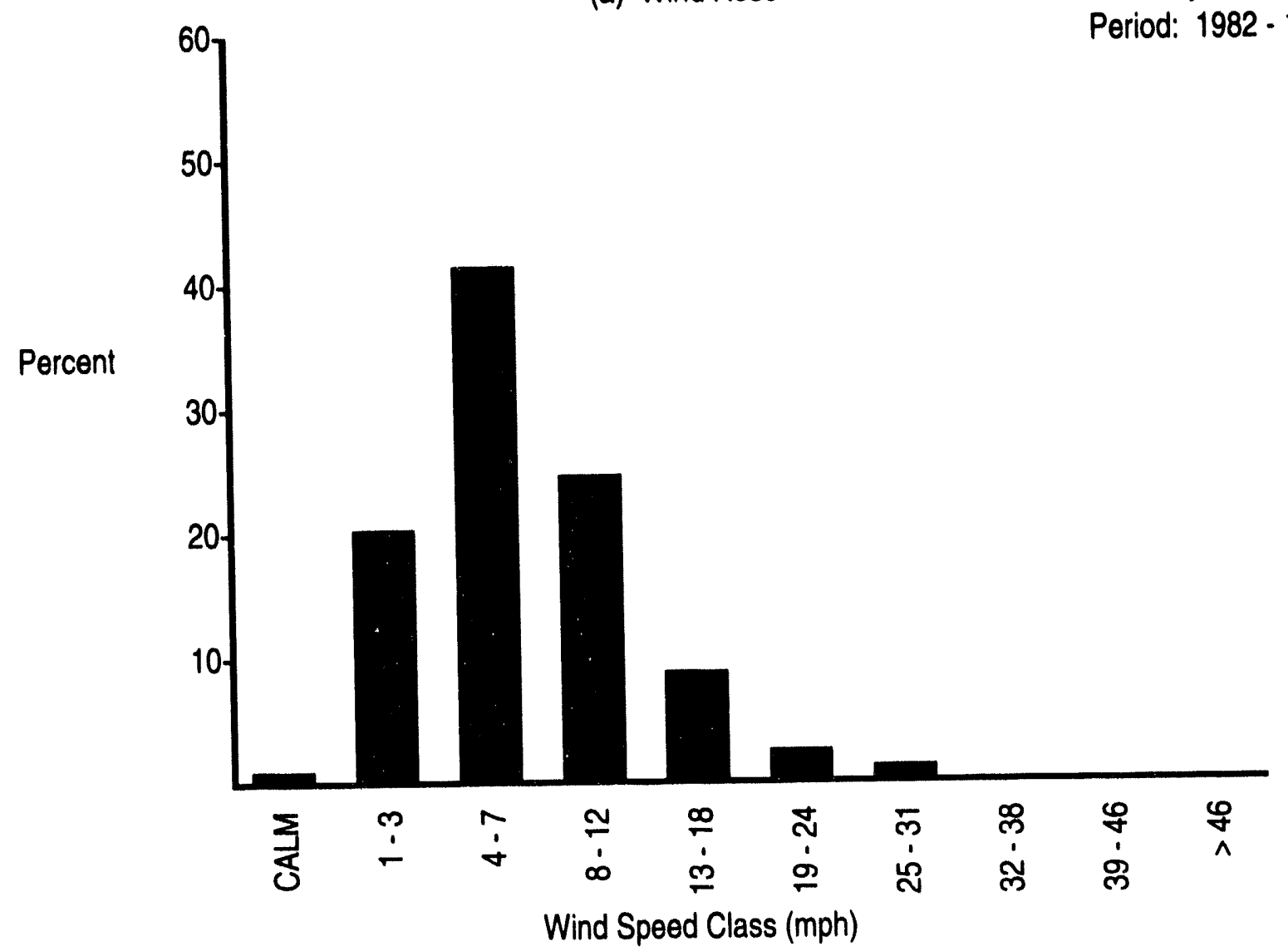

(b) Wind Speed Histogram

FIGURE B.1. (contd)
February Data

Period: 1982 - 1993

\section{B.43}




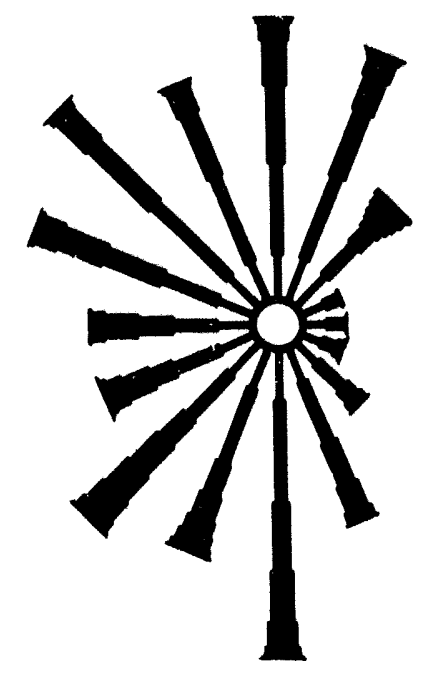

(a) Wind Rose

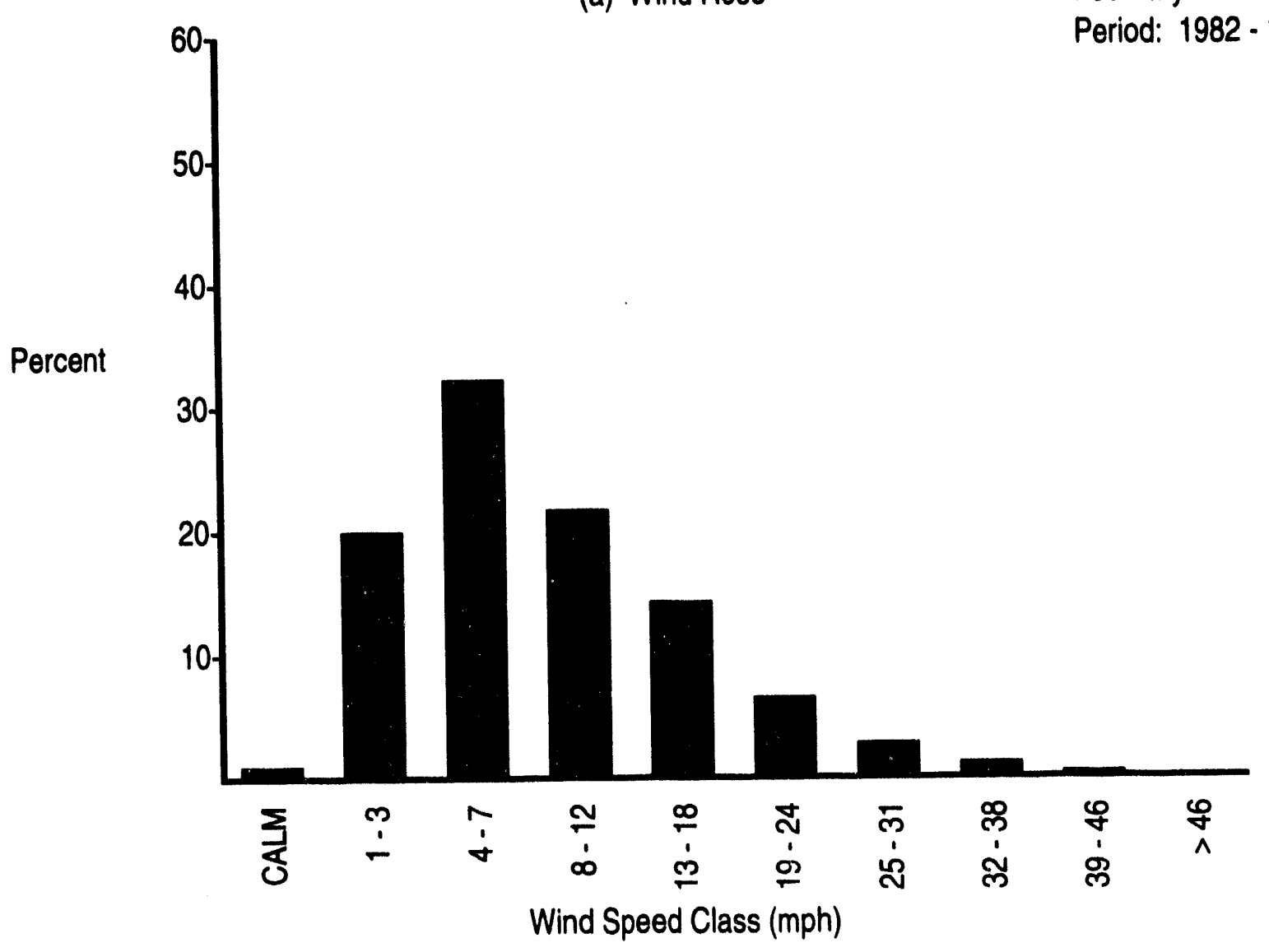

(b) Wind Speed Histogram
February Data

Period: $1982 \cdot 1993$

\section{FIGURE B.1. (contd)}



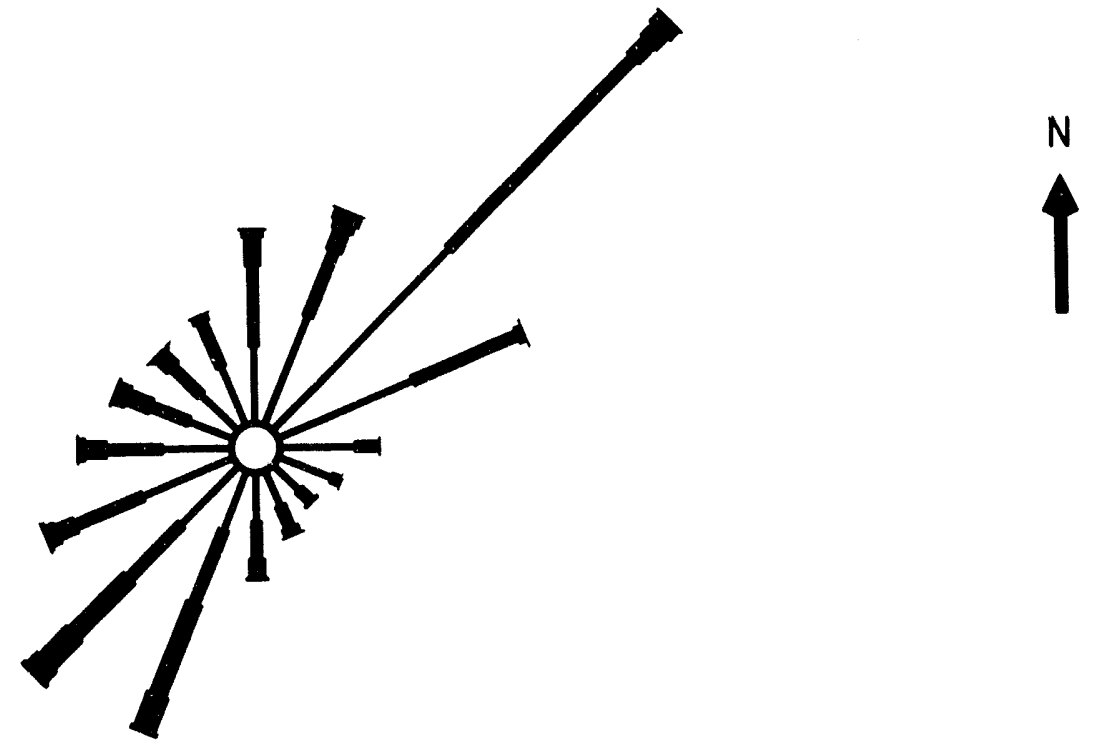

(a) Wind Rose

February Data

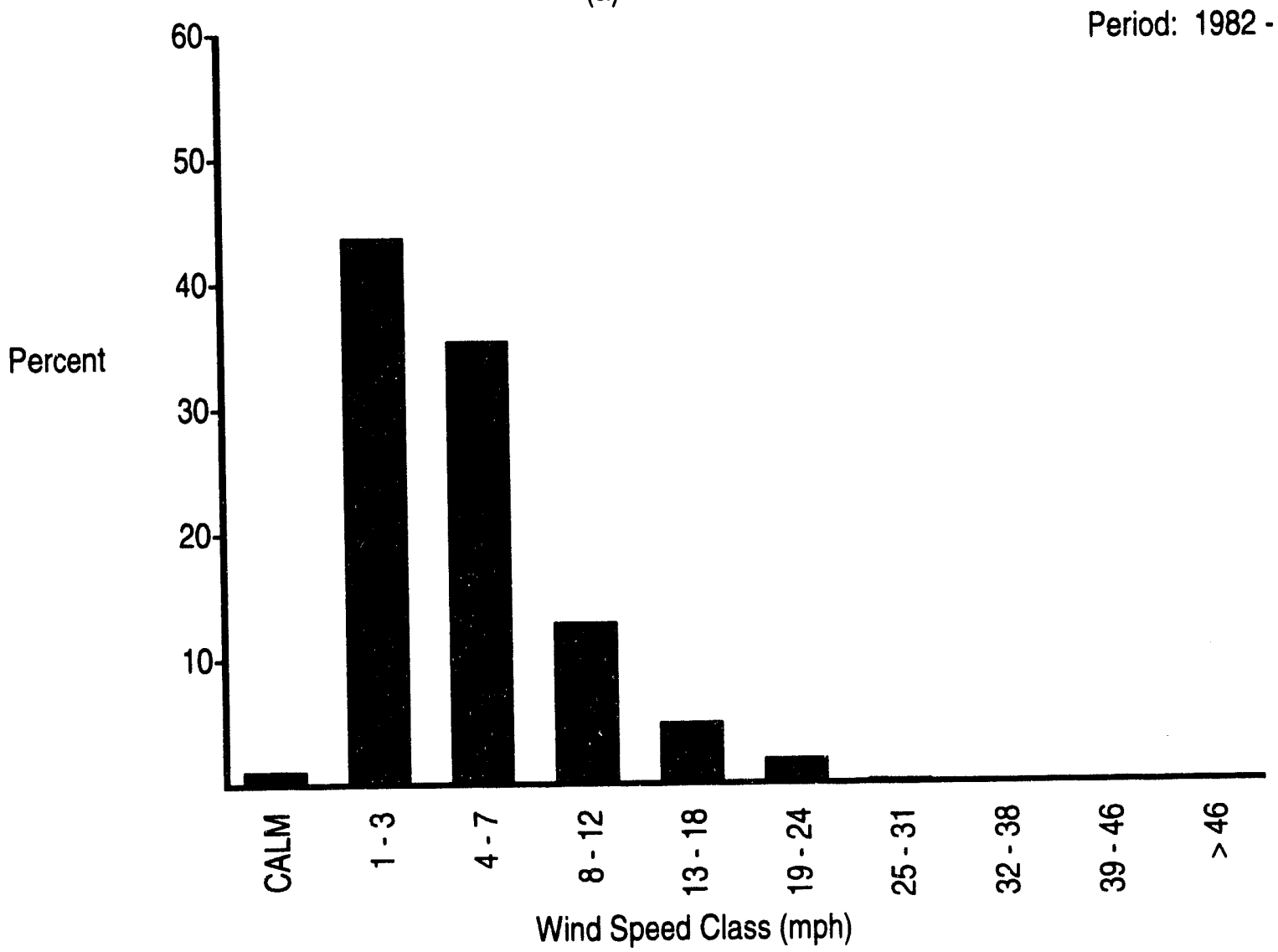

(b) Wind Speed Histogram

FIGURE B.1. (contd) 


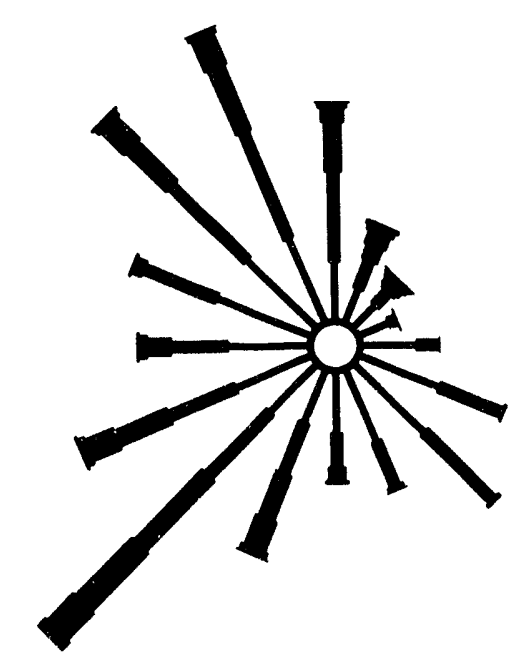

(a) Wind Rose

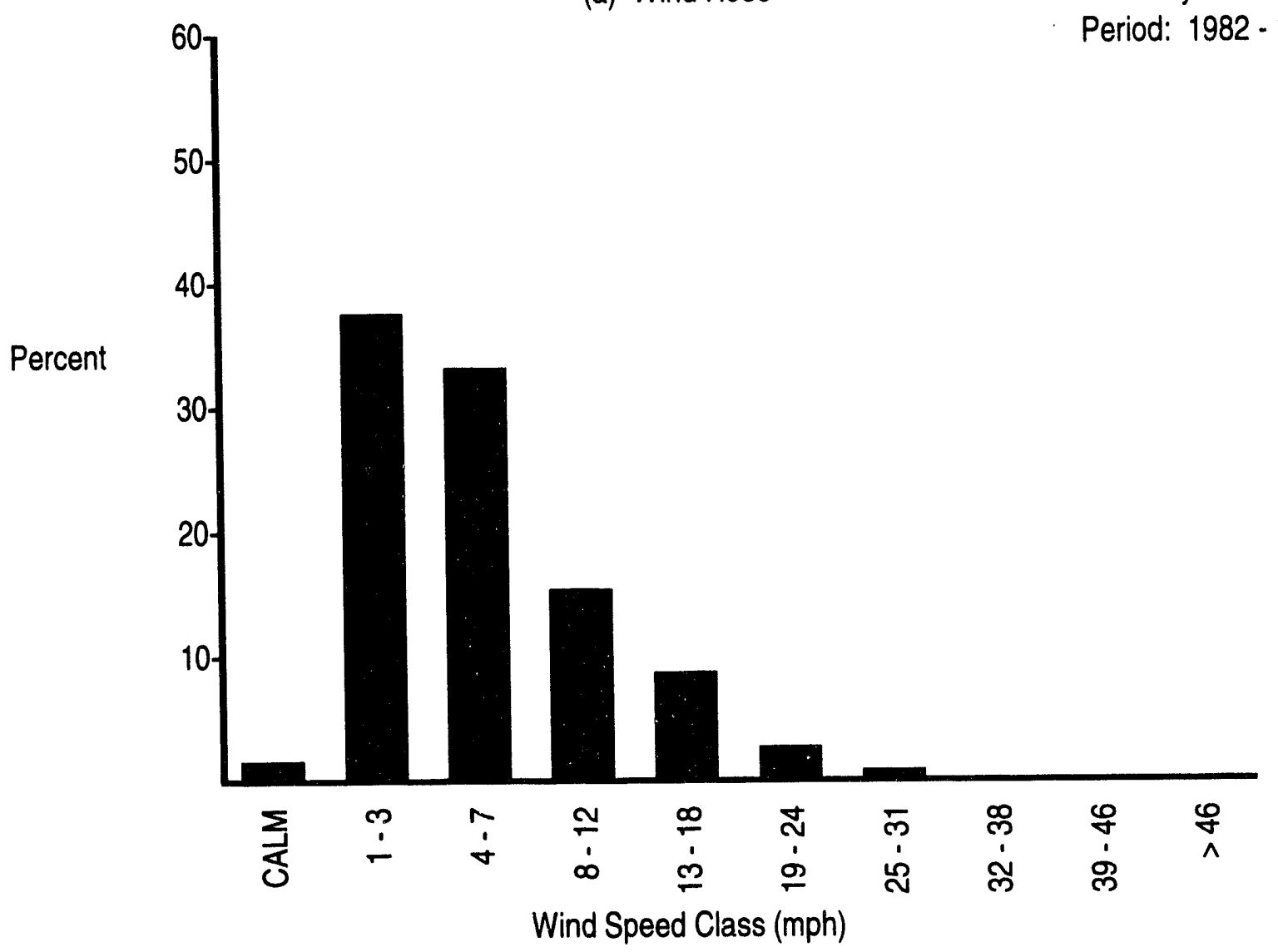

(b) Wind Speed Histogram

FIGURE B.1. (contd)
February Data

Period: 1982 - 1993

\section{B.46}




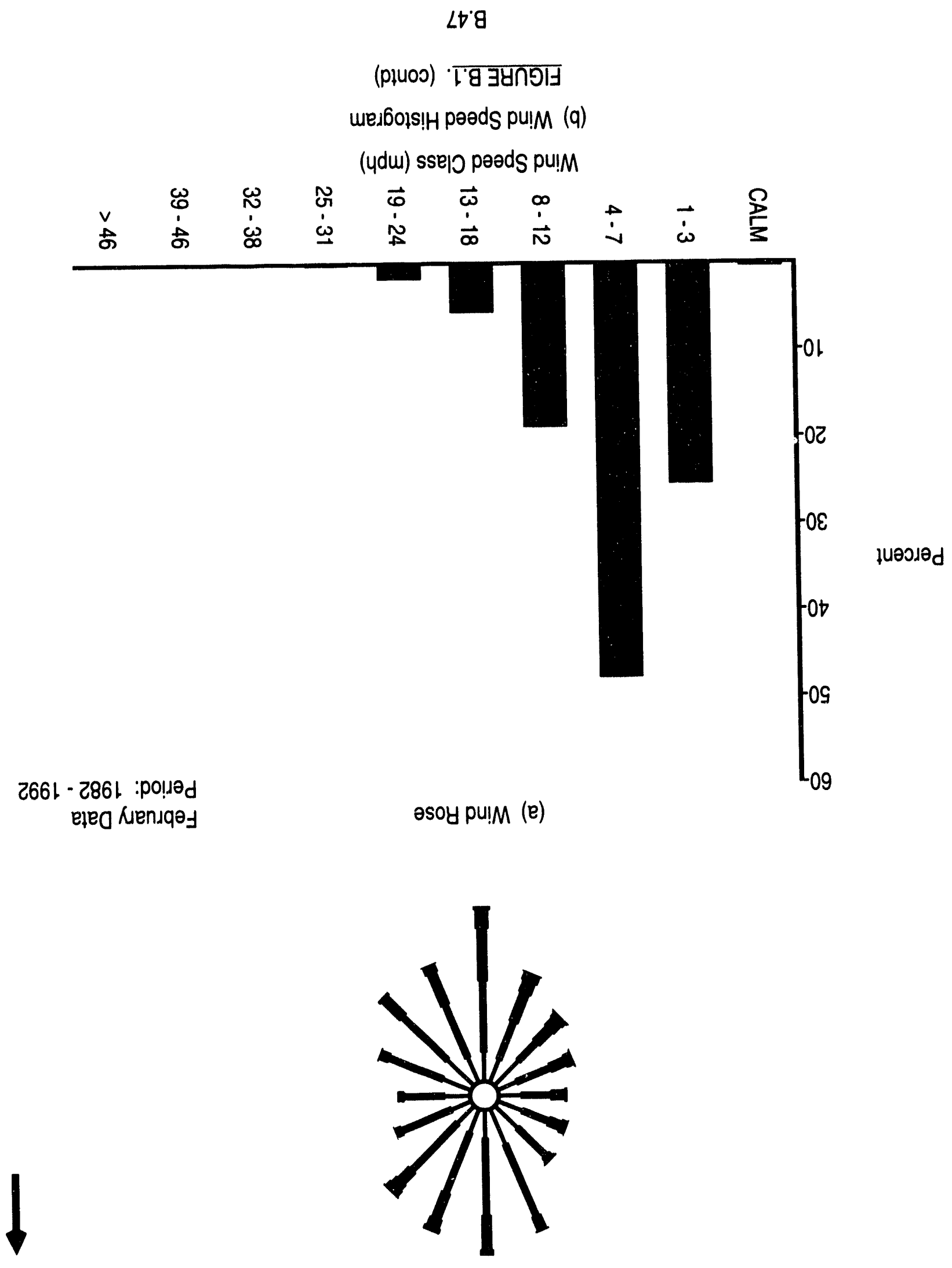

N 
$87^{\circ} 8$

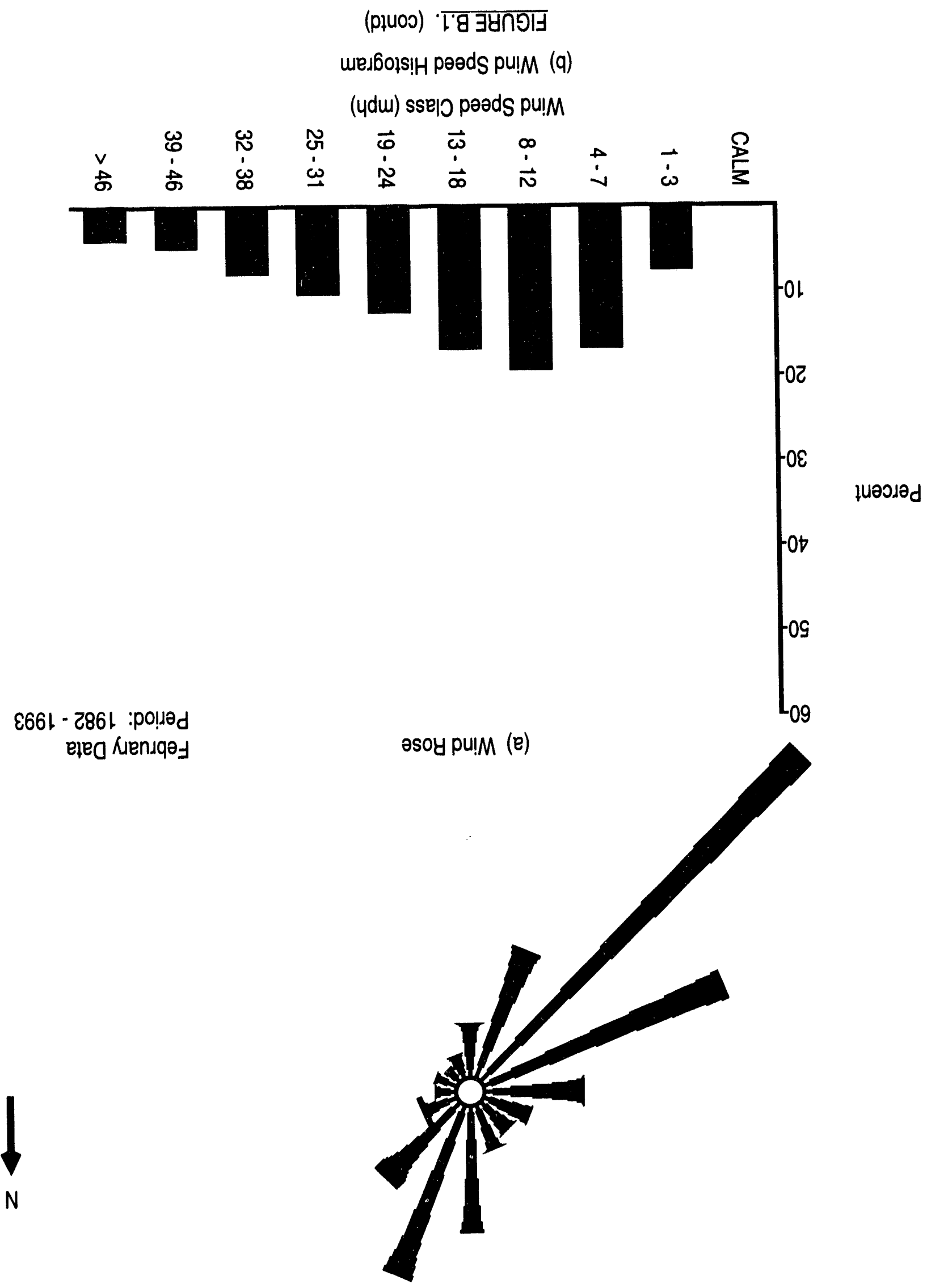




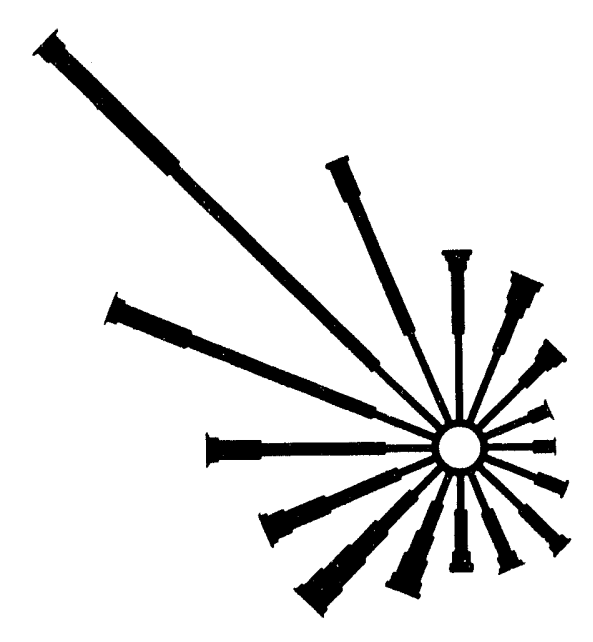

(a) Wind Rose

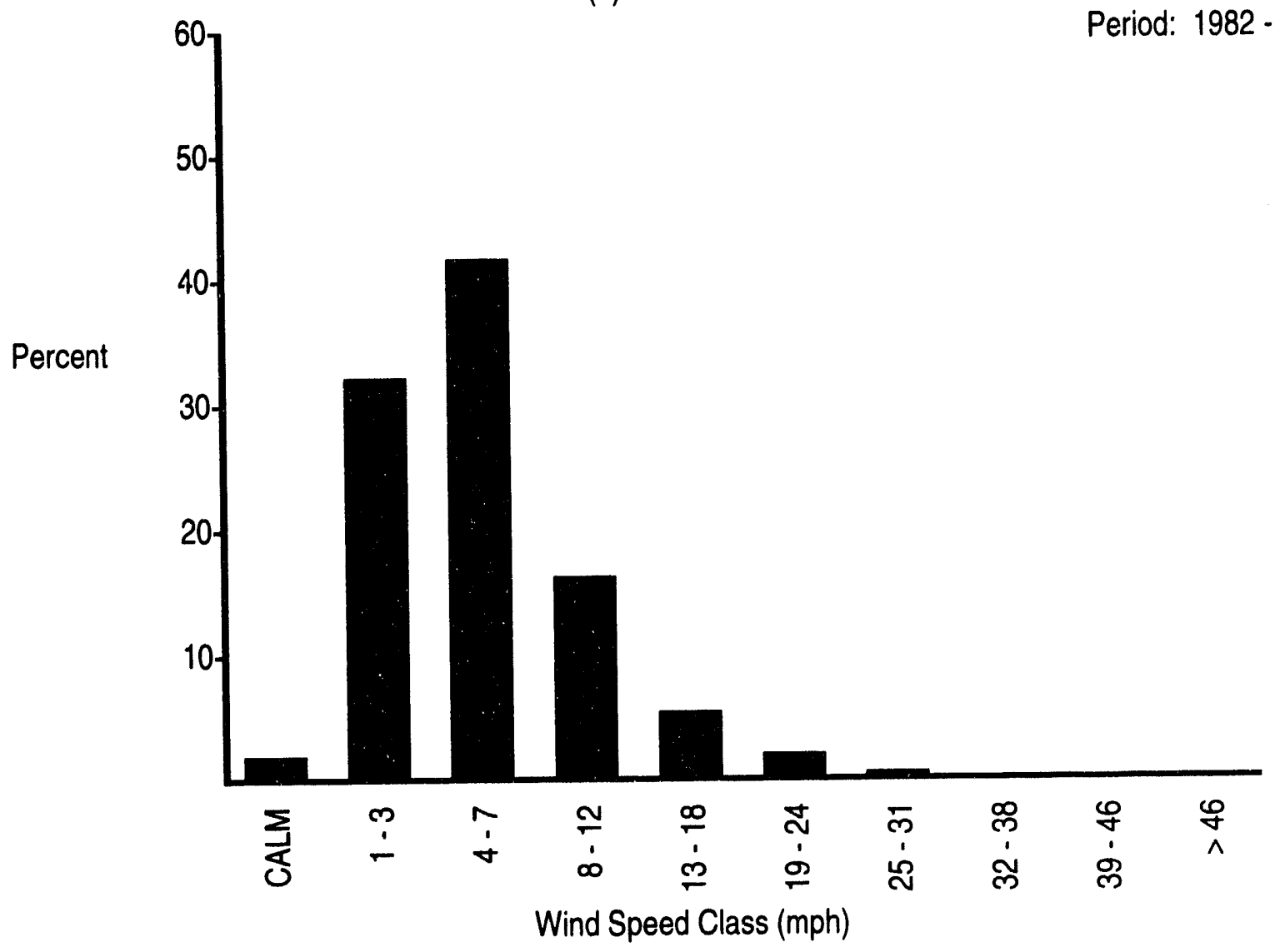

(b) Wind Speed Histogram

FIGURE B.1. (contd)
February Data

Period: 1982 - 1993 


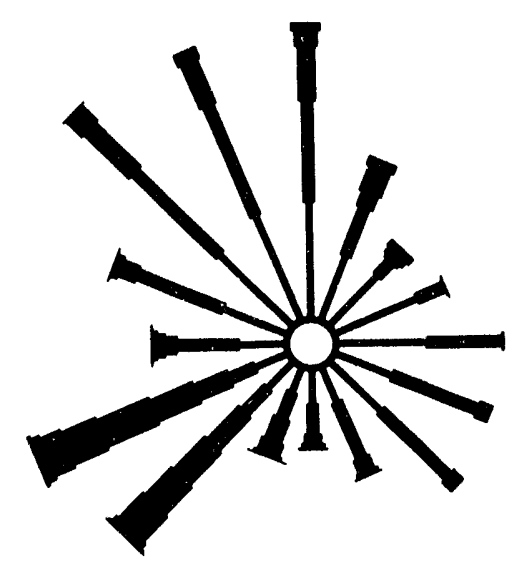

(a) Wind Rose

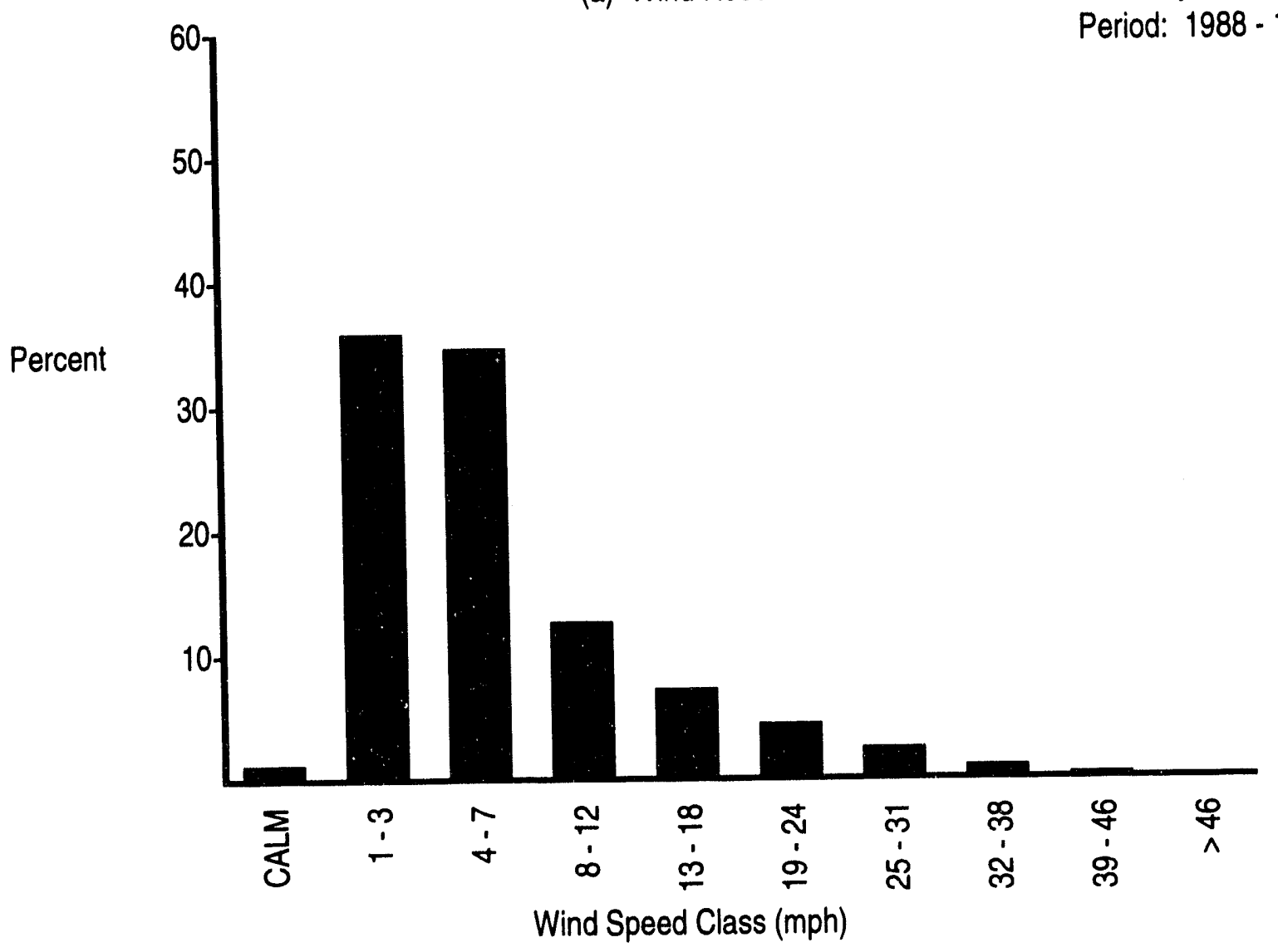

(b) Wind Speed Histogram

FIGURE B.1. (contd)
February Data

Period: 1988 - 1993 


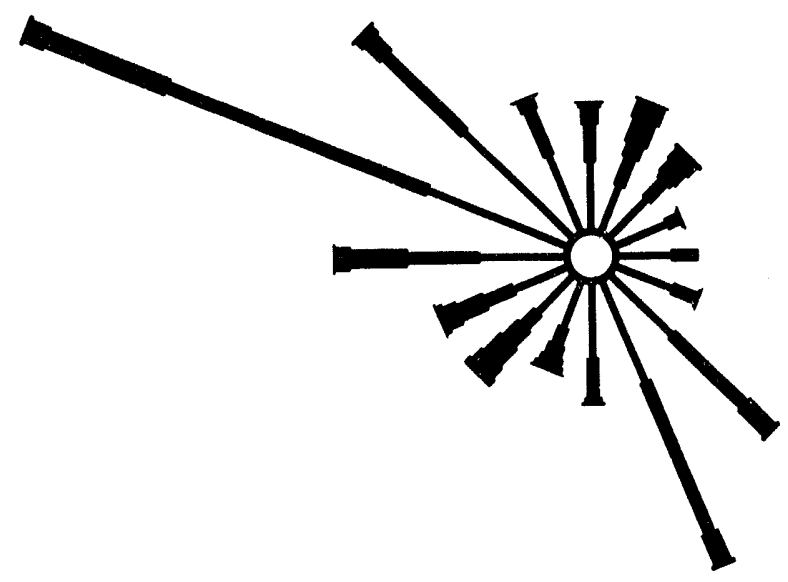

(a) Wind Rose

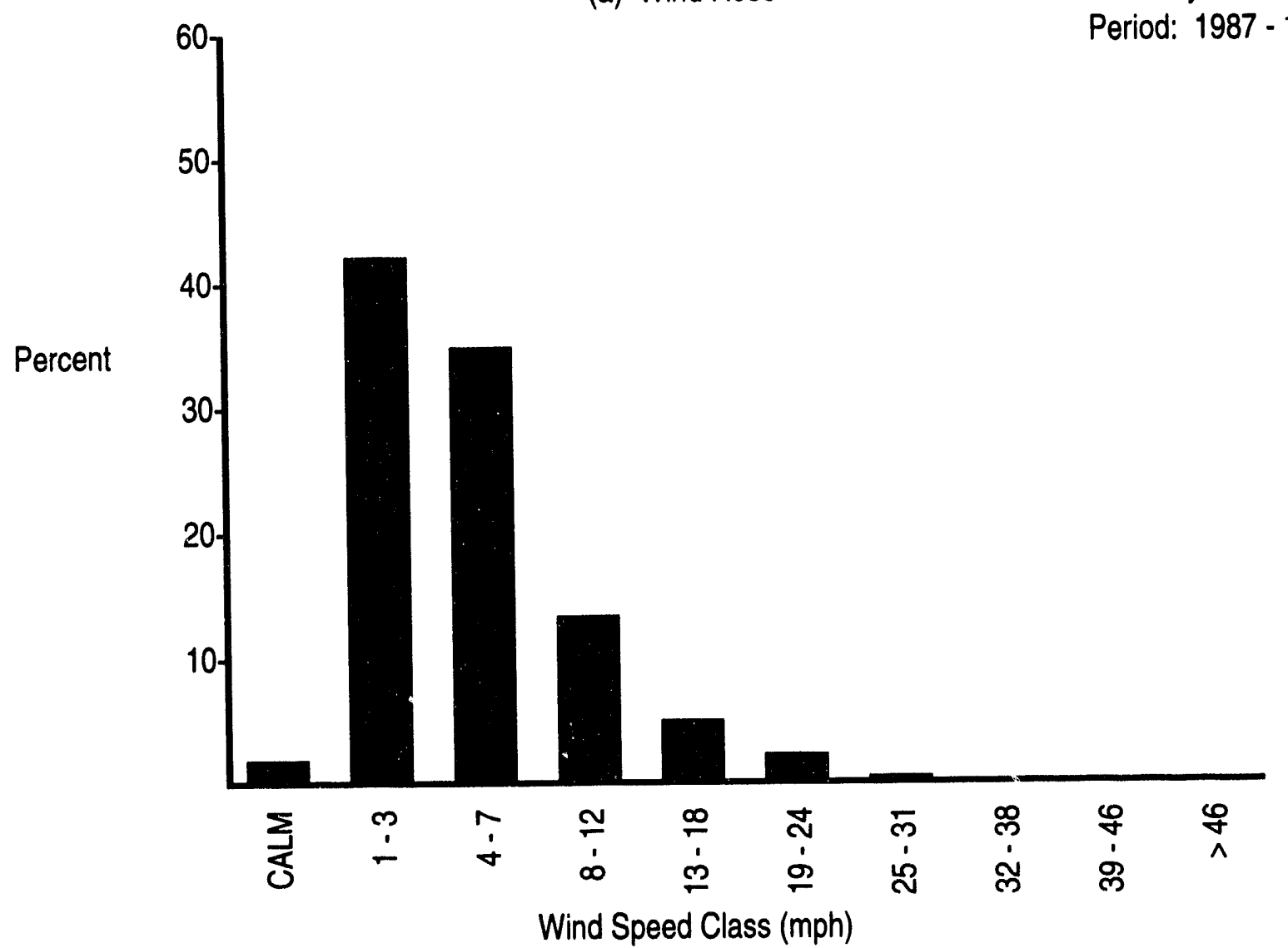

(b) Wind Speed Histogram

FIGURE B.1. (contd) 


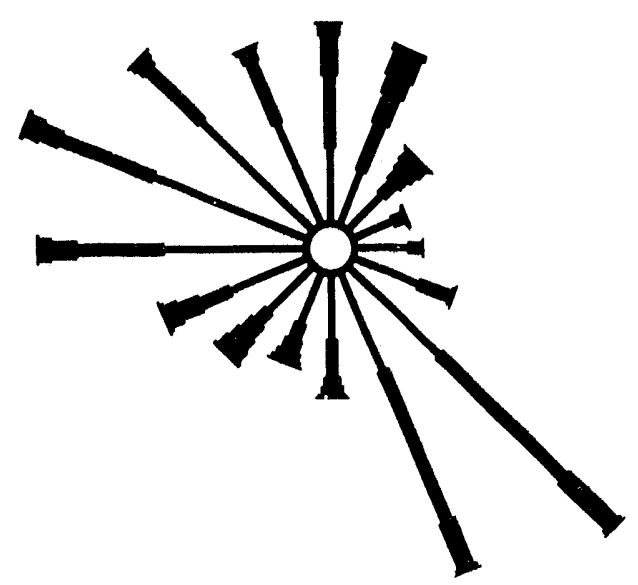

(a) Wind Rose

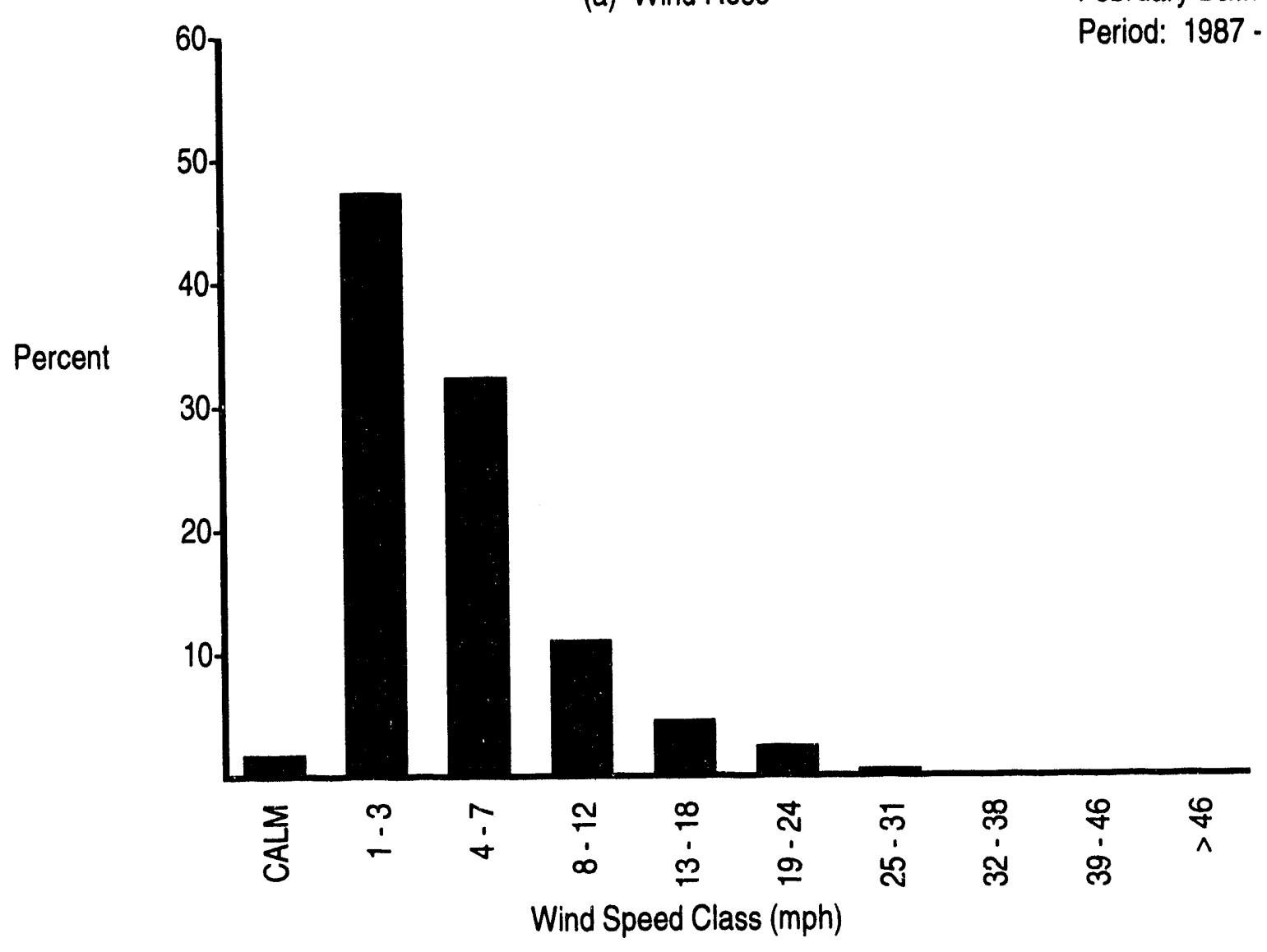

(b) Wind Speed Histogram

FIGURE B.1. (contd)
February Data

Period: 1987 - 1993 


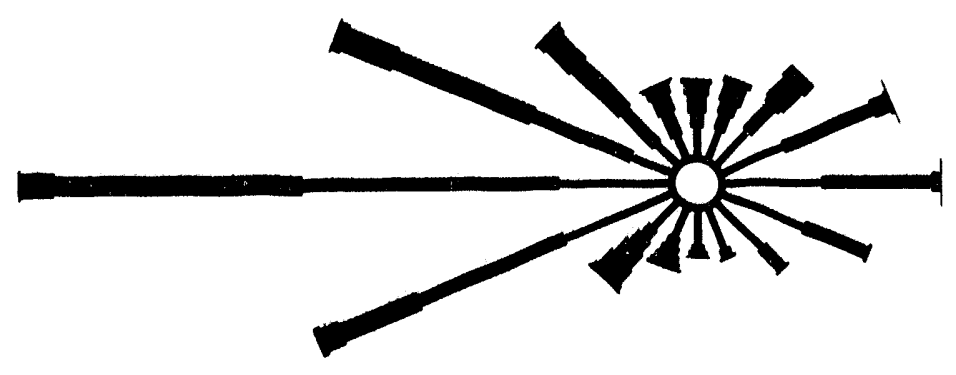

February Data

(a) Wind Rose

Period: 1988 - 1993

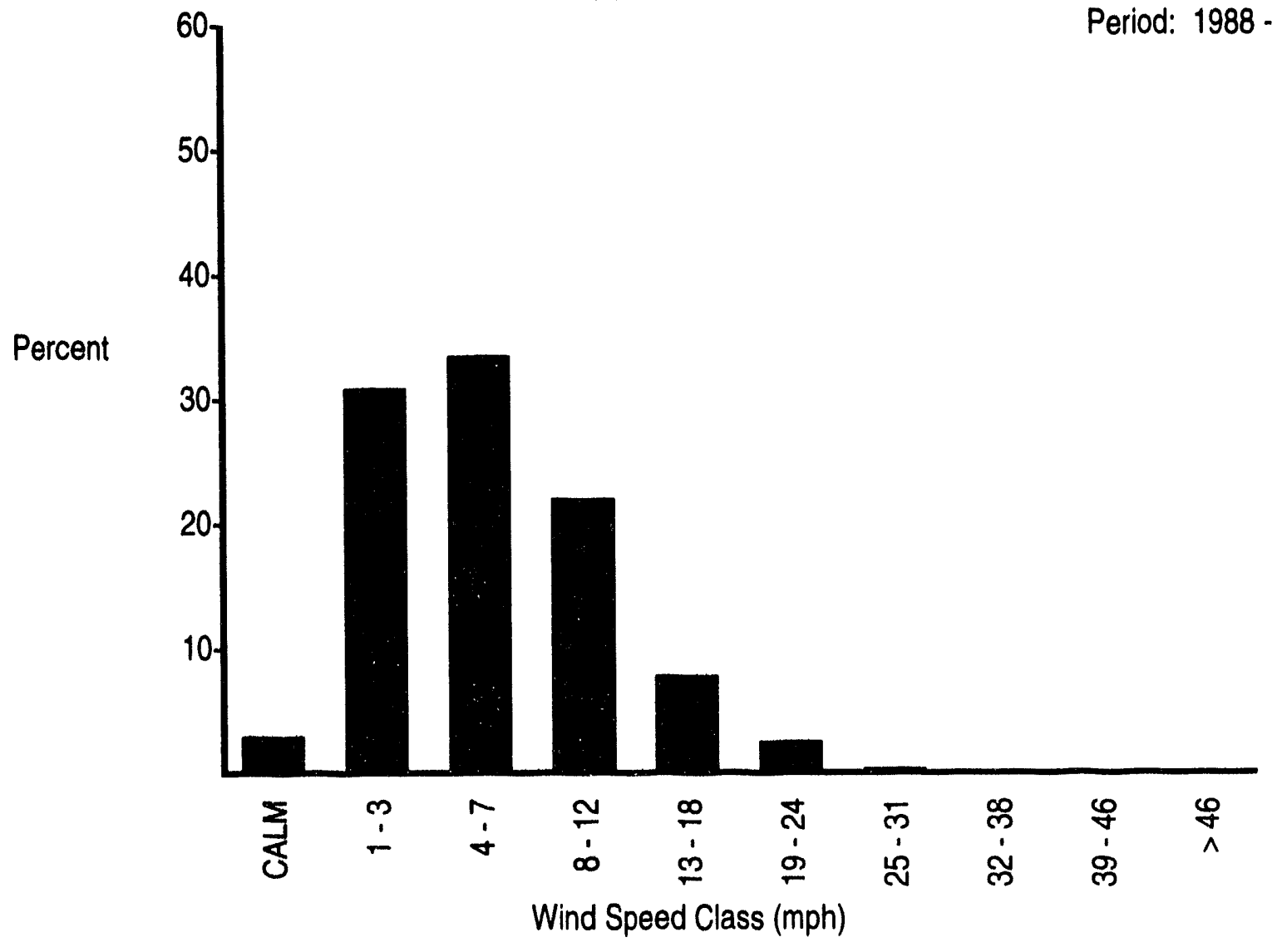

(b) Wind Speed Histogram

FIGURE B.1. (contd) 


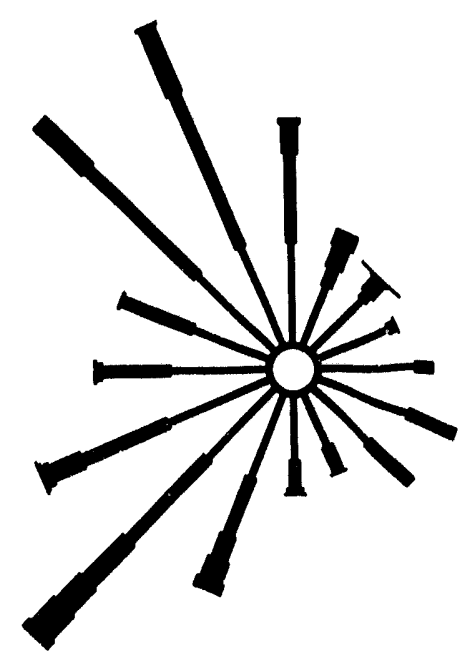

(a) Wind Rose

February Data

Period: 1991 - 1993

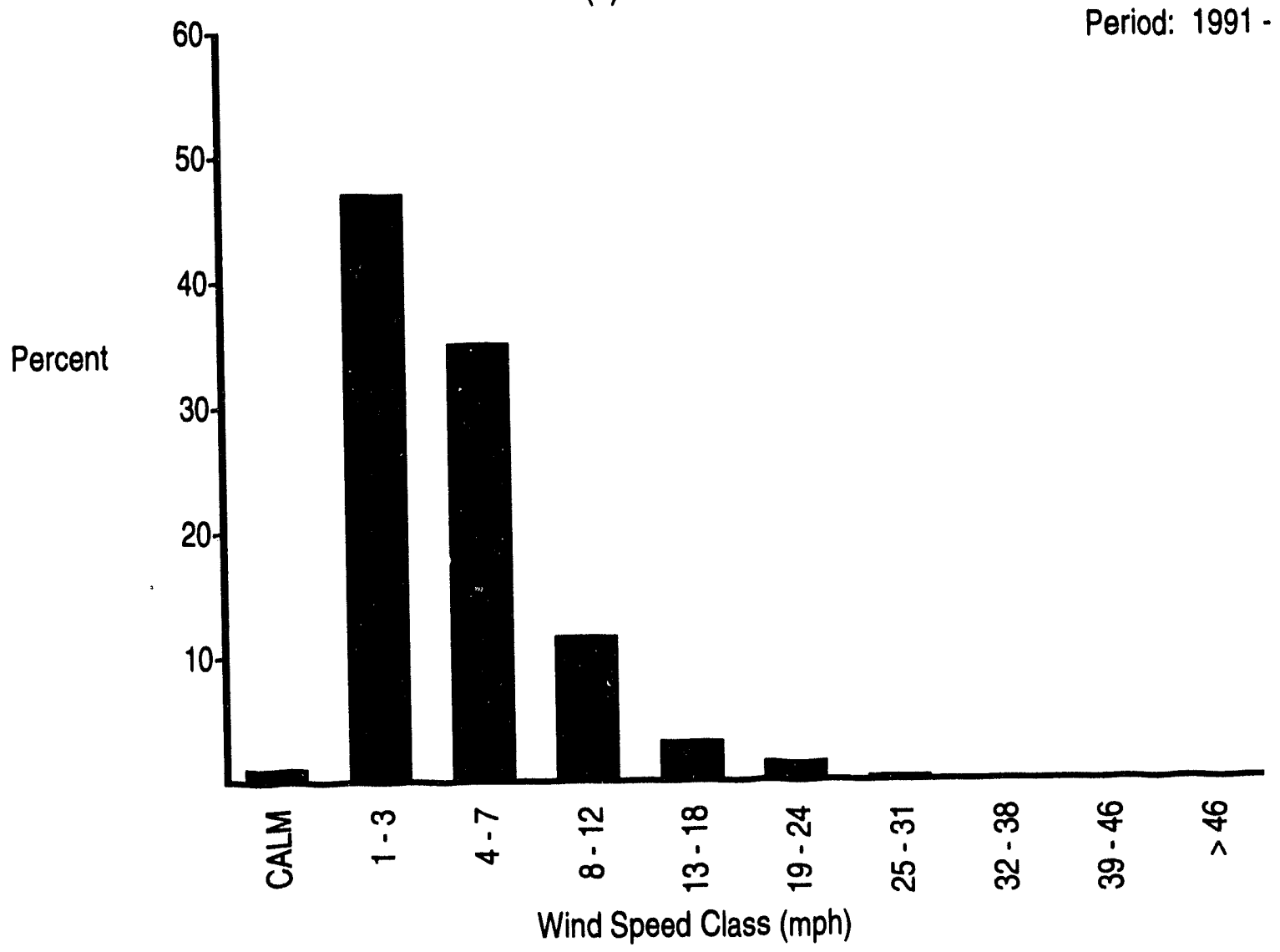

(b) Wind Speed Histogram

FIGURE B.1. (contd) 


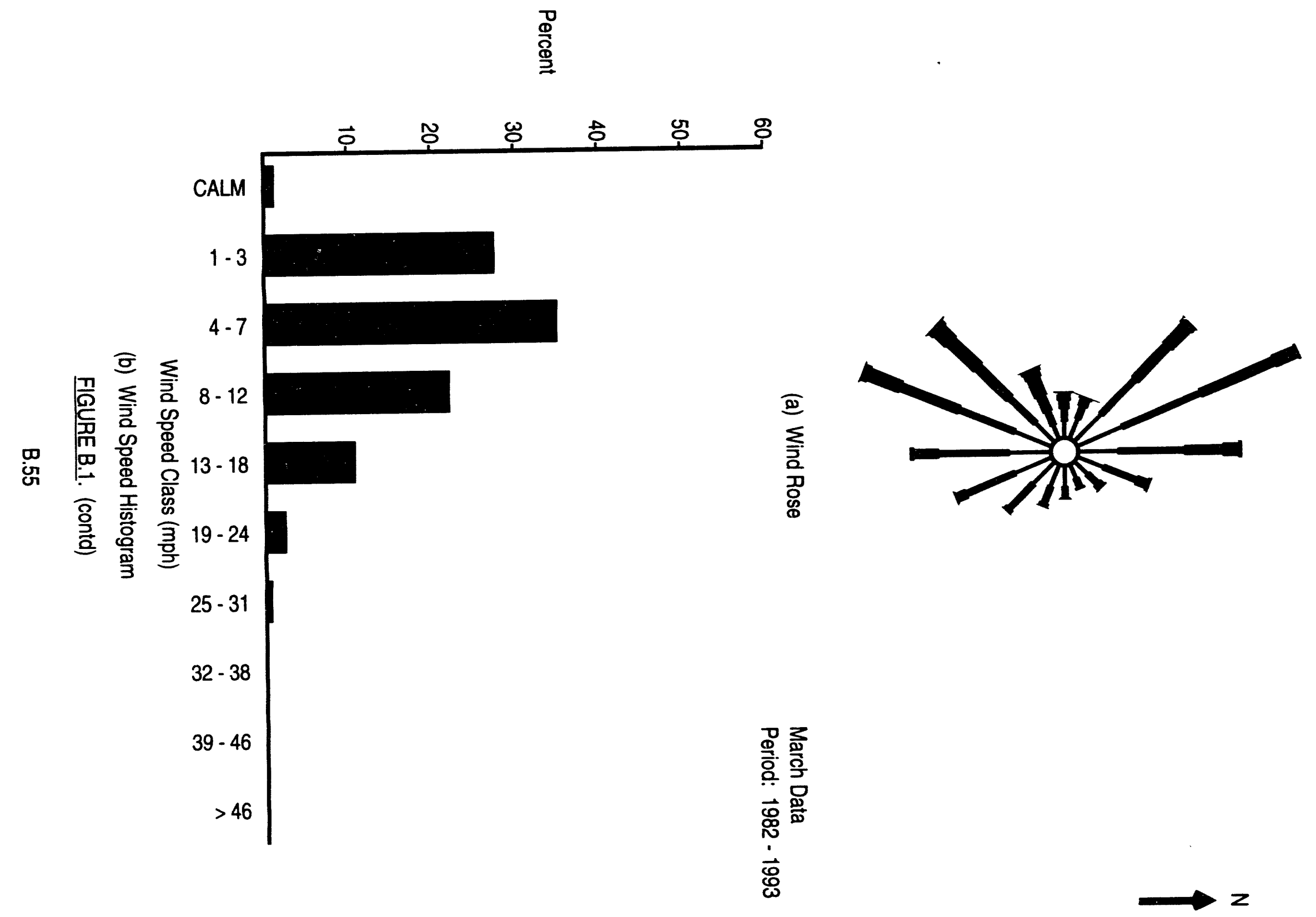




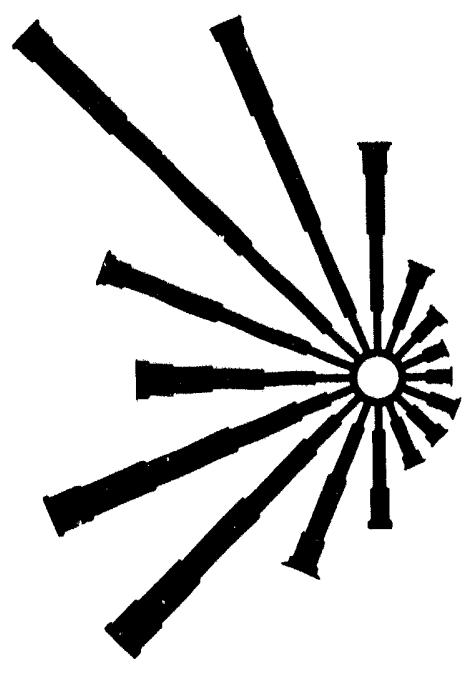

(a) Wind Rose

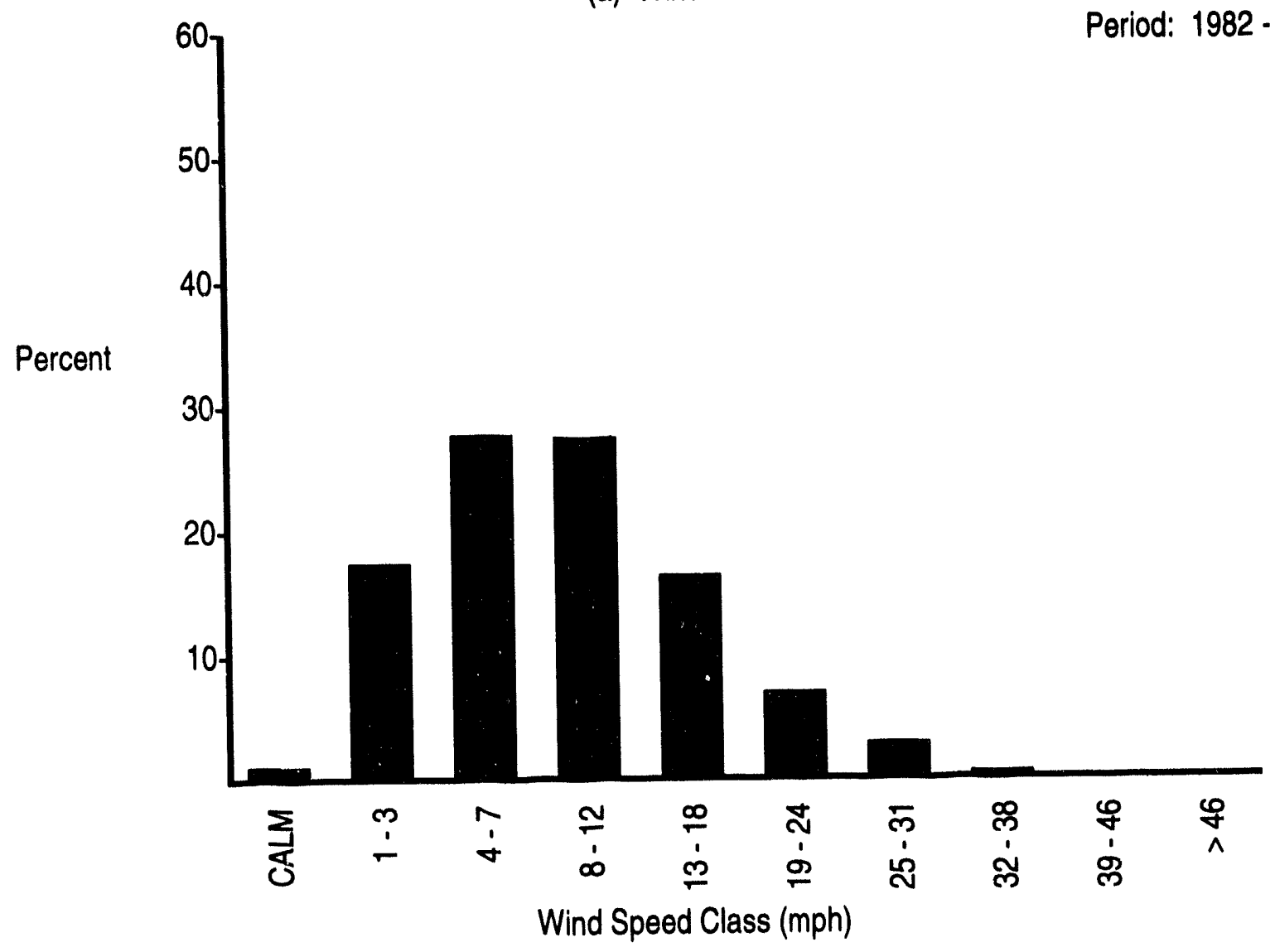

(b) Wind Speed Histogram

FIGURE B.1. (contd) 


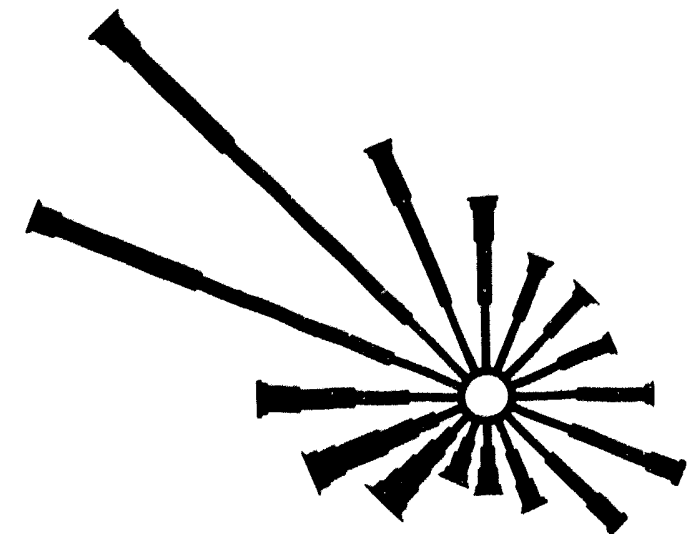

(a) Wind Rose

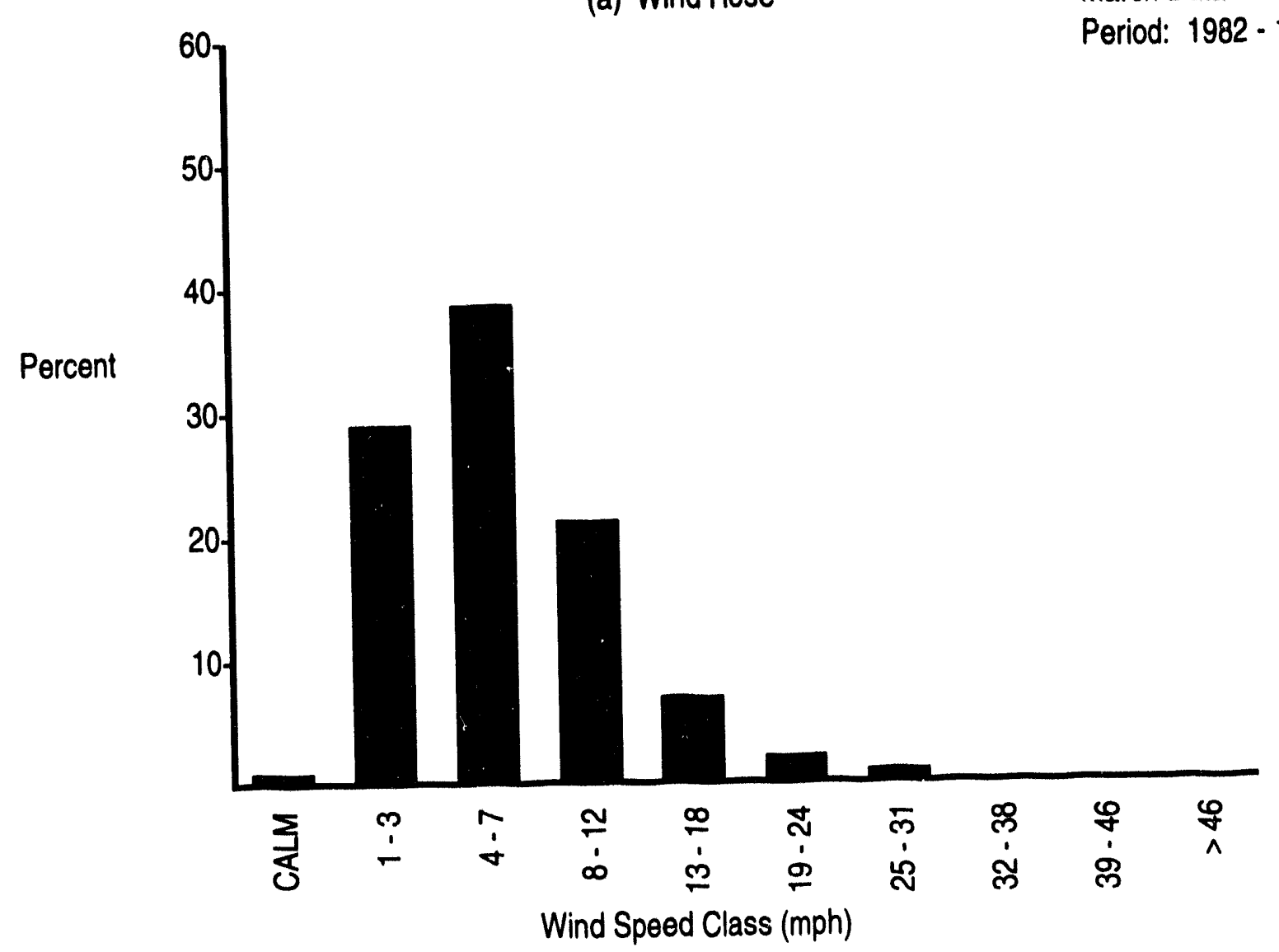

(b) Wind Speed Histogram

FIGURE B.1. (contd)
March Data

Period: 1982 - 1993 


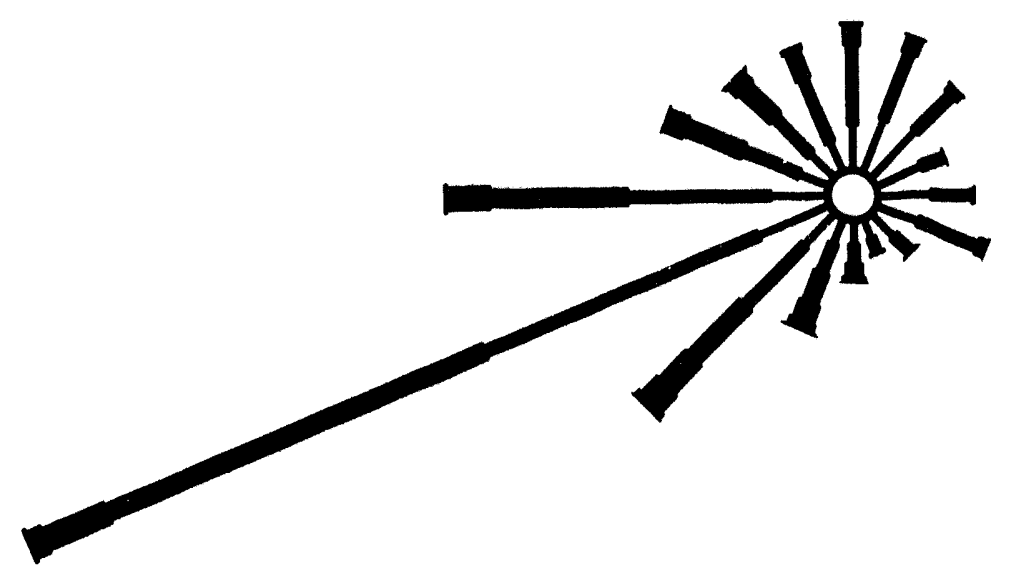

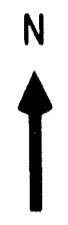

(a) Wind Rose

March Data

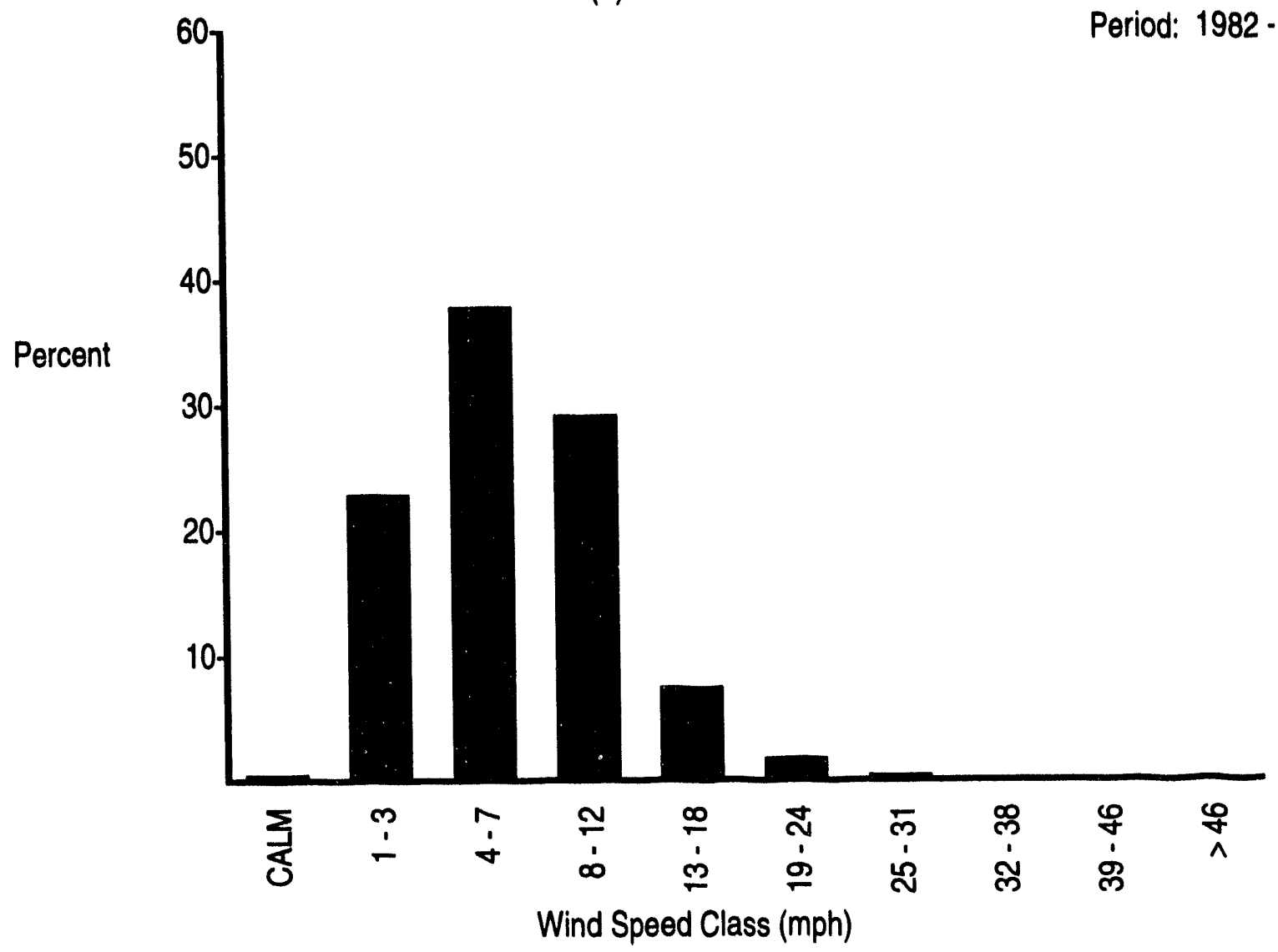

(b) Wind Speed Histogram

FIGURE B.1. (contd) 


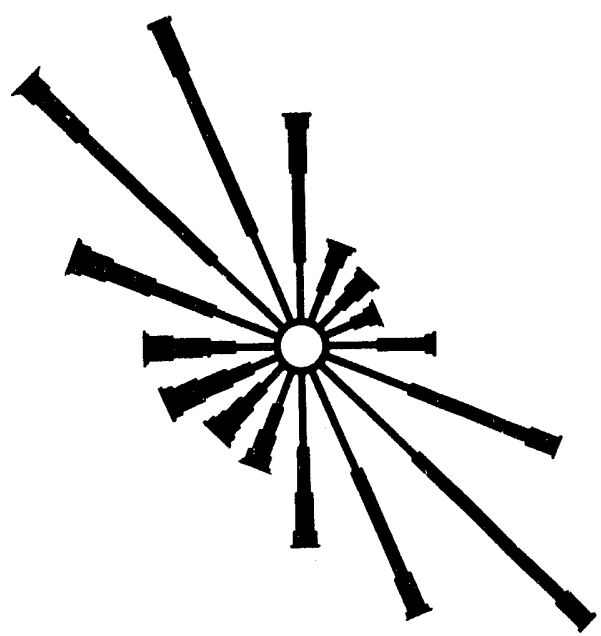

(a) Wind Rose

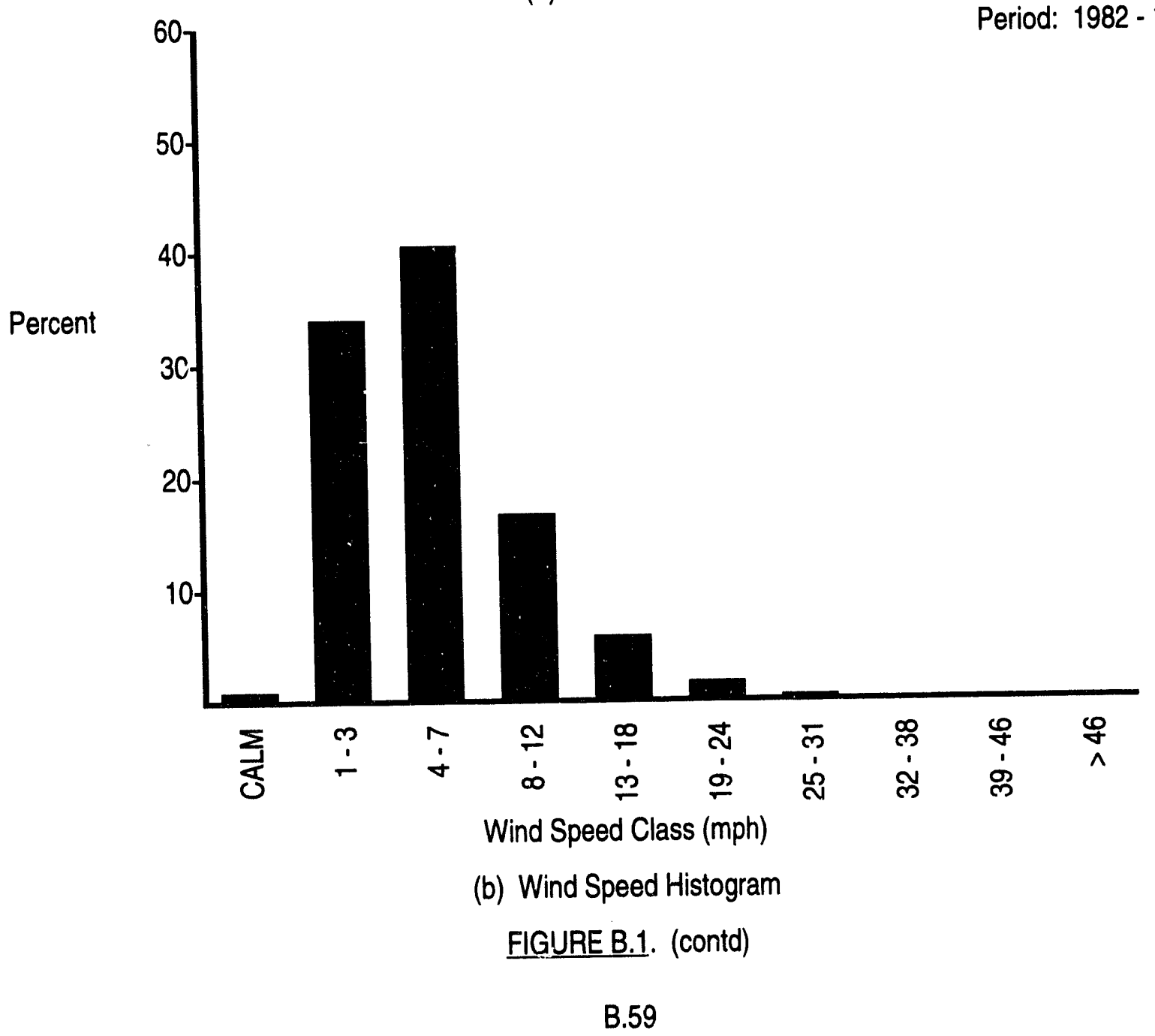

March Data

Period: 1982 - 1993 


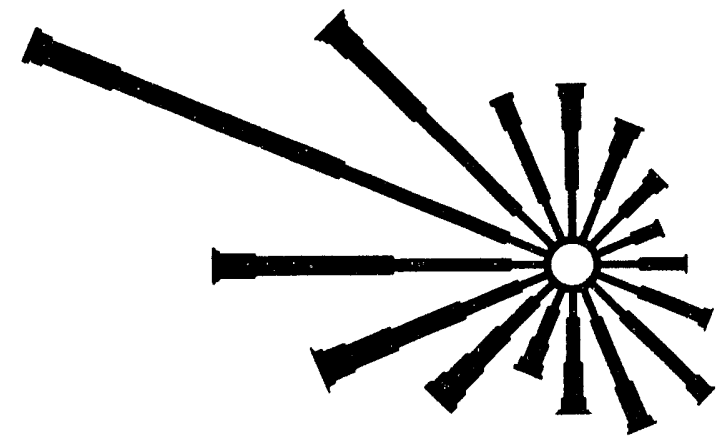

(a) Wind Rose

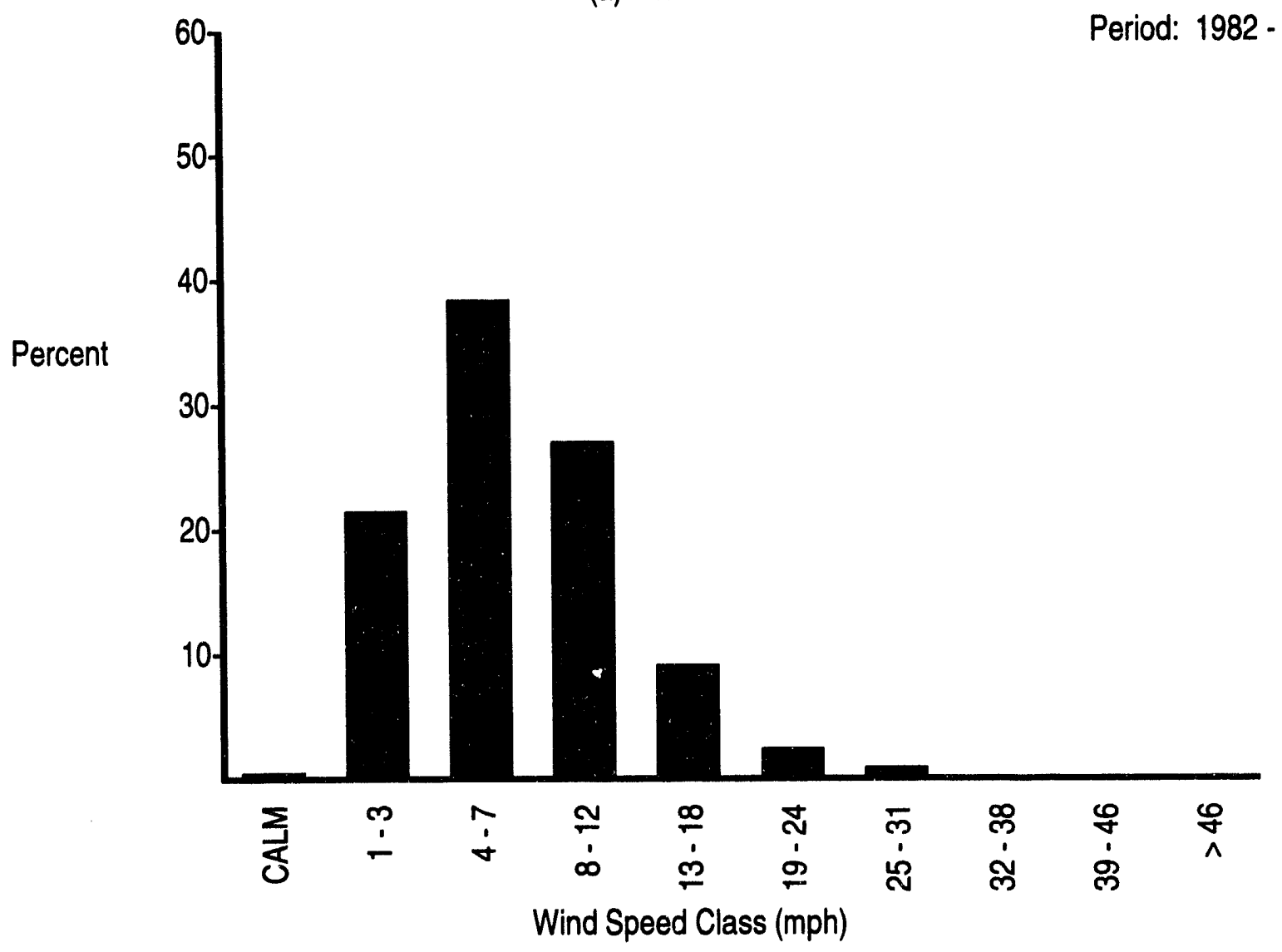

(b) Wind Speed Histogram

FIGURE B.1. (contd) 

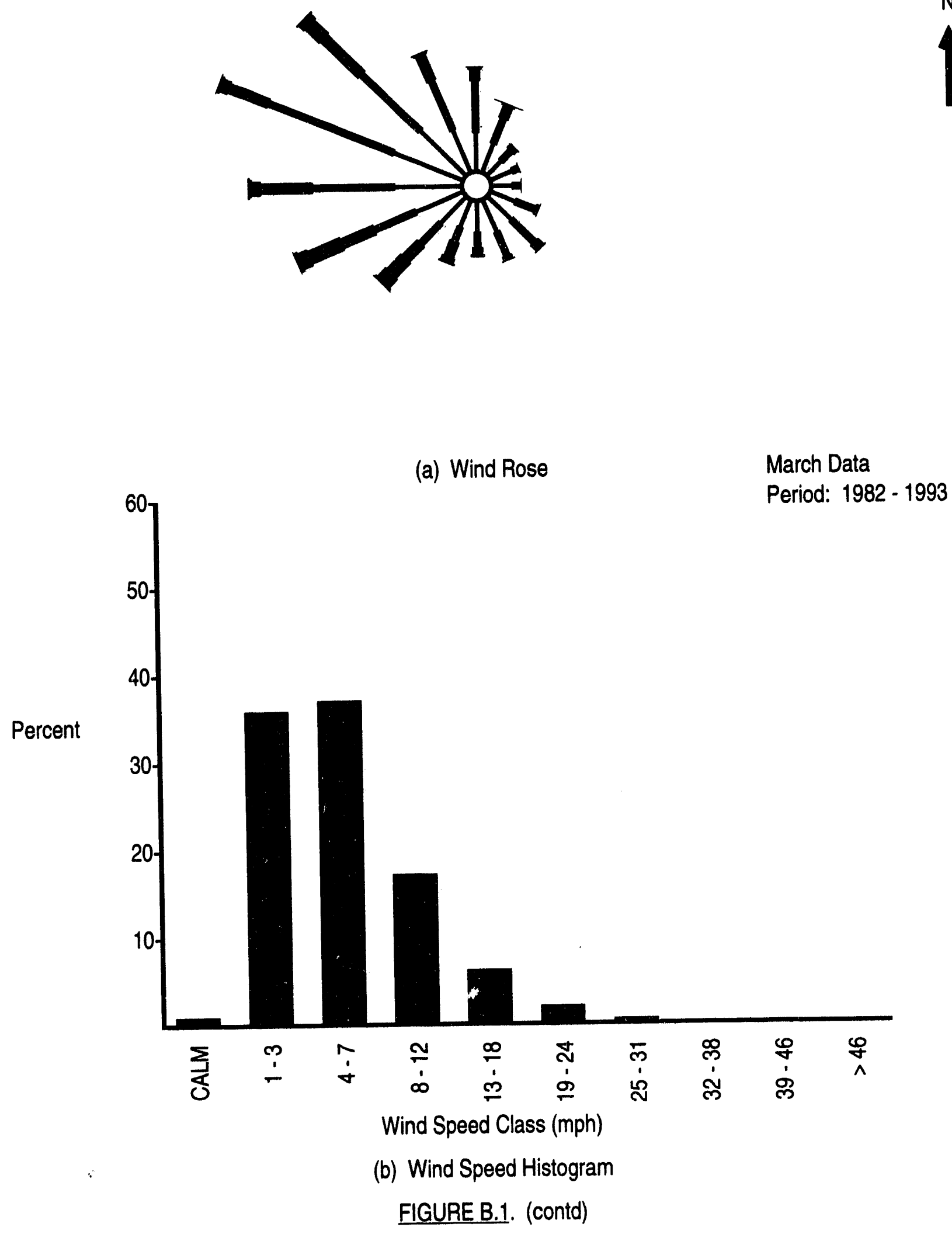

B.61 


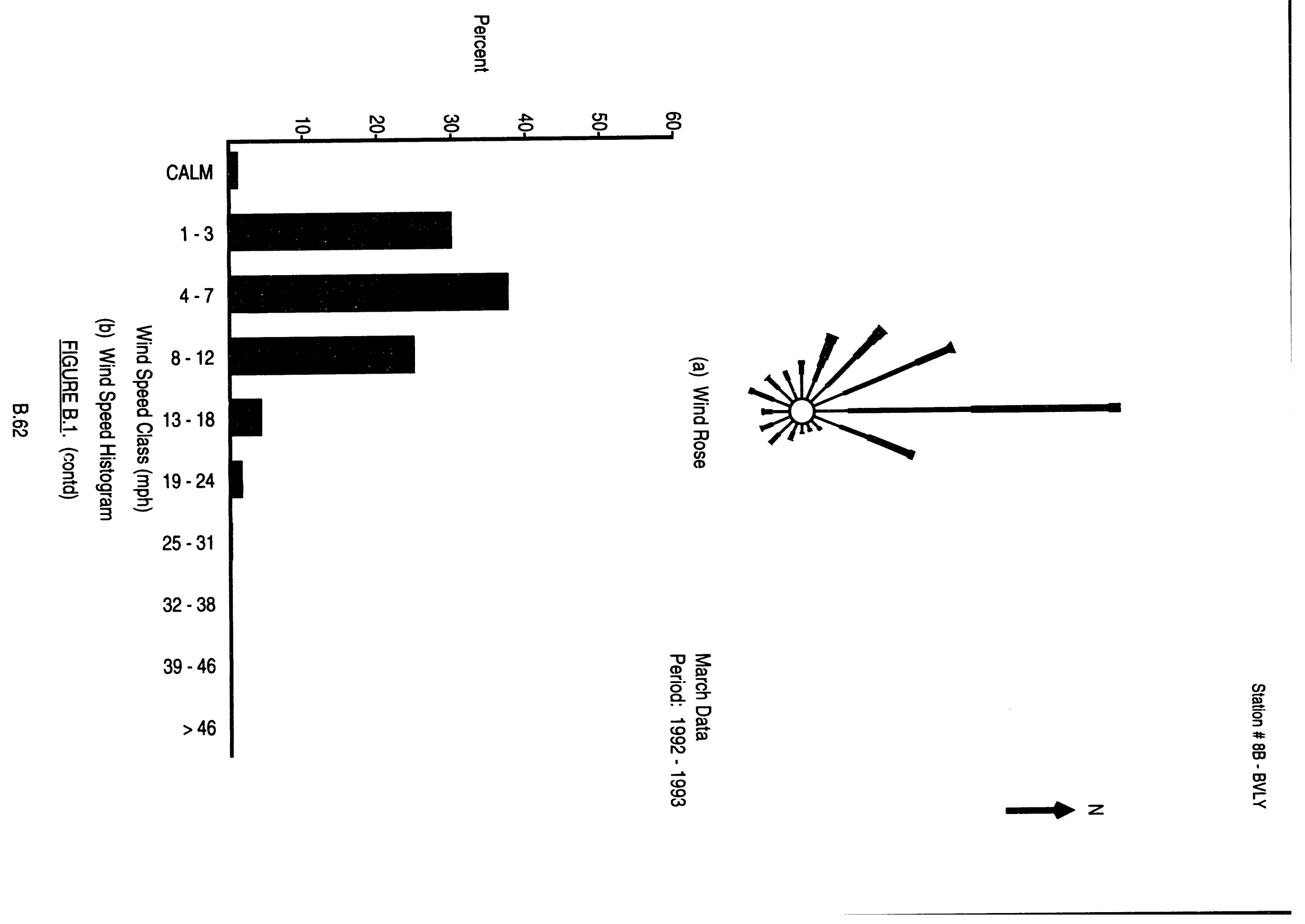




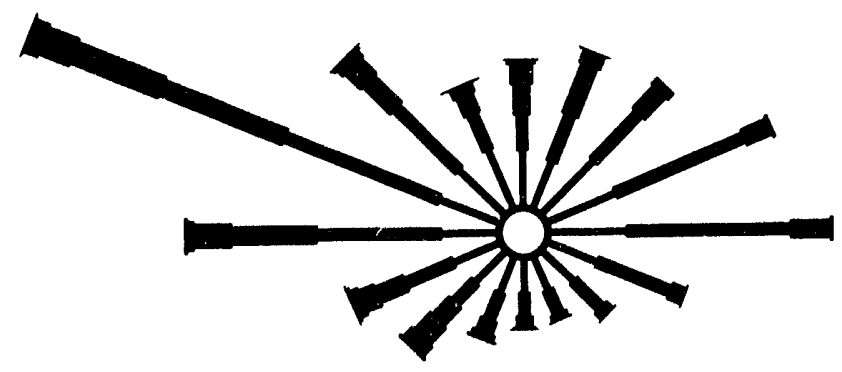

(a) Wind Rose

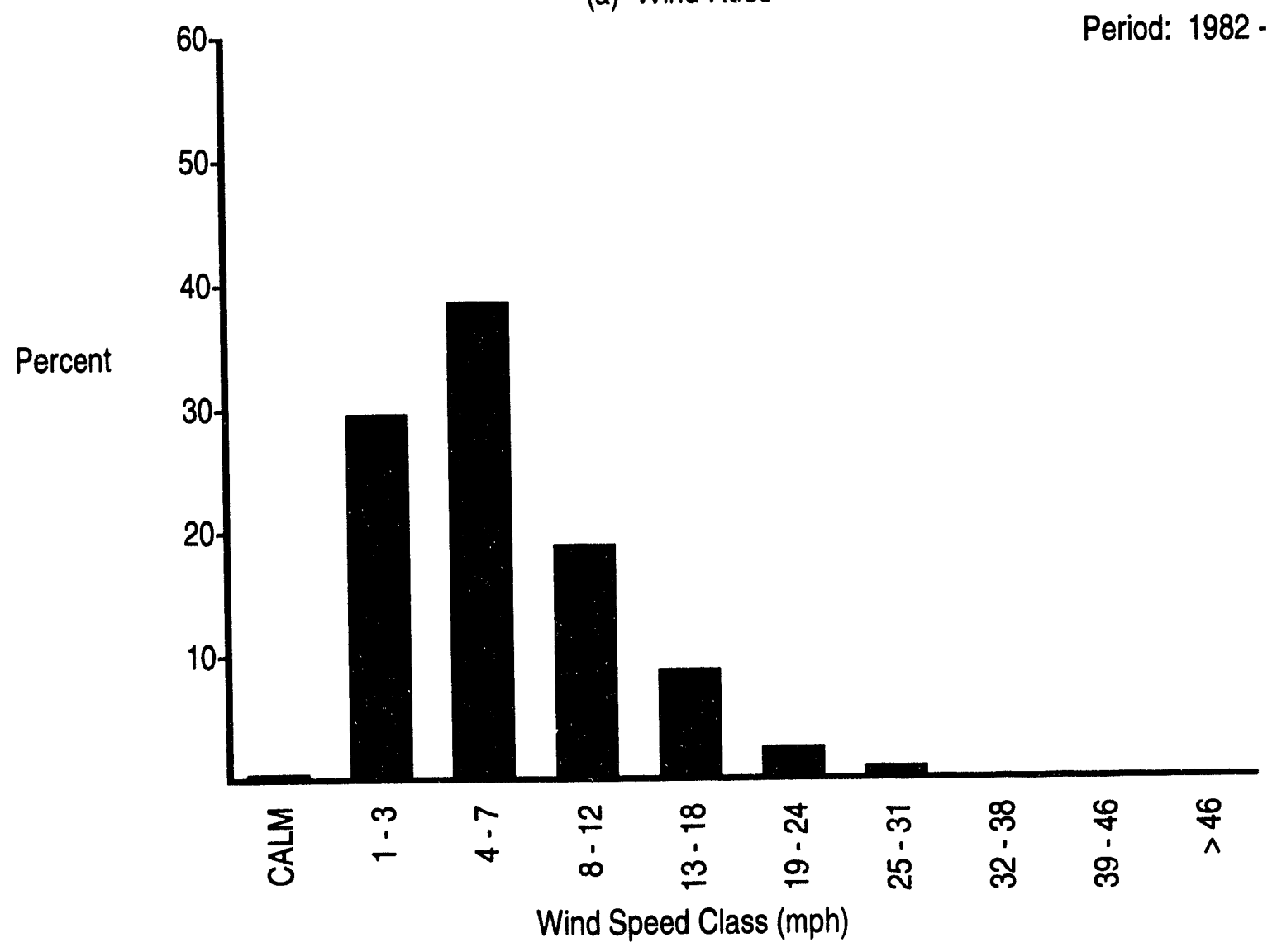

(b) Wind Speed Histogram

FIGURE B.1. (contd)
March Data

Period: 1982 - 1991 


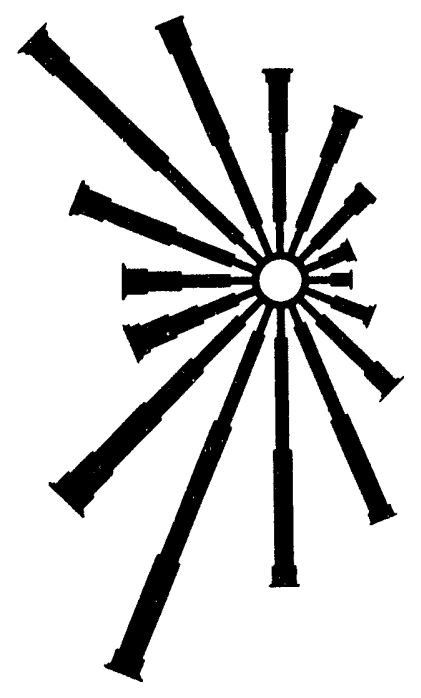

(a) Wind Rose

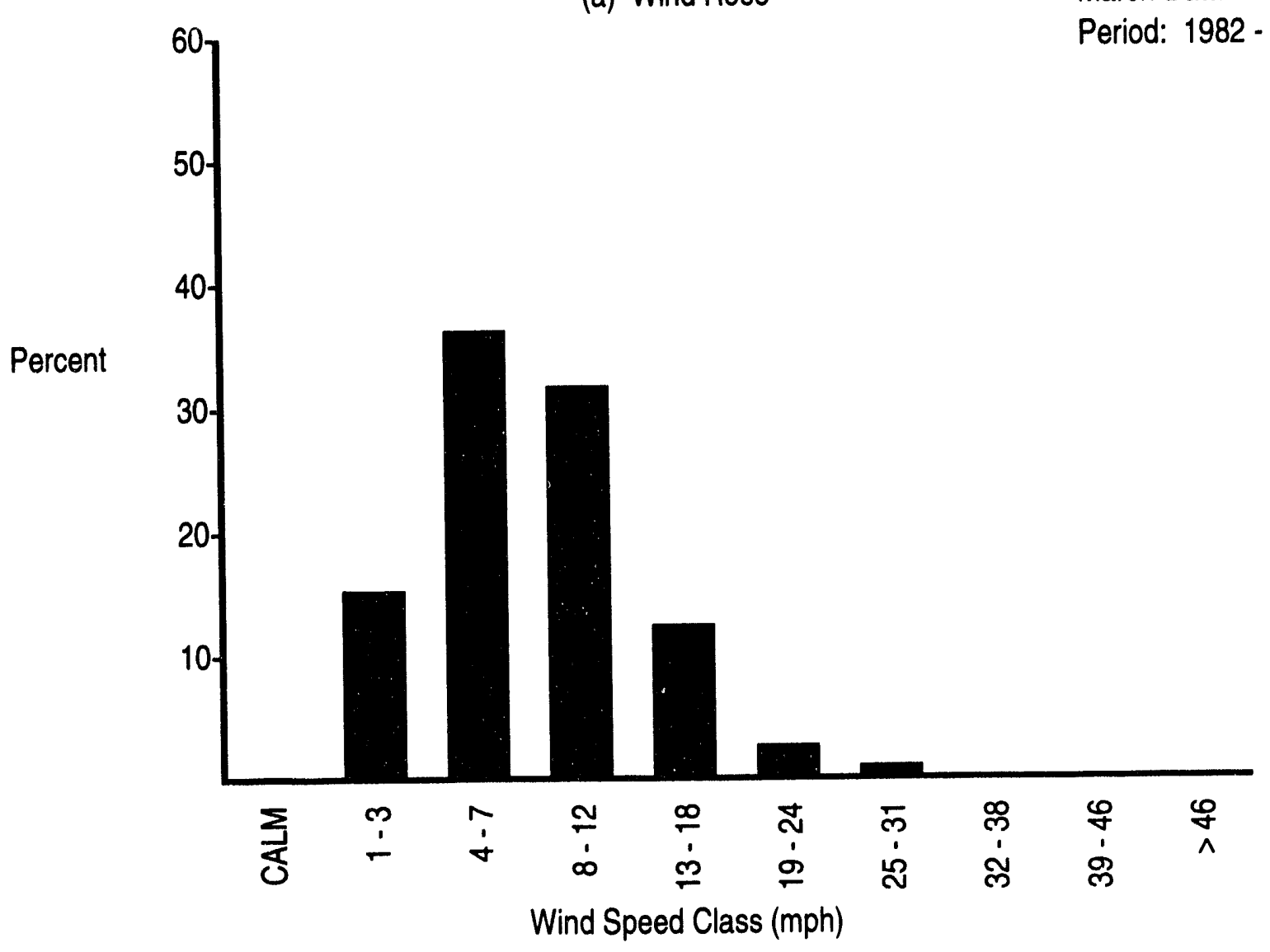

(b) Wind Speed Histogram

FIGURE B.1. (contd)
March Data

Period: 1982 - 1993

\section{B.64}




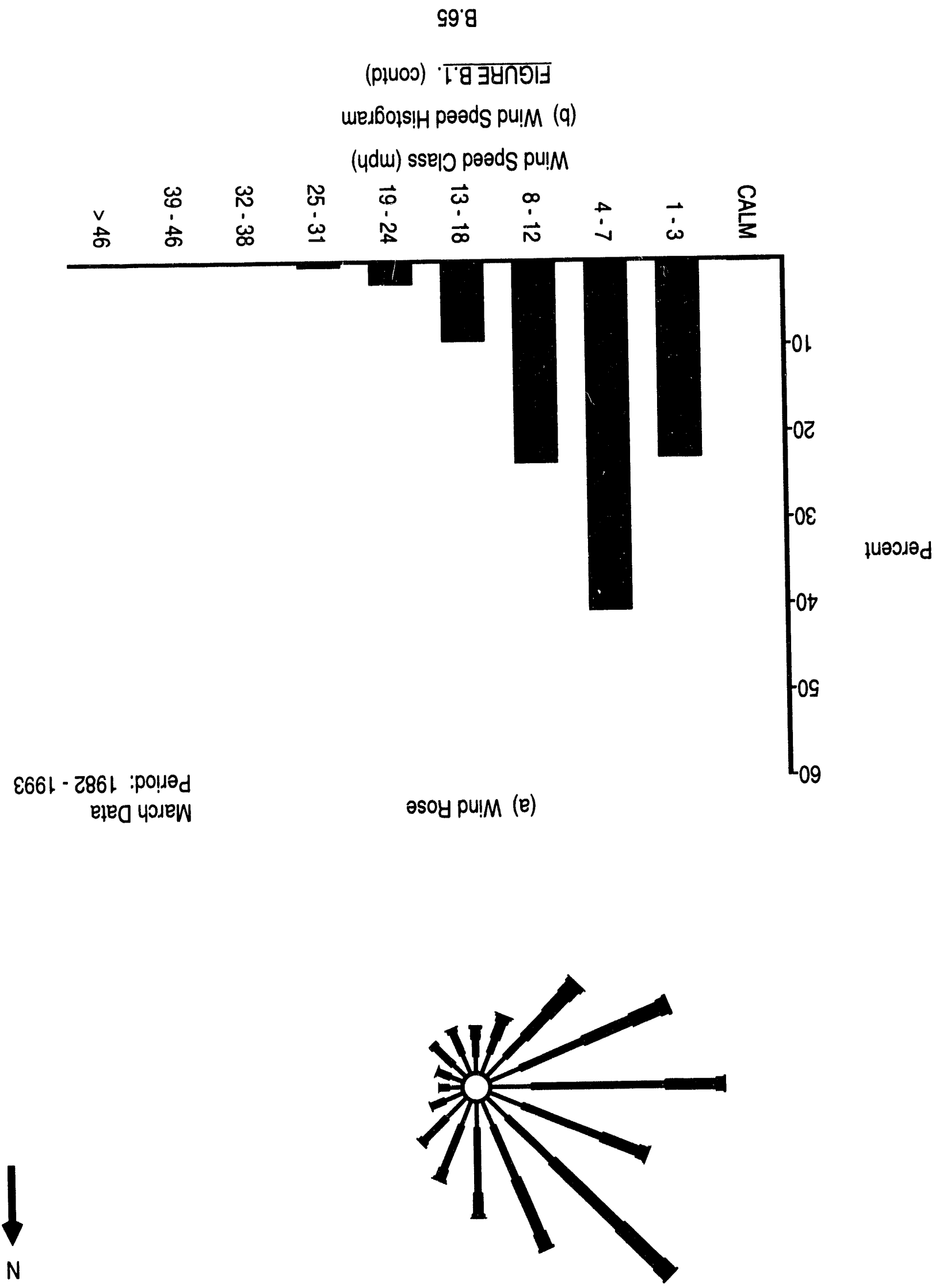




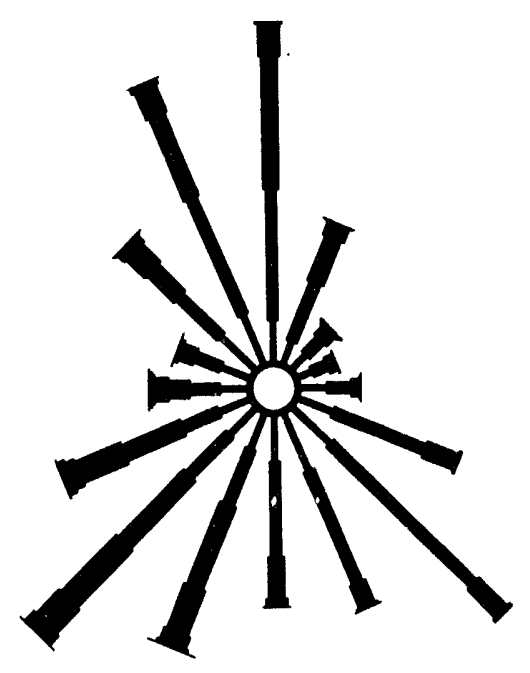

(a) Wind Rose

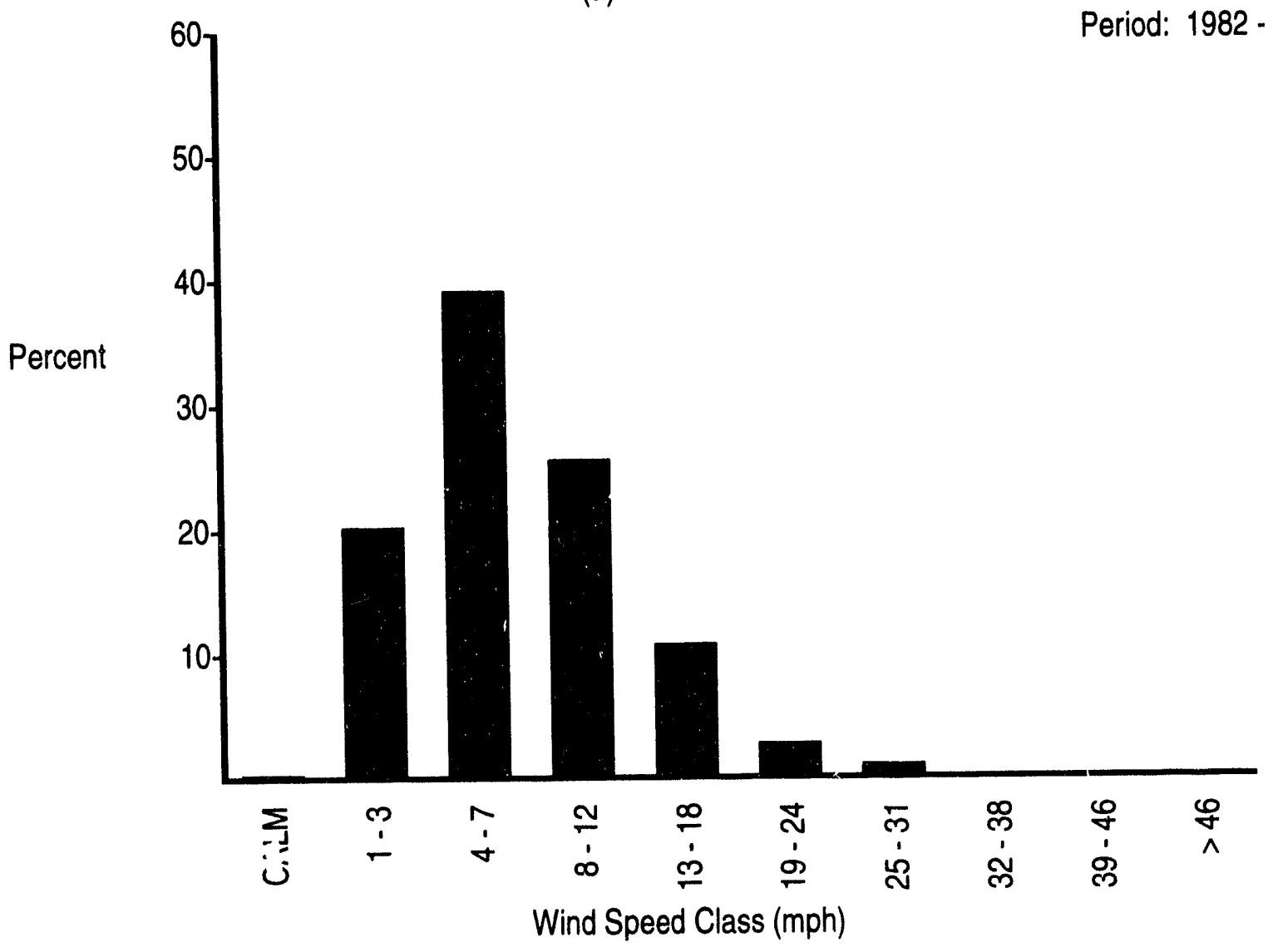

(b) Wind Speed Histogram

FIGURE B.1. (contd)
March Data

Period: 1982 - 1993

\section{B.66}




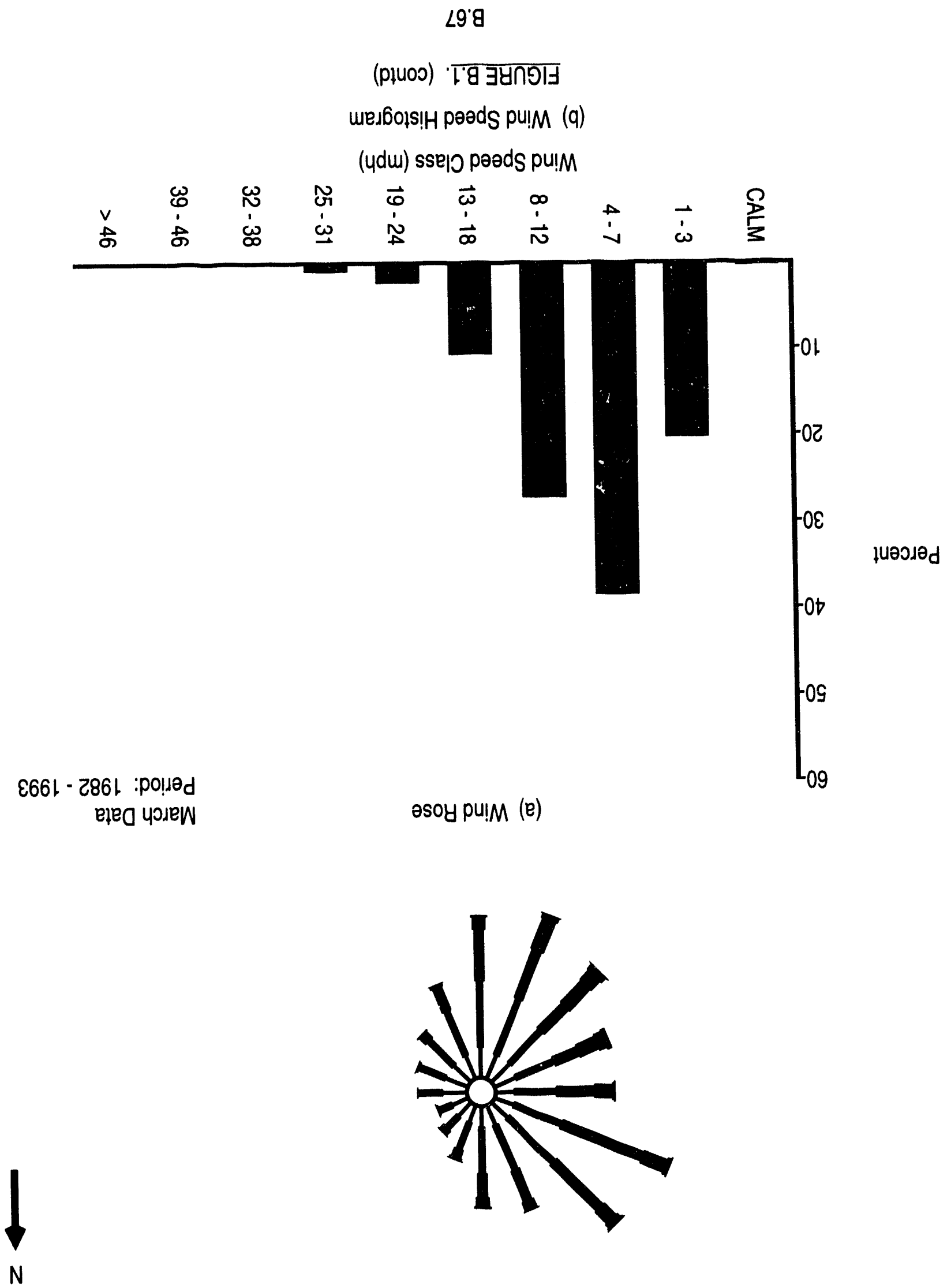




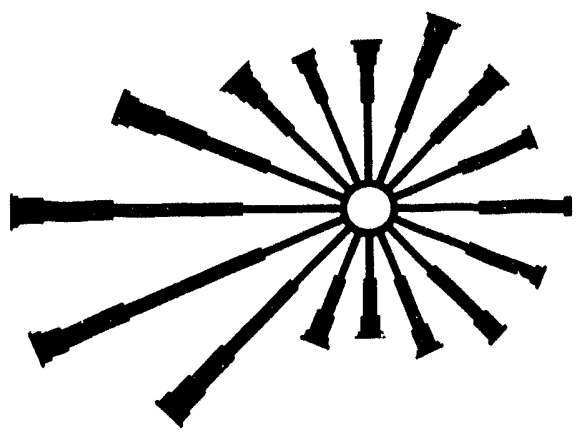

(a) Wind Rose

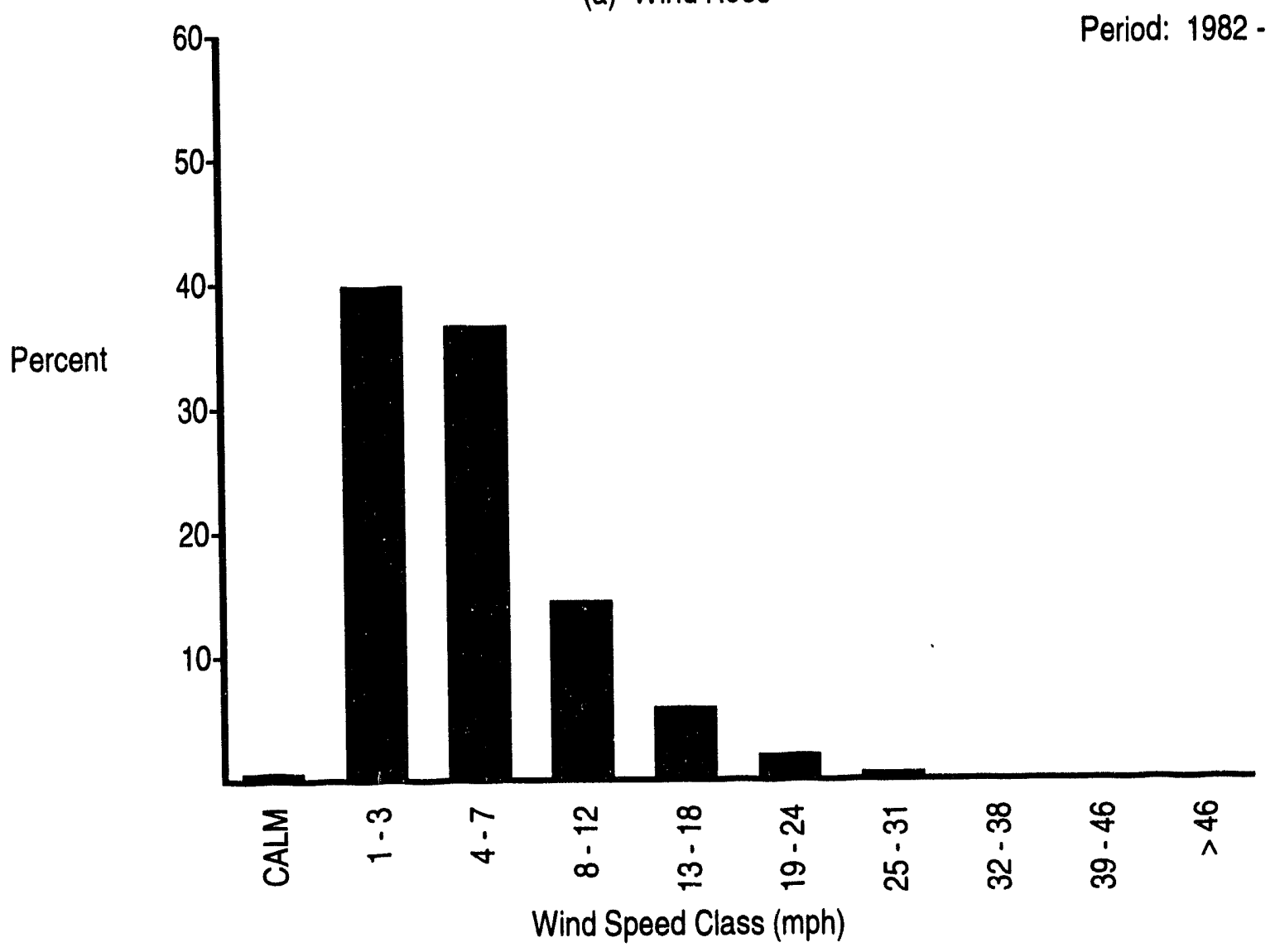

(b) Wind Speed Histogram

FIGURE B.1. (contd) 

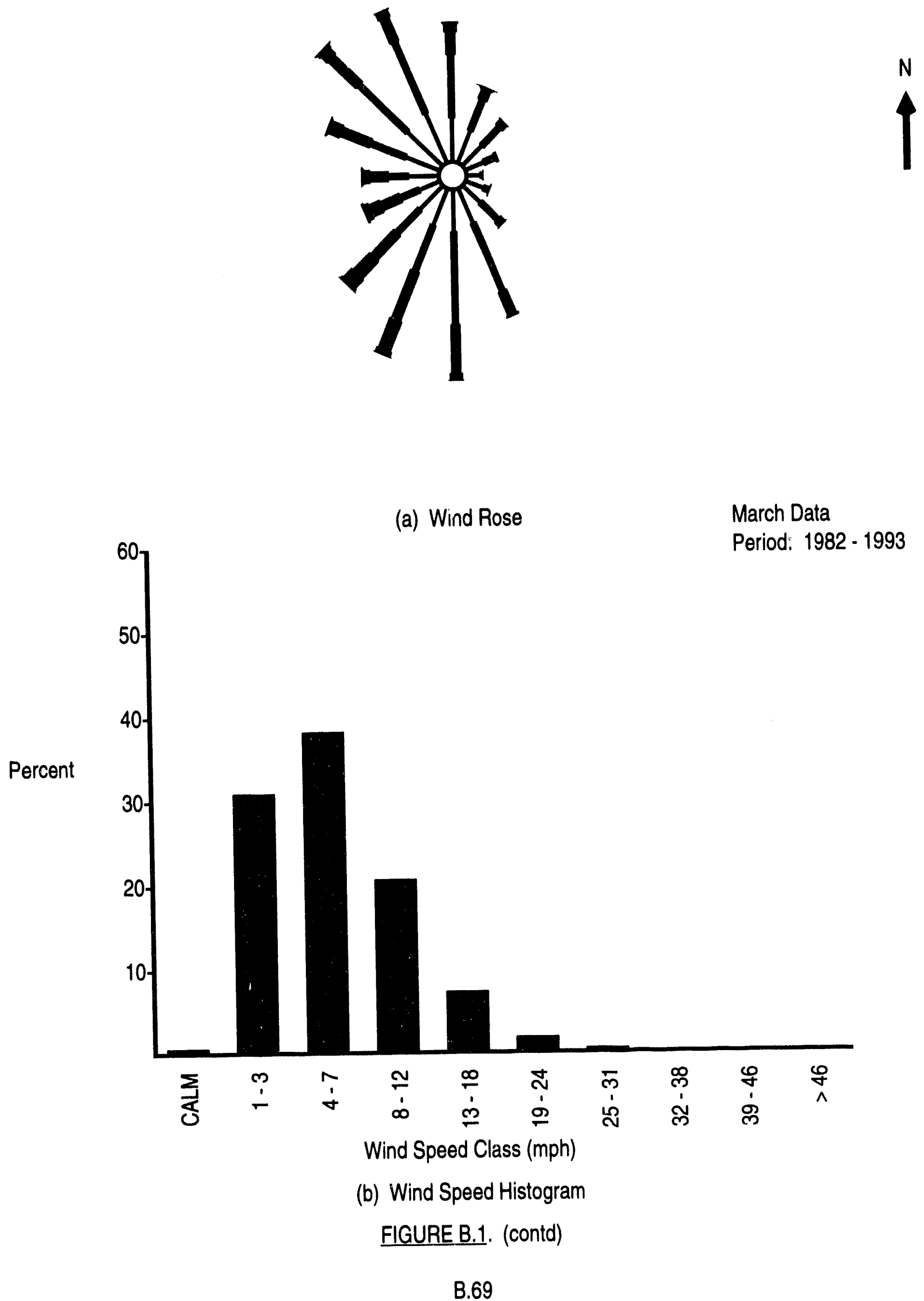

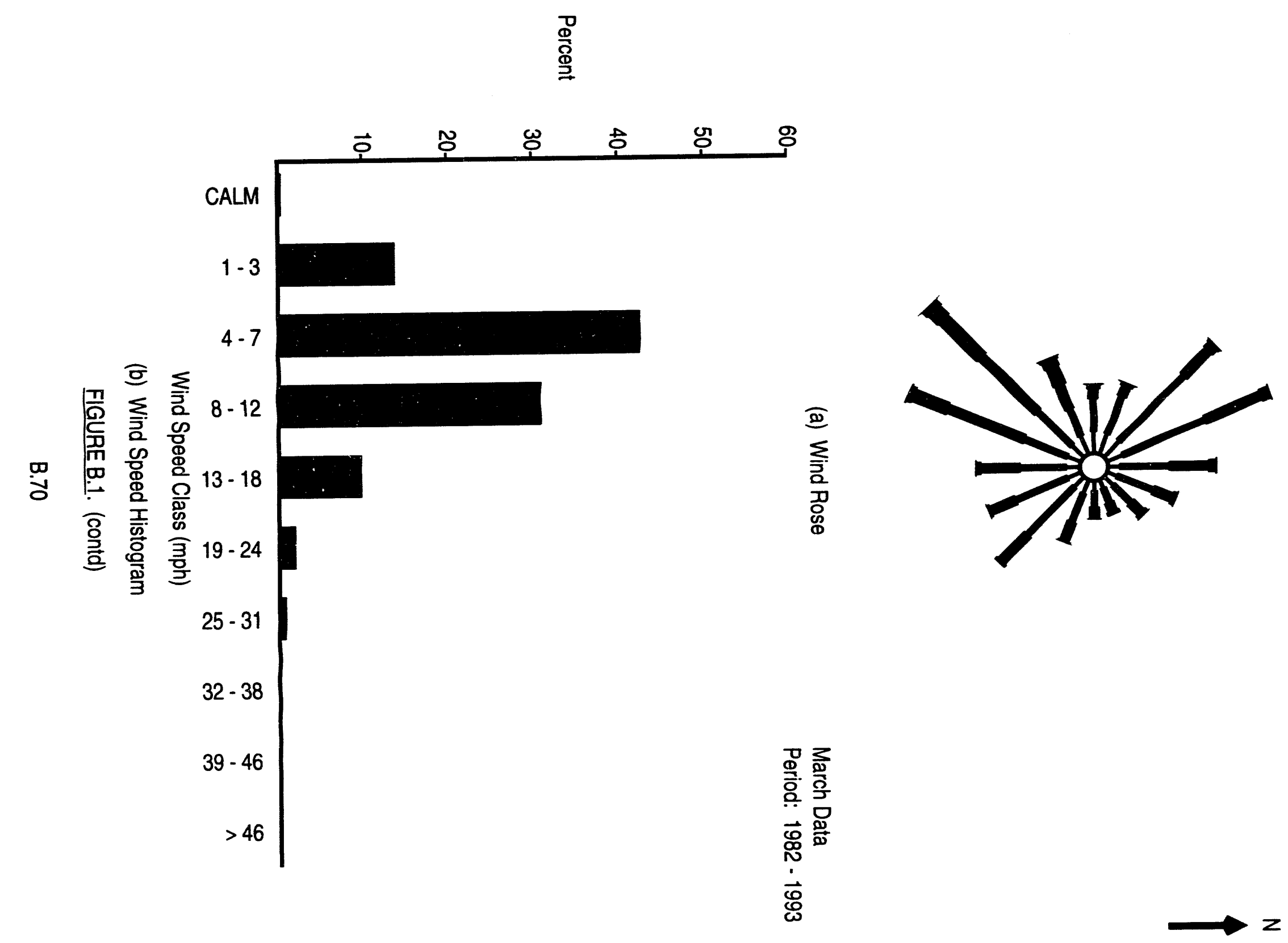


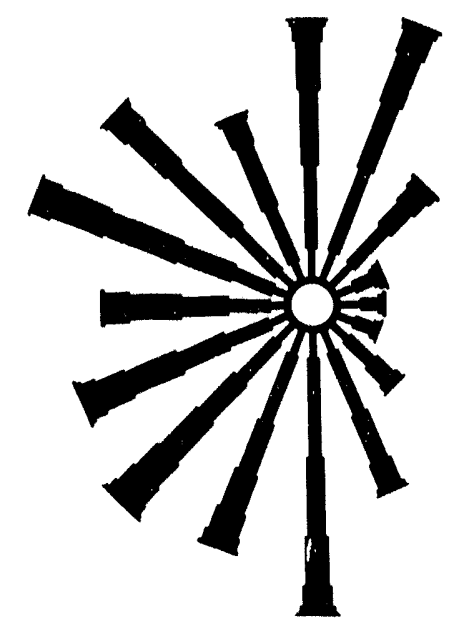

(a) Wind Rose

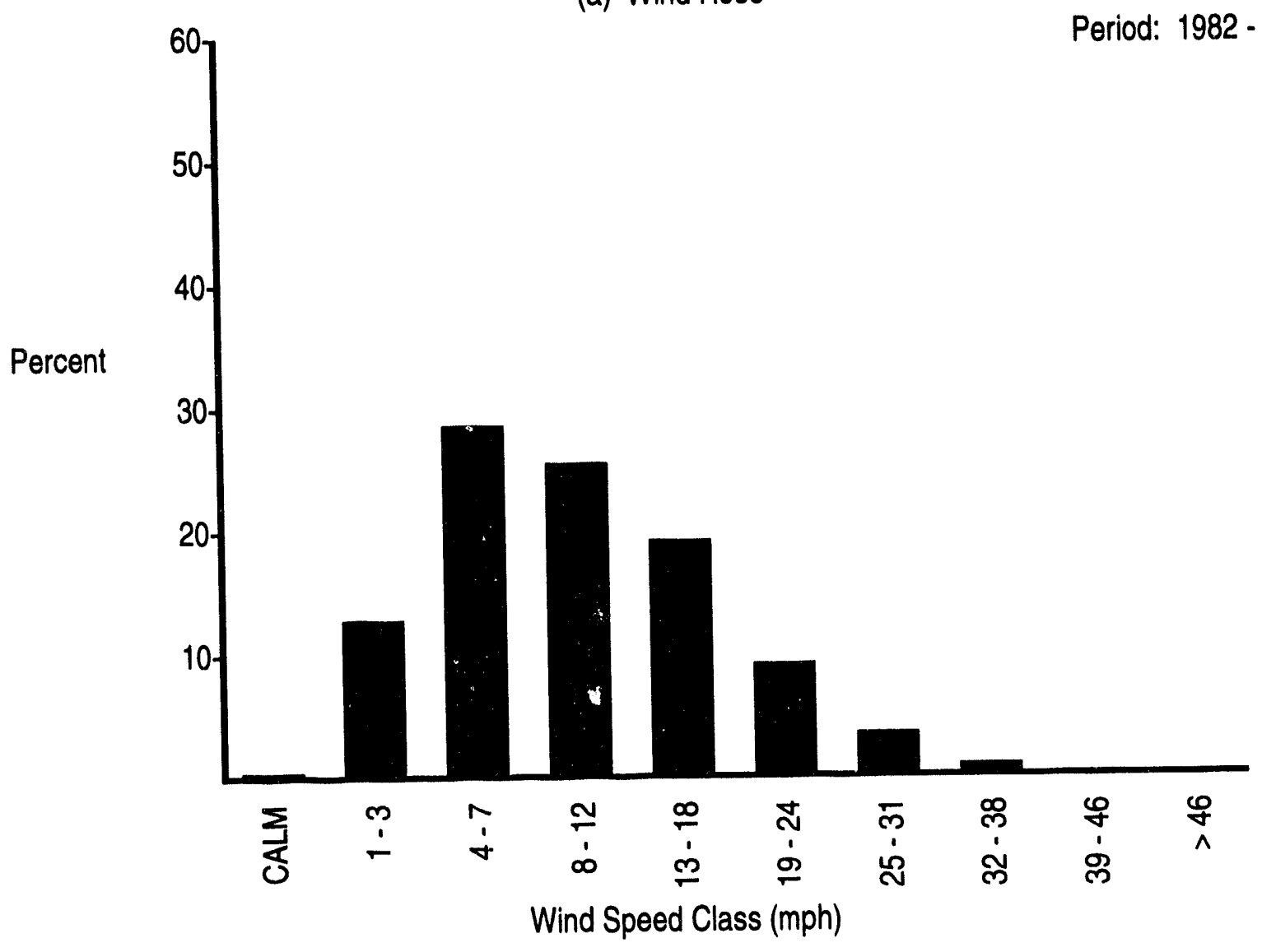

(b) Wind Speed Histogram

FIGURE B.1. (contd)
March Data

Period: 1982 - 9993 


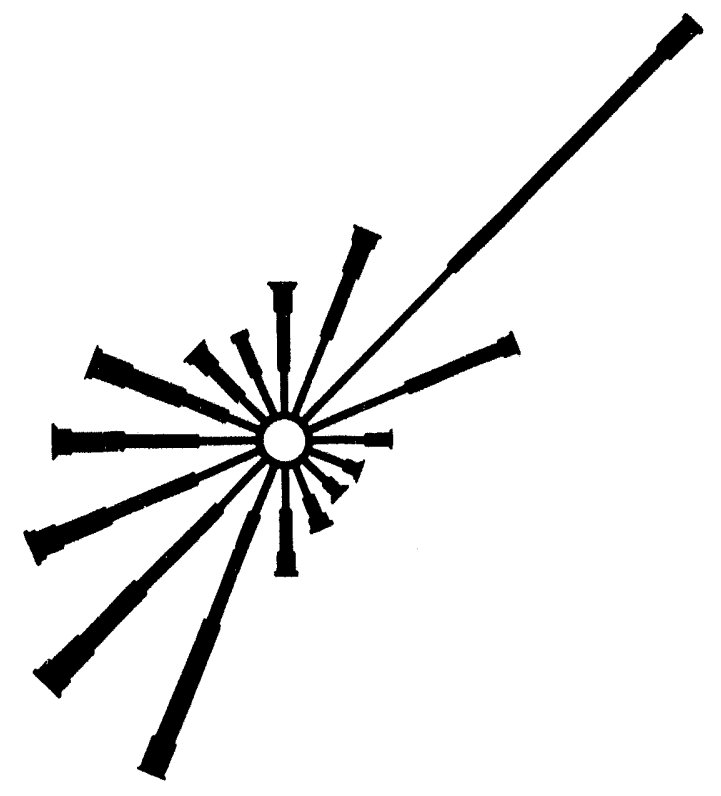

(a) Wind Rose

March Data

Period: 1982 - 1993

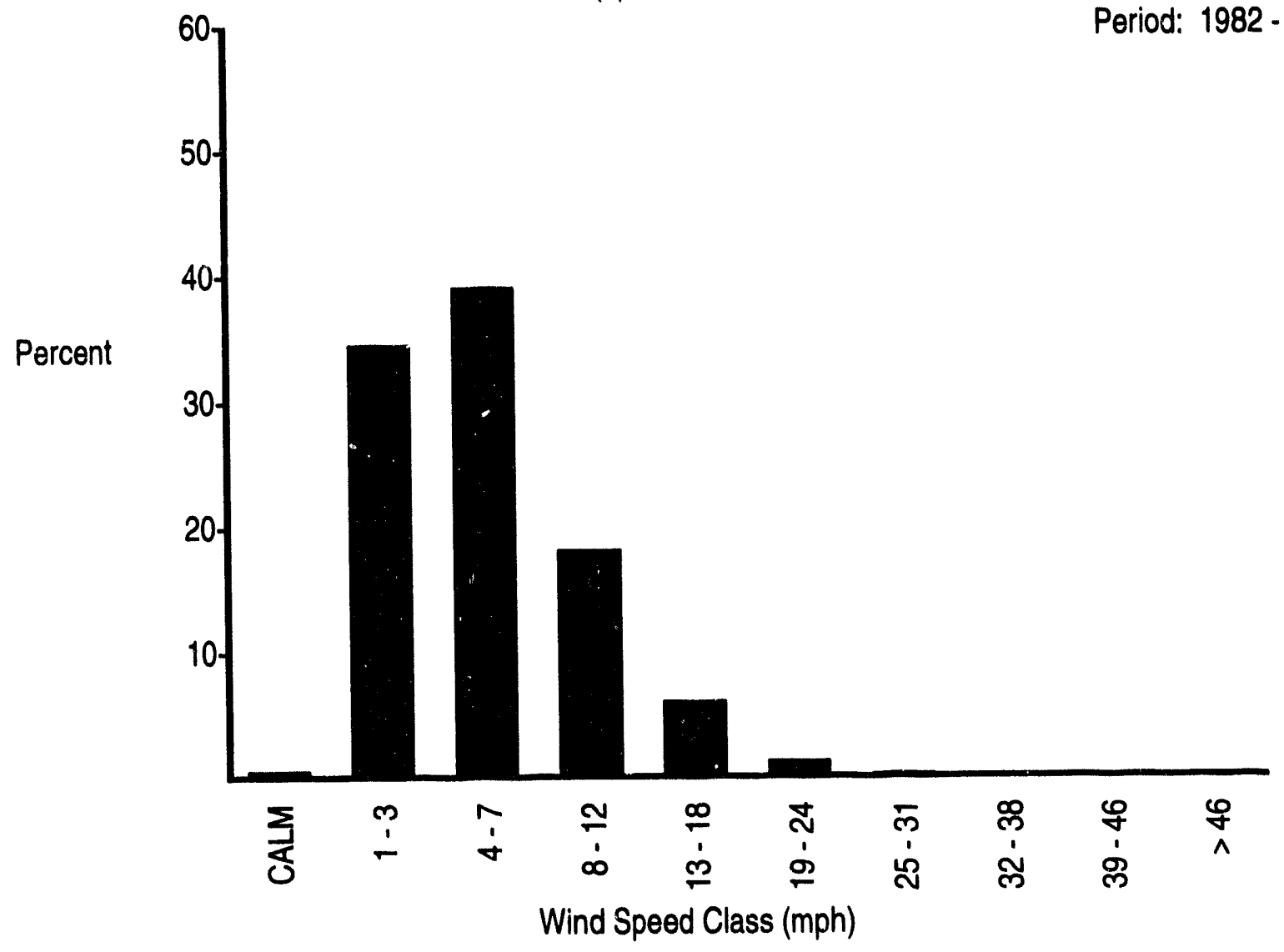

(b) Wind Speed Histogram

FIGURE B.1. (contd) 

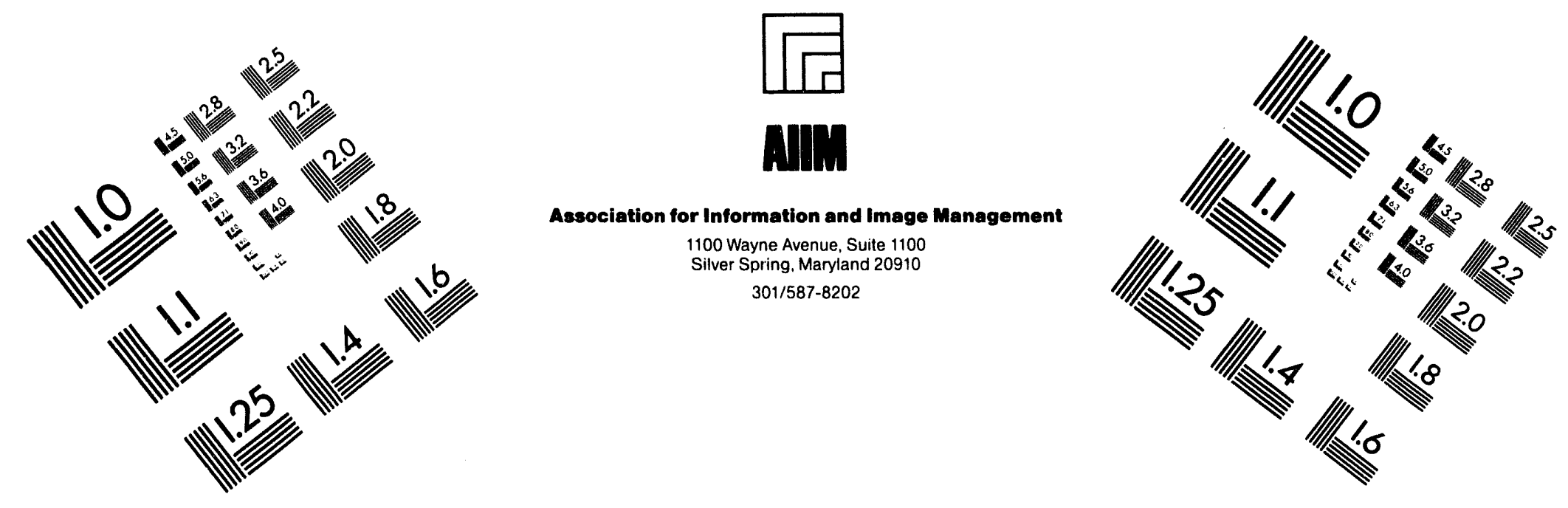

\section{Centimeter}

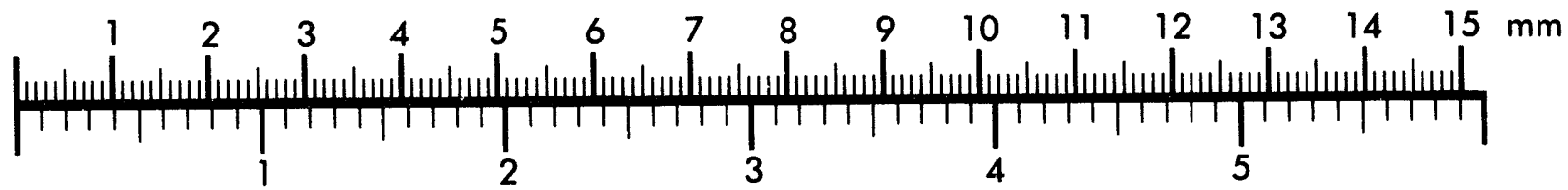
Inches
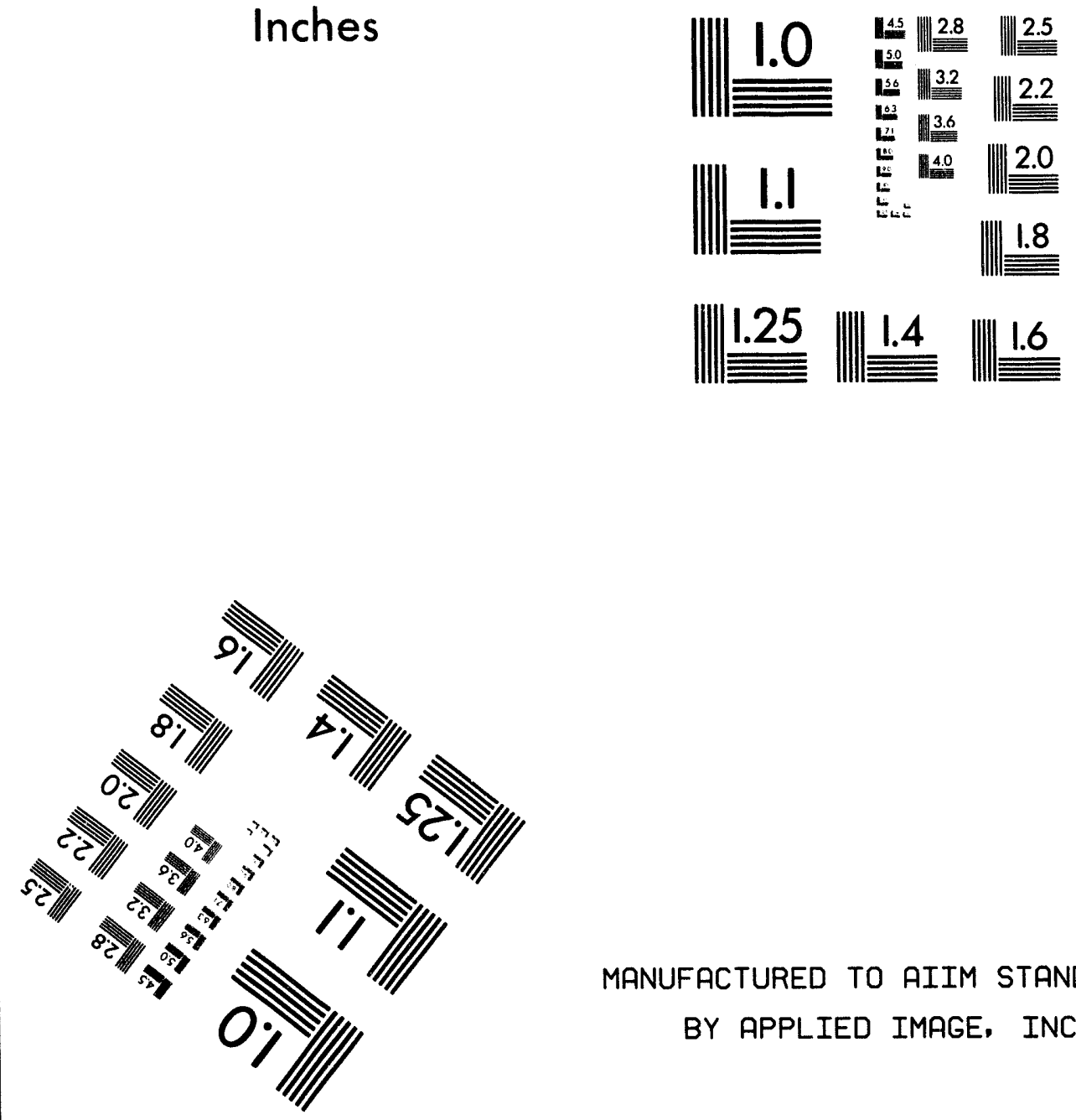

MANUFACTURED TO AIIM STANDARDS

BY APPLIED IMAGE, INC.

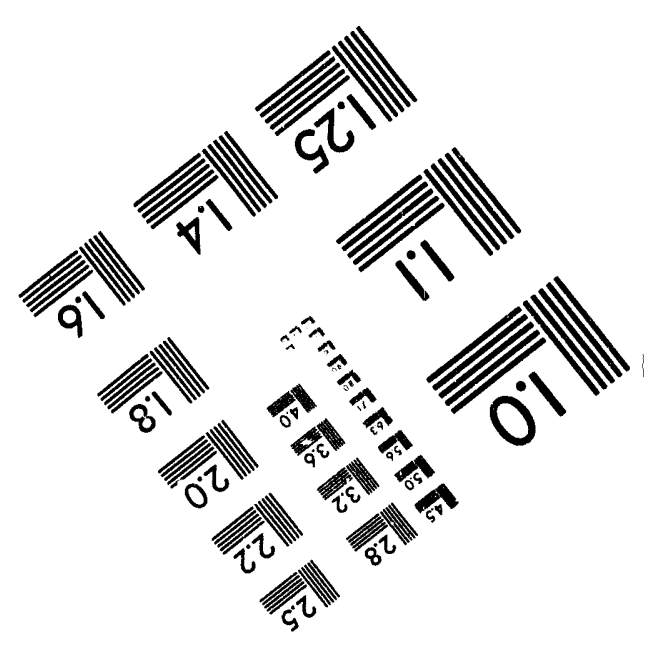



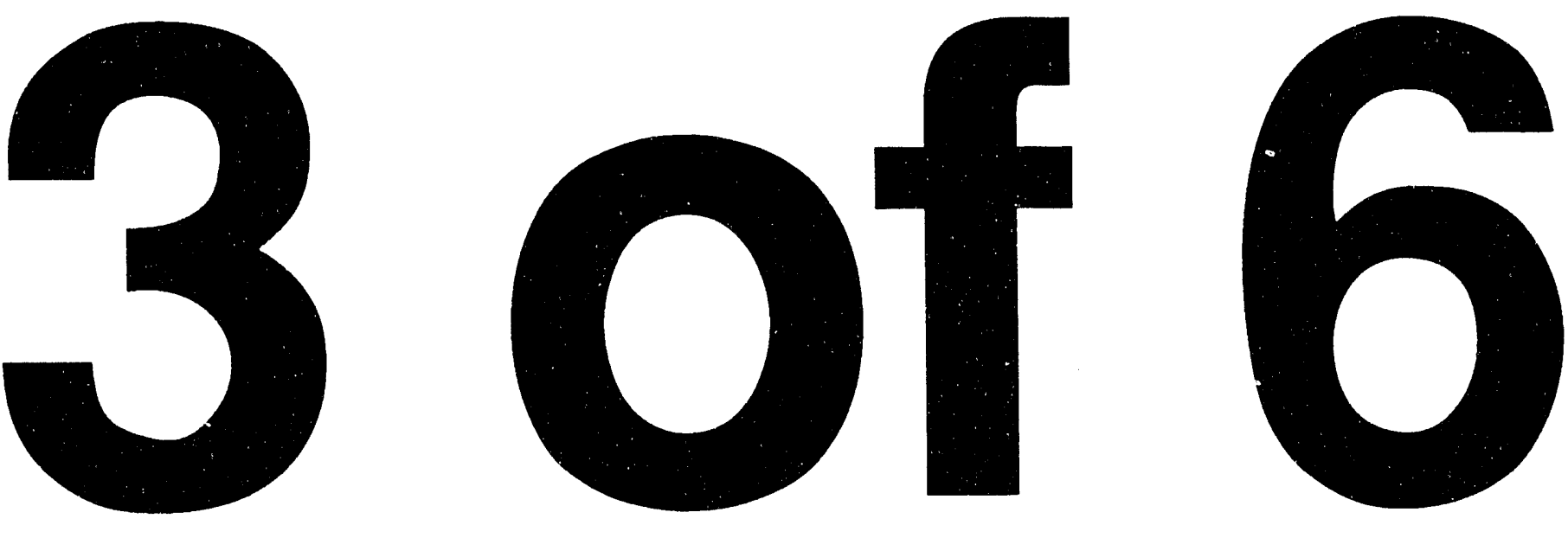

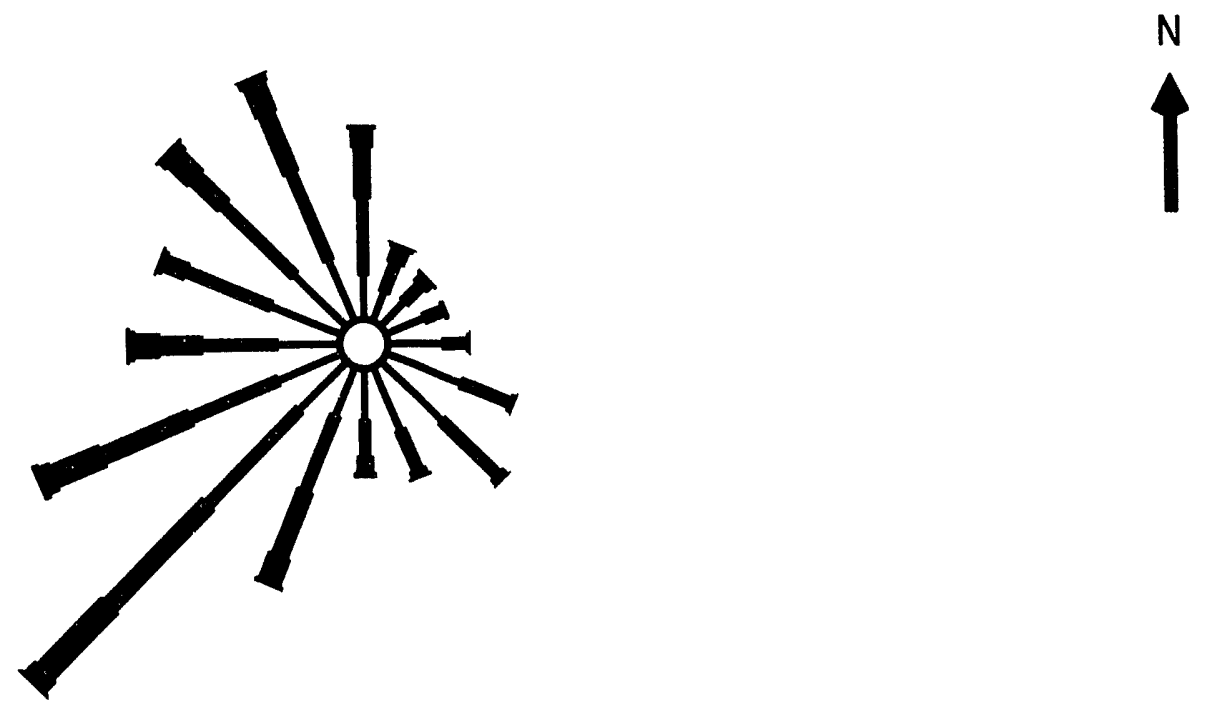

(a) Wind Rose

March Data

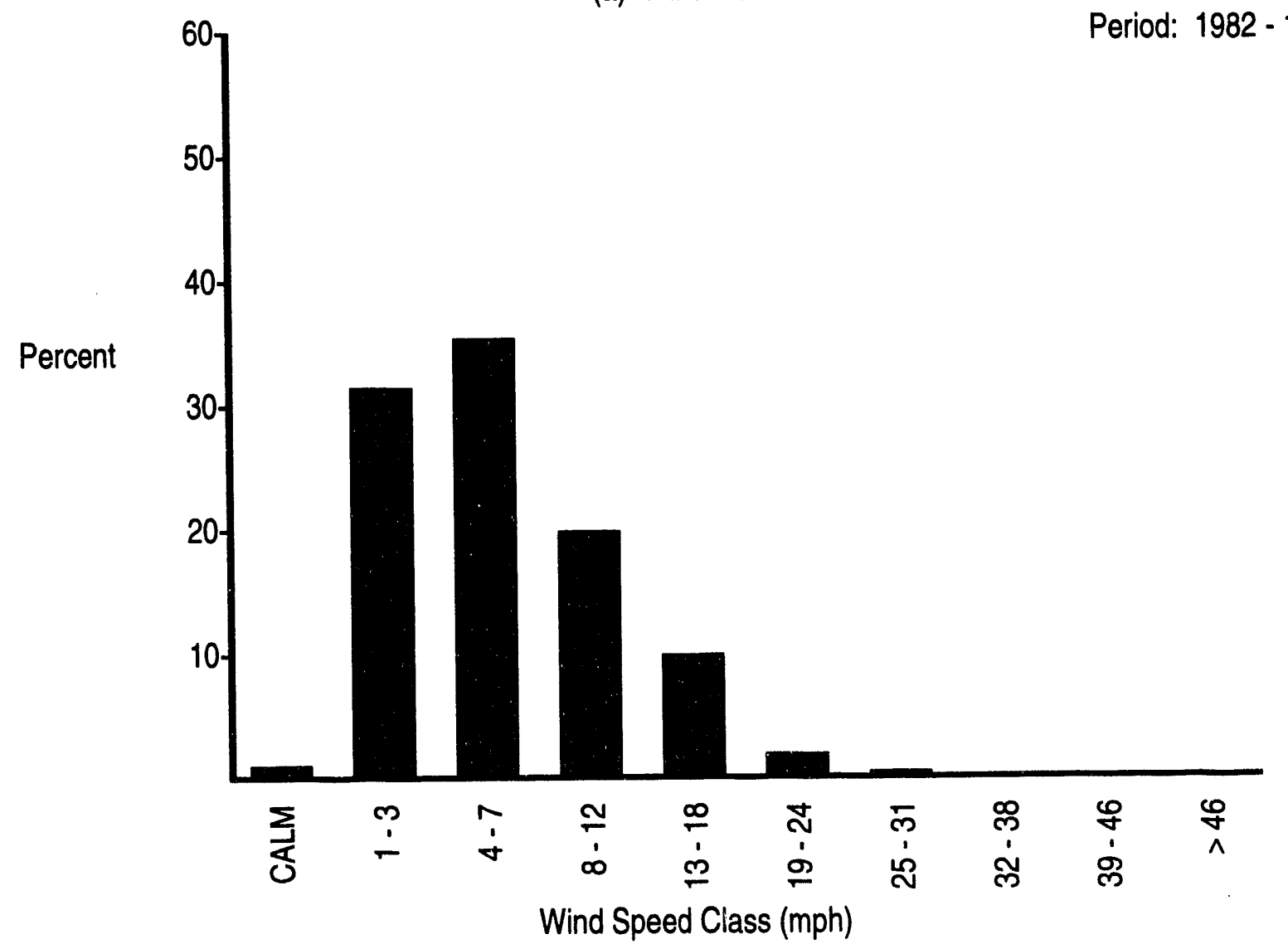

(b) Wind Speed Histogram

FIGURE B.1. (contd) 


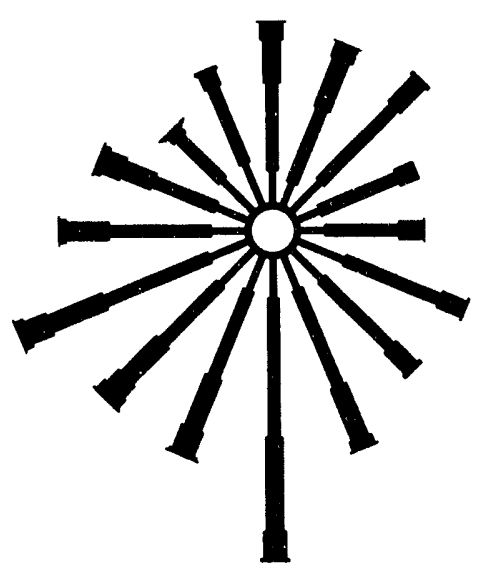

(a) Wind Rose

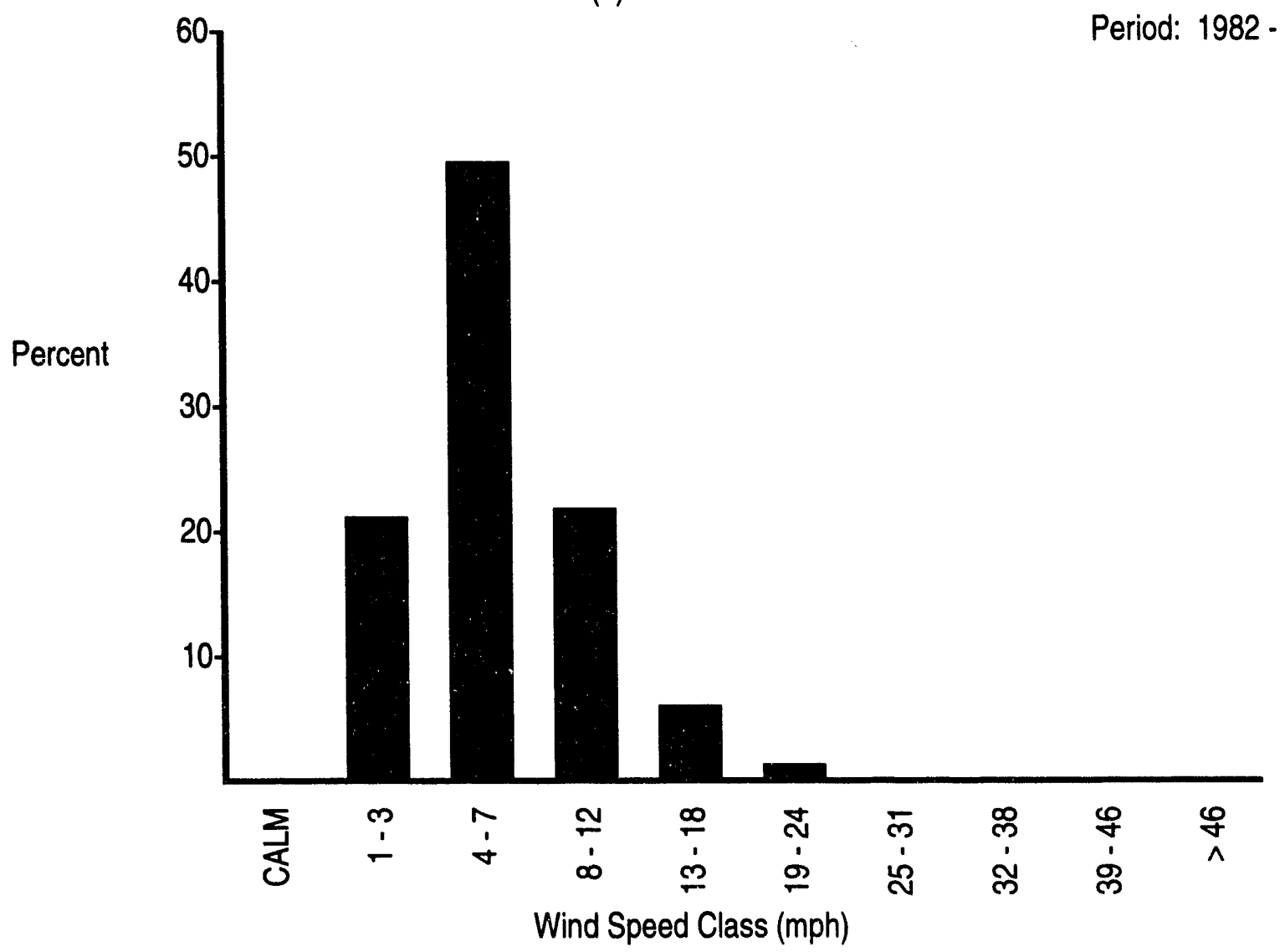

(b) Wind Speed Histogram

FIGURE B.1. (contd)
March Data

Period: 1982 - 1992 


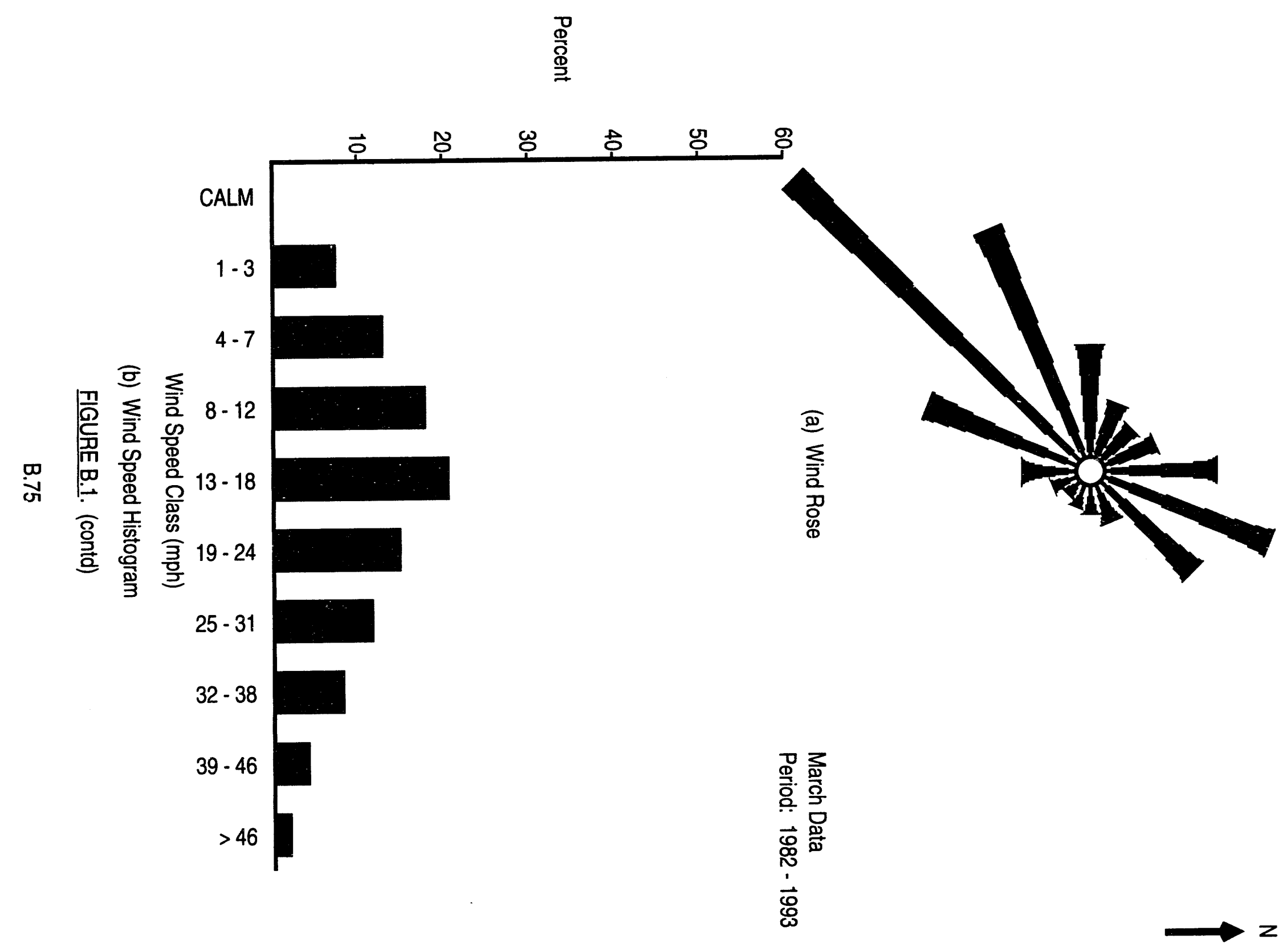



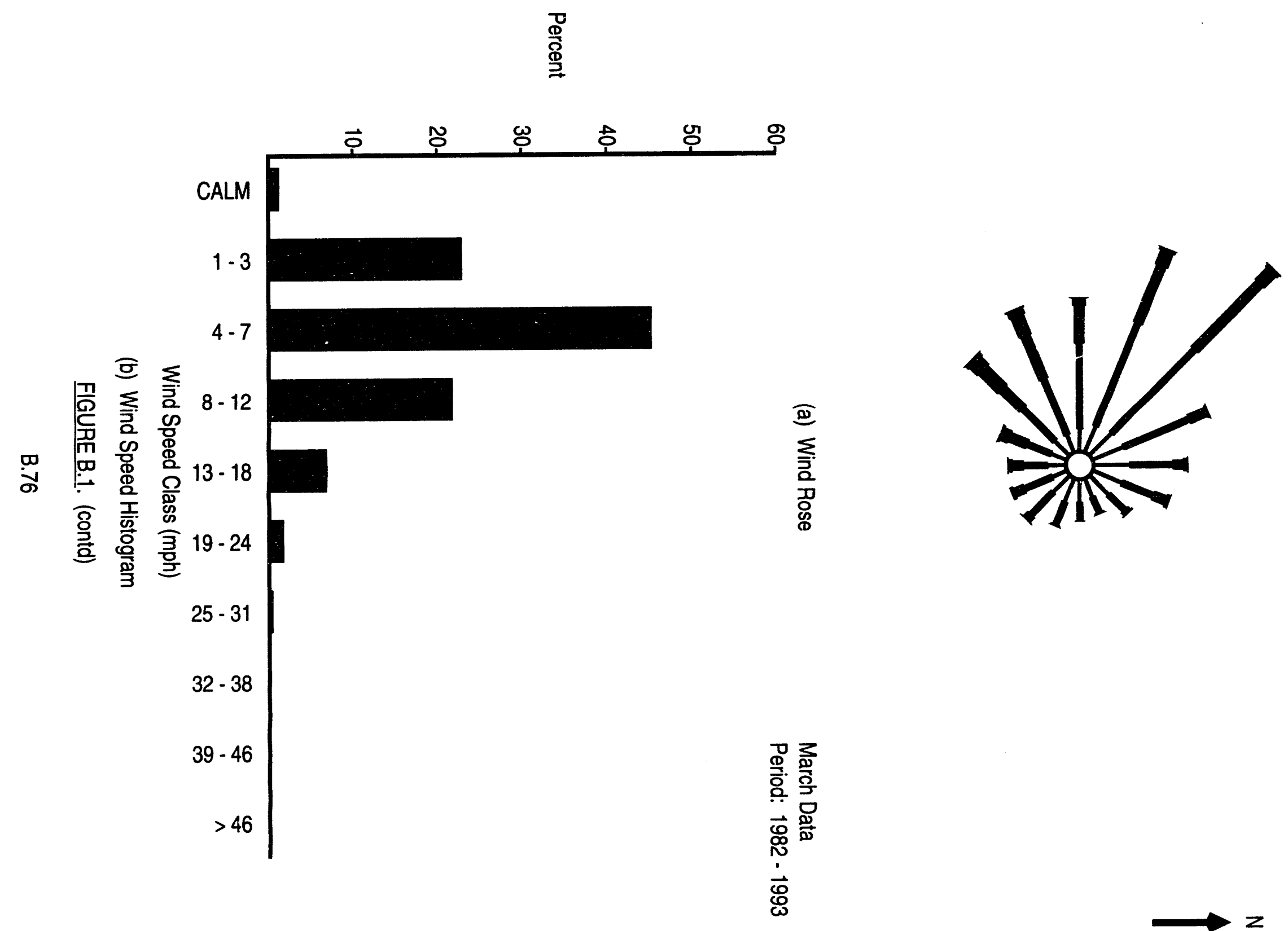

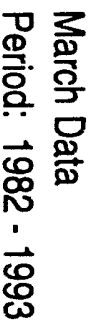




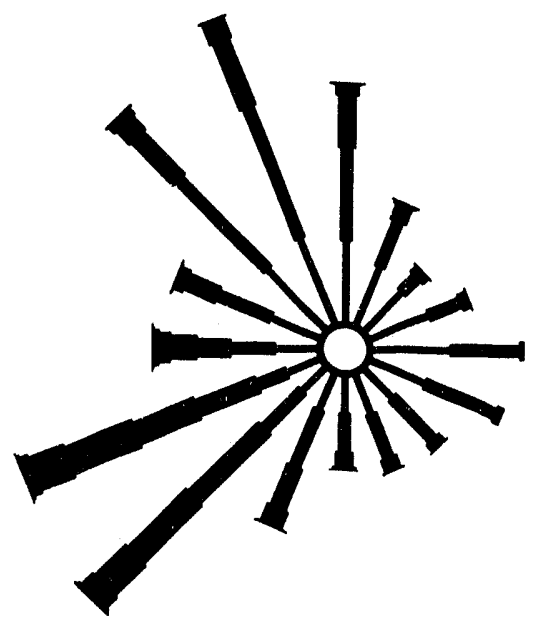

(a) Wind Rose

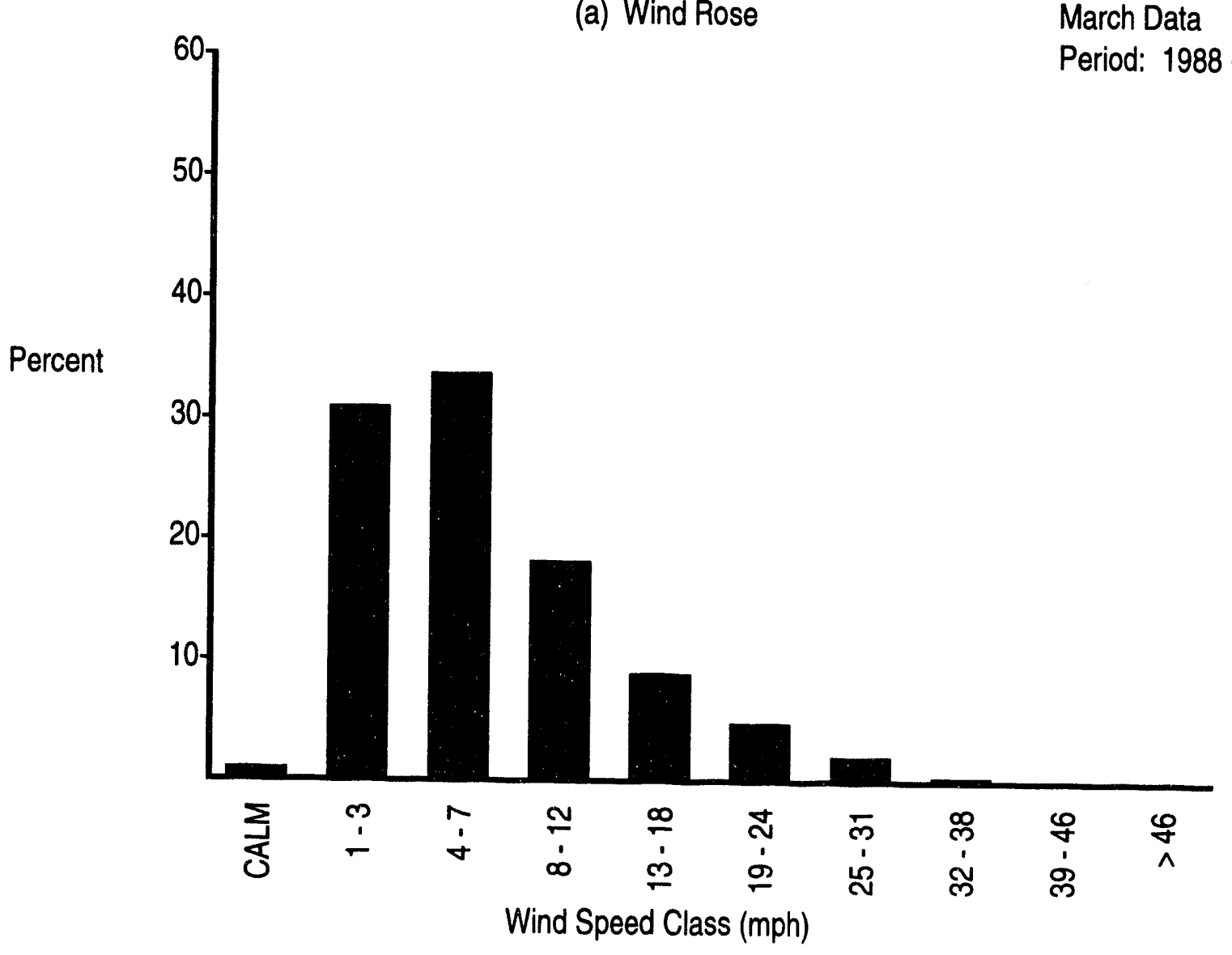

(b) Wind Speed Histogram

FIGURE B.1. (contd) 


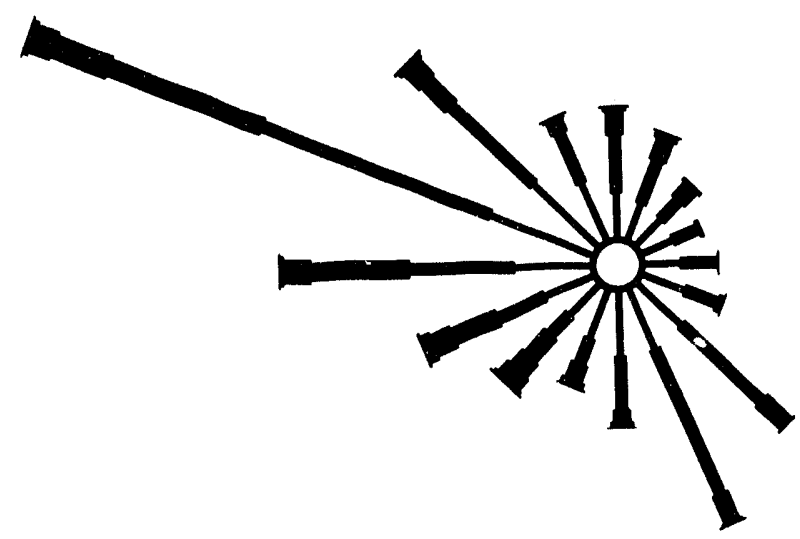

(a) Wind Rose

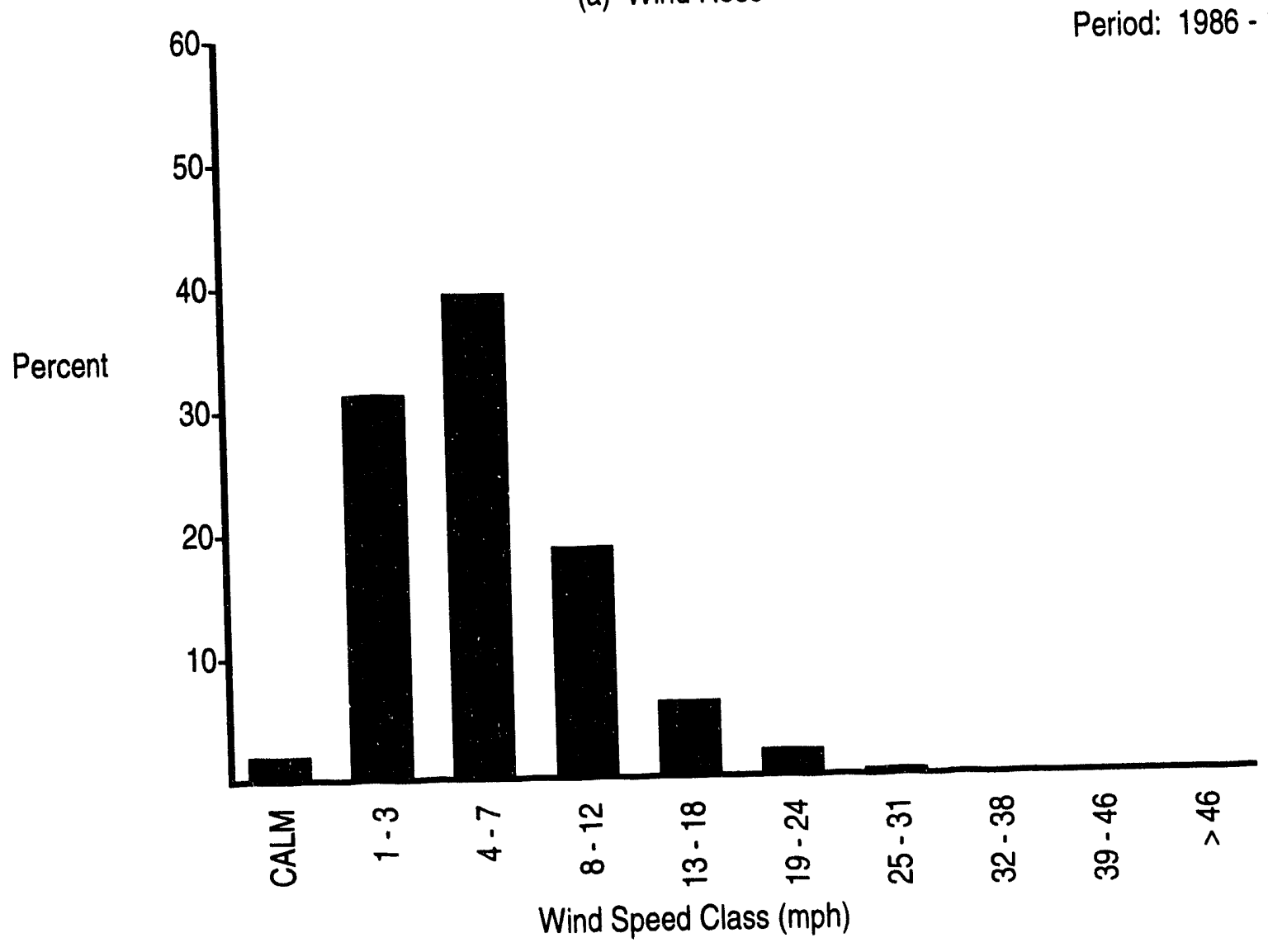

(b) Wind Speed Histogram

FIGURE B.1. (contd)
March Data

Period: 1986 - 1993

\section{B.78}




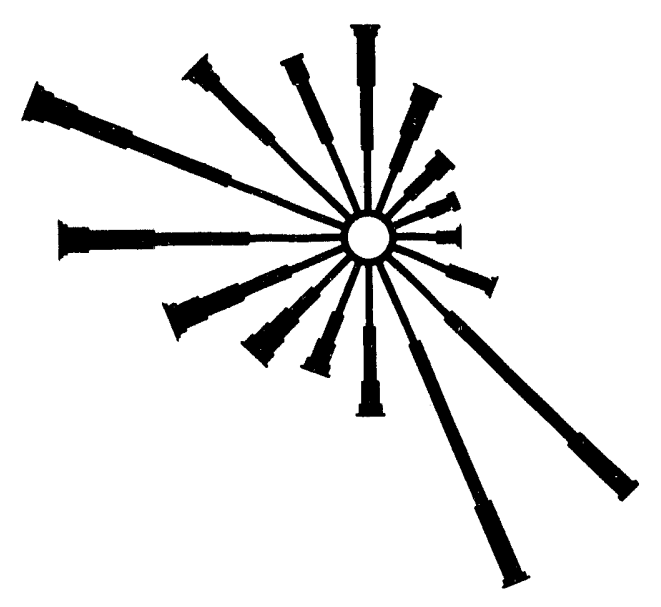

(a) Wind Rose

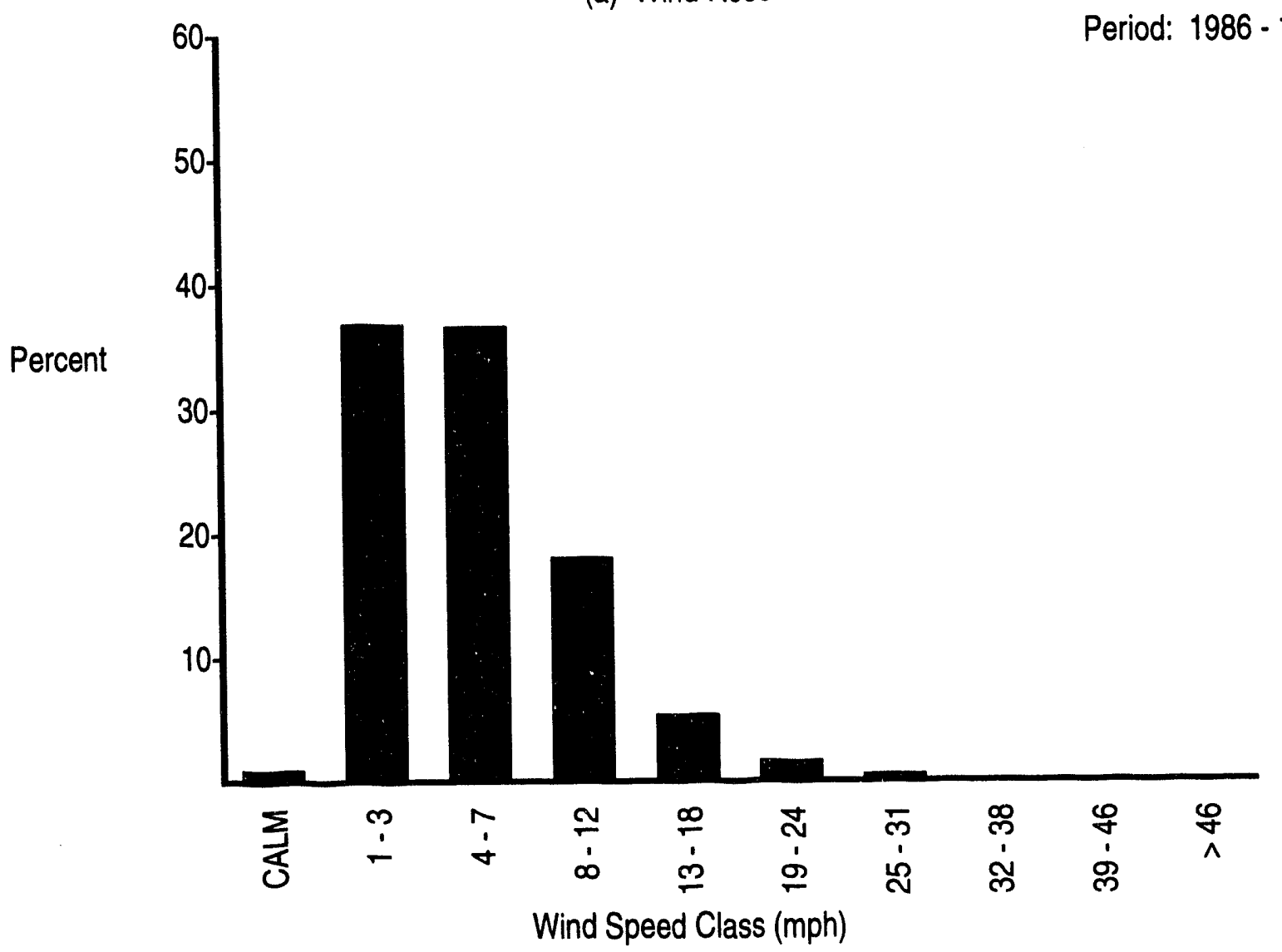

(b) Wind Speed Histogram

FIGURE B.1. (contd) 

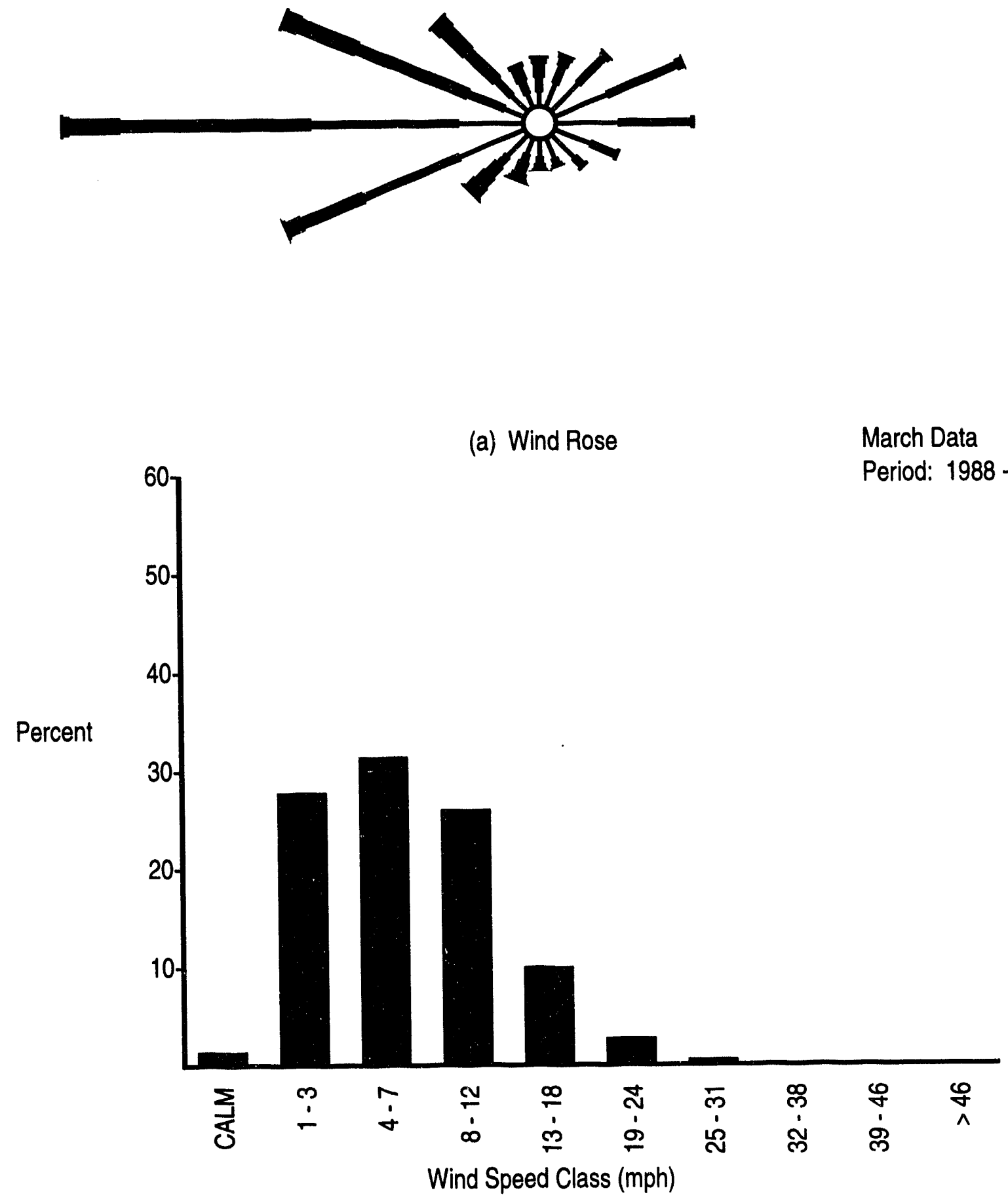

(b) Wind Speed Histogram

FIGURE B.1. (contd) 


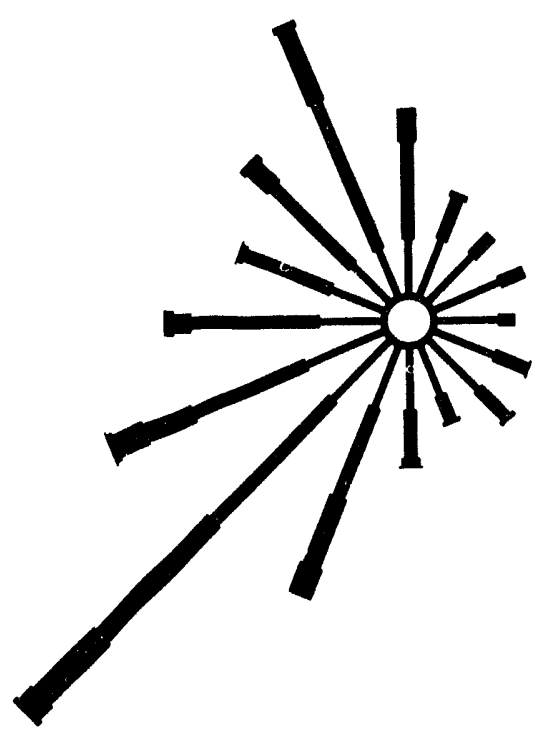

(a) Wind Rose

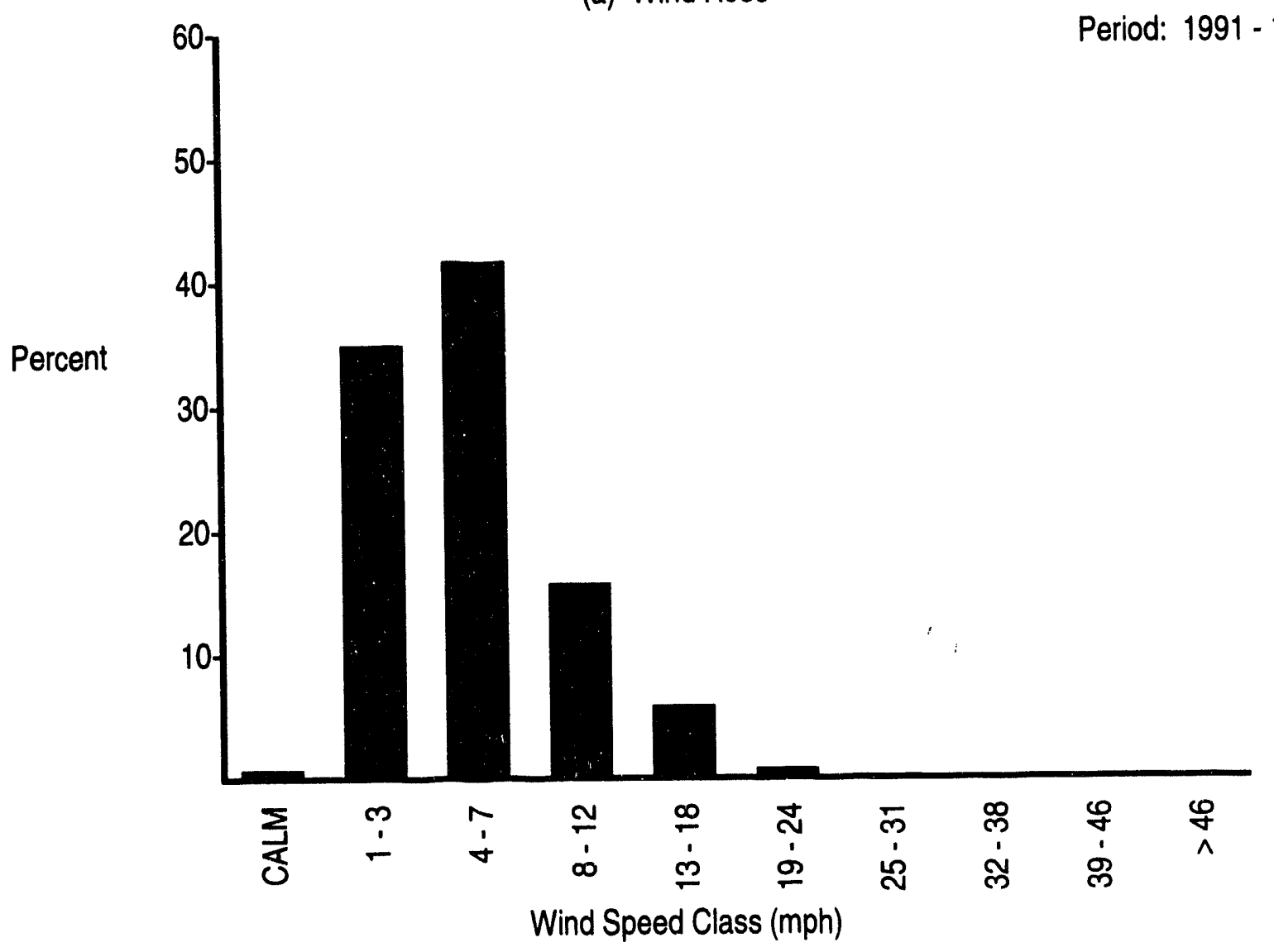

(b) Wind Speed Histogram

FIGURE B.1. (contd)
March Data

Period: 1991 - 1993 


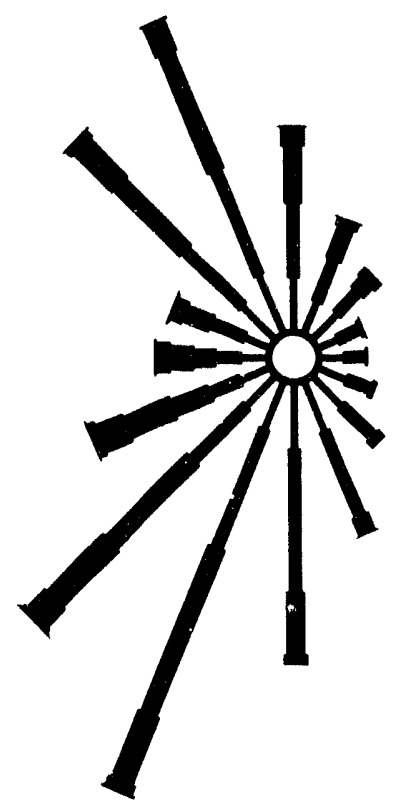

(a) Wind Rose

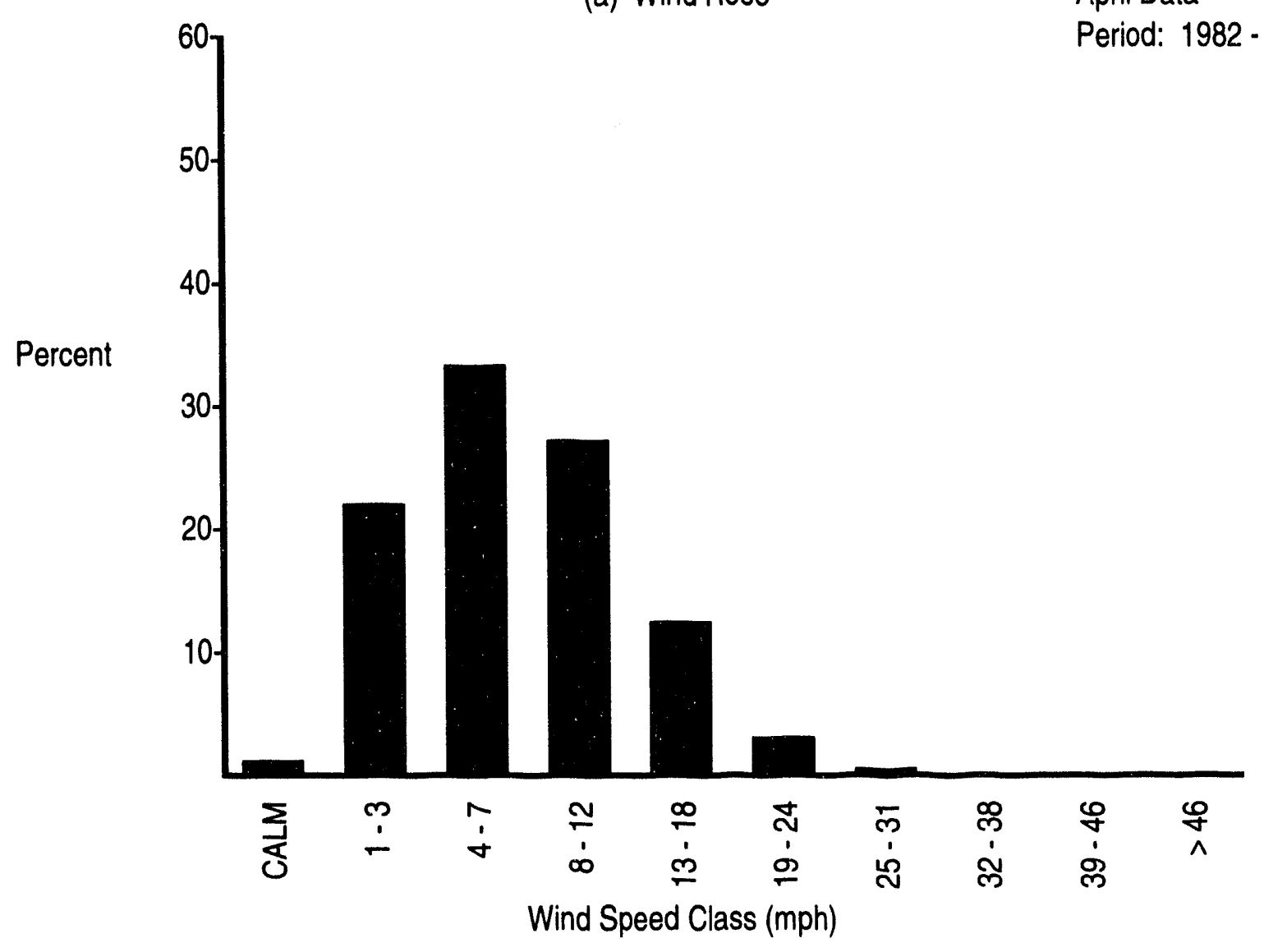

(b) Wind Speed Histogram

FIGURE B.1. (contd)
April Data

Period: $1982-1993$ 


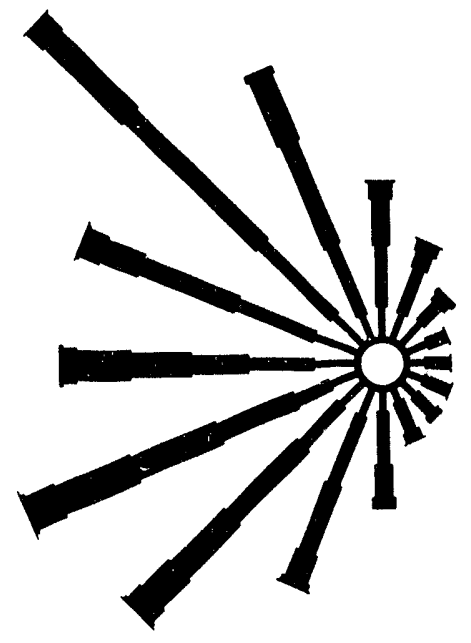

(a) Wind Rose

April Data

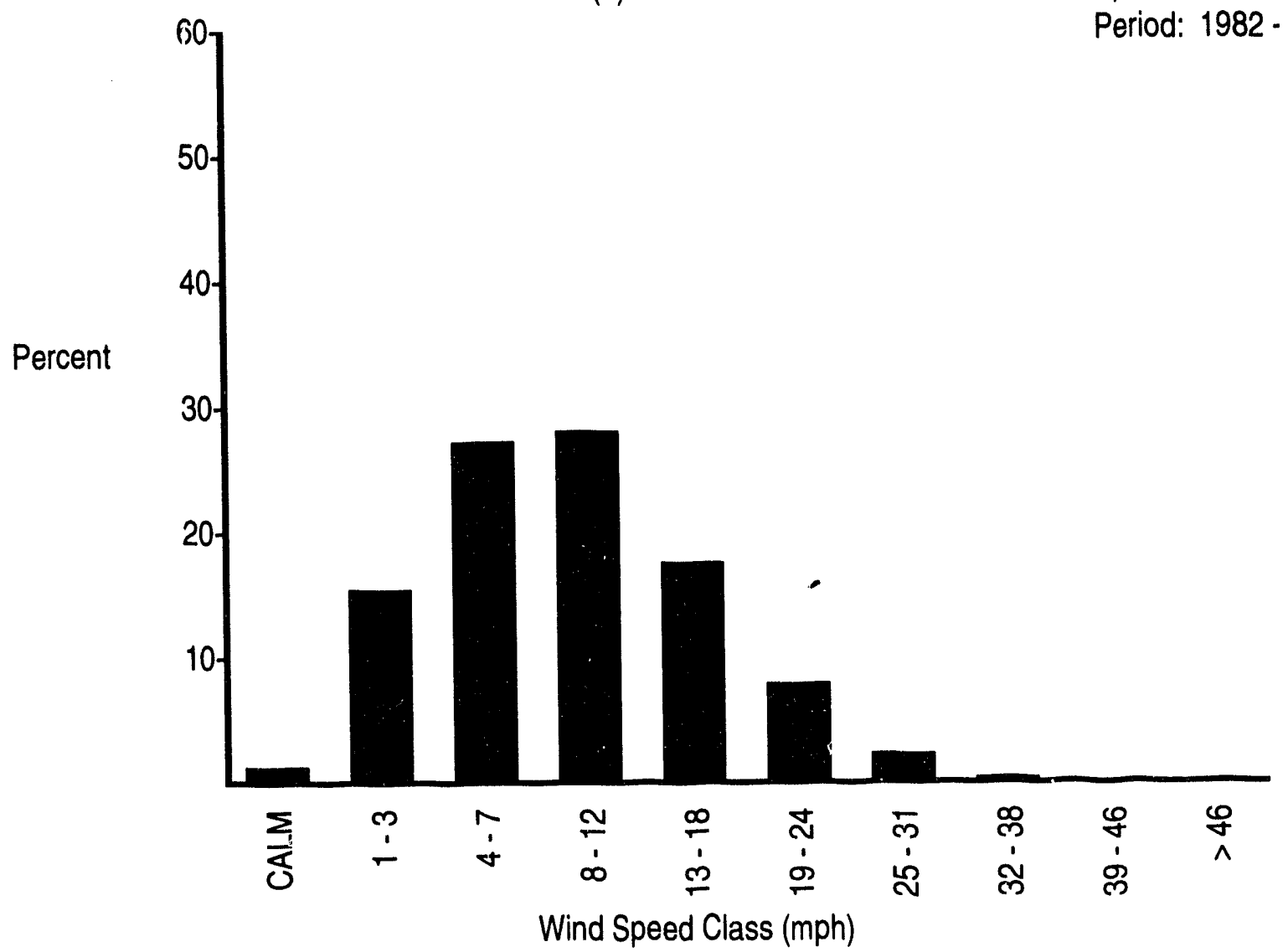

(b) Wind Speed Histogram

FIGURE B.1. (contd) 


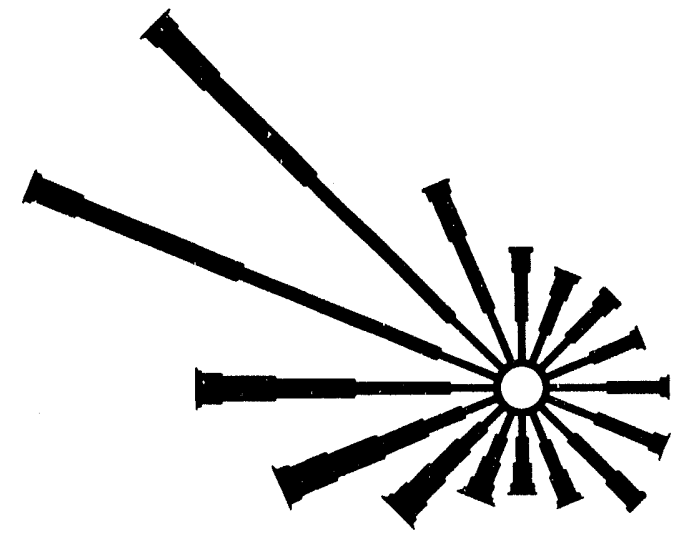

(a) Wind Rose

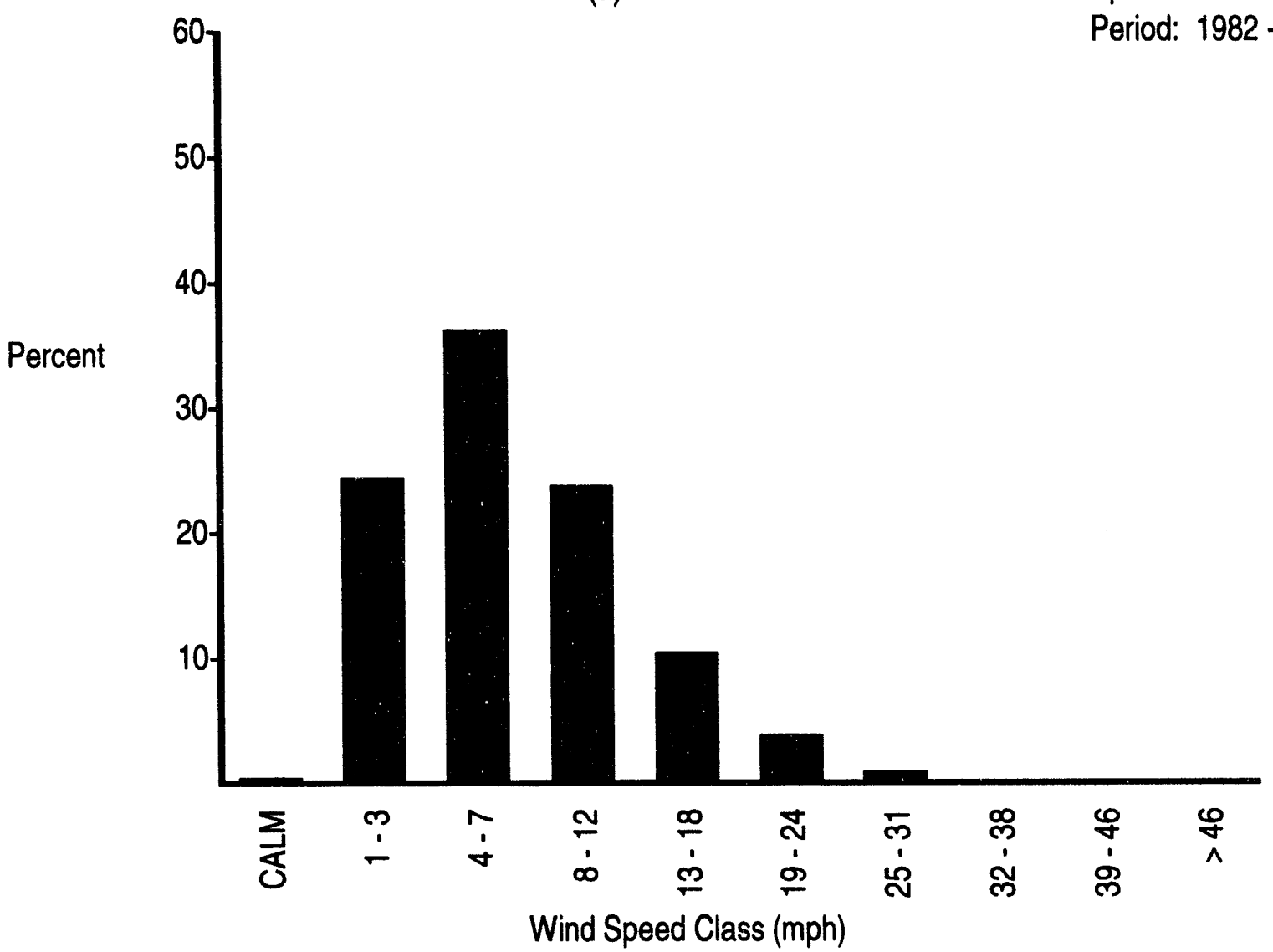

(b) Wind Speed Histogram

FIGURE B.1. (contd)
April Data

Period: 1982 - 1993

\section{B.84}



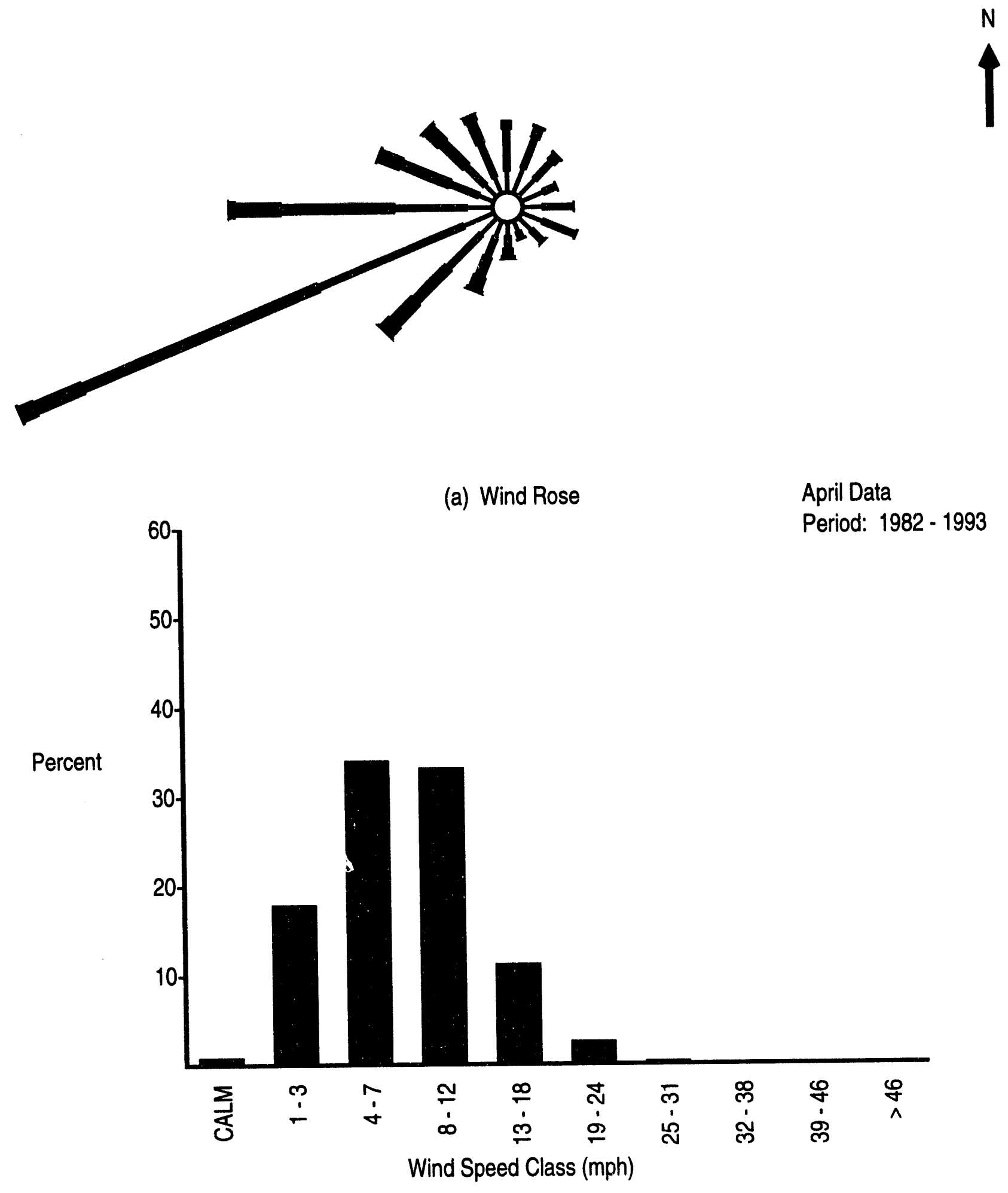

(b) Wind Speed Histogram

FIGURE B.1. (contd) 


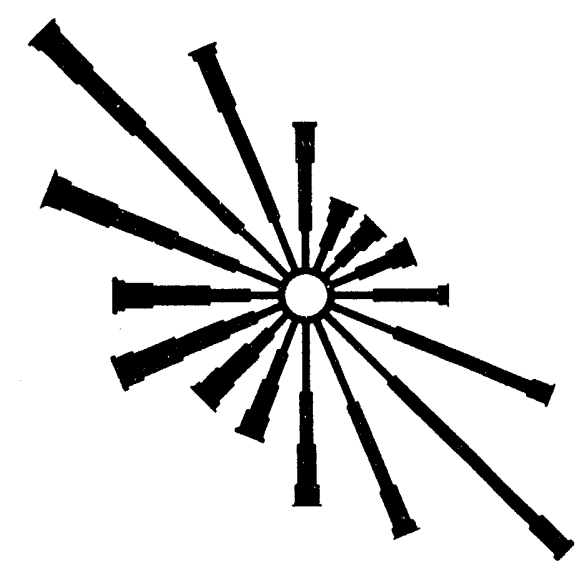

(a) Wind Rose

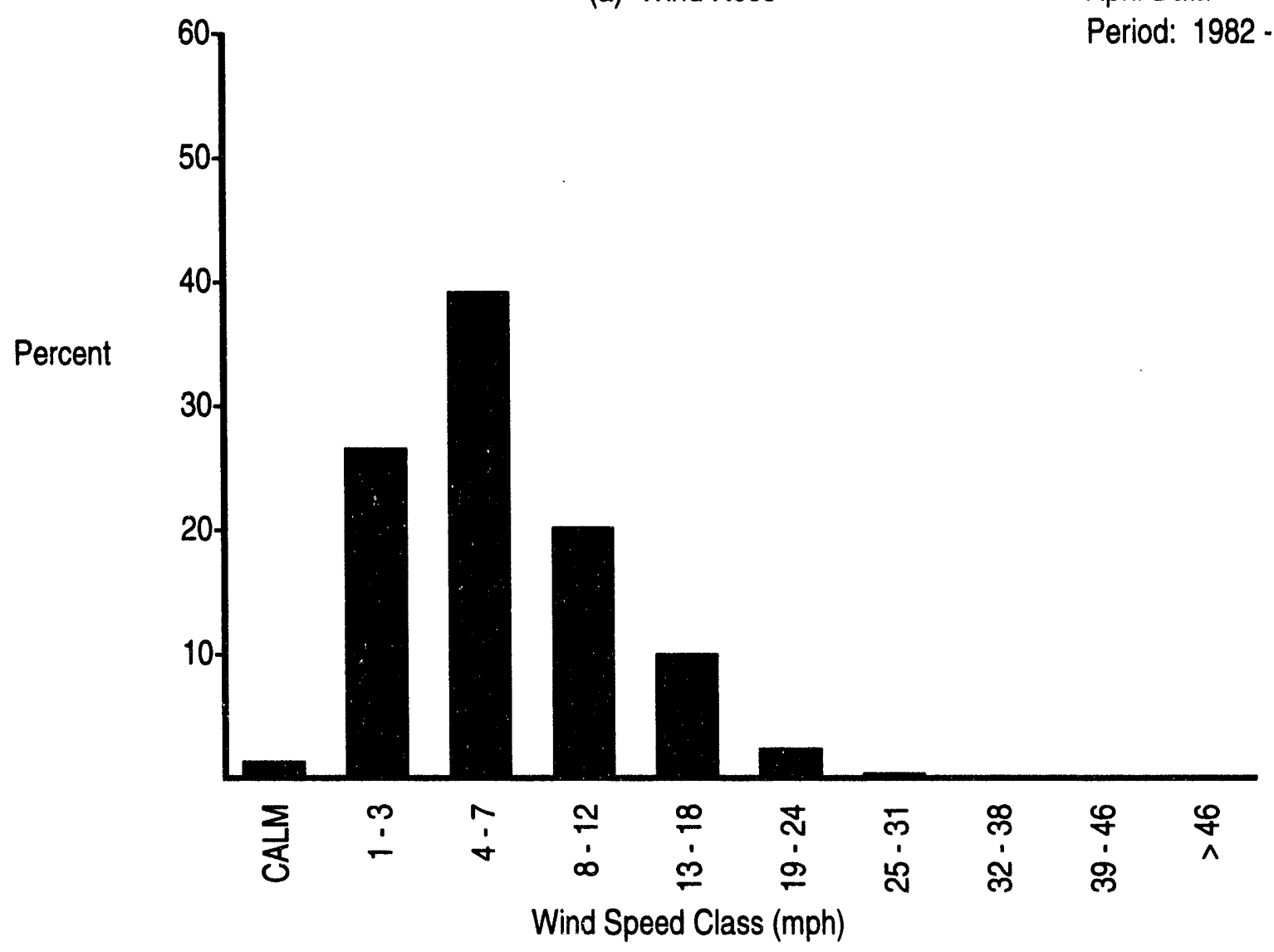

(b) Wind Speed Histogram

FIGURE B.1. (contd)
April Data

Period: $1982-1993$

\section{B.86}




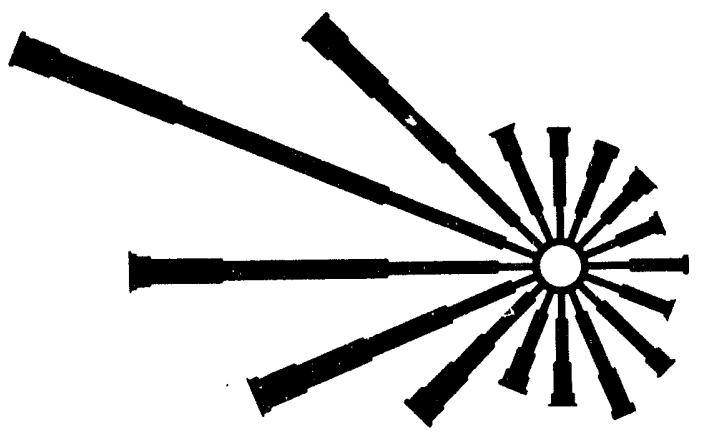

(a) Wind Rose

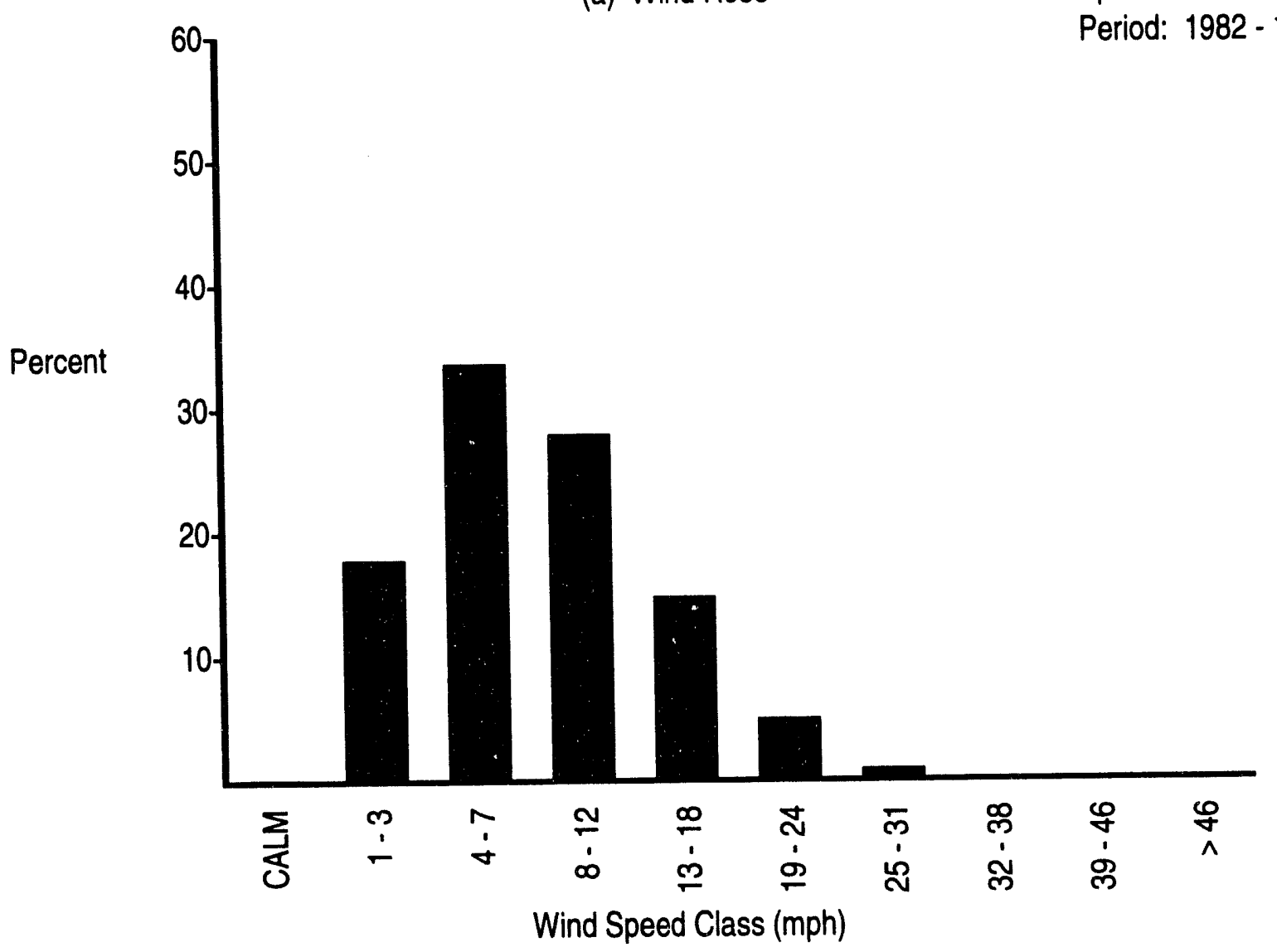

(b) Wind Speed Histogram

FIGURE B.1. (contd) 


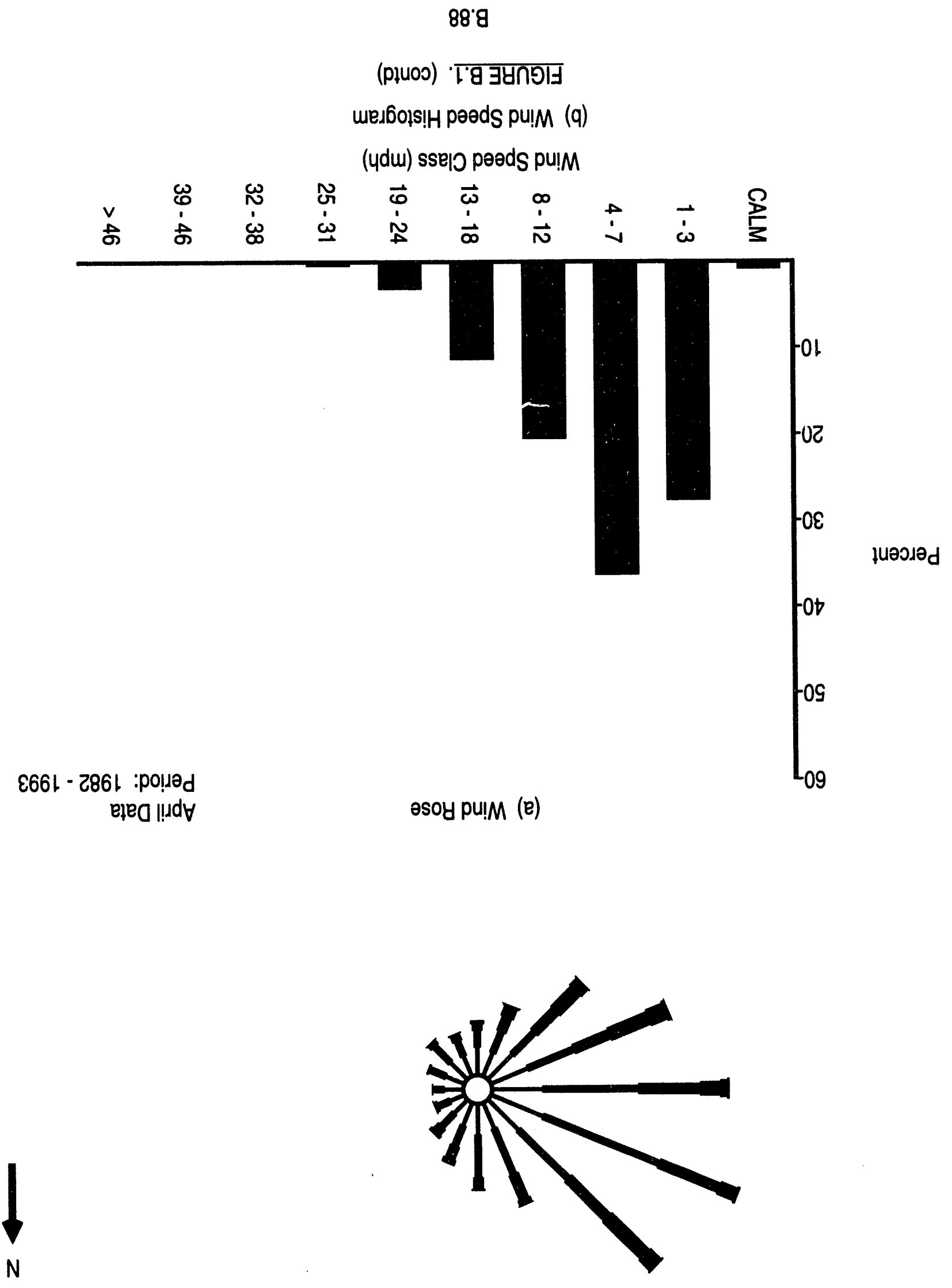




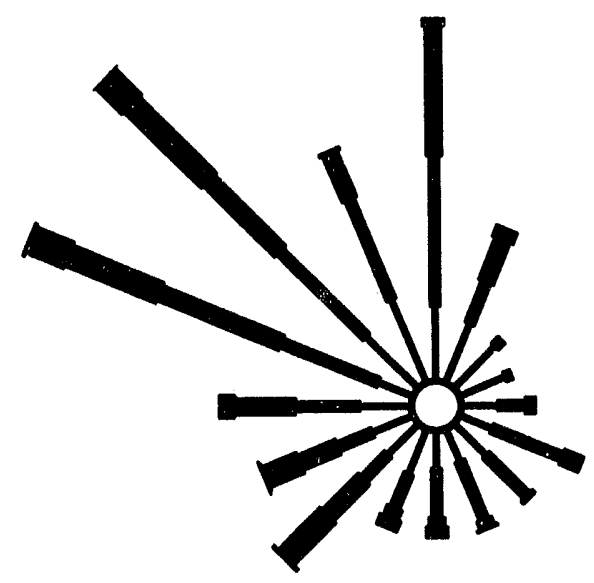

(a) Wind Rose

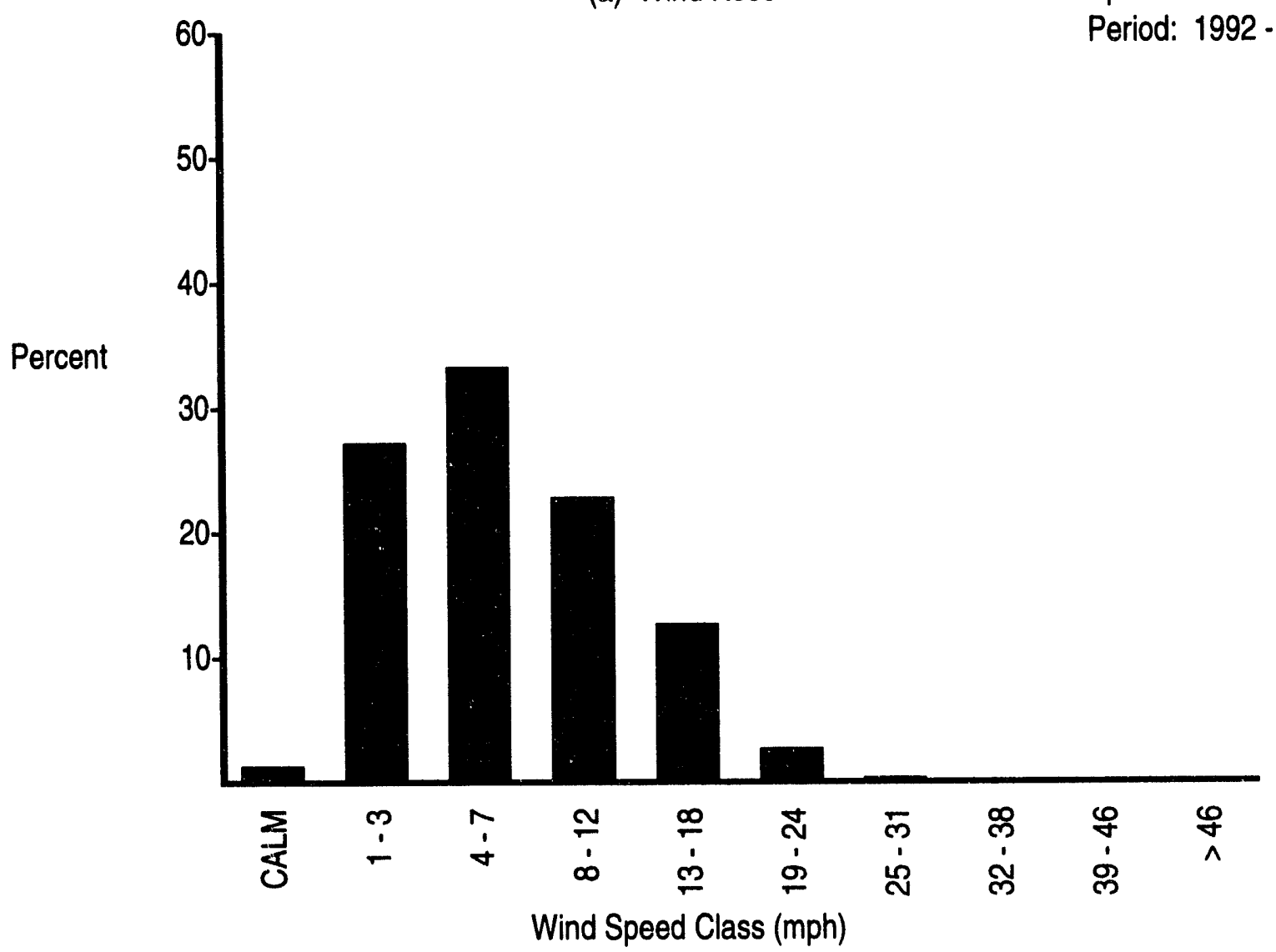

April Data

Period: 1992 - 1993

(b) Wind Speed Histogram

FIGURE B.1. (contd) 


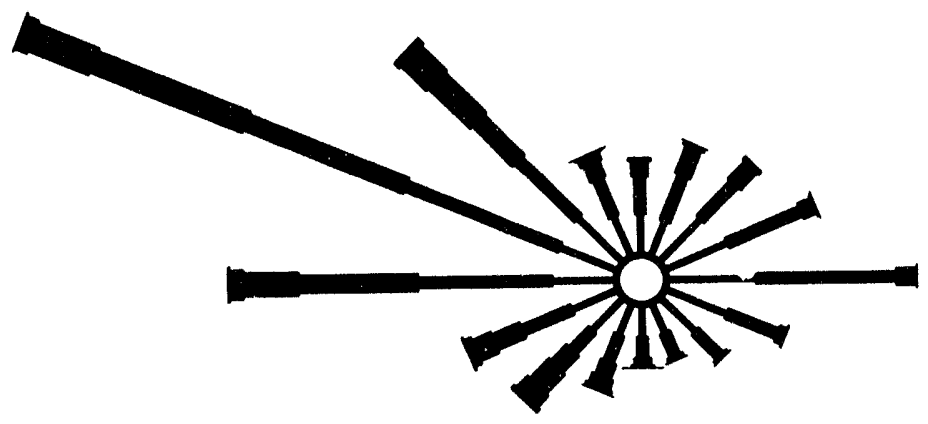

(a) Wind Rose

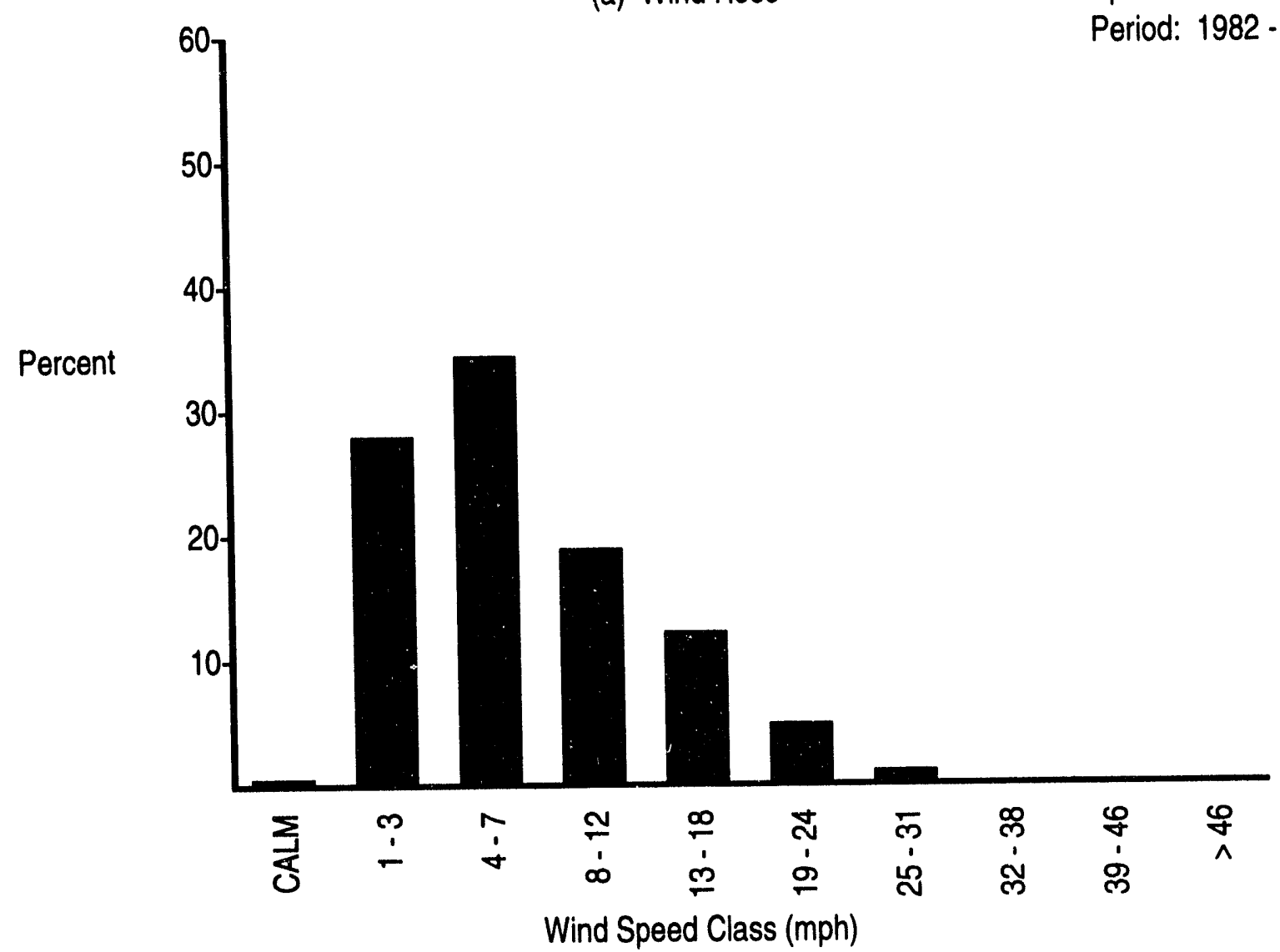

(b) Wind Speed Histogram

FIGURE B.1. (contd) 


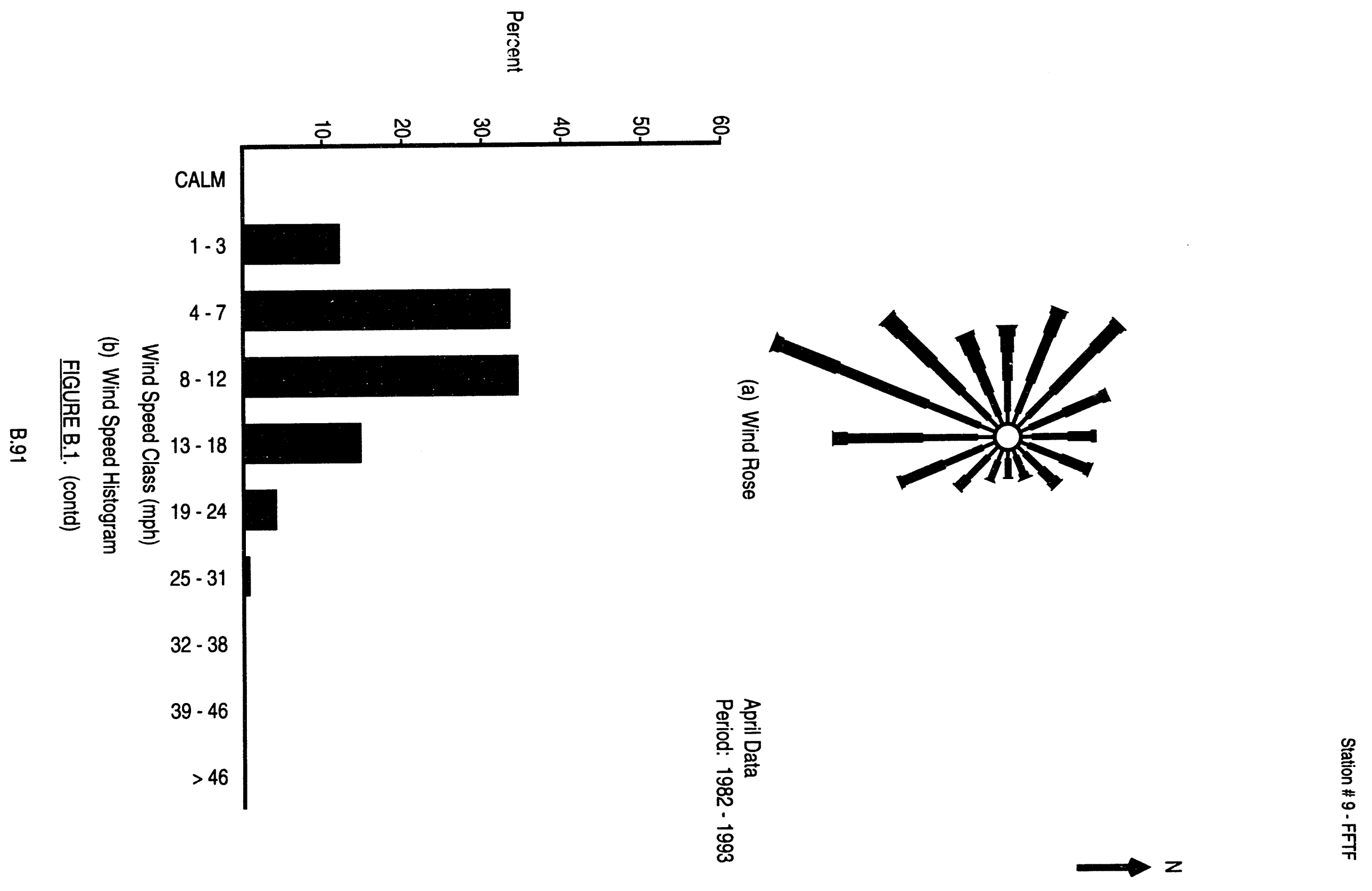




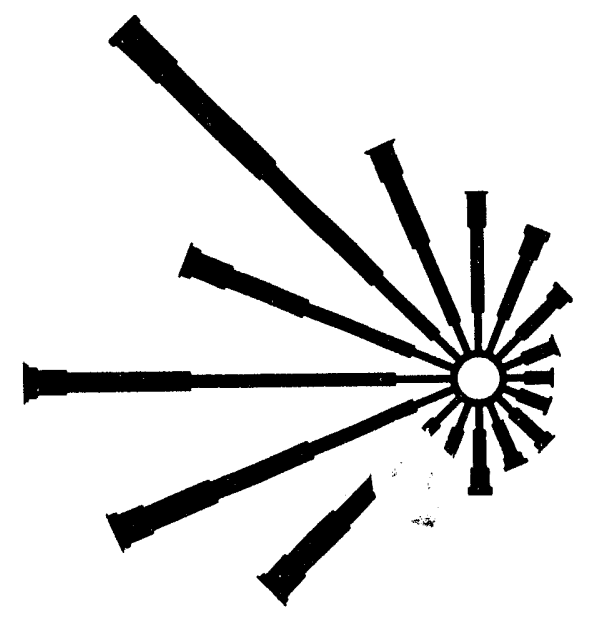

(a) Wind Rose

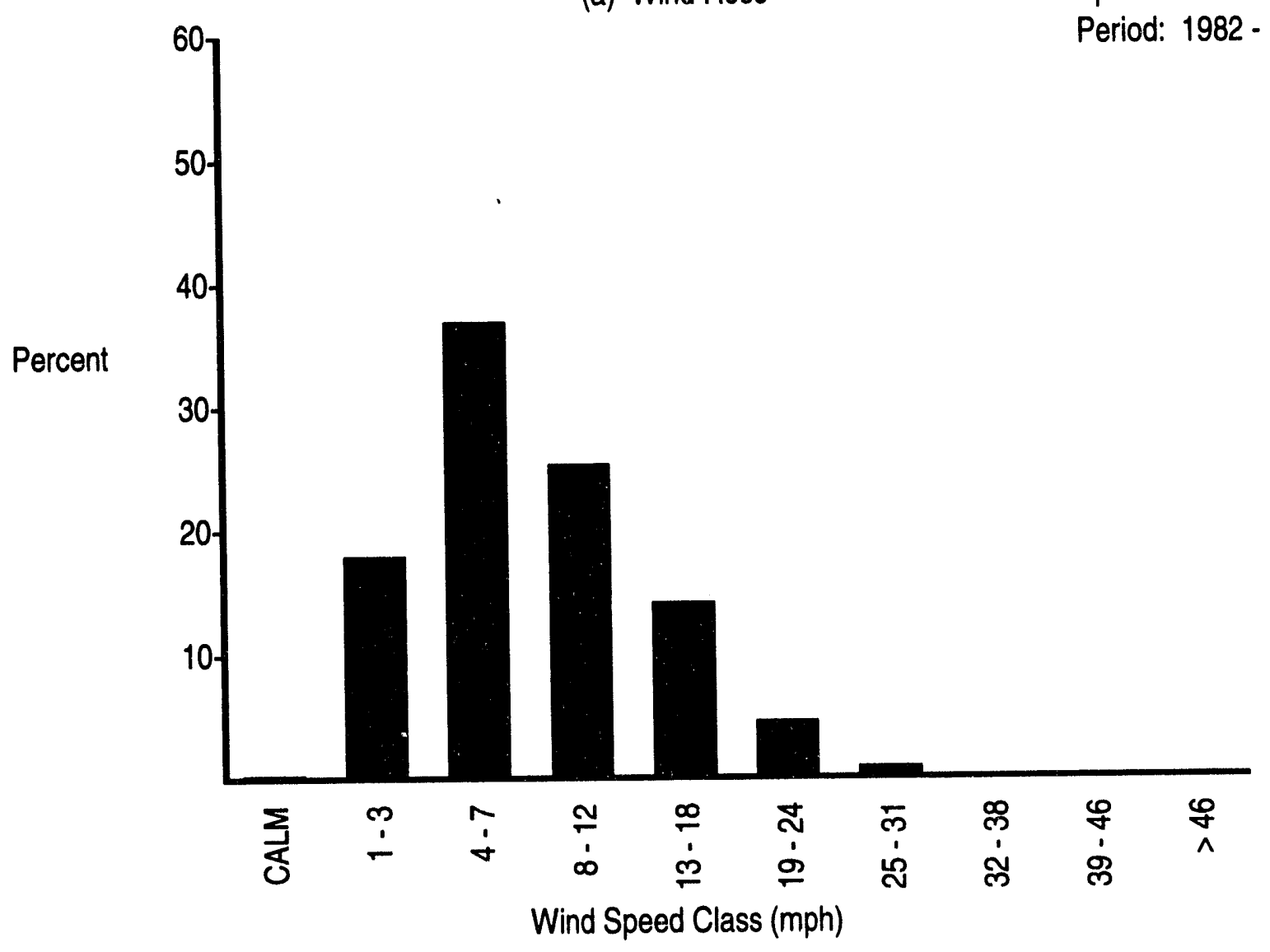

(b) Wind Speed Histogram

FIGURE B.1. (contd) 


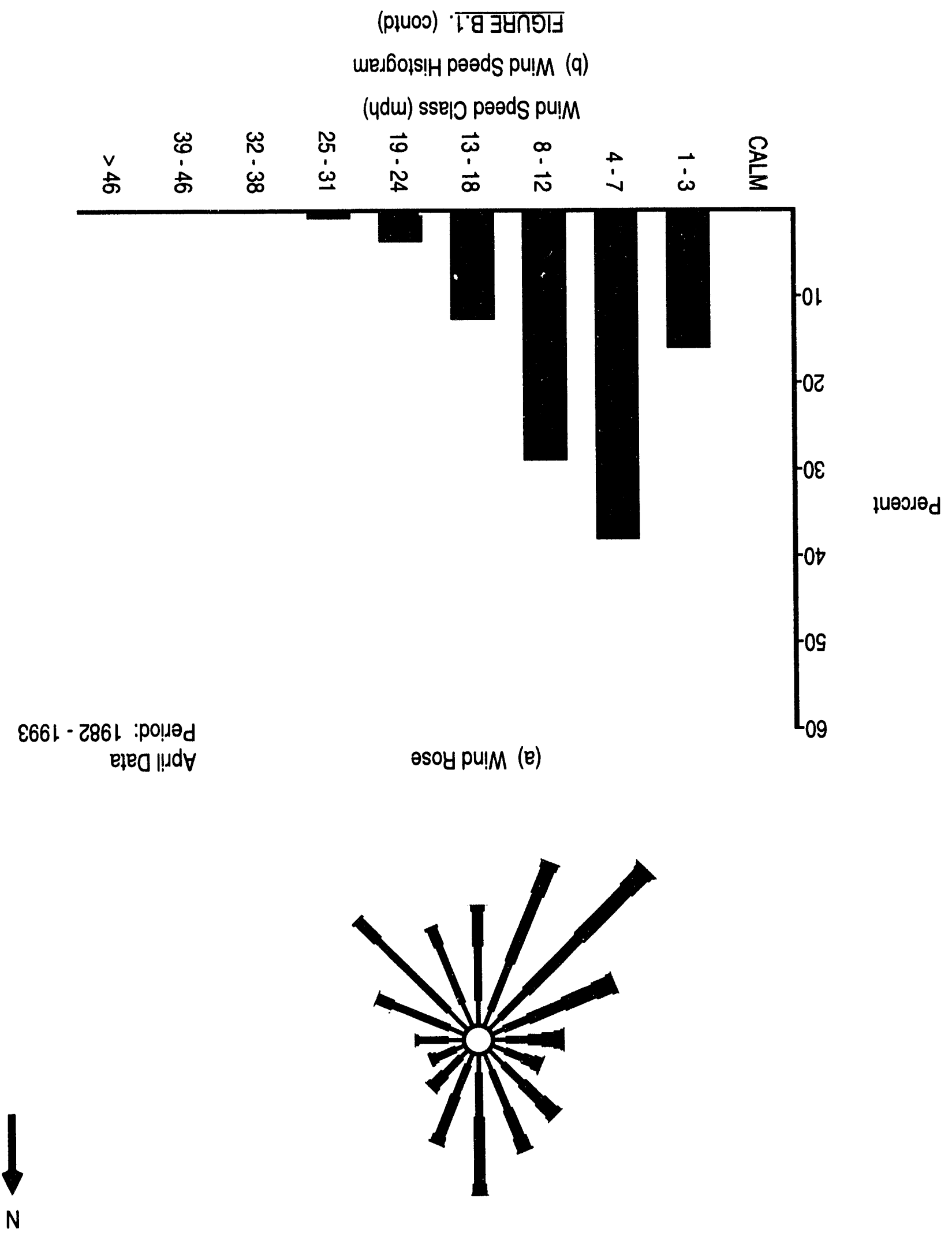




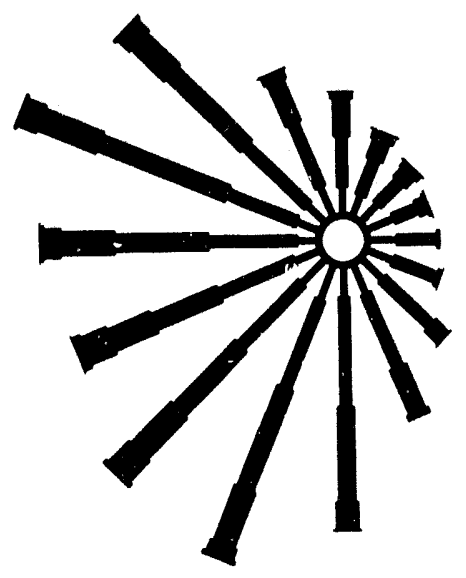

(a) Wind Rose

April Data

Period: $1982 \cdot 1993$

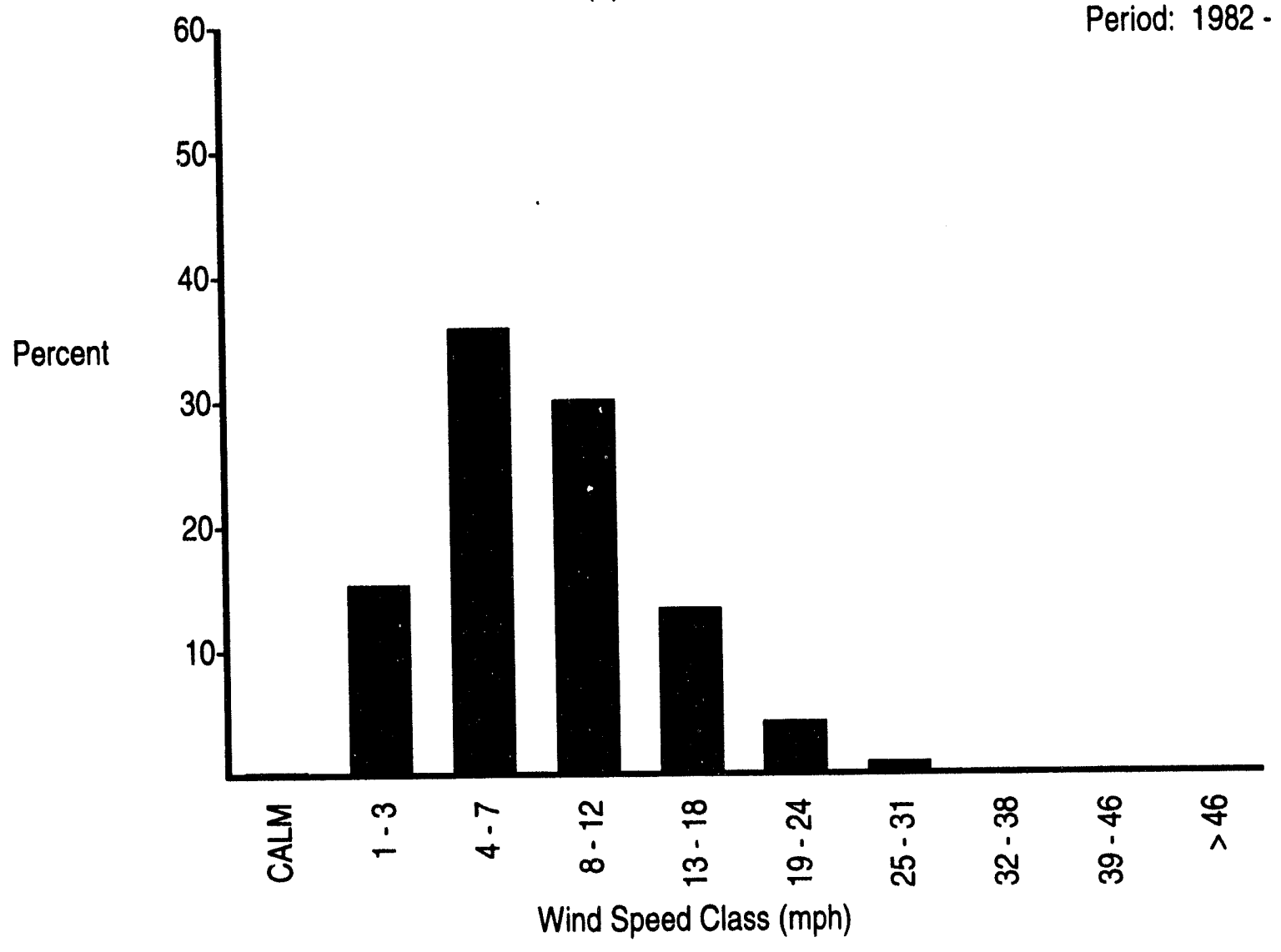

(b) Wind Speed Histogram

FIGURE B.1. (contd) 


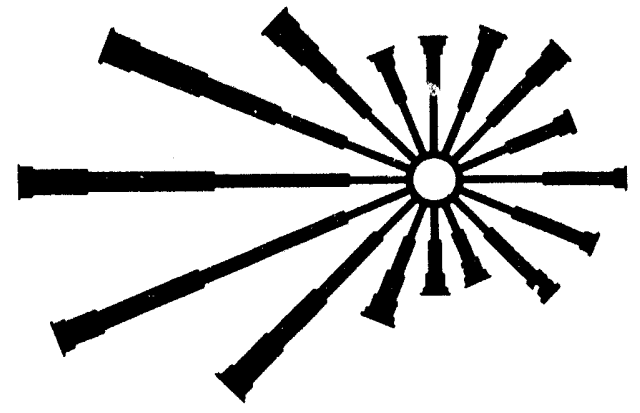

(a) Wind Rose

April Data

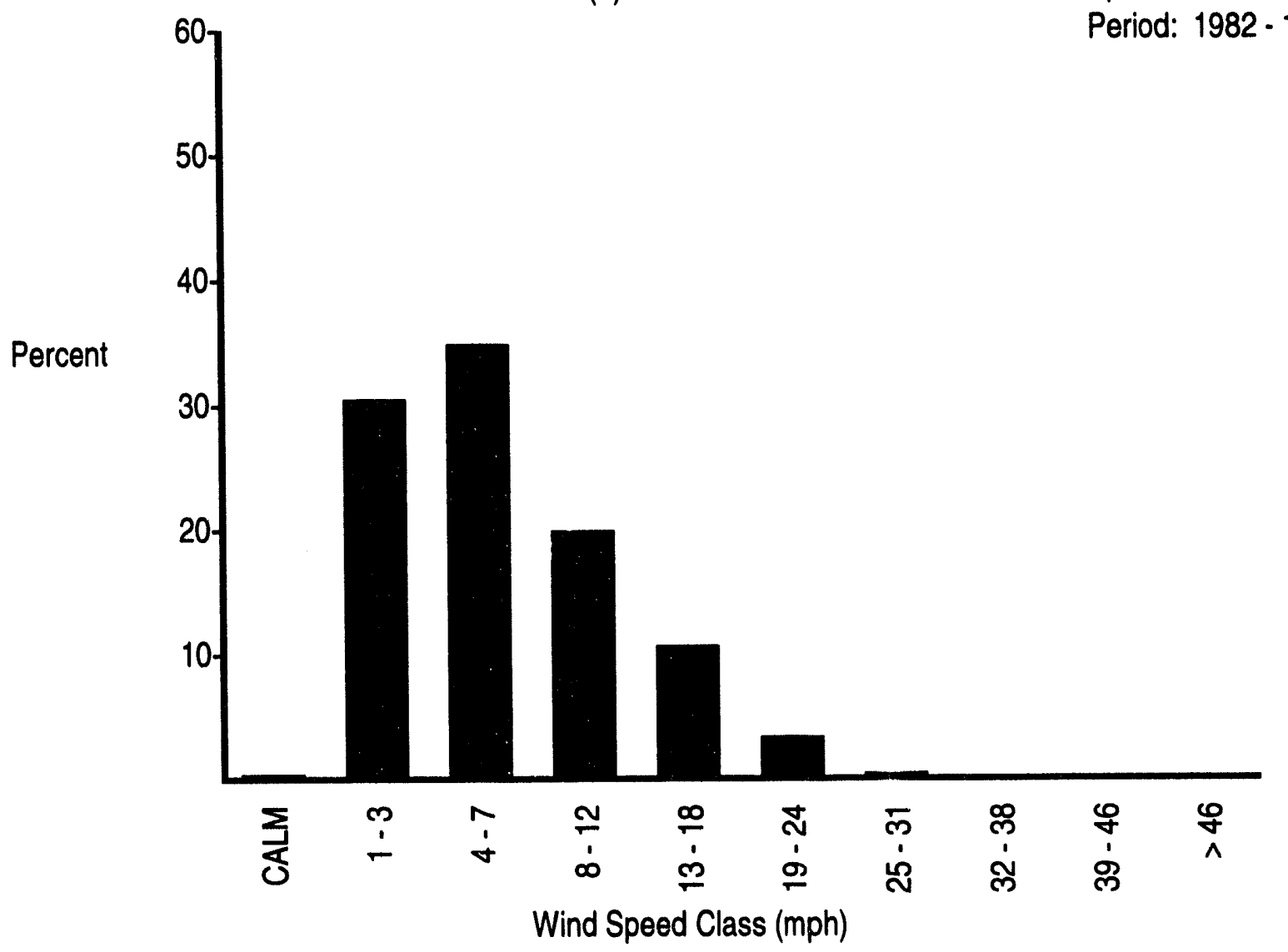

(b) Wind Speed Histogram

FIGURE B.1. (contd) 

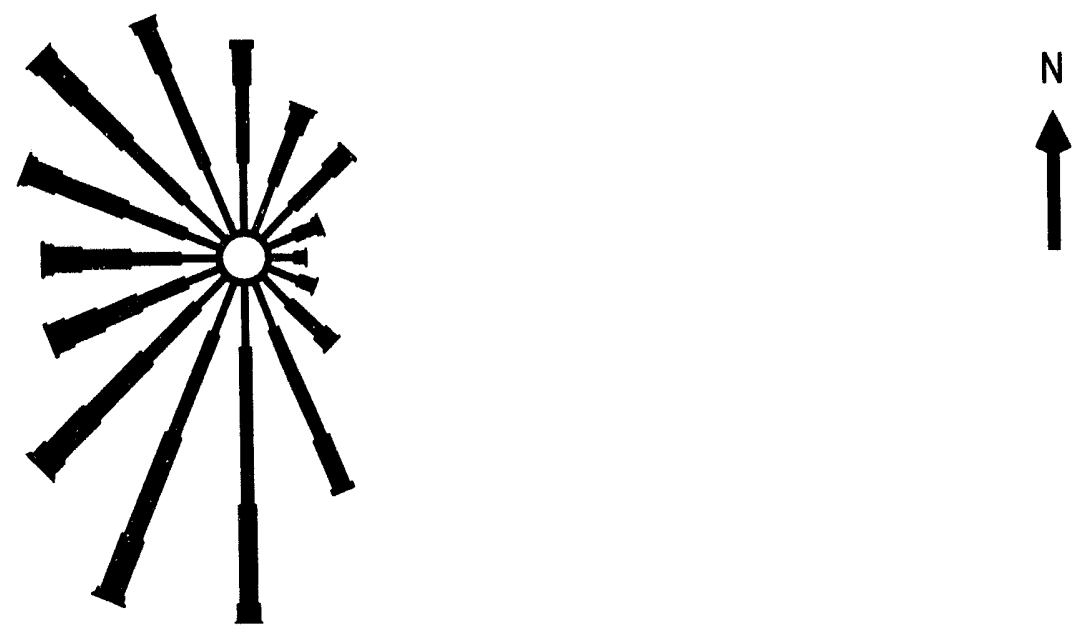

(a) Wind Rose

April Data

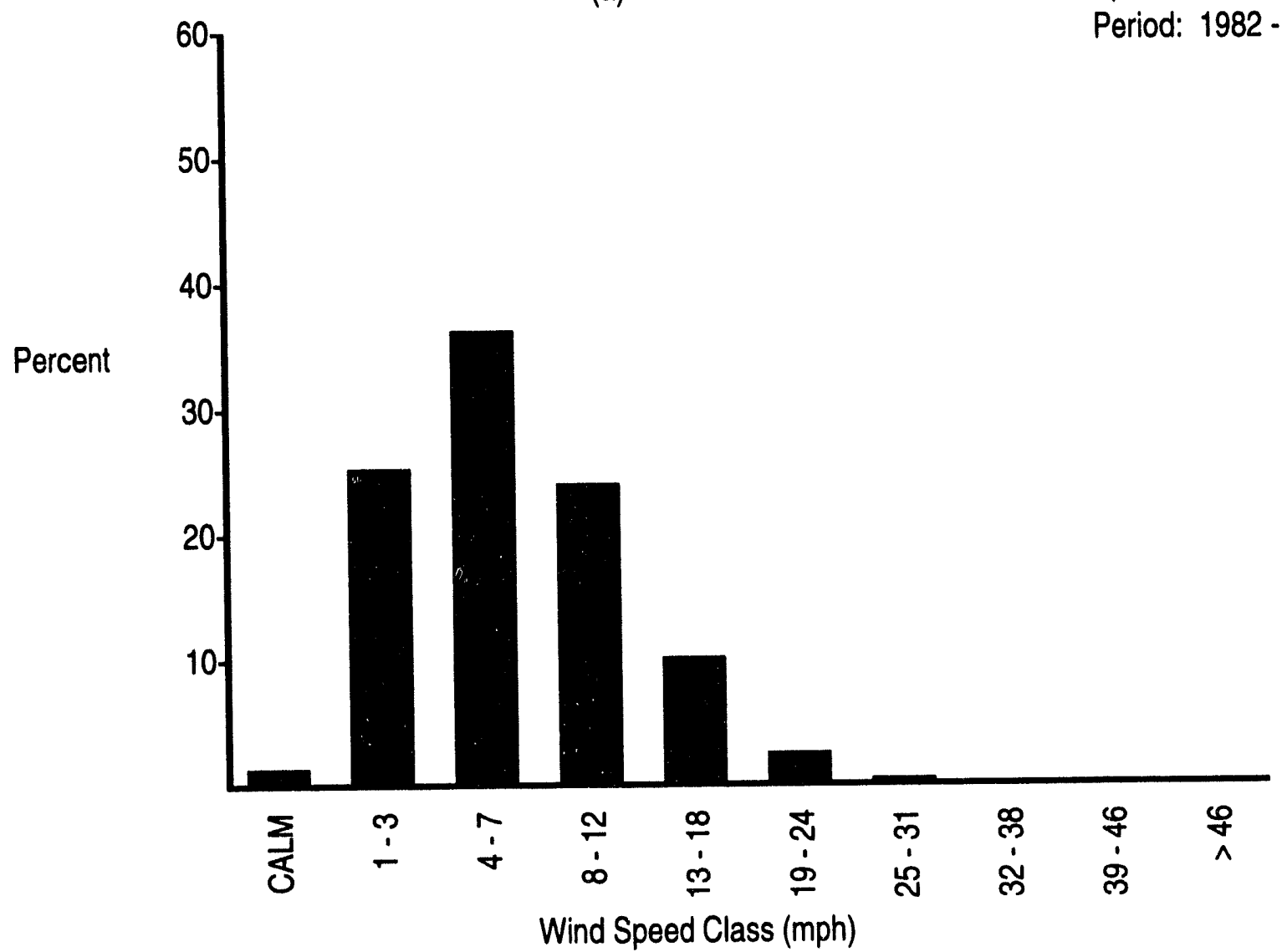

(b) Wind Speed Histogram

FIGURE B.1. (contd) 


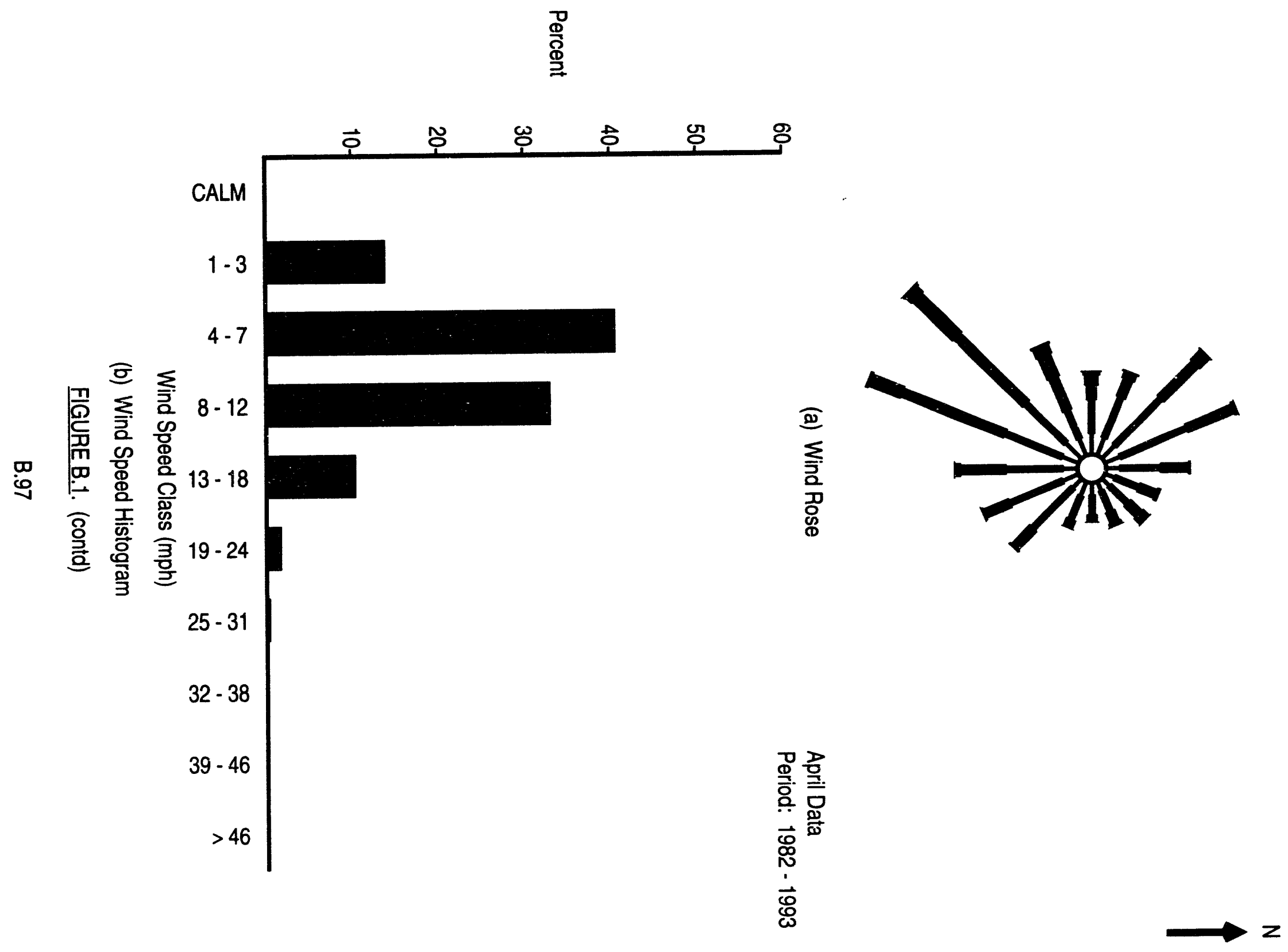




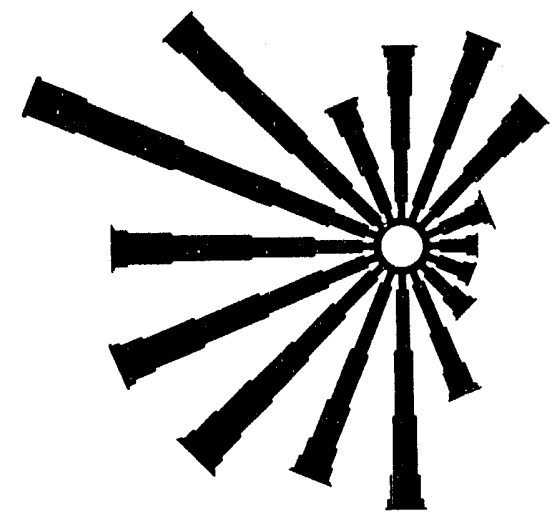

(a) Wind Rose

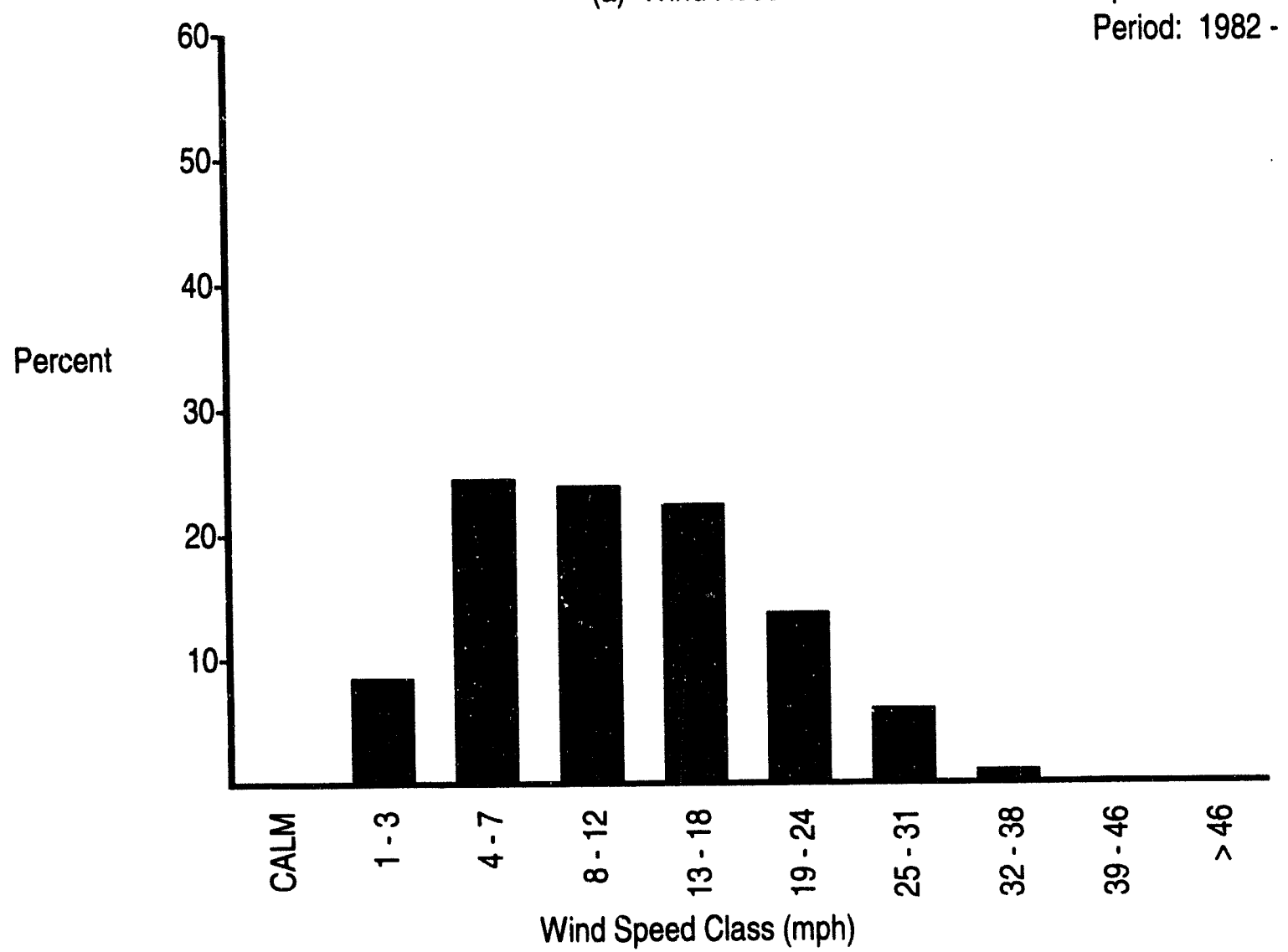

April Data

Period: $1982 \cdot 1993$

(b) Wind Speed Histogram

FIGURE B.1. (contd) 


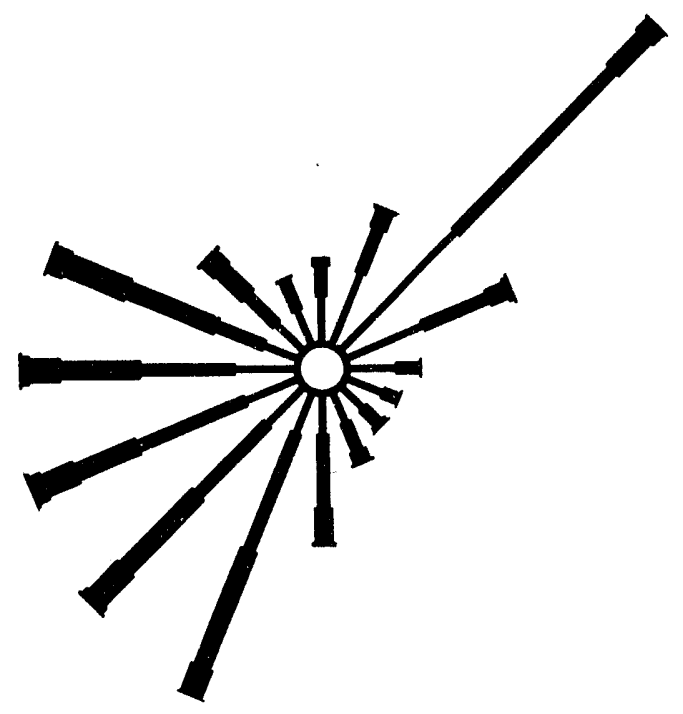

(a) Wind Rose

April Data

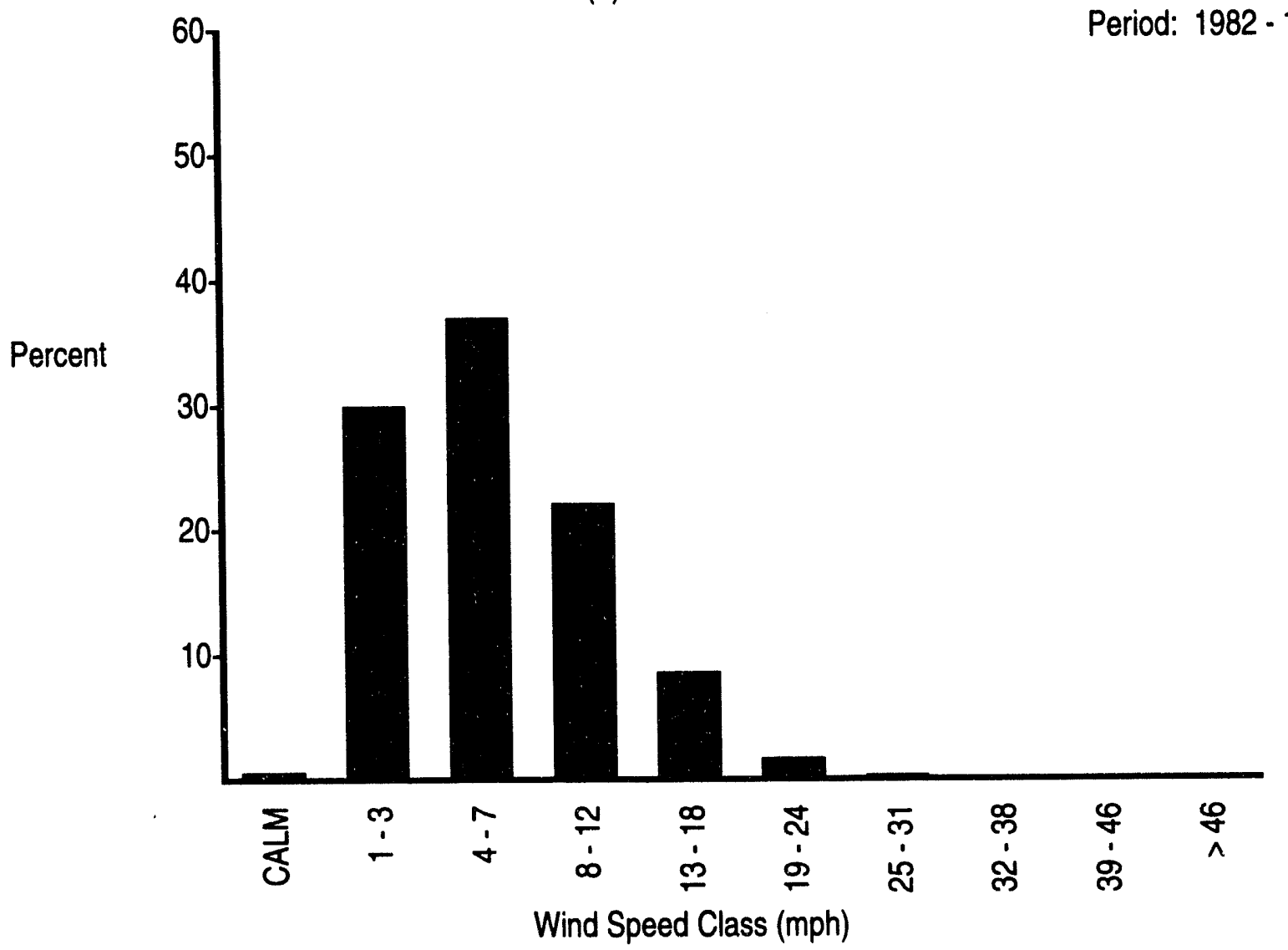

(b) Wind Speed Histogram

FIGURE B.1. (contd) 


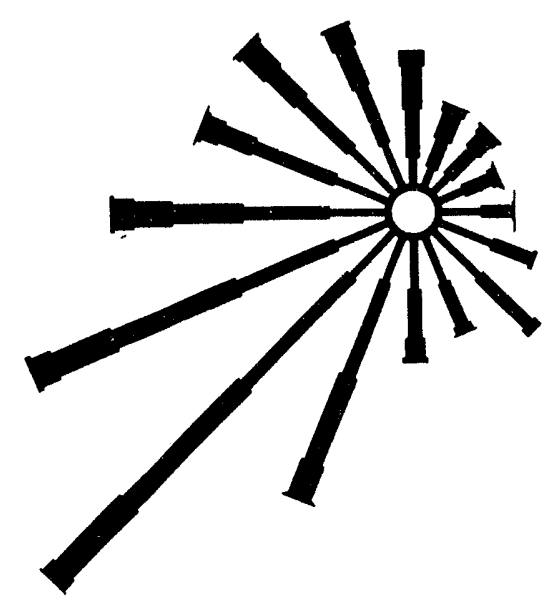

(a) Wind Rose

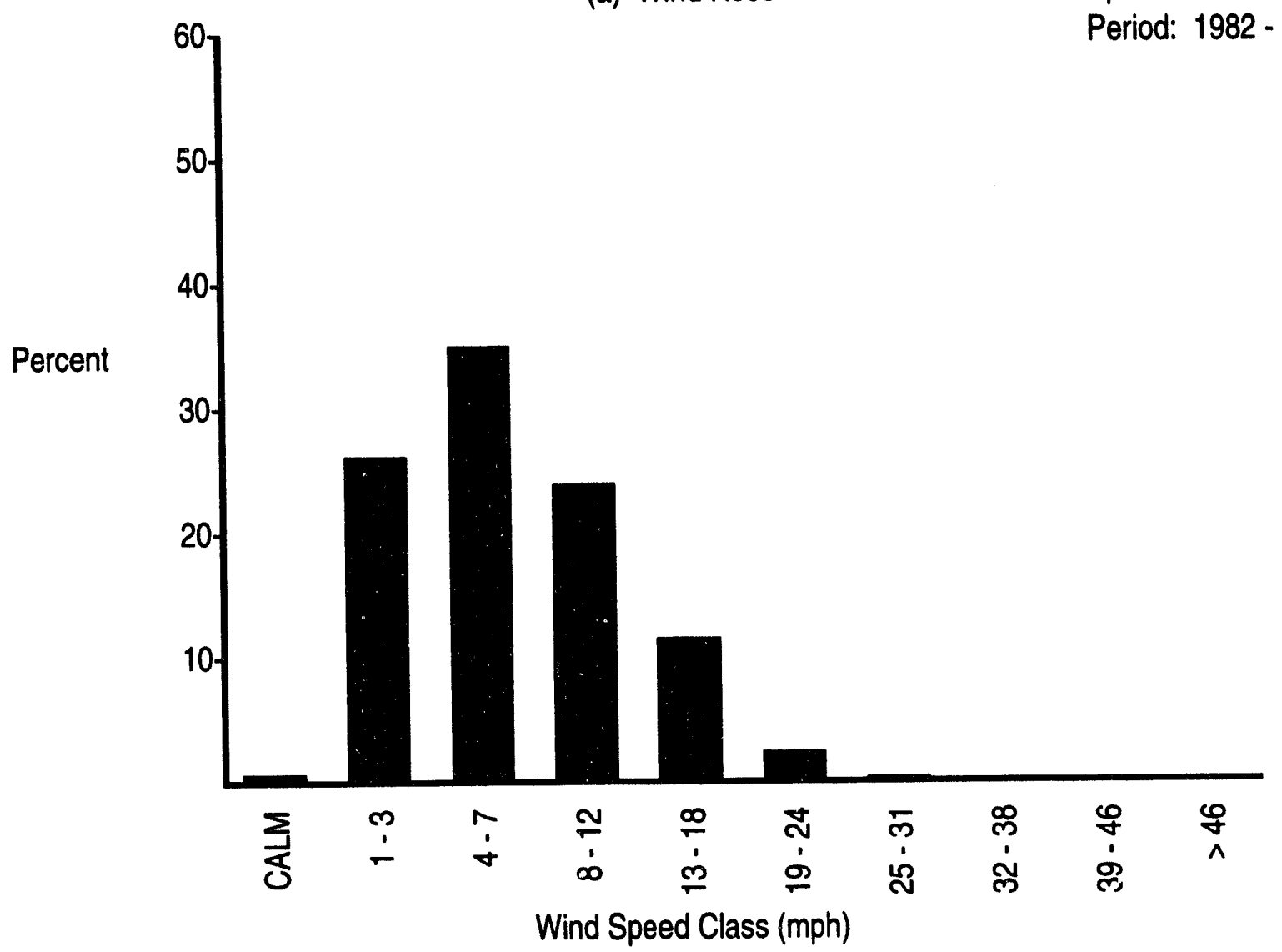

(b) Wind Speed Histogram

FIGURE B.1. (contd)

B. 100 


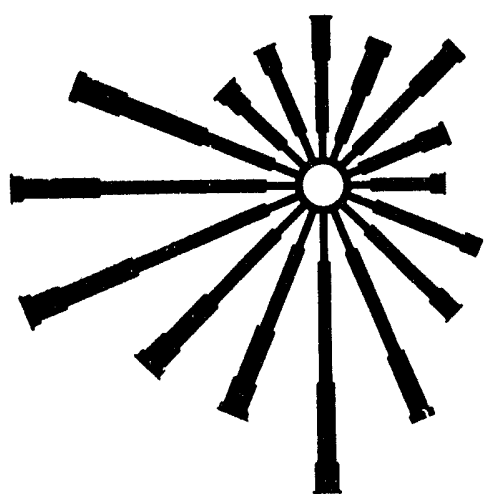

(a) Wind Rose

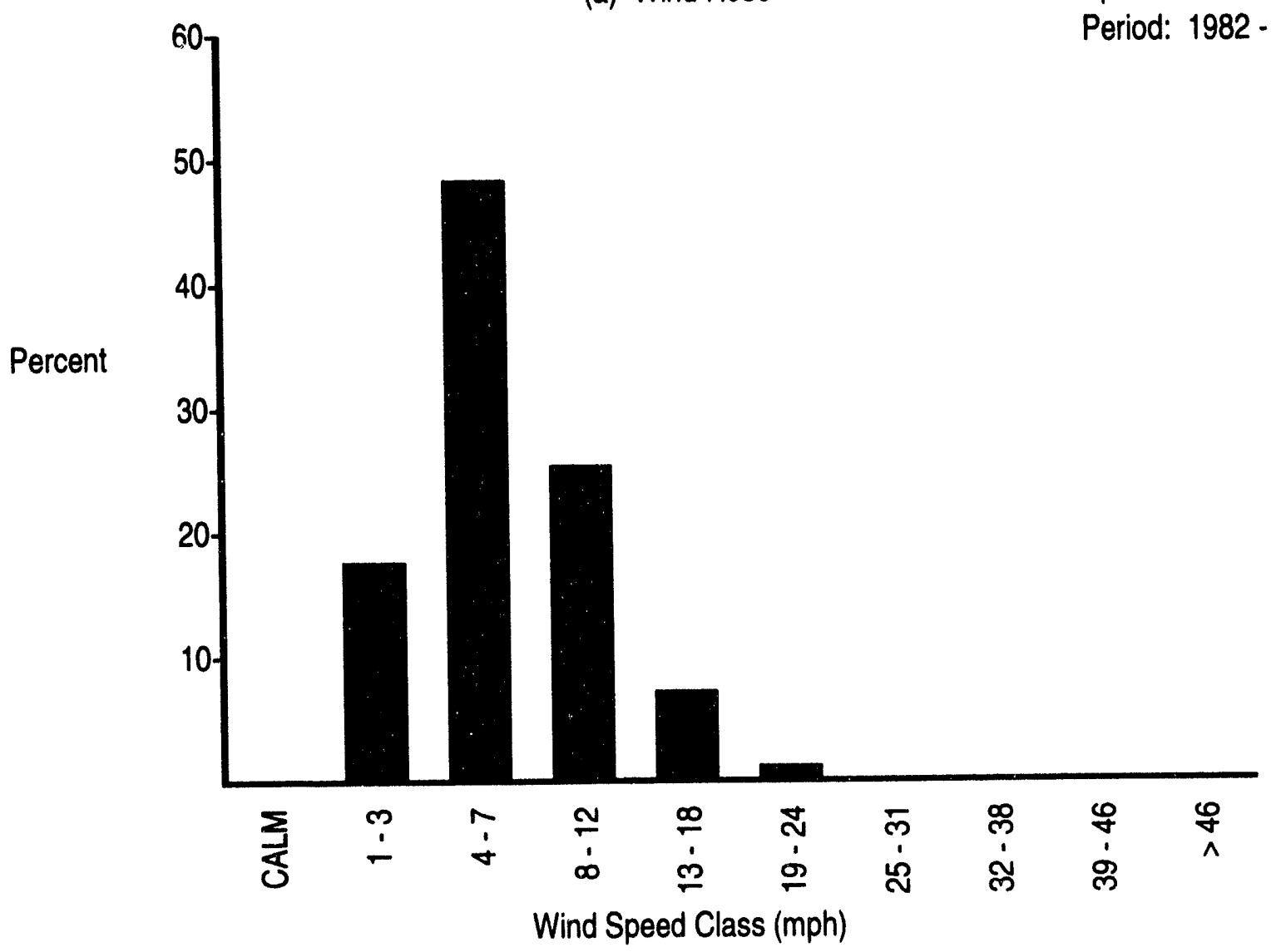

(b) Wind Speed Histogram

FIGURE B.1. (contd)
April Data

Period: 1982 - 1992 


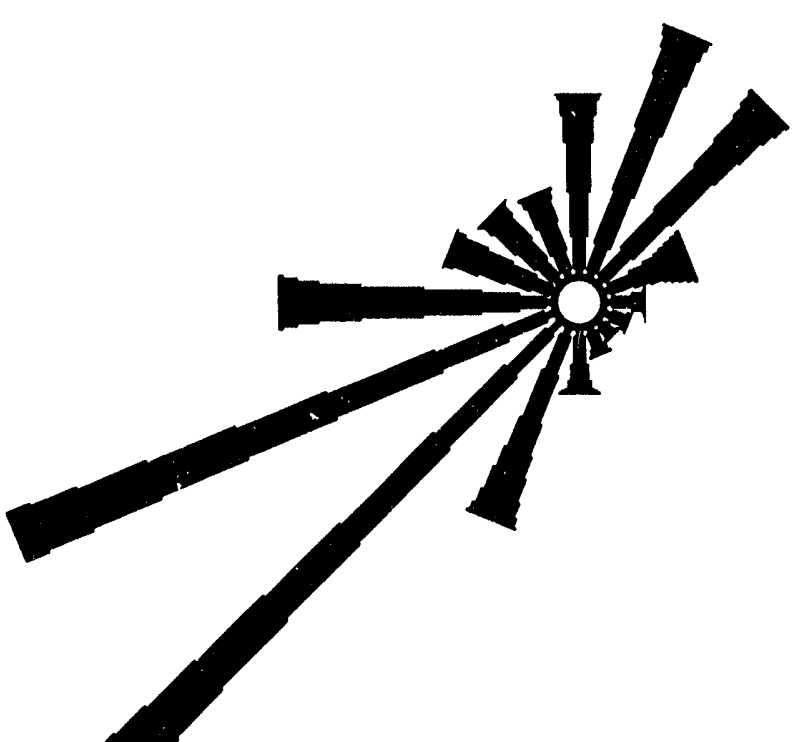

(a) Wind Rose

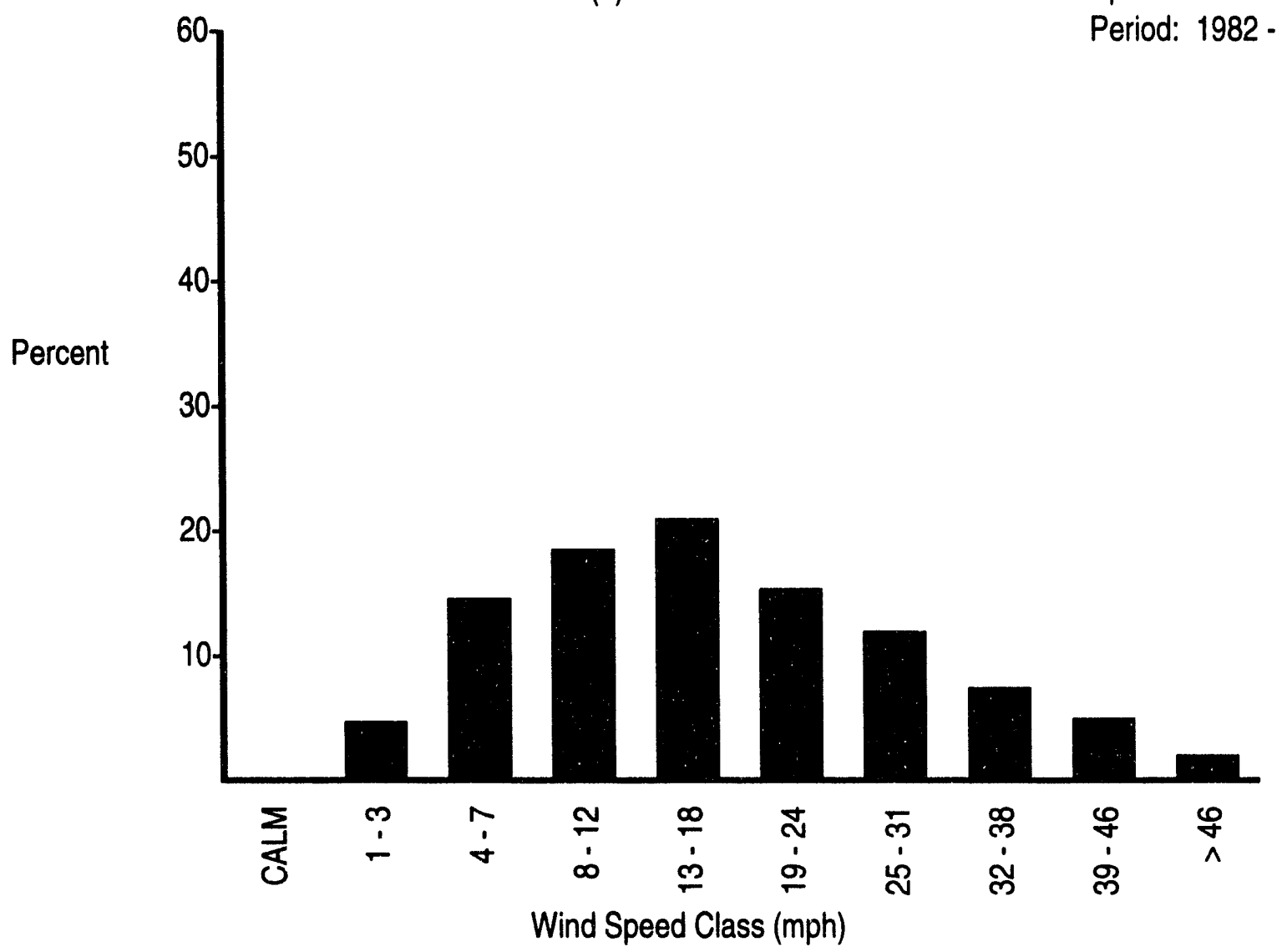

(b) Wind Speed Histogram
April Data

Period: $1982-1993$

\section{FIGURE B.1. (contd)}



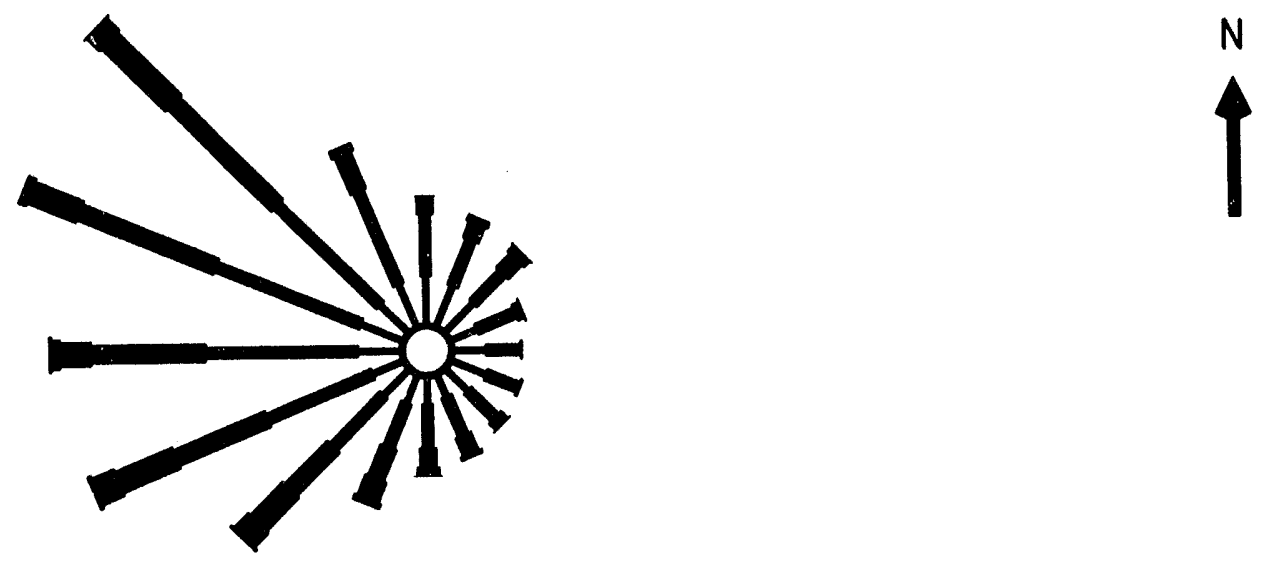

(a) Wind Rose

April Data

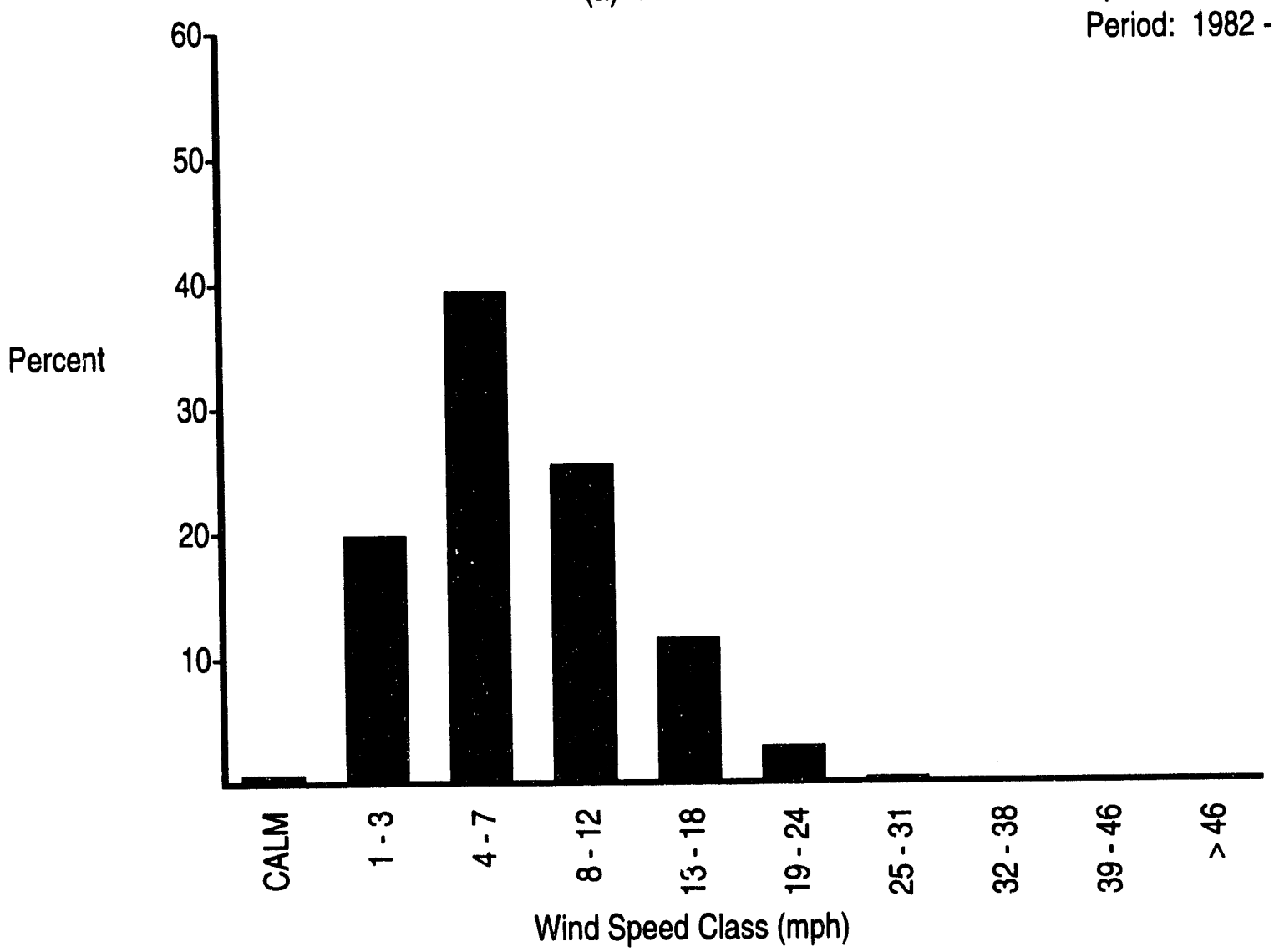

(b) Wind Speed Histogram

FIGURE B.1. (contd) 


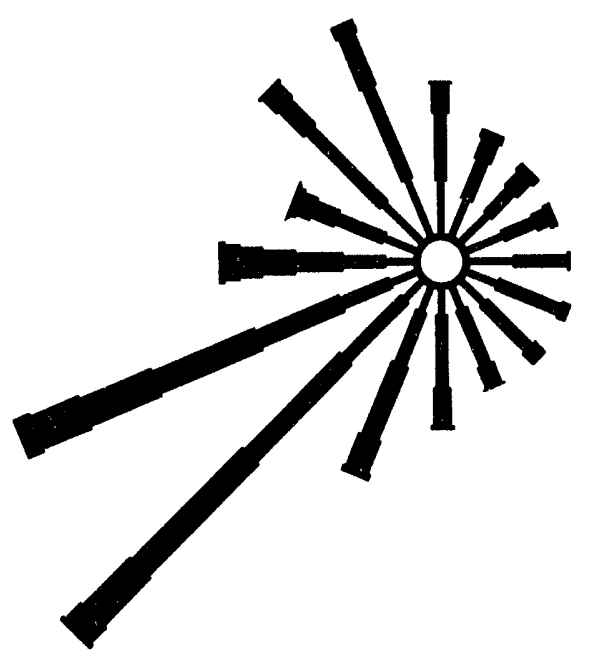

(a) Wind Rose

April Data

Period: 1988 - 1993

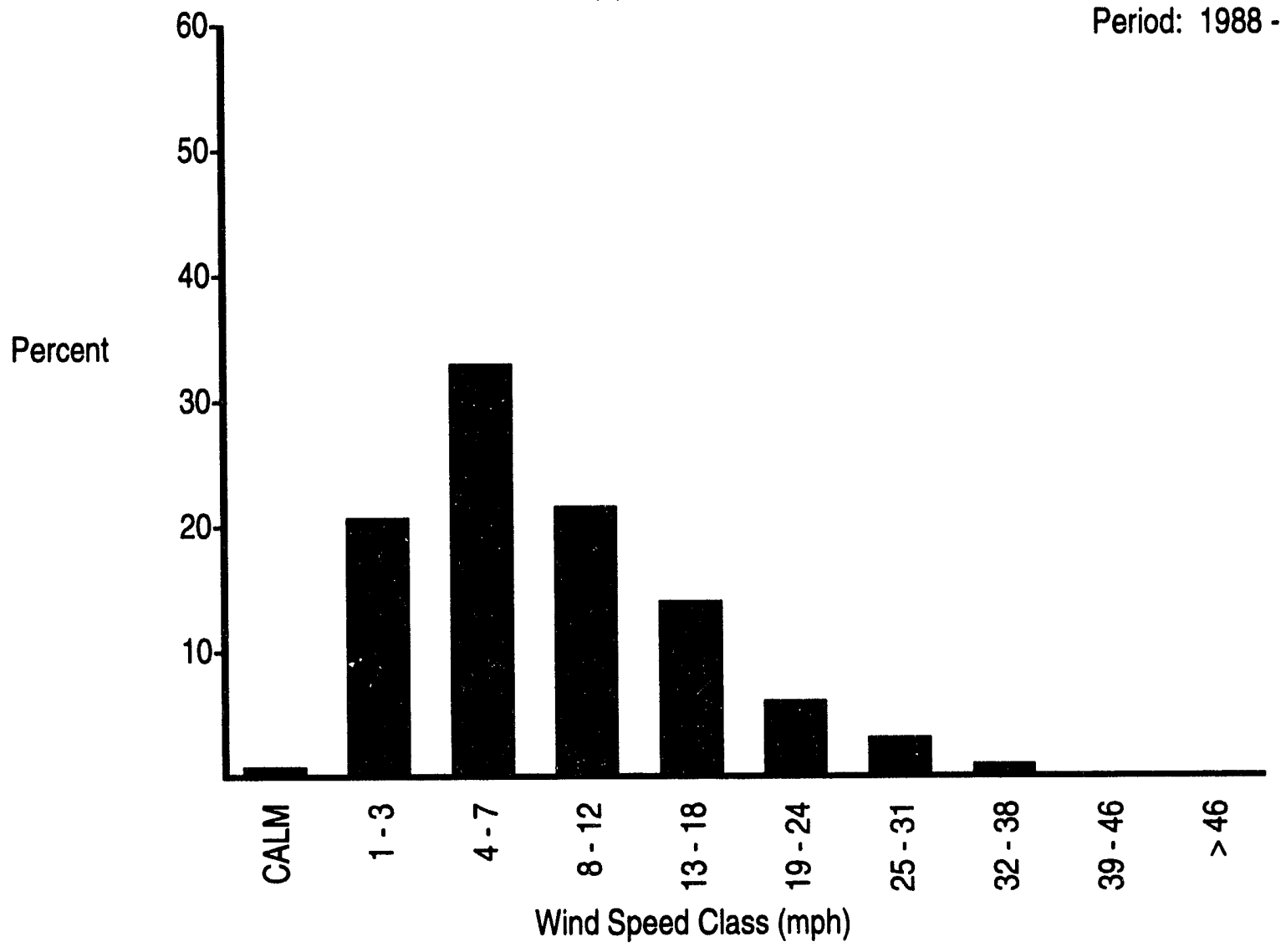

(b) Wind Speed Histogram

FIGURE B.1. (contd)

B.104 


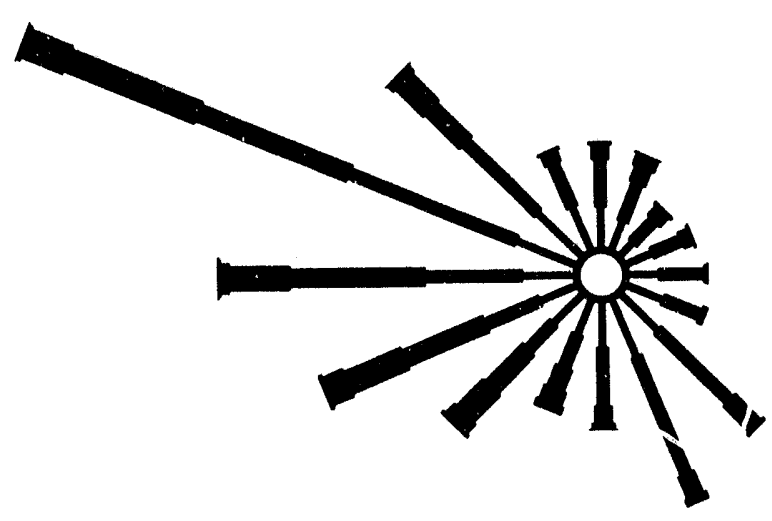

N

(a) Wind Rose

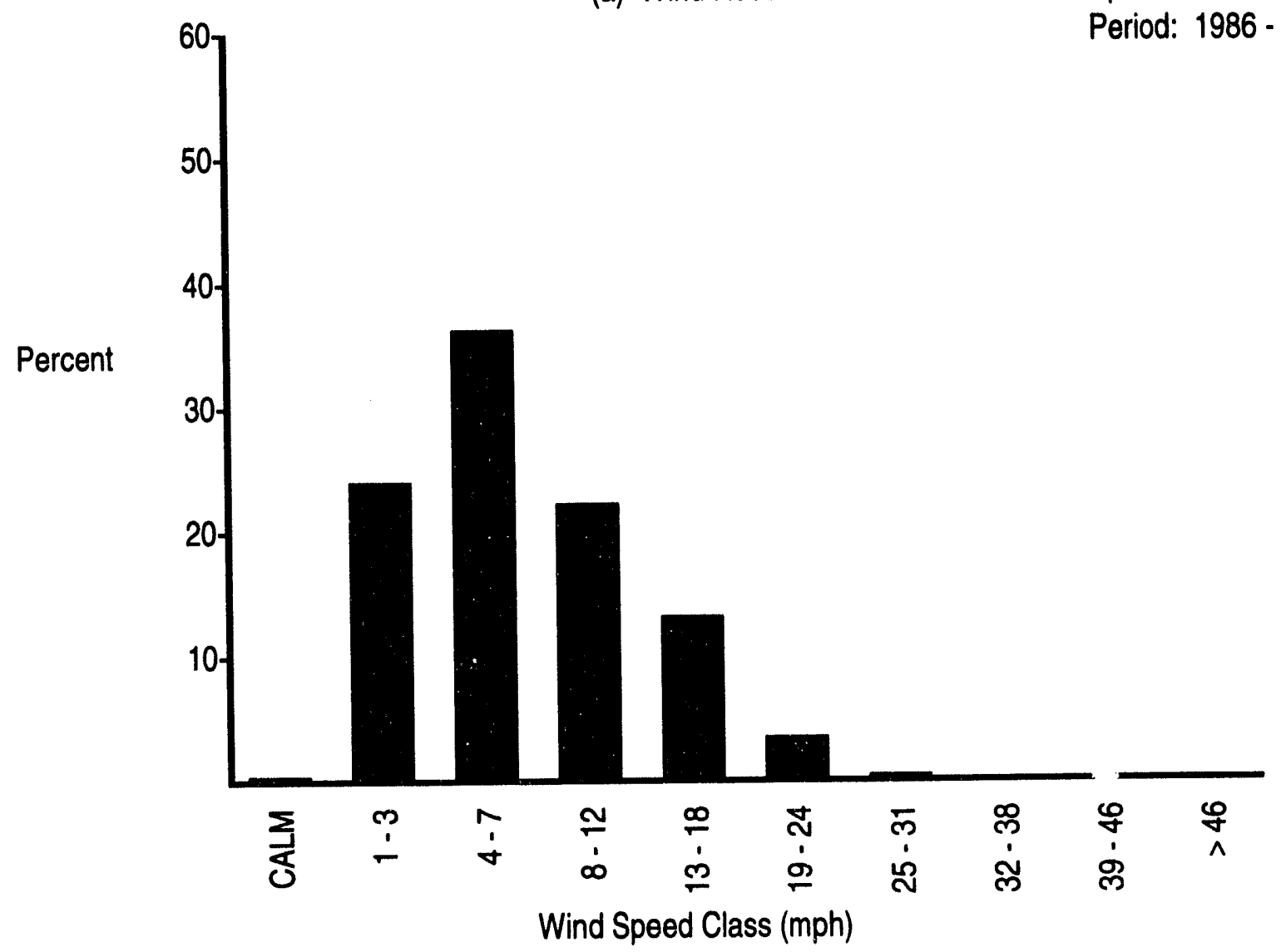

(b) Wind Speed Histogram

FIGURE B.1. (contd)

\section{B.105}




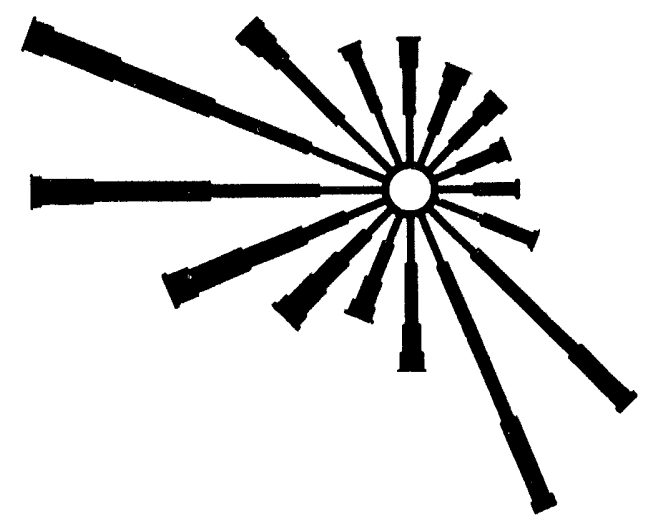

(a) Wind Rose

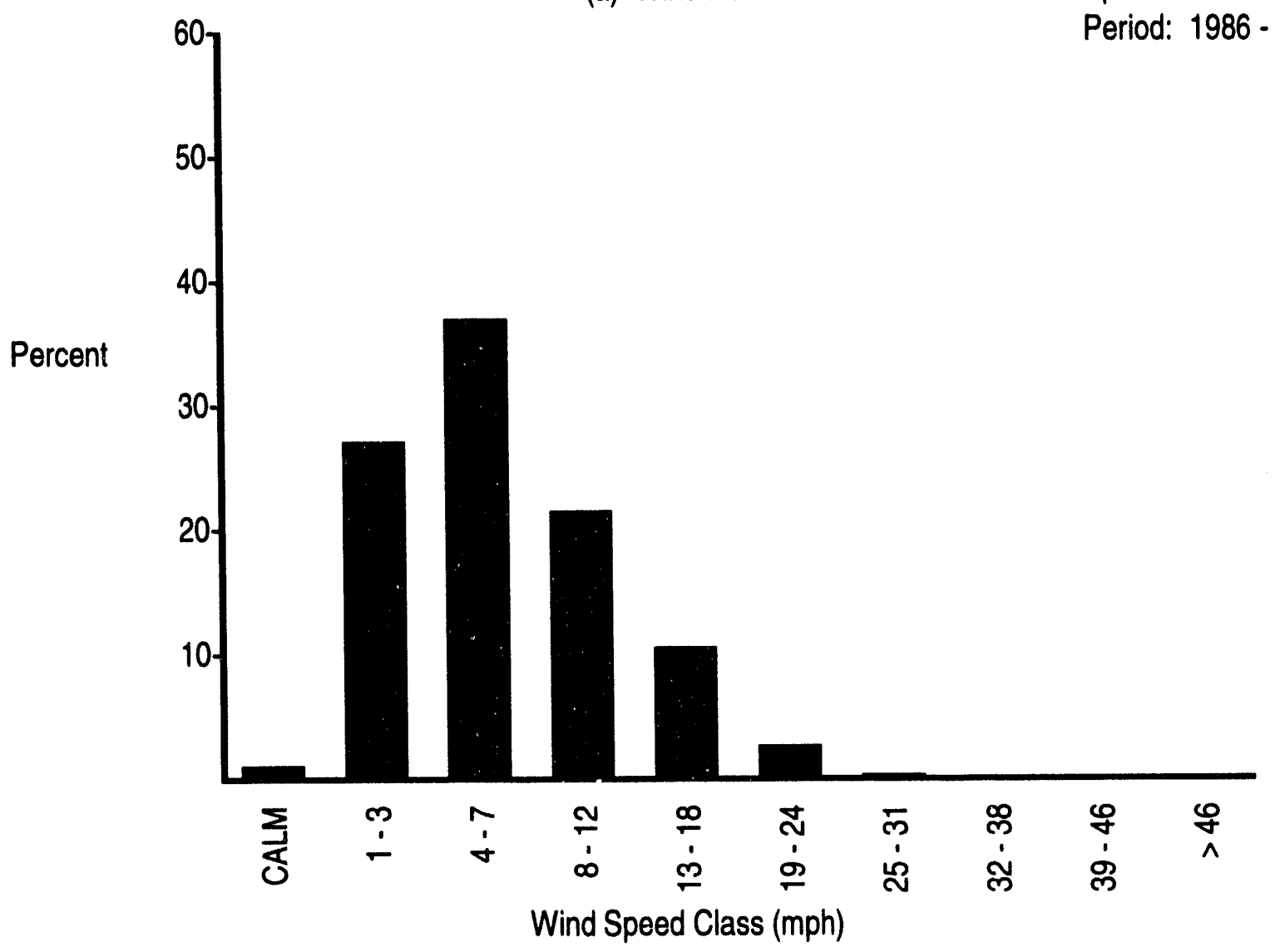

(b) Wind Speed Histogram

FIGURE B.1. (contd) 


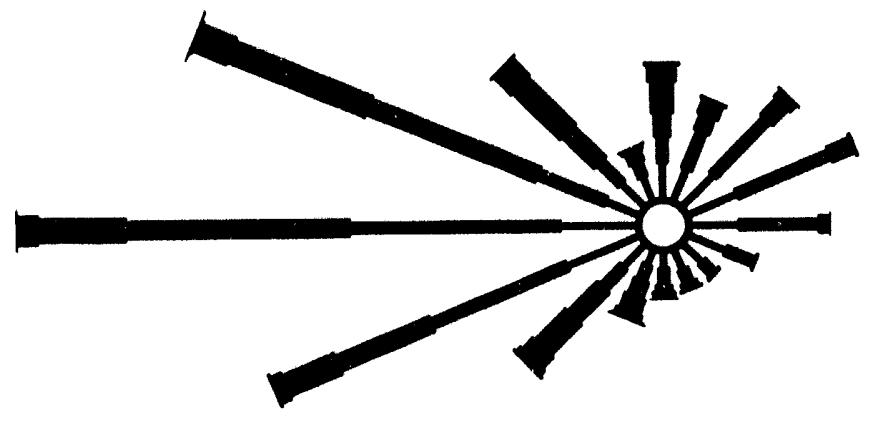

(a) Wind Rose

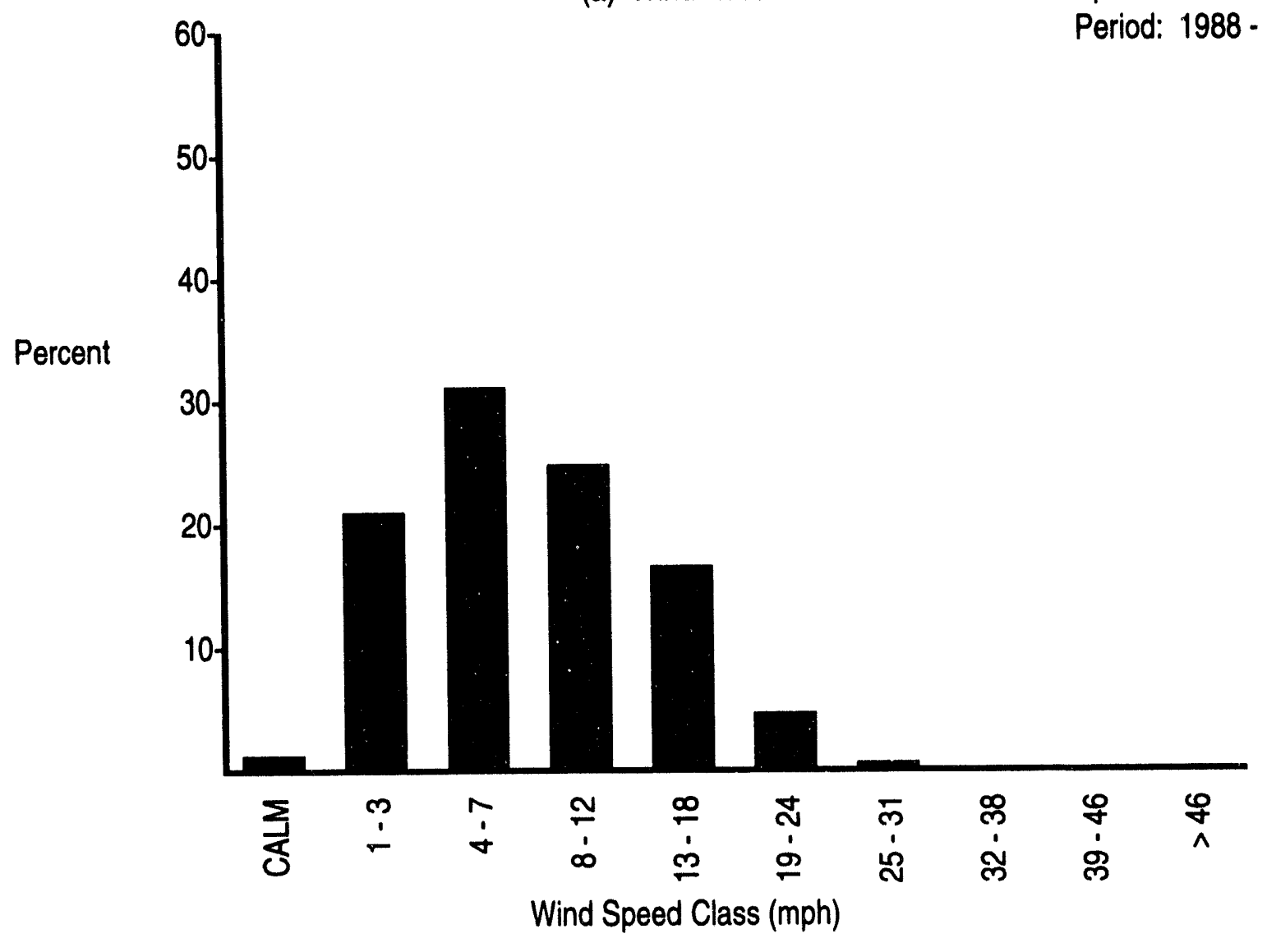

(b) Wind Speed Histogram

FIGURE B.1. (contd) 


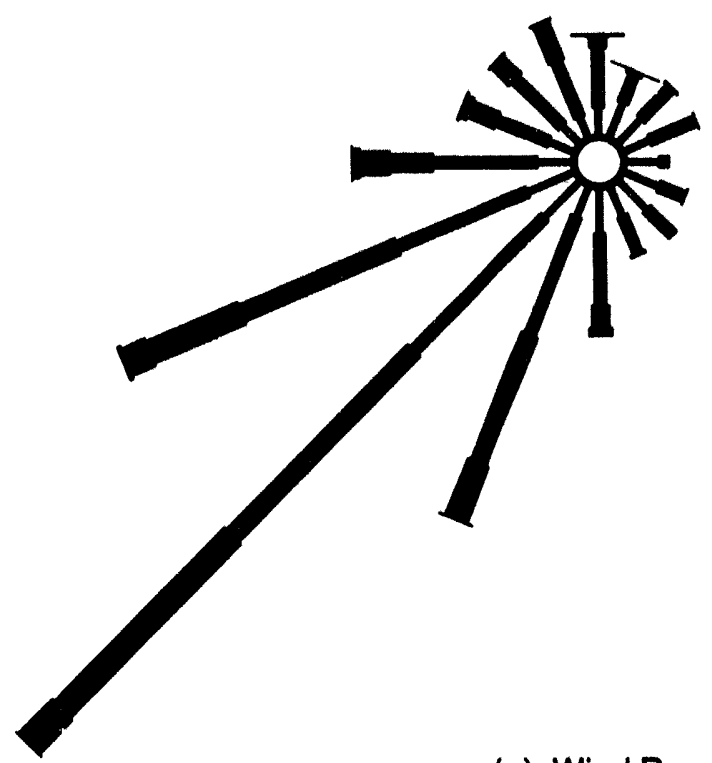

(a) Wind Rose

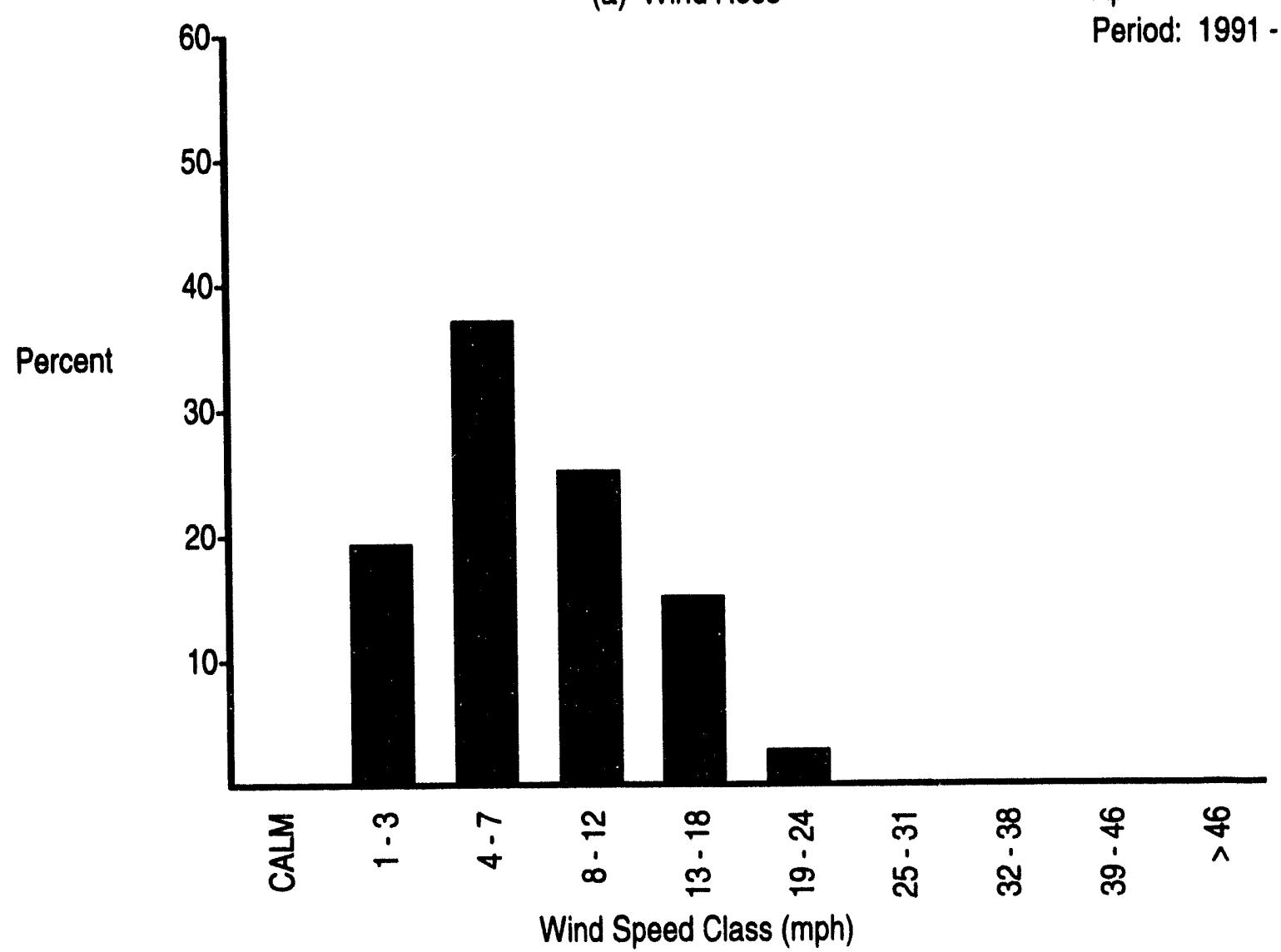

(b) Wind Speed Histogram

FIGURE B.1. (contd) 


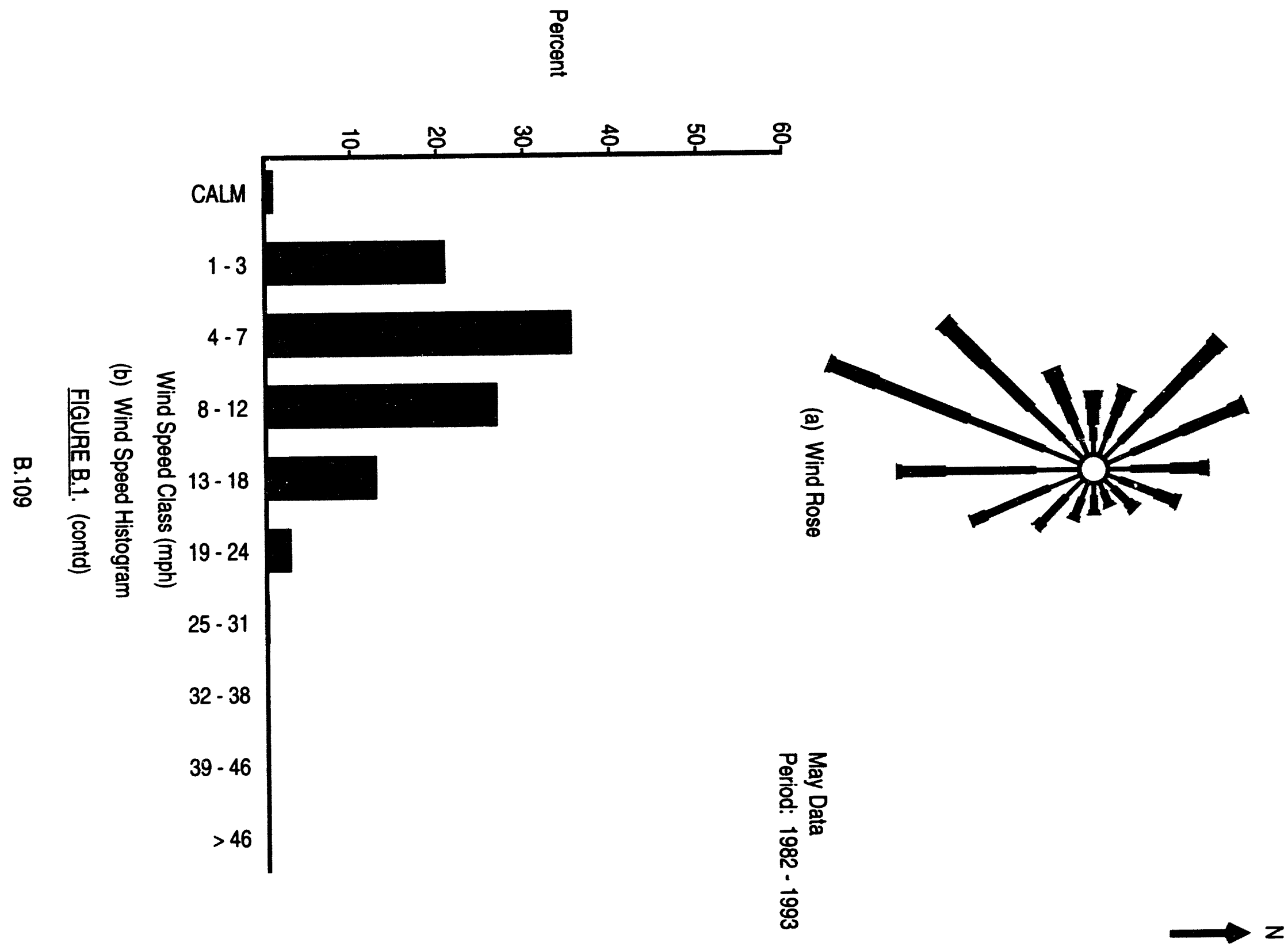




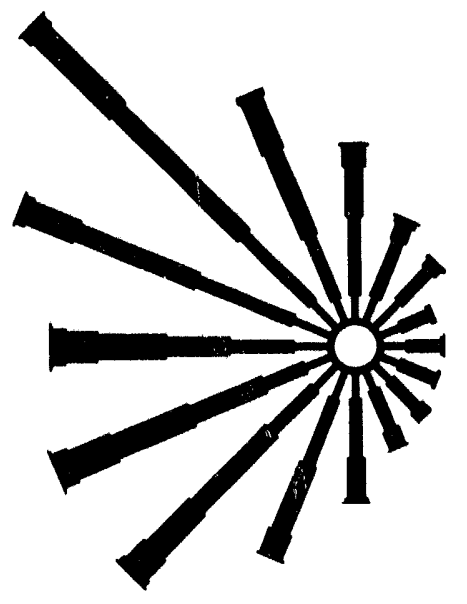

(a) Wind Rose

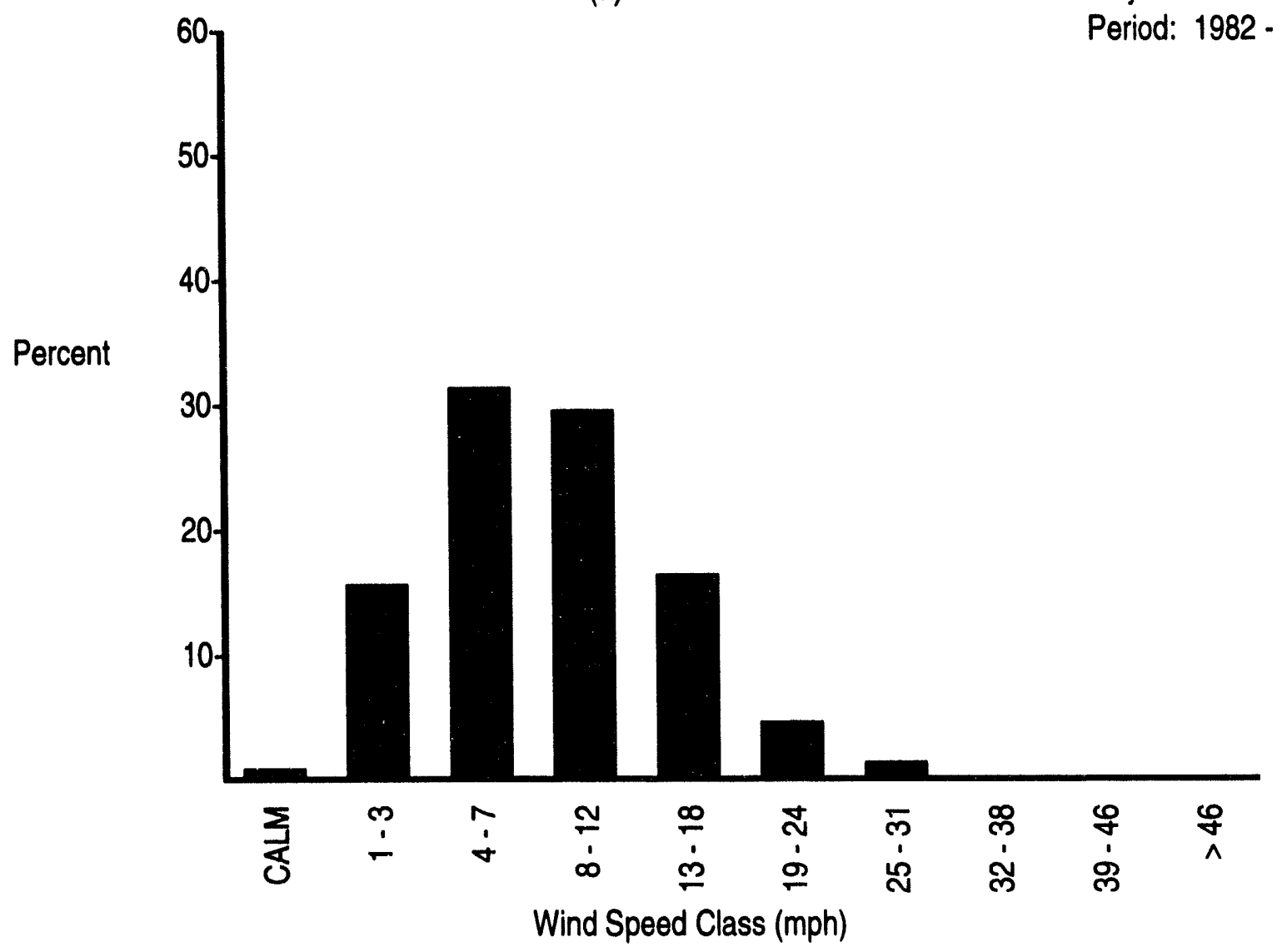

(b) Wind Speed Histogram

FIGURE B.1. (contd)
May Data

Period: 1982 - 1993

\section{B. 110}




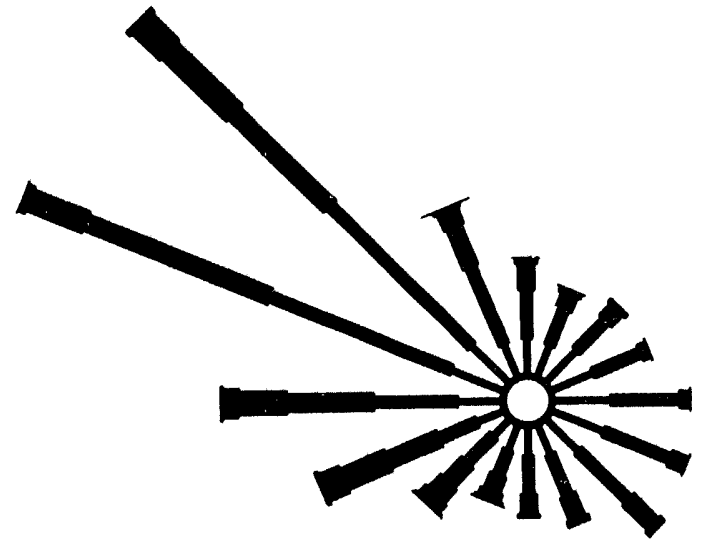

(a) Wind Rose

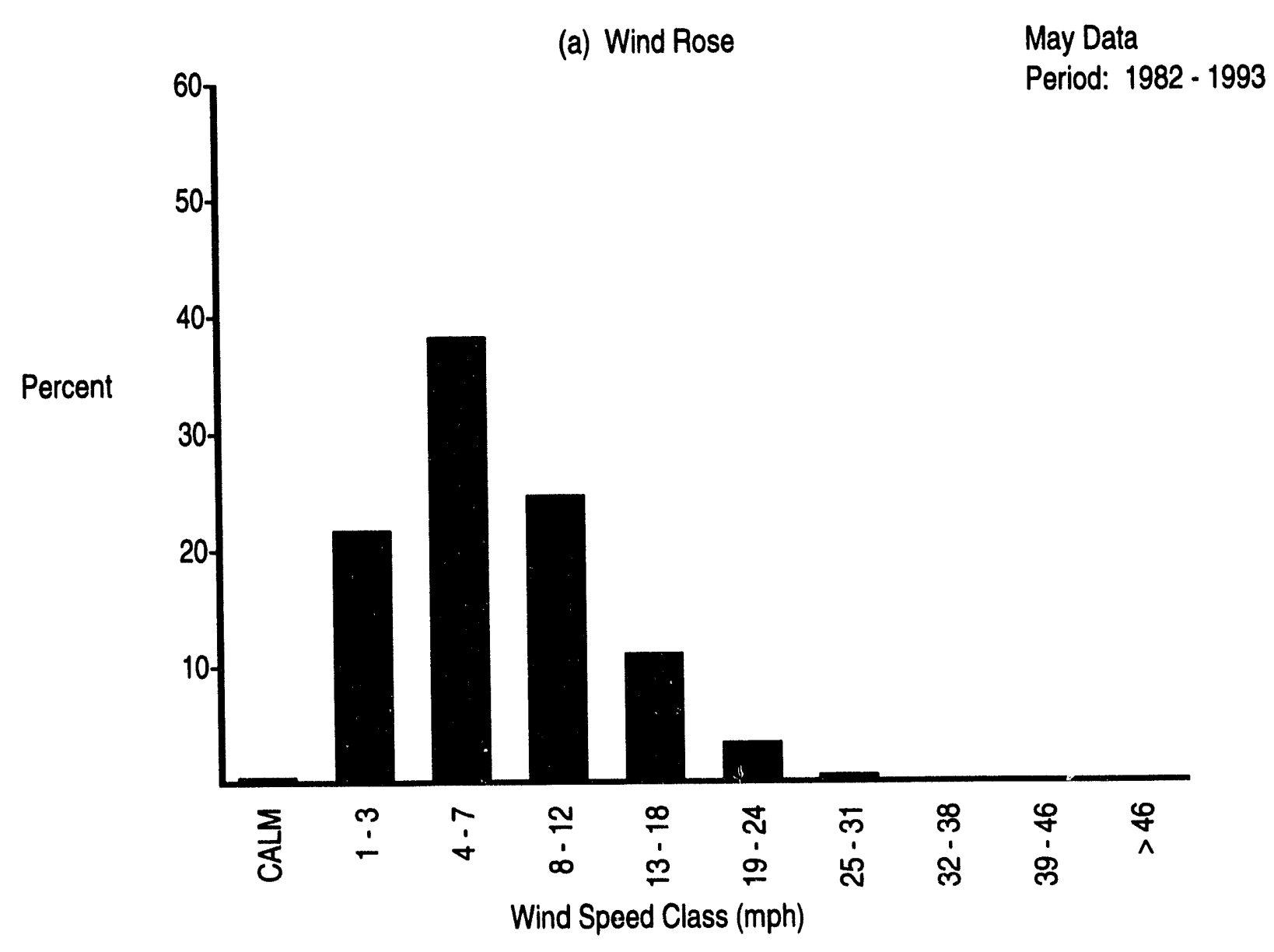

(b) Wind Speed Histogram

FIGURE B.1. (contd)

B.111 


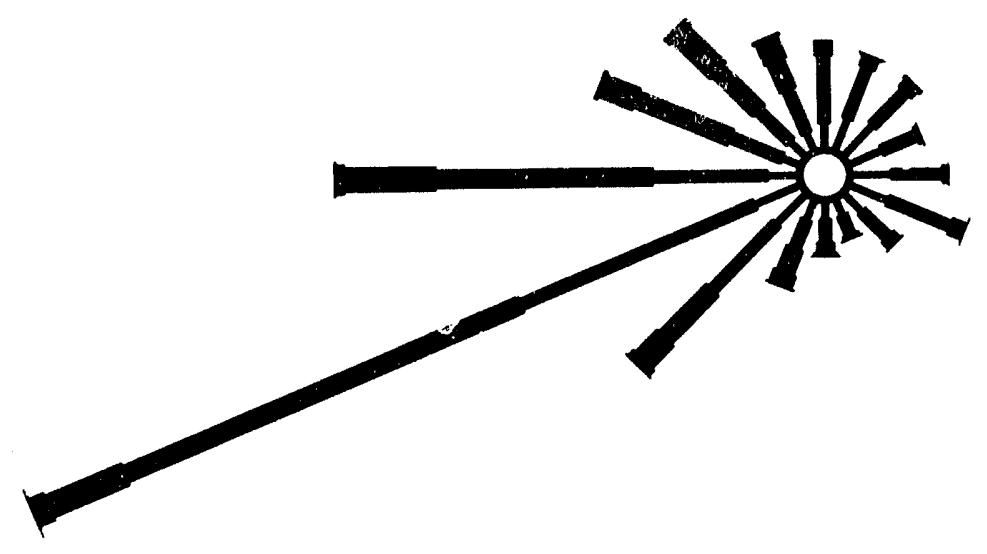

May Data

(a) Wind Rose

Period: 1982 - 1993

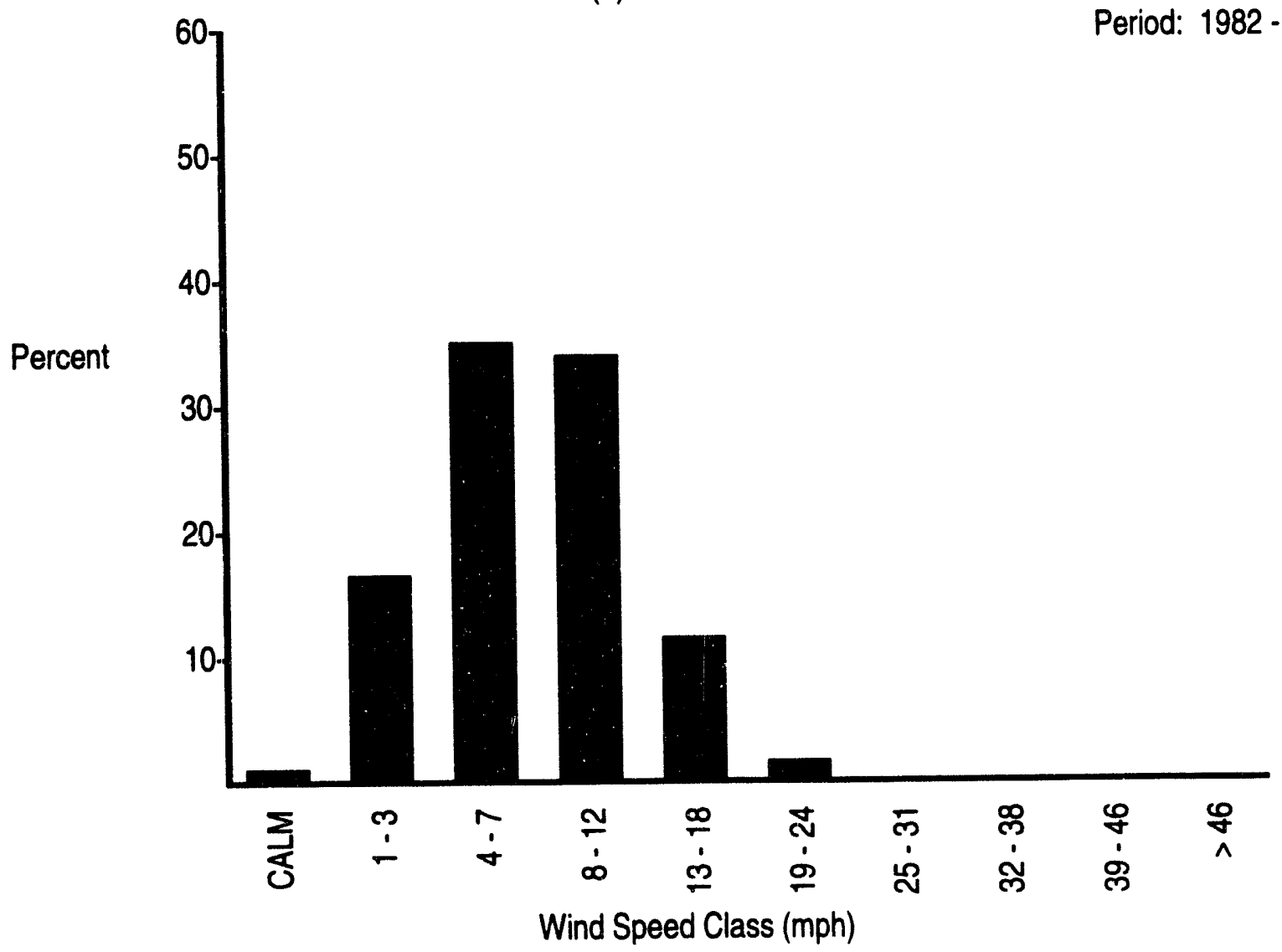

(b) Wind Speed Histogram

FIGURE B.1. (contd)

\section{B.112}




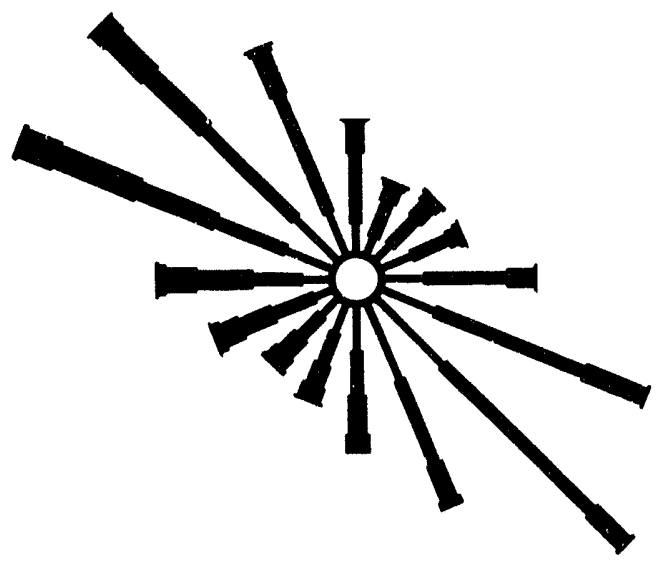

(a) Wind Rose

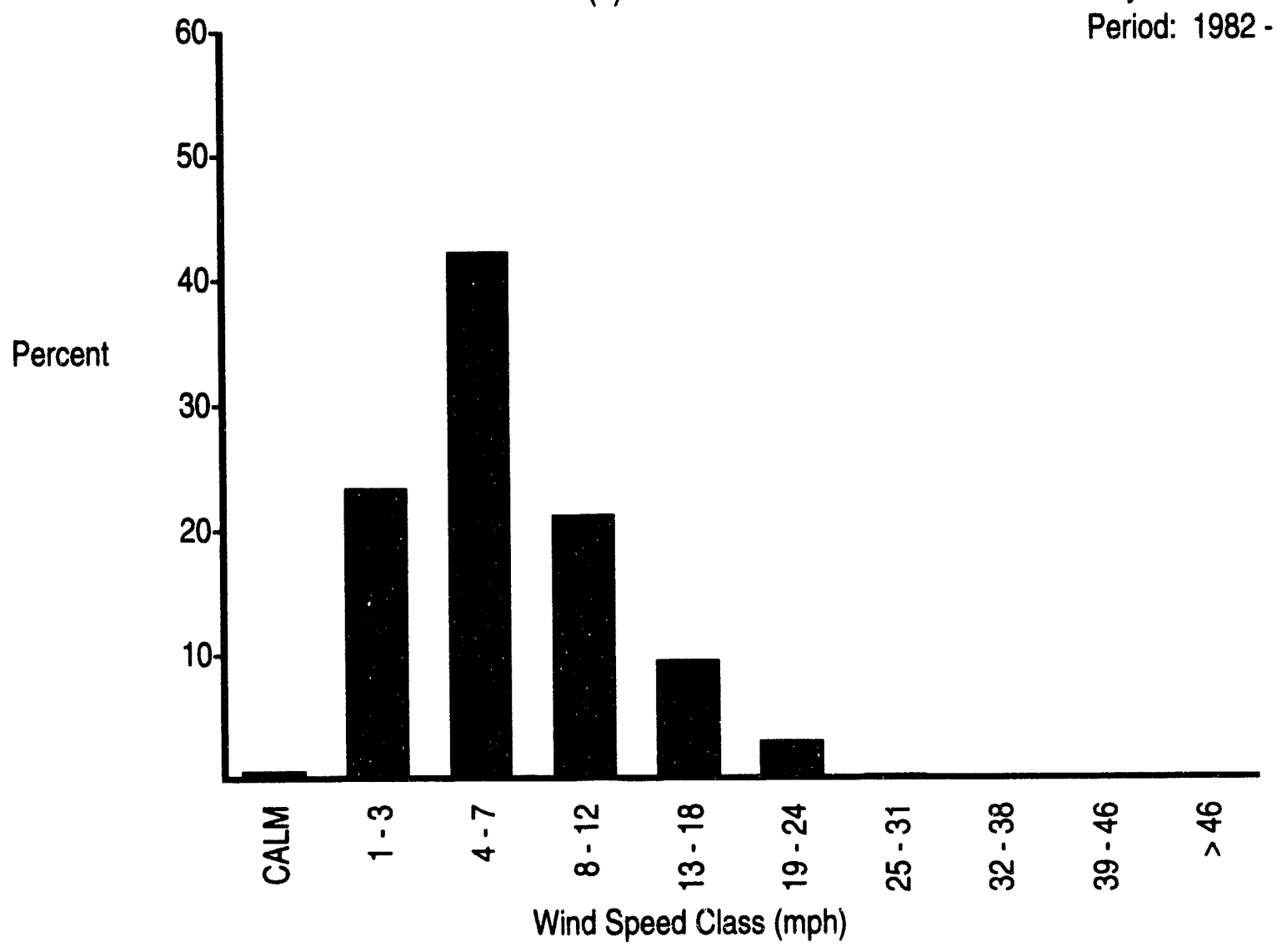

(b) Wind Speed Histogram
May Data

Period: 1982 - 1993

\section{FIGURE B.1. (contd)}




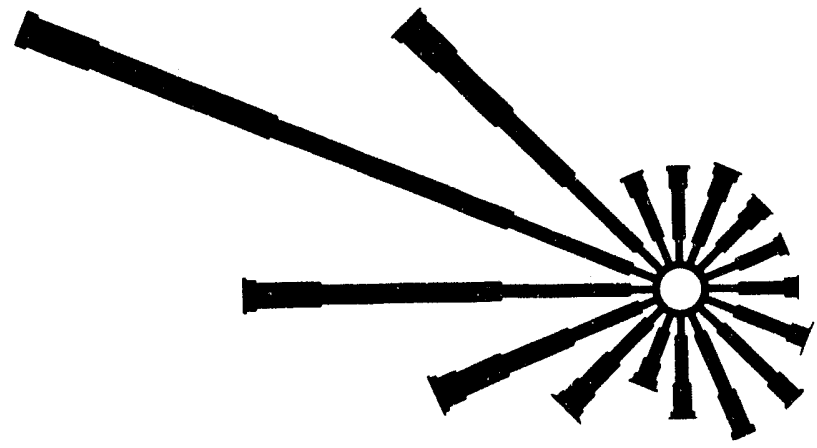

N

(a) Wind Rose

May Data

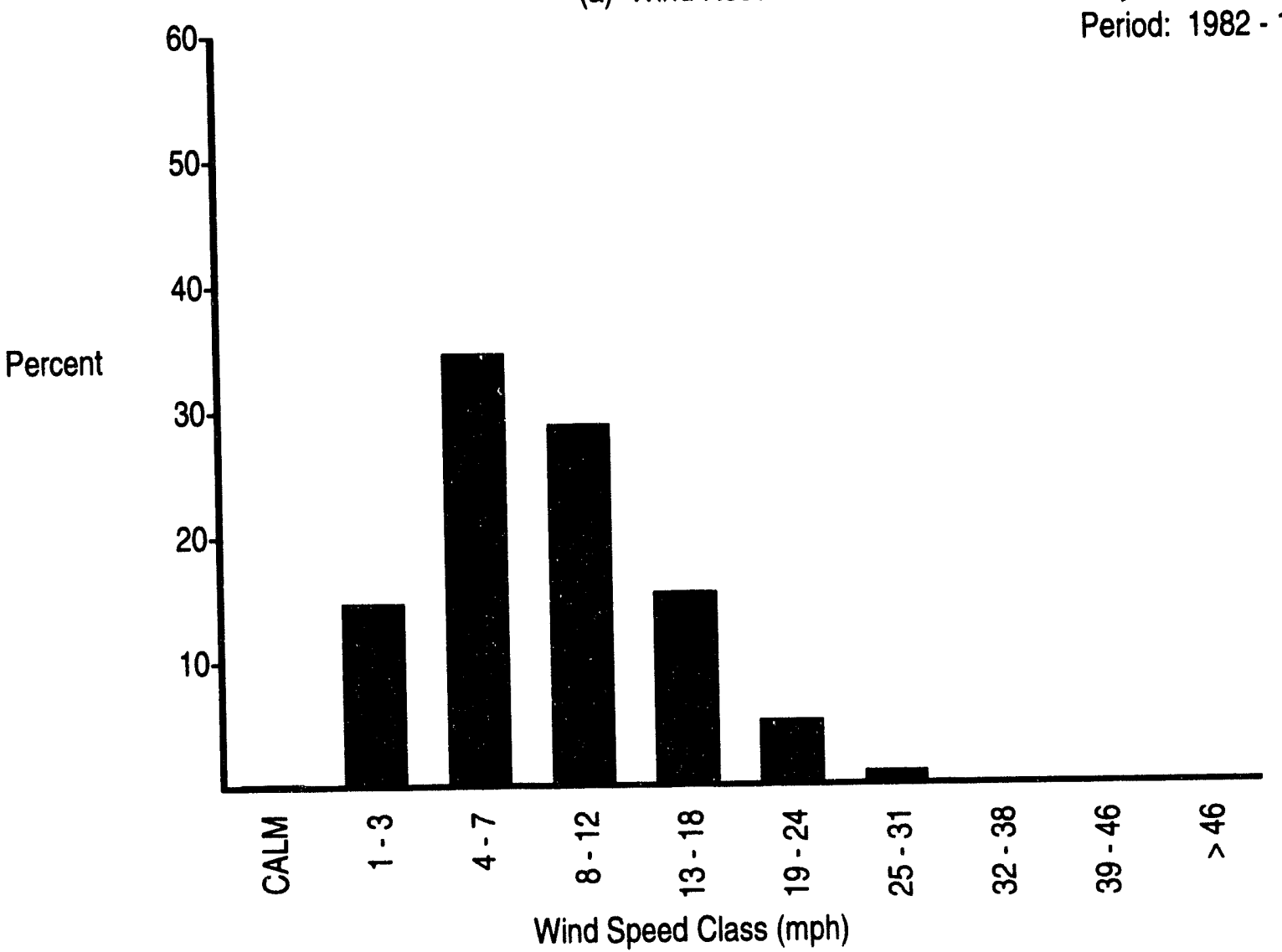

(b) Wind Speed Histogram

FIGURE B.1. (contd)

B.114 


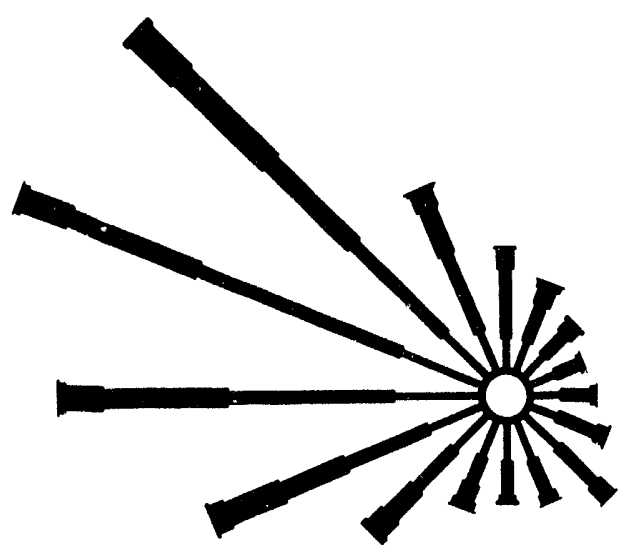

(a) Wind Rose

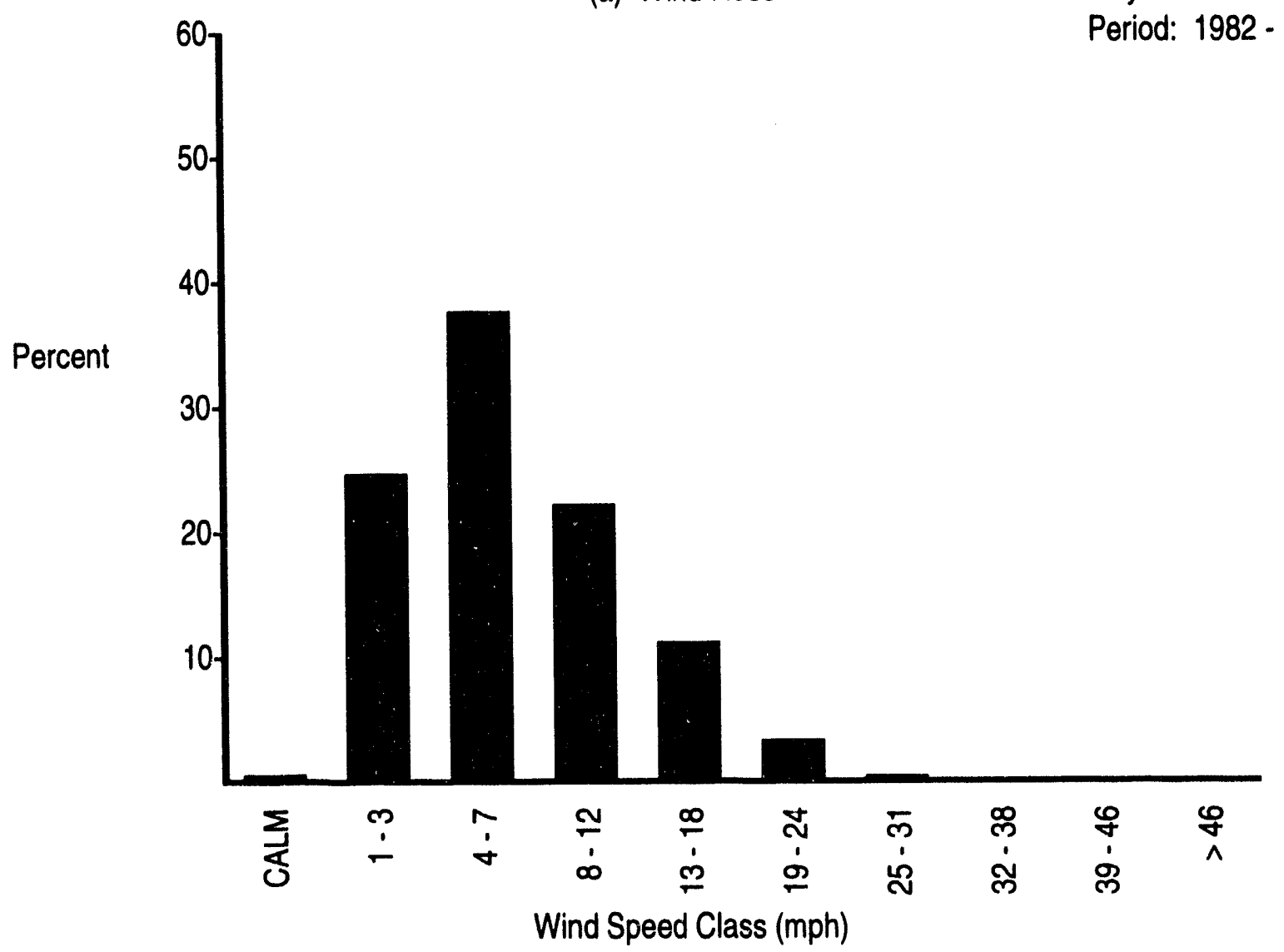

(b) Wind Speed Histogram

FIGURE B.1. (contd)

B.115 


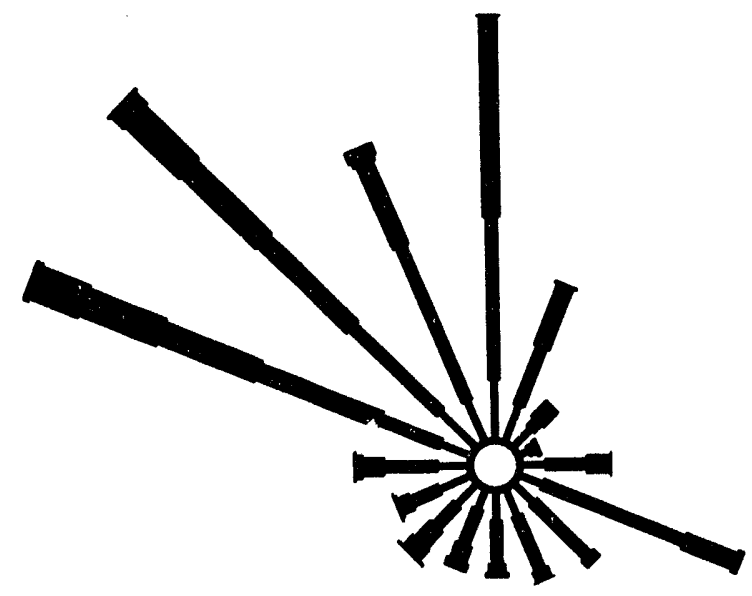

(a) Wind Rose

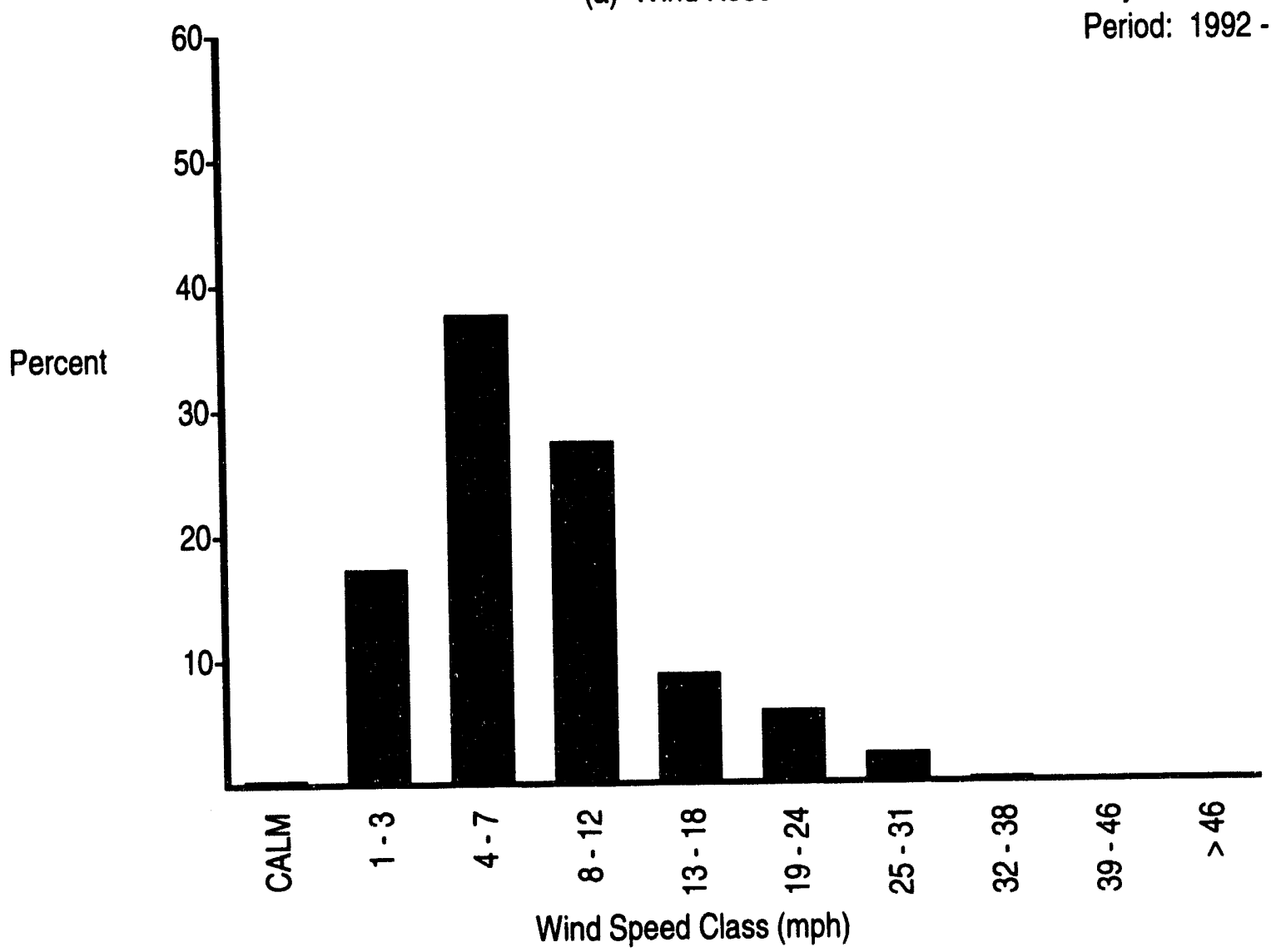

(b) Wind Speed Histogram

FIGURE B.1. (contd)

B.116 


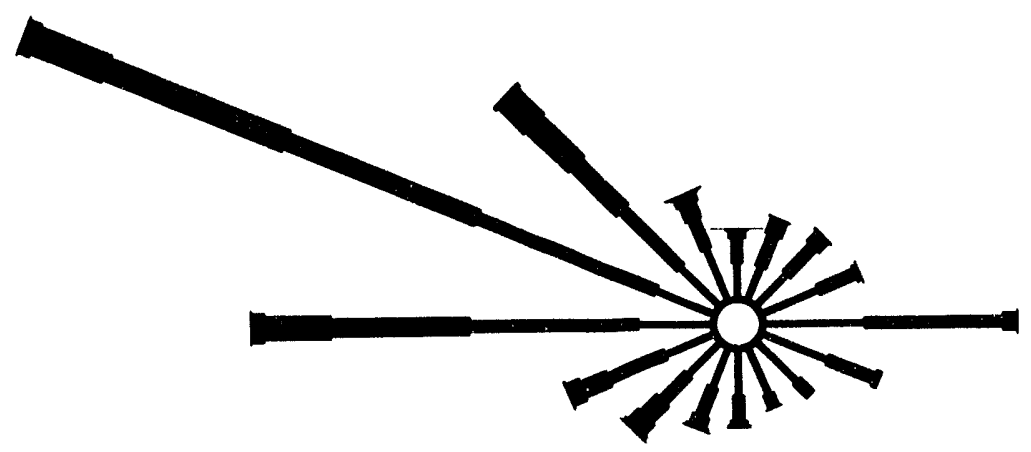

(a) Wind Rose

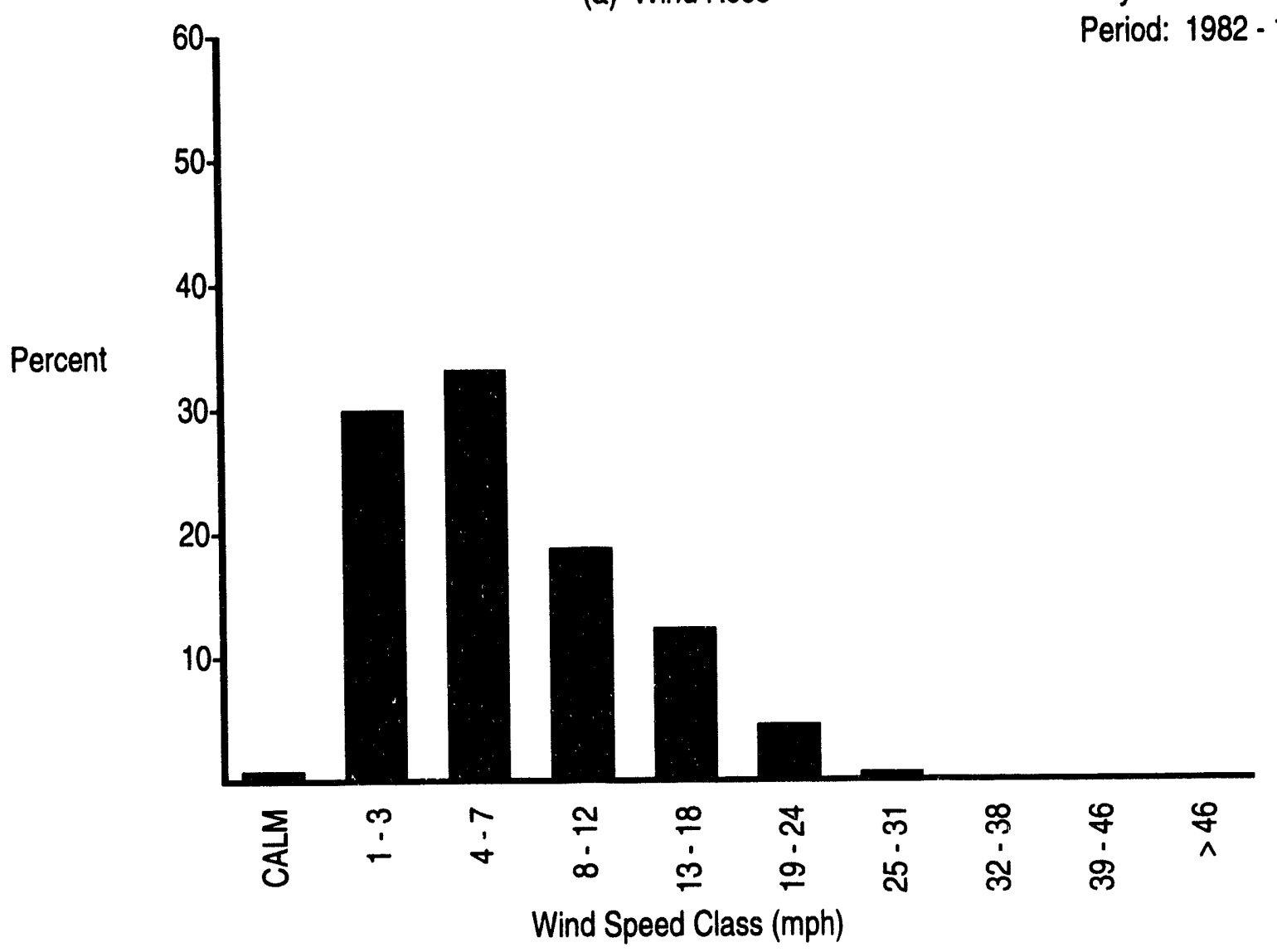

(b) Wind Speed Histogram

\section{FIGURE B.1. (contd)}

May Data

Period: 1982 - 1991

\section{B.117}




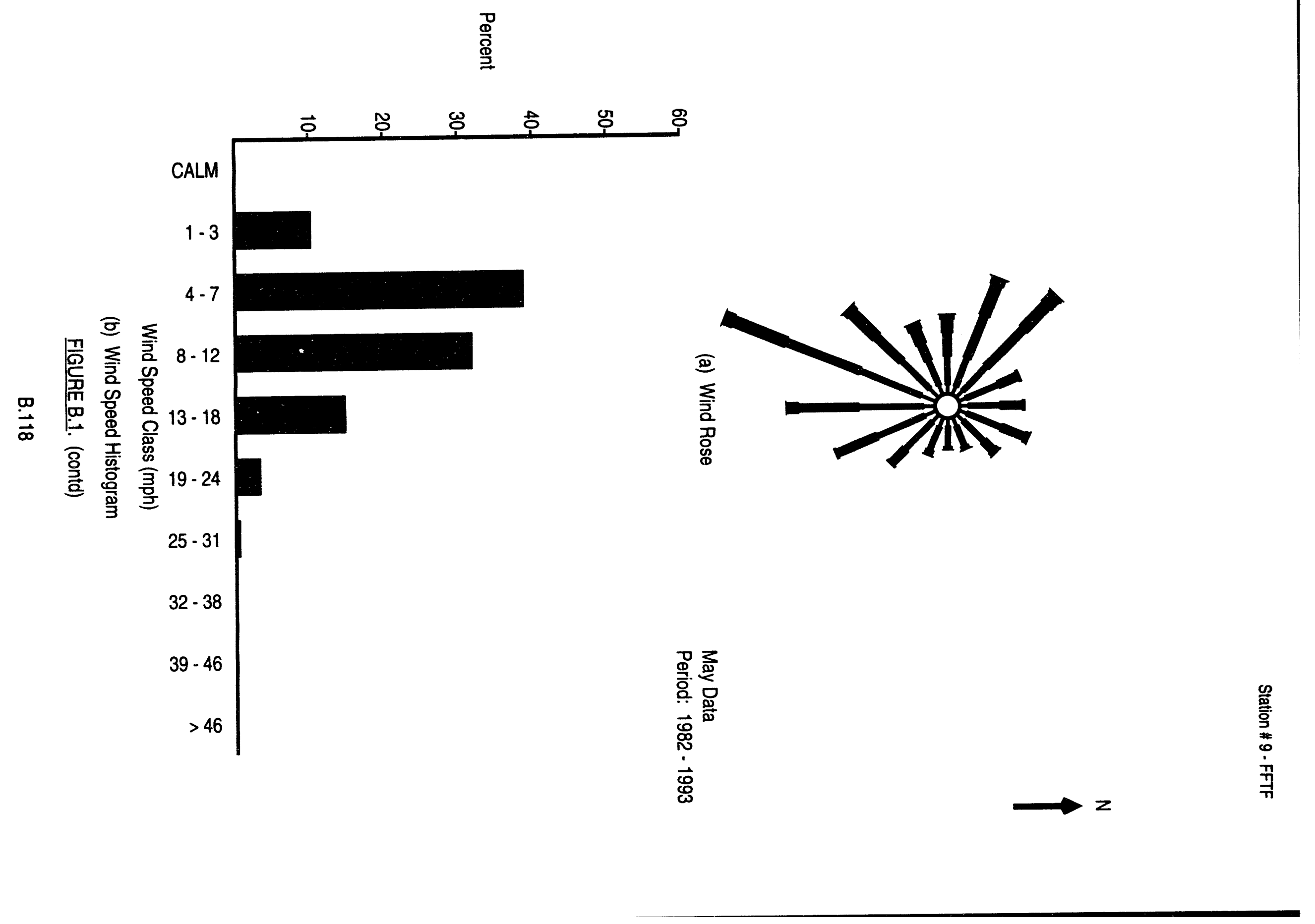




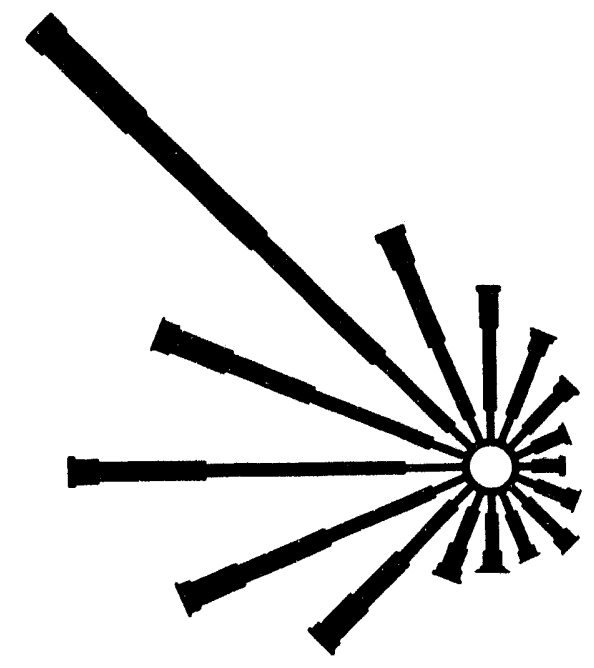

(a) Wind Rose

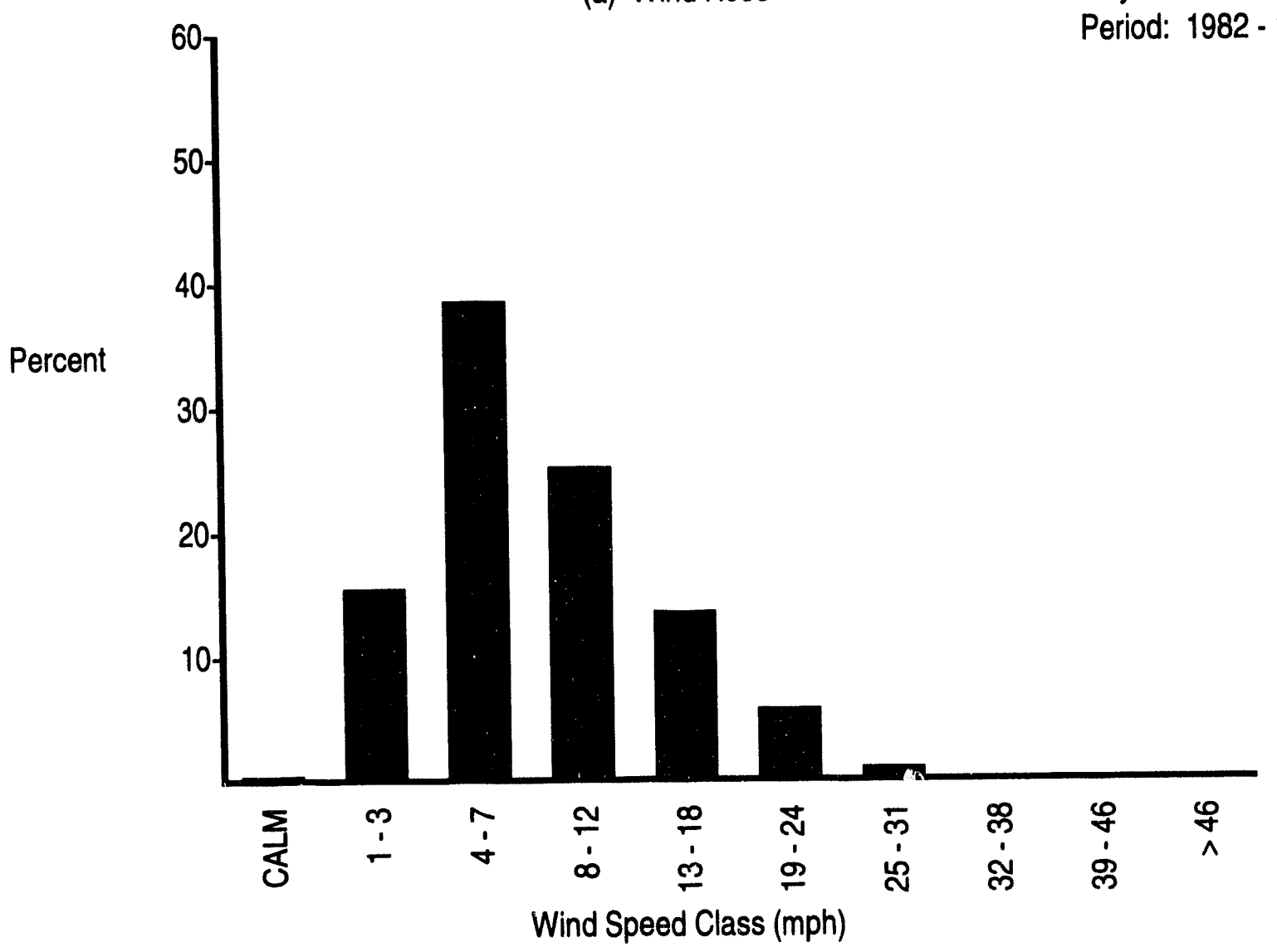

(b) Wind Speed Histogram

B.119 


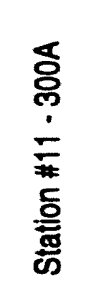

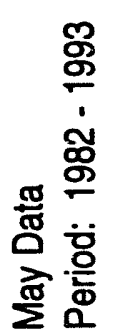

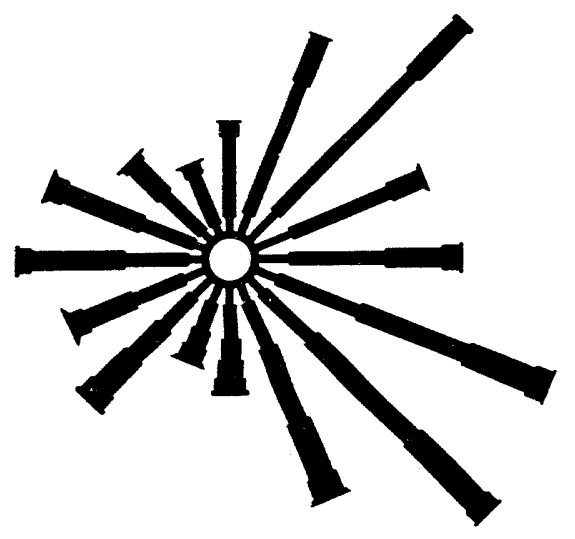

\$
0
0
을
즈

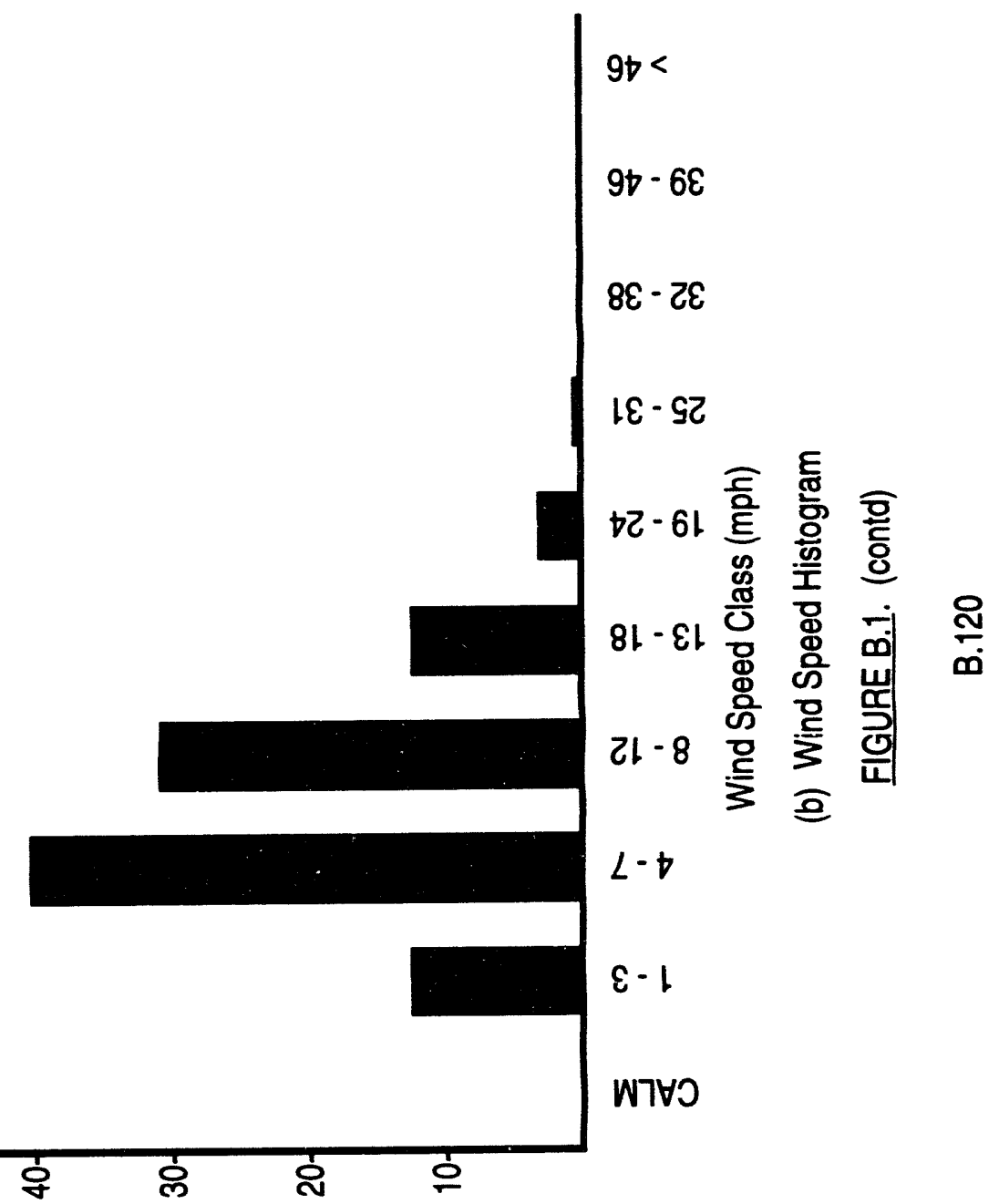

్ㅗㅇ
임 


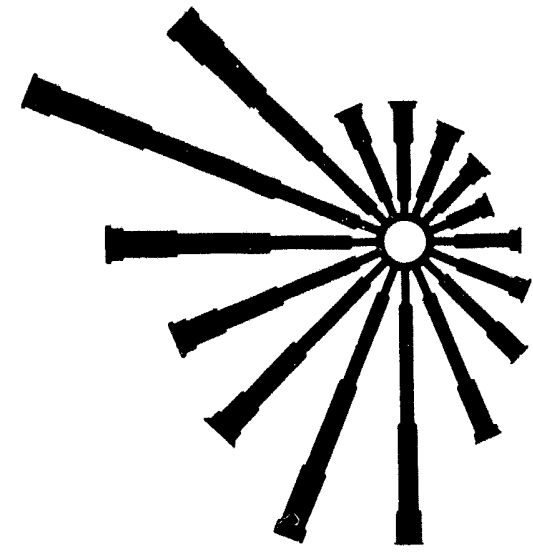

$\hat{N}$

(a) Wind Rose

Maj Data

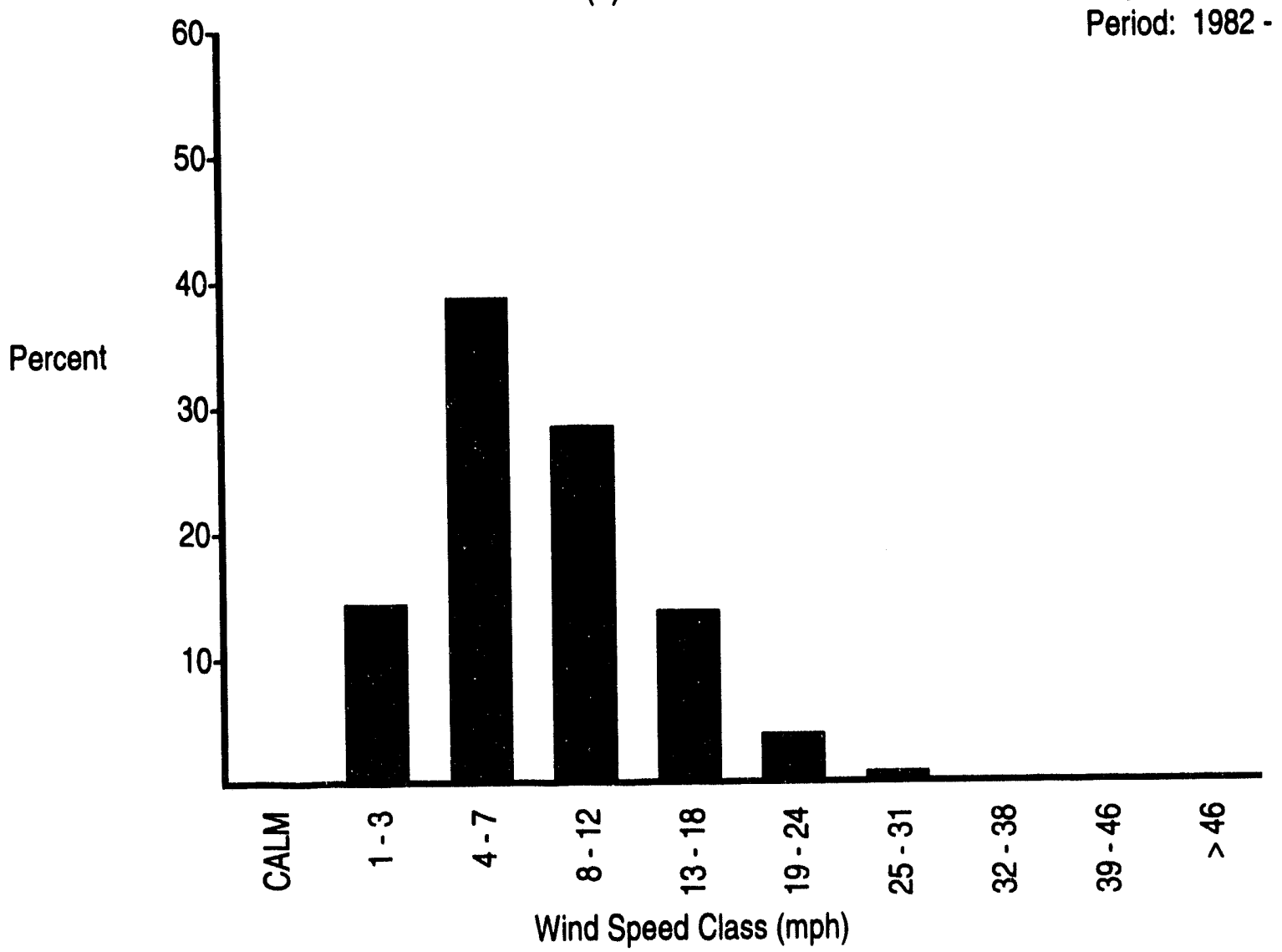

(b) Wind Speed Histogram

FIGURE B.1. (contd) 


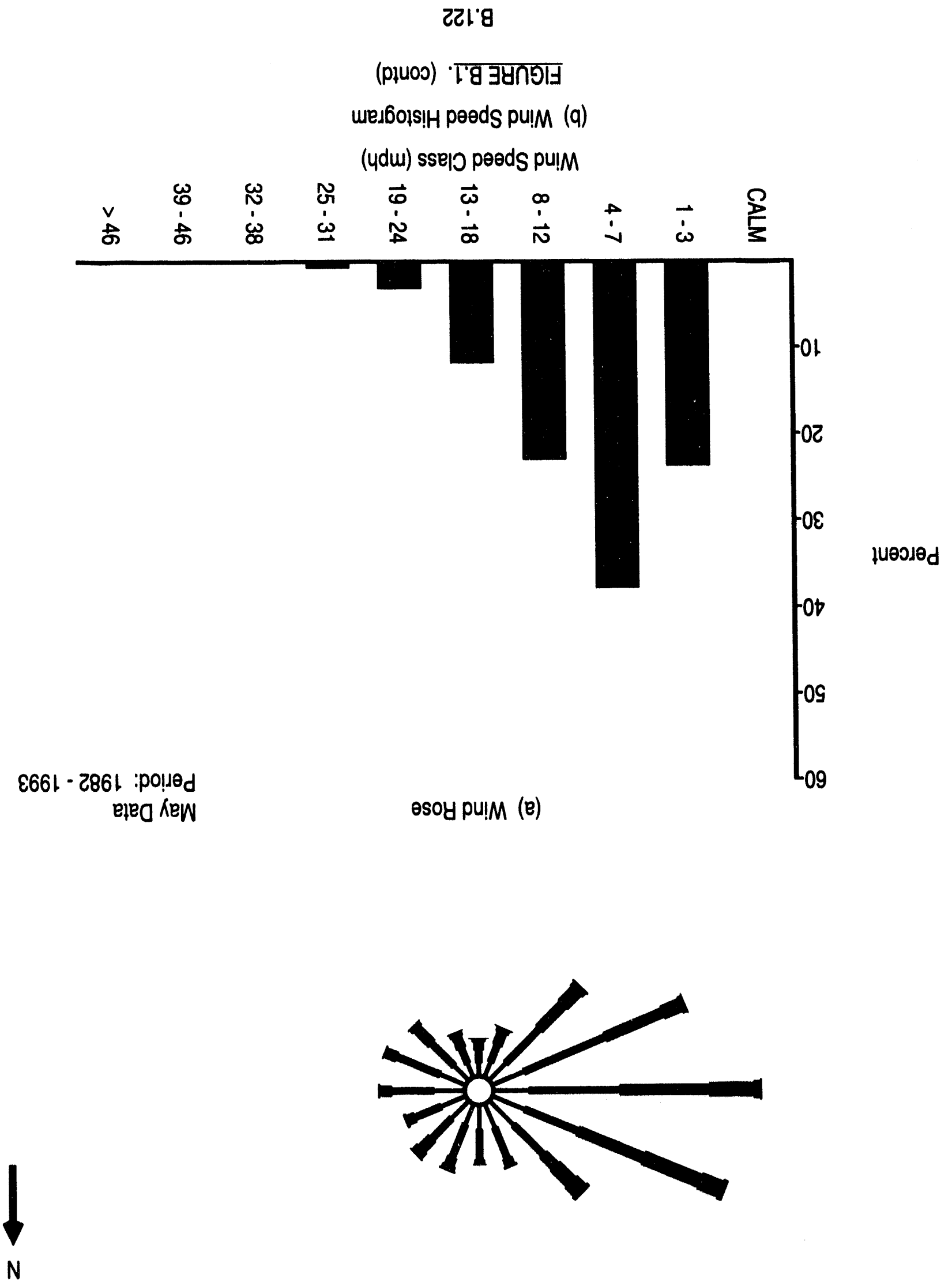




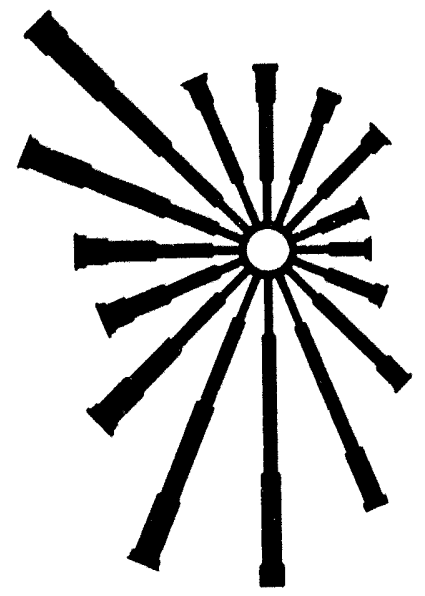

(a) Wind Rose

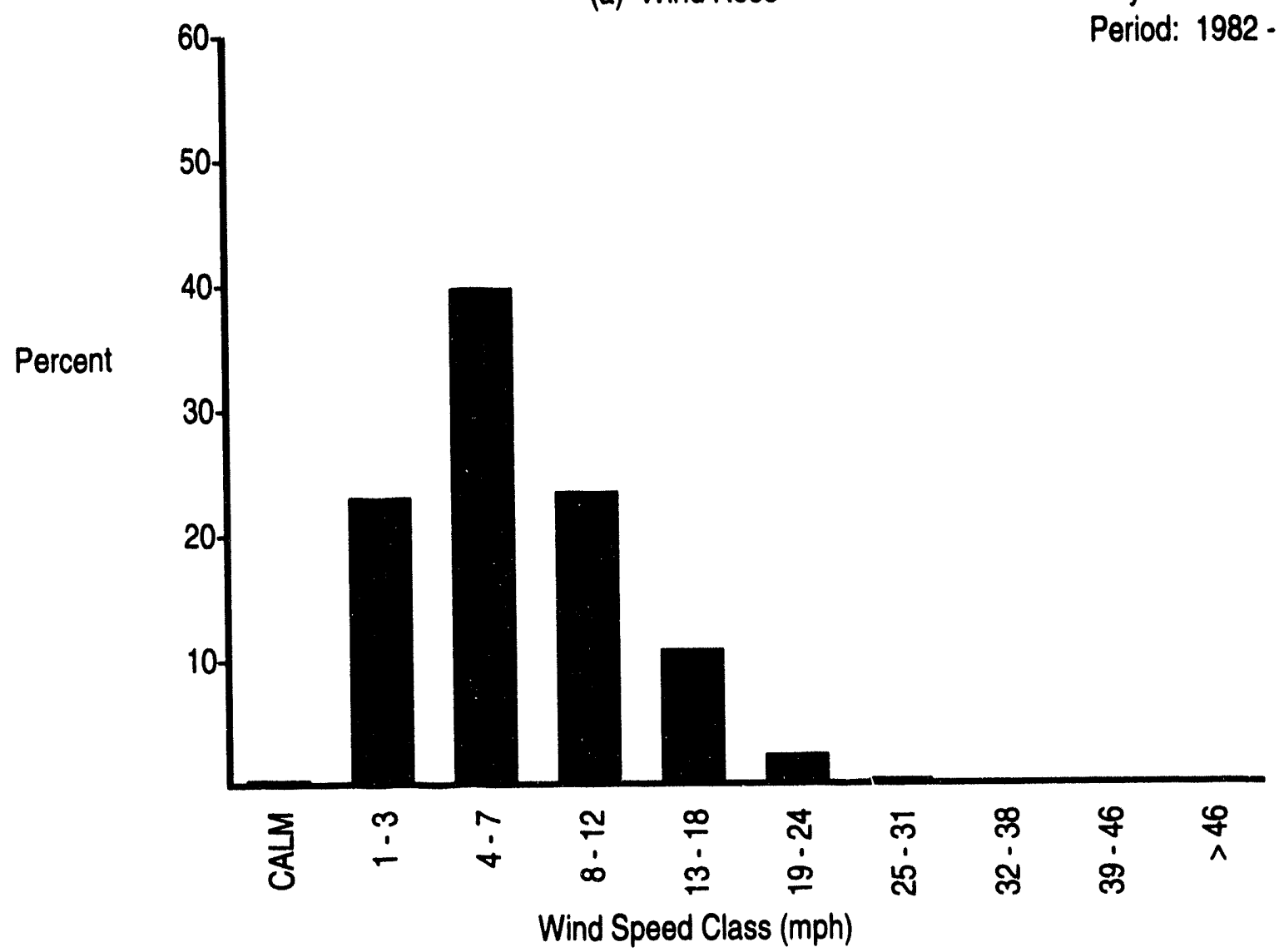

May Data

Period: 1982 - 1993

(b) Wind Speed Histogram

FIGURE B.1. (contd)

B.123 


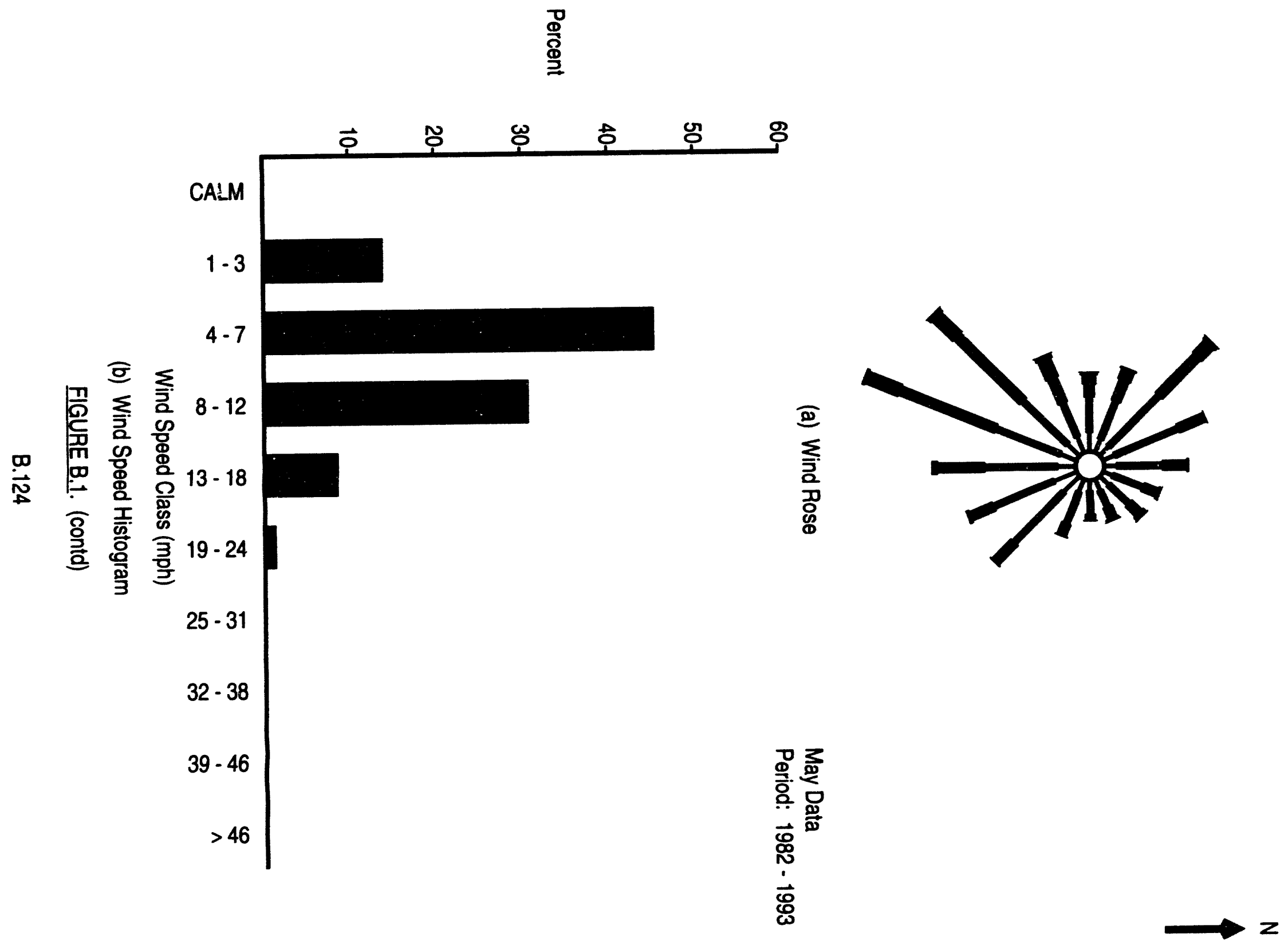



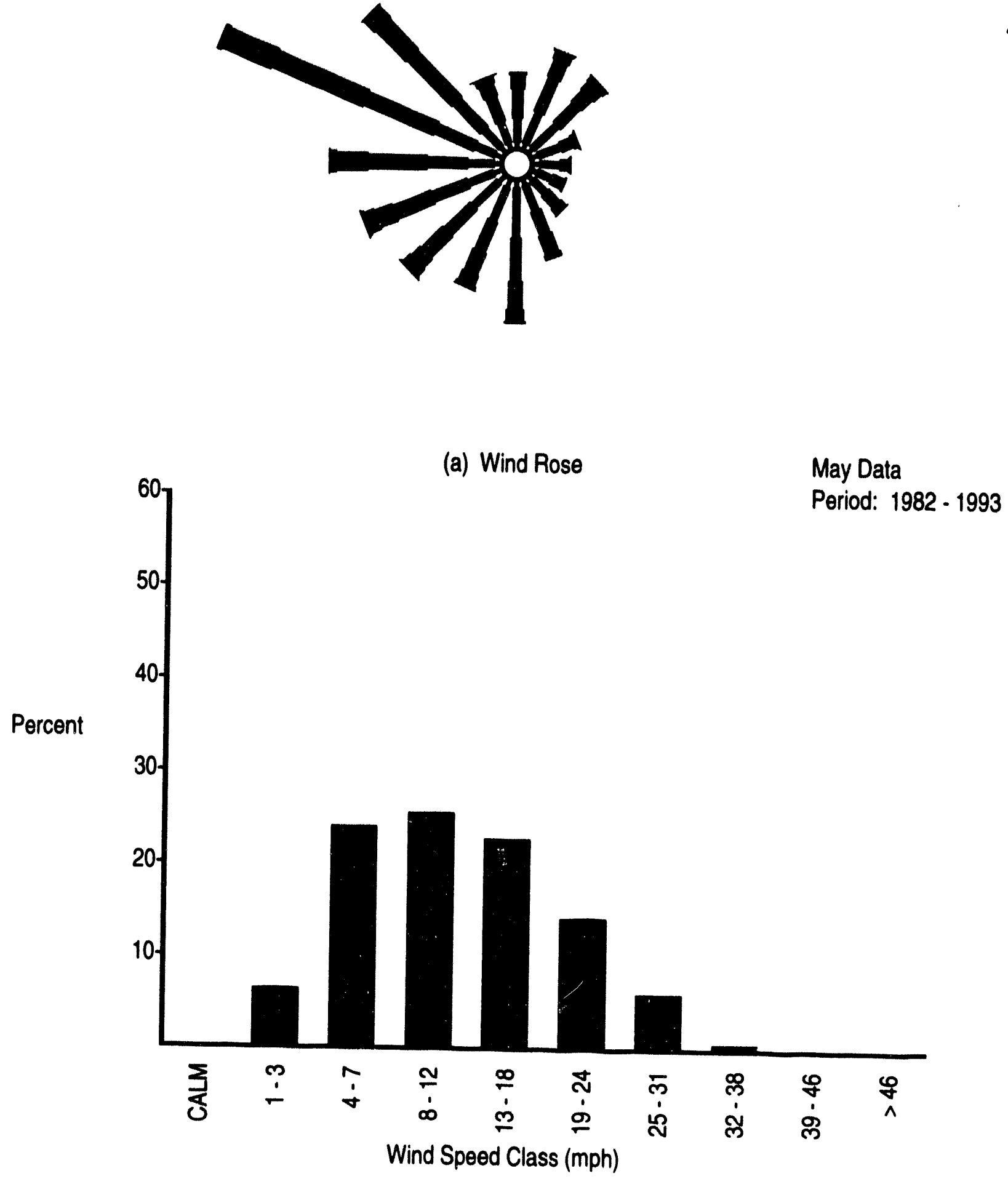

(b) Wind Speed Histogram

FIGURE B.1. (contd)

B.125 

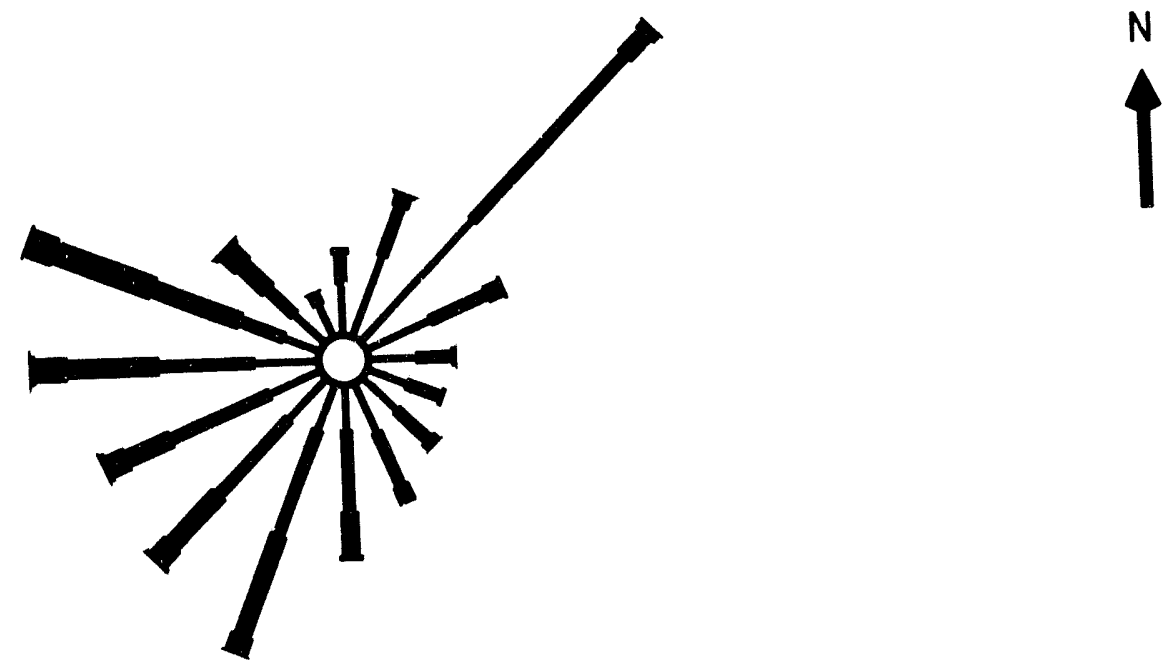

(a) Wind Rose

May Data

Period: $1982-1993$

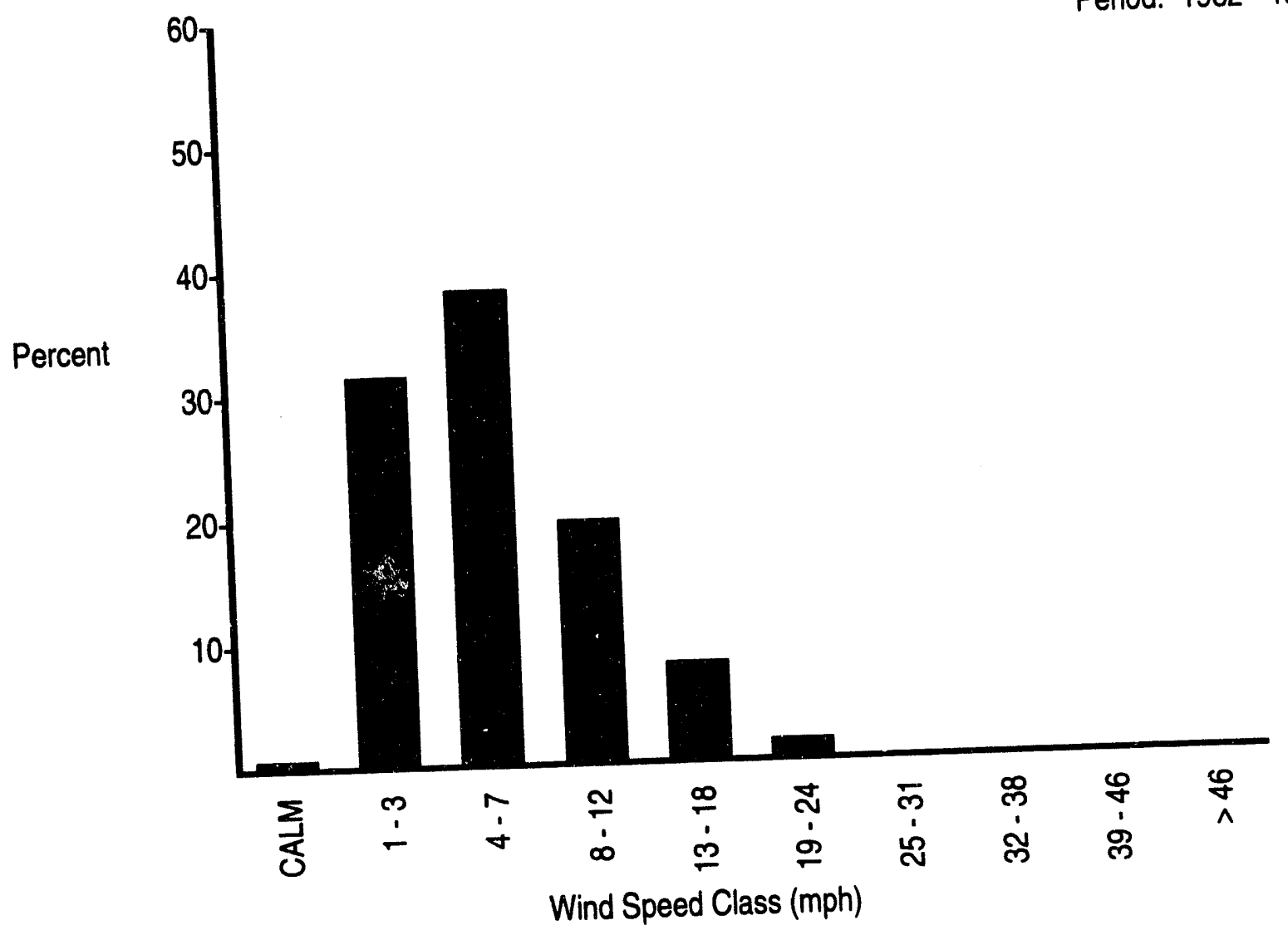

(b) Wind Speed Histogram

FIGURE B.1. (contd)

B.126 
$\angle Z I^{\prime} \theta$

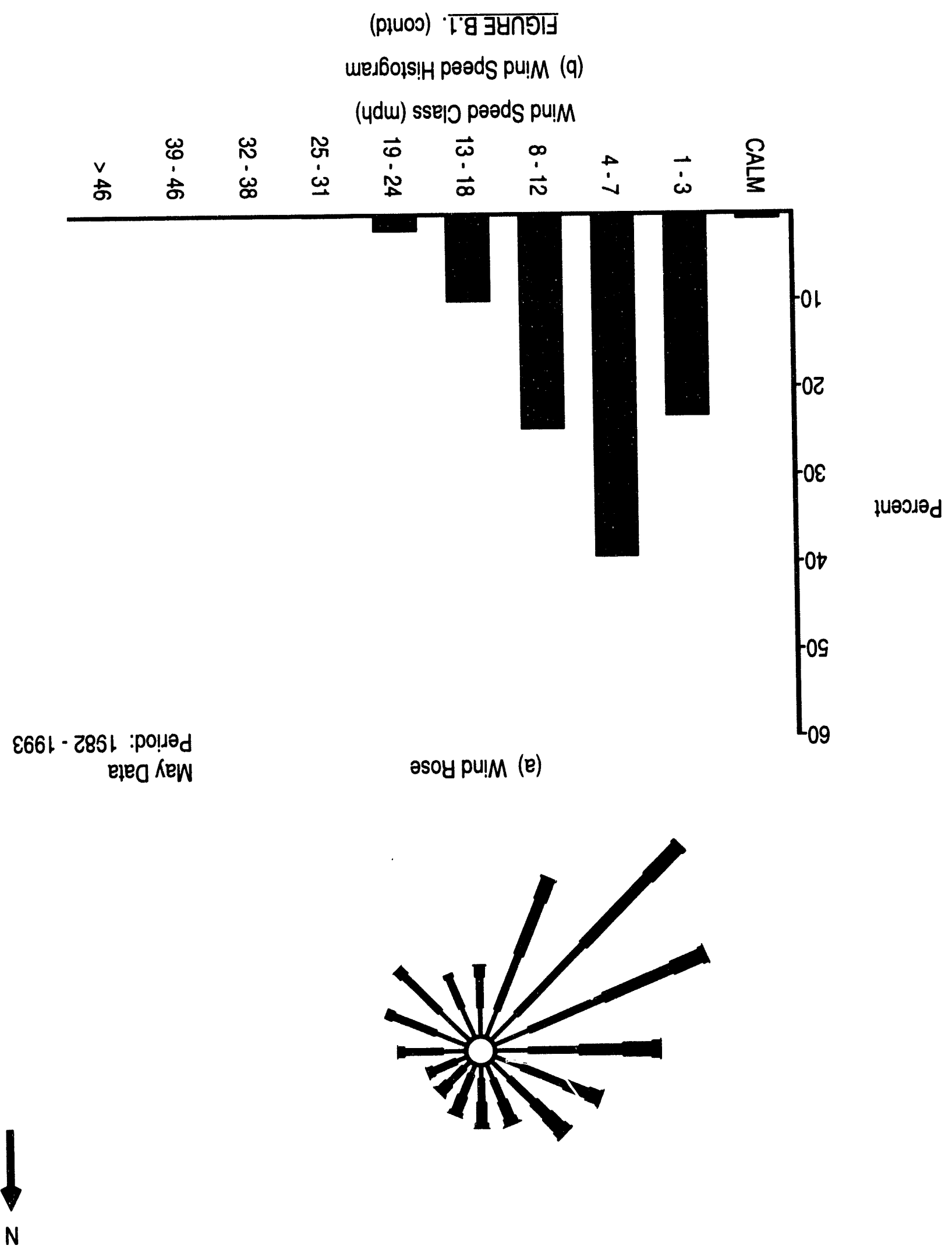




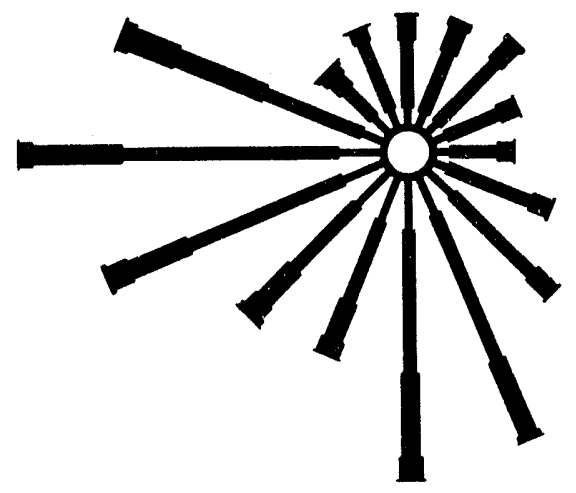

(a) Wind Rose

Percent
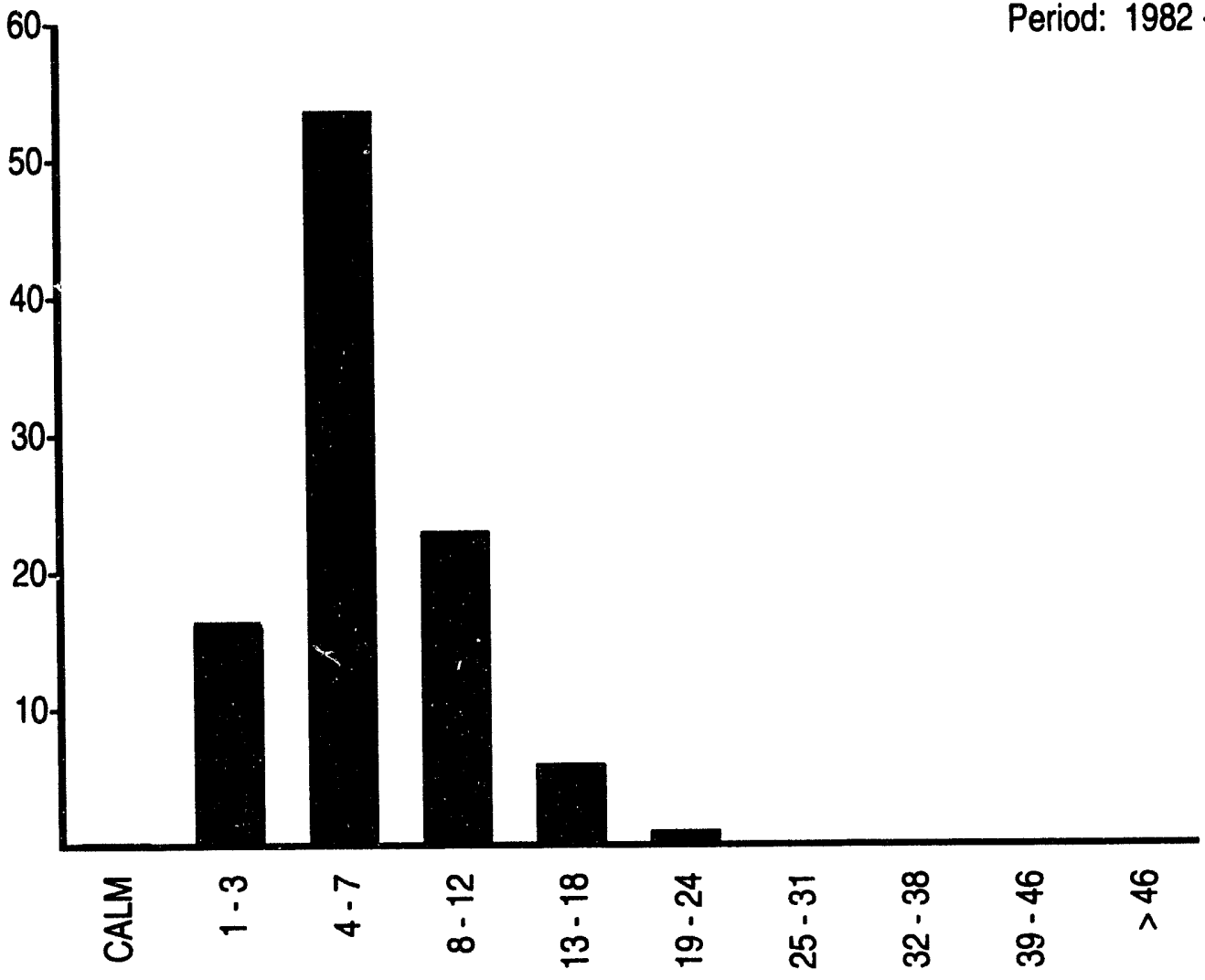

Wind Speed Class (mph)

(b) Wind Speed Histogram

FIGURE B.1. (contd)

B.128 


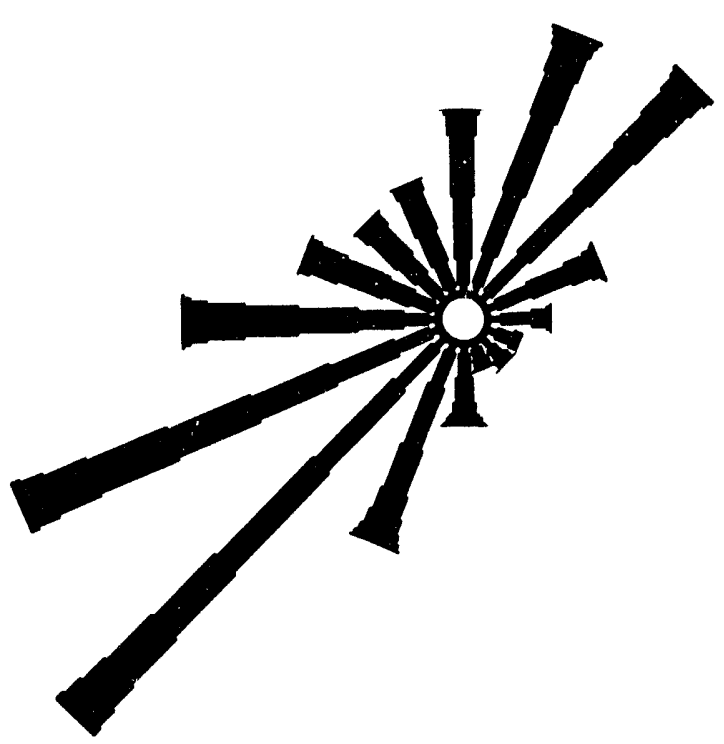

(a) Wind Rose

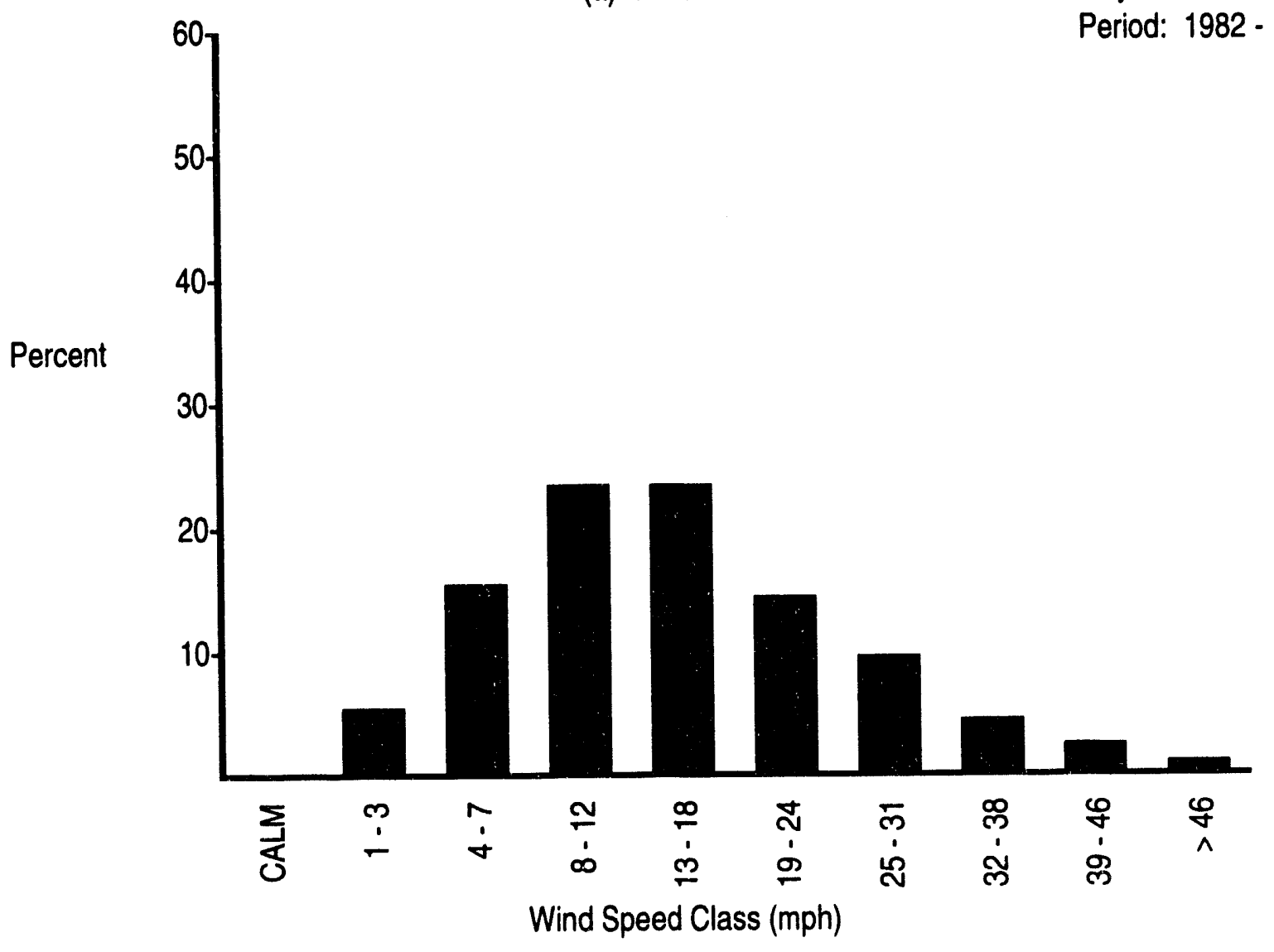

(b) Wind Speed Histogram

FIGURE B.1. (contd)
May Data

Period: 1982 - 1993

\section{B.129}



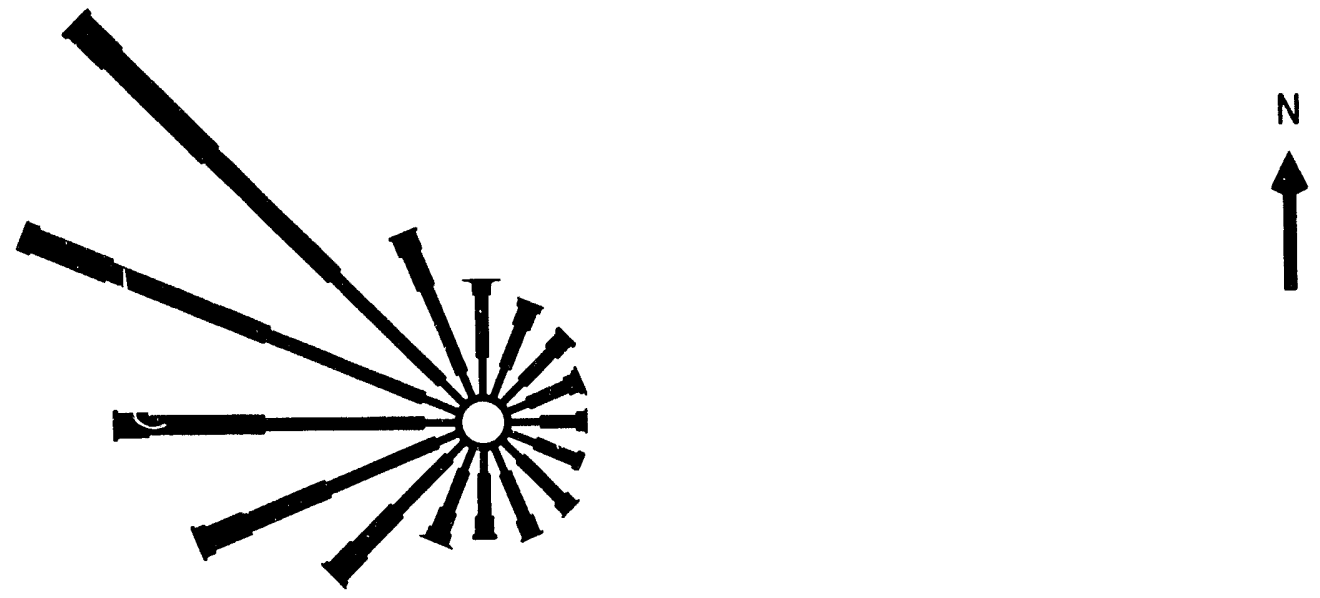

(a) Wind Rose

May Data

Percent

60
50

Period: 1982 - 1993

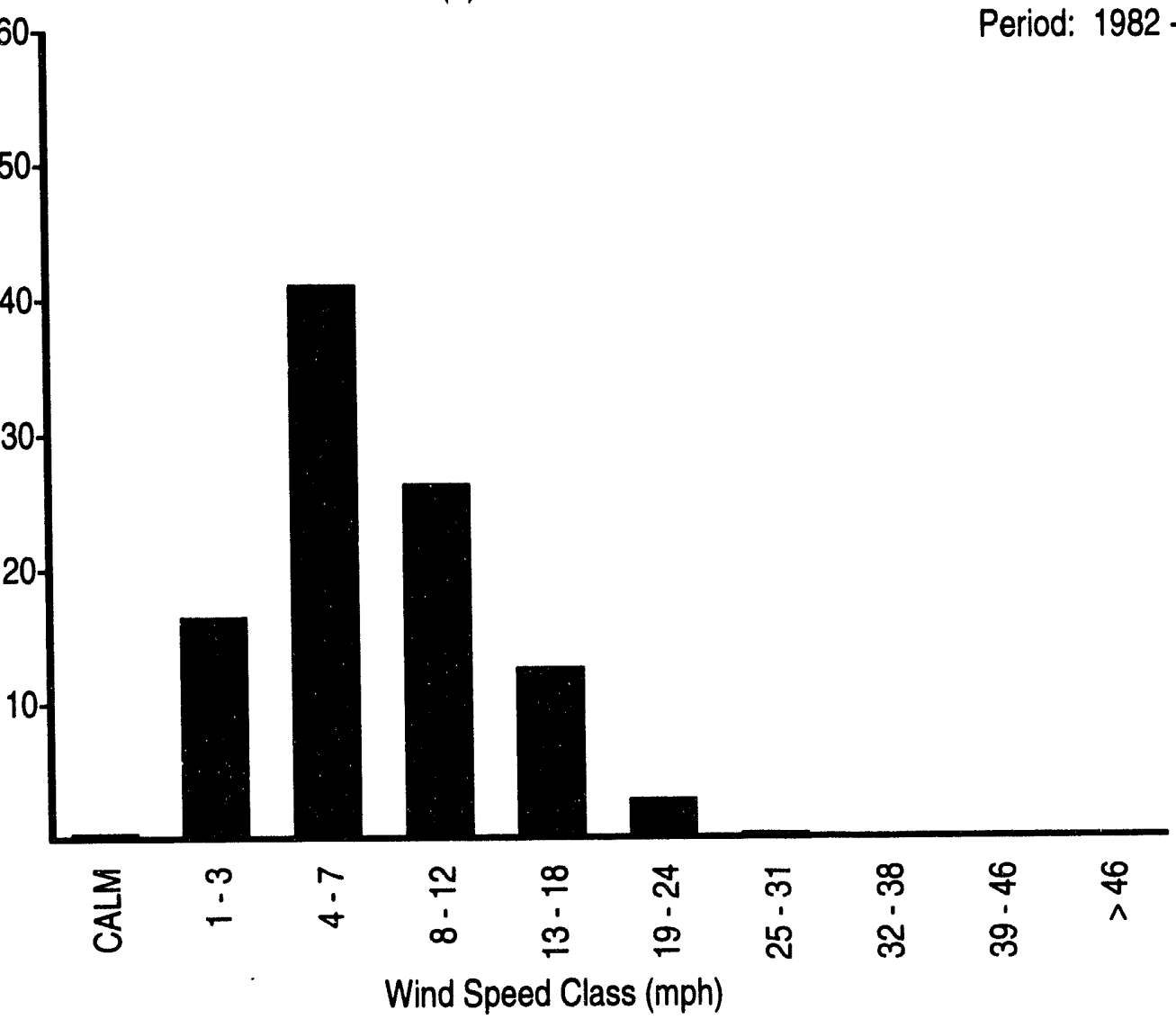

(b) Wind Speed Histogram

FIGURE B.1. (contd)

B. 130 

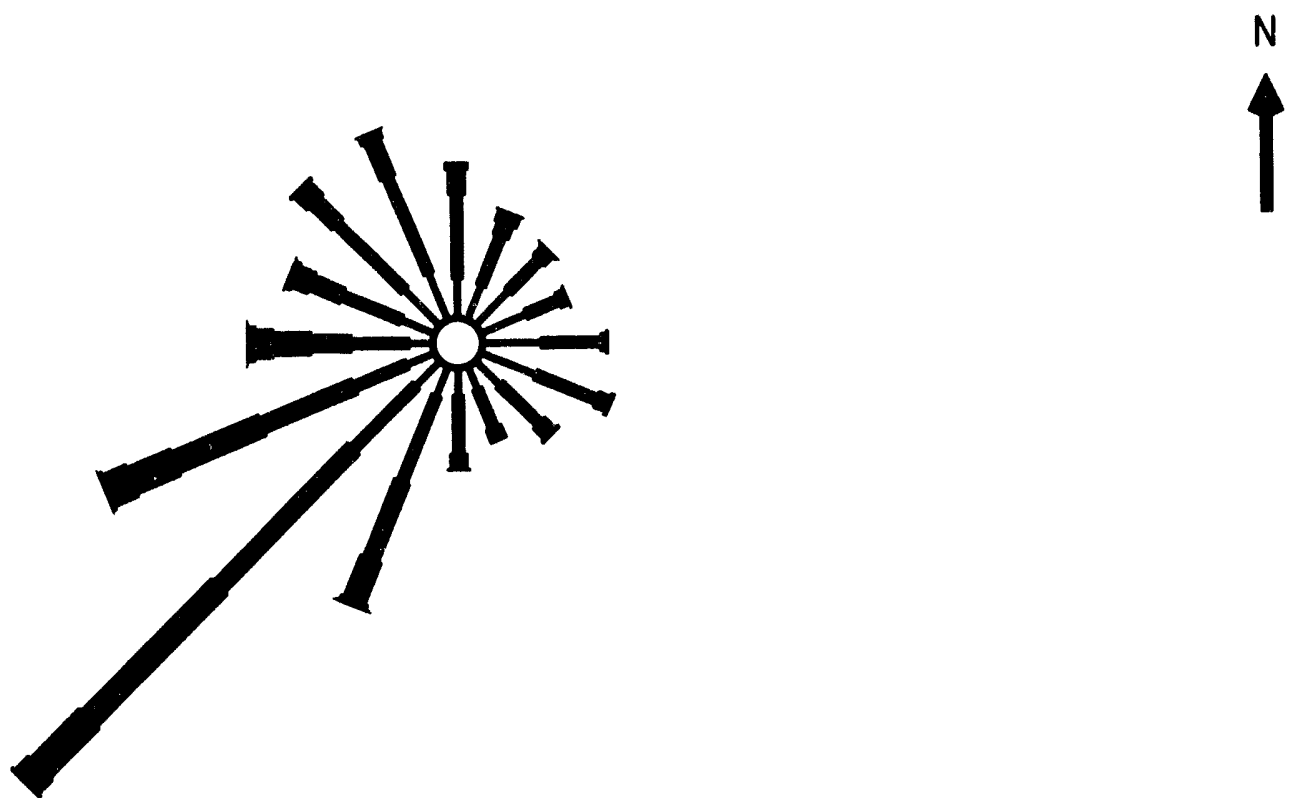

(a) Wind Rose

May Data

Period: 1988 - 1993

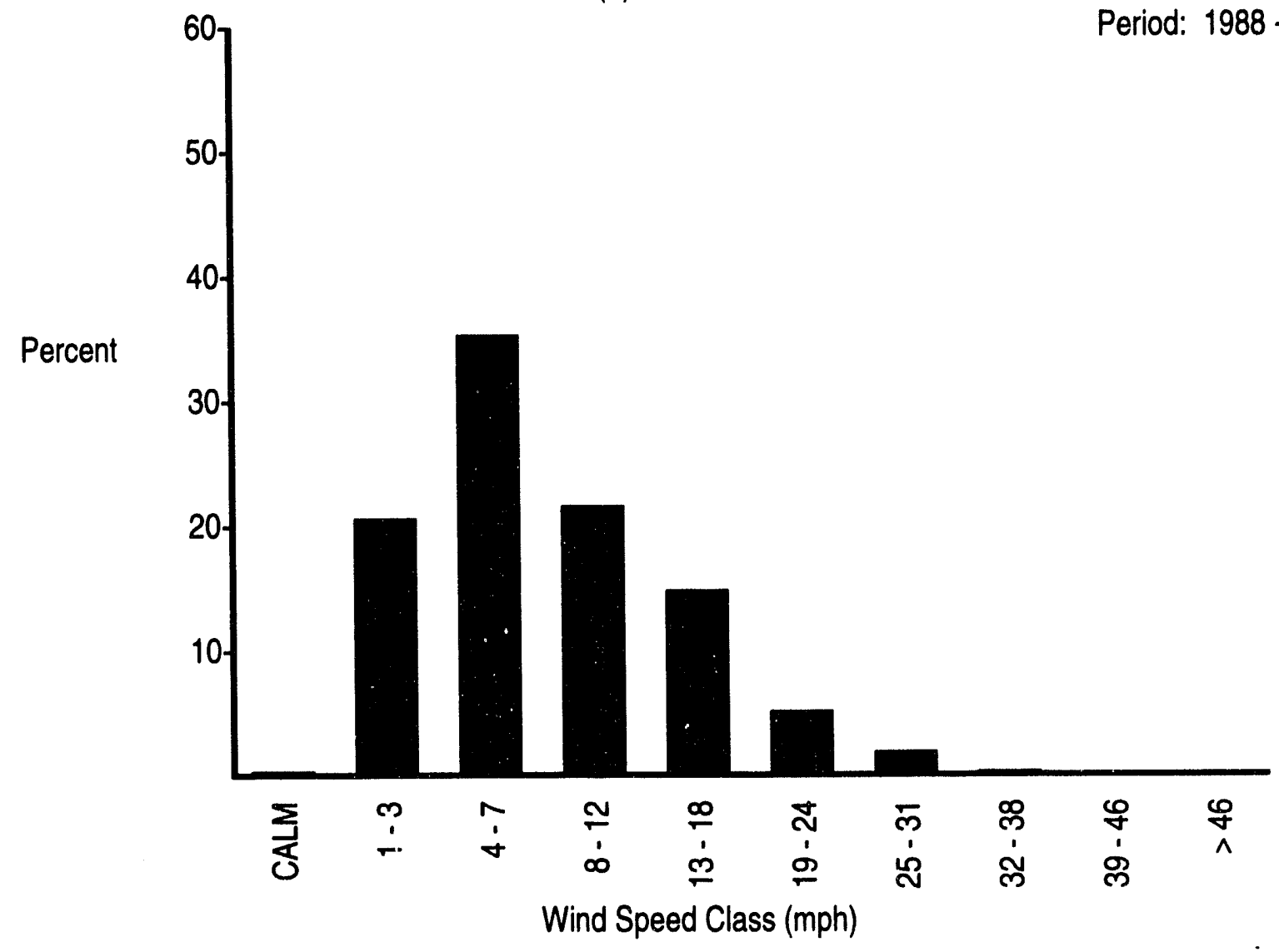

(b) Wind Speed Histogram

FIGURE B.1. (contd) 


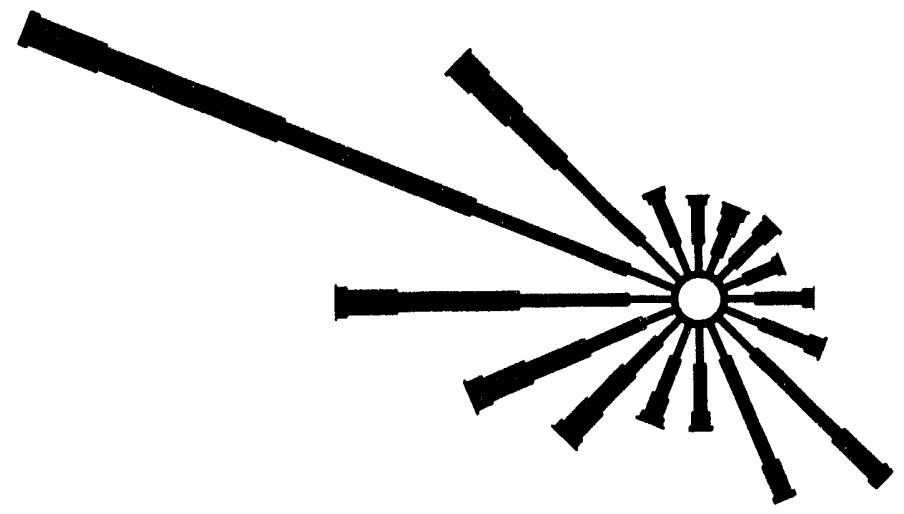

(a) Wind Rose

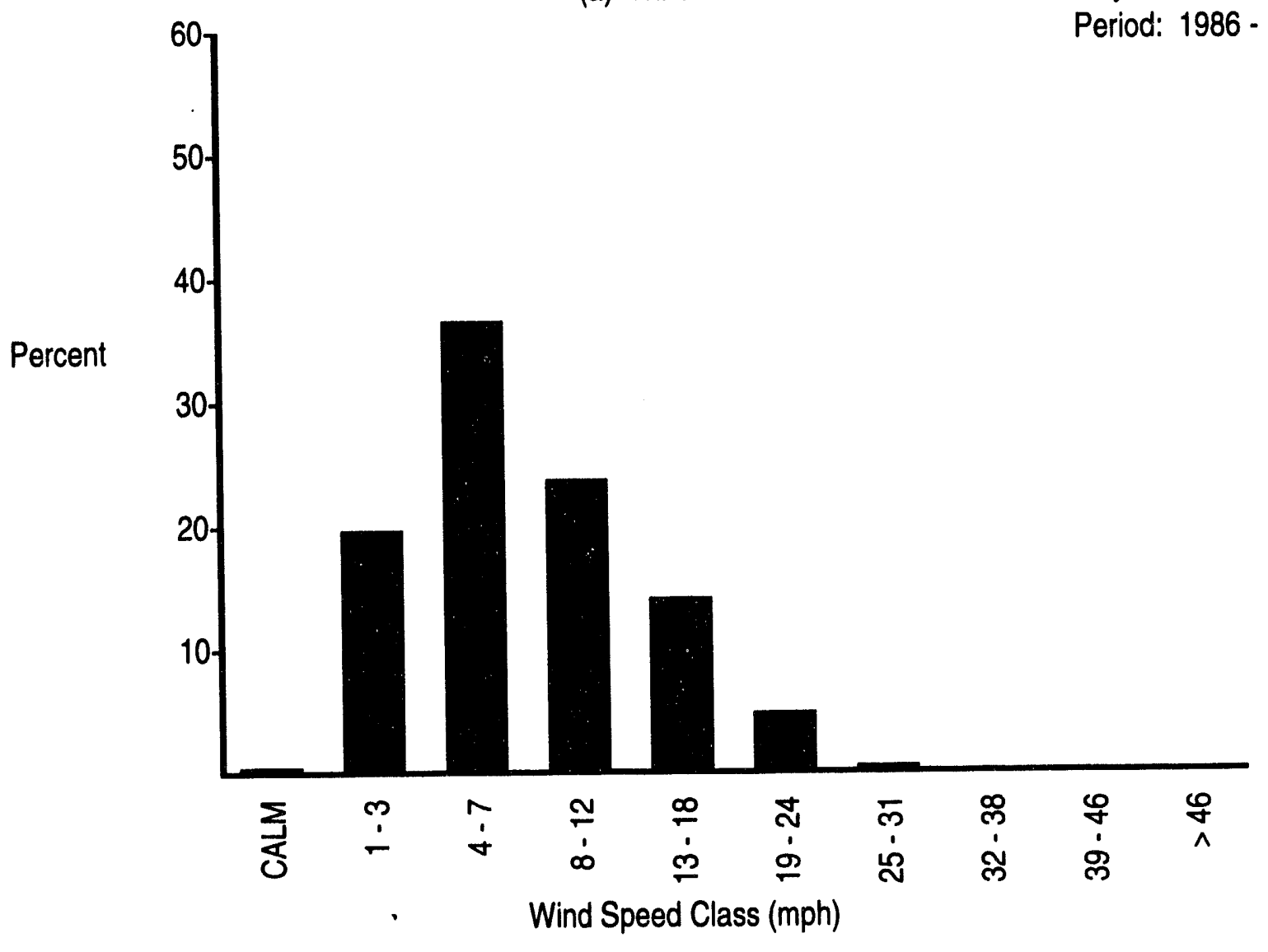

(b) Wind Speed Histogram

FIGURE B.1. (contd) 


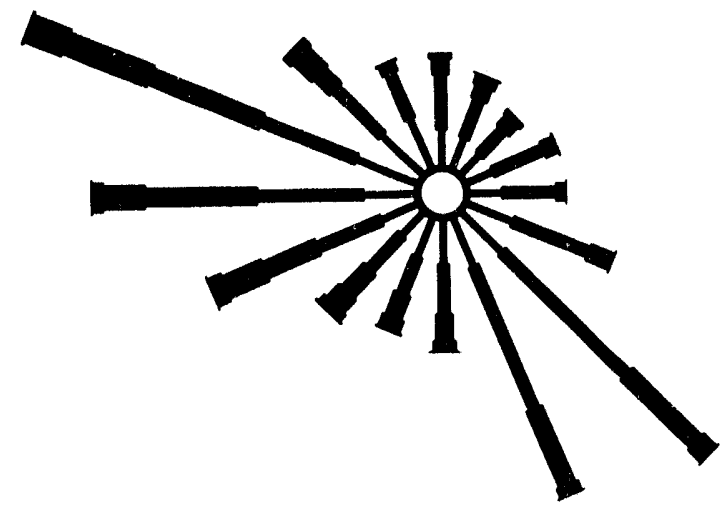

(a) Wind Rose

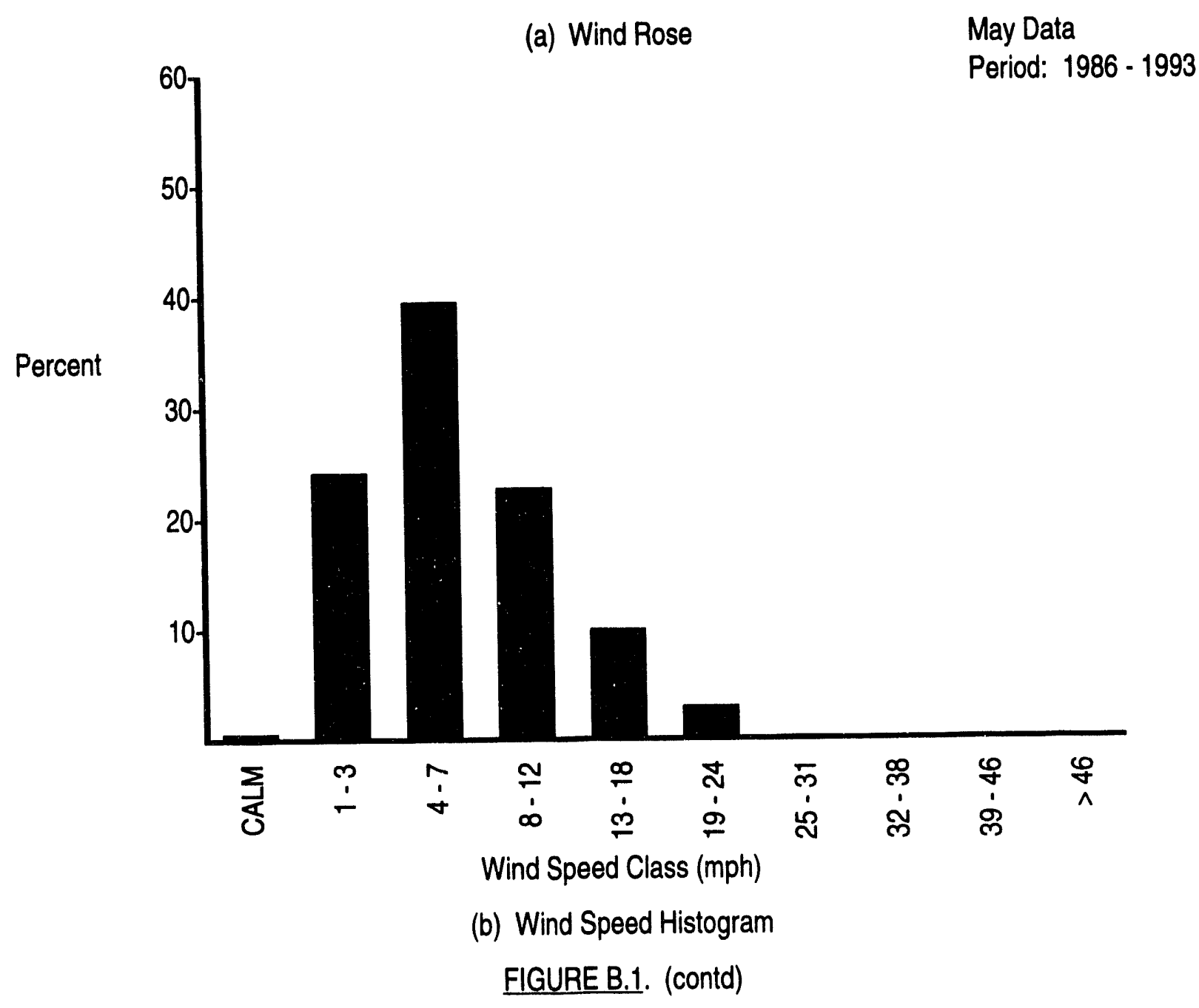

B. 133 

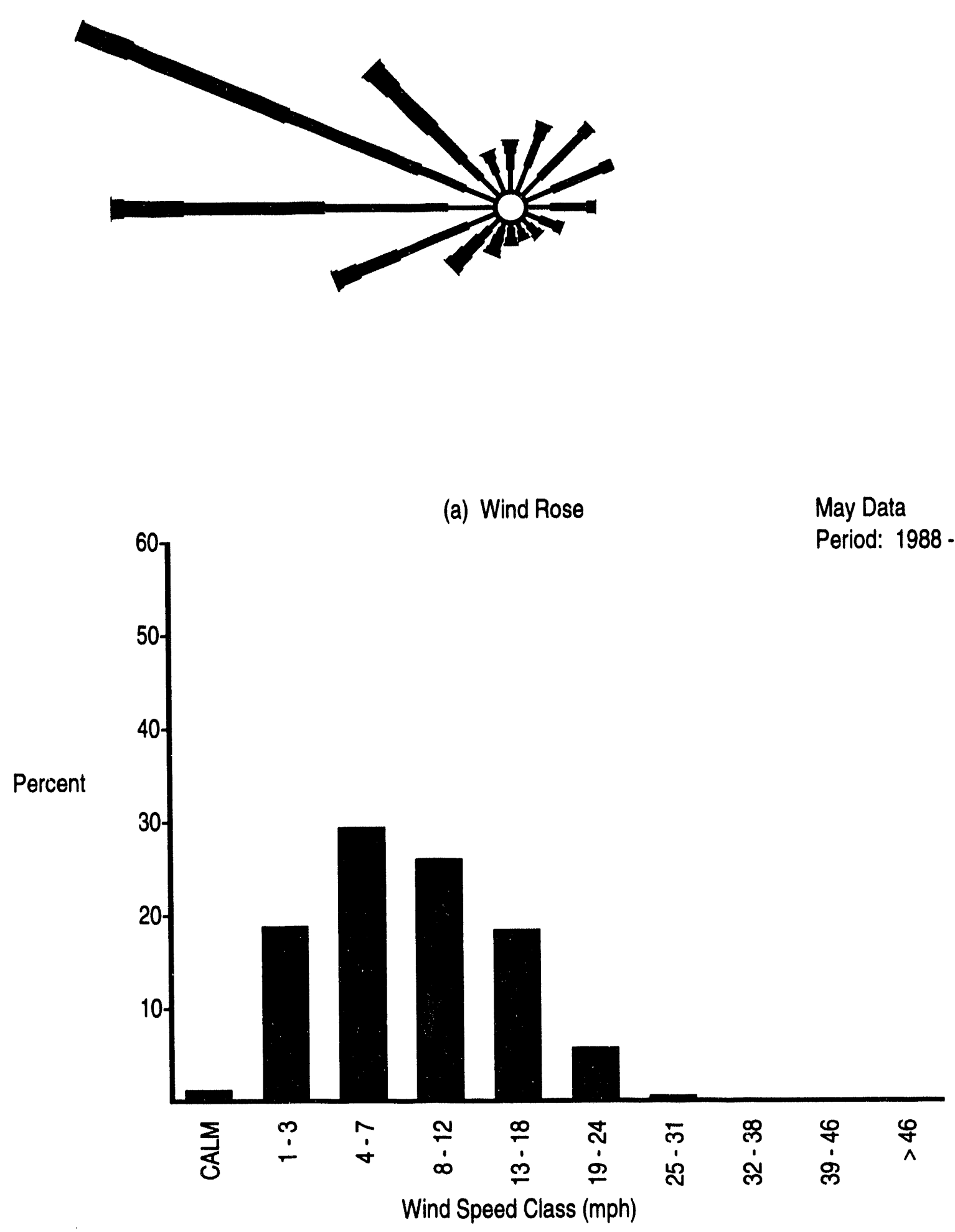

(b) Wind Speed Histogram

FIGURE B.1. (contd)

\section{B.134}




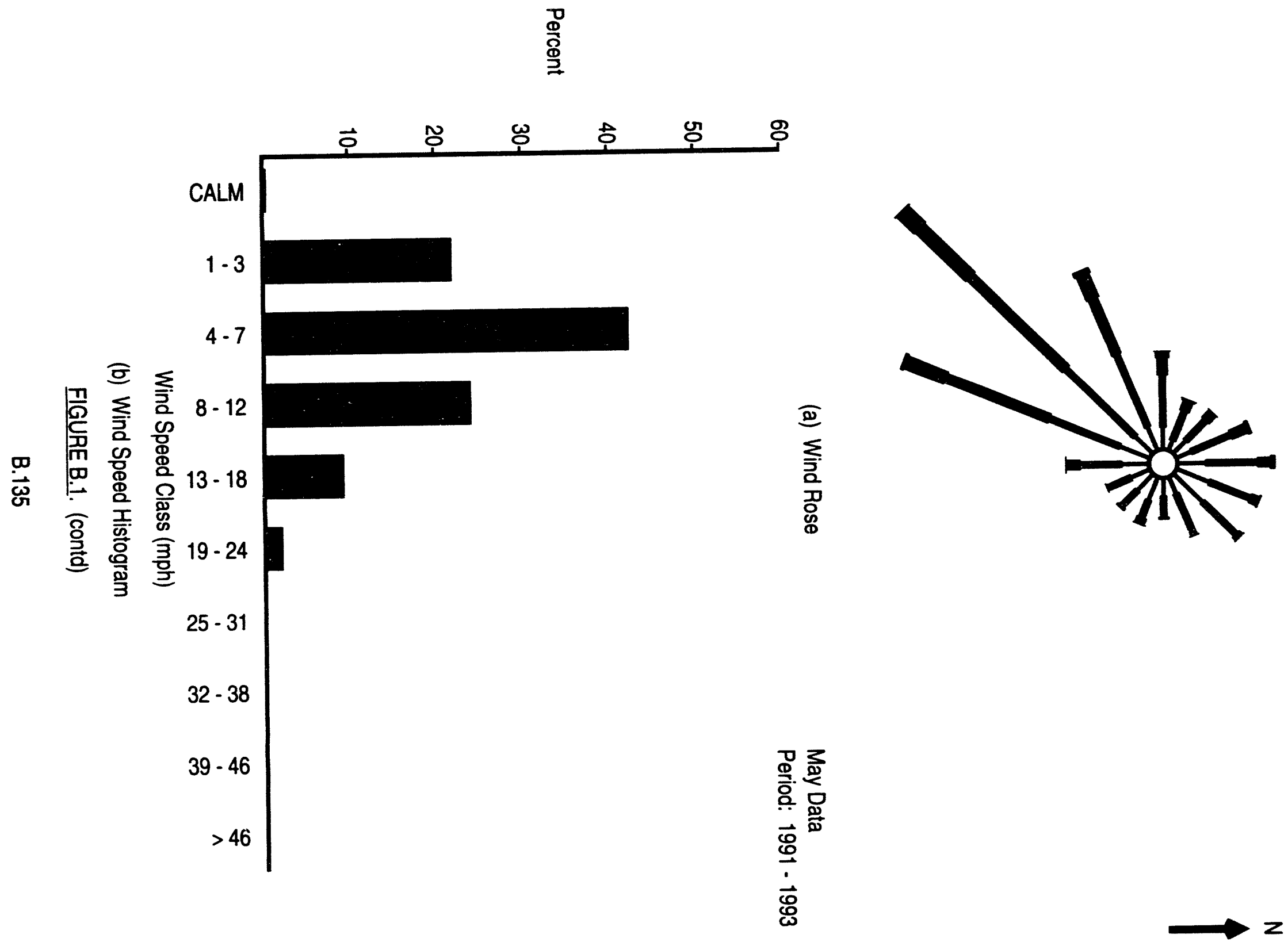



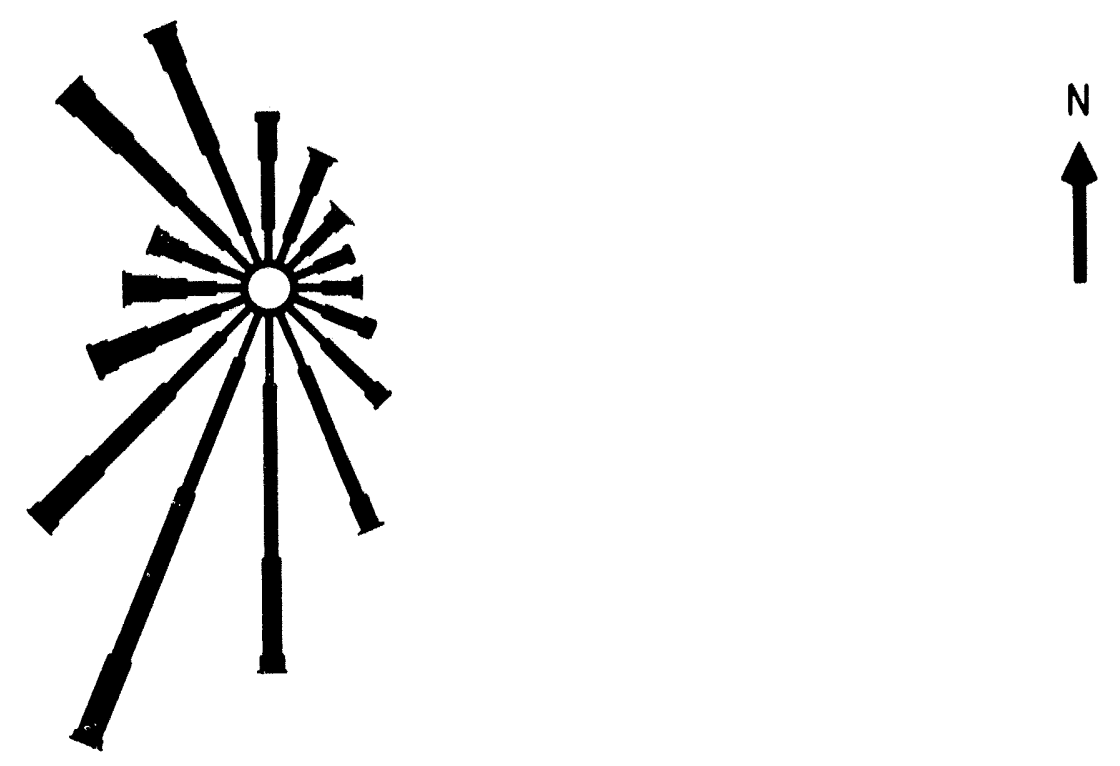

(a) Wind Rose

June Data

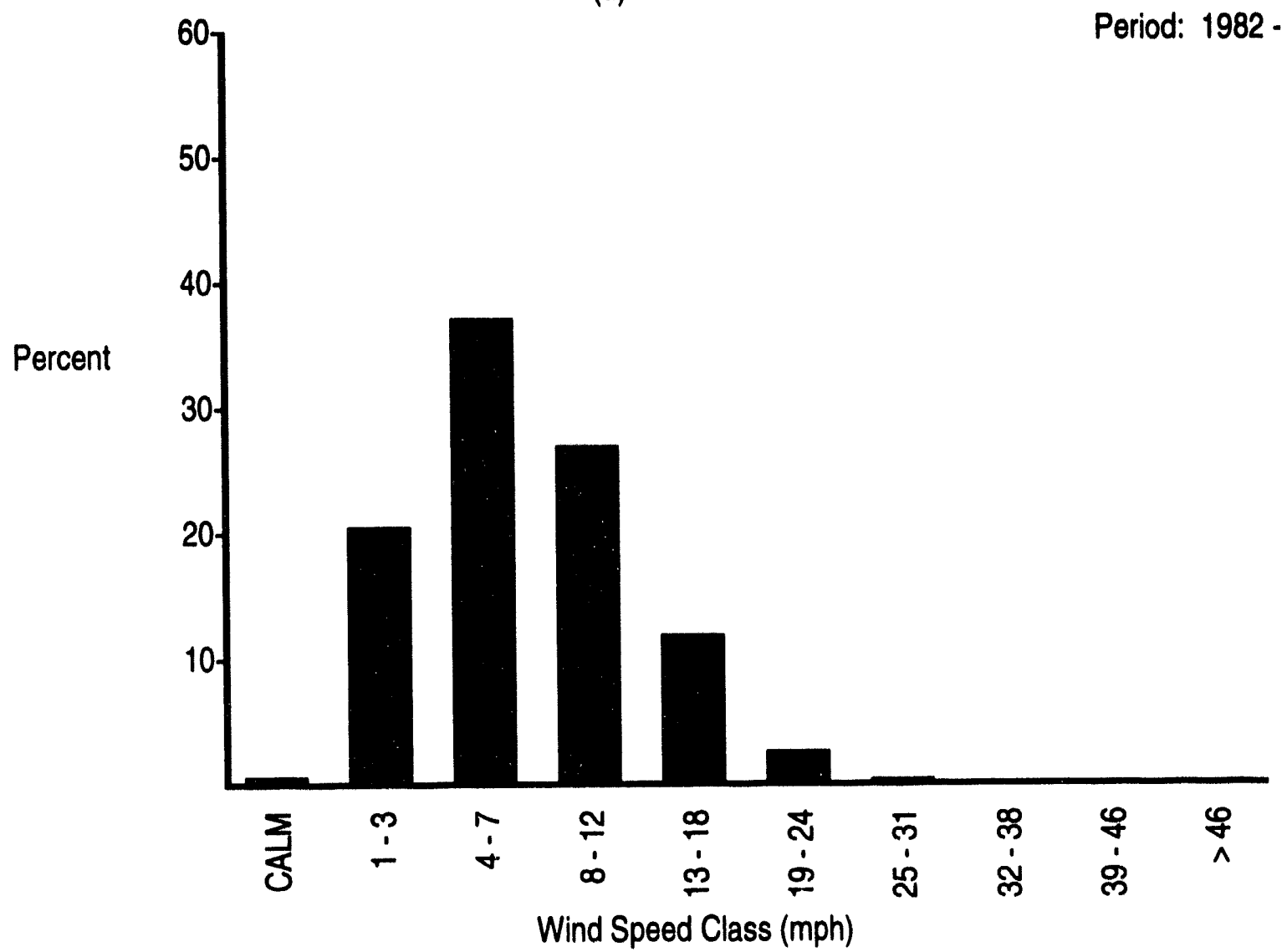

(b) Wind Speed Histogram

FIGURE B.1. (contd) 


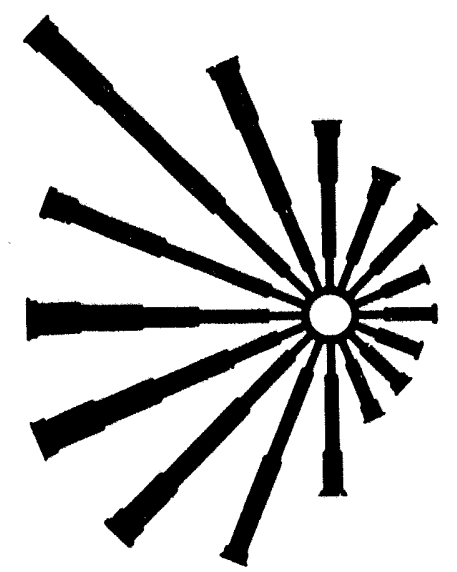

(a) Wind Rose

June Data

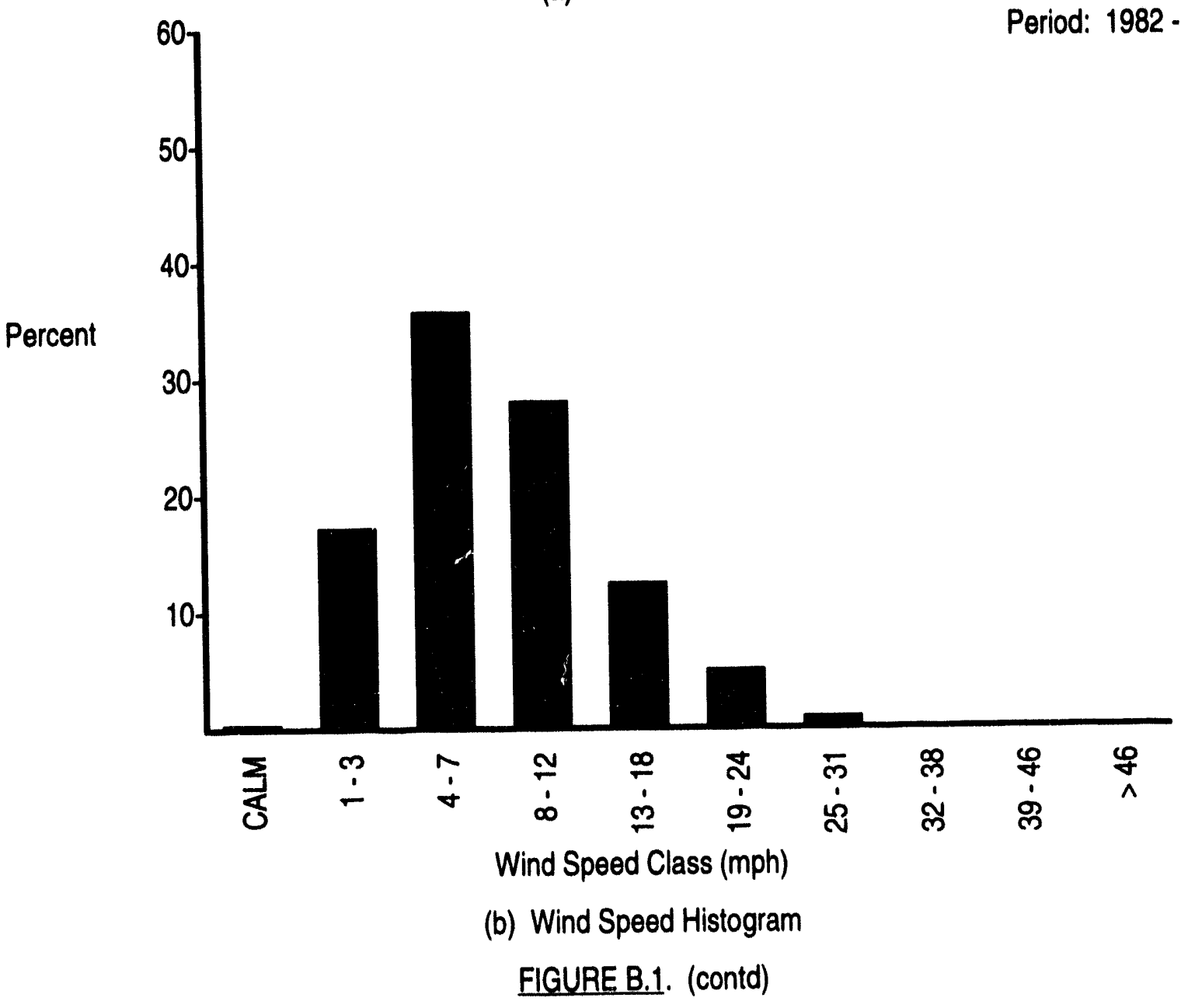

B. 137 


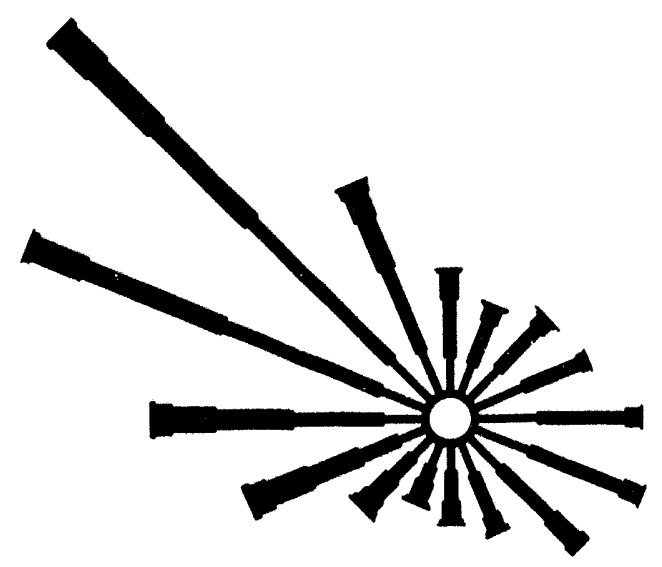

(a) Wind Rose

June Data

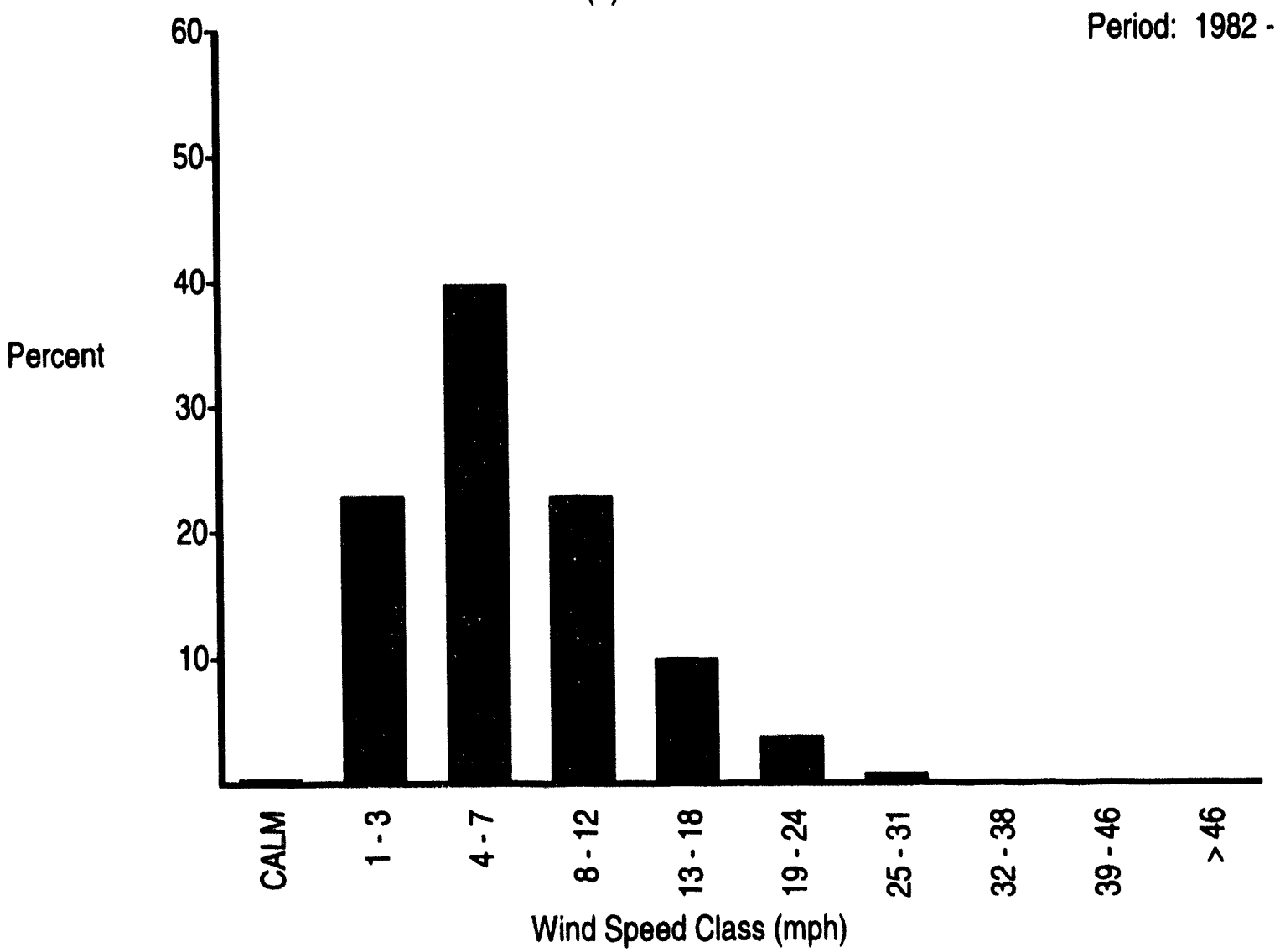

(b) Wind Speed Histogram

FIGURE B.1. (contd) 


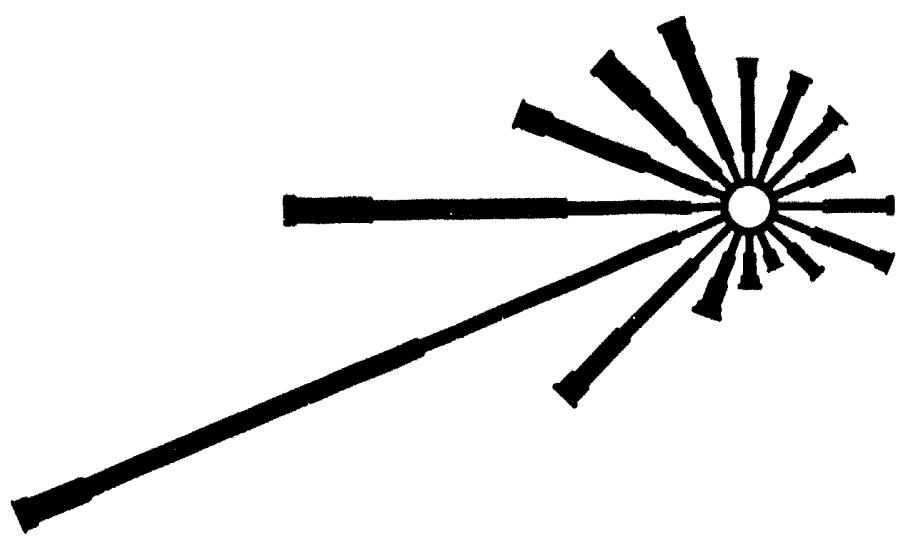

(a) Wind Rose

June Data

Period: 1982 - 1993

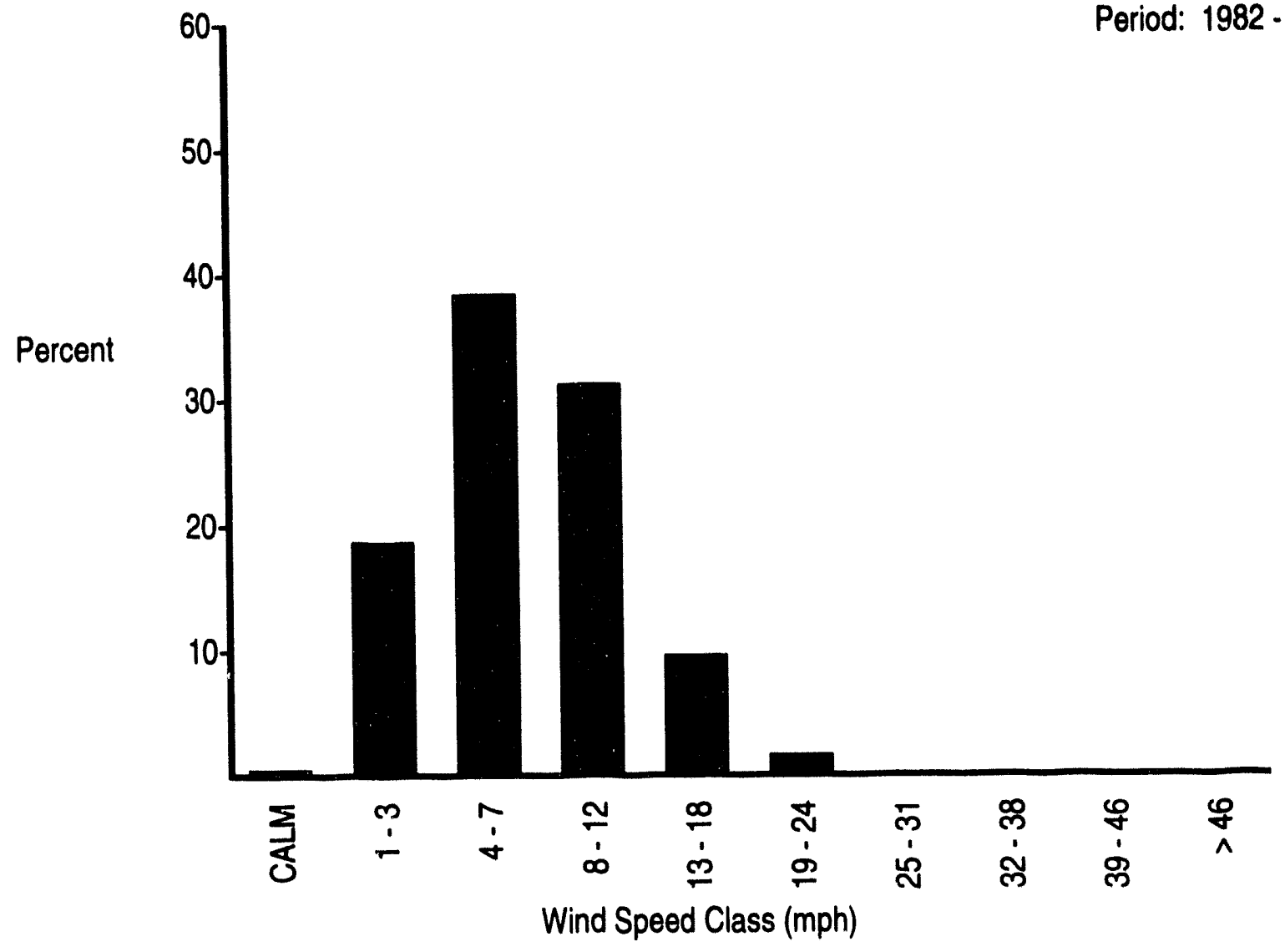

(b) Wind Speed Histogram

FIGURE B.1. (contd) 


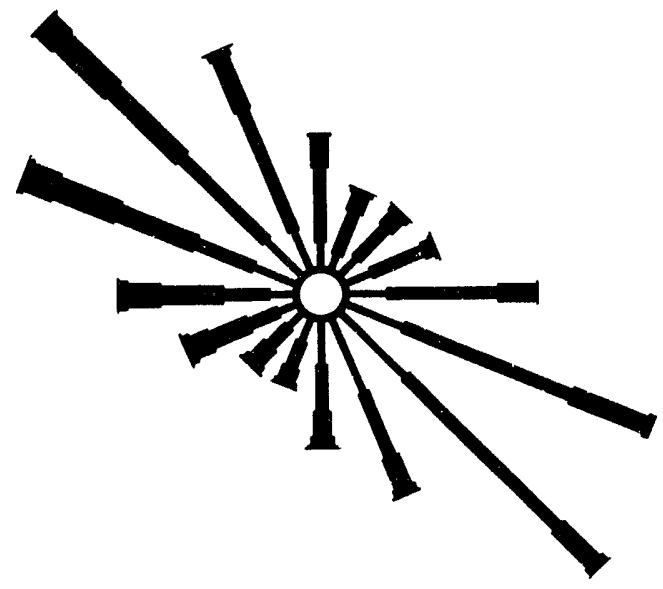

(a) Wind Rose

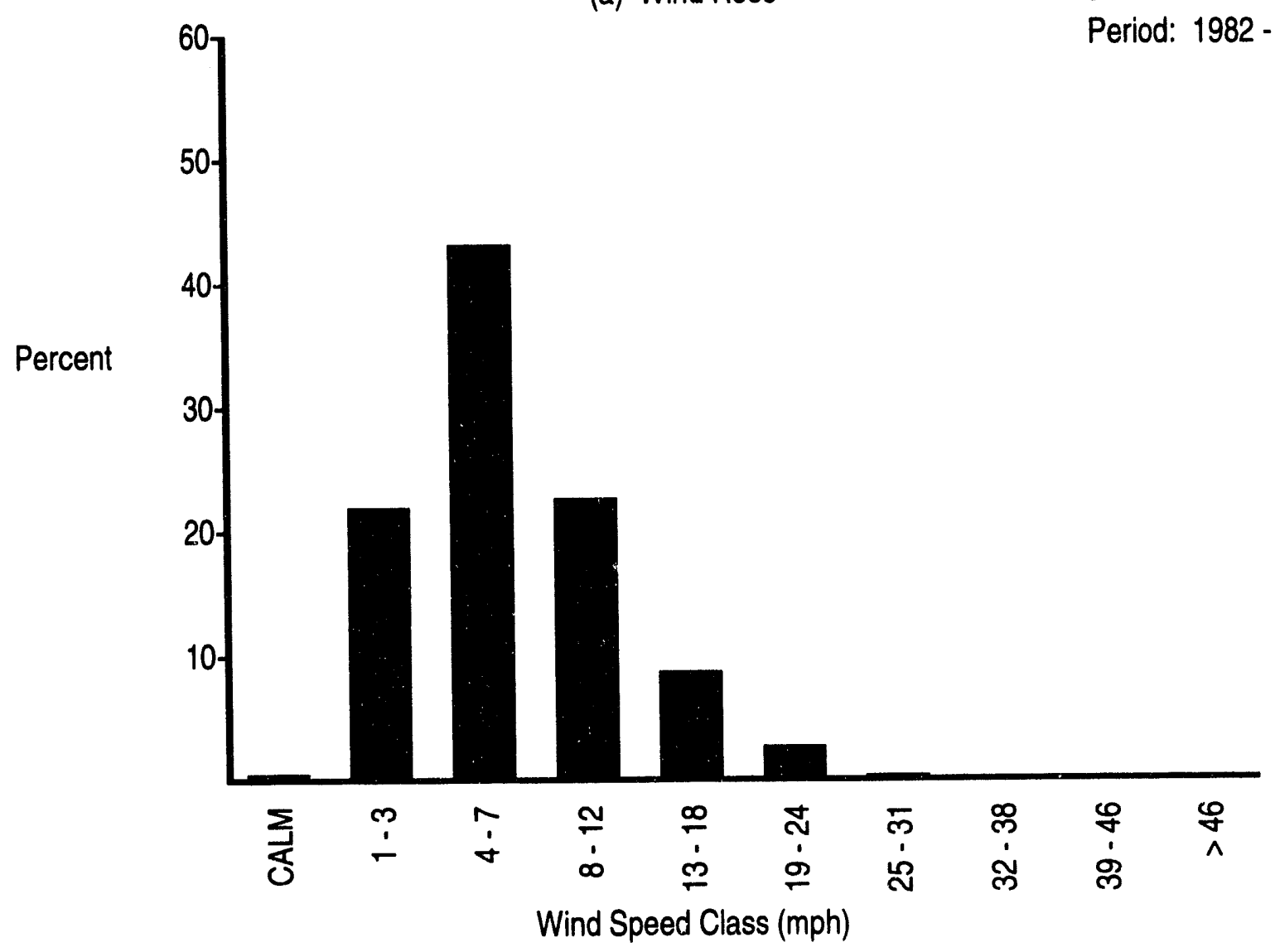

(b) Wind Speed Histogram

FIGURE B.1. (contd)
June Data

Period: 1982 - 1993

\section{B. 140}




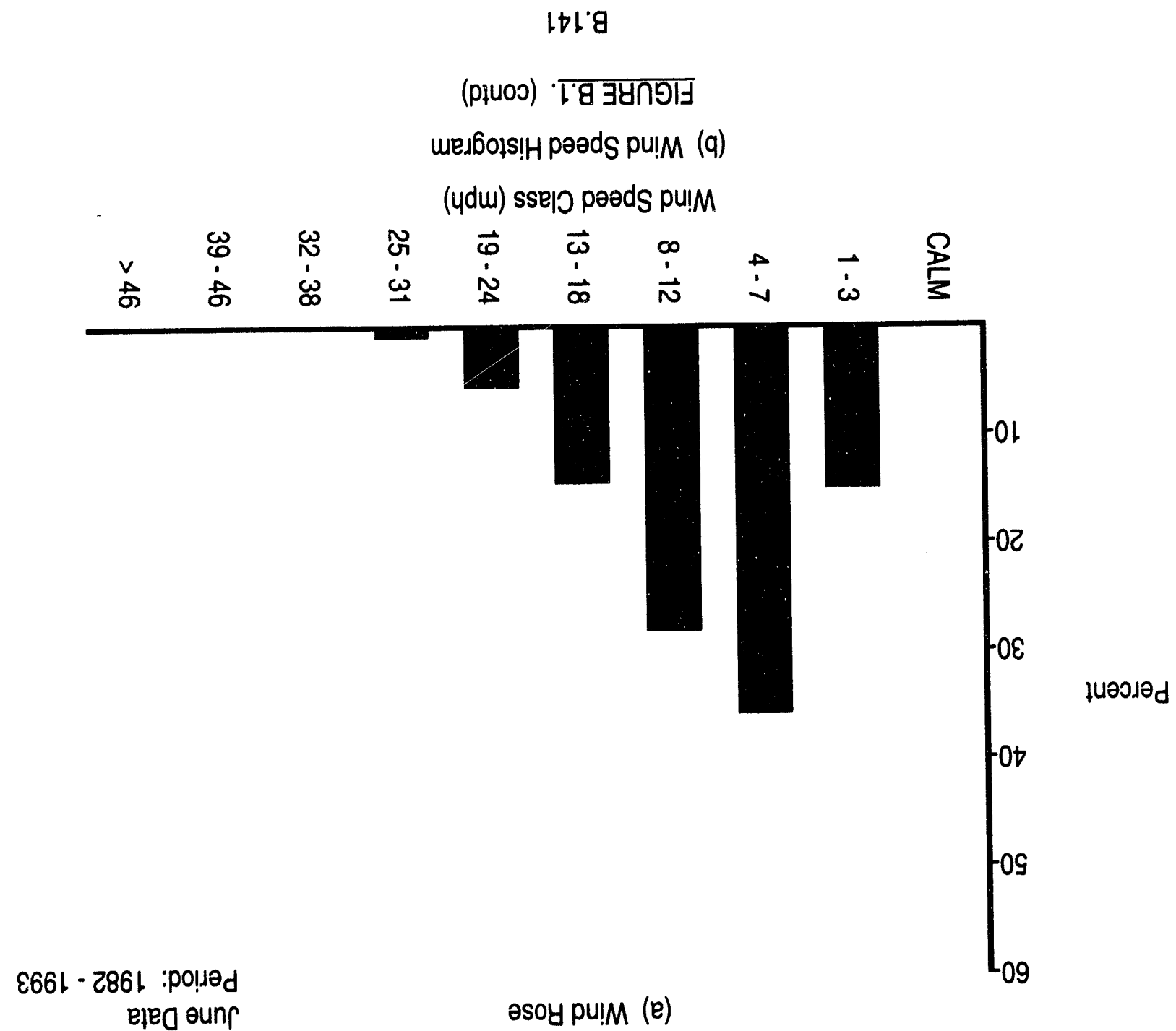

efeg oun

әsoy pu!M (e)

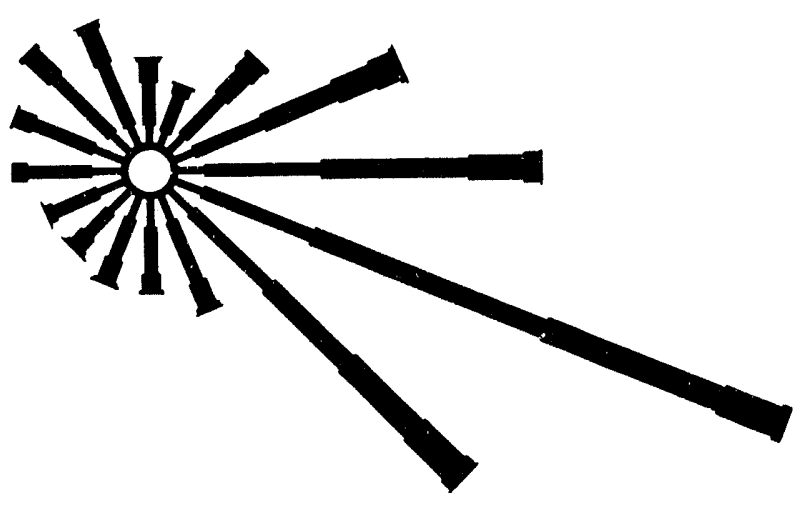



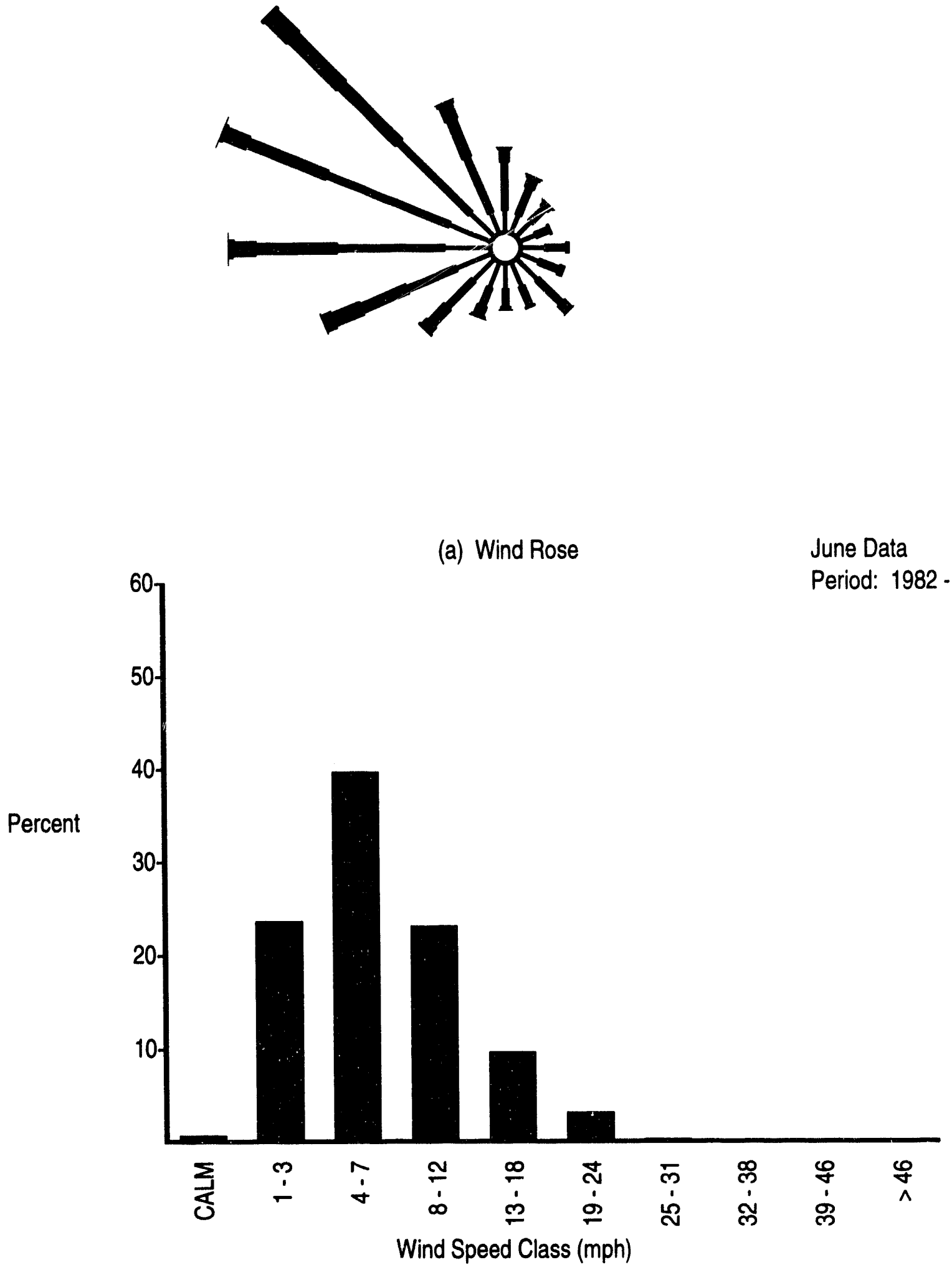

(b) Wind Speed Histogram

FIGURE B.1. (contd) 

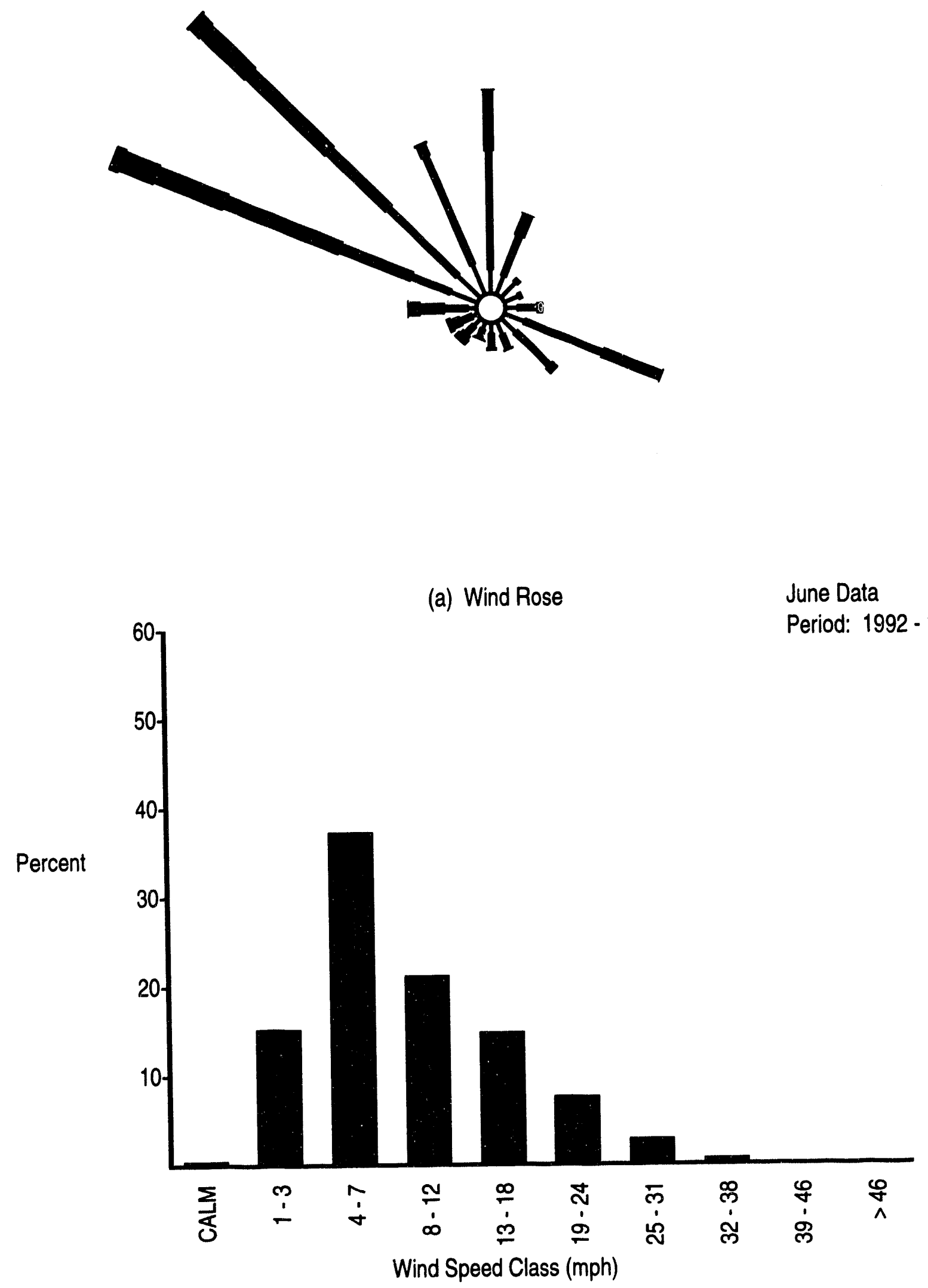

(b) Wind Speed Histogram

FIGURE B.1. (contd)

B. 143 


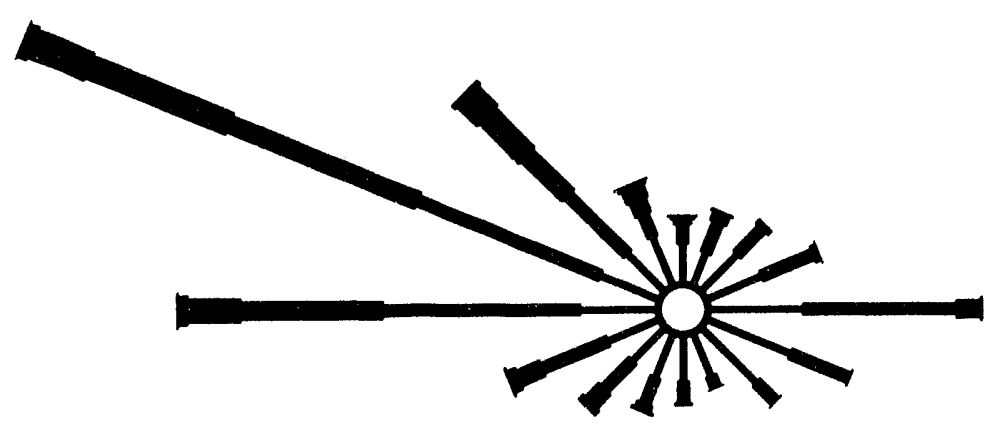

(a) Wind Rose

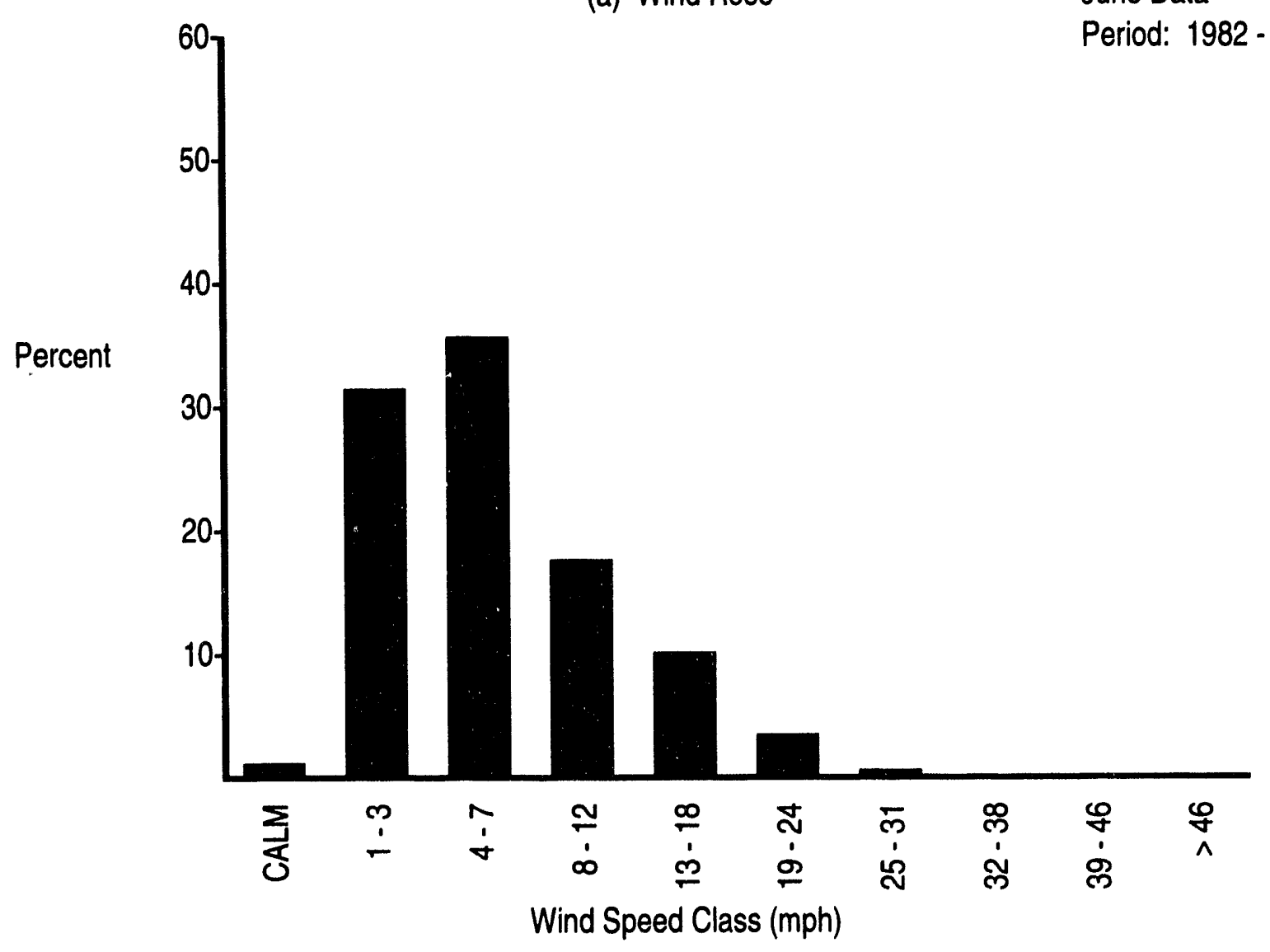

(b) Wind Speed Histogram
June Data

Period: 1982 - 1991

FlGURE B.1. (contd)

B. 144 


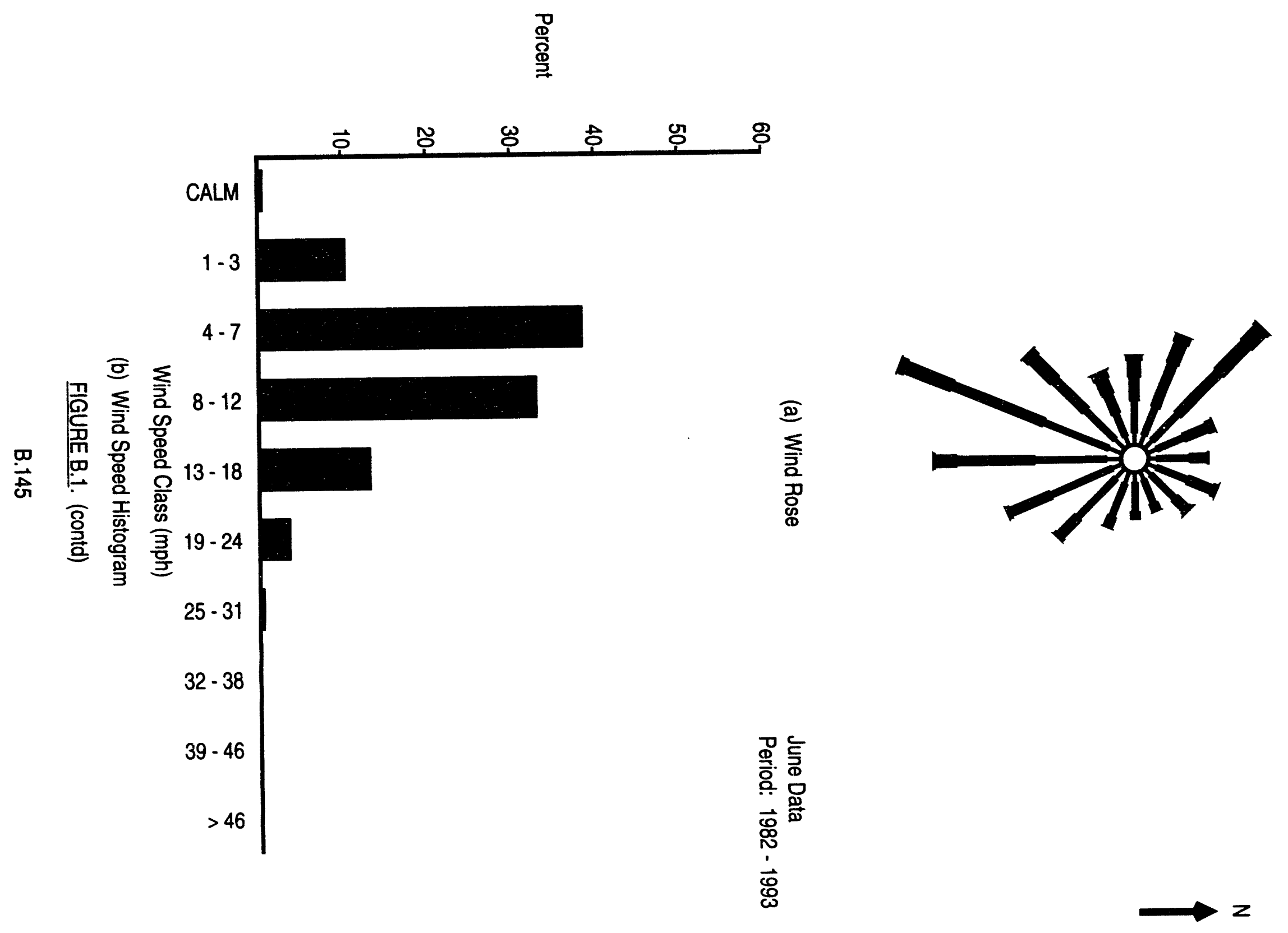



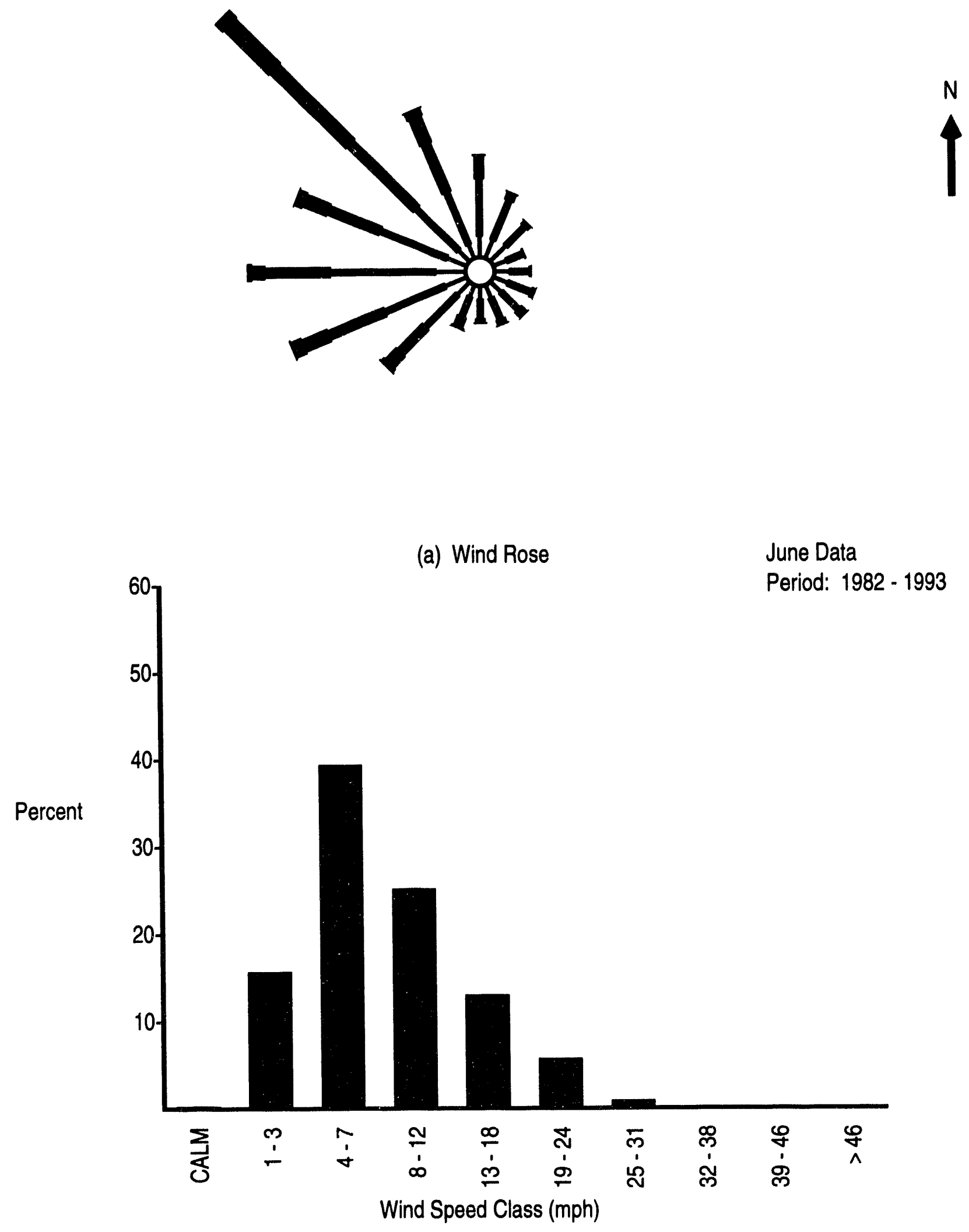

(b) Wind Speed Histogram

FIGURE B.1. (contd)

\section{B.146}



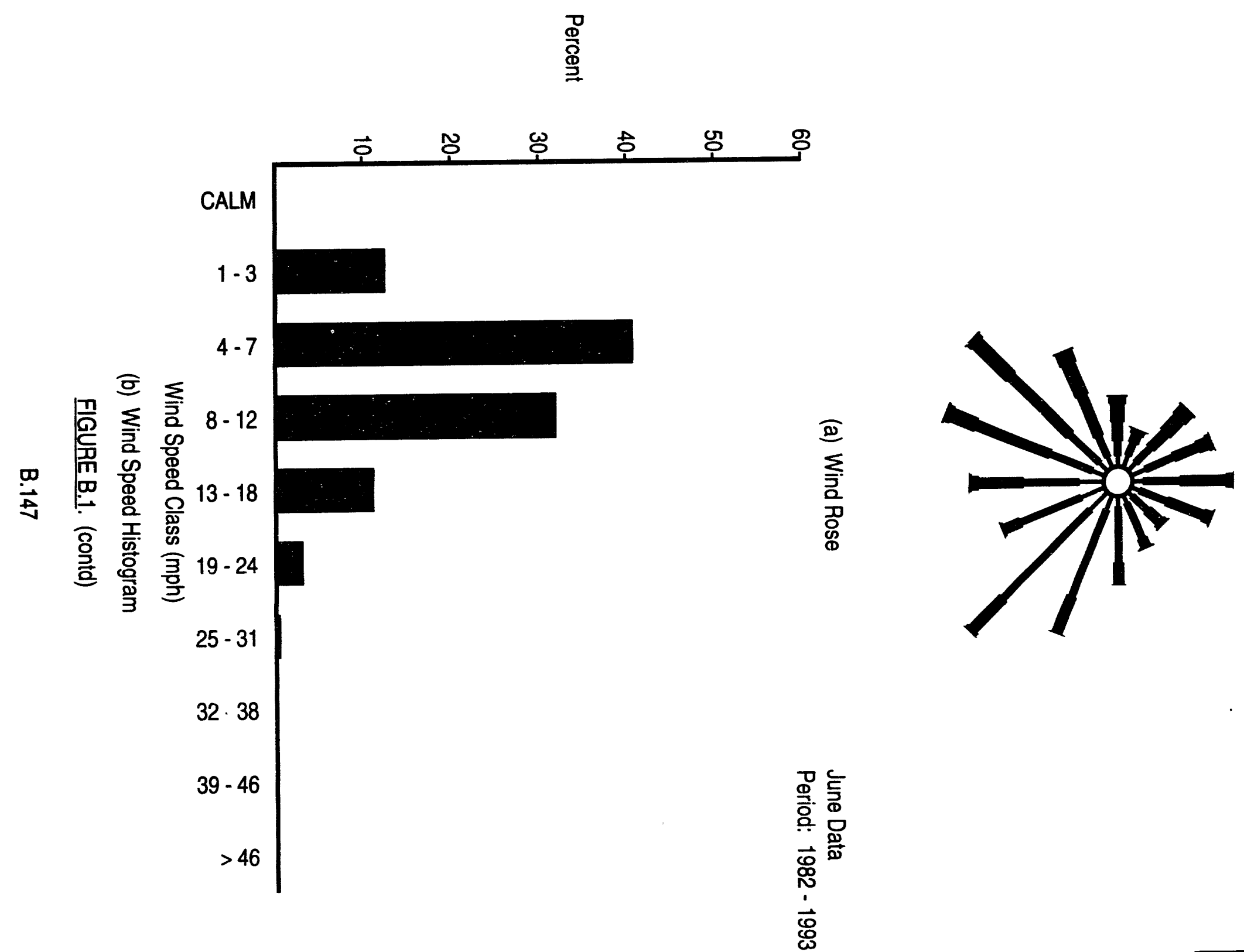

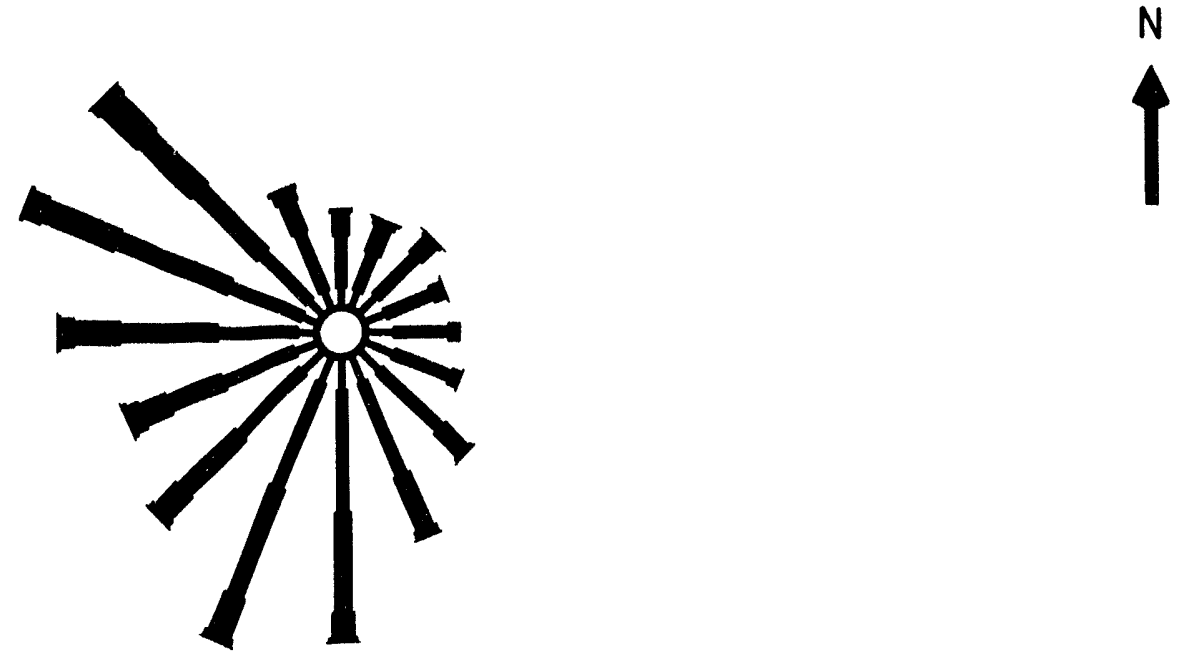

(a) Wind Rose

June Data

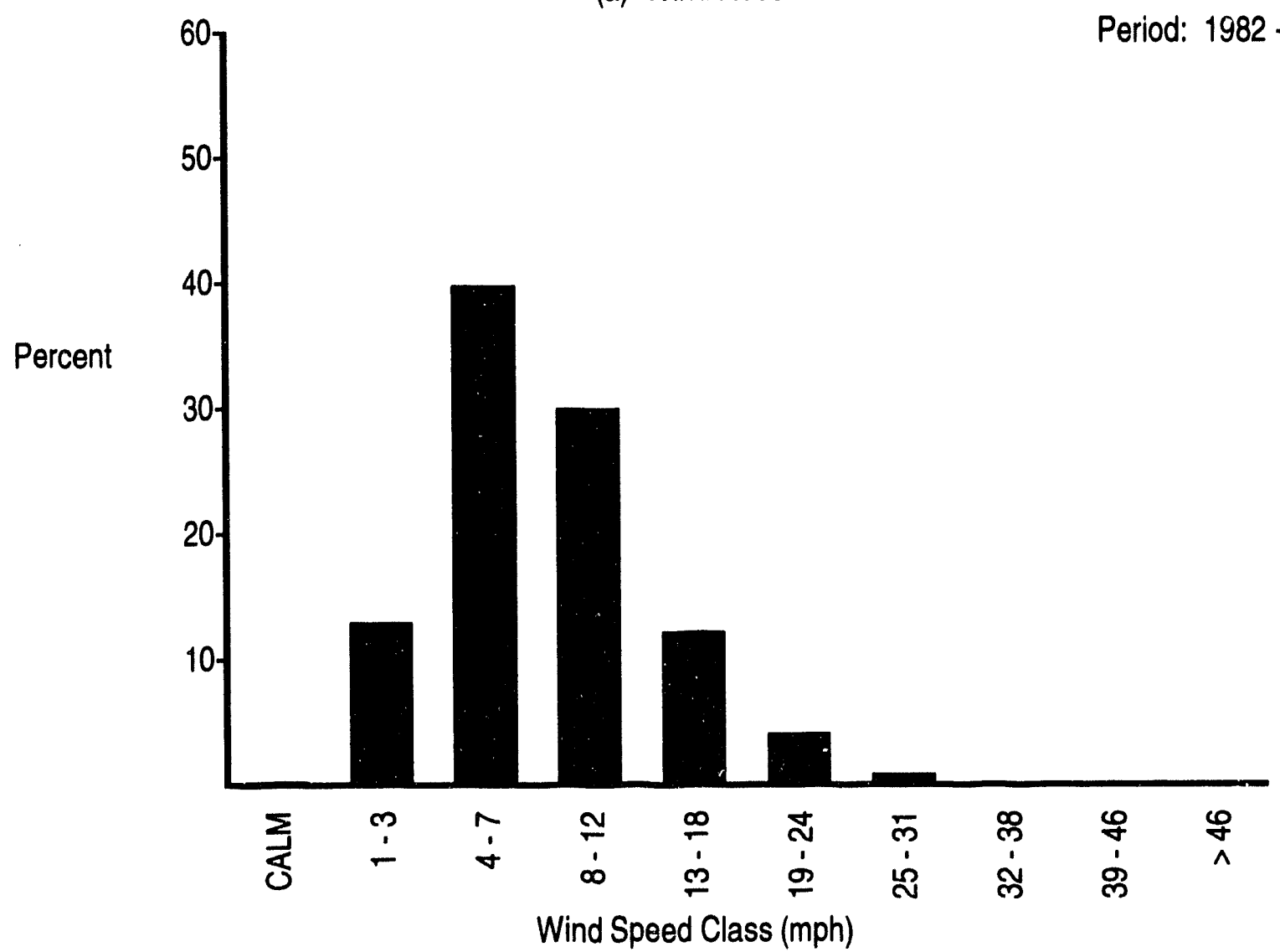

(b) Wind Speed Histogram

FIGURE B.1. (contd)

B.148 


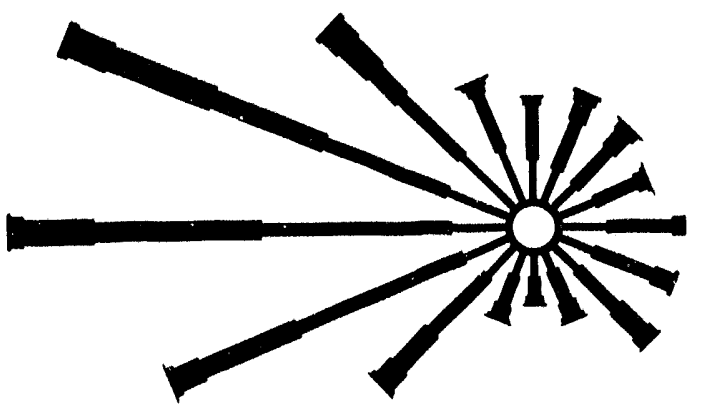

(a) Wind Rose

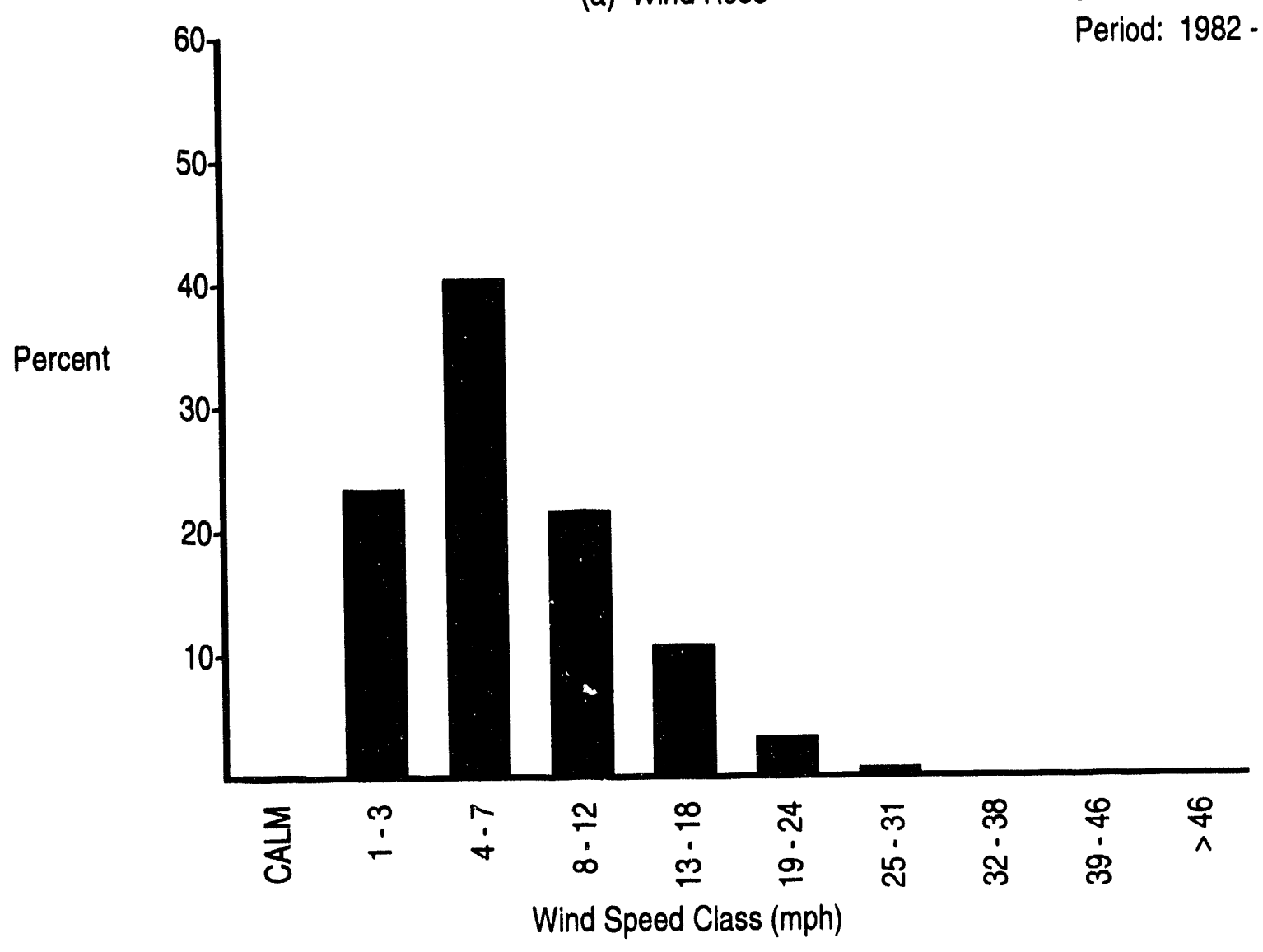

(b) Wind Speed Histogram

FIG!'RE B.1. (contd)

\section{B.149}



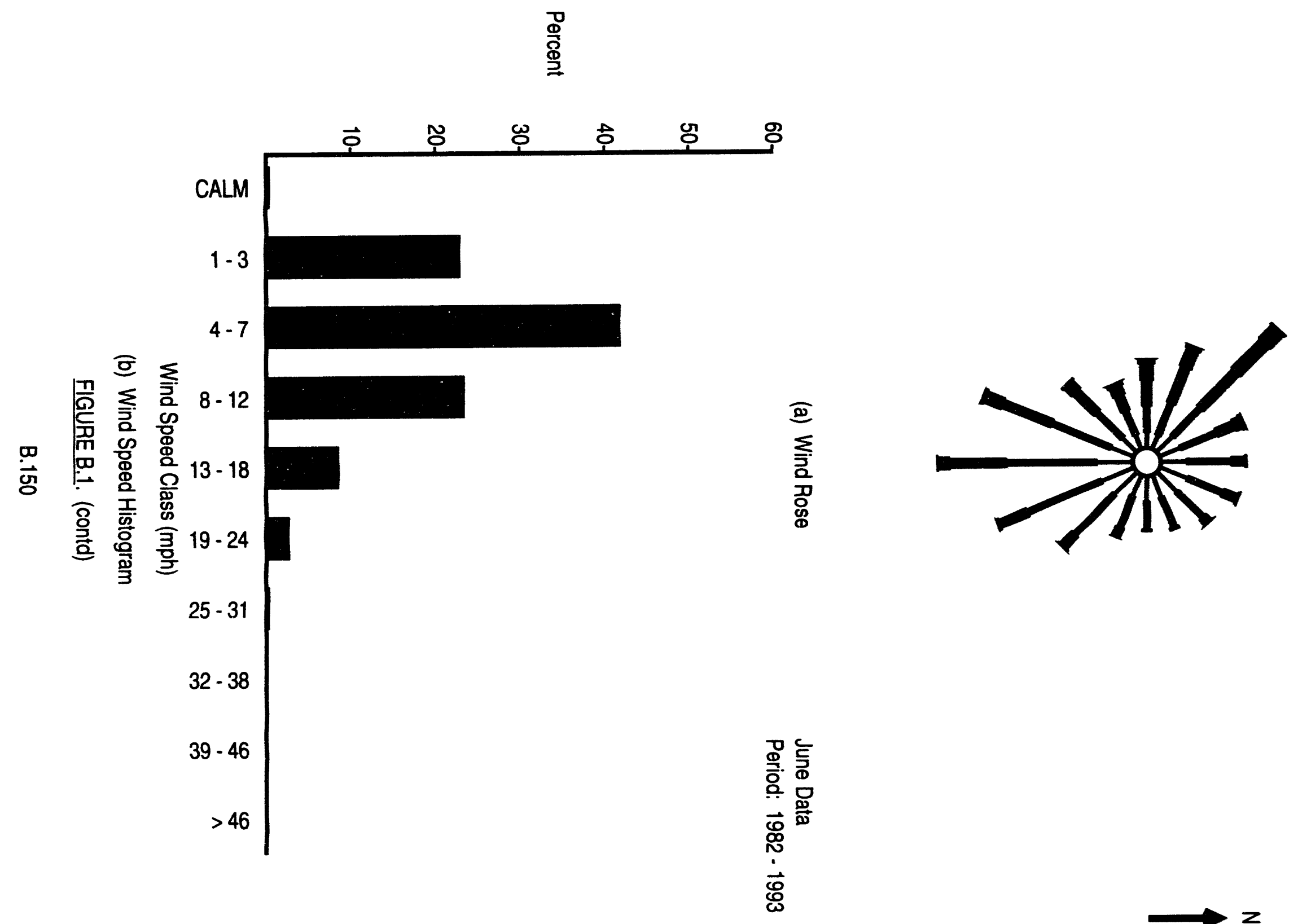

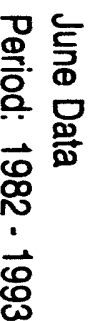




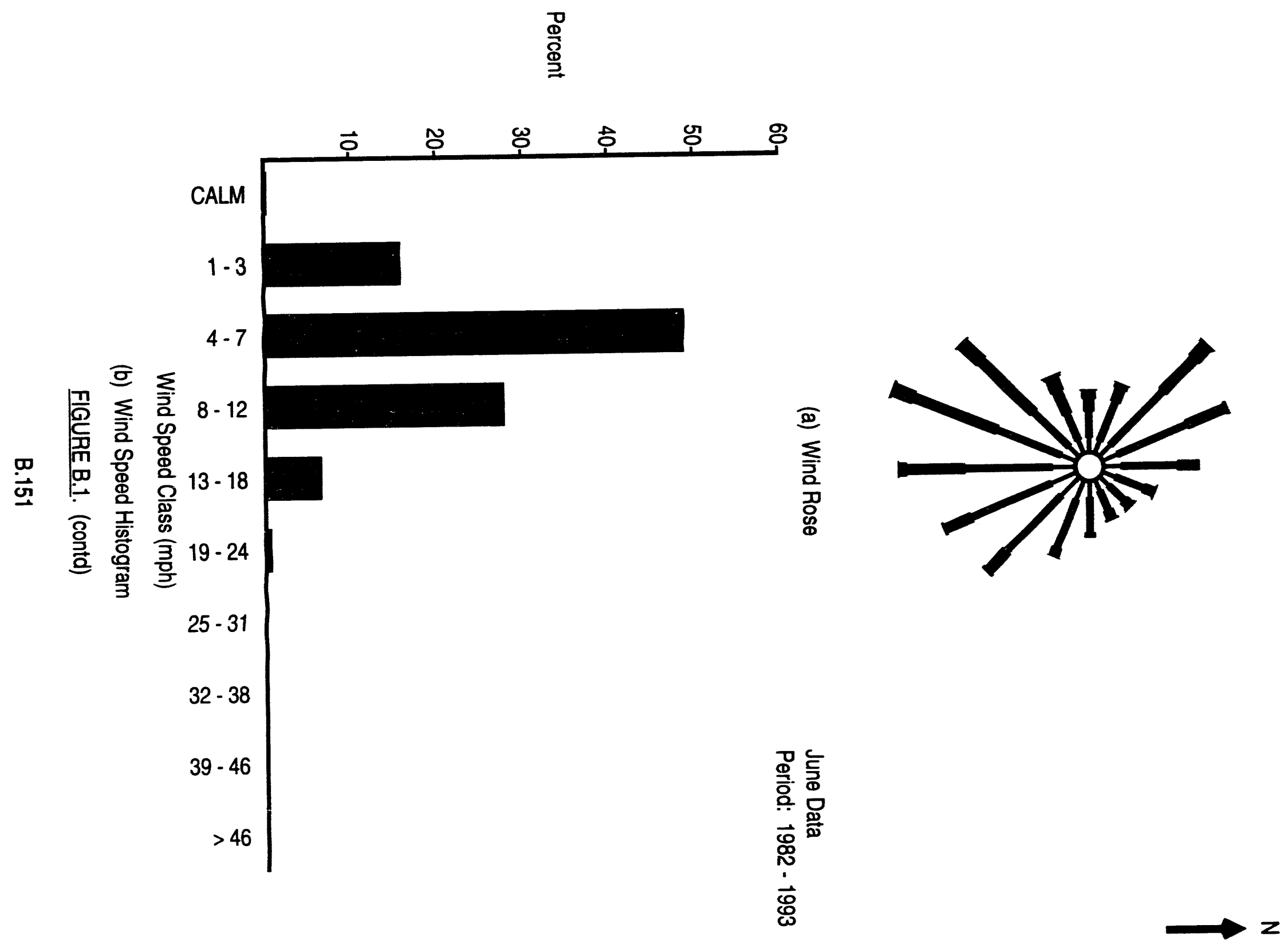




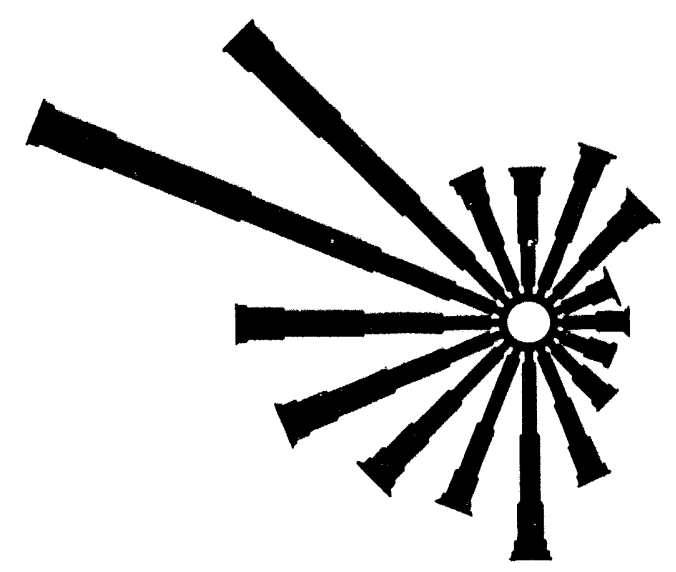

(a) Wind Rose

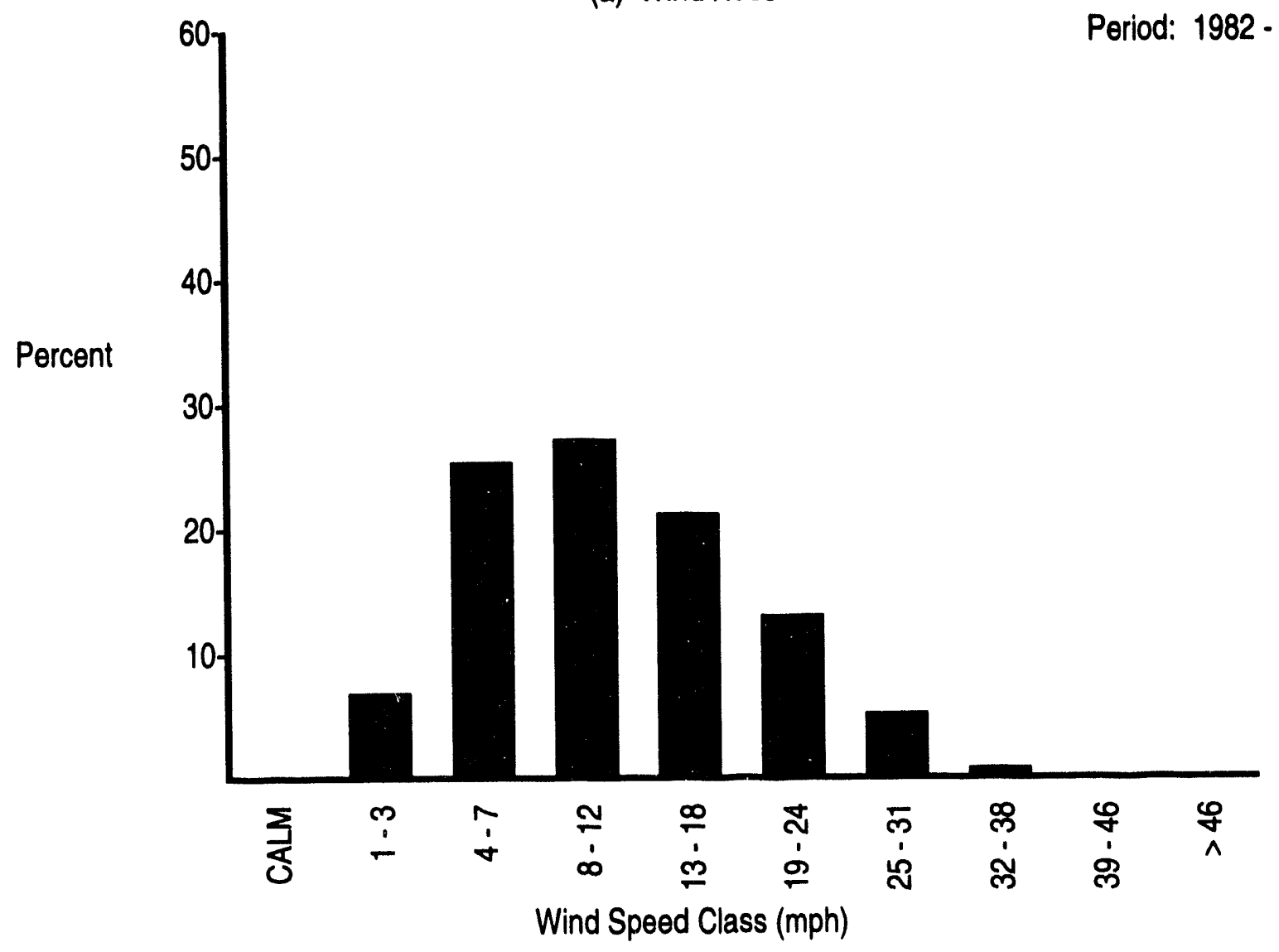

(b) Wind Speed Histogram

FIGURE B.1. (contd)
June Data

Period: 1982 - 1993

\section{B. 152}




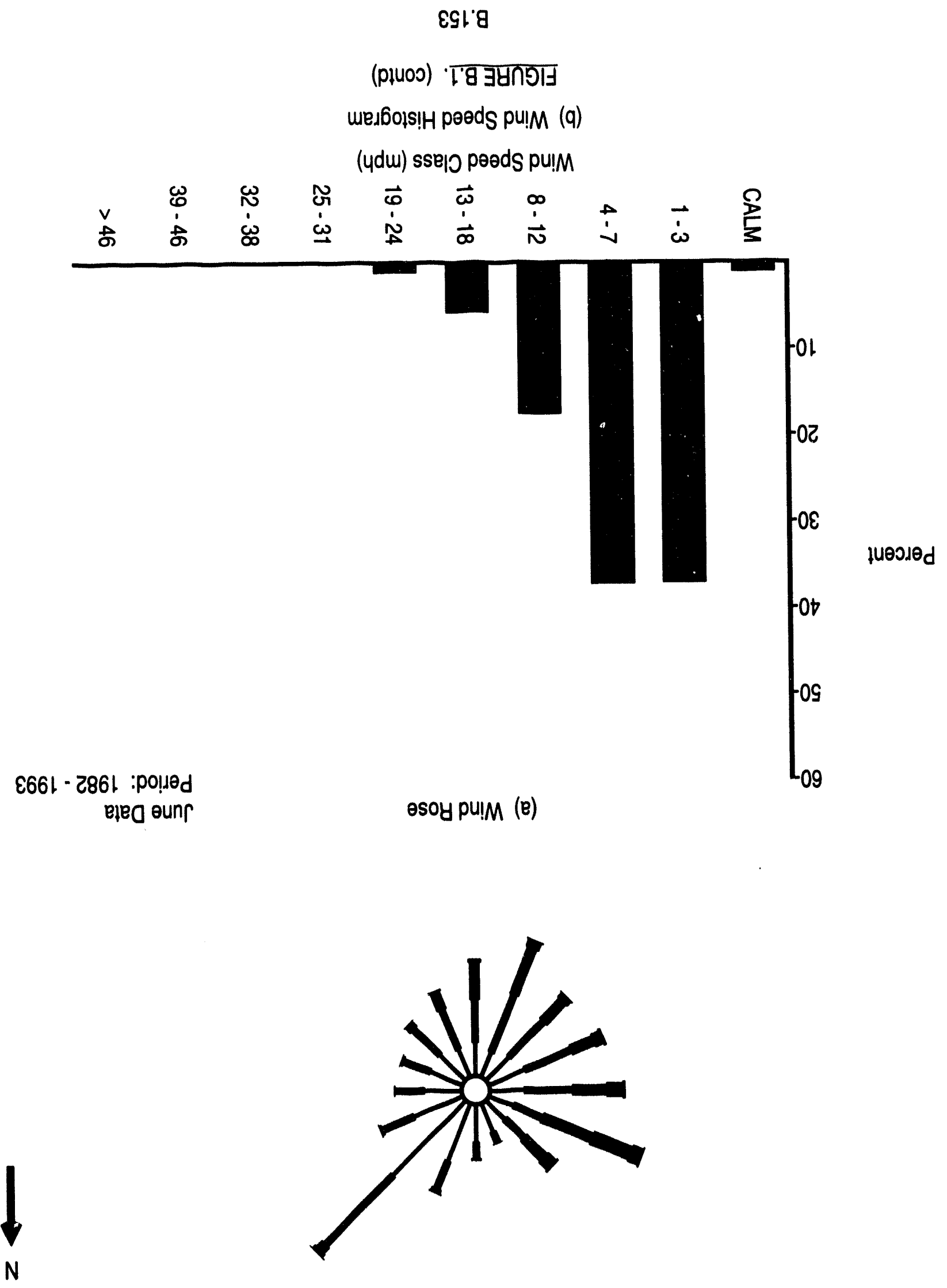


†รเ

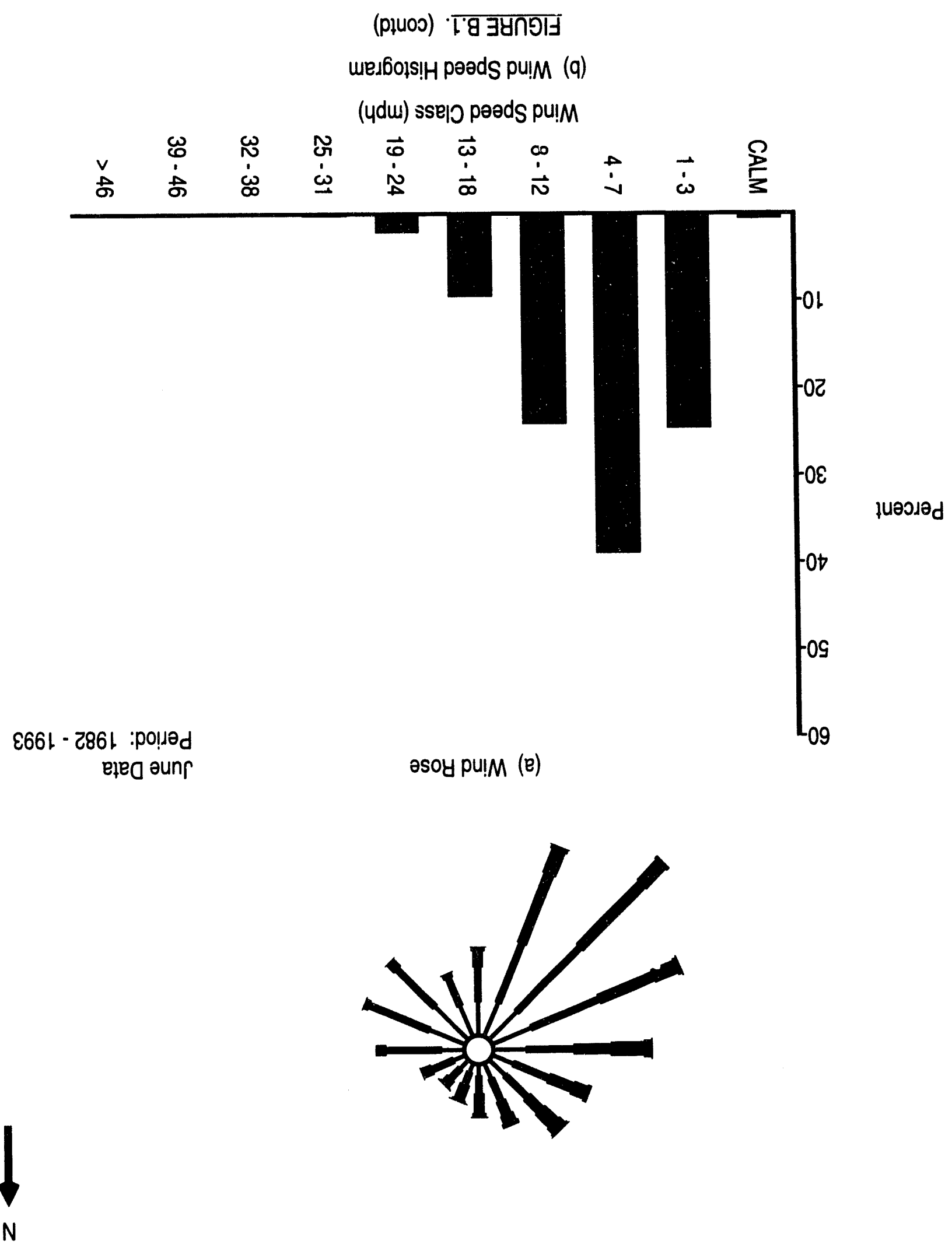




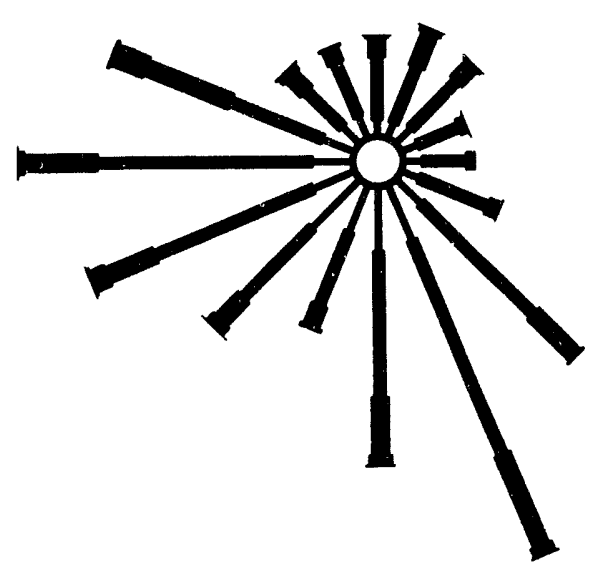

(a) Wind Rose

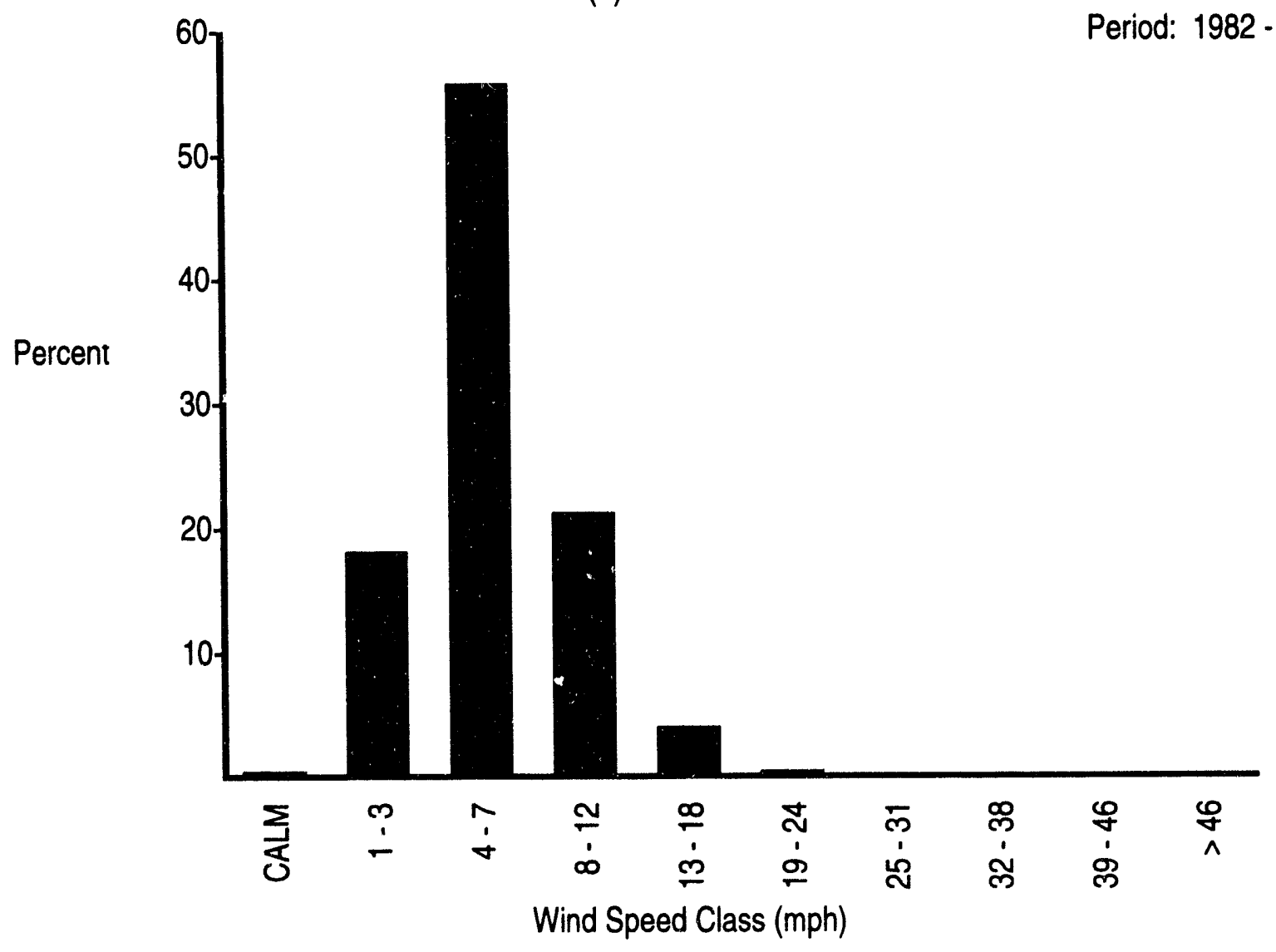

(b) Wind Speed Histogram

FIGURE B.1. (contd)
June Data

Period: 1982 - 1992

\section{B. 155}




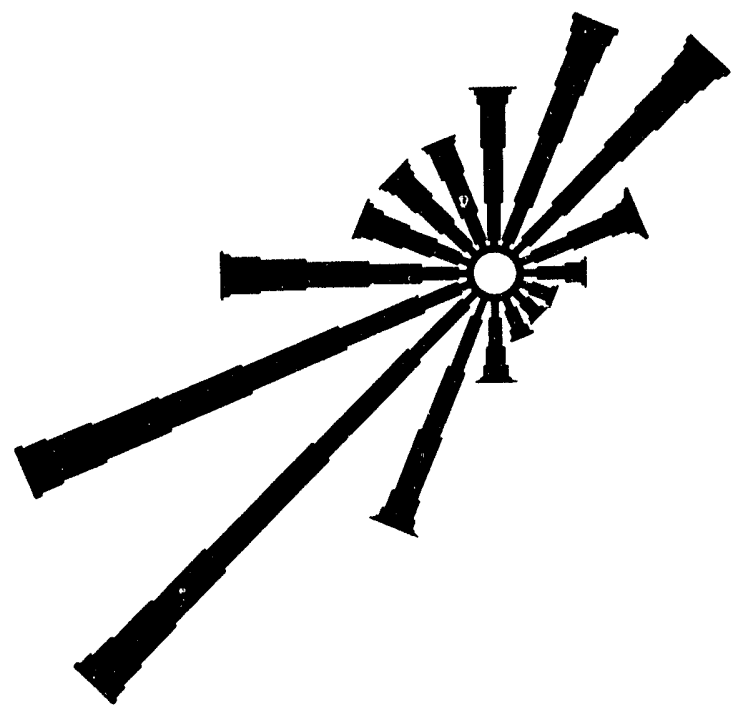

(a) Wind Rose

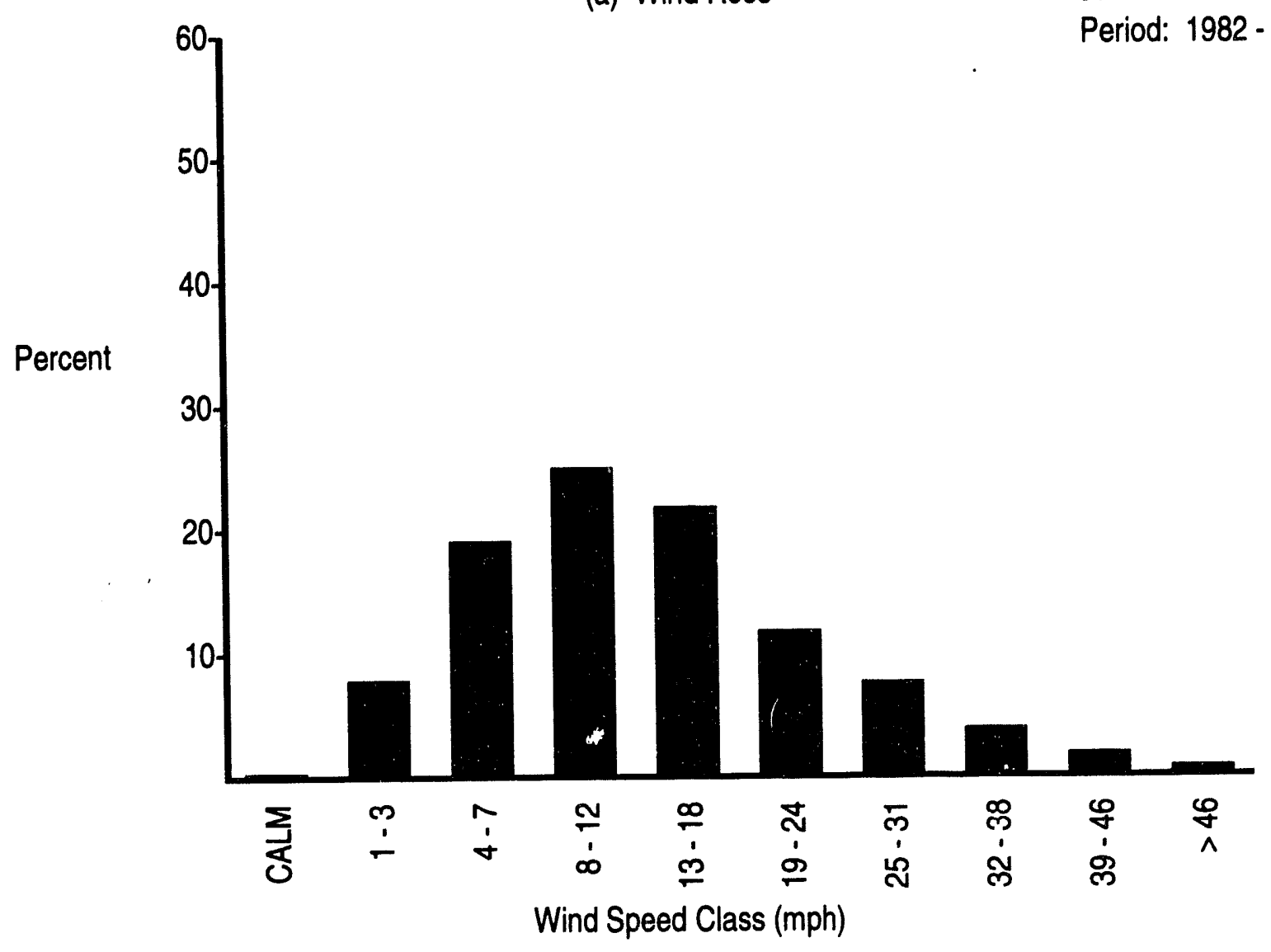

(b) Wind Speed Histogram

FIGURE B.1. (contd)
June Data

Period: 1982 - 1993

\section{B.156}



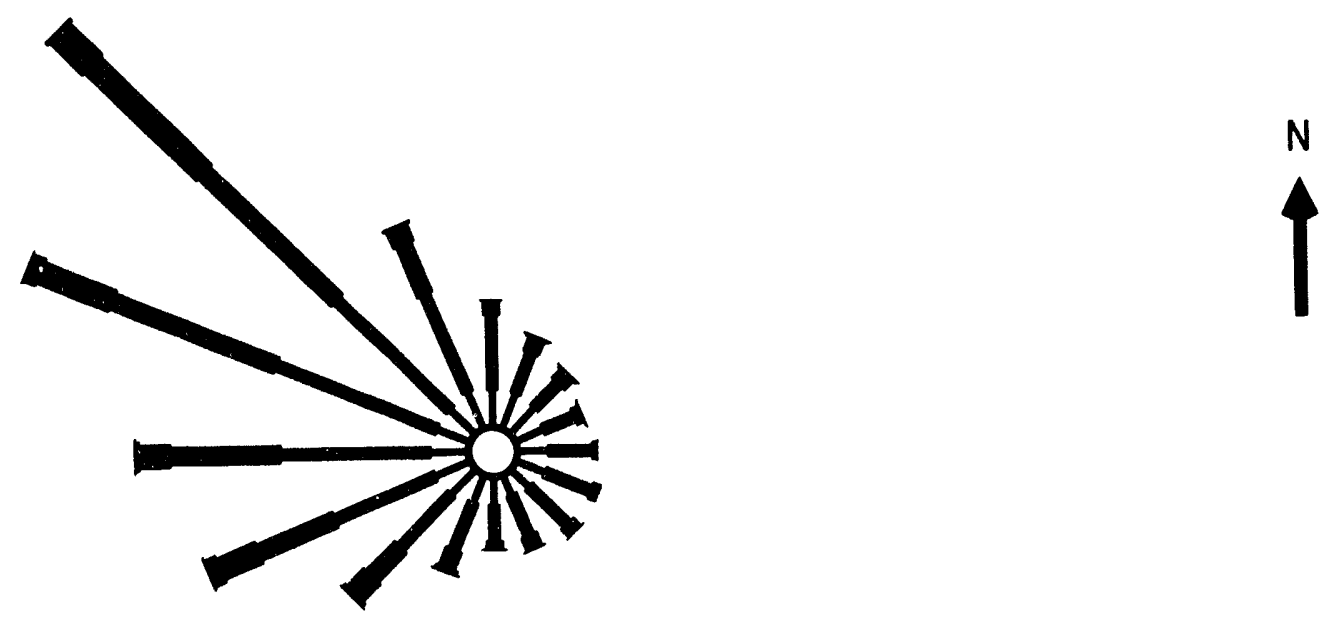

(a) Wind Rose

June Data

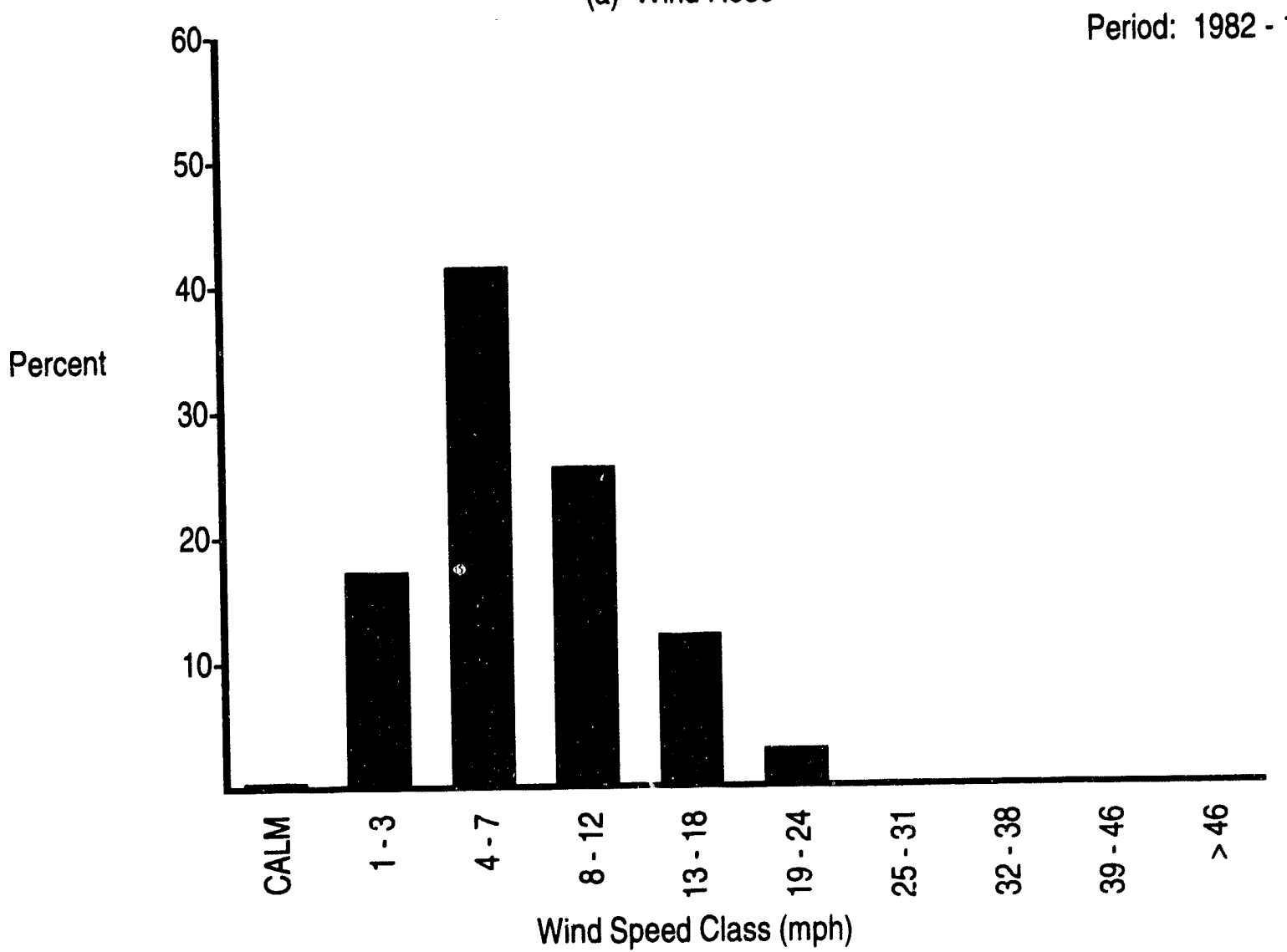

(b) Wind Speed Histogram

FIGURE B.1. (contd) 


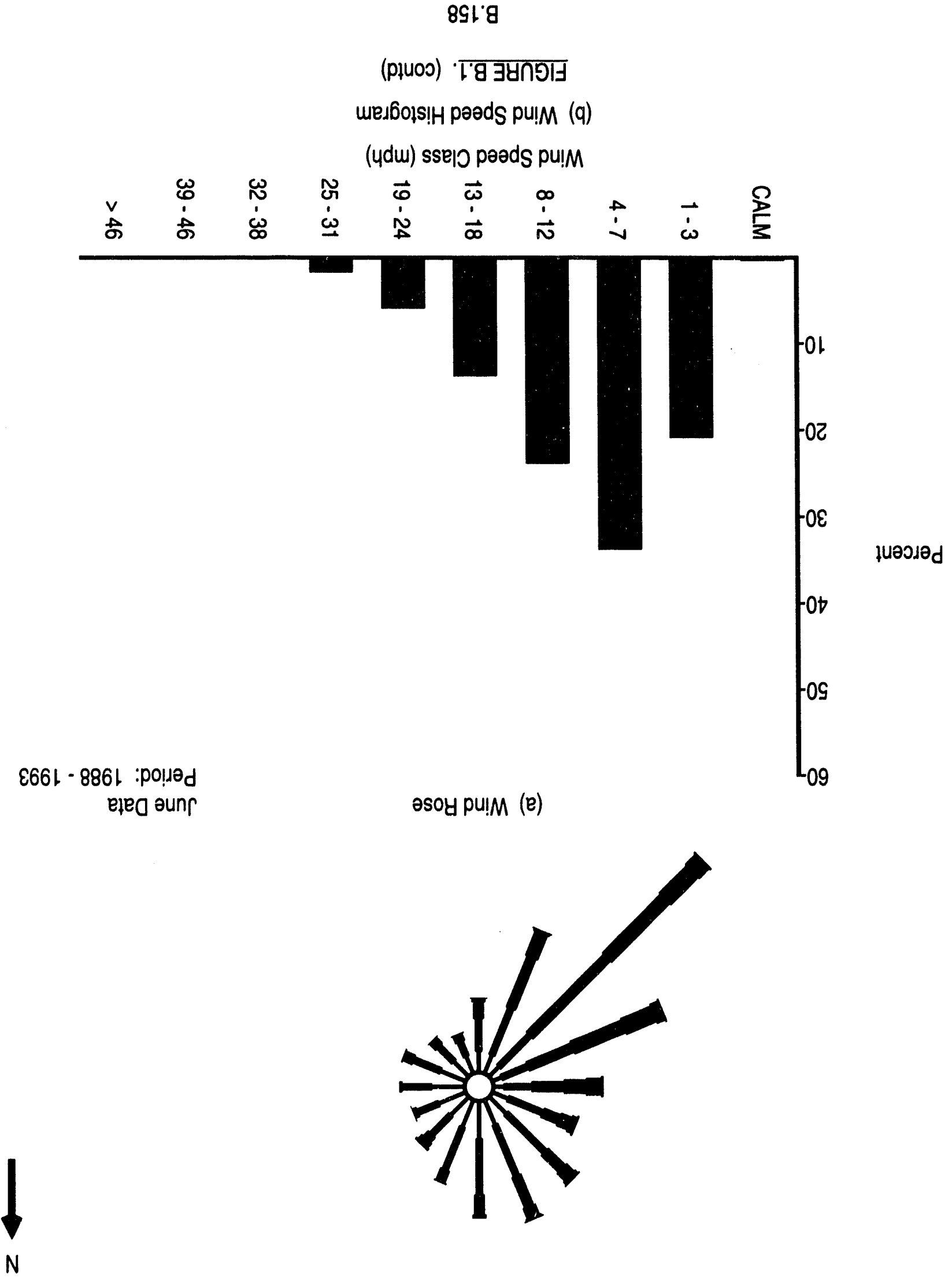




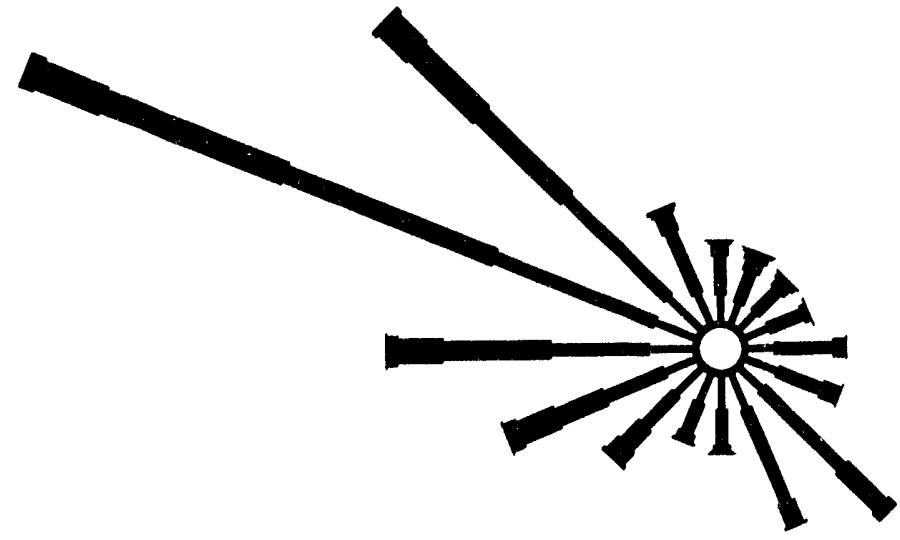

(a) Wind Rose

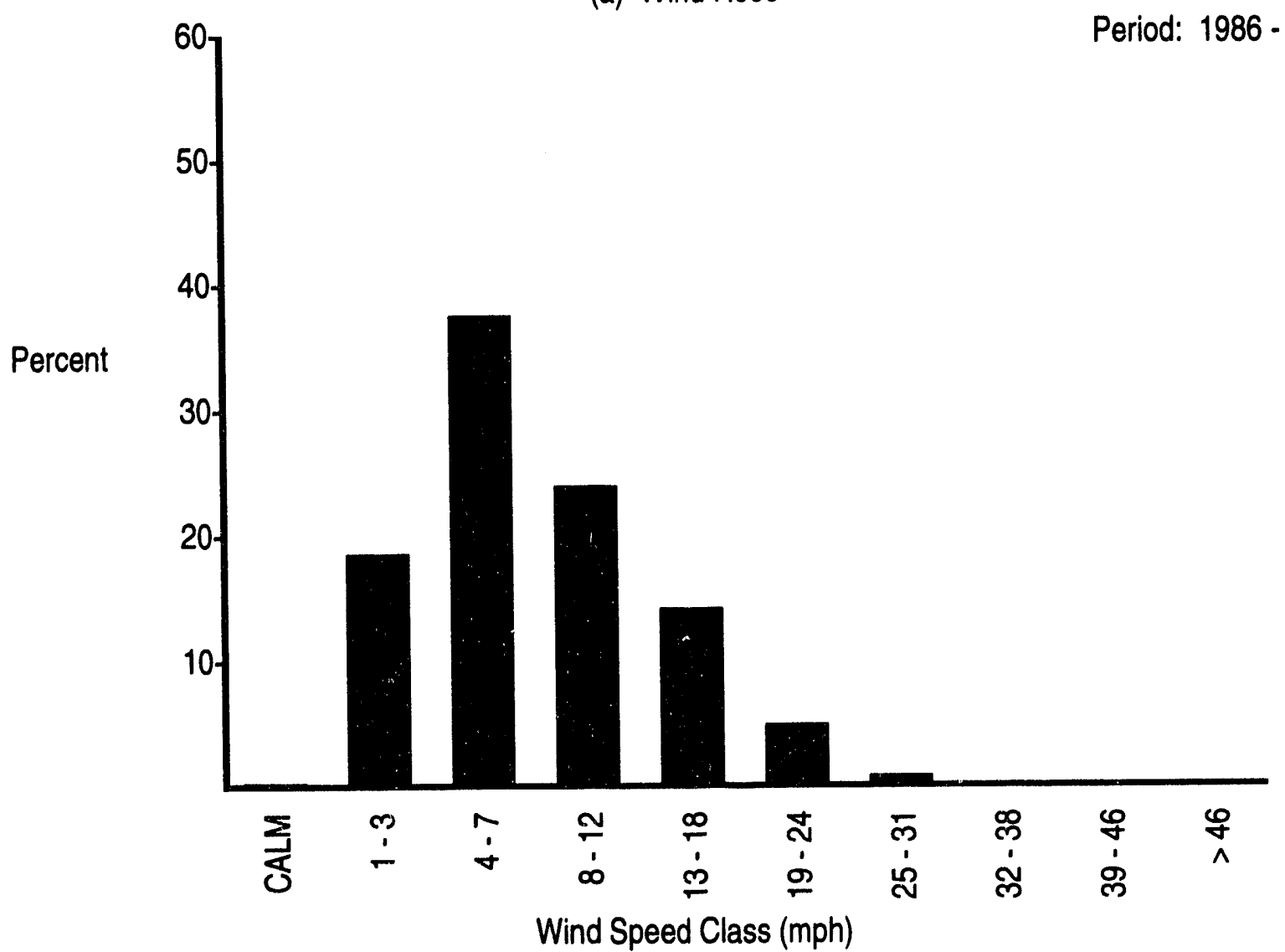

June Data

Period: $1986-1993$

(b) Wind Speed Histogram

FIGURE B.1. (contd)

B.159 


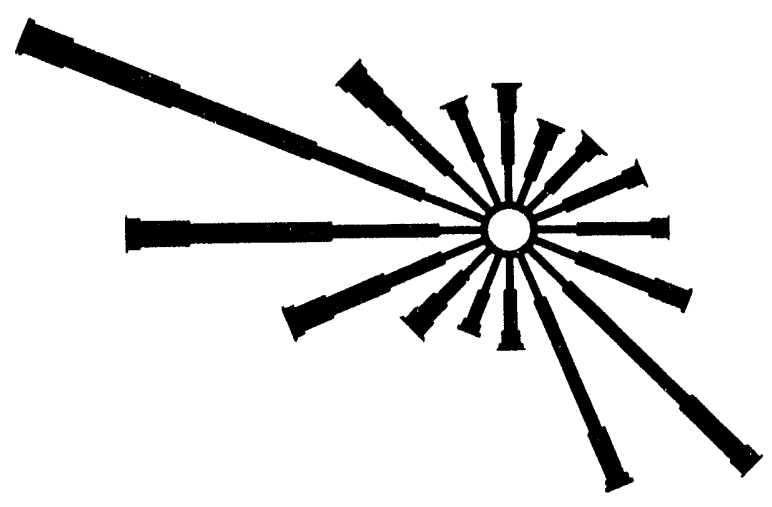

(a) Wind Rose

June Data

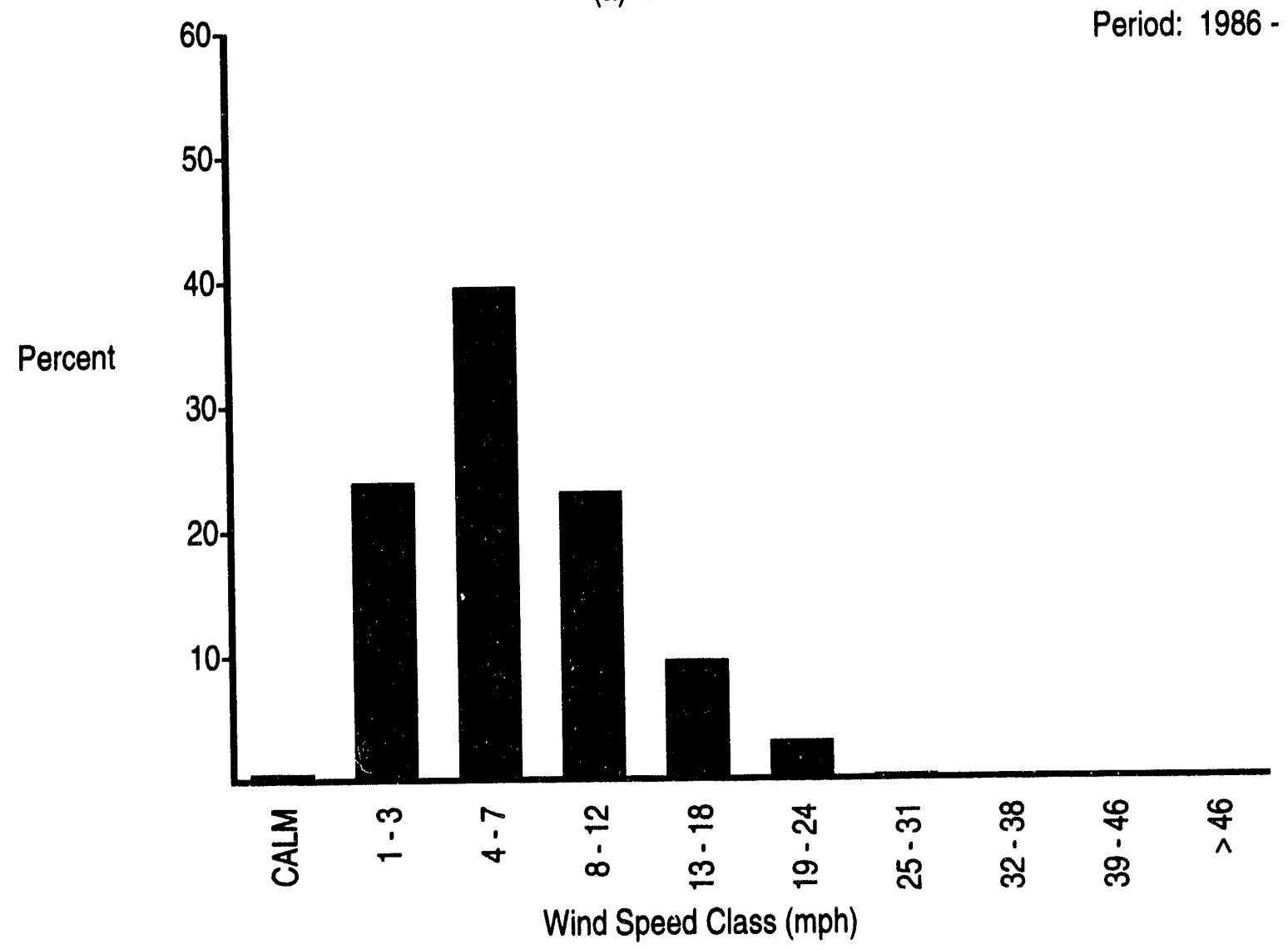

(b) Wind Speed Histogram

FIGURE B.1. (contd) 


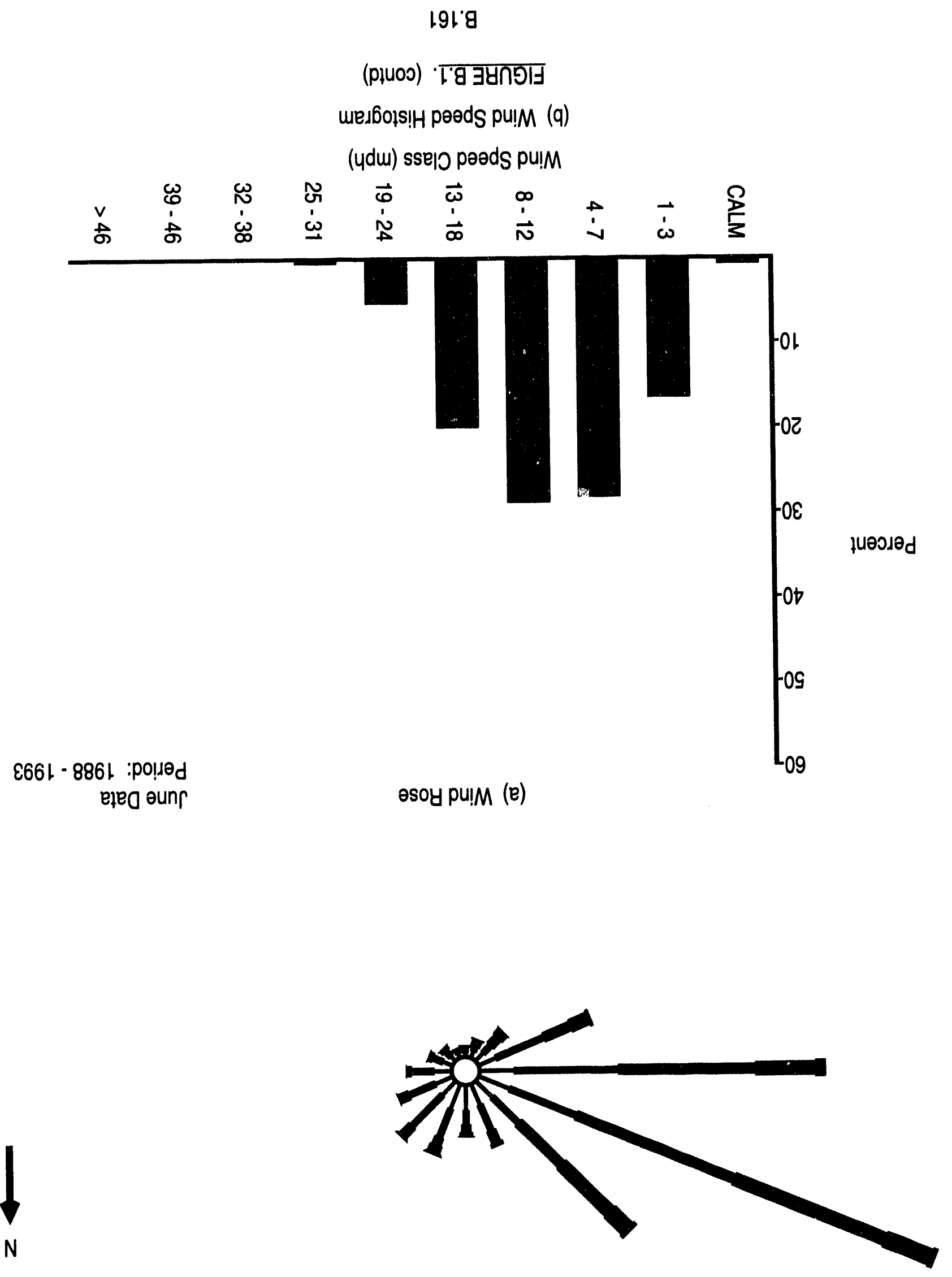




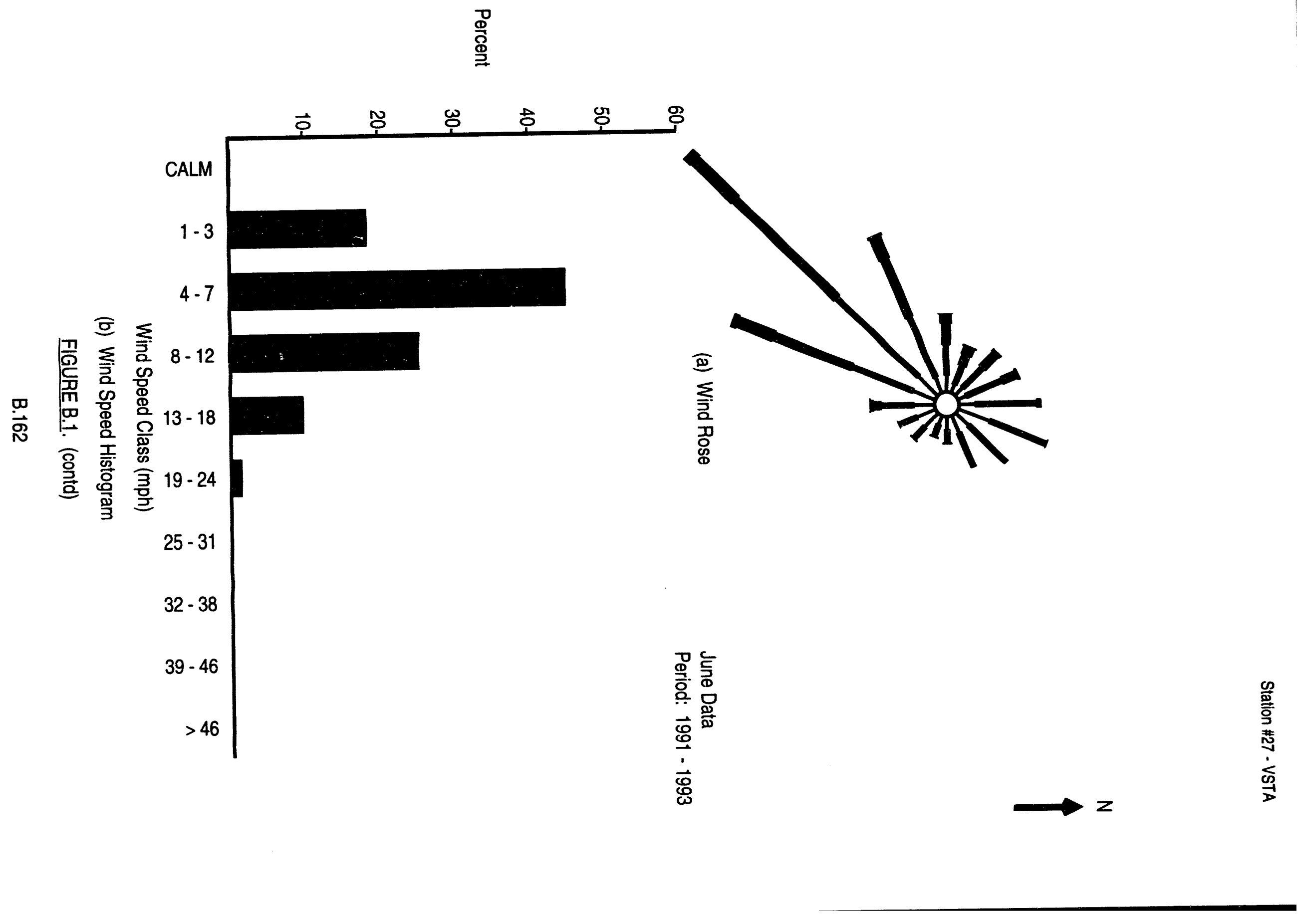




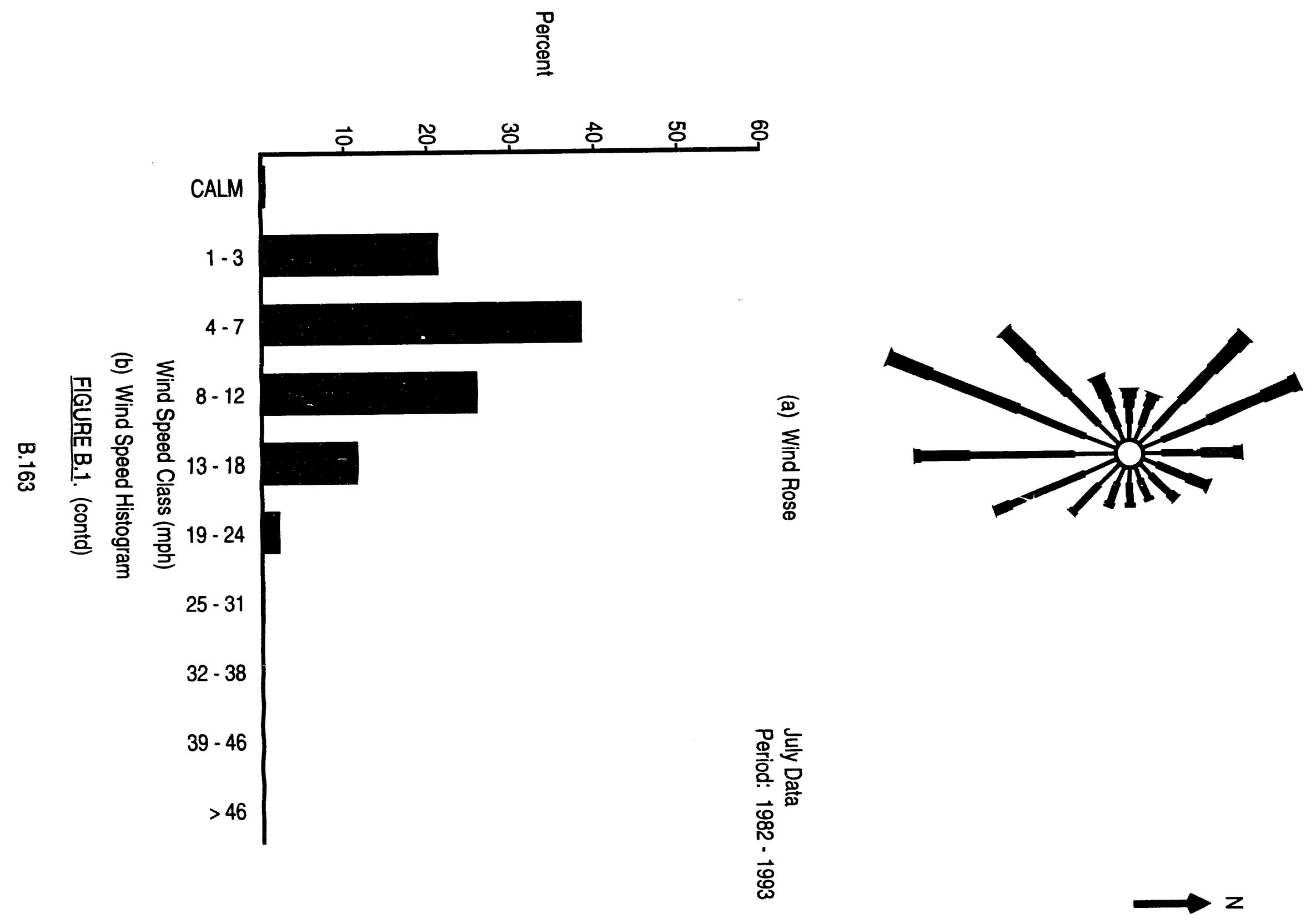




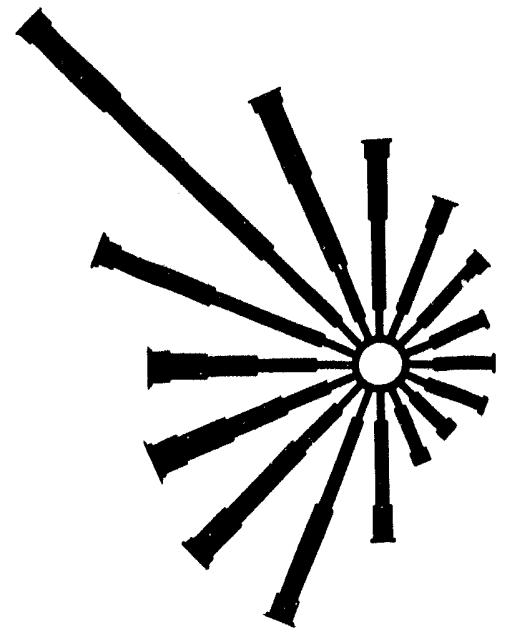

(a) Wind Rose

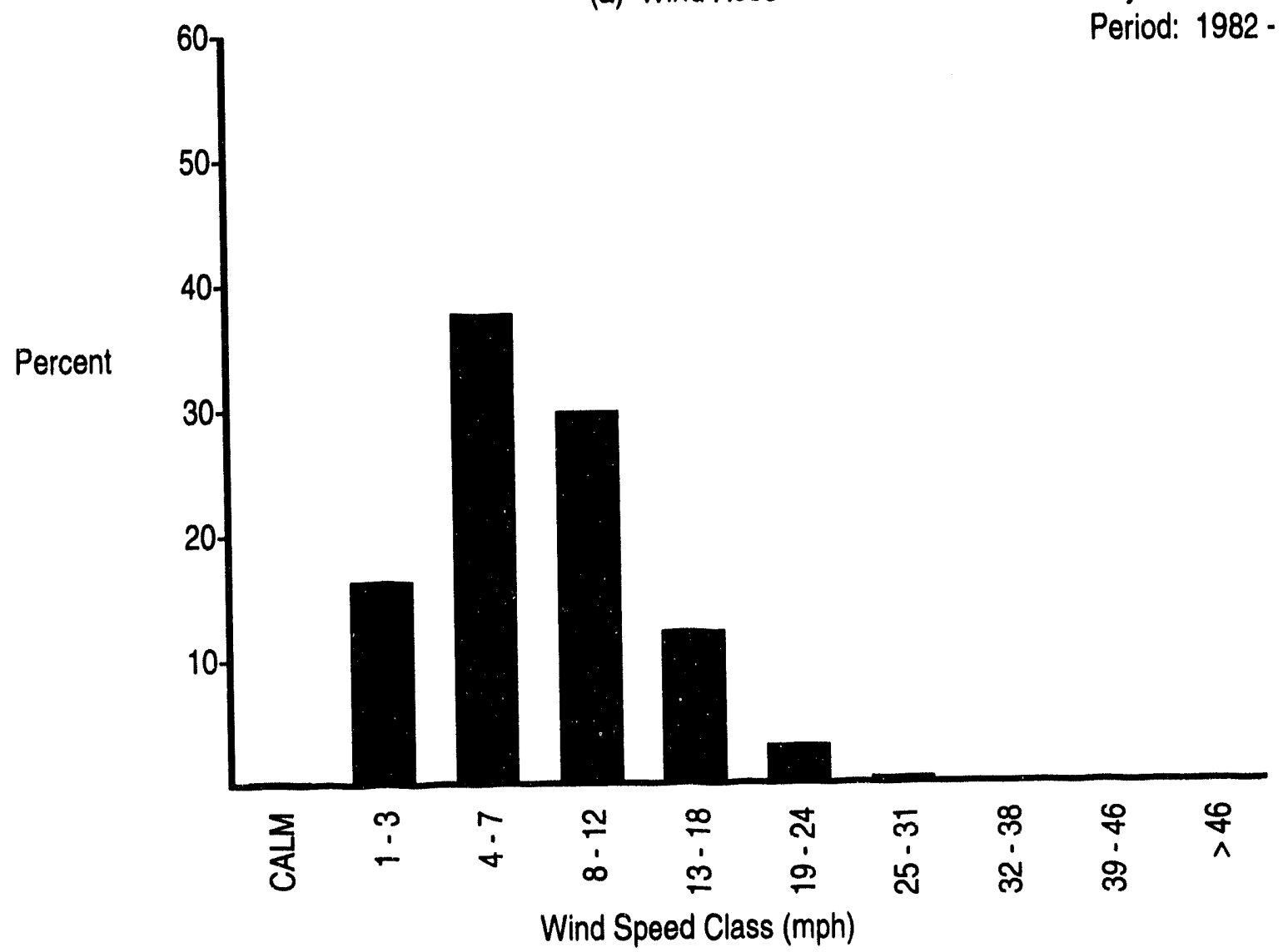

(b) Wind Speed Histogram
July Data

Period: $1982-1993$

\section{FIGURE B.1. (contd)}

\section{B.164}




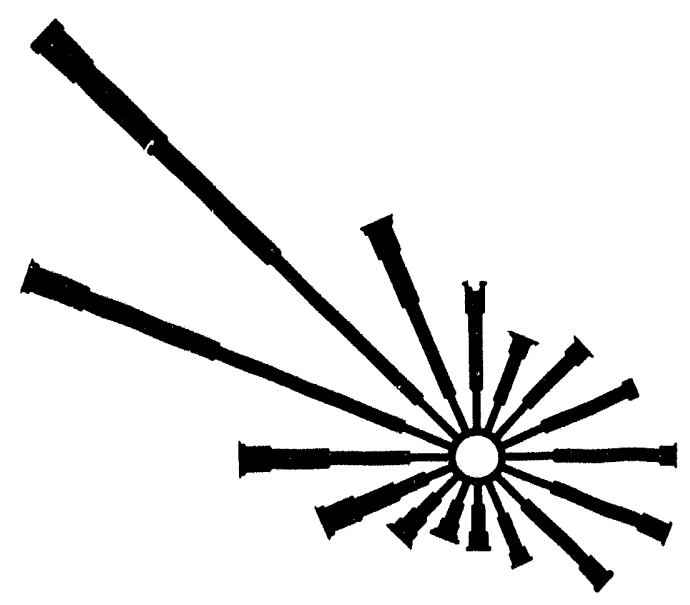

(a) Wind Rose

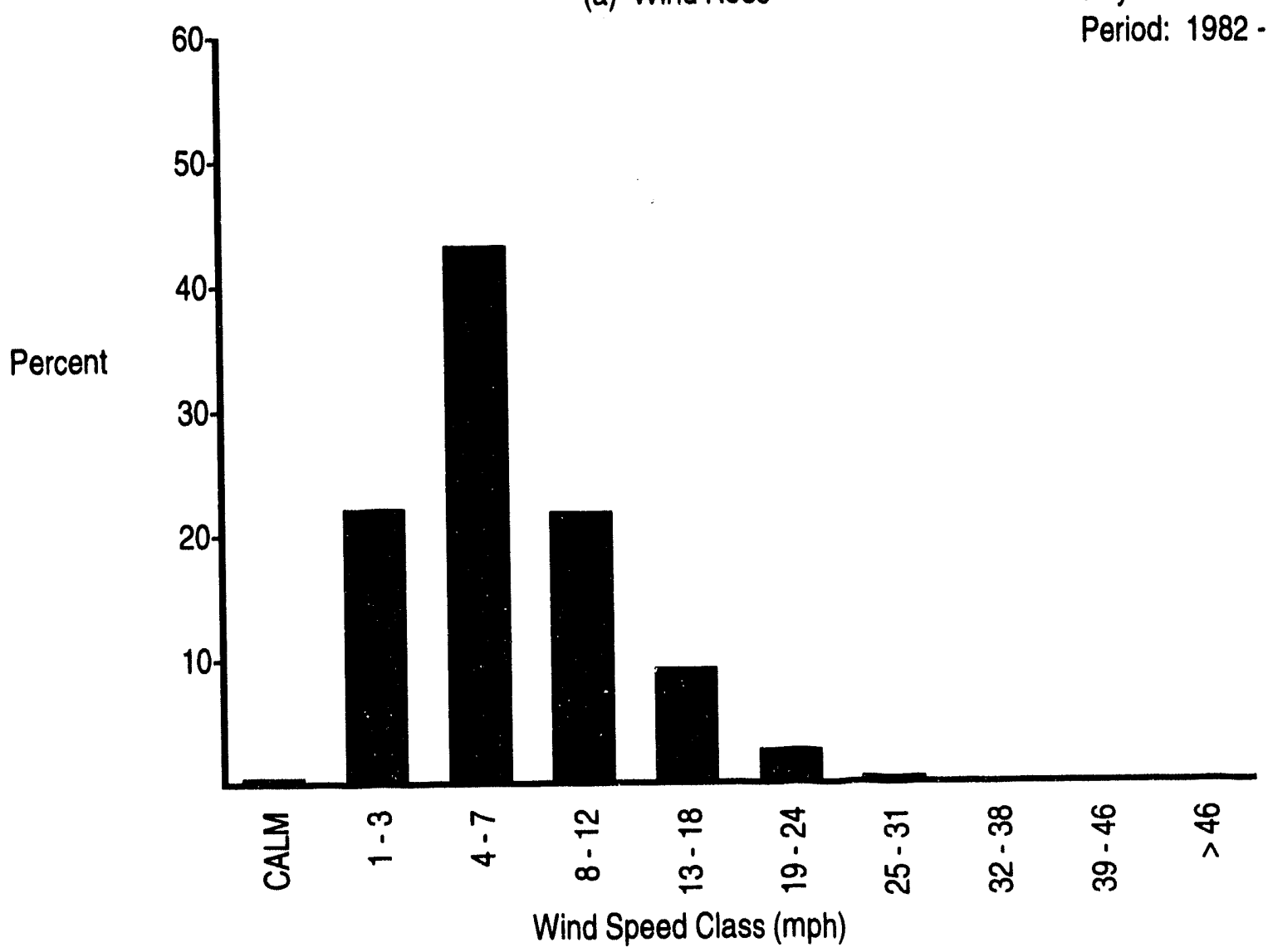

(b) Wind Speed Histogram

FIGURE B.1. (contd) 

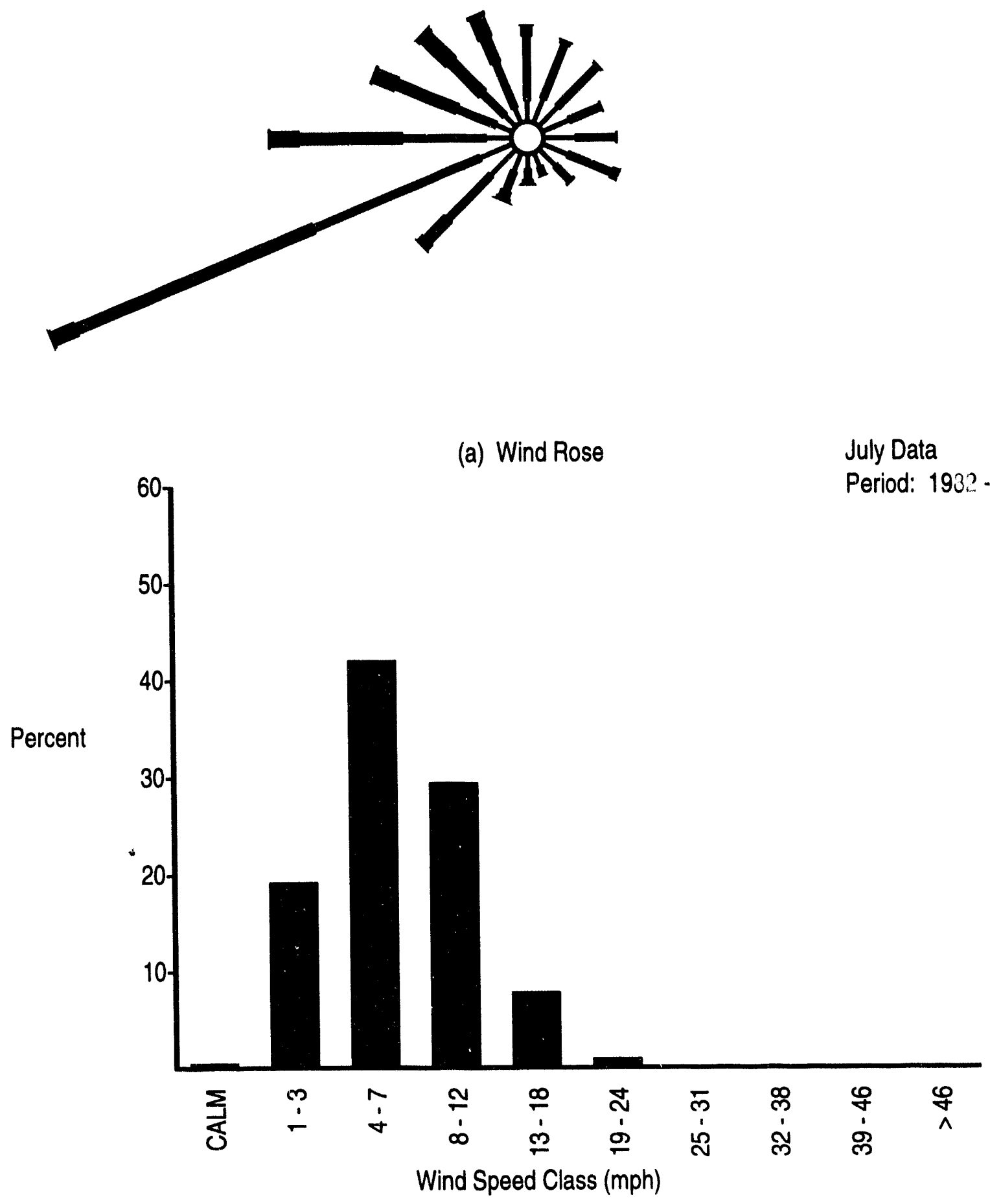

(b) Wind Speed Histogram

FIGURE B.1. (contd)

B.166 


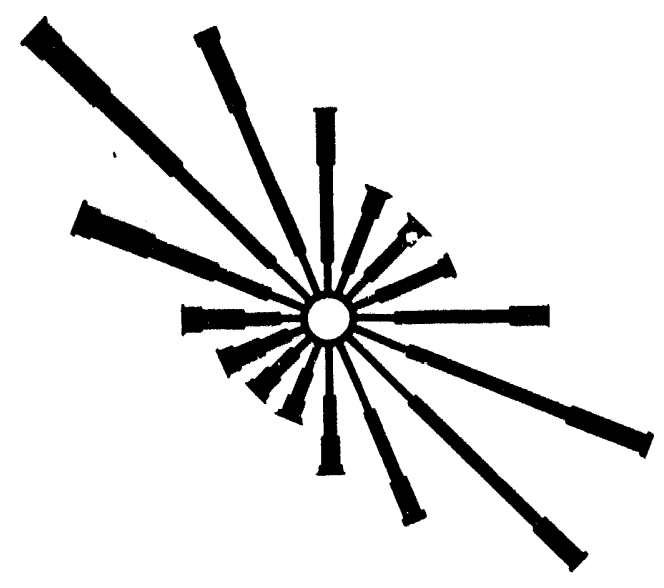

(a) Wind Rose

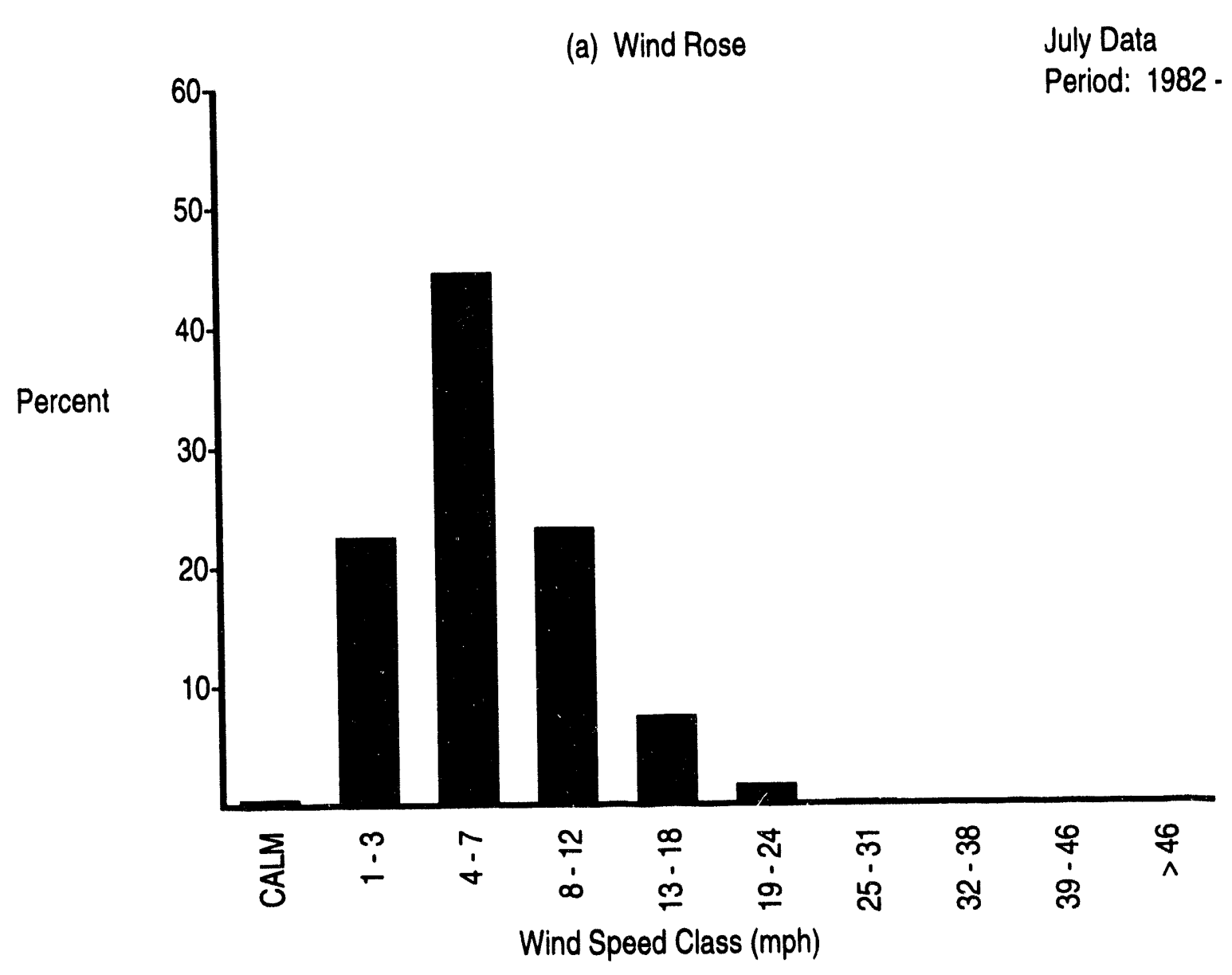

(b) Wind Speed Histogram

FIGURE B.1. (contd) 

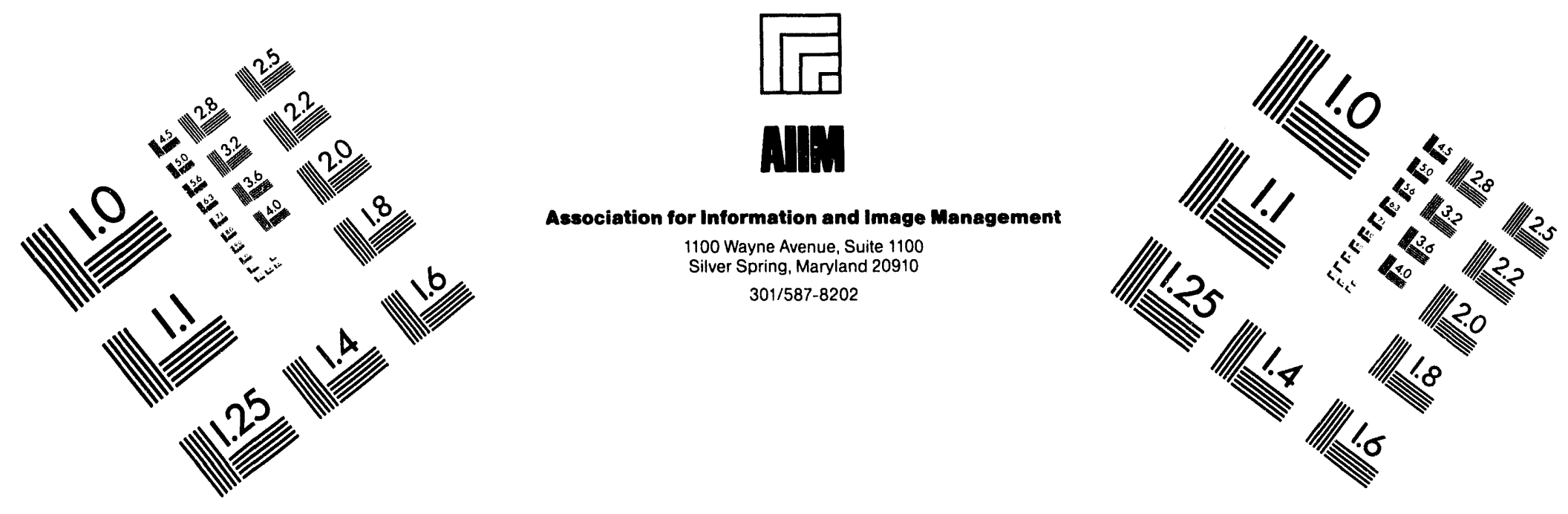

\section{Centimeter}

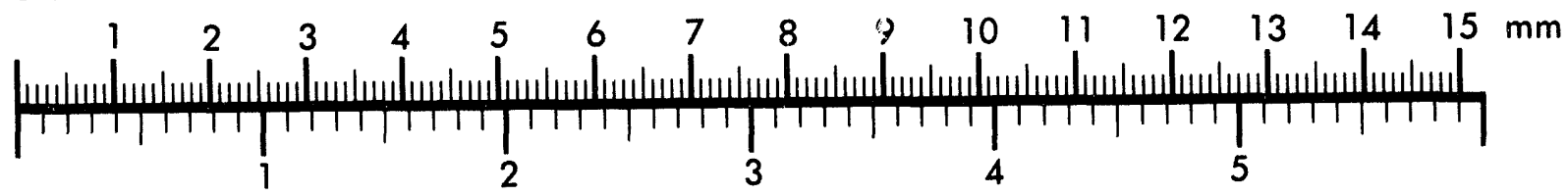
Inches
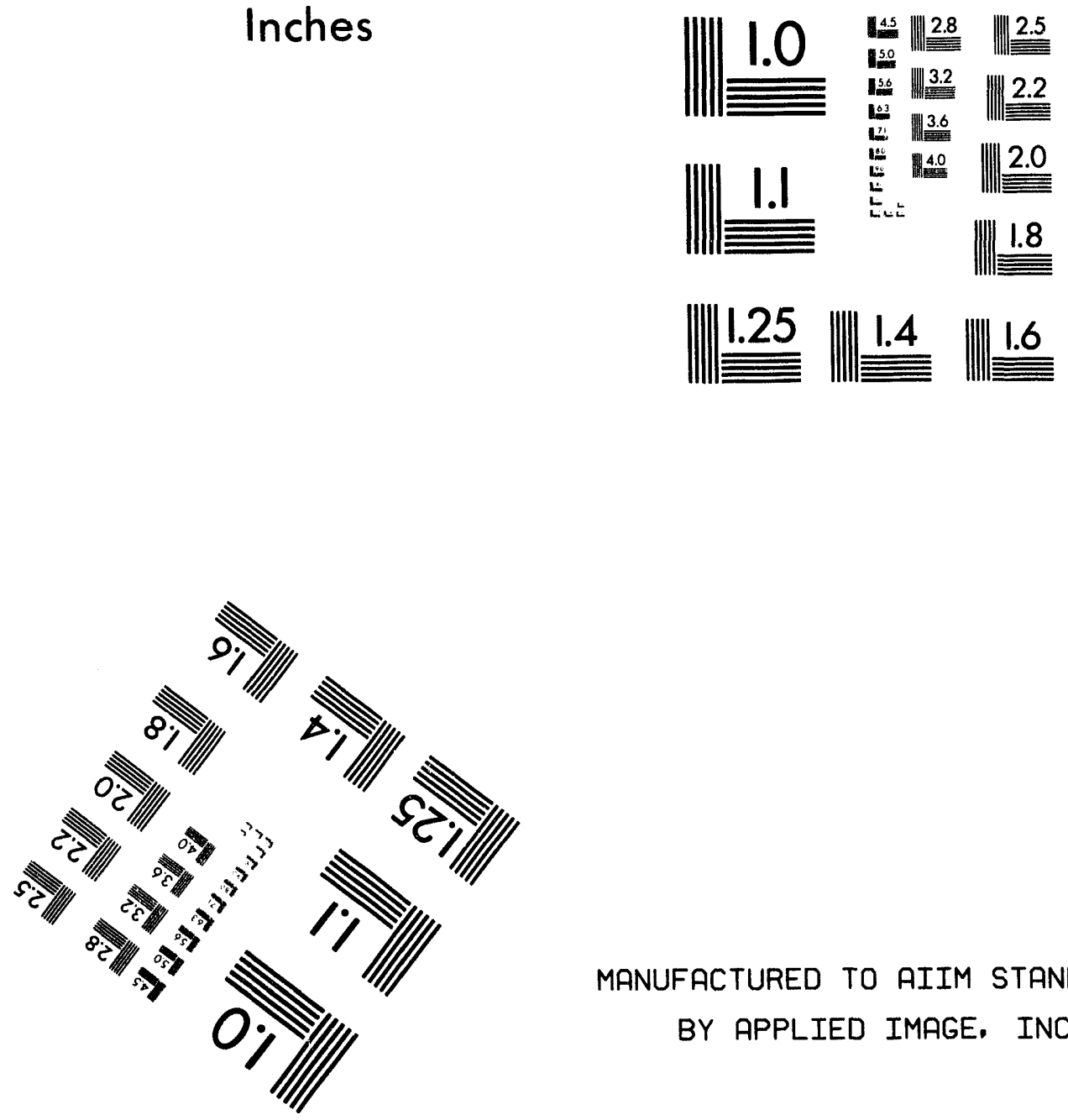

MANUFACTURED TO AIIM STANDARDS

BY APPLIED IMAGE. INC.

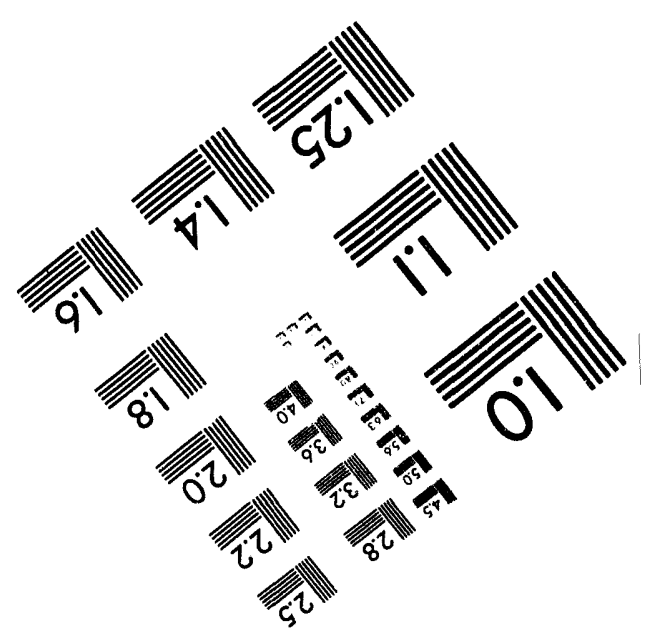



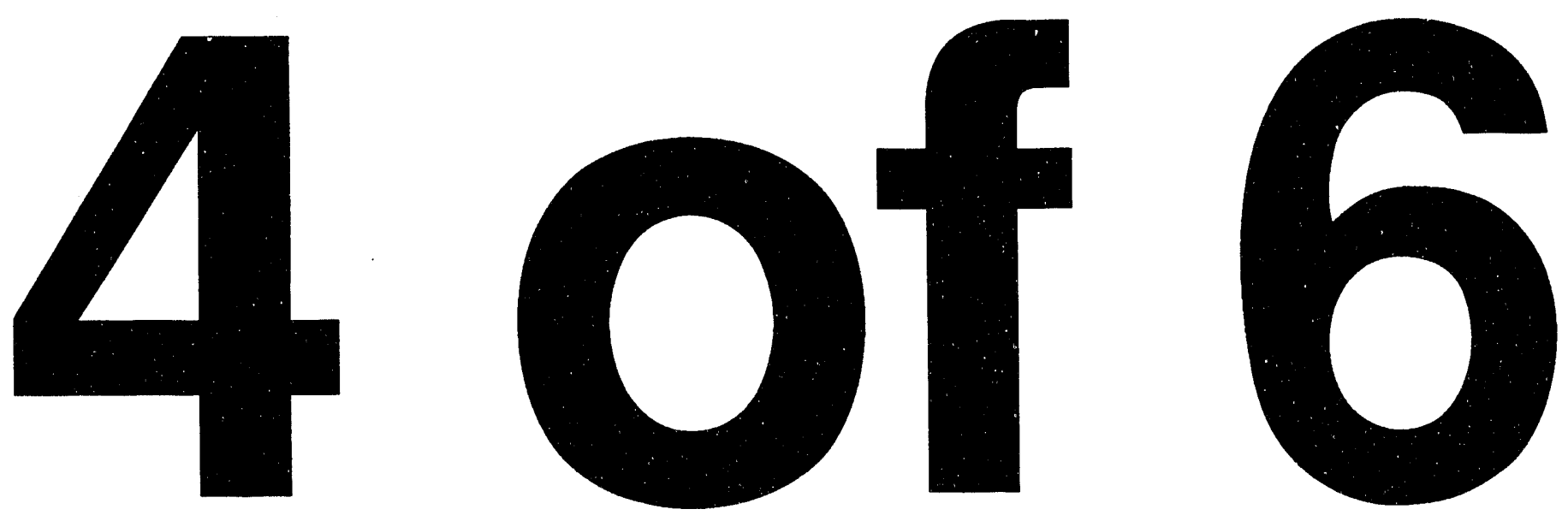


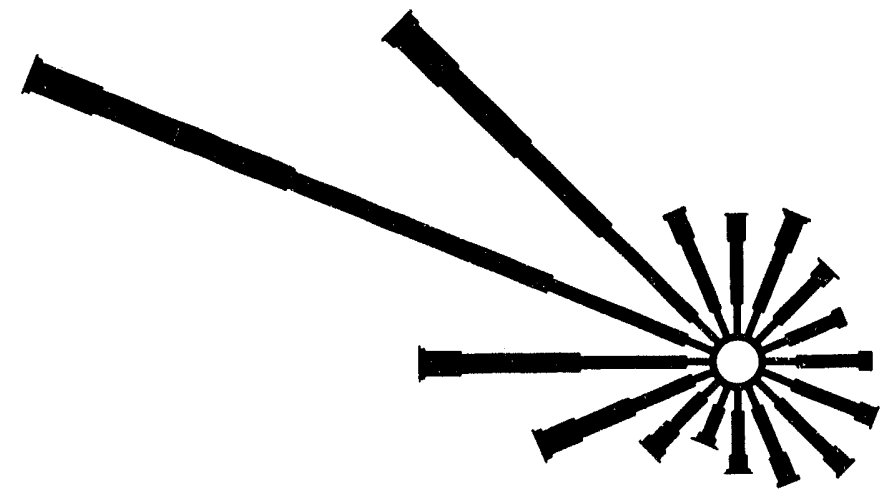

(a) Wirld Rose

July Data

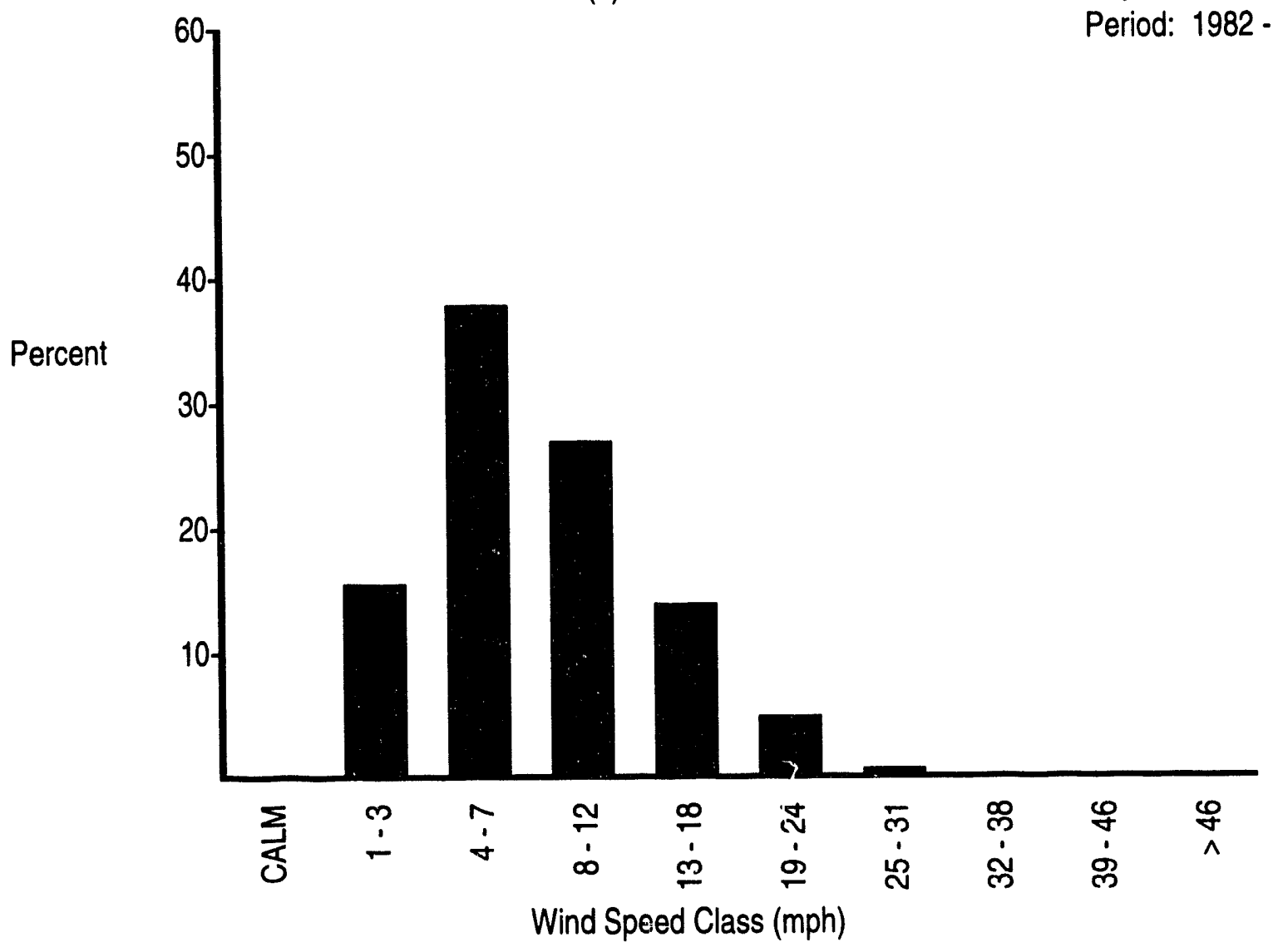

(b) Wind Speed Histogram

FIGURE B.1. (contd) 

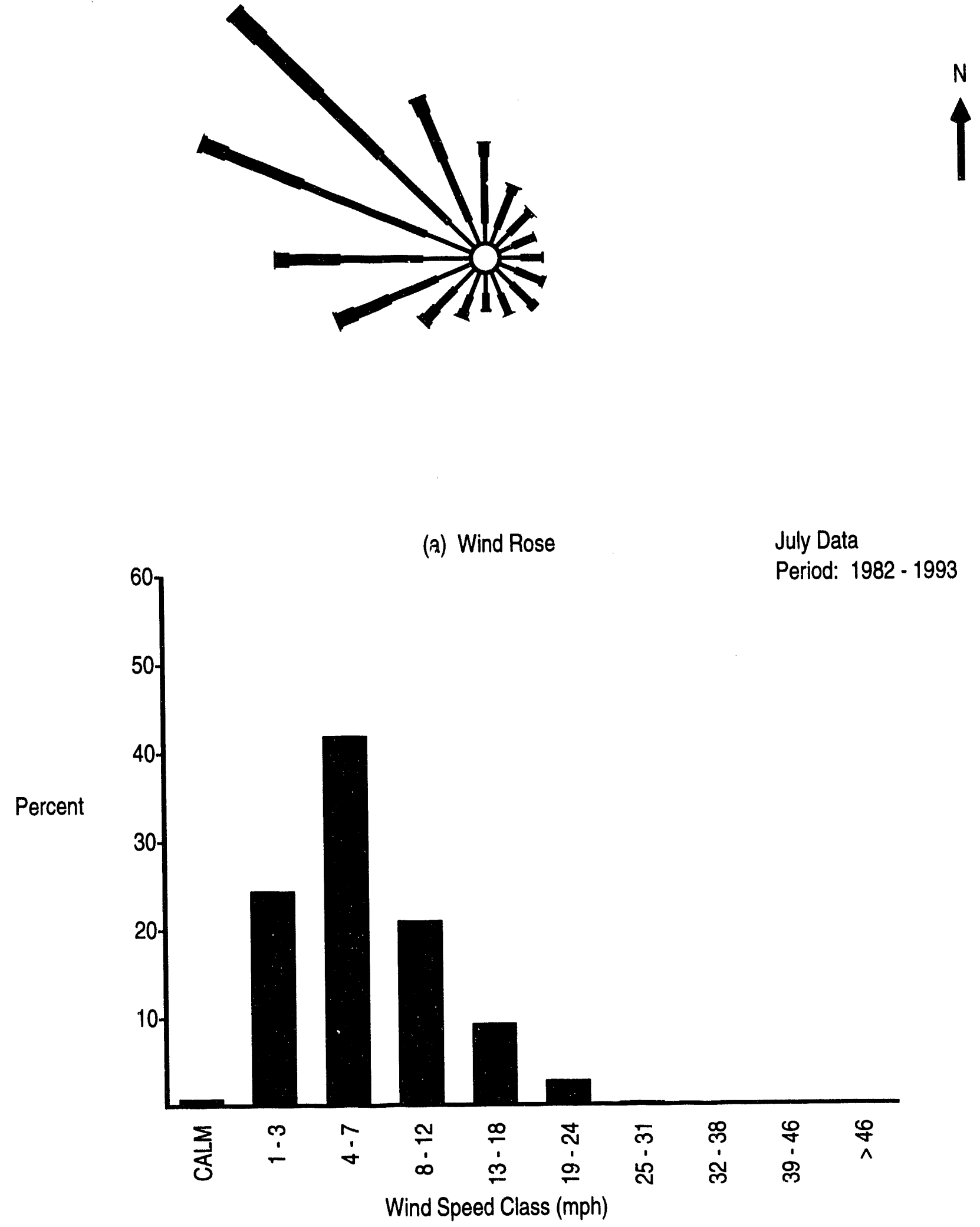

(b) Wind Speed Histogram

FIGURE B.1. (contd) 


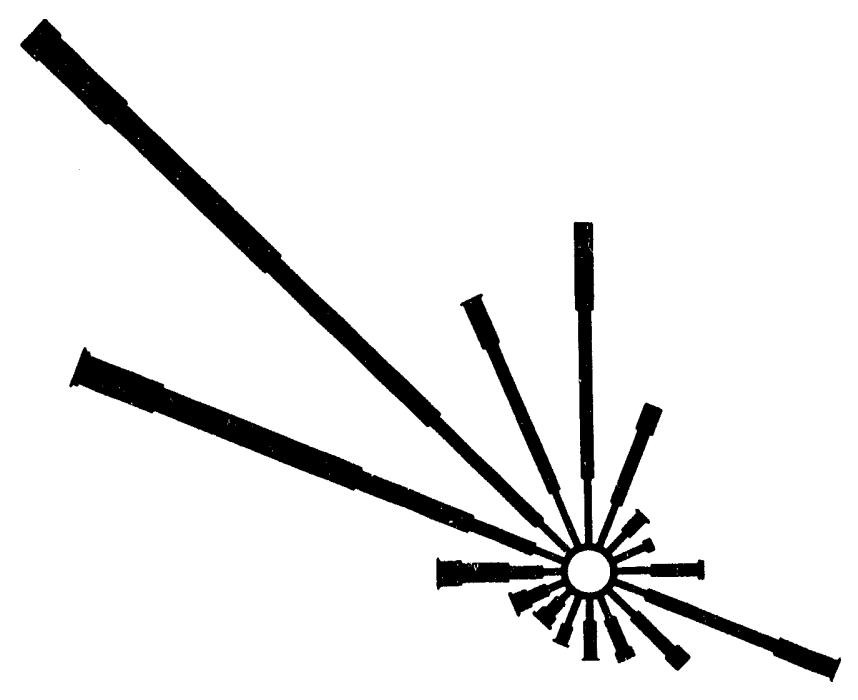

(a) Wind Rose

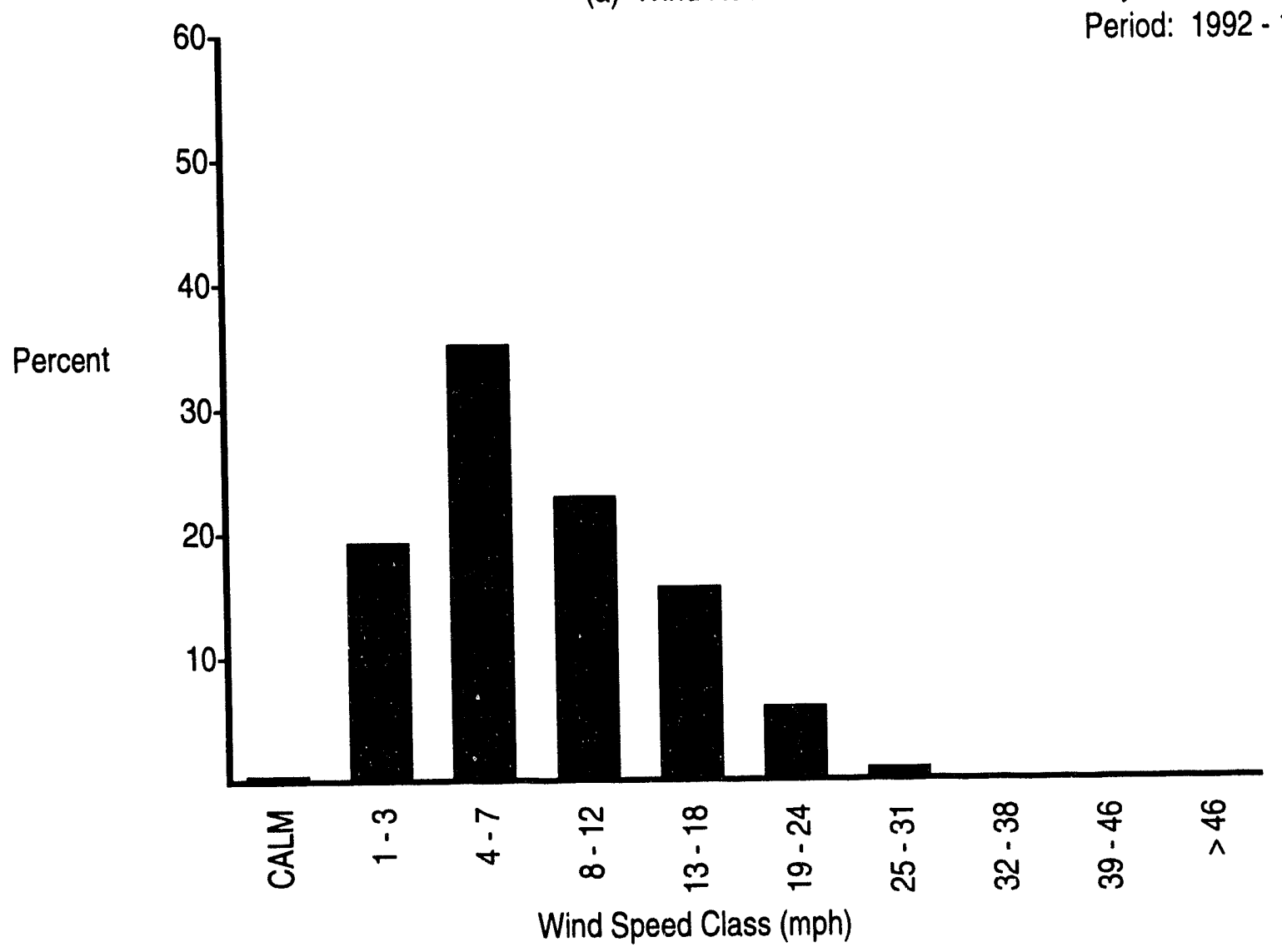

(b) Wind Speed Histogram
July Data

Period: 1992 - 1993

\section{FIGURE B.1. (contd)}




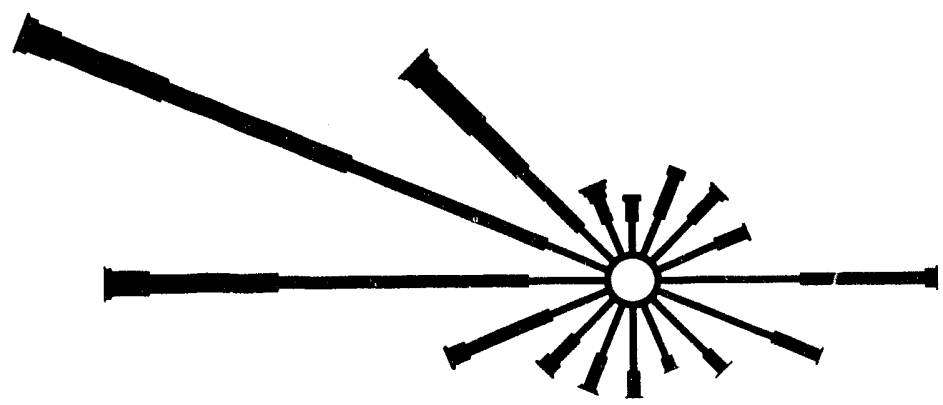

(a) Wind Rose

July Data

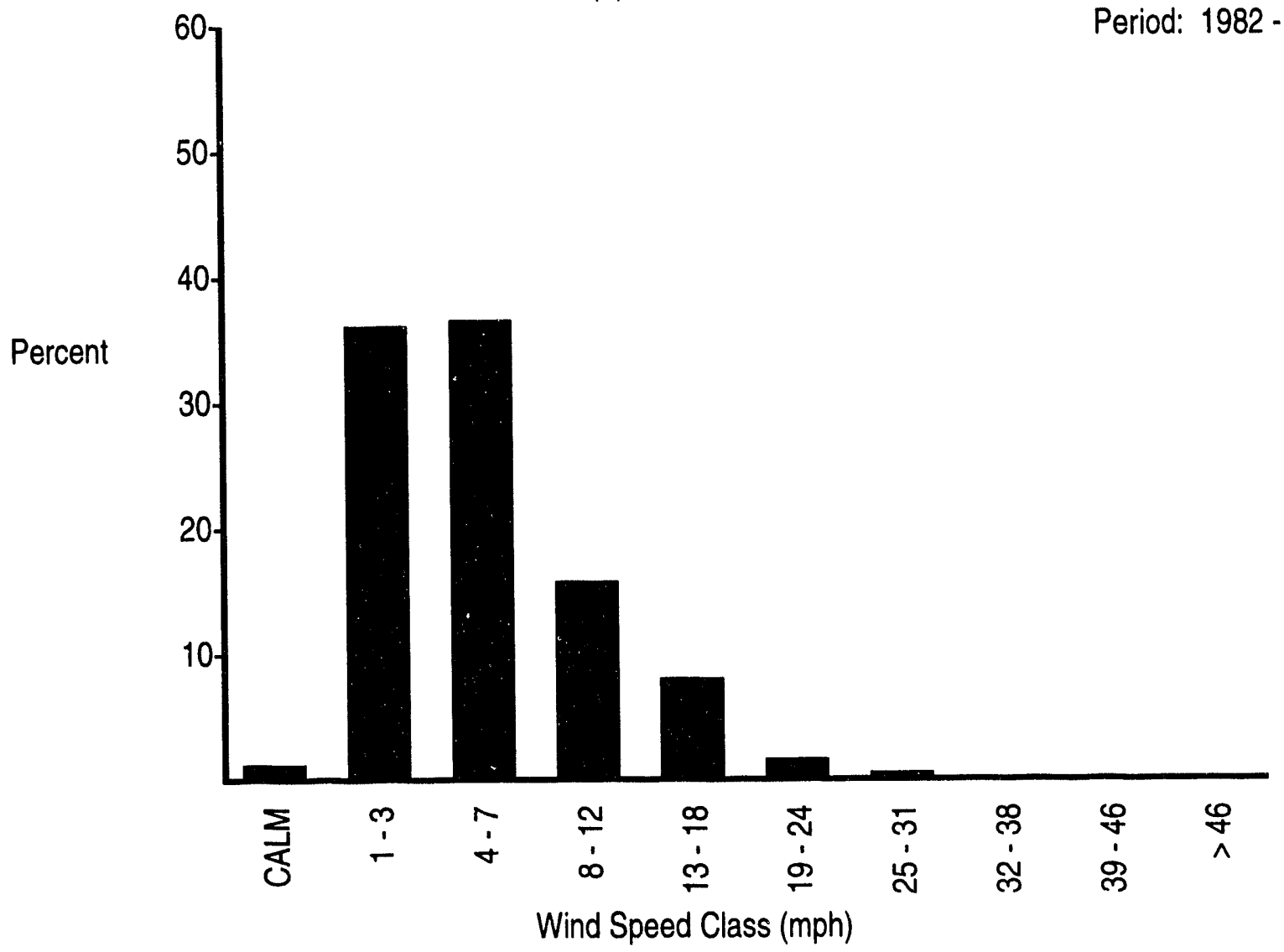

(b) Wind Speed Histogram

FIGURE B.1. (contd) 


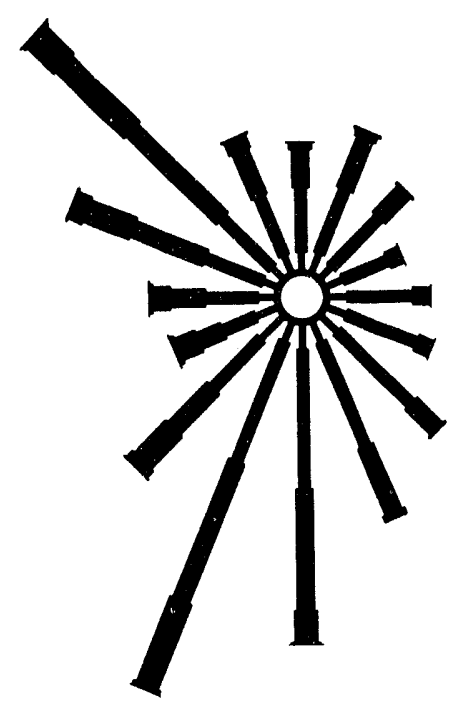

(a) Wind Rose

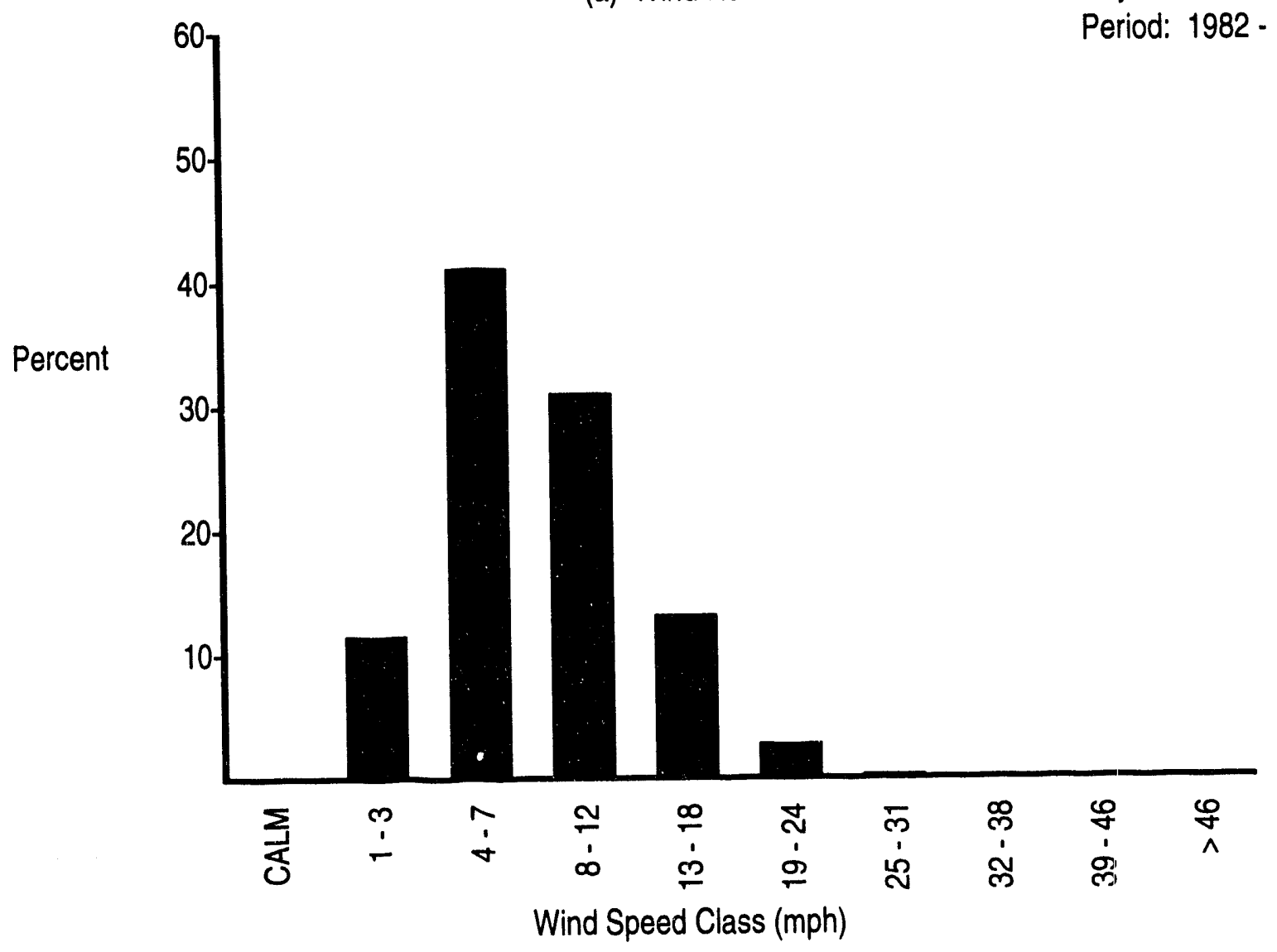

(b) Wind Speed Histogram

FIGURE B.1. (contd)
July Data

Period: 1982 - 1993

\section{B. 172}



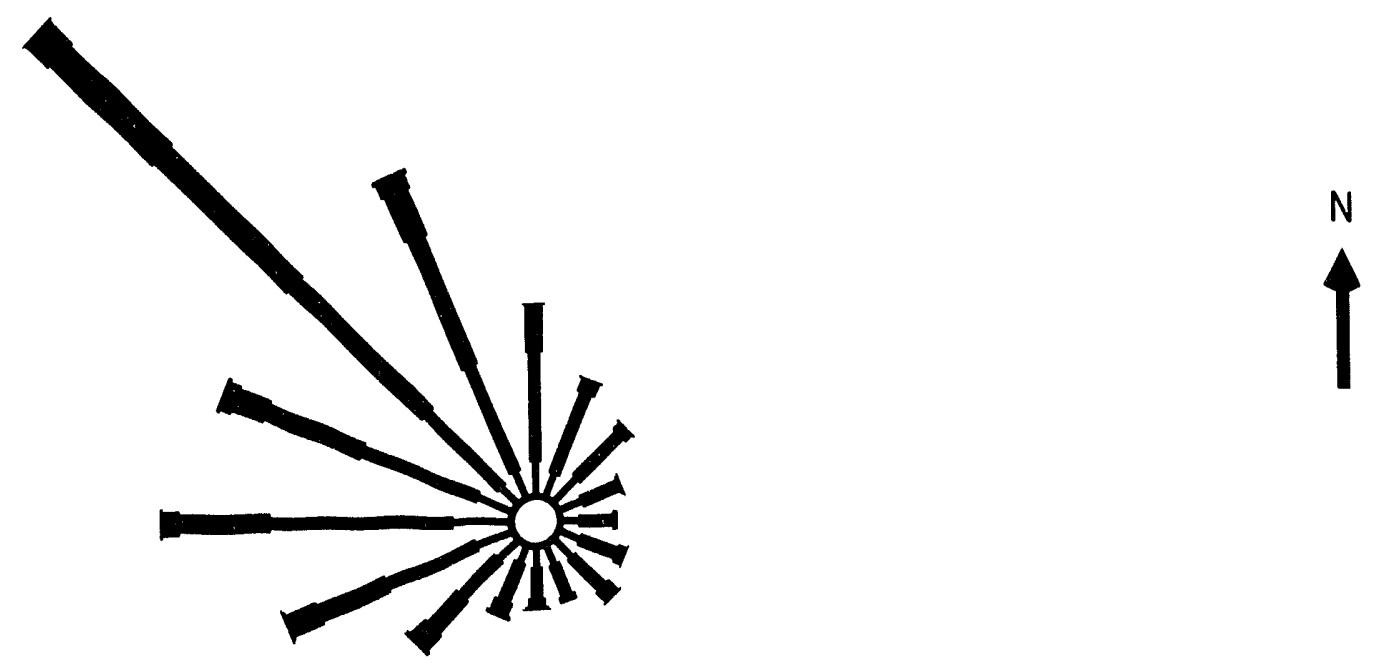

(a) Wind Rose

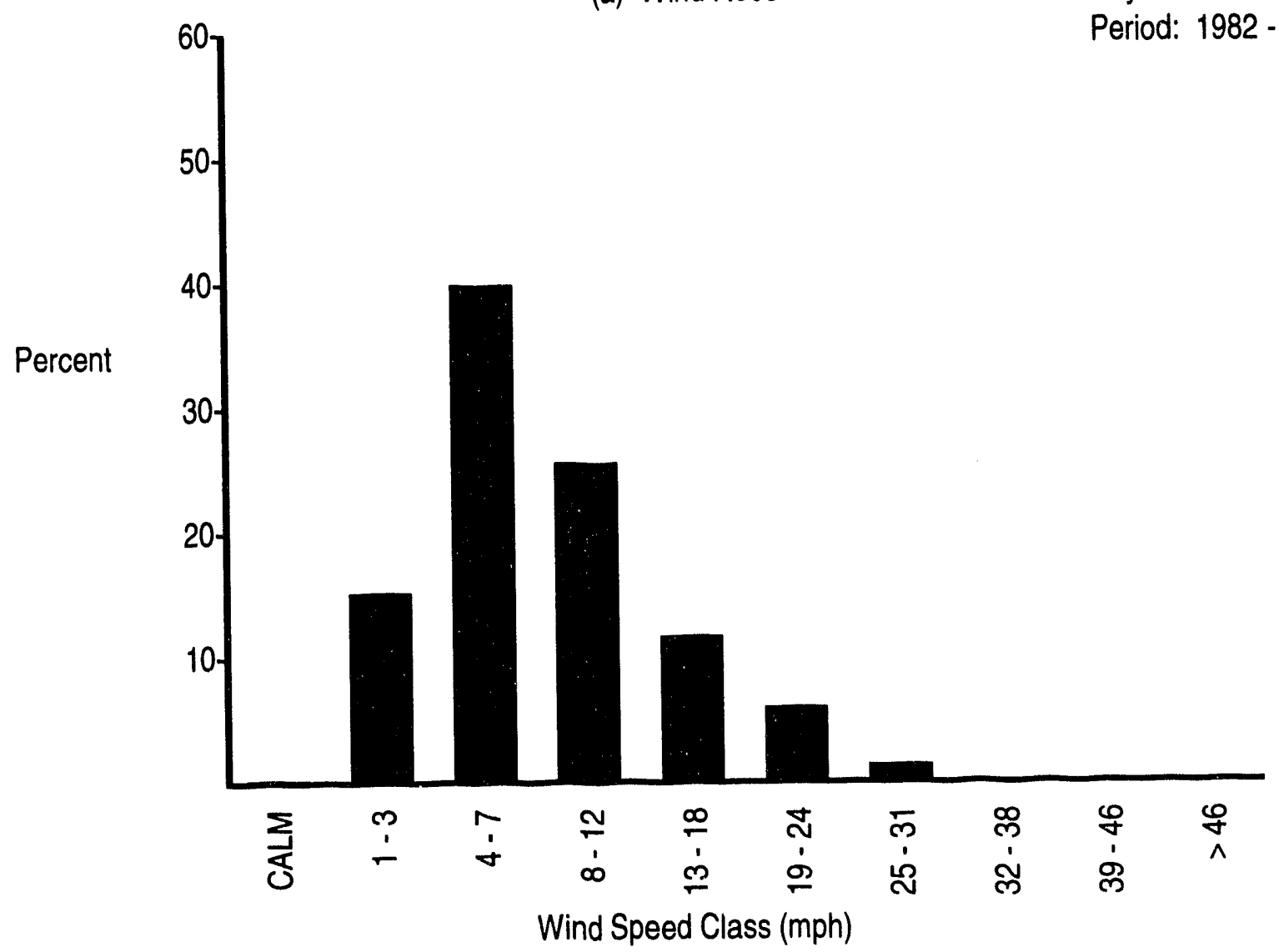

(b) Wind Speed Histogram

FIGURE B.1. (contd) 


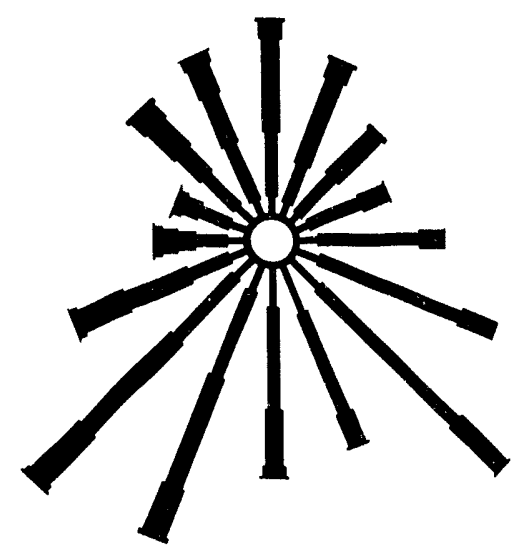

(a) Wind Rose

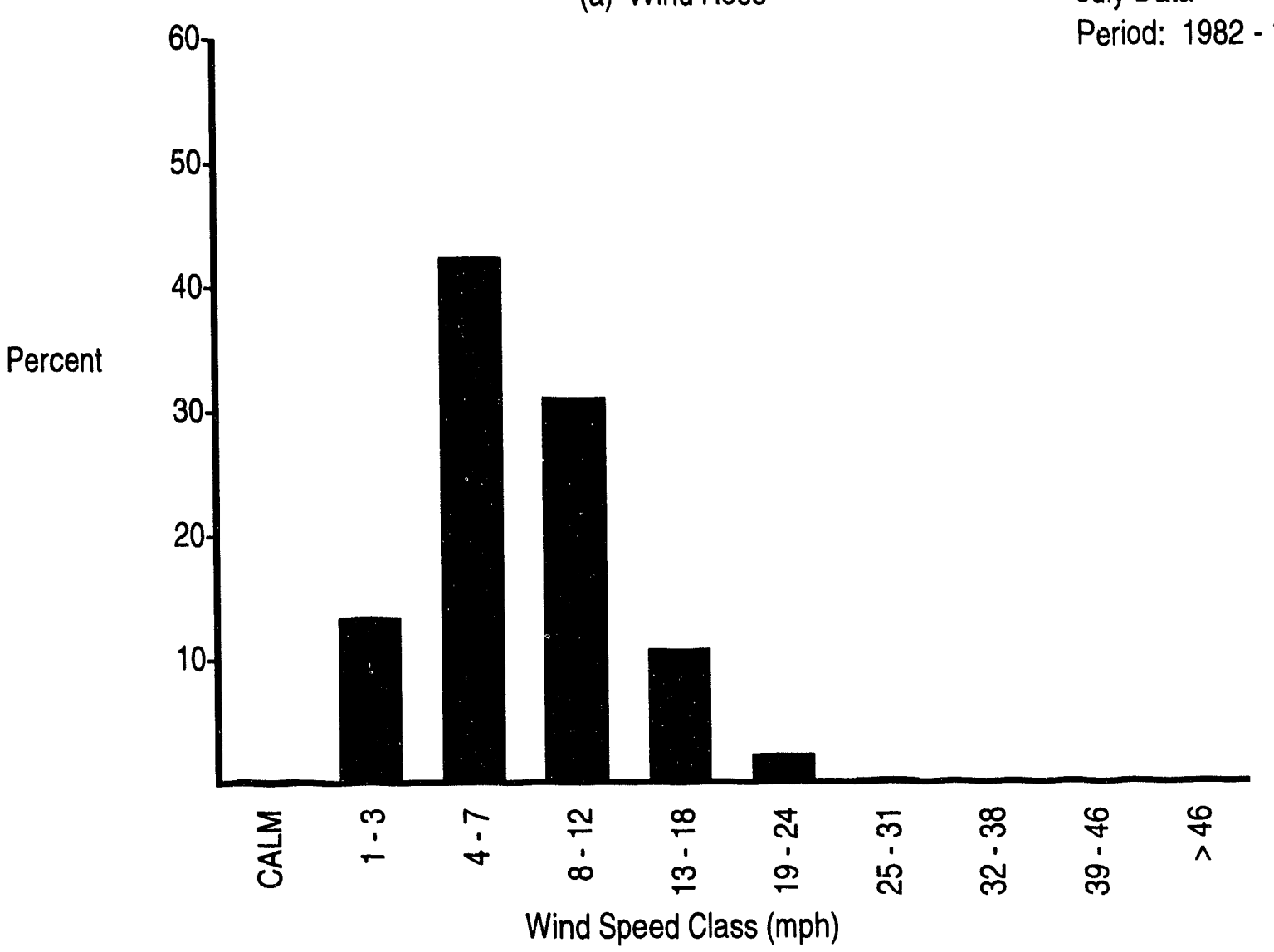

(b) Wind Speed Histogram

FIGURE B.1. (contd) 


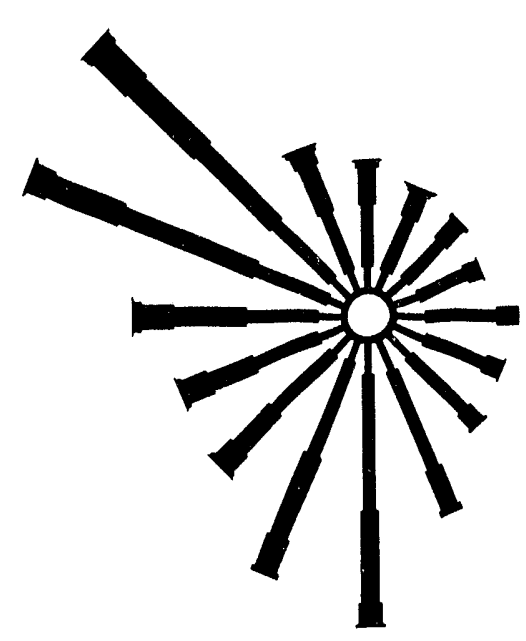

(a) Wind Rose

July Data

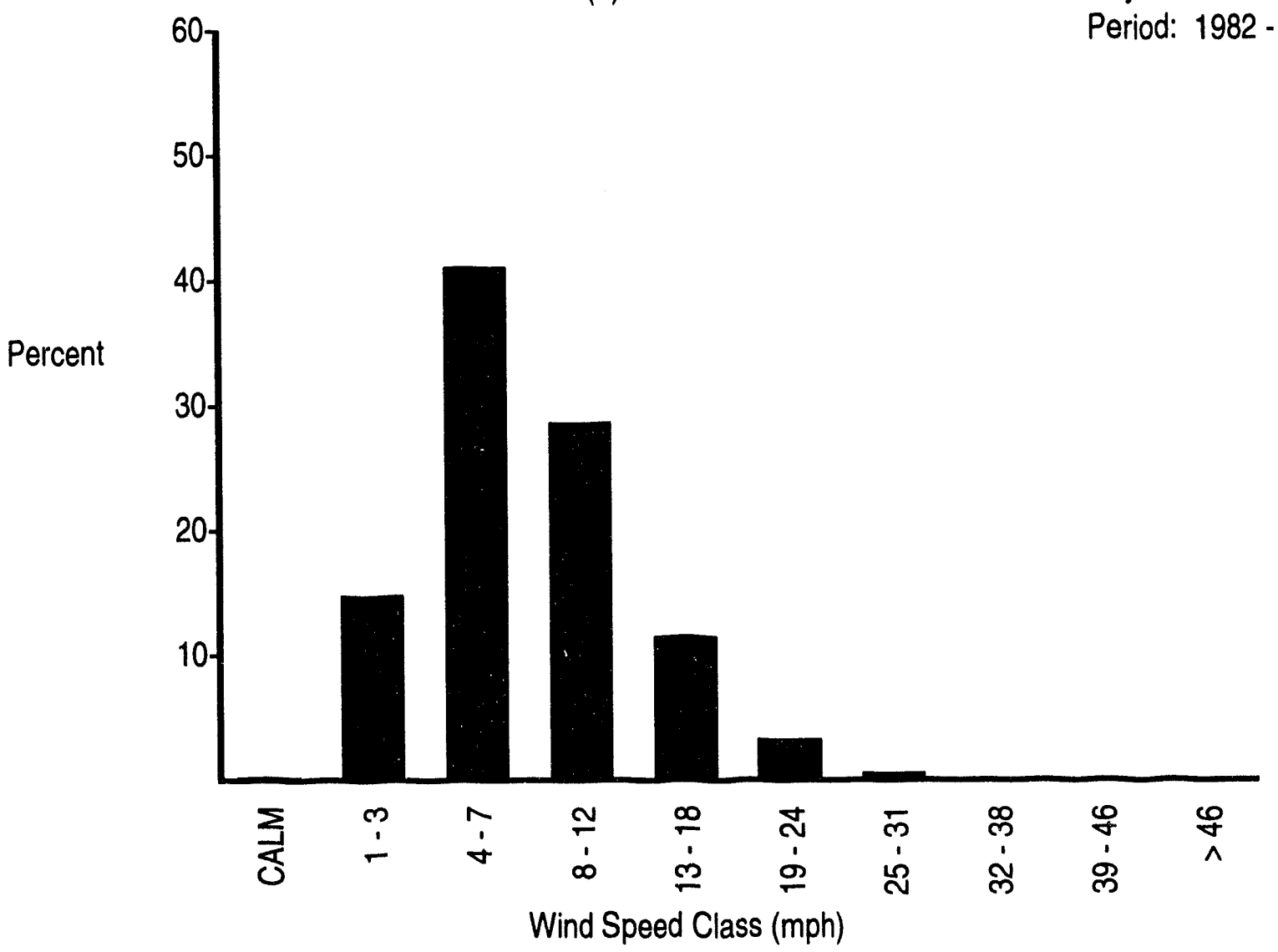

(b) Wind Speed Histogram

FIGURE B.1. (contd) 


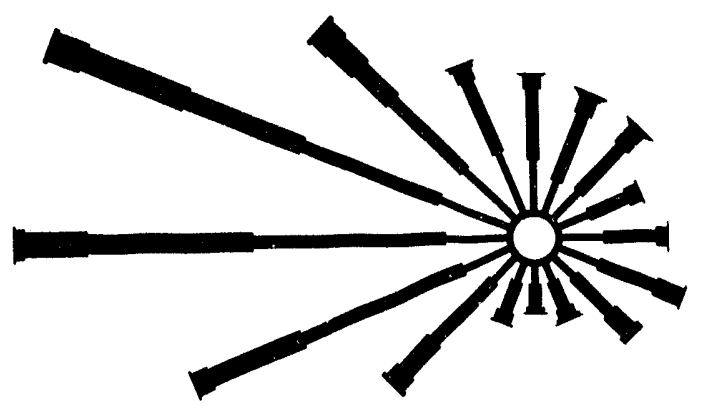

(a) Wind Rose

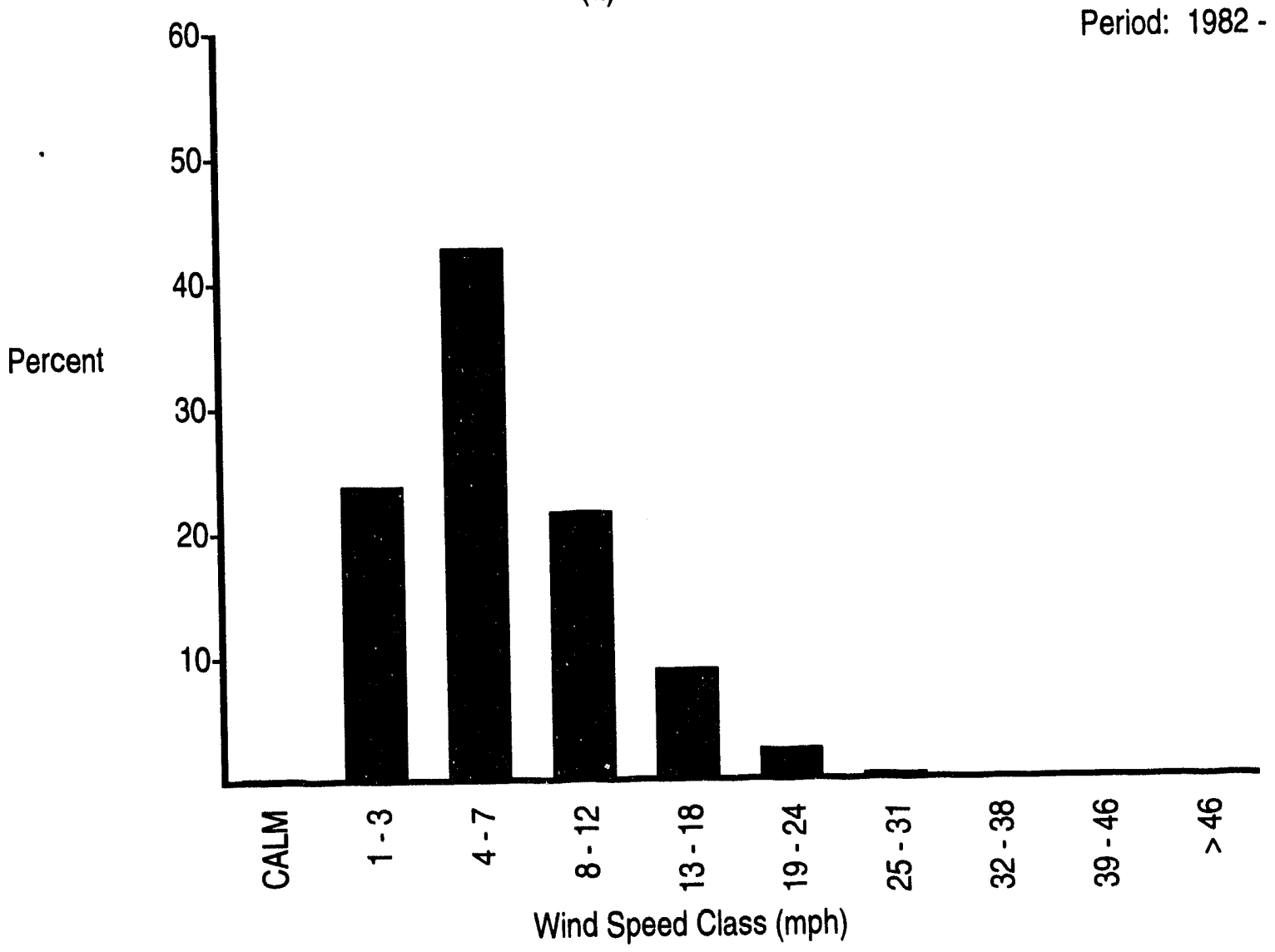

(b) Wind Speed Histogram

FIGURE B.1. (contd)
July Data

Period: 1982 - 1993

\section{B.176}



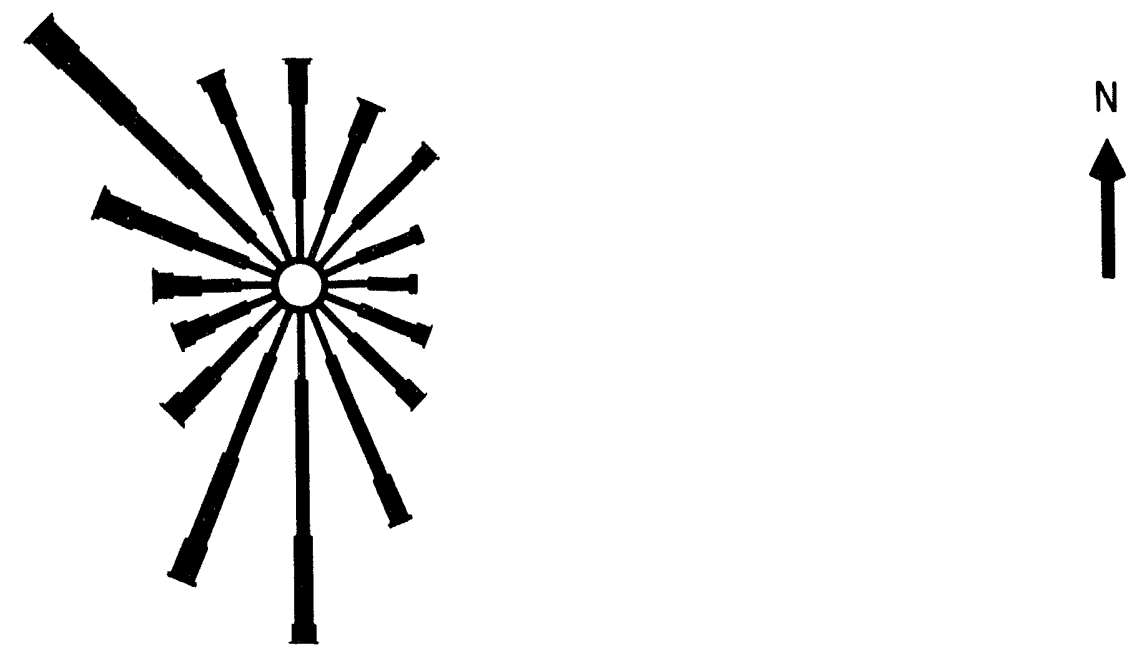

(a) Wind Rose

July Data

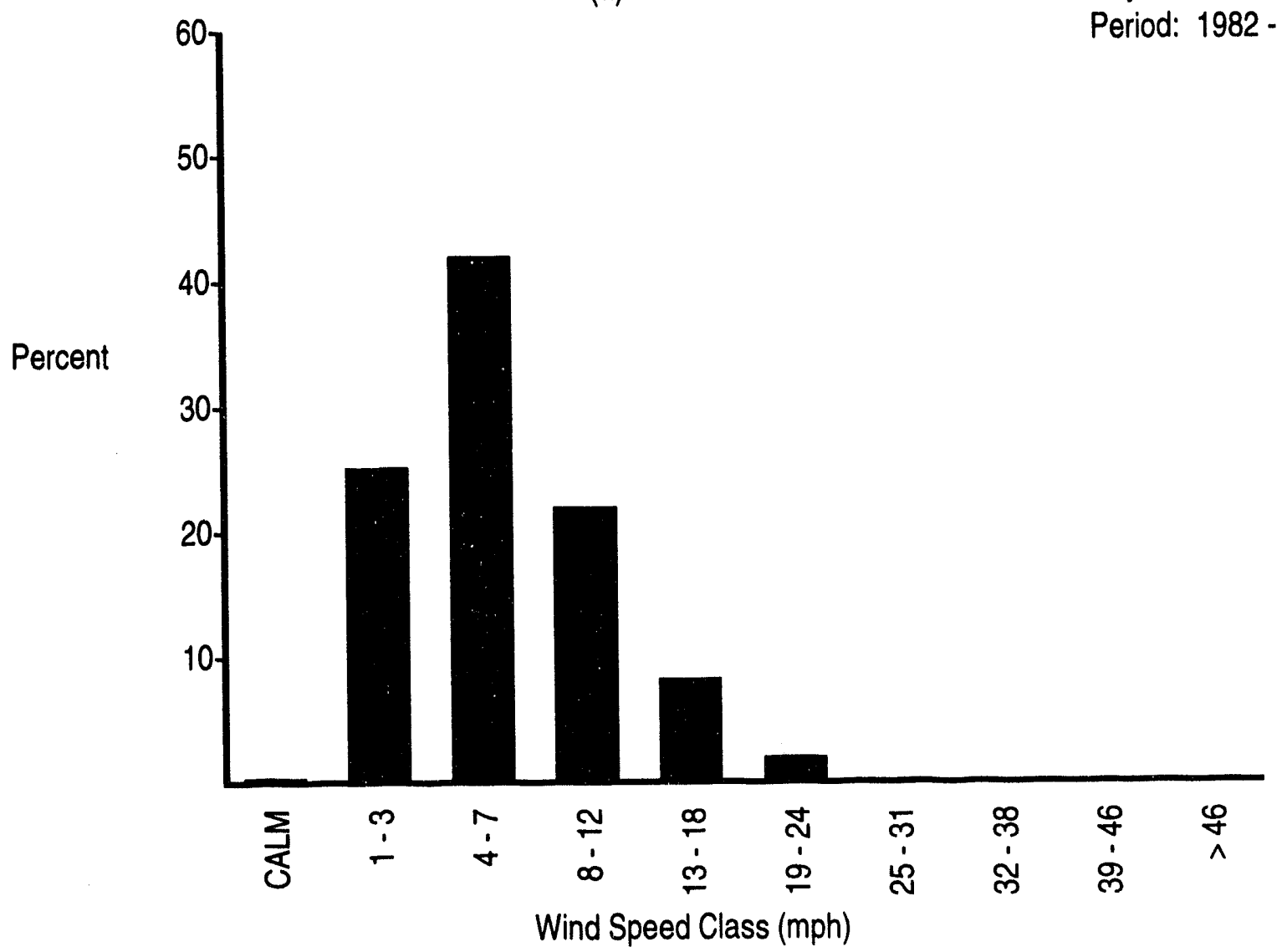

(b) Wind Speed Histogram

FIGURE B.1. (contd)

B.177 


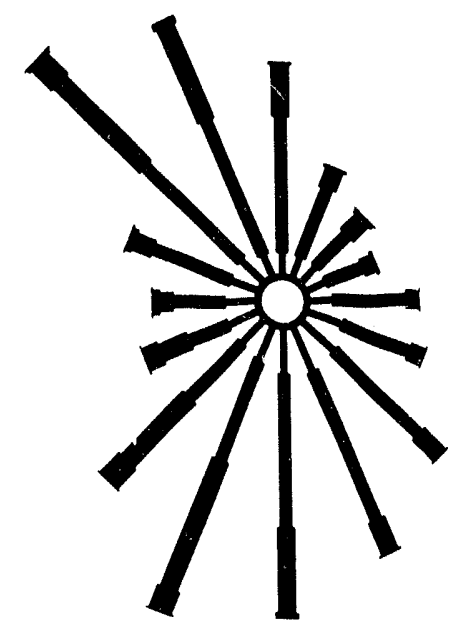

(a) Wind Rose

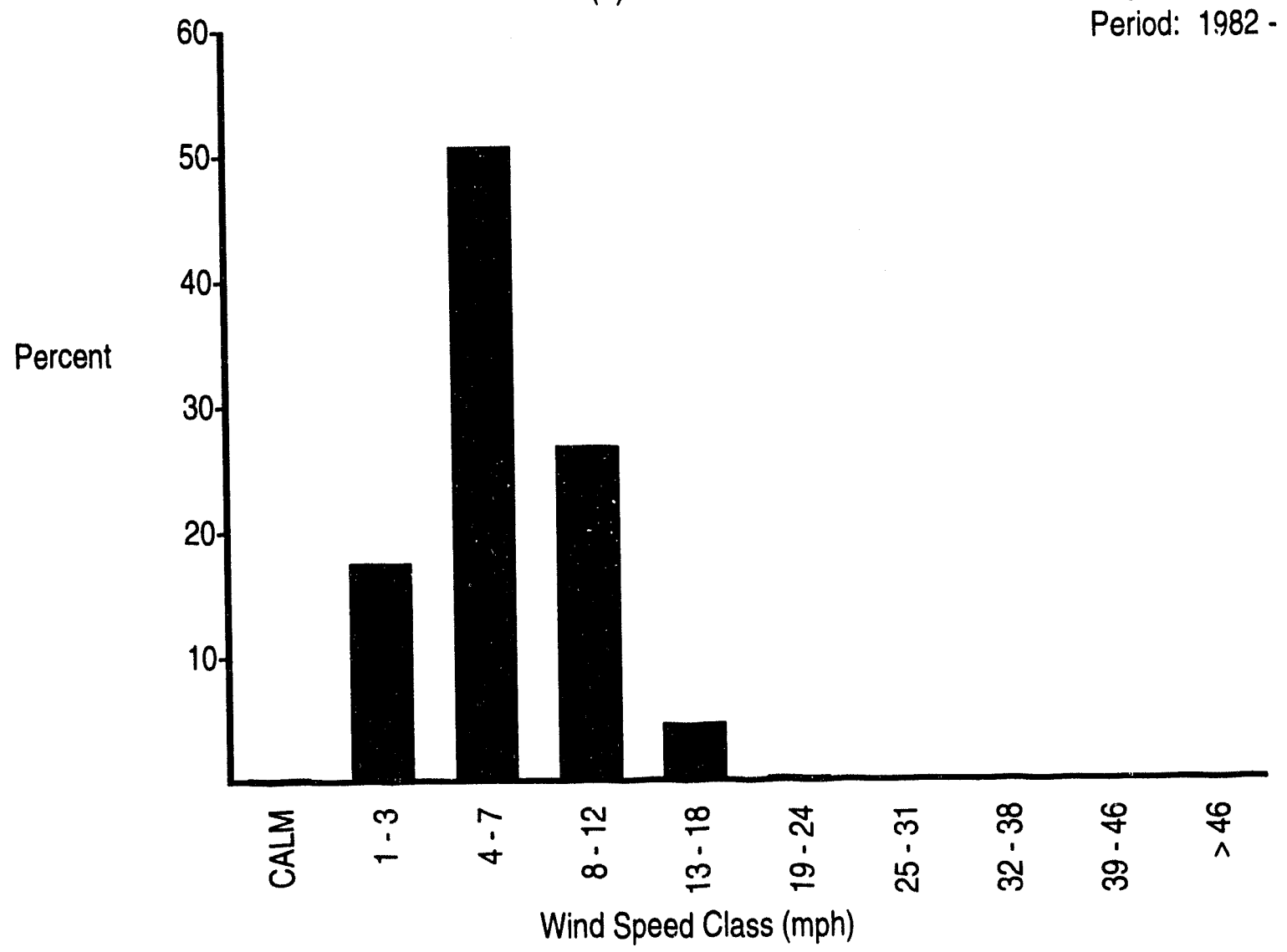

(b) Wind Speed Histogram

FIGURE B.1. (contd)
July Data

Period: $1.982-1993$ 


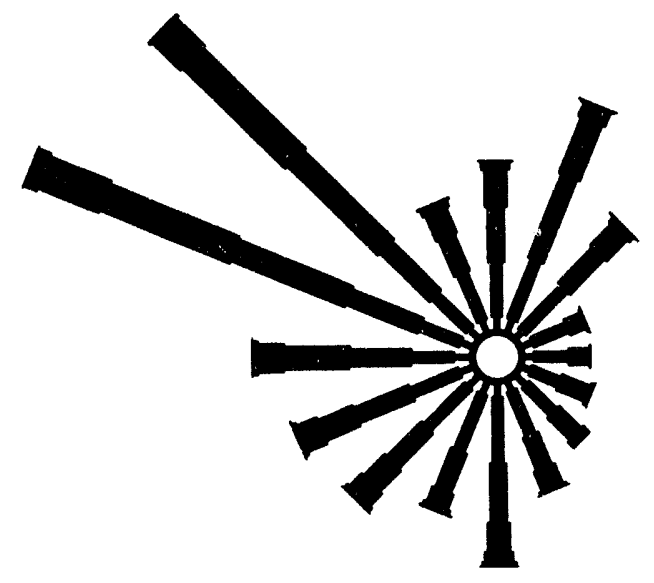

(a) Wind Rose

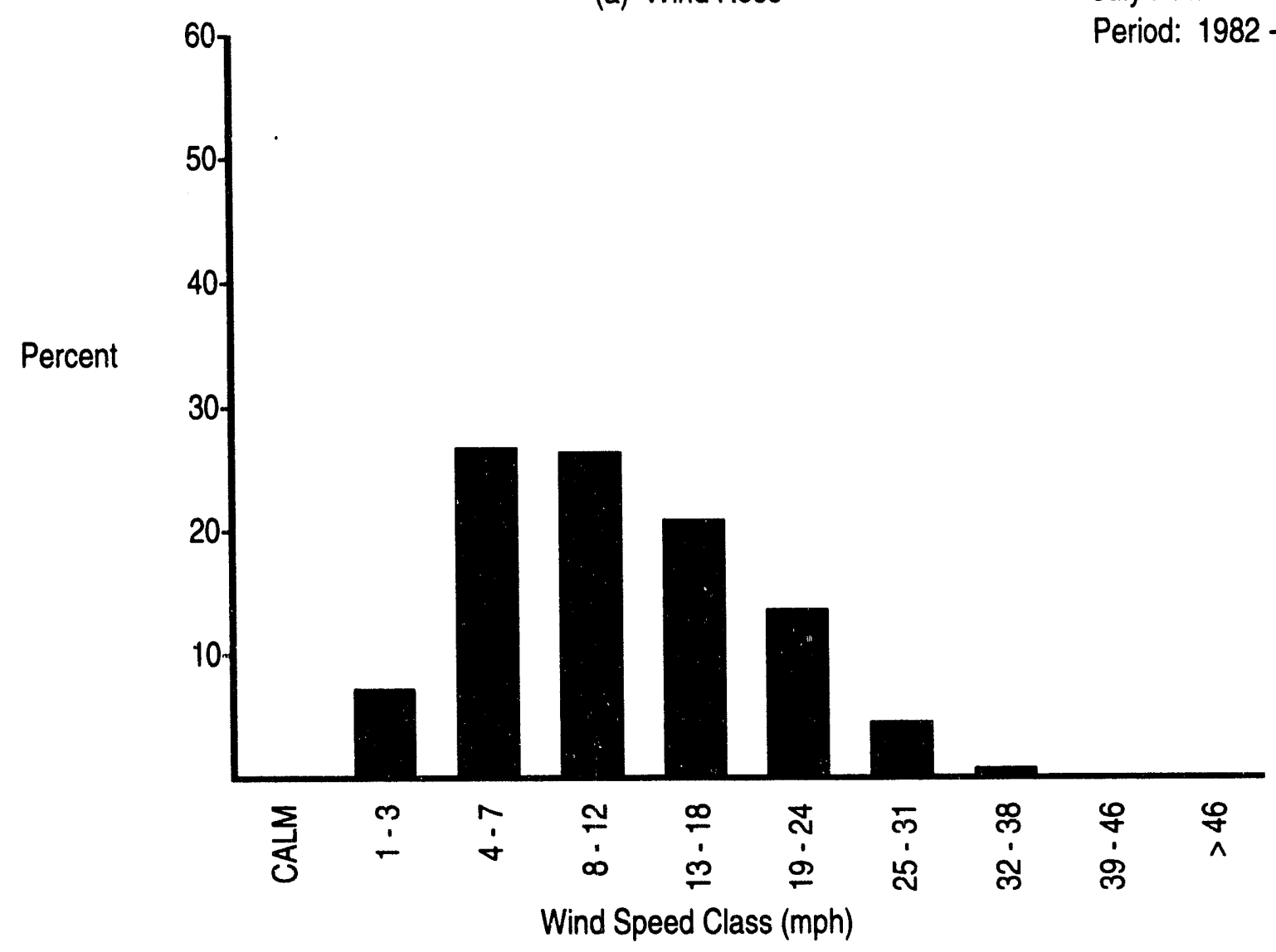

(b) Wind Speed Histogram

FIGURE B.1. (contd) 
$081^{\circ} \mathrm{g}$

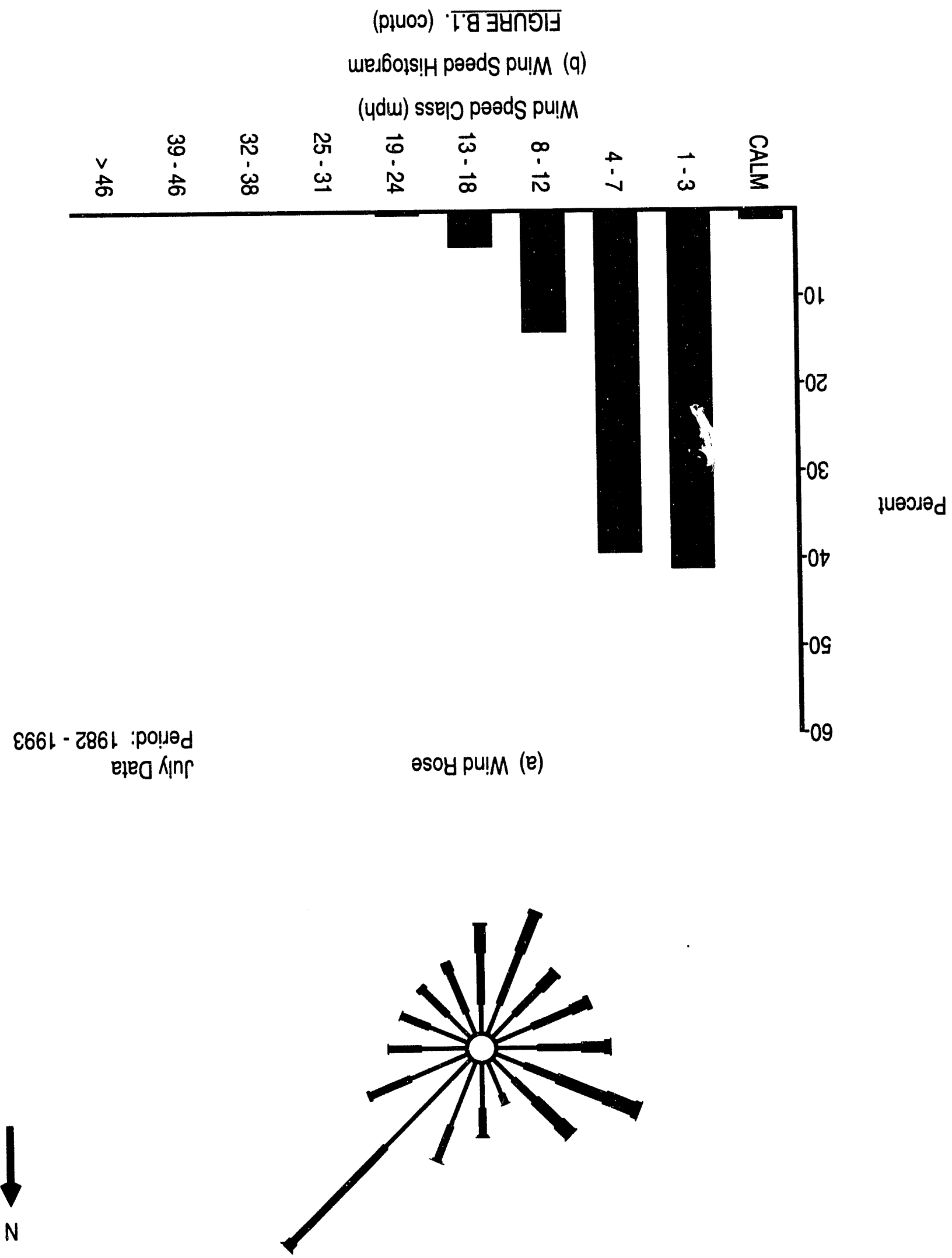



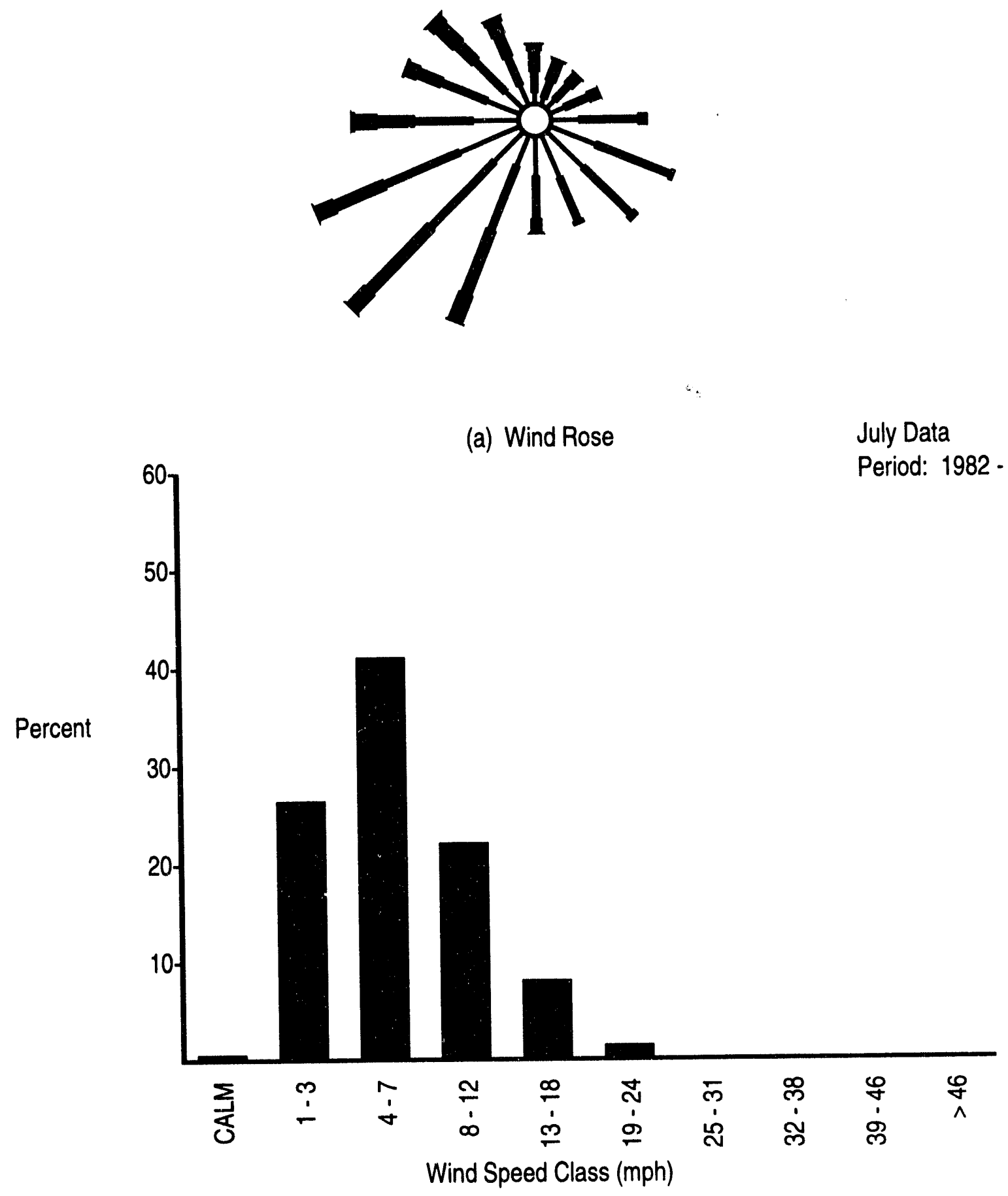

(b) Wind Speed Histogram

FIGURE B.1. (contd) 


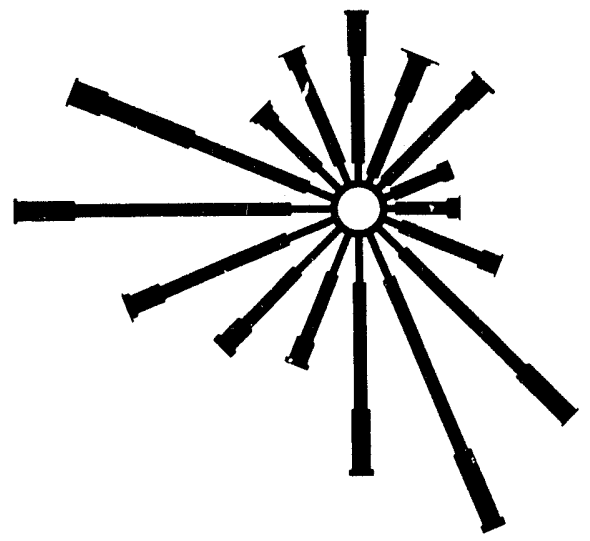

(a) Wind Rose

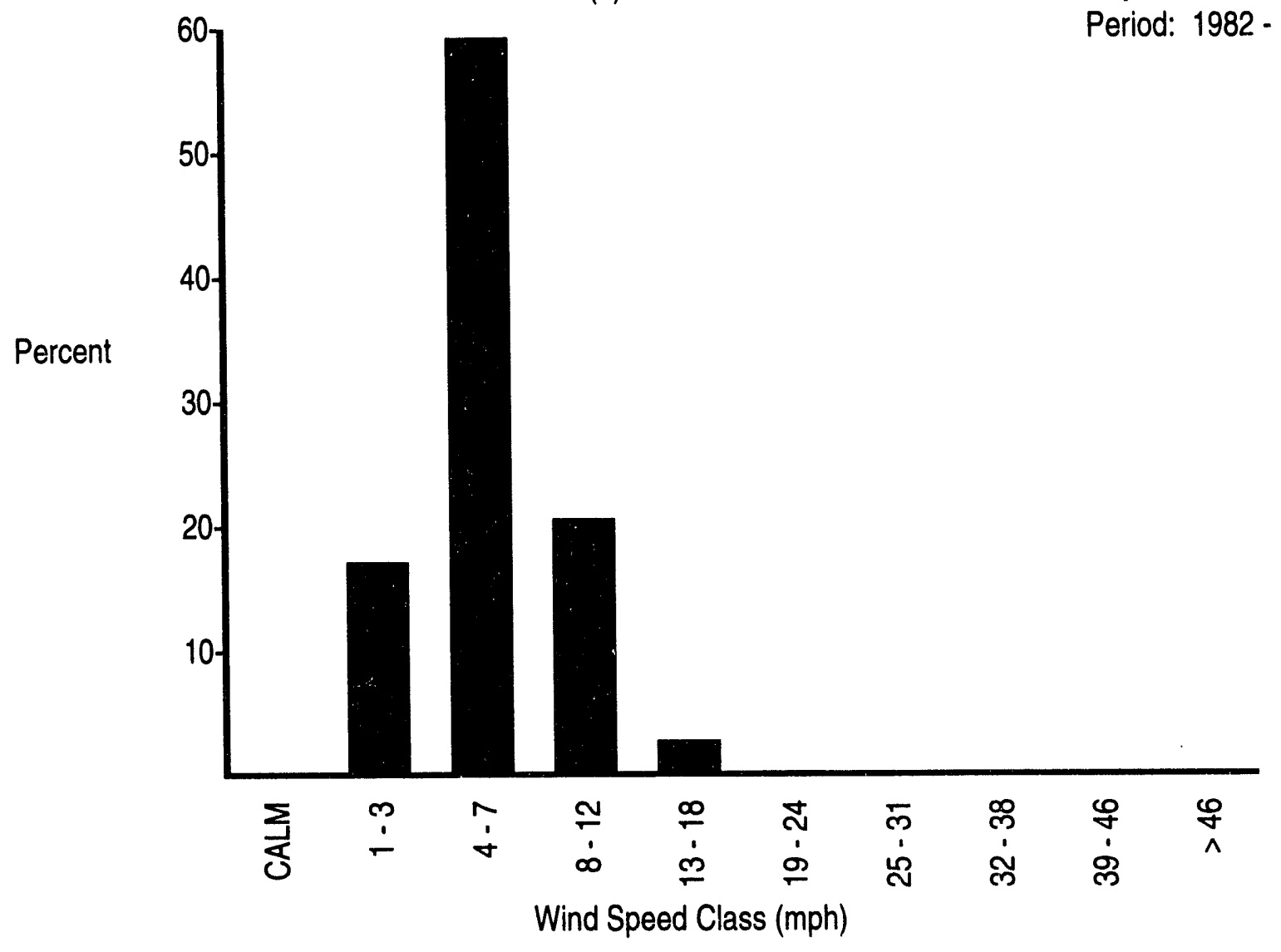

(b) Wind Speed Histogram

FIGURE B.1. (contd)
July Data

Period: 1982 - 1992

\section{B.182}




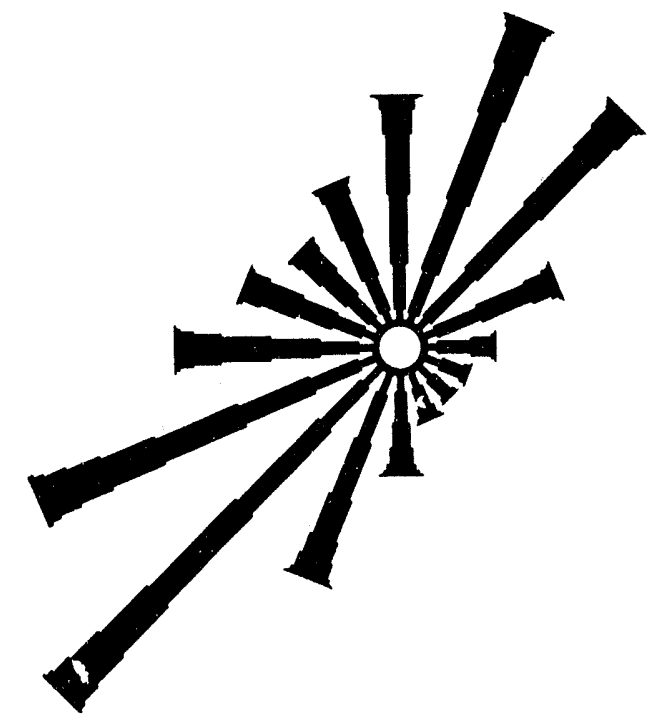

(a) Wind Rose

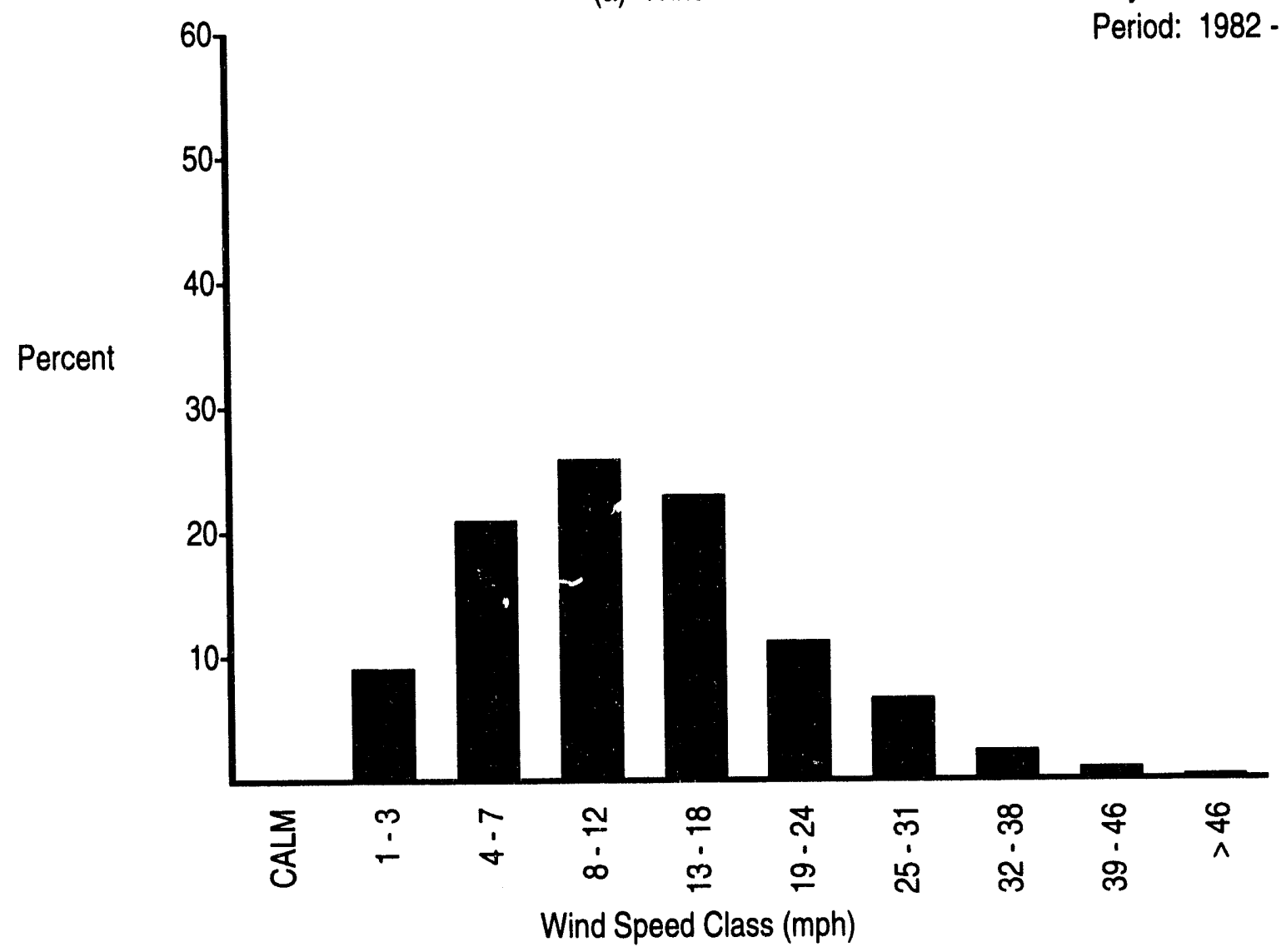

(b) Wind Speed Histogram
July Data

Period: 1982 - 1993

\section{FIGURE B.1. (contd)}




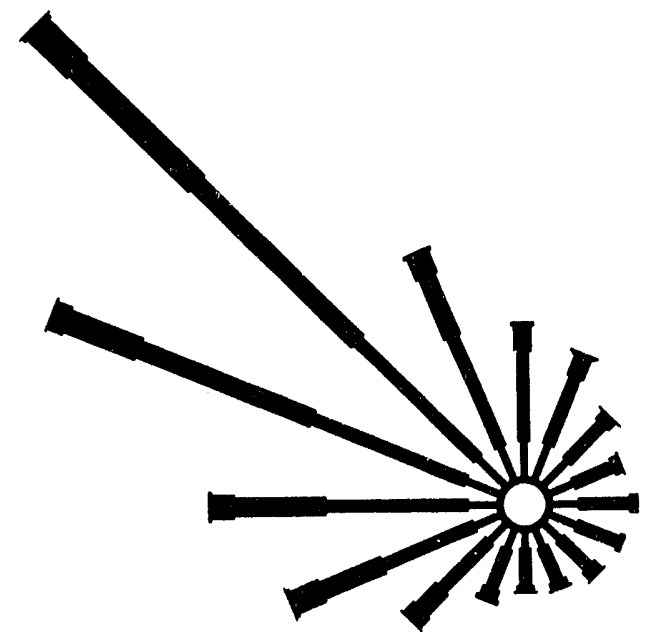

(a) Wind Rose

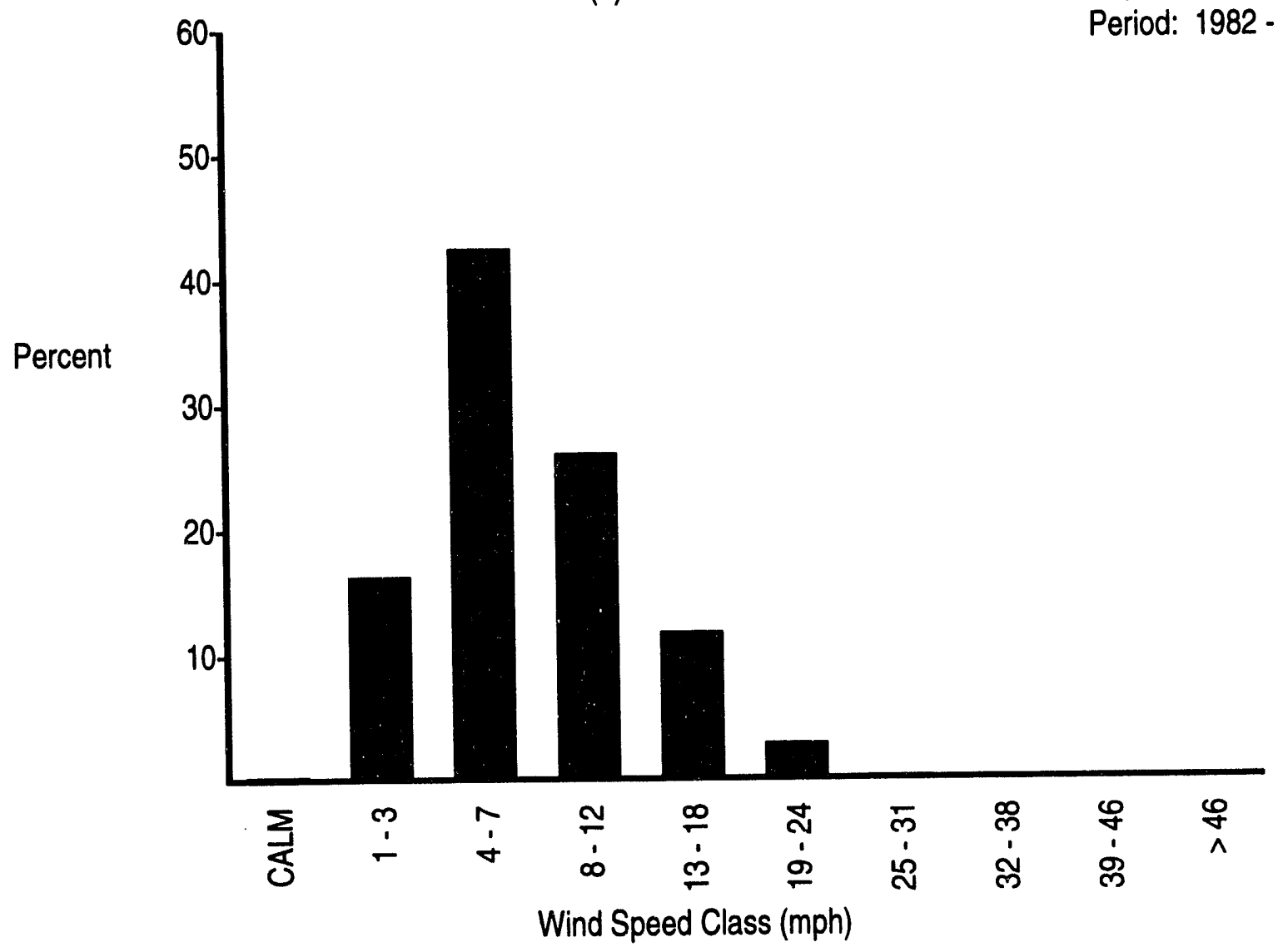

(b) Wind Speed Histogram

FIGURE B.1. (contd)
July Data

Period: 1982 - 1993 

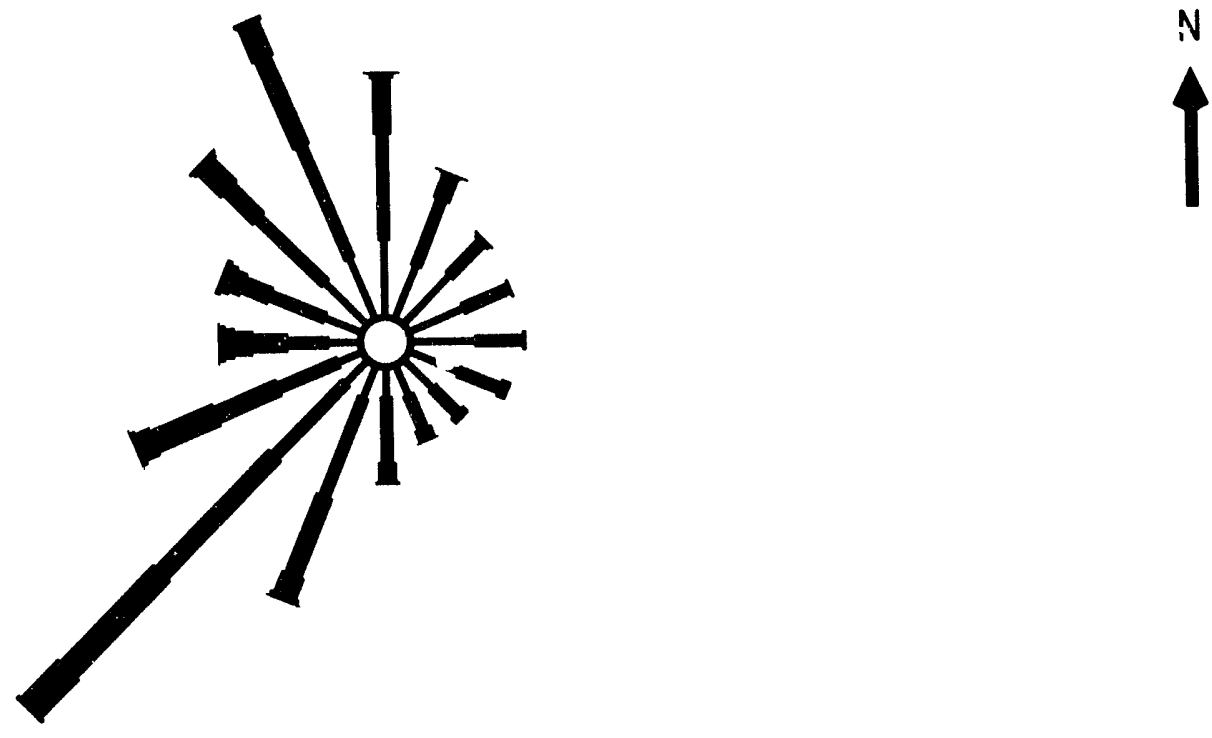

(a) Wind Rose

July Data

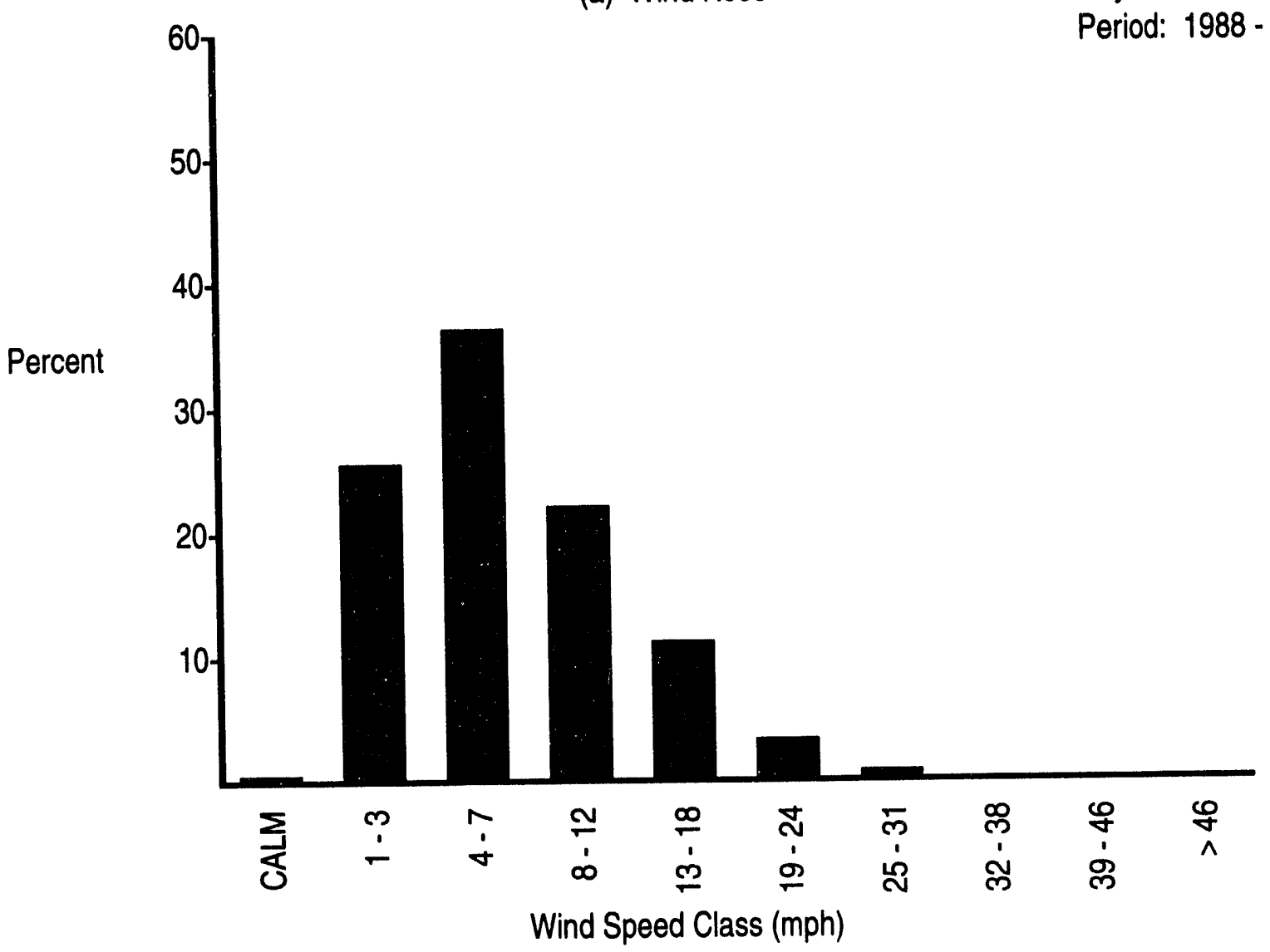

(b) Wind Speed Histogram

FIGURE B.1. (contd)

B.185 


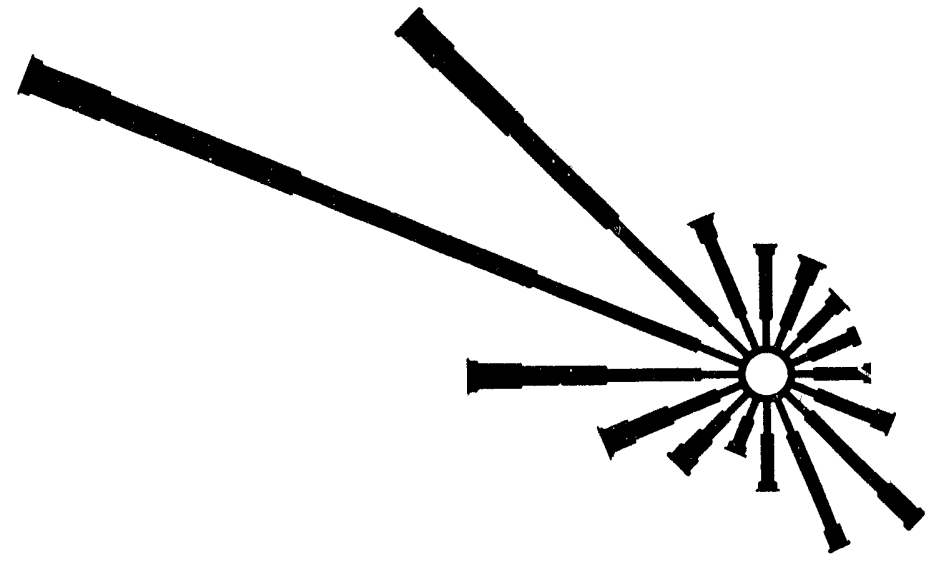

(a) Wind Rose

July Data

Percent

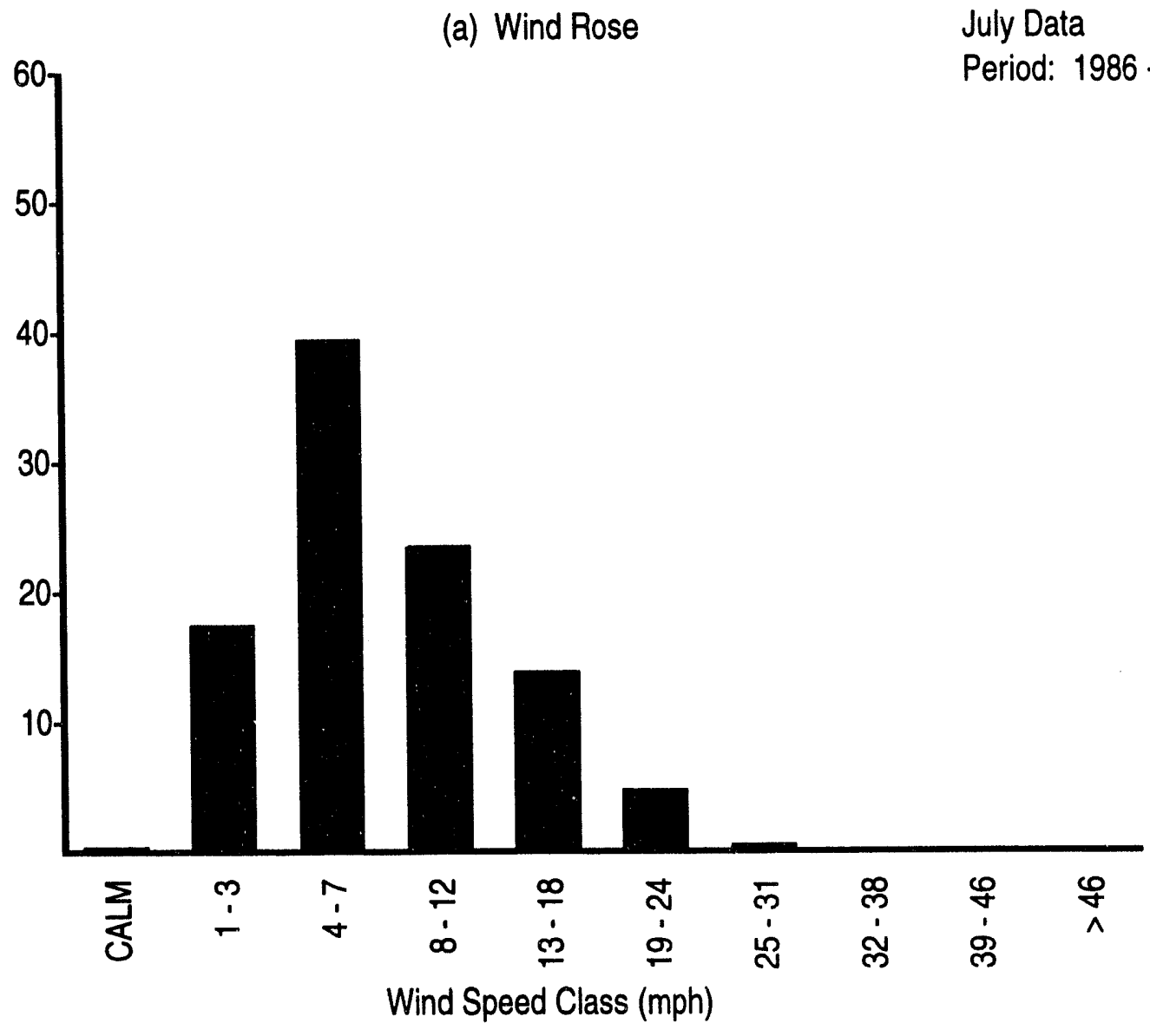

(b) Wind Speed Histogram

FIGURE B.1. (contd)

\section{B.186}




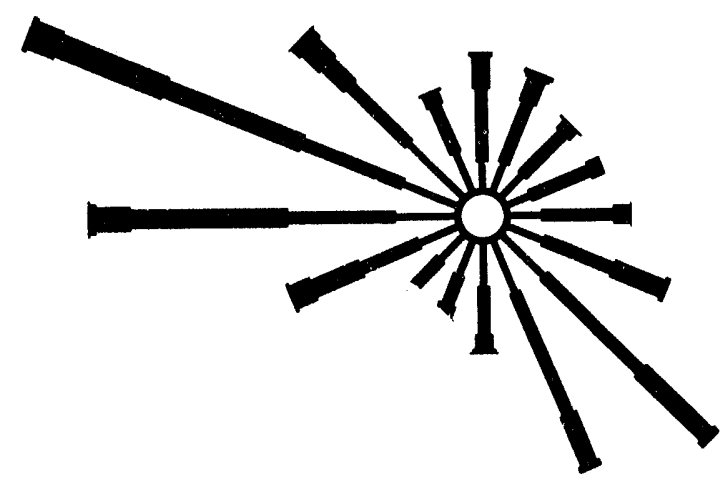

(a) Wind Rose

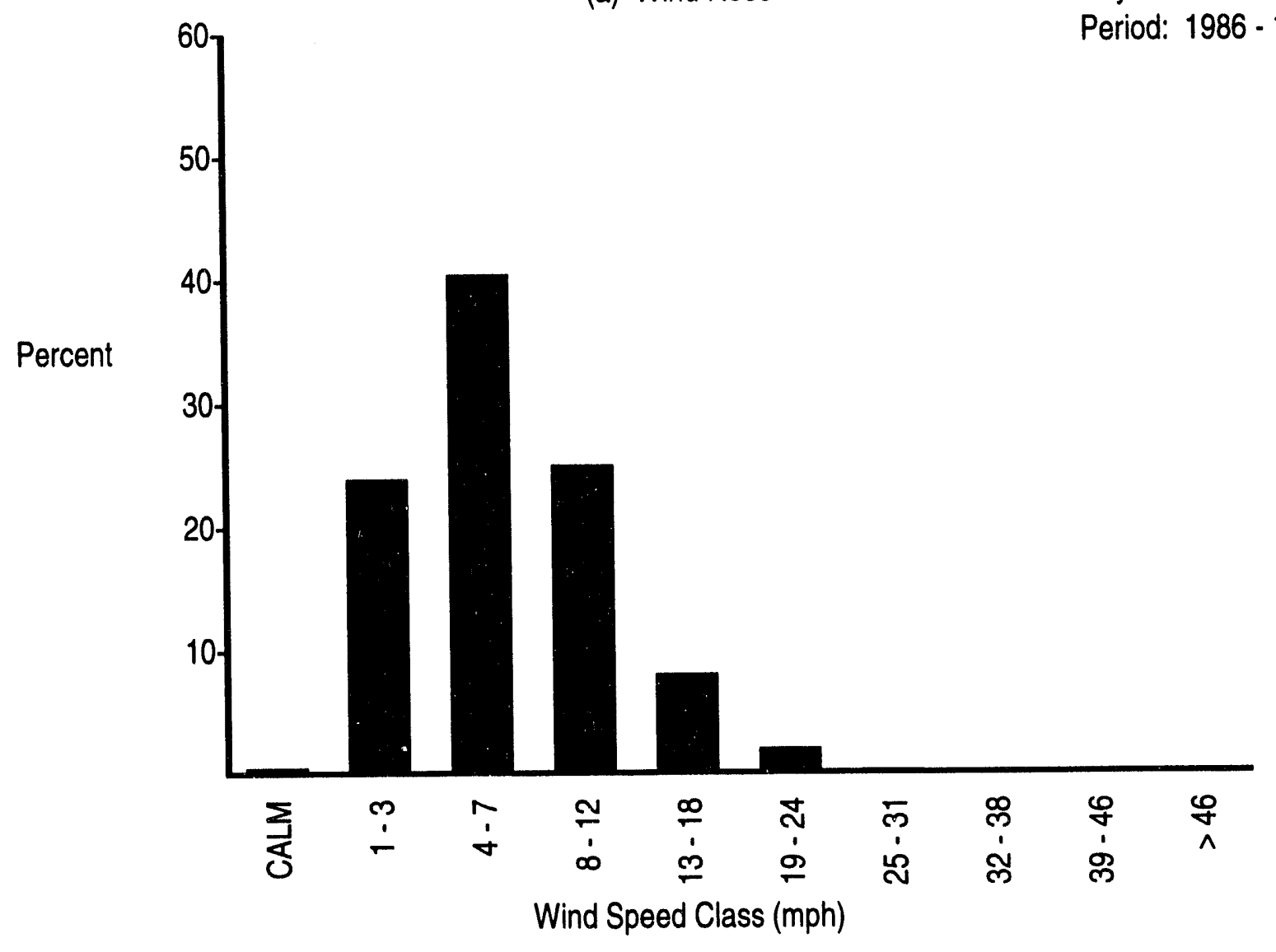

July Data

Period: 1986 - 1993

(b) Wind Speed Histogram

FIGURE B.1. (contd) 

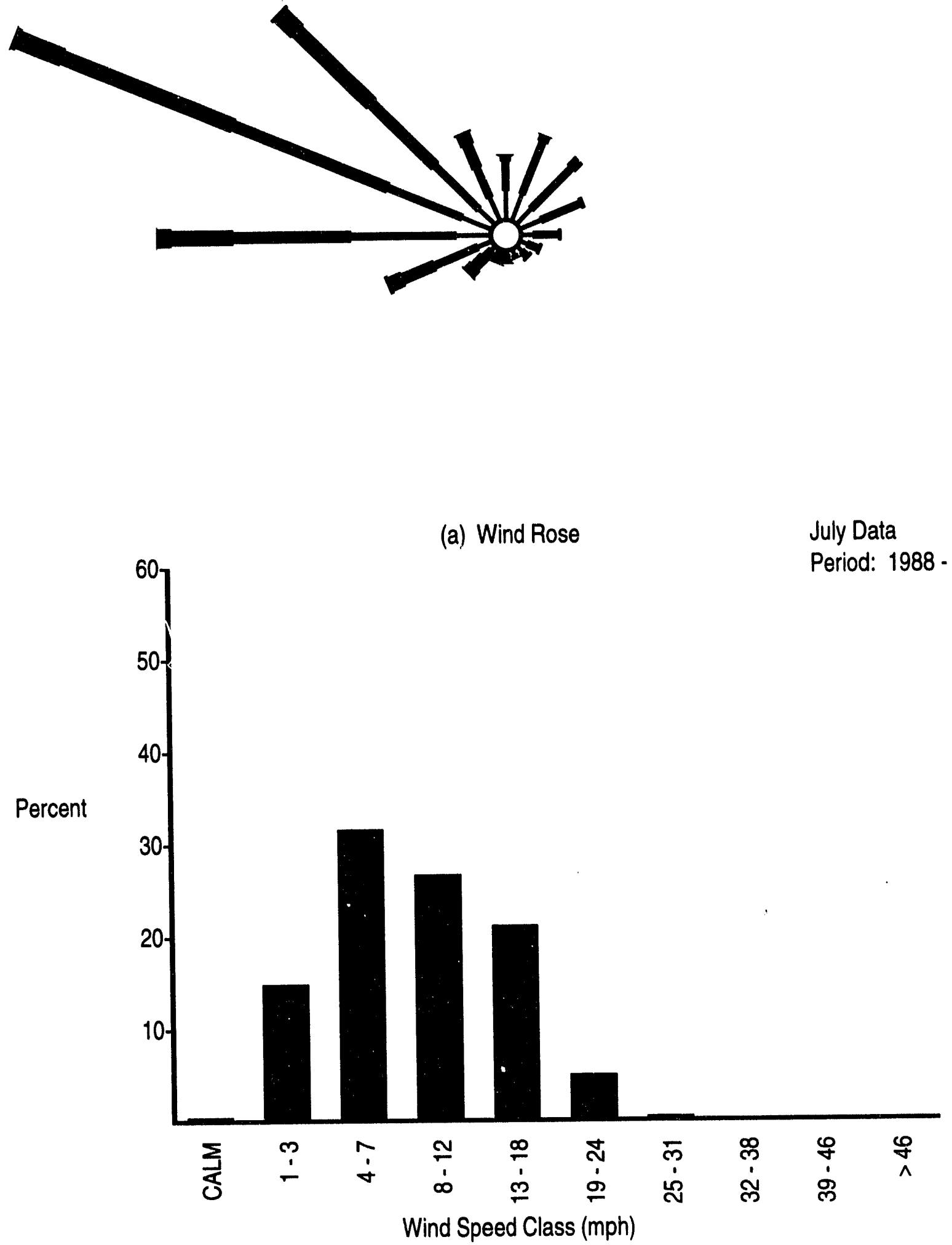

(b) Wind Speed Histogram

FIGURE B.1. (contd) 

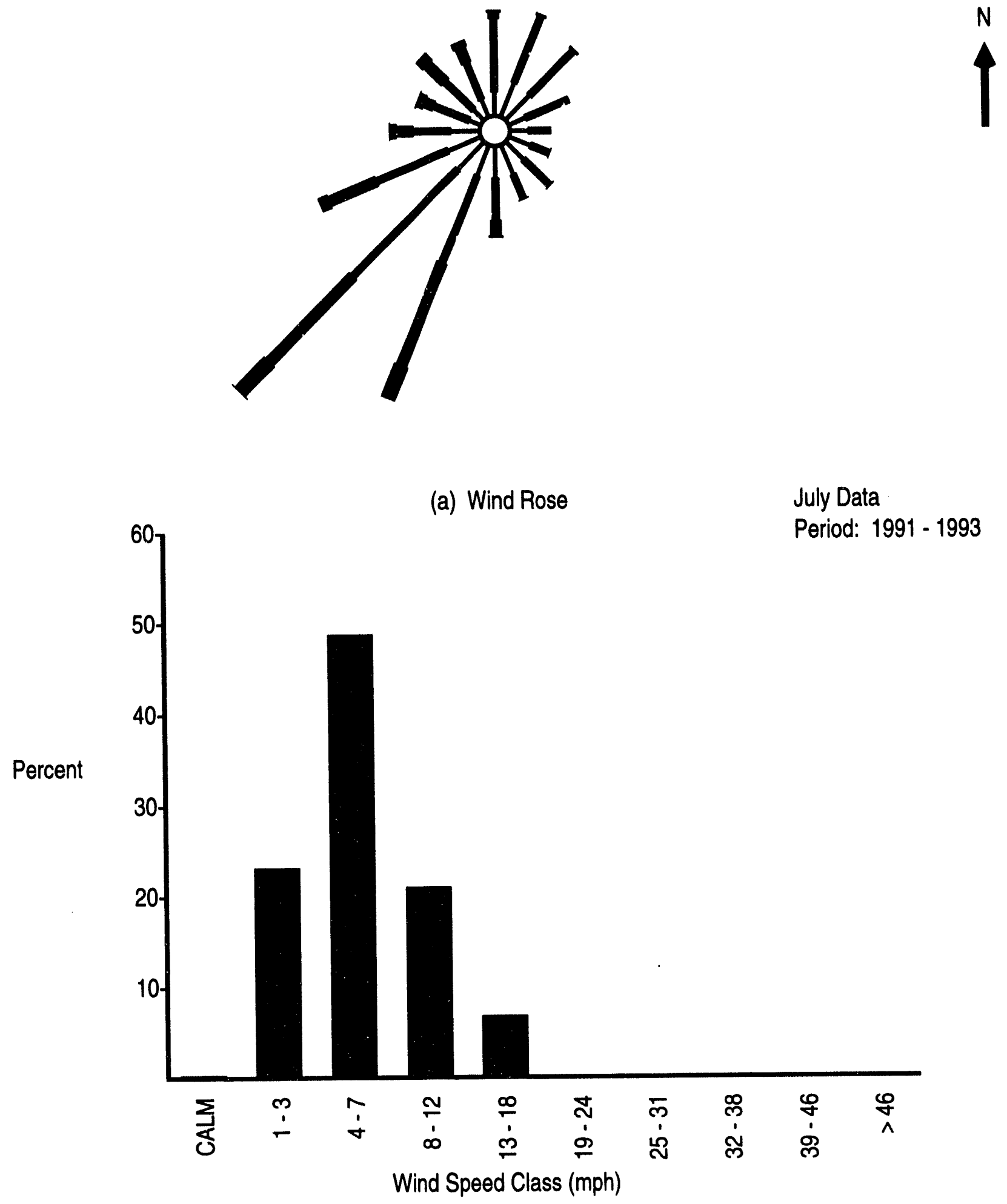

(b) Wind Speed Histogram

FIGURE B.1. (contd)

\section{B. 189}



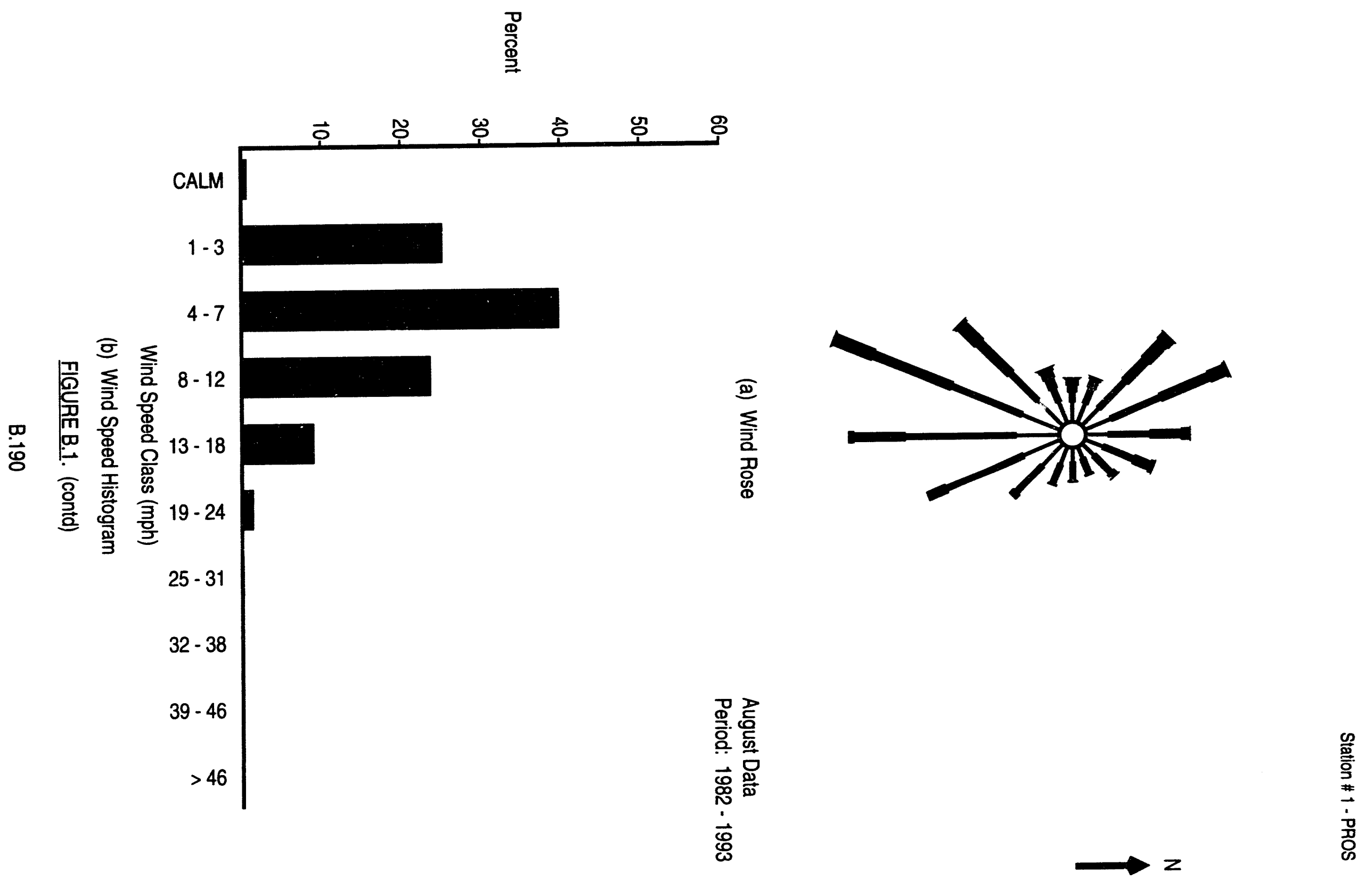

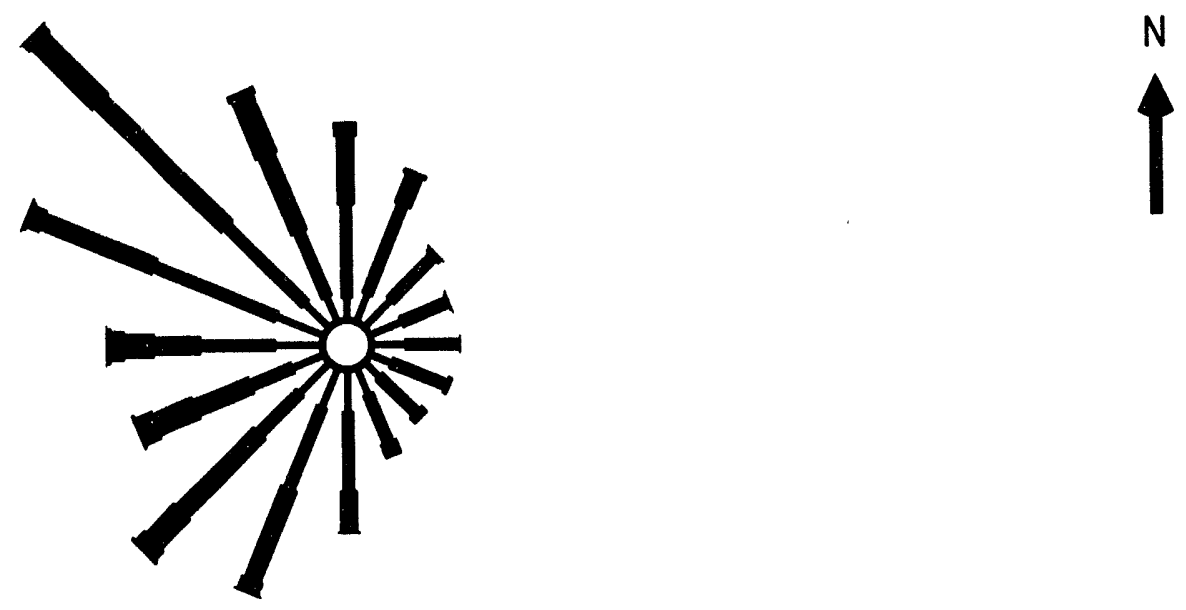

(a) Wind Rose

August Data

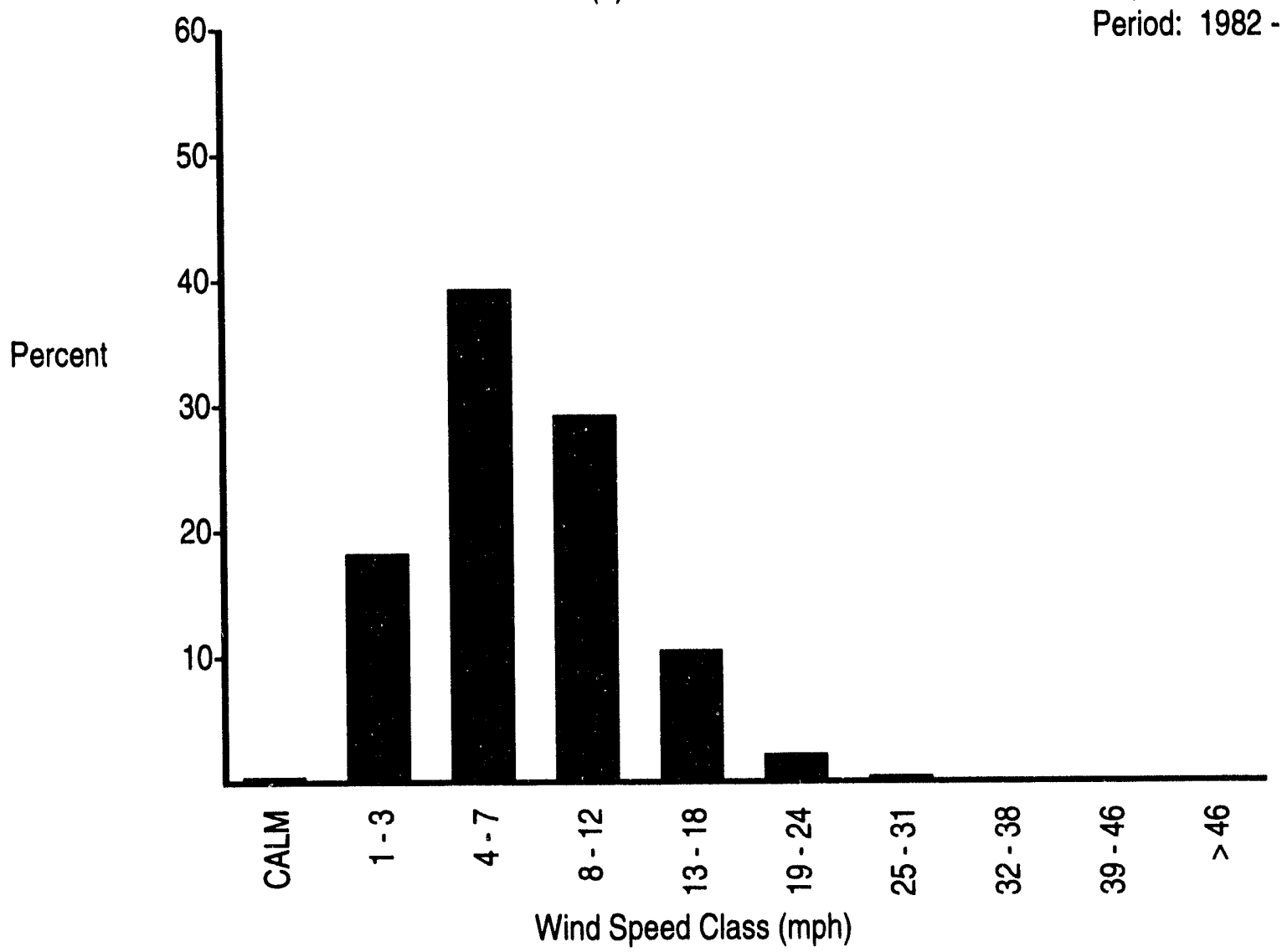

(b) Wind Speed Histogram

FIGURE B.1. (contd) 


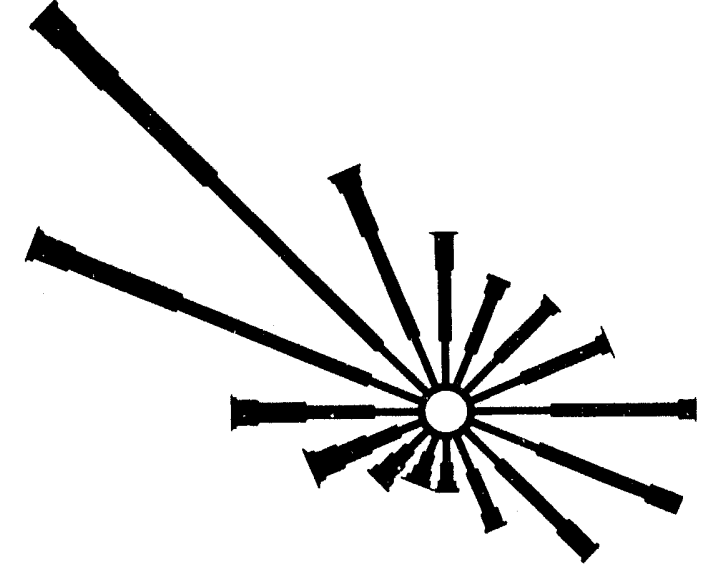

(a) Wind Rose

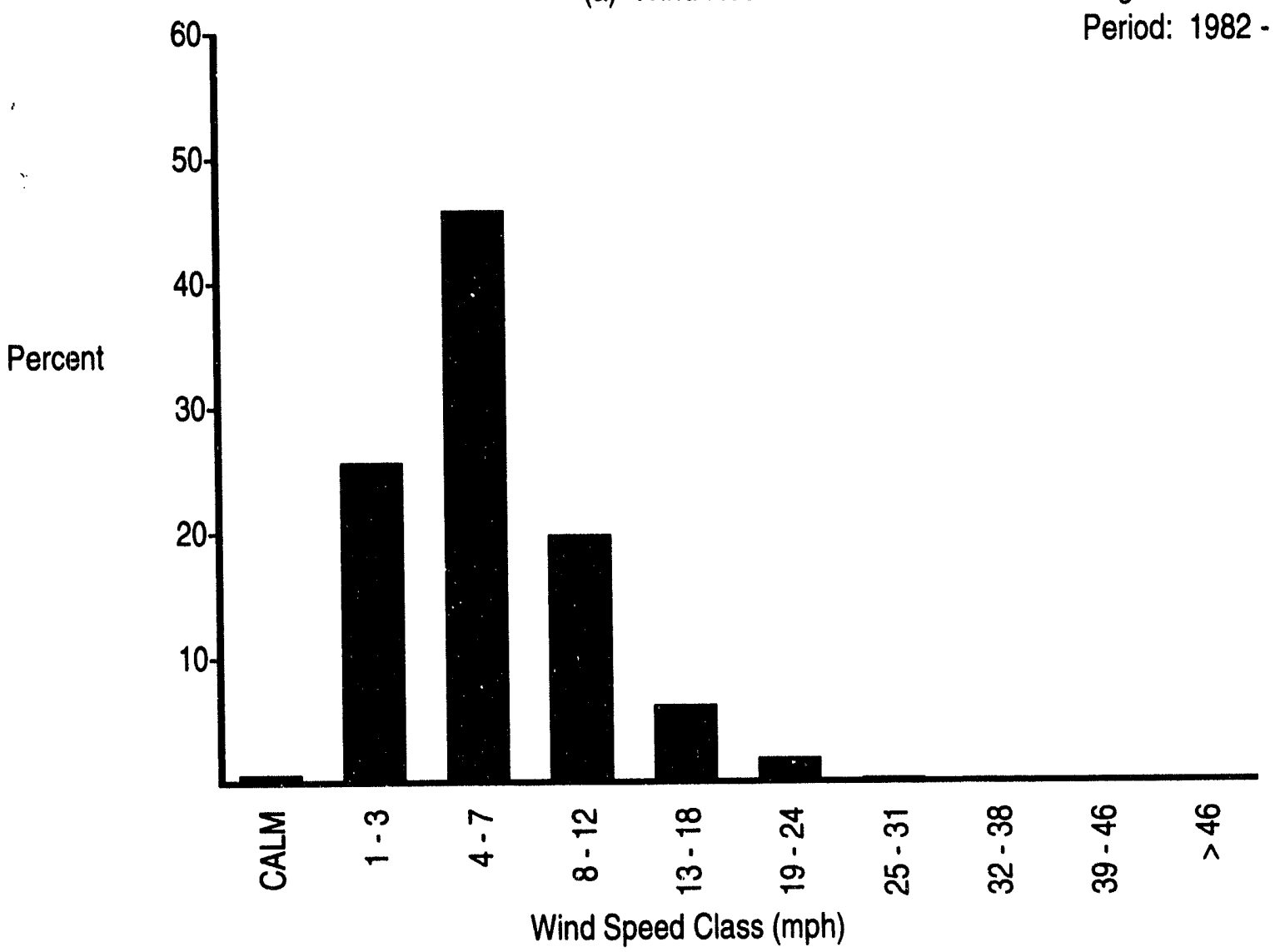

(b) Wind Speed Histogram

FIGURE B.1. (contd) 
E6!' 8

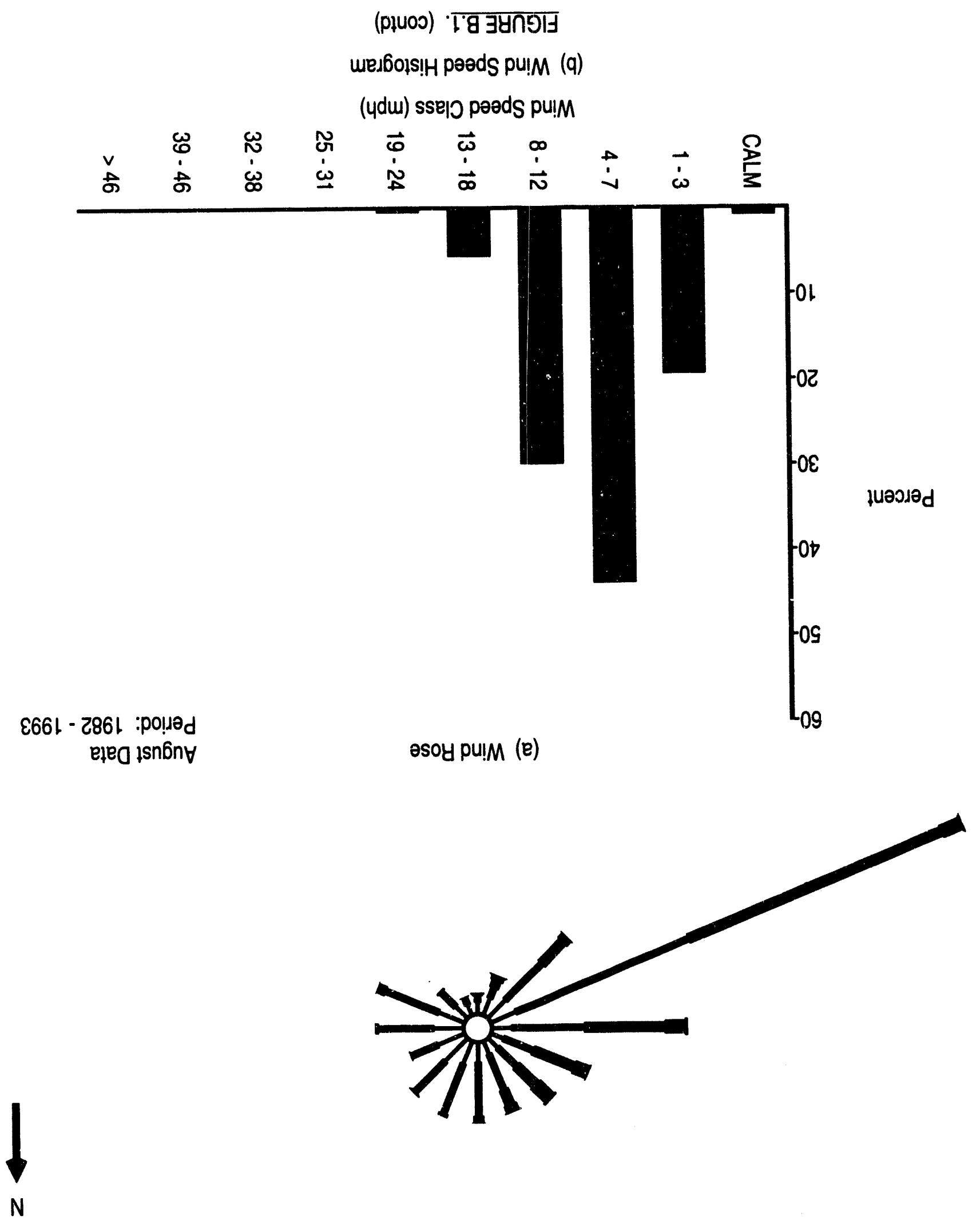




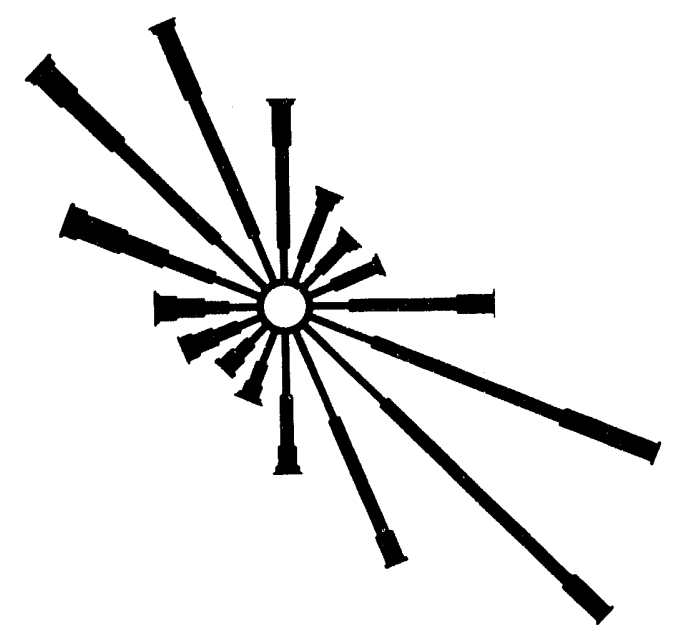

(a) Wind Rose

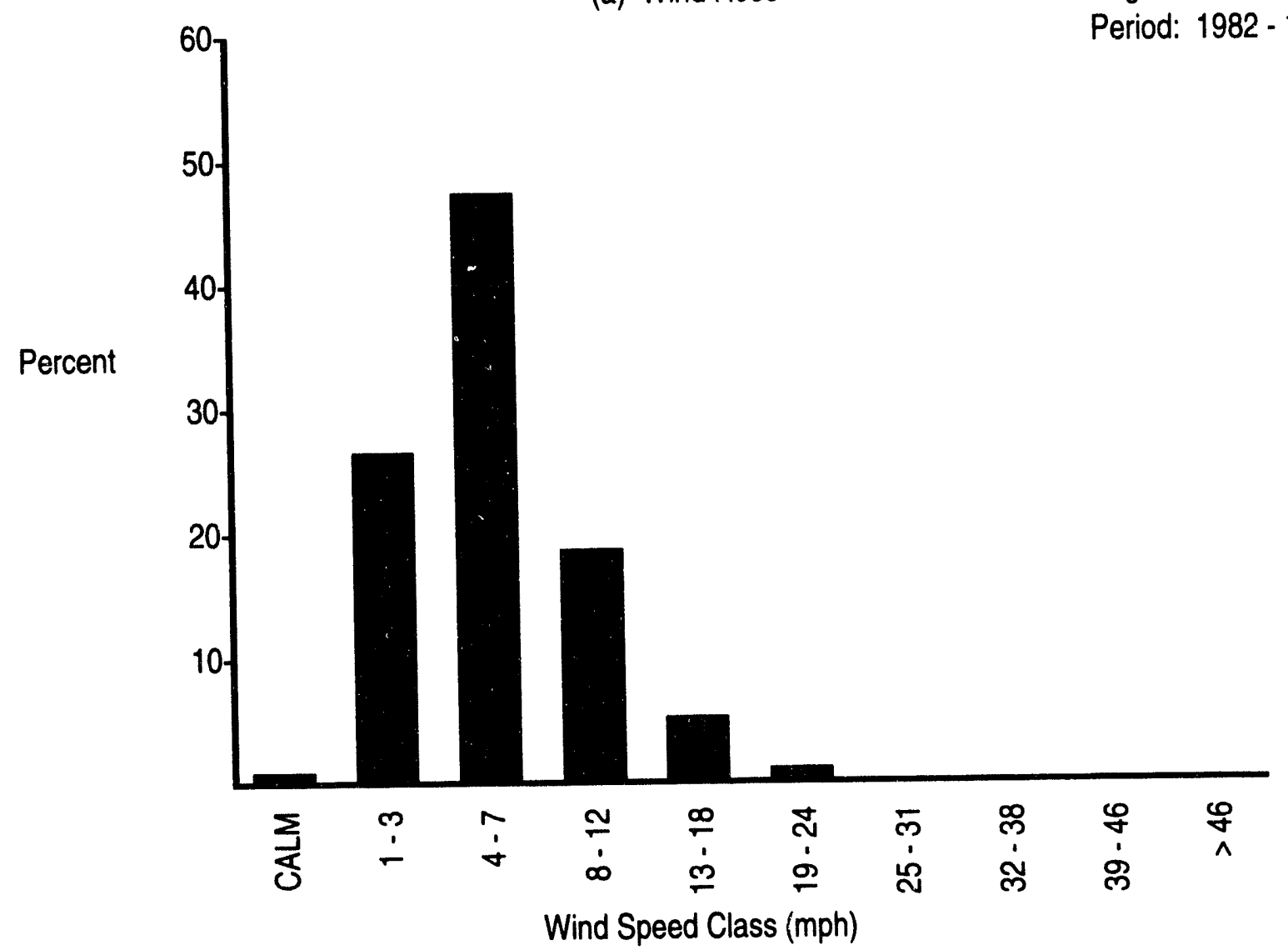

(b) Wind Speed Histogram

FIGURE B.1. (contd)
August Data

Period: 1982 - 1993 


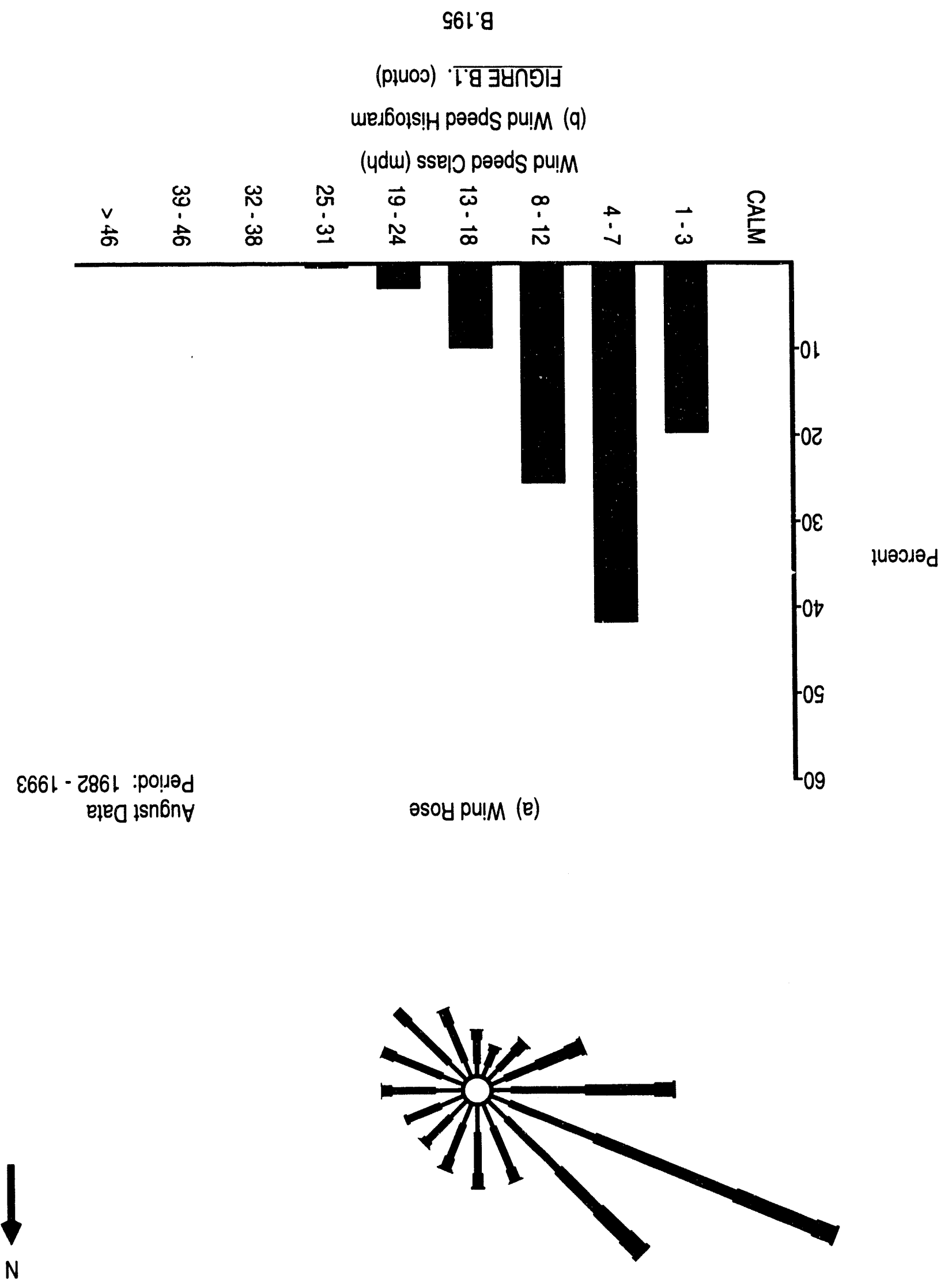




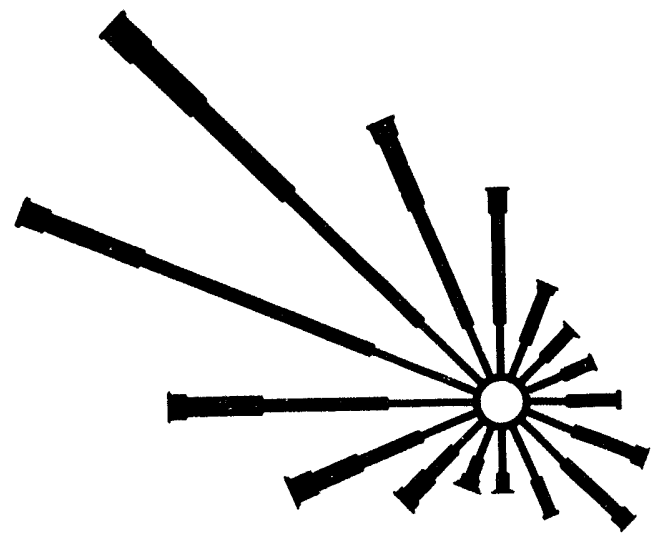

(a) Wind Rose

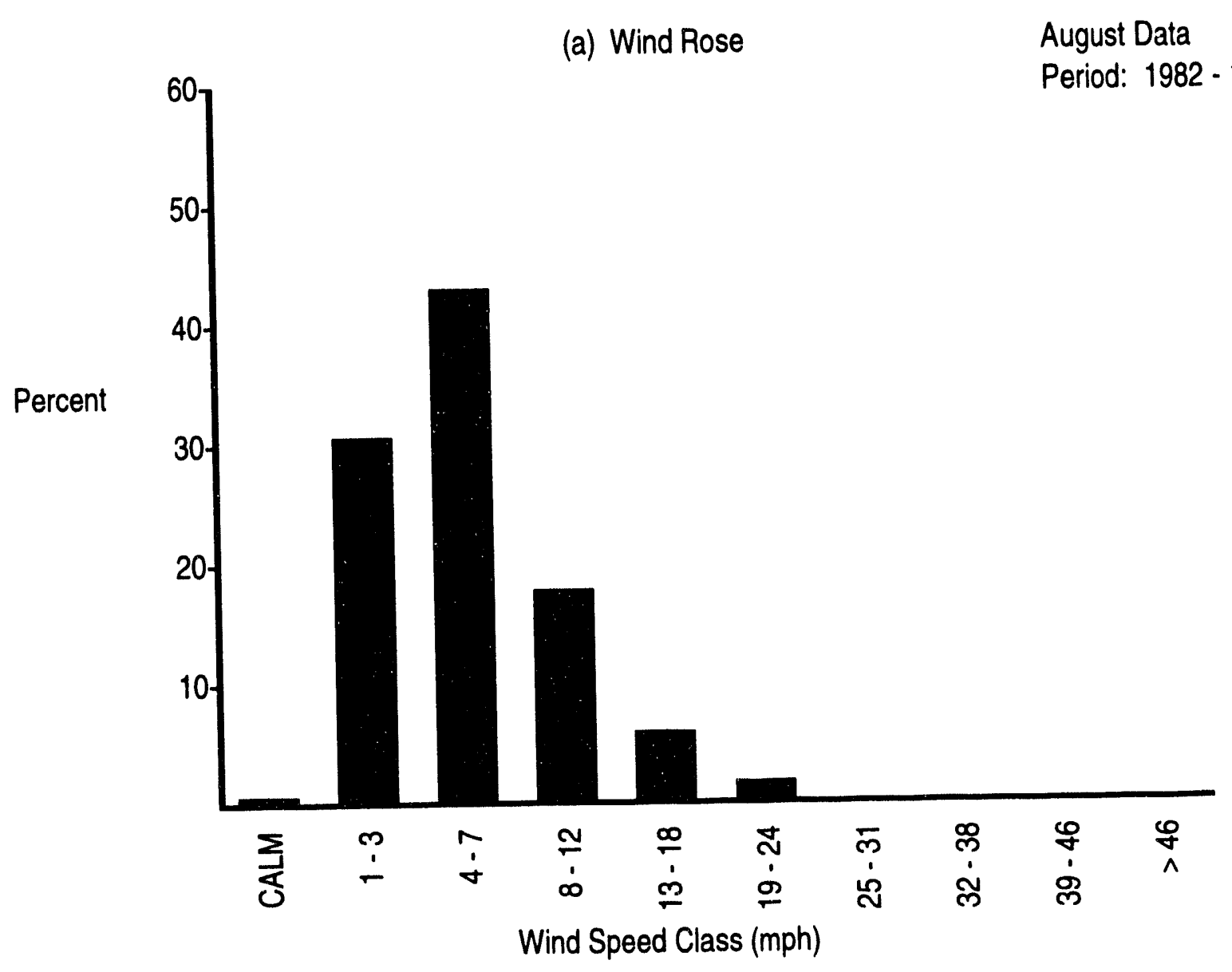

(b) Wind Speed Histogram

FIGURE B.1. (contd)

B.196 


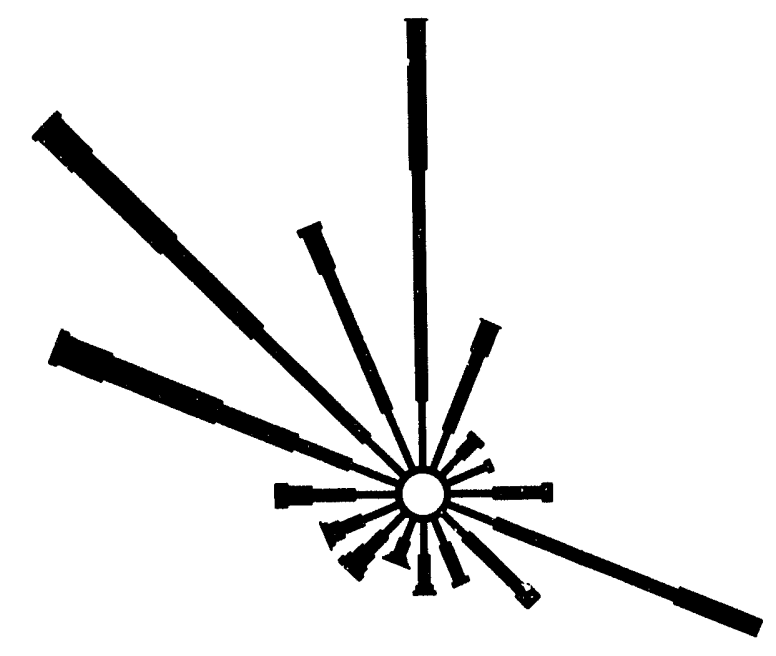

(a) Wind Rose

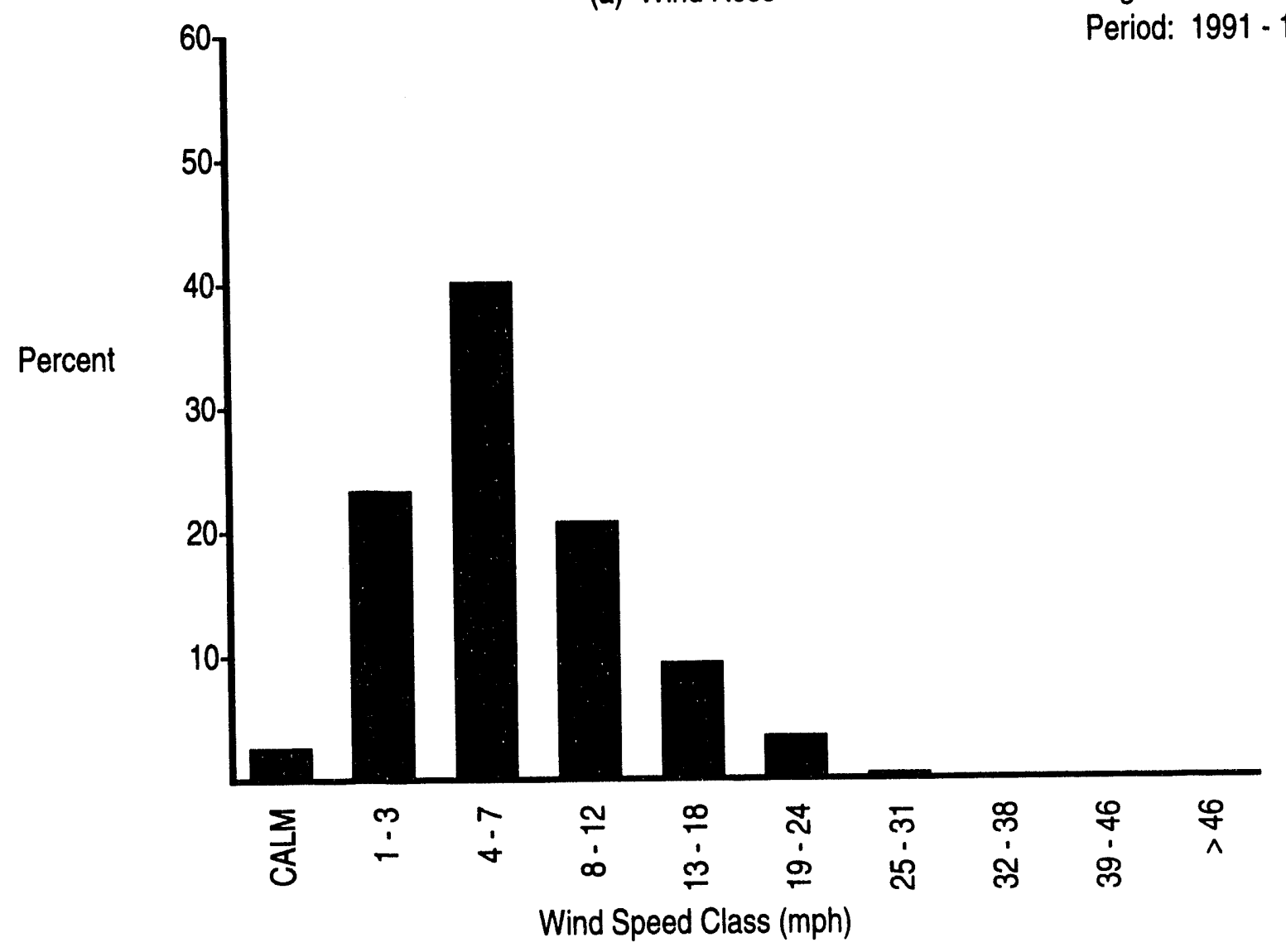

(b) Wind Speed Histogram

FIGURE B.1. (contd)
August Data

Period: 1991 - 1993

\section{B.197}




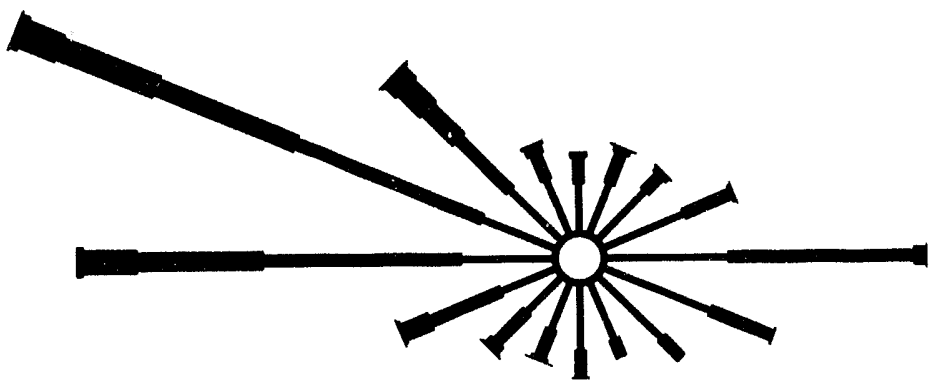

(a) Wind Rose

Percent

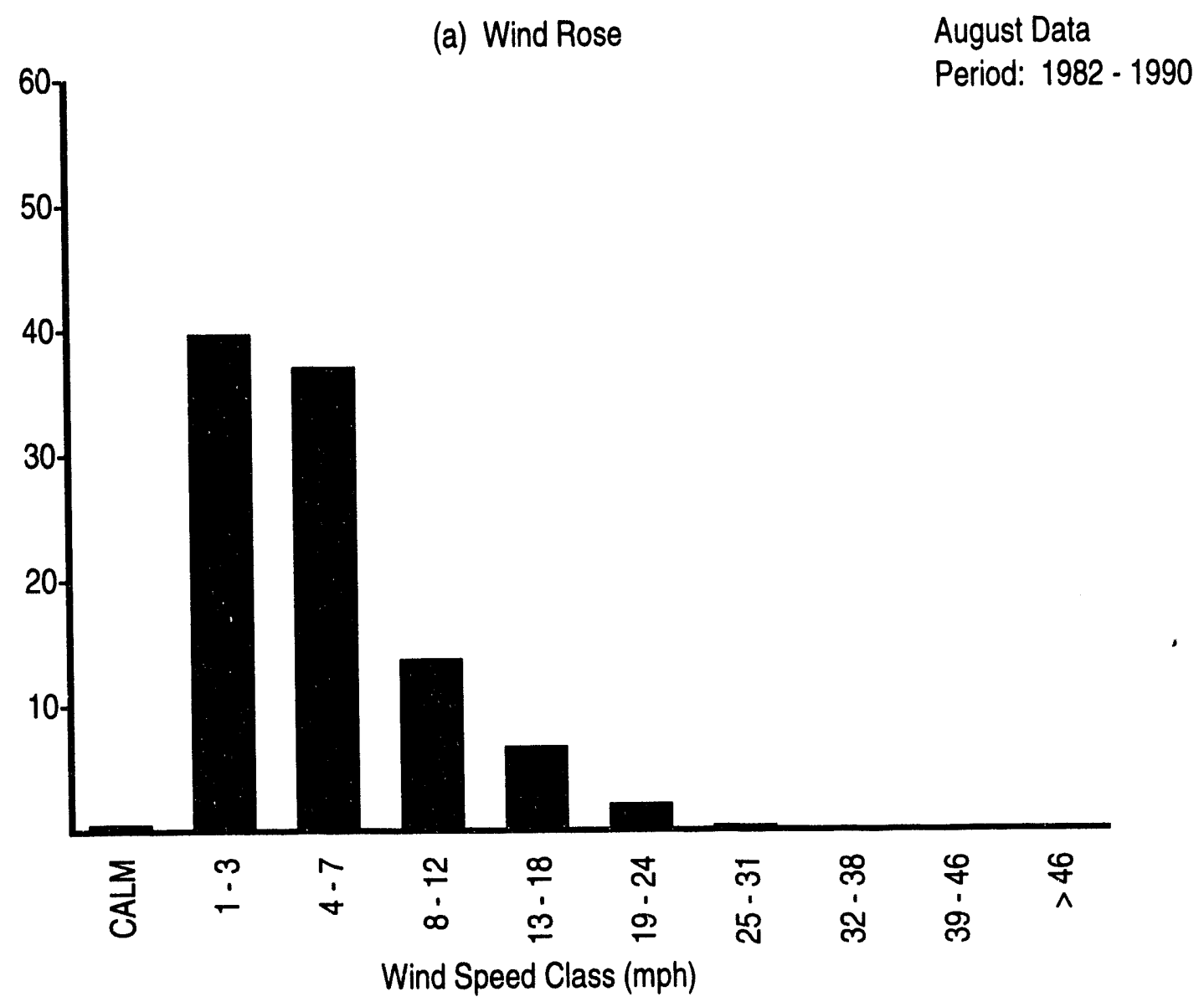

(b) Wind Speed Histogram

FIGURE B.1. (contd)

\section{B.198}




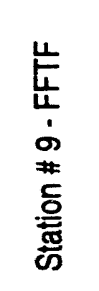

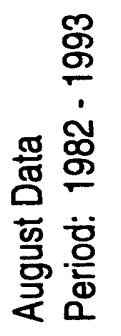

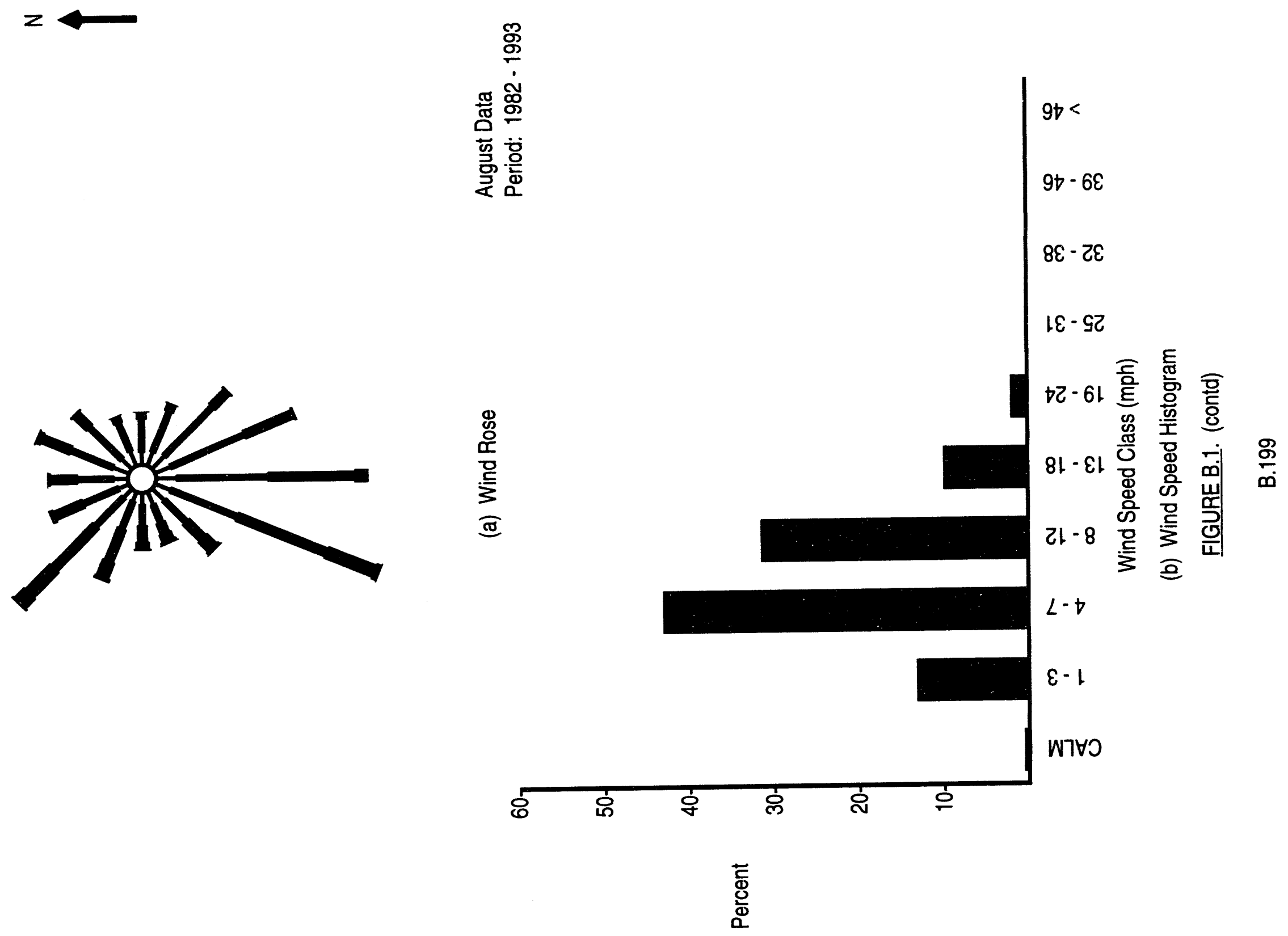



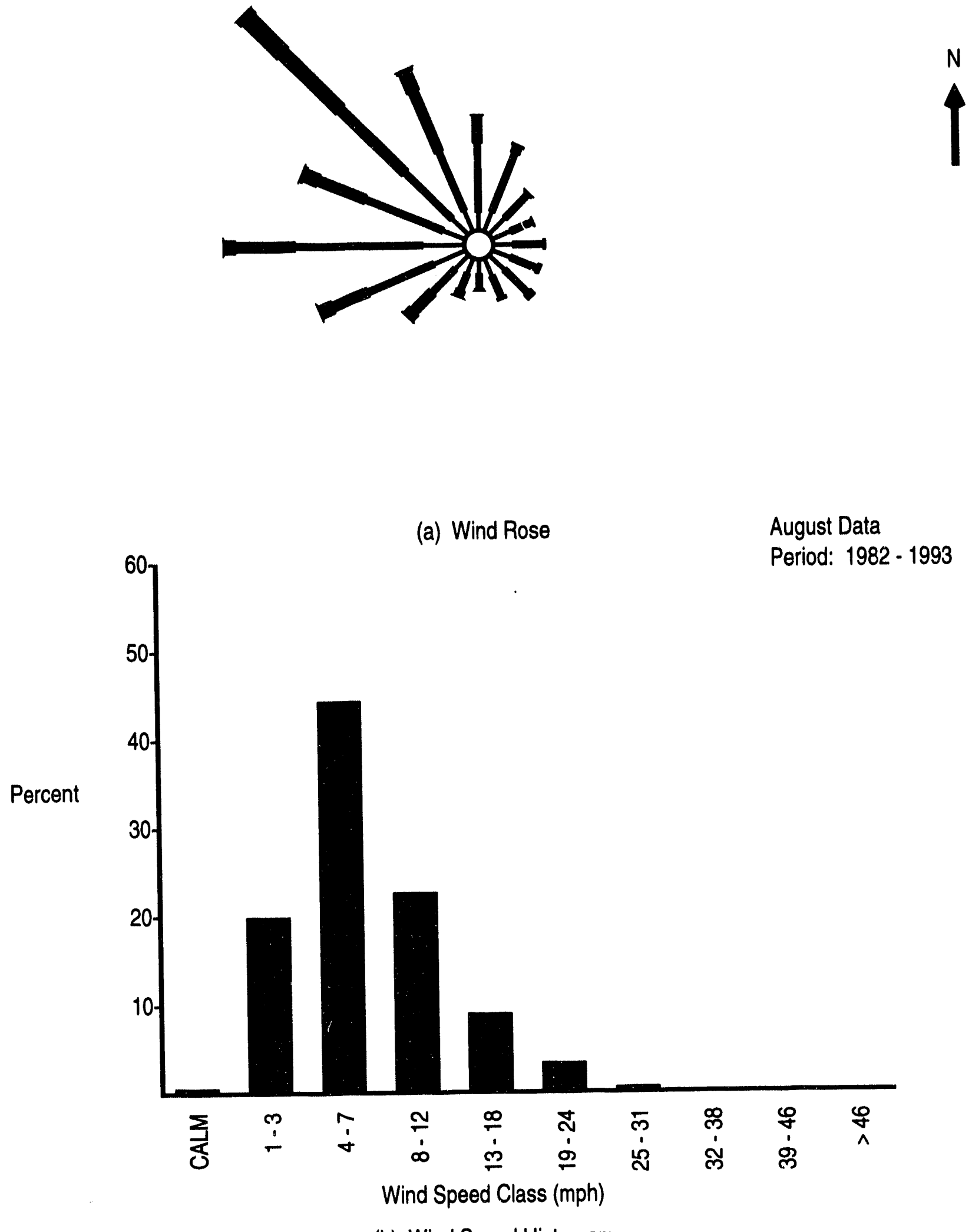

(b) Wind Speed Histogram

FIGURE B.1. (contd) 


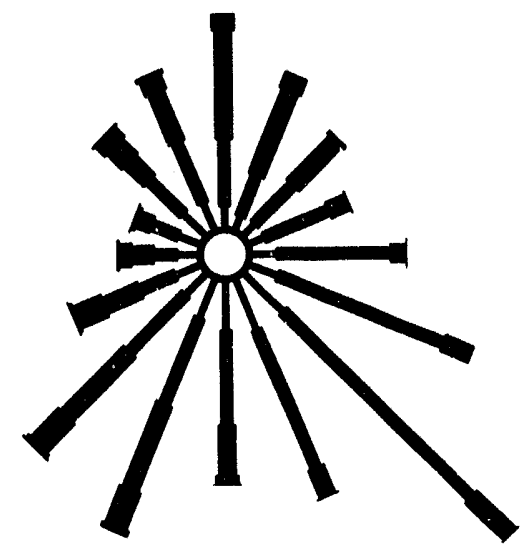

(a) Wind Rose

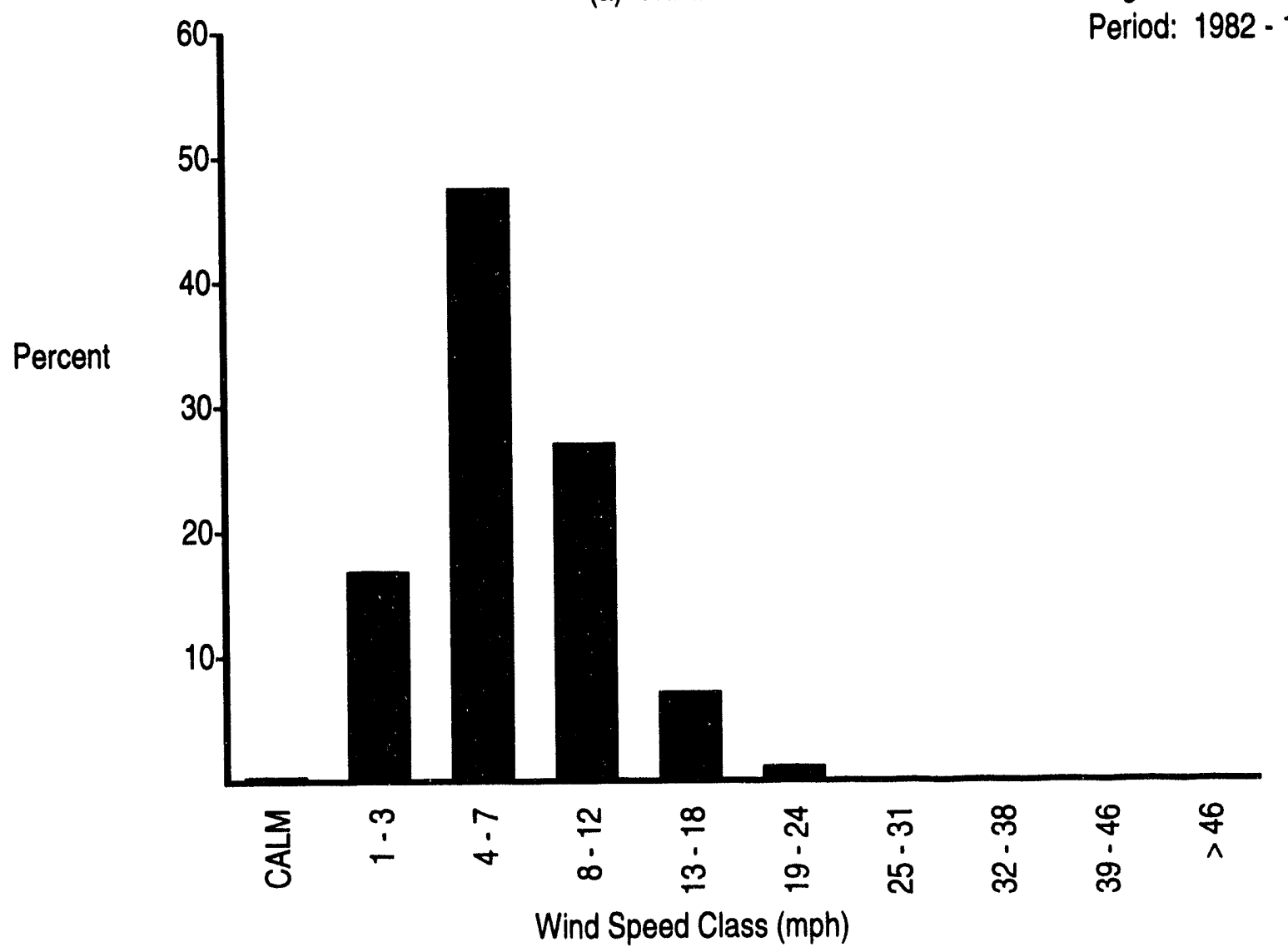

(b) Wind Speed Histogram

FIGURE B.1. (contd)
August Data

Period: 1982 - 1993 


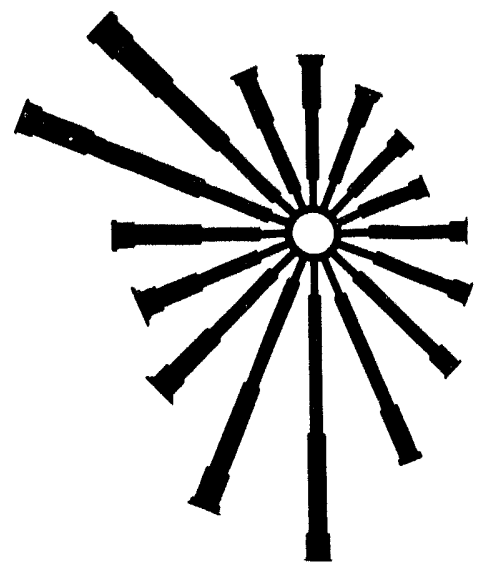

(a) Wind Rose

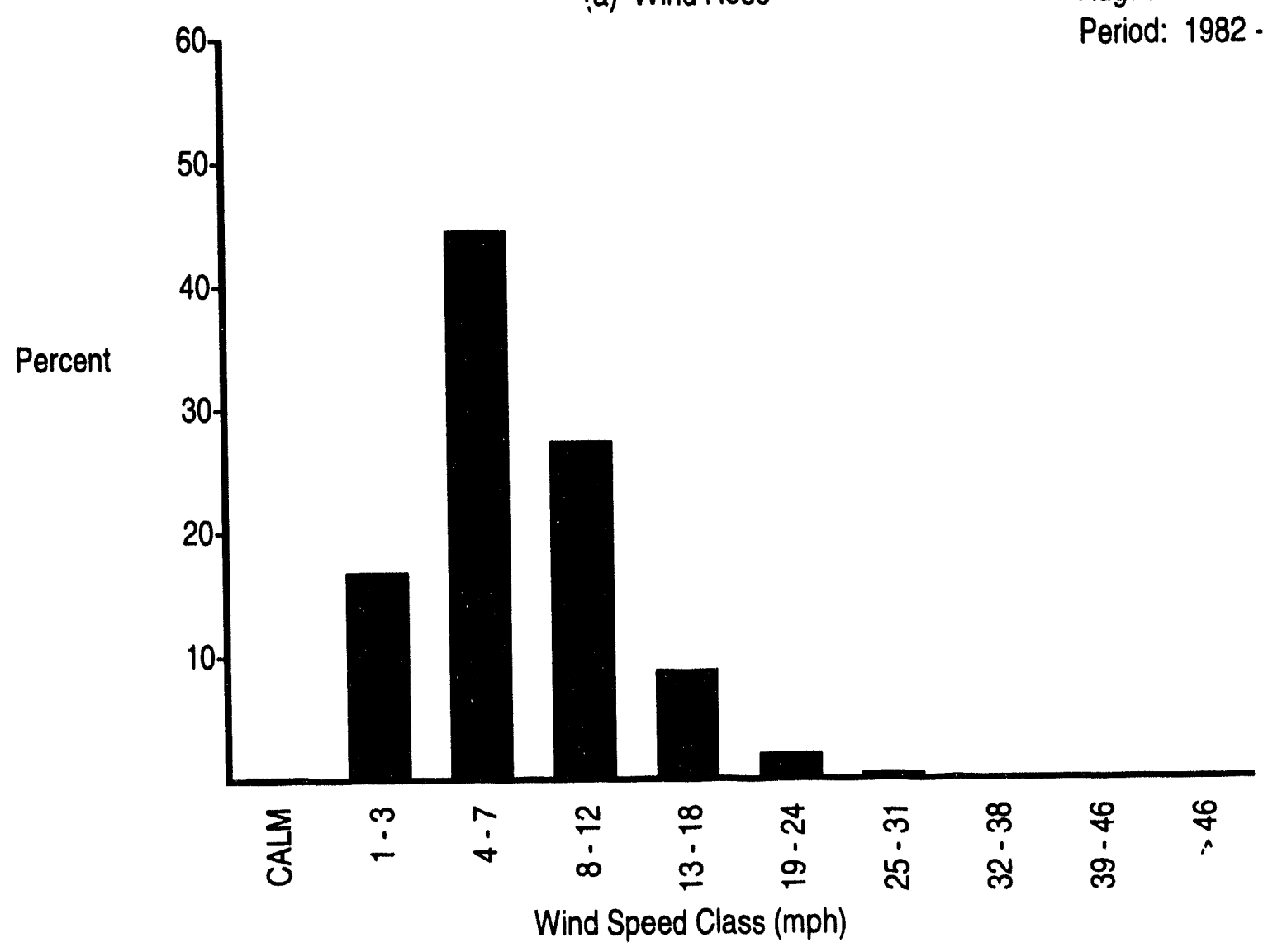

(b) Wind Speed Histogram

FIGURE B.1. (contd)
August Data

Period: 1982 - 1993 


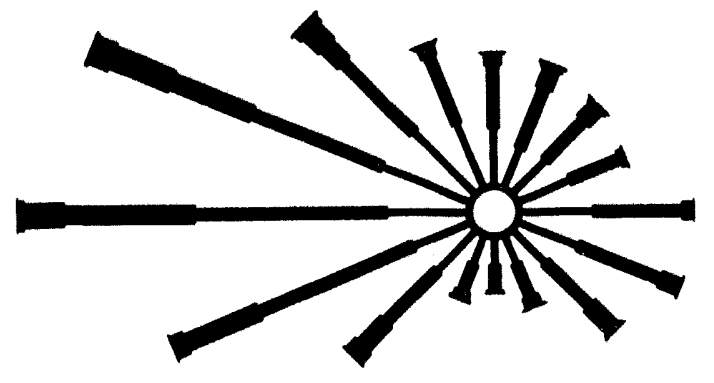

(a) Wind Rose

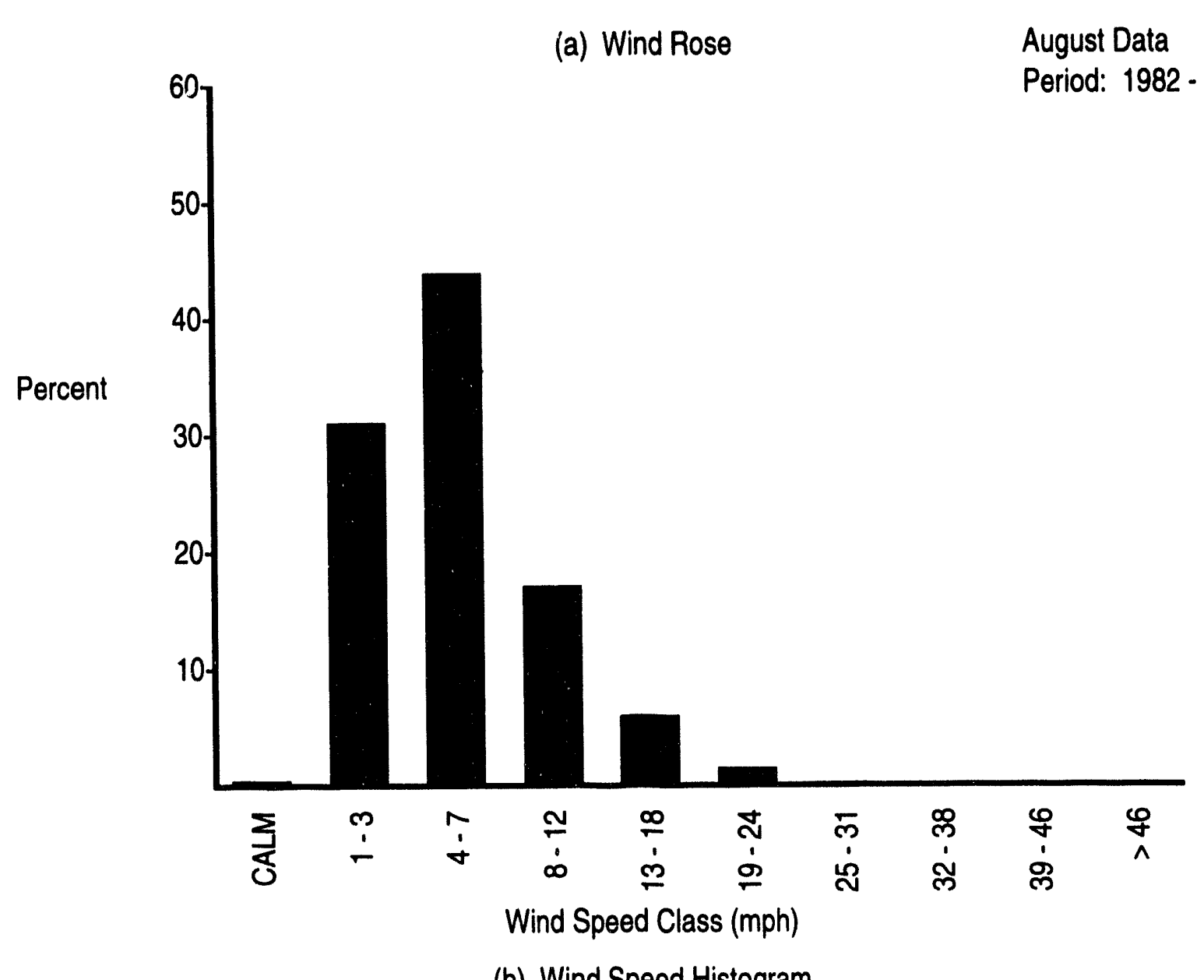

(b) Wind Speed Histogram

FIGURE B.1. (contd) 


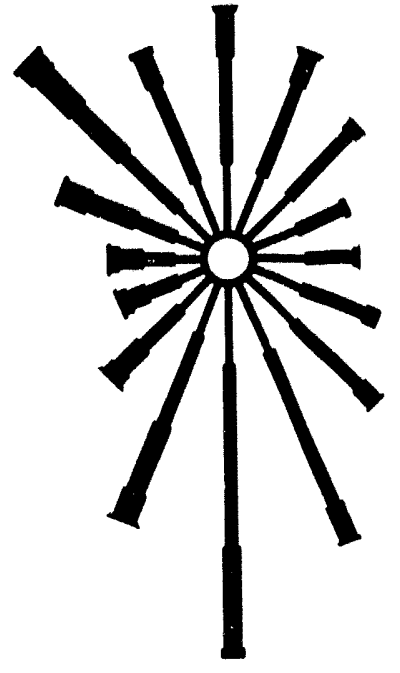

(a) Wind Rose

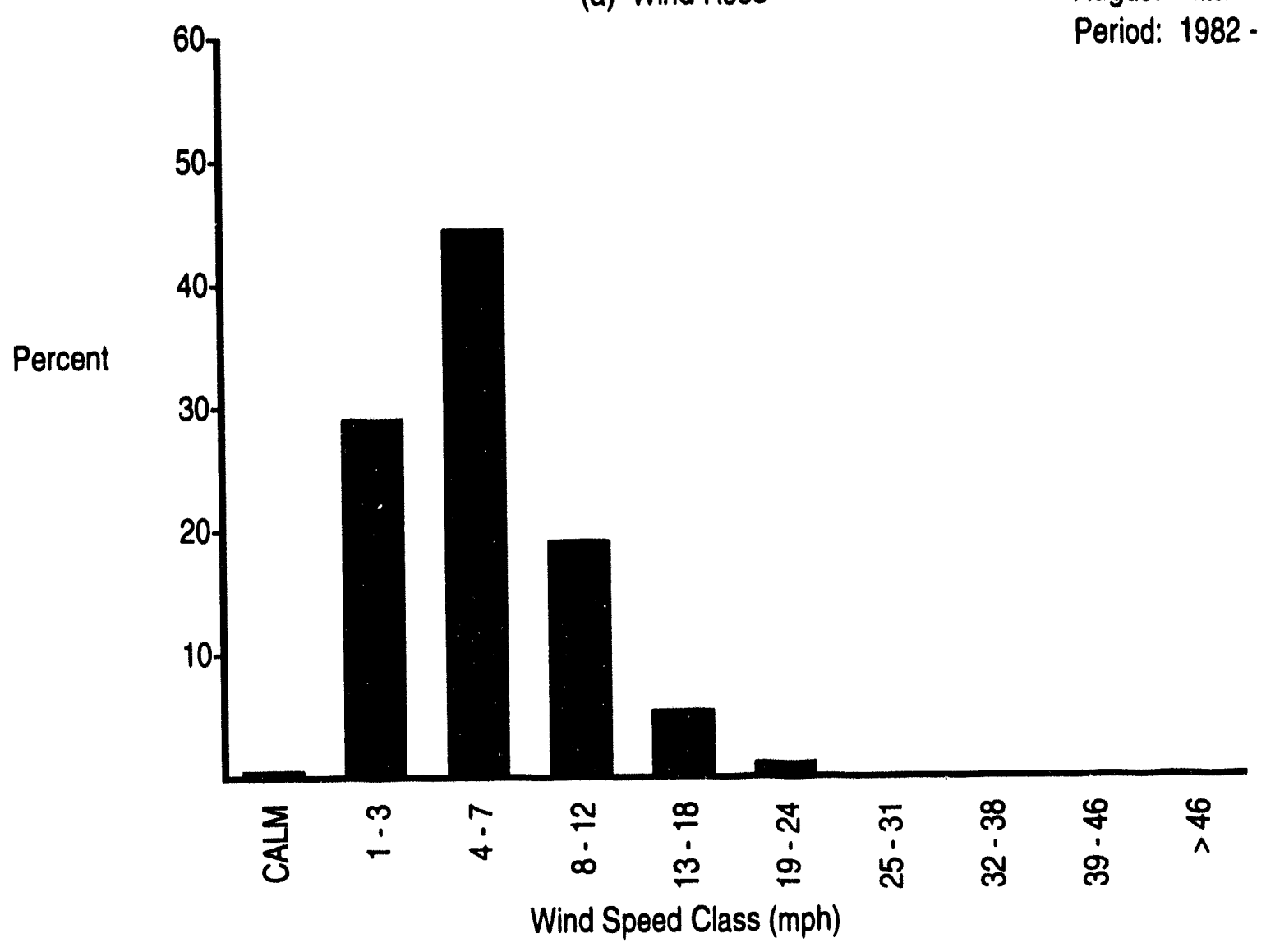

(b) Wind Speed Histogram

FIGURE B.1. (contd)
August Data

Period: $1982-1993$ 

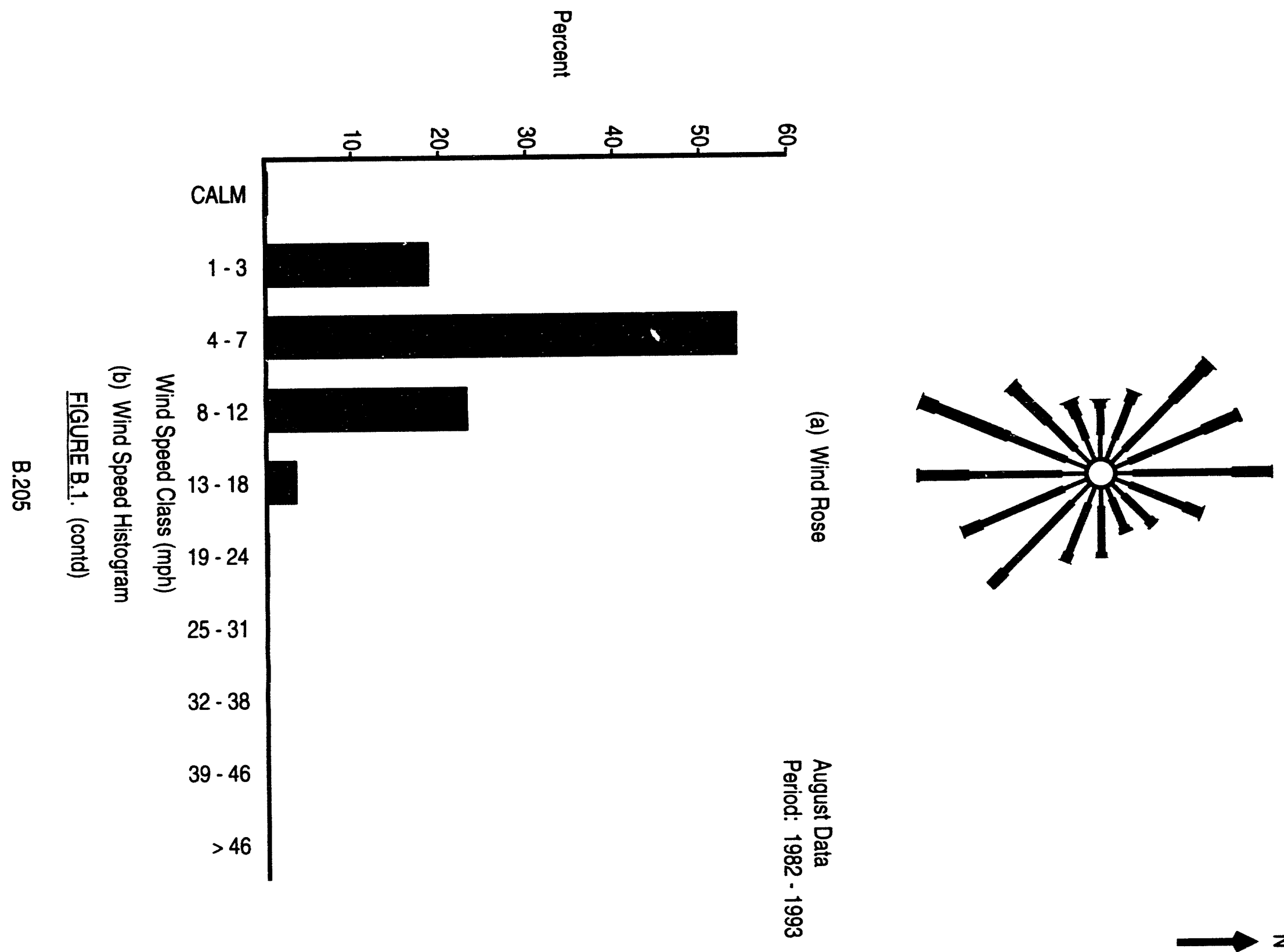

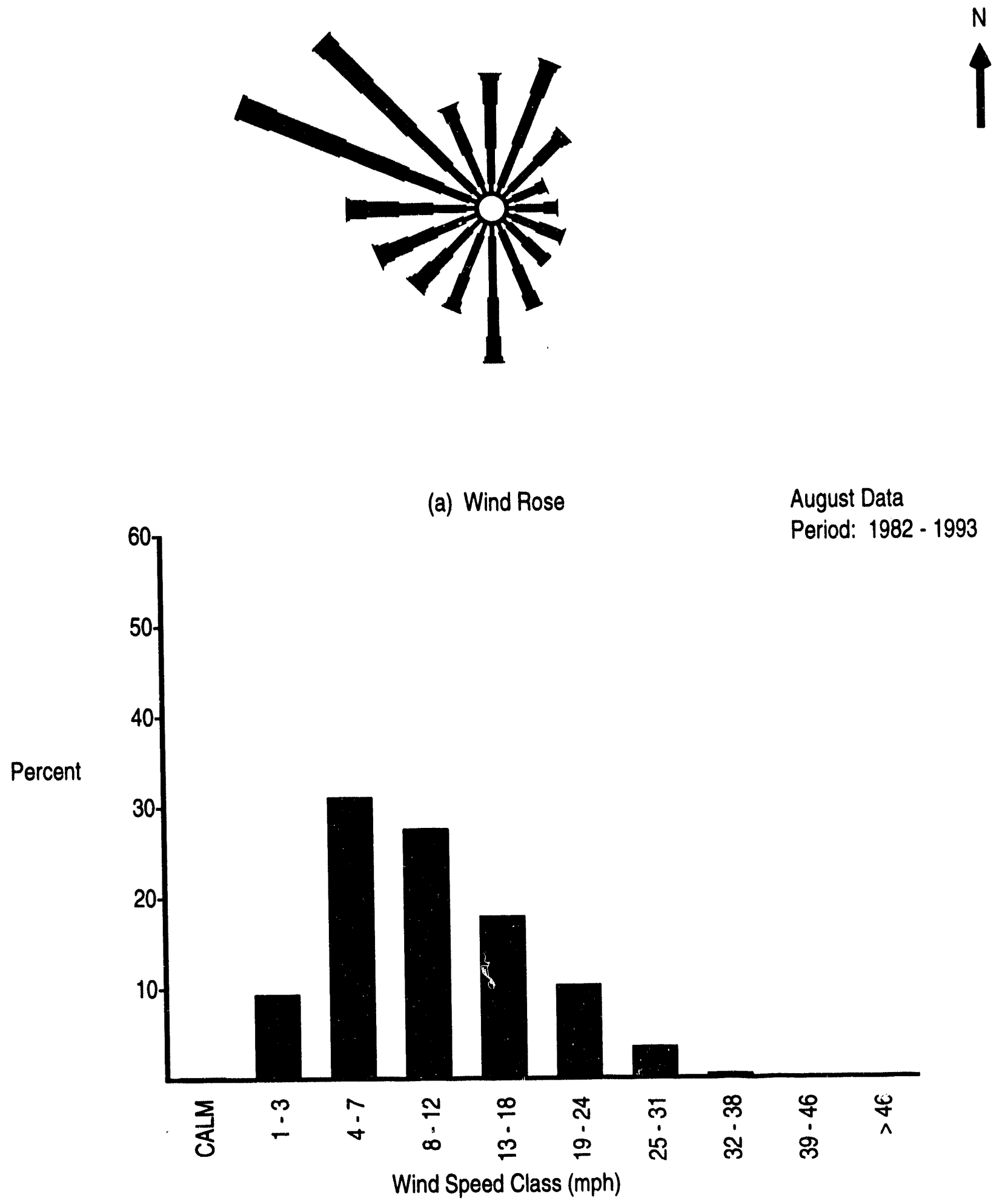

(b) Wind Speed Histogram

FIGURE B.1. (contd) 

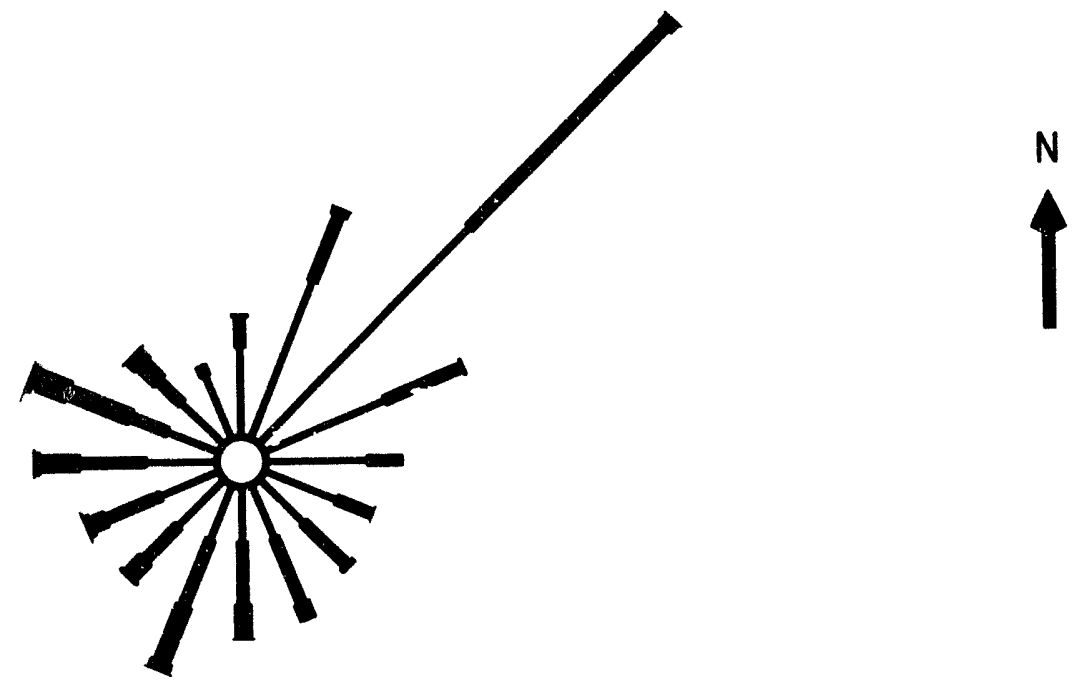

(a) Wind Rose

August Data

Period: $1982-1993$

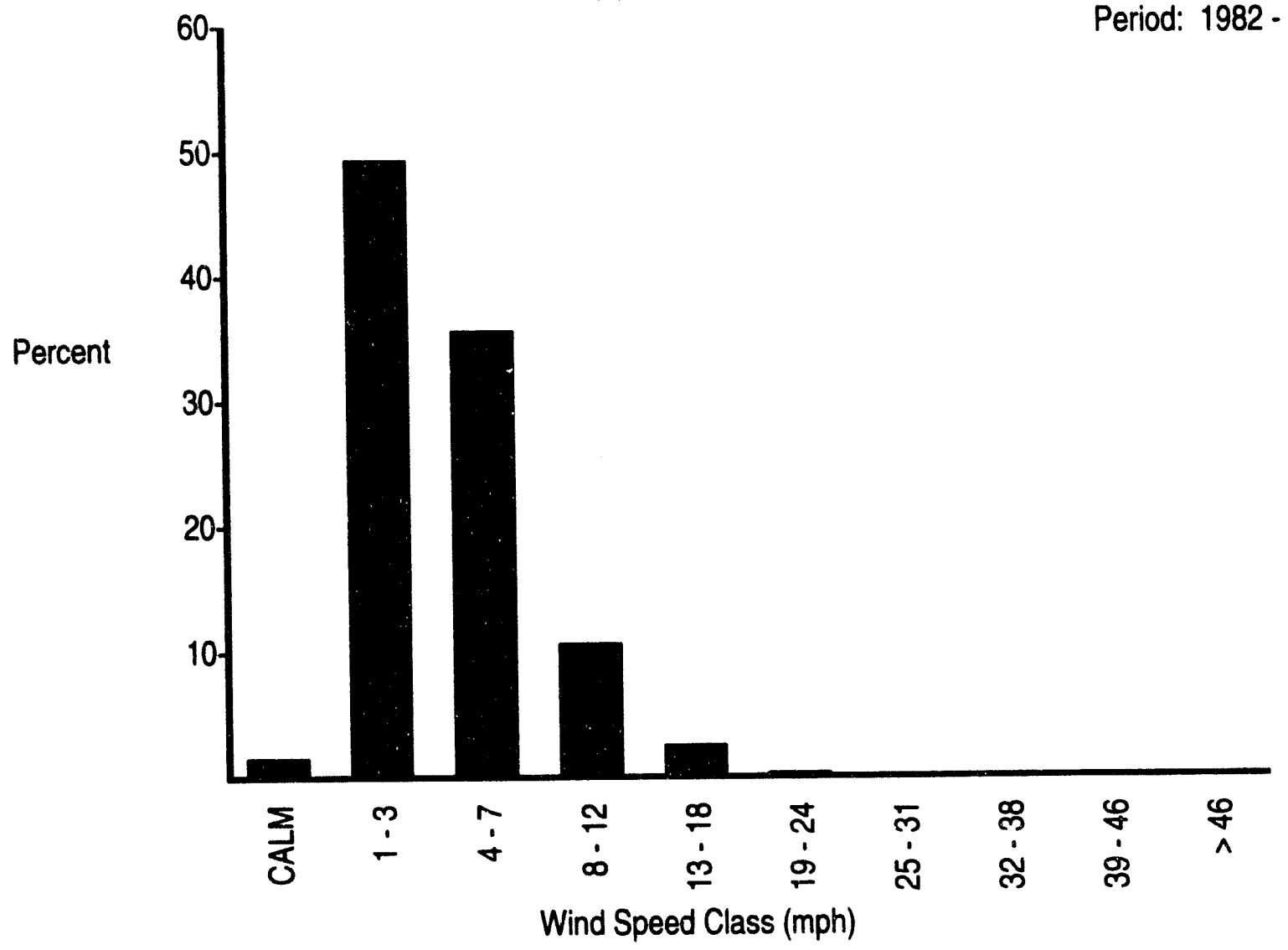

(b) Wind Speed Histogram

FIGURE B.1. (contd) 


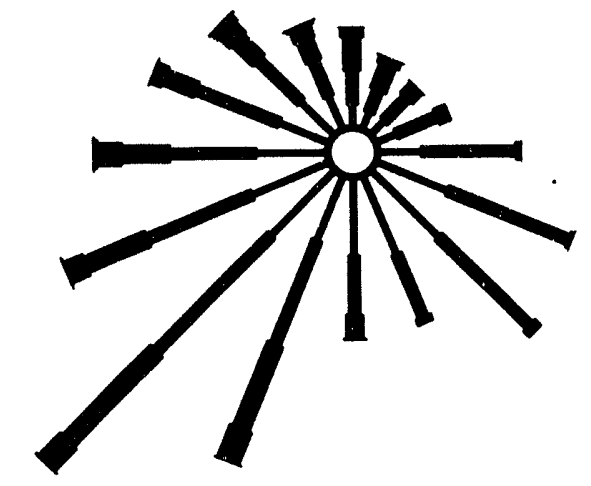

(a) Wind Rose

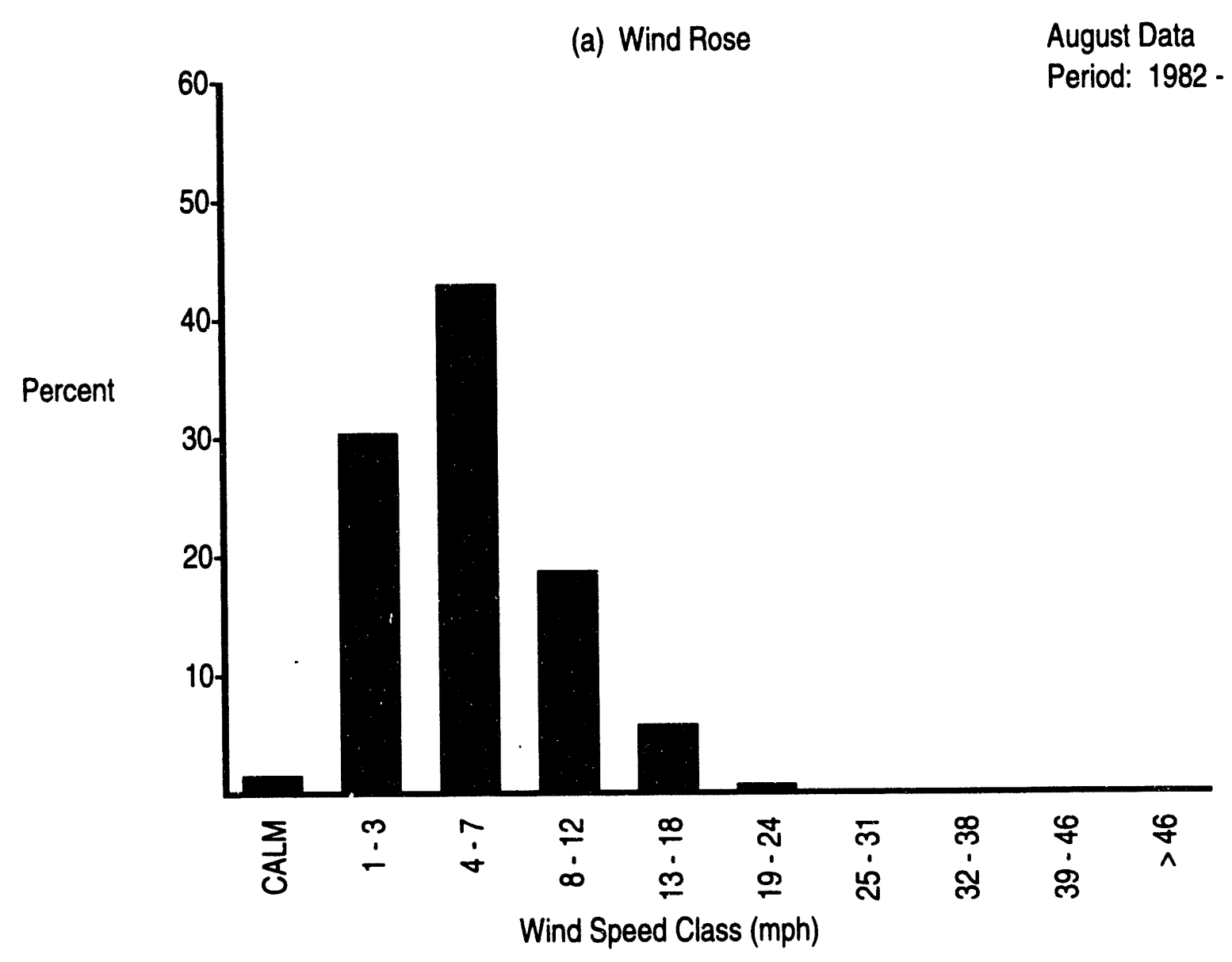

(b) Wind Speed Histogram

FIGURE B.1. (contd) 


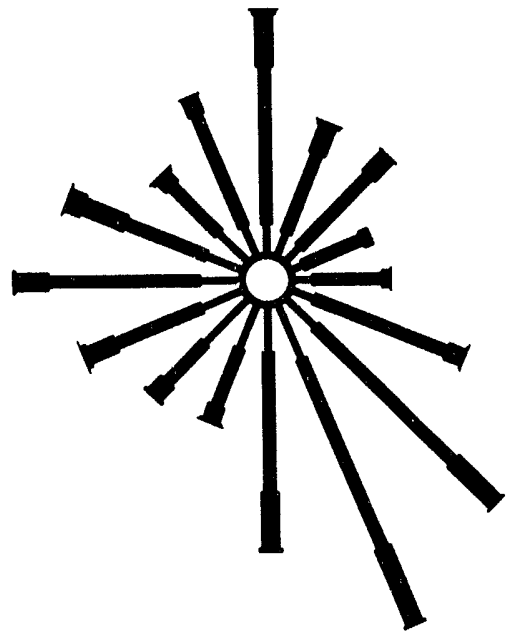

(a) Wind Rose

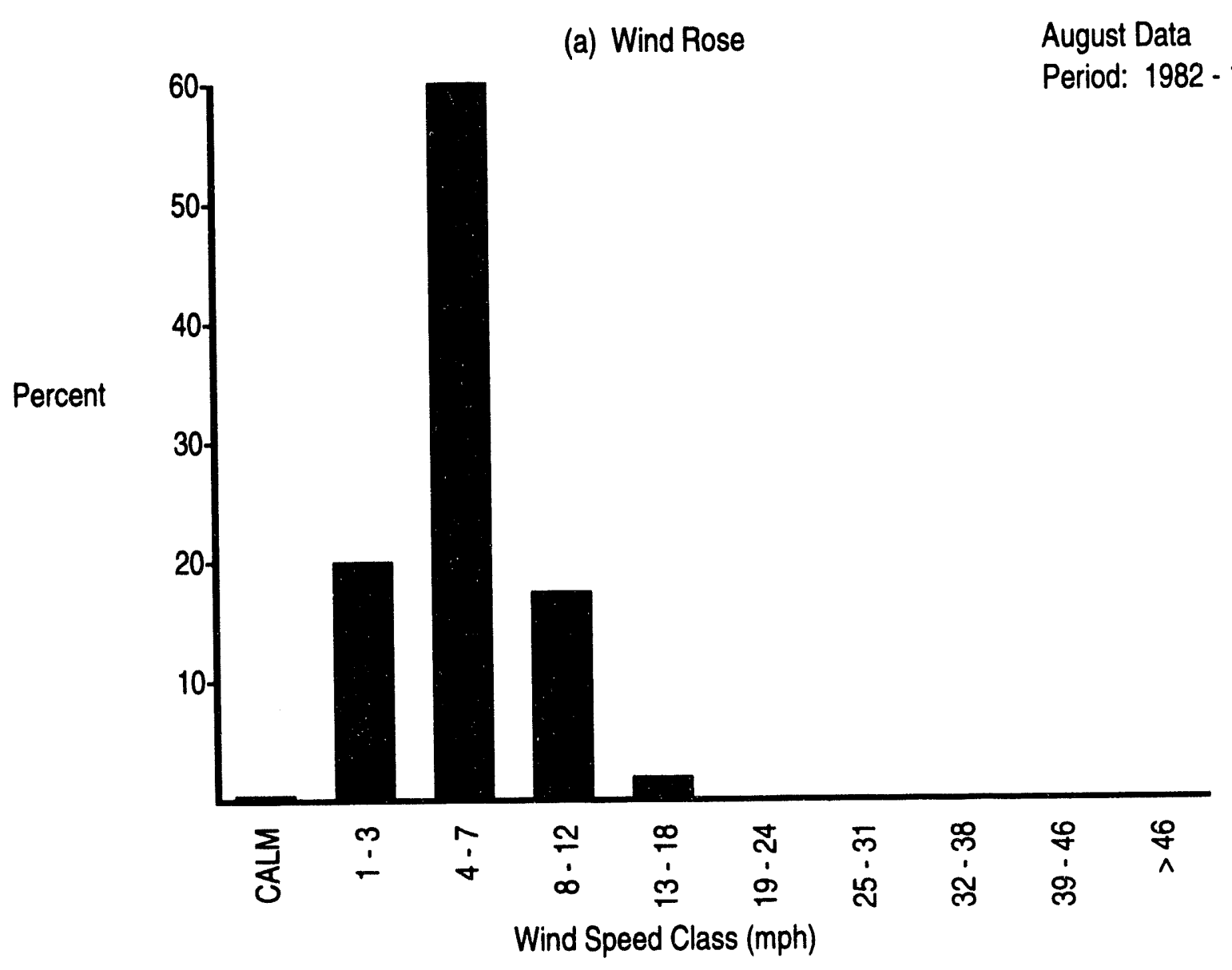

(b) Wind Speed Histogram

FIGURE B.1. (contd) 


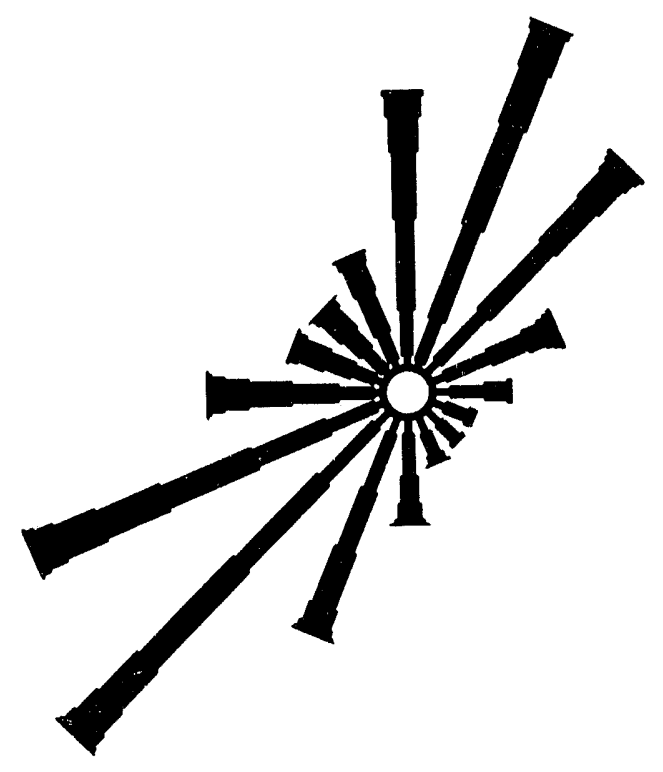

(a) Wind Rose

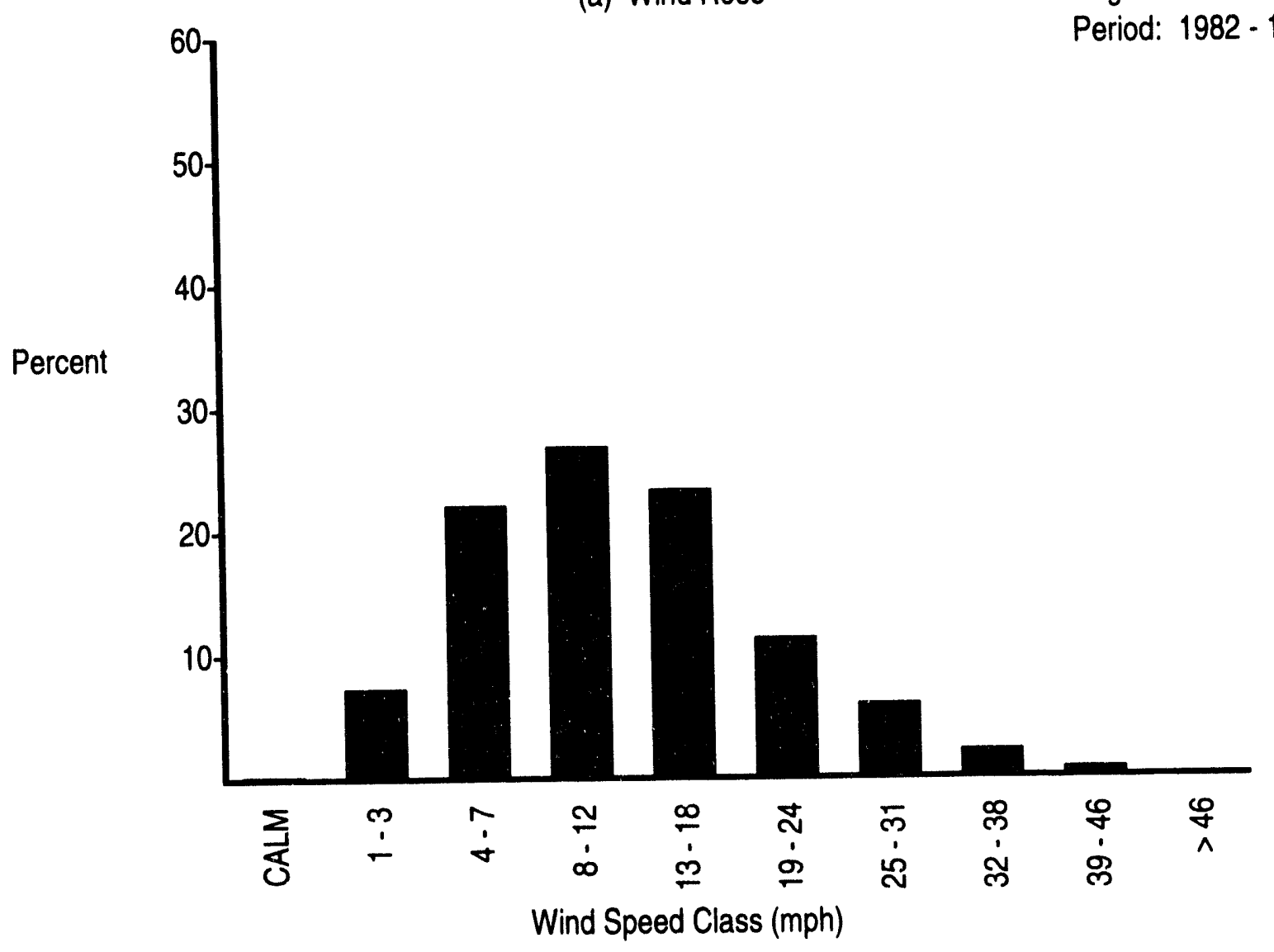

(b) Wind Speed Histogram

FIGURE B.1. (contd)
August Data

Period: 1982 - 1993

\section{B.210}



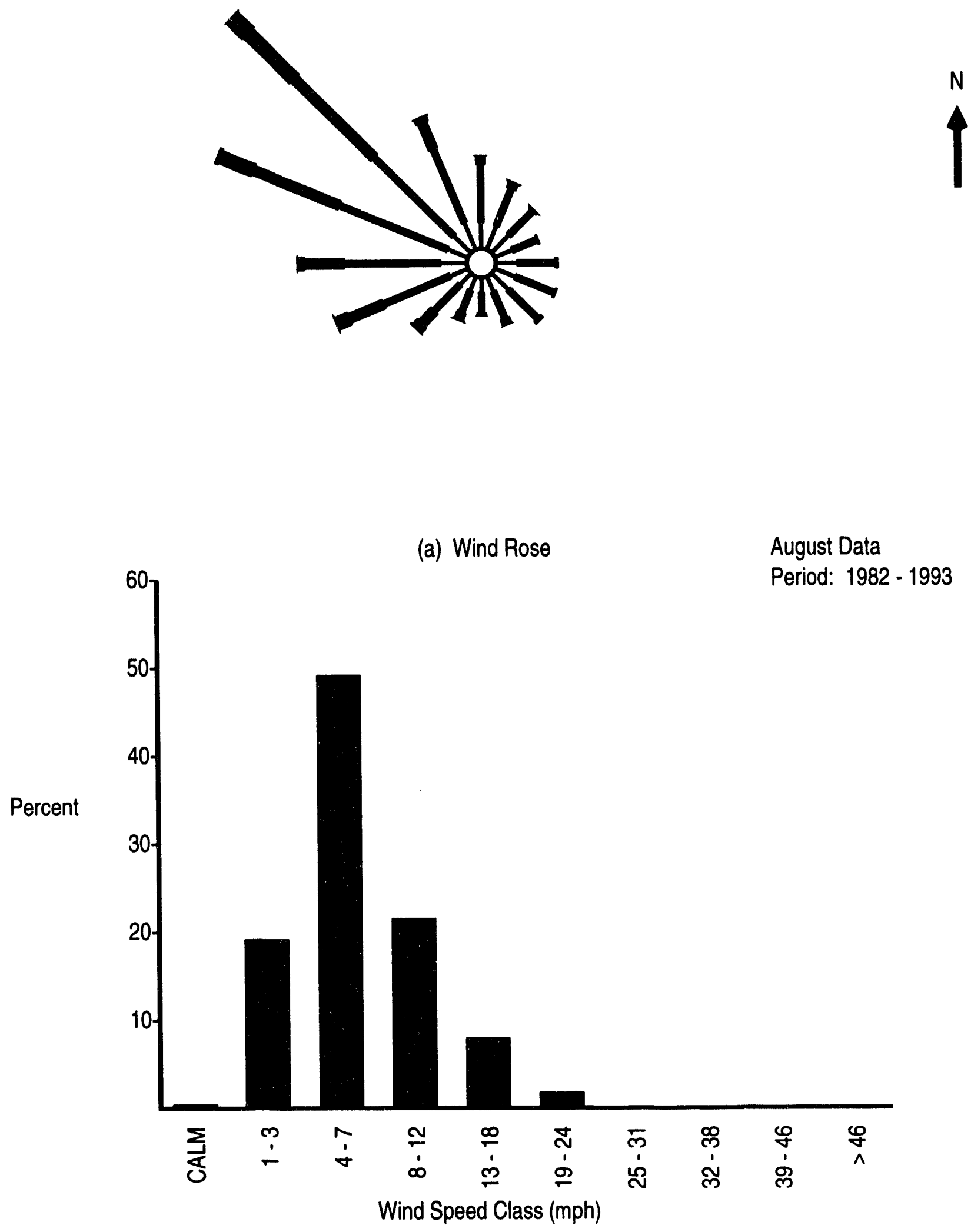

(b) Wind Speed Histogram

FIGURE B.1. (contd) 

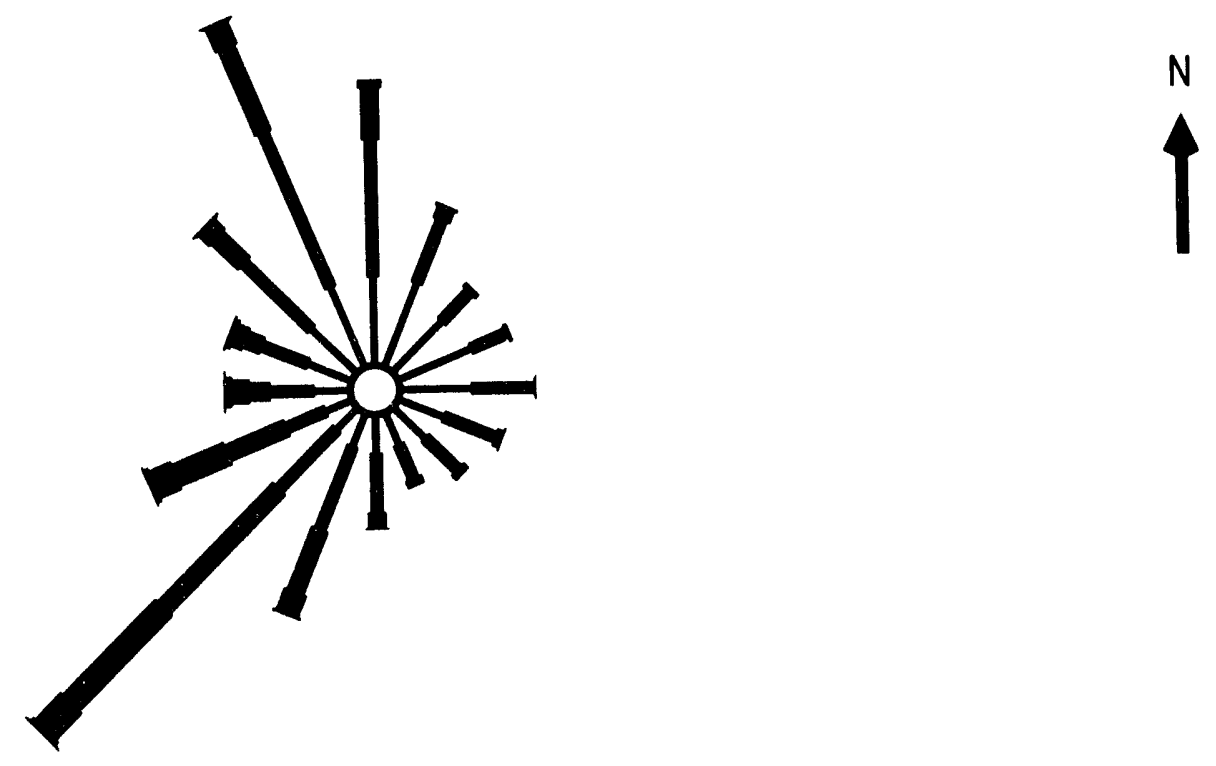

(a) Wind Rose

August Data

Period: $1988-1993$

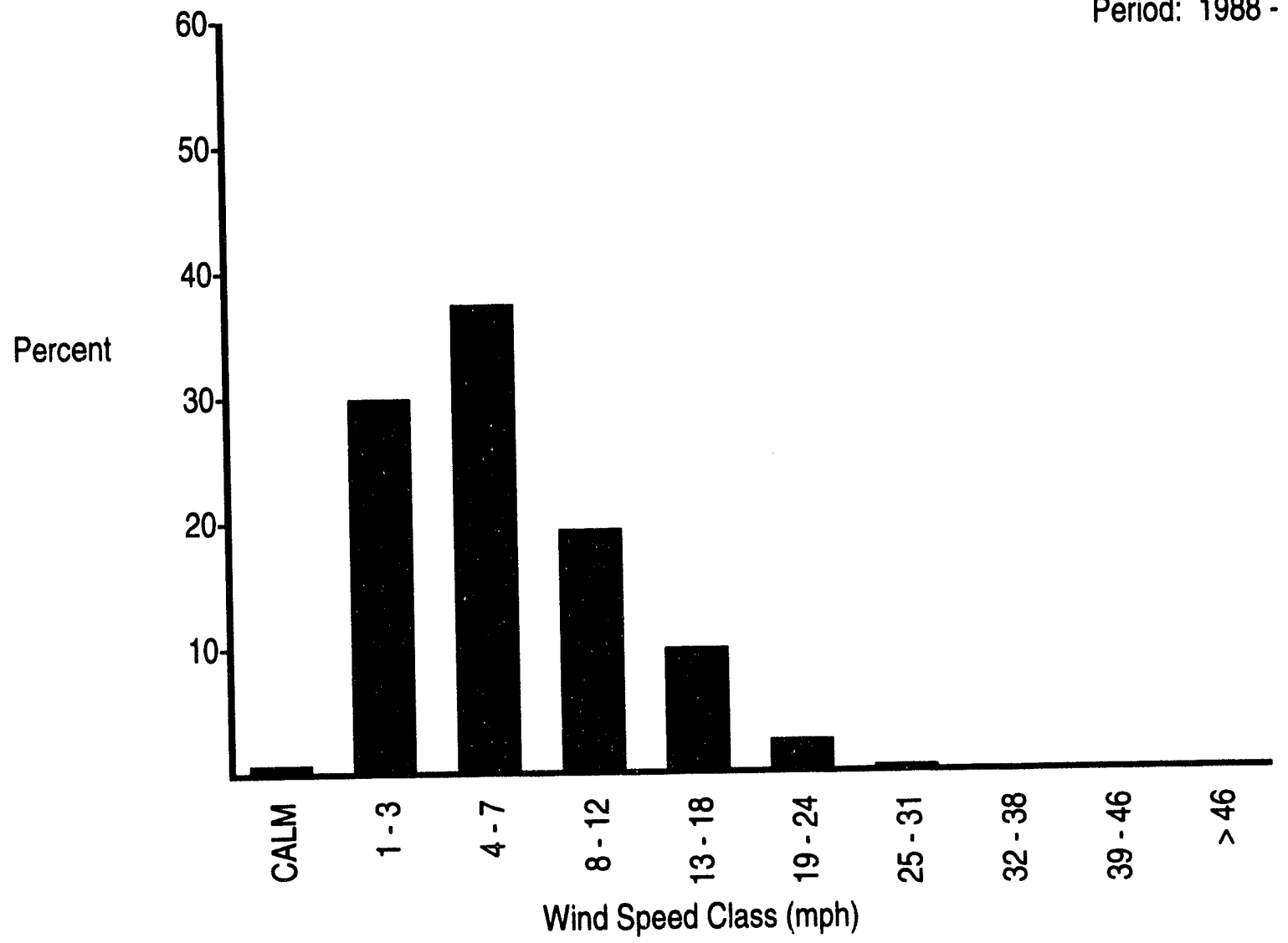

(b) Wind Speed Histogram

FIGURE B.1. (contd) 

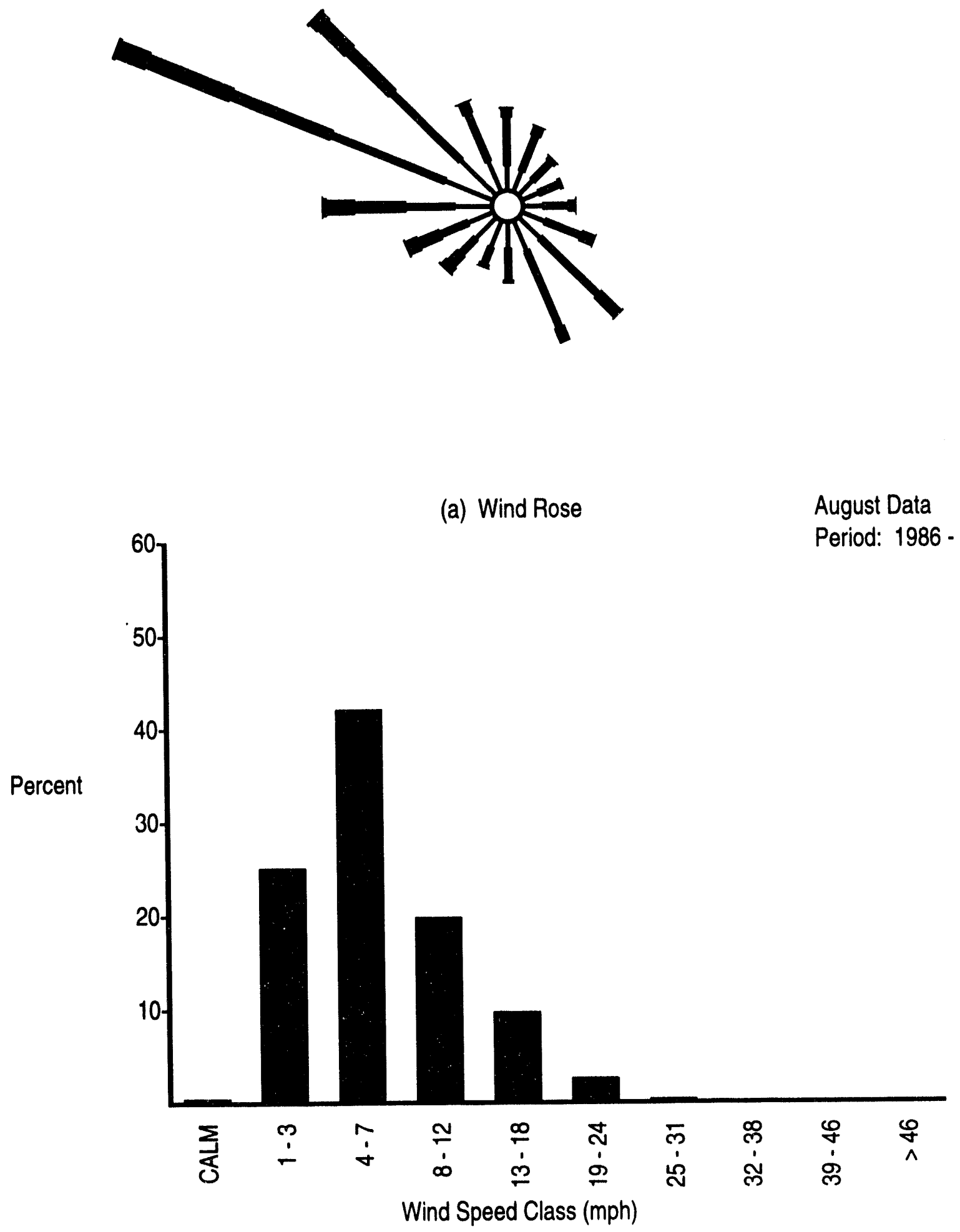

(b) Wind Speed Histogram

FIGURE B.1. (contd) 


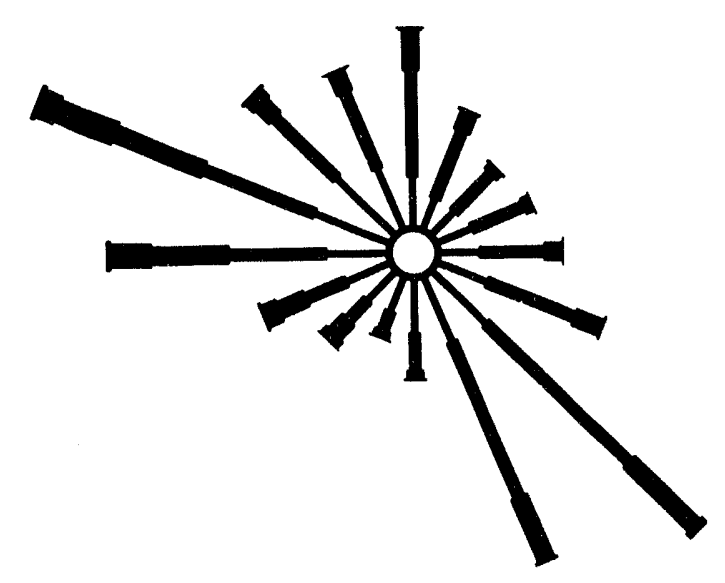

(a) Wind Rose

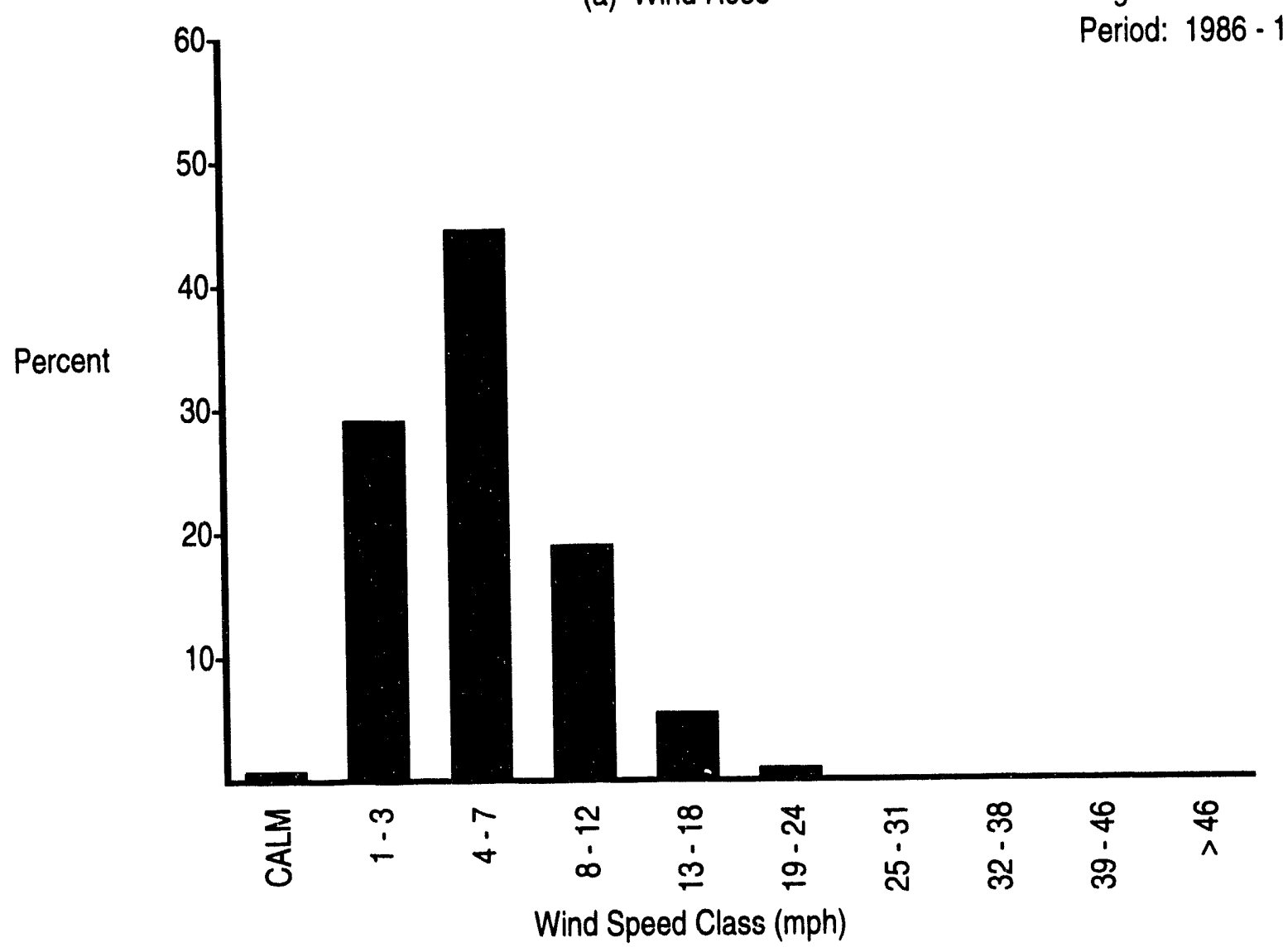

(b) Wind Speed Histogram

FIGURE B.1. (contd)
August Data

Period: 1986 - 1993

\section{B.214}



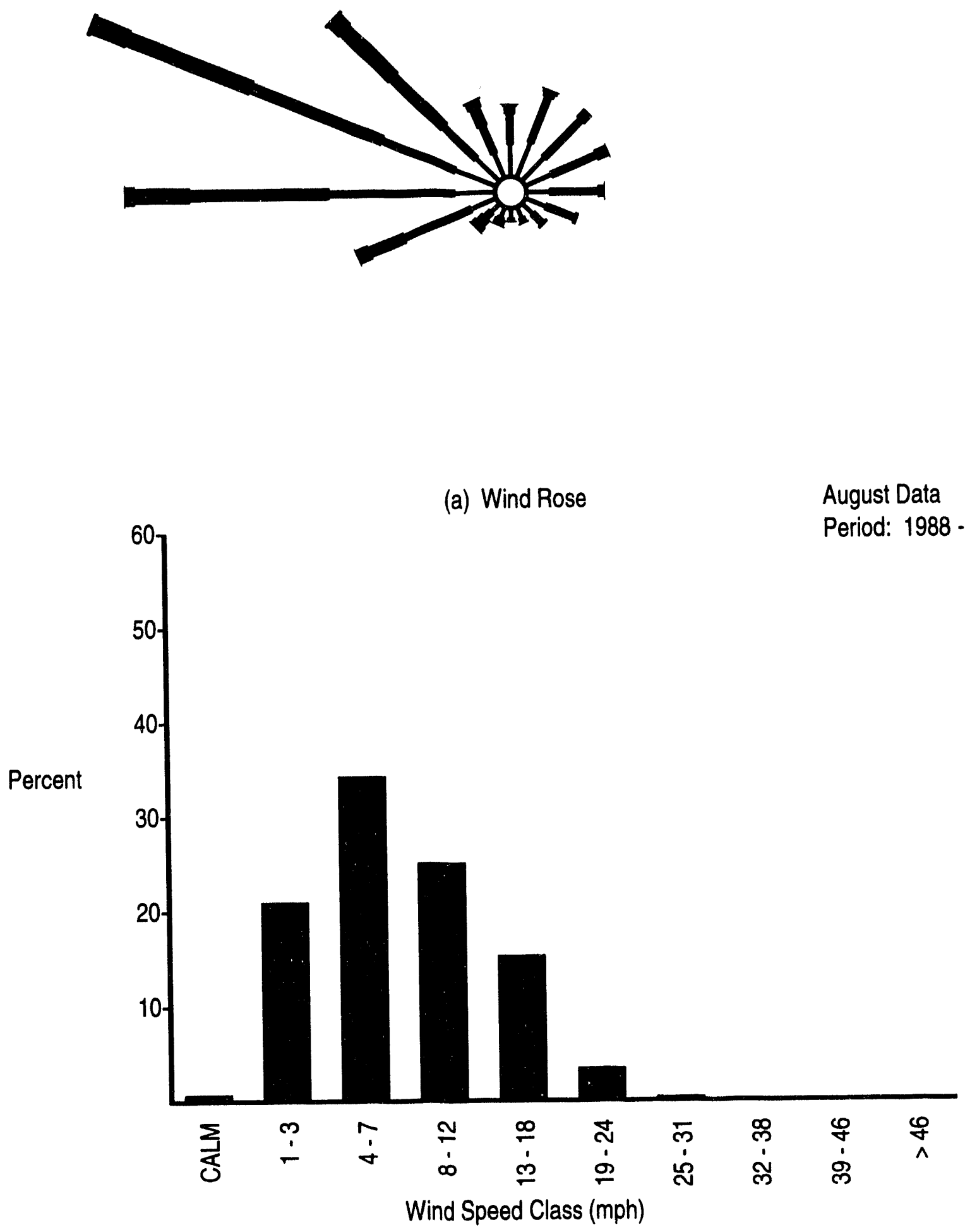

(b) Wind Speed Histogram

FIGURE B.1. (contd) 


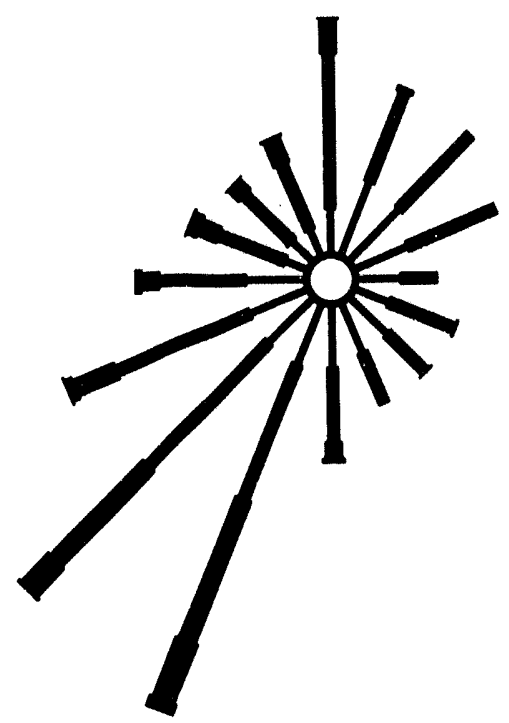

(a) Wind Rose

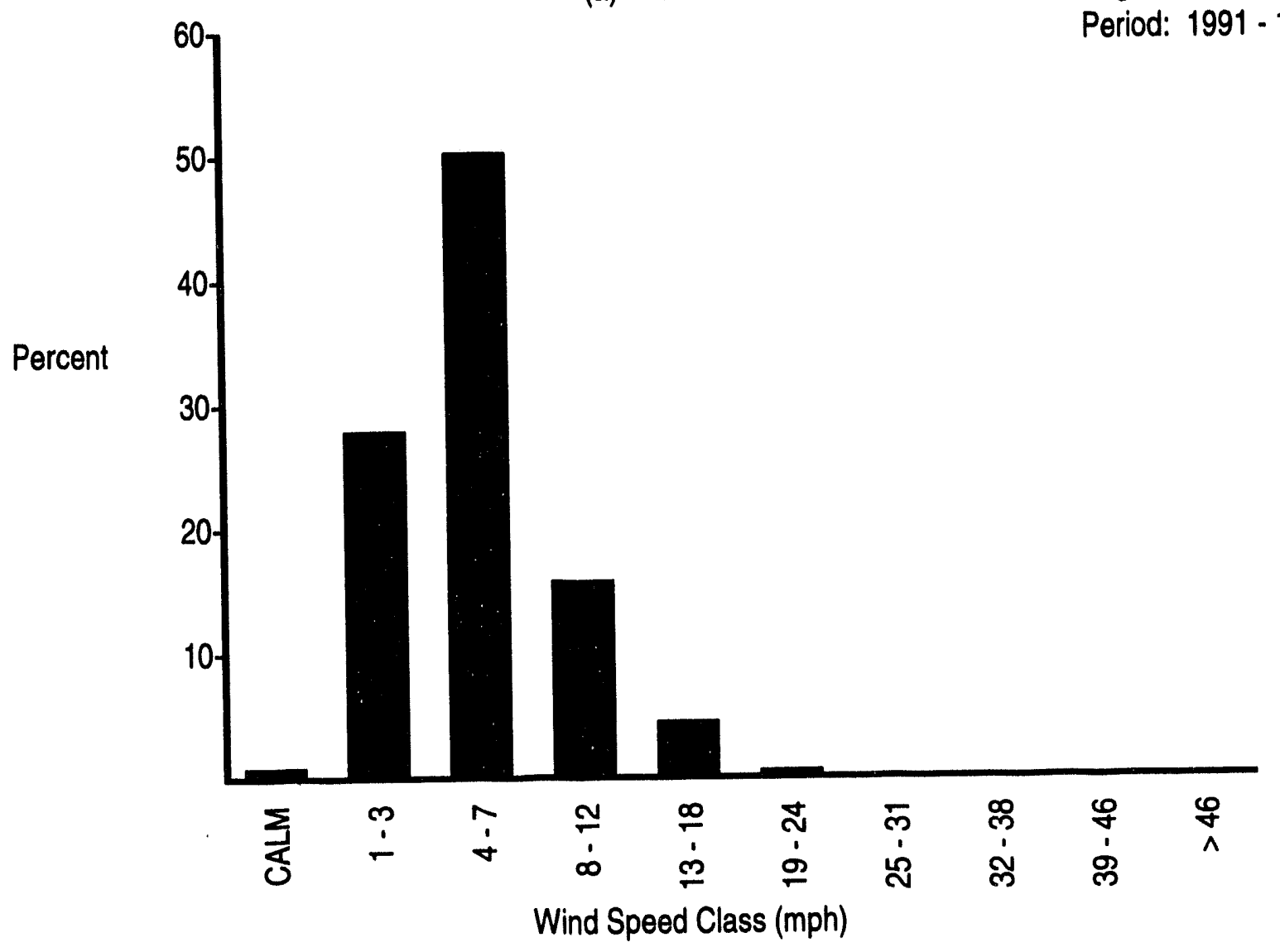

(b) Wind Speed Histogram

FIGURE B.1. (contd)
August Data

Period: 1991 - 1993 


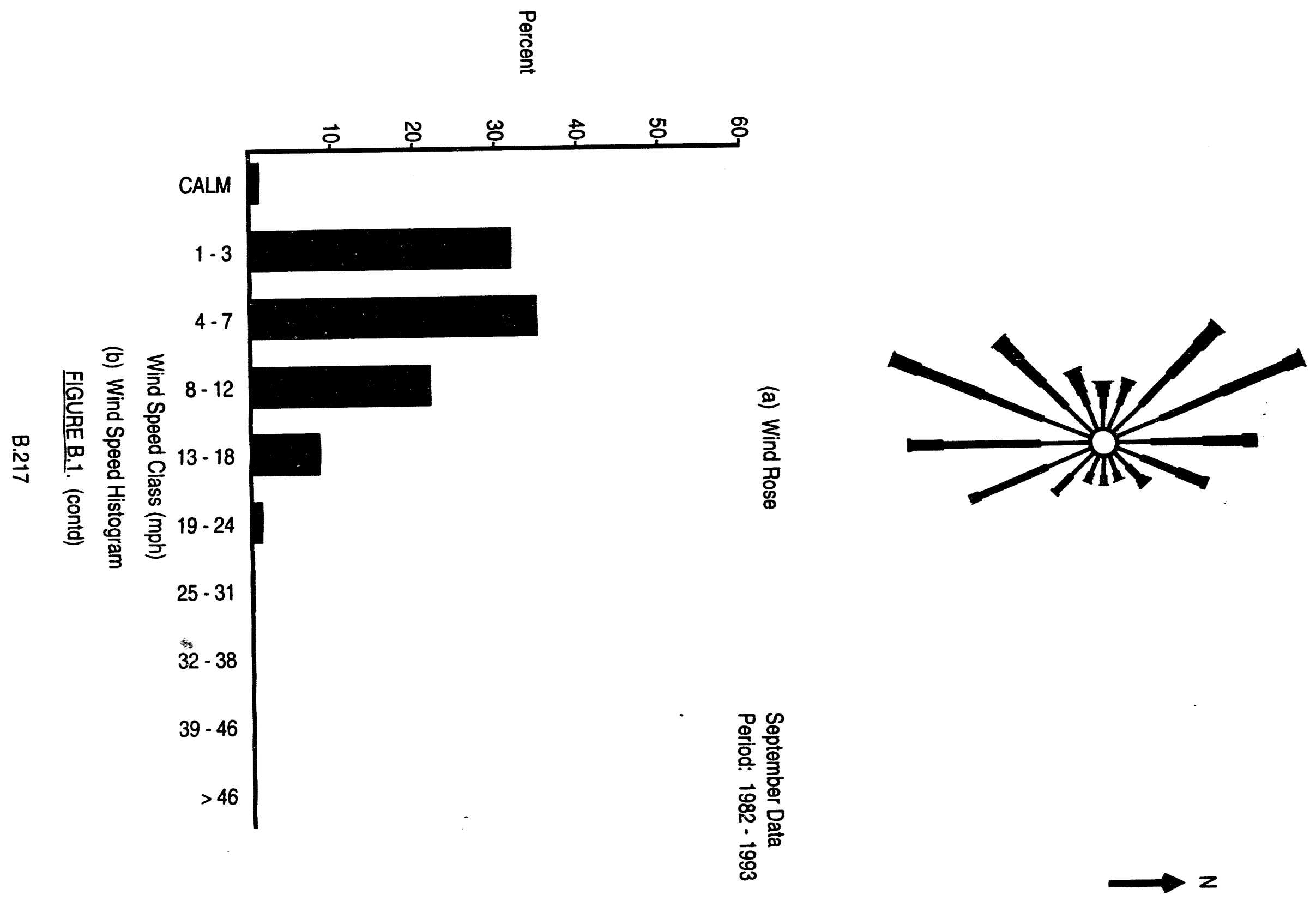



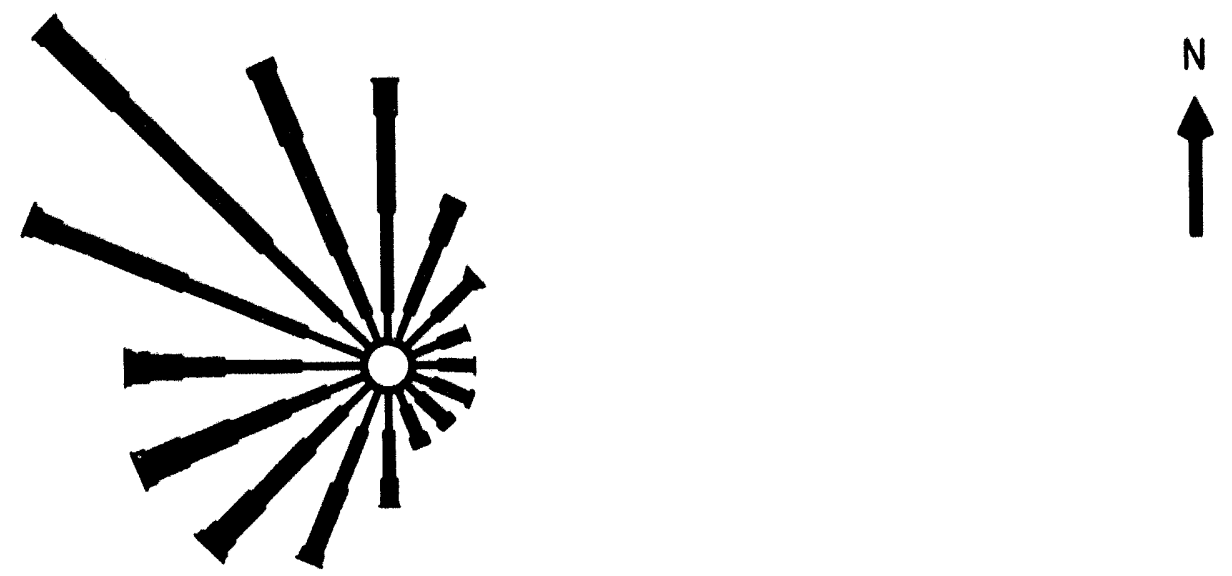

(a) Wind Rose

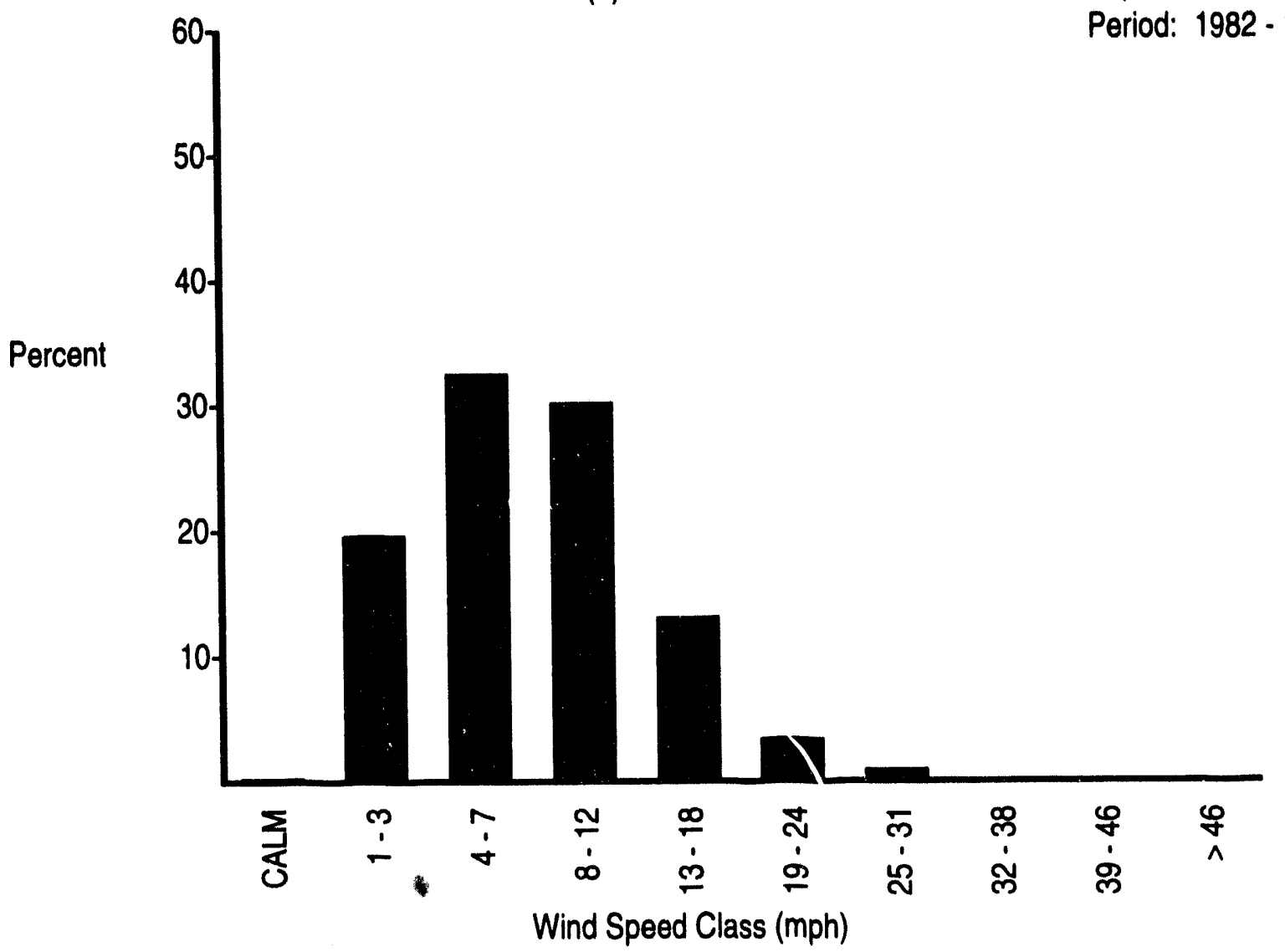

(b) Wind Speed Histogram

FIGURE B.1. (contd) 

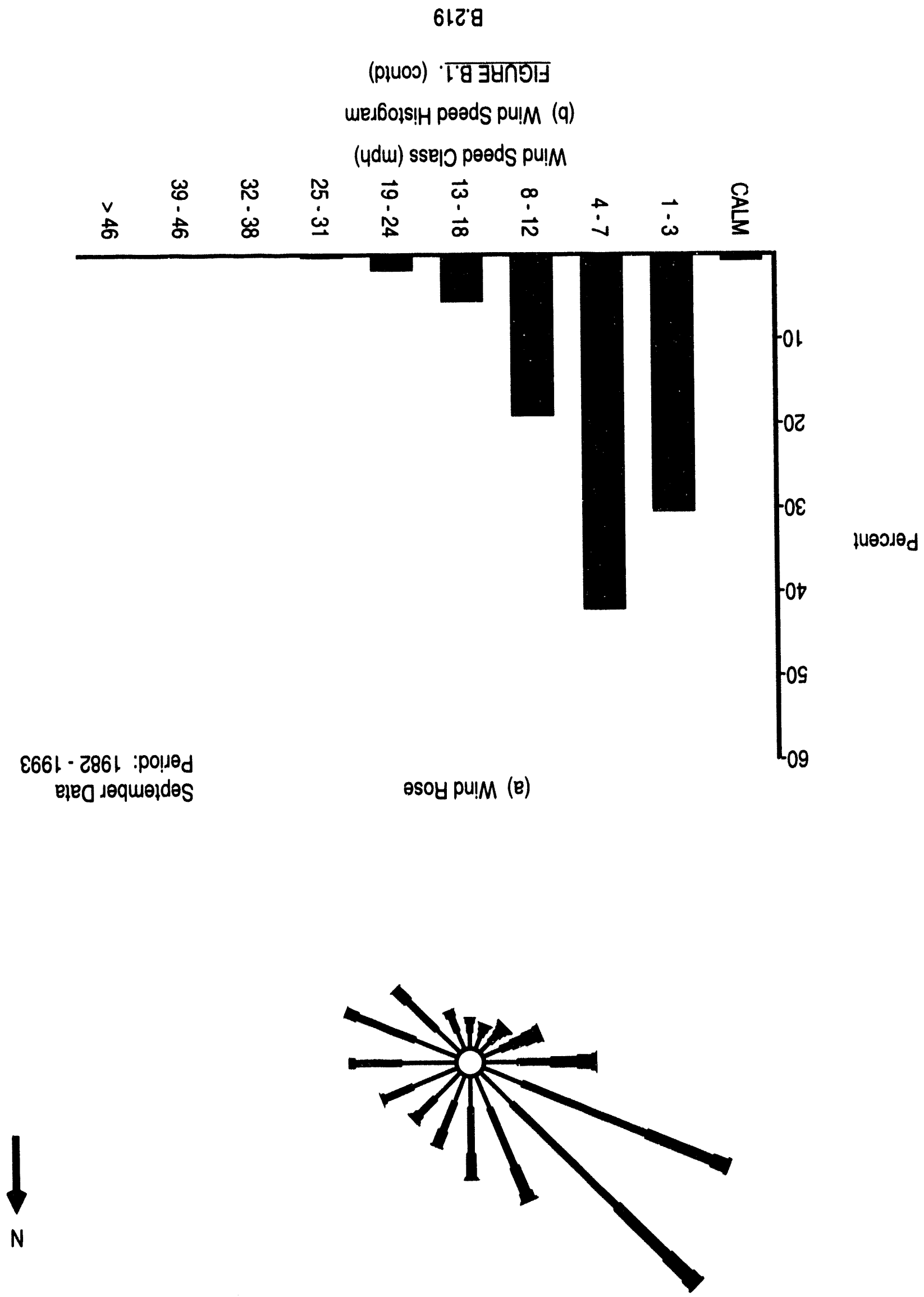


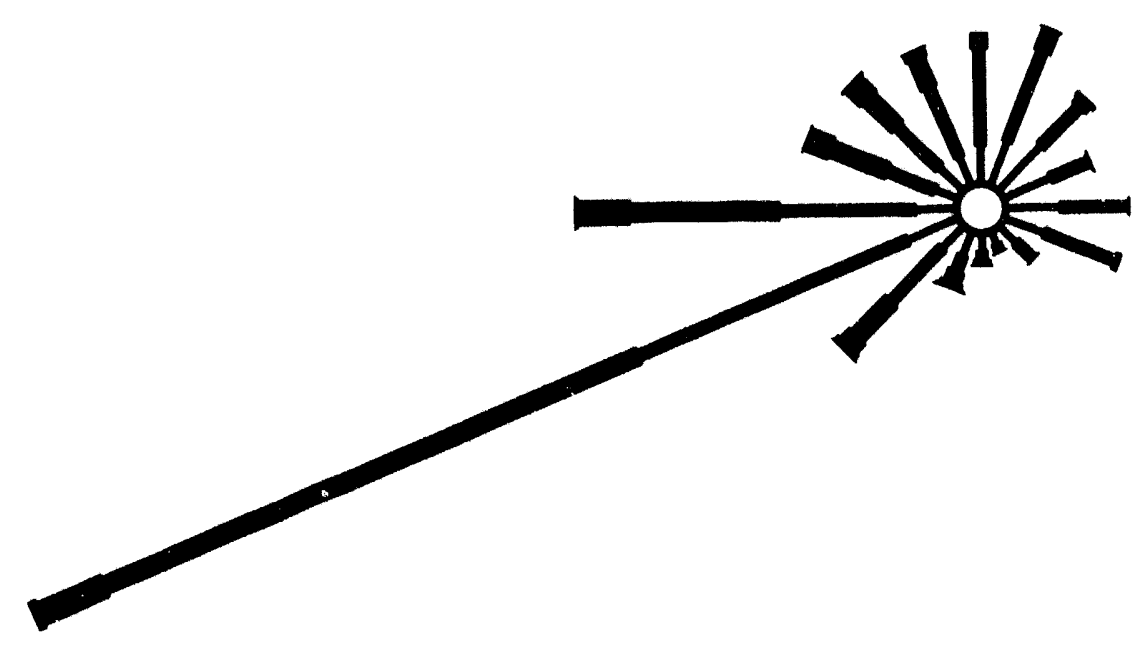

(a) Wind Rose

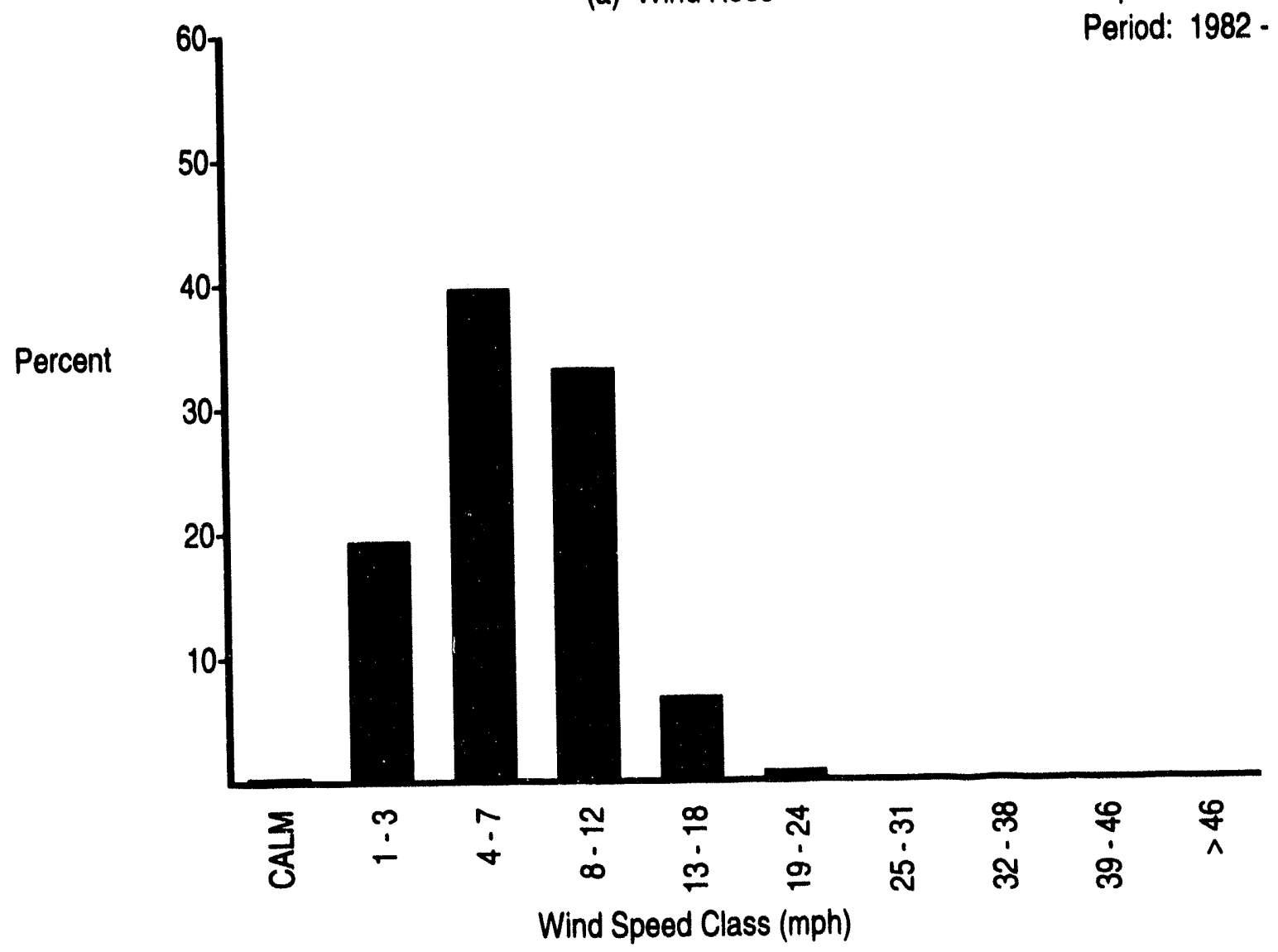

(b) Wind Speed Histogram

FIGURE B.1. (contd) 


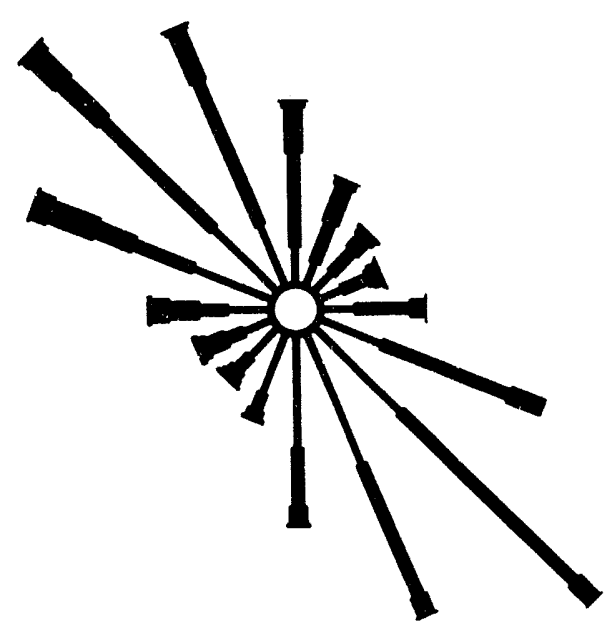

(a) Wind Rose

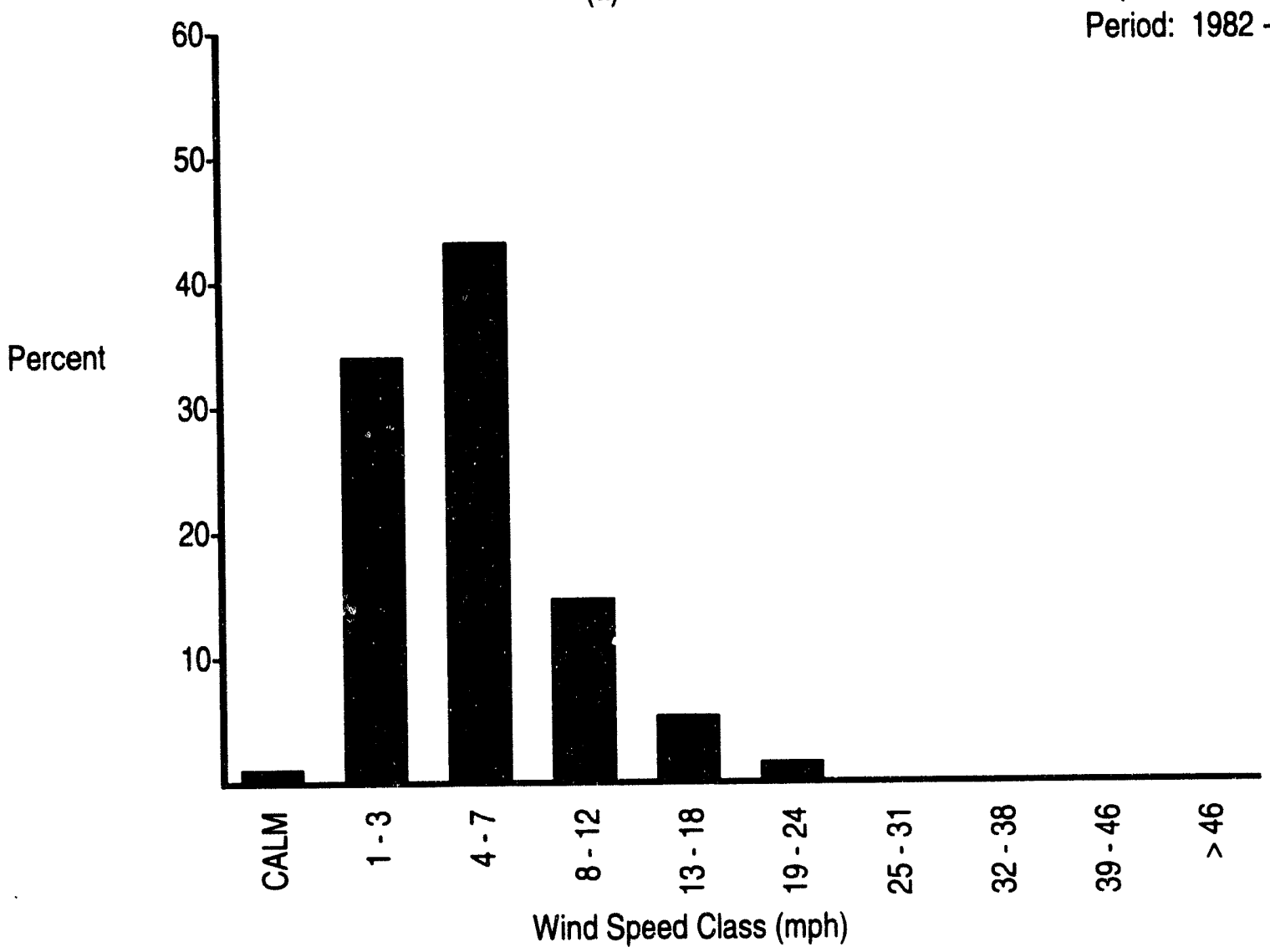

(b) Wind Speed Histogram

FIGURE B.1. (contd)
September Data

Period: 1982 - 1993 


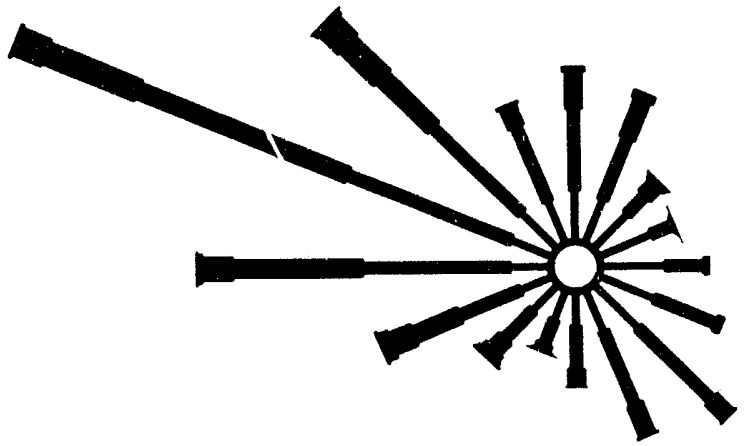

(a) Wind Rose

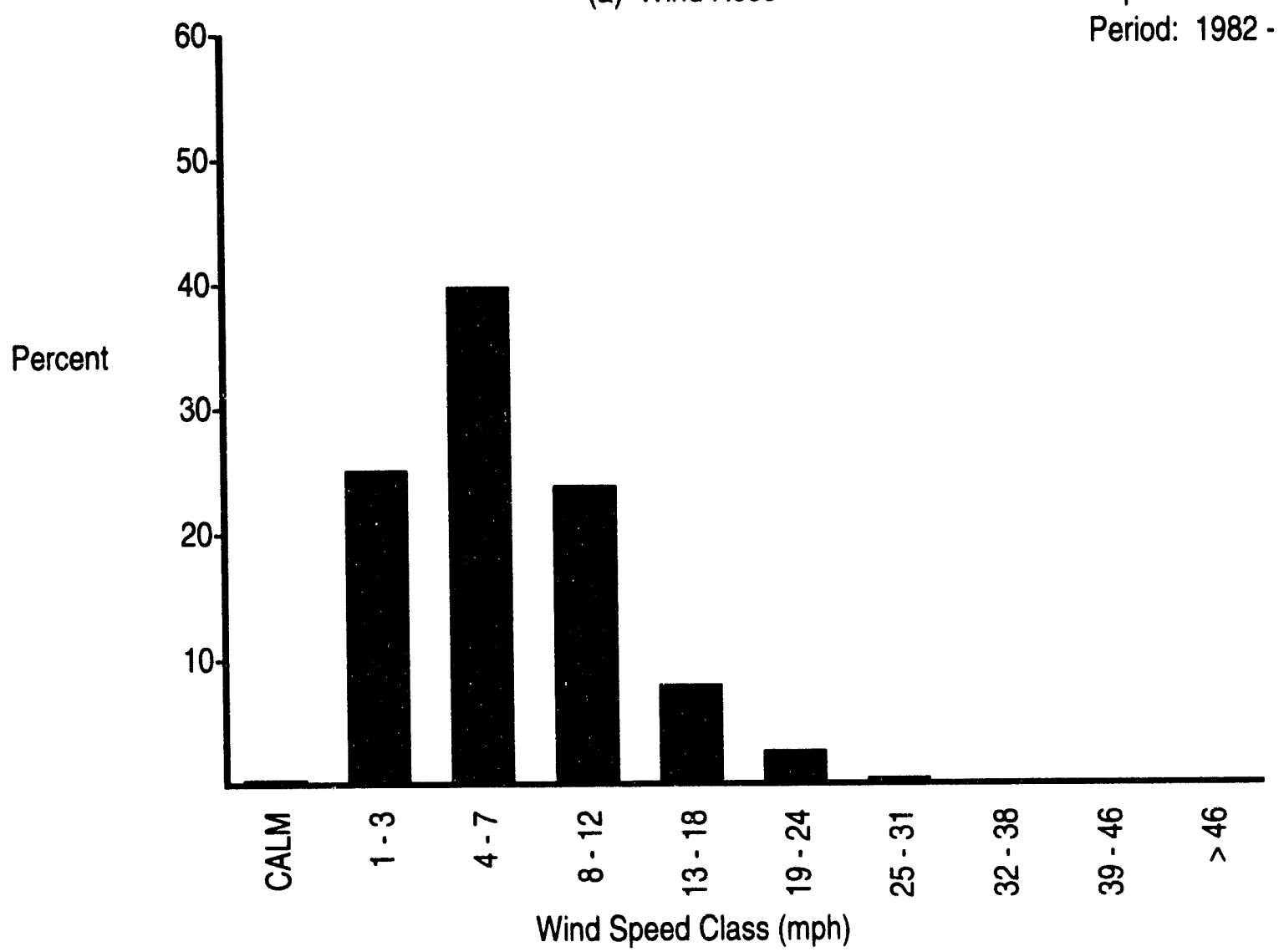

(b) Wind Speed Histogram
September Data

Period: $1982-1993$

\section{FIGURE B.1. (contd)}




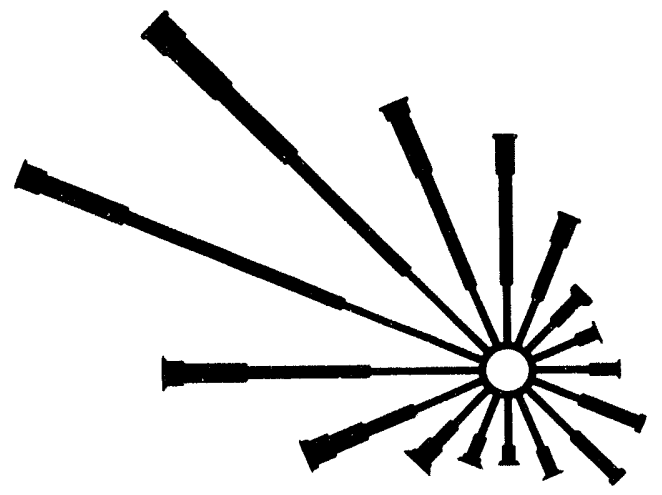

(a) Wind Rose

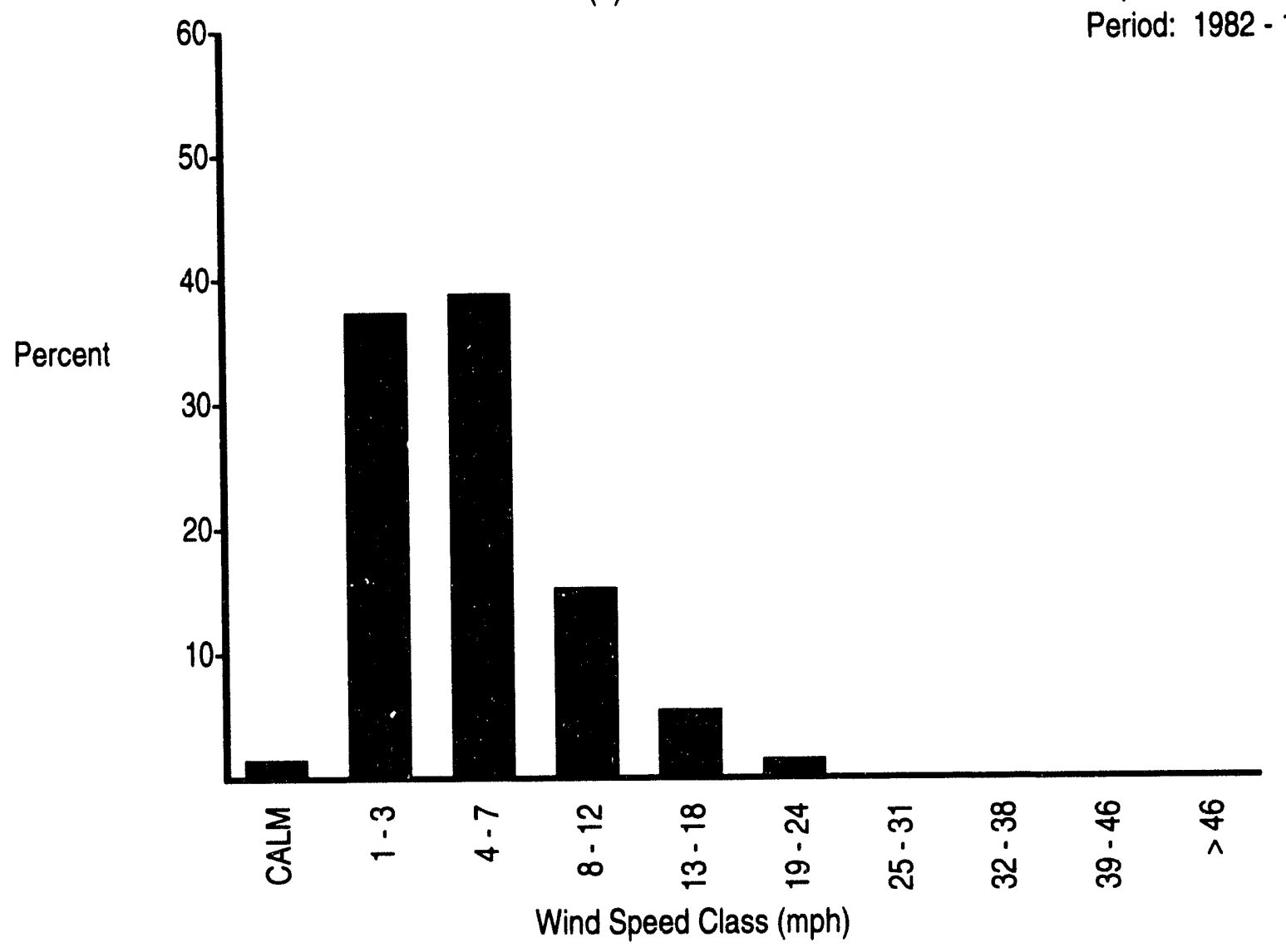

(b) Wind Speed Histogram

FIGURE B.1. (contd) 


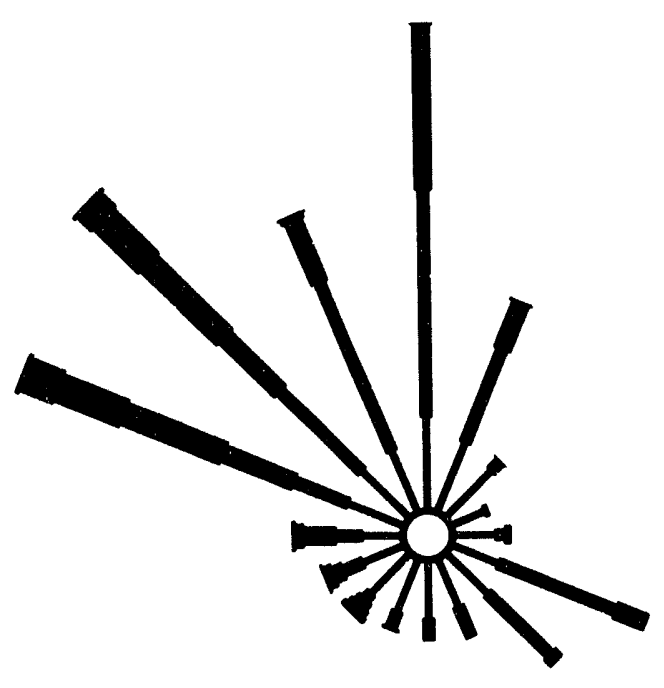

(a) Wind Rose

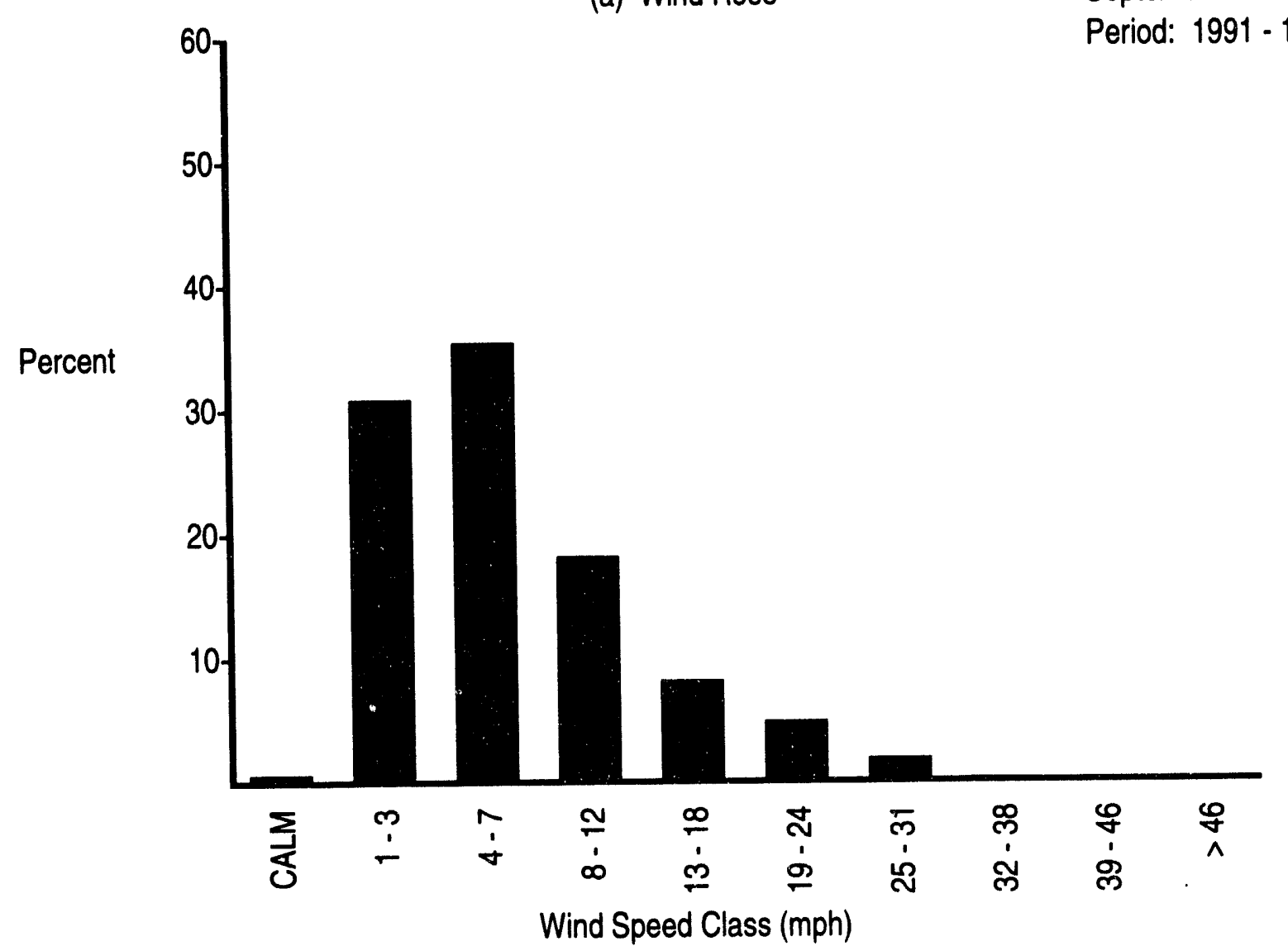

(b) Wind Speed Histogram

FIGURE B.1. (contd)
September Data

Period: 1991 - 1993 
S२2'8

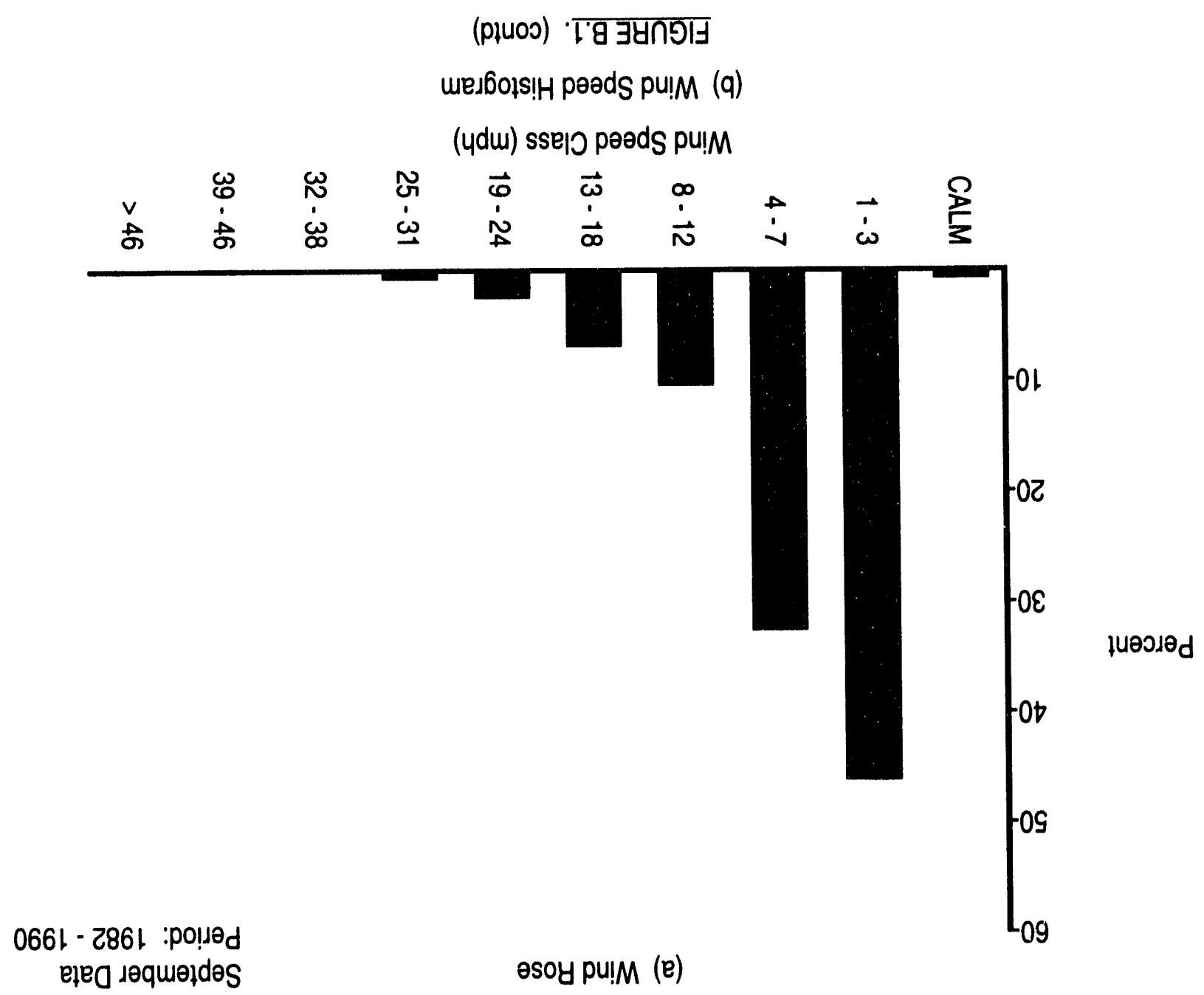

eleg dequejdes

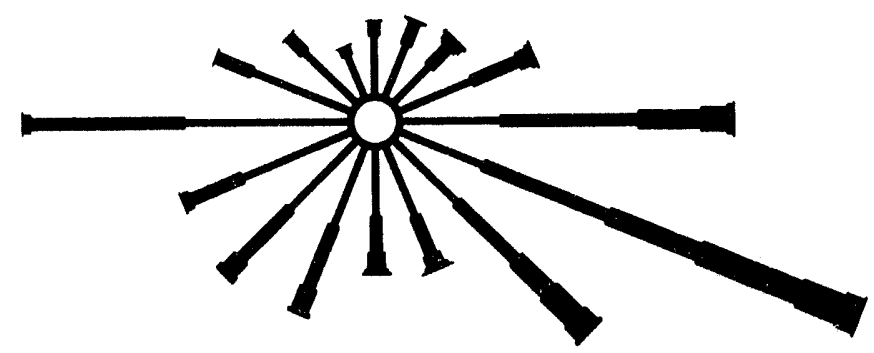

N 


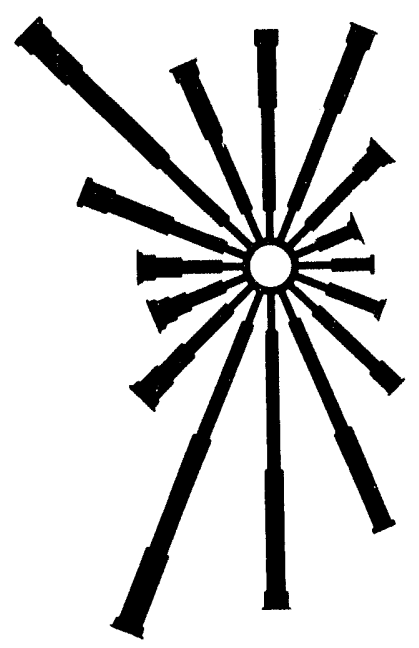

(a) Wind Rose

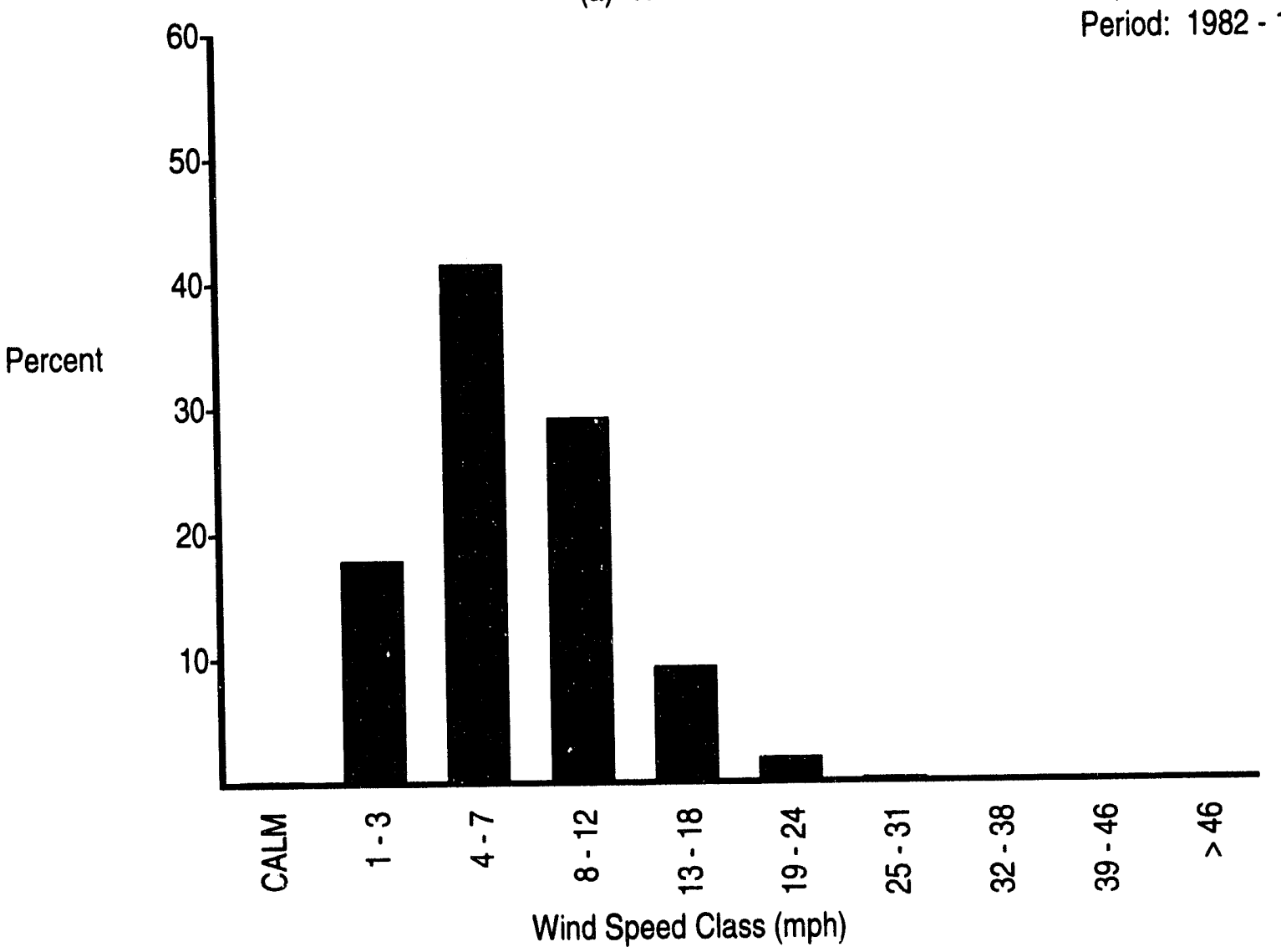

(b) Wind Speed Histogram

FIGURE B.1. (contd) 


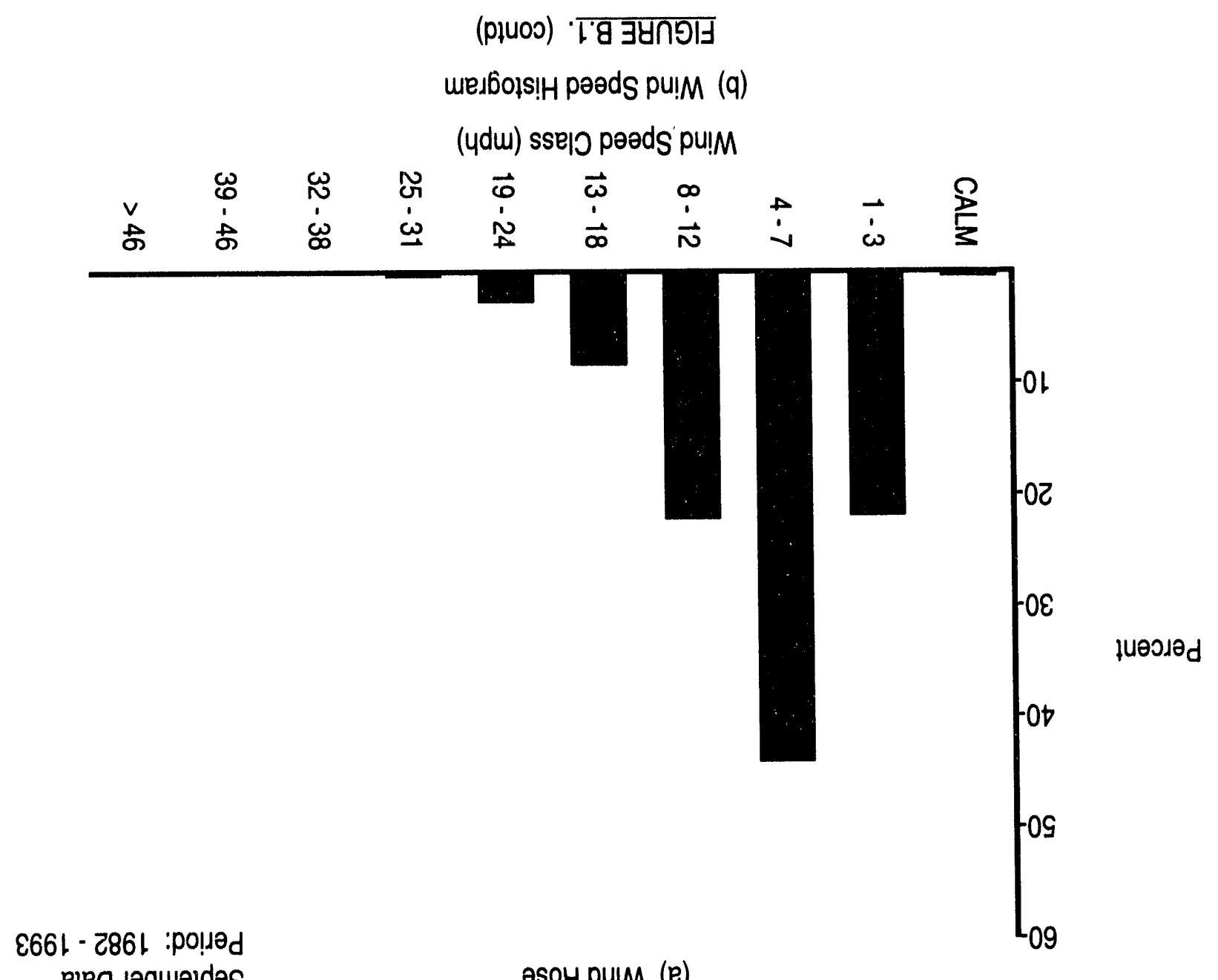

eleo səqueides 


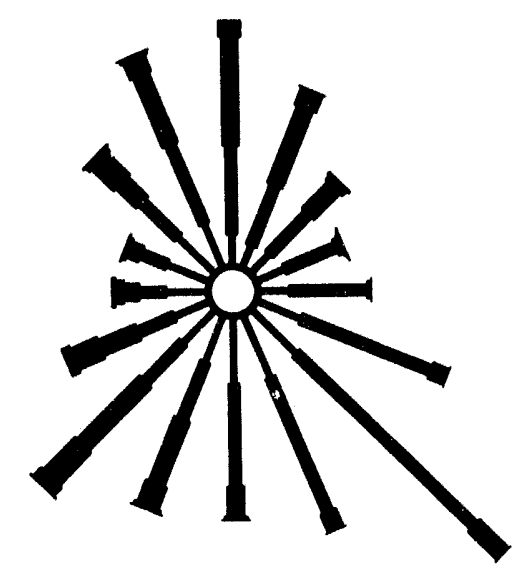

(a) Wind Rose

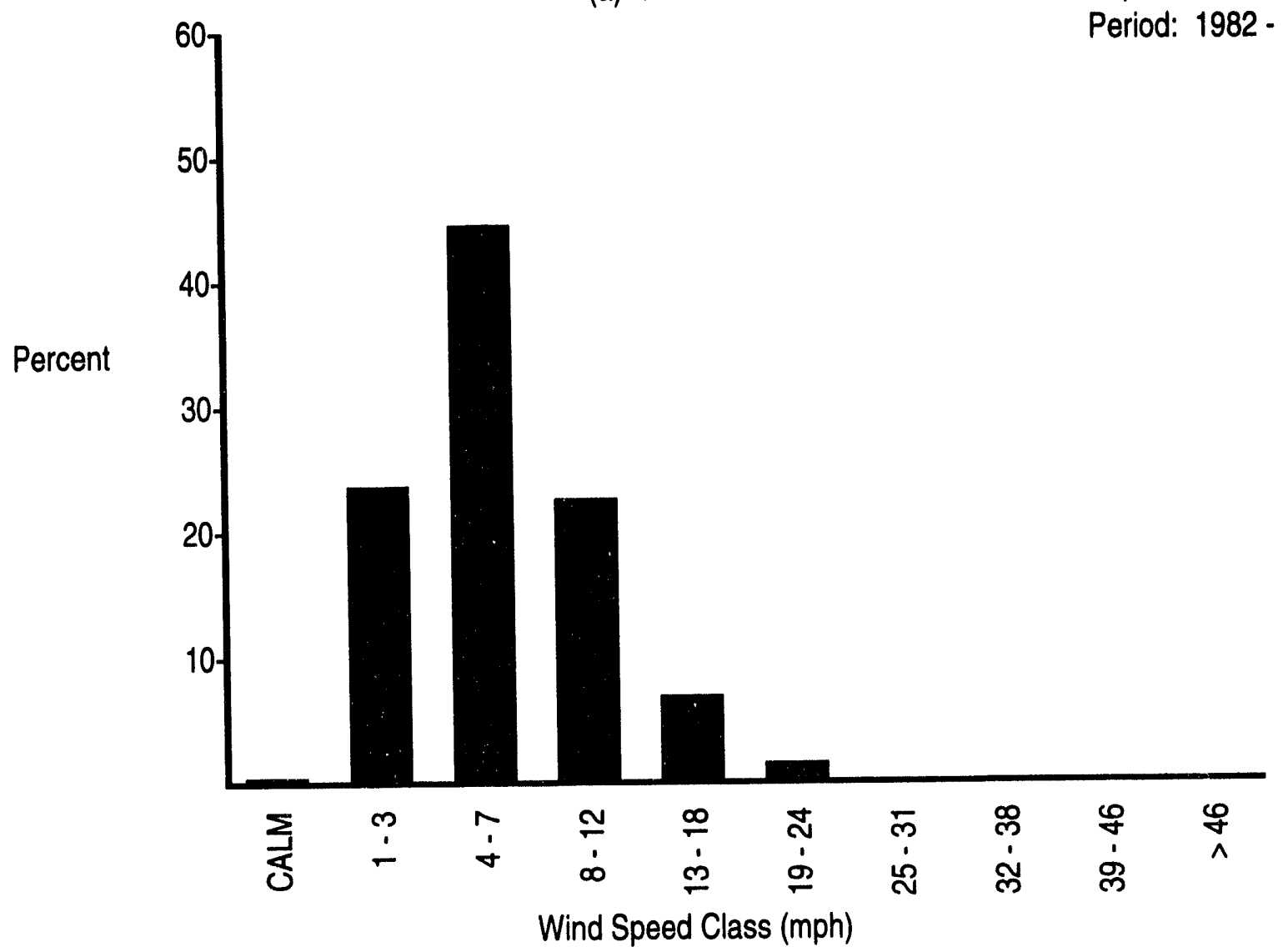

(b) Wind Speed Histogram

FIGURE B.1. (contd)
September Data

Period: $1982-1993$ 


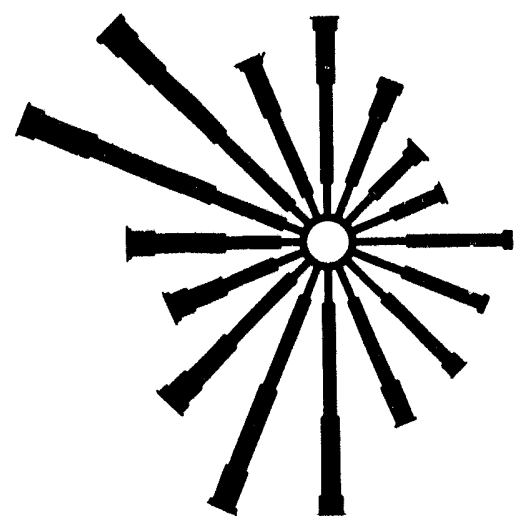

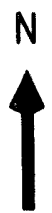

(a) Wind Rose

September Data

Period: 1982 - 1993

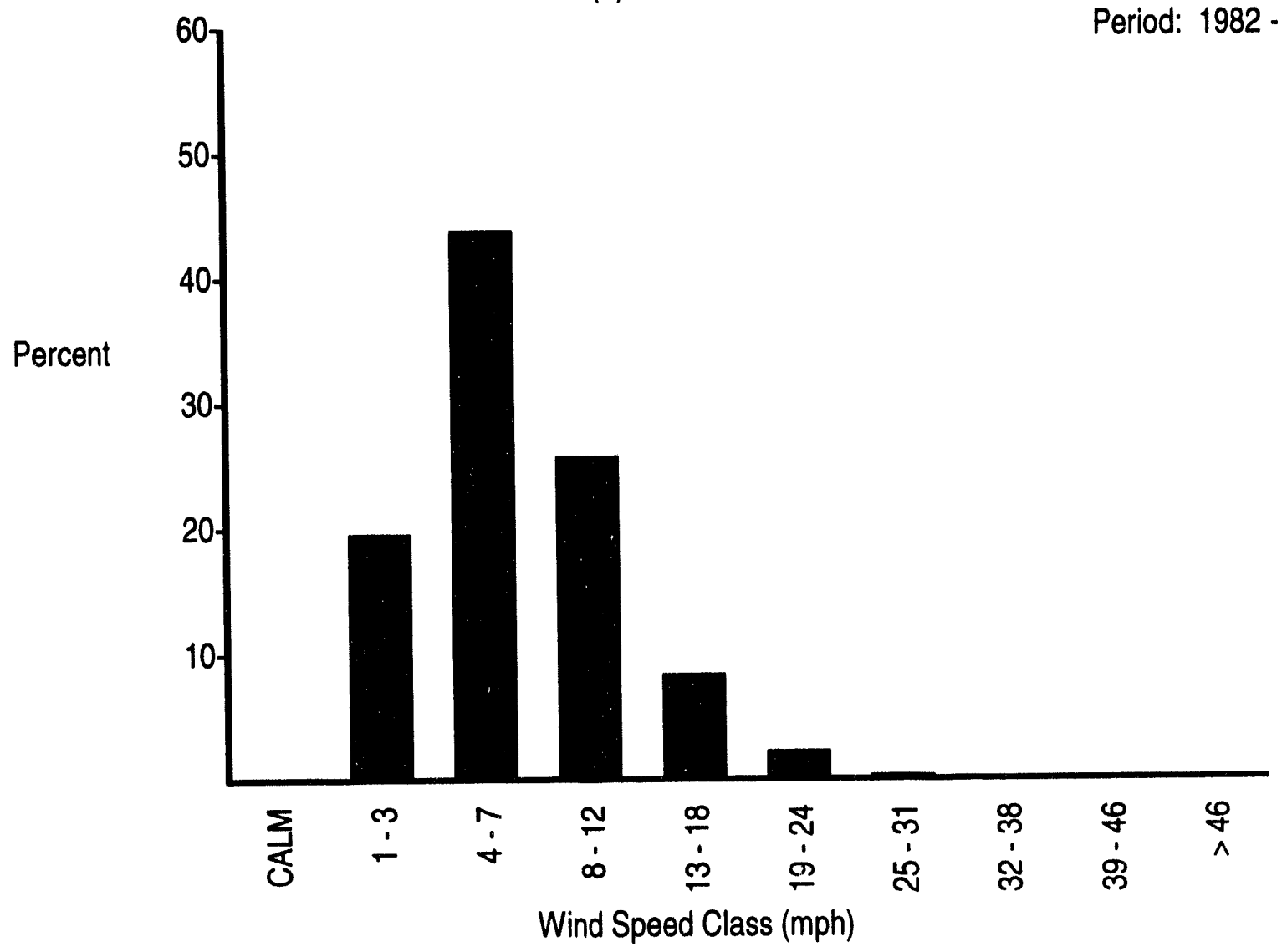

(b) Wind Speed Histogram

FIGURE B.1. (contd) 


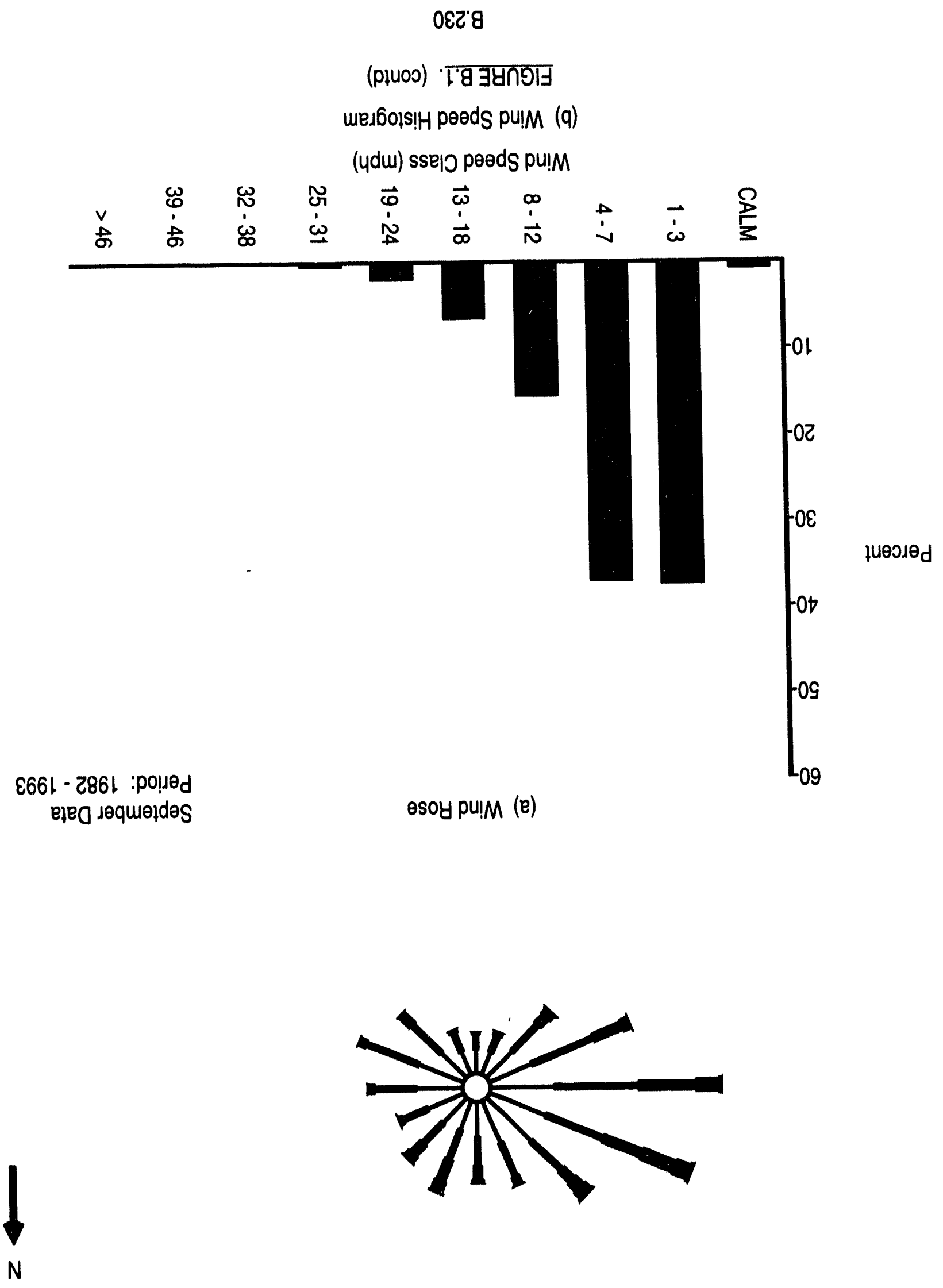




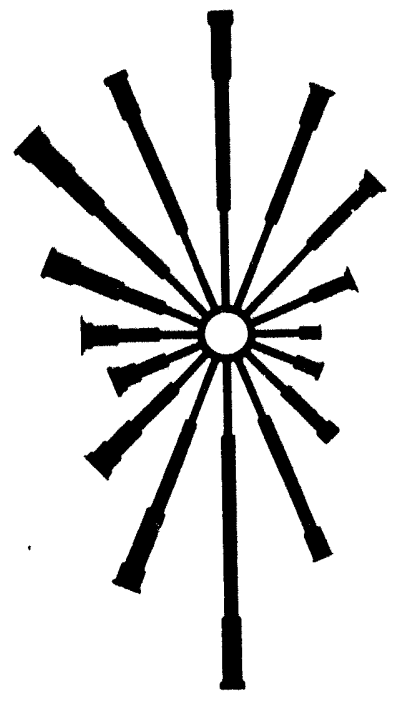

(a) Wind Rose

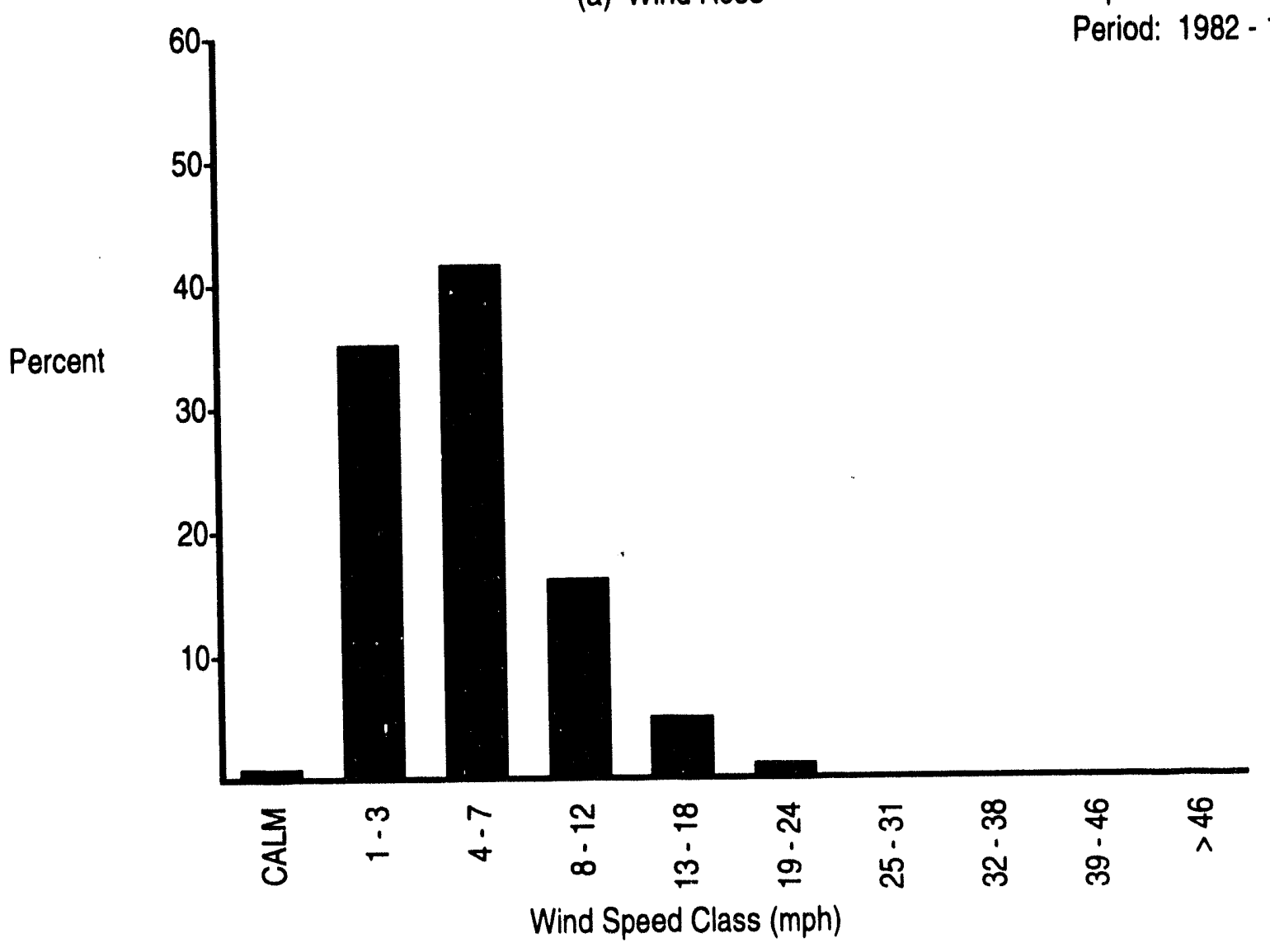

(b) Wind Speed Histogram

FIGURE B.1. (contd)
September Data

Period: 1982 - 1993 


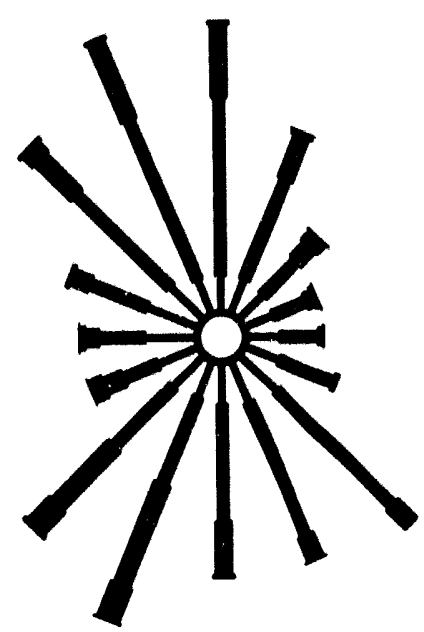

(a) Wind Rose

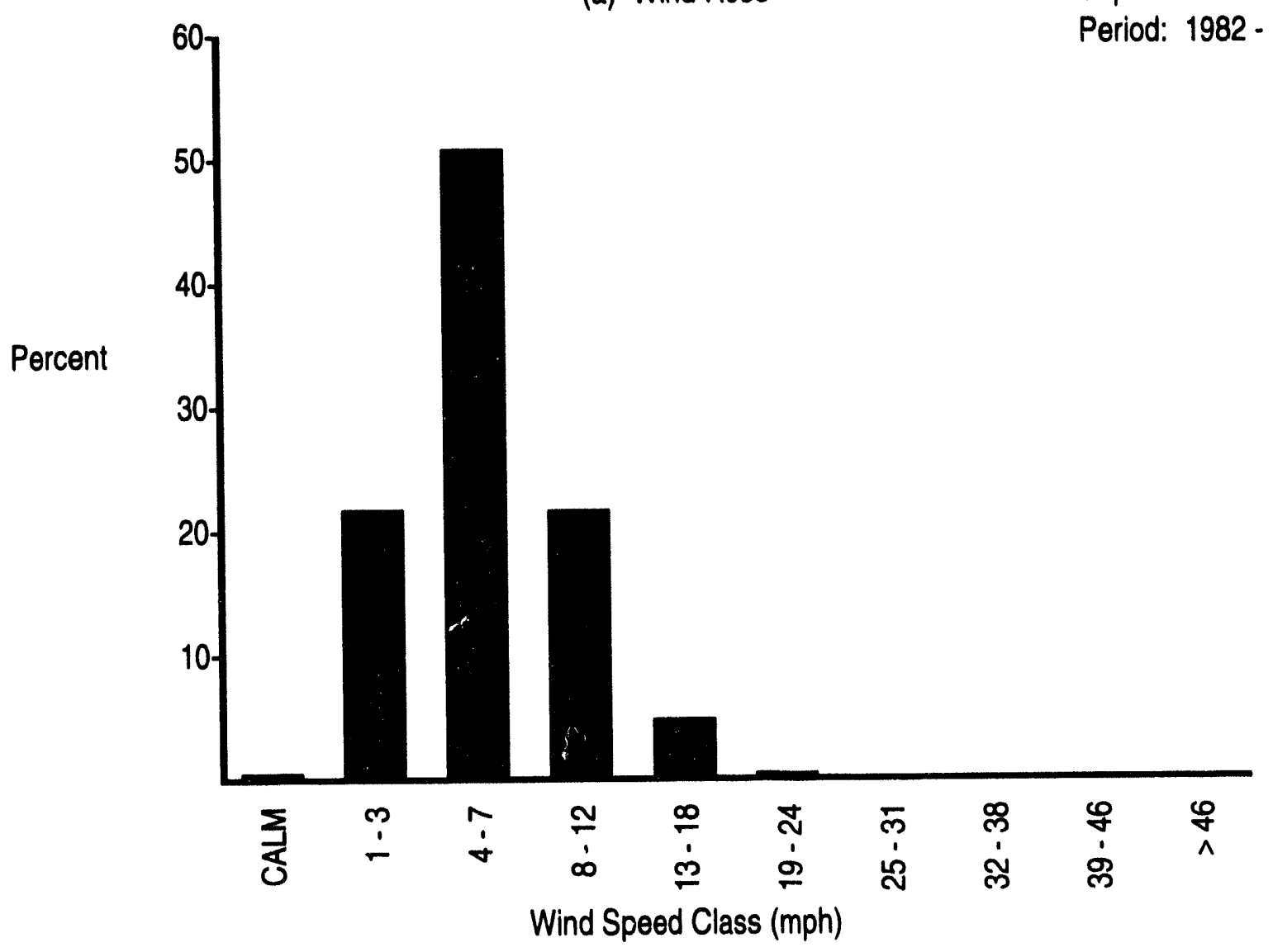

(b) Wind Speed Histogram

FIGURE B.1. (contd)
September Data

Period: 1982 - 1993

\section{B.232}




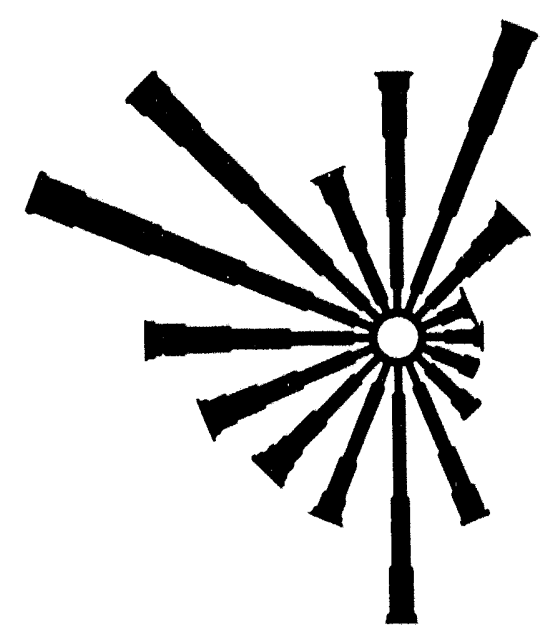

(a) Wind Rose

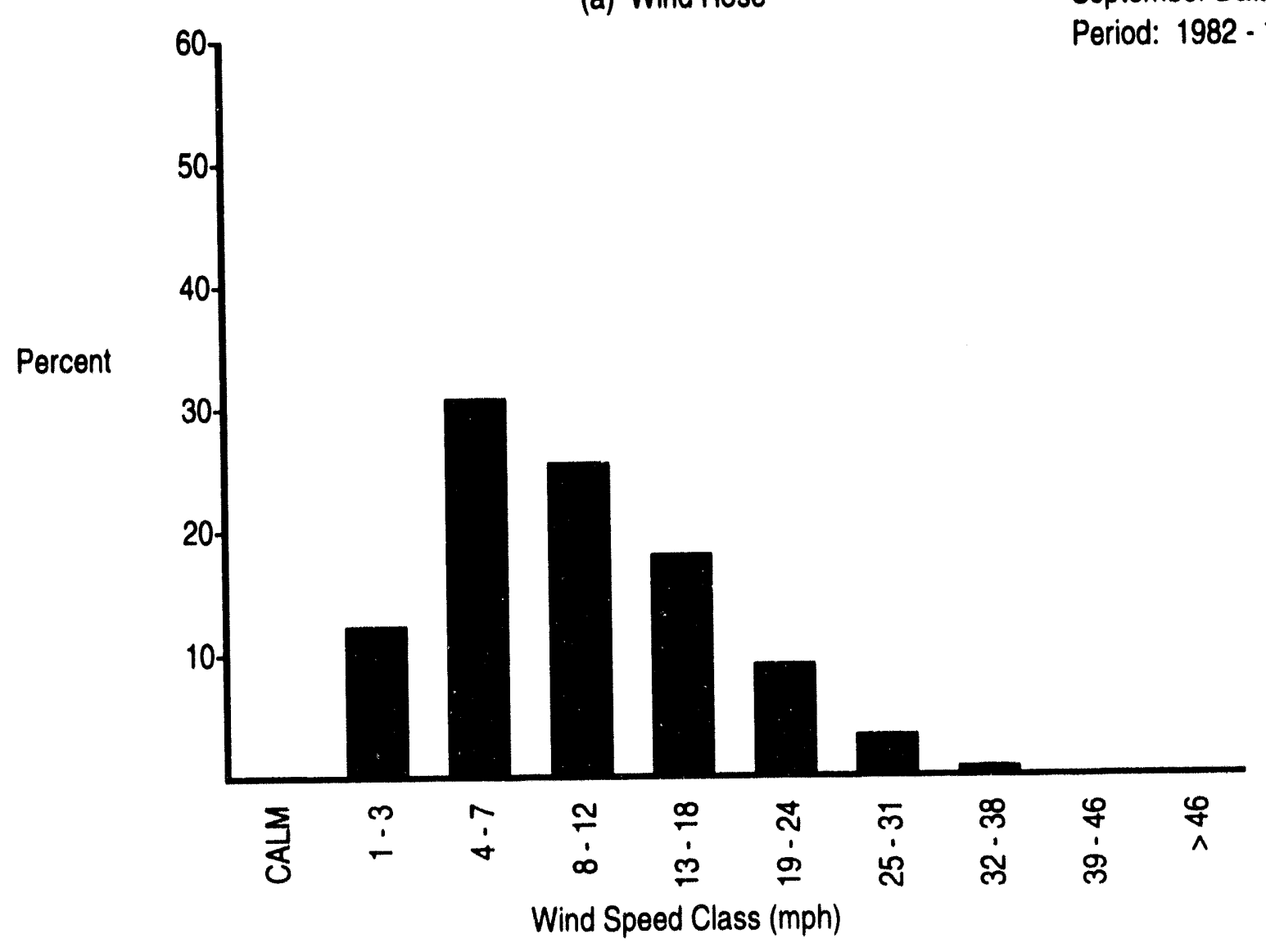

(b) Wind Speed Histogram

FIGURE B.1. (contd)
September Data

Period: 1982 - 1993 


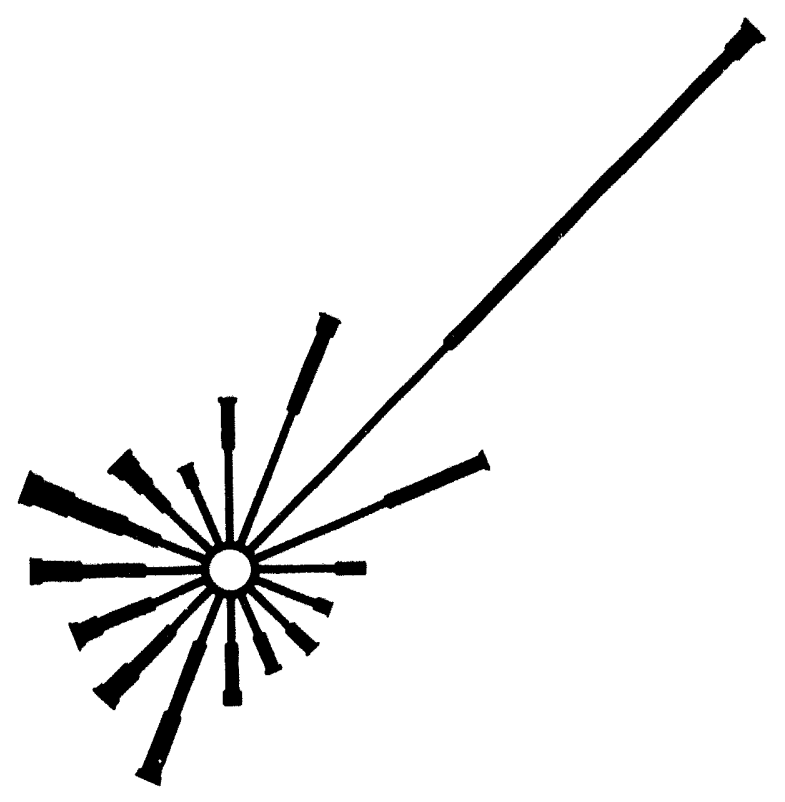

$\hat{N}$

(a) Wind Rose

September Data

Percent

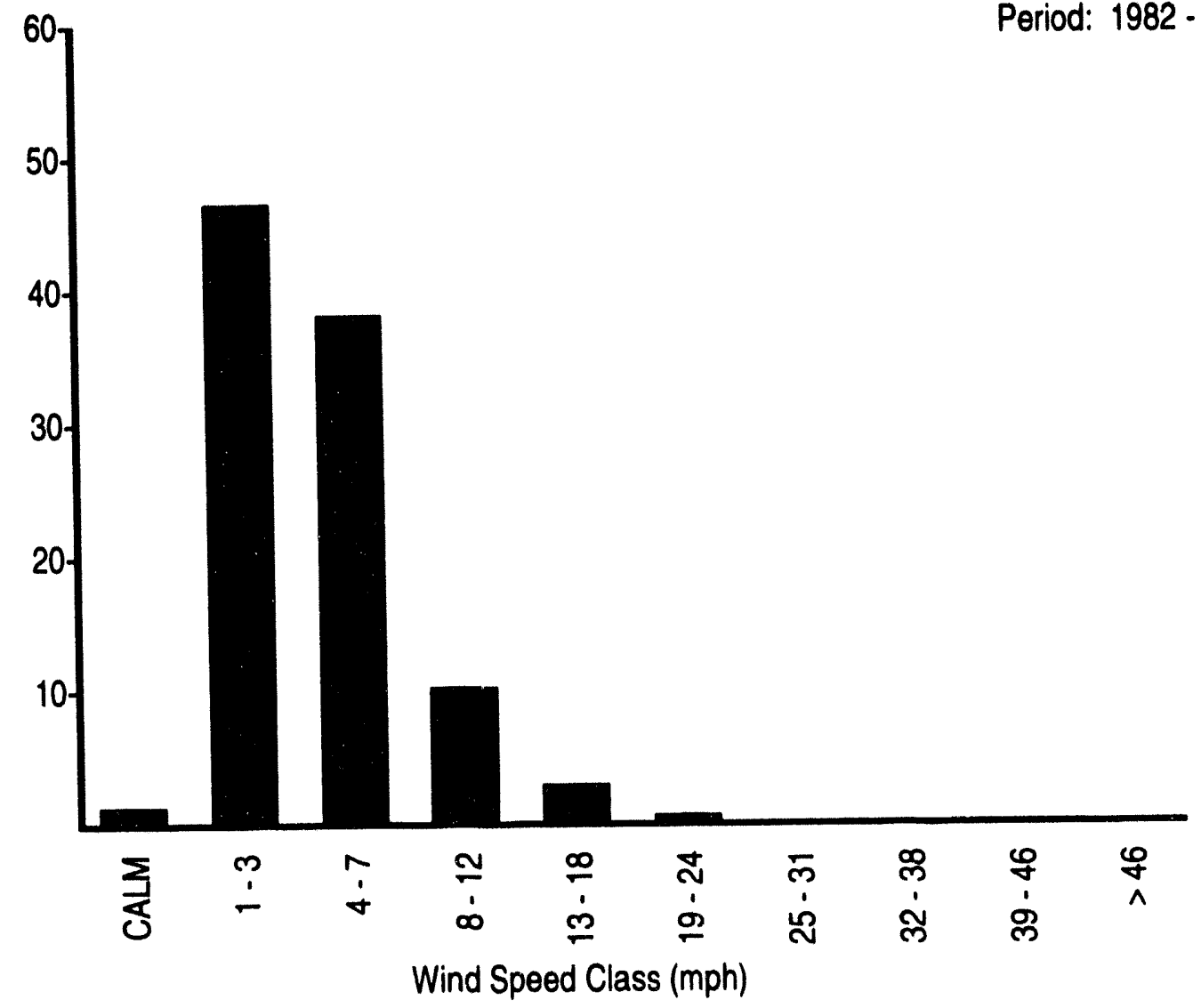

(b) Wird Speed Histogram

FIGURE B.1. (contd) 


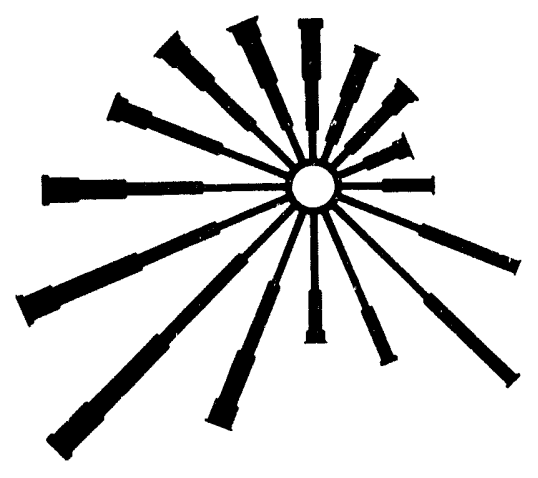

N

(a) Wind Rose

September Data

Period: $1982-1993$

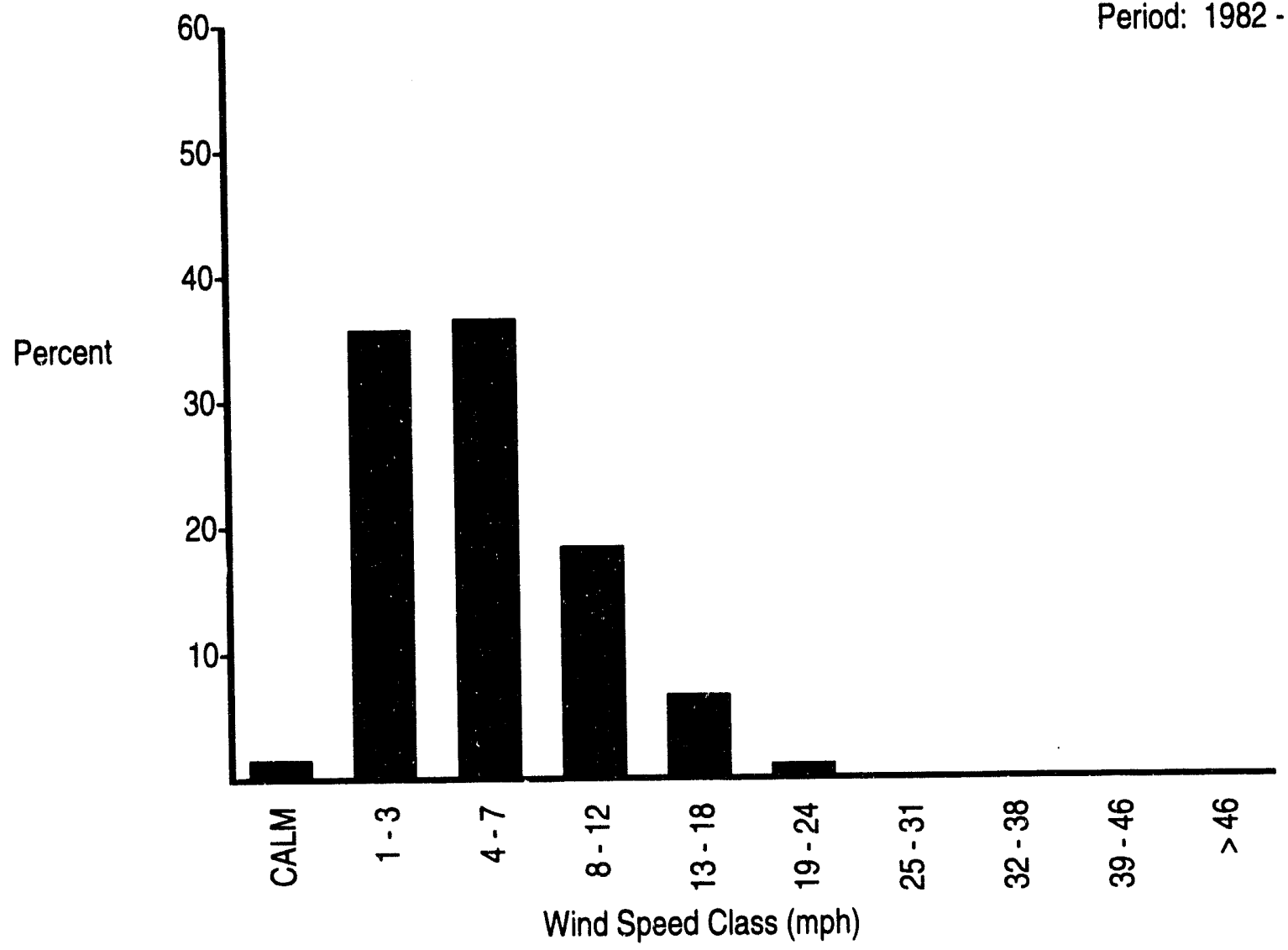

(b) Wind Speed Histogram

FIGURE B.1. (contd) 


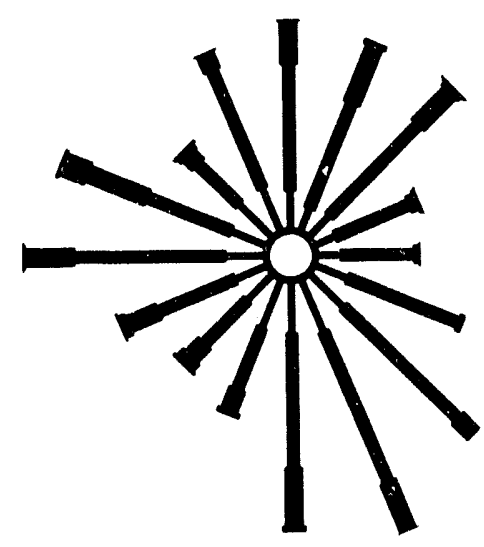

(a) Wind Rose

September Data

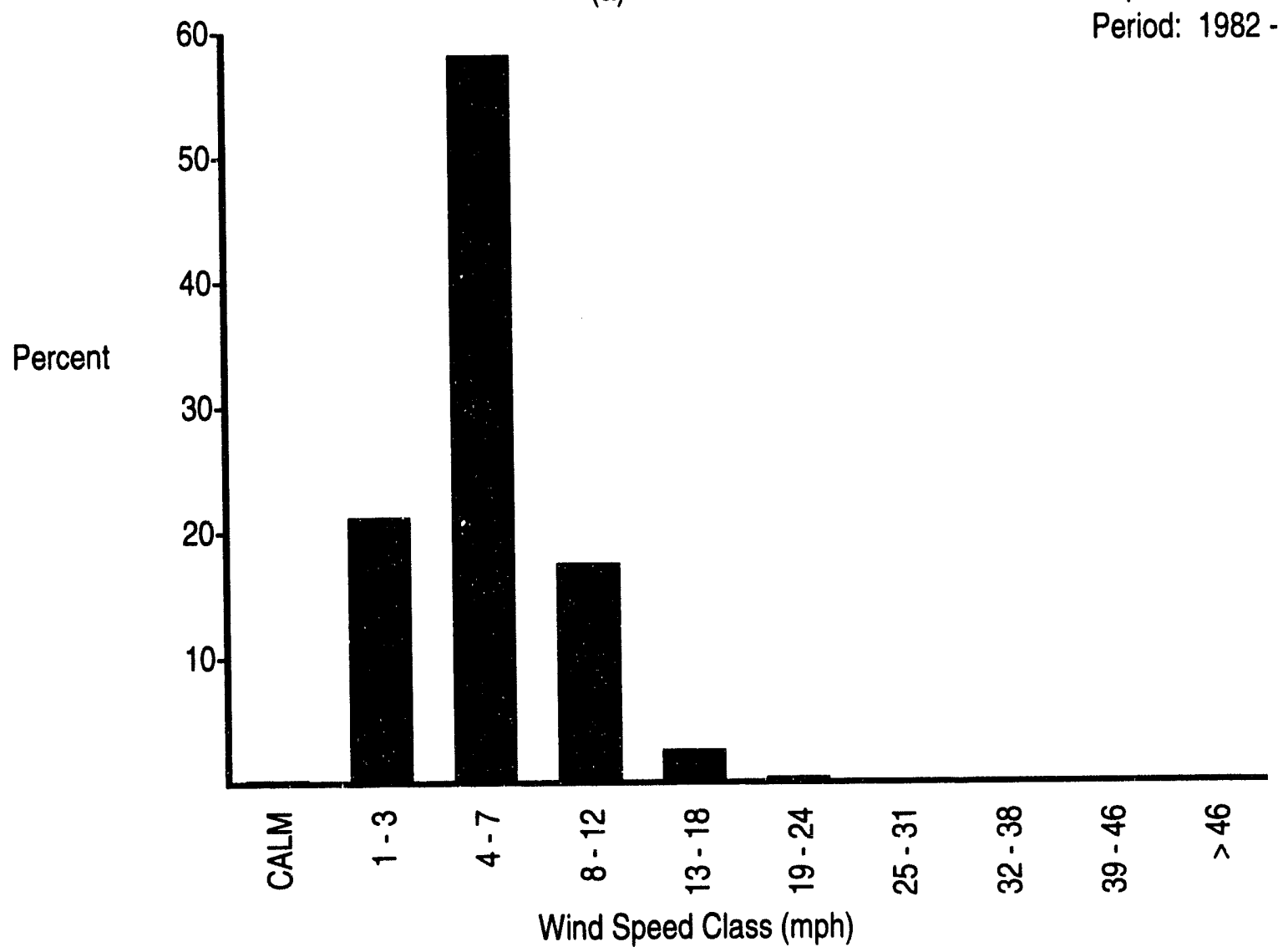

(b) Wind Speed Histogram

FIGURE B.1. (contd) 


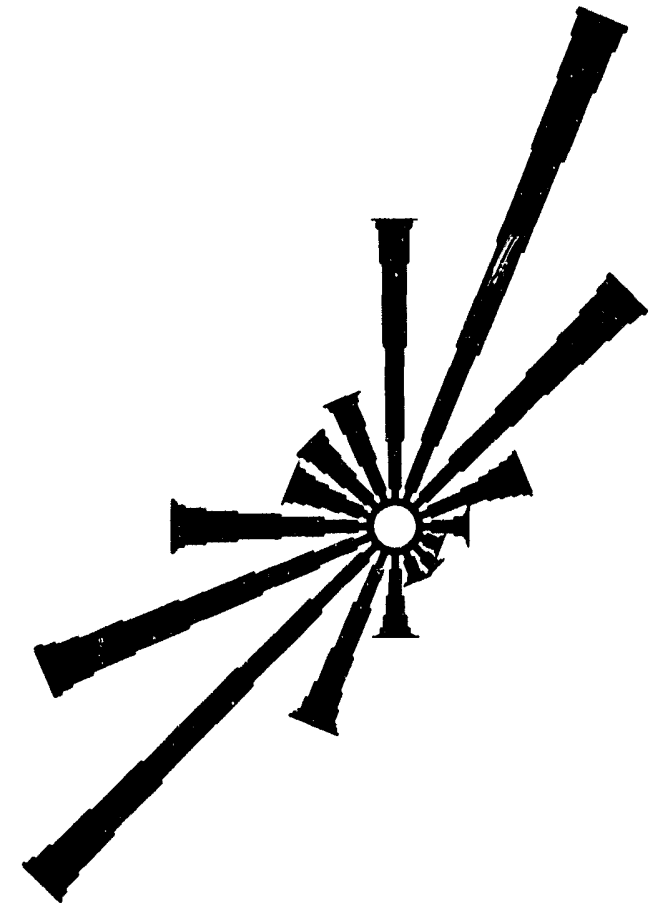

(a) Wind Rose

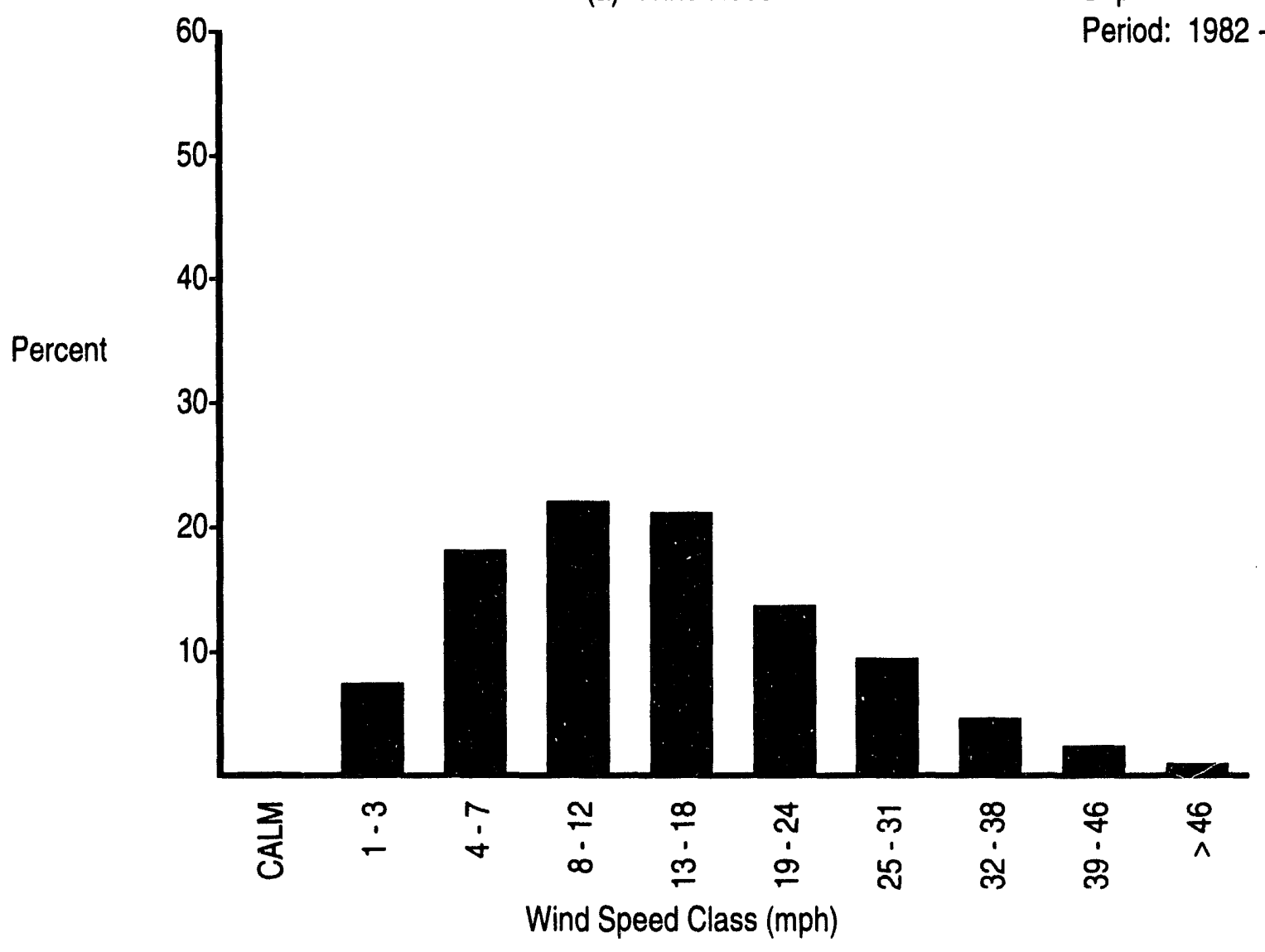

(b) Wind Speed Histogram

FIGURE B.1. (contd)
September Data

Period: 1982 - 1993 


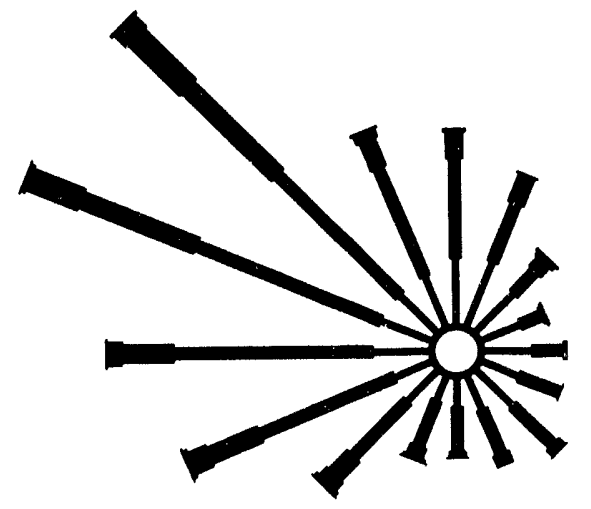

(a) Wind Rose

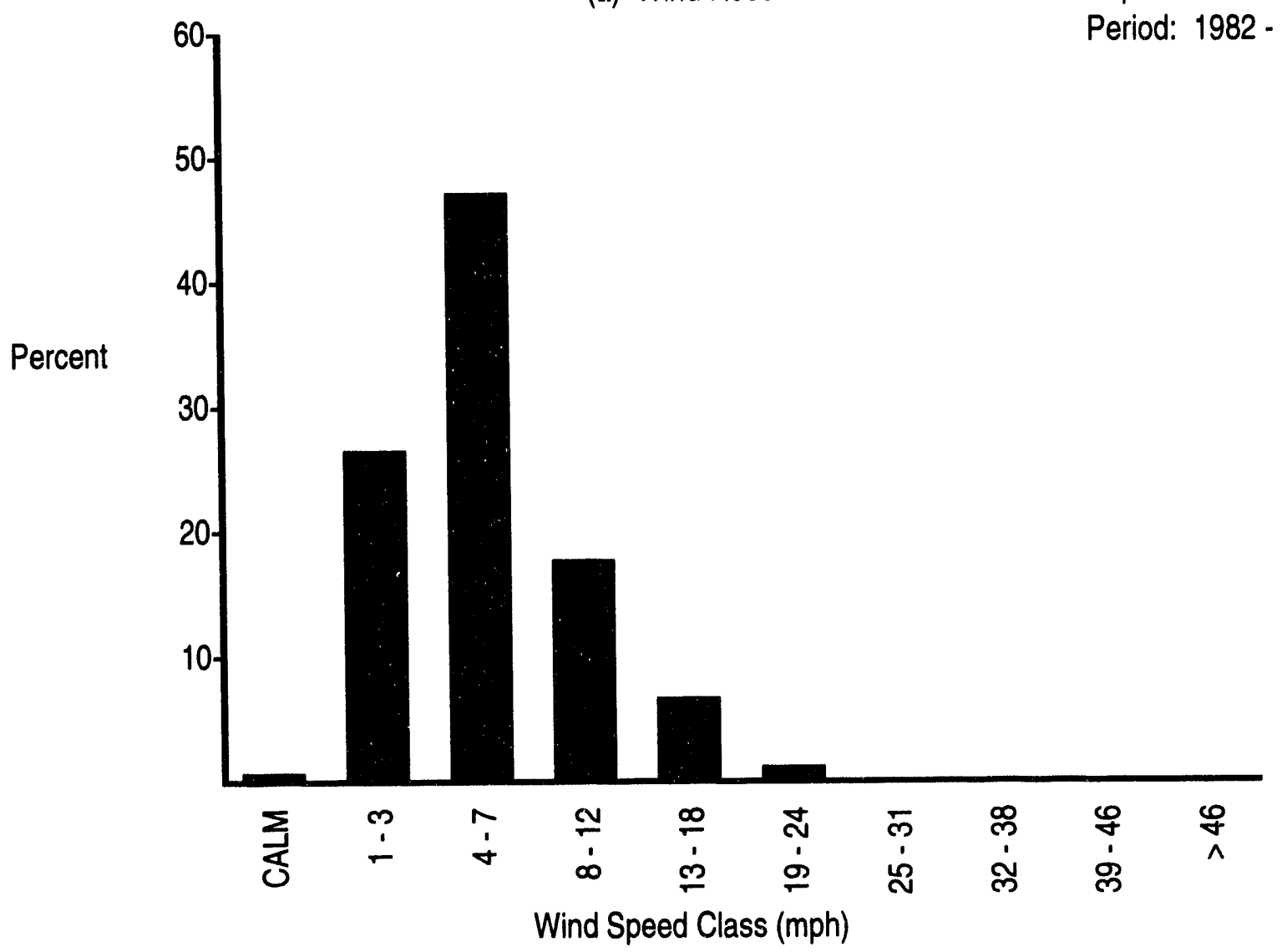

(b) Wind Speed Histogram
September Data

Period: 1982 - 1993

\section{FIGURE B.1. (contd)}




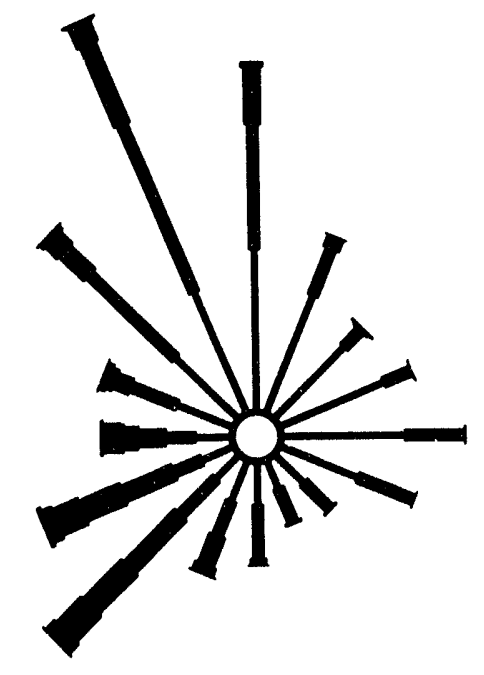

(a) Wind Rose

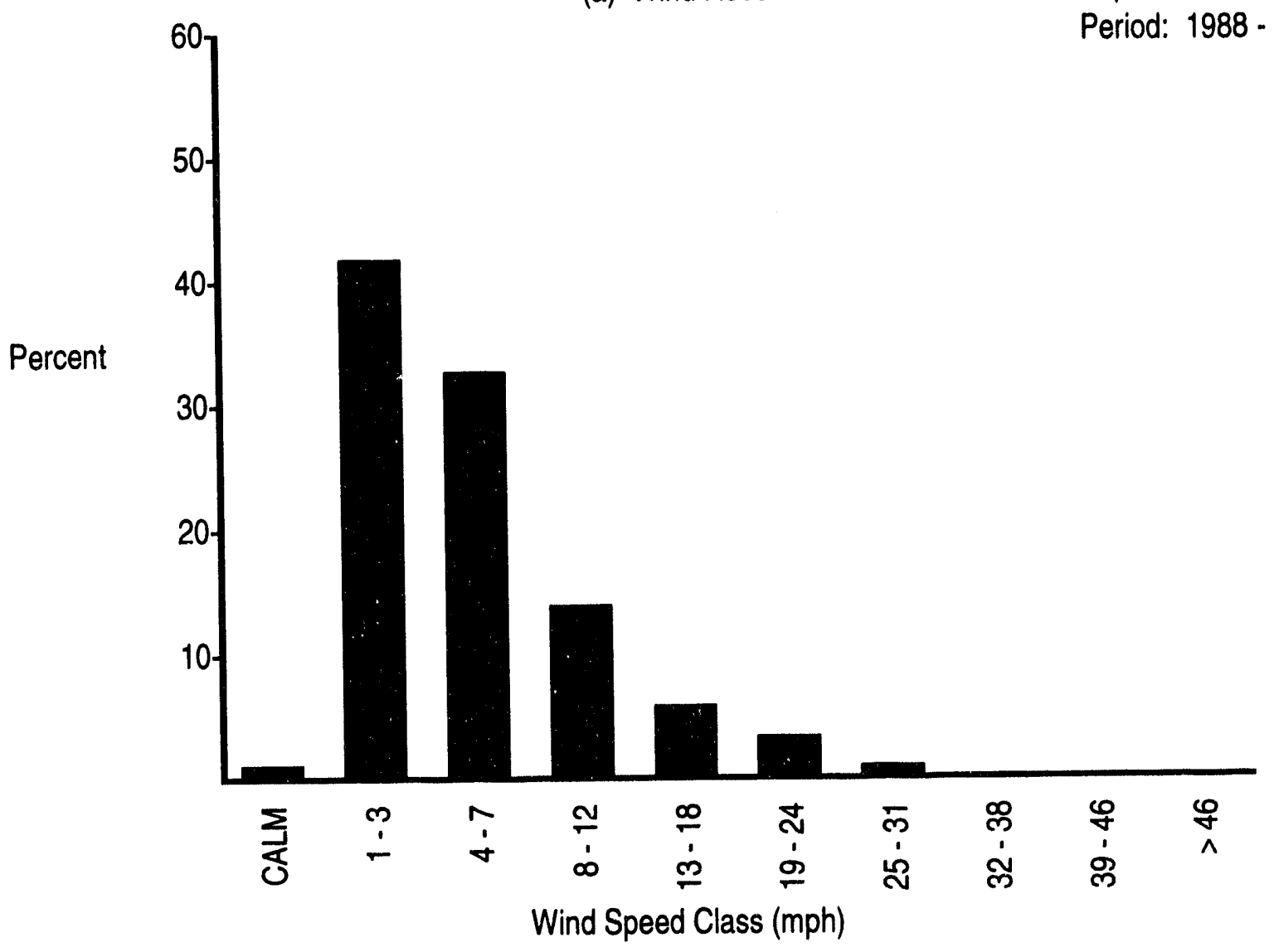

(b) Wind Speed Histogram
September Data

Period: 1988 - 1993

FIGURE B.1. (contd) 


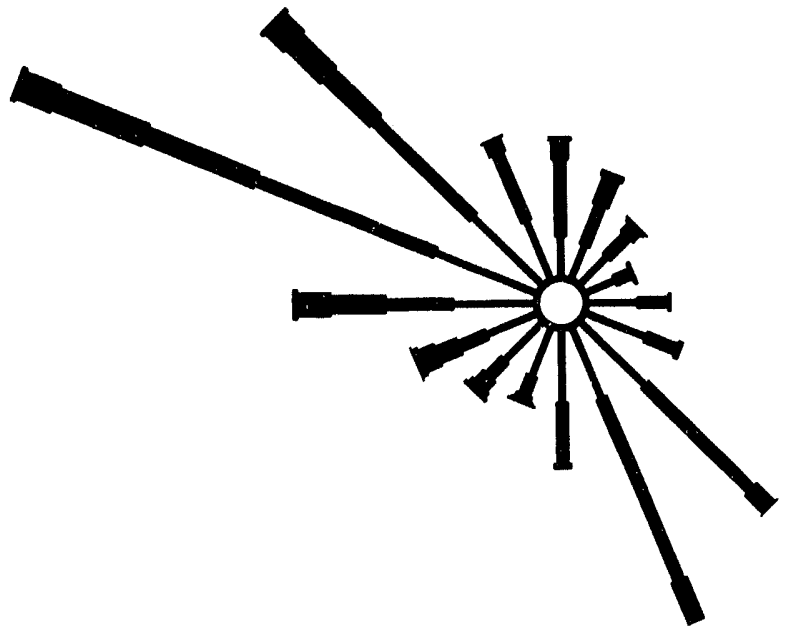

(a) Wind Rose

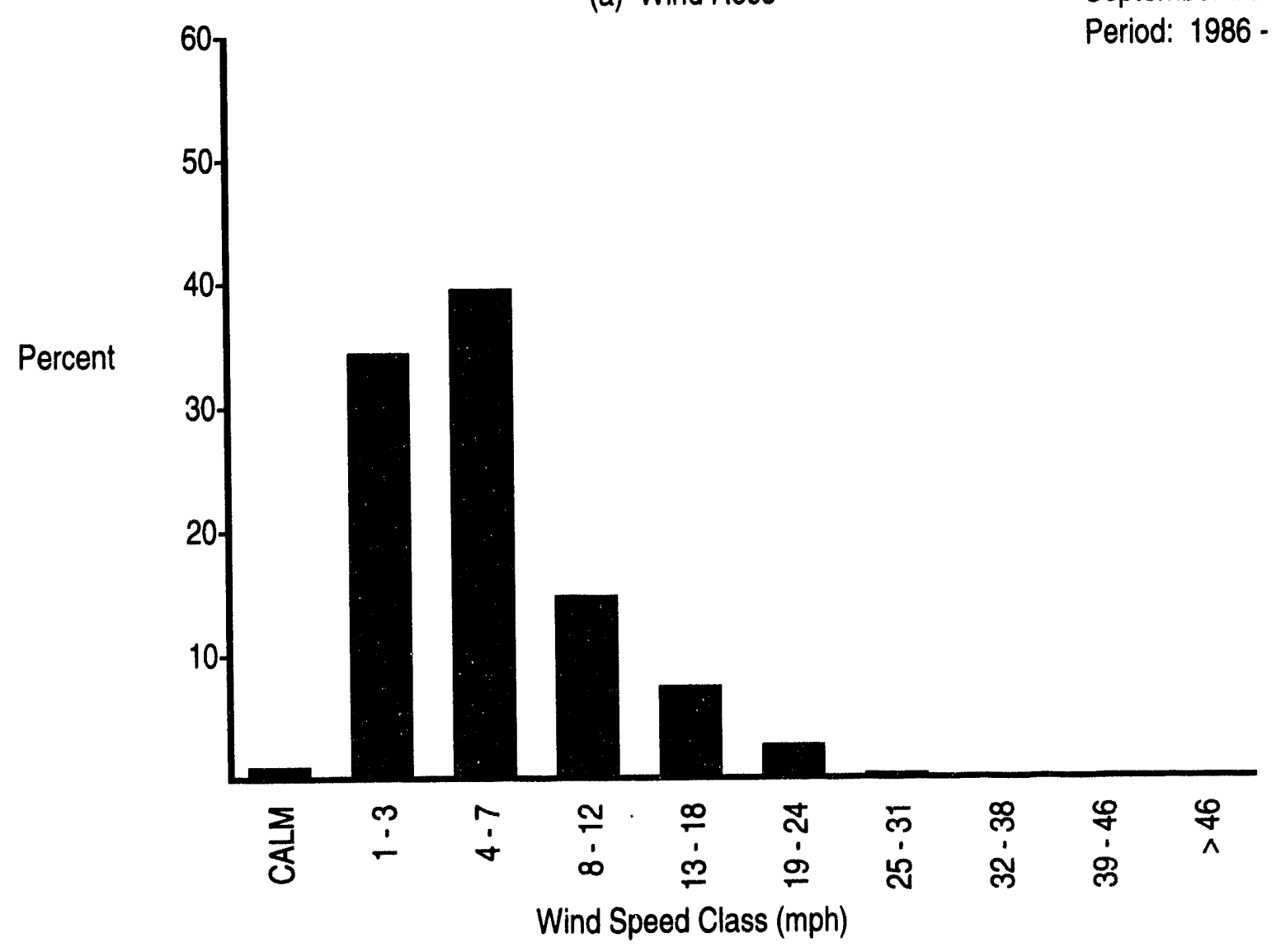

(b) Wind Speed Histogram

FIGURE B.1. (contd)
September Data

Period: 1986 - 1993 


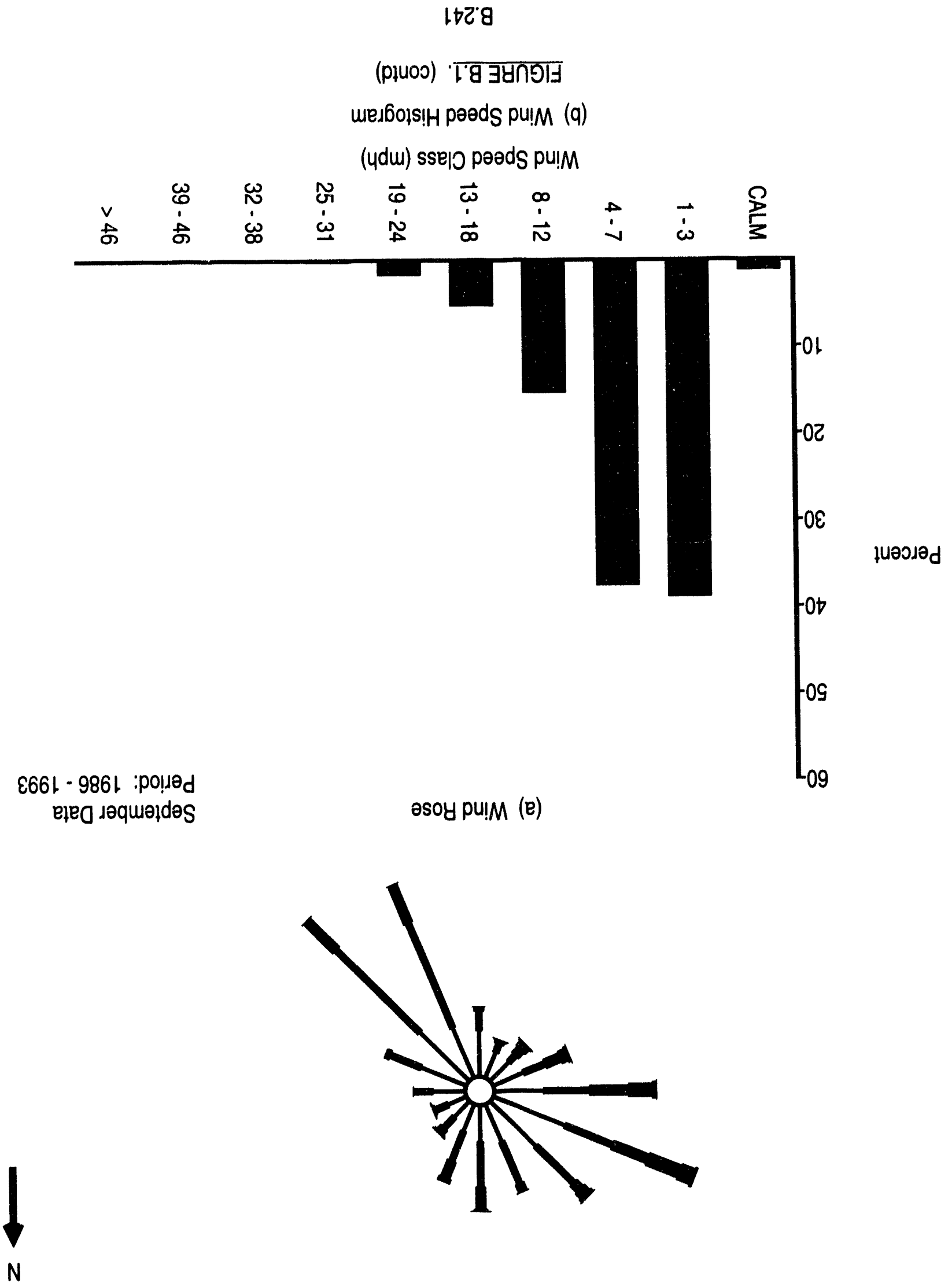



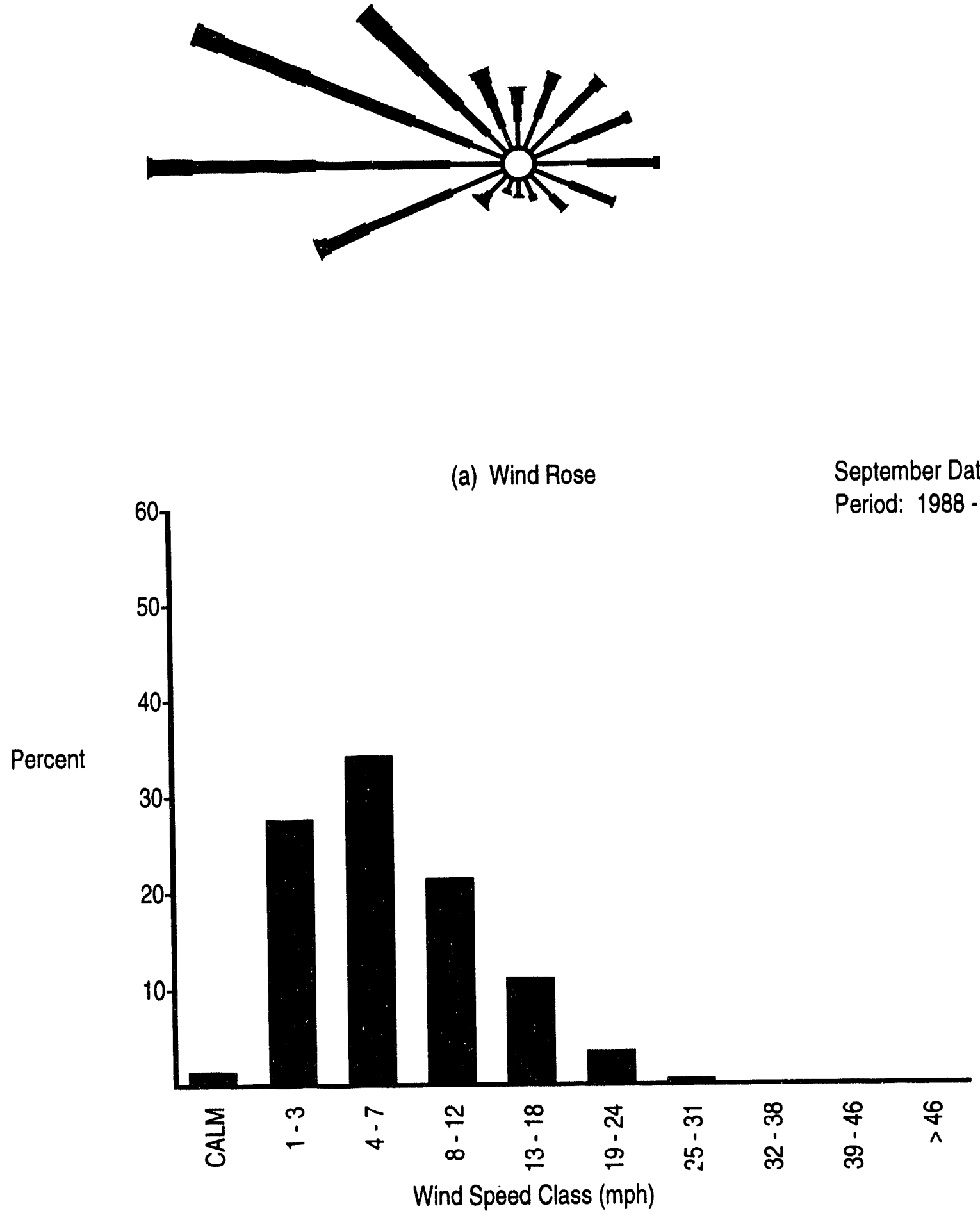

(b) Wind Speed Histogram

FIGURE B.1. (contd) 


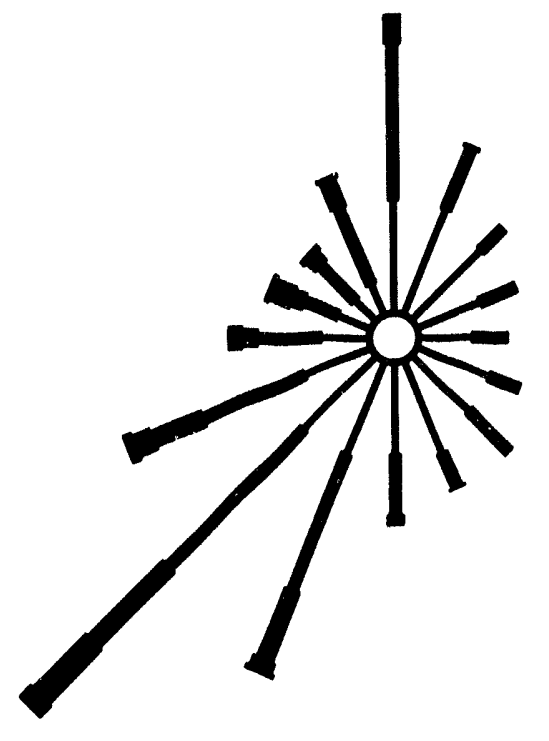

(a) Wind Rose

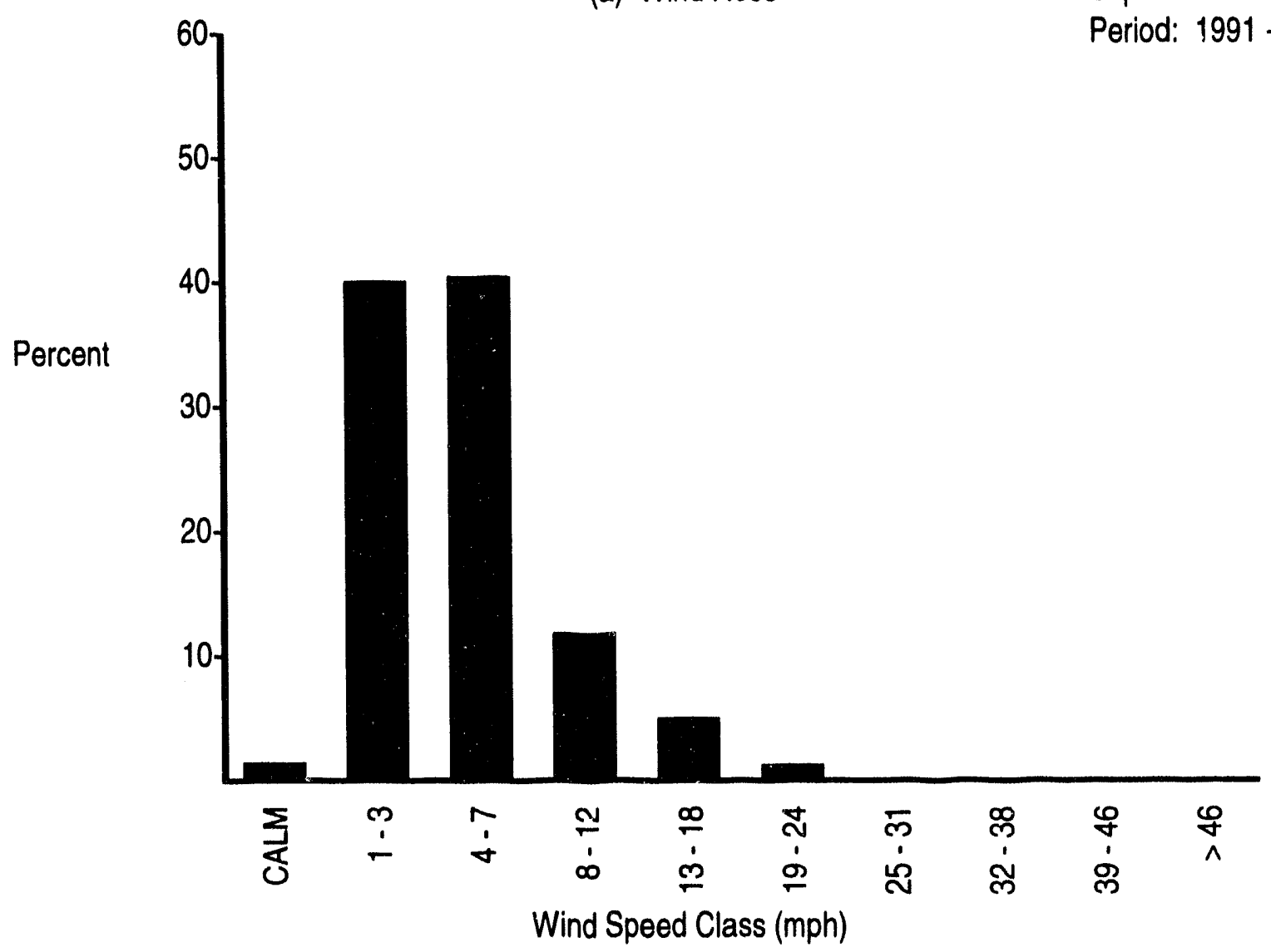

(b) Wind Speed Histogram

FIGURE B.1. (contd)
September Data

Period: 1991 - 1993

\section{B.243}




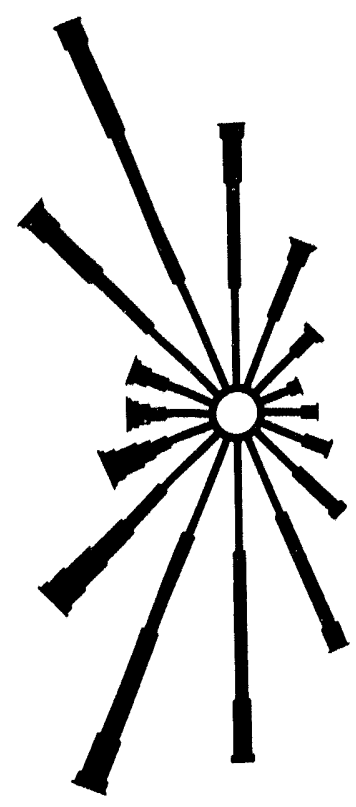

(a) Wind Rose

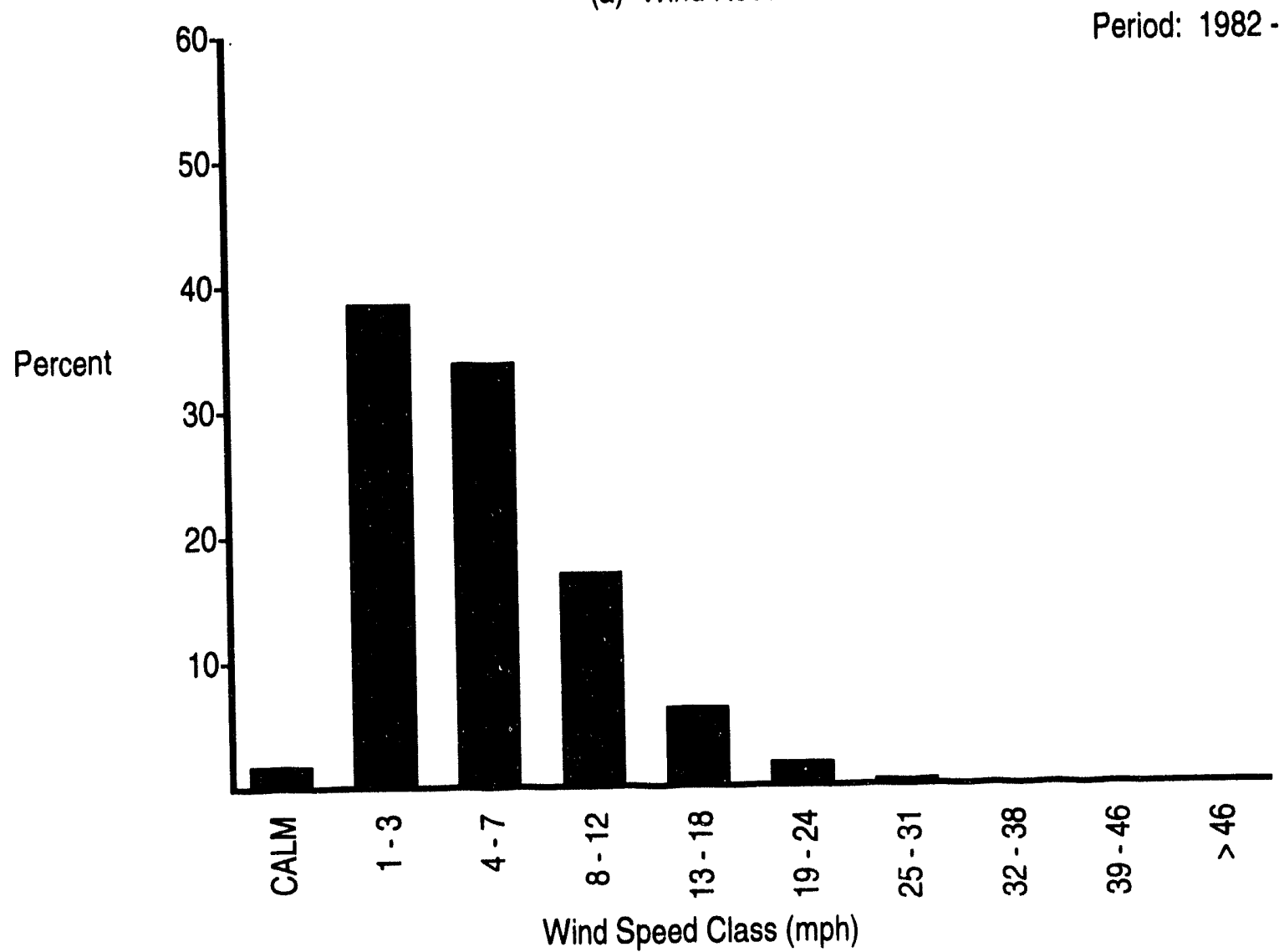

(b) Wind Speed Histogram

FIGURE B.1. (contd)
October Data

Period: $1982-1993$ 

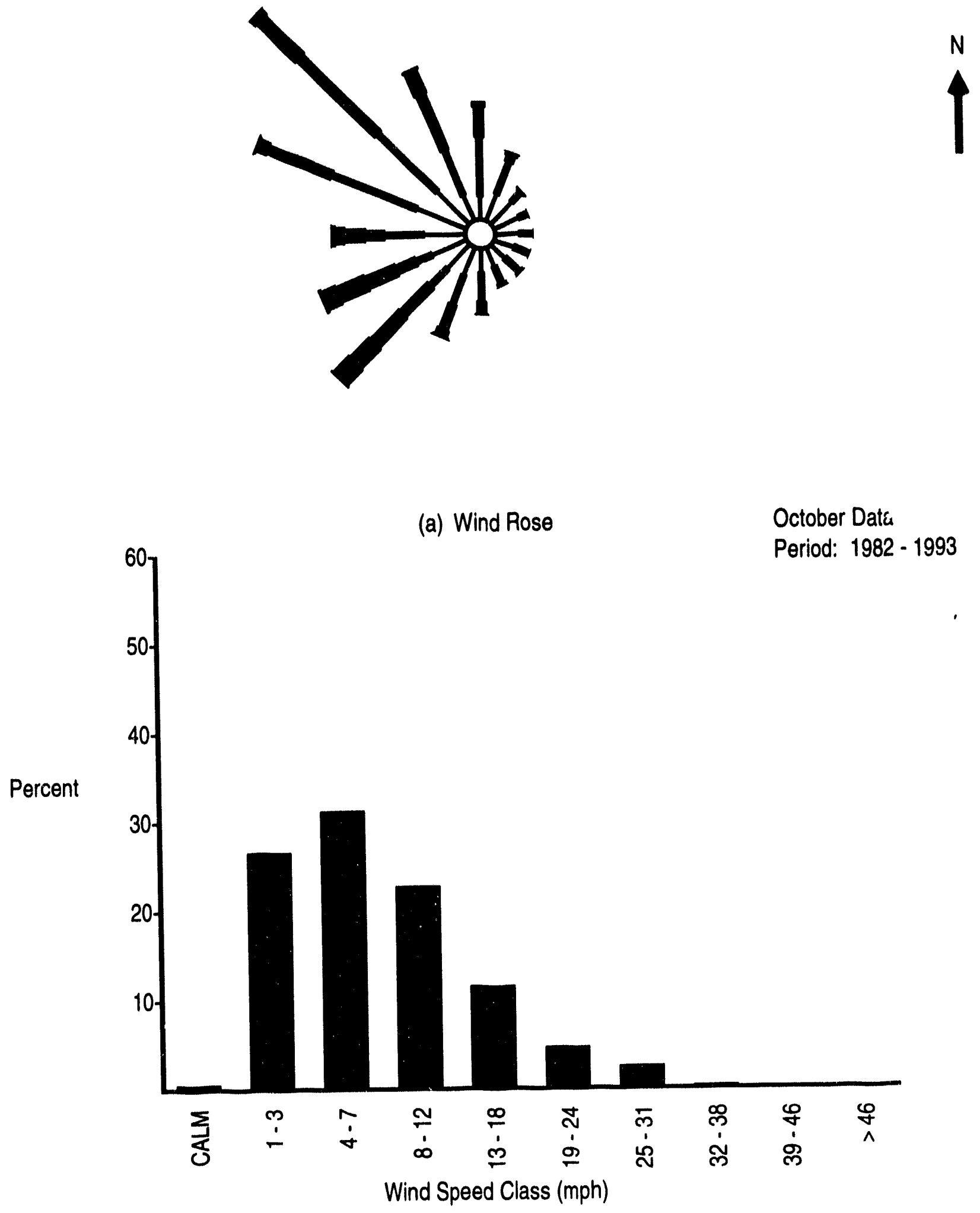

(b) Wind Speed Histogram

FIGURE B.1. (contd) 


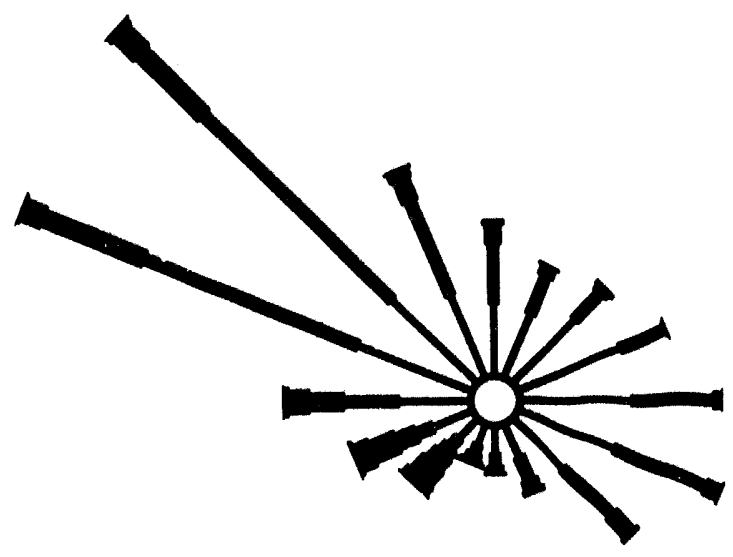

(a) Wind Rose

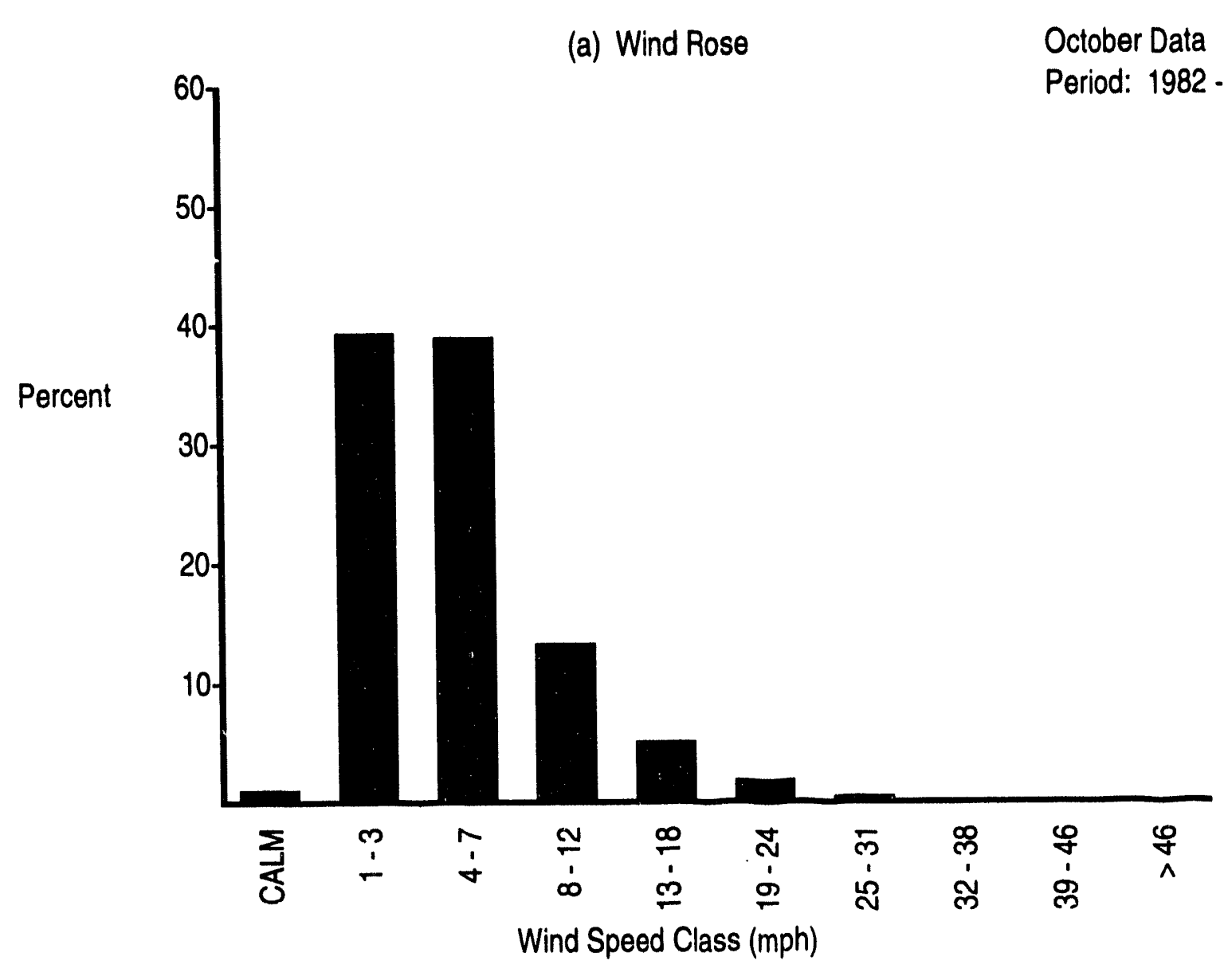

(b) Wind Speed Histogram

FIGURE B.1. (contd) 


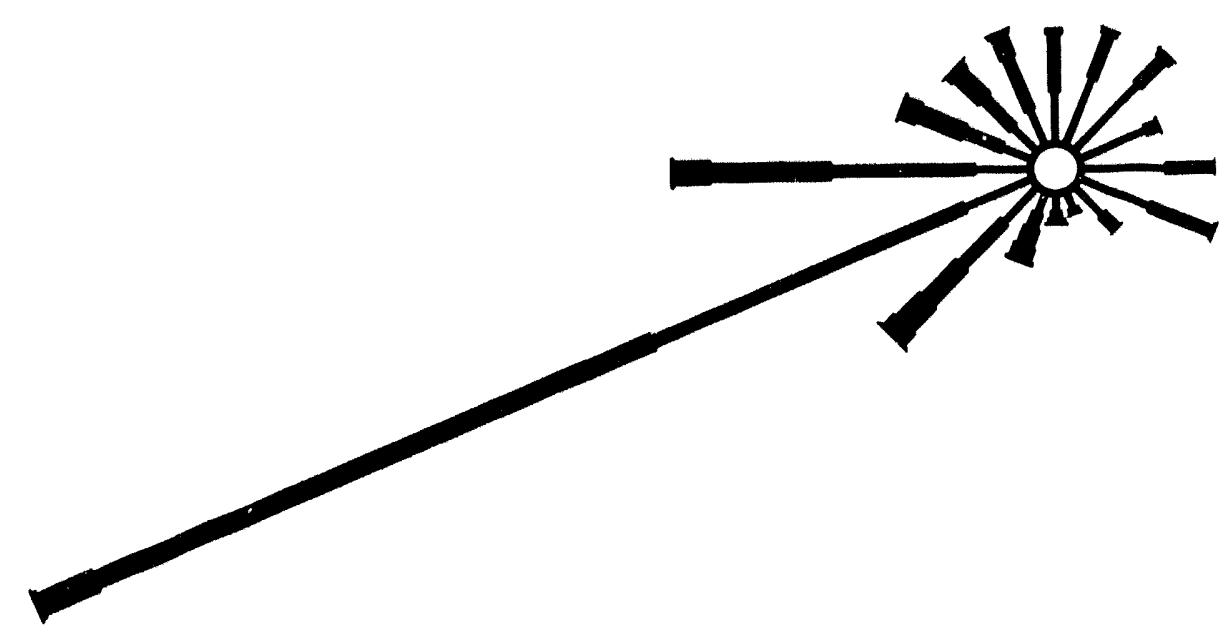

(a) Wind Rose

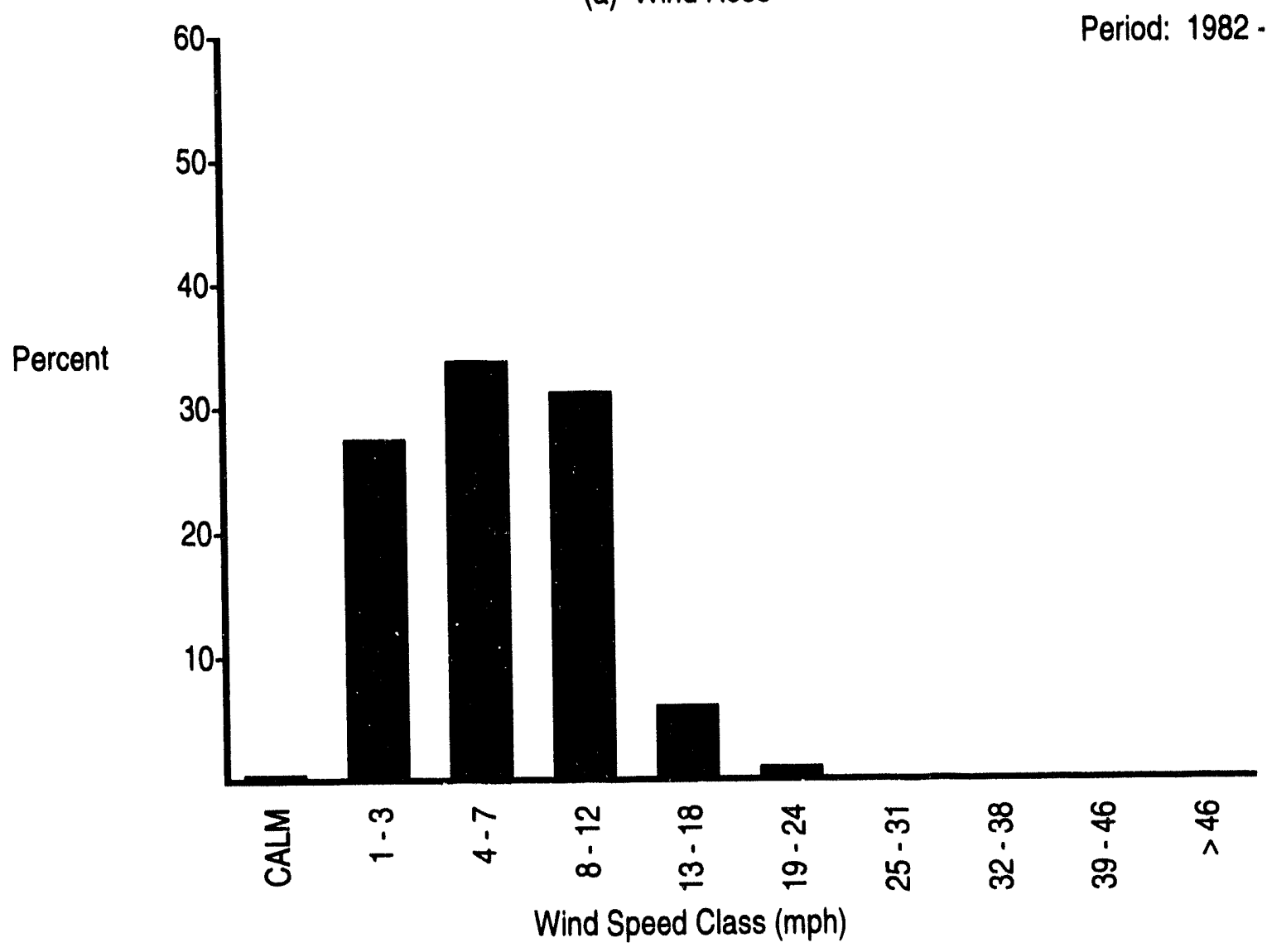

(b) Wind Speed Histogram

FIGURE B.1. (contd)
October Data

Period: 1982 - 1993 


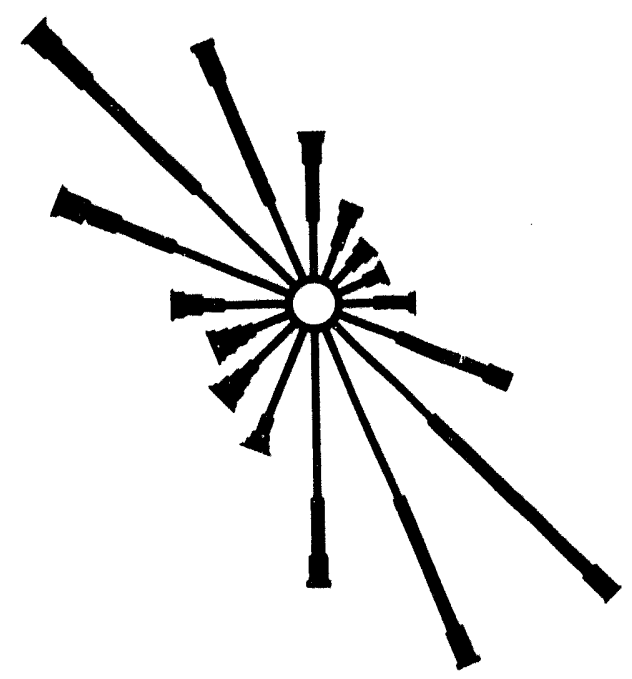

(a) Wind Rose

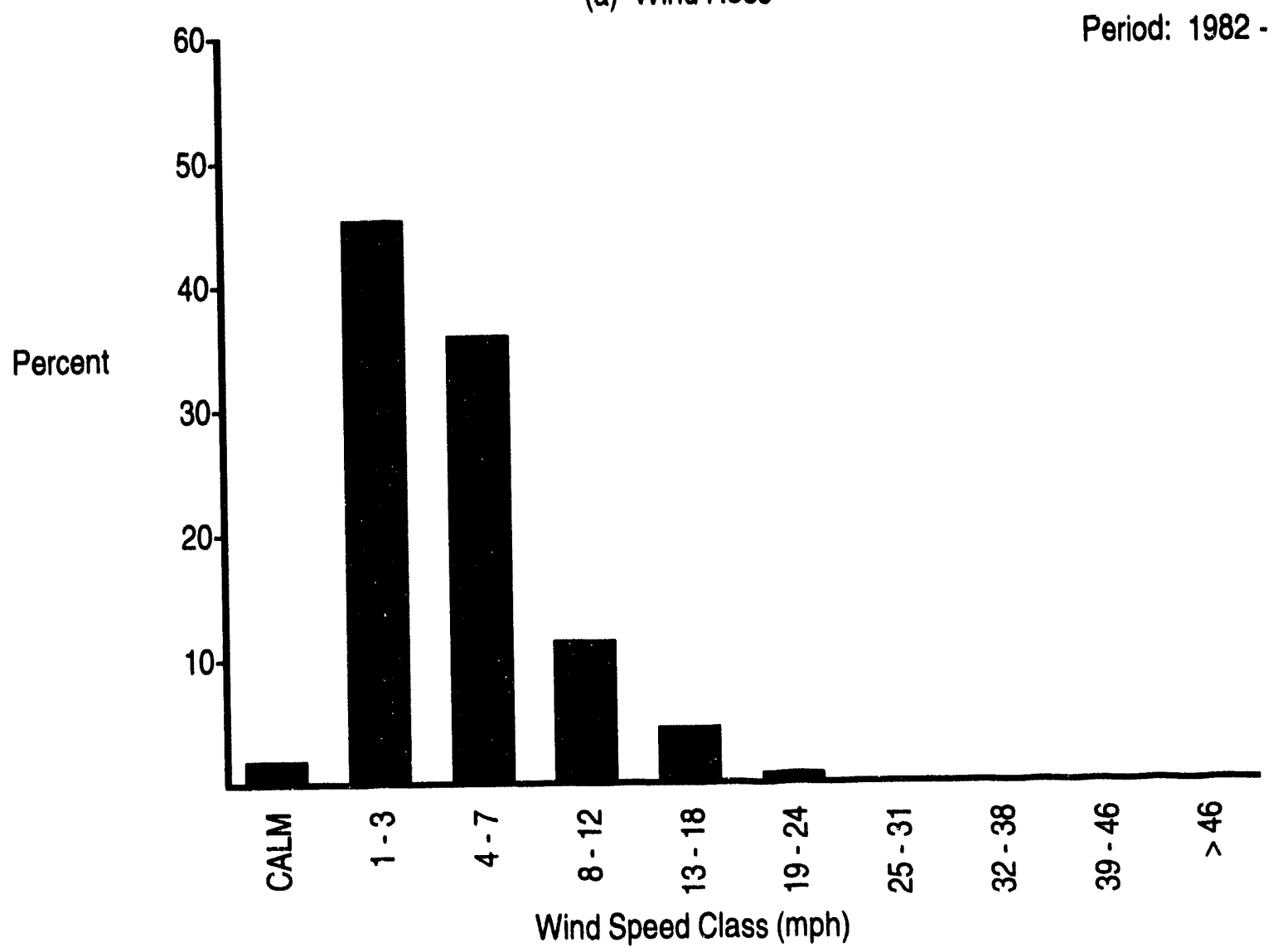

(b) Wind Speed Histogram

FIGURE B.1. (contd)
October Data

Period: 1982 - 1993 


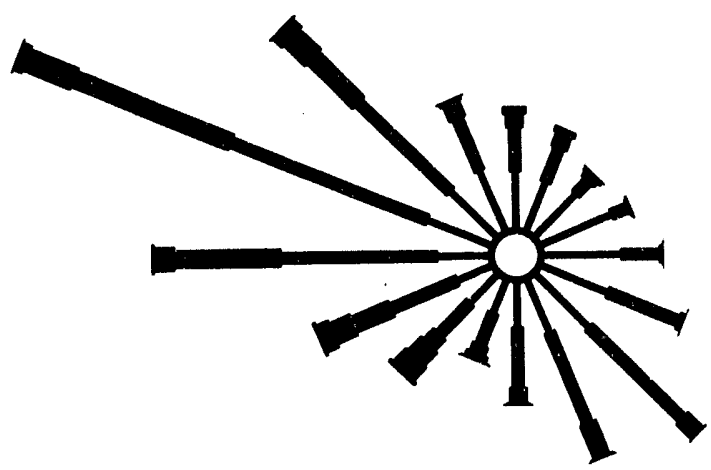

(a) Wind Rose

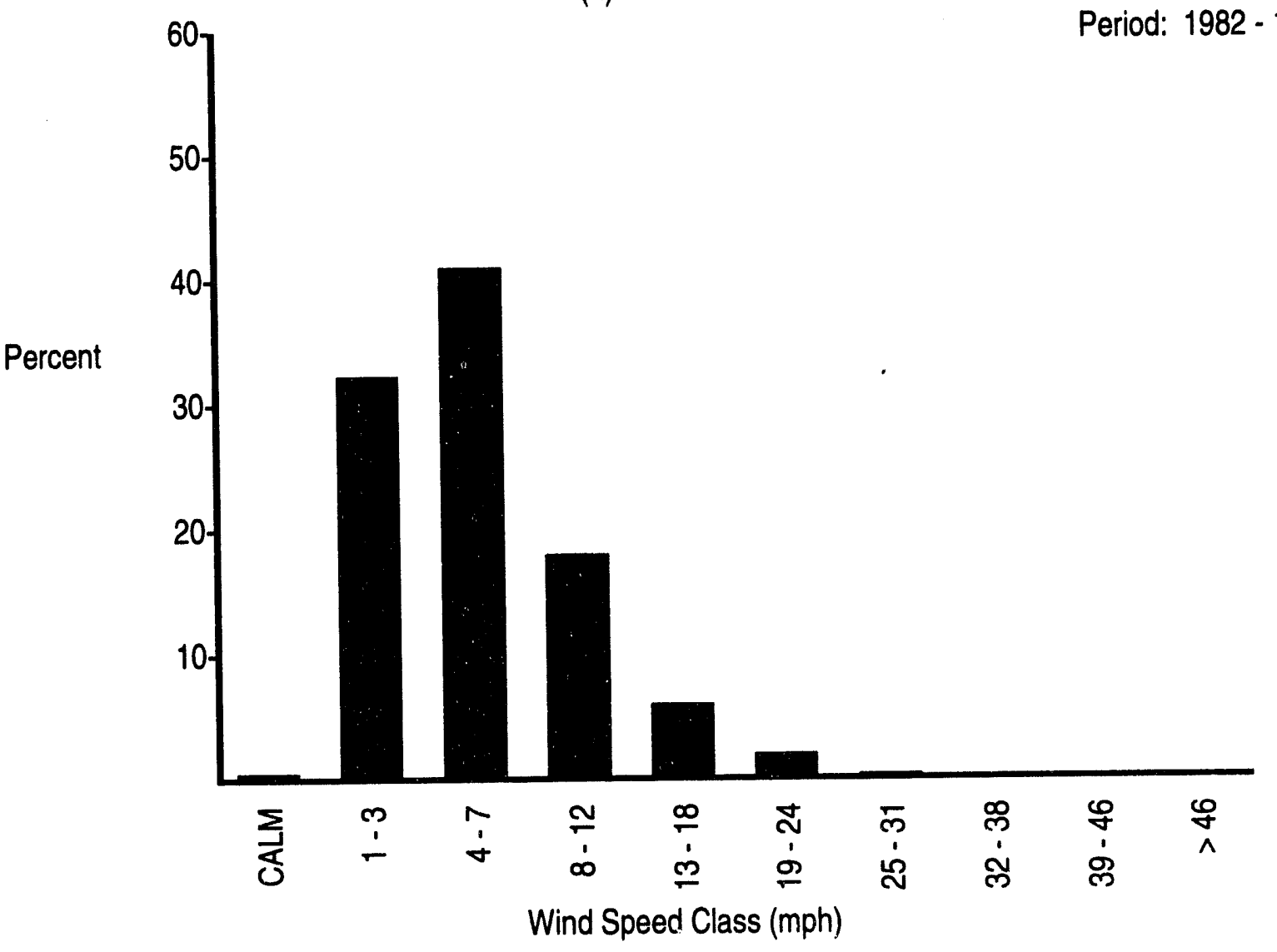

(b) Wind Speed Histogram

FIGURE B.1. (contd)
October Data

Period: 1982 - 1993 


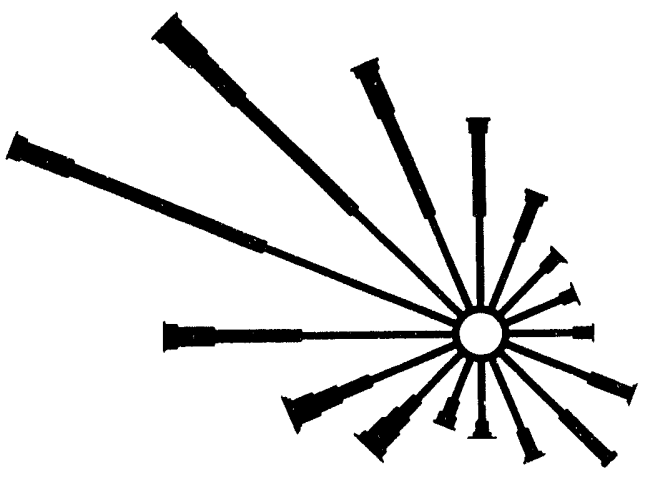

N

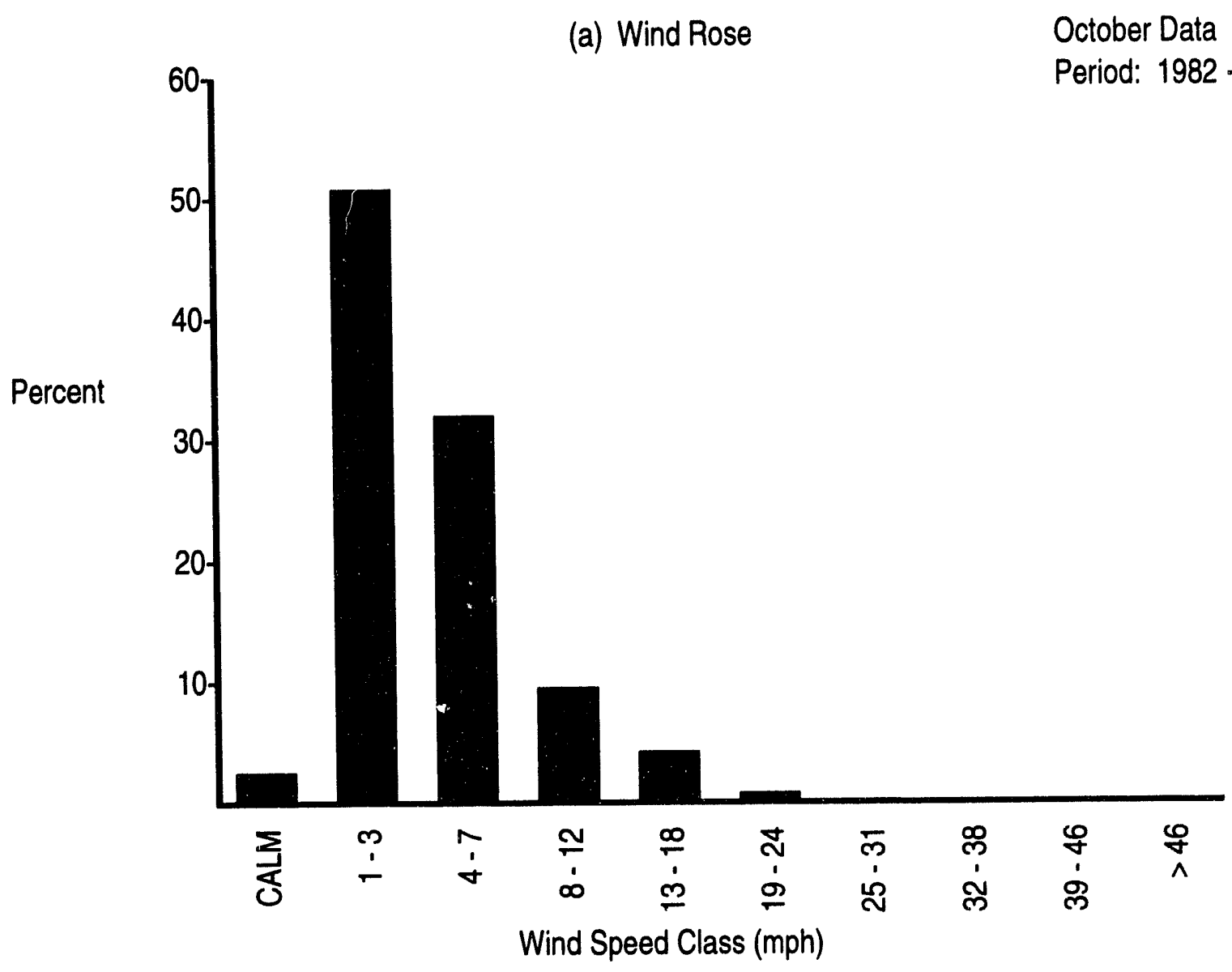

(b) Wind Speed Histogram

FIGURE B.1. (contd) 


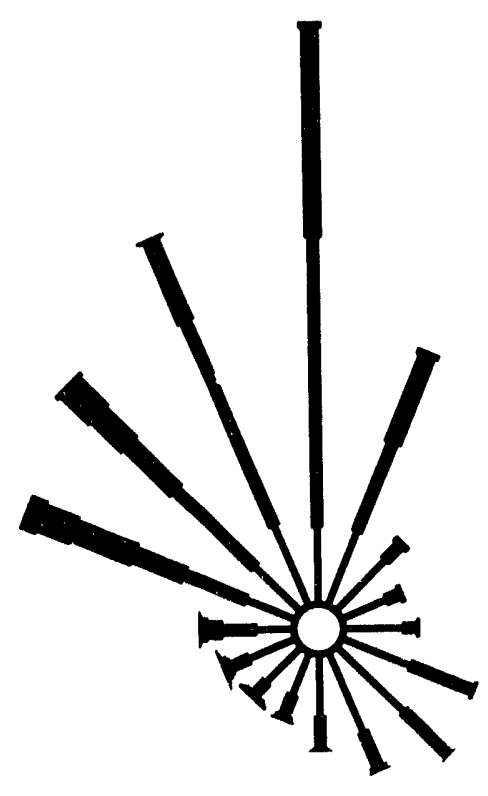

(a) Wind Rose

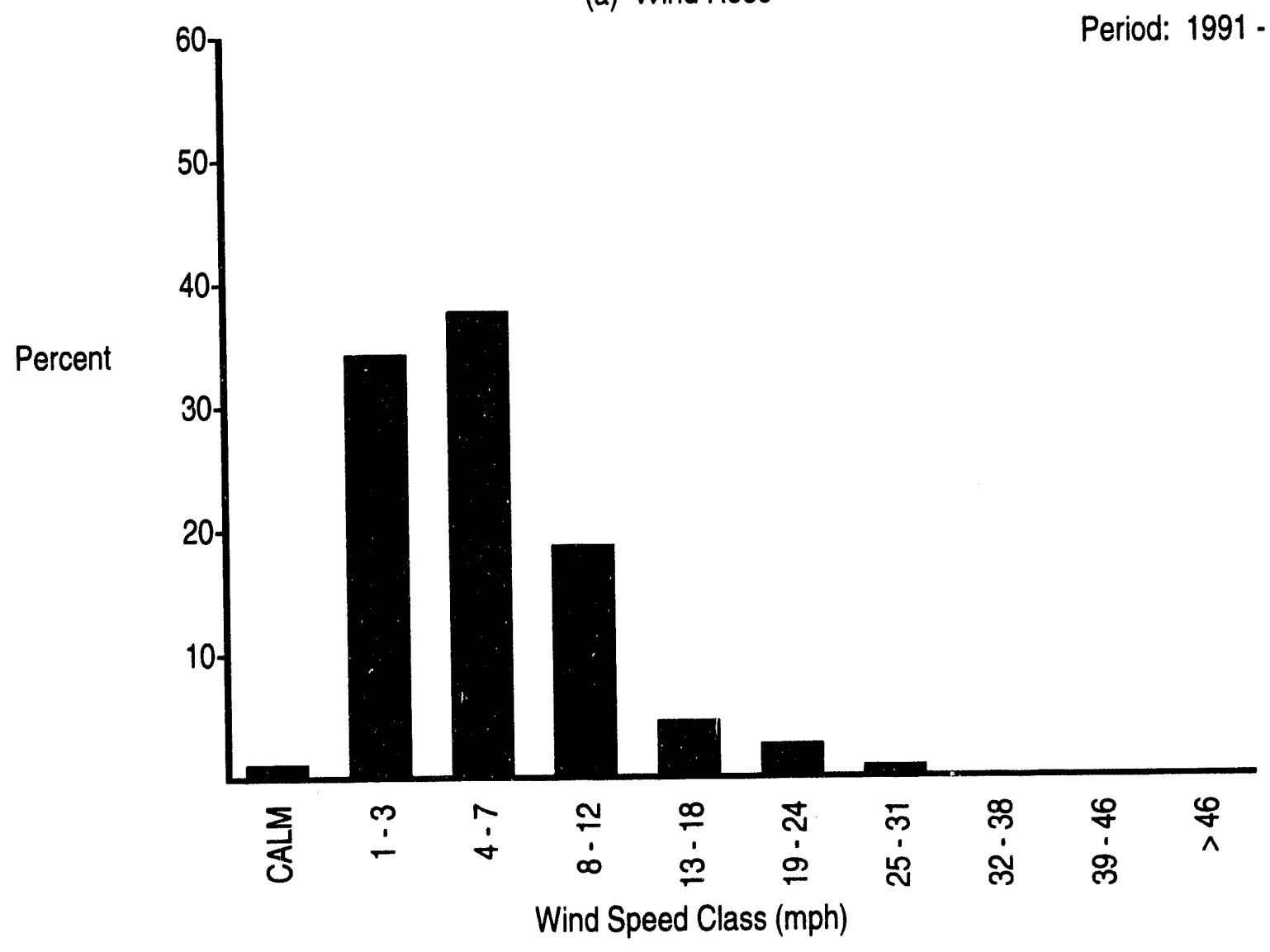

(b) Wind Speed Histogram

FIGURE B.1. (contd)
October Data

Period: 1991 - 1993 


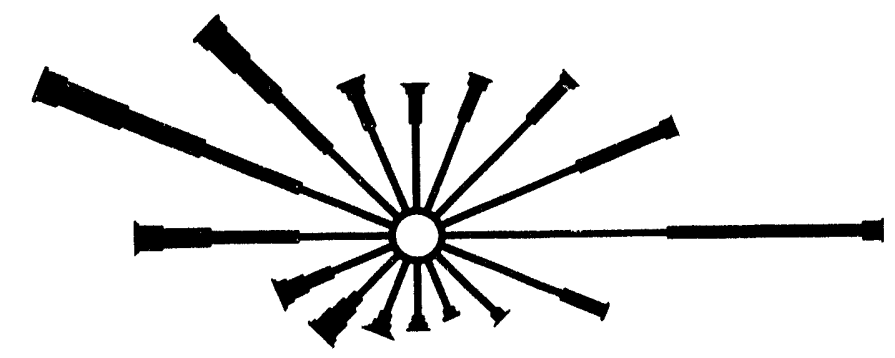

(a) Wind Rose

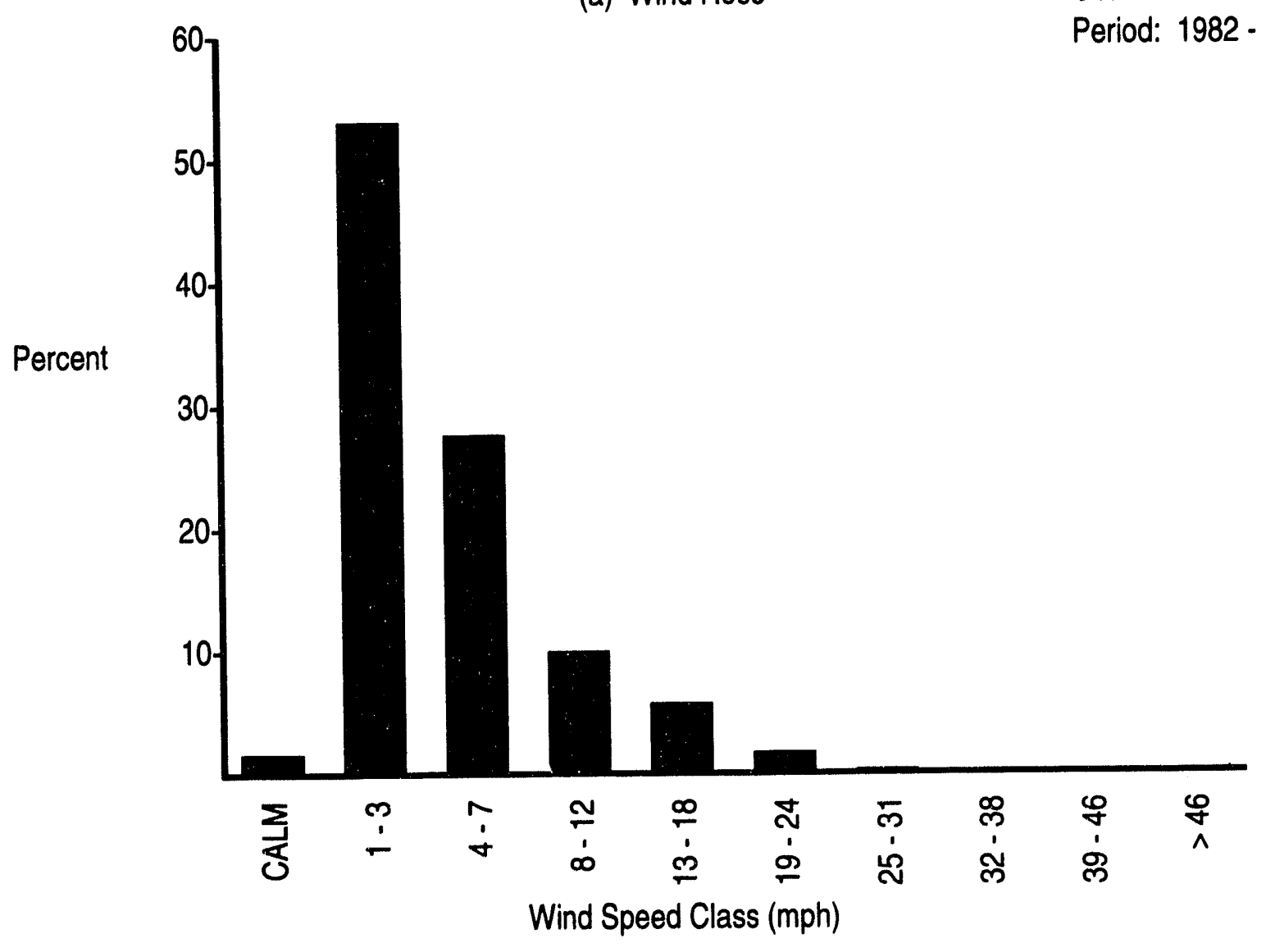

(b) Wind Speed Histogram

FIGURE B.1. (contd)
October Data

Period: 1982 - 1990

\section{B.252}



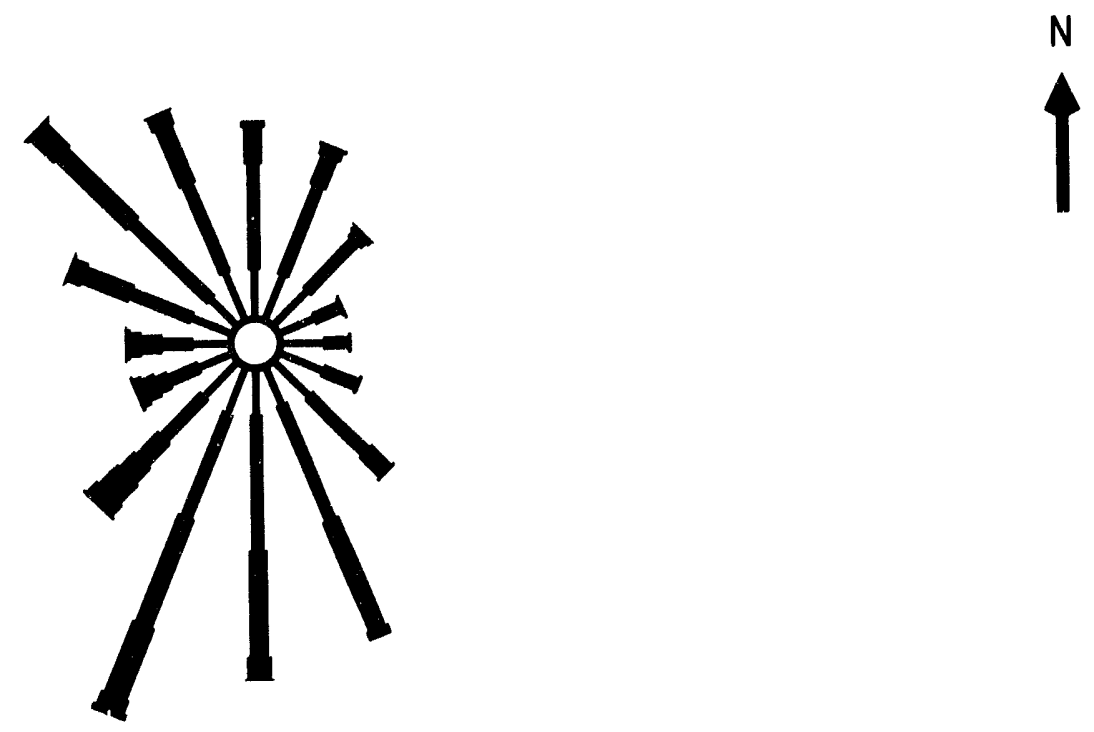

(a) Wind Rose

October Data

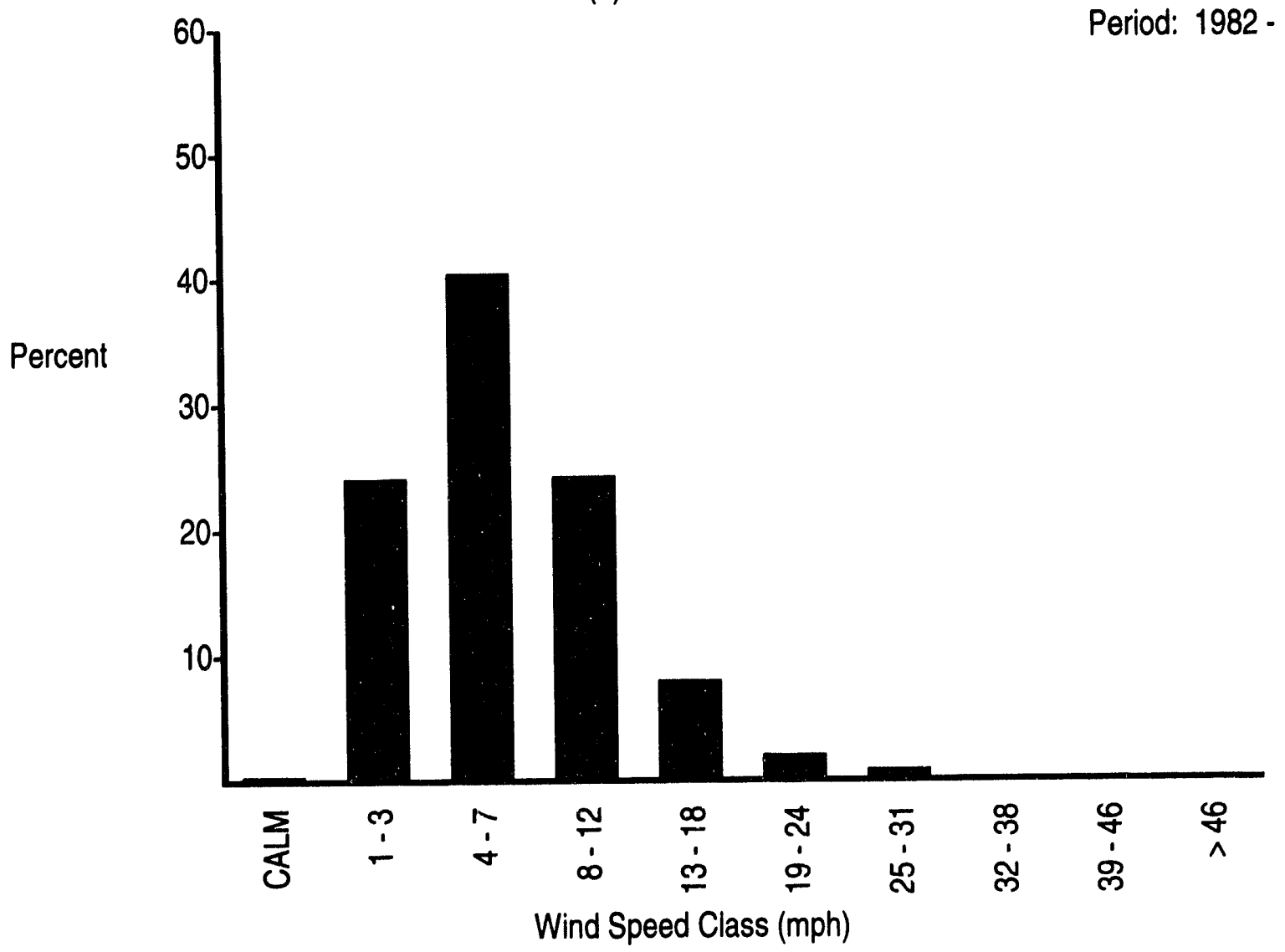

(b) Wind Speed Histogram

FIGURE B.1. (contd) 


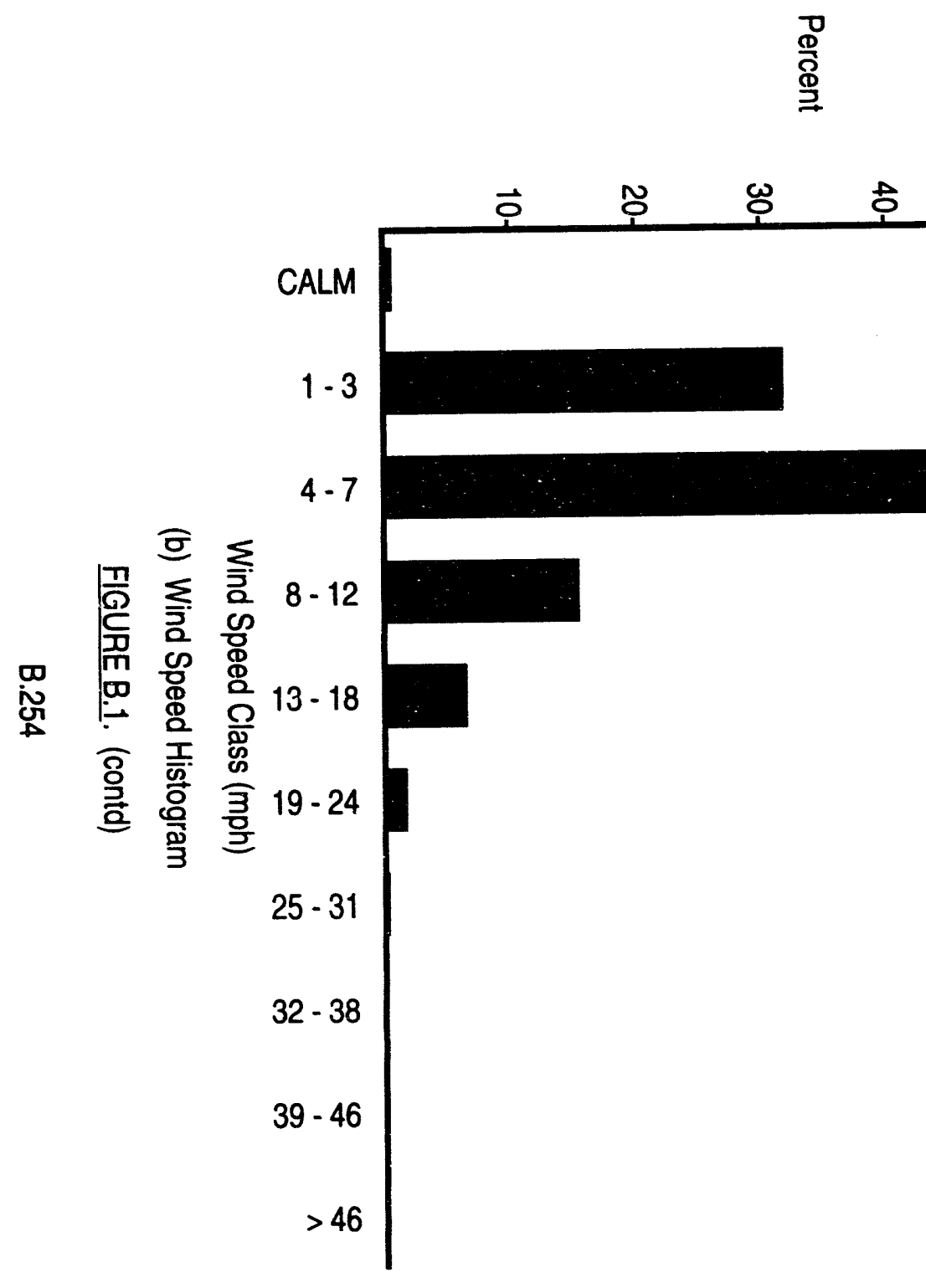

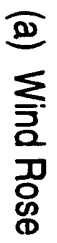

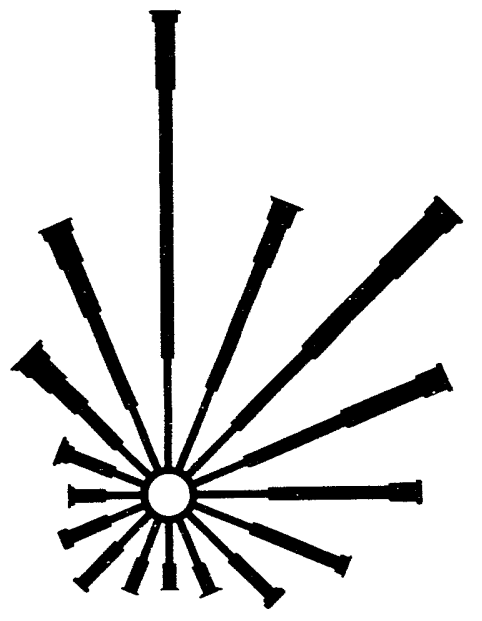

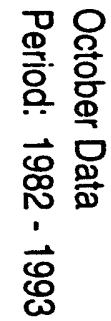




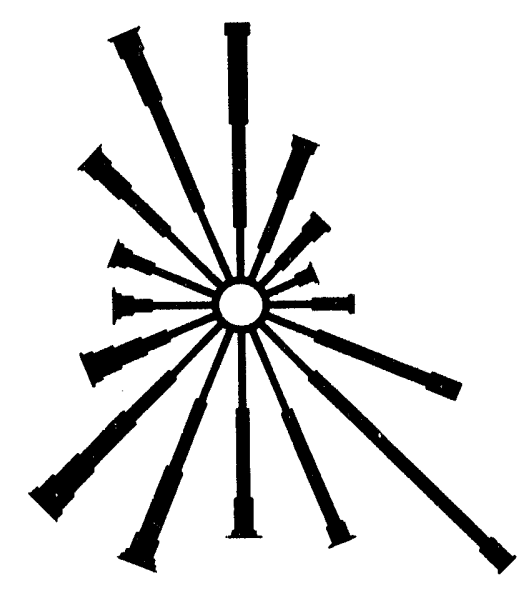

(a) Wind Rose

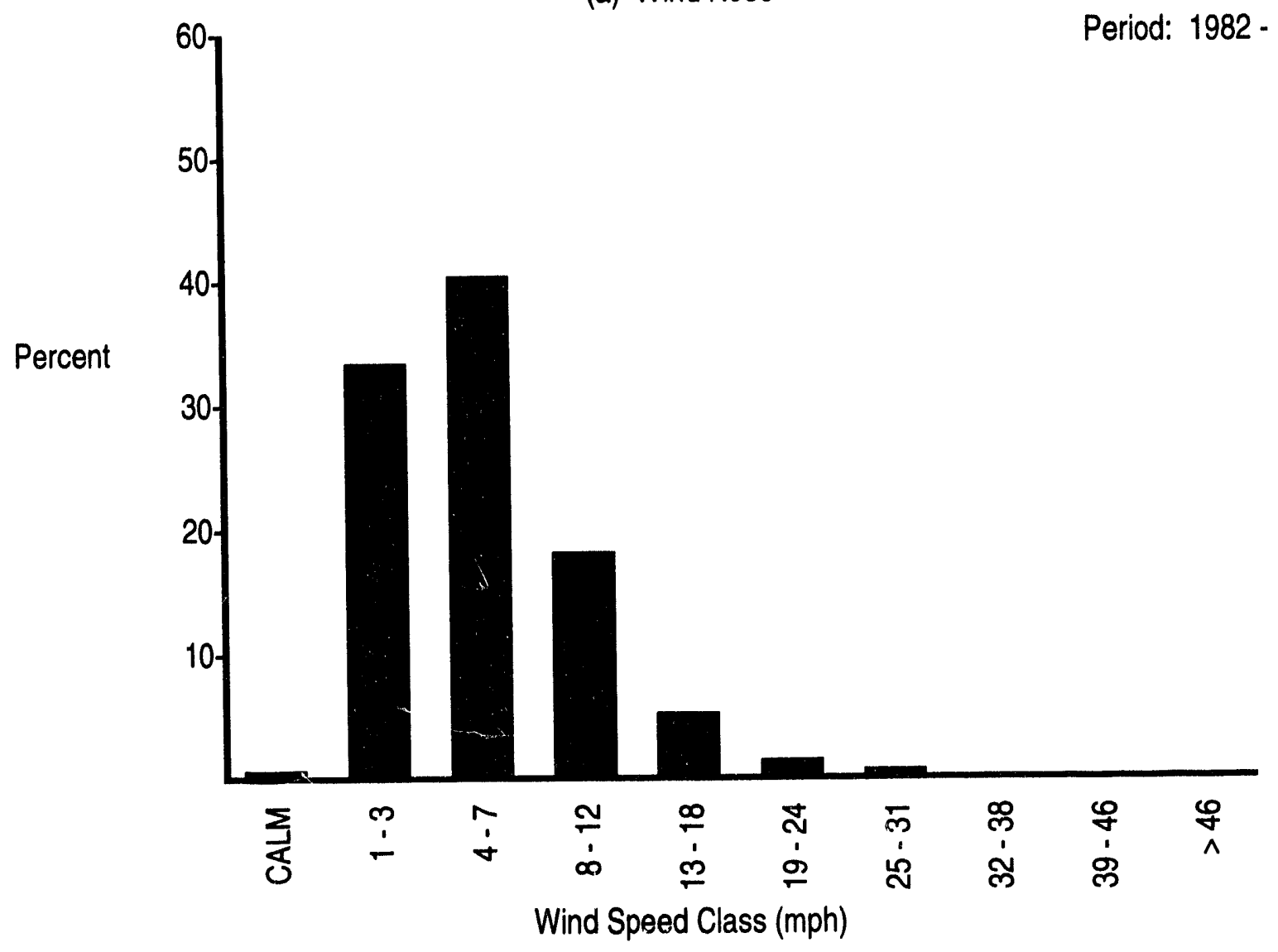

(b) Wind Speed Histogram

FIGURE B.1. (contd)
October Data

Period: 1982 - 1993

\section{B.255}




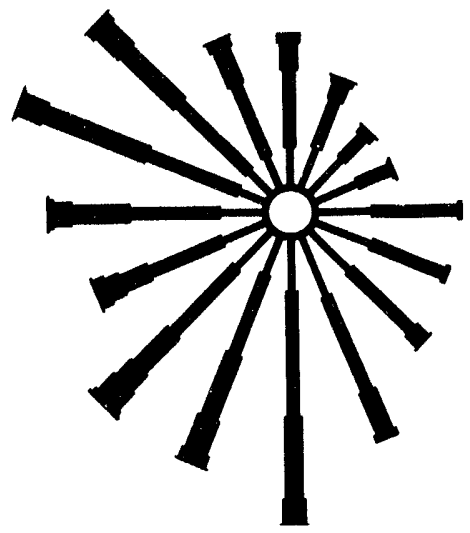

N

(a) Wind Rose

October Data

Period: 1982 - 1993

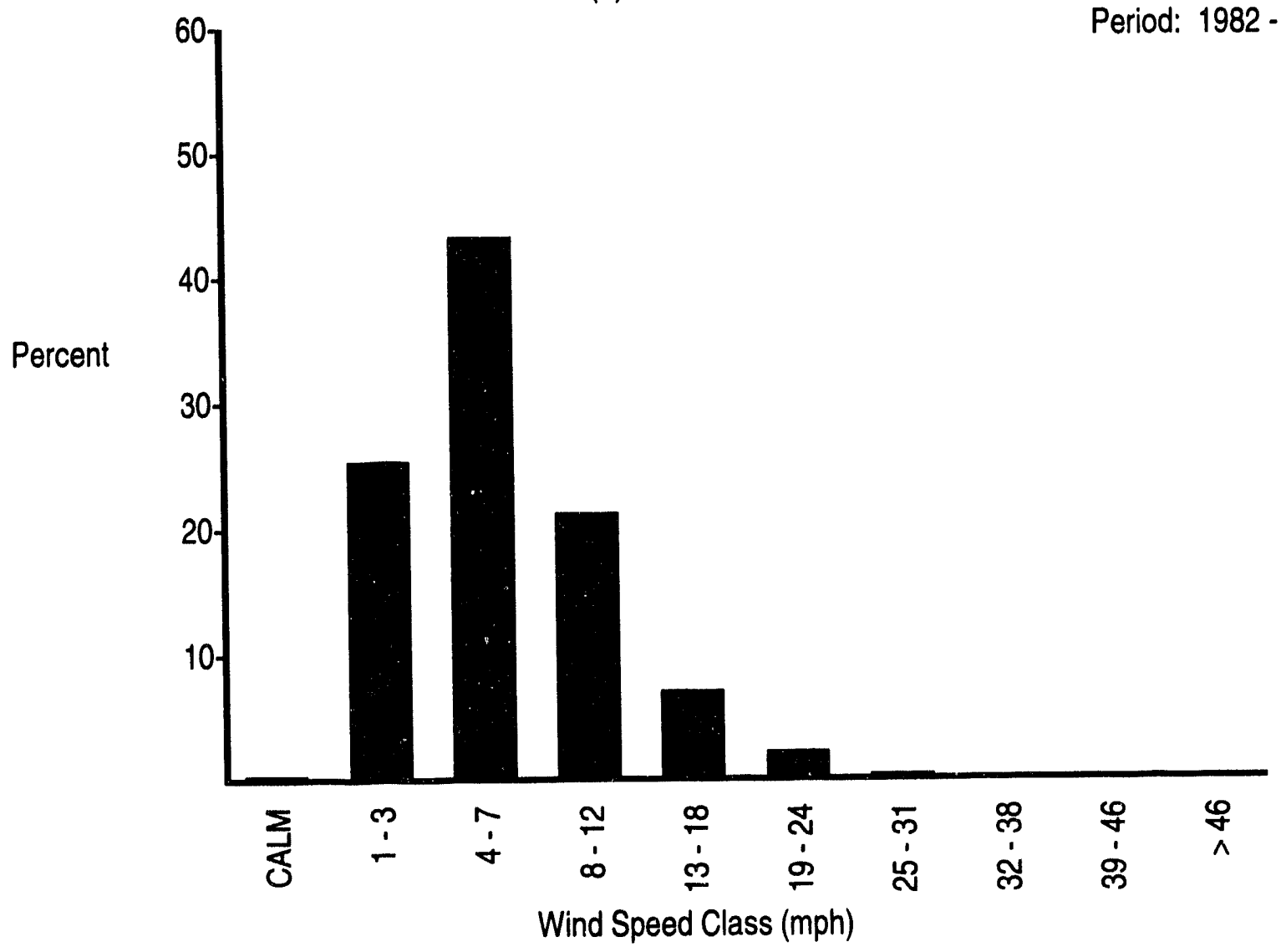

(b) Wind Speed Histogram

FIGURE B.1. (contd) 


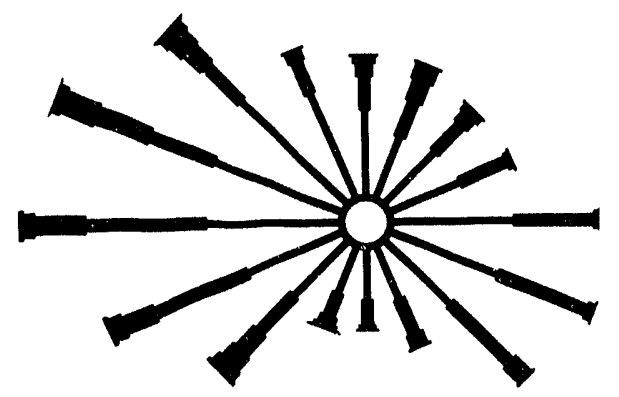

(a) Wind Rose

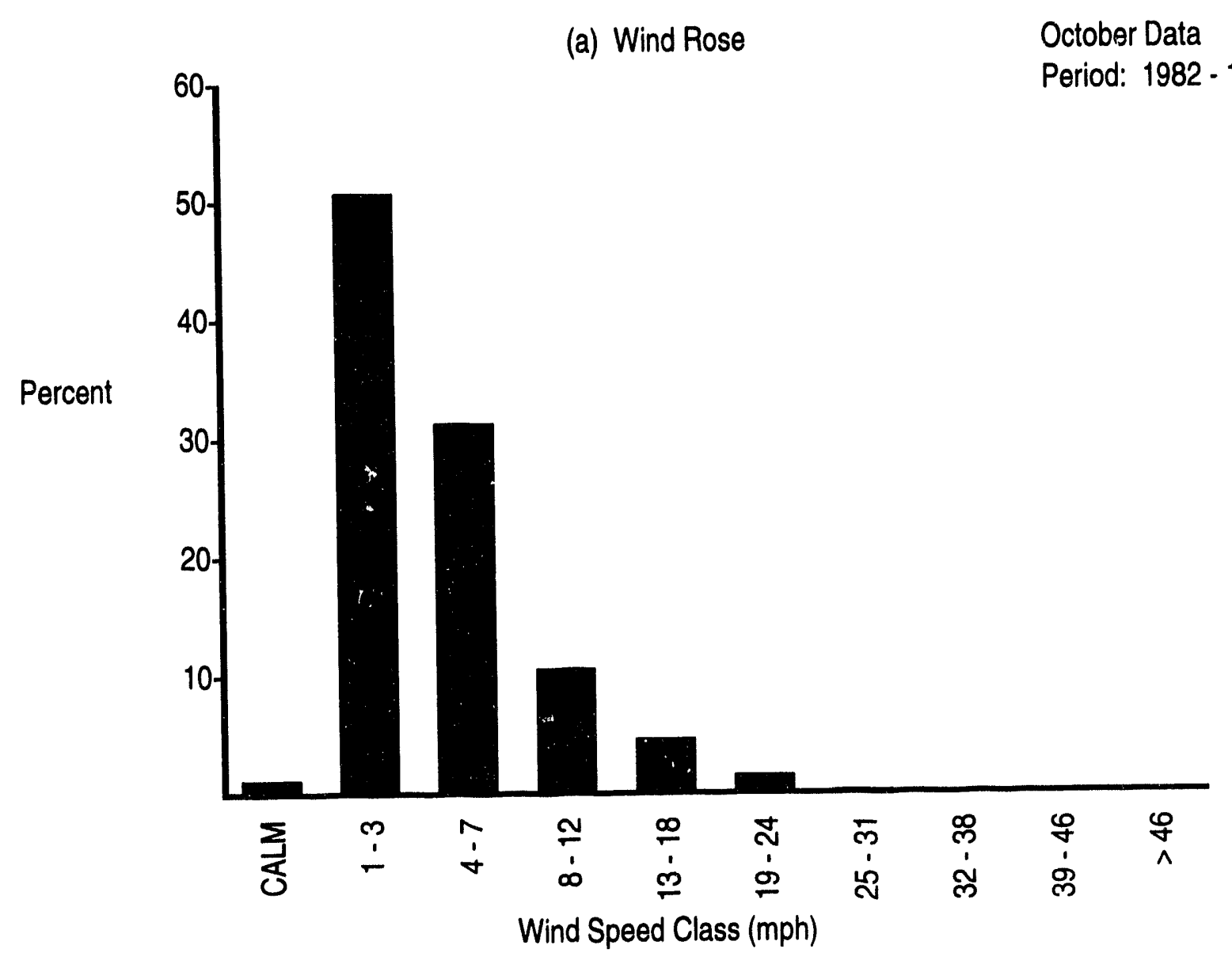

(b) Wind Speed Histogram

FIGURE B.1. (contd) 


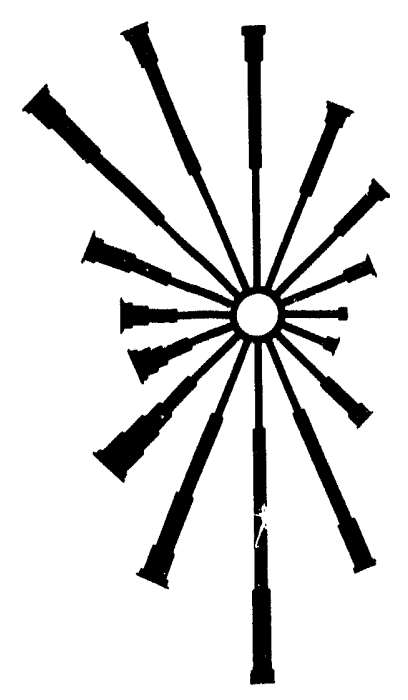

(a) Wind Rose

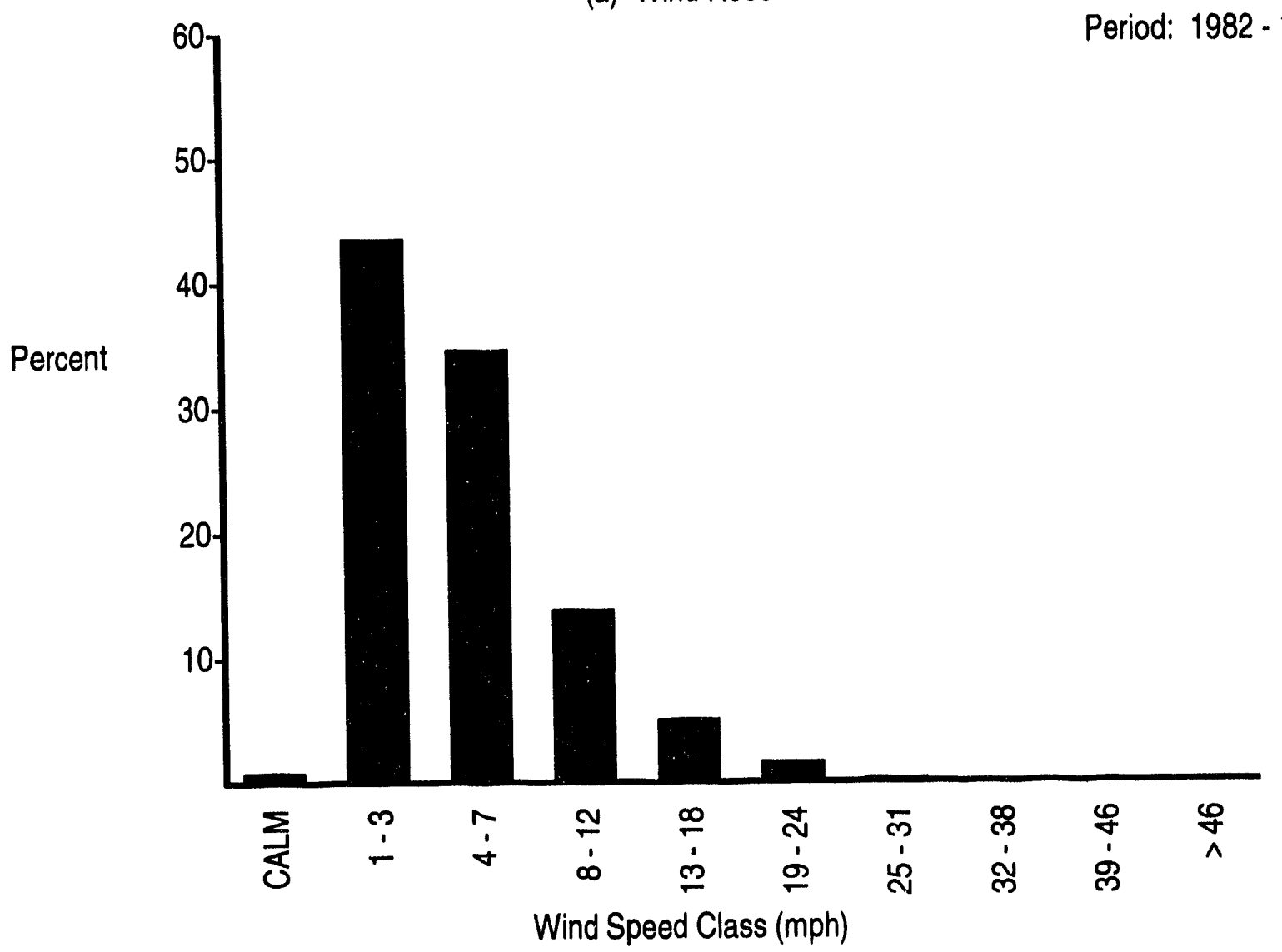

October Data

Period: $1982-1993$

(b) Wind Speed Histogram

FIGURE B.1. (contd) 


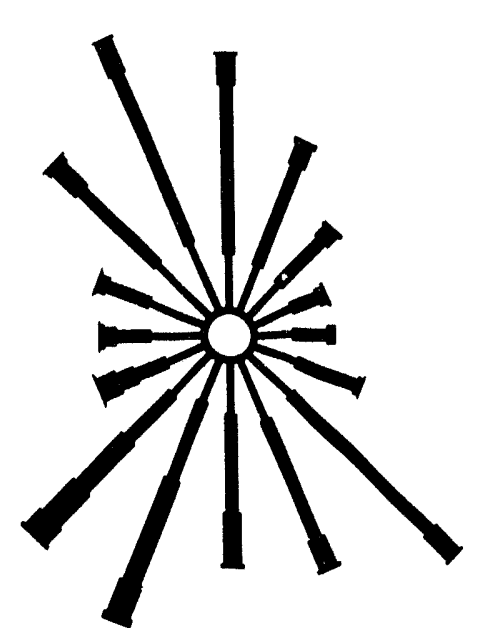

(a) Wind Rose

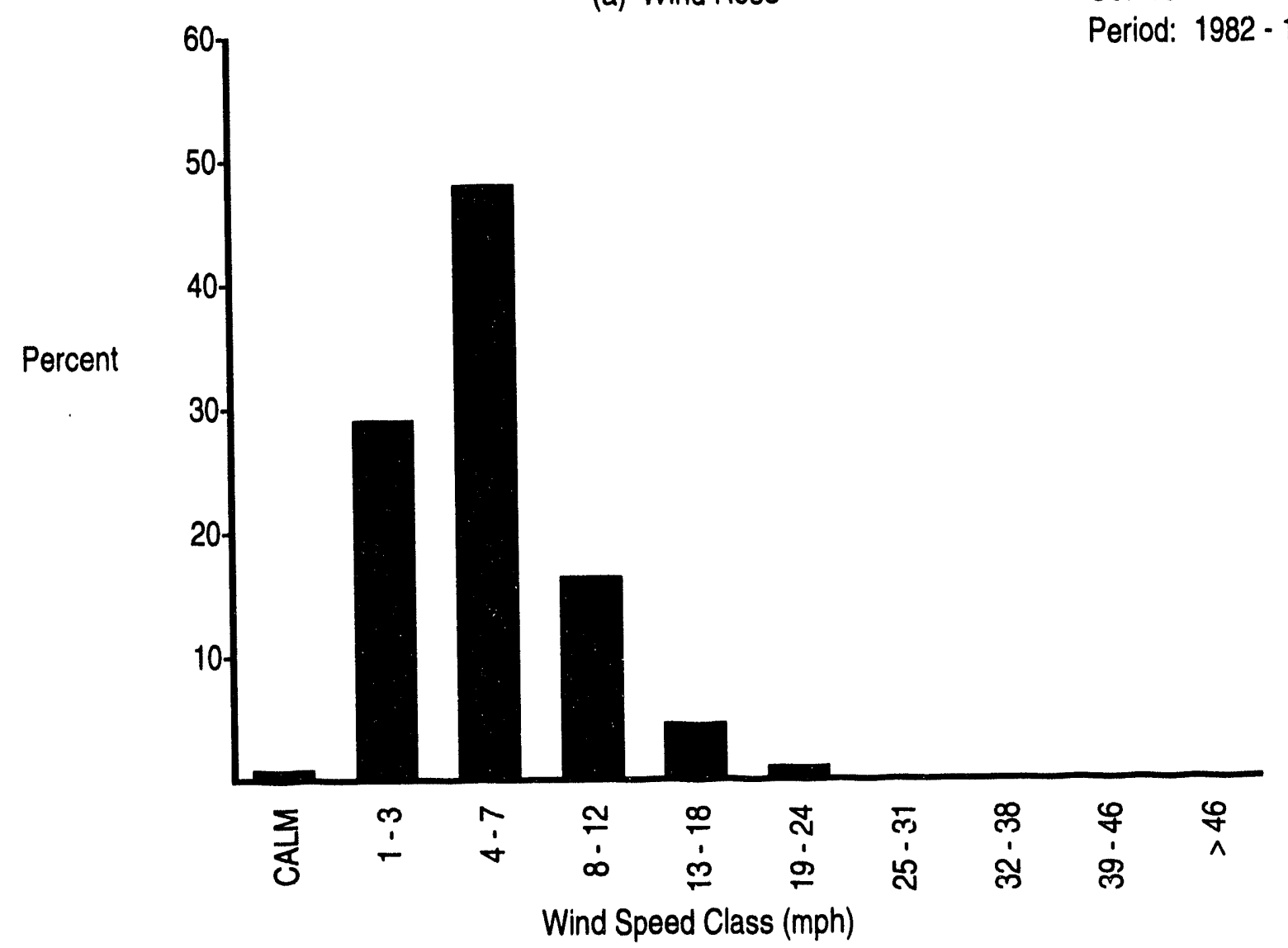

(b) Wind Speed Histogram

FIGURE B.1. (contd)
October Data

Period: $1982-1993$ 

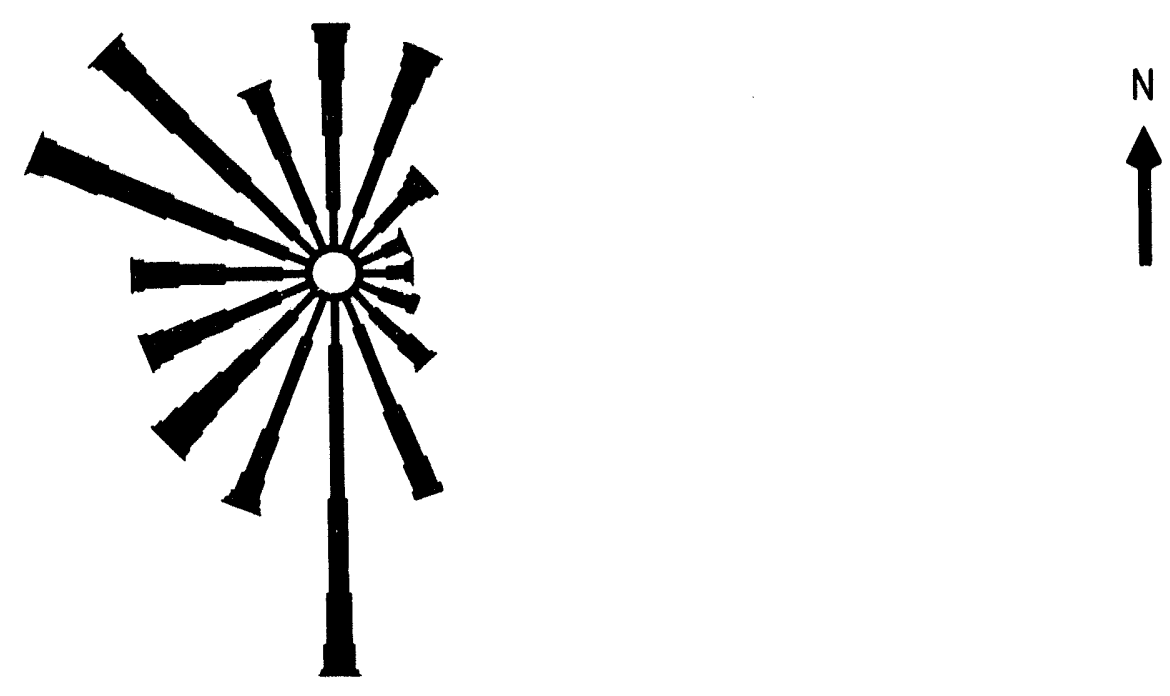

(a) Wind Rose

October Data

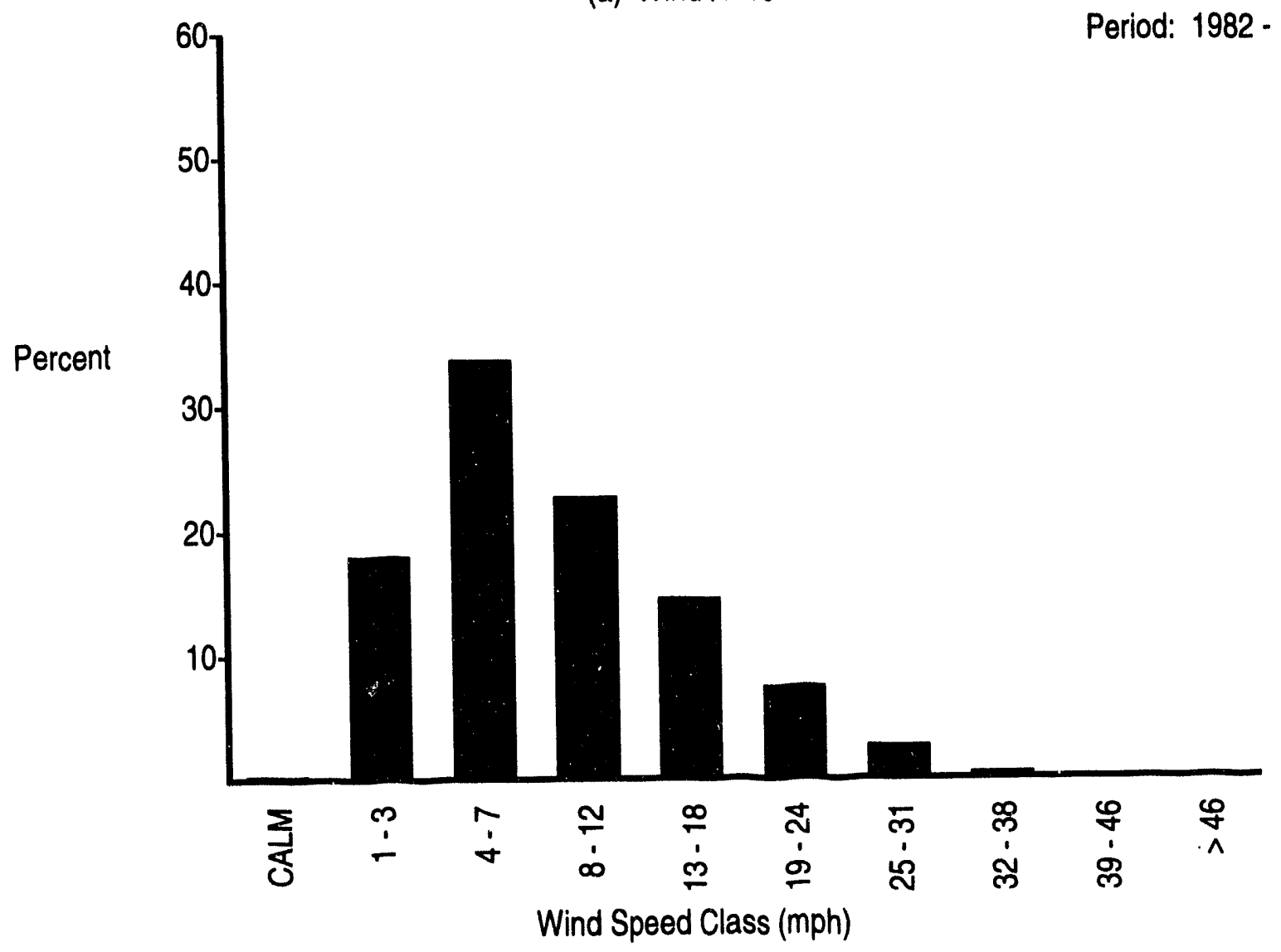

(b) Wind Speed Histogram

FIGURE B.1. (contd) 

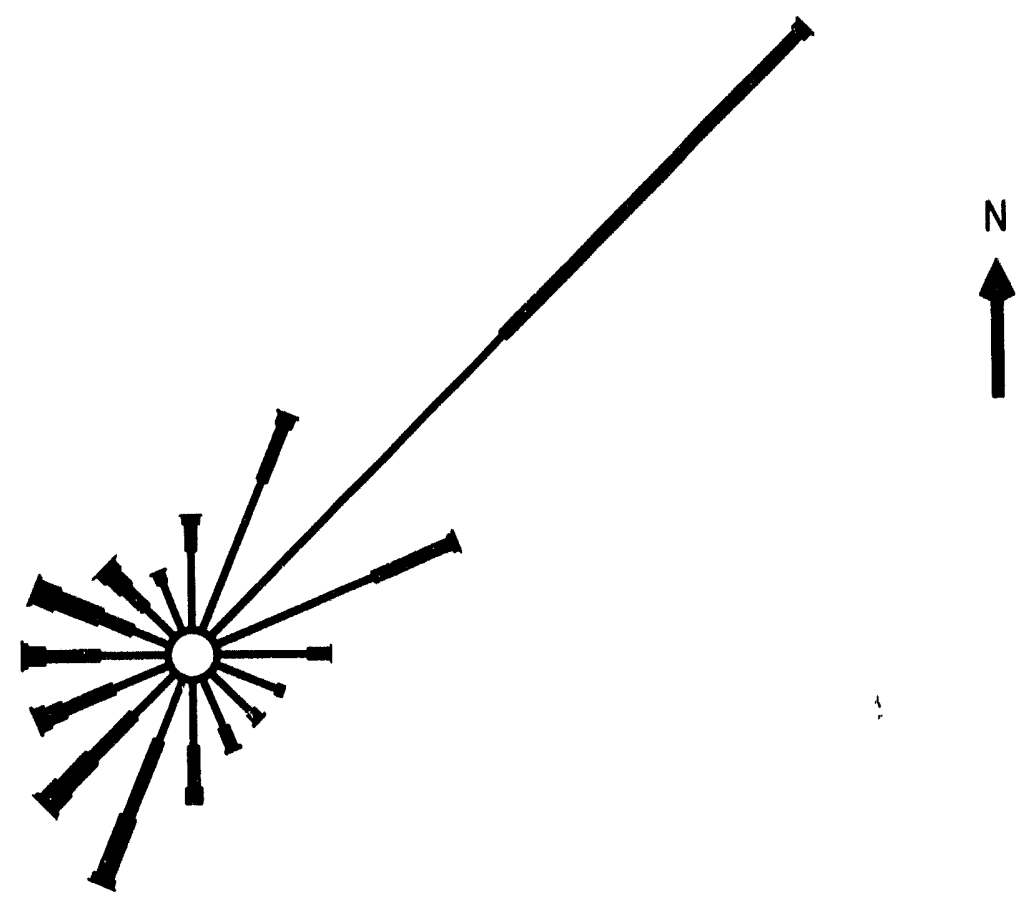

(a) Wind Rose

October Data

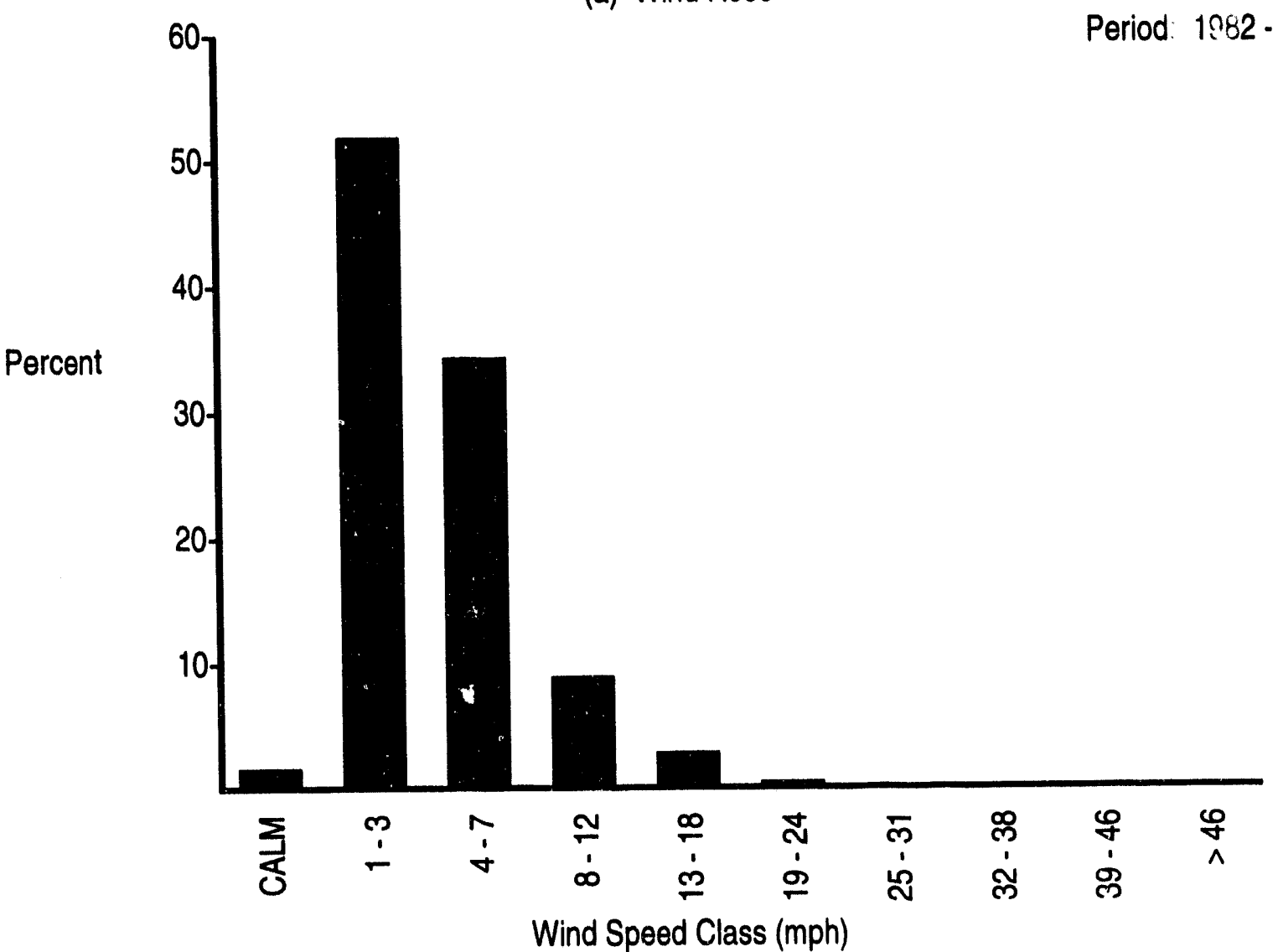

(b) Wind Speed Histogram

FIGURE B.1. (contd) 


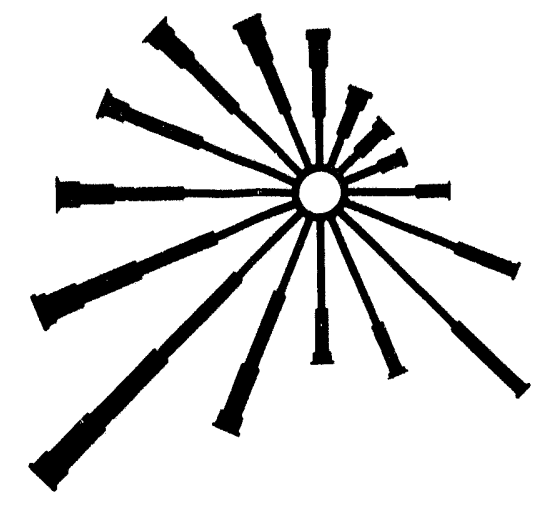

(a) Wind Rose

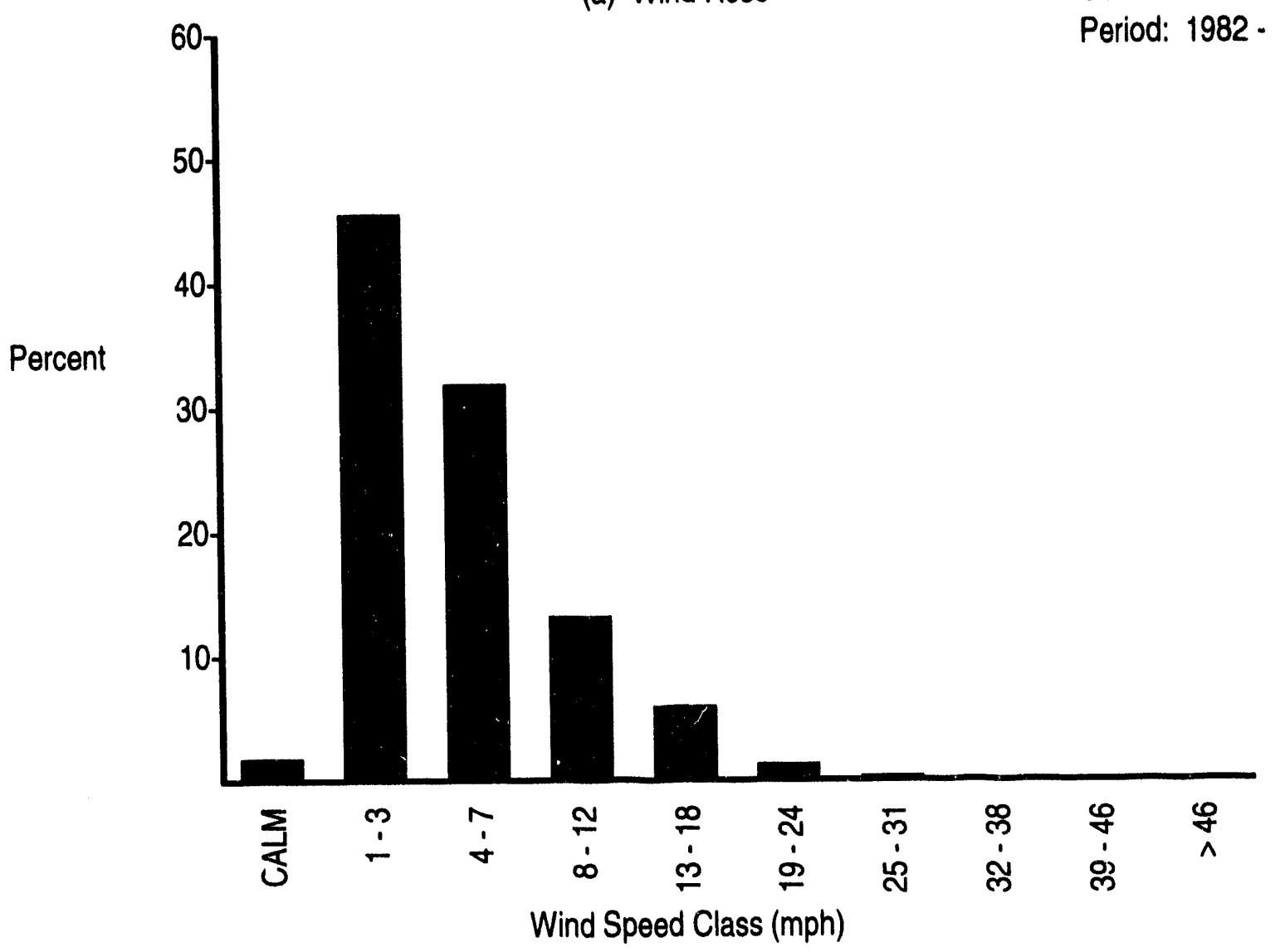

(b) Wind Speed Histogram

FIGURE B.1. (contd)
October Data

Period: 1982 - 1993 

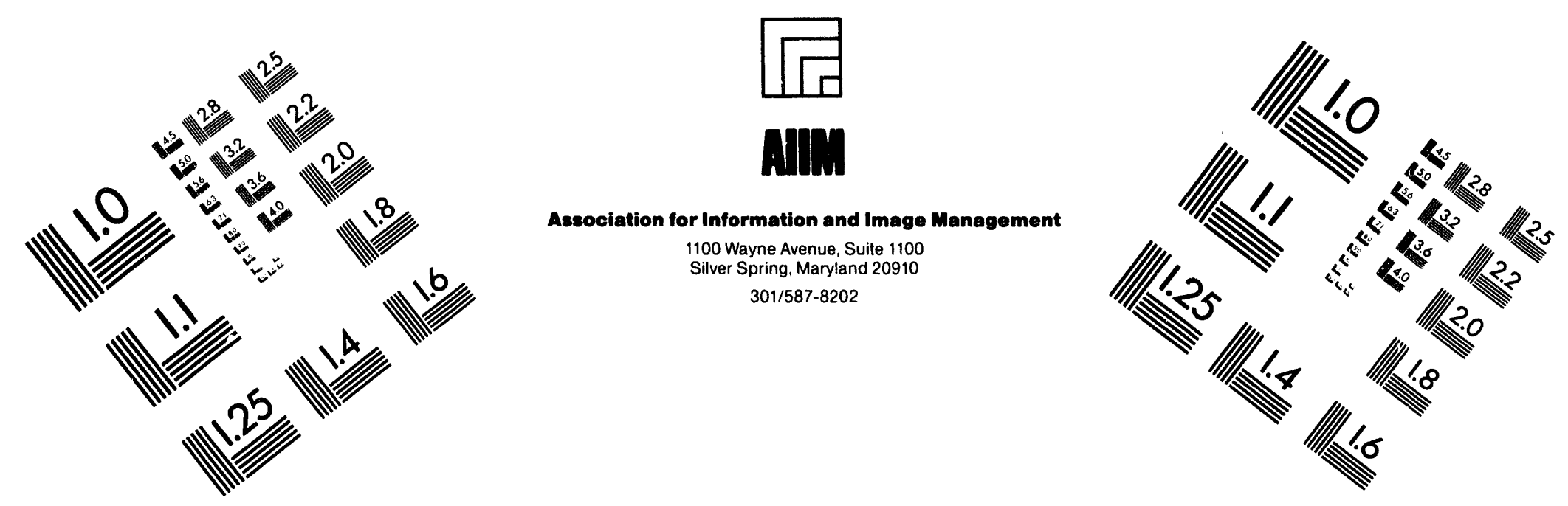

\section{Centimeter}

2
1 Inches
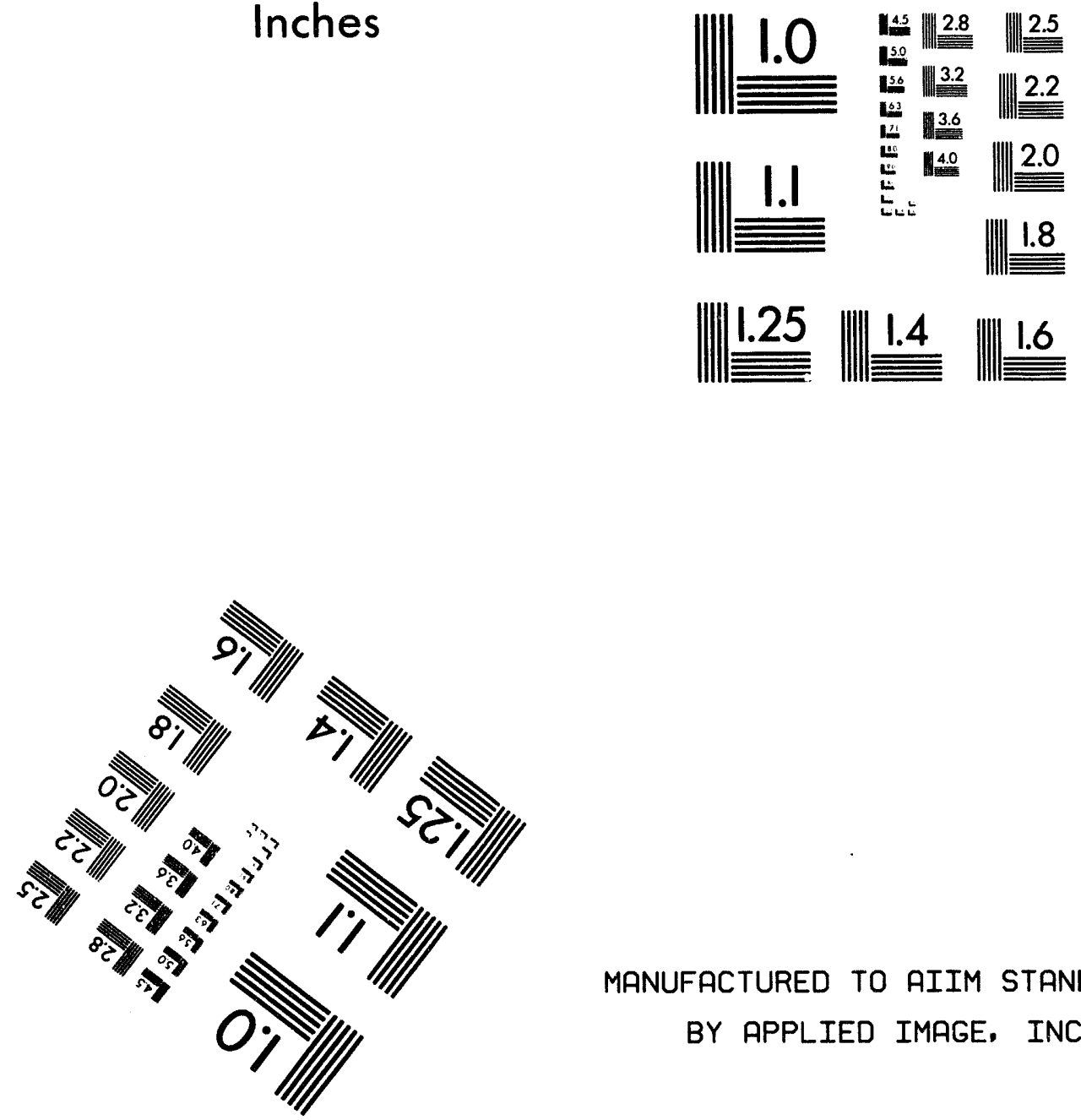

MANUFACTURED TO AIIM STANDARDS BY APPLIED IMAGE, INC.

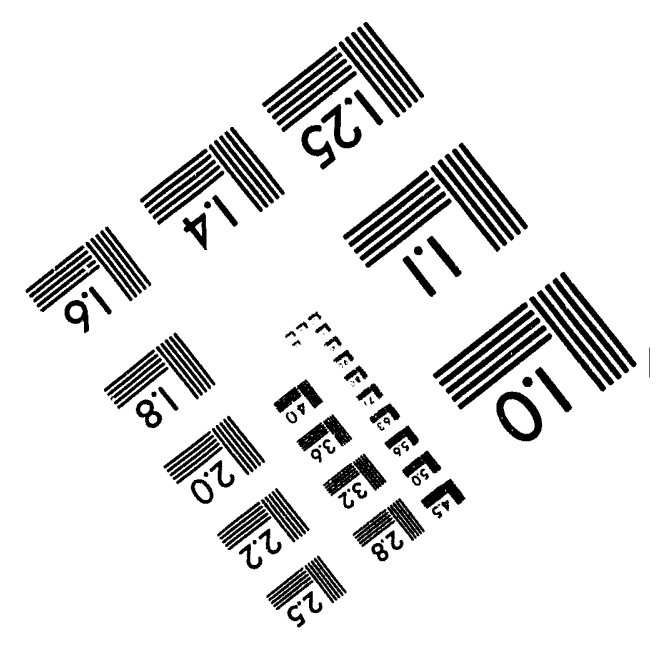





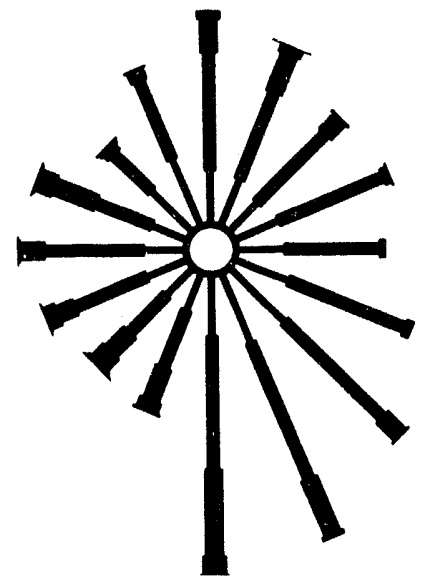

(a) Wind Rose

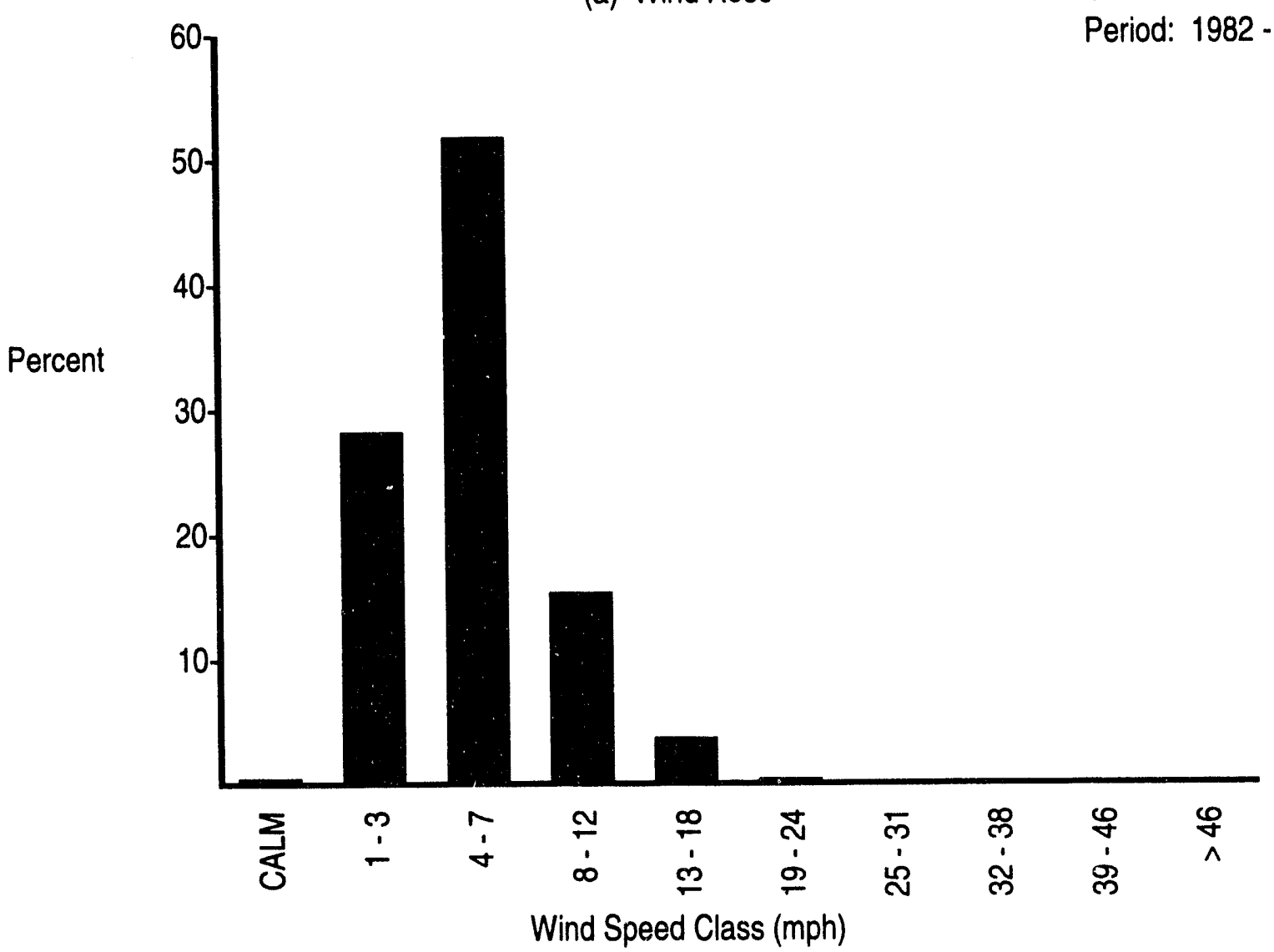

(b) Wind Speed Histogram

FIGURE B.1. (contd)
October Data

Period: 1982 - 1992 


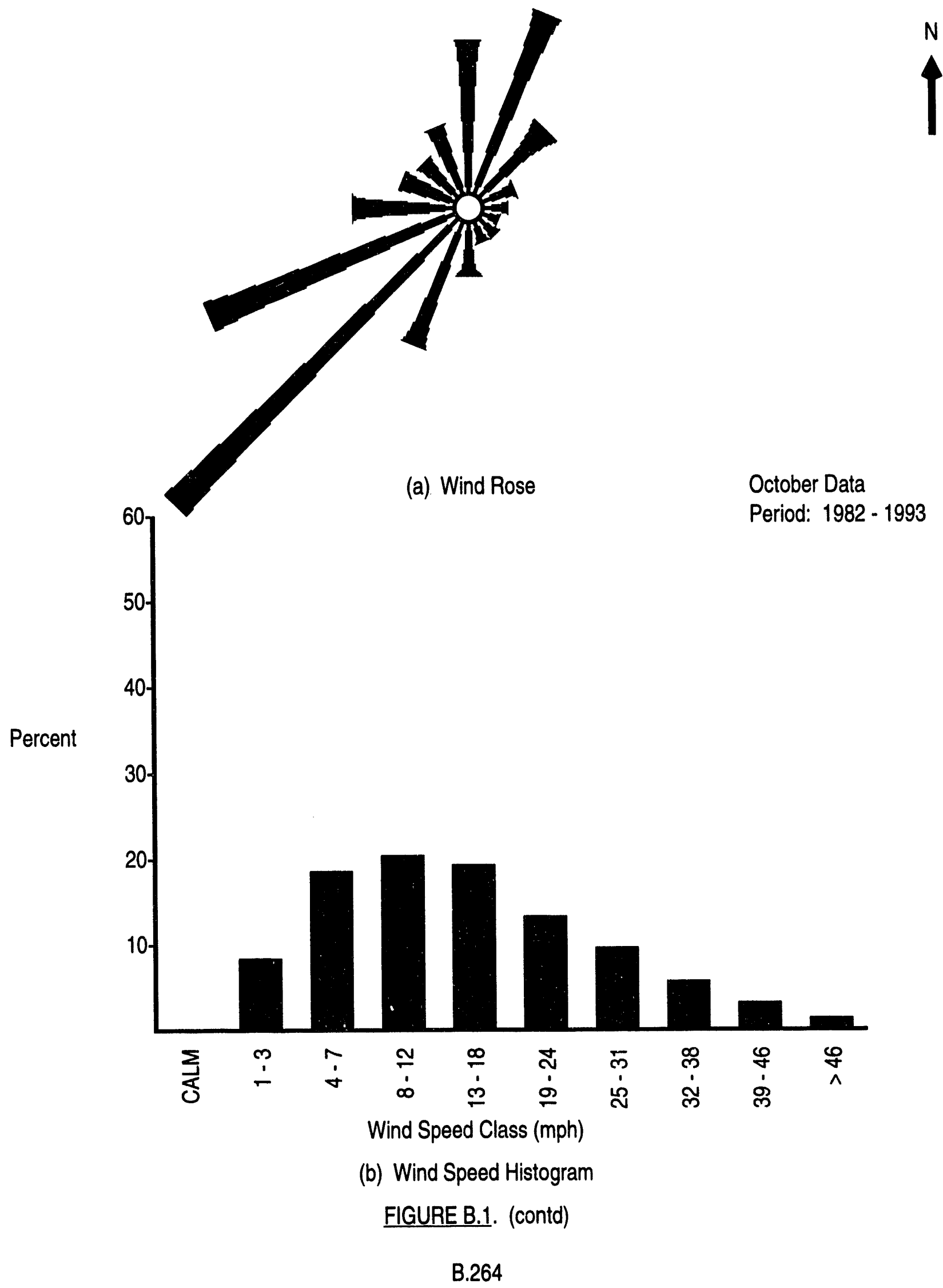



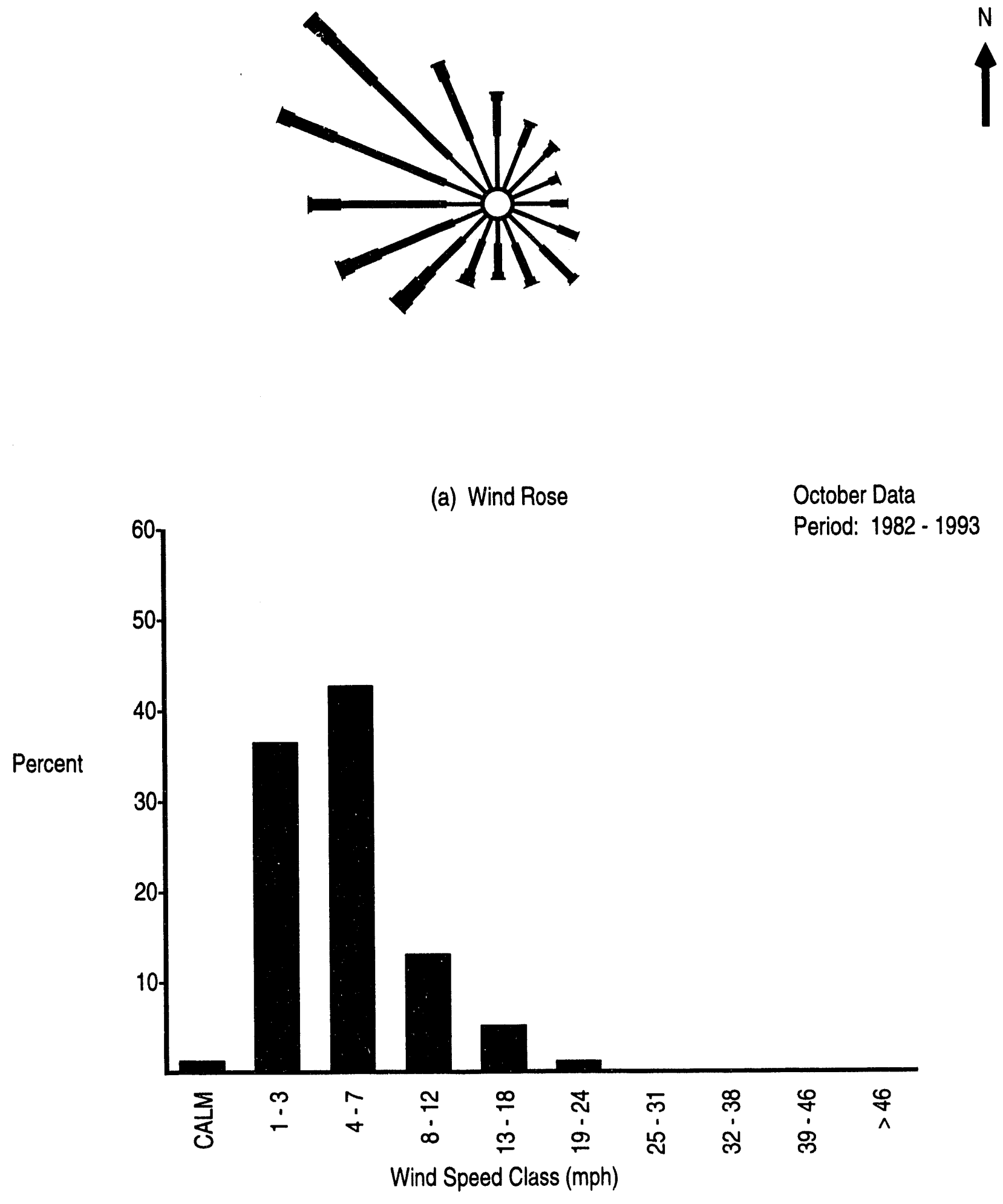

(b) Wind Speed Histogram

FIGURE B.1. (contd) 


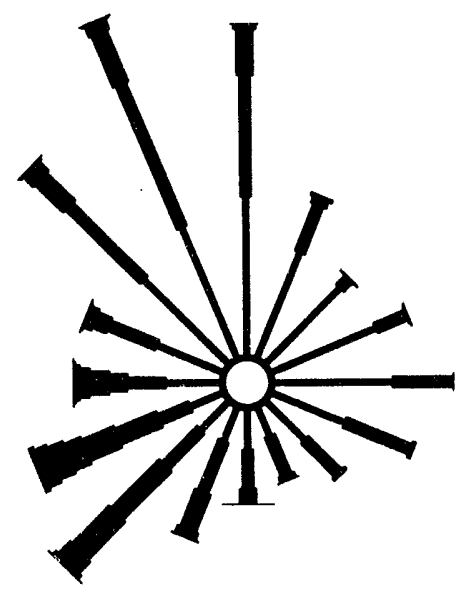

(a) Wind Rose

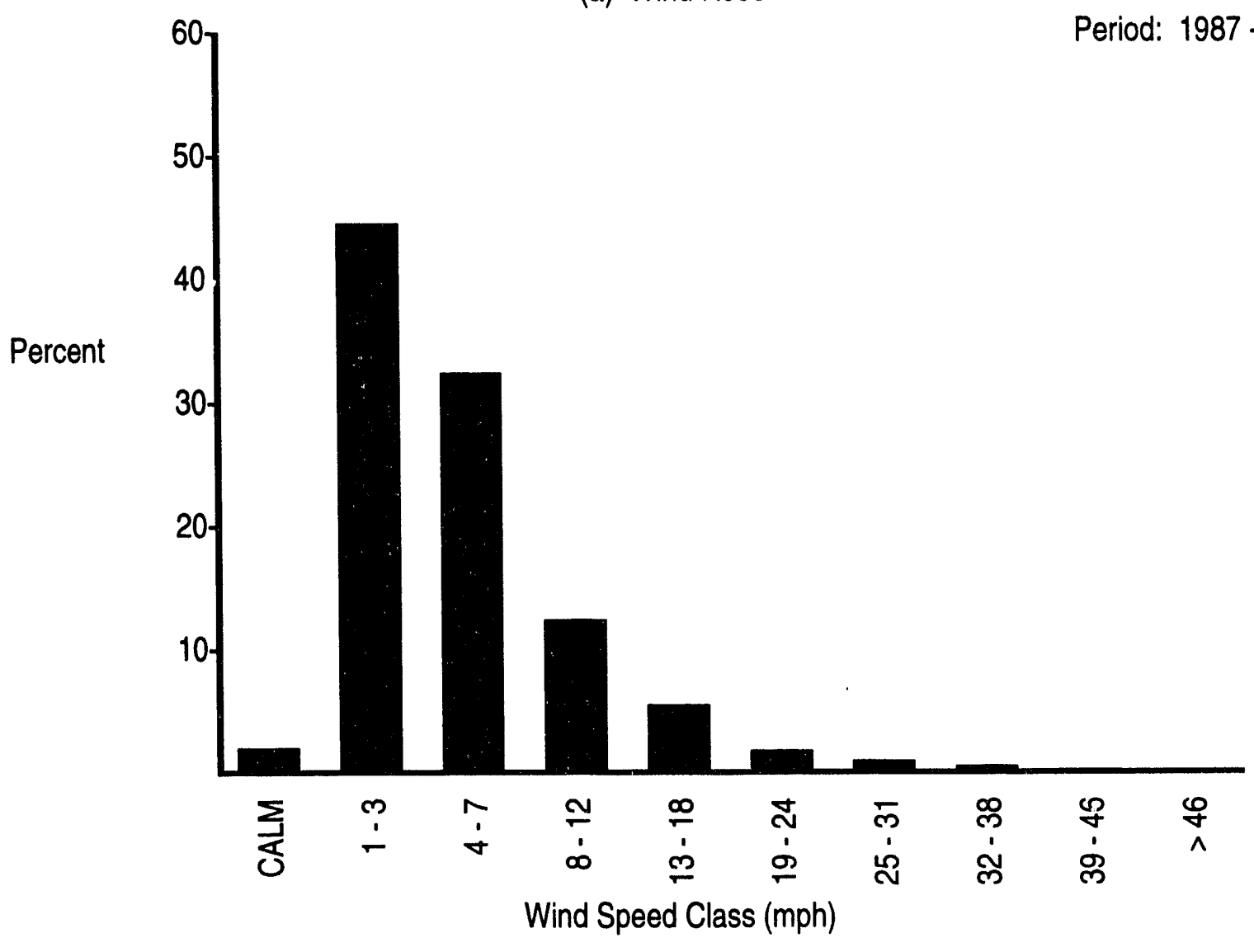

(b) Wind Speed Histogram

FIGURE B.1. (contd) 


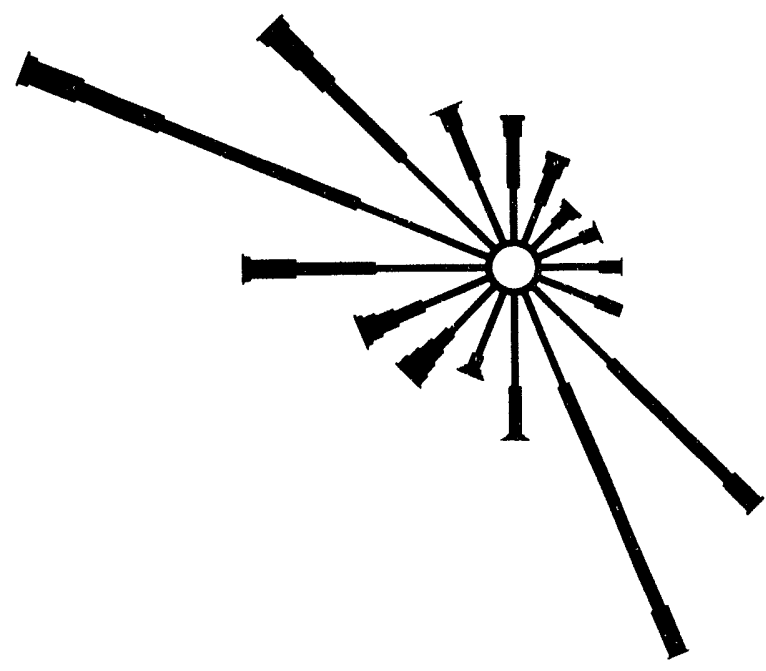

(a) Wind Rose

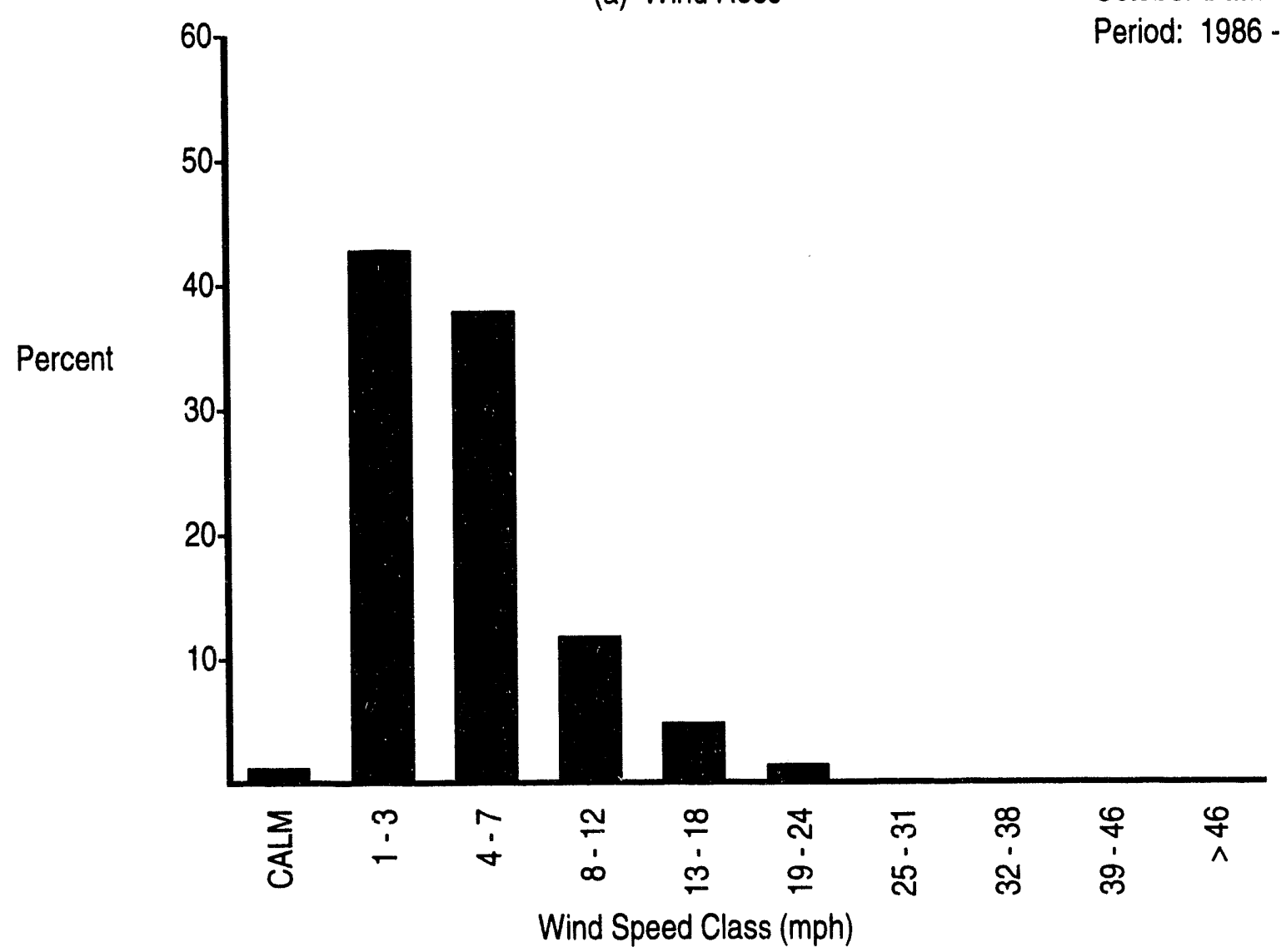

(b) Wind Speed Histogram

FIGURE B.1. (contd)
October Data

Period: 1986 - 1993 


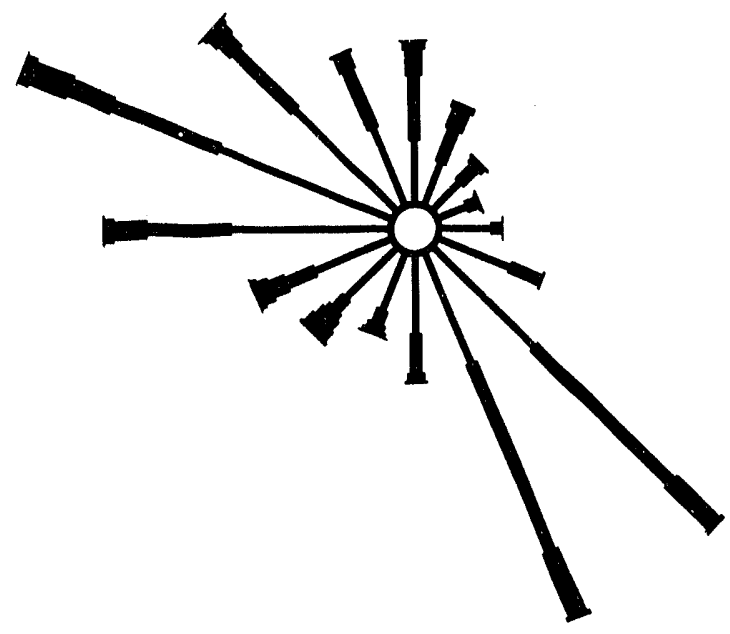

(a) Wind Rose

October Data

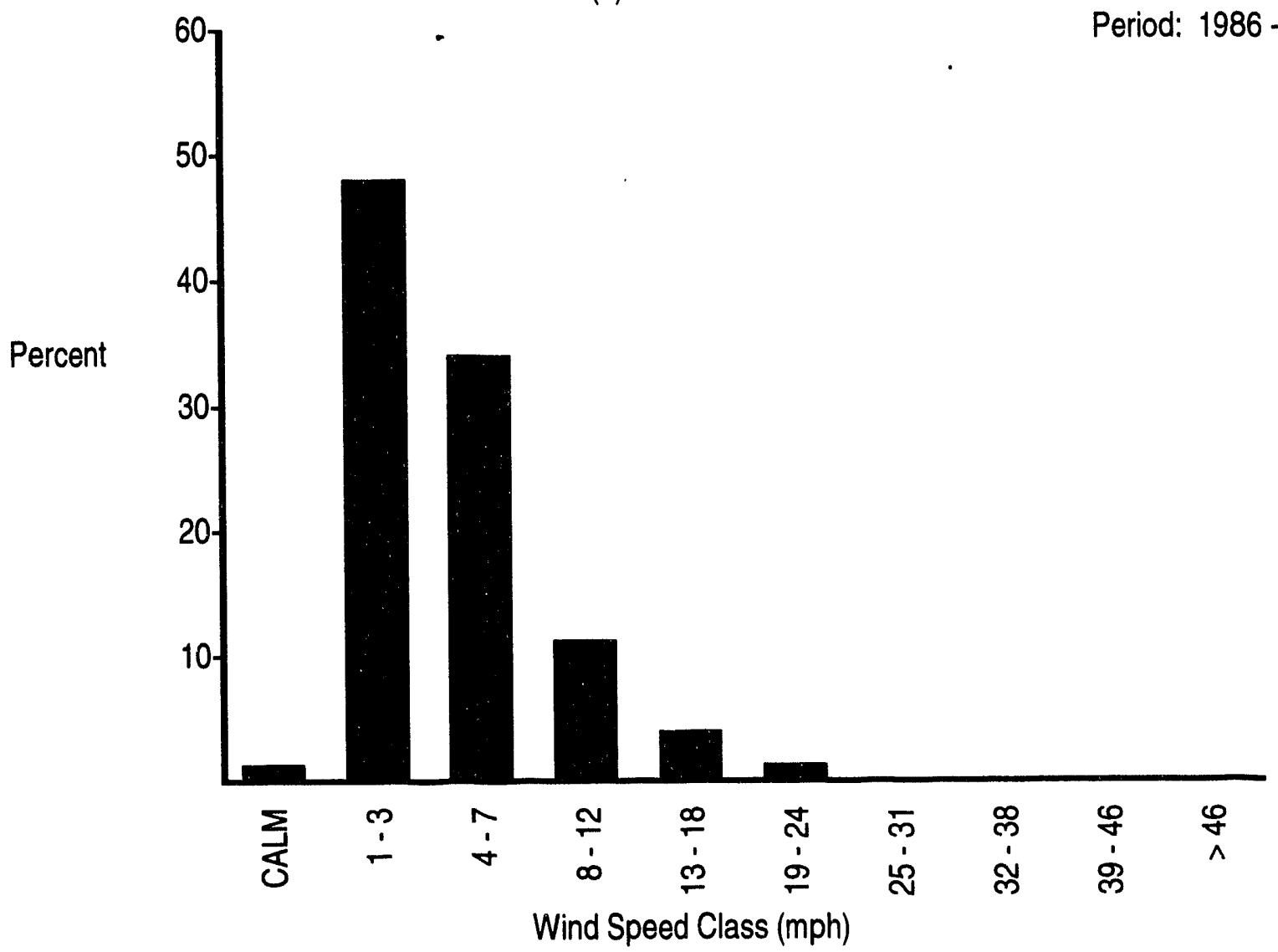

(b) Wind Speed Histogram

FIGURE B.1. (contd) 

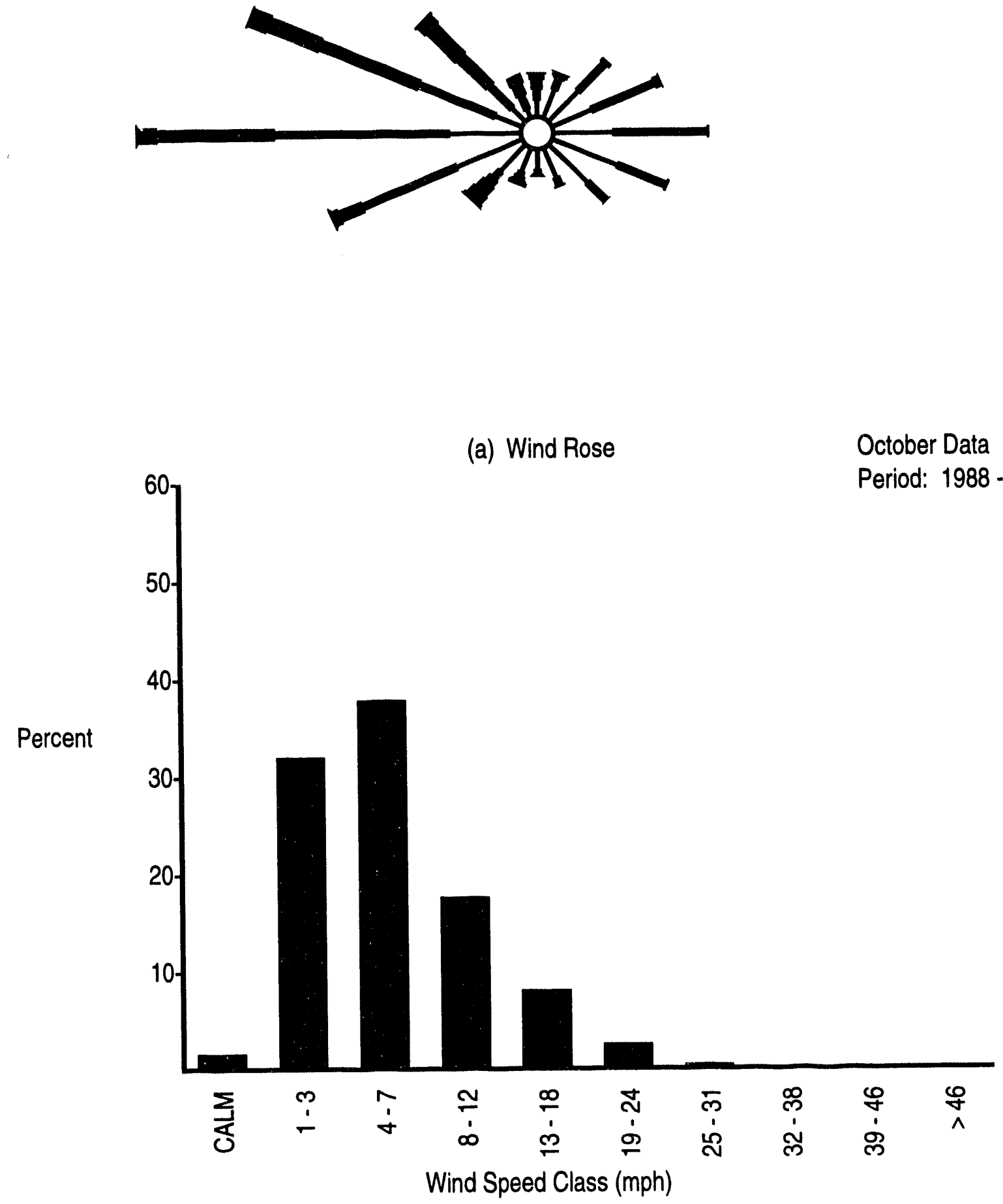

(b) Wind Speed Histogram

FIGURE B.1. (contd) 

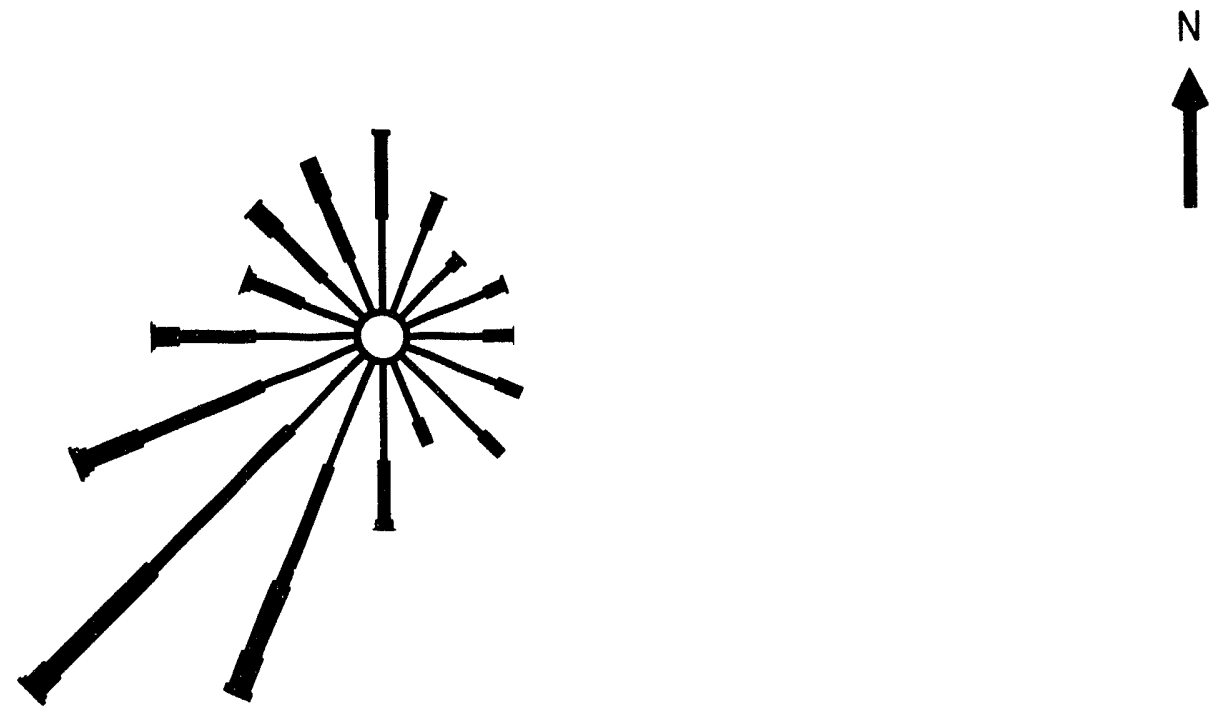

(a) Wind Rose

October Data

Percent

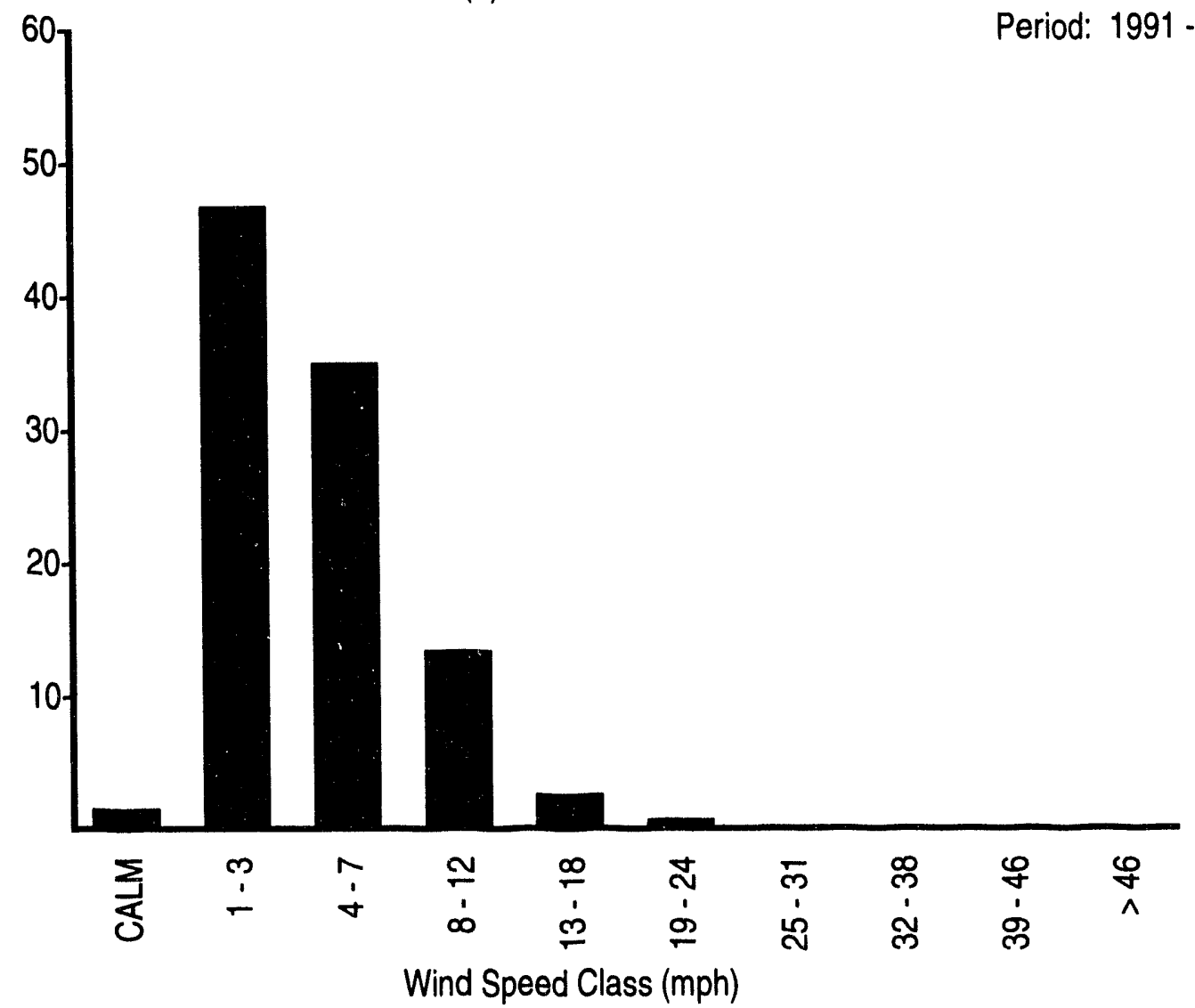

(b) Wind Speed Histogram

FIGURE B.1. (contd) 


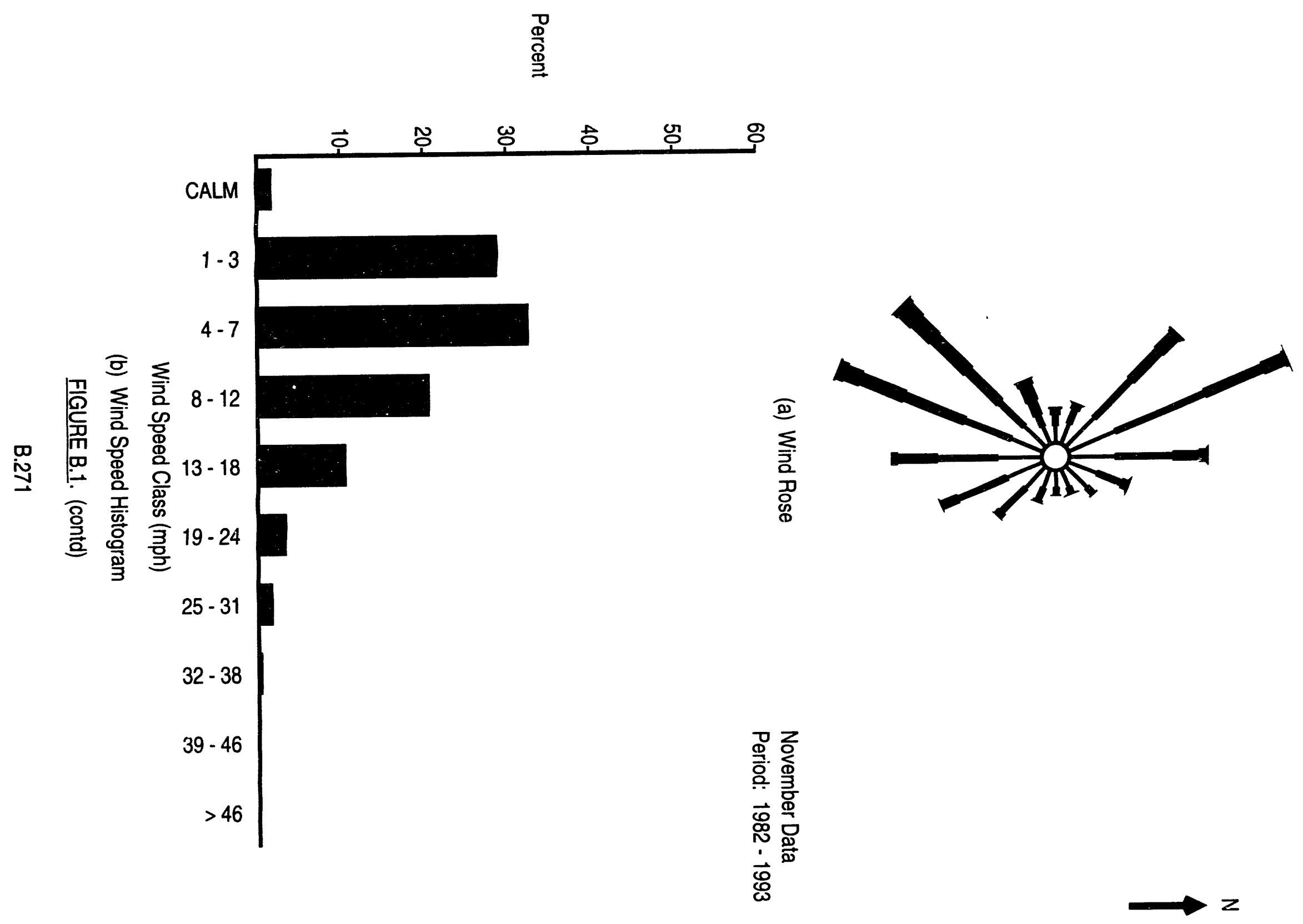




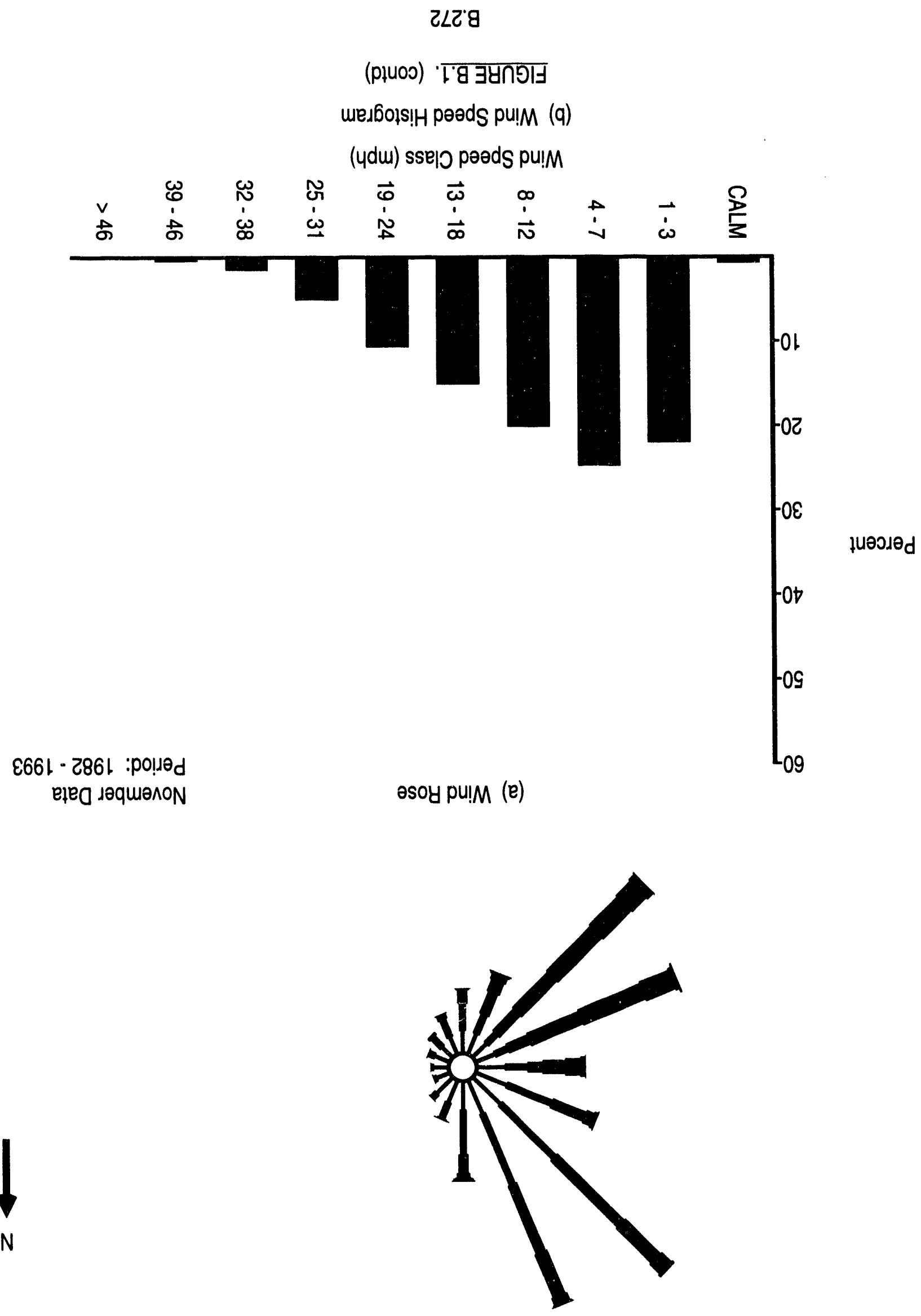



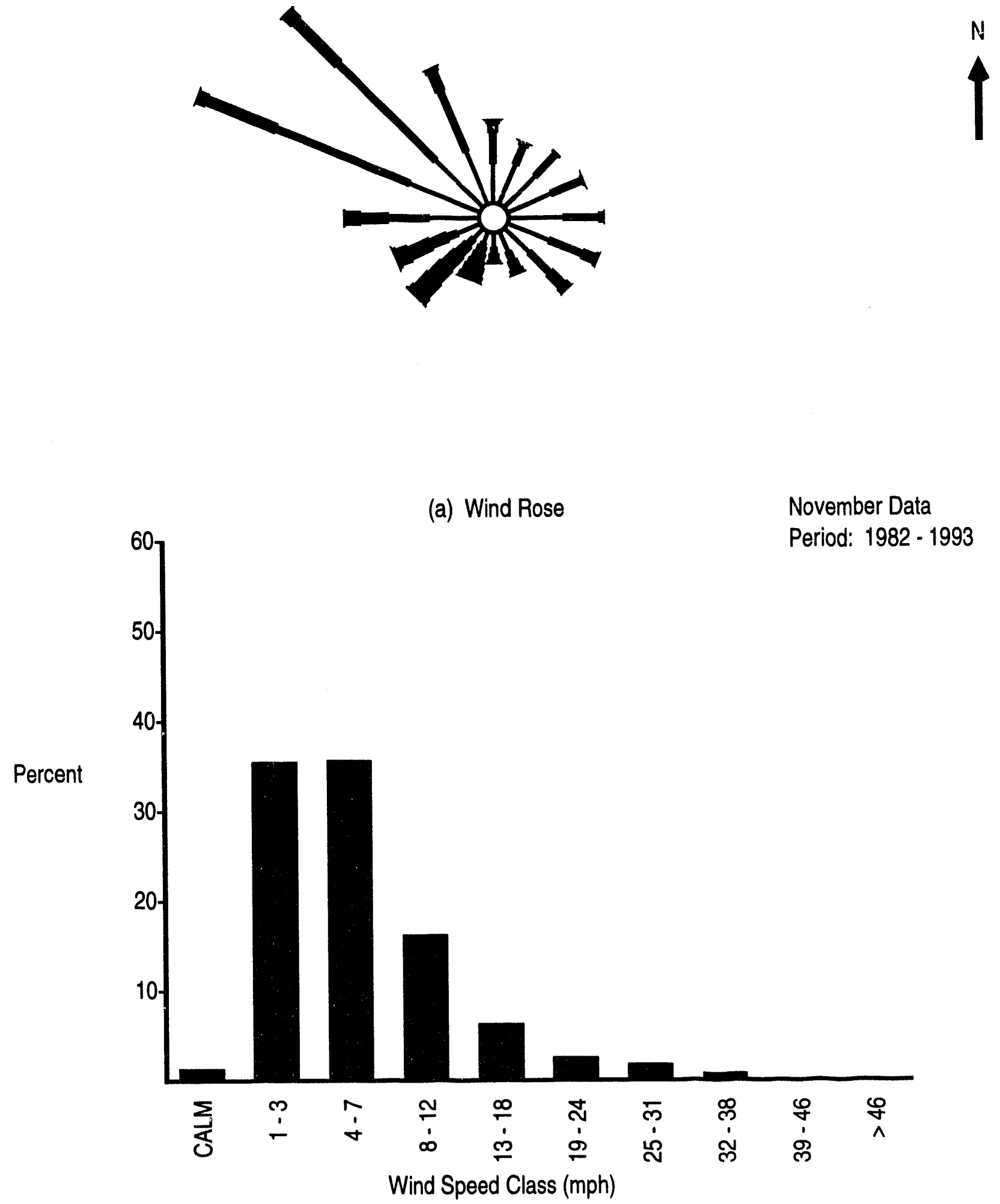

(b) Wind Speed Histogram

FIGURE B.1. (contd) 


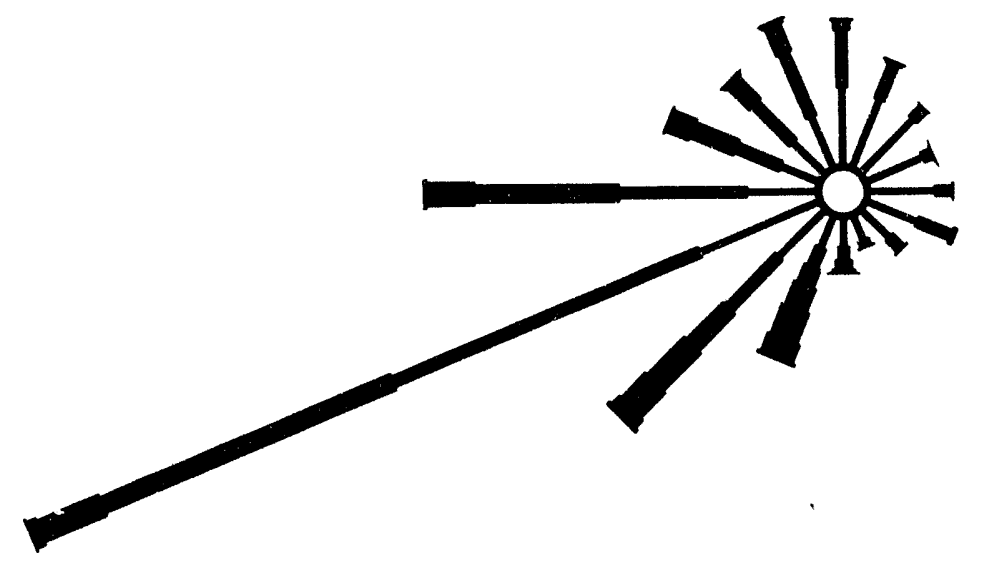

(a) Wind Rose

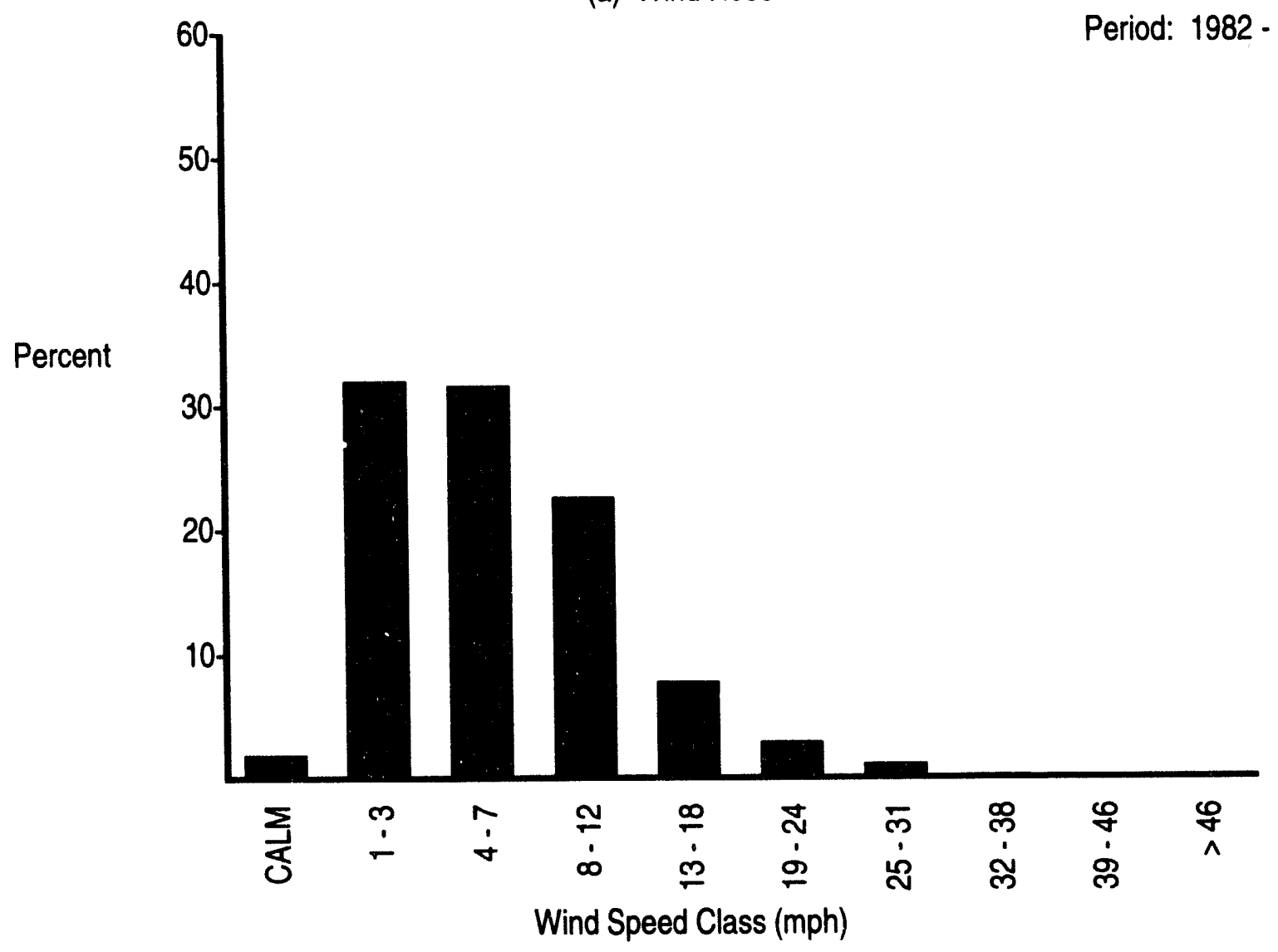

(b) Wind Speed Histogram

FIGURE B.1. (contd)
November Data

Period: 1982 - 1993 


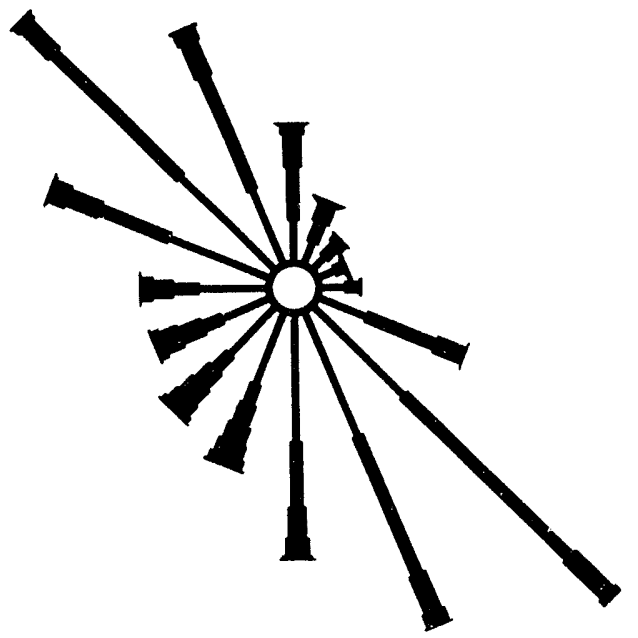

(a) Wind Rose

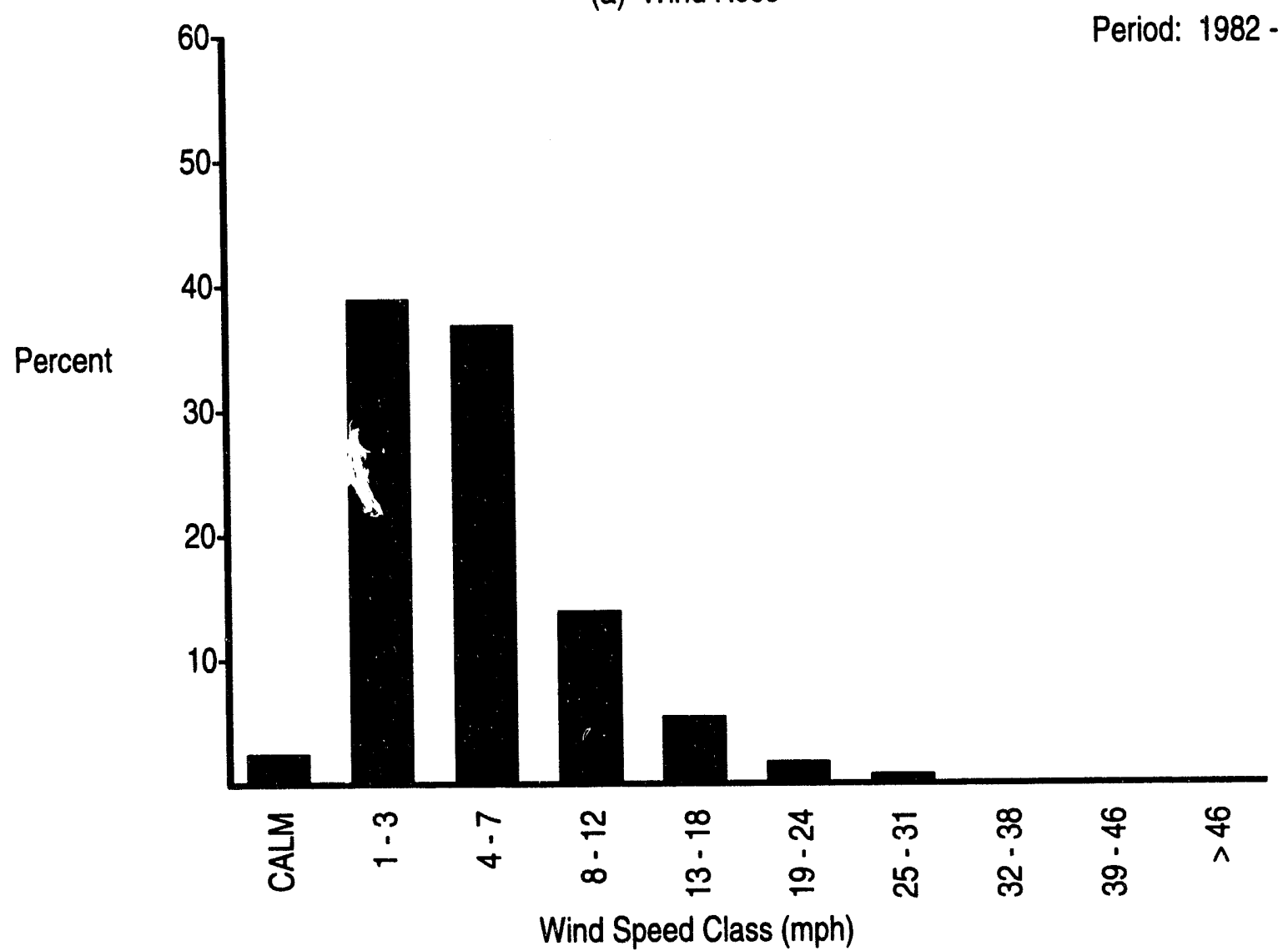

(b) Wind Speed Histogram

FIGURE B.1. (contd)
November Data

Period: 1982 - 1993 


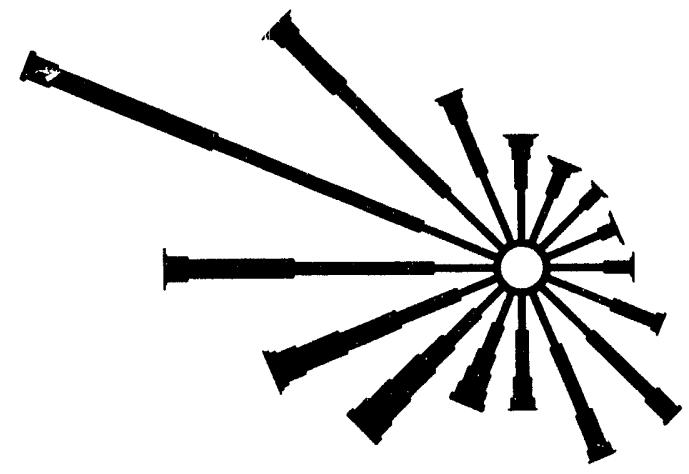

(a) Wind Rose

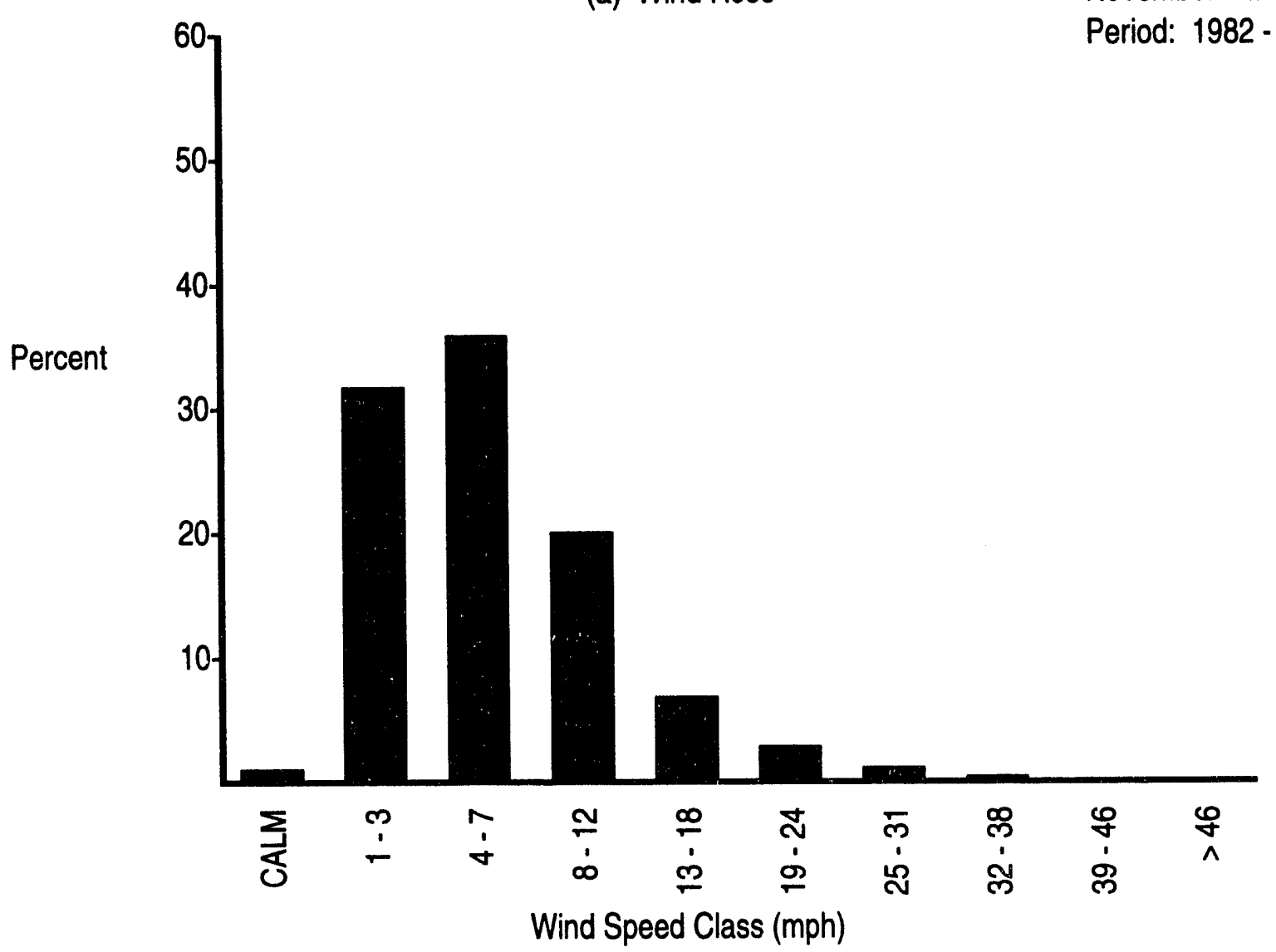

(b) Wind Speed Histogram

FIGURE B.1. (contd) 


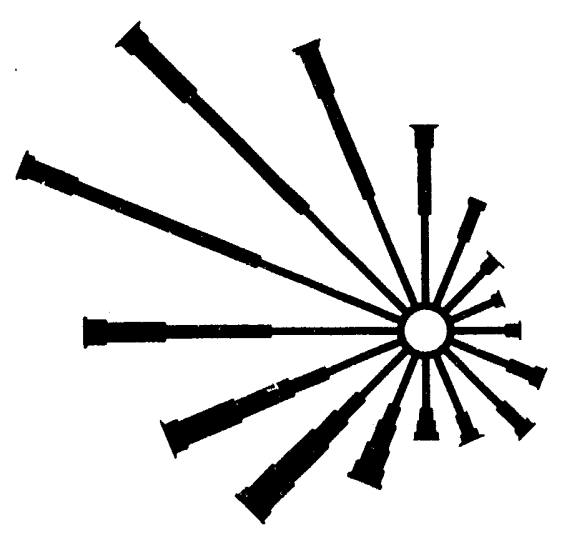

(a) Wind Rose

November Data

Period: 1982 - 1993

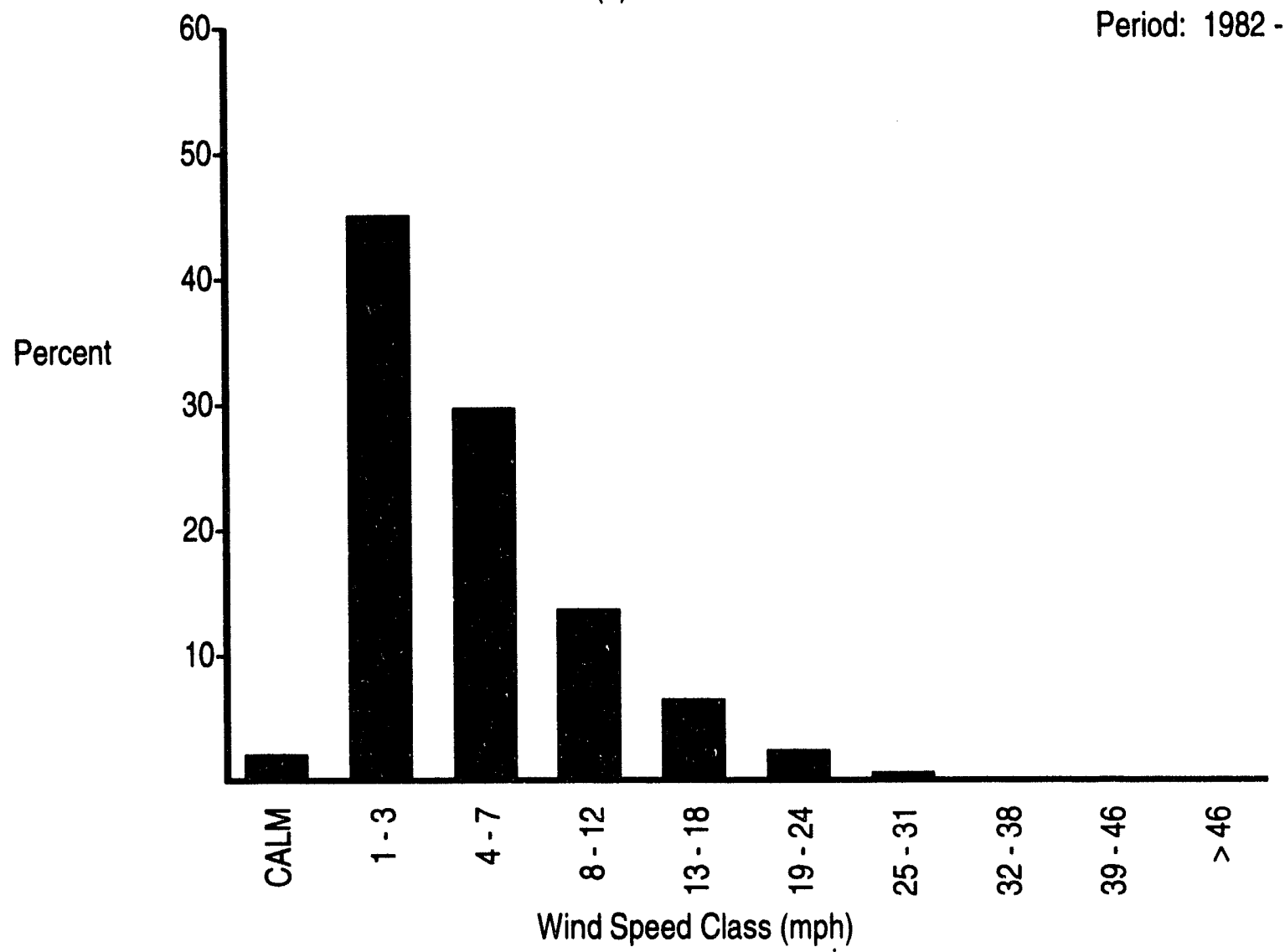

(b) Wind Speed Histogram

FIGURE B.1. (contd) 


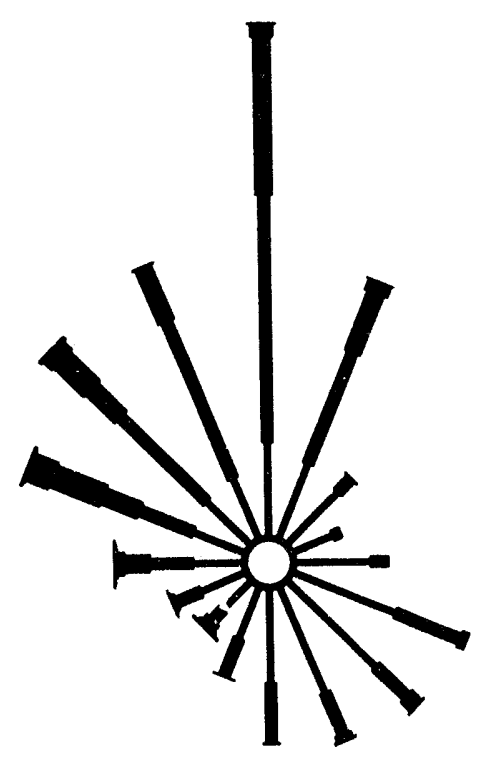

(a) Wind Rose

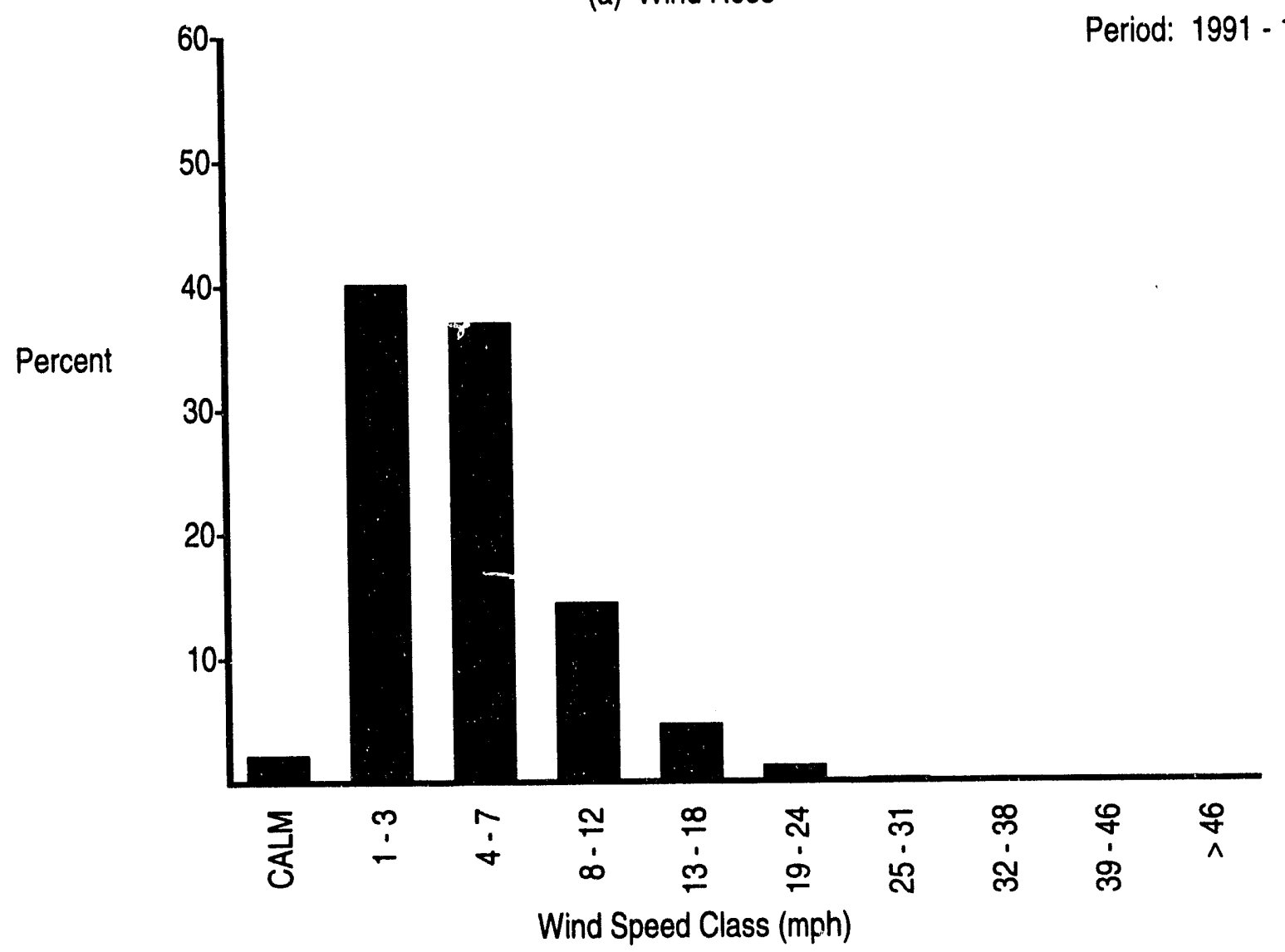

(b) Wind Speed Histogram

FIGURE B.1. (contd)
November Data

Period: 1991 - 1993 


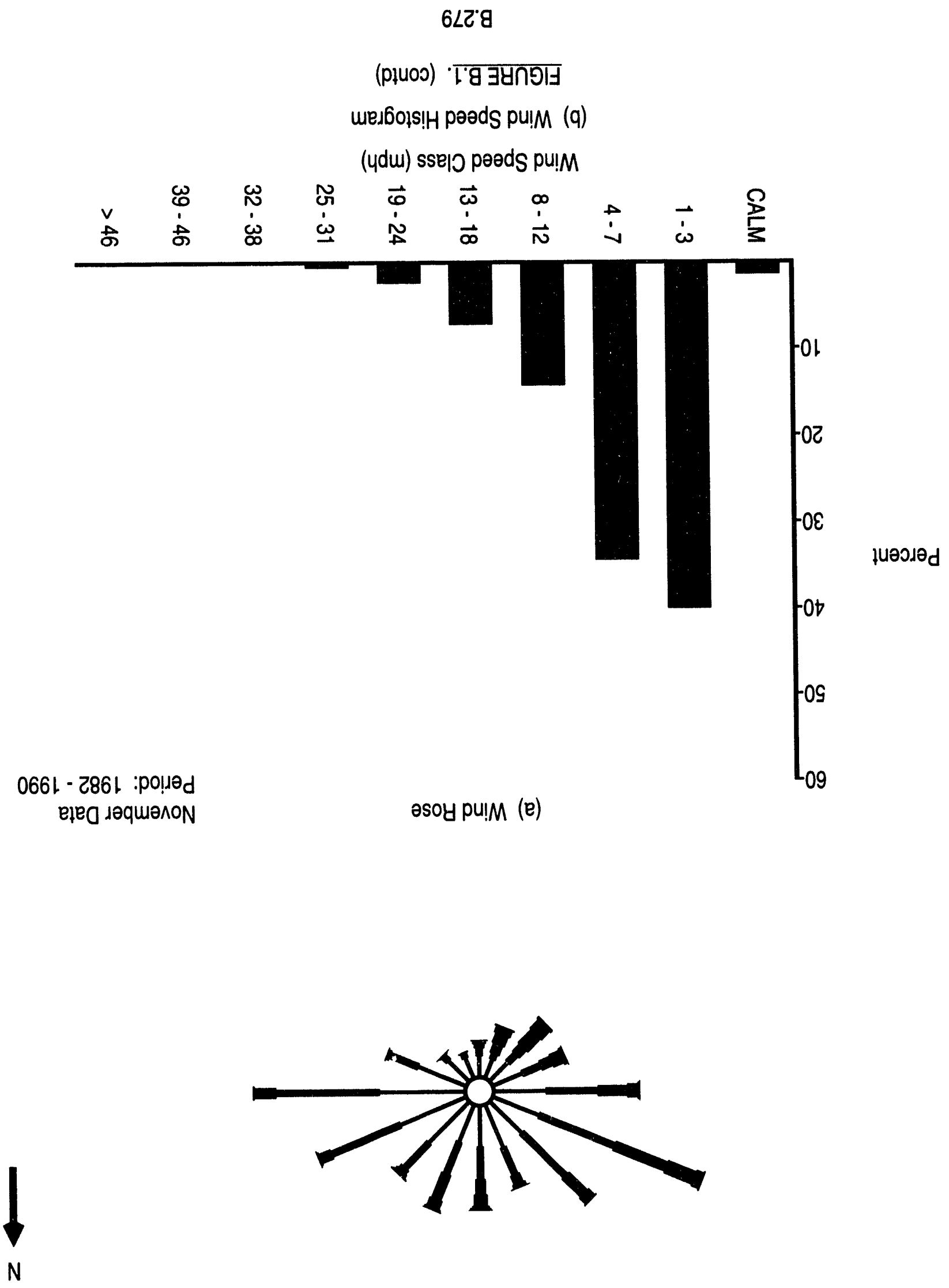




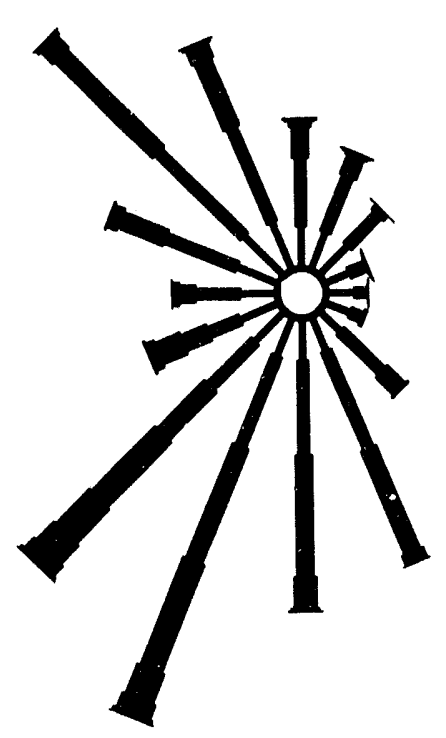

(a) Wind Rose

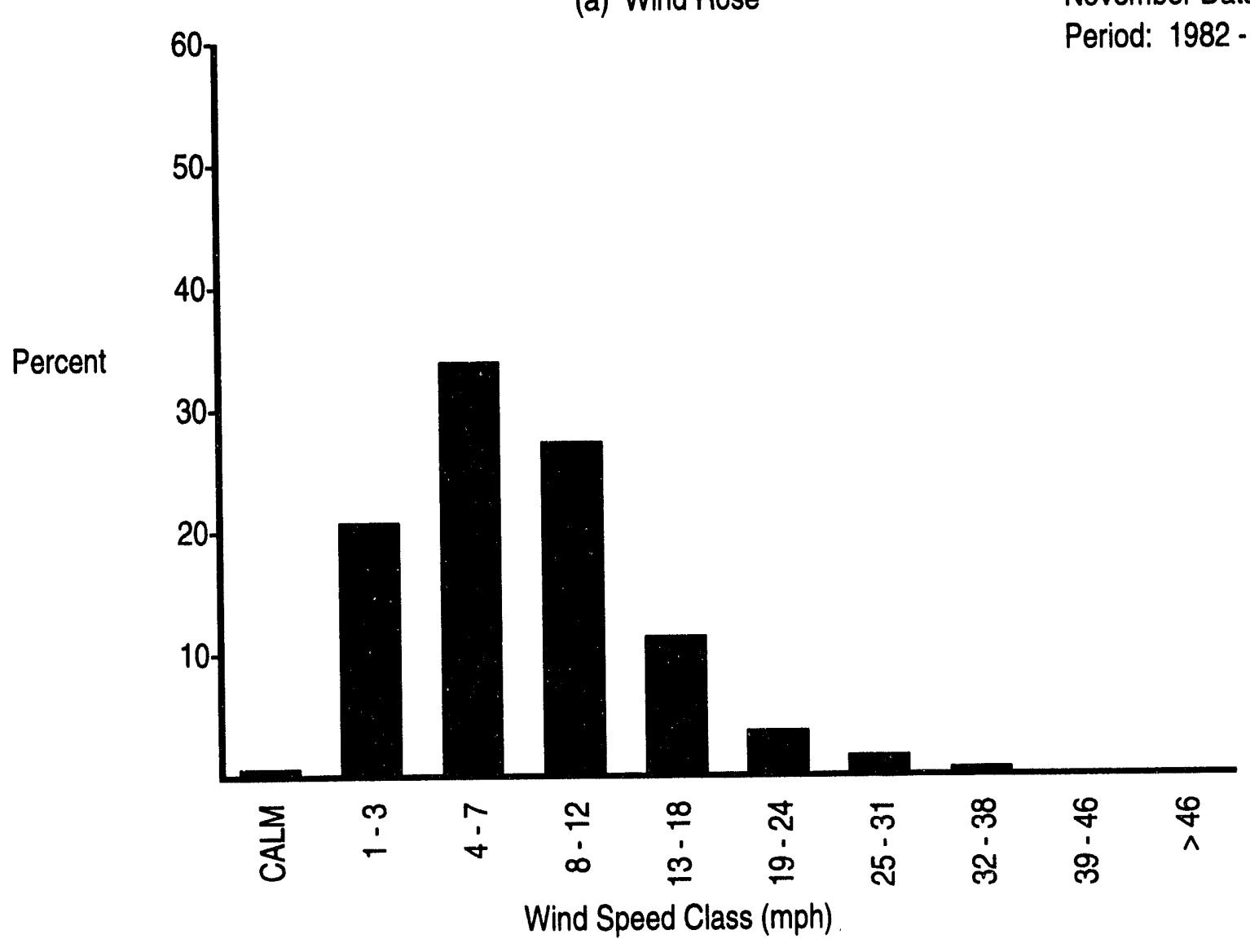

(b) Wind Speed Histogram
November Data

Period: $1982-1993$

FIGURE B.1. (contd) 


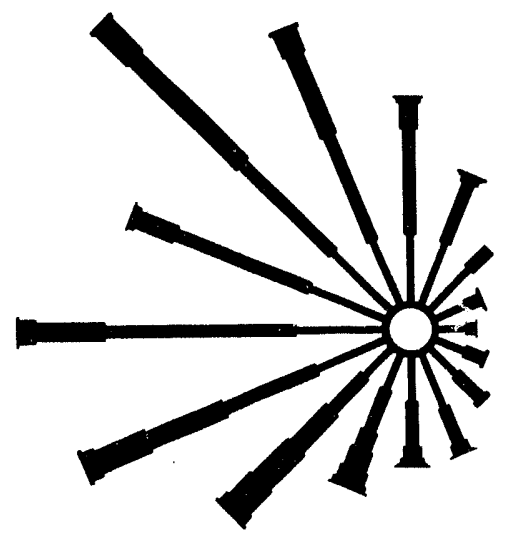

(a) Wind Rose

November Data

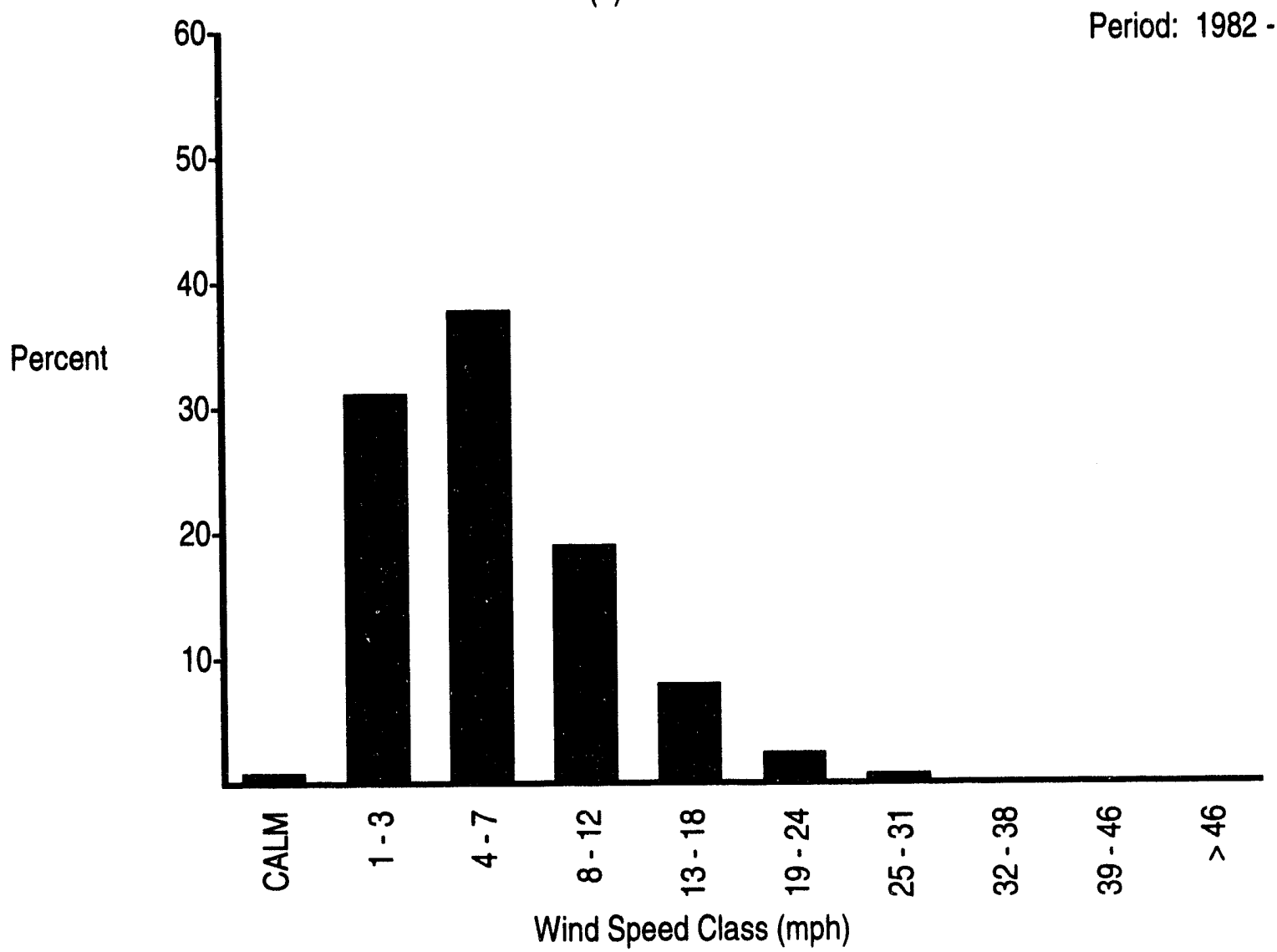

(b) Wind Speed Histogram

FIGURE B.1. (contd) 

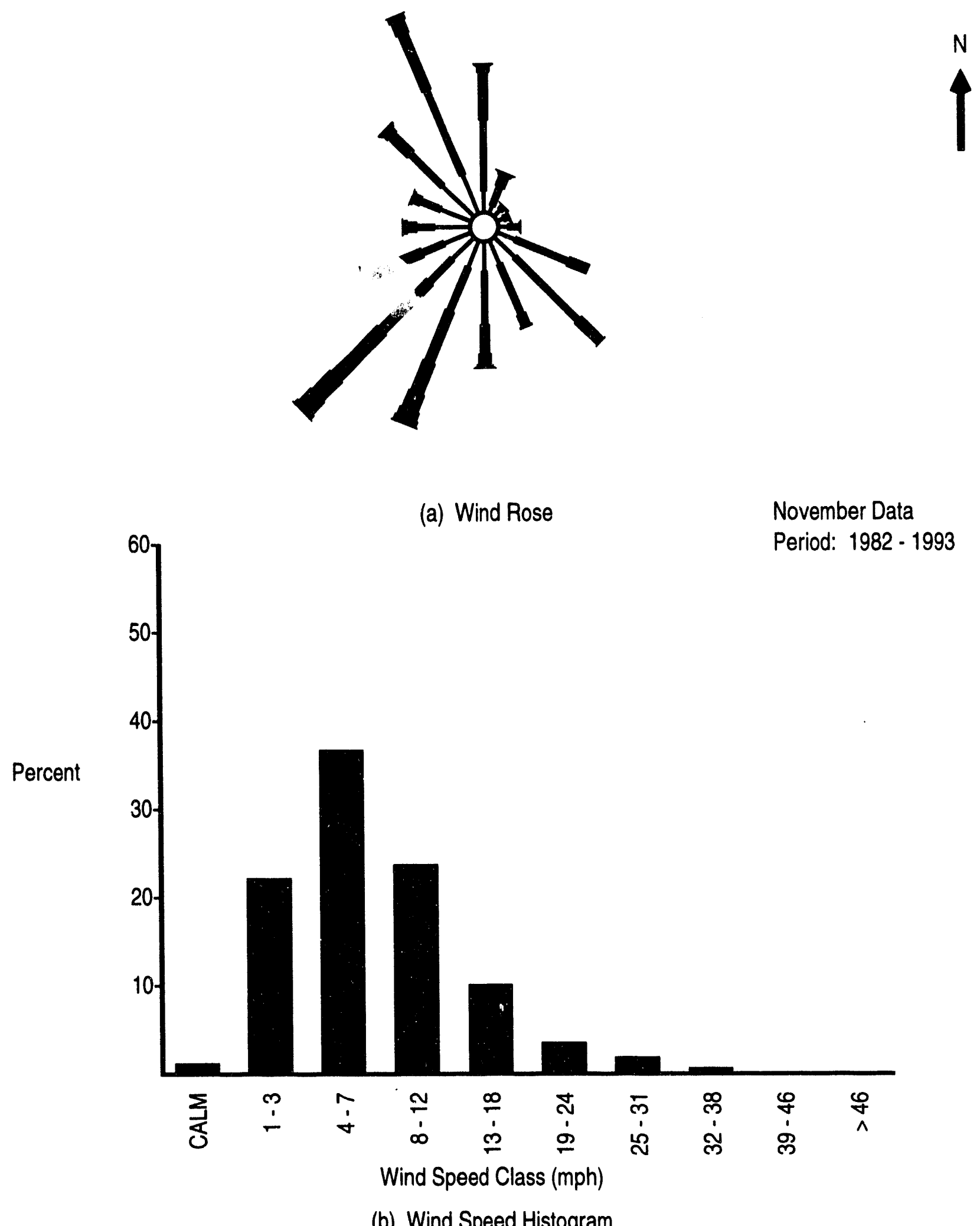

(b) Wind Speed Histogram

FIGURE B.1. (contd) 

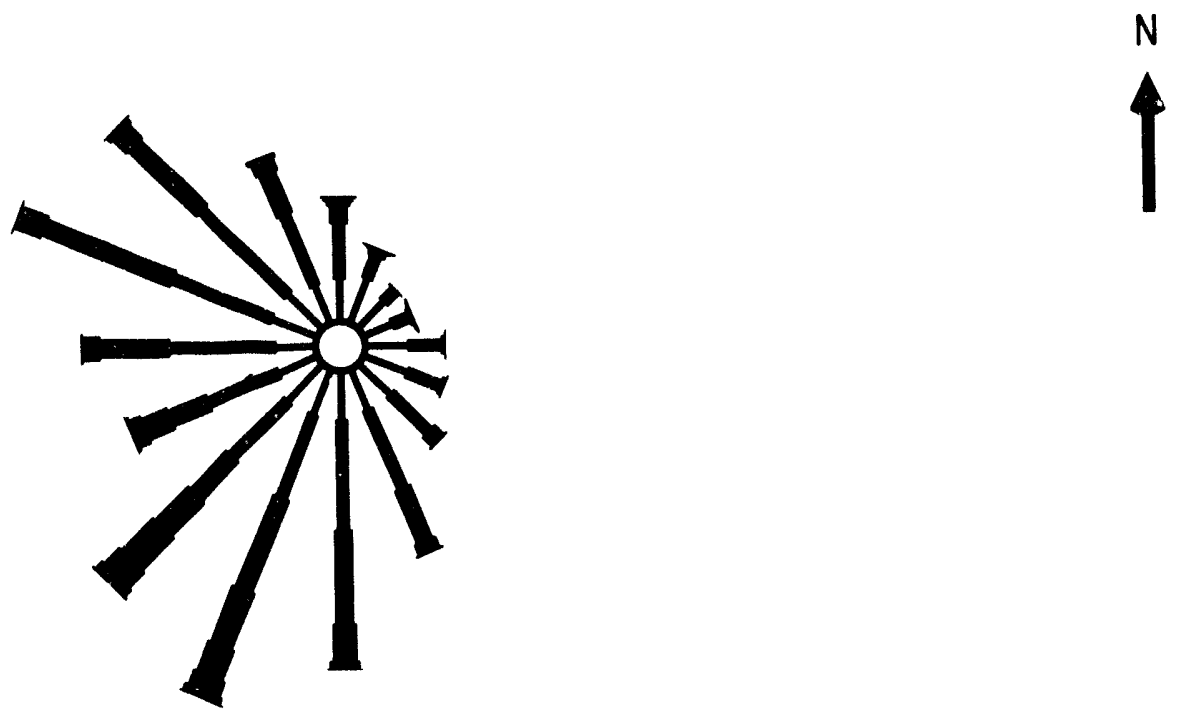

(a) Wind Rose

November Data

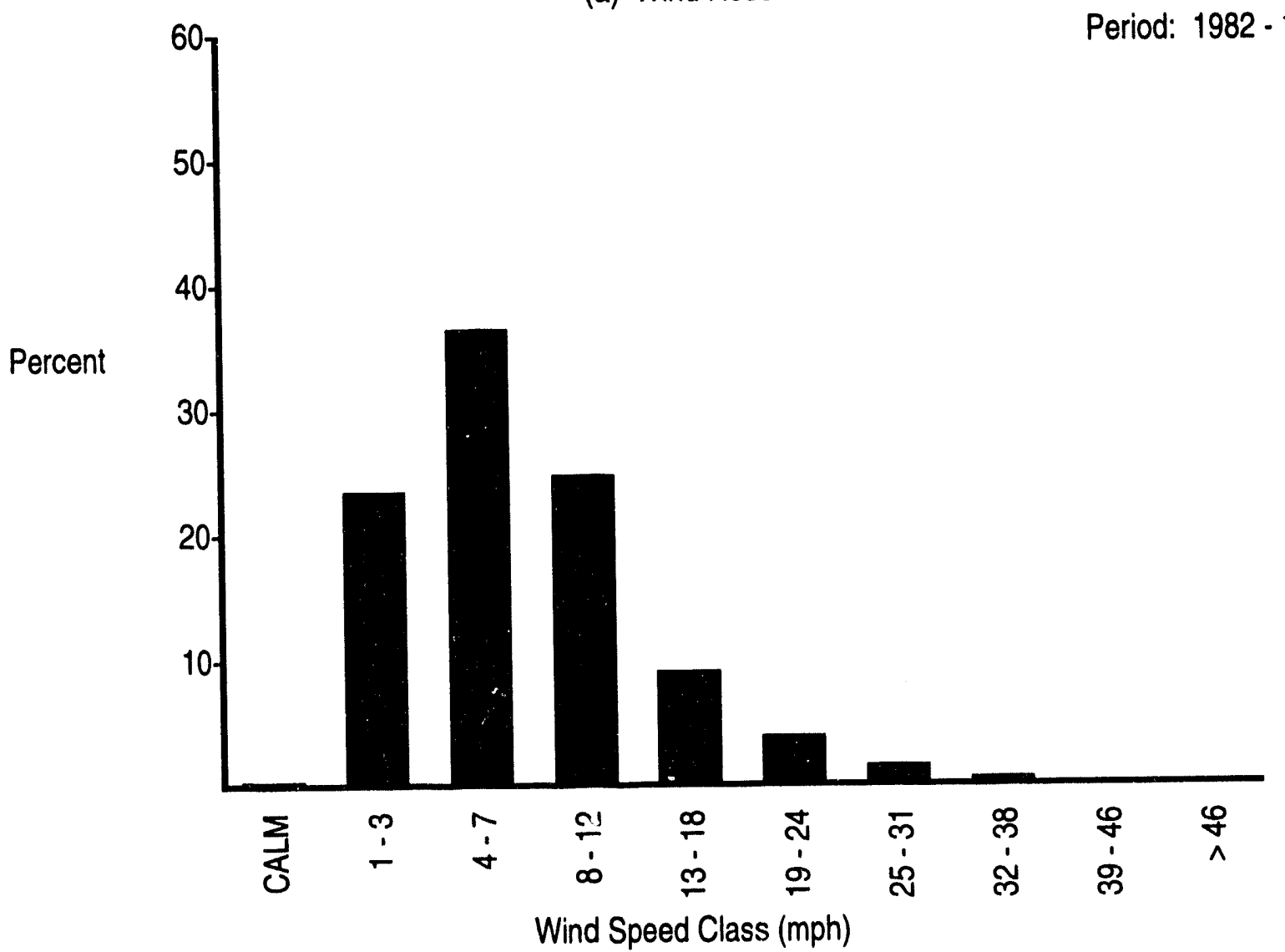

(b) Wind Speed Histogram

FIGURE B.1. (contd) 

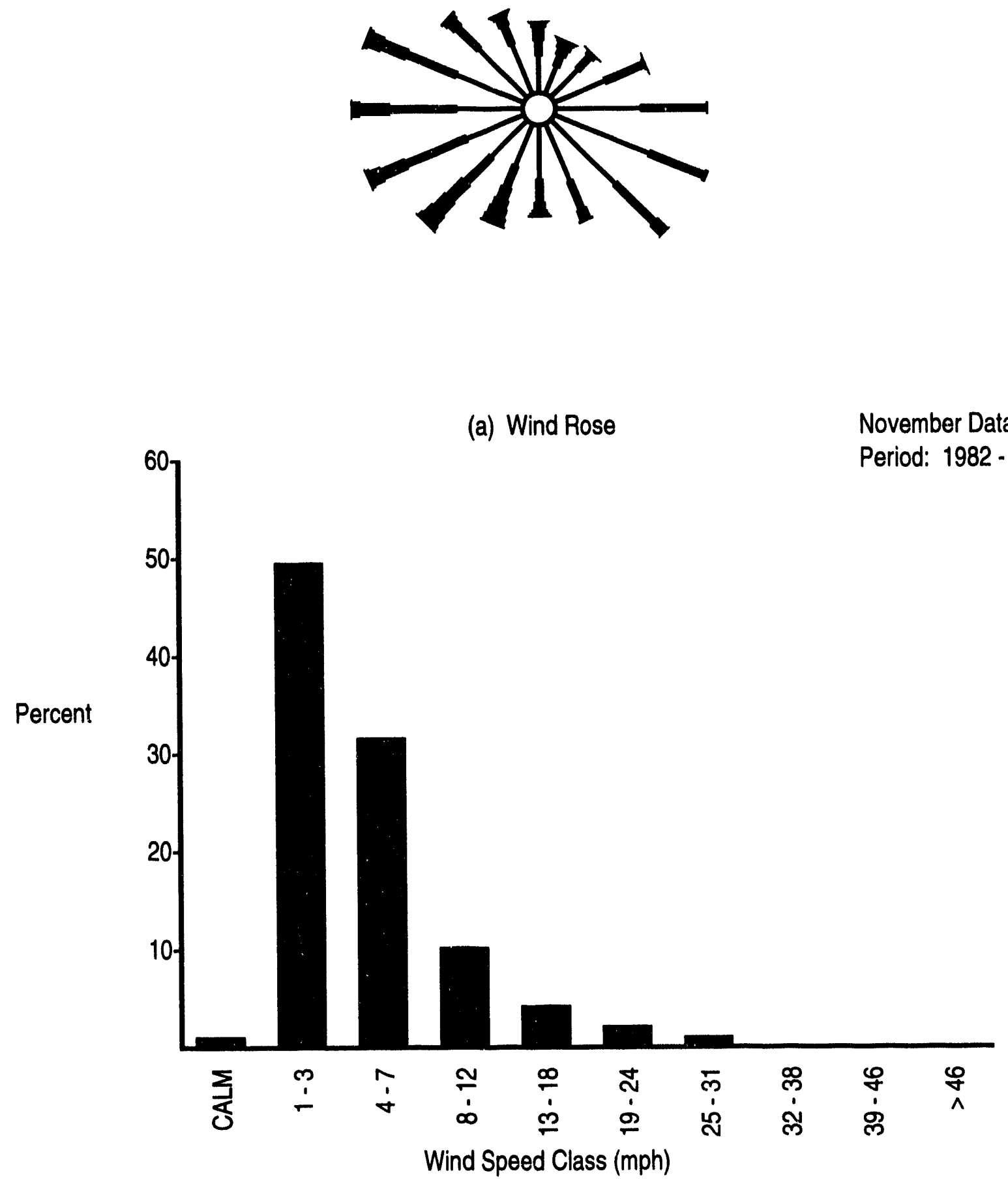

(b) Wind Speed Histogram

FIGURE B.1. (contd) 


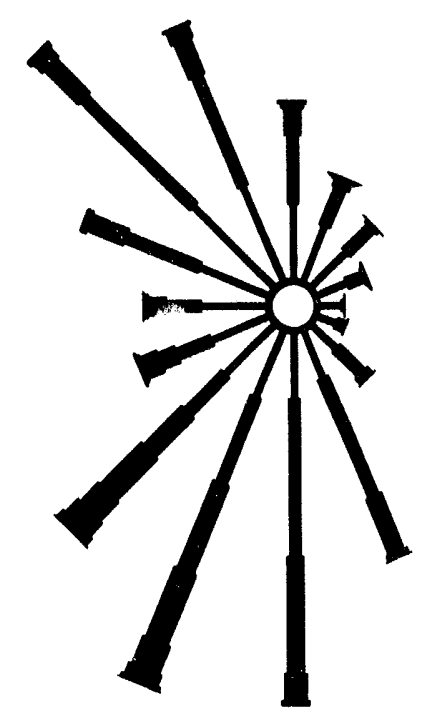

(a) Wind Rose

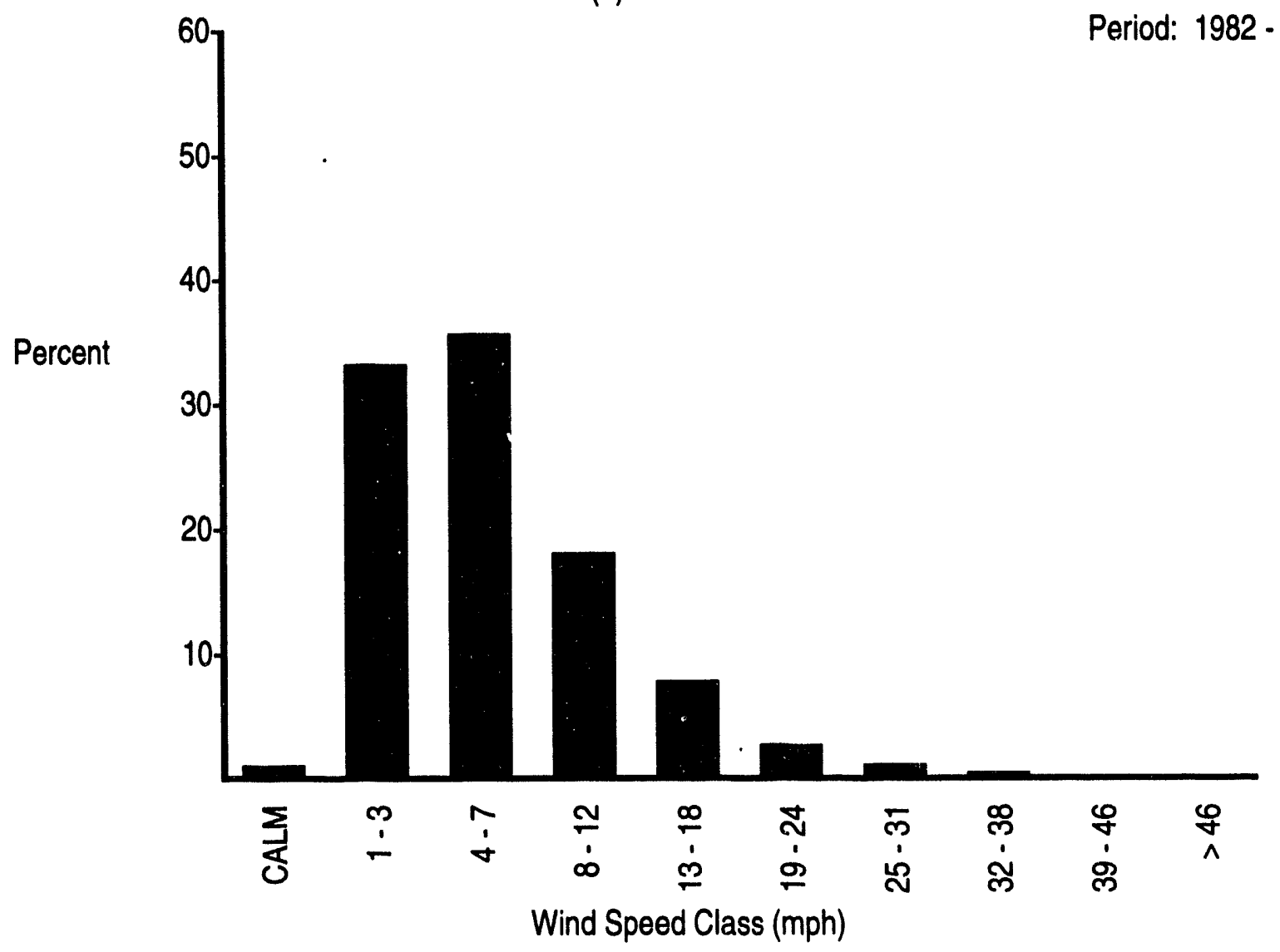

(b) Wind Speed Histogram

FIGURE B.1. (contd) 


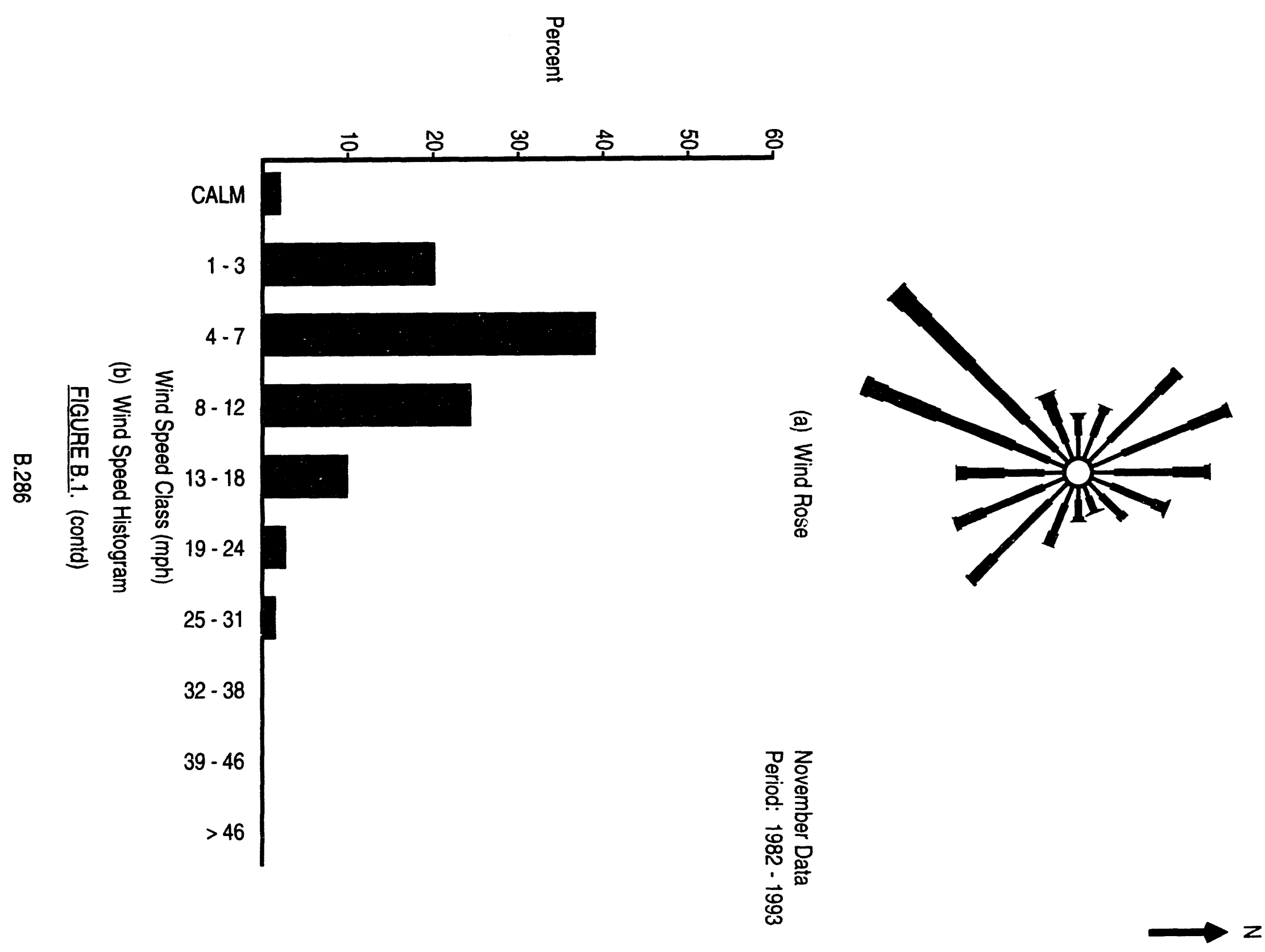



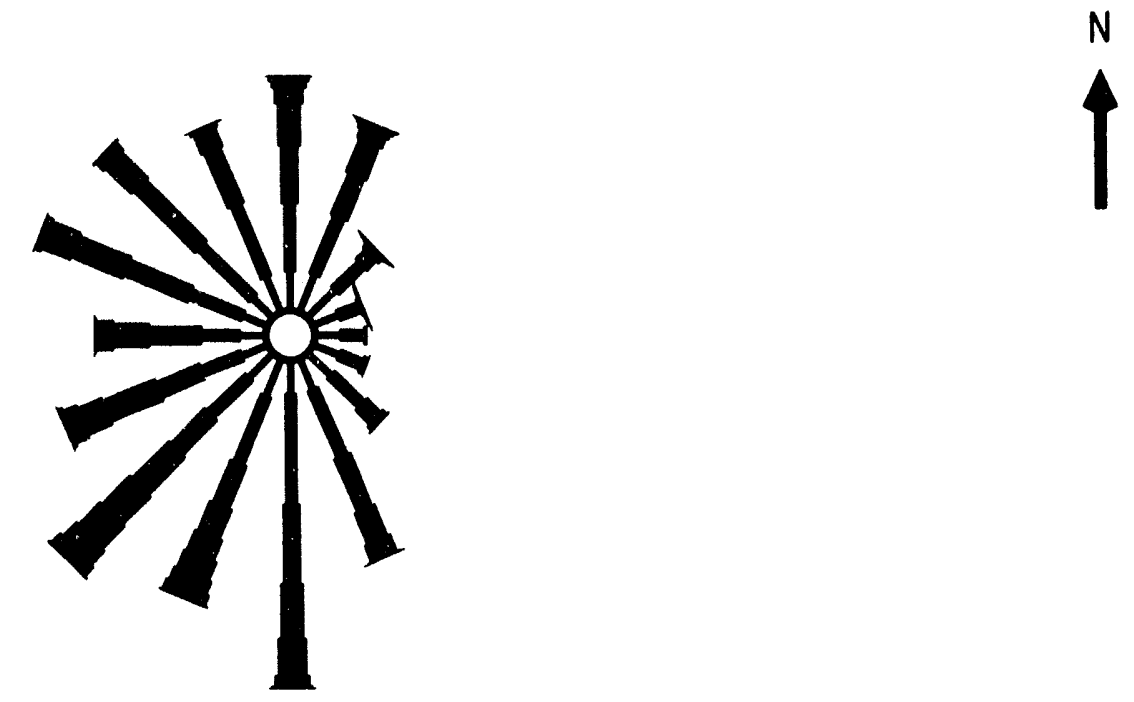

(a) Wind Rose

November Data

Period: 1982 - 1993

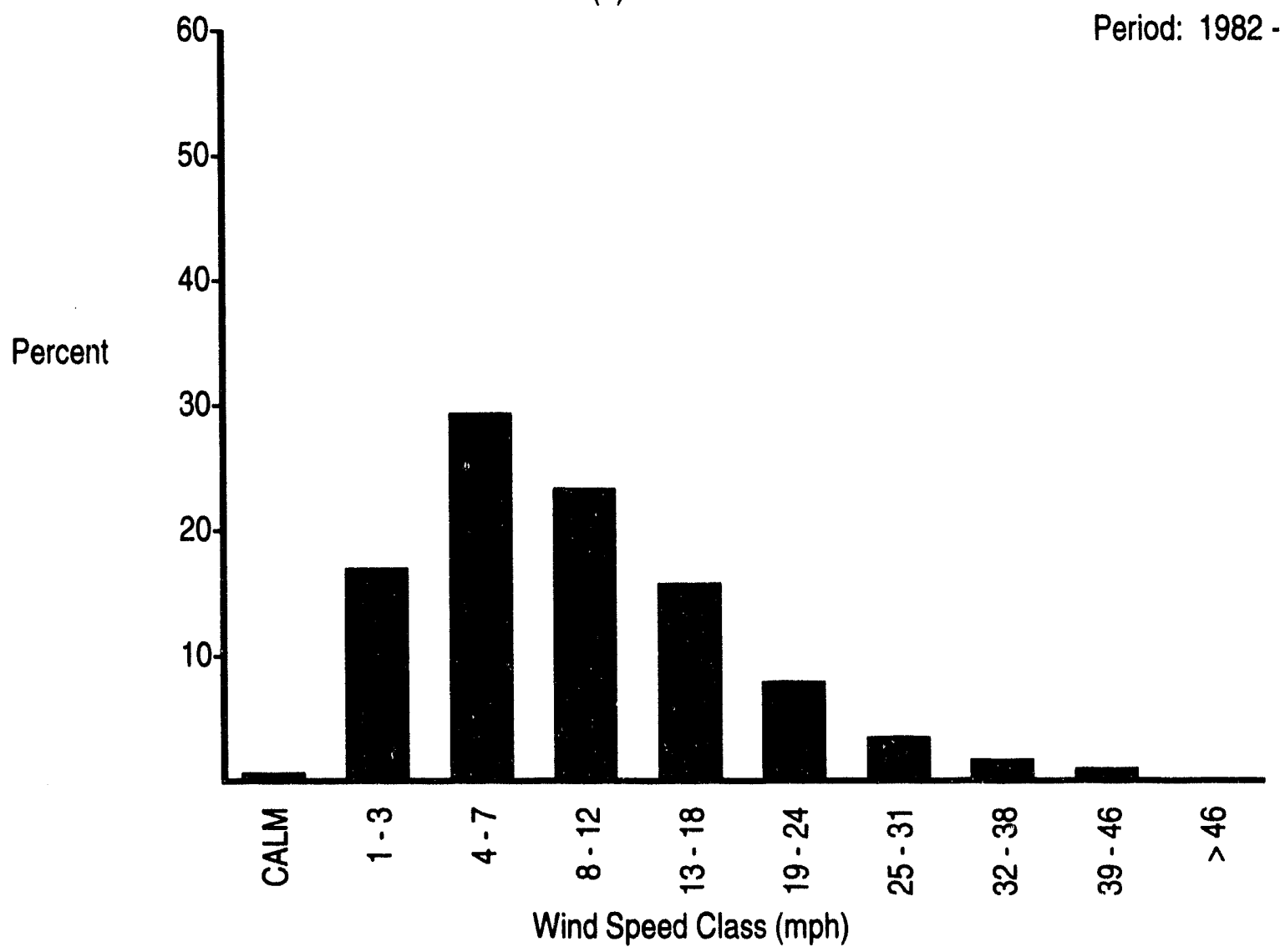

(b) Wind Speed Histogram

FIGURE B.1. (contd) 


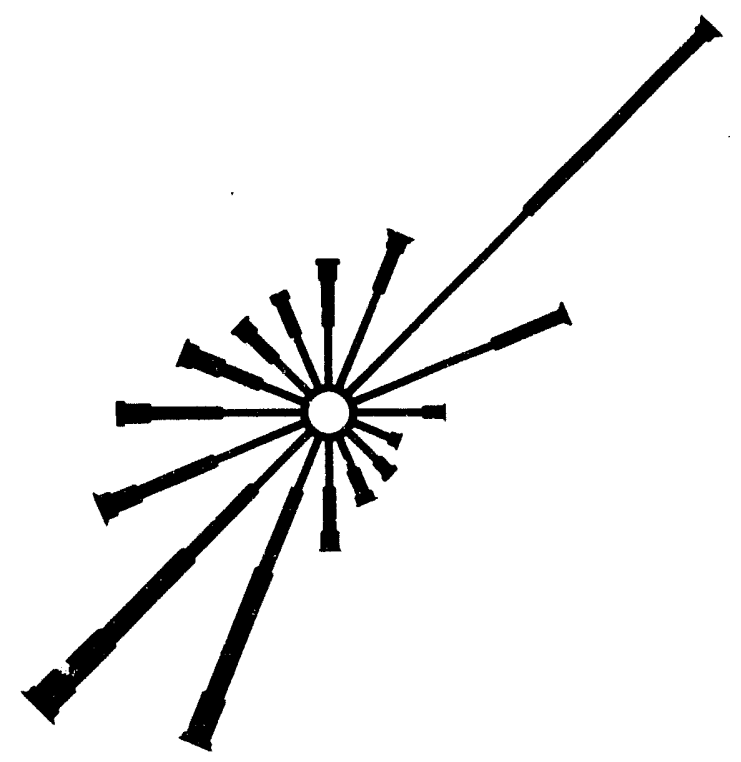

(a) Wind Rose

November Data

Period: 1982 - 1993

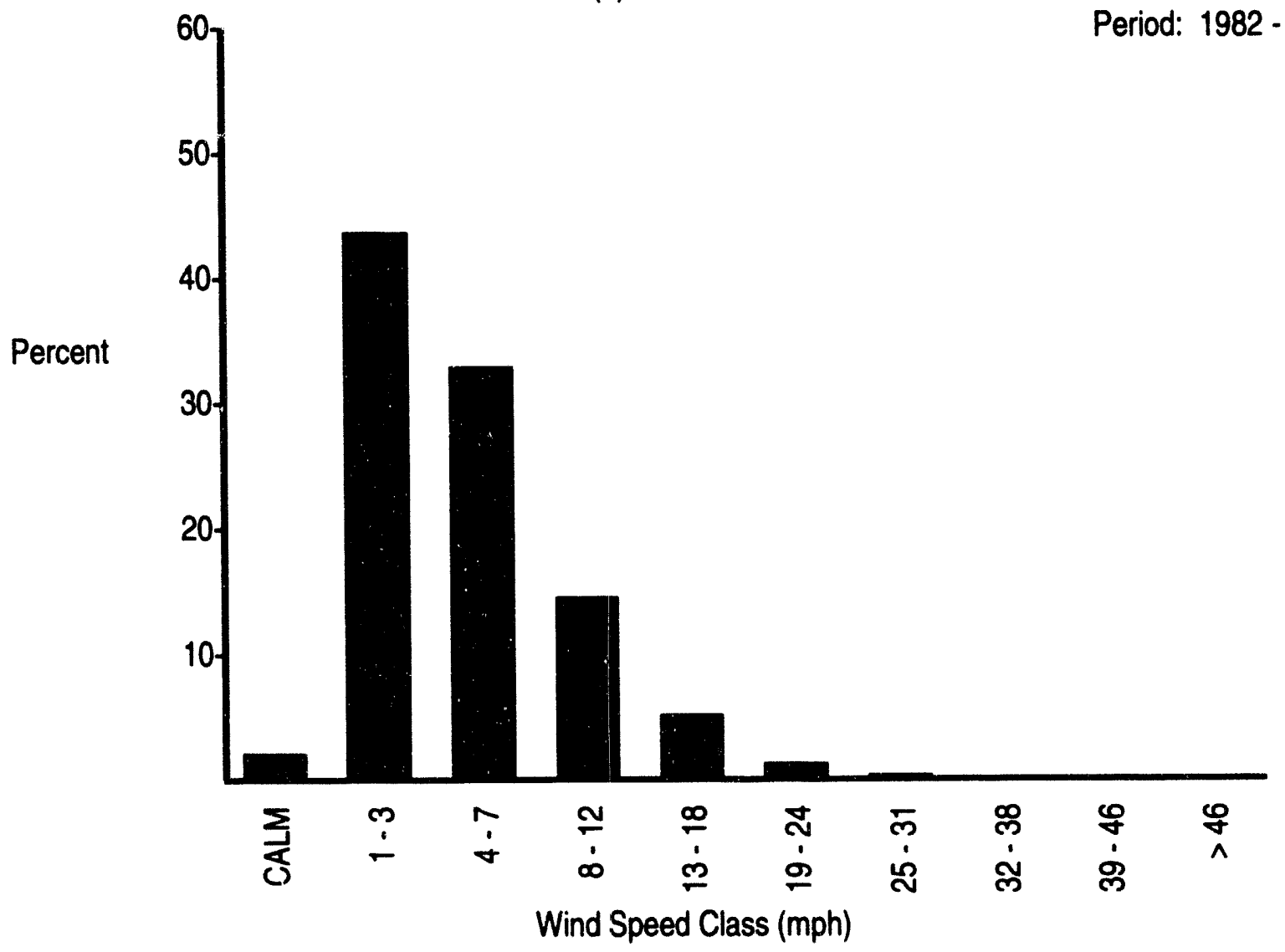

(b) Wind Speed Histogram

FIGURE B.1. (contd) 
$682^{\prime} 9$

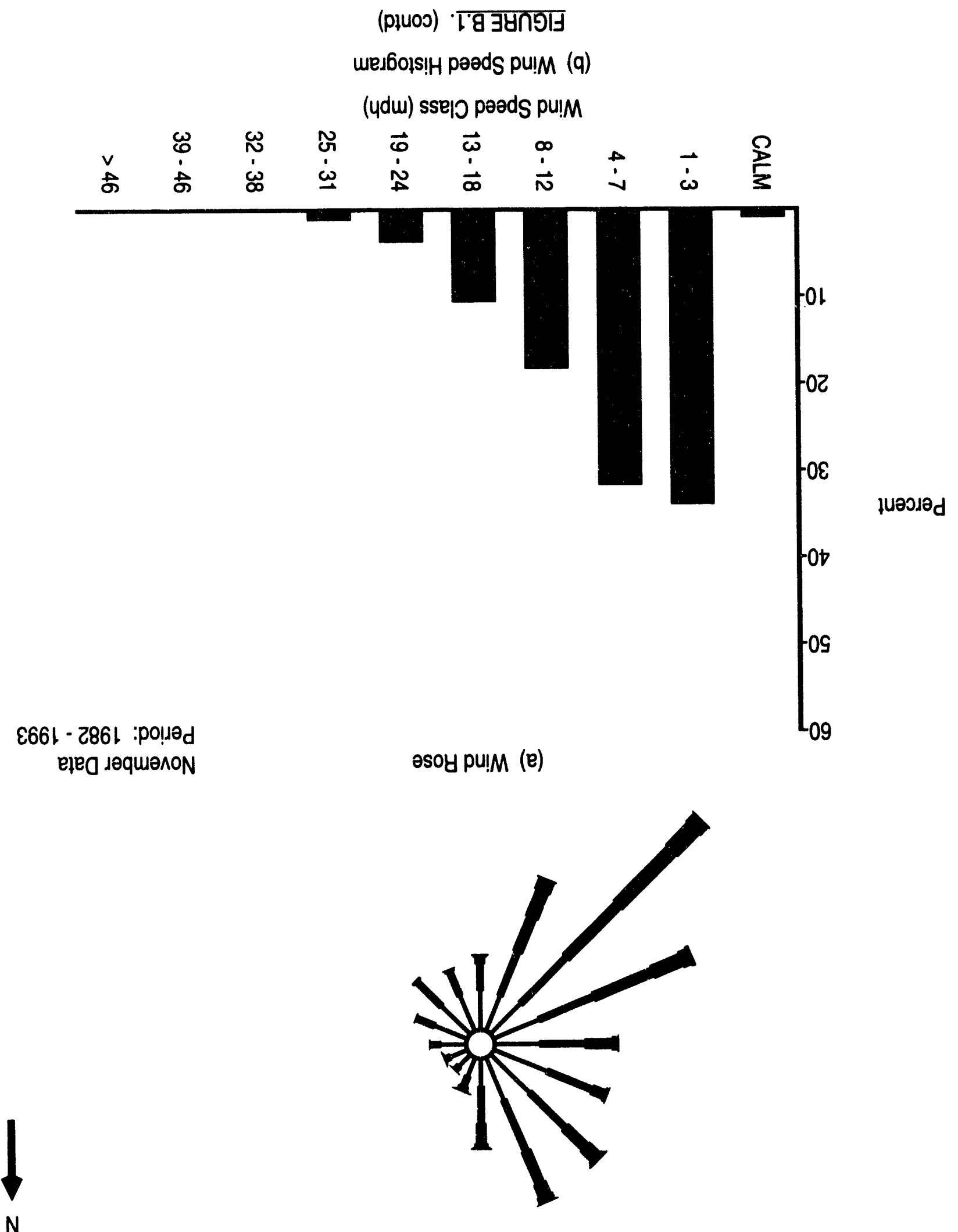




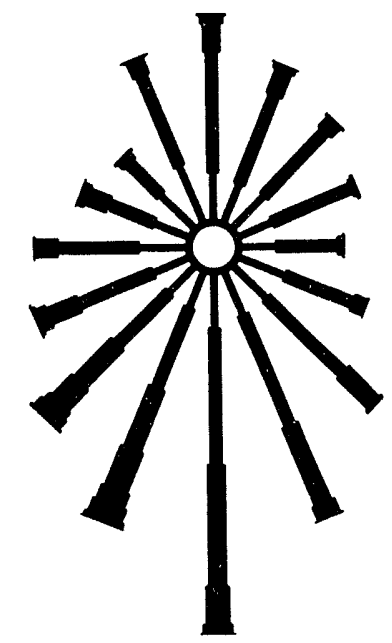

(a) Wind Rose

November Data

Period: 1982 - 1992

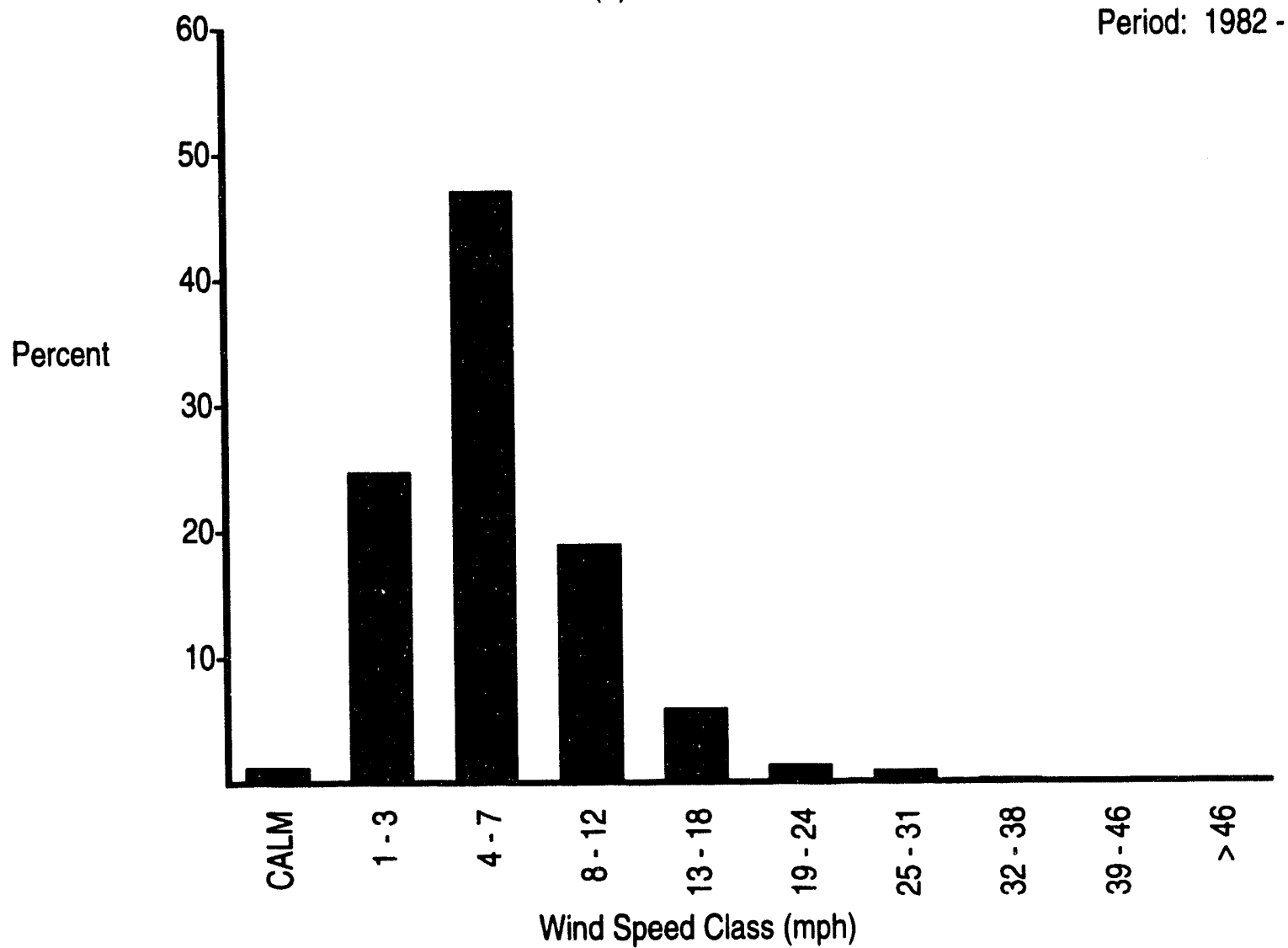

(b) Wind Speed Histogram

FIGURE B.1. (contd) 


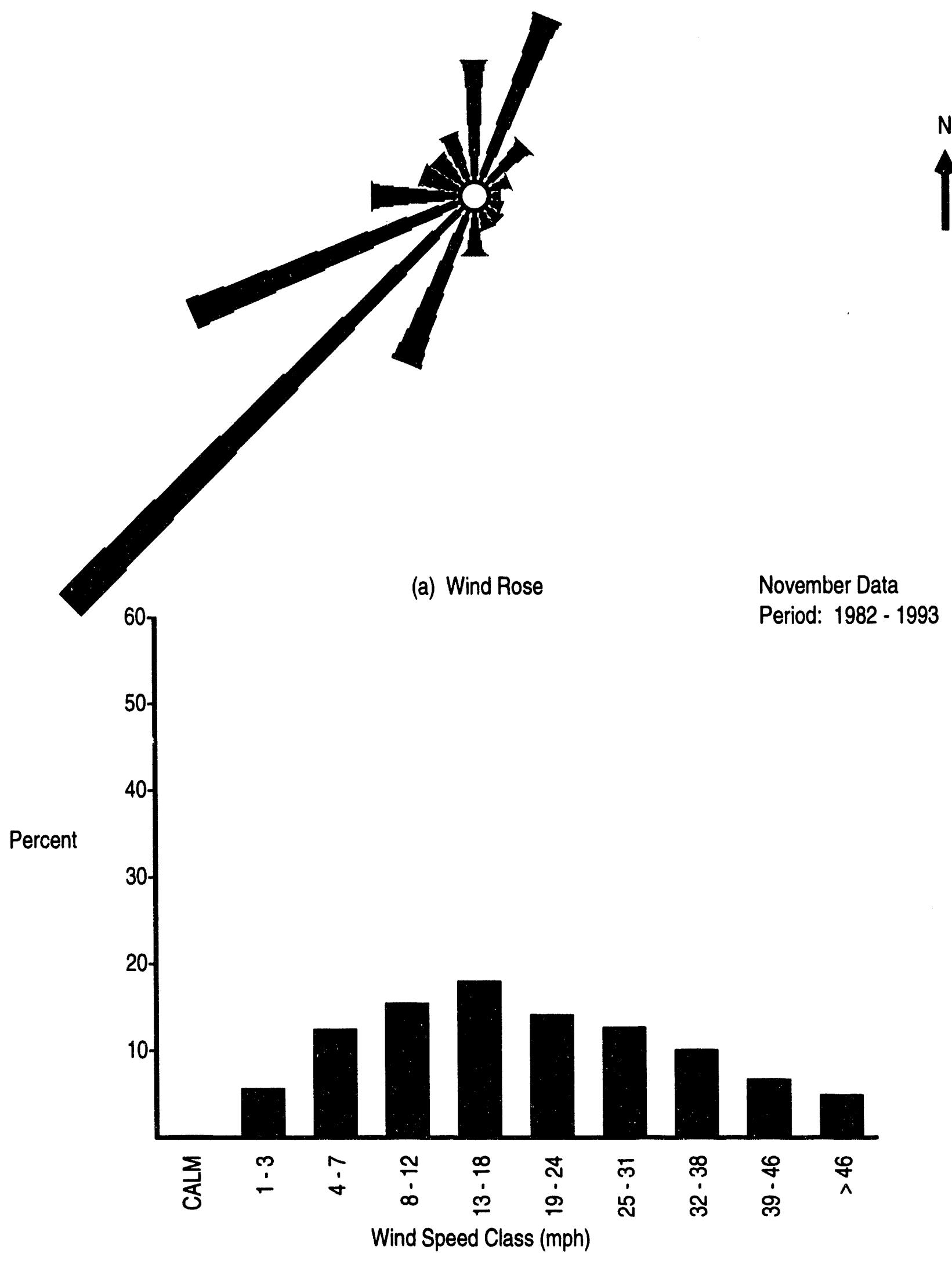

(b) Wind Speed Histogram

FIGURE B.1. (contd) 

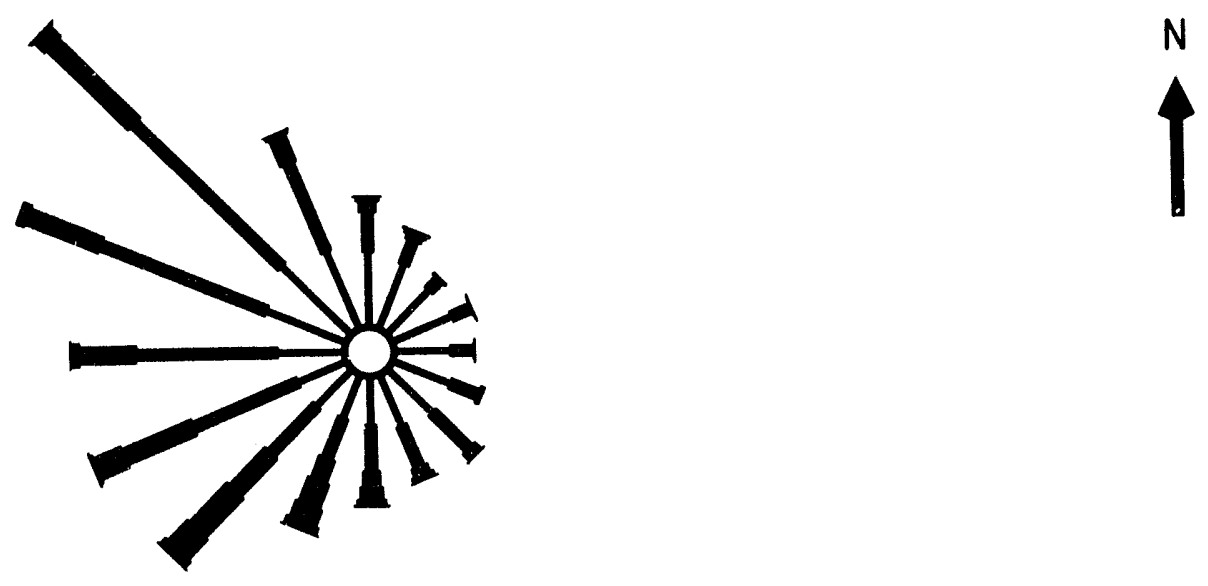

(a) Wind Rose

November Data

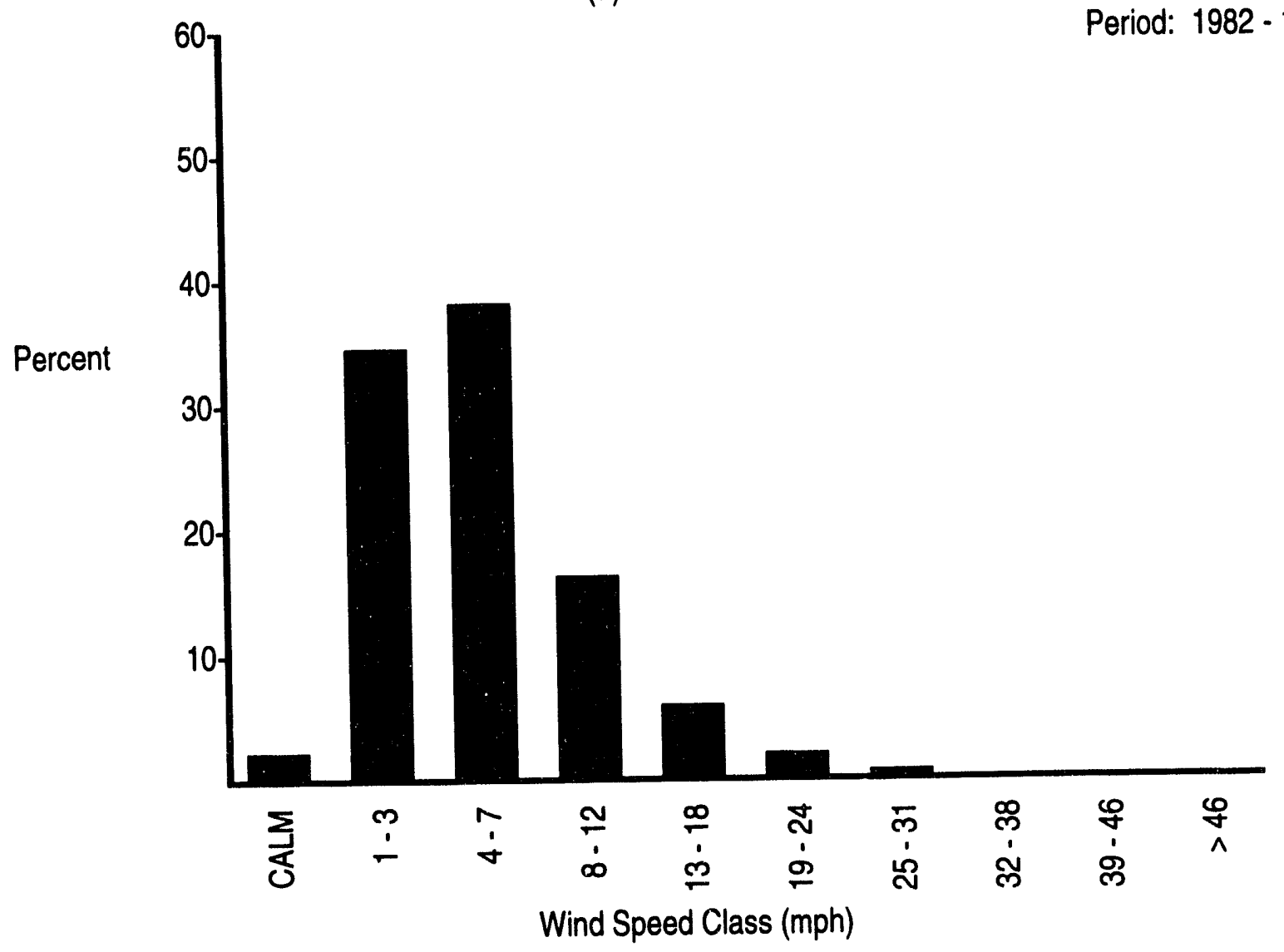

(b) Wind Speed Histogram

FIGURE B.1. (contd) 


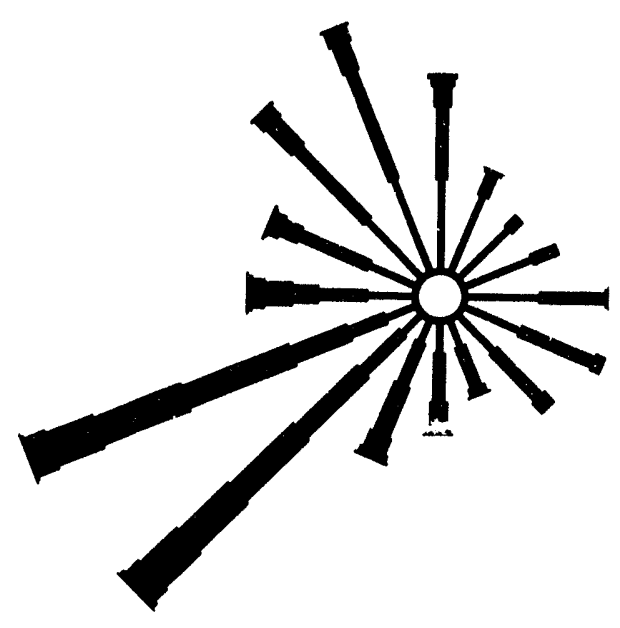

N

(a) Wind Rose

November Data

Period: 1987 - 1993

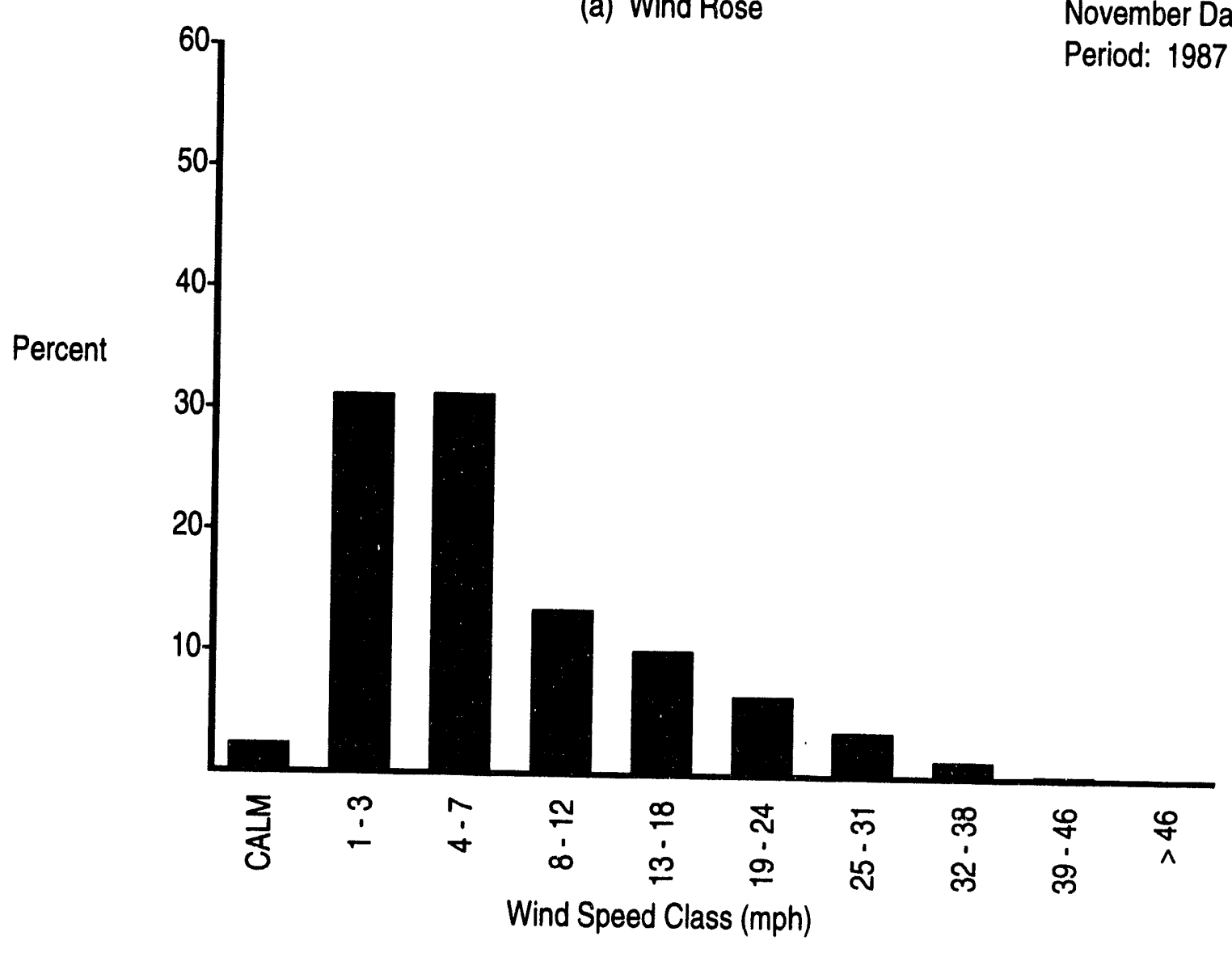

(b) Wind Speed Histogram

FIGURE B.1. (contd) 


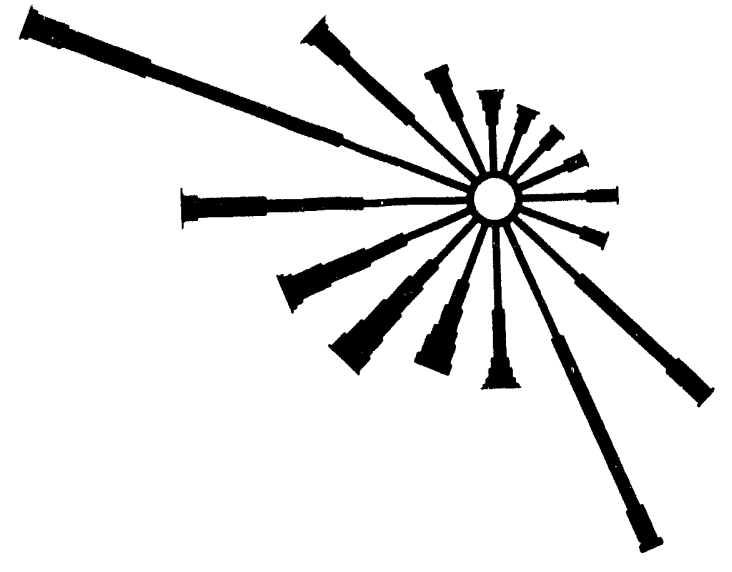

(a) Wind Rose

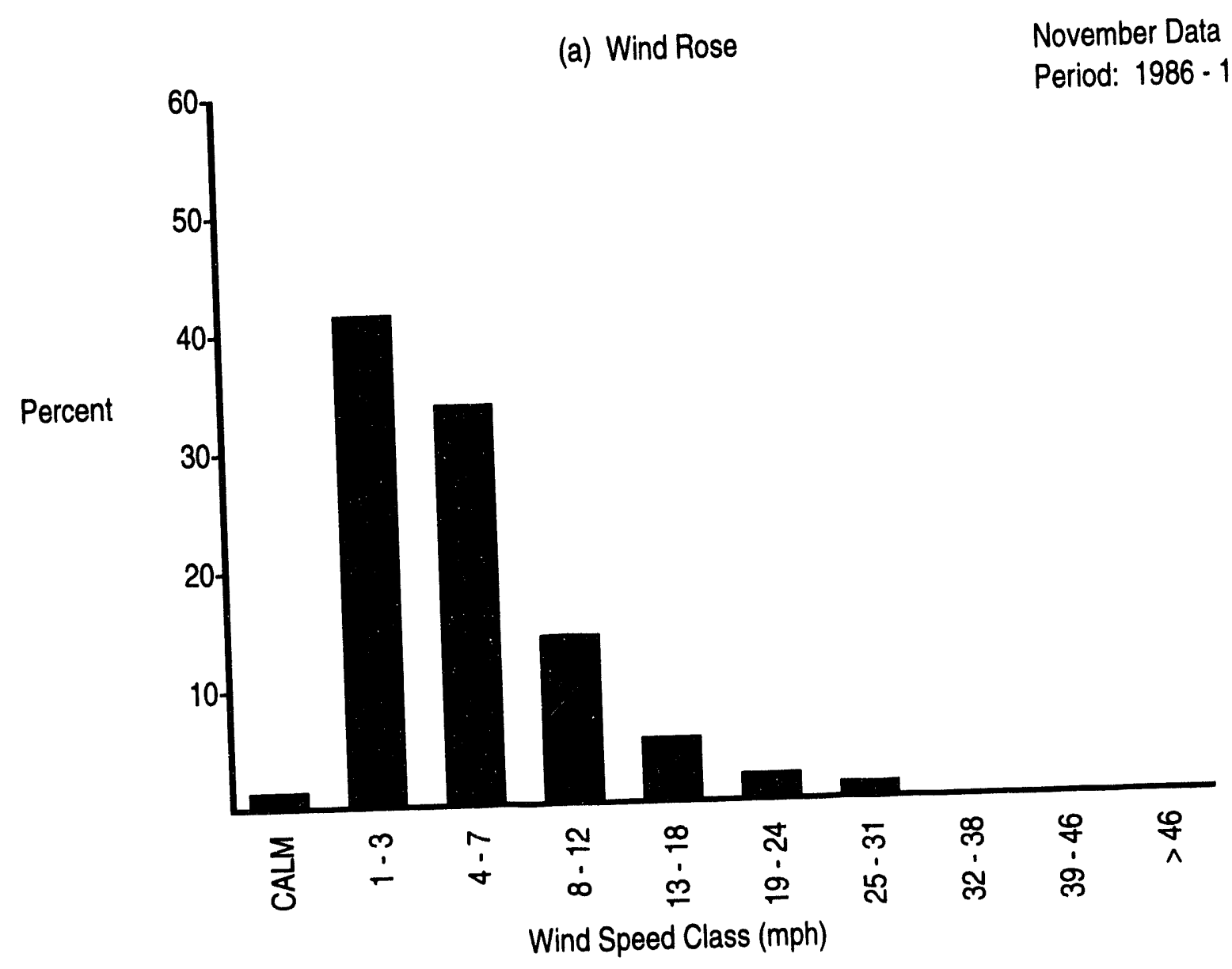

(b) Wind Speed Histogram

FIGURE B.1. (contd) 


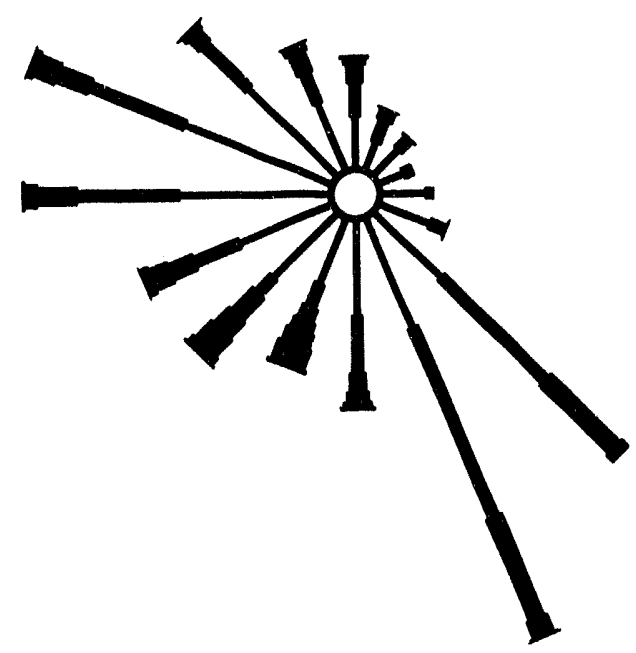

(a) Wind Rose

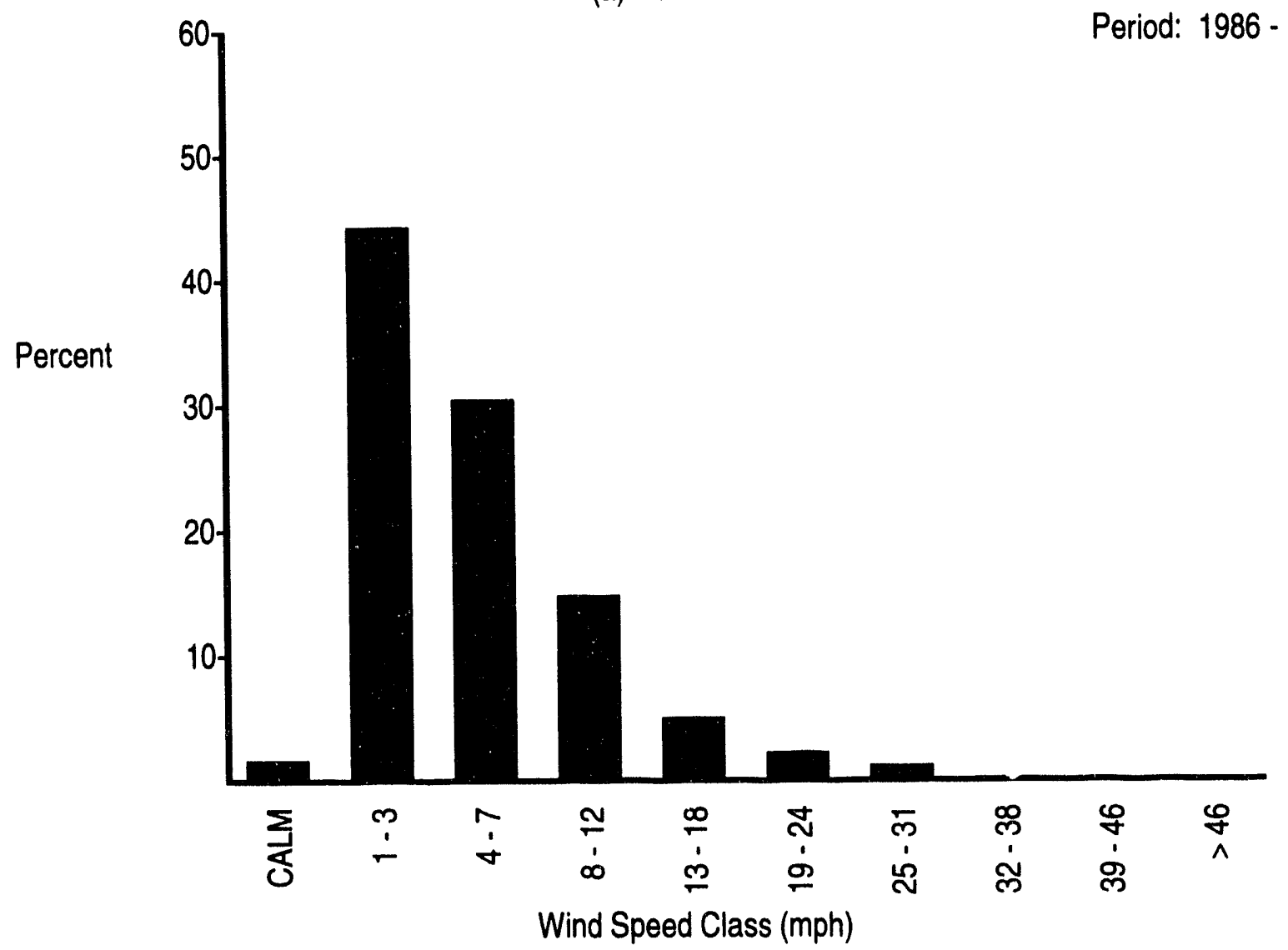

(b) Wind Speed Histogram

FIGURE B.1. (contd)
November Data

Period: 1986 - 1993 


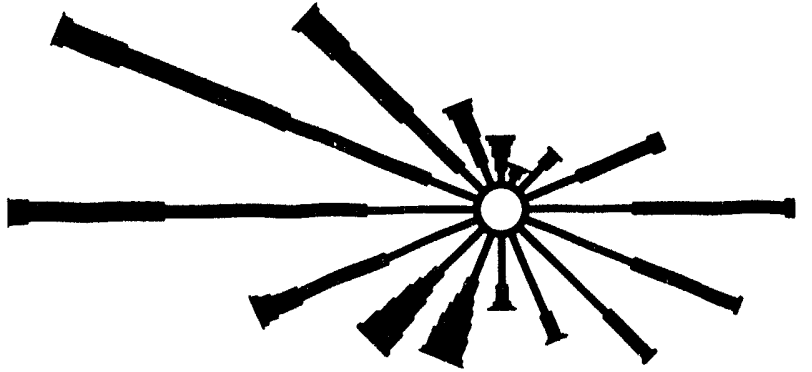

(a) Wind Rose

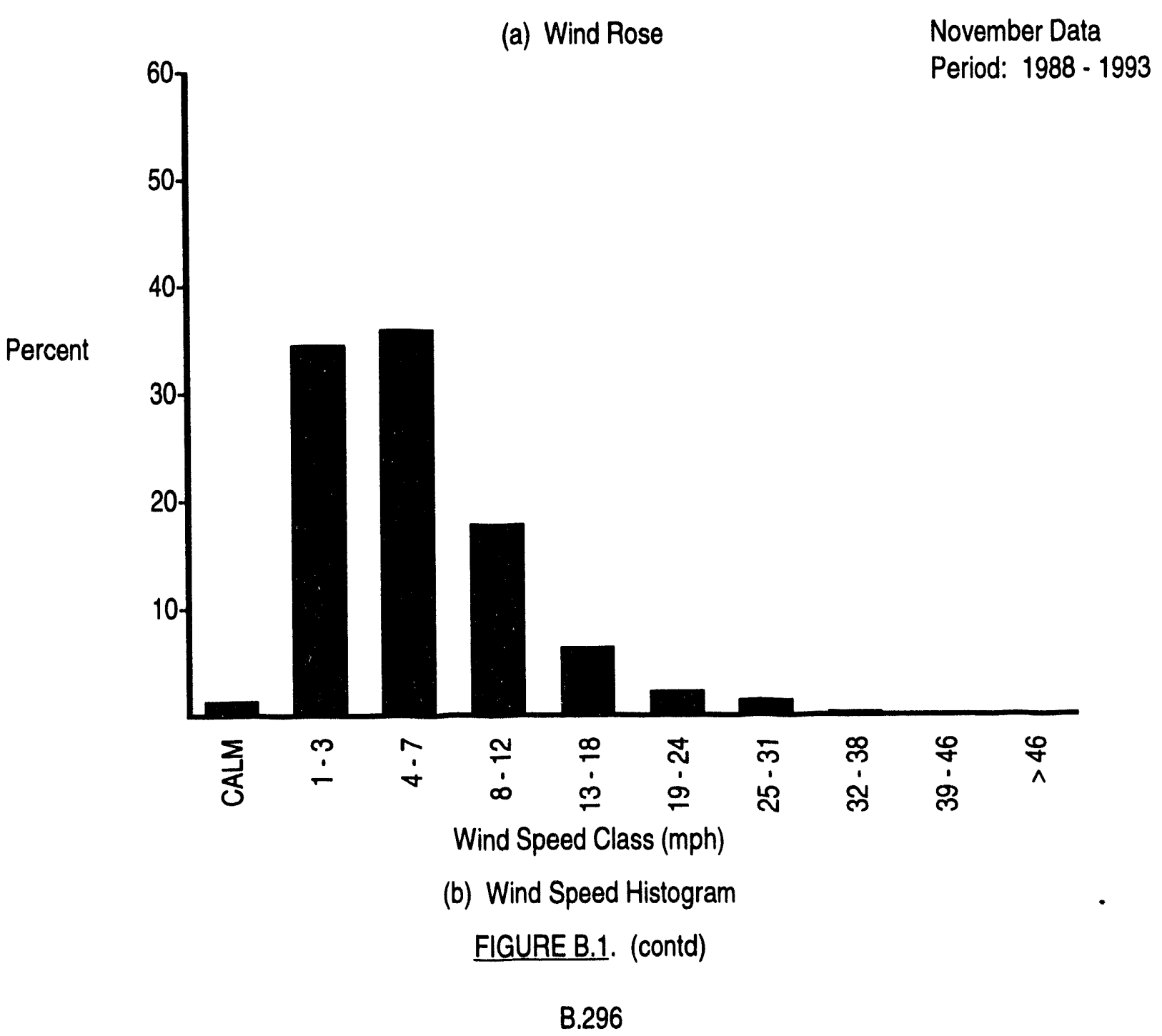




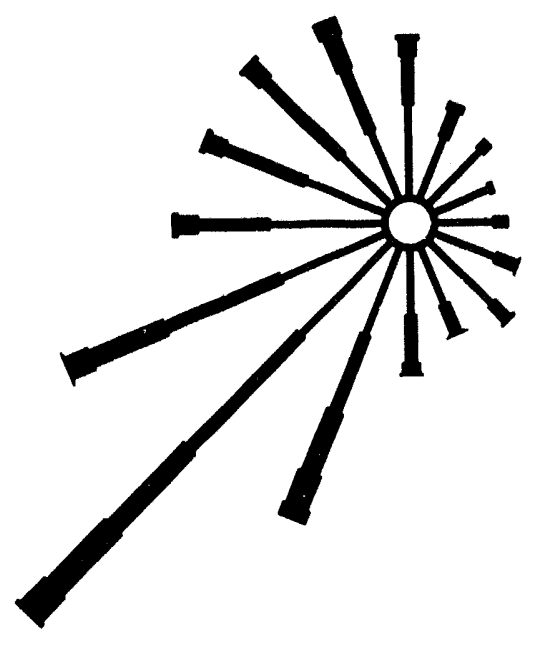

(a) Wind Rose

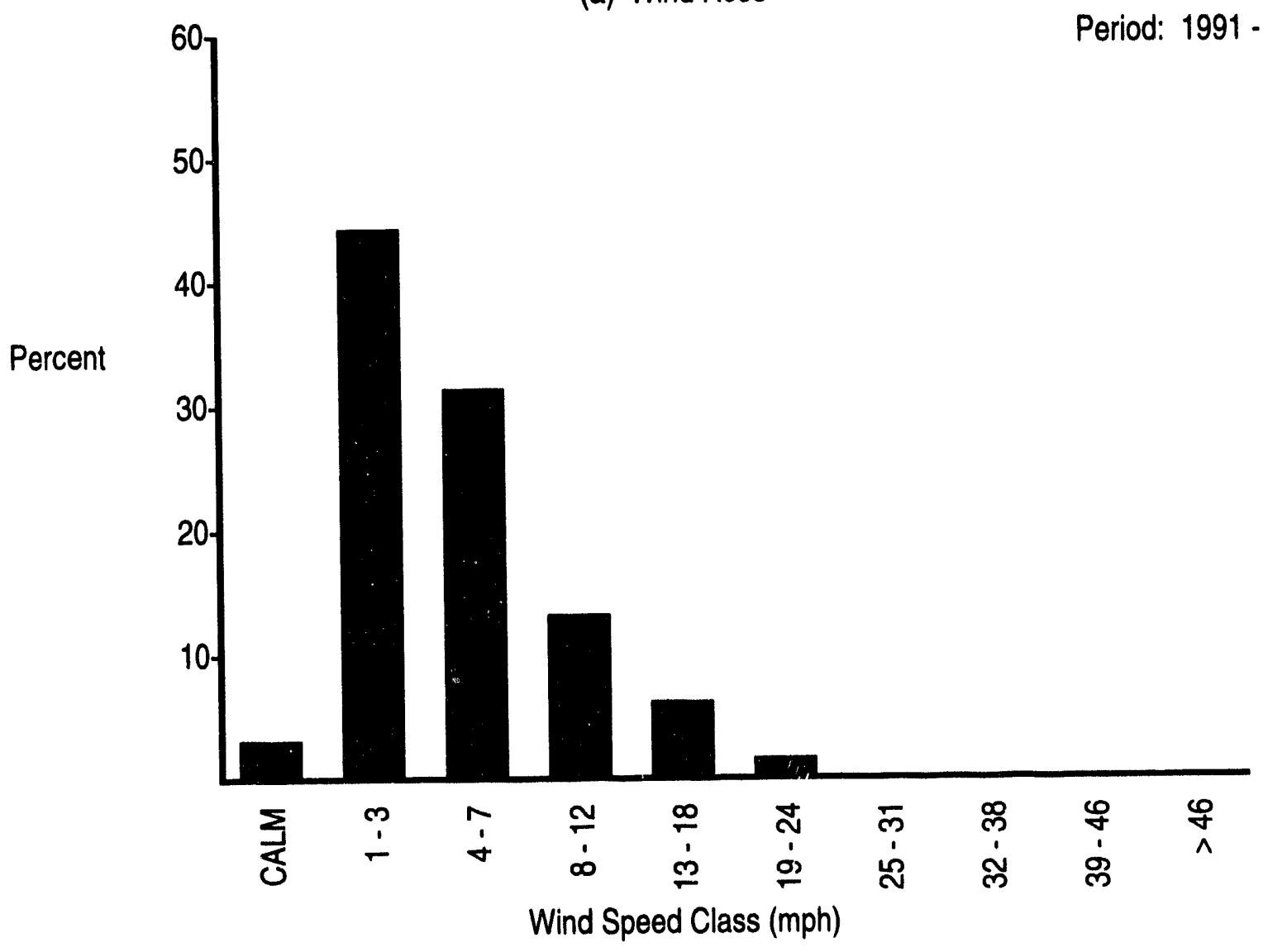

(b) Wind Speed Histogram

FIGURE B.1. (contd)
November Data

Period: 1991 - 1993 

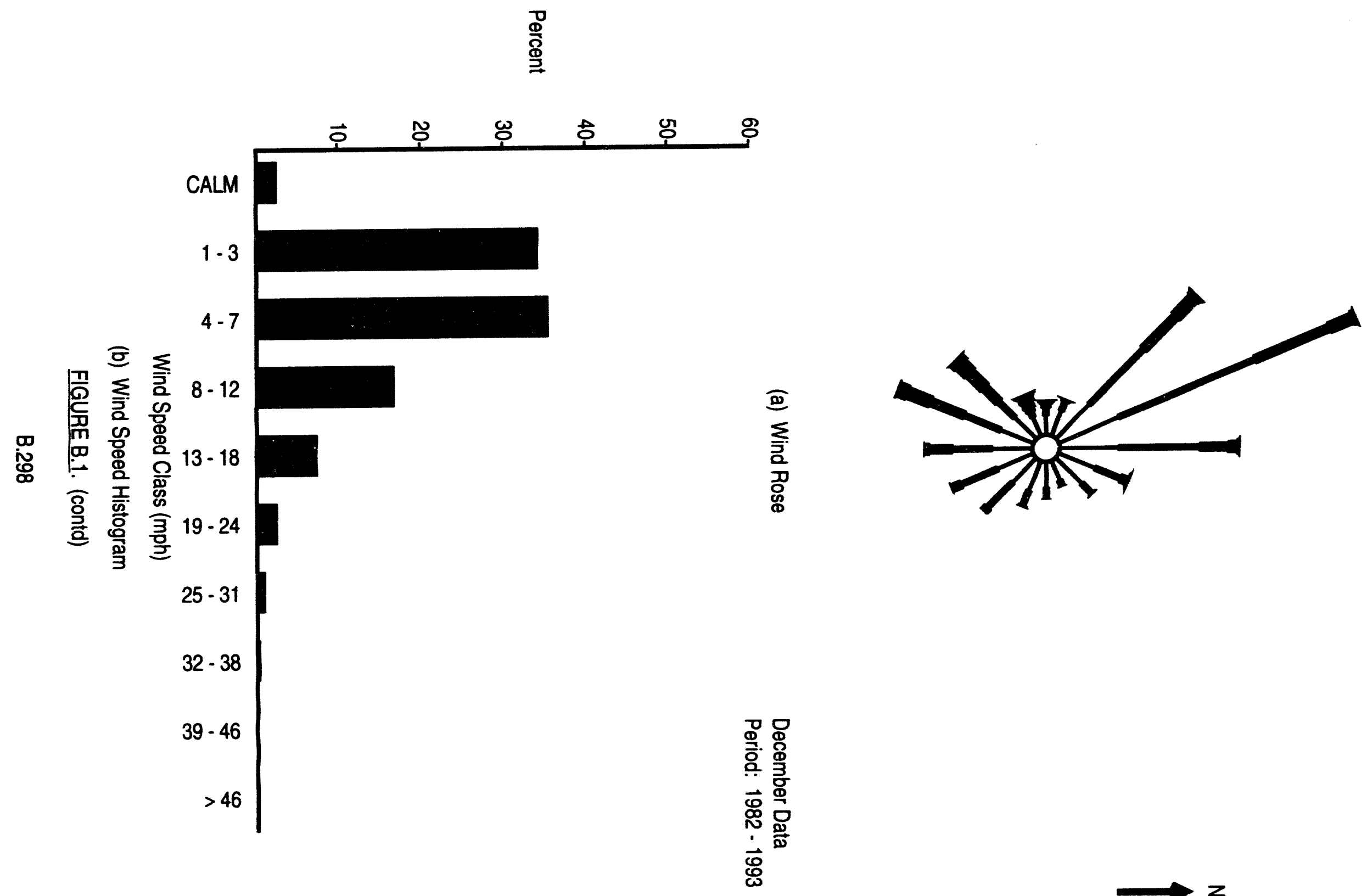

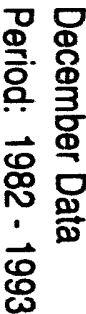

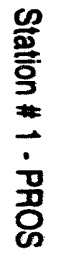




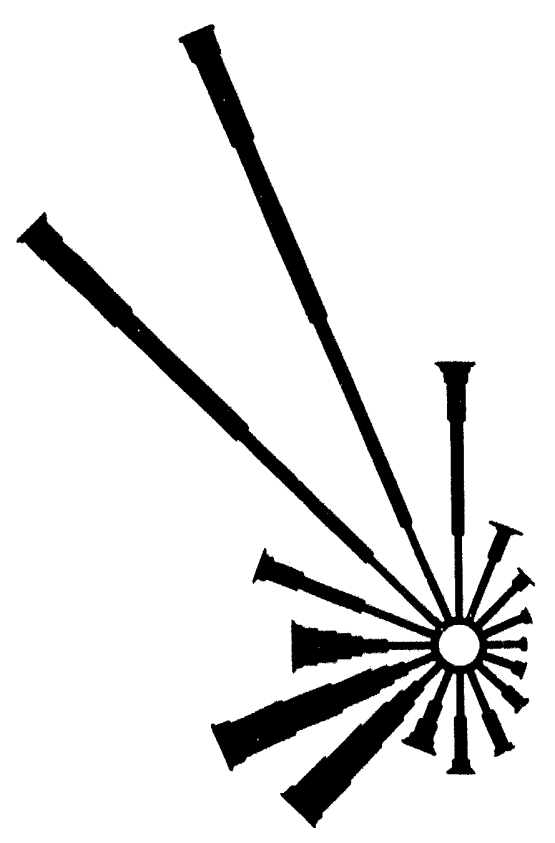

Station \# 2 - EOC

(a) Wind Rose

December Data

Period: $1982 \cdot 1993$

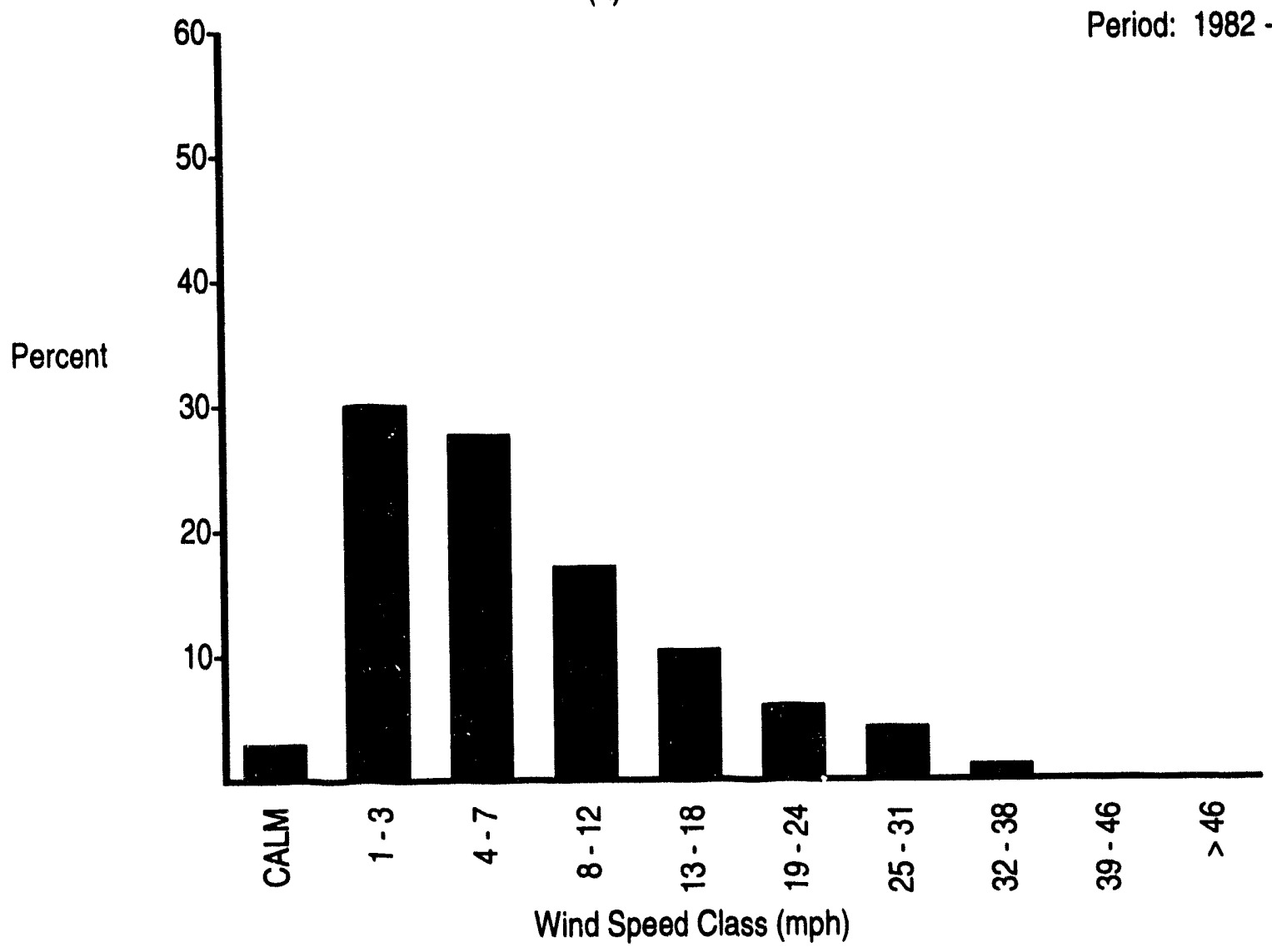

(b) Wind Speed Histogram

FIGURE B.1. (contd) 

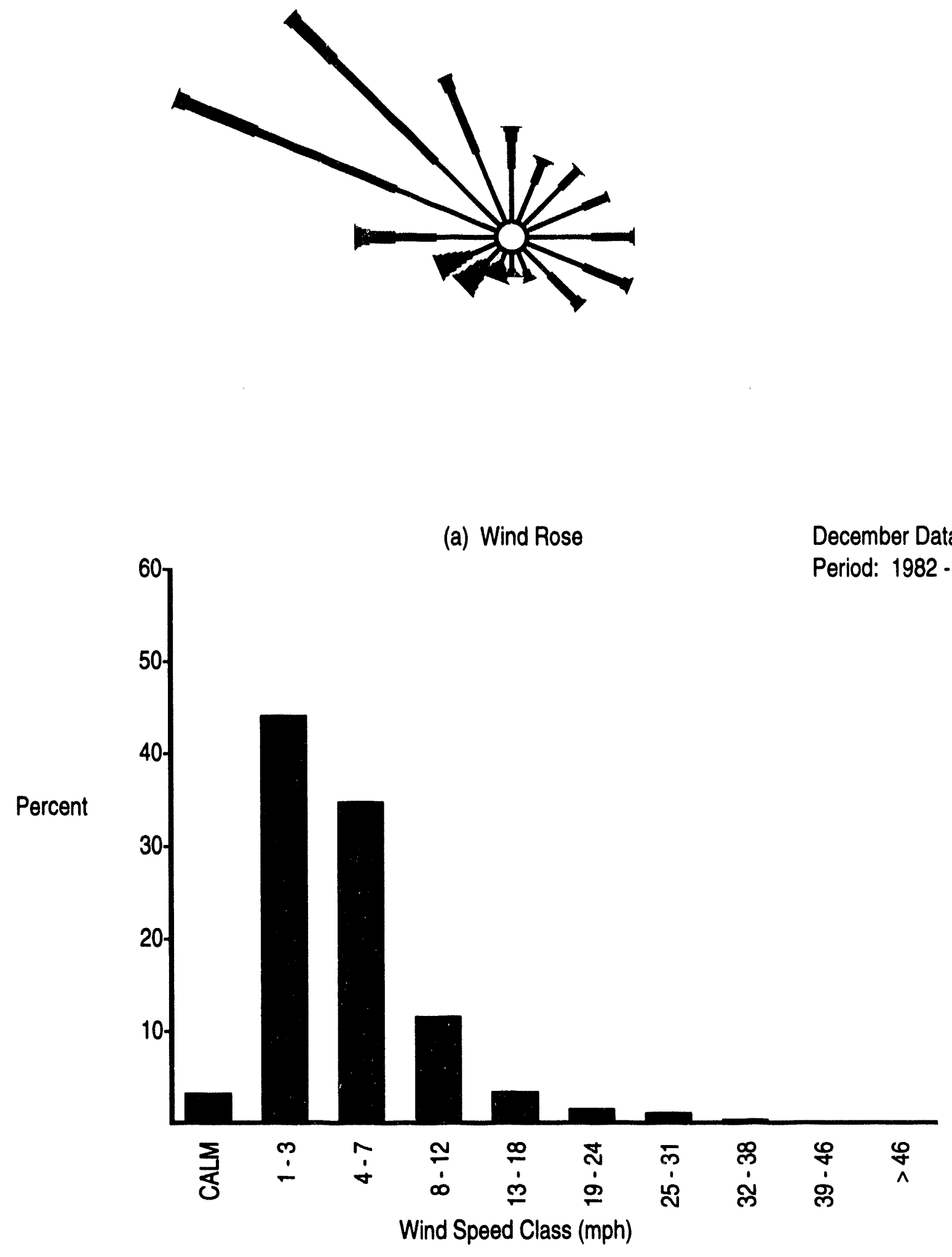

(b) Wind Speed Histogram

FIGURE, B.1. (contd) 


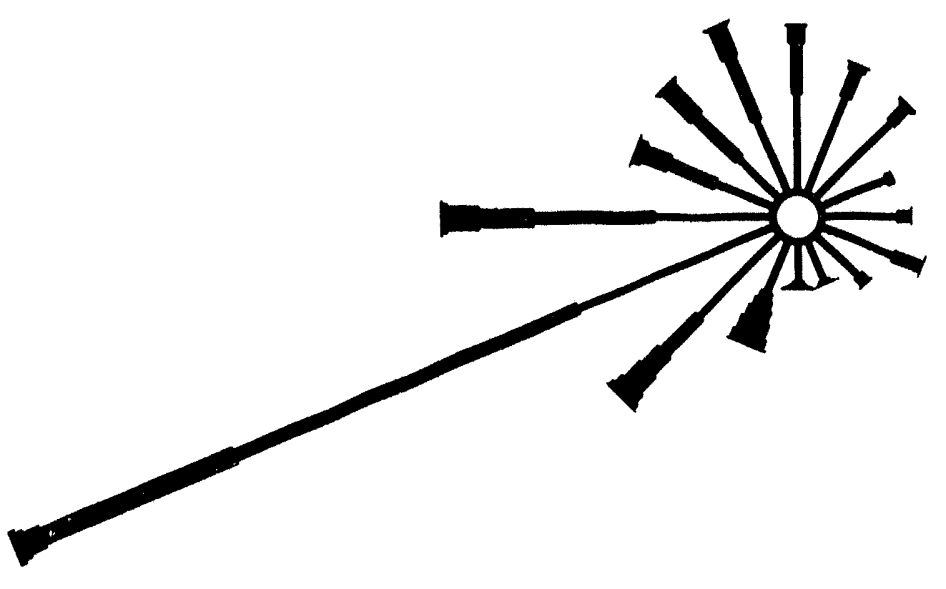

(a) Wind Rose

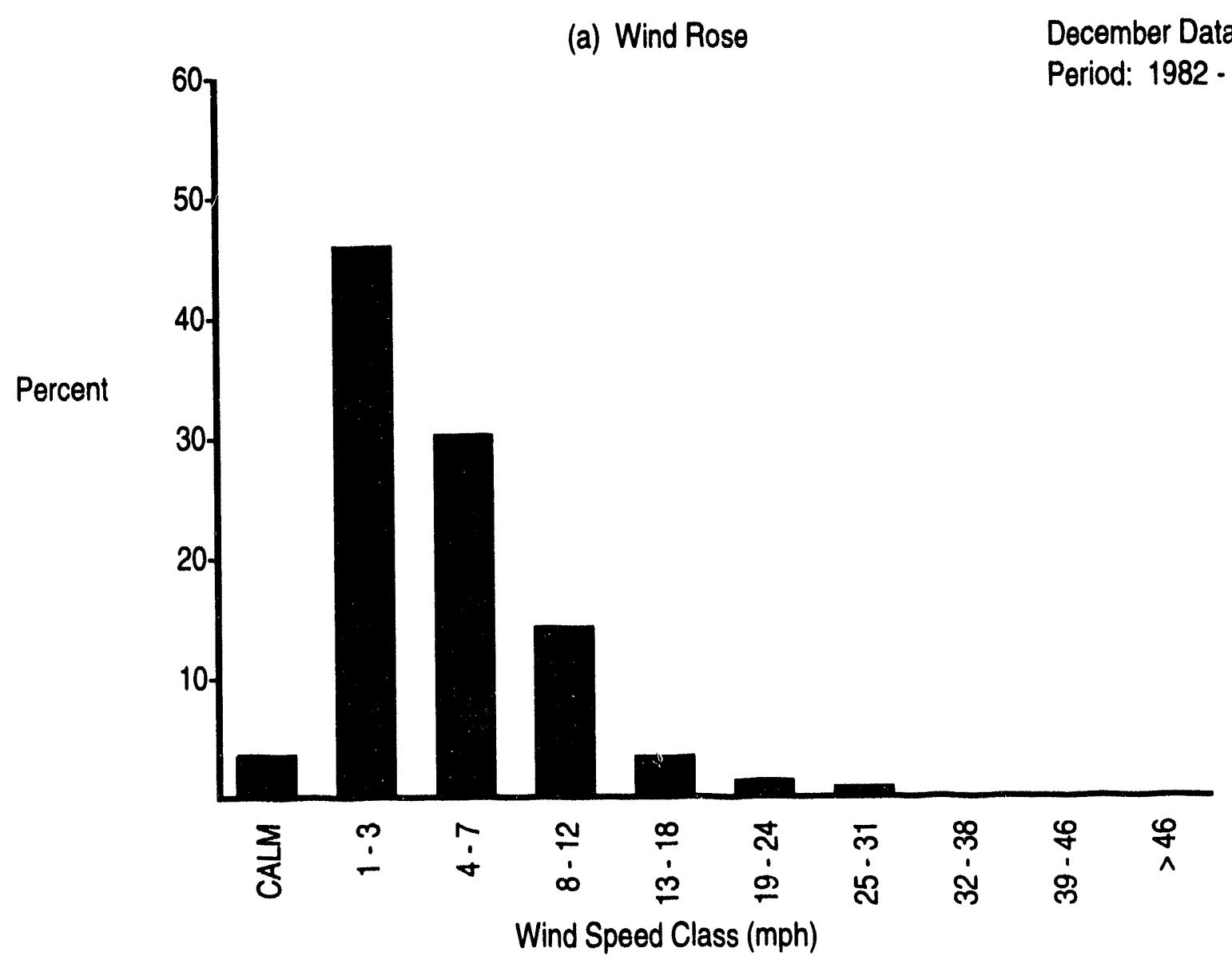

(b) Wind Speed Histogram

FIGURE B.1. (contd) 


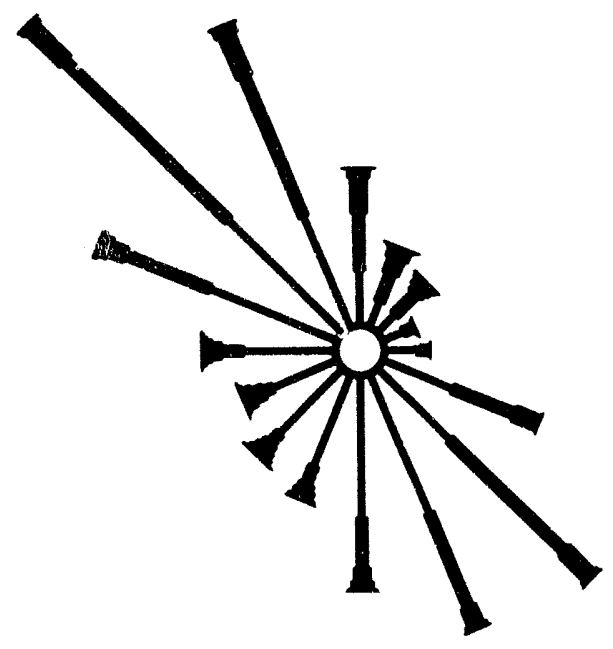

(a) Wind Rose

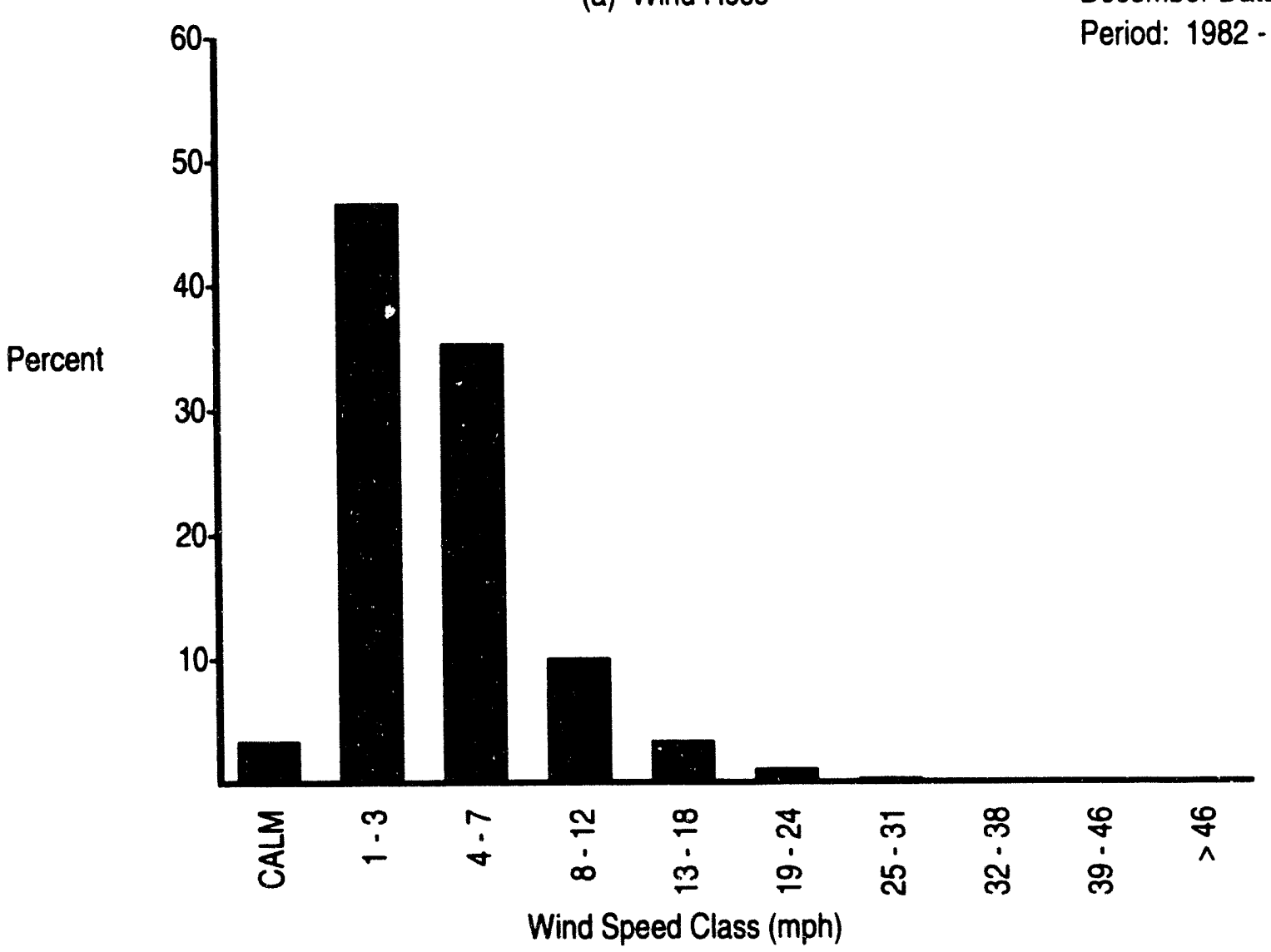

(b) Wind Speed Histogram

FIGURE B.1. (contd) 


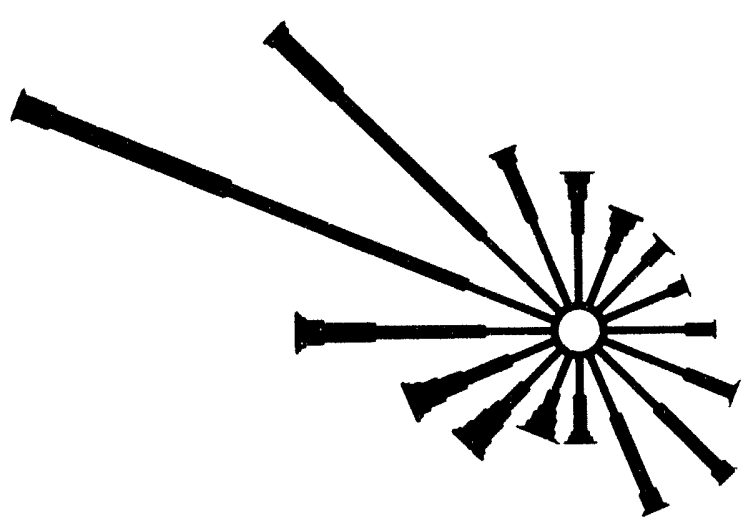

(a) Wind Rose

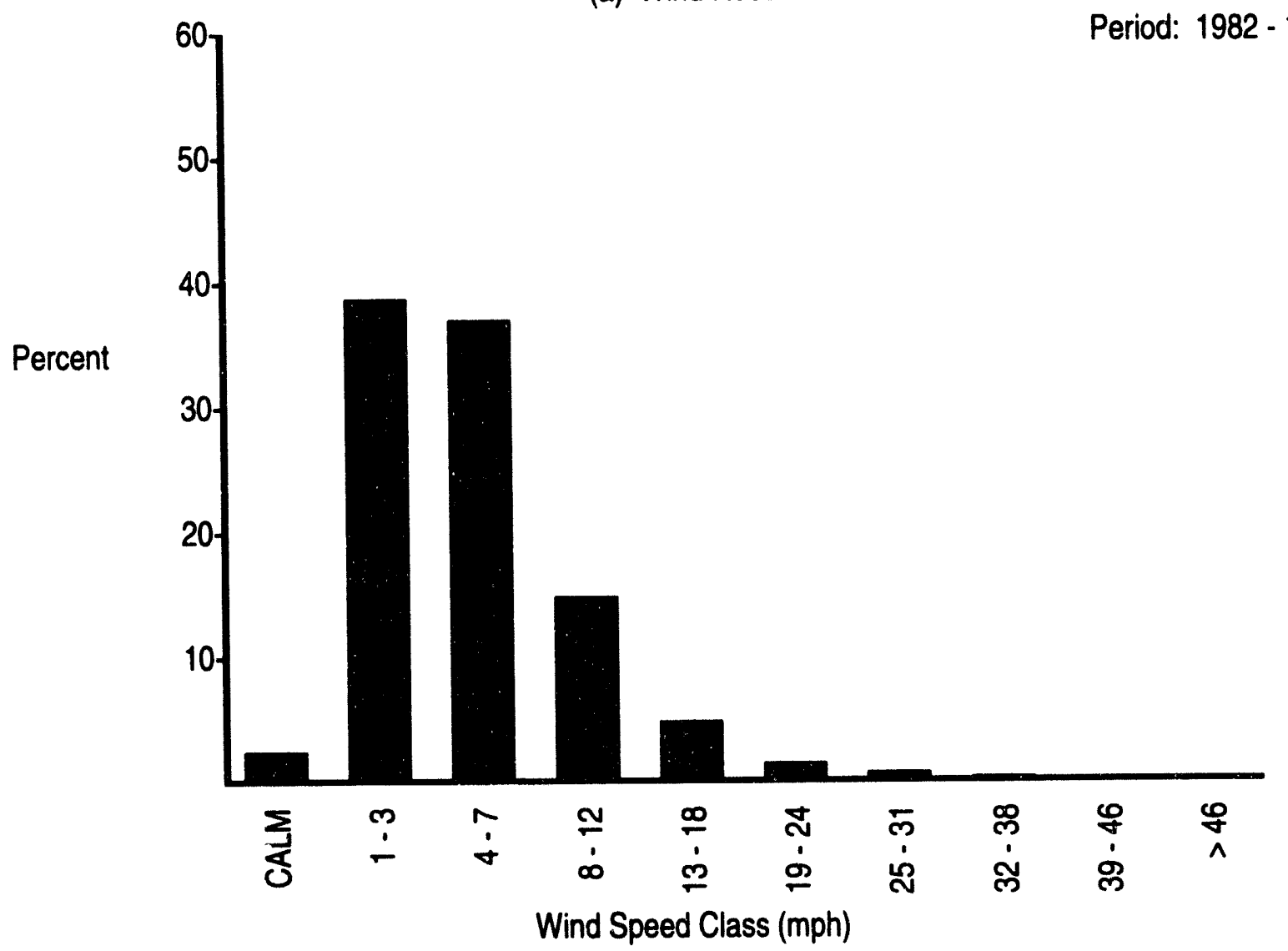

December Data

1993

(b) Wind Speed Histogram

FIGURE B.1. (contd) 


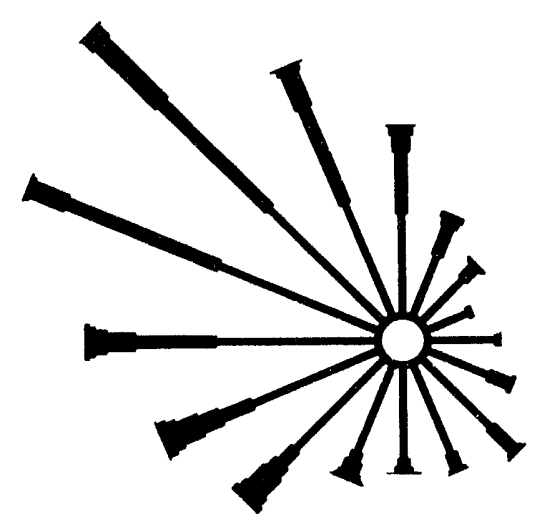

(a) Wind Rose

December Data

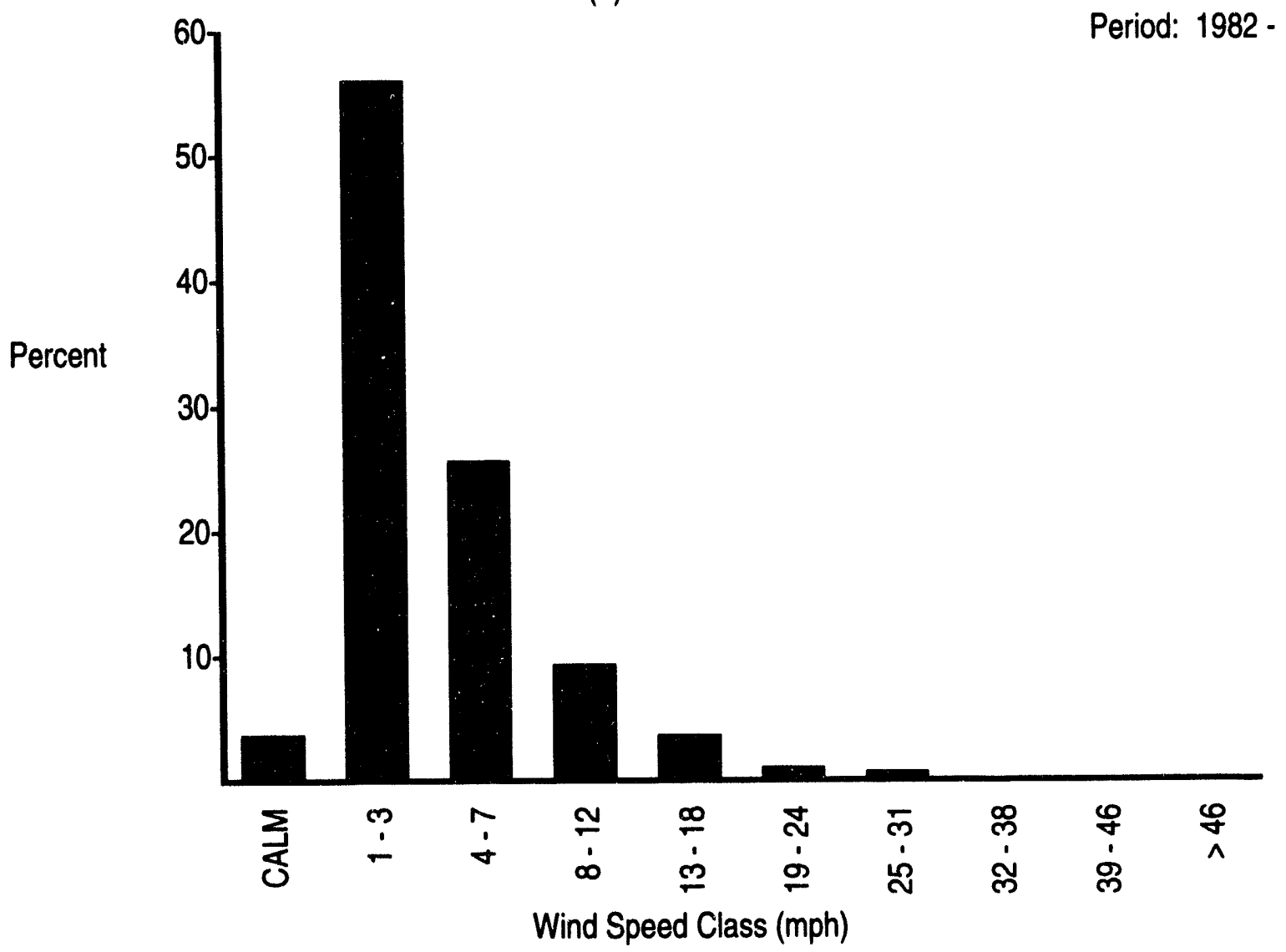

(b) Wind Speed Histogram

FIGURE B.1. (contd) 


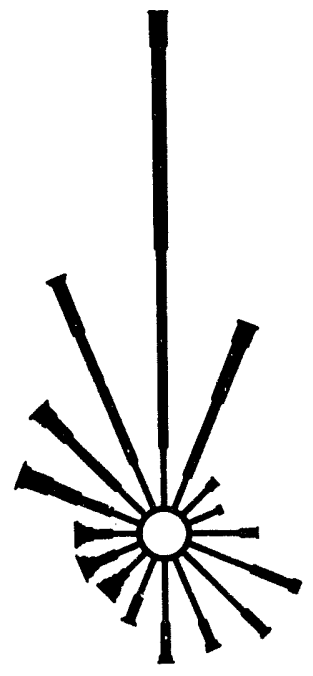

(a) Wind Rose

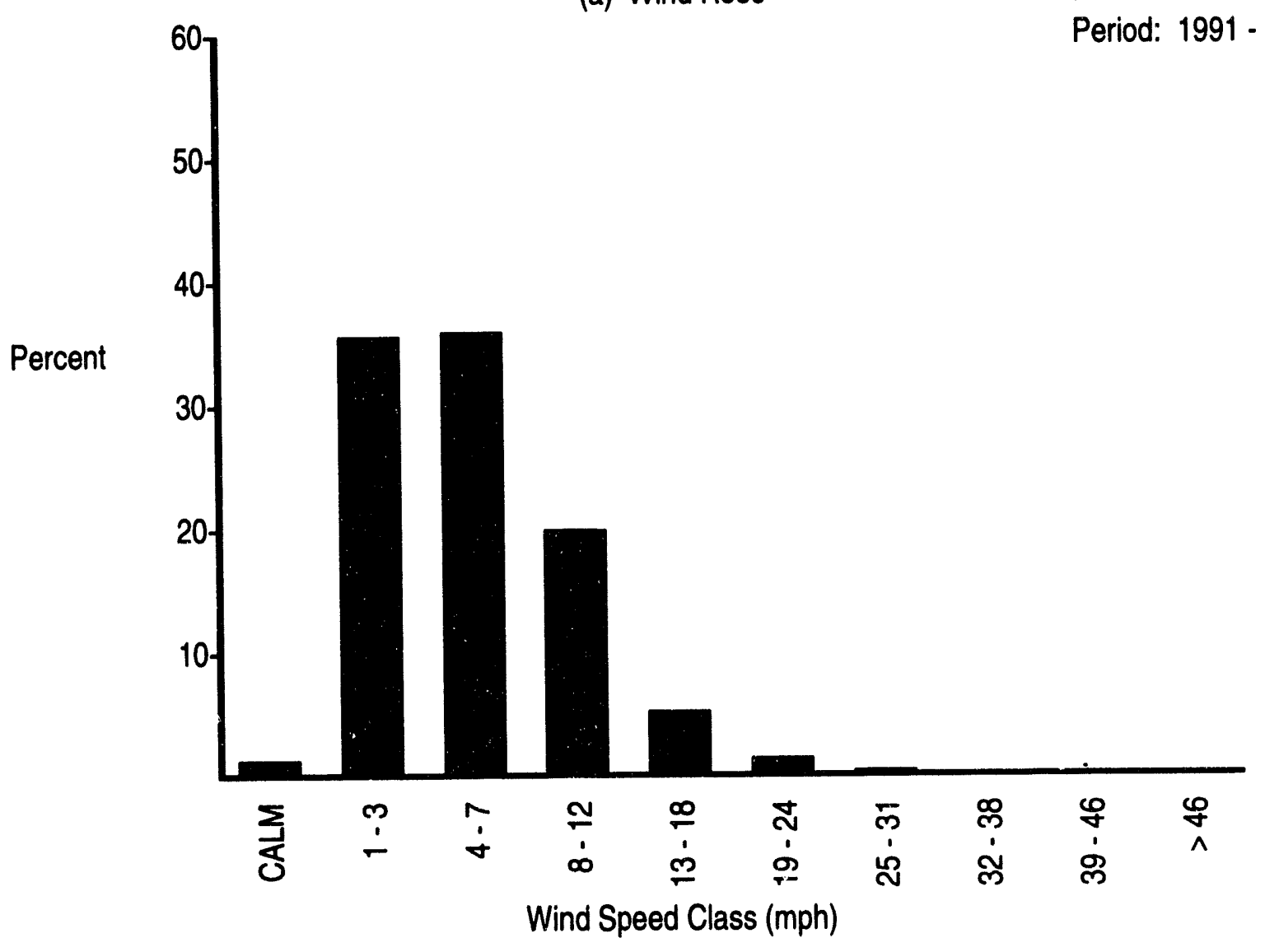

(b) Wind Speed Histogram

FIGURE B.1. (contd)
December Data

Period: 1991 - 1993

\section{B.305}




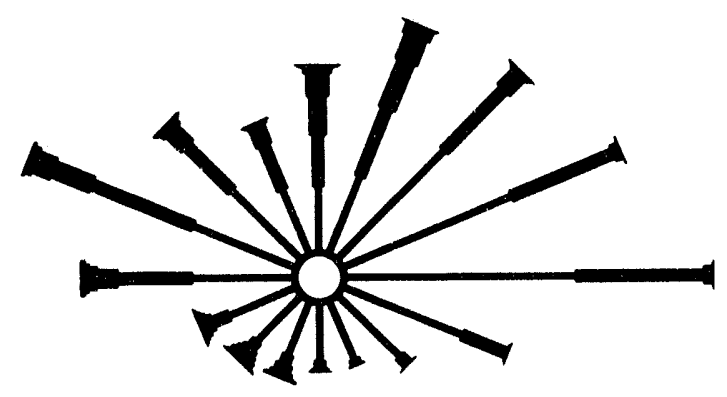

(a) Wind Rose

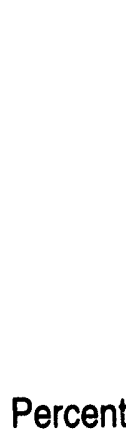

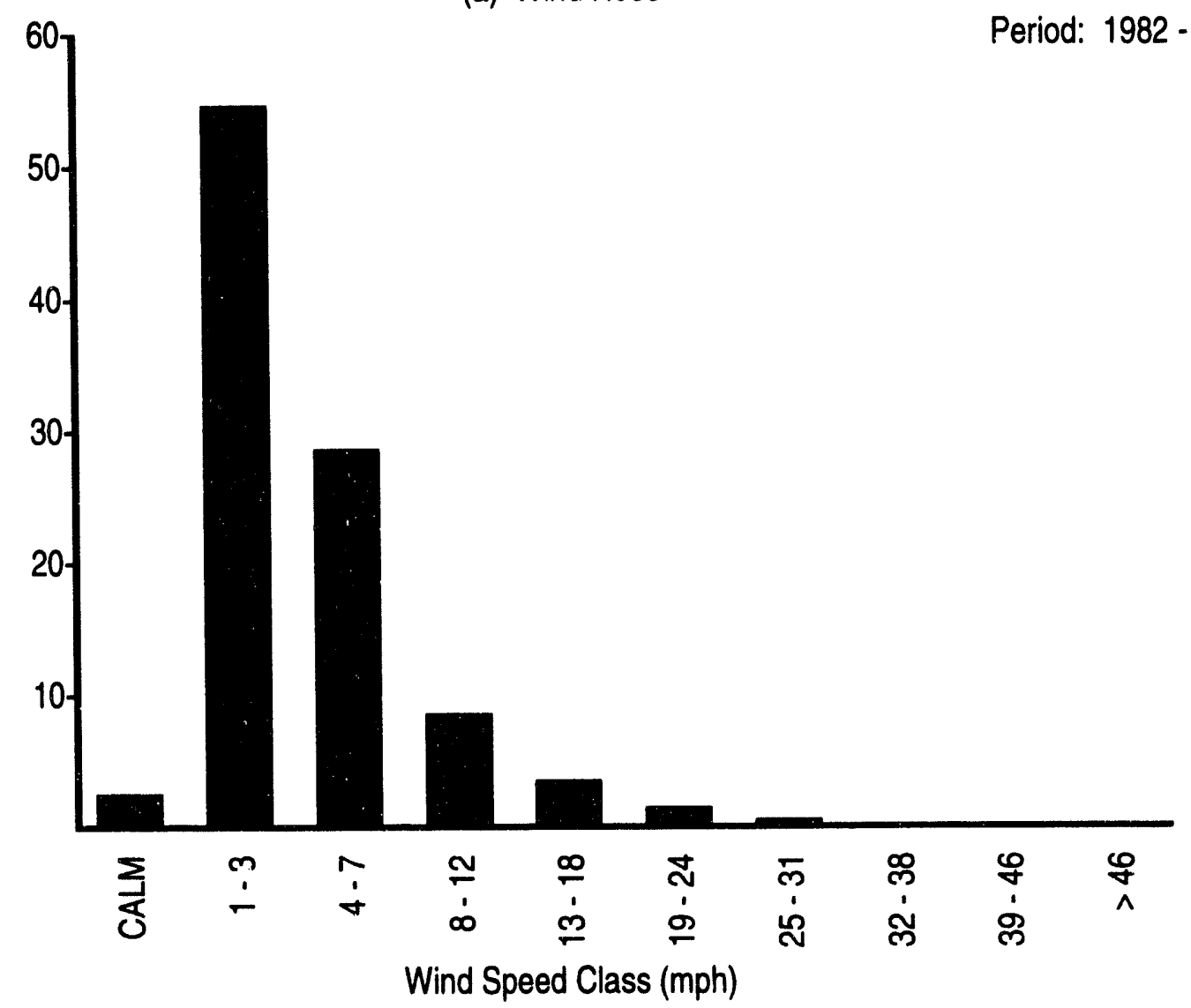

(b) Wind Speed Hislogram

FIGURE B.1. (contd) 


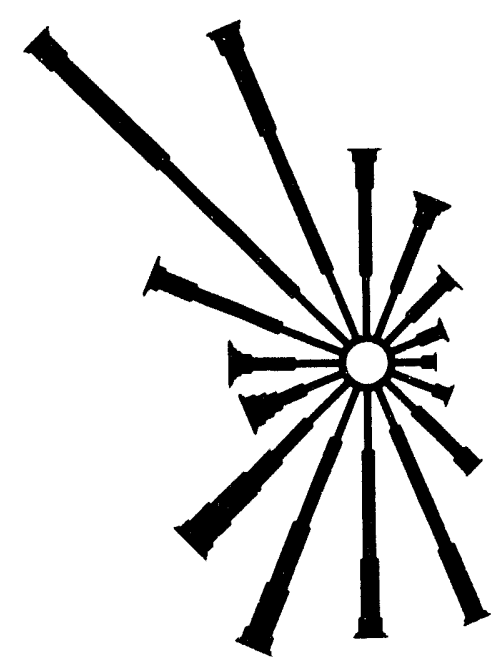

N

(a) Wind Rose

December Data

Period: $1982-1993$

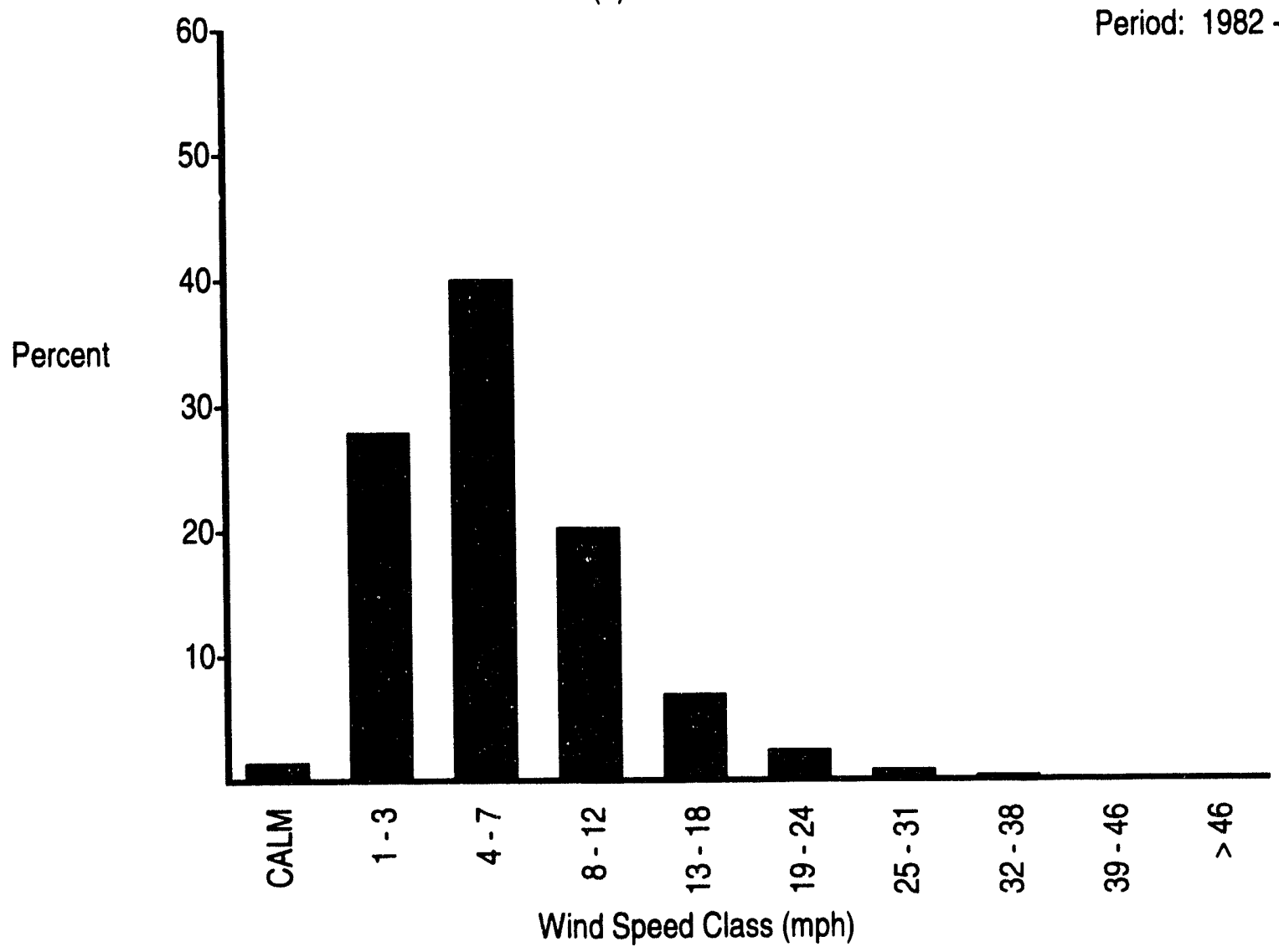

(b) Wind Speed Histogram

FIGURE B.1. (contd) 


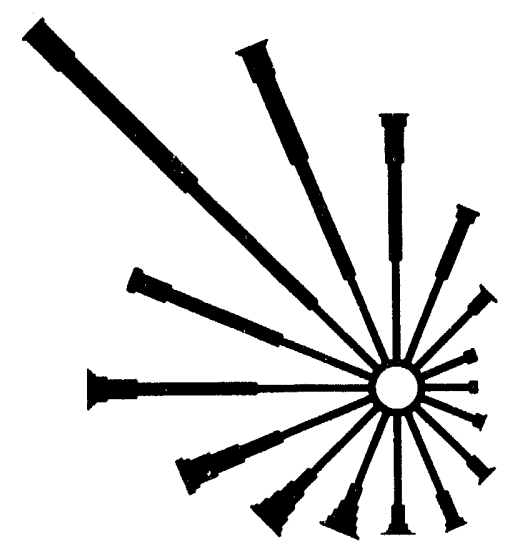

(a) Wind Rose

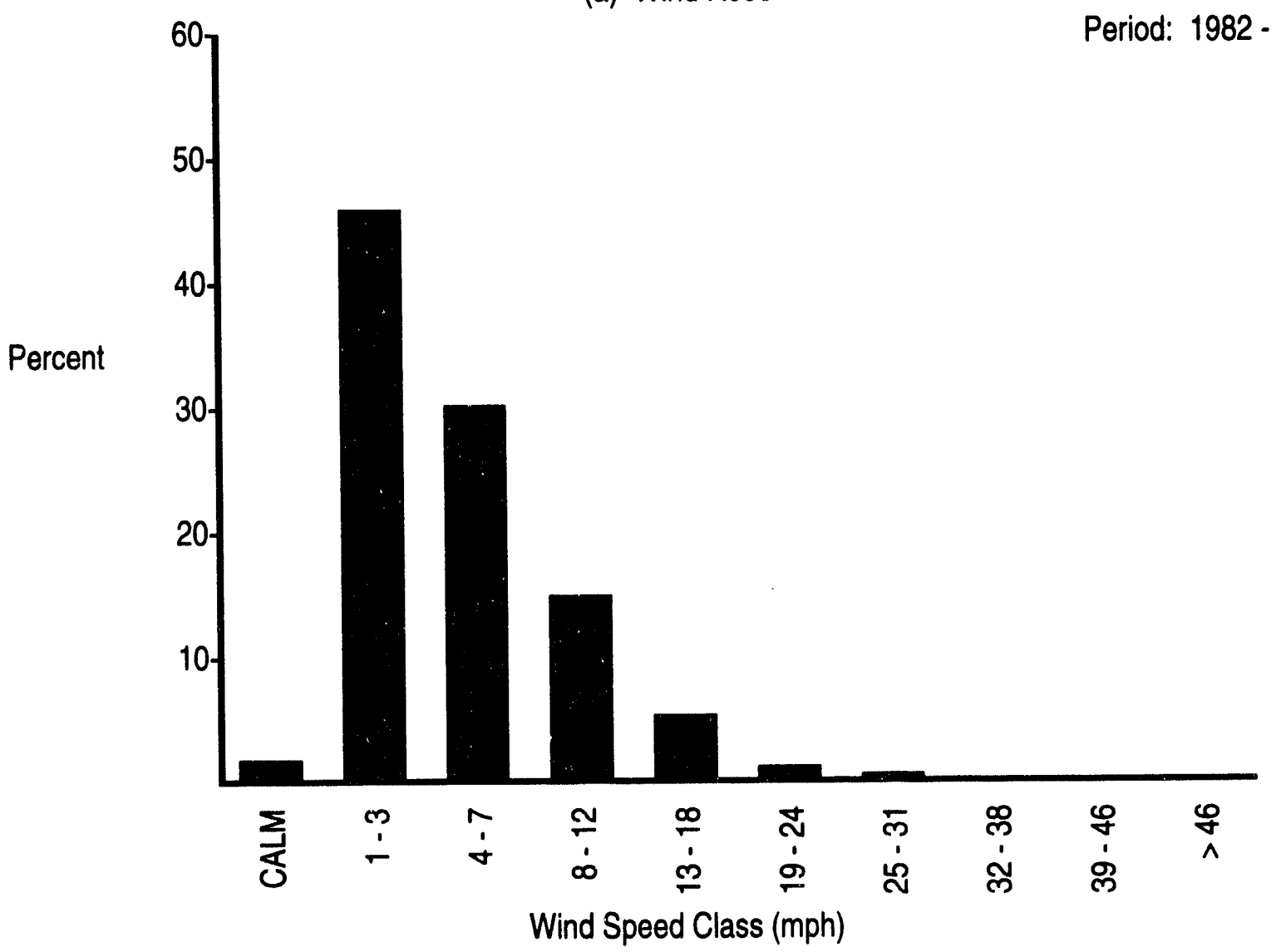

(b) Wind Speed Histogram

FIGURE B.1. (contd) 

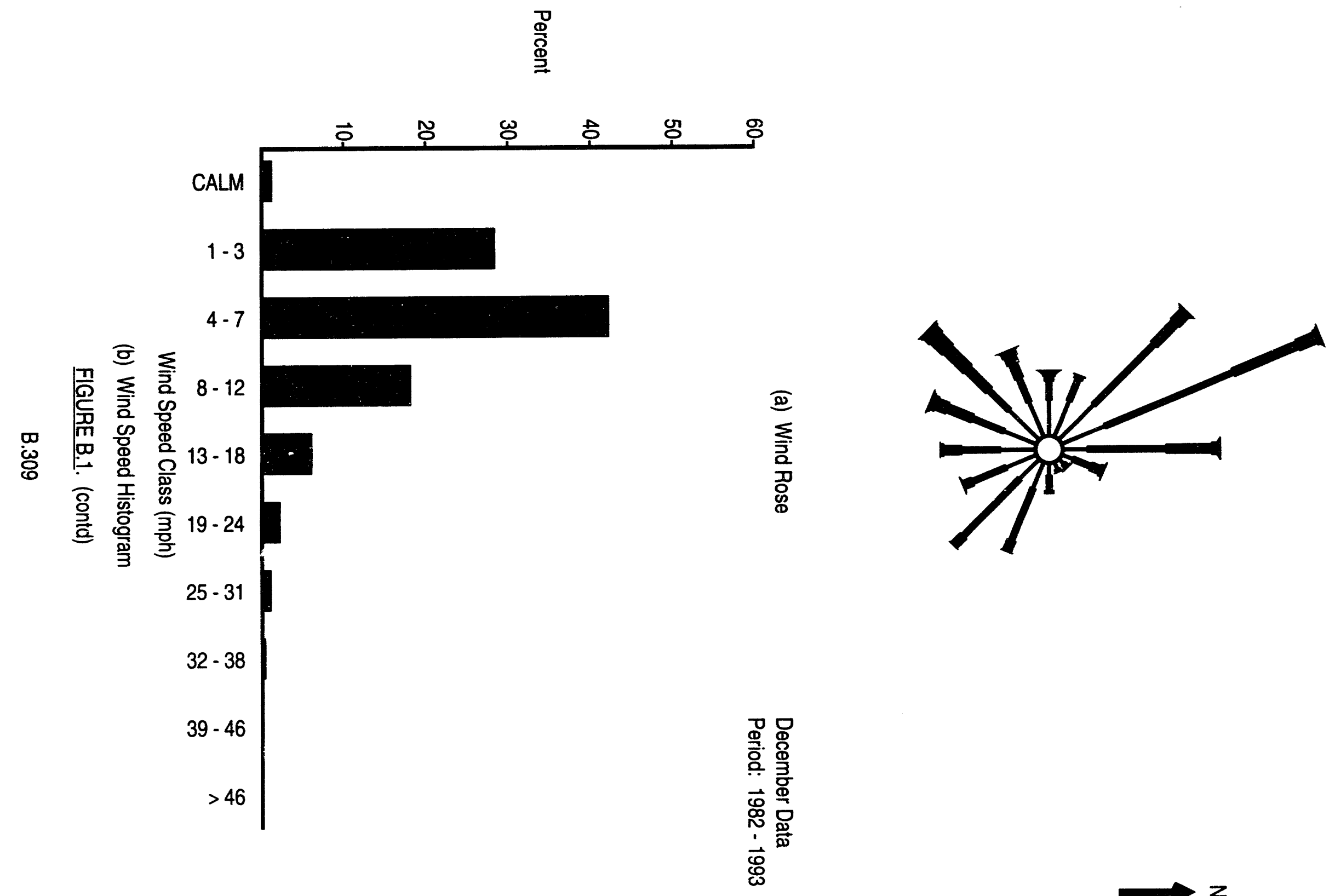


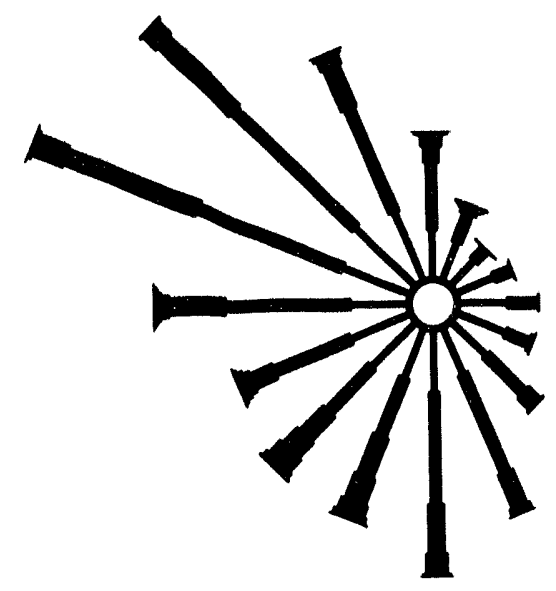

N

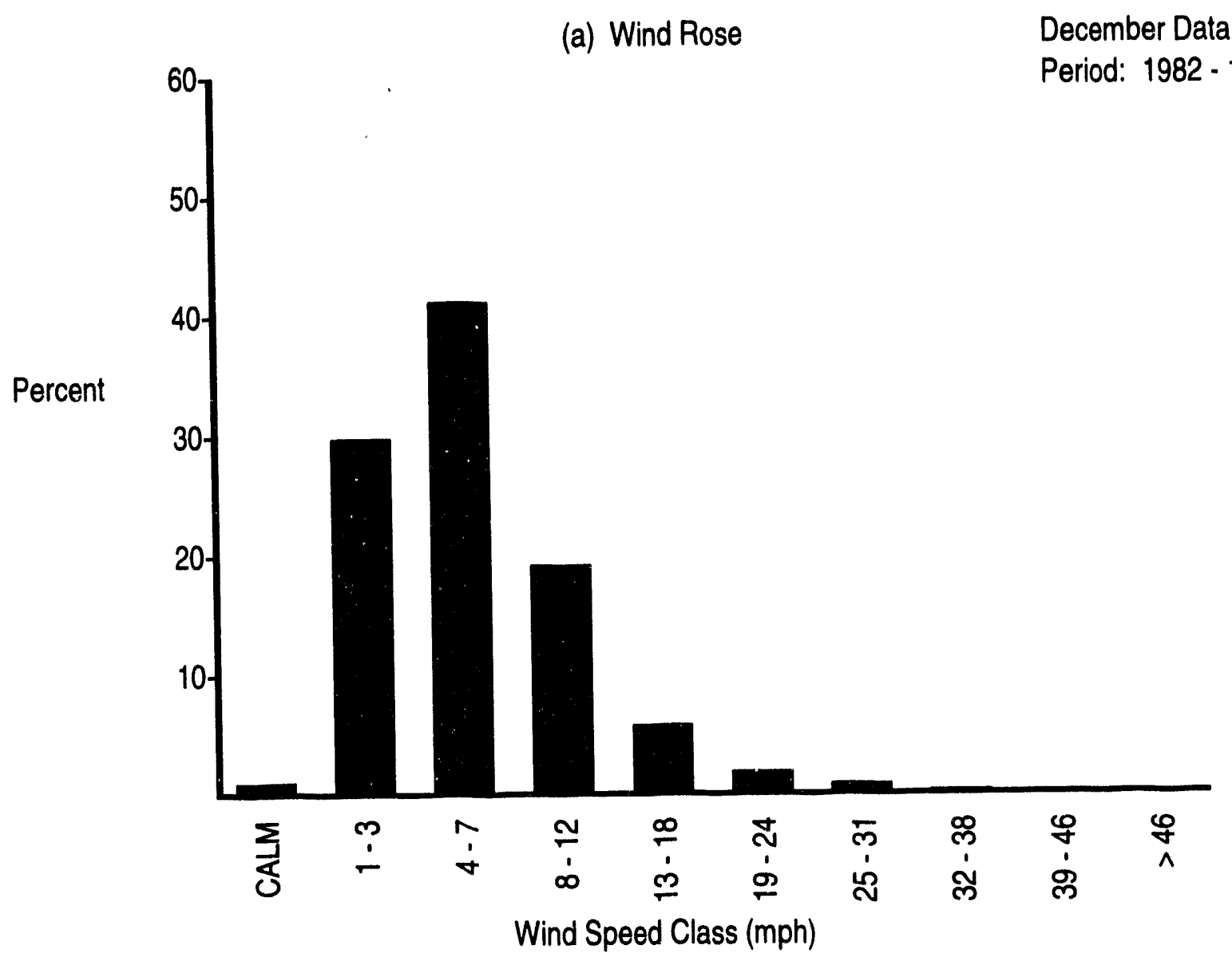

(b) Wind Speed Histogram

FIGURE B.1. (contd) 


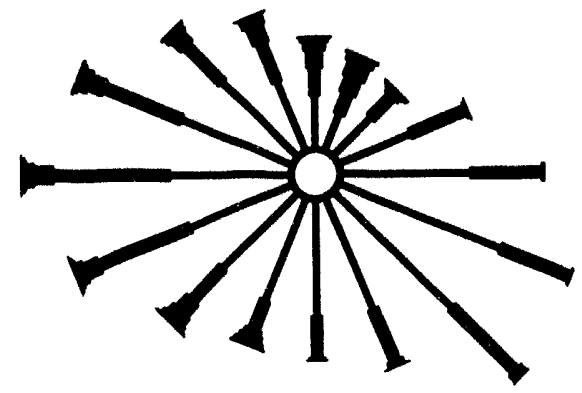

(a) Wind Rose

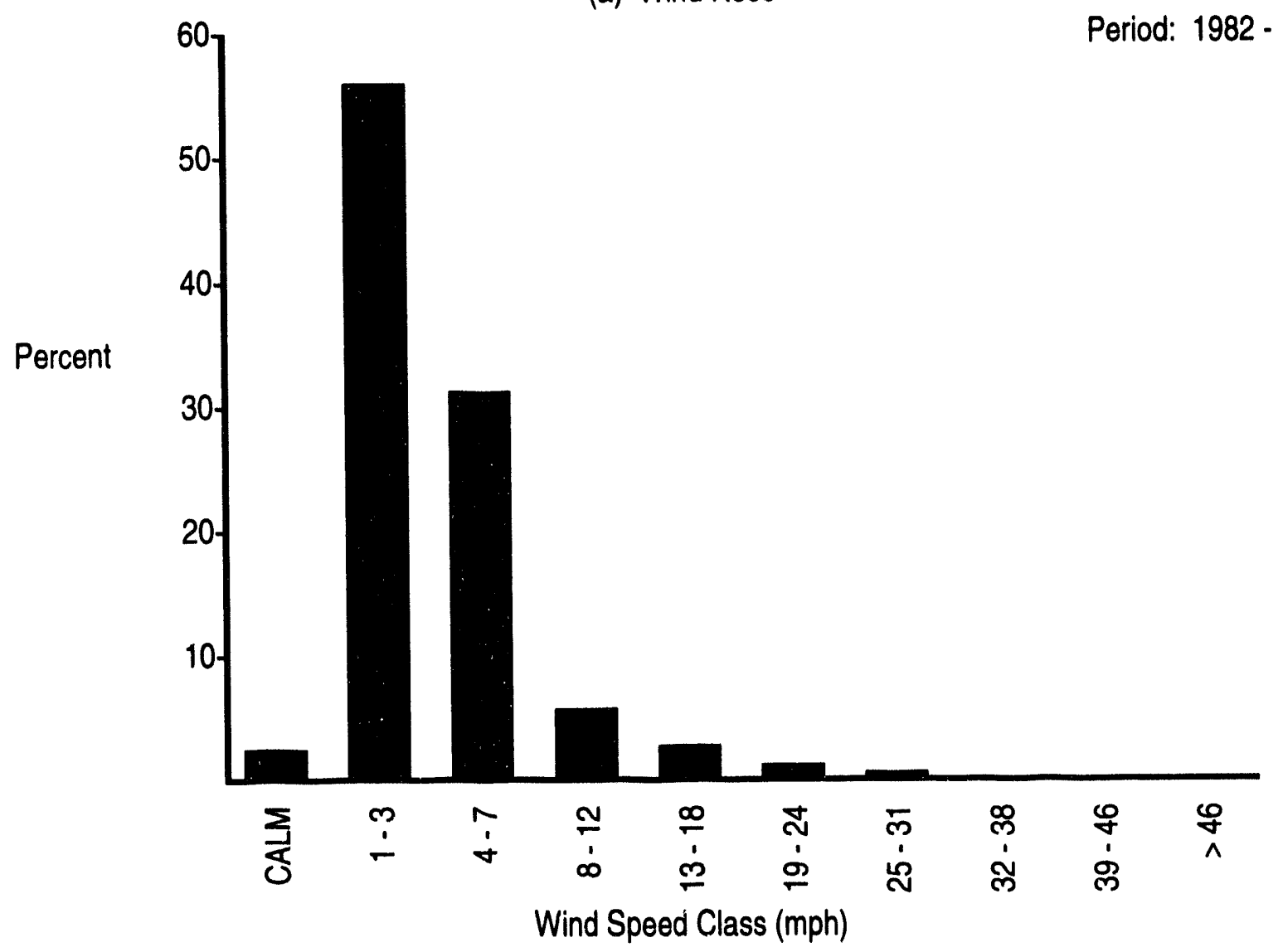

(b) Wind Speed Histogram

FIGURE B.1. (contd)
December Data

Period: 1982 - 1993 


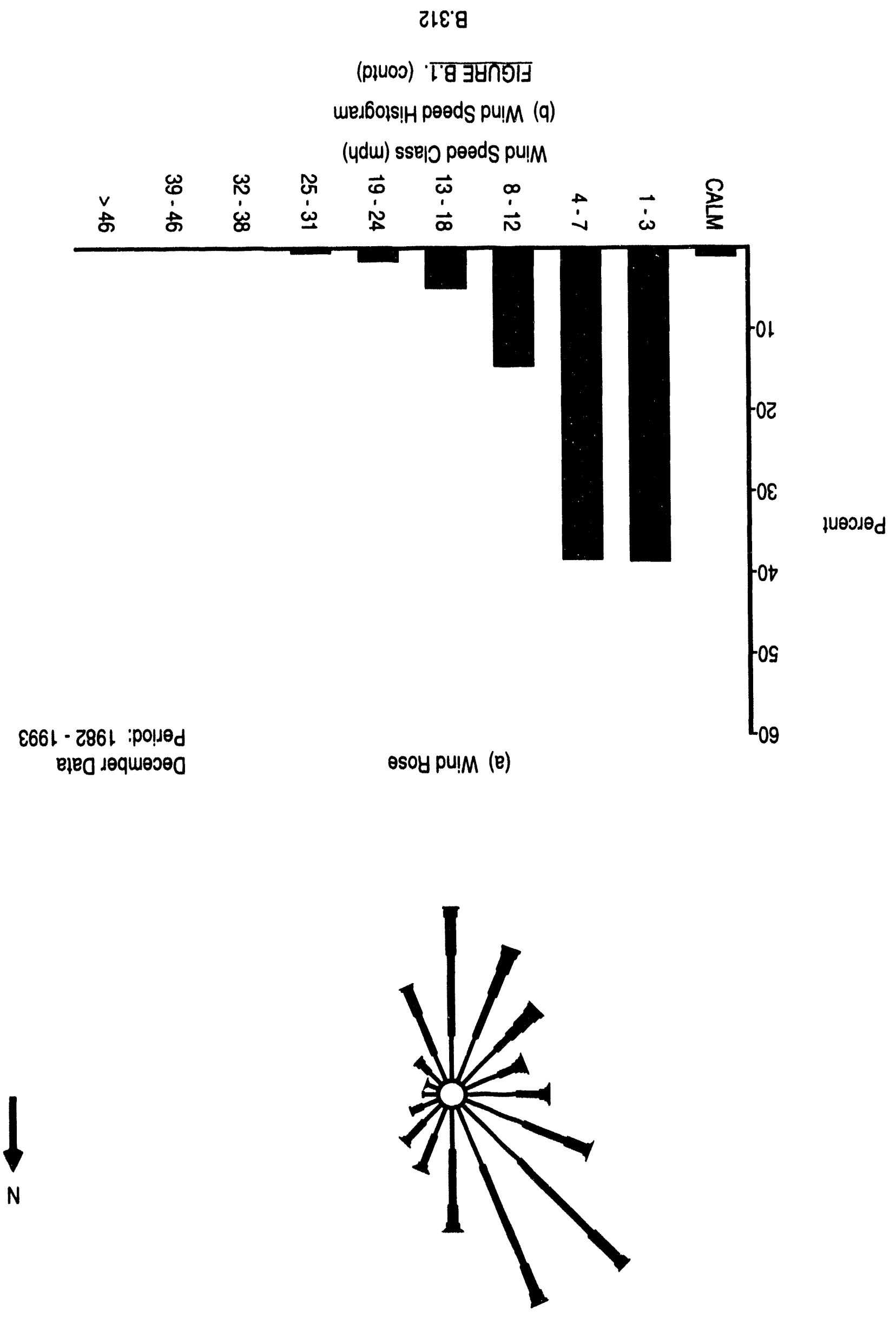




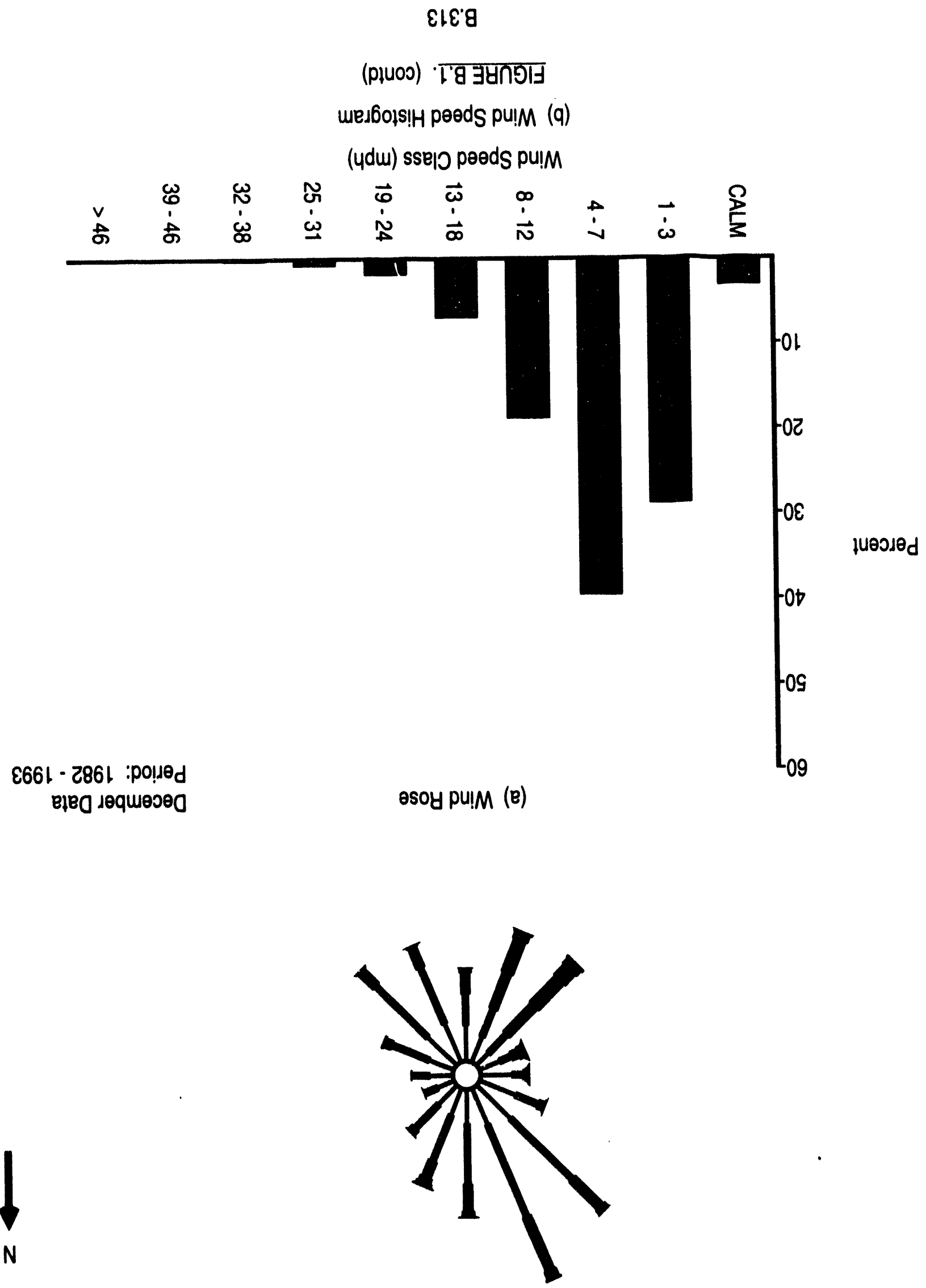




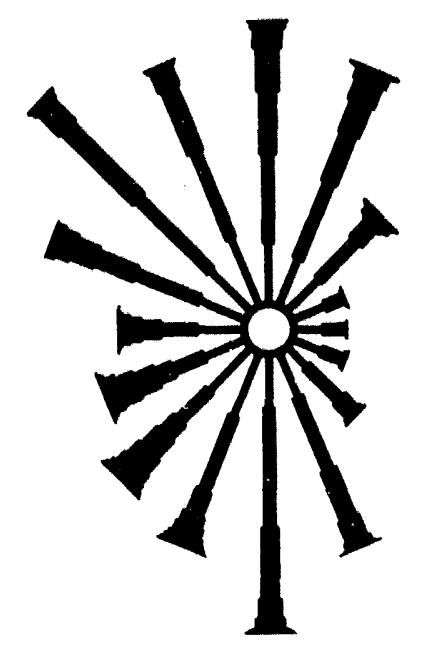

(a) Wind Rose

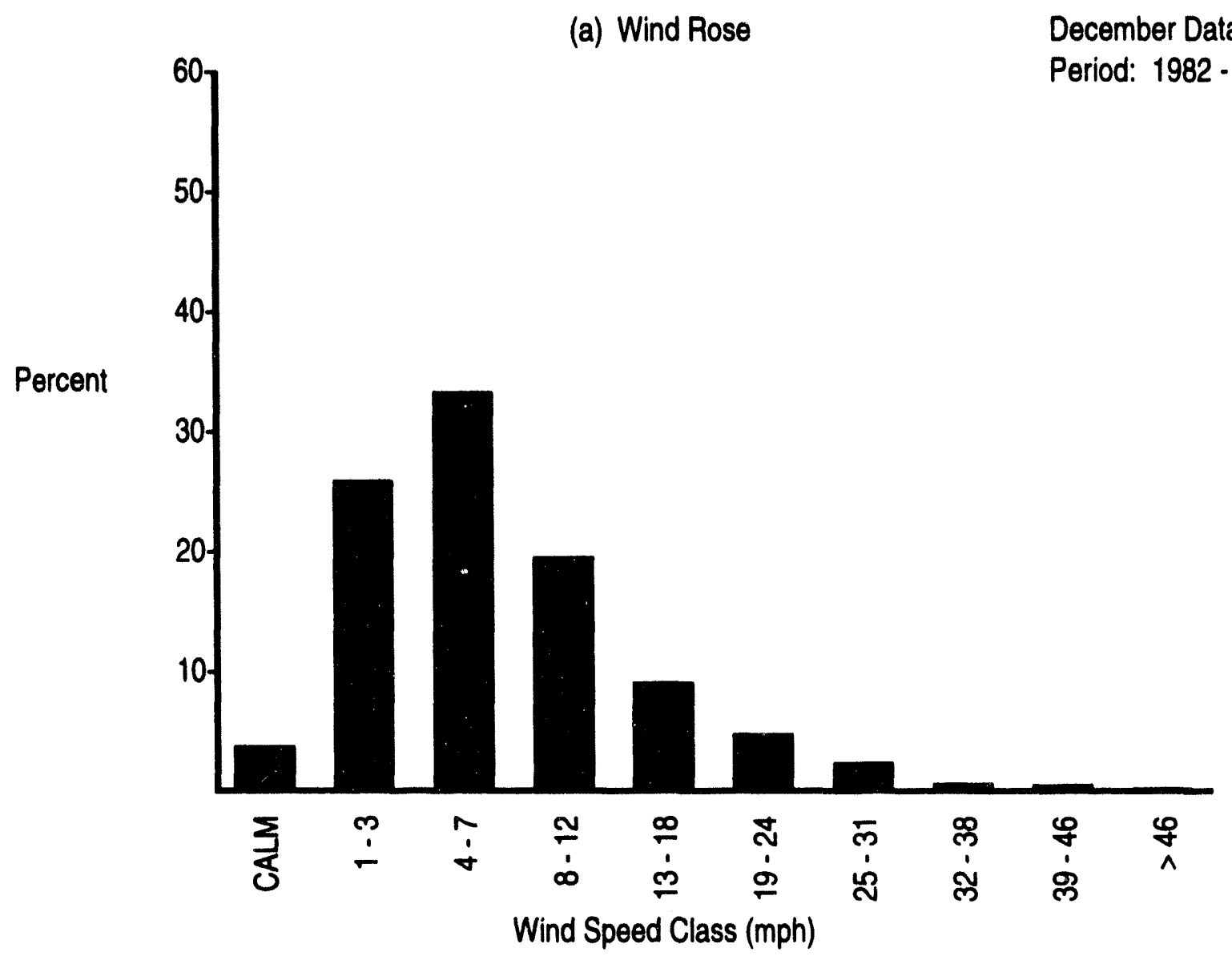

(b) Wind Speed Histogram

FIGURE B.1. (contd) 

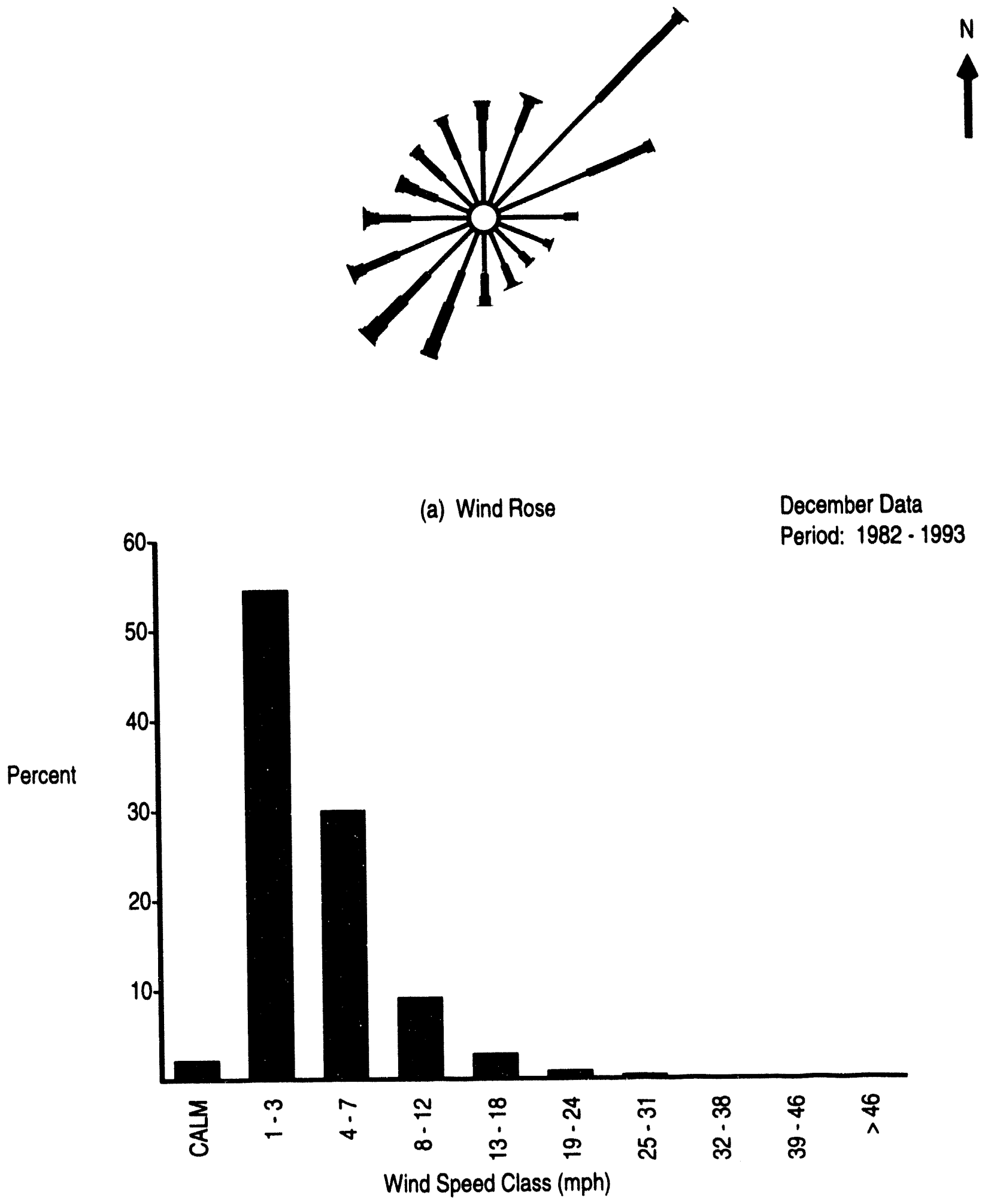

(b) Wind Speed Histogram

FIGURE B.1. (contd)

B.3" 


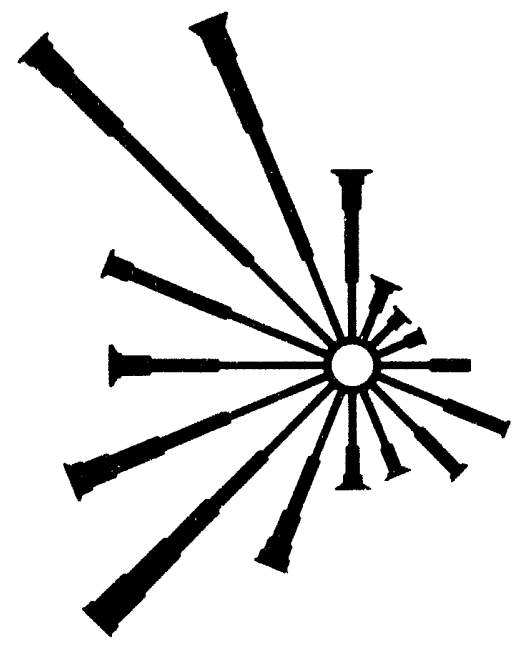

(a) Wind Rose

December Data

Percent

60
50
$40-$
$30-$
$20-$
$10-$

Period: 1982 - 1993

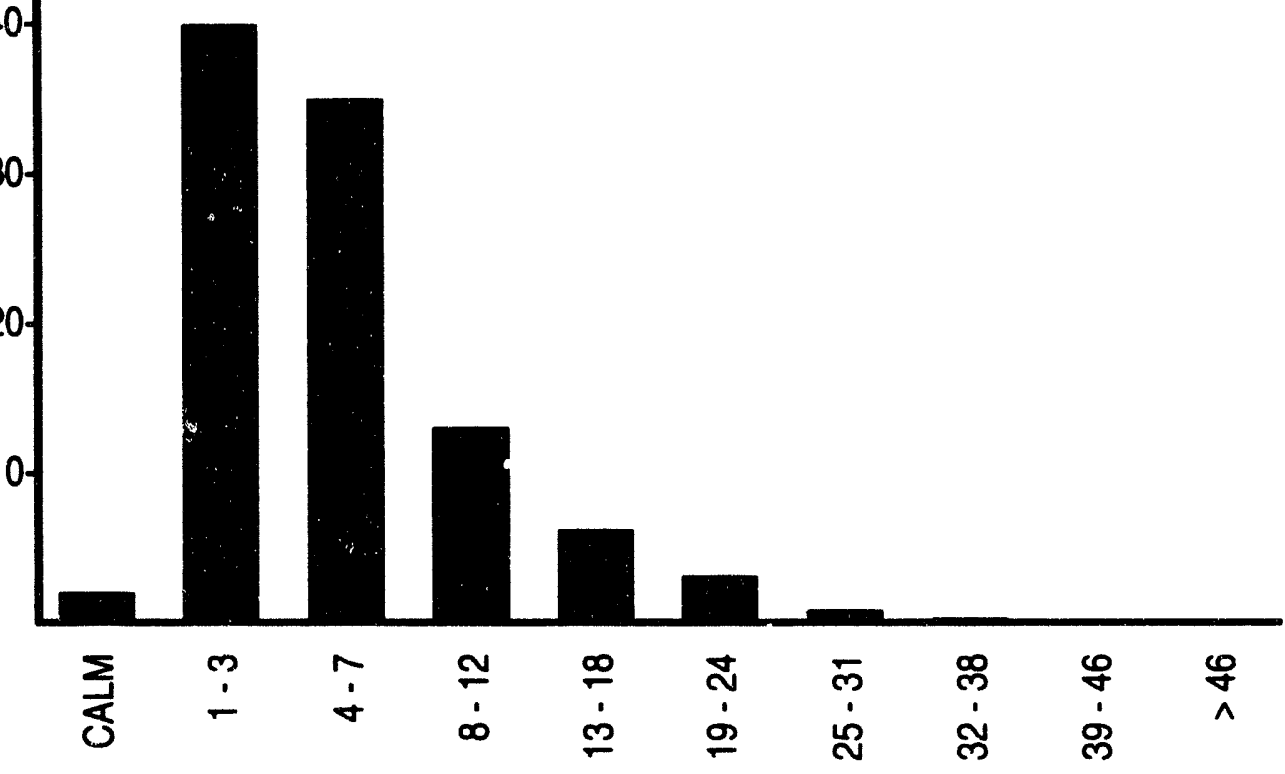

Wind Speed Class (mph)

(b) Wind Speed Histogram

FIGURE B.1. (contd) 


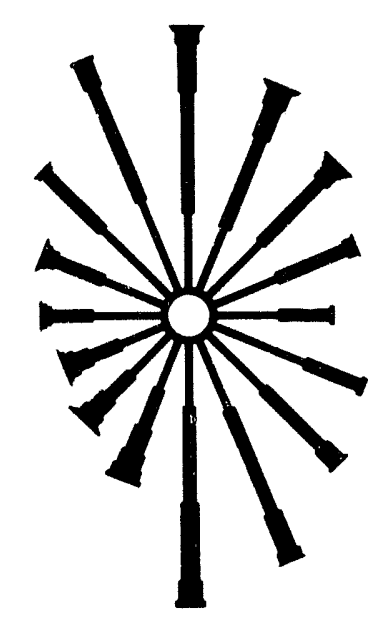

(a) Wind Rose

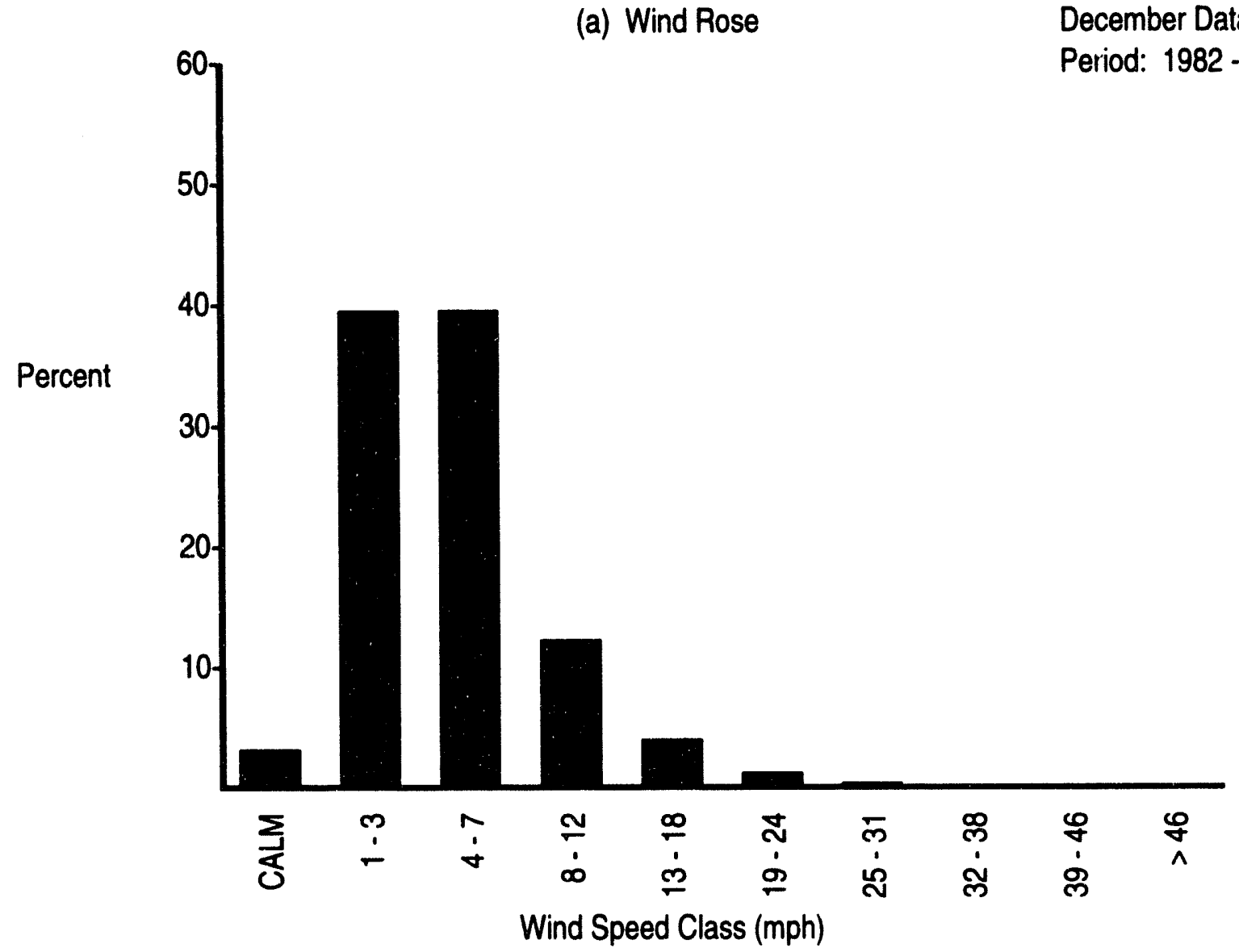

(b) Wind Speed Histogram

FIGURE B.1. (contd) 


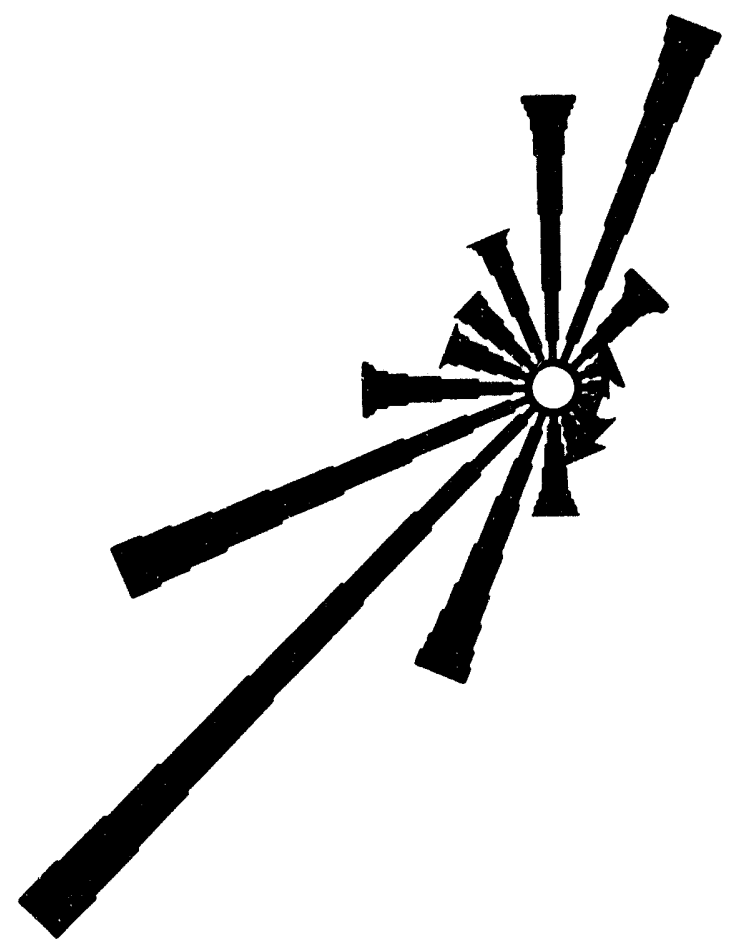

1

(a) Wind Rose

December Data

Period: 1982 - 1993

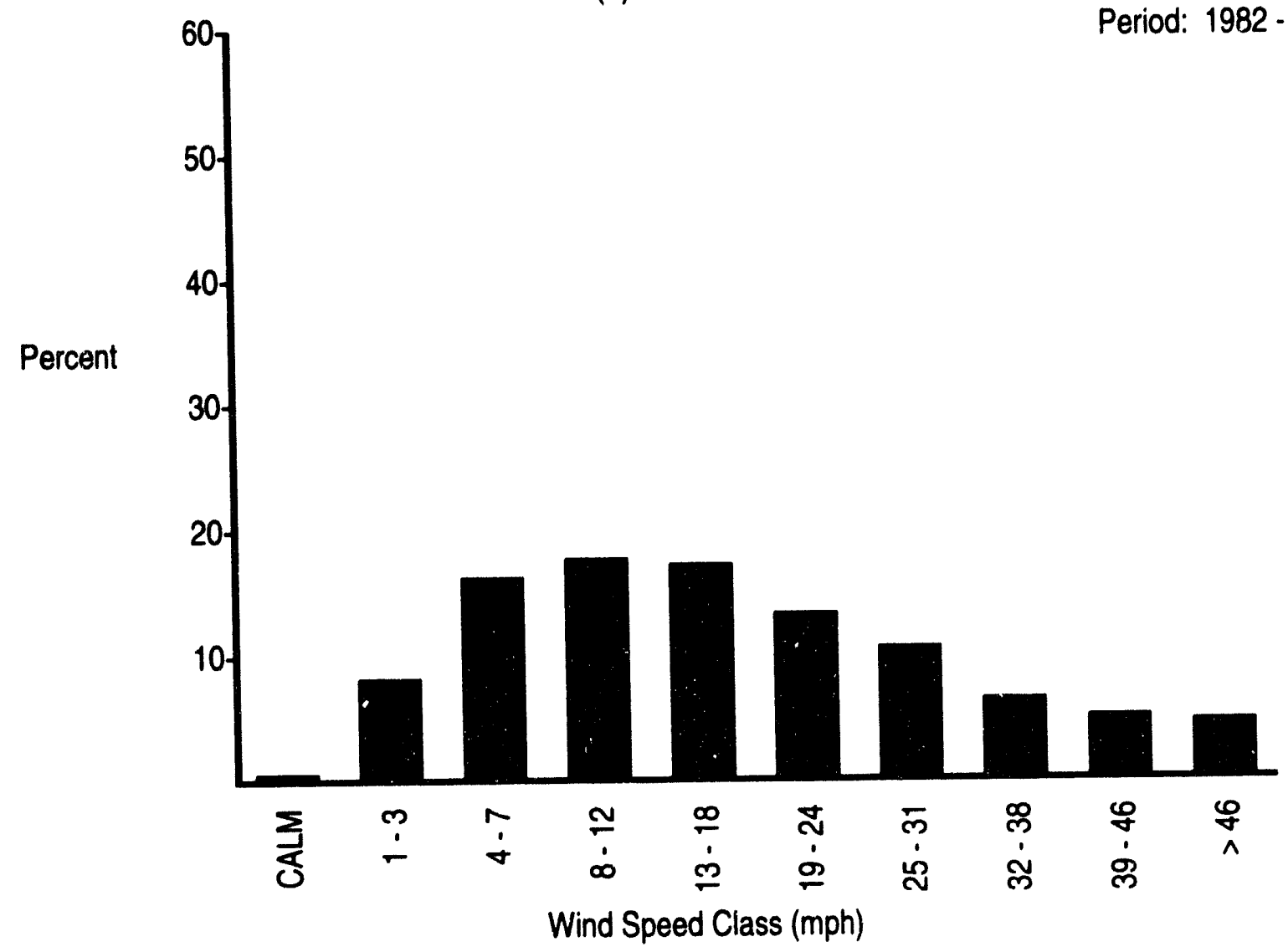

(b) Wind Speed Histogram

FIGURE B.1. (contd) 

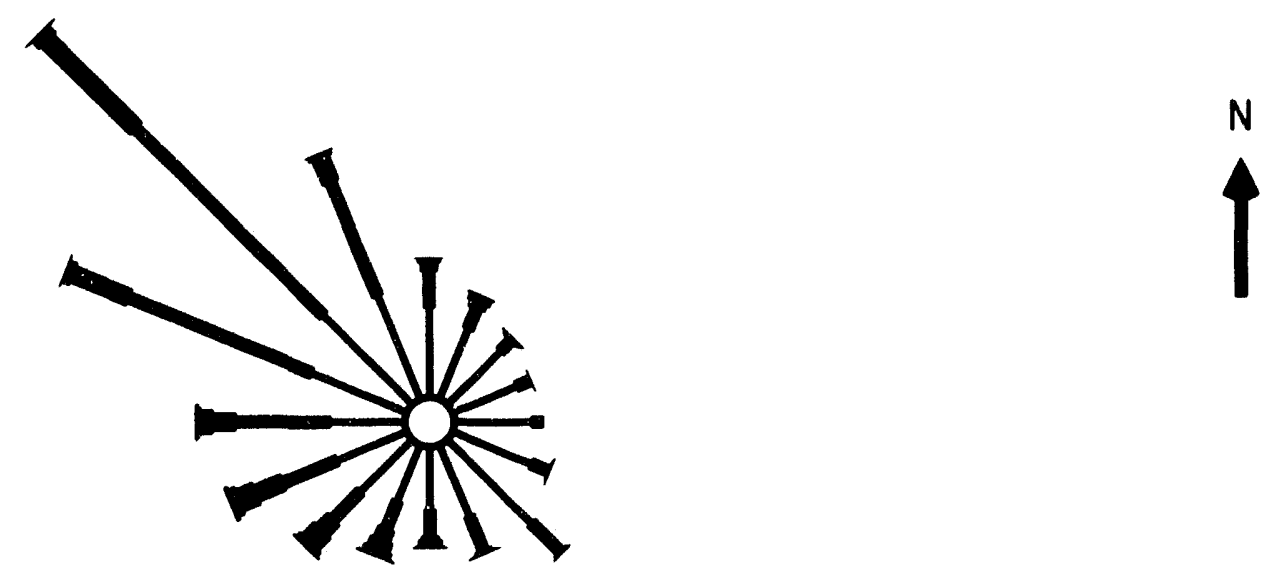

(a) Wind Rose

December Data

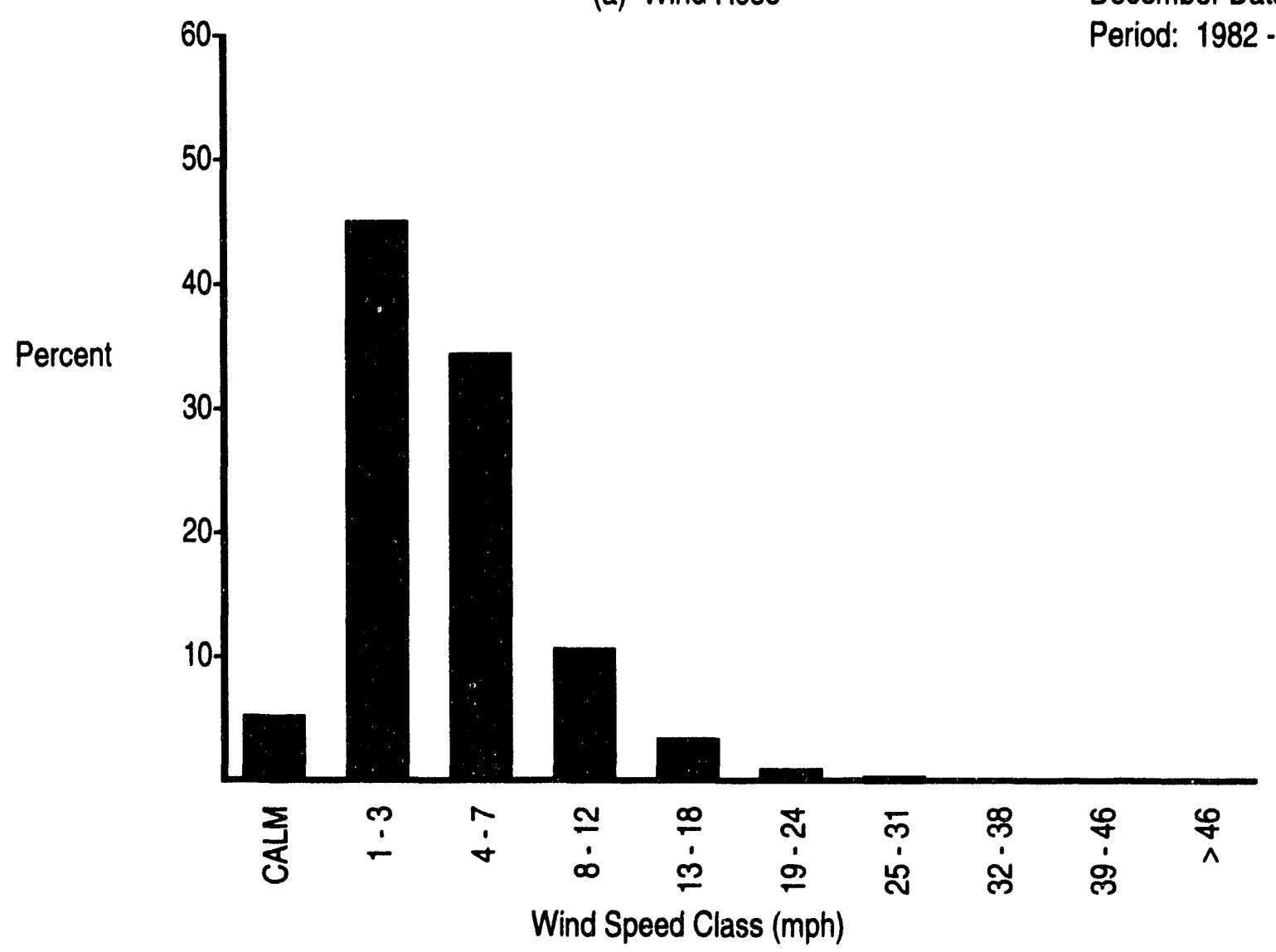

(b) Wind Speed Histogram

FIGURE B.1. (contd) 


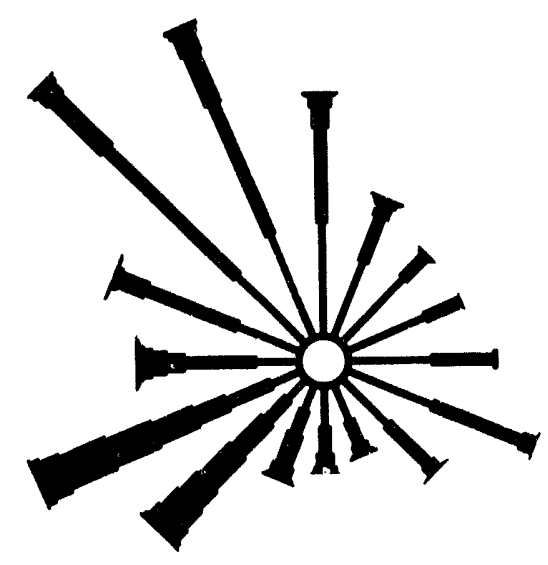

(a) Wind Rose

December Data

Period: 1987 - 1993

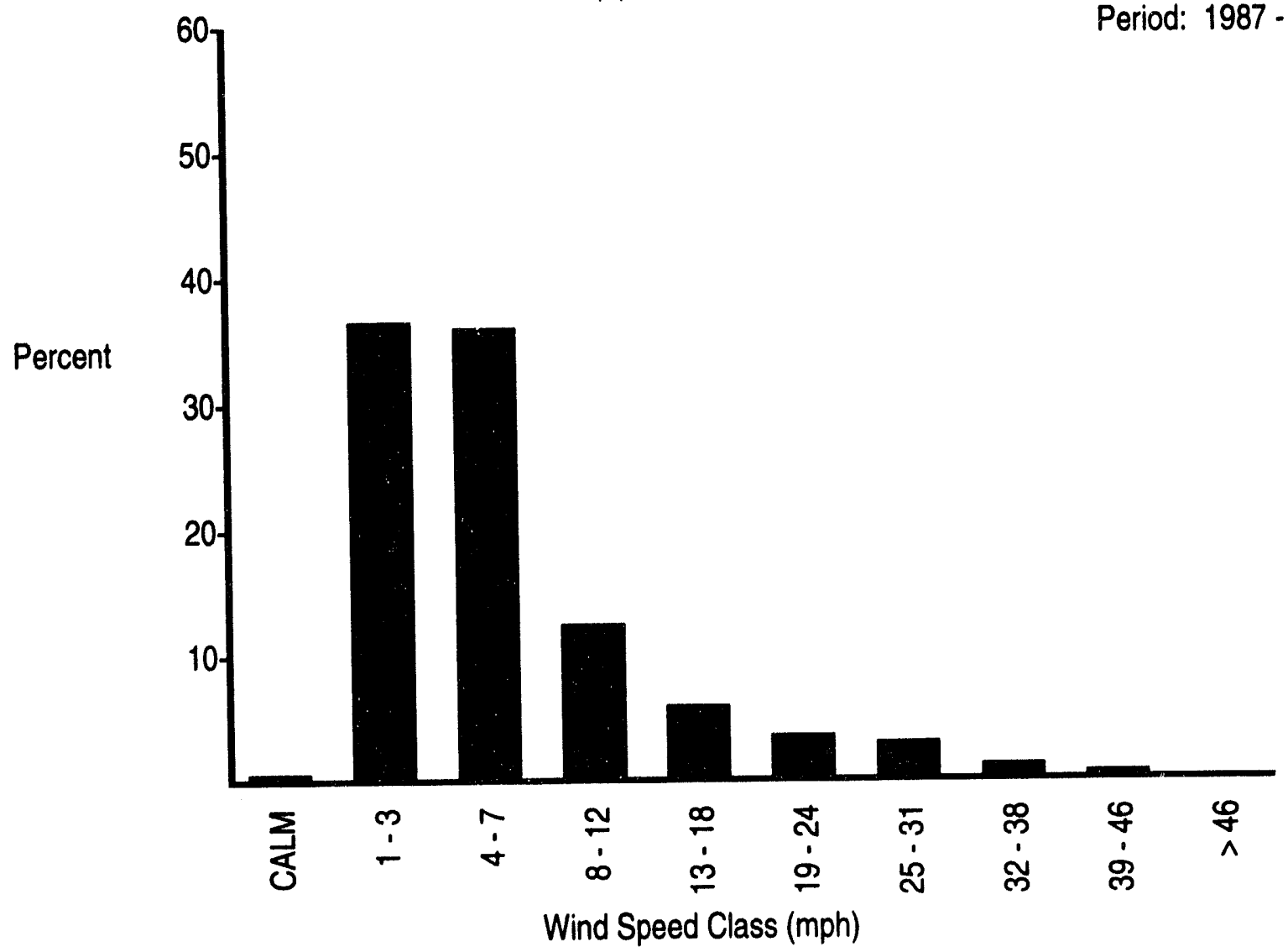

(b) Wind Speed Histogram

FIGURE B.1. (contd) 


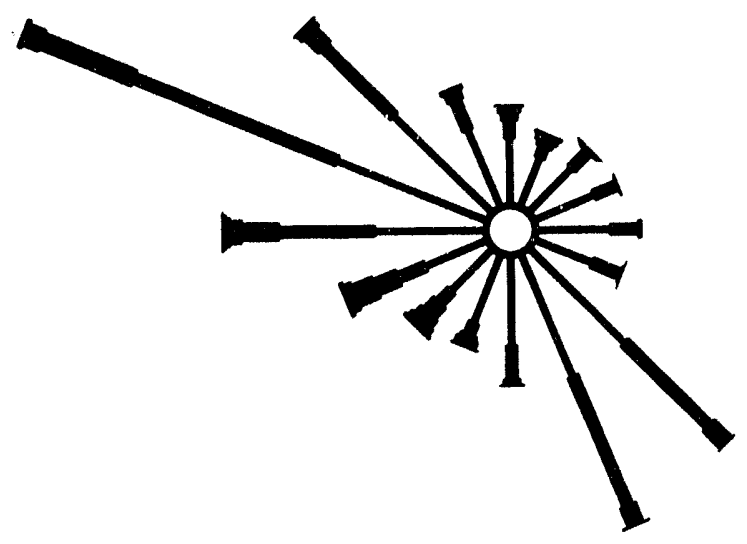

(a) Wind Rose

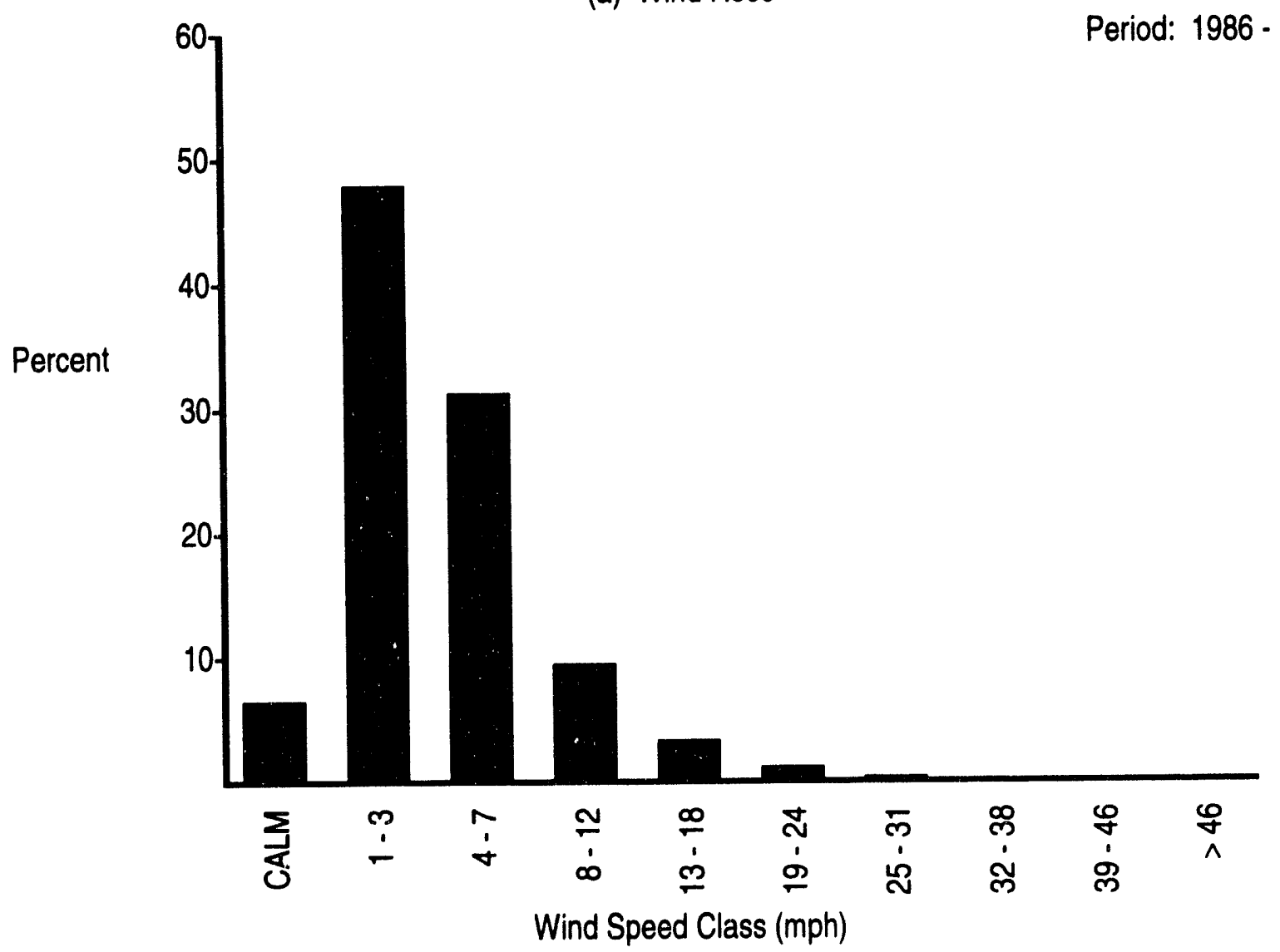

(b) Wind Speed Histogram

FIGURE B.1. (contd) 
टఒ६'g

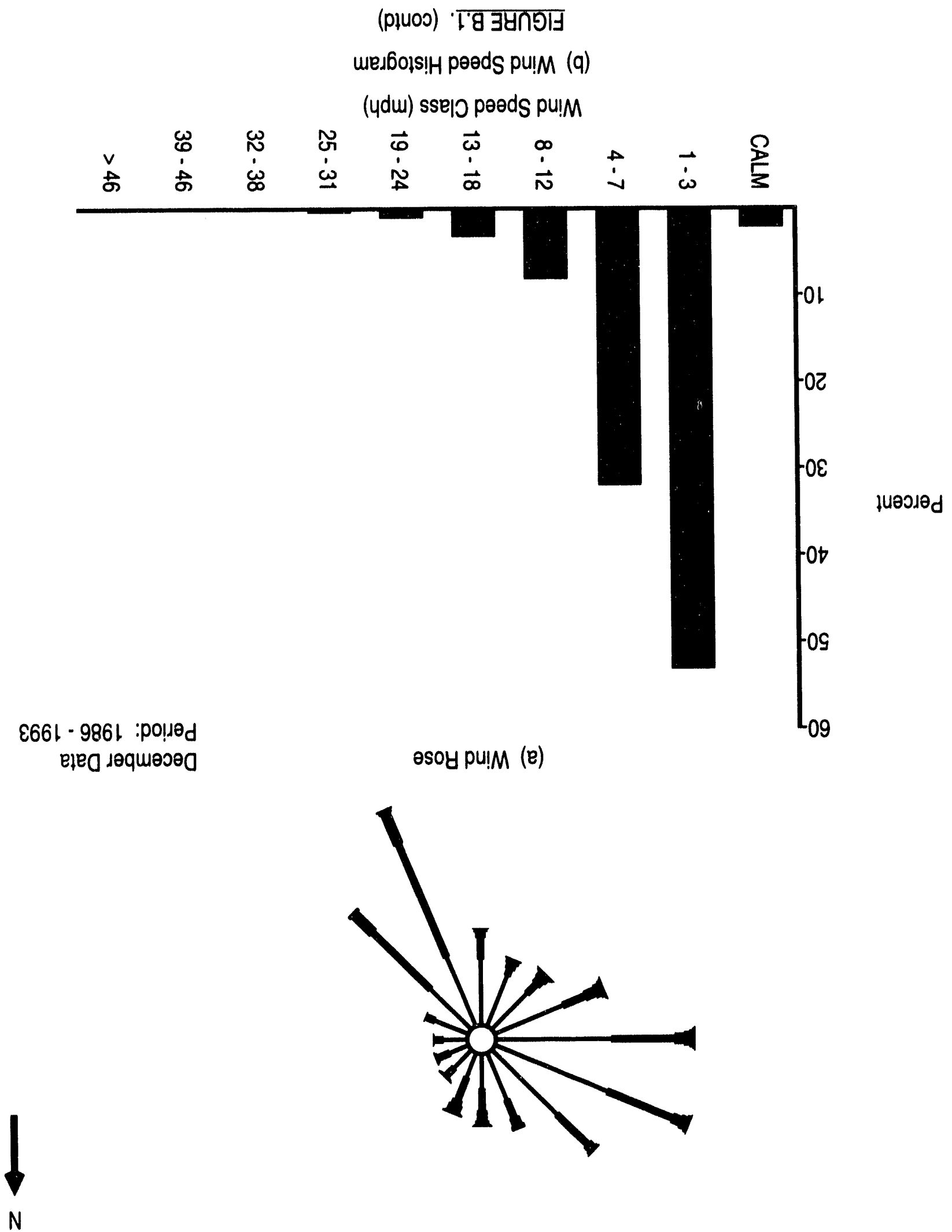




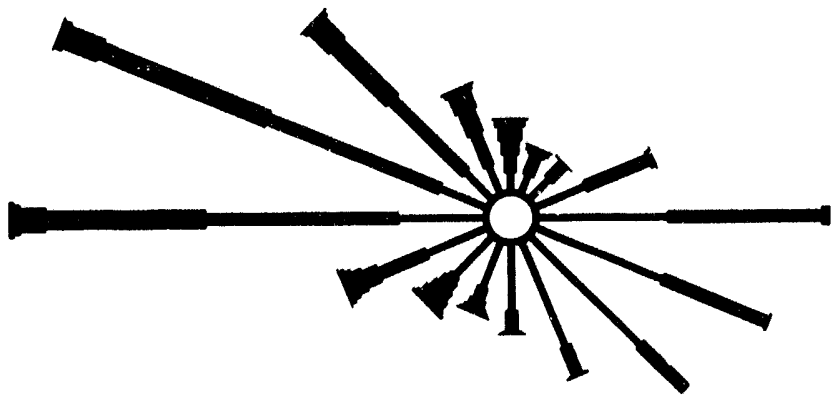

(a) Wind Rose

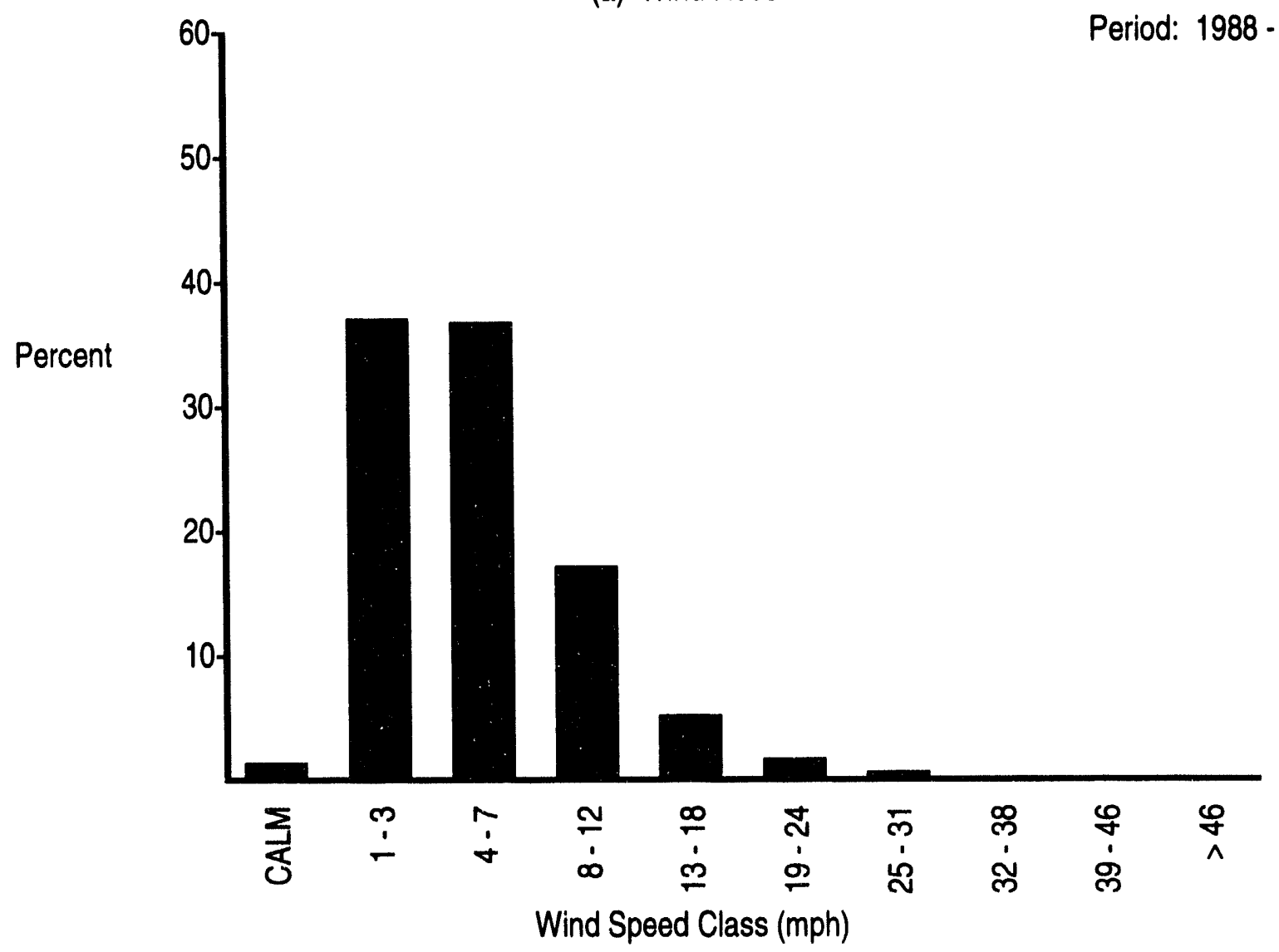

(b) Wind Speed Histogram

FIGURE B.1. (contd)
December Data

Period: 1988 - 1993 


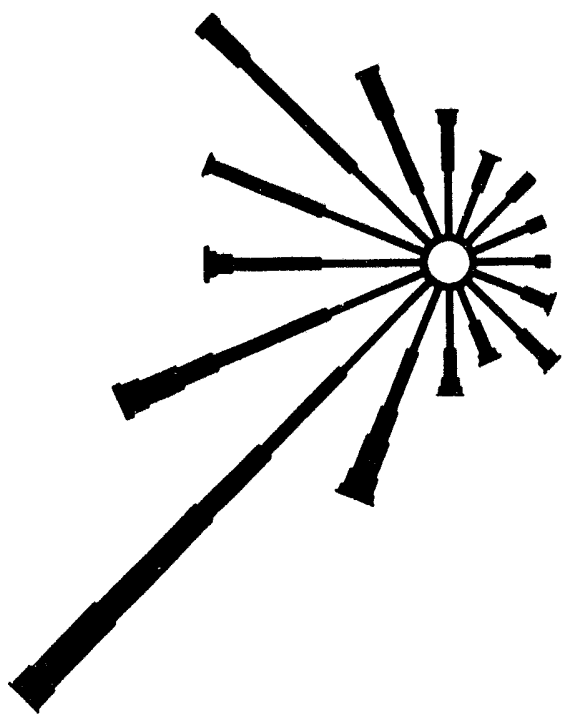

N

(a) Wind Rose

December Data

Period: 1991 - 1993

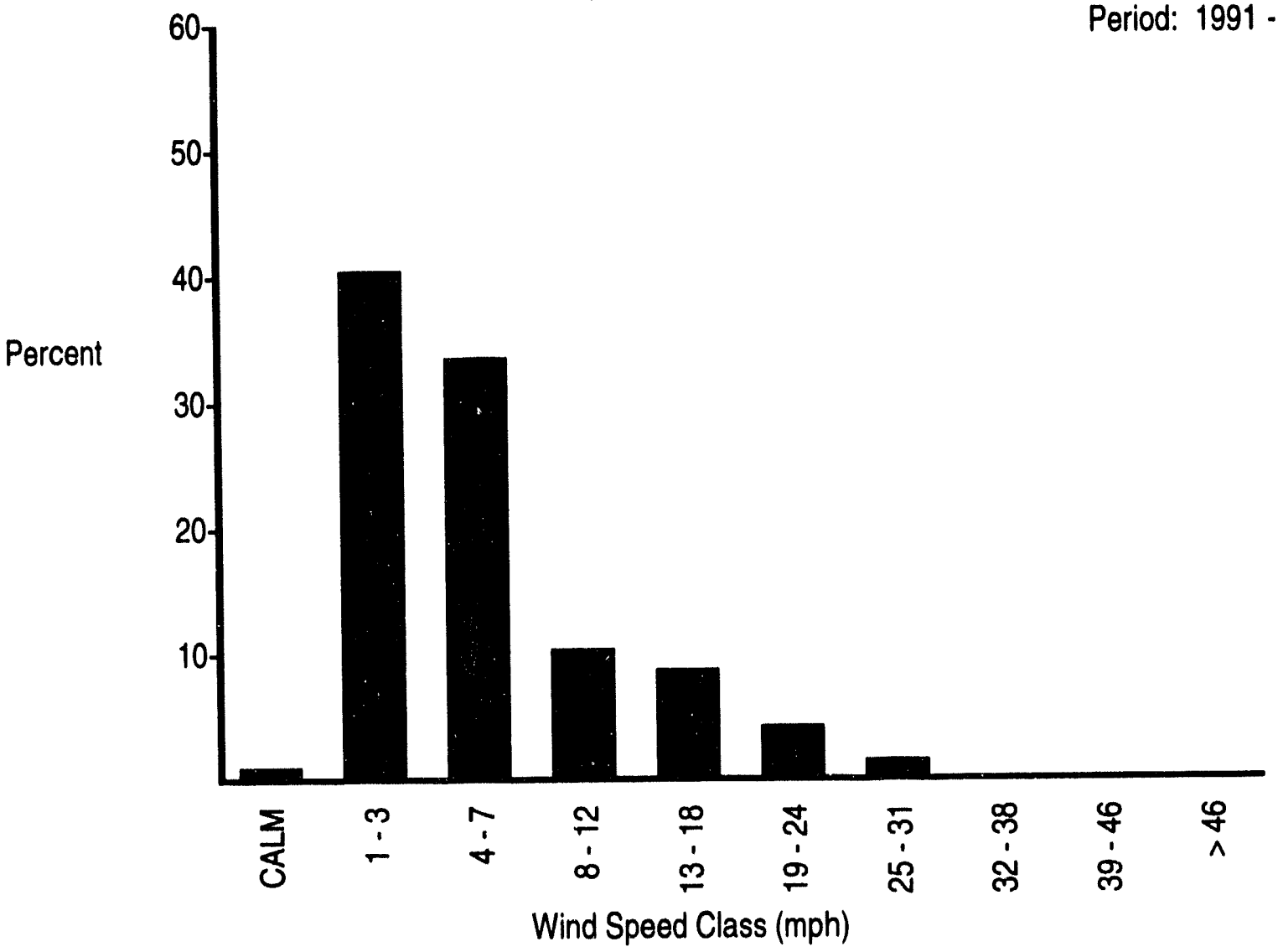

(b) Wind Speed Histogram

FIGURE B.1. (contd) 


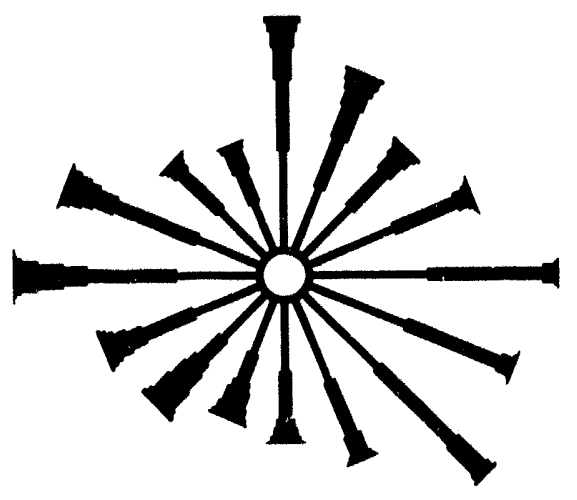

(a) Wind Rose

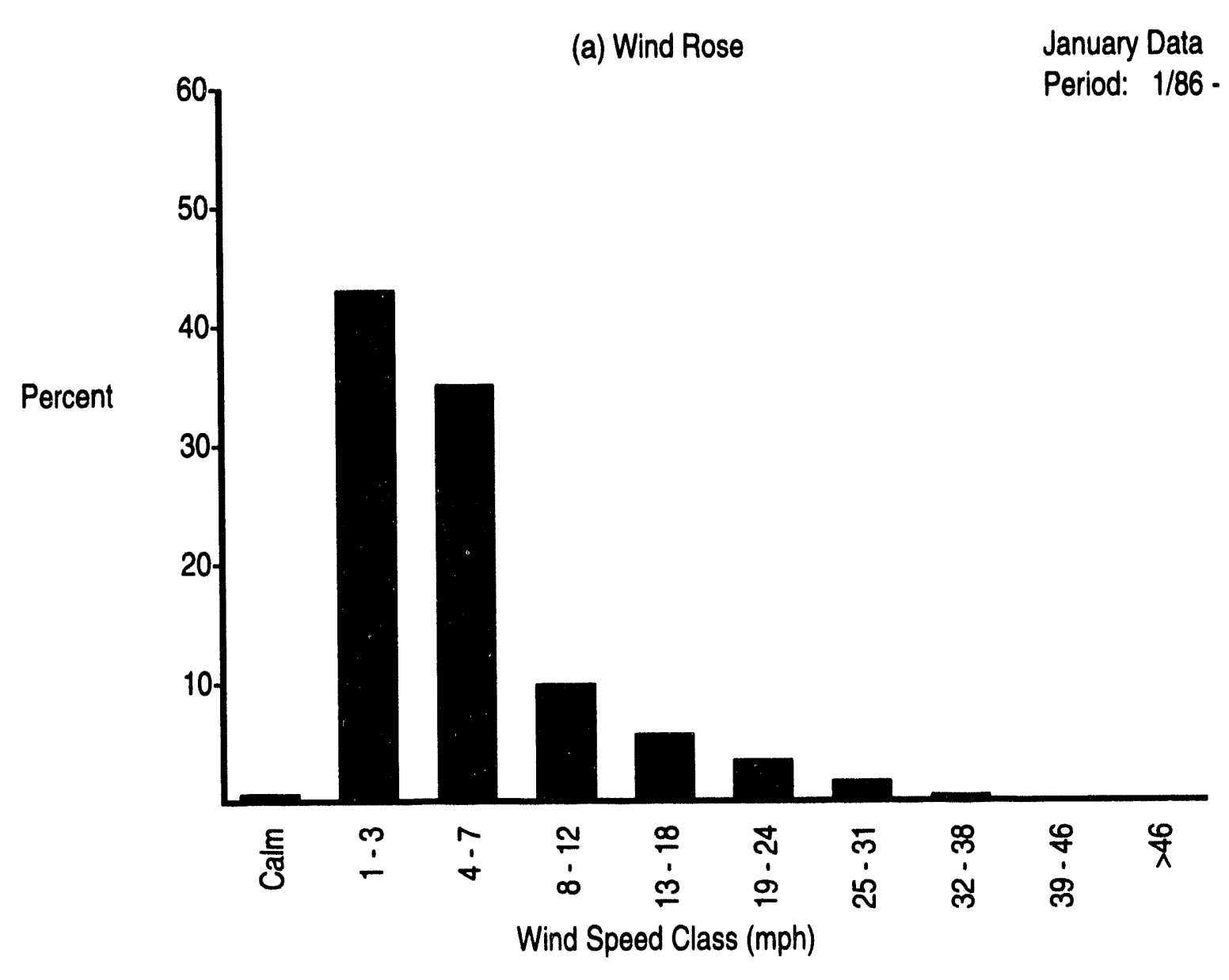

(b) Wind Speed Histogram

FIGURE B.2. Wind Rose and Wind Speed Histogram (60 m) 


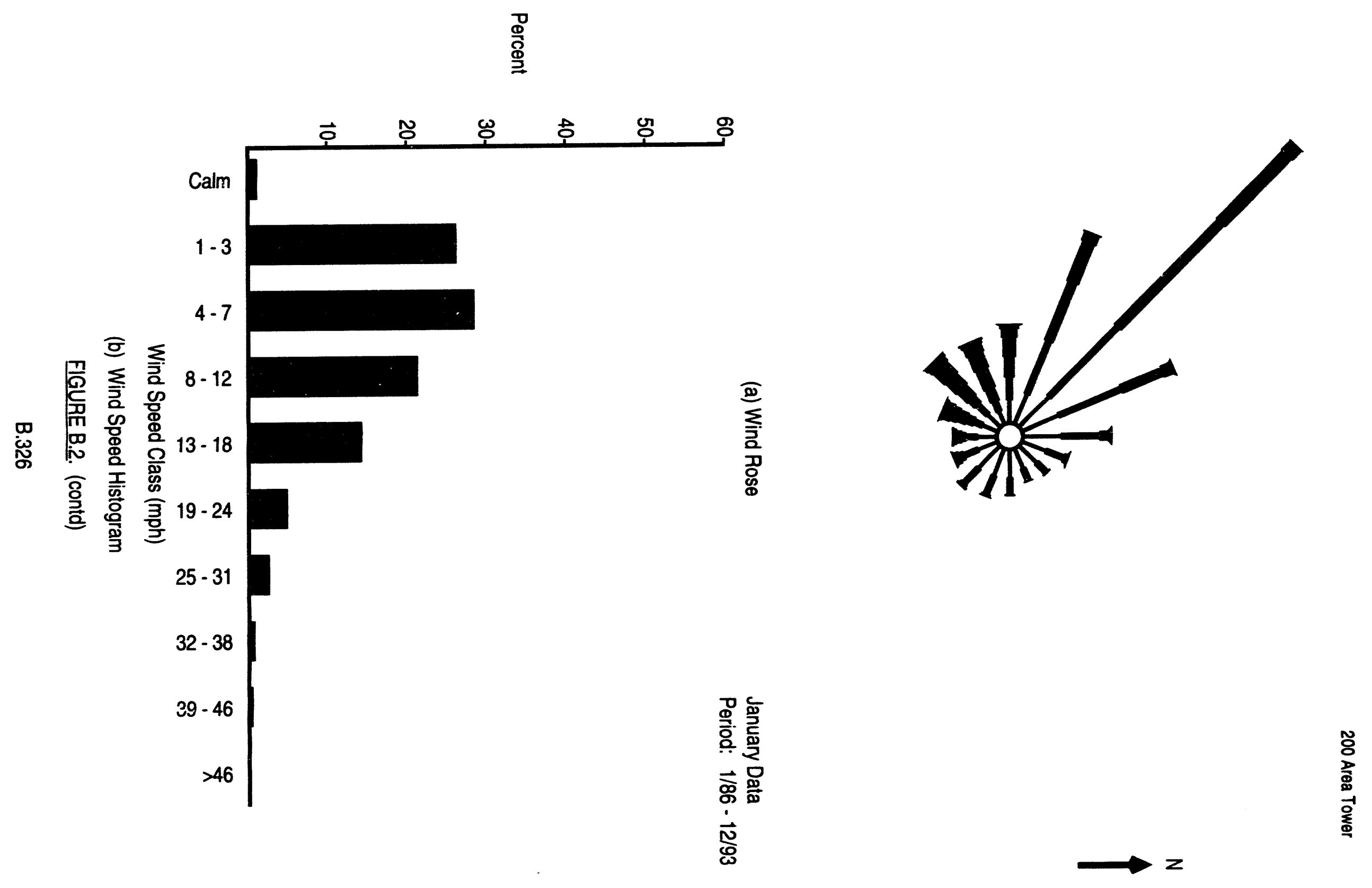




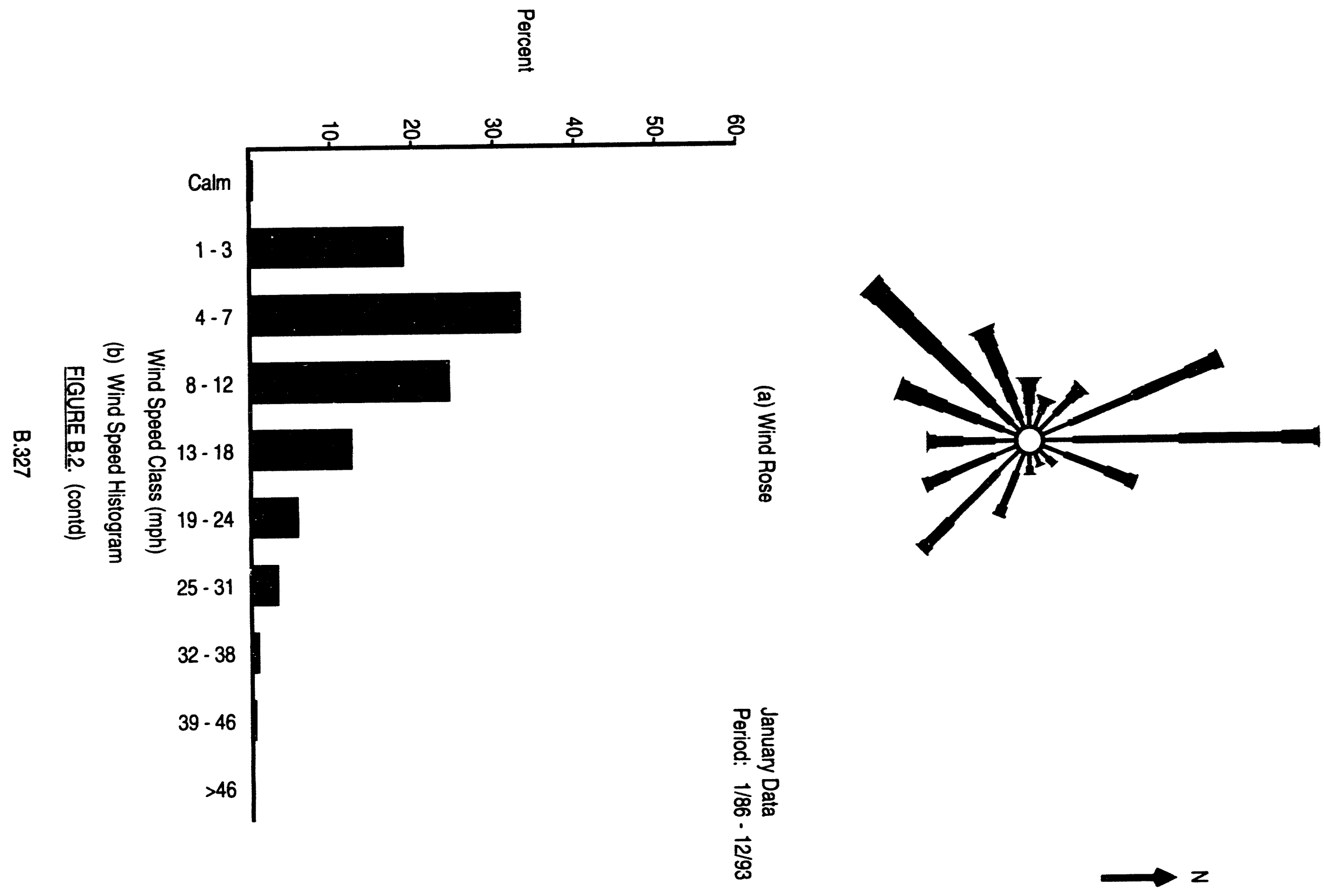




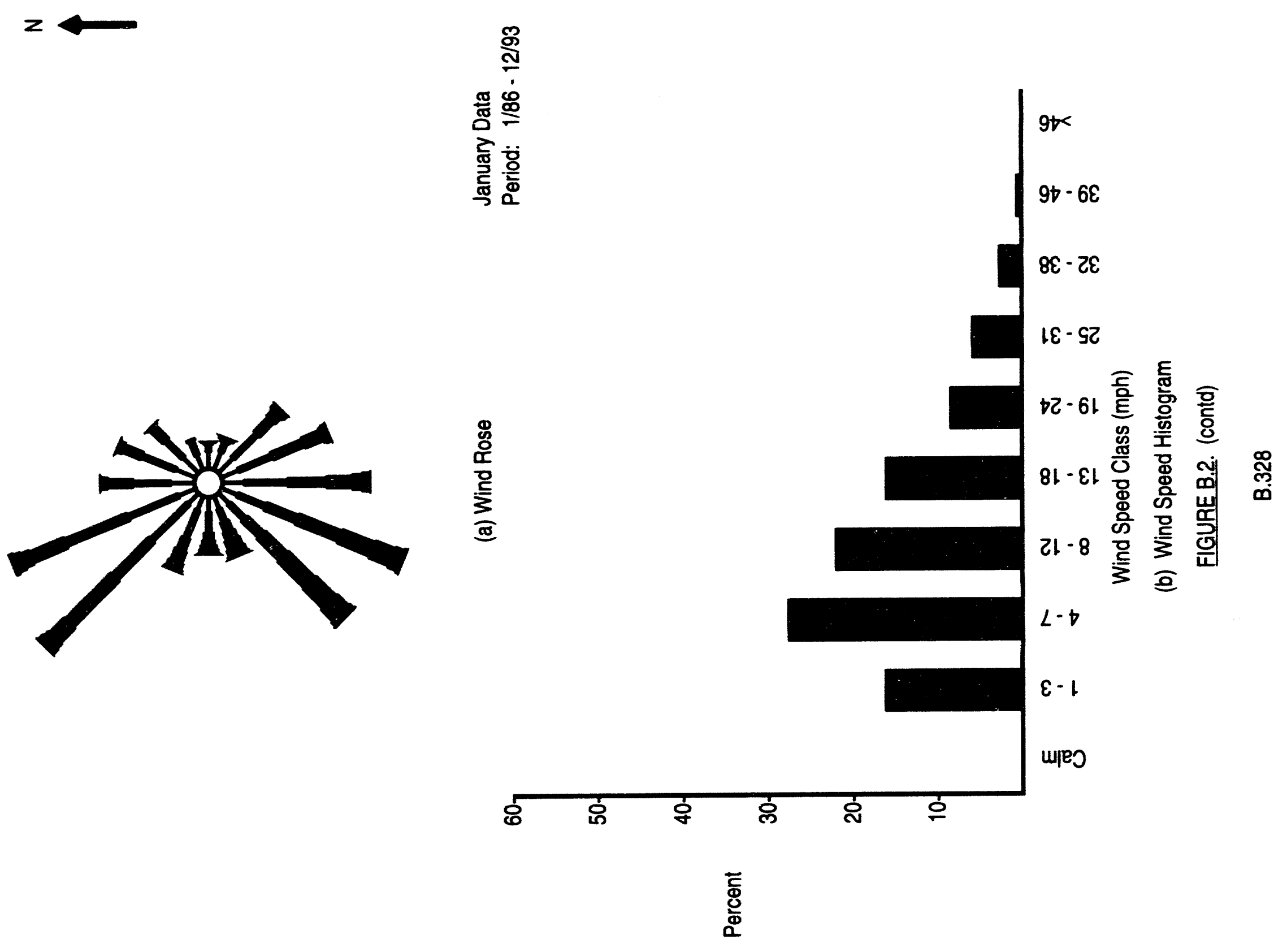




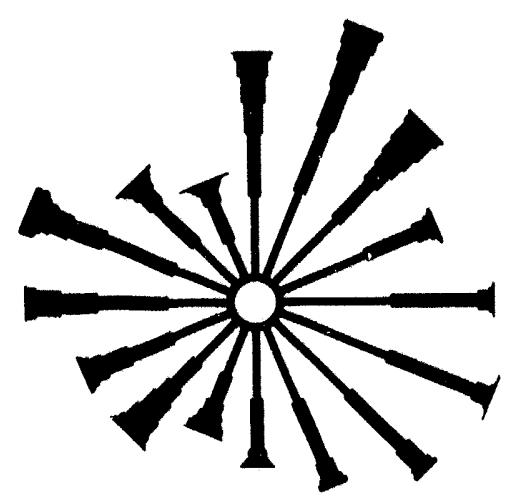

(a) Wind Rose

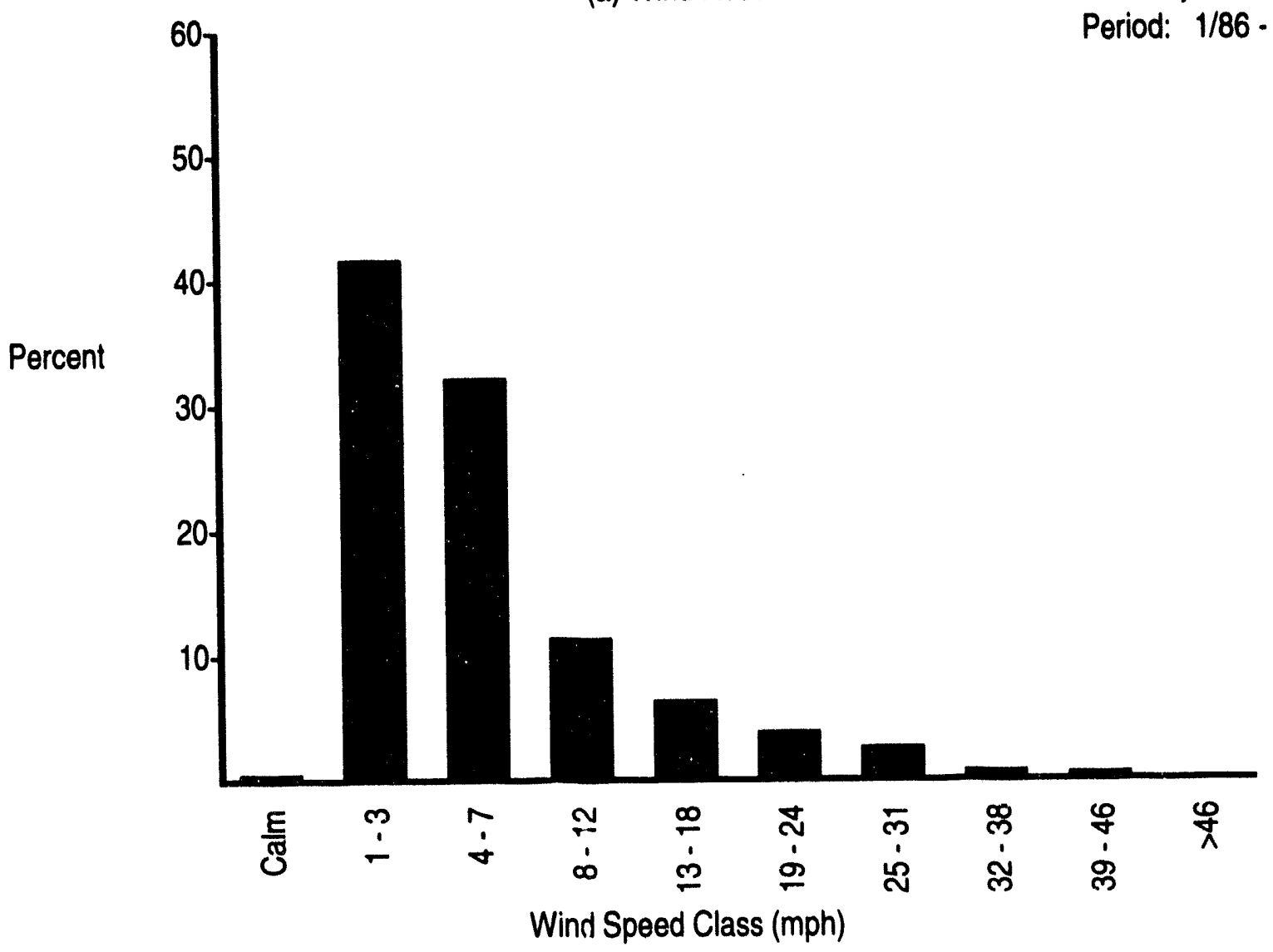

(b) Wind Speed Histogram

FIGURE B.2. (contd)
February Data

Period: 1/86 - 12/93 


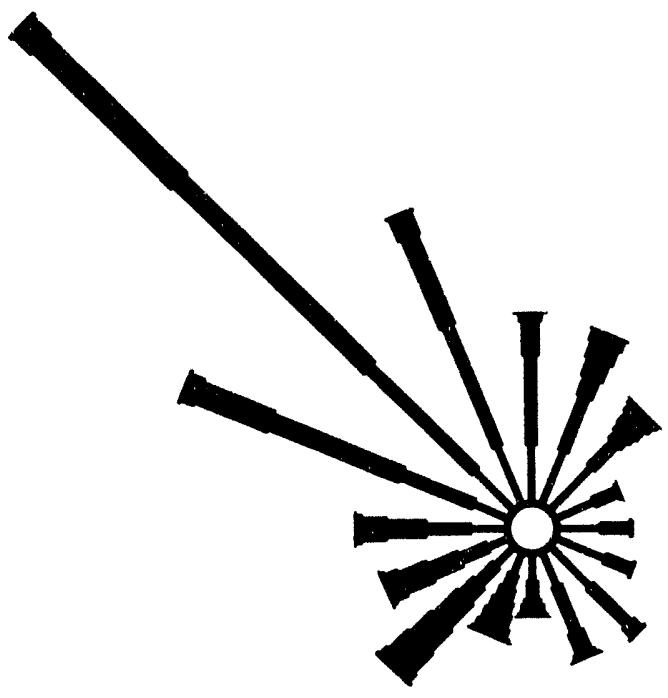

(a) Wind Rose

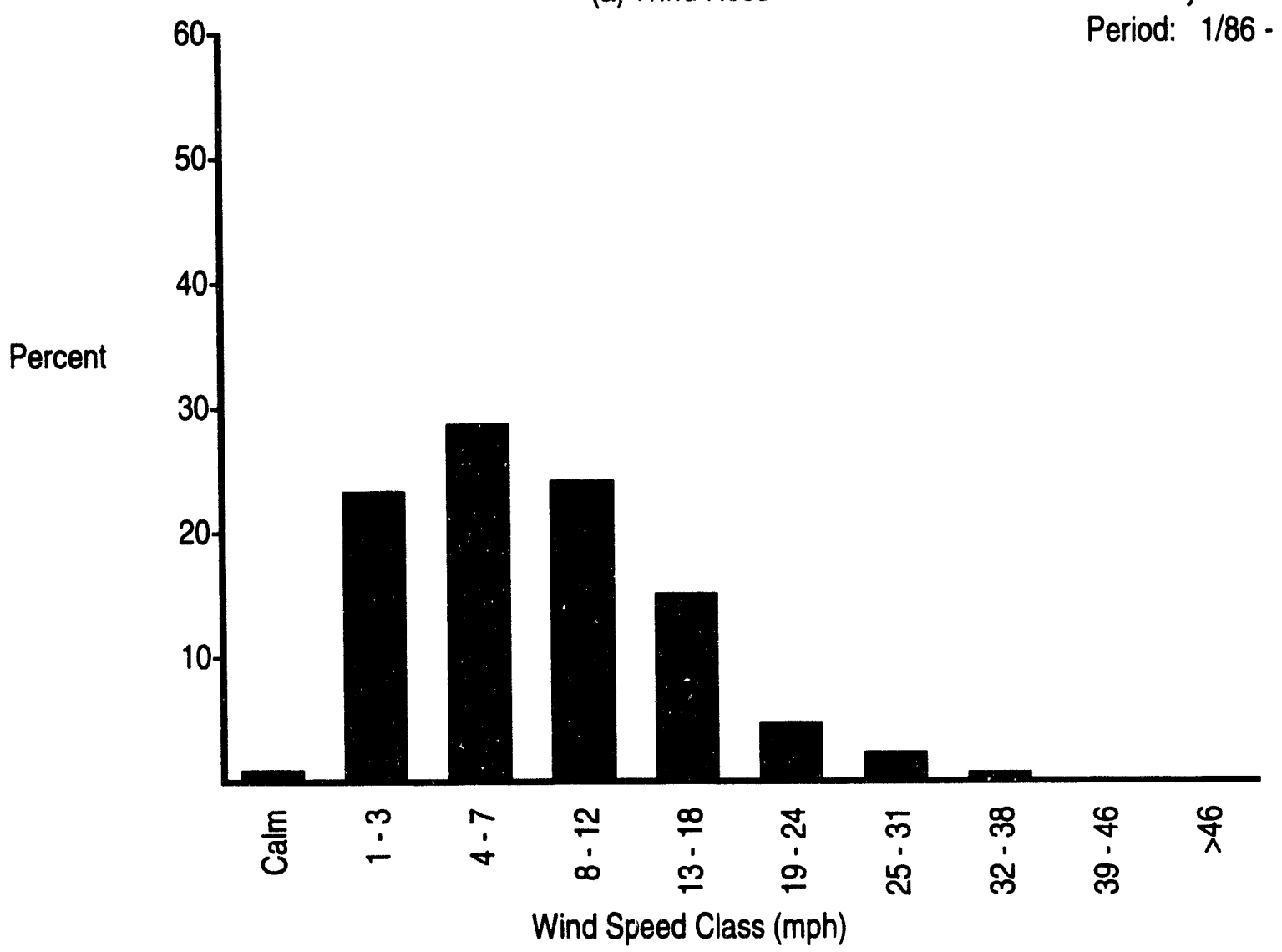

(b) Wind Speed Histogram

FIGURE B.2. (contd) 


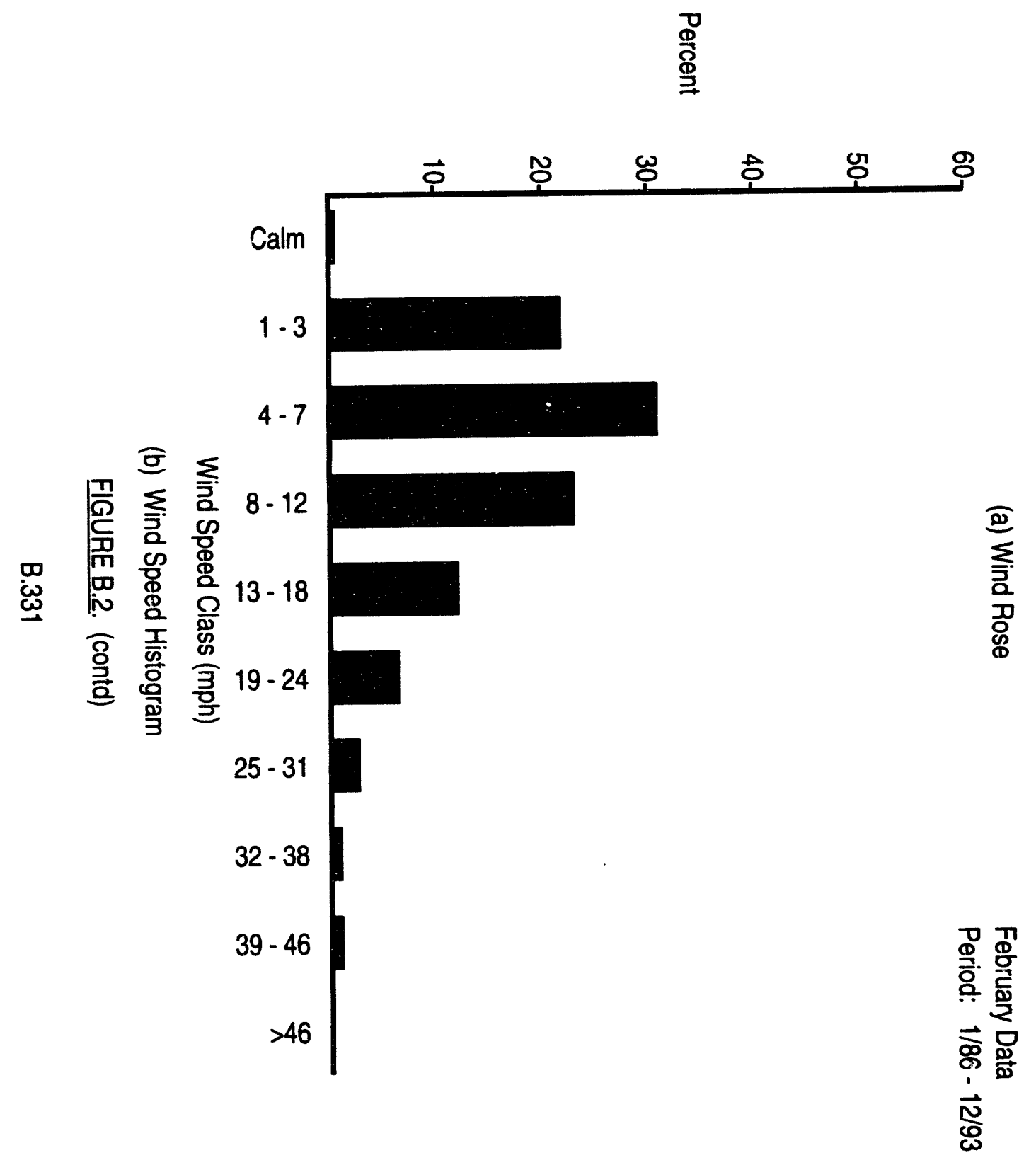




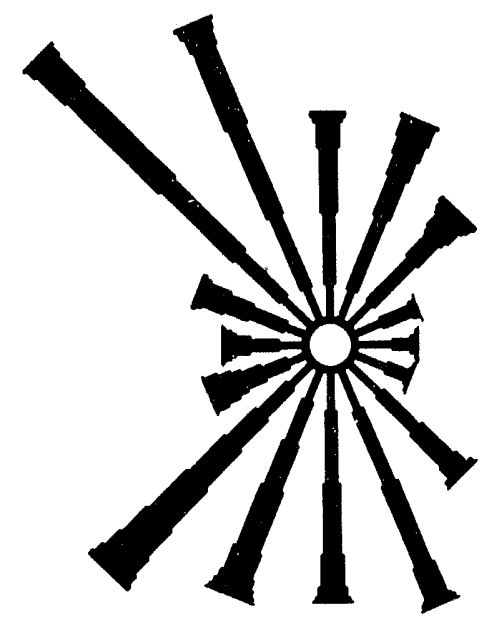

N

(a) Wind Rose

February Data

Period: $1 / 86 \cdot 12 / 93$

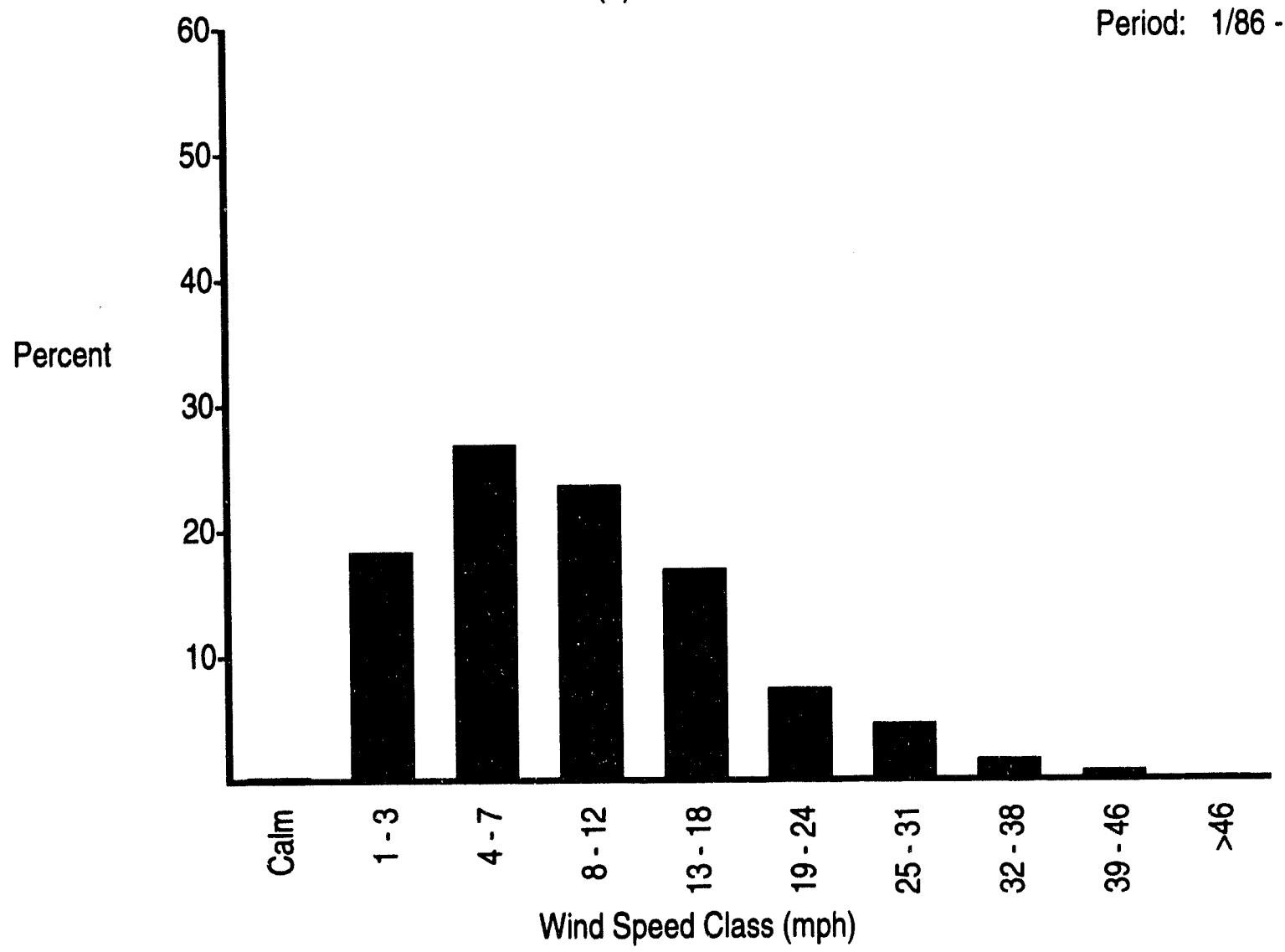

(b) Wind Speed Histogram

FIGURE B.2. (contd) 


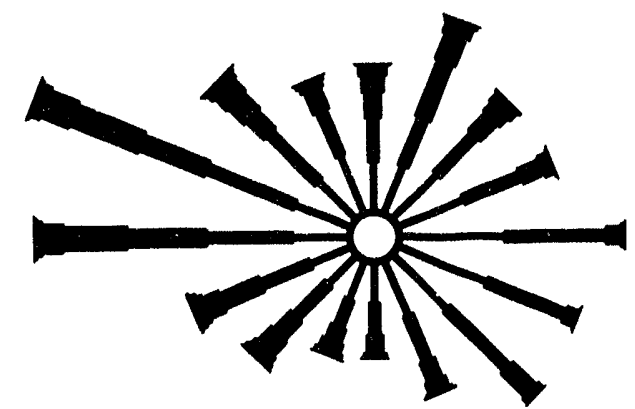

(a) Wind Rose

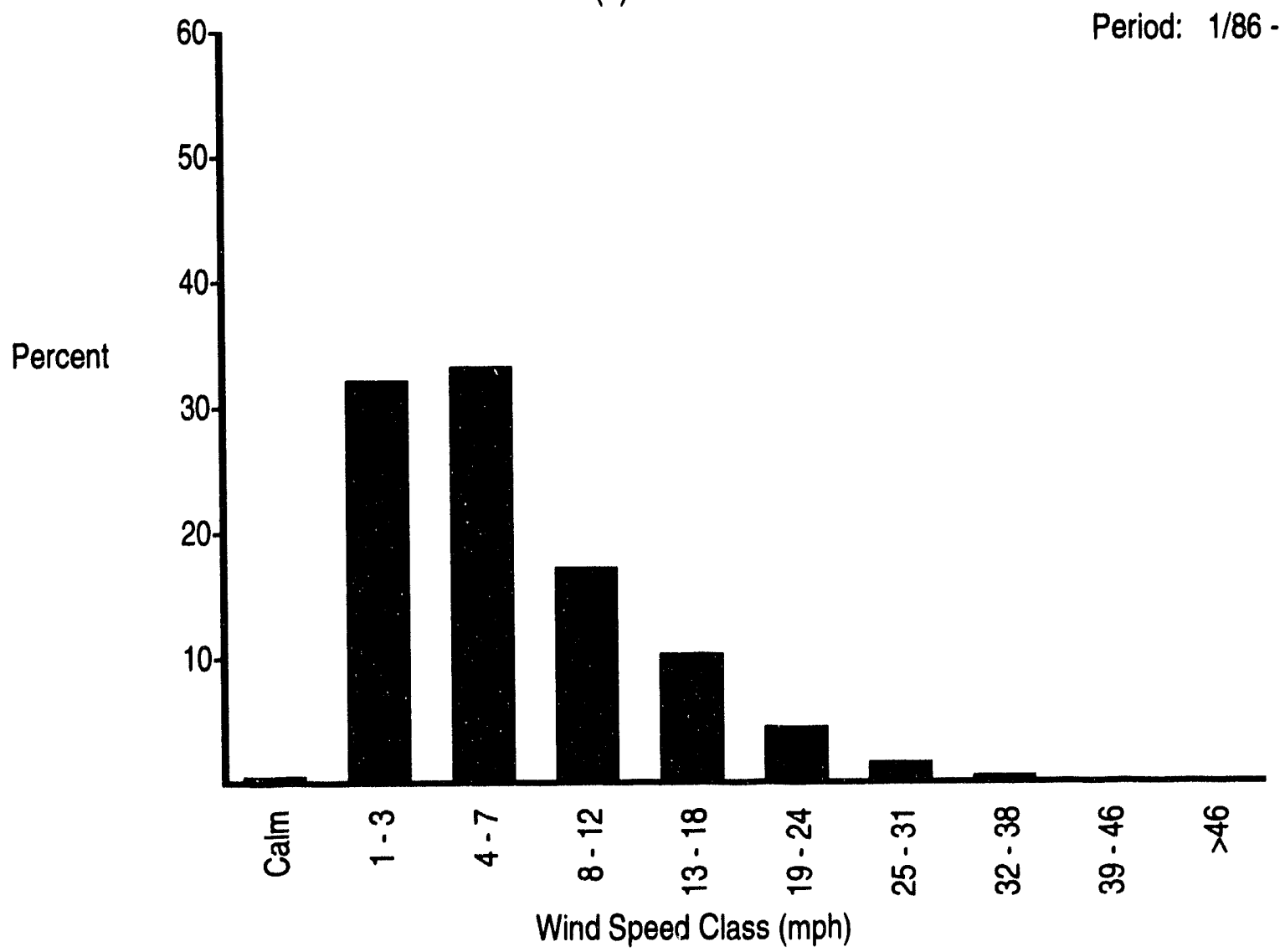

(b) Wind Speed Histogram

FIGURE B.2. (contd) 

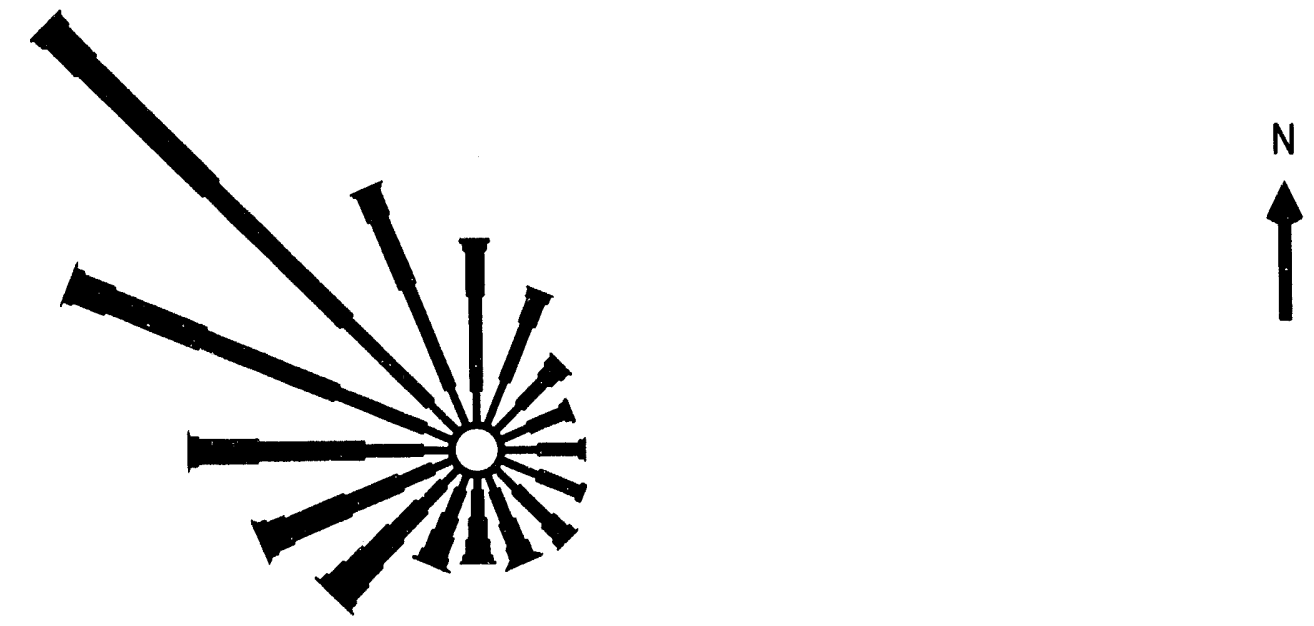

(a) Wind Rose

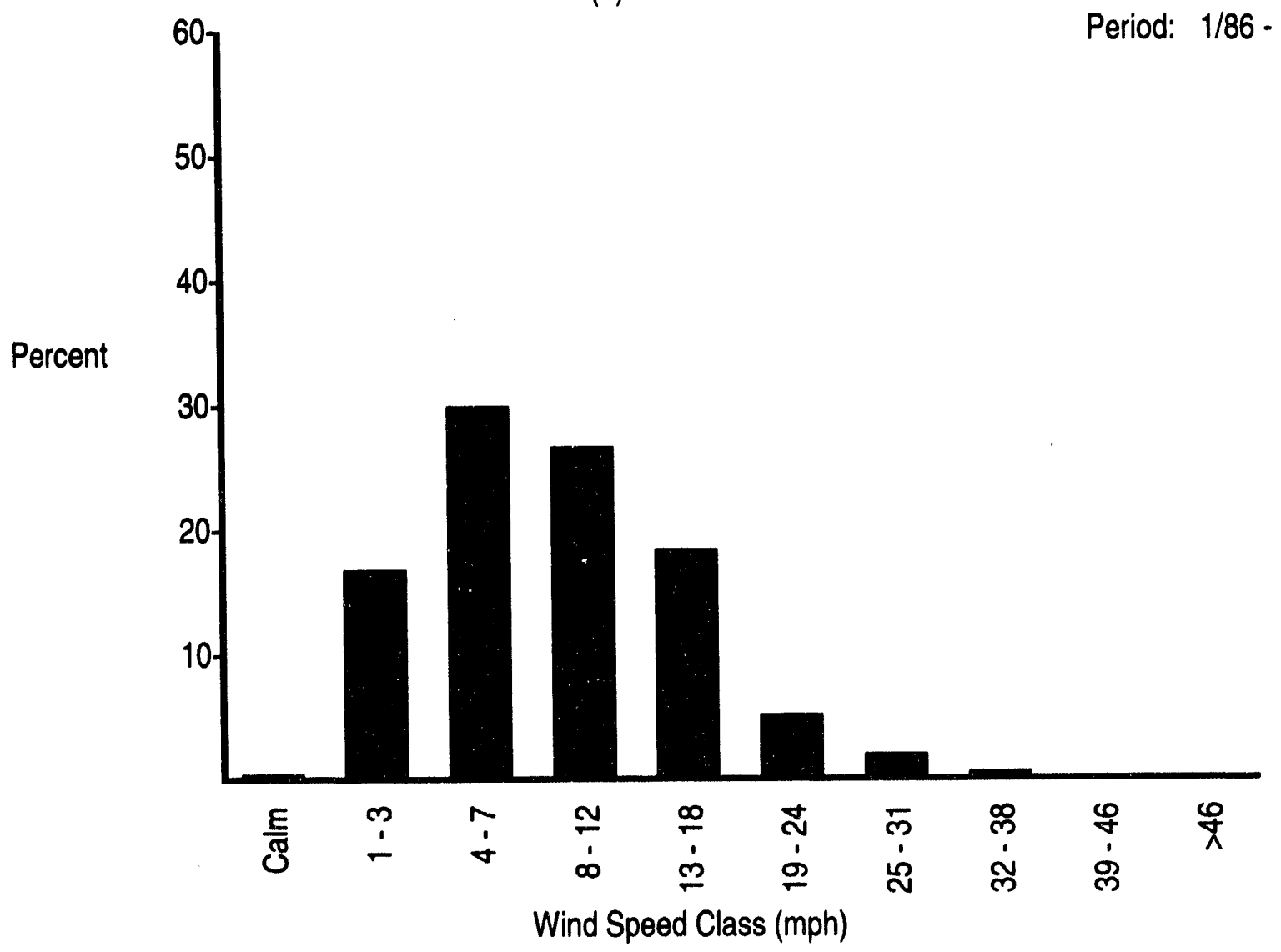

(b) Wind Speed Histogram

FIGURE B.2. (contd) 


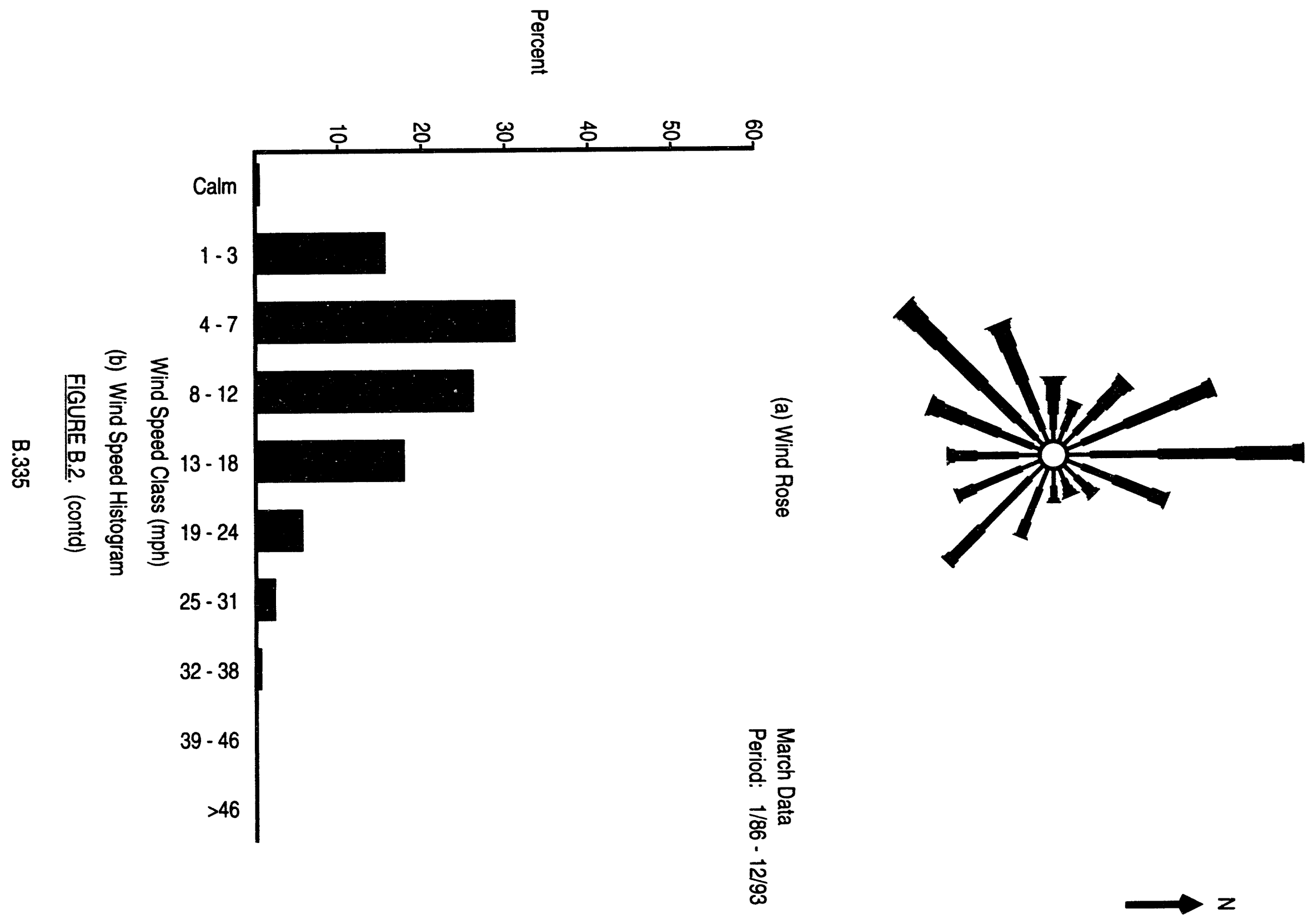




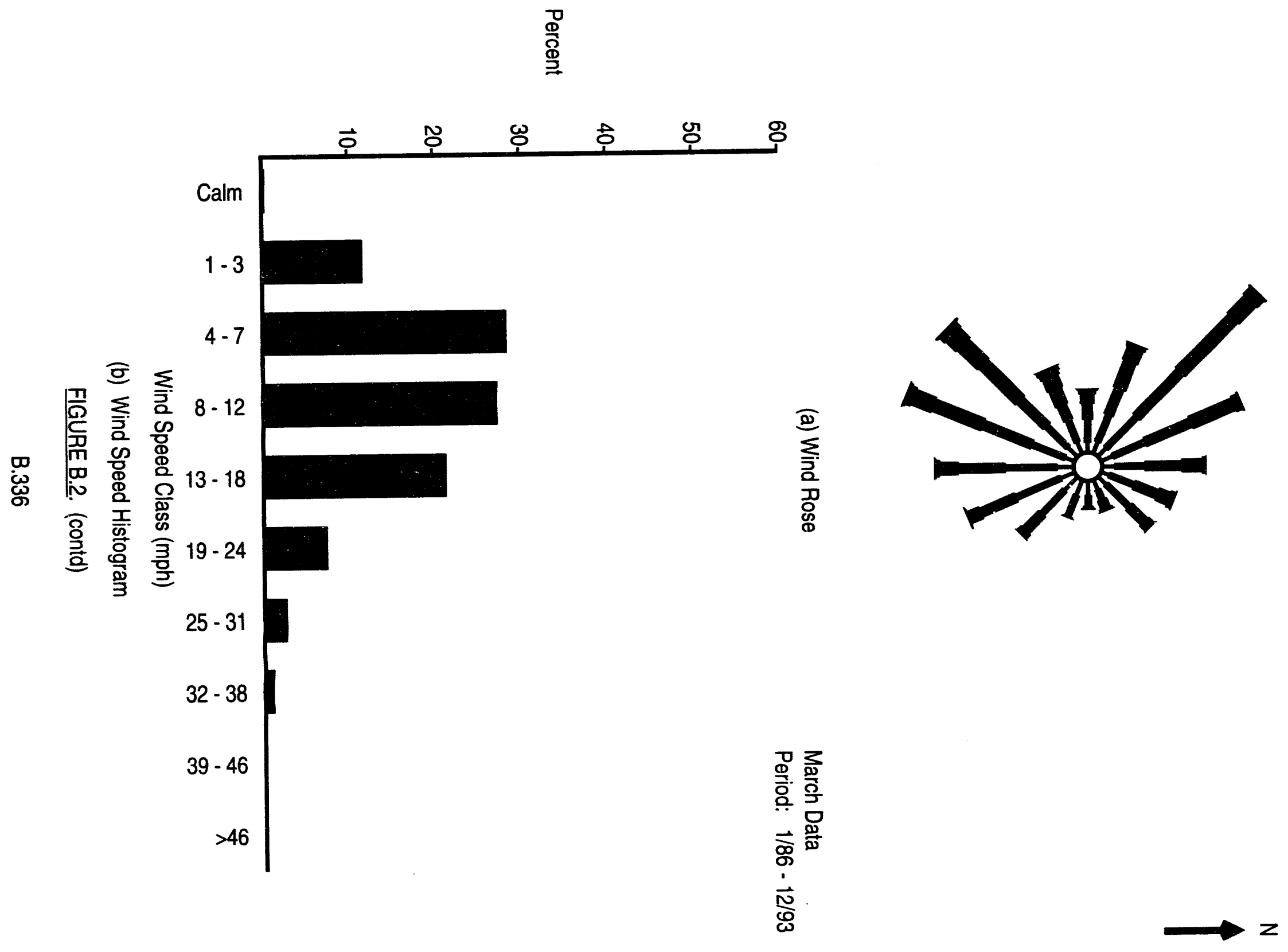




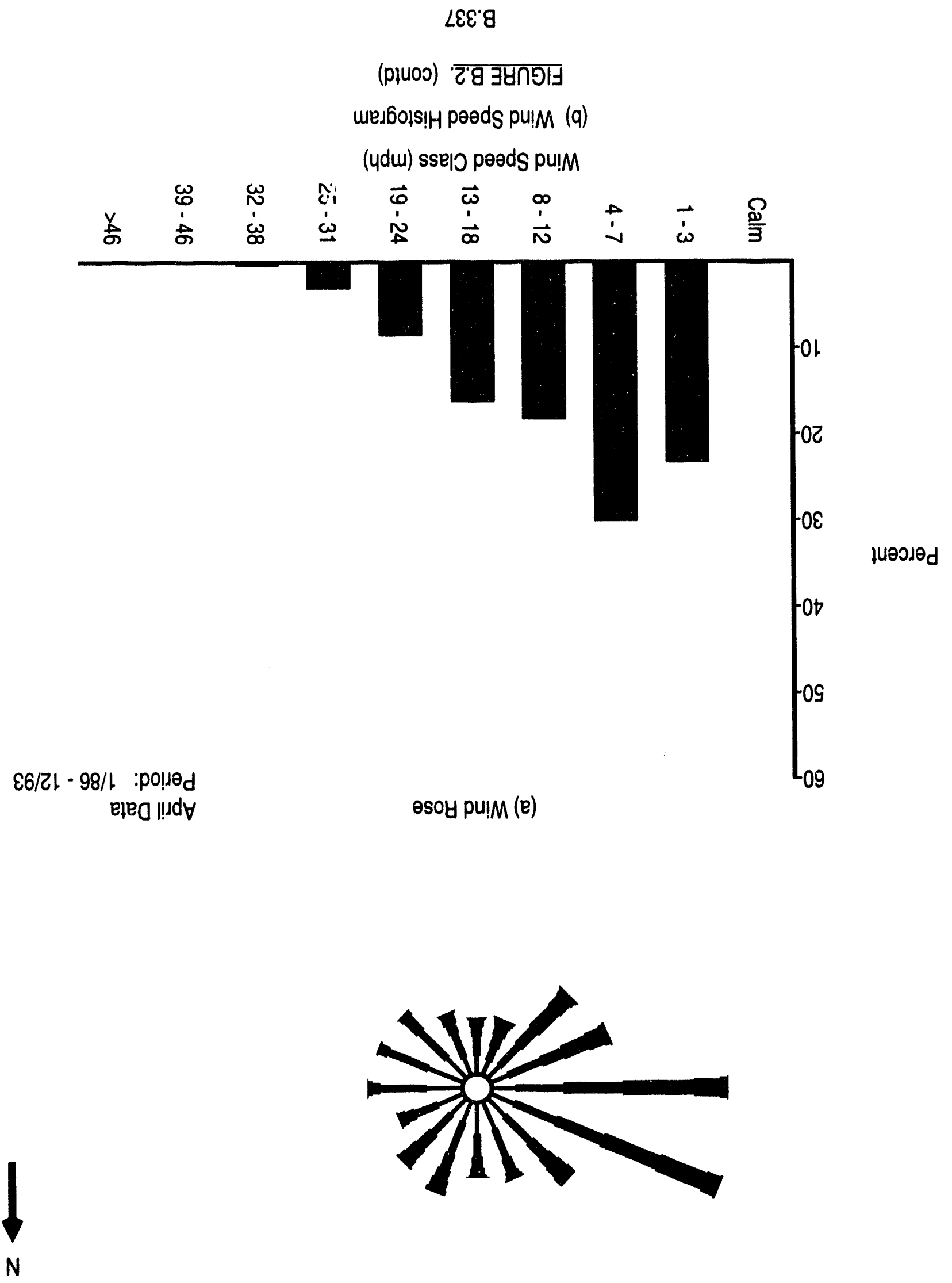




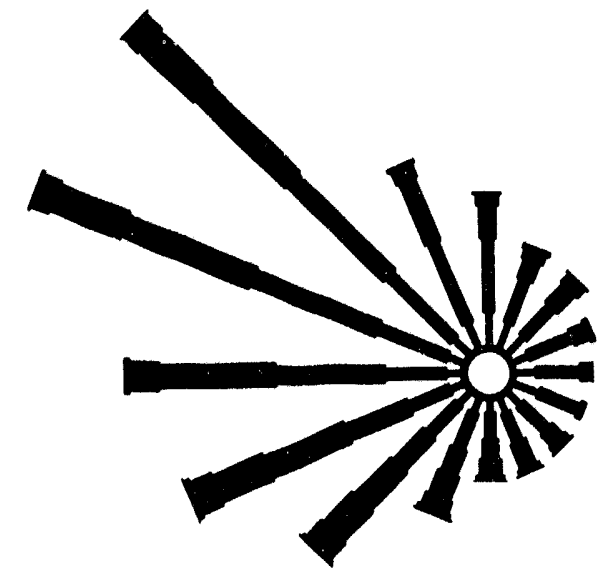

N

(a) Wind Rose

April Data

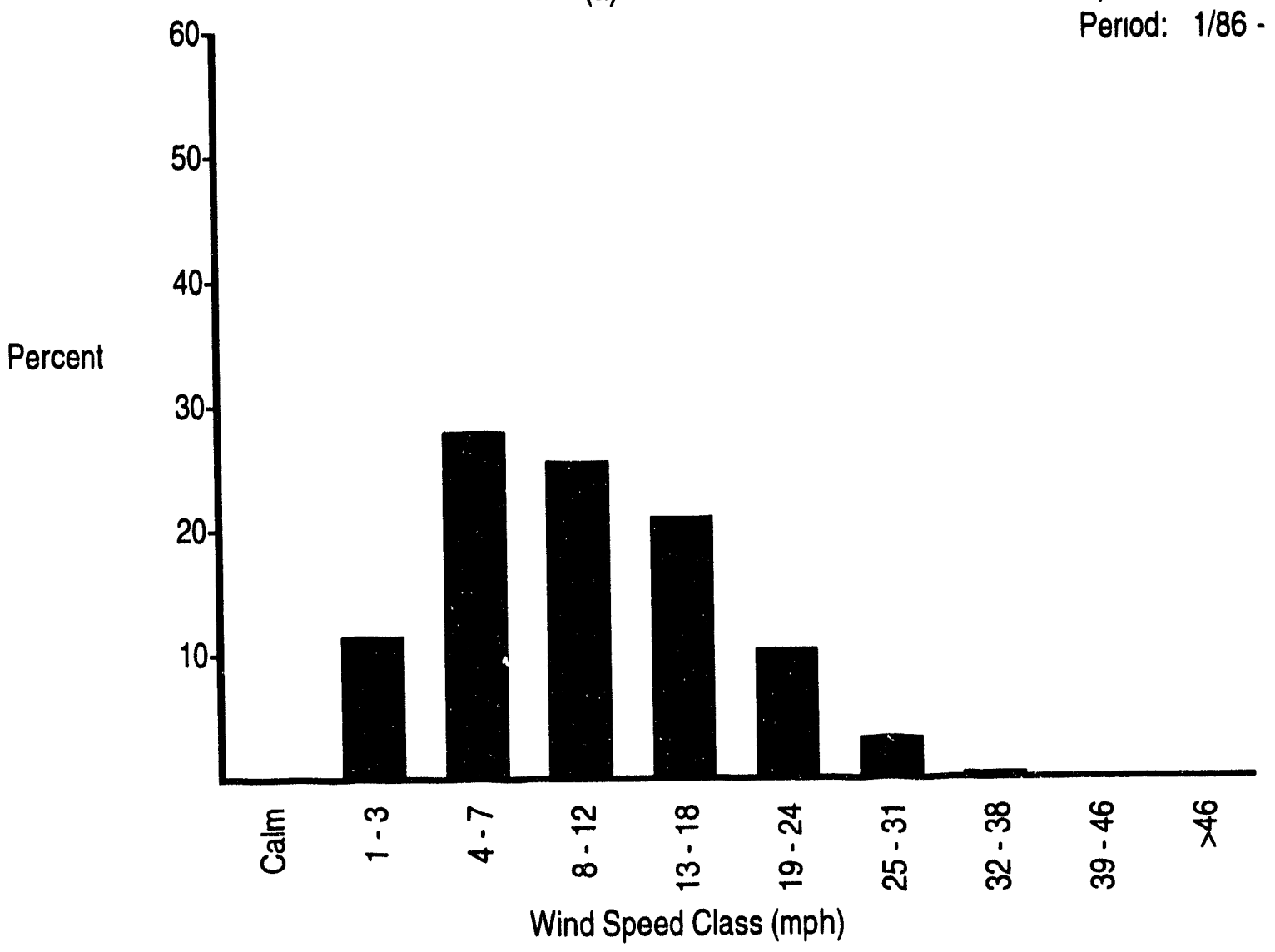

(b) Wind Speed Histogram

FIGURE B.2. (contd) 


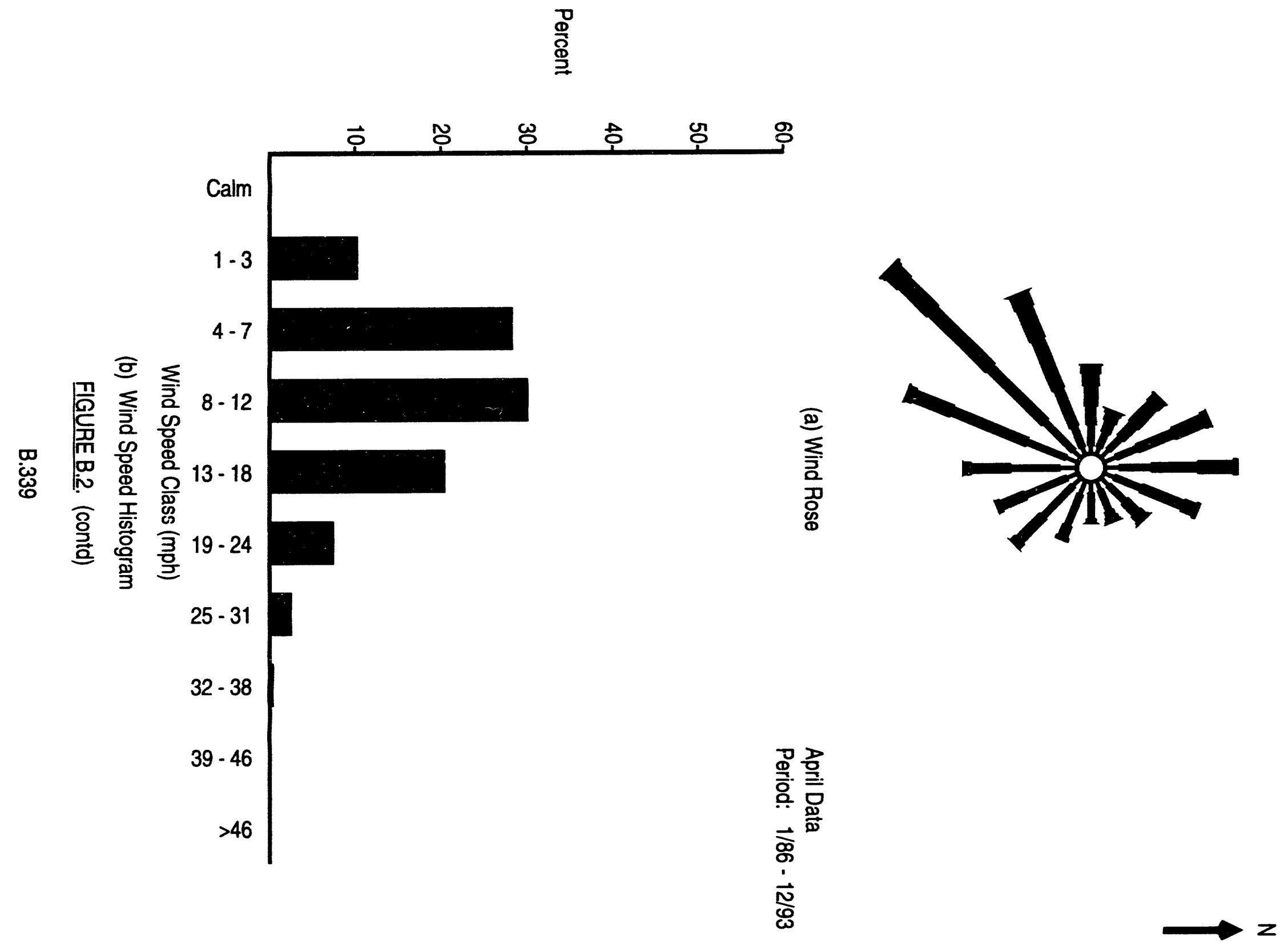




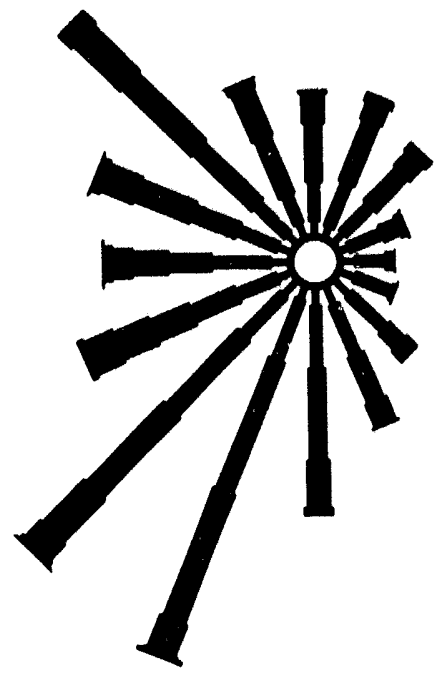

(a) Wind Rose

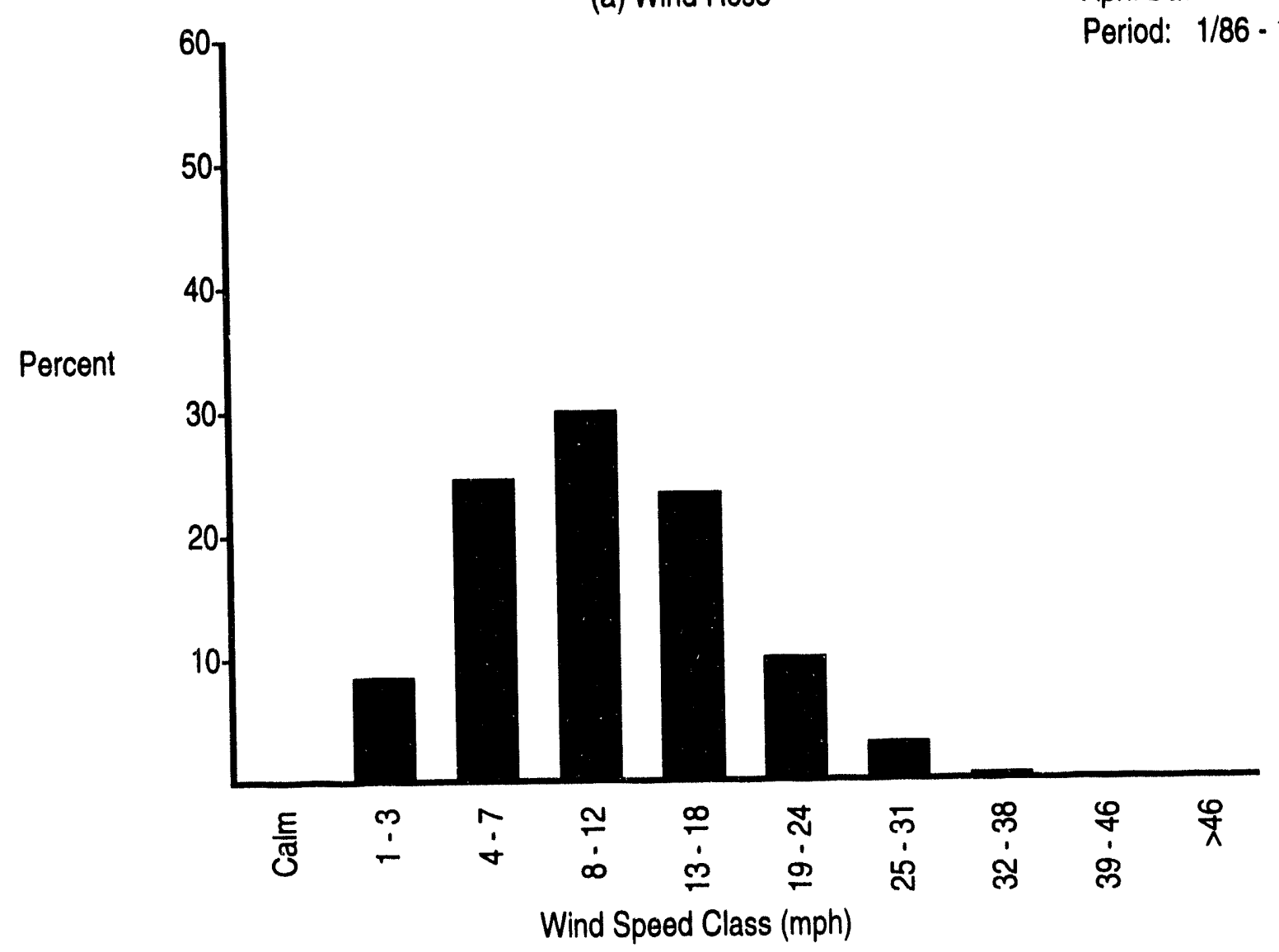

(b) Wind Speed Histogram

FIGURE B.2. (contd)
April Data

Period: $1 / 86 \cdot 12 / 93$ 


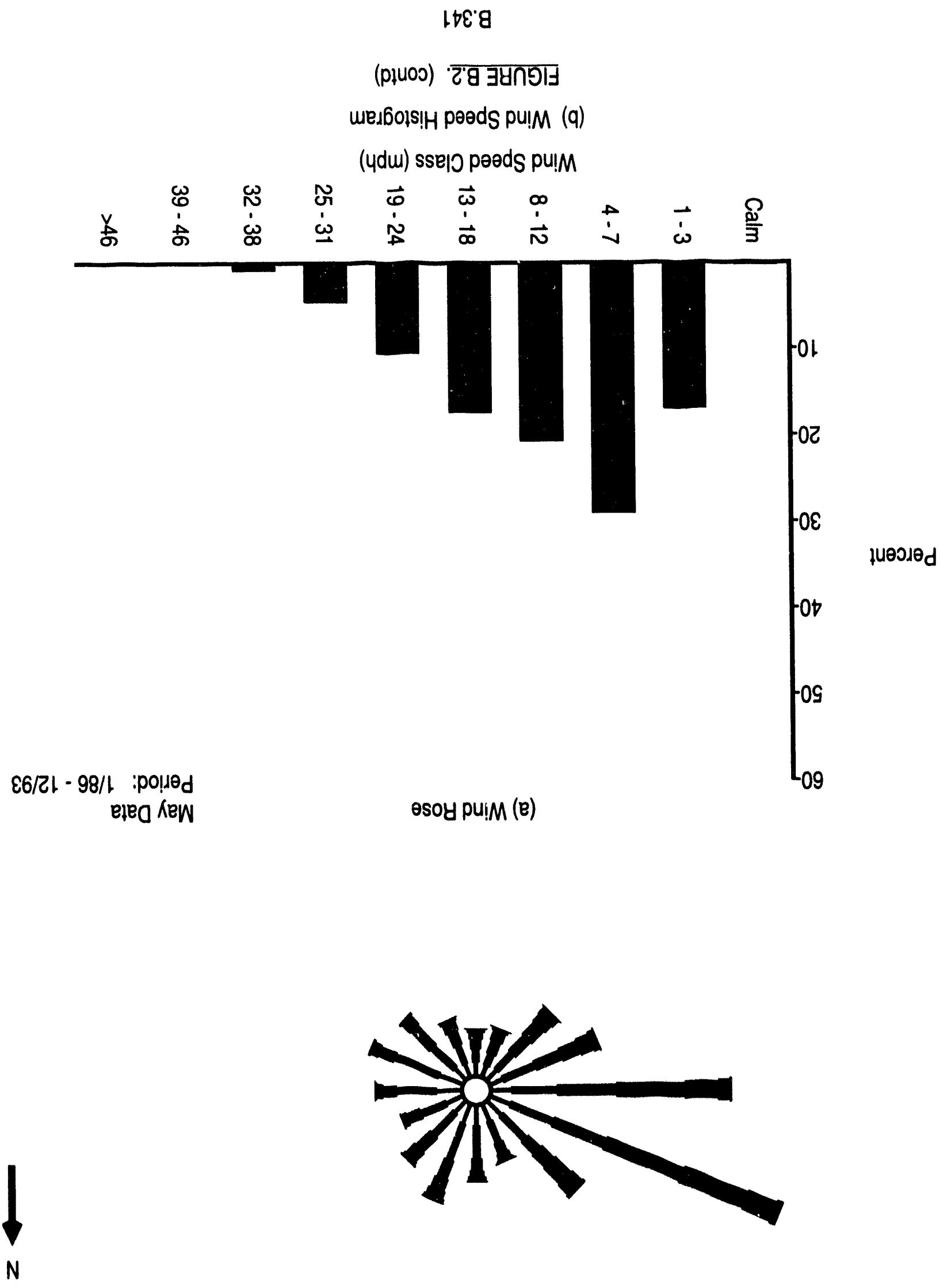



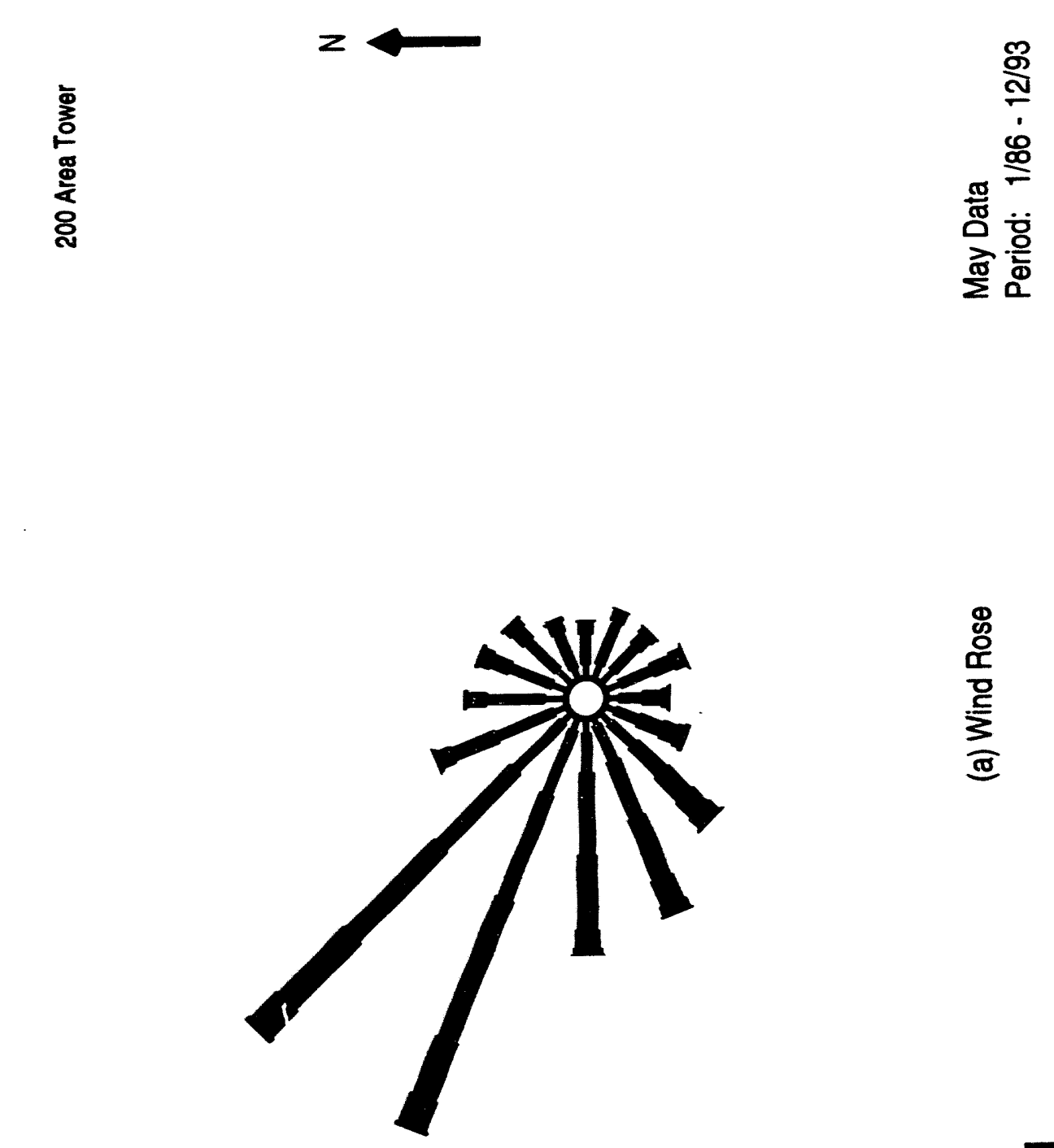

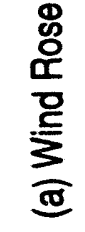

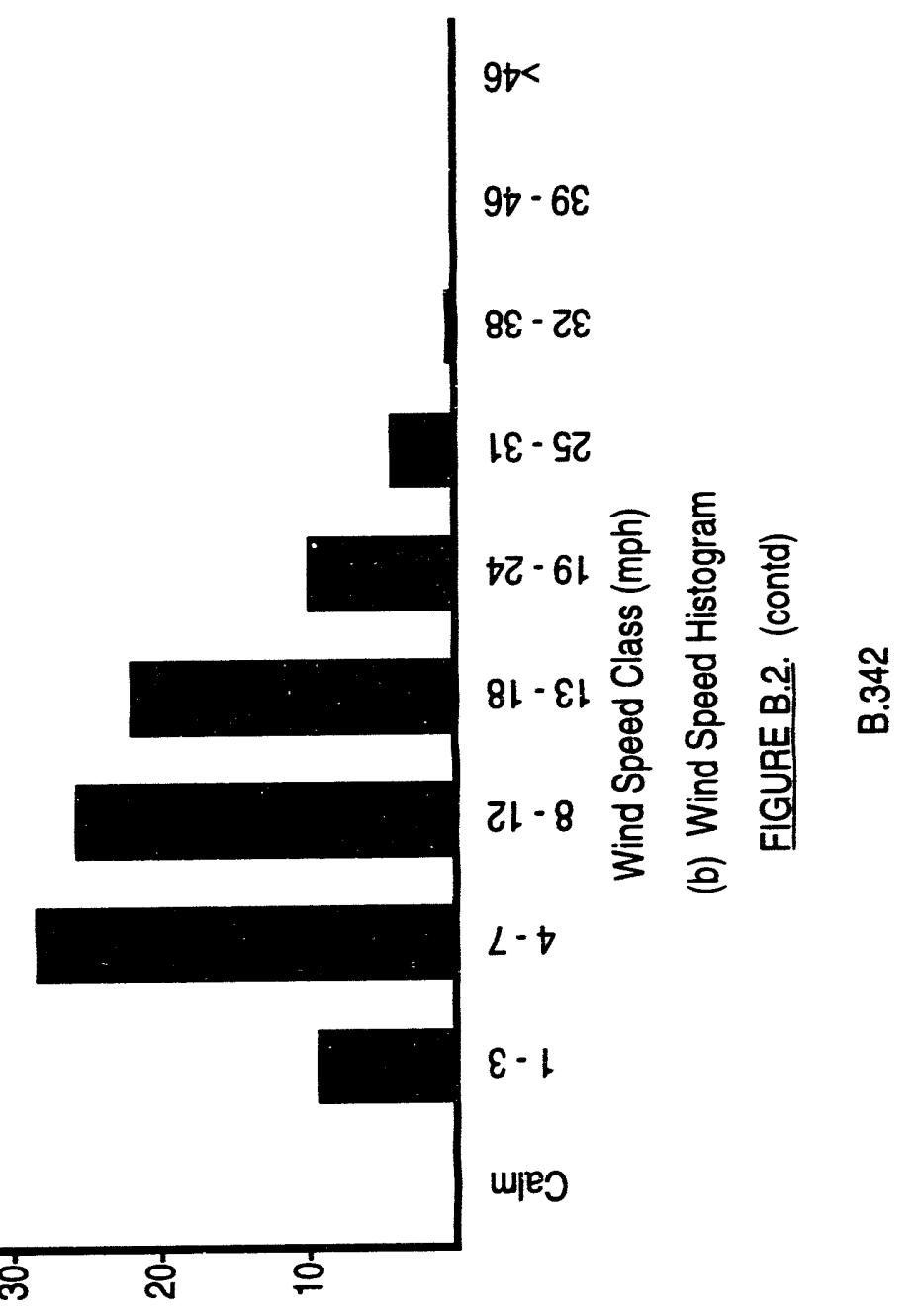

్ㅗㅇ
잉 


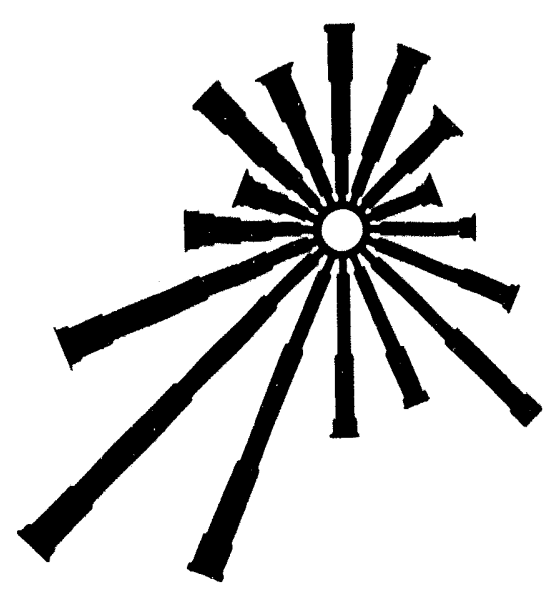

(a) Wind Rose

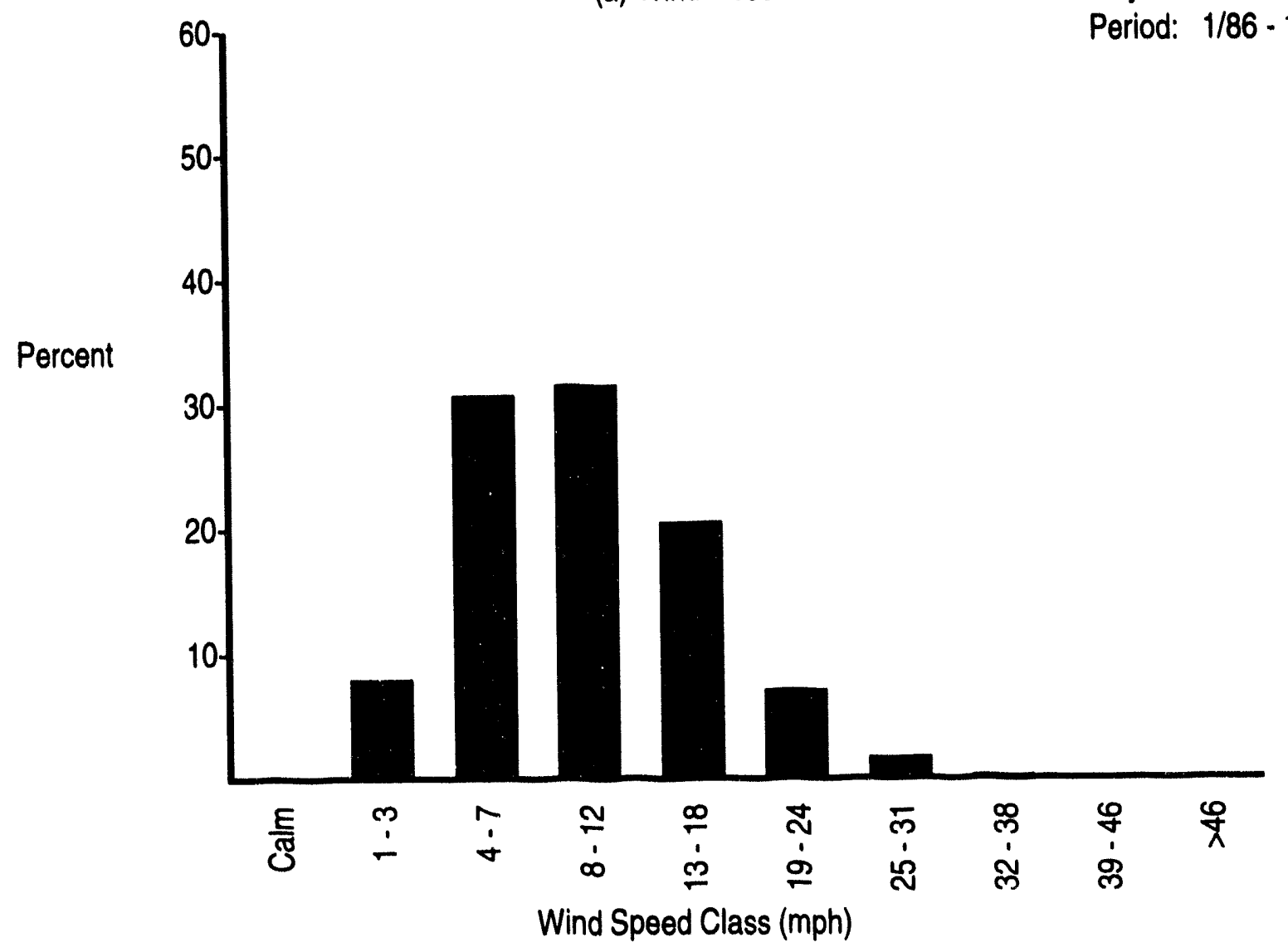

(b) Wind Spegd Histogram

FIGURE B.2. (contd)
May Data

Period: 1/86 - 12/93 


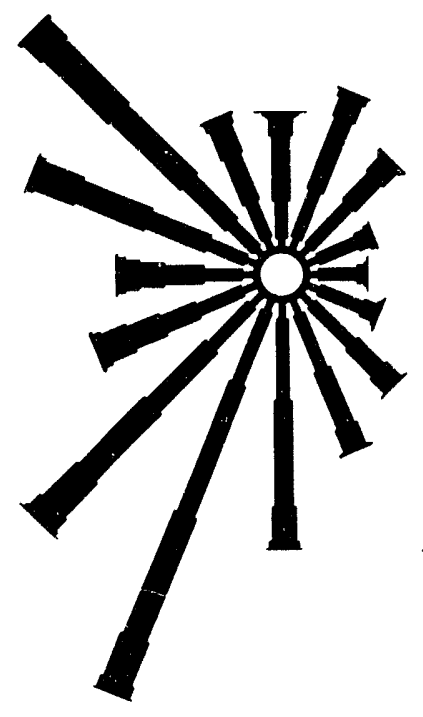

(a) Wind Rose

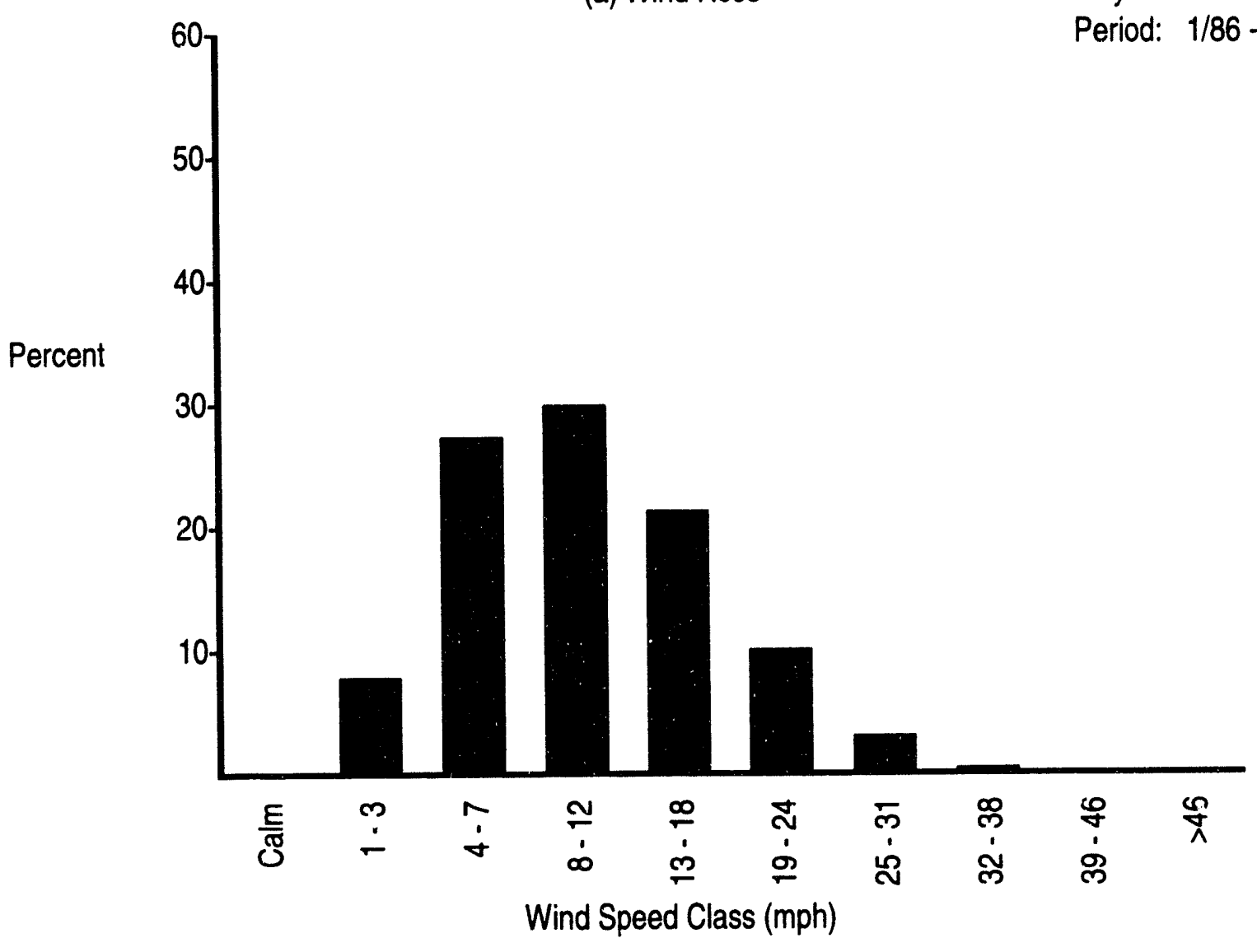

(b) Wind Speed Histogram

FIGURE B.2. (contd)
May Data

Period: $1 / 86-12 / 93$ 

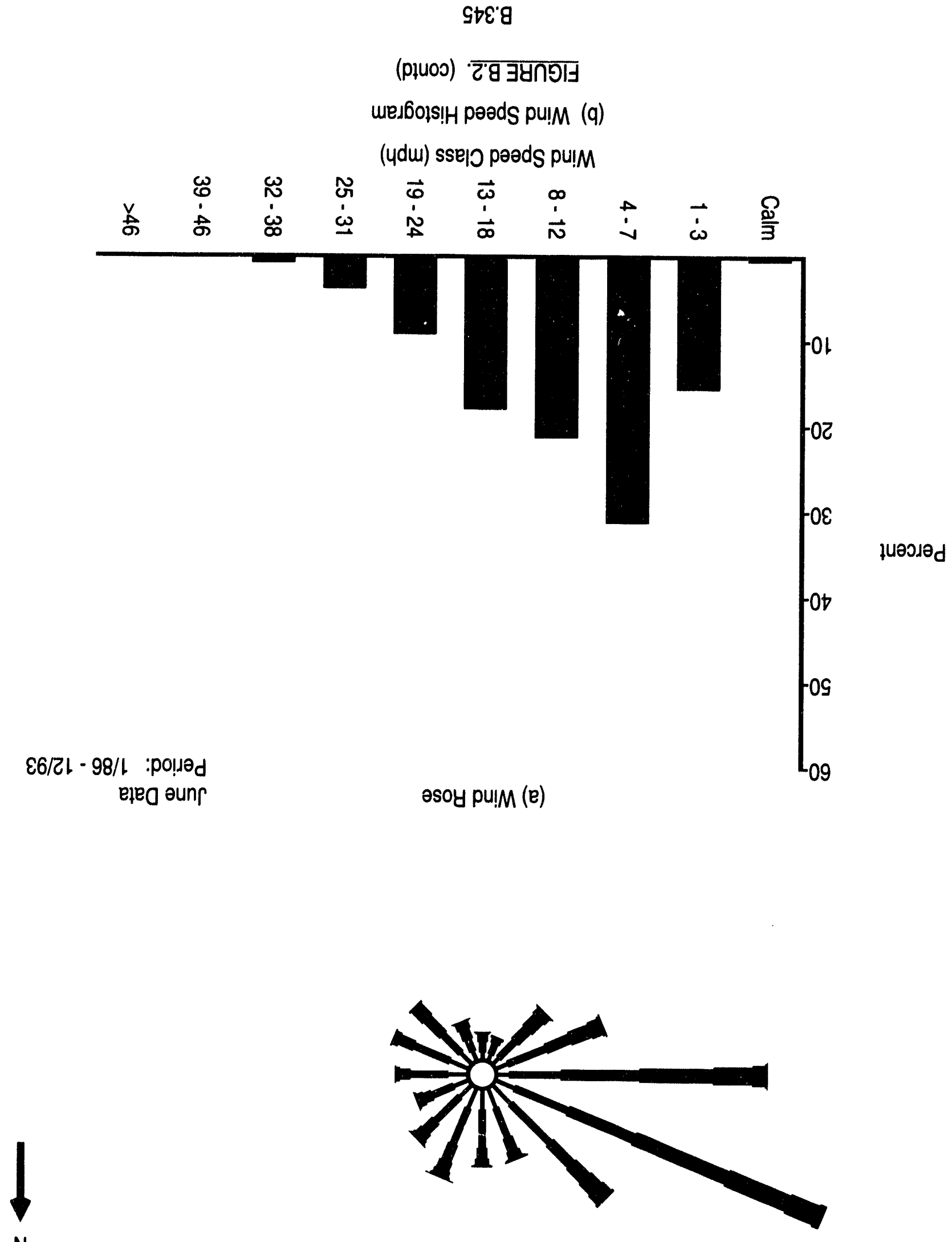

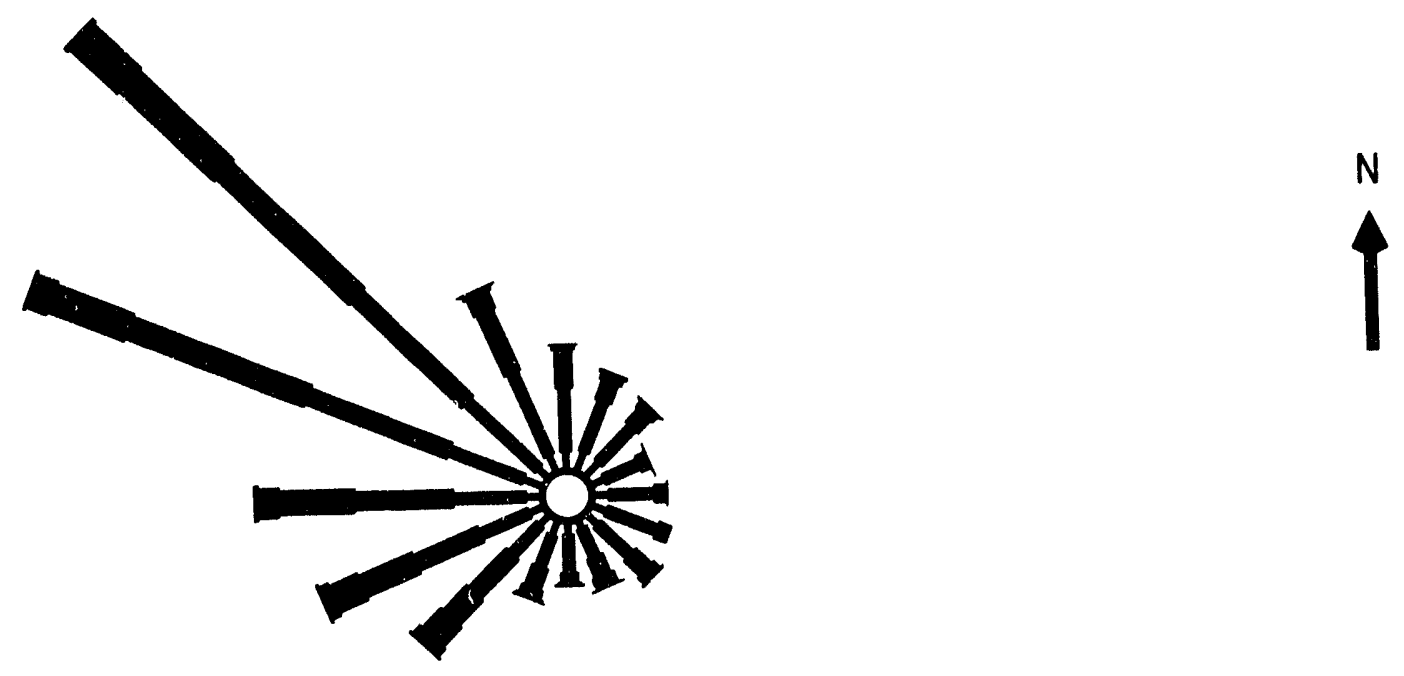

(a) Wind Rose

June Data

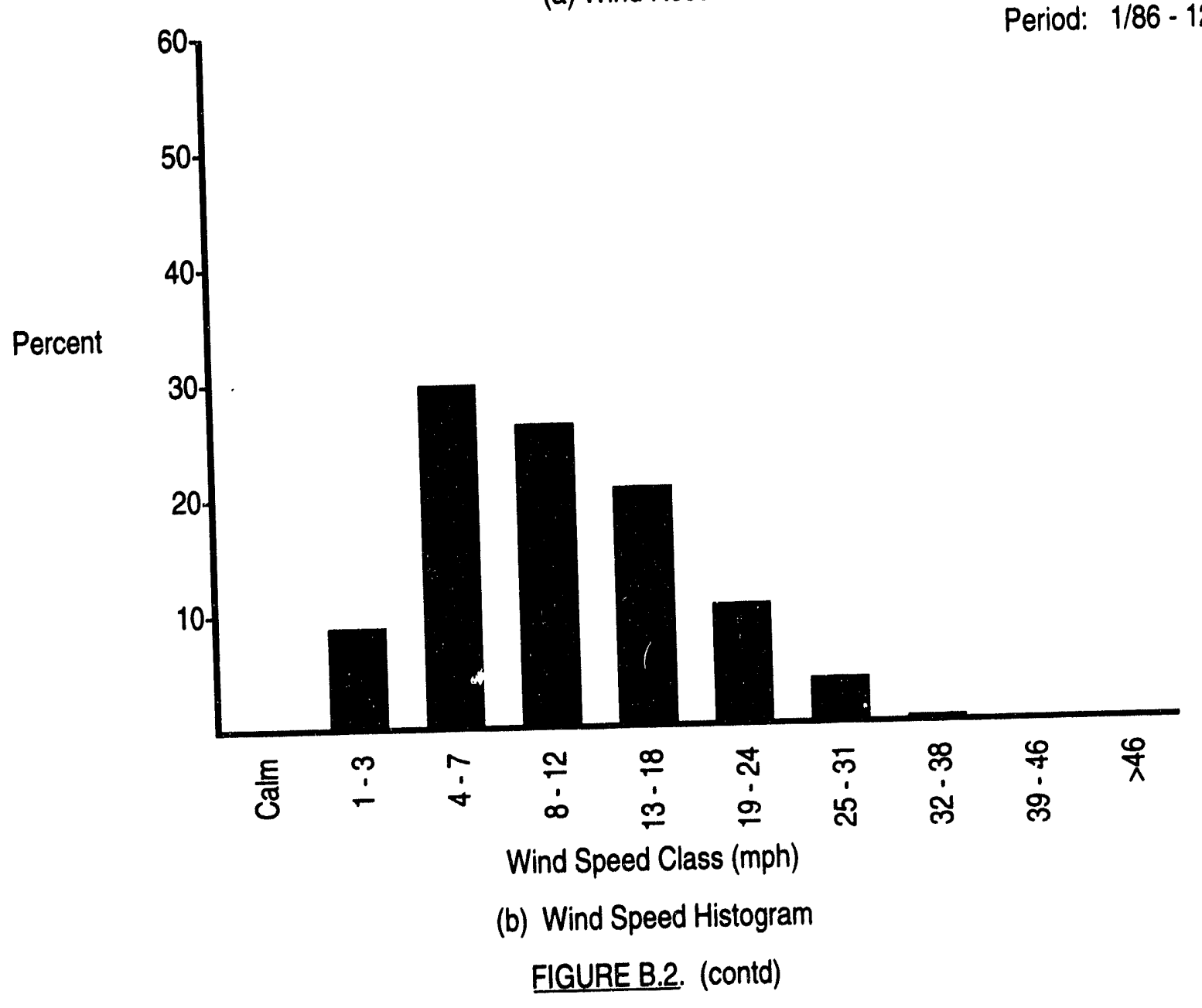

B.346 


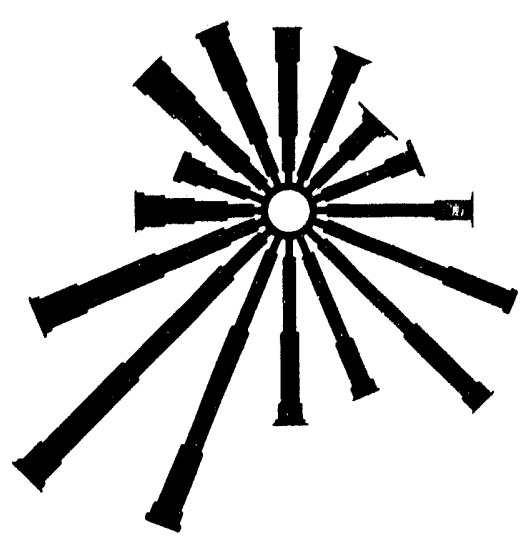

(a) Wind Rose

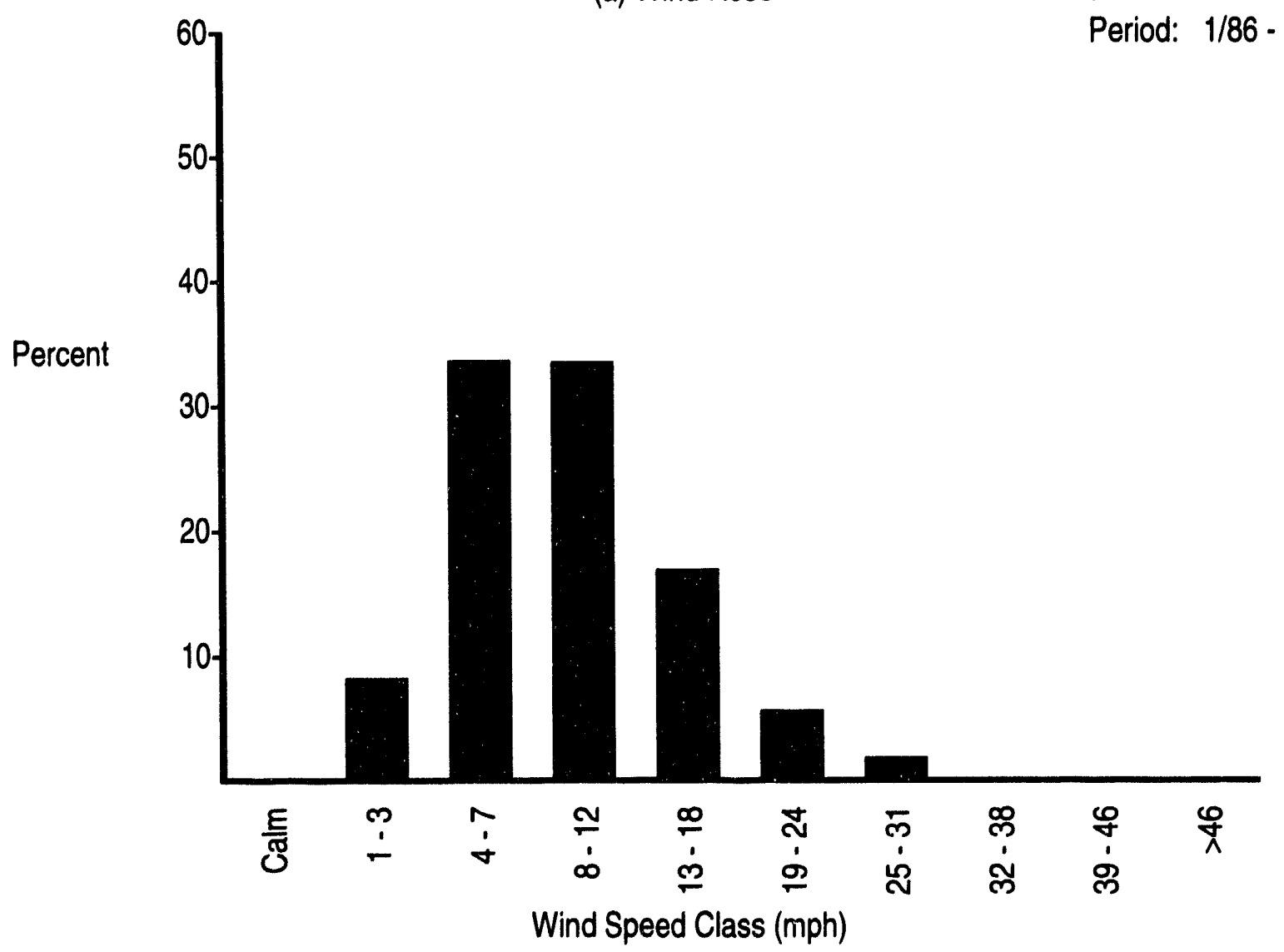

(b) Wind Speed Histogram

FIGURE B.2. (contd)
June Data

Period: $1 / 86 \cdot 12 / 93$ 


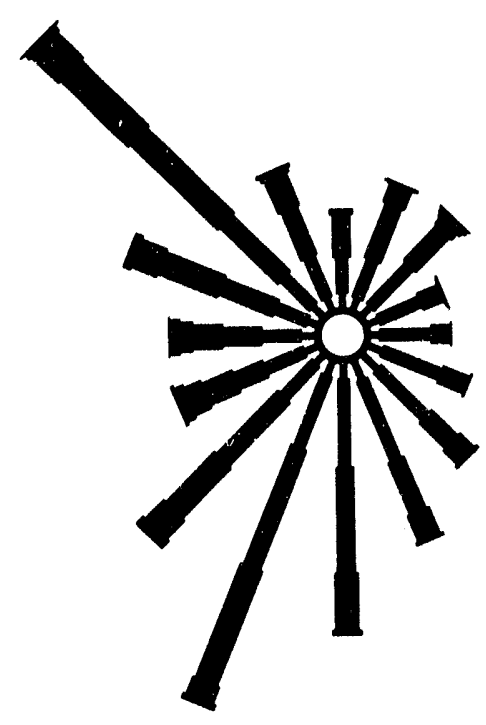

N

(a) Wind Rose

June Data

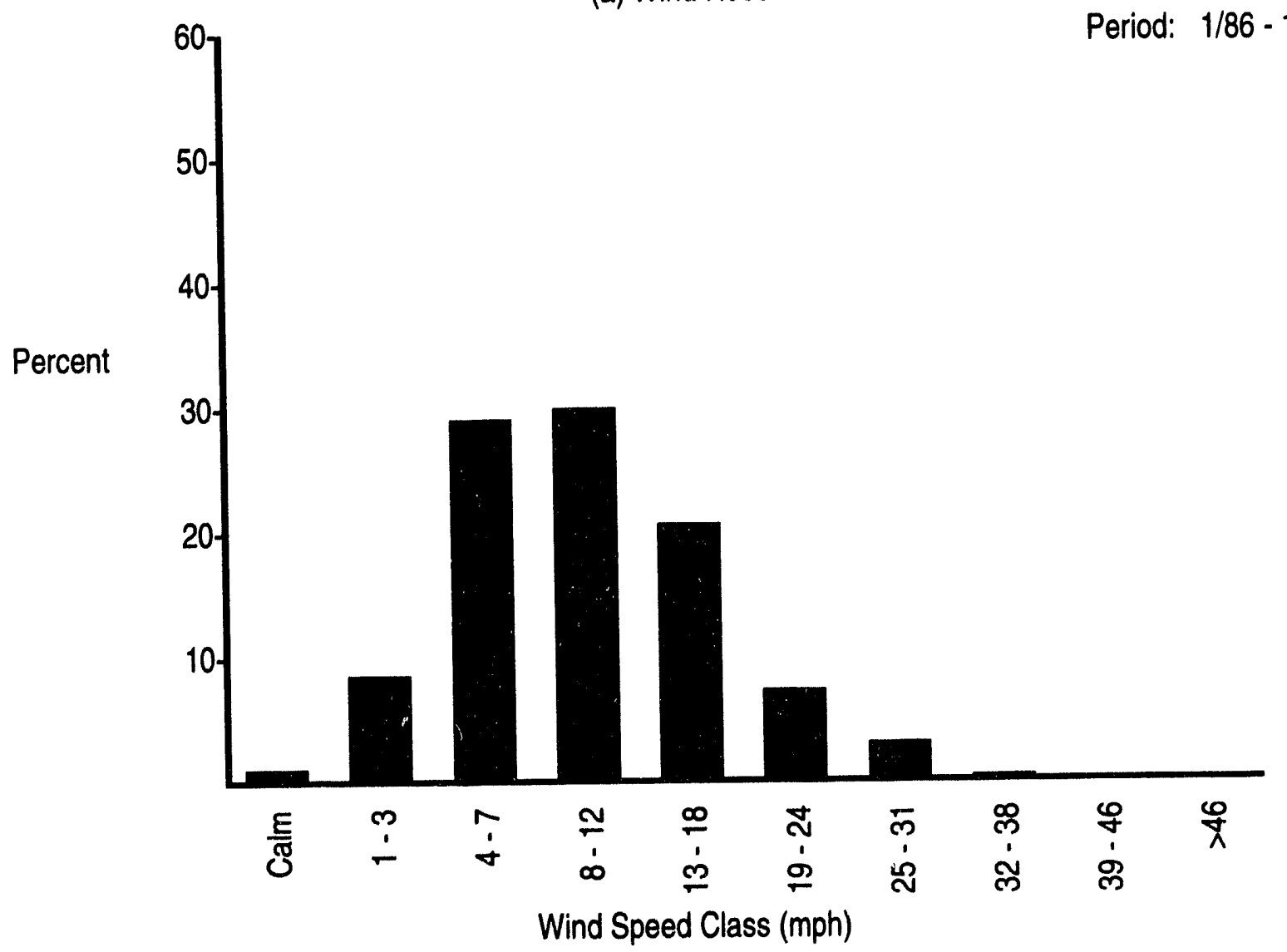

(b) Wind Speed Histogram

FIGURE B.2. (contd) 


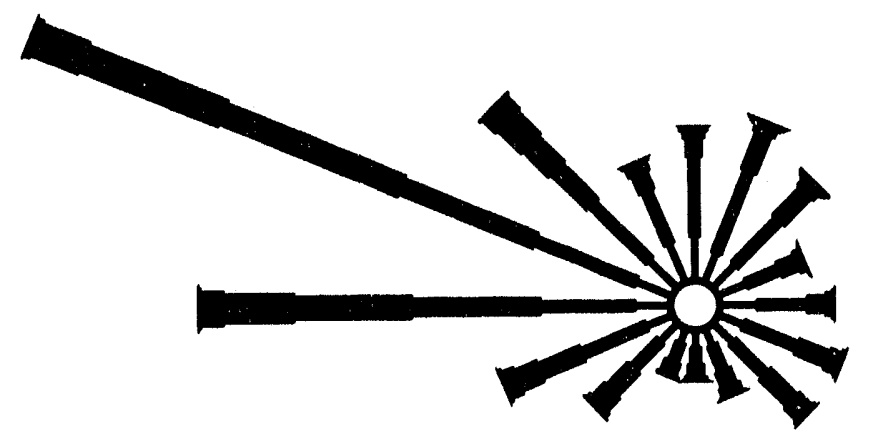

(a) Wind Rose

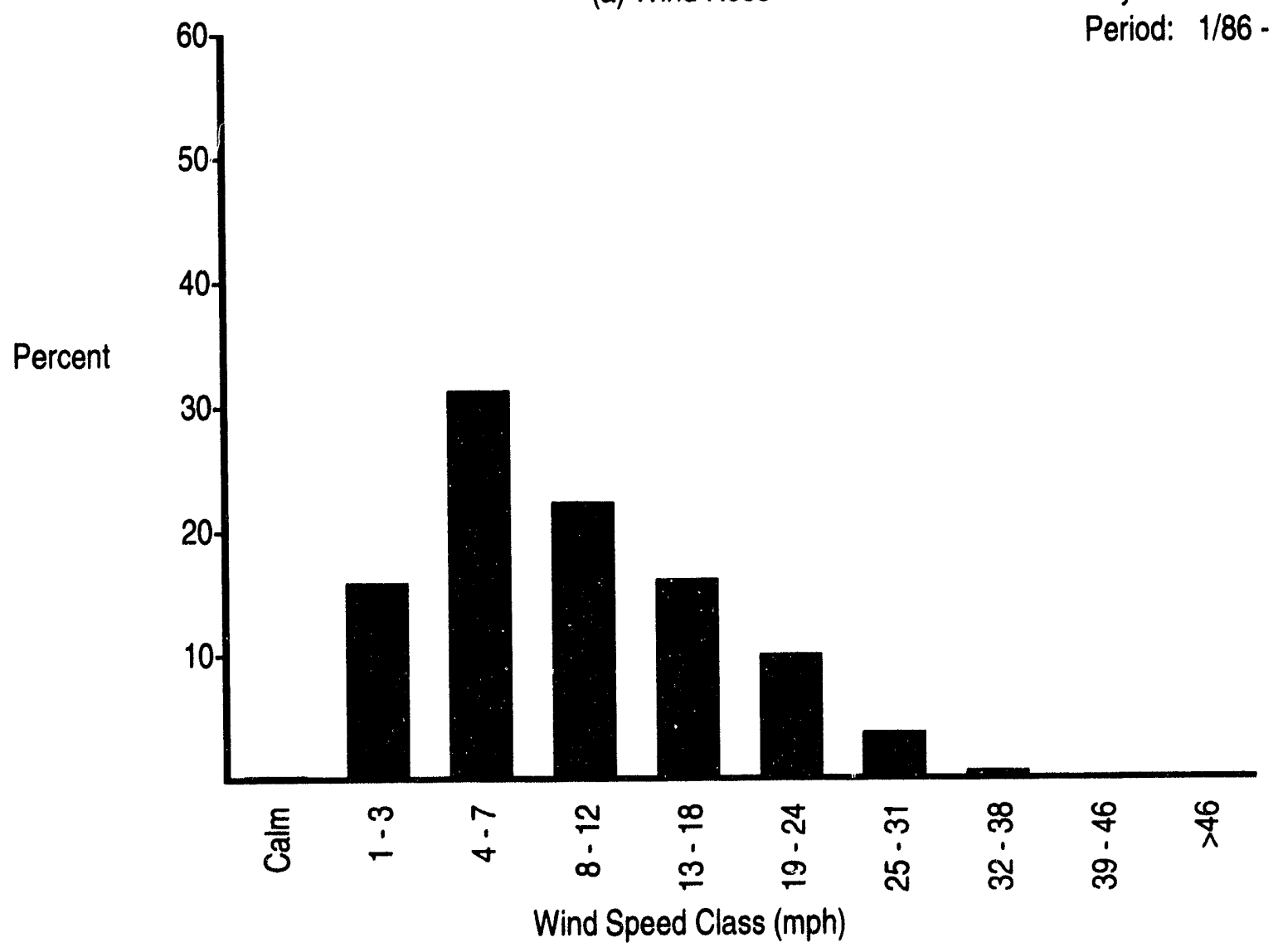

(b) Wind Speed Histogram
July Data

Period: $1 / 86-12 / 93$

\section{FIGURE B.2. (contd)}




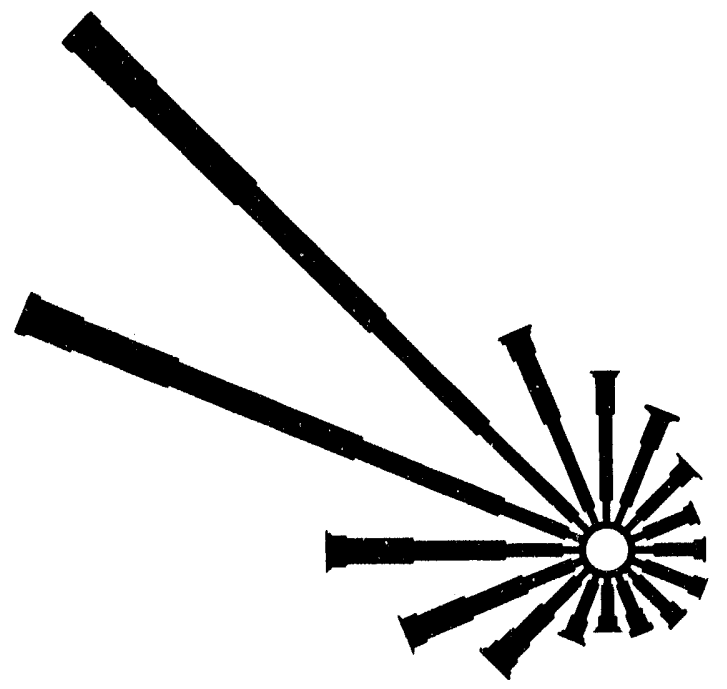

(a) Wind Rose

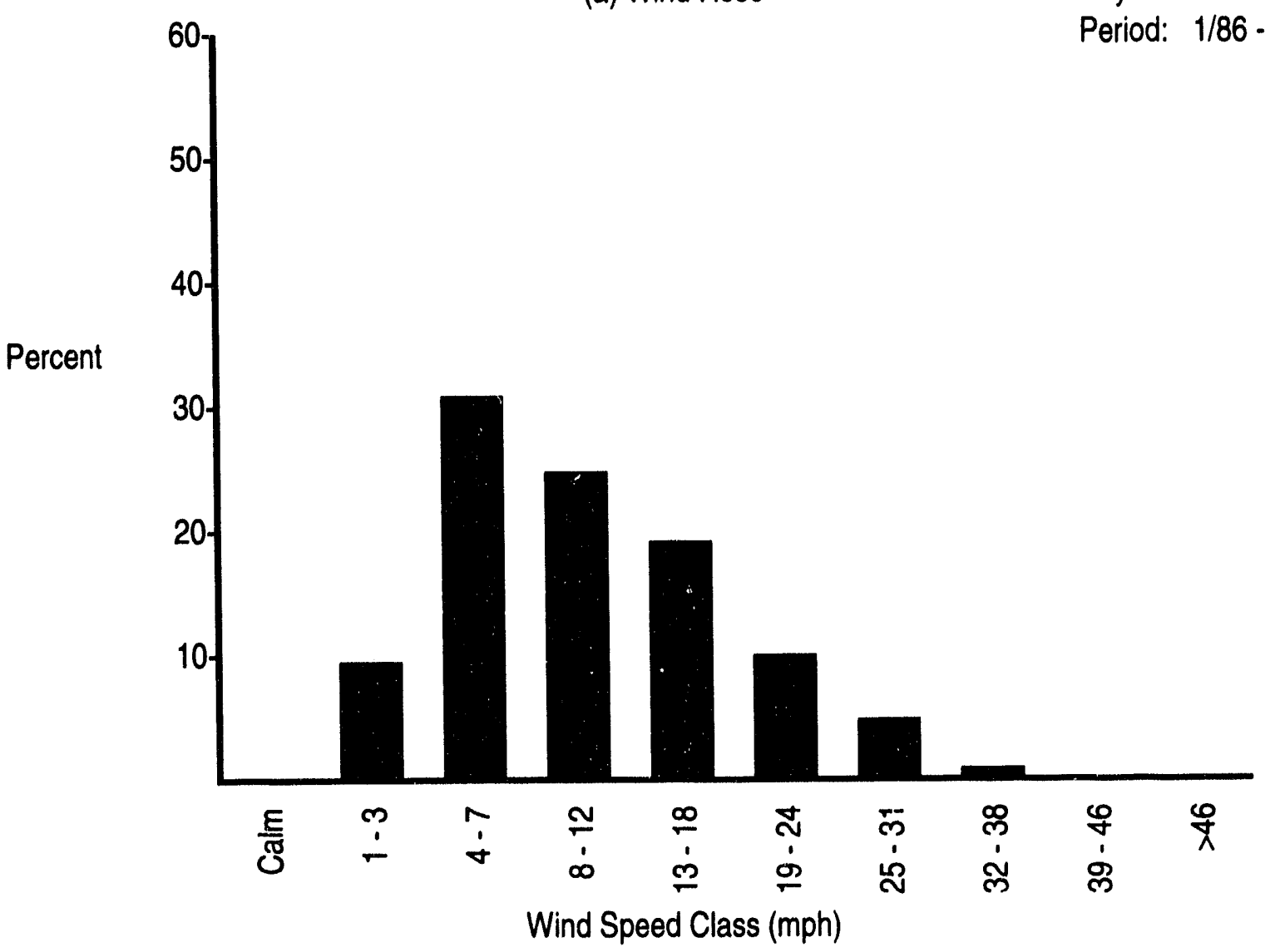

(b) Wind Speed Histogram

FIGURE B.2. (contd) 


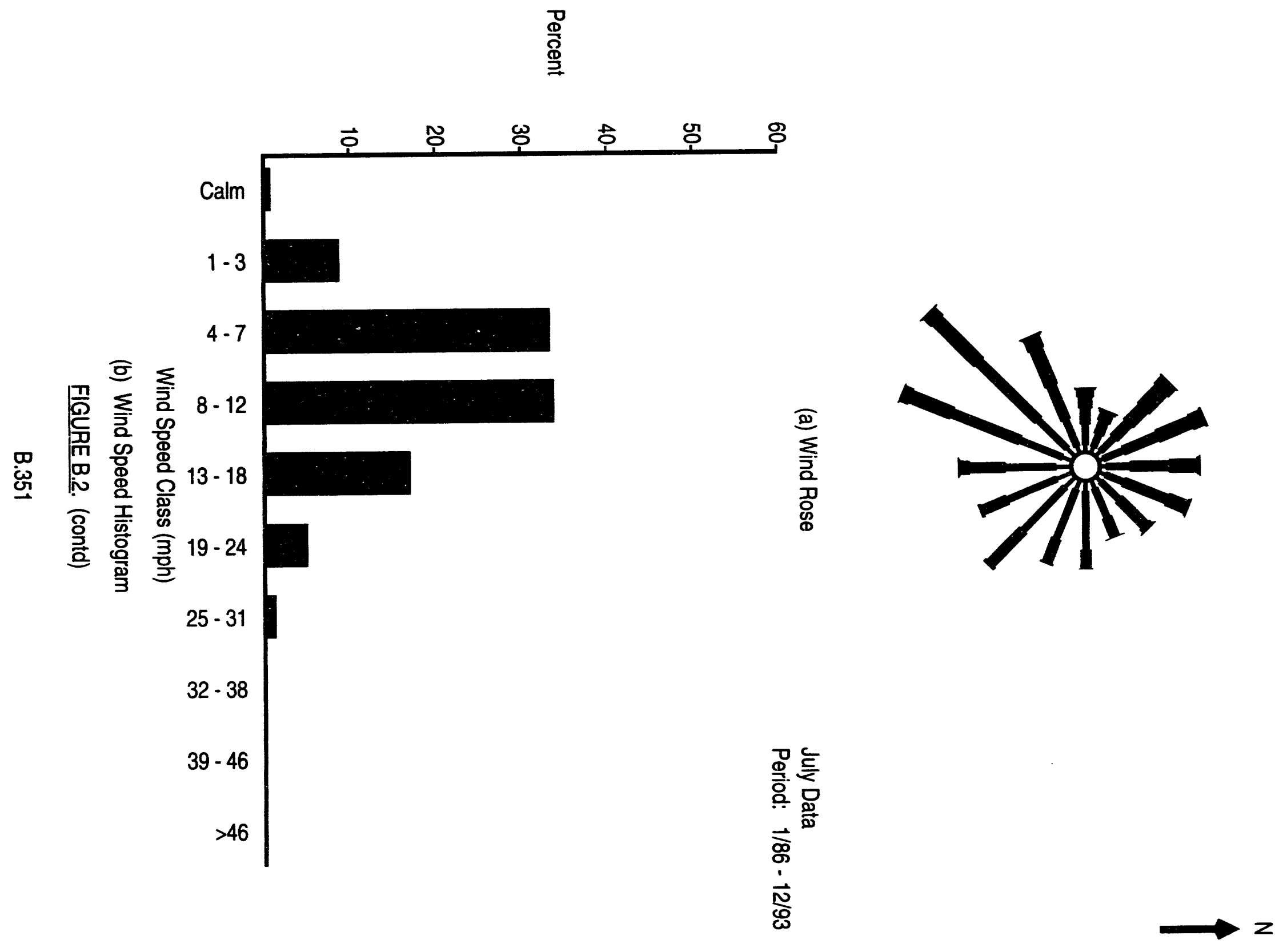




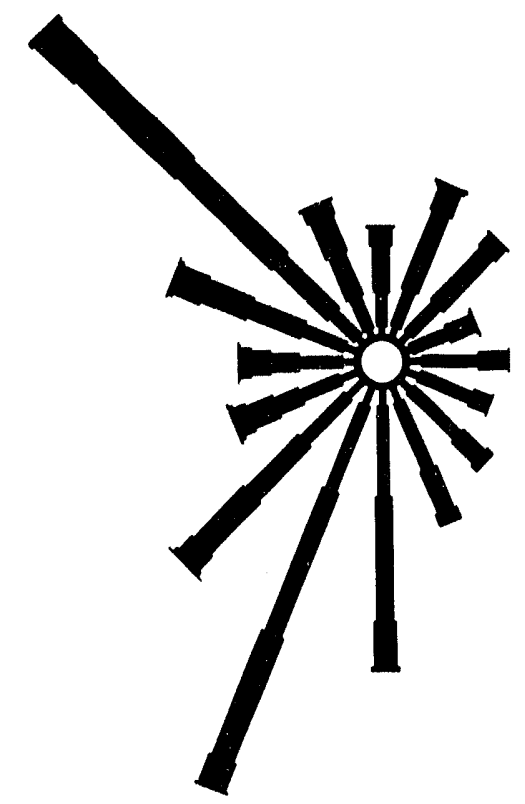

(a) Wind Rose

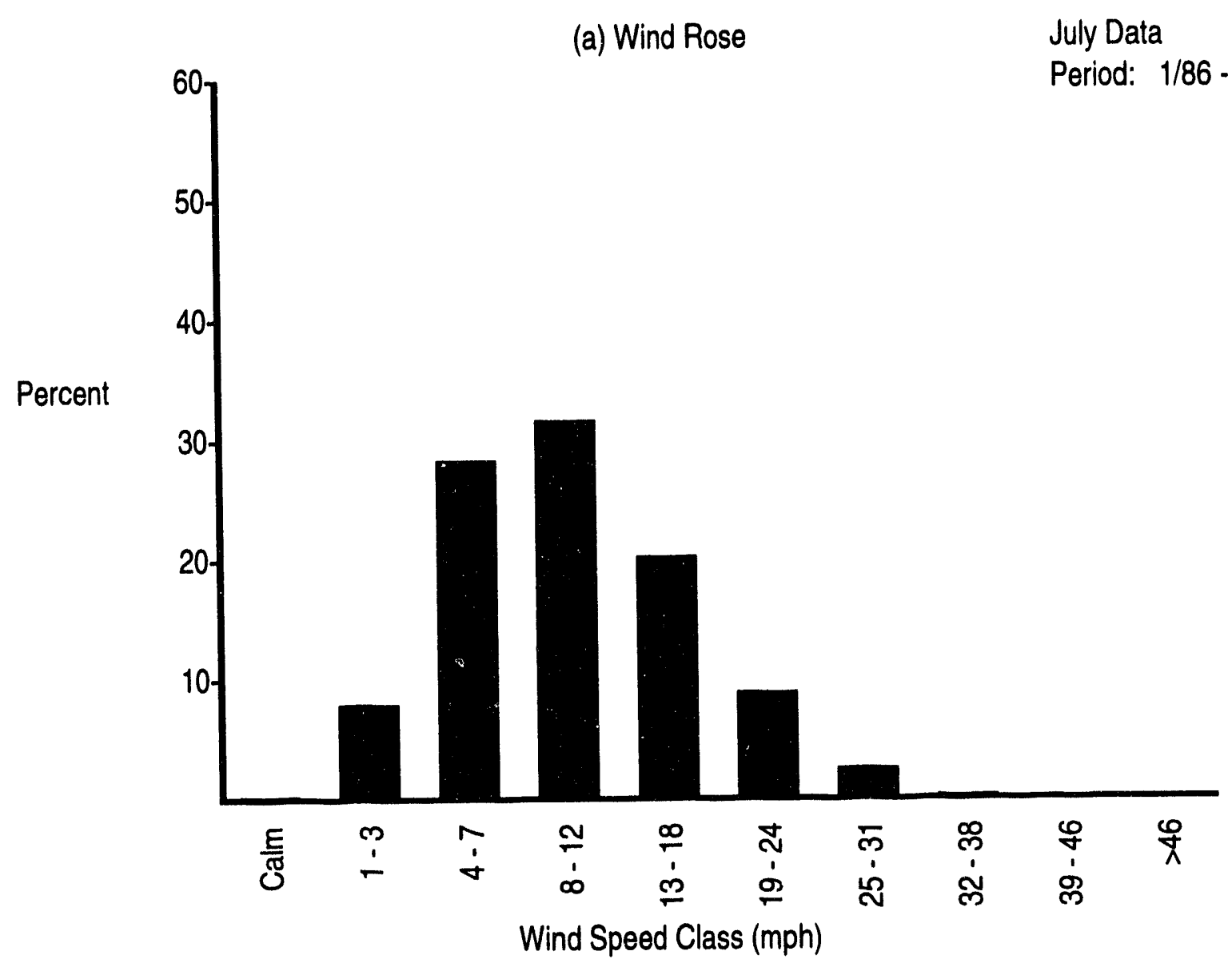

(b) Wind Speed Histogram

FIGURE B.2. (contd) 

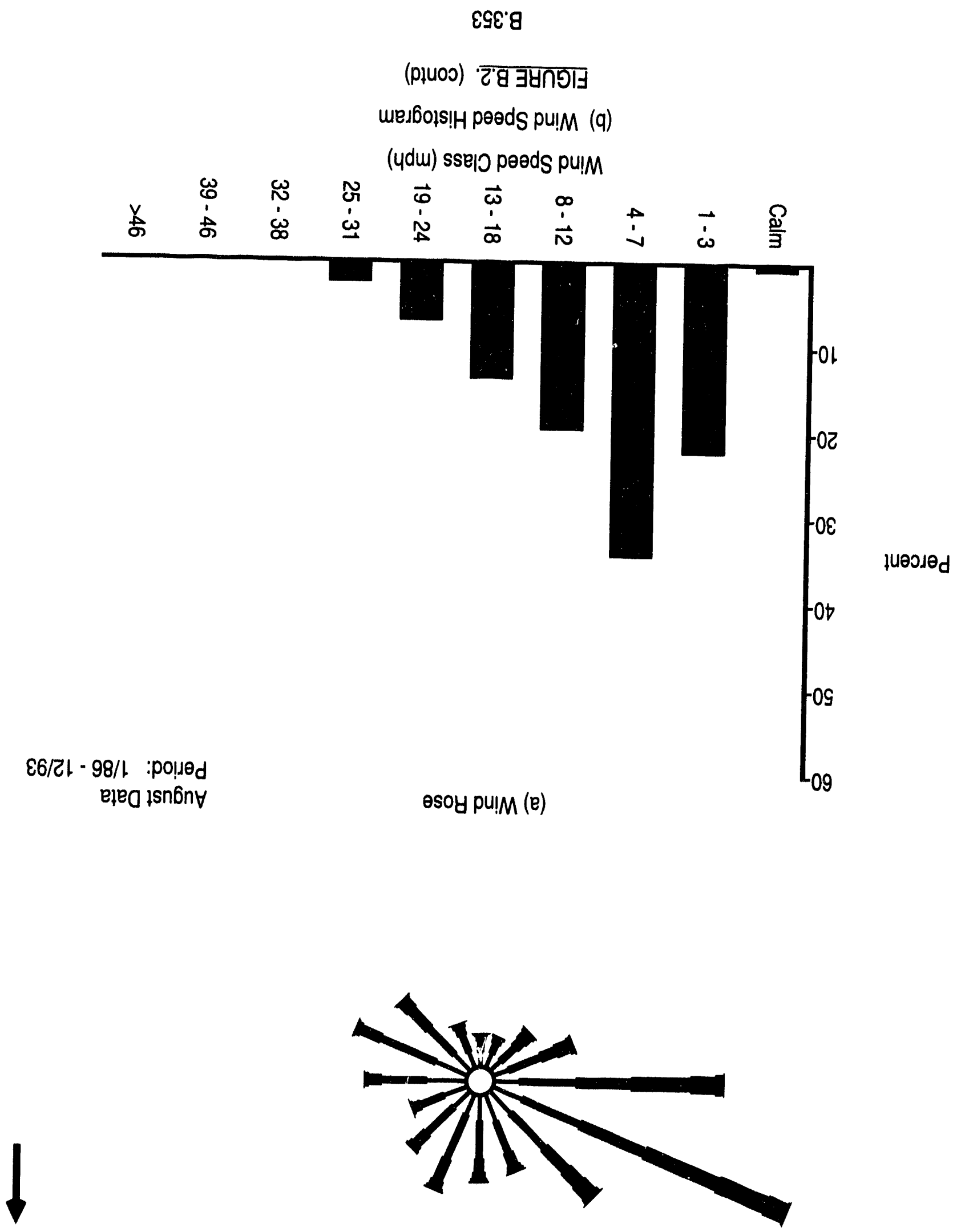

$\mathrm{N}$ 

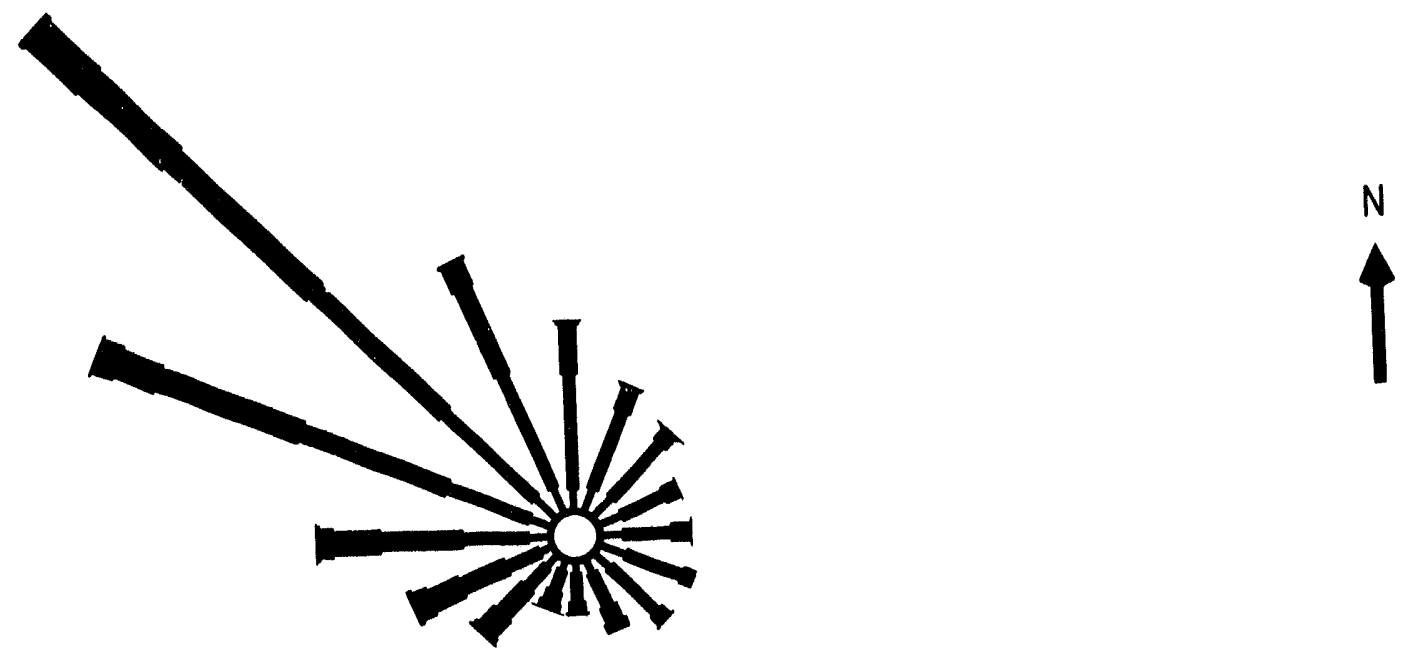

(a) Wind Rose

August Data

Period: 1/86 - 12/93

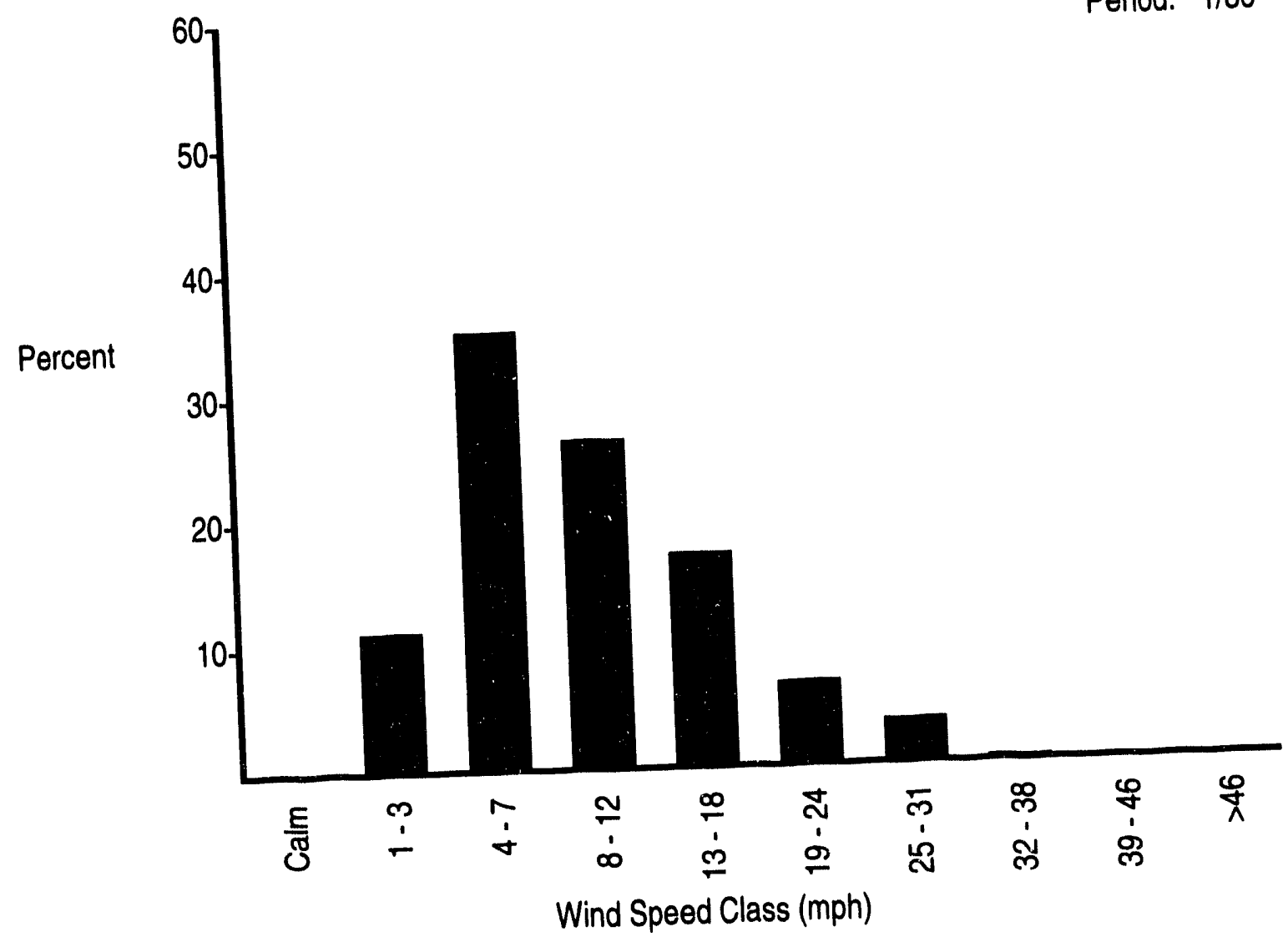

(b) Wind Speed Histogram

FIGURE B.2. (contd) 


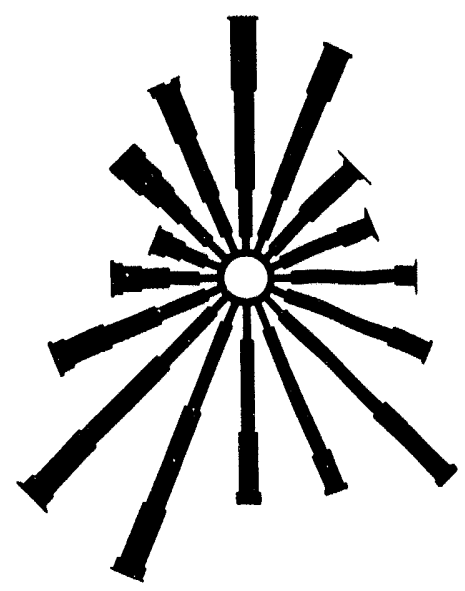

(a) Wind Rose

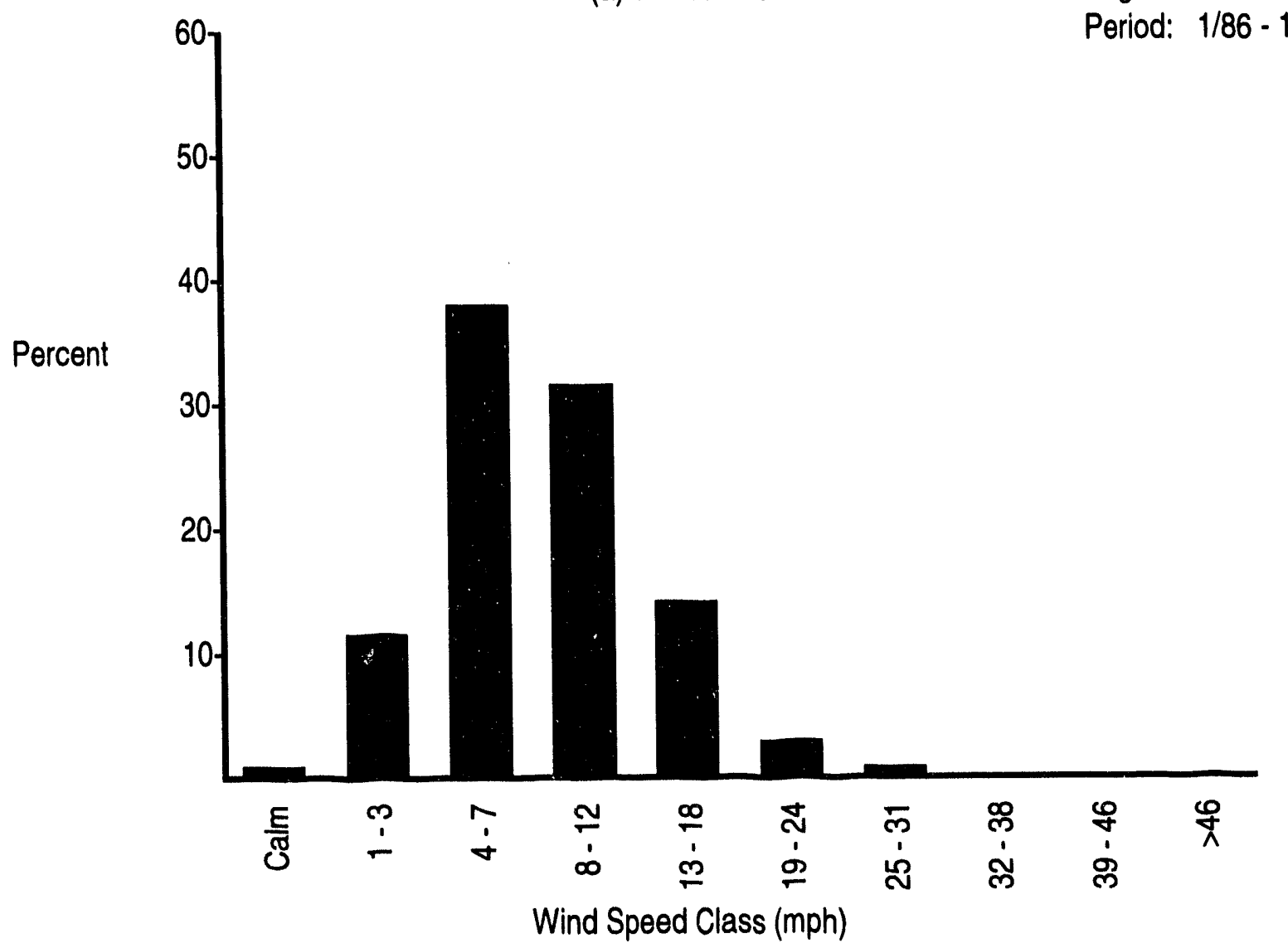

(b) Wind Speed Histogram

FIGURE B.2. (contd) 


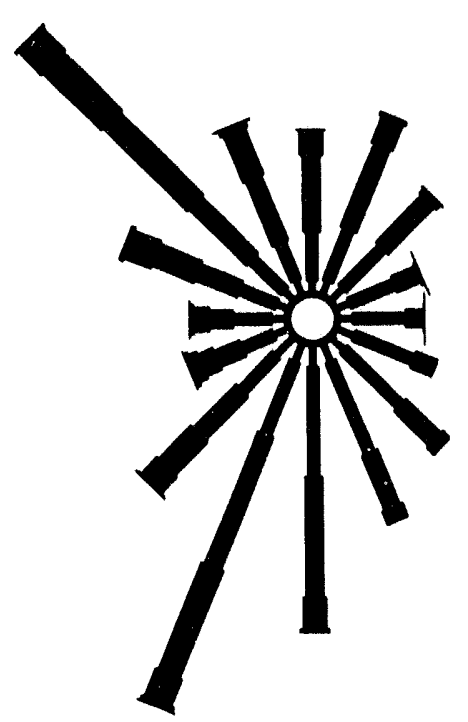

(a) Wind Rose

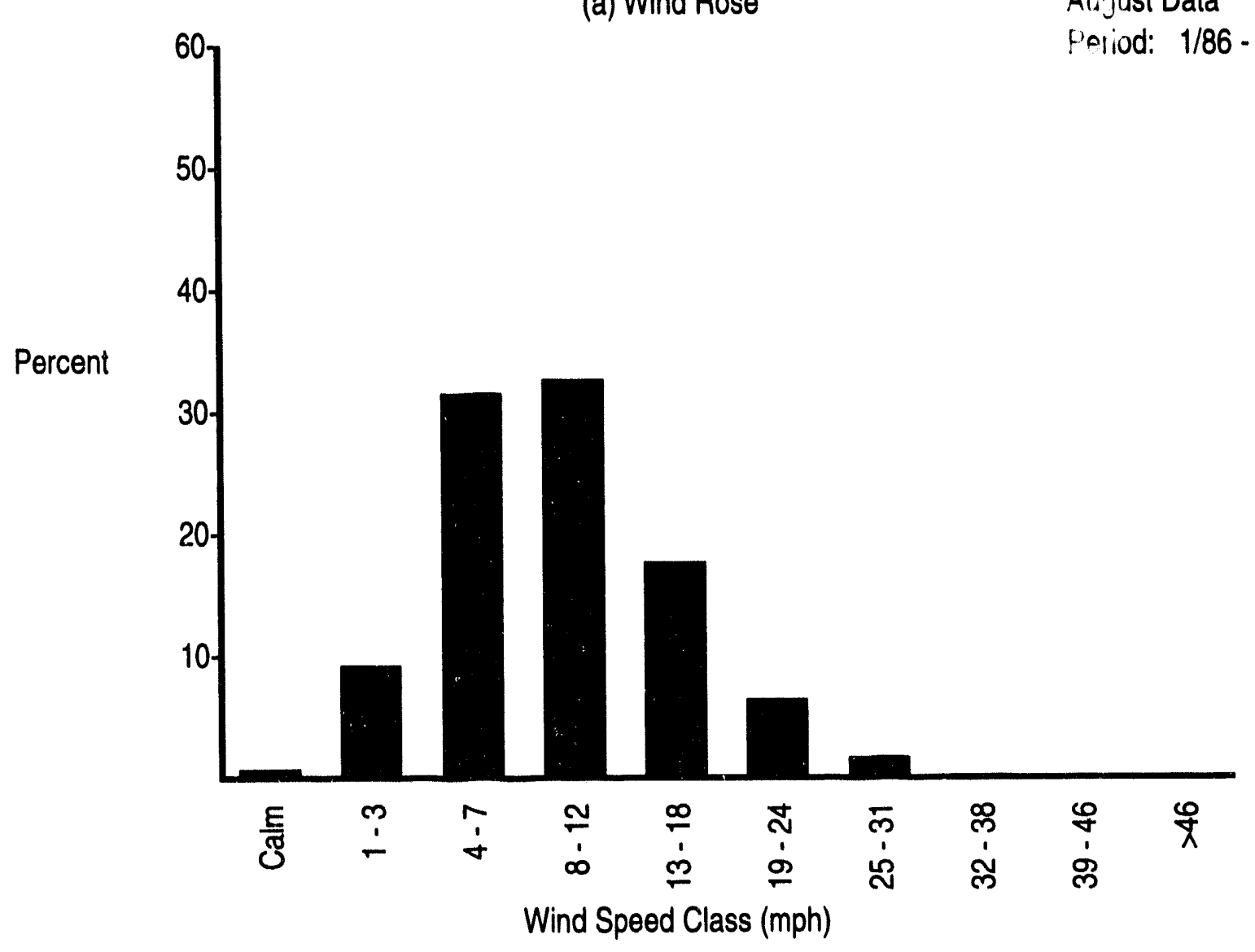

(b) Wind Speed Histogram

FIGURE B.2. (contd)
Au'just Data

Peliod: $1 / 86-12 / 93$ 


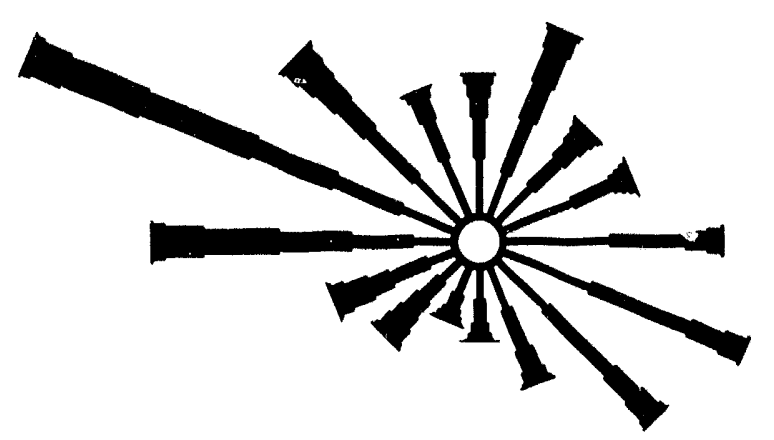

(a) Wind Rose

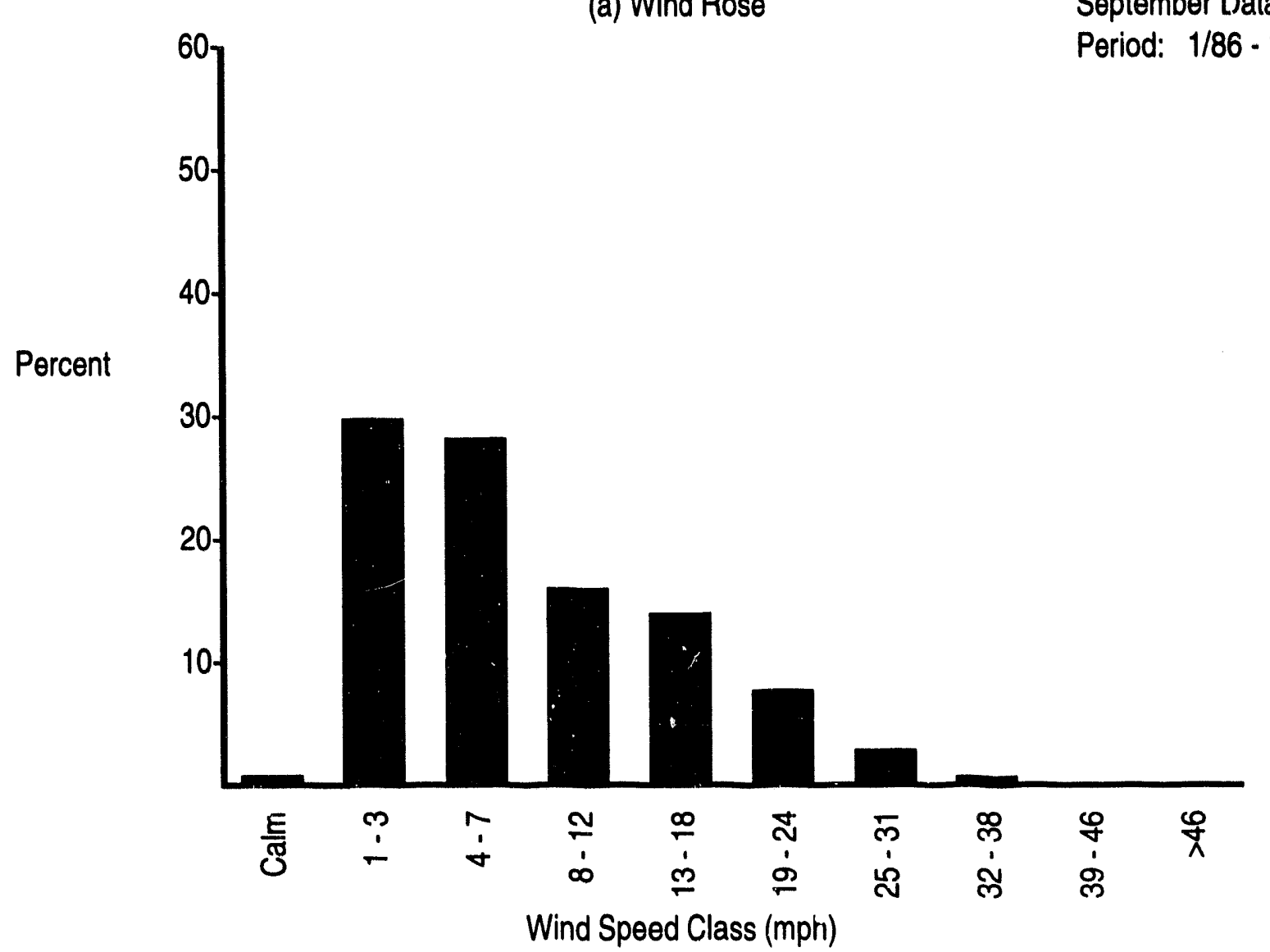

(b) Wind Speed Histogram
September Data

Period: 1/86-12/93

\section{FIGURE B.2. (contd)}

\section{B.357}



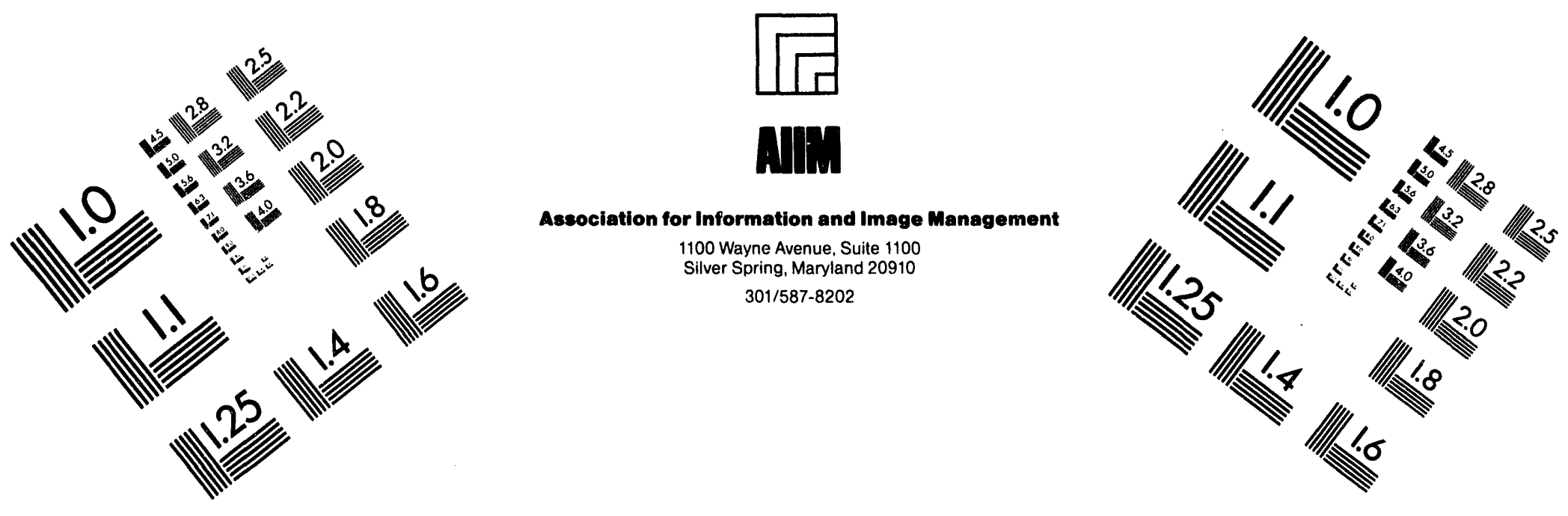

\section{Centimeter}

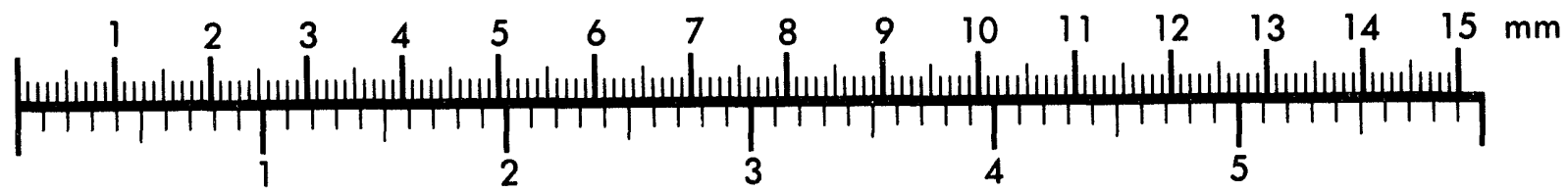
Inches
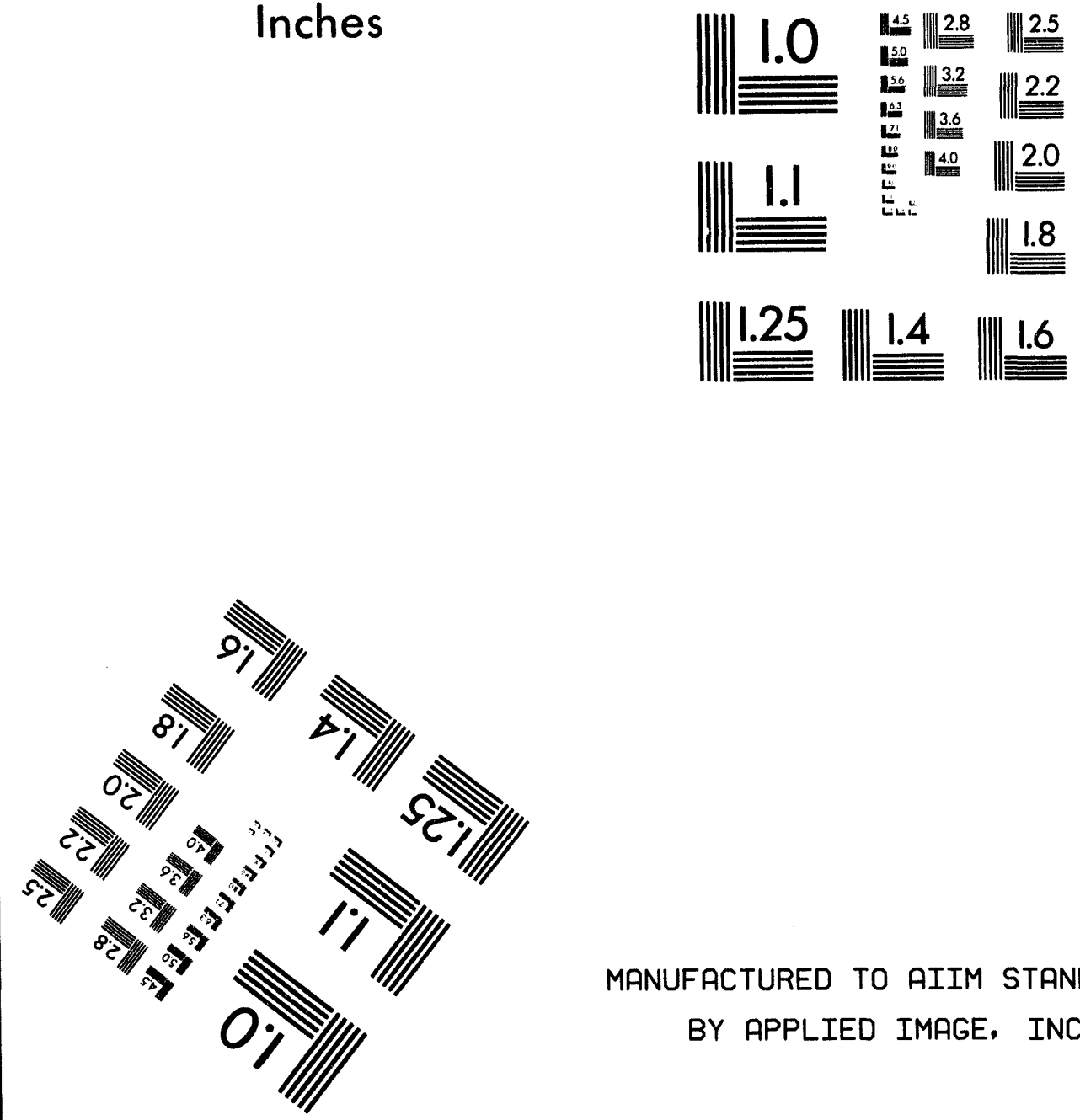

MANUFACTURED TO AIIM STANDARDS BY APPLIED IMAGE, INC.

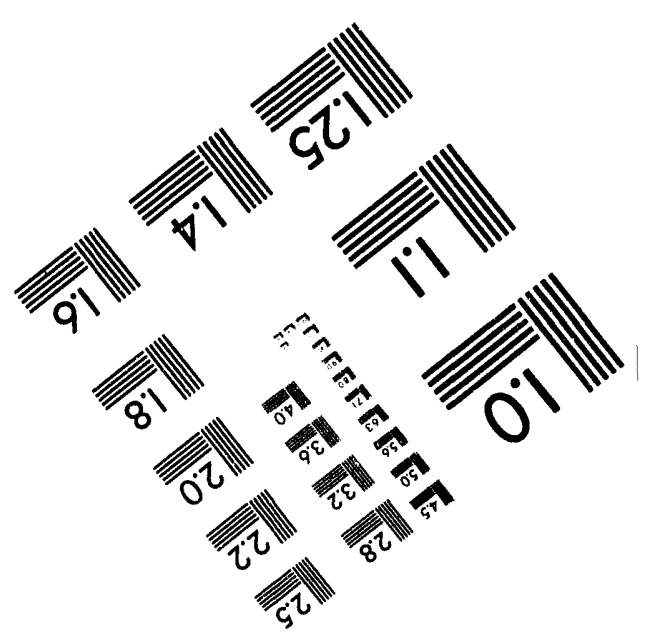



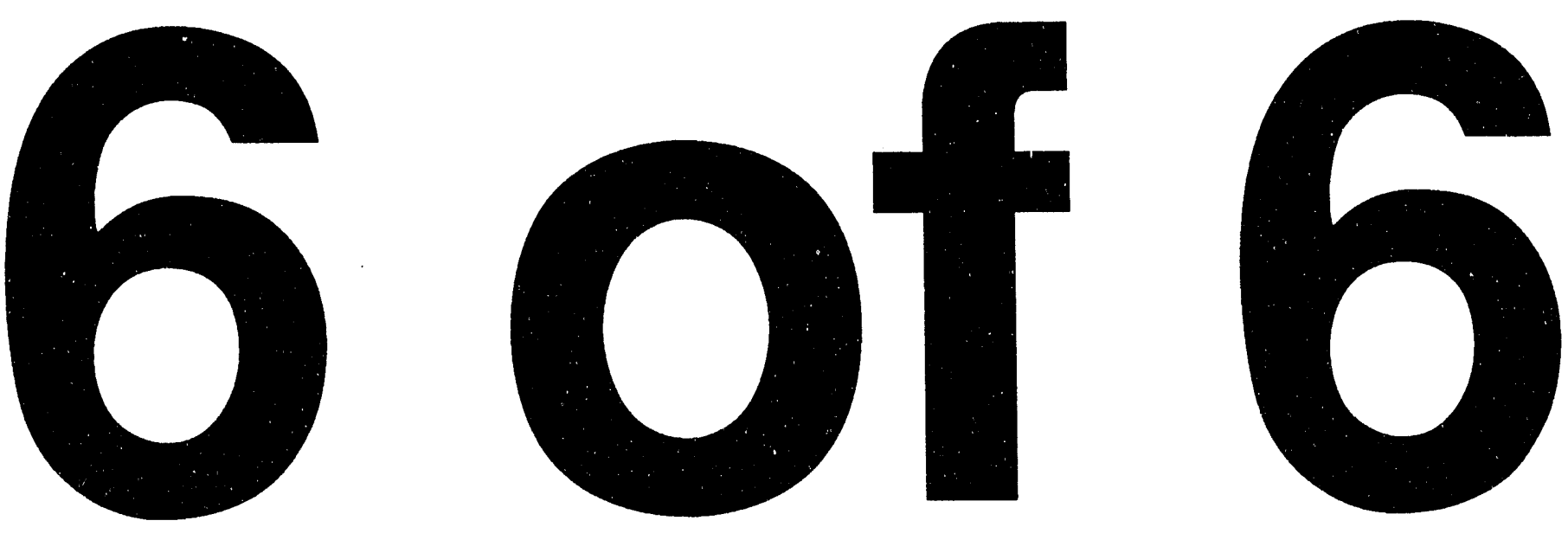

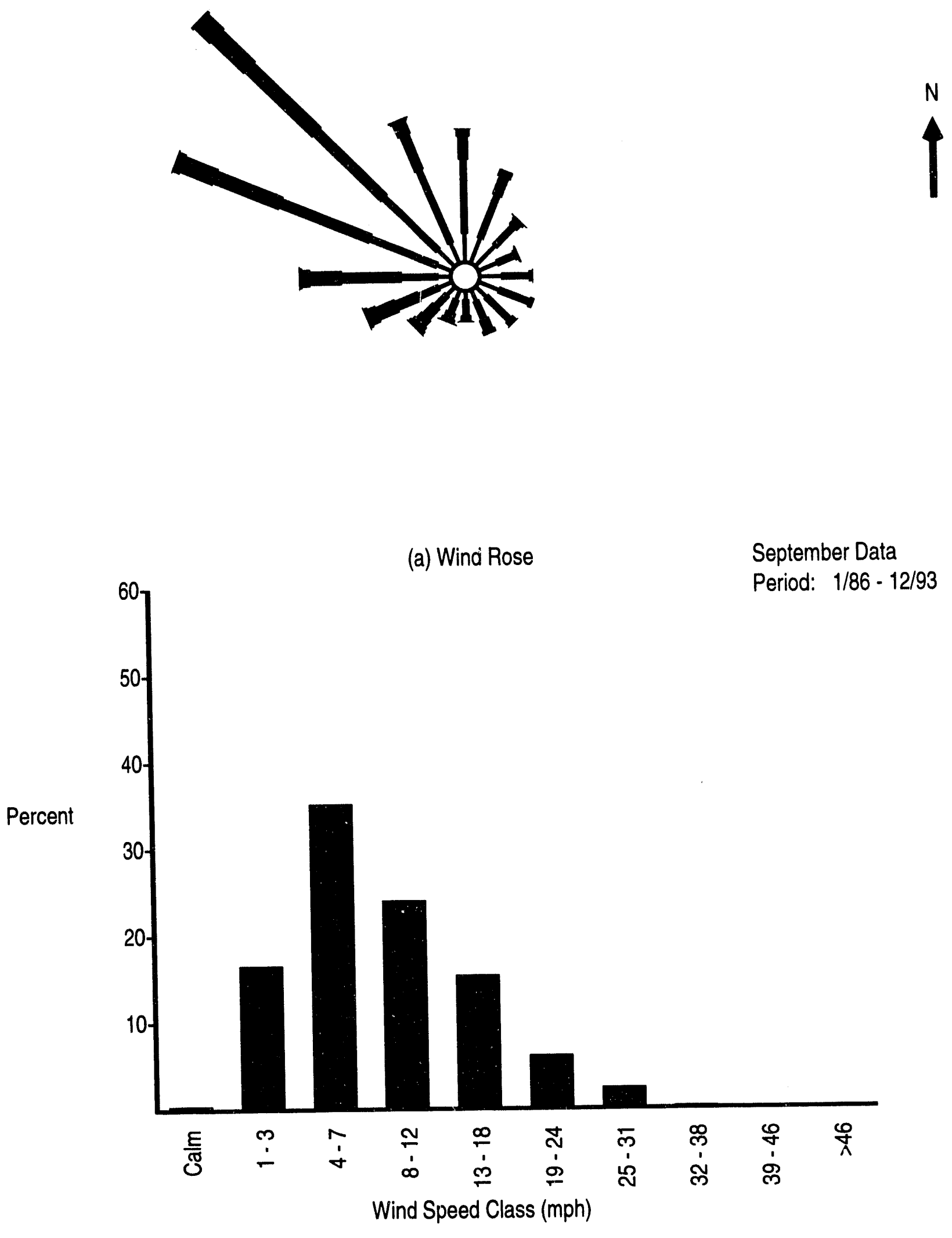

(b) Wind Speed Histogram

FIGURE B.2. (contd) 


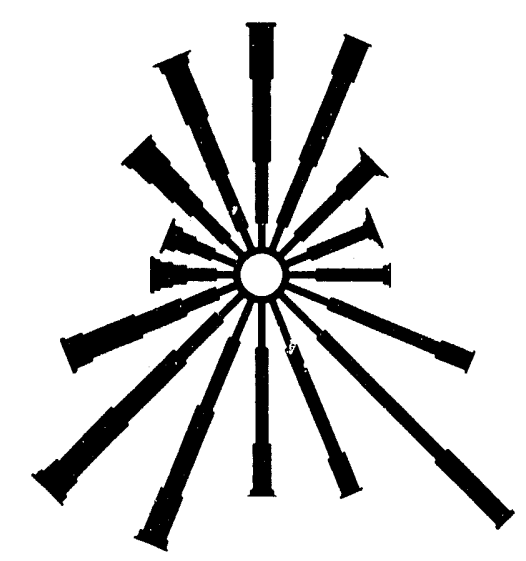

(a) Wind Rose

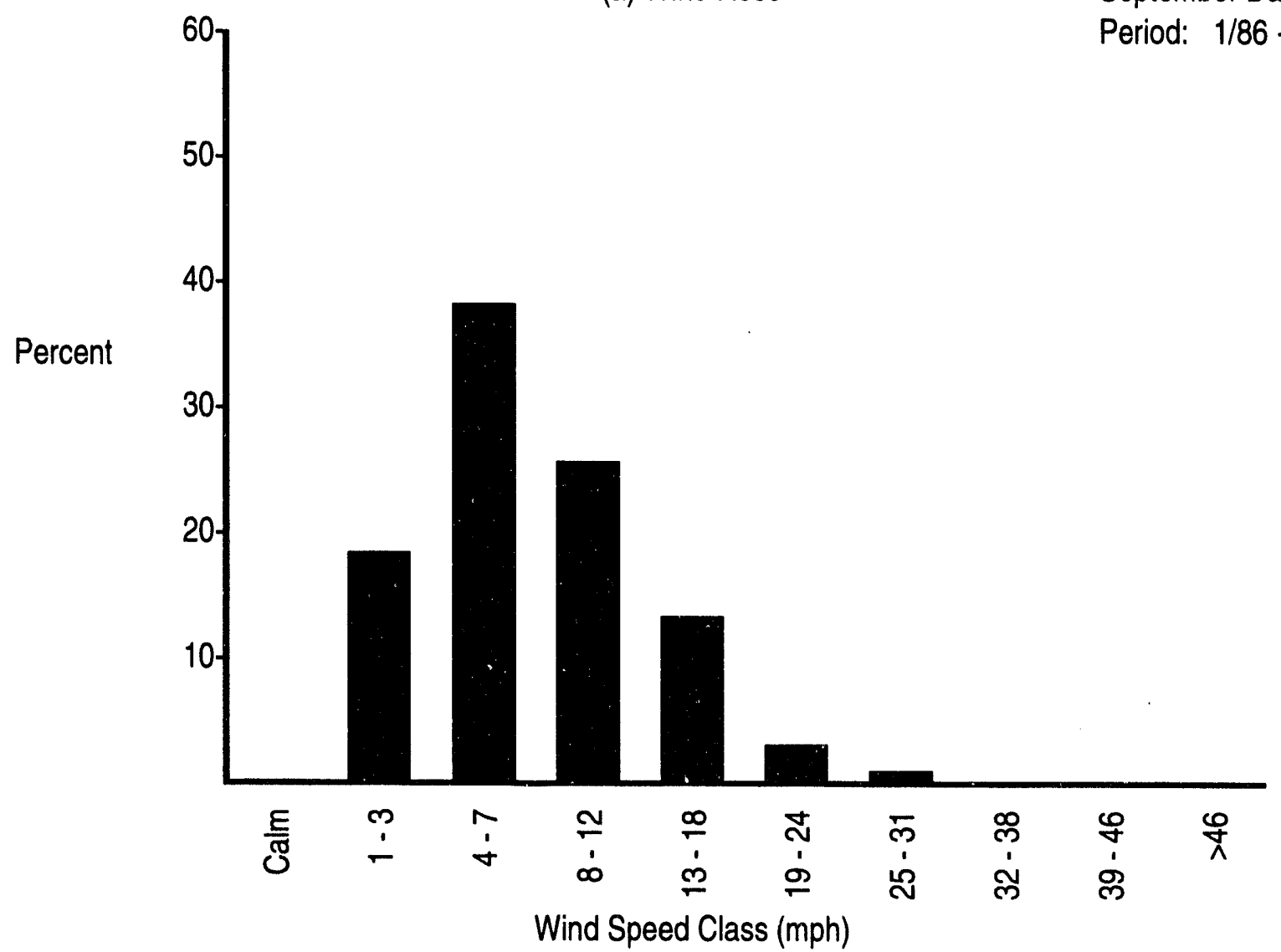

(b) Wind Speed Histogram

FIGURE B.2. (contd)
September Data

Period: 1/86 - 12/93 


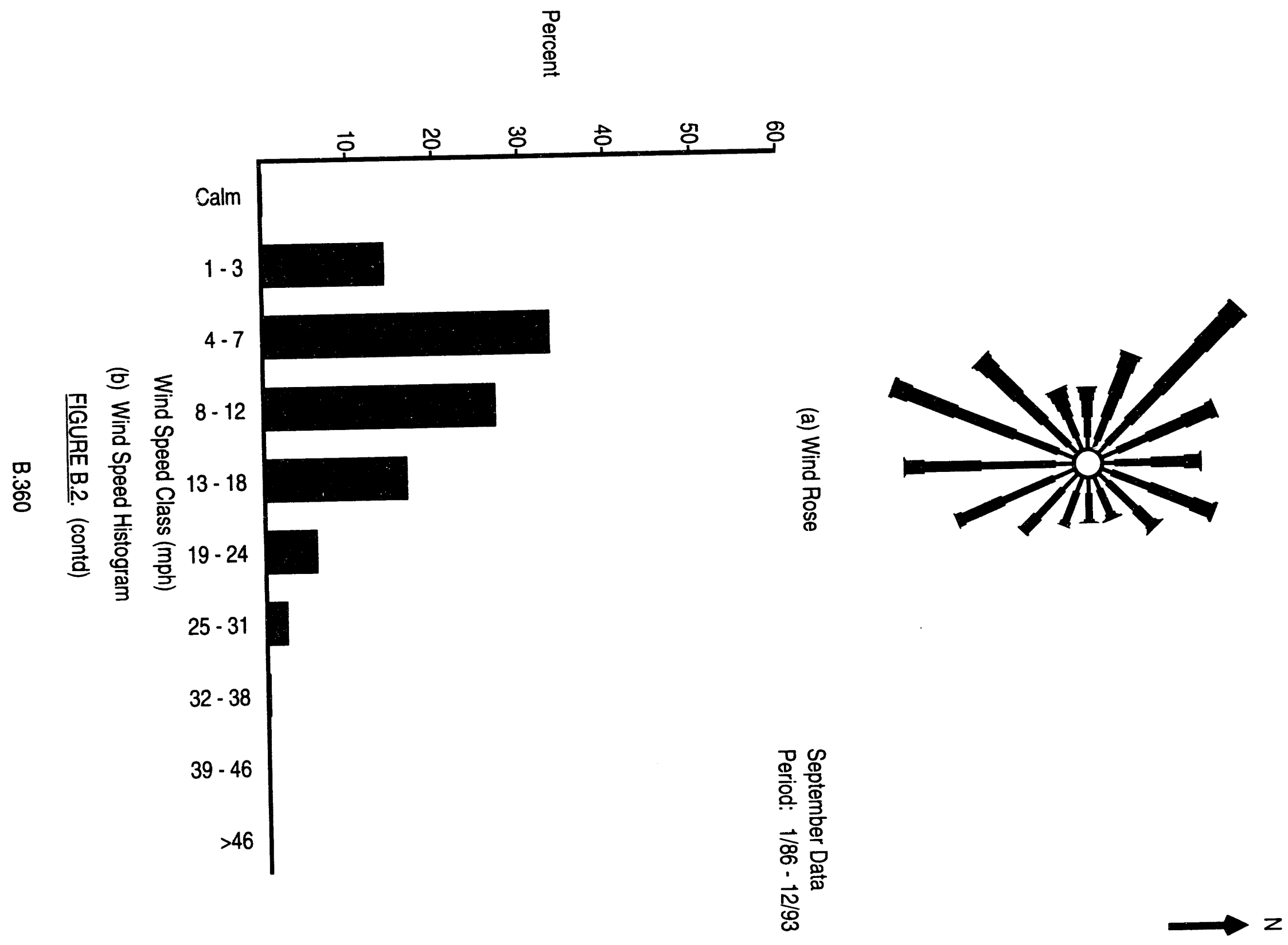




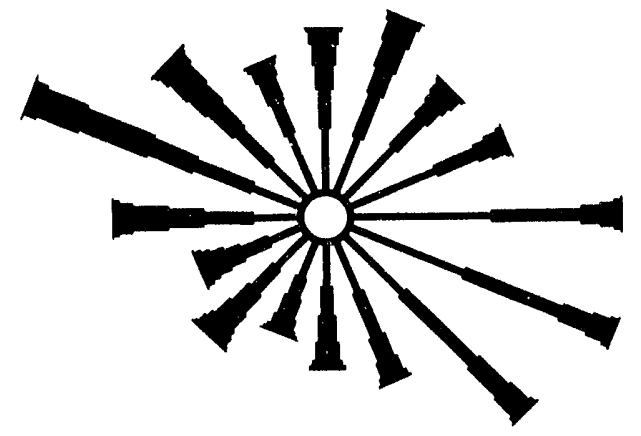

(a) Wind Rose

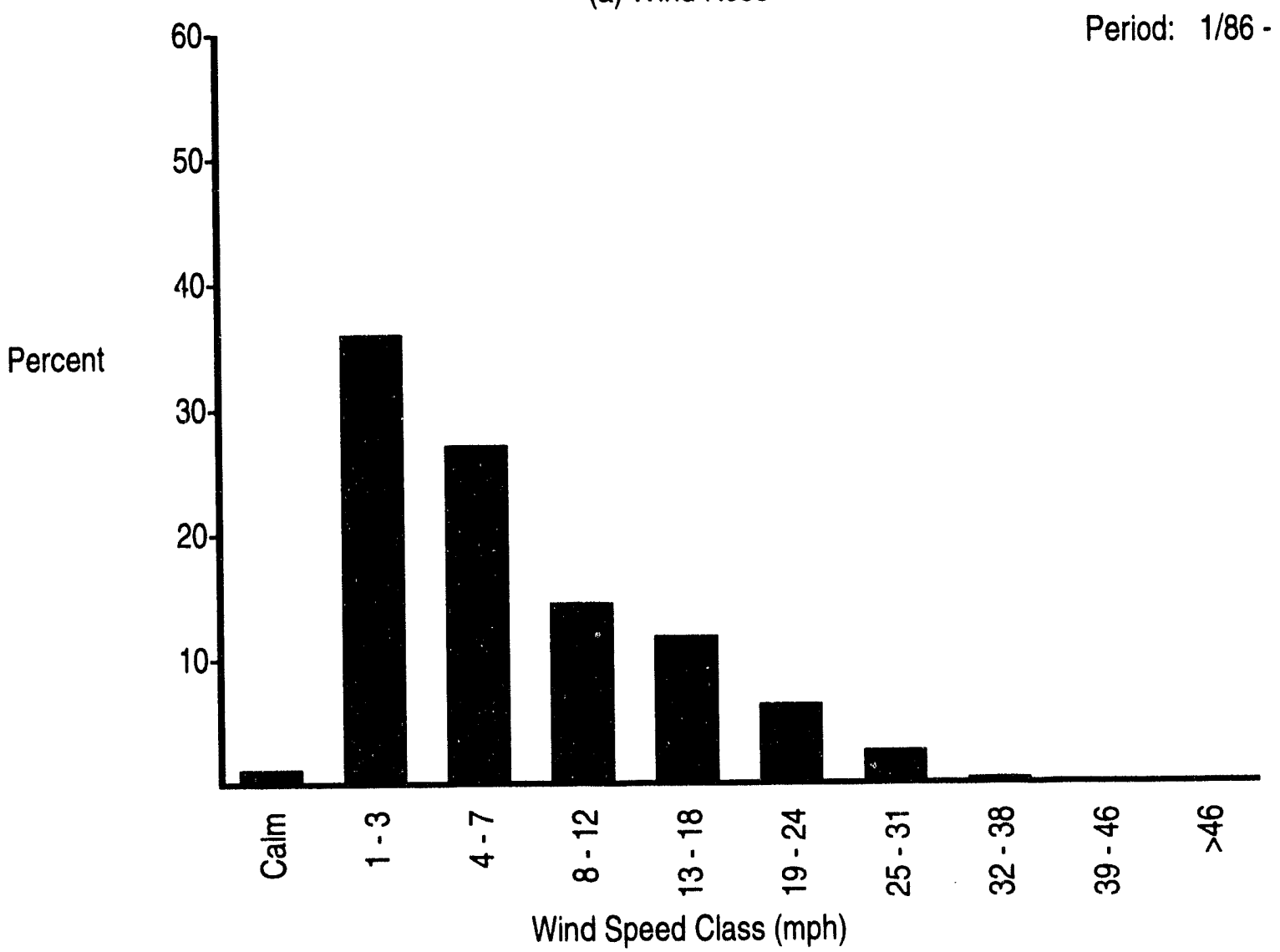

(b) Wind Speed Histogram
October Data

Period: 1/86-12/93

\section{FIGURE B.2. (contd)}



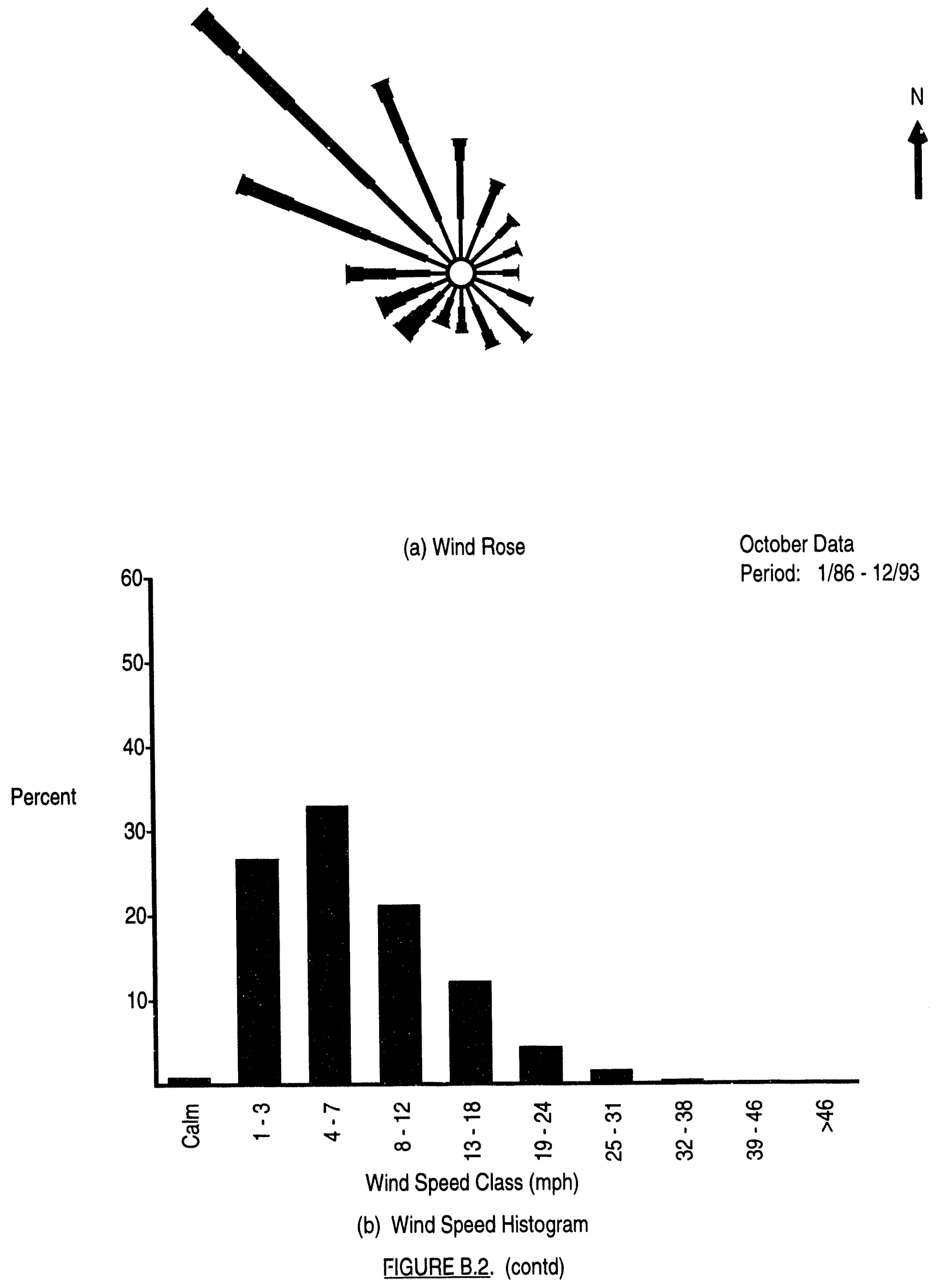


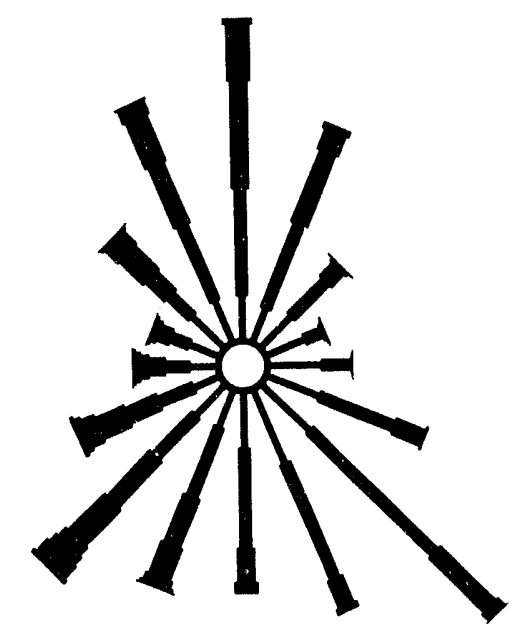

(a) Wind Rose

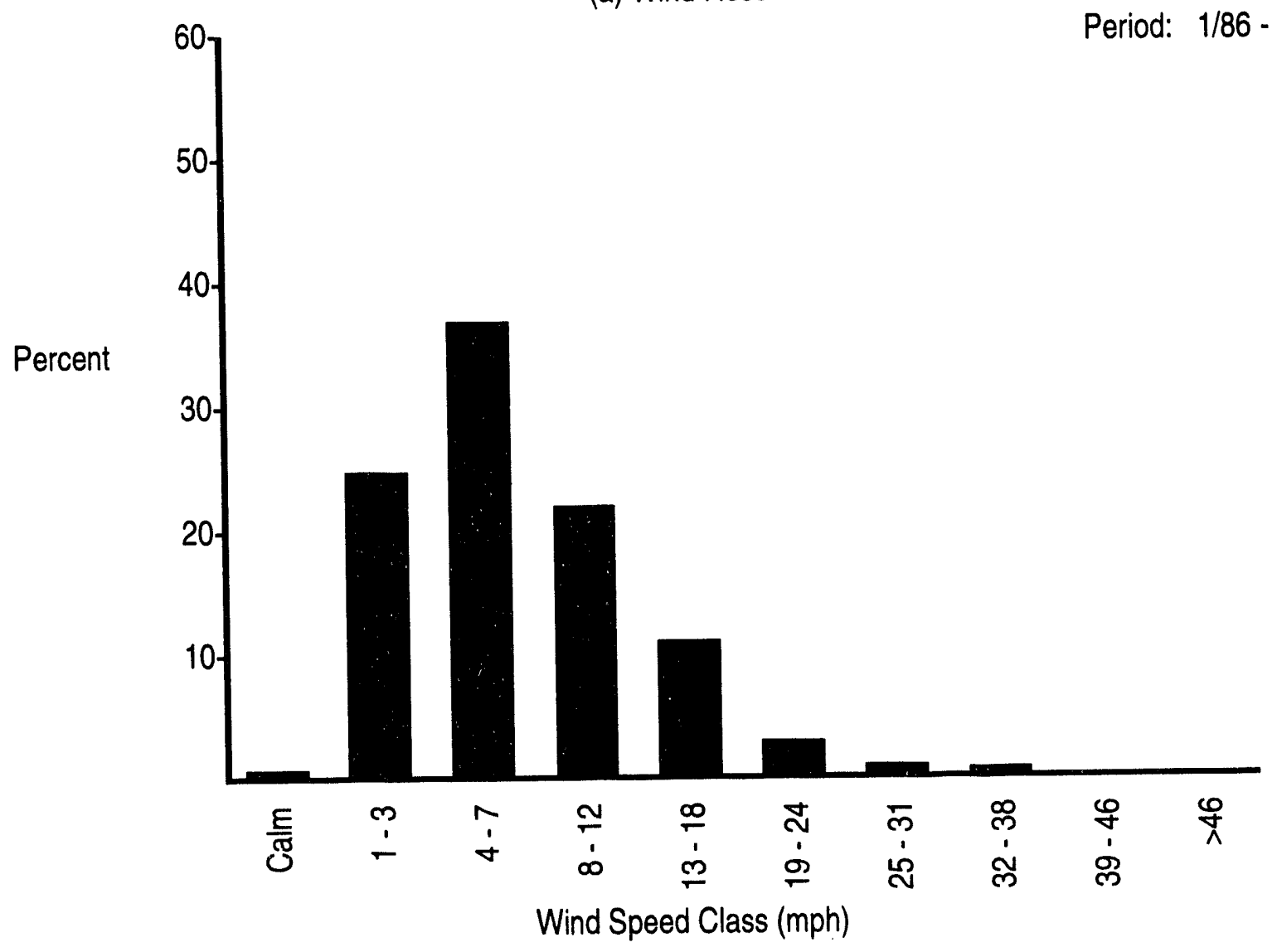

(b) Wind Speed Histogram

FIGURE B.2. (contd)
October Data

Period: 1/86-12/93 

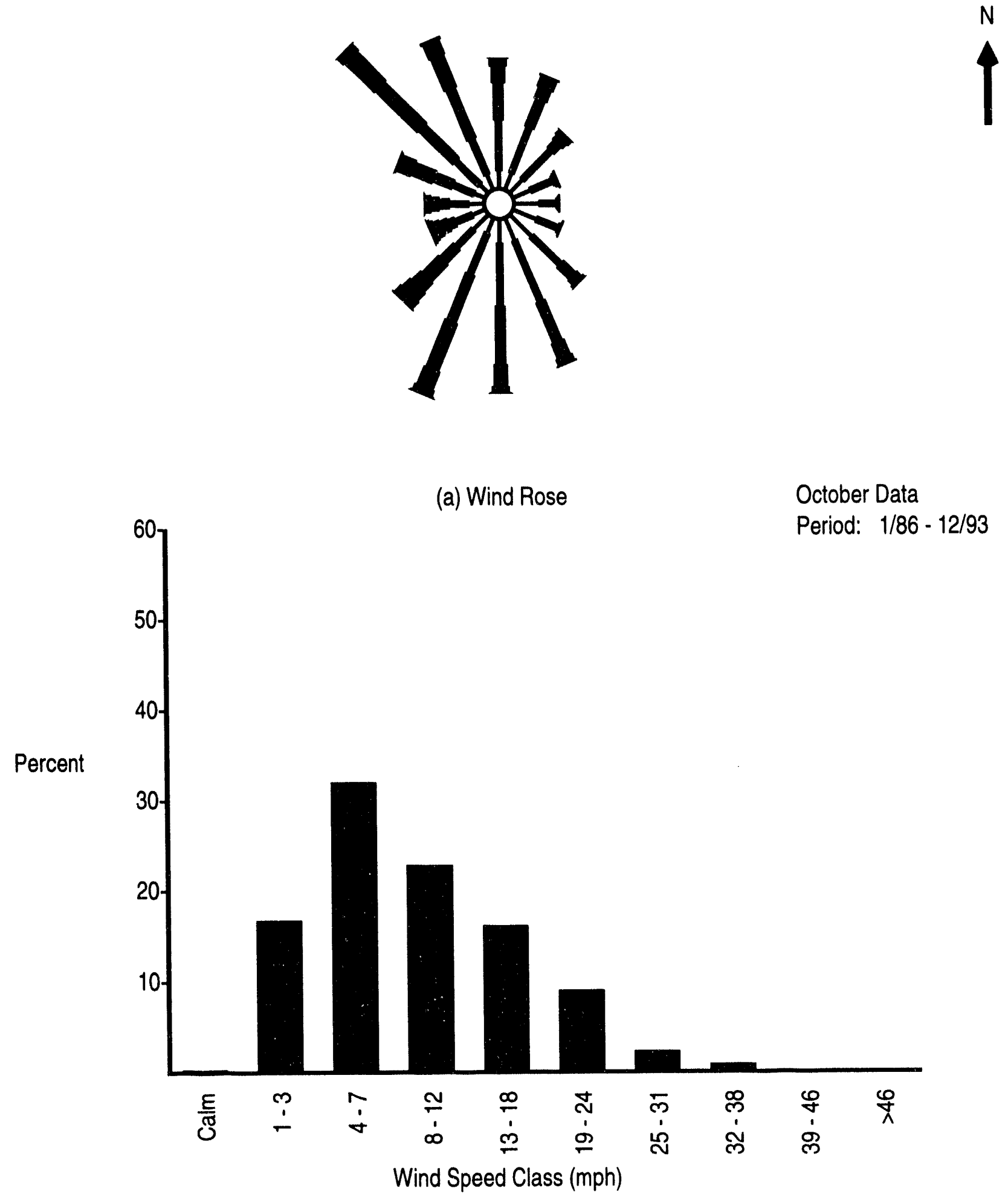

(b) Wind Speed Histogram

FIGURE B.2. (contd) 


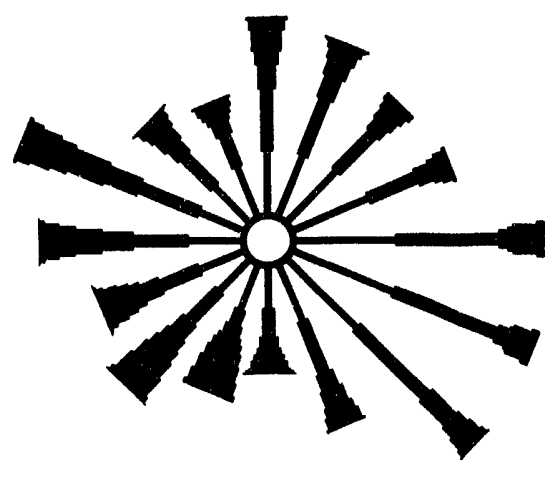

(a) Wind Rose

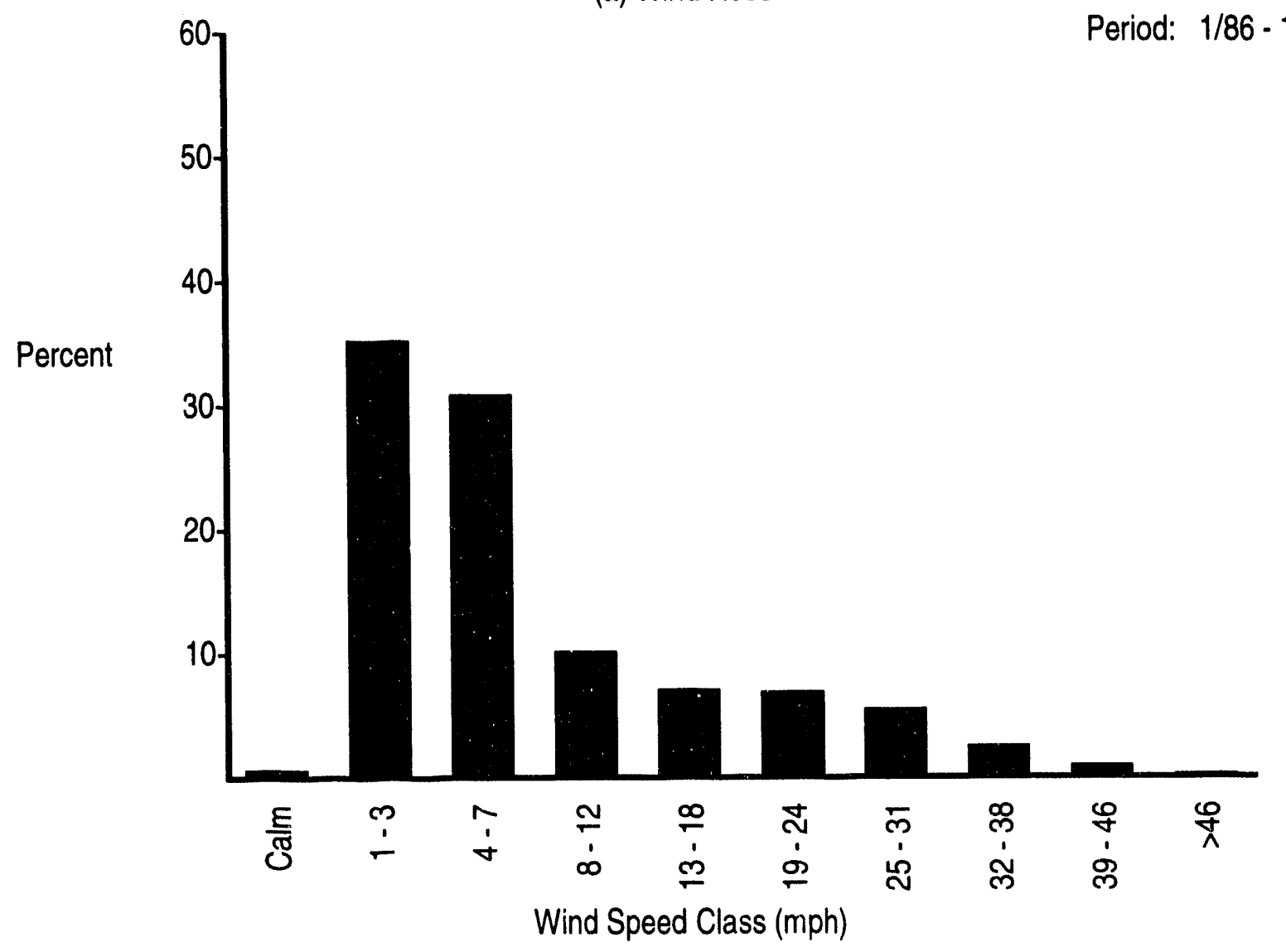

(b) Wind Speed Histogram
November Data

Period: $1 / 86-12 / 93$

\section{FIGURE B.2. (contd)}




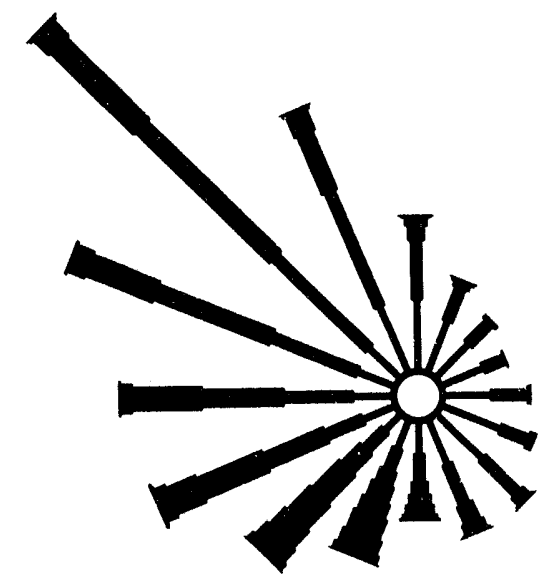

(a) Wind Rose

November Data

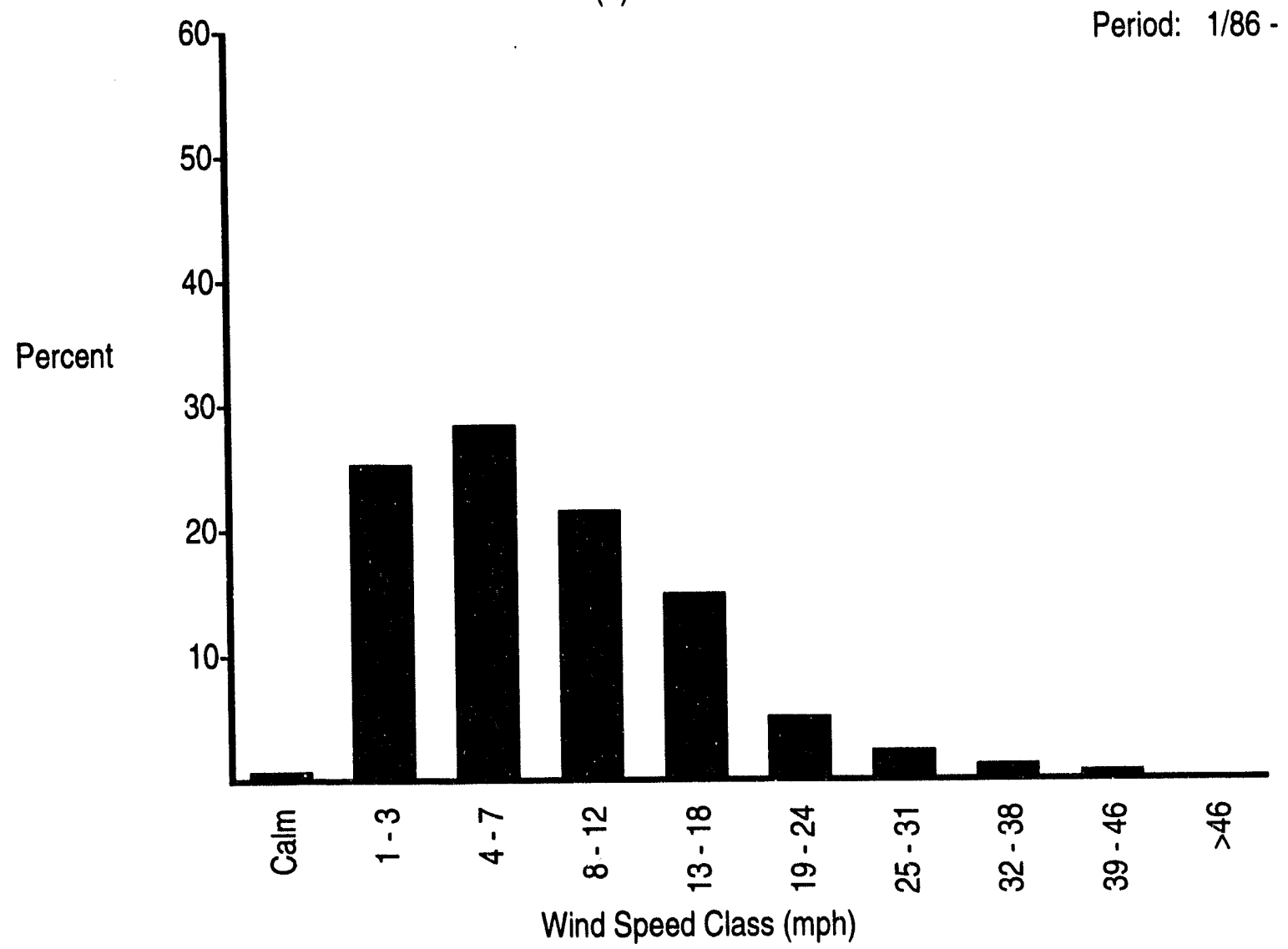

(b) Wind Speed Histogram

FIGURE B.2. (contd) 


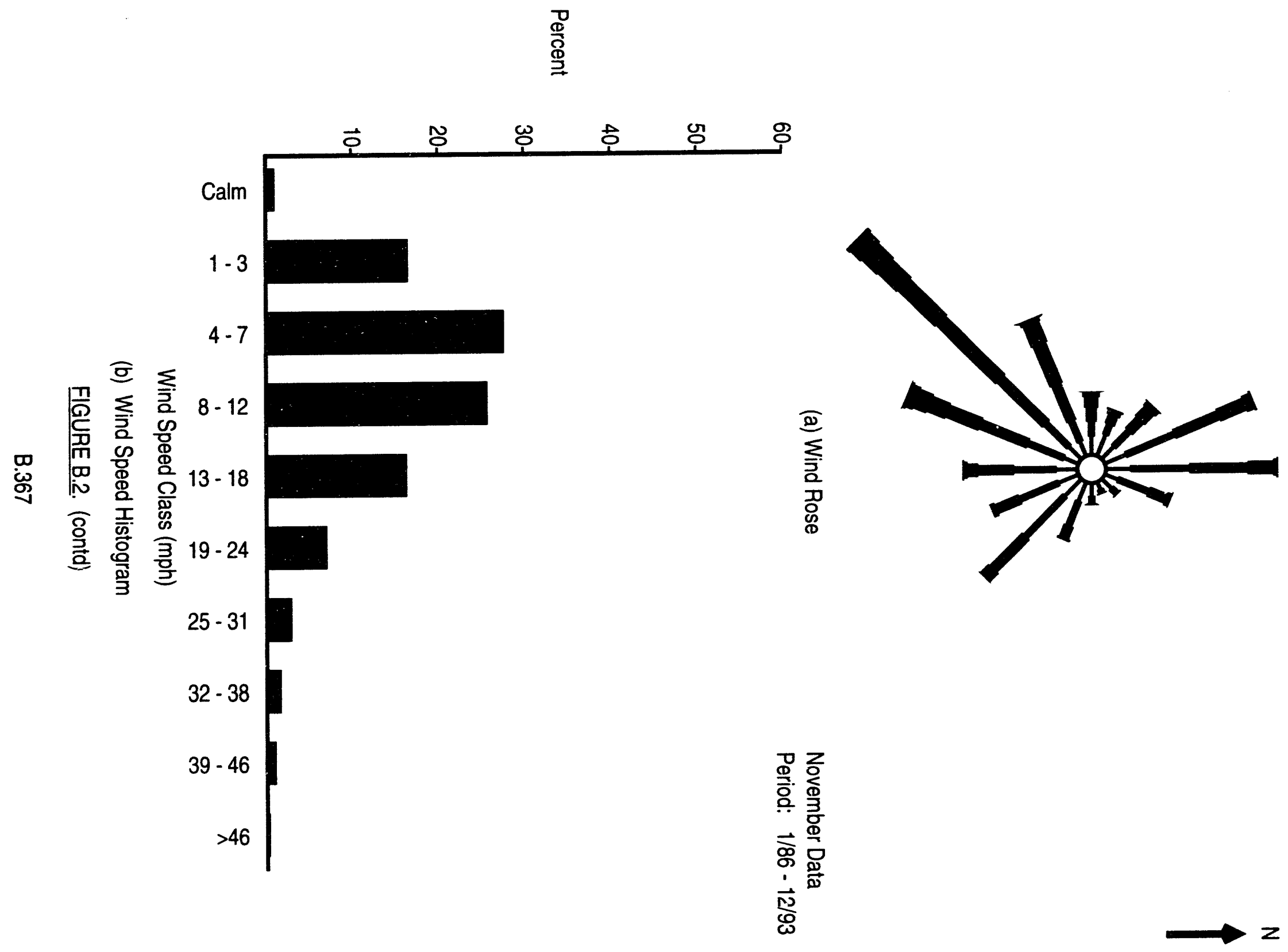




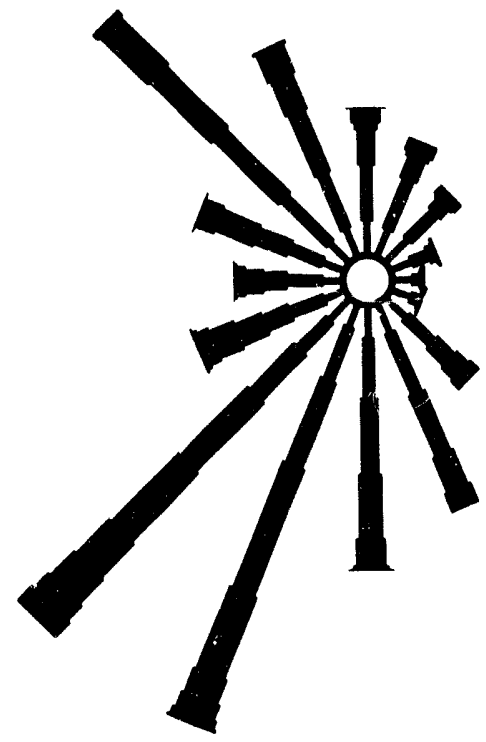

(a) Wind Rose

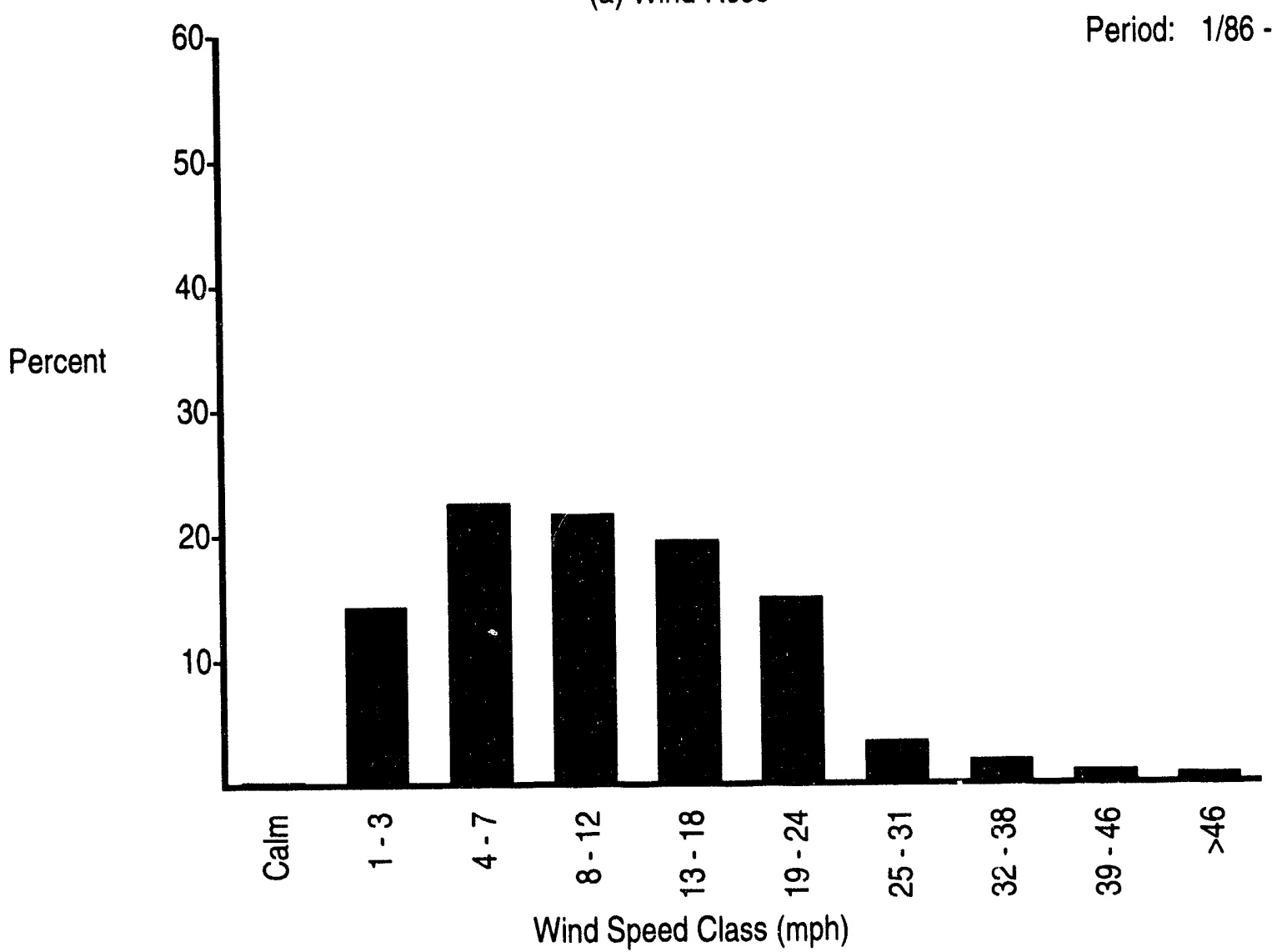

(b) Wind Speed Histogram

FIGURE B.2. (contd)
November Data

Period: 1/86 - 12/93 


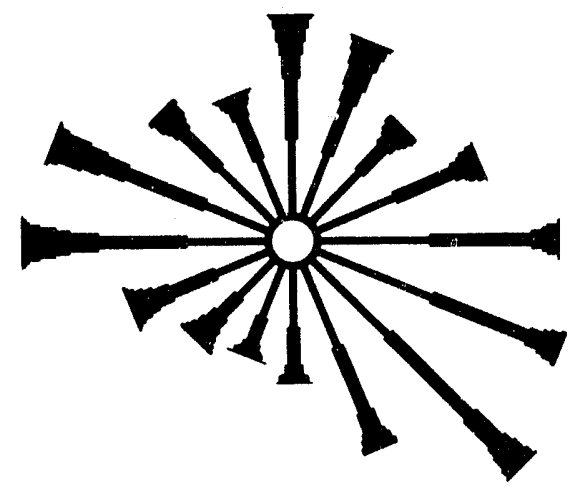

(a) Wind Rose

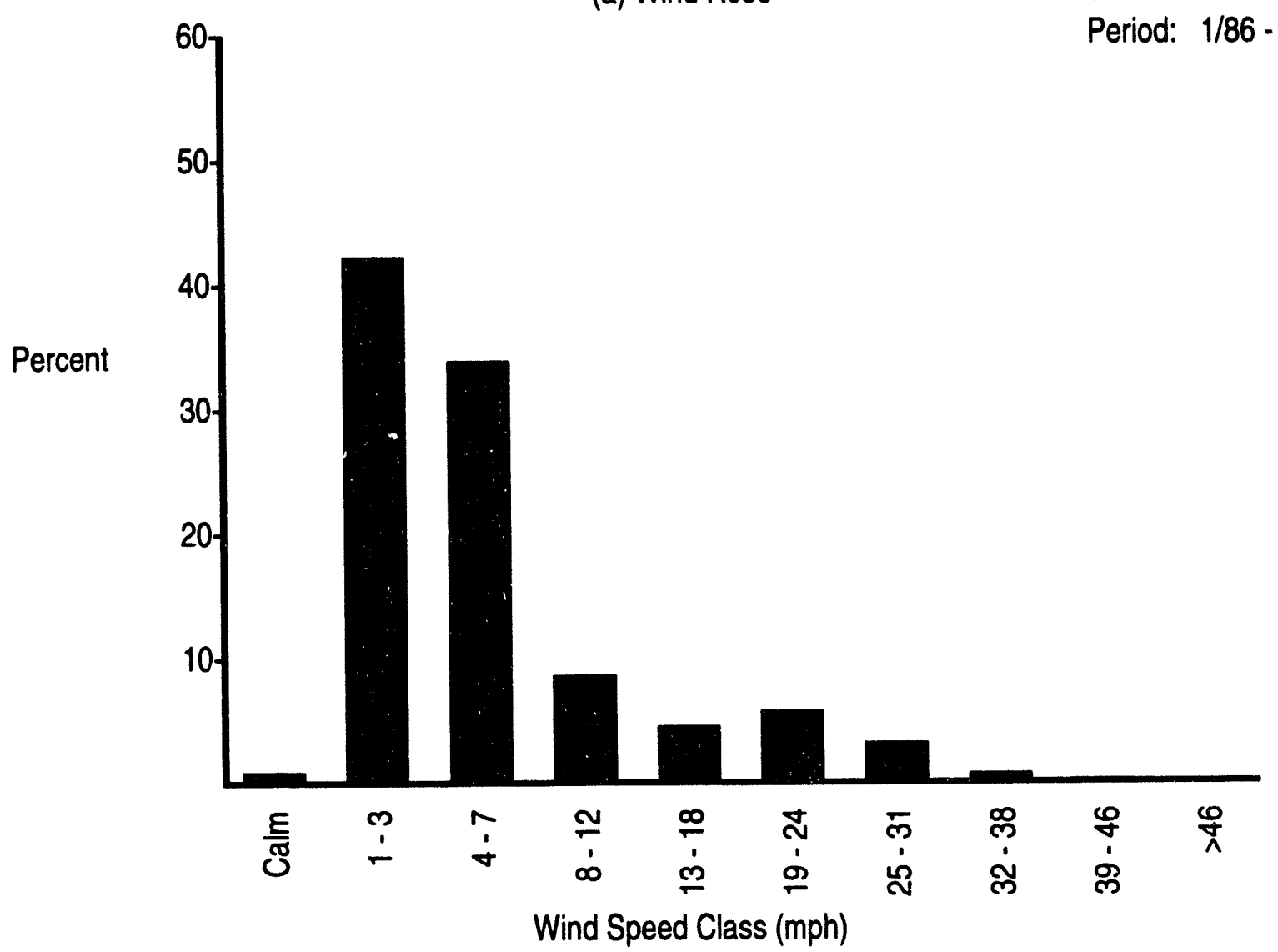

(b) Wind Speed Histogram

FIGURE B.2. (contd)
December Data

Period: $1 / 86-12 / 93$ 

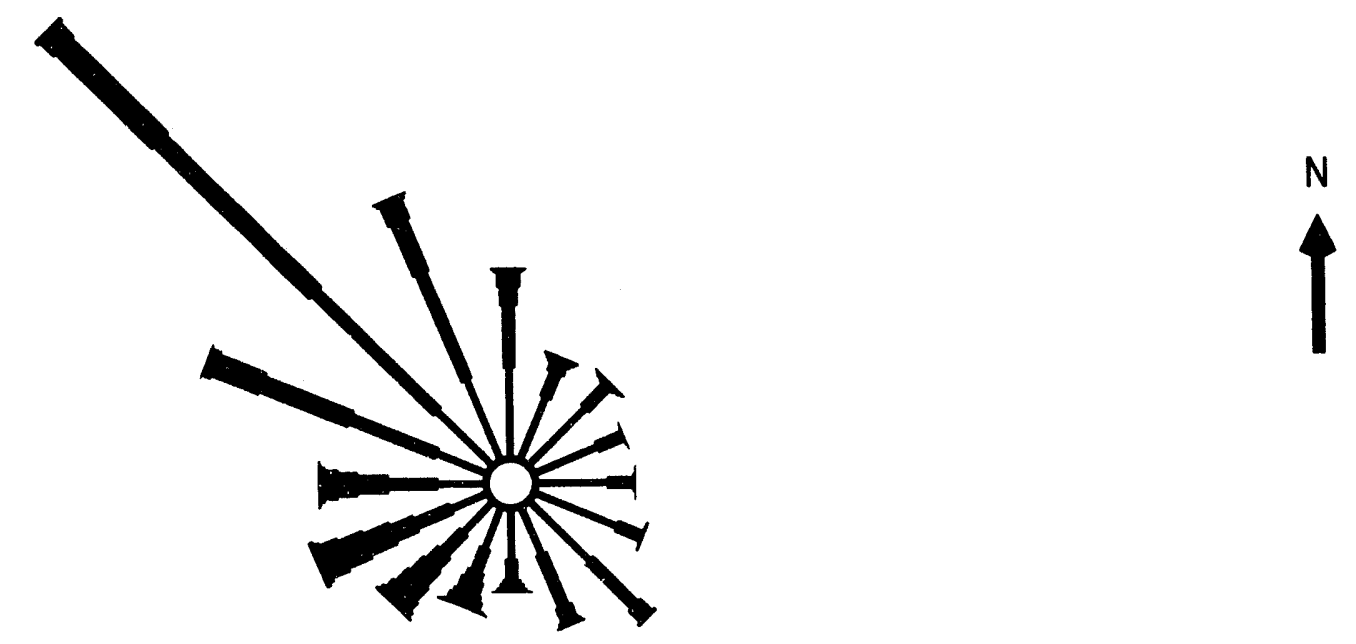

(a) Wind Rose

December Data

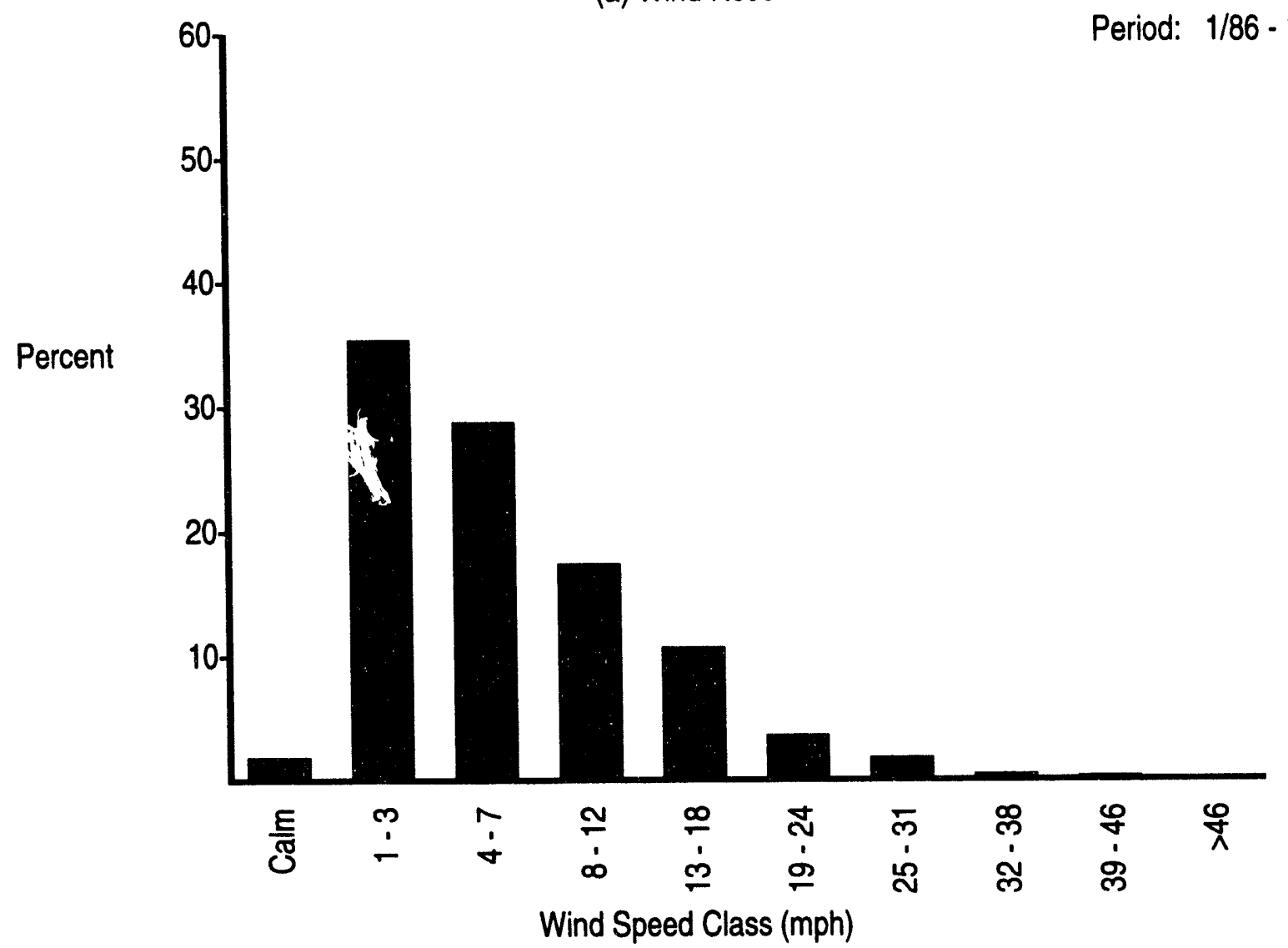

(b) Wind Speed Histogram

FIGURE B.2. (contd) 


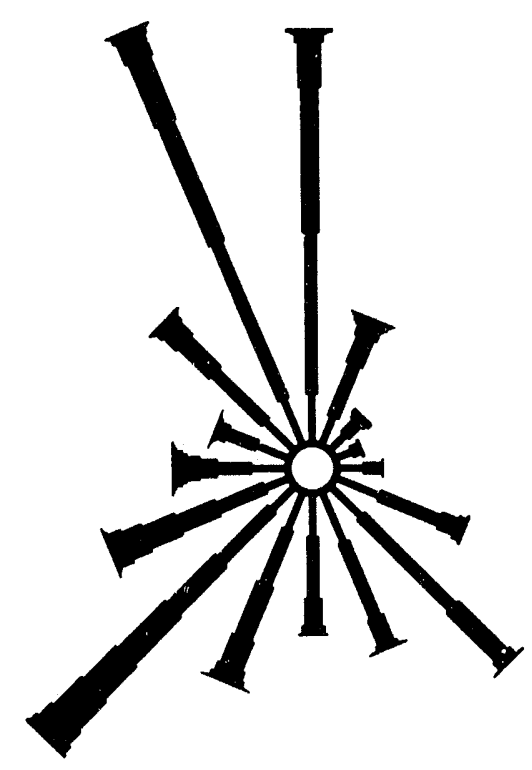

(a) Wind Rose

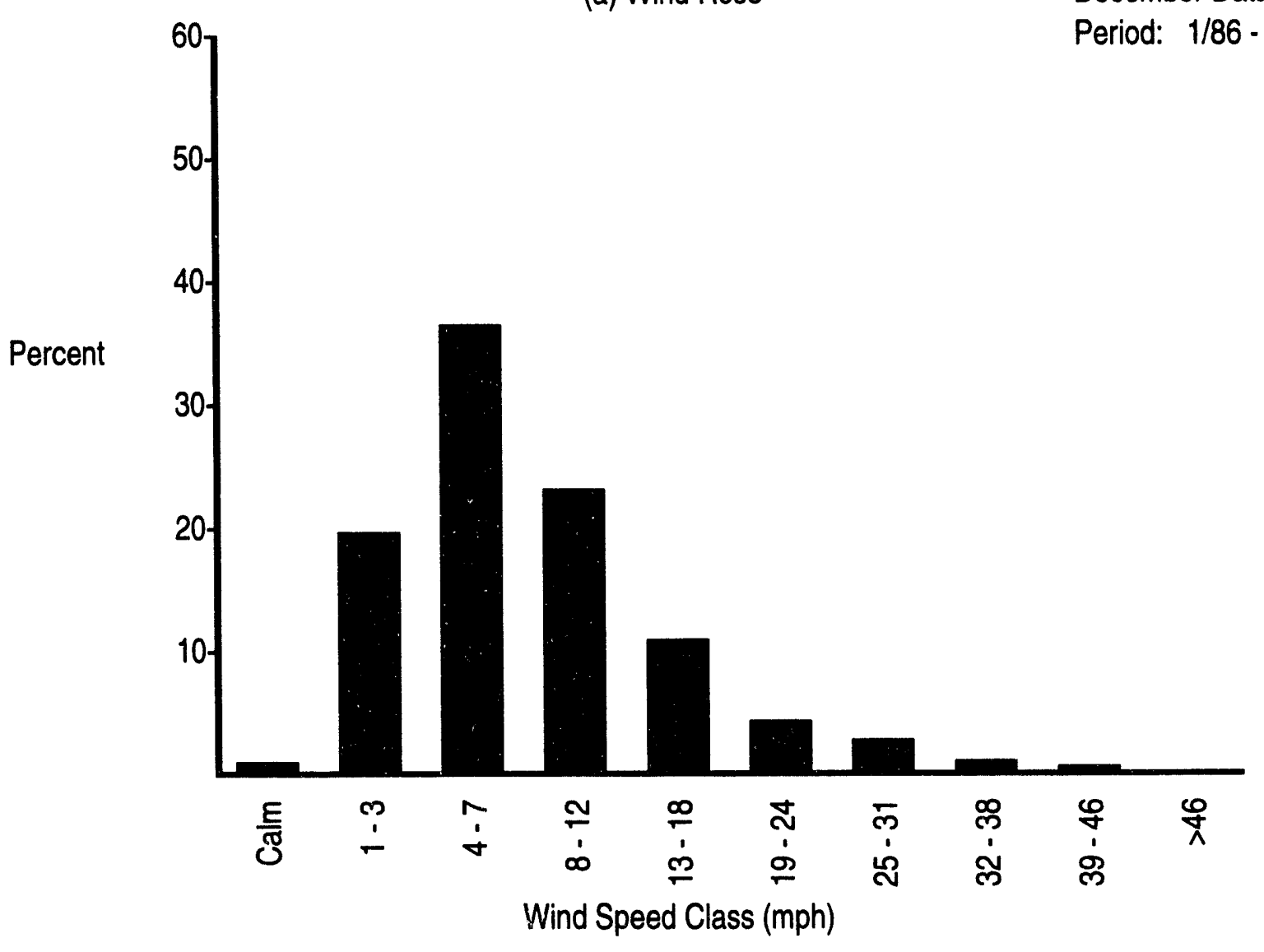

(b) Wind Speed Histogram

FIGURE B.2. (contd)

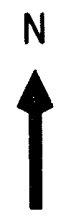

December Data

Period: 1/86-12/93

\section{B.371}



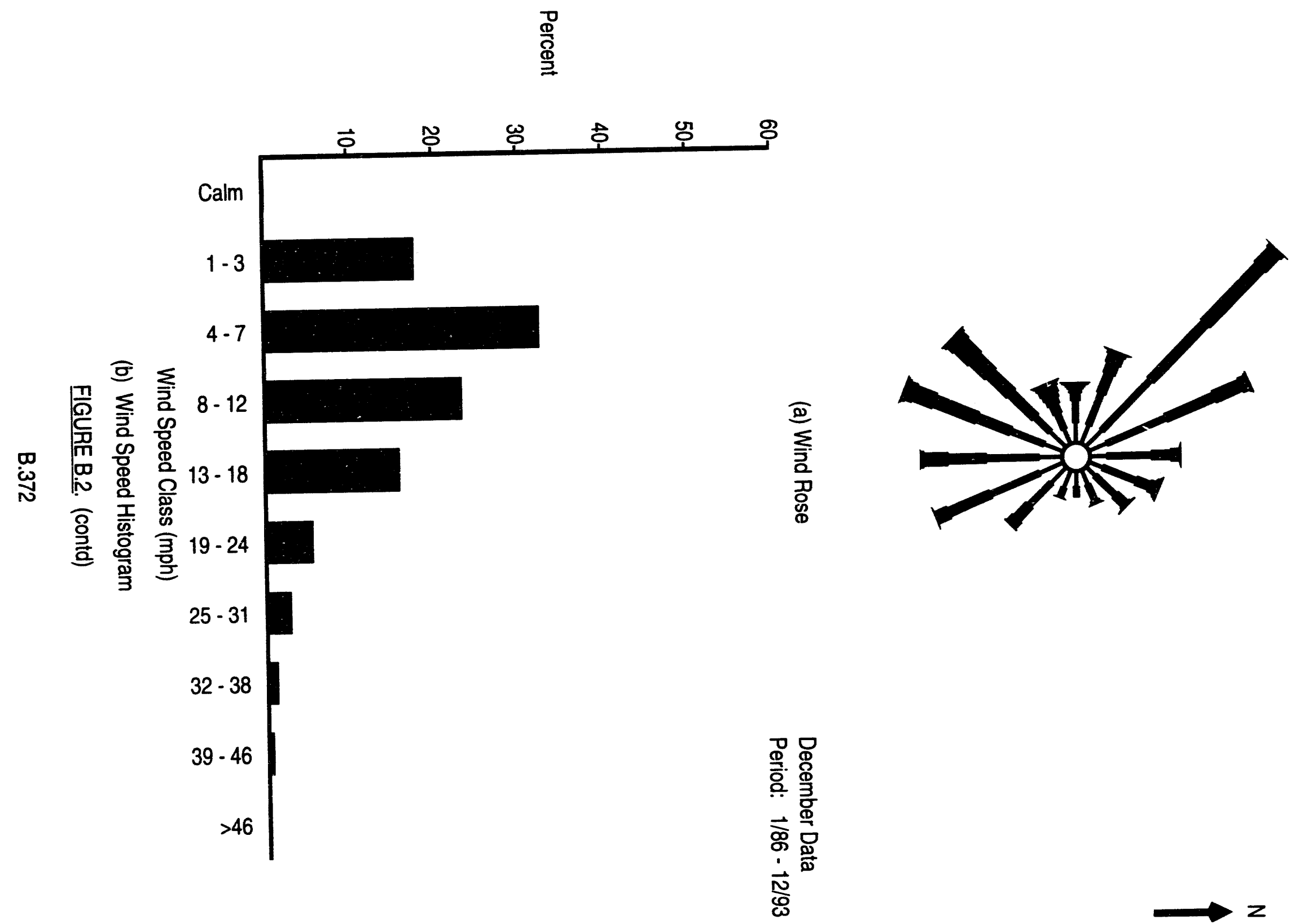
TABLE B.1. 1982-1993 Joint Frequency Distributions for Meteorological Monitoring Network Wind Stations

Station: (1) PROS

Begin: $1 / 82$ DIRECTION End: $12 / 93$

Total Hours: 100901

\begin{tabular}{|c|c|c|c|c|c|c|c|c|c|c|c|c|c|c|c|c|c|c|}
\hline $\begin{array}{r}\text { CALM } \\
1-3 \\
4-7 \\
8-12\end{array}$ & $\begin{array}{r}N \\
.0 \\
2.3 \\
3.4 \\
1.9 \\
.6 \\
.1 \\
.0 \\
.0 \\
.0 \\
.0 \\
8.3\end{array}$ & $\begin{array}{c}\text { NNE } \\
.0 \\
1.6 \\
2.0 \\
.9 \\
.2 \\
.1 \\
.0 \\
.0 \\
.0 \\
.0 \\
4.8\end{array}$ & $\begin{array}{l}\text { NE } \\
.0 \\
1.4 \\
1.0 \\
.3 \\
.1 \\
.0 \\
.0 \\
.0 \\
.0 \\
.0 \\
2.8\end{array}$ & $\begin{array}{c}\text { ENE } \\
.0 \\
1.0 \\
.7 \\
.1 \\
.0 \\
.0 \\
.0 \\
.0 \\
.0 \\
.0 \\
1.8\end{array}$ & $\begin{array}{r}E \\
.0 \\
1.1 \\
.8 \\
.1 \\
.0 \\
.0 \\
.0 \\
.0 \\
.0 \\
.0 \\
2.0\end{array}$ & $\begin{array}{c}\text { ESE } \\
.0 \\
1.3 \\
1.0 \\
.2 \\
.0 \\
.0 \\
.0 \\
.0 \\
.0 \\
.0 \\
2.6\end{array}$ & $\begin{array}{r}\text { SE } \\
.0 \\
1.8 \\
2.0 \\
.4 \\
.0 \\
.0 \\
.0 \\
.0 \\
.0 \\
.0 \\
4.2\end{array}$ & $\begin{array}{c}\text { SSE } \\
.0 \\
2.3 \\
3.8 \\
.9 \\
.0 \\
.0 \\
.0 \\
.0 \\
.0 \\
.0 \\
7.0\end{array}$ & $\begin{array}{r}S \\
.0 \\
2.6 \\
4.6 \\
2.2\end{array}$ & $\begin{array}{r}\text { SSW } \\
.0 \\
2.3 \\
3.7 \\
4.4 \\
2.2 \\
.4 \\
.1 \\
.0 \\
.0 \\
.0 \\
13.1\end{array}$ & $\begin{array}{r}S W \\
.0 \\
1.8 \\
2.1 \\
2.5 \\
2.0 \\
.7 \\
.3 \\
.1 \\
.0 \\
.0 \\
9.5\end{array}$ & $\begin{array}{c}\text { WSW } \\
.0 \\
1.2 \\
.8 \\
.8 \\
.9 \\
.4 \\
.1 \\
.0 \\
.0 \\
.0 \\
4.2\end{array}$ & $\begin{array}{r}.0 \\
1.1 \\
.7\end{array}$ & $\begin{array}{c}\text { WNW } \\
.0 \\
1.3 \\
.9 \\
.5 \\
.2 \\
.0 \\
.0 \\
.0 \\
.0 \\
.0 \\
3.0\end{array}$ & $\begin{array}{r}\text { NW } \\
.0 \\
2.1 \\
2.9 \\
2.5 \\
1.5\end{array}$ & $\begin{array}{c}\text { NNW } \\
.0 \\
2.7 \\
5.0 \\
3.9 \\
1.2 \\
.2\end{array}$ & $\begin{array}{l}\text { CALM } \\
1.4 \\
.0\end{array}$ & $\begin{array}{r}\text { TOTAL } \\
1.4 \\
27.9 \\
35.4 \\
22.2 \\
9.7 \\
2.4\end{array}$ \\
\hline
\end{tabular}

Station: ( 2) EOC

Begin: $1 / 82$ End: $12 / 93$

Total Hours: 101797

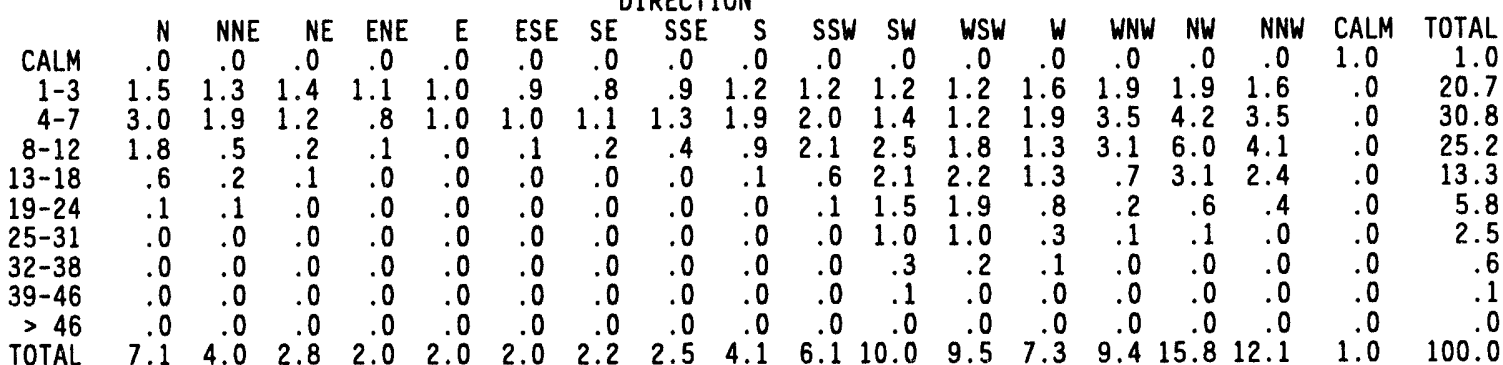

Station: ( 3) ARMY

Begin: 1/82 End: $12 / 93$

Total Hours: 101661

$\begin{array}{rrrrrrrrrrrrrrrrrrr} & \text { N } & \text { NNE } & \text { NE } & \text { ENE } & \text { E } & \text { ESE } & \text { SE } & \text { SSE } & \text { S } & \text { SSW } & \text { SW } & \text { WSW } & \text { W } & \text { WNW } & \text { NW } & \text { NNW } & \text { CALM } & \text { TOTAL } \\ \text { CALM } & .0 & .0 & .0 & .0 & .0 & .0 & .0 & .0 & .0 & .0 & .0 & .0 & .0 & .0 & .0 & .0 & 1.1 & 1.1 \\ 1-3 & 1.9 & 1.7 & 2.1 & 2.4 & 2.7 & 2.5 & 1.9 & 1.2 & .9 & .8 & .9 & 1.2 & 2.1 & 3.2 & 3.1 & 2.4 & .0 & 31.1 \\ 4-7 & 2.1 & 1.5 & 1.6 & 1.9 & 2.6 & 2.8 & 2.2 & 1.0 & .6 & .5 & .6 & .9 & 2.4 & 7.1 & 7.6 & 3.5 & .0 & 38.7 \\ 8-12 & .8 & .5 & .3 & .2 & .4 & .7 & .9 & .5 & .4 & .4 & .6 & 1.1 & 1.8 & 4.5 & 4.0 & 1.4 & .0 & 18.5 \\ 13-18 & .2 & .1 & .1 & .0 & .0 & .1 & .2 & .1 & .2 & .2 & .6 & 1.1 & 1.0 & 1.1 & 1.6 & .4 & .0 & 7.0 \\ 19-24 & .0 & .0 & .0 & .0 & .0 & .0 & .0 & .0 & .0 & .1 & .5 & .6 & .3 & .2 & .6 & .1 & .0 & 2.5 \\ 25-31 & .0 & .0 & .0 & .0 & .0 & .0 & .0 & .0 & .0 & .1 & .3 & .2 & .1 & .0 & .1 & .0 & .0 & .9 \\ 32-38 & .0 & .0 & .0 & .0 & .0 & .0 & .0 & .0 & .0 & .0 & .1 & .0 & .0 & .0 & .0 & .0 & .0 & .0 \\ 39-46 & .0 & .0 & .0 & .0 & .0 & .0 & .0 & .0 & .0 & .0 & .0 & .0 & .0 & .0 & .0 & .0 & .0 & .0 \\ >46 & .0 & .0 & .0 & .0 & .0 & .0 & .0 & .0 & .0 & .0 & .0 & .0 & .0 & .0 & .0 & .0 & .0 & .0 \\ \text { TOTAL } & 5.1 & 3.9 & 4.1 & 4.5 & 5.7 & 6.1 & 5.2 & 2.9 & 2.1 & 2.2 & 3.6 & 5.0 & 7.6 & 16.0 & 17.0 & 7.9 & 1.1 & 100.0\end{array}$

Station: ( 4) RSPG

Begin: $1 / 82$ End: $12 / 93$

Total Hours: 101623

$\begin{array}{rrrrrrrrrrrrrrrrrrr} & \text { N } & \text { NNE } & \text { NE } & \text { ENE } & \text { E } & \text { ESE } & \text { SE } & \text { SSE } & \text { S } & \text { SSW } & \text { SW } & \text { WSW } & \text { W } & \text { WNW } & \text { NW } & \text { NNW } & \text { CALM } & \text { TOTAL } \\ \text { CALM } & .0 & .0 & .0 & .0 & .0 & .0 & .0 & .0 & .0 & .0 & .0 & .0 & .0 & .0 & .0 & .0 & 1.2 & 1.2 \\ 1-3 & 1.8 & 2.0 & 2.2 & 1.8 & 1.9 & 1.9 & 1.2 & .8 & .8 & 1.1 & 2.0 & 3.0 & 1.9 & 1.1 & 1.2 & 1.3 & .0 & 26.2 \\ 4-7 & 2.5 & 2.1 & 1.5 & .9 & 1.6 & 2.0 & .8 & .4 & .5 & .8 & 2.6 & 10.3 & 4.3 & 1.8 & 1.8 & 2.2 & .0 & 36.1 \\ 8-12 & .5 & .4 & .2 & .1 & .1 & .3 & .1 & .1 & .2 & .6 & 1.8 & 13.3 & 4.6 & 2.2 & 1.5 & 1.1 & .0 & 27.0 \\ 13-18 & .0 & .1 & .1 & .0 & .0 & .0 & .0 & .0 & .1 & .5 & 1.0 & 1.8 & 1.7 & .8 & .8 & .4 & .0 & 7.4 \\ 19-24 & .0 & .0 & .0 & .0 & .0 & .0 & .0 & .0 & .0 & .3 & .5 & .4 & .2 & .1 & .1 & .1 & .0 & 1.7 \\ 25-31 & .0 & .0 & .0 & .0 & .0 & .0 & .0 & .0 & .0 & .1 & .1 & .1 & .0 & .0 & .0 & .0 & .0 & .4 \\ 32-38 & .0 & .0 & .0 & .0 & .0 & .0 & .0 & .0 & .0 & .0 & .0 & .0 & .0 & .0 & .0 & .0 & .0 & .0 \\ 39-46 & .0 & .0 & .0 & .0 & .0 & .0 & .0 & .0 & .0 & .0 & .0 & .0 & .0 & .0 & .0 & .0 & .0 & .0 \\ >46 & .0 & .0 & .0 & .0 & .0 & .0 & .0 & .0 & .0 & .0 & .0 & .0 & .0 & .0 & .0 & .0 & .0 & .0 \\ >\text { TOTAL } & 4.8 & 4.6 & 4.0 & 2.8 & 3.6 & 4.2 & 2.2 & 1.3 & 1.7 & 3.5 & 8.0 & 29.0 & 12.8 & 6.1 & 5.3 & 5.1 & 1.2 & 100.0\end{array}$ 
TABLE B.1. (contd)

Station: (5) EDNA

Begin: $1 / 82$ End: $12 / 93$

Total Hours: 101997

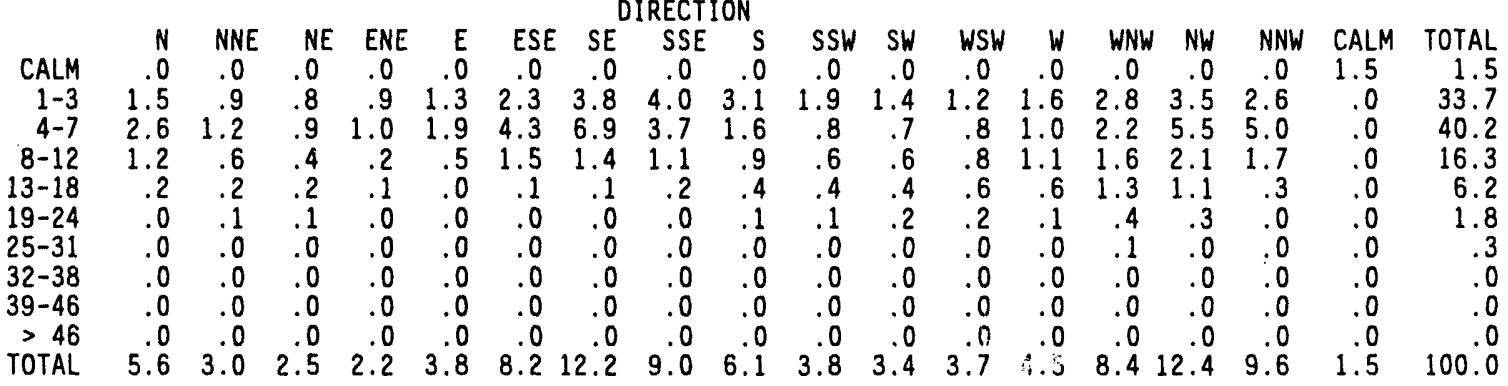

Station: ( 6) $200 E$

Begin: $1 / 82$ End: 12/93

Total Hours: 101495

\begin{tabular}{|c|c|c|c|c|c|c|c|c|c|c|c|c|c|c|c|c|c|c|}
\hline & & & & & & & & IRECTI & ION & & & & & & & & & \\
\hline & N & NNE & NE & ENE & $E$ & ESE & SE & SSE & $\mathrm{S}$ & SSW & SW & WSW & $W$ & WNW & NW & NNW & CALM & TOTAL \\
\hline CALM & .0 & .0 & .0 & .0 & .0 & .0 & 0 & .0 & .0 & .0 & 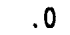 & .0 & 0 & .0 & .0 & .0 & & \\
\hline $1-3$ & 1.6 & 1.5 & 1.6 & 1.7 & 1.8 & 1.8 & 1.7 & 1.3 & 1.1 & 1.0 & 1.0 & 1.1 & 1.5 & 1.9 & 2.1 & 1. & & \\
\hline 7 & 1.7 & 1.5 & 1.1 & 1.1 & 1.5 & 2.1 & 3.0 & 2.4 & 1.5 & 1.0 & 1. & 2.1 & 4.1 & 6.2 & 4. & 2. & & \\
\hline $3-1$ & .7 & .7 & .3 & .1 & .2 & .3 & .8 & 1.1 & & .4 & .5 & 1.9 & 4 & 7.6 & 3. &. & & \\
\hline $3-$ & .2 & .2 & .1 & .0 & .0 & .0 & .1 & .2 & .2 & 3 & .7 & 1.2 & 1. & 3 & 1. & . & & \\
\hline & .0 & 1 & .0 & 0 & .0 & .0 & 0 & 8 & .0 & .1 & .4 & .0 &. & $y$ & $\cdot$ & .0 & & 0 \\
\hline & .0 & 0 & .0 & 0 & 0 & 0 & 0 & 0 & 0 & 0 & 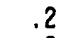 & , & & , & & . & & \\
\hline & .0 & .0 & .0 & .0 & .0 & .0 & .0 & .0 & .0 & .0 & .0 & & & & & . & & \\
\hline $39-$ & .0 & 0 & .0 & .0 & & .0 & .0 & .0 & & 0 & & .0 & & .0 & .0 & .0 & & \\
\hline & .0 & .0 & .0 & .0 & .0 & .0 & .0 & .0 & .0 & .0 & f & .0 & & .0 & .0 & .0 & & \\
\hline DTh & 4.3 & 3.9 & 3.2 & 2.9 & 3.6 & 4.3 & 5.5 & 5.0 & 3.4 & 2.9 & 4.8 & 7.21 & 11.0 & 20.2 & 12.3 & 4.9 & .7 & 100.0 \\
\hline
\end{tabular}

Station: (7) 200W

Begin: $1 / 82$ EIRECTION End: 12/93

Total Hours: 87866

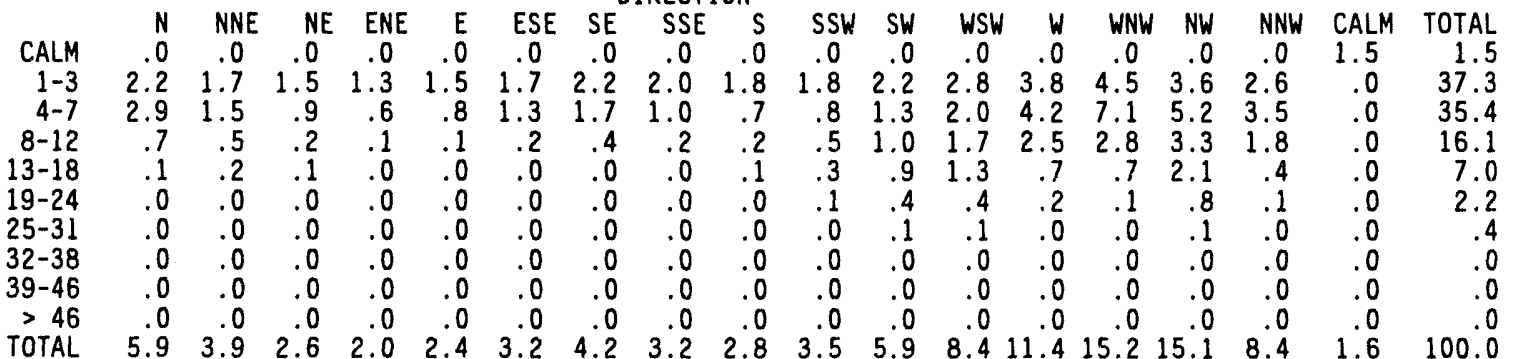

Station: ( 8 B) BVLY

Begin: 8/91 End: $12 / 93$

Total Hours: 20720

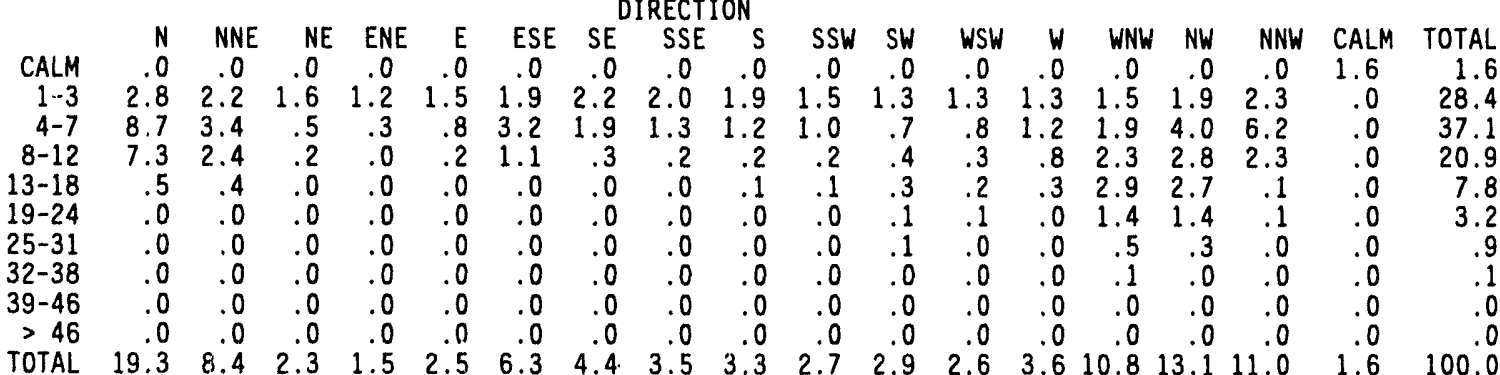


TABLE B.1. (contd)

Station: ( $8 W$ W WAHL

Begin: $1 / 82$

Total Hours:

79579

\begin{tabular}{|c|c|c|c|c|c|c|c|c|c|c|c|c|c|c|c|c|c|c|}
\hline $\begin{array}{l}1-3 \\
4-7\end{array}$ & $\begin{array}{r}N \\
.0 \\
1.9 \\
1.3 \\
.6 \\
.3 \\
.1 \\
.0 \\
.0 \\
.0 \\
.0 \\
4.0\end{array}$ & $\begin{array}{c}\text { NNE } \\
.0 \\
2.0 \\
1.7 \\
.9 \\
.3 \\
.1 \\
.0 \\
.0 \\
.0 \\
.0 \\
5.0\end{array}$ & $\begin{array}{c}\text { NE } \\
.0 \\
2.9 \\
1.8 \\
.5 \\
.2 \\
.0 \\
.0 \\
.0 \\
.0 \\
.0 \\
5.3\end{array}$ & $\begin{array}{c}\text { ENE } \\
.0 \\
3.4 \\
2.8 \\
.5 \\
.1 \\
.0 \\
.0 \\
.0 \\
.0 \\
.0 \\
6.7\end{array}$ & $\begin{array}{r}E \\
.0 \\
4.6 \\
5.2 \\
.8 \\
.1 \\
.0 \\
.0 \\
.0 \\
.0 \\
.0 \\
10.7\end{array}$ & $\begin{array}{c}\text { ESE } \\
.0 \\
3.1 \\
1.9 \\
.1 \\
.0 \\
.0 \\
.0 \\
.0 \\
.0 \\
.0 \\
5.2\end{array}$ & $\begin{array}{r}\text { SE } \\
.0 \\
2.2 \\
.8 \\
.1 \\
.0 \\
.0 \\
.0 \\
.0 \\
.0 \\
.0 \\
3.1\end{array}$ & $\begin{array}{r}\text { SSE } \\
.0 \\
1.6 \\
.5 \\
.1 \\
.0 \\
.0 \\
.0 \\
.0 \\
.0 \\
.0 \\
2.2\end{array}$ & $\begin{array}{r}5 \\
.0 \\
1.7 \\
.6 \\
.2 \\
.1 \\
.0 \\
.0 \\
.0 \\
.0 \\
.0 \\
2.5\end{array}$ & $\begin{array}{c}\text { SSW } \\
.0 \\
1.7 \\
.8 \\
.3 \\
.2 \\
.1 \\
.0 \\
.0 \\
.0 \\
.0 \\
3.0\end{array}$ & $\begin{array}{r}\text { SW } \\
.0 \\
1.8 \\
.9 \\
.5 \\
.5 \\
.2 \\
.1 \\
.0 \\
.0 \\
.0 \\
4.0\end{array}$ & $\begin{array}{c}\text { WSW } \\
.0 \\
2.1 \\
1.8 \\
.7 \\
.4 \\
.1 \\
.0 \\
.0 \\
.0 \\
.0 \\
5.2\end{array}$ & $\begin{array}{r}W \\
.0 \\
2.8 \\
4.7 \\
2.9 \\
1.2 \\
.2\end{array}$ & $\begin{array}{r}\text { WNW } \\
.0 \\
2.7 \\
5.3 \\
4.4 \\
3.3 \\
1.2 \\
.3 \\
.0 \\
.0 \\
.0 \\
17.2\end{array}$ & $\begin{array}{r}\text { NW } \\
.0 \\
2.4 \\
2.7 \\
1.5 \\
1.2\end{array}$ & $\begin{array}{r}\text { NNW } \\
. U \\
2.1 \\
1.2 \\
.5 \\
.2 \\
.1 \\
.0 \\
.0 \\
.0 \\
.0 \\
4.1\end{array}$ & $\begin{array}{l}.0 \\
.0 \\
.0 \\
.0 \\
.0 \\
.0 \\
1.3\end{array}$ & OT \\
\hline
\end{tabular}

Station: (9) FFTF

Begin: $1 / 82$ End: $12 / 93$

Total Hours: 101168

CALM N NNE NE ENE E ESE SE SSE S SSW SW WSW W WNW NW NNW CALM TOTAL

$\begin{array}{lllllllllllllllllllll}1-3 & 1.0 & .0 & .0 & .0 & .0 & .0 & .0 & .0 & .0 & .0 & .0 & .0 & .0 & .0 & .0 & .0 & .4 & .4\end{array}$

$\begin{array}{lllllllllllllllllll}4-7 & 2.7 & 2.5 & 2.0 & 1.1 & 1.1 & 1.4 & 2.6 & 3.8 & 4.1 & 3.6 & 2.0 & 1.2 & 1.5 & 2.2 & 3.4 & 3.1 & .0 & 38.4\end{array}$

$\begin{array}{lllllllllllllllllll}8-12 & 1.4 & 1.3 & .7 & .2 & .2 & .2 & 1.0 & 3.1 & 3.8 & 4.5 & 1.8 & .8 & 1.0 & 2.1 & 4.0 & 2.4 & .0 & 28.6\end{array}$

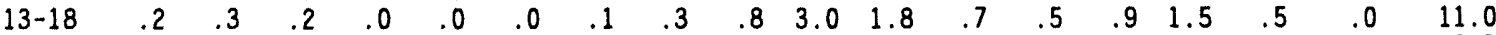

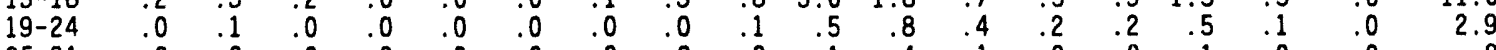

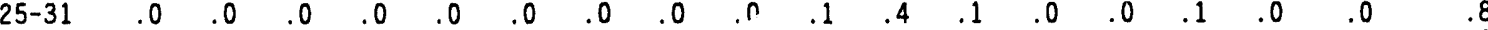

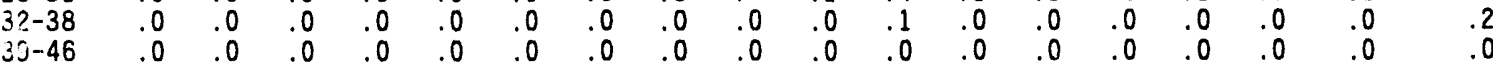

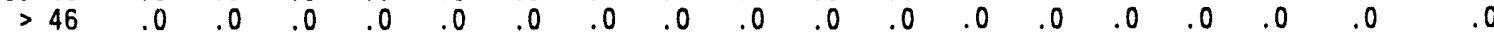

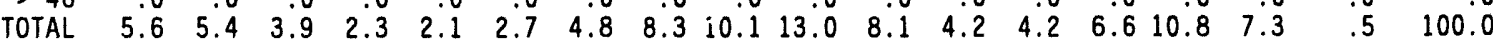

Station: (10) YAKB

Begin: $1 / 82$ End: $12 / 93$

Total Hours: 101819

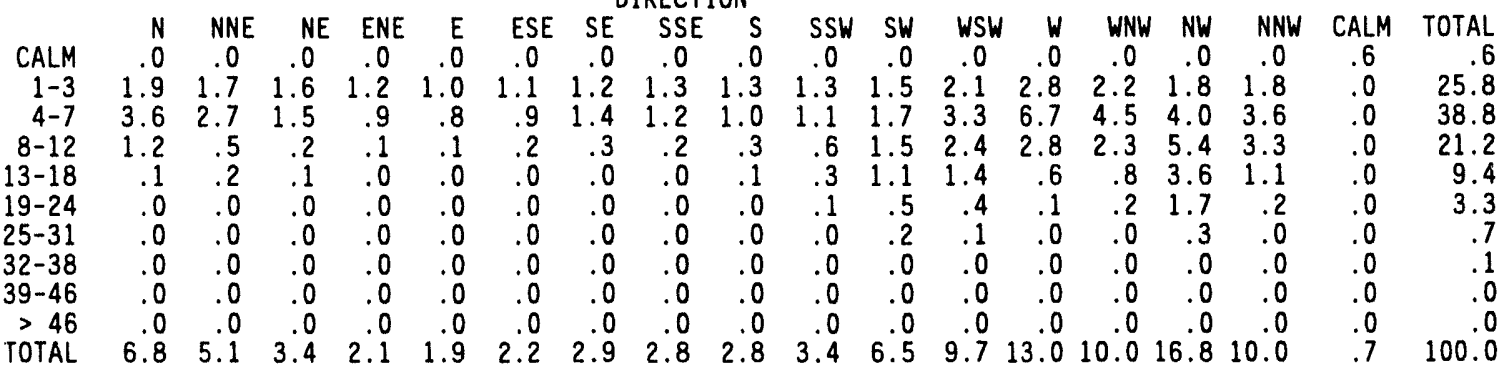

Station: (11) 300A

Begin: $1 / 82$

End: $12 / 93$

Total Hours:

88577

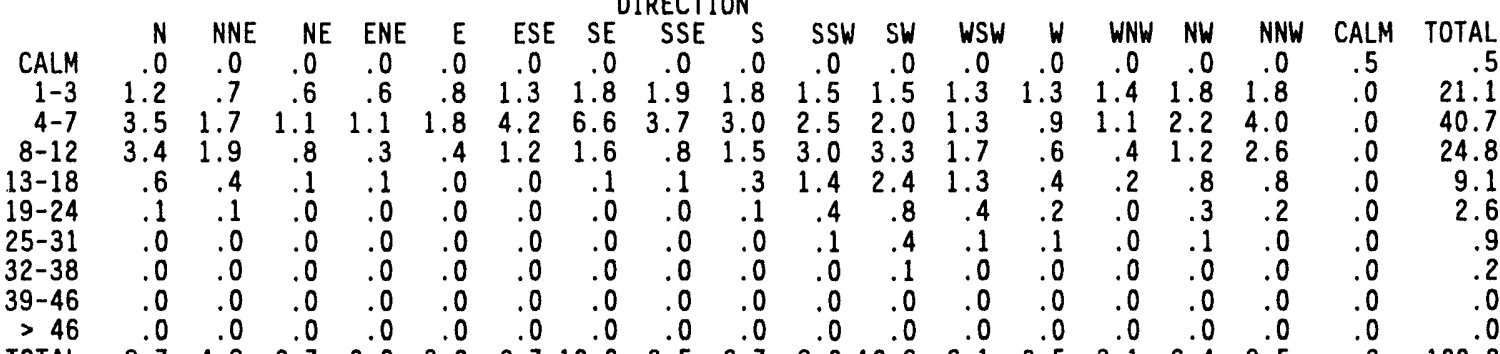

$\begin{array}{lllllllllllllllllll}\text { TOTAL } & 8.7 & 4.8 & 2.7 & 2.0 & 3.0 & 6.7 & 10.0 & 6.5 & 6.7 & 9.0 & 10.6 & 6.1 & 3.5 & 3.1 & 6.4 & 9.5 & .6 & 100.0\end{array}$ 
TABLE B.1. (contd)

Station: (12) WYEB

Begin: $1 / 82$ End: $12 / 93$

Total Hours: 101150

$\begin{array}{rrrrrrrrrrrrrrrrrrr} & \text { N } & \text { NNE } & \text { NE } & \text { ENE } & \text { E } & \text { ESE } & \text { SE } & \text { SSE } & \text { S } & \text { SSW } & \text { SW } & \text { WSW } & \text { W } & \text { WNW } & \text { NW } & \text { NNW } & \text { CALM } & \text { TOTAL } \\ \text { CALM } & .0 & .0 & .0 & .0 & .0 & .0 & .0 & .0 & .0 & .0 & .0 & .0 & .0 & .0 & .0 & .0 & .4 & .4 \\ 1-3 & 1.2 & 1.1 & 1.1 & 1.1 & 1.3 & 1.5 & 1.4 & 1.4 & 1.5 & 1.3 & 1.4 & 1.3 & 1.2 & 1.2 & 1.3 & 1.3 & .0 & 20.5 \\ 4-7 & 2.3 & 1.5 & 1.2 & 1.1 & 1.9 & 1.8 & 2.4 & 3.1 & 3.9 & 3.1 & 2.7 & 2.5 & 2.9 & 3.3 & 3.7 & 2.8 & .0 & 40.3 \\ 8-12 & 1.1 & .6 & .3 & .2 & .3 & .3 & .7 & 1.6 & 2.8 & 2.8 & 1.9 & 1.5 & 2.3 & 4.3 & 3.2 & 1.5 & .0 & 25.2 \\ 13-18 & .3 & .2 & .1 & .0 & .0 & .0 & .1 & .2 & .8 & 1.5 & 1.1 & .8 & .9 & 1.7 & 1.4 & .4 & .0 & 9.6 \\ 19-24 & .0 & .1 & .0 & .0 & .0 & .0 & .0 & .0 & .1 & .4 & .6 & .4 & .2 & .4 & .6 & .1 & .0 & 3.0 \\ 25-31 & .0 & .0 & .0 & .0 & .0 & .0 & .0 & .0 & .0 & .1 & .3 & .1 & .1 & .1 & .2 & .0 & .0 & .9 \\ 32-38 & .0 & .0 & .0 & .0 & .0 & .0 & .0 & .0 & .0 & .0 & .1 & .0 & .0 & .0 & .0 & .0 & .0 & .1 \\ 39-46 & .0 & .0 & .0 & .0 & .0 & .0 & .0 & .0 & .0 & .0 & .0 & .0 & .0 & .0 & .0 & .0 & .0 & .0 \\ >46 & .0 & .0 & .0 & .0 & .0 & .0 & .0 & .0 & .0 & .0 & .0 & .0 & .0 & .0 & .0 & .0 & .0 & .0 \\ \text { TOTAL } & 4.9 & 3.5 & 2.7 & 2.5 & 3.4 & 3.6 & 4.5 & 6.4 & 9.1 & .0 .3 & \mathbf{8 . 1} & 6.5 & 7.6 & 10.9 & 10.3 & 6.1 & .4 & 100.0\end{array}$

Station: (13) 100N

Begin: $1 / 82$

End: $12 / 93$

Total Hours: 101552

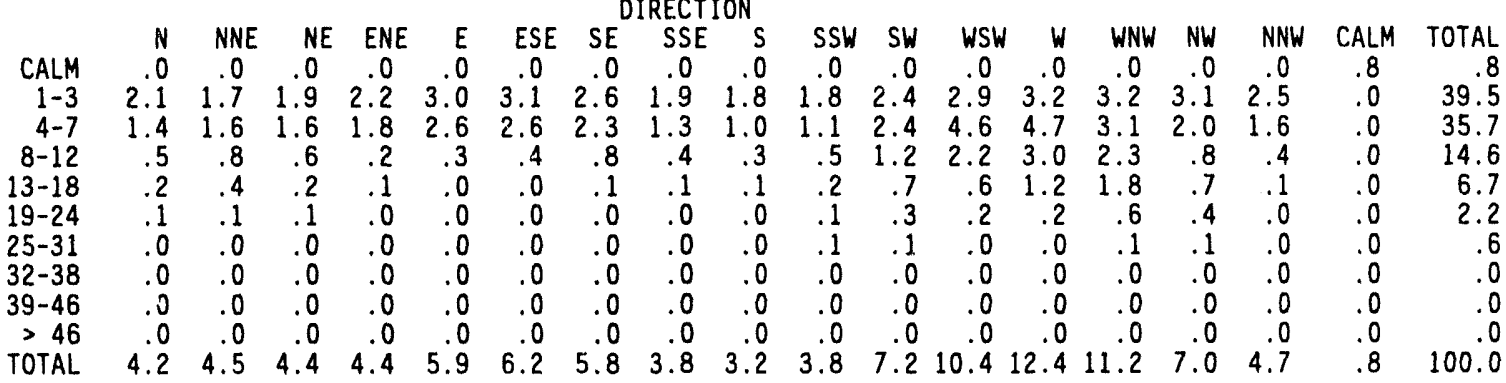

Station: (14) WPPS

\begin{tabular}{|c|c|c|c|c|c|c|c|c|c|c|c|c|c|c|c|c|c|c|}
\hline & & & & & Begi & $n: 1$ & /82 & דתחם & $E$ & nd: 1 & $2 / 93$ & & & & Tot & a) $\mathrm{H}$ & s: & 101932 \\
\hline & & & & ENE & $E$ & ESE & $S E$ & SSE & S & SSW & SW & WSW & $W$ & WNW & NW & NNW & CALM & TOTAL \\
\hline $\begin{array}{c}\text { CALM } \\
1-3\end{array}$ & .0 & .0 & .0 & .0 & .0 & .0 & .0 & .0 & .0 & 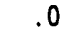 & .0 & .0 & .0 & .0 & .0 & .0 & .8 & \\
\hline $1-3$ & 2.6 & 2.2 & 2.0 & 1.4 & 1.2 & 1.2 & 1.6 & 2.1 & 2.5 & 2.2 & 1.9 & 1.6 & 1.7 & 2.0 & 2.9 & 3. & & 3 \\
\hline $4-$ & 3.3 & 2.2 & 2.0 & 1.1 & .7 & .9 & 1.8 & 4.1 & 5.2 & 3. & 1.7 & 1.2 & 1.3 & 2.0 & 3 & 4. & & \\
\hline $3-1$ & 1.2 & .7 & .4 & .2 & .1 & .2 & .6 & 1.6 & 3.0 & & 1. & .8 & 0 & 1.7 & & 1.3 & & 1) \\
\hline $3-1$ & 3 & .2 & .1 & .0 & .0 & .0 & .0 & .2 & .6 & 1. & 1. & .5 & .4 & .8 & 1. & . & & 7. \\
\hline .2 & & .1 & .0 & .0 & .0 & .0 & .0 & .0 & .0 &. & .5 & .2 & .1 & .1 & . & . & . & 2. \\
\hline & & 0 & 0 & .0 & 0 & .0 & 0 & 0 & & 1 & 2 & .1 & 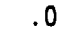 &. & & . & & \\
\hline & & 0 & .0 & .0 & 0 & .0 & .0 & .0 & & 0 & .0 & .0 & .0 &. & .0 & . & . & \\
\hline & & & .0 & .0 & 0 & .0 & 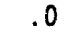 & .0 & & .0 & . & .0 & .0 & .0 & .0 &. & . & \\
\hline & .0 & .0 & .0 & .0 & .0 & .0 & .0 & .0 & .0 & .0 & .0 & .0 & .0 & .0 & &. & . & \\
\hline TTA & 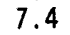 & 5.3 & 4.5 & 2.7 & 2.0 & 2.3 & 4.0 & 8.0 & 11.3 & 9.9 & 6.9 & 4.3 & 4.5 & 6.6 & 10.6 & 8.8 & .8 & 100.0 \\
\hline
\end{tabular}

Station: (15) FRNK

Begin: $1 / 82$ End: $12 / 93$

Total Hours: 101408.

\begin{tabular}{|c|c|c|c|c|c|c|c|c|c|c|c|c|c|c|c|c|c|c|}
\hline & & & & & & & & IRECT & ION & & & & & & & & & \\
\hline CAl & $\begin{array}{l}N \\
0\end{array}$ & NNE & & & & ESE & SE & SSE & $\begin{array}{l}S \\
0\end{array}$ & SSW & & WSW & & WNW & NW & NNW & CALM & L \\
\hline 1 - & 1.2 & 1.0 & 1.0 & 8 & 9 & 1.1 & 1.5 & 1.6 & 13 & 1.1 & 12 & 1.2 & 1.4 & 14 & 1.6 & 1.0 & 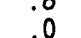 & $19^{\circ}$ \\
\hline $4-7$ & .1 & 2.7 & 1.7 & 1.1 & 1.4 & 2.2 & 4.6 & 4.1 & 3. & 2. & 2. & 1. & 1.4 & 2.0 & 4.4 & 5 & & \\
\hline $8-1$ & .8 & 1.0 & .6 & .3 & .3 & .6 & 1.5 & 1.4 & 2. & 4. & 3. & 1. & .5 & .0 & 2.2 & 2. & & \\
\hline $3-1$ & .2 & .2 & .2 & .1 & • & .0 & .1 & .1 & .4 & 1.9 & 2. & .7 & & & .5 & .2 & & \\
\hline $9-2$ & .0 & 2 & .0 & .0 & .0 & .0 & .0 & 0 & 0 & .3 & t & .2 &. &. & 1 & & & \\
\hline $5-$ & .0 & 0 & .0 & 0 & $c$ & .0 & .0 & .0 & .0 & .1 & & .1 & ( &. & .1 & & & \\
\hline & 0 & 0 & & .0 & .0 & .0 & .0 & .0 & .0 & 0 & .0 &. & .0 & . & .8 & . & & \\
\hline-7 & .0 & .0 & 0 & .0 & 0 & .0 & .0 & .0 & .0 & & 0 & .0 & .0 & .0 & .0 & & & \\
\hline דט & 3 & 50 & 3.5 & 2.4 & $?$ & 3.9 & 7 & 71 & 7.5 & 10 & 0.3 & 4.6 & 3.6 & 4.3 & 8.9 & 9.5 & $y$ & \\
\hline
\end{tabular}


TABLE B.1. (contd)

Station: (16) GABL

Begin: 1/82 End: 12/93

Total Hours: 100922

\begin{tabular}{|c|c|c|c|c|c|c|c|c|c|c|c|c|c|c|c|c|c|c|}
\hline & $\mathrm{N}$ & NNE & NE & ENE & $E$ & ESE & SE & SSE & $S$ & SSW & SW & WSW & $W$ & WNW & NW & NNI & CALM & TOTAL \\
\hline CALM & .0 & .0 & .0 & .0 & .0 & .0 & .0 & .0 & .0 & .0 & 0 & .0 & .0 & .0 & .0 & .0 & .6 & \\
\hline $1-3$ & 1.0 & 1.0 & .9 & .7 & .7 & .6 & .7 & .8 & 1.1 & 1.1 & 1.0 & .9 & .8 & .8 & .9 & 1. & .0 & 14 \\
\hline & 2.3 & 2.4 & 1.6 & .9 & .9 & .9 & 1.3 & 2.1 & 3.2 & 2.5 & 1.8 & 1.6 & 1.6 & 1.7 & 2.2 & 2. & & \\
\hline 8- & 2.1 & 2.2 & 1.0 & .3 & .3 & .5 & .8 & 1.5 & 2. & 1.5 & 1.4 & 1.5 & 1.7 & 2.2 & 2.8 & 1. & & \\
\hline 13- & 1.3 & 1.4 & .5 & .1 & .0 & .1 & .3 & .9 & 1.4 & .9 & 1.3 & 1.5 & 1.7 & 2.8 & 2.5 & .7 & & \\
\hline & .4 & .5 & .3 & .0 & .0 & .0 & .0 & .3 &. & A & . & .8 & .9 & 2.7 & 1. & .2 & & \\
\hline & 1 & 2 & .2 & .0 & .0 & .0 & .0 & .0 & & .3 &. & .4 & .2 & 1.2 & & & & \\
\hline & 0 & .0 & .1 & .0 & .0 & .0 & .0 & .0 & & .1 & .3 & .1 & .0 & 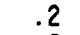 & .0 & . & & \\
\hline & 0 & & .0 & .0 & .0 & .0 & .0 & 0 & .0 & .1 & .1 & .0 & .0 & & .0 & . & & \\
\hline & 0 & .0 & .0 & .0 & .0 & .0 & .0 & .0 & .0 & .0 & .0 & .0 & .0 & 0 & 0 & .0 & .0 & \\
\hline$T$ & 3 & 7.8 & 6 & 2 & 0 & 1 & 0 & 5. & 19 & 68 & 7.3 & 6.9 & 0 & 6 & .6 & 6.0 & 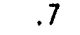 & \\
\hline
\end{tabular}

Station: (17) RING

$$
\text { Begin: 1/82 End: 12/93 }
$$

Total Hours: 101063

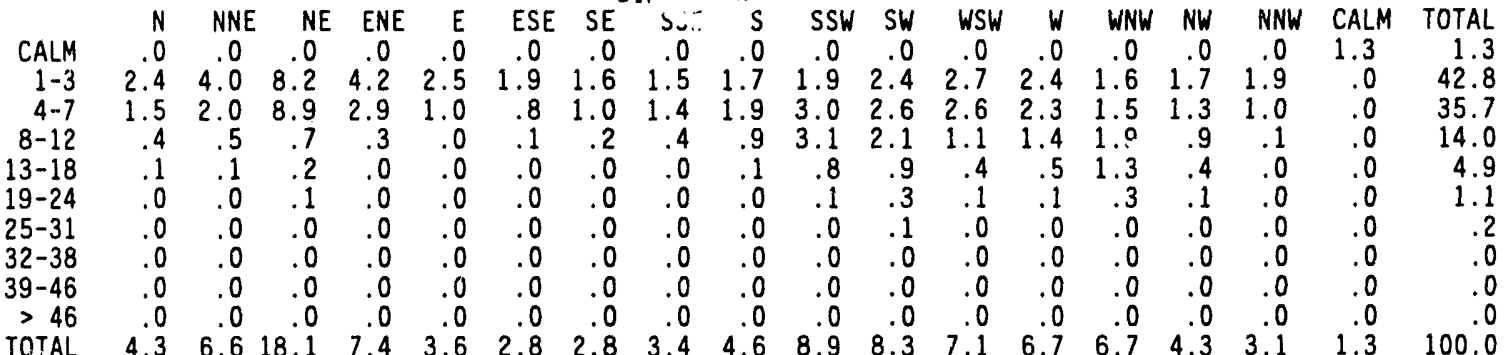

Station: (18) RICH

$$
\text { Begin: } 1 / 82 \text { DIRECTION End: } 12 / 93
$$

Total Hours: 102070

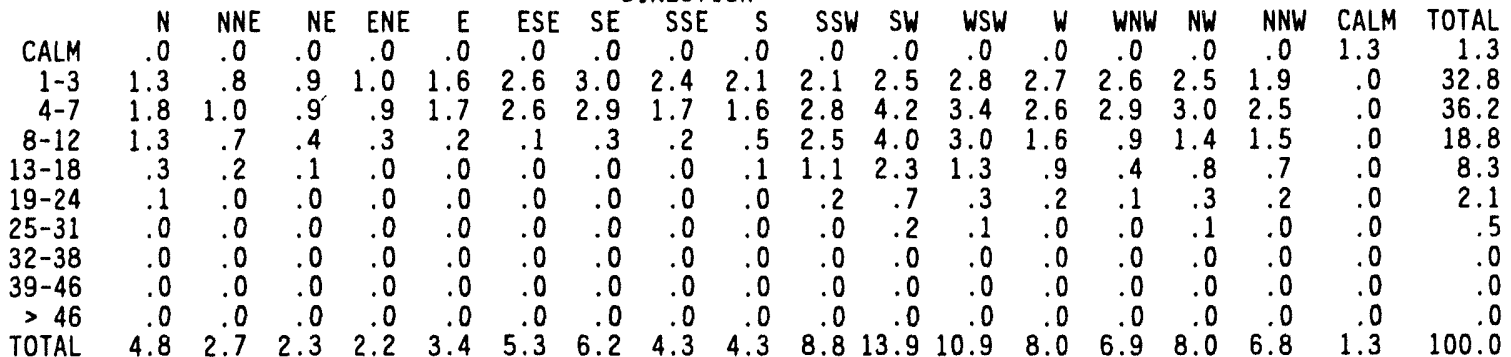

Station: (19) SAGE

$$
\text { Begin: } 3 / 82
$$

End: $12 / 92$

Total Hours

91756

$\begin{array}{rrrrrrrrrrrrrrrrrrr} & \text { N } & \text { NNE } & \text { NEE } & \text { ENE } & \text { E } & \text { ESE } & \text { SE } & \text { SSE } & \text { S } & \text { SSW } & \text { SW } & \text { WSW } & \text { W } & \text { WNW } & \text { NW } & \text { NNW } & \text { CALM } & \text { TOTAL } \\ \text { CALM } & .0 & .0 & .0 & .0 & .0 & .0 & .0 & .0 & .0 & .1 & .0 & .0 & .0 & .0 & .0 & .0 & .7 & .7 \\ 1-3 & 1.3 & 1.1 & 1.2 & 1.0 & 1.1 & 1.4 & 1.7 & 1.9 & 1.8 & 1.1 & 1.7 & 1.5 & 1.5 & 1.3 & 1.6 & 1.6 & .0 & 23.3 \\ 4-7 & 3.6 & 2.9 & 3.3 & 2.5 & 2.1 & 3.2 & 4.2 & 4.9 & 4.3 & 2.4 & 2.2 & 3.4 & 4.4 & 2.5 & 1.9 & 3.4 & .0 & 51.2 \\ 8-12 & 1.4 & 1.3 & .9 & .4 & .4 & .5 & 1.3 & 2.3 & 2.7 & 1.3 & 1.1 & 1.3 & 1.5 & 1.8 & .4 & .6 & .0 & 19.1 \\ 13-18 & .2 & .3 & .1 & .0 & .0 & .0 & .1 & .3 & .7 & .6 & .5 & .5 & .2 & .8 & .2 & .1 & .0 & 4.5 \\ 19-24 & .0 & .0 & .1 & .0 & .0 & .0 & .0 & .0 & .1 & .2 & .2 & .1 & .0 & .1 & .0 & .0 & .0 & .9 \\ 25-31 & .0 & .0 & .0 & .0 & .0 & .0 & .0 & .0 & .0 & .1 & .1 & .0 & .0 & .0 & .0 & .0 & .0 & .2 \\ 32-38 & .0 & .0 & .0 & .0 & .0 & .0 & .0 & .0 & .0 & .0 & .0 & .0 & .0 & .0 & .0 & .0 & .0 & .0 \\ 39-46 & .0 & .0 & .0 & .0 & .0 & .0 & .0 & .0 & .0 & .0 & .0 & .0 & .0 & .0 & .0 & .0 & .0 & .0 \\ >46 & .0 & .0 & .0 & .0 & .0 & .0 & .0 & .0 & .0 & .0 & .0 & .0 & .0 & .0 & .0 & .0 & .0 & .0 \\ \text { TOTAL } & 6.6 & 5.7 & 5.6 & 3.9 & 3.6 & 5.2 & 7.2 & 9.3 & 9.6 & 6.1 & 5.9 & 6.8 & 7.5 & 6.5 & 4.1 & 5.7 & .7 & 100.0\end{array}$




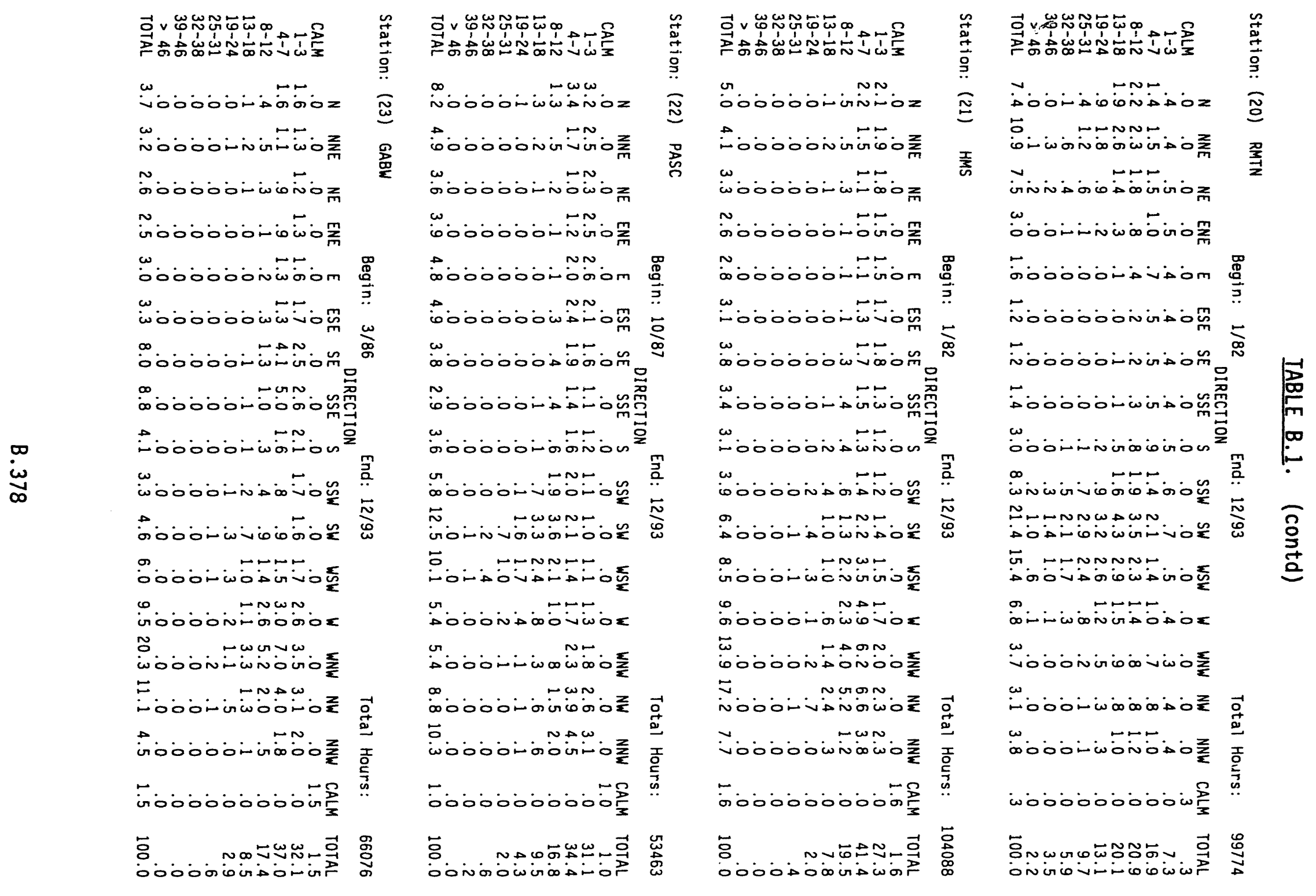




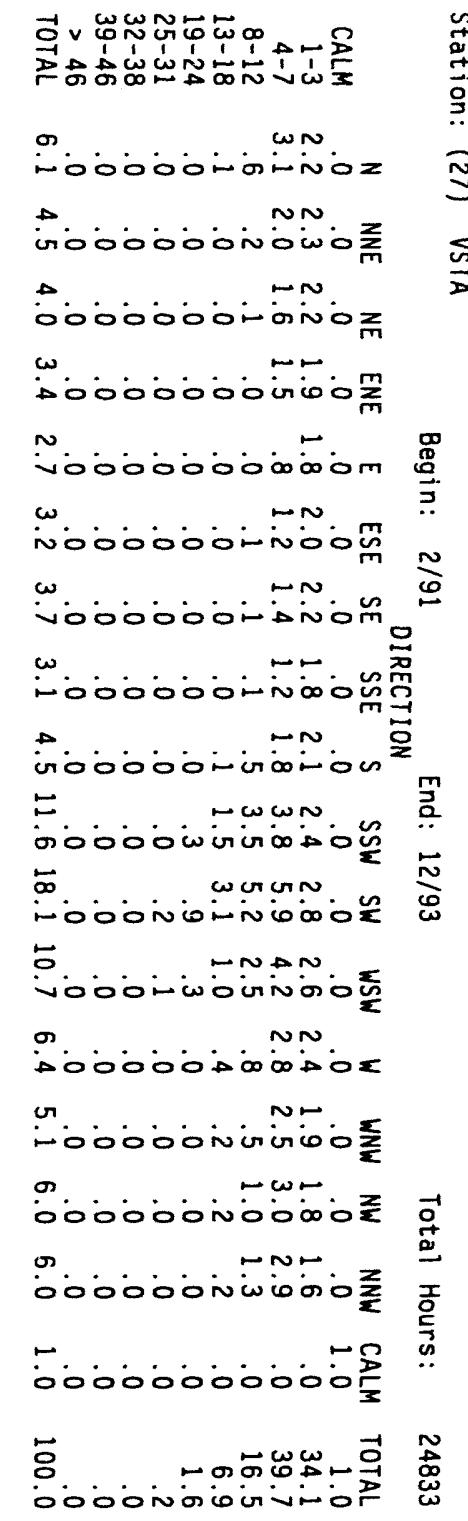

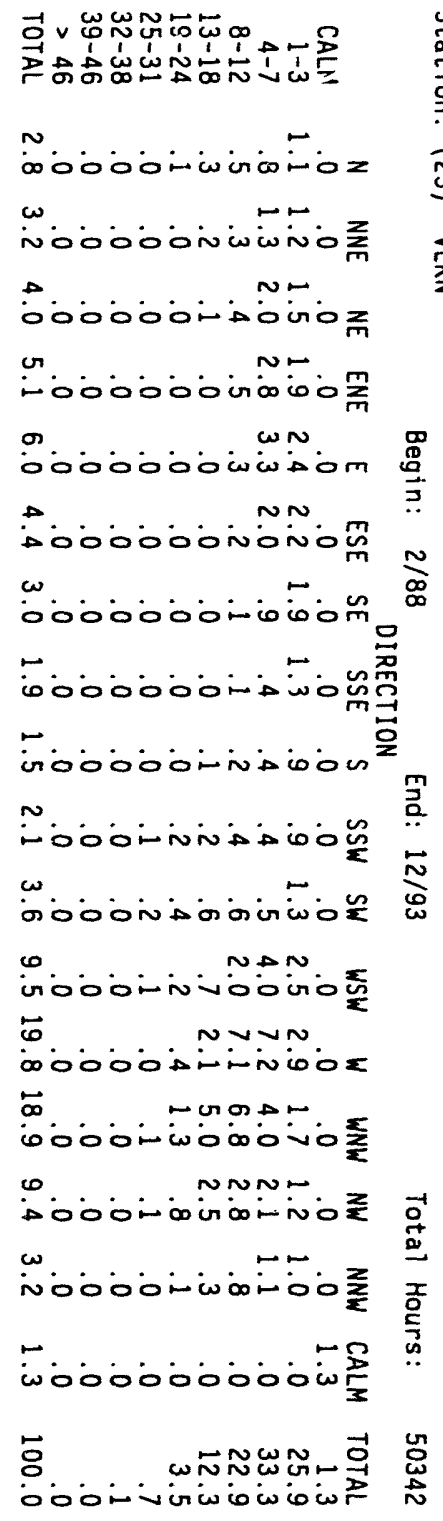

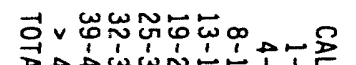

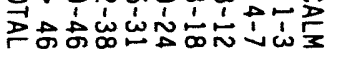

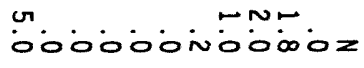

wododivivi

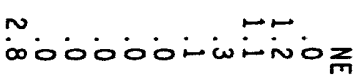

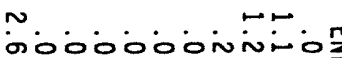

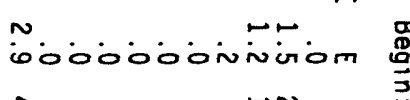

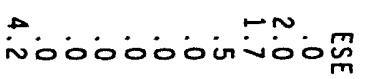

F.... Nuw m

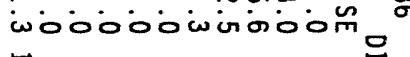

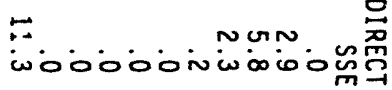

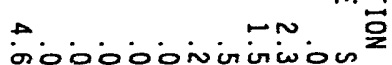

w

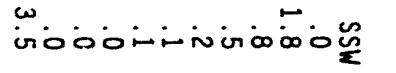

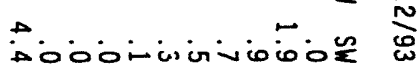

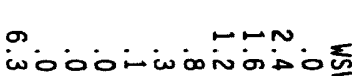

5. . . . F w w.

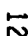

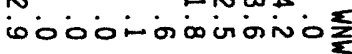

$\therefore \ldots . . . . N \omega$.

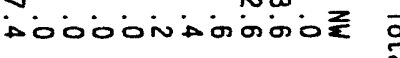

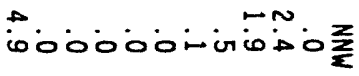

Nododododón?

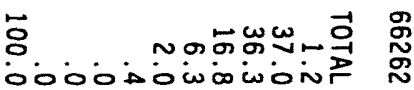


No. of

Copies

OFFSITE

12 DOE/Office of Scientific and Technical Information

All Seasons Heating \& Air Conditioning, Inc. 302 S. 3rd Avenue Yakima, WA 98902

Attn: T. Poe

B. Allen

611 Vaughan Road

Yakima, WA 98908

J. Akers

UNOCAL

P.0. Box 5797

Kennewick, WA 99336

Benton County PUD

P.0. Box 6270

Kennewick, WA 99336

Attn: K. Mey

L. A. Bisping

Siemens Power Corporation 2101 Horn Rapids Road

Richland, WA 99352

R. C. Blewett

4409 Arlington Drive

West Richland, WA 99352

T. Brazil

Cascade Natural Gas

P.0. Box 6899

Kennewick, WA 99336

\section{DISTRIBUTION}

No. of

Copies

\section{OFFSITE}

R. Campbe11

UNOCAL

P.0. Box 5797

Kennewick, WA 99336

E. Charvet

MeGregor Company

5251 Eltopia West Road

Eltopia, WA 99330

City of Benton City

P.0. Box 70

Benton City, WA 99320

Attn: City Clerk

W. Clore

1317 Paterson Road

Prosser, WA 99350

W. T. Cobb

Cobb Consulting Services

815 S. Kellogg

Kennewick, WA 99336

Columbia Basin College Library

Reference Librarian

2600 N. 20th

Pasco, WA 99301

Columbia National Wildl ife Refuge

U.S. Fish \& Wildlife Service

U.S. Department of the Interior

P.0. Box $F$

Othello, WA 99344

K. Corliss

Stimson Lane Ltd.

P.0. Box 580

Grandview, WA 98930 
No. of

Copies

\section{OFFSITE}

H. Critchfield

Washington State Climatologist Western Washington University

Bellingham, WA 98225

J. Denkers

Washington State Department of Ecology

P.0. Box 47600

Olympia, WA 98504

L. D. Dunbar

City of Richland

P.0. Box 190

Richland, WA 99352

J. Erickson

Division of Radiation Protection

P.0. Box 47827

Olympia, WA 98504

D. Fiskum

Kadlec Medical Center

888 Swift Boulevard

Richland, WA 99352

G. Freeman

$M / D$ PE-12

3000 George Washington Way

Richland, WA 99352

B. Hachey

Mid-Columbia Library

1320 W. Hopkins

Pasco, WA 99301
No. of

Copies

\section{OFFSITE}

P. M. Hamilton, Jr.

Assistant Director

Physical Plant

Central Washington University

Ellensburg, WA 98926

D. Harper

Wilber-Ellis Company

P.0. Box 764

Pasco, WA 99301

A. Haymaker

1721 Cottonwood Drive

Pasco, WA 99301

T. Holdren

City of Richland - Accounting

P.0. Box 190

Richland, WA 99352

J. Hollis

TRIDEC

901 N. Colorado

Kennewick, WA 99336

Jacob and Rhodes

P.0. Box 6881

Kennewick, WA 99336

E. Johns

Bureau of Reclamation

Denver Federal Center

P.0. Box 25007 - Code D5752

Denver, CO 80225

B. Johnson

556 Columbia Center

Kennewick, WA 99336 
No. of

Copies

OFFSITE

B. Kelley

614 S. Kellogg Street

Kennewick, WA 99336

K. Ker

Lamb-Weston

2005 Saint Street

Richland, WA 99352

J. Kleckner

D. Hittle Associates

757 Columbia Drive, SE

Richland, WA 99352

M. W. Kroeger

Rt. 2, Box 2953A

Prosser, WA 99350

R. LaFramboise

Central Washington Farm Crops Assoc.

P.0. Box 202

Yakima, WA 98907

D. A. Lauer

BFWWC APCA

650 George Washington Way

Richland, WA 99352

K. Lettau

George Grant Construction Co.

P.0. Box 789

Richland, WA 99352

M. E. Lippold

Water and Waste Utilities

P.0. Box 190

Richland, WA 99352
No. of

Copies

OFFSITE

J. Lund

622 N. Kellogg

Kennewick, WA 99336

J. Mannix

Kennewick School District

622 N. Kellogg

Kennewick, WA 99336

R. Mazurkiewicz

Bonneville Power Administration

P.0. Box 968, MD-399

Richland, WA 99352

W. A. McCullough

2402 Lariat Lane

Richland, WA 99352

National Weather Service Office

2406 W. Washington Avenue

Yakima, WA 98903

R. Okarma

Kennewick High School

500 S. Dayton

Kennewick, WA 99336

M. Orgill

904 Lenox

Las Cruces, NM 88005

R. Overman

Electrical Dispatch

P.0. Box 190

Richland, WA 99352

Distr.3 
No. of

Copies

OFFSITE

Pasco School District

Tri-Cities Airnort B1dg. 210

Pasco, WA 9930i

Attn: K. Gage-Raska

Pioneer $\mathrm{Hi}$-Bred

1040 Settler Road

Connel1, WA 99326

Potato Growers of Washington

1807 W. Bonneville

Pasco, WA 99301-4913

D. C. Powell

2110 Hudson

Richland, WA 99352

Professional Ag Services

P.0. Box 3053

Pasco, WA 99302

F. D. Quinn

Washington Public Power

Supply System

P.0. Box 968, MD- 1020

Richland, WA 99352

Raekes, Rettig, Osborne

6725 W. Clearwater

Kennewick, WA 99336

Attn: D. Aust in

J. T. Raese

Tree Fruit Research Laboratory

1104 N. Western Avenue

Wenatchee, WA 98801

H. Ramsey

Mobay Chemical Corporation

290 W. Taylor Road

Selah, WA 98942
No. of

Copies

\section{OFFSITE}

K. Redmond

Western Regional Climate Ctr.

Desert Research Institute

P.0. Box 60220

Reno, NV 89506

R. R. Reid

1884 Rustic Road

Walla Walla, WA 99362

Richland Public Library

Reference Department

955 Northgate

Richland, WA 99352

W. Sahli

P.0. Box 912

Mattawa, WA 99344

D. Sexton

Vogel Popcorn Company

5401 Eltopia Road, W.

Eltopia, WA 99330

M. Sheeran

Benton County PUD

P.0. Box 6270

Kennewick, WA 99336

C. L. Simpson

Route 1 Box 5896

Richland, WA 99352

Soil Conservation Service

1620 Road 44, North

Pasco, WA 99301-2667

N. Sorick

Frankl in County PUD

P.0. Box 2407

Pasco, WA 99302 
No. of

Copies

OFFSITE

L. Stewart

Benton Rural Electric Assoc.

P.0. Box 1150

Prosser, WA 99350

K. Tanaka

Siemans Power Corporation

2101 Horn Rapids Road

Richland, WA 99352

G. Taylor

Oregon Climate Service

Strand 326

Oregon State University

Corvalis, OR 97331-2209

J. Thompson

CENEX Land $0^{\prime}$ Lakes

Rt. 7, Box 430

Kennewick, WA 99337

K. Topham

Unocal Petroleum Products and Chemicals Division

P.0. Box 5797

Kennewick, WA 99336

M. Troutman

1207 Sunnyvale Road

Kennewick, WA 99337

U.S. Army Corp Energy

Hydrology Branch

Walla Walla, WA 99362-9265

J. L. Walker

Kennewick School District

200 S. Dayton

Kennewick, WA 99336
No. of

Copies

OFFSITE

R. W. Wallace

89 Waldron Street

Richland, WA 99352

Washington State Department of Agriculture

Agriculture Chemical Branch

2015 S. First Street

Yakima, WA 98903

J. Watson

County Extension Agent

1121 Dudley Avenue

Prosser, WA 99350

R. N. Wing

727 Airway Avenue

Lewiston, ID 83501

L. Zeiher

405 South Dayton

Kennewick, WA 99336

W. Ziebell

Prosser School District

823 Park Avenue

Prosser, WA 99350

\section{ONSITE}

2 U.S. Department of Energy, Richland Operations Office

R. F. Brich

A5-55

J. B. Hall

A5-55

Boeing Computer Services, Richl and

J. H. Bingham

H1-64 
No. of

Copies

ONSITE

U.S. Environmental Protection Agency

D. R. Einain

B5-01

33 Westinghouse Hanford Company
E. G. Allen
G6-02
T. E. Arndt
L5-07
D. B. Barnett
H6-06
F. J. Bich1
S2-12
J. A. Caggiano
H6-06
F. T. Calapristi
B2-35
D. R. Cowen
S2-01
L. L. Daniels
L6-75
EPIC
H6-08
J. E. Eucker
S2-12
L. A. Fort
J. D. Fulcher
W. P. Gilles
D. R. Hall
D. G. Horton
A. R. Johnson
D. F. Landeen
A. G. Law
C. L. Looney
V. D. Maupin
M. J. McCarthy
J. D. McCormack
R. D. Mills
K. L. Petersen
M. R. Sackschewsky
J. D. Schaffer
J. W. Strege
R. W. Szelmeczka
R. F. Thornton
R. Tranbarger
T. L. Traub
D. J. Washenfelder
V. E. Winston
L7-09
B5-25
T6-02

No. of

Copies

ONSITE

8 ICF Kaiser Hanford Company
P. G. Beaudet
E2 -30
L. A. Gaddis
E6-31
J. H. Mortimer
E2 -70
T. L. Ostrander
E2 -50
R. C. Savard
G7 -52
L. H. Smith
E6-36
G. W. Snyder
$\mathrm{T} 7-28$
P. L. Taylor
B4-65

572 Pacific Northwest Laboratory

0. B. Abbey

K6-08

D. L. Anderson

P7 -60

G. L. Andrews

P7-41

D. A. Baker

K3-54

W. R. Barchet

$\mathrm{K} 6-11$

J. C. Barnard

$\mathrm{K} 6-07$

L. E. Bisping

K6-61

W. J. Bjorklund

P7 -68

J. L. Brimhall

$P 8-16$

K. W. Burk (5)

K6 - 55

L. L. Cadwe11

P7-54

M. D. Campbe11

K6-77

W. C. Cliff

P7-35

D. A. Cochran

P7 -25

T. S. Cress

K1 -74

C. E. Cushing

P7-59

M. T. Dana

K6-08

E. C. DeBattista

P7 -63

J. C. Doran

K6-11

C. E. Elderkin

K6-03

D. L. Elliott

K6- 07

D. Felmy

K6-77

N. D. Foote

K7 -10

H. D. Freeman

P8-38

G. W. Gee

K6-77

E. R. Gerk

P7-63

C. S. Glantz

K5-55

G. L. Gower 
No. of

Copies

\section{ONSITE}

R. H. Gray

D. L. Hadley

R. K. Hadlock

D. R. Haffner

J. C. Hail

D. J. Hoitink (500)

M. G. Horstman

R. R. Kirkham

N. R. Larson

N. S. Laulainen

R. N. Lee

M. W. Ligotke

D. B. Mackey

J. P. McKinley

P. J. Mellinger

M. C. Miller

J. M. Nelson

T. L. Page

J. V. Ramsde 11

R. B. Richman
No. of

Copies

\section{ONSITE}

K1-33

K5-08

K6-55

K7-97

P7-70

K6-55

K4-10

K6-77

K6-84

K6-11

P8- 19

P7 -54

P8-44

K3-61

B1-34

P7 -39

P8 -47

B1 -40

K6-55

P7 -78
L. E. Rogers

W. J. Shaw

K. L. Soldat

D. L. Stewart

G. M. Stokes

J. M. Thorp

L. L. Wendell

C. D. Whiteman

C. Williams

R. K. Woodruff

Publishing Coordination

Technical Report Files (5)

Routing

R. M. Ecker

M. J. Graham

P. M. Irving

C. S. Sloane

P. C. Hays - last
P7-54

K6-11

K3-57

K6-96

$\mathrm{K} 1-74$

K6-08

K6-07

K6-11

P7-60

K6-13

Distr. 7 

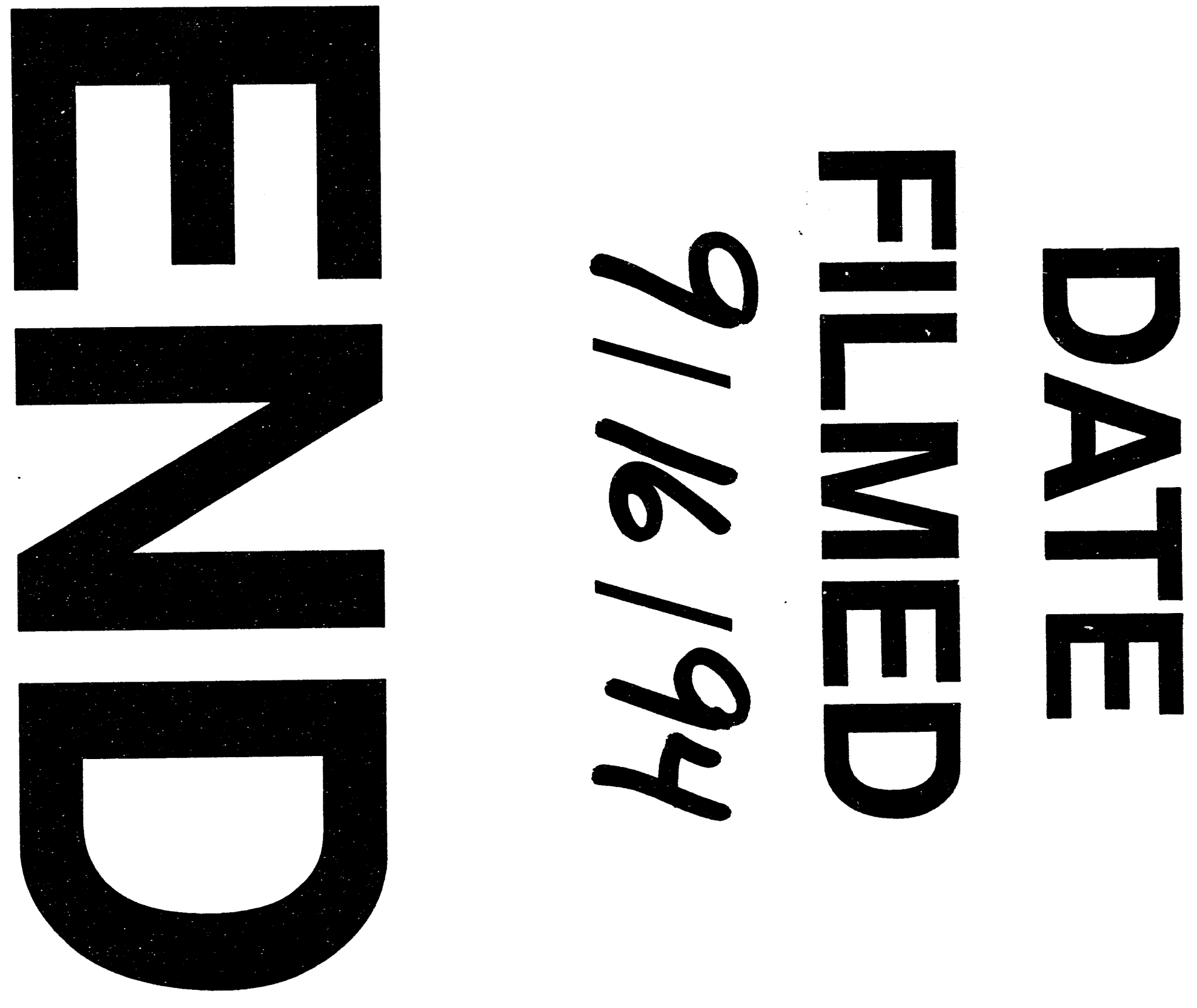
